*ak RMIS View/Frint Document Cover Sheet tow

This document was retrieved from the Documentation and Records Manaqement (DRM) ISEARCH System. It is intended for Information only and may not be the most recent or updated version. Contact a Document Service Center (see Hanford Info for locations) if you need additional retrieval information.

Accession \#: D195061617

Document \#: SD-WM-TRP-246

Title/Desc:

SOLID WASTE DRUM ARRAY FIRE PERFORMANCE [SEC 1 OF 7]

Pages: 151 
THIS DOCUMENT WAS TOO LARGE TO SCAN AS A SINGLE

DOCUMENT. IT REQUIRED BREAKING INTO SMALLER SECTIONS.

DOCUMENT NUMBER: SD-WM-TRP-2H6

SECTION: $\quad 1$ OF 7

TITLE: slid waste Drum Array five Performance

AUTHOR: Louie PL

RECIPIENT:

DATE: $11 / 07 / 95$

KEYWORDS:

REFERENCES: EDT -612232 
Blank 


\begin{tabular}{|c|c|}
\hline $\begin{array}{l}\text { 2. To: (Receivino Oromization) } \\
\text { Sol id Waste Projects }\end{array}$ & $\begin{array}{l}\text { 3. From: (originating orgmization) } \\
\text { Solid Waste Projects }\end{array}$ \\
\hline $\begin{array}{l}\text { 5. Proj./Pros./Dept./Oiv.: } \\
\text { SWP/Solid Waste Disposal }\end{array}$ & $\begin{array}{l}\text { 6. Cos. Enor.: } \\
\text { RL Louie }\end{array}$ \\
\hline
\end{tabular}

\section{Originator Remarks:}

For Release

11. Receiver Remarks:

\begin{tabular}{|c|c|}
\hline $\begin{array}{l}\text { 3rom: (Originating organization) } \\
\text { Solid Waste Projects }\end{array}$ & $\begin{array}{l}\text { 4. Reloted EDT No.: } \\
612232\end{array}$ \\
\hline $\begin{array}{l}\text { Coo. Enor.: } \\
\text { IL Louie }\end{array}$ & $\begin{array}{l}\text { 7. Purchase Ordor Mo.: } \\
\text { MTM-SWV-164570 }\end{array}$ \\
\hline
\end{tabular}

9. Equip./Component Mo.:

N/A

10. Syeter/etdo./Fecility:

$N / A$

12. Najor Aaem. Ow. No.: N/A

13. Pormit/Pernit Applicotion Ho.: N/A

14. Required Reapense Date: $N / A$

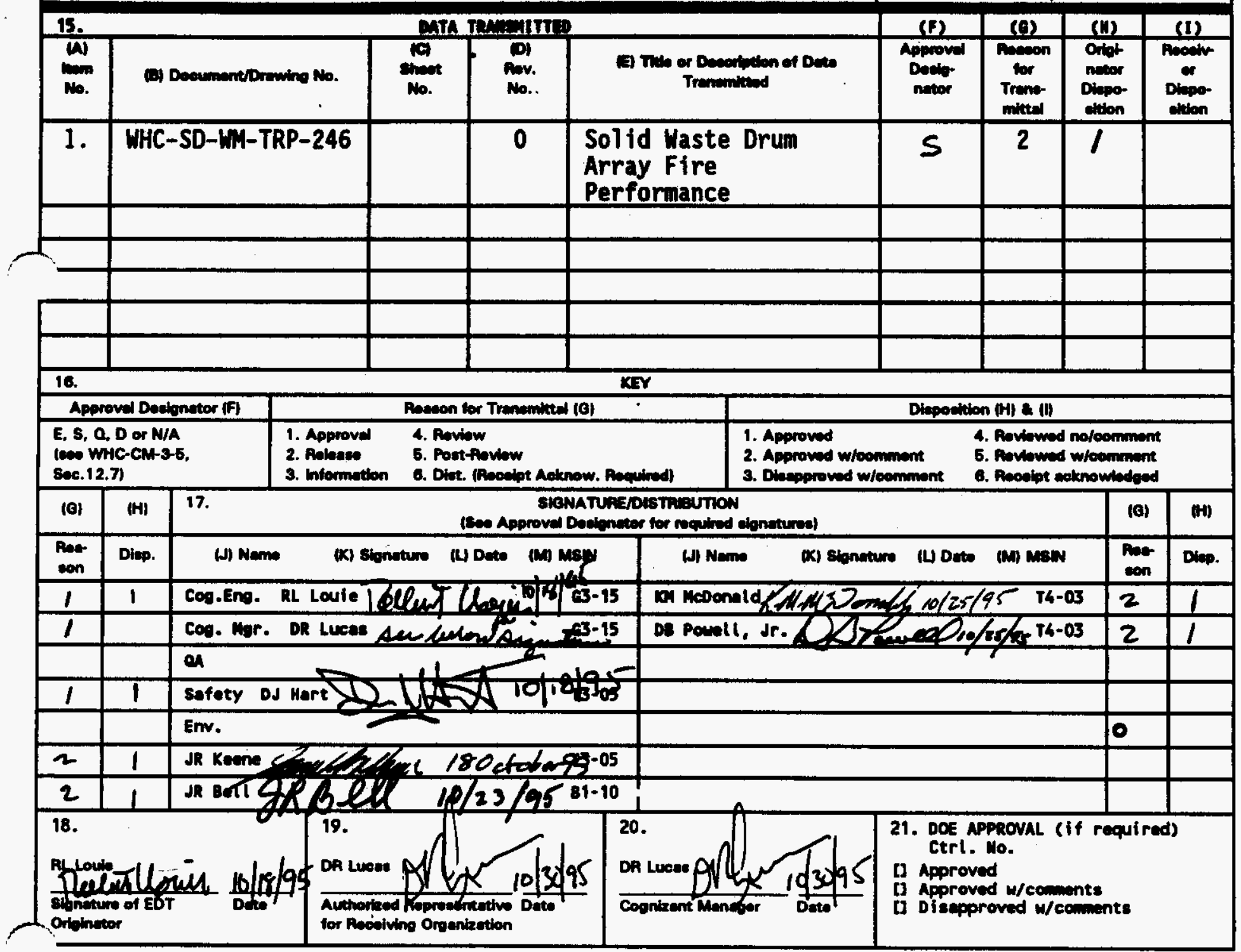




\section{INSTRUCTIONS FOR COMPLETION OF THE ENGINEERING DATA TRANSMITTAL}

(USE BLACK INK OR TYPE)

\begin{tabular}{|c|c|}
\hline$\frac{B L O C K}{(1)^{*}}$ & $\frac{\text { TITLE }}{\text { EDT }}$ \\
\hline (2) & To: ARoceiving Orgenizstion) \\
\hline (3) & From: OOriginating Orgenization \\
\hline (4) & Related EDT No. \\
\hline$(5)^{\bullet}$ & Proj./Proo./Dopt./Div. \\
\hline$(6)^{\circ}$ & Coonizant Engineer \\
\hline (7) & Purchace Order No. \\
\hline$(8)^{\bullet}$ & Originetor Remark: \\
\hline (9) & Equipment/Component No. \\
\hline (10) & Syotom/Bidg./Fecility \\
\hline (11) & Receiver Pomarke \\
\hline 112$\}$ & Mujor Asem. Dwg. No. \\
\hline (13) & Permit/Permit Application No. \\
\hline (14) & Required Response Dato \\
\hline
\end{tabular}

(15) - Date Trenemittod
(A)* Kern Number
(B) * Document/Drowing No.
(C) $)^{-}$Shoot No.
(D)* Rov. No.
(E) Title or Description of Deta Transmitted
(F)* Approval Designator
(G) Rescon for Transmittel
(H) Originator Disposition
(I) Roceiver Disposition

116) KoY

(17) Signature/Distribution
(G) Renson
(H) Dieposition
(J) Name
(K) - Signature
(L)* Date
(M) * MSIN

(18)

Signature of EDT Originator

(19) Authorized Representative

(20) Cognizant Maneger

1211. DOE Approval
- Pre-assigned EDT number.

- Enter the individual's name, titlo of the organization, or entity (0.9.. Distribution) that the EDT is being tranamitted to.

- Enter the title of the organization originating and transmitting the EDT.

- Enter EDT numbers which reiato to the data being trensmitted.

- Enter the Project/Program/Depertment/Division title or Project/Program ecronym or Project Number. Work Order Number or Organization Code.

- Enter the name of the individual identified es being responaible for coordinating disposition of the EDT.

- Enter ralated Purchase Onder (P.0.I Number, if available.

- Enter special or additional commente concerning transmittal, or "Key" rotrioval worde may bo entered.

- Enter equipmont/component number of affected itom, if eppropriate.

- Enter applicable system, building or facility number, if appropriate.

- Enter special or edditional comments concerning tranemittal.

- Enter applicable drawing number of major assembly, if appropriate.

- Enter applicable permit or permit application number, if appropriate.

- Enter the date a response is required from individuals identified in Block 17 (Signaturo/Distribution).

- Enter soquential number, boginning with 1, of the information listed on EDT.

- Enter the unique identification number aceigned to the document or drawing being tranemitted.

- Enter the sheet number of the information being transmitted. If no sheot number, leave blank.

- Enter the revision number of the information being trensmitted. If no revieion number, beve biank.

- Enter the title of the document or drewing or a brief description of the eubject if no titb is identifiod.

- Enter the eppropriate Approvel Designator (Block 15). Also, indicate the appropriate approvale for each itom listod, i.e., SO. ESO. otc.

- Enter the appropriste code to identify the purpose of the data tranamittal (soe Block 16).

- Enter the appropriate disposition code (eee Block 16).

- Enter the sppropriate disposition code (see Block 16).

- Number codes used in completion of Blocks 15 (G). (H), and (I), and 17 (G), (H) (Signaturo/Distribution).

- Enter tho code of the reason for transmittat (Block 16).

- Enter the code for the dispocition (Block 16).

- Enter the signature of the individual completing the Disposition $17 \mathrm{HH}$ and the Tranamittal.

- Obtain appropriate signature(s).

- Enter date signature is obtainod

- Enter MSIN. Note: If Distribution Sheot is used, show ontire distribution fincluding that indicated on Page 1 of the EOTI on the Distribution Shest.

- Enter the signature and date of the individual originating the EOT lentered prior to transmittal to feceiving Orgenizetion). If the EDT originator is the cognizant engineer, sign both Blocks 17 and 18.

- Enter the signature and date of the individual identified by the Receiving Organization as authorized to approve disposition of the EDT and acceptance of the data tranemitted, as applicable.

- Enter the signature and date of the cognizant manager. (This signature is authorization for relesse.1 - Enter DOE approval fif required) by signature or control number that tracks the approval to $\bullet$ signature,
and indicate DOE action. 


\section{RELEASE AUTHORIZATION}

Document Number: - WHC-SD-WH-TRP-246, Rev. 0

Document Thide: Solid Waste Drum Array Fire Performance

Rclease Date: $\quad 11 / 7 / 95$

This document was reviewed following the procedures described in WHC-CM-3-4 and is:

APPROVED FOR PUBLIC RELEASE

WHC Information Release Administration Specialist:

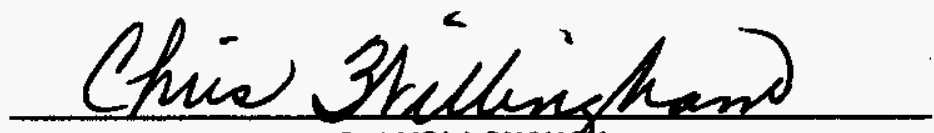

$11 / 7 / 95$

c. WILLINGHSH

TRADEMRK DISCLAIMER. Reference herein to any specific commercial product, process, or service by trade nane, trademark, manufacturer, or otherwise, does not necessarily constitute or inply its endorsement, recomendation, or favoring by the United States Government or any agency thereof or its contractors or subcontractors.

This report has been reproduced from the best available copy. Available in paper copy. Printed in the United States of Anerica. To obtain copies of this report, contact:

Westinghouse Hanford Company - Document Control Services

P.0. Box 1970, Mailstop H6-08, Richland, WA 99352

Telephone: (509) 372-2420; Fax: (509) 376-4989 
Blanter 


\begin{tabular}{|c|c|c|}
\hline $\begin{array}{l}\text { 2. Title } \\
\text { SOLID WASTE DRUM ARRAY FIRE PERFORMANCE }\end{array}$ & $\begin{array}{l}\text { 3. Mubor } \\
\text { NHC-SD-MN-TRP-246 }\end{array}$ & $\begin{array}{l}\text { 4. Rev No. } \\
0 \\
\end{array}$ \\
\hline $\begin{array}{l}\text { 5. KoY Wrot } \\
\text { FIRE TESTING } \\
\text { SOLID WASTE DRUN STORAGE } \\
\text { FIRE PROPAGATION } \\
\text { DRUM ARRAY } \\
\text { PALLET } \\
\text { RACK }\end{array}$ & $\begin{array}{l}\text { 6. Muthor } \\
\text { Mame: RL Louie } \\
\text { Tloelent Llouil } \\
\text { oreanizotionveneres codo }\end{array}$ & KO50/A4612 \\
\hline
\end{tabular}

\section{Abstrect}

Fire hazards associated with drum storage of radioactively contaminated wastes are a major concern in DOE waste storage facilities. This report is the second of two reports on fire testing designed to provide data relative to the propagation of a fire anong storage drum arrays. The first report covers testing of individual drums subjected to an initiating fire and the development of the analytical wethodology to predict fire propagation anong storage drum arrays. This report is the second report, which documents the results of drum array fire tests. The purpose of the array tests was to confirm the analytical methodology developed by Phase I fire testing. These tests provide conclusive evidence that fire will not propagate from drum to drum unless an continuous fuel source other than drum contents is provided.

OFFICIAL RELEASE
BYWHC
DATE NOV 07 190
Dtatin 31


blank 
WHC-SD-WM-TRP-246 REV. 0

\title{
Solid Waste Drum Array Fire Performance
}

\author{
Volume I
}

$-$

Prepared for

Westinghouse Henford Company

Richland, WA 99352

\author{
Propared by \\ Chris F. Haecker \\ Los Alamos Technical Associates, Inc. \\ Kennewick, WA 99306
}

Jesse J. Beitel, Daniel T. Gottuk, Brian T. Rhodes, and Craig L. Beyler Hughes Associates, Inc.

Baltimore, MD 21227 
Blank 
WHC-SD-WM-TRP-246 REV. 0

SOLID WASTE DRUM ARRAY FIRE PERFORMANCE

Prepared by

Hughes Associates, Inc. and Los Alamos Technical Associates, Inc.

Technical Peer Review Conducted by

Dr. James A. Mike

APPROVED

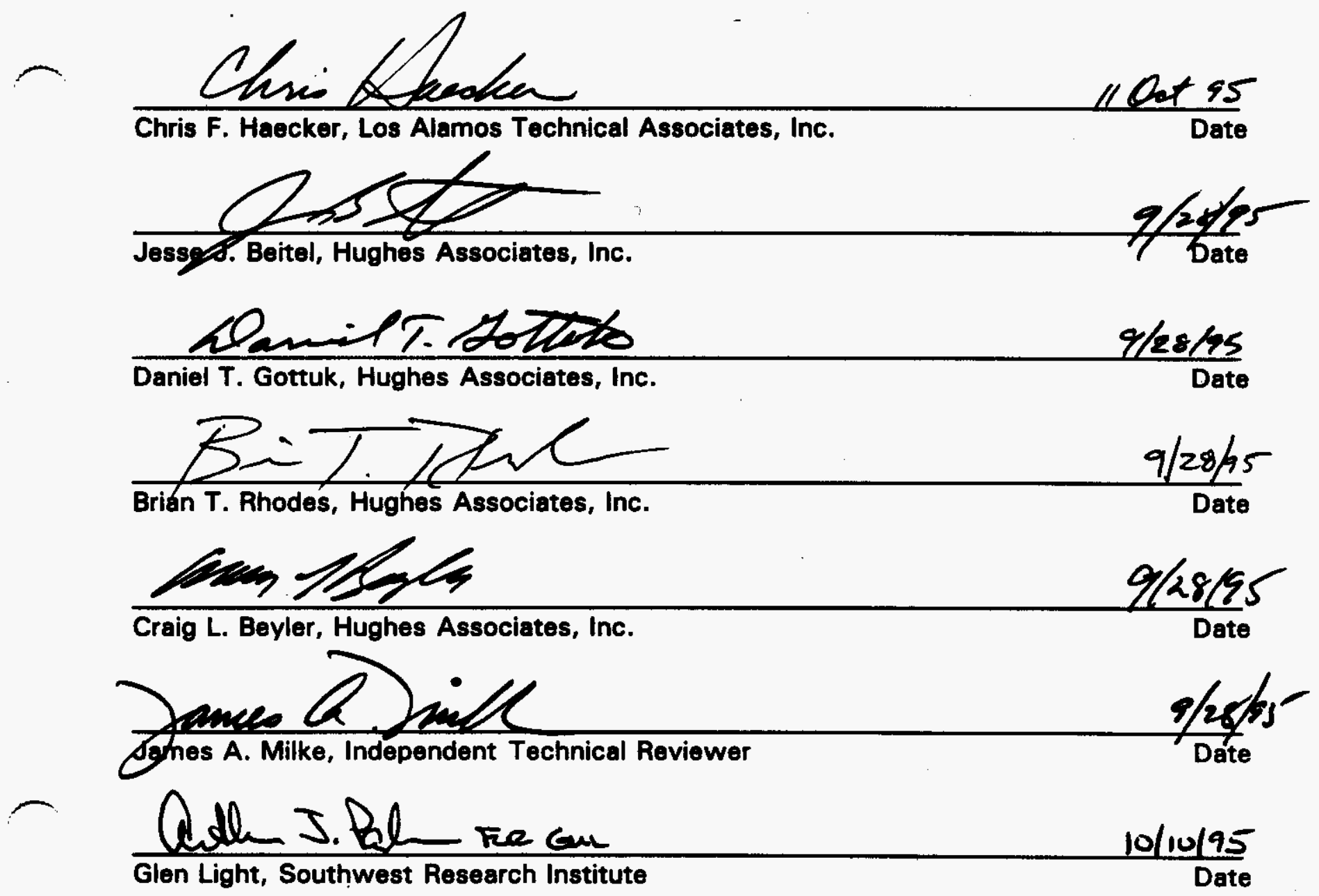


Blank 


\section{CONTENTS}

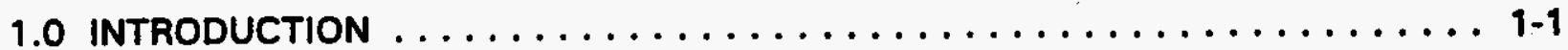

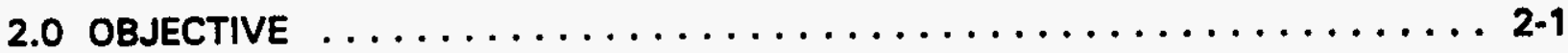

3.0 PREDICTION OF DRUM PERFORMANCE IN FULL-SCALE FIRE TESTS $\ldots \ldots \ldots$ 3-1

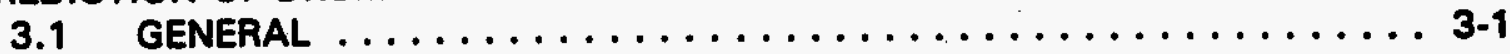

3.2 RESPONSE OF DRUMS TO FIRE EXPOSURES $\ldots \ldots \ldots \ldots \ldots \ldots \ldots$ 3-1

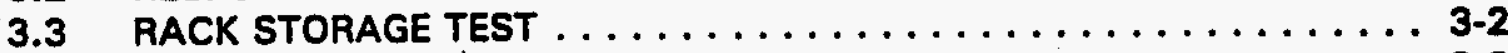

3.3.1 Description of Fire Exposure $\ldots \ldots \ldots \ldots \ldots \ldots \ldots \ldots \ldots .3 .2$

3.3.2 Predicted Response of Rack Storage Drums .......... 3-8

3.4 PALlet STORAGE TEST $\ldots \ldots \ldots \ldots \ldots \ldots \ldots \ldots \ldots \ldots \ldots \ldots \ldots \ldots \ldots$

3.4.1 Description of Fire Exposure ............... 3-13

3.4.2 Predicted Response of Pallet Storage Drums ......... 3.13

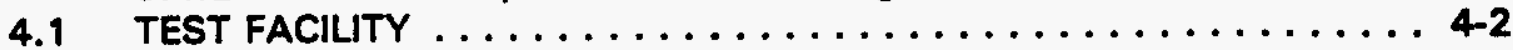

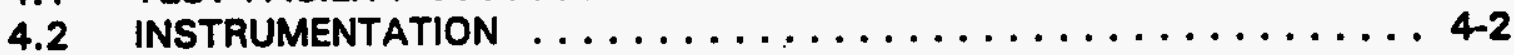

4.2.1 Temperatures $\ldots \ldots \ldots \ldots \ldots \ldots \ldots \ldots \ldots \ldots \ldots .4 .2$

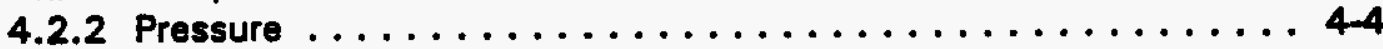

4.2.3 Heat Fiux . . . . . . . . . . . . . . . . .

4.2.4 Mass Loss .......................4 4-4

4.3 DATA ACQUISITION .................... 4-4

4.4 VISUAL DOCUMENTATION $\ldots \ldots \ldots \ldots \ldots \ldots \ldots \ldots \ldots \ldots \ldots \ldots$

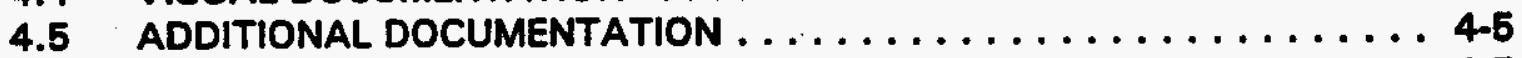

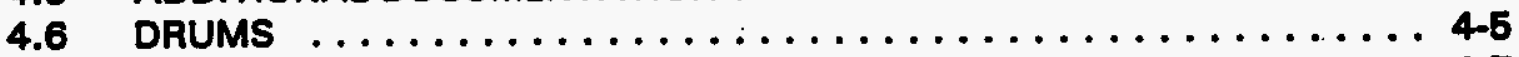

4.6.1 Water-filled Drums .................... 4.7

4.6.2 Standard Load Drums $\ldots \ldots \ldots \ldots \ldots \ldots \ldots \ldots \ldots \ldots$ 4.7

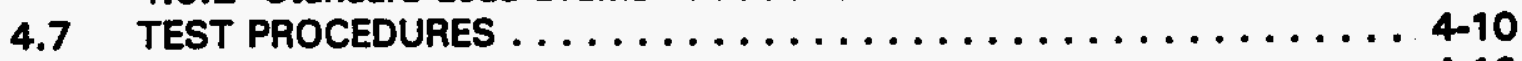

4.8 TEST TERMINATION $\ldots \ldots \ldots \ldots \ldots \ldots \ldots \ldots \ldots \ldots \ldots \ldots$ 4-10

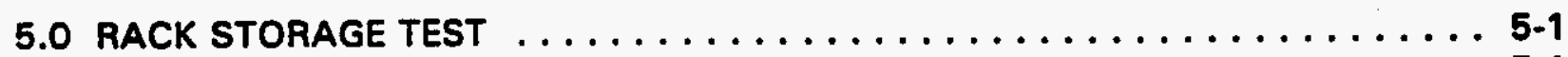

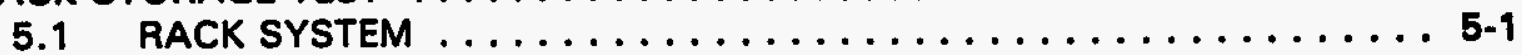

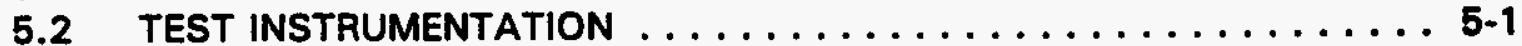

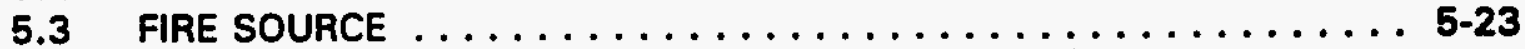

6.0 PAlletized storage test $\ldots \ldots \ldots \ldots \ldots \ldots \ldots \ldots \ldots \ldots \ldots \ldots$ 6-1

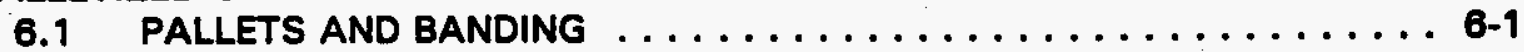

6.2 TEST INSTRUMENTATION $\ldots \ldots \ldots \ldots \ldots \ldots \ldots \ldots \ldots \ldots \ldots, 6 \ldots \ldots$

6.3 FIRE SOURCE $\ldots \ldots \ldots \ldots \ldots \ldots \ldots \ldots \ldots \ldots \ldots \ldots \ldots \ldots, 1$

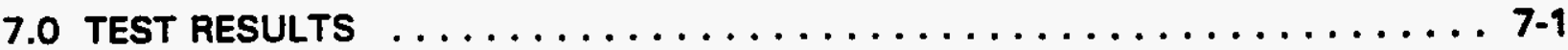

7.1 RACK STORAGE TEST $\ldots \ldots \ldots \ldots \ldots \ldots \ldots \ldots \ldots \ldots \ldots$ 7-1

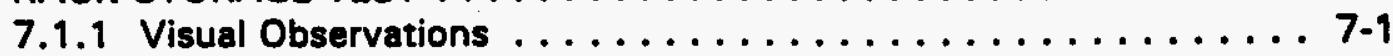

7.1 .2 Pressure Data ................... 7-2

7.1 .3 Total Heat Flux Data ................. 7-2

7.1.4 Drum Surface and Tree Temperatures $\ldots \ldots \ldots \ldots \ldots \ldots$ 7-5

7.1.5 Drum and Rack Inspection $\ldots \ldots \ldots \ldots \ldots \ldots \ldots \ldots \ldots$ 7-6 
blank 
7.2 PALletIZEd STORAGE TEST $\ldots \ldots \ldots \ldots \ldots \ldots \ldots \ldots \ldots \ldots 7-11$

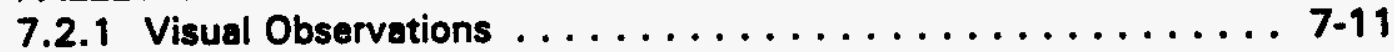

7.2 .2 Pressure Data .................. 7-14

7.2.3 Total Heat Flux Data . . . . . . . . . . . . . 7-14

7.2.4 Drum Surface and Tree Temperatures $\ldots \ldots \ldots \ldots \ldots \ldots$ 7-17

7.2.5 Post-Test Pallet Inspection .............. 7-17

8.0 ANALYSIS OF RESULTS $\ldots \ldots \ldots \ldots \ldots \ldots \ldots \ldots \ldots \ldots \ldots$ 8-1

8.1 GENERAL $\ldots \ldots \ldots \ldots \ldots \ldots \ldots \ldots \ldots \ldots \ldots \ldots \ldots \ldots \ldots$ 8-1

8.2 RACK STORAGE TEST $\ldots \ldots \ldots \ldots \ldots \ldots \ldots \ldots \ldots \ldots \ldots \ldots \ldots \ldots \ldots .2$

8.2.1 Drums Within The Flame $\ldots \ldots \ldots \ldots \ldots \ldots \ldots \ldots \ldots$ 8-5

8.2.2 Drums Outside The Flame $\ldots \ldots \ldots \ldots \ldots \ldots \ldots \ldots \ldots$ 8-10

8.2.3 Thermal Response of Rack Structure ........... 8-13

8.3 PALlet STORAGE TEST $\ldots \ldots \ldots \ldots \ldots \ldots \ldots \ldots \ldots \ldots .6 \ldots \ldots$

8.3.1 Drums Within The Flame $\ldots \ldots \ldots \ldots \ldots \ldots \ldots \ldots, 8,18$

8.3.2 Drums Outside The Flame $\ldots \ldots \ldots \ldots \ldots \ldots \ldots \ldots .8 .20$

8.3.3 Impact of Wood Pallets . . . . . . . . . . . . . .

8.3.4 Assessment of Drum Stack Stability Model . . . . . . . 8 8-24

8.4 REASSESSMENT OF THE MODEL PERFORMANCE $\ldots \ldots \ldots \ldots \ldots .8-25$

9.0 CONCLUSIONS AND RECOMMENDATIONS $\ldots \ldots \ldots \ldots \ldots \ldots \ldots \ldots .9 .1$

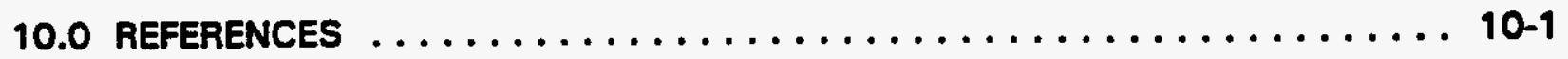

Appendix A - Rack Storage and Palletized Storage Pre-test Checklist . . . . . . A A-1

Appendix B - Rack Storage and Palletized Storage Test Procedure Checklist ..... B-1

Appendix $\mathrm{C}$ - Rack Storage Test Data $\ldots \ldots \ldots \ldots \ldots \ldots \ldots \ldots \ldots \ldots$ C-1

Appendix D - Rack Storage Drum Data Sheets ................ D-1

Appendix E - Palletized Storage Test Data . . . . . . . . . . . . . E-1

Appendix F - Palletized Storage Drum Data Sheets $\ldots \ldots \ldots \ldots \ldots \ldots \ldots \ldots$ F-1

Appendices $C$ and $D$ are located in Volume II of this report, and Appendices $E$ and $F$ are located in Volume III of this report. 
Blank 


\section{FIGURES}

1. Drum Wall Temperatures - Full Perimeter $\ldots \ldots \ldots \ldots \ldots \ldots$

2. Drum Wall Temperatures - Half Perimeter . . . . . . . . . . . . . 34

3. Planned Overall Rack Storage Experimental Setup . . . . . . . . . . . . 3-5

4. Rack Storage Drum Location Diagram . . . . . . . . . . . . . . . 3-6

5. Predicted Rack Storage Lid Losses . . . . . . . . . . . . . . . 3-10

6. Planned Overall Rack Storage Experimental Setup . . . . . . . . . . . . 3-11

7. Pallet Storage Drum Location Diagram $\ldots \ldots \ldots \ldots \ldots \ldots \ldots \ldots$. . . . . . . . . 12

8. Predicted Pallet Storage Lid Losses $\ldots \ldots \ldots \ldots \ldots \ldots \ldots \ldots$. . . . . . . . . .

9. Drum with Temperature and Pressure Instrumentation $\ldots \ldots \ldots \ldots \ldots \ldots$

10. Drum Data Sheet $\ldots \ldots \ldots \ldots \ldots \ldots \ldots \ldots \ldots \ldots \ldots \ldots \ldots \ldots \ldots \ldots$

11. Overall Rack Storage Experimental Setup $\ldots \ldots \ldots \ldots \ldots \ldots \ldots \ldots$

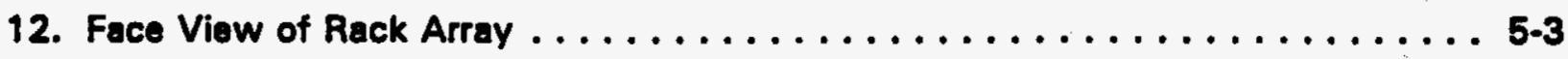

13. Rack Storage Drum Location Diagram . . . . . . . . . . . . . . . . . . 5-4

14. Pre-test Rack Storage Array . . . . . . . . . . . . . . . . . . 5-5

15. Pre-test Rack Storage Array, View Looking Northwest . . . . . . . . . . . . 5-6

16. Drum Spacing and Seismic Event Bar Location $\ldots \ldots \ldots \ldots \ldots \ldots \ldots$

17. Side View of Rack and Pan on Beam Supports (S-hooks not shown for clarity) .. 5-8

18. End View of a Rack $\ldots \ldots \ldots \ldots \ldots \ldots \ldots \ldots \ldots \ldots \ldots \ldots \ldots$

19. Rack Storage Experimental Setup and Instrumentation Layout . . . . . . . . 5-10

20. Thermocouple Tree - Rack $1-$ TC Layout $\ldots \ldots \ldots \ldots \ldots \ldots$

21. Thermocouple Tree - Rack 2 - TC Layout $\ldots \ldots \ldots \ldots \ldots \ldots$

22. Thermocouple Tree - Rack 3 - TC Layout $\ldots \ldots \ldots \ldots \ldots$

23. Thermocouples on Rack $1 \ldots \ldots \ldots \ldots \ldots \ldots \ldots \ldots \ldots \ldots$

24. Thermocouples on Rack $2 \ldots \ldots \ldots \ldots \ldots \ldots \ldots \ldots \ldots \ldots \ldots \ldots$ 
Blante 


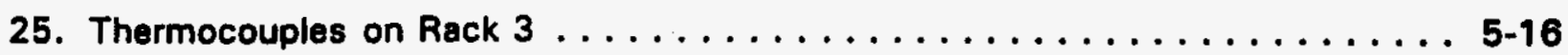

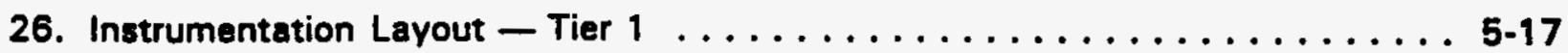

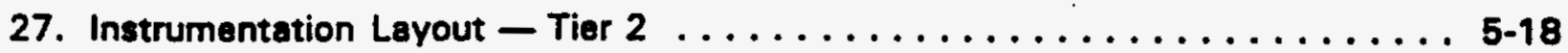

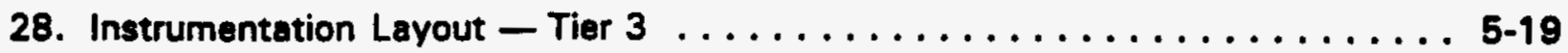

29. Instrumentation Layout - Tier $4 \ldots \ldots \ldots \ldots \ldots \ldots \ldots \ldots \ldots \ldots \ldots .5 \ldots \ldots$

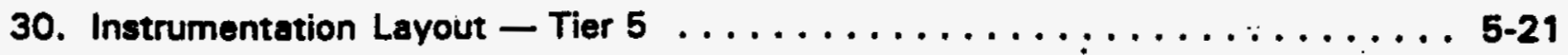

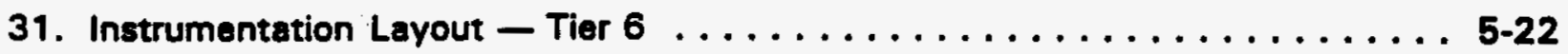

32. Overall Pallet Storage Setup $\ldots \ldots \ldots \ldots \ldots \ldots \ldots \ldots \ldots \ldots \ldots .6 \ldots \ldots .2$

33. Steel Pallet Construction $\ldots \ldots \ldots \ldots \ldots \ldots \ldots \ldots \ldots \ldots \ldots \ldots$ 6.3

34. Wood Pallet Construction $\ldots \ldots \ldots \ldots \ldots \ldots \ldots \ldots \ldots \ldots \ldots \ldots .6 \ldots \ldots$

35. Drum Arrangement on Pallet $\ldots \ldots \ldots \ldots \ldots \ldots \ldots \ldots \ldots \ldots \ldots .6 \ldots \ldots$

36. Pallet Storage Drum Location Diagram $\ldots \ldots \ldots \ldots \ldots \ldots \ldots \ldots \ldots$ 6-6

37. Pre-test Pallet Storage Array, View Looking Northwest $\ldots \ldots \ldots \ldots \ldots$ 6.7

38. Pre-test Pallet Storage Array, View Looking Southwest $\ldots \ldots \ldots \ldots \ldots \ldots$ 6-8

39. Pallet Storage Experimental Setup and Instrumentation Layout $\ldots \ldots \ldots \ldots$ 6-9

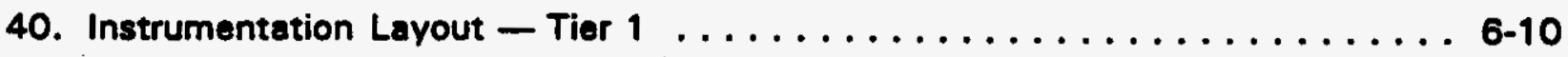

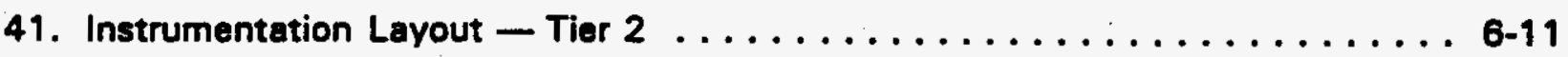

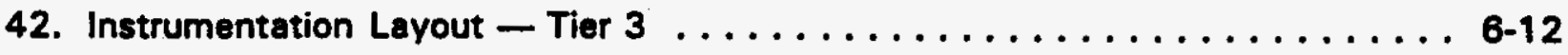

43. Rack Storage Test after Pool Fire Extinguishment $\ldots \ldots \ldots \ldots \ldots \ldots \ldots$

44. Post-test Drum Assessment $\ldots \ldots \ldots \ldots \ldots \ldots \ldots \ldots \ldots \ldots \ldots \ldots \ldots$ 7-8

45. Weight Loss Data - Rack Storage Test $\ldots \ldots \ldots \ldots \ldots \ldots \ldots \ldots \ldots$ 7-9

46. Post-test Damage, End of Rack $3 \ldots \ldots \ldots \ldots \ldots \ldots \ldots \ldots \ldots \ldots \ldots$ 7-10

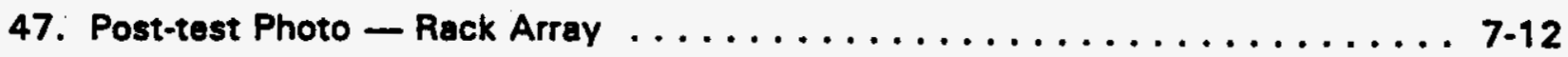

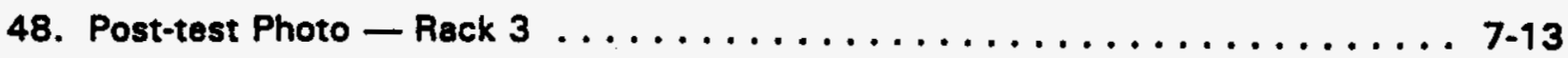


Blante 
WHC-SD-WM-TRP-246 REV. 0

FIGURES (Concluded)

49. Burning Progressing through Pallet Array $\ldots \ldots \ldots \ldots \ldots \ldots \ldots \ldots$

50. Photo - Lid Rupture $\ldots \ldots \ldots \ldots \ldots \ldots \ldots \ldots \ldots \ldots \ldots$

51. Photo - Bulging Drum $\ldots \ldots \ldots \ldots \ldots \ldots \ldots \ldots \ldots \ldots \ldots \ldots \ldots$

52. Photo - Seam Failure $\ldots \ldots \ldots \ldots \ldots \ldots \ldots \ldots \ldots \ldots \ldots \ldots \ldots$. . . . . . . . . . .

53. Pallet Storage Drum Location Diagram . . . . . . . . . . . . . . . 7-22

54. Weight Loss Data - Pallet Array Test . . . . . . . . . . . . . . 7-23

55. Photo of Post-test Pallet Array . . . . . . . . . . . . . . . . . 7-24

56. Photo of Post-test Pallet Array . . . . . . . . . . . . . . . . .

57. Predicted Drum Performance - Rack Storage Test $\ldots \ldots \ldots \ldots \ldots$

58. Actual and Predicted Drum Performance - Rack Storage Test . . . . . . . 8-4

59. Approximate Heat Flux to Drum No. $\times 226$ (Cal\#16) . . . . . . . . . 8-7

60. Drum Wall Temperature for Drum No. $\times 226 \ldots \ldots \ldots \ldots \ldots$

61. Rack Storage Test - Transducer Heat Flux Measurement along North Edge of Pool ................................ 8-12

62. Rack Storage Test - Transducer Heat Flux Measurements along West Edge of Pool (Cal \#1) . . . . . . . . . . . . . . . . . . . . . . . 8-14

63. Predicted Drum Performance - Pallet Storage Test . . . . . . . . . . 8-16

64. Predicted and Actual Drum Performance - Pallet Storage Test . . . . . . . 8-17

65. Pallet Storage Test - Transducer Heat Flux Measurement along West Edge of Pool $(C a l \# 4) \ldots \ldots \ldots \ldots$. . . . . 8-21

66. Pallet Storage Test - Transducer Heat Flux Measurement along North Edge of Pool (Cal $\# 10) \ldots \ldots \ldots \ldots \ldots \ldots \ldots \ldots \ldots \ldots \ldots \ldots \ldots \ldots . \ldots \ldots$. . . . . . . . . . 
Blank 
1. Drum Loading Breakdown $\ldots \ldots \ldots \ldots \ldots \ldots \ldots \ldots \ldots \ldots \ldots \ldots \ldots \ldots$

2. Standard Combustible Loading $\ldots \ldots \ldots \ldots \ldots \ldots \ldots \ldots \ldots \ldots \ldots$ 4-8

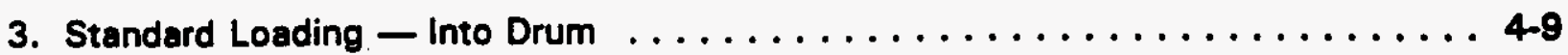

4. Peak Pressures Measured For Each Drum $\ldots \ldots \ldots \ldots \ldots \ldots \ldots \ldots \ldots$

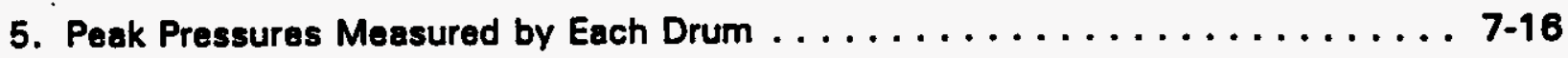

6. Summary of Experimental Data for Pressure Instrumented Rack Storage Drums Within the Flame $\ldots \ldots \ldots \ldots \ldots \ldots \ldots \ldots \ldots \ldots \ldots \ldots, 8 \ldots \ldots$

7. Comparison of Drum Wall Temperatures at Lid and Seal Failure for the Rack Test with Prior Individual Drum Tests $\ldots \ldots \ldots \ldots \ldots \ldots \ldots \ldots \ldots \ldots .8 .9$

8. Summary of Experimental Data for Pressure Instrumented Rack Storage Drums Outside the Flame .........................8 8-11

9. Summary of Experimental Data for Pressure Instrumented Pallet Storage Drums Within the Flame ........................... 8-18

10. Comparison of Drum Wall Temperatures at Lid and Seal Failure for the Pallet Test with Prior Individual Drum Tests . . . . . . . . . . . . . . . 8-19

11. Summary of Experimental Data for Pressure Instrumented Pallet Storege Drums Outside the Flame $\ldots \ldots \ldots \ldots \ldots \ldots \ldots \ldots \ldots \ldots \ldots \ldots . .6 .23$

12. Comparison of Heat-induced Lid Failures in the Pretest Predictions, the Revised Predictive Method, and the Drum Array Test ............. 8-26 
Blank 


\section{Solid Waste Drum Array Fire Performance}

\subsection{INTRODUCTION}

In earlier work [Rhodes et al., 1995], a methodology was developed which can be used to predict the performance of drums in various storage configurations during a fire. The performance of drums under fire conditions and the potential for fire propagation was assessed. In general, two failure modes were identified for the drums: (1) lid loss, and (2) lid seal failure. The basis for the analyses included both analytical and experimental results for drums exposed to specified fire conditions. Based on these results, predictions as to the extent of fire propagation and the performance of drums in storage configurations were made for several fire initiating events. It is believed that the results represent worst case conditions and serve as a conservative estimate of drum performance in an actual fire event.

The drum response model was developed to support FHA/SAR analyses for solid waste drum storage facilities. It has already been applied to a limited number of storage configurations including rack and stacked pallet drum arrays. The drum array fire tests, reported herein, were intended to validate the drum response model and show that it is a conservative tool which can be used to predict drum failures.

While the earlier drum testing was an essential element of establishing the validity of the models, it is also necessary to assess the models by comparison with drum array tests. Individual drum tests as performed earlier were instrumented thoroughly, the fire environment was controlled well, and a relatively large number of tests were performed. Array tests cannot be so thoroughly instrumented or controlled, and a very limited number of tests is possible. Array tests do, however, allow the various component phenomena to interact. This can give rise to phenomena which cannot occur in individual drum tests. An obvious example is drum array stability which inherently cannot be studied in single drum tests. Thus, the drum array testing was needed to validate the models developed. Another benefit of the drum array testing was to provide a more quantitative measure of the amount of drum contents expelled during the tests. 
Blank 


\subsection{OBJECTIVE}

The purpose of the drum array fire tests was to demonstrate that the analytical methods developed in earlier work are predictive and yet somewhat conservative. The fullscale tests, which included both rack storage and palletized drum array configurations, were conducted. Using the methodologies developed, predictions of drum performance and extent of fire propagation were made for the full-scale tests. A comparison of these predictions with the actual test results would serve as a validation of the models generated in previous work efforts.

The following section provides the results of the predictions made prior to the drum array tests based on the test arrangements proposed in the test plan. Following that section, the details of the drum array experimental setups, the tests results, and discussion of the . results are provided. 
Blank 


\subsection{PREDICTION OF DRUM PERFORMANCE IN FULL-SCALE FIRE TESTS}

\subsection{GENERAL}

Based on the earlier work, the following analysis and predictions of the drum array test results were made. The methodologies that were used were expected to provide somewhat conservative drum performance estimates. These predictions were made and finalized prior to the conduct of the rack storage and pallet storage array tests. No changes to the analysis in this section were made based on the actual tests.

\subsection{RESPONSE OF DRUMS TO FIRE EXPOSURES}

As a result of the earlier drum fire tests, criteria were developed to indicate lid loss failure for a drum exposed to a fire. It was found that the steol drum wall temperature was in the range of $600-800^{\circ} \mathrm{C}$ for all of the drums at the time of lid loss failure. In no cases did lid loss occur when wall temperatures were below $600^{\circ} \mathrm{C}$. Although eil drums which experienced lid loss were in this temperature range, not all of the drums which achieved these temperatures experienced lid loss. Several drums in the range of $600^{\circ} .750^{\circ} \mathrm{C}$ resulted in lid seal failure only. However, for temperatures exceeding $750^{\circ} \mathrm{C}$, all of the drums resulted in lid loss failure.

All of the orums which experienced lid loss did so in less than three minutes. After this time, even if drum wall temperatures exceeded $600^{\circ} \mathrm{C}$, lid loss did not occur. This resulted from the gasket seal decomposing and venting the drum prior to lid loss. Although criteria were established for lid loss, no systematic trend was observed for lid seal failure.

The thermal response of the drums was calculated using the Semi-Infinite Solid Contents Analyses presented in the earlier report. This model accounted for the packing density of the drum and provided temperature and pressure data for a drum exposed to an external heat flux. Based on the lid loss criteria above, it was determined that if an exposed drum wali section was heated to at least $600^{\circ} \mathrm{C}$ in less than approximately 180 seconds, then lid loss was possible. Further, if the drum was heated to above $750^{\circ} \mathrm{C}$ in less than 180 seconds, than lid loss failure was almost certain.

Based on the drum and fuel configurations in the rack and pallet storage tests, it was expected that the drums will generally either be completely exposed to the fire or they will be exposed on only one side. The thermal properties of the standard load were based on a material having a similar packing density. In this case, properties of regranulated cork were used. The thermal properties needed for the model were as follows:

$$
\begin{aligned}
& \rho=120 \mathrm{~kg} / \mathrm{m}^{3} \quad \text { (packing density of standard load drum is about } 110 \mathrm{~kg} / \mathrm{m}^{3} \text { ), } \\
& k=0.045 \mathrm{E}-03 \mathrm{~kW} / \mathrm{m}^{\circ} \mathrm{C} \text {, and } \\
& C_{p}=1.88 \mathrm{~kJ} / \mathrm{kg}-{ }^{\circ} \mathrm{C} .
\end{aligned}
$$

These values were based upon the packing density of the drum and literature data for materials with this bulk density. 
Figures 1 and 2 show the calculated drum wall temperature results for a full and half perimeter exposure to a drum. For the full perimeter exposure, if the incident heat flux was less than about $40 \mathrm{~kW} / \mathrm{m}^{2}$, lid loss would not occur. For incident fluxes greater than about $70 \mathrm{~kW} / \mathrm{m}^{2}$, lid loss failure was expected. Between 40 and $70 \mathrm{~kW} / \mathrm{m}^{2}$, the failure mode of the drum would vary resulting in some lid loss drums and some lid seal failures. For the half perimeter exposure, the minimum critical flux was approximately $45 \mathrm{~kW} / \mathrm{m}^{2}$, and for incident heat fluxes greater than $75 \mathrm{~kW} / \mathrm{m}^{2}$, lid loss would be expected. Based on the calculated responses of drums to specified incident heat fluxes, the response of the drums during the fire tests was predicted.

\subsection{RACK STORAGE TEST}

Figures 3 and 4 provide diagrams of the proposed test arrangement for the rack storage test configuration. The configuration consisted of three rows of storage with each row being six tiers high $(7.54 \mathrm{~m})$. Two rows were back-to-back, and one row was separated from the other rows by a $1.57 \mathrm{~m}(5.2 \mathrm{ft})$ aisle. The rack system was anchored to the concrete floor of the test facility.

A total of 144 drums were used in the test. The overall weight of each drum was 79.6 $\mathrm{kg}(175.6 \mathrm{lb})$, and it contained both combustible and noncombustible materials. The drums were mounted in the racks using the containments pan and mesh arrangement. Seismic bars were also in place. The fire source consisted of approximately $208 \mathrm{~L}$ (53 gal) diesel fuel spilled into an area of about $16.6 \mathrm{~m}^{2}\left(179 \mathrm{ft}^{2}\right)$ as shown in Figure 3 . The methodology developed in the earlier work was applied to this fire scenario in order to predict the number of drum failures and the extent of fire propagation.

\subsubsection{Description of Fire Exposure}

In order to determine the exposure to the drums, it was necessary to determine the burning behavior of the liquid pool fire. Based on the configuration of the fuel shown in Figure 3, estimates of the fire growth rate, maximum heat release rate, flame height, and burning duration were calculated.

Typically, flammable/combustible liquid pool fires grow to their maximum intensity very quickly. Since the fire growth time would be small compared to the overall burning duration, it was assumed that the pool fire burned at its maximum heat release rate for the duration of the experiment. The maximum heat release rate for the diesel pool fire was estimated as

$$
\dot{Q}=\dot{m}^{\prime \prime} A \Delta H_{c}
$$

\footnotetext{
where $\dot{0} \quad=$ the heat release rate $(\mathrm{kW})$,

$\dot{\mathrm{m}}^{-} \quad=$ the steady mass burning rate per unit area $\left(\mathrm{kg} / \mathrm{m}^{2}-\mathrm{s}\right)$,
} 


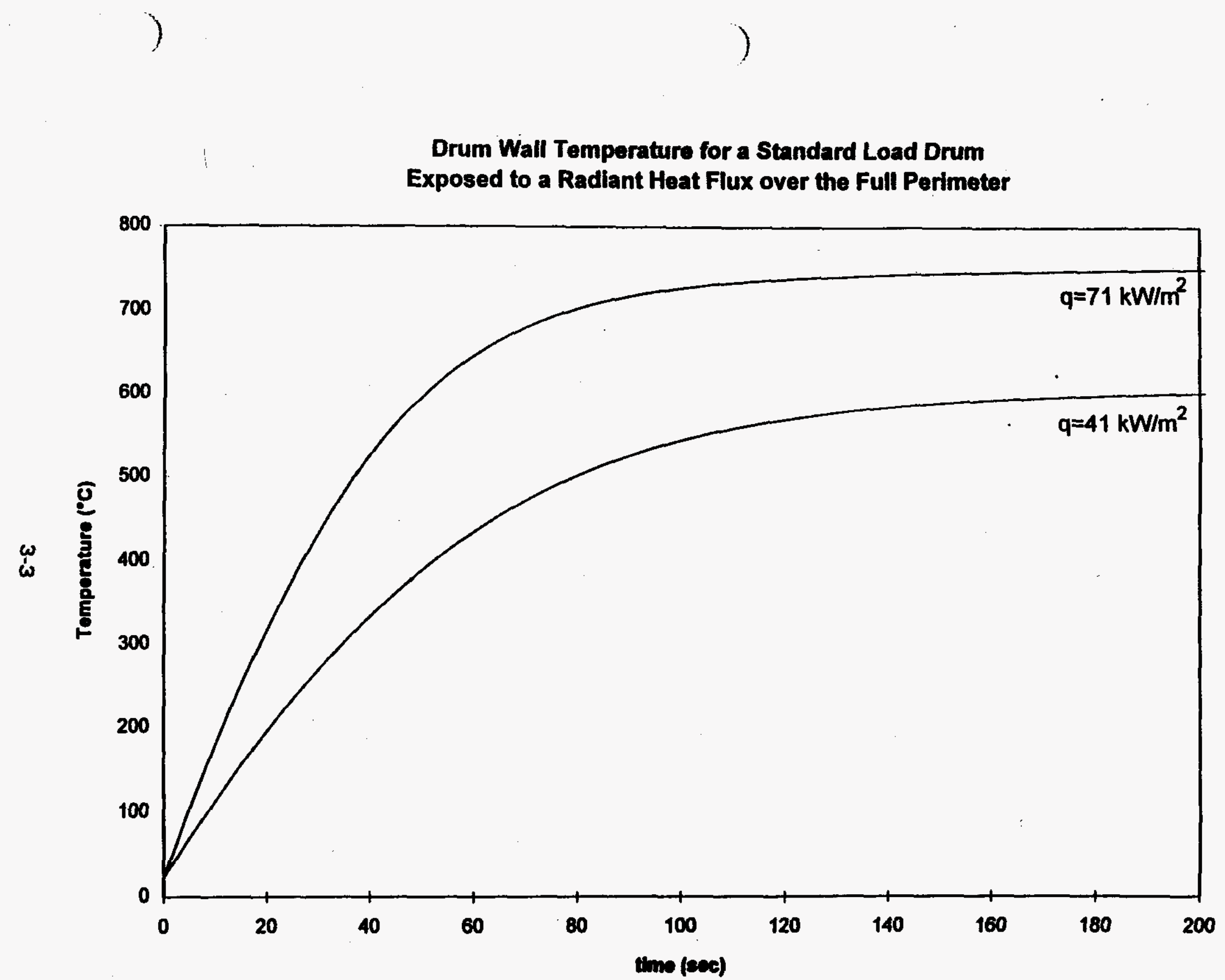

Figure 1. Drum Wall Temperatures - Full Perimeter 
Drum Wall Temperature for a Standard Load Drum

Exposed to a Radiant Heat Flux over One-Half the Perimeter

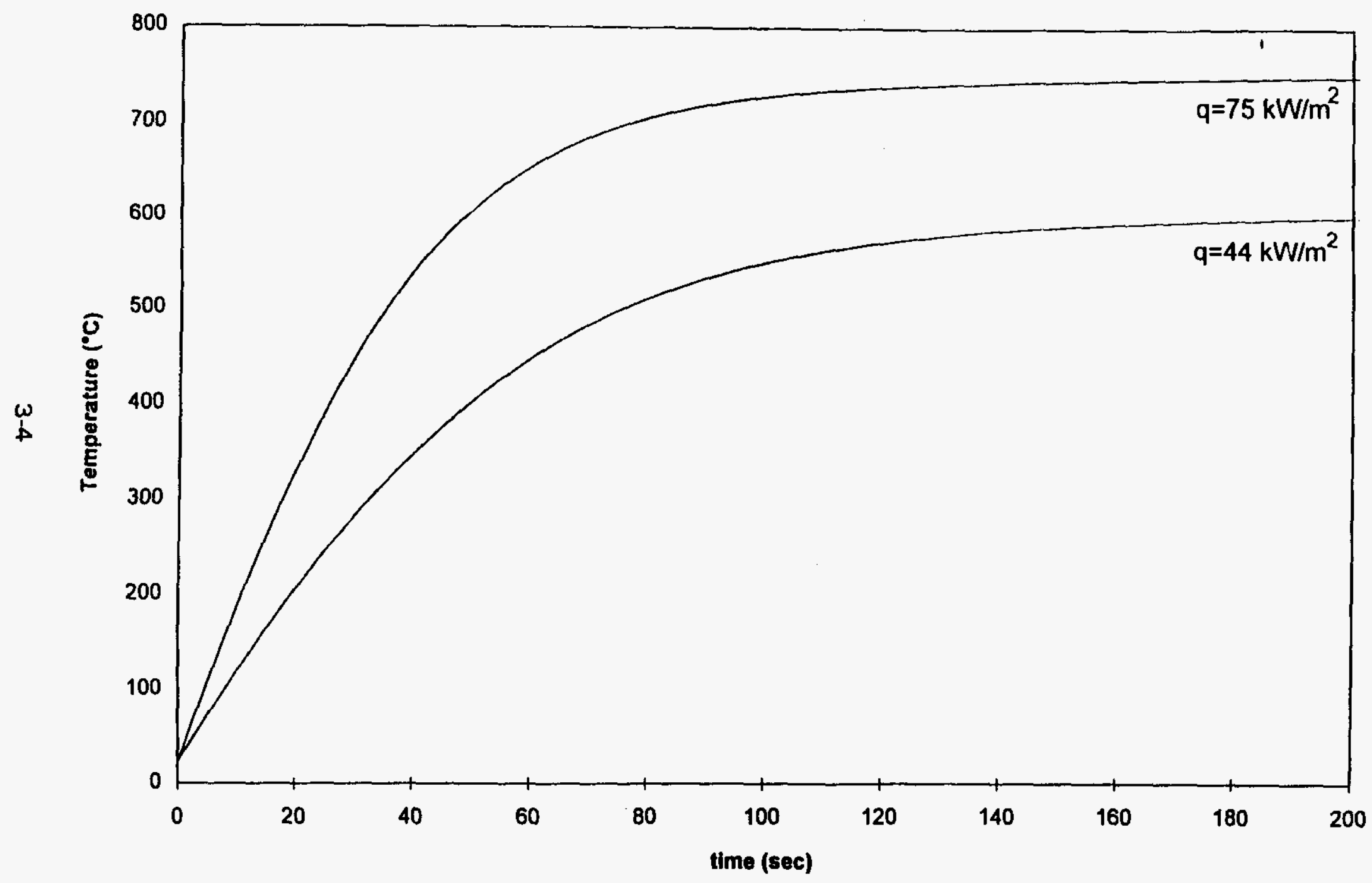

Figure 2. Drum Wall Temperatures - Half Perimeter 

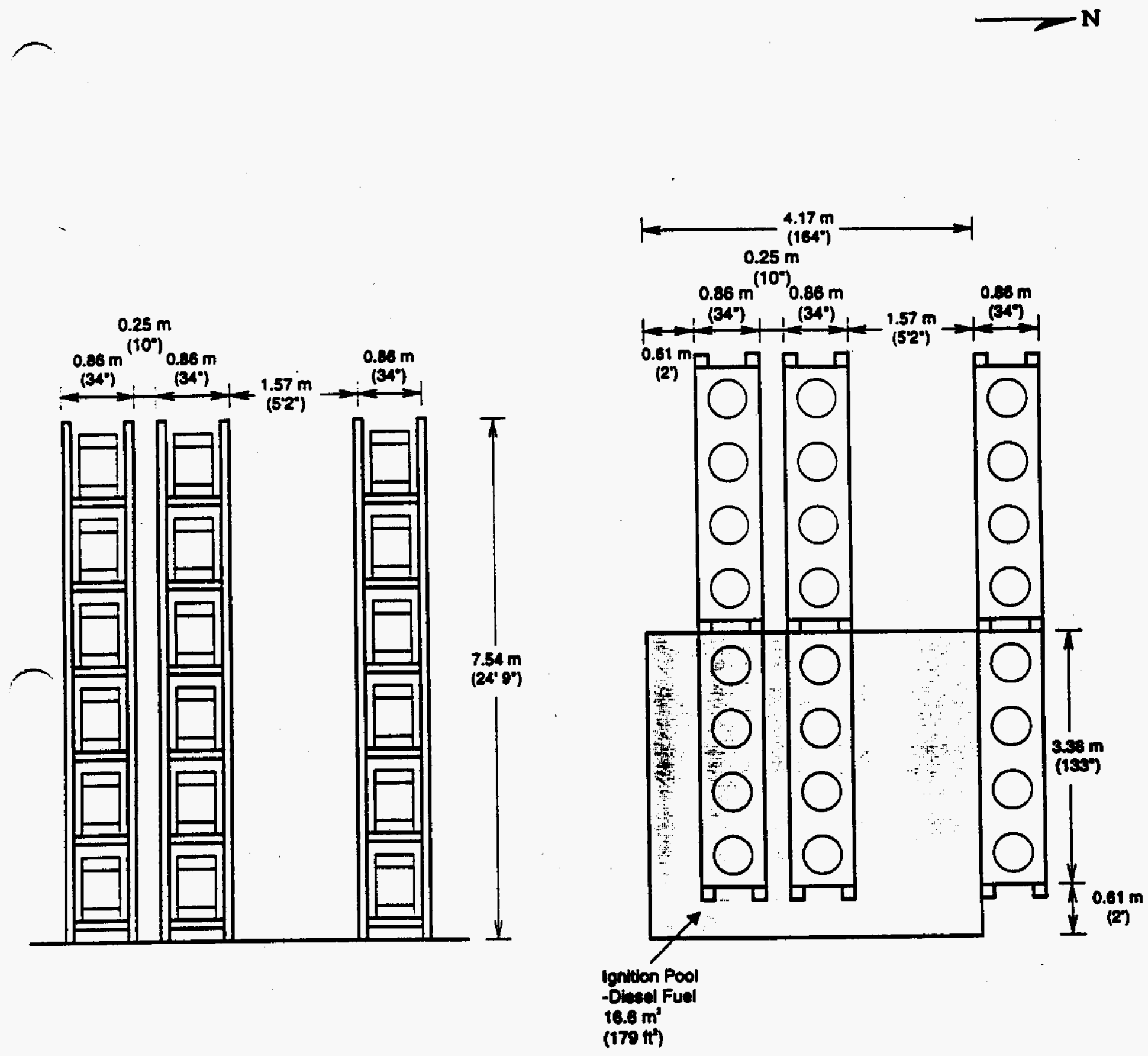

Side View

Plan View

Figure 3. Planned Overall Rack Storage Experimental Setup 
WHC-SD-WM-TRP-246 REV. 0

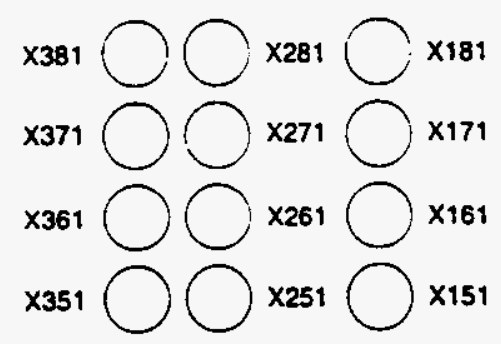

Tier 1
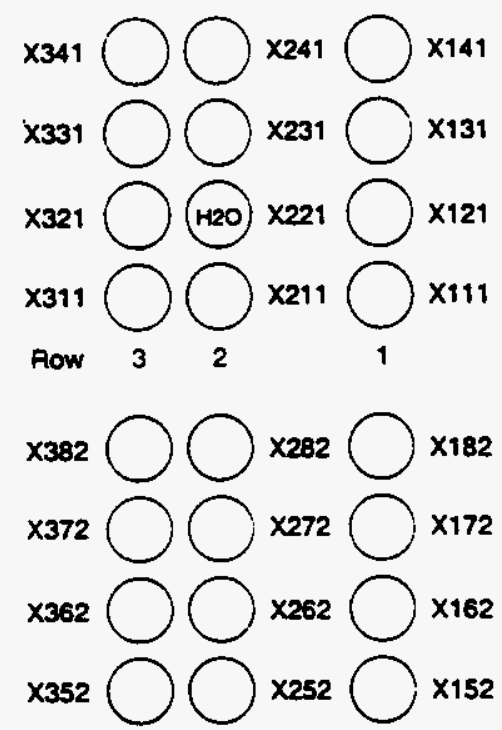

Tier 2
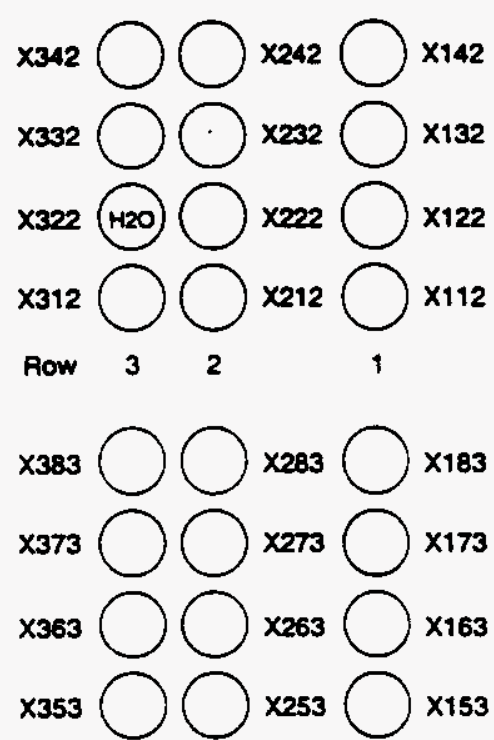

Tier 3

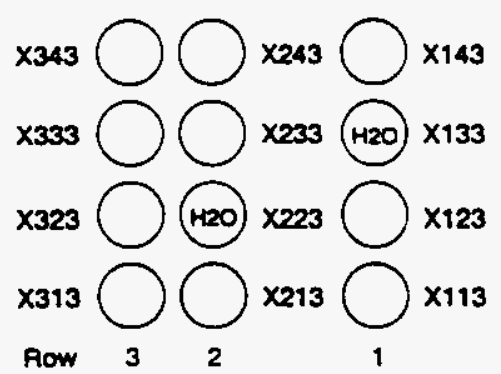

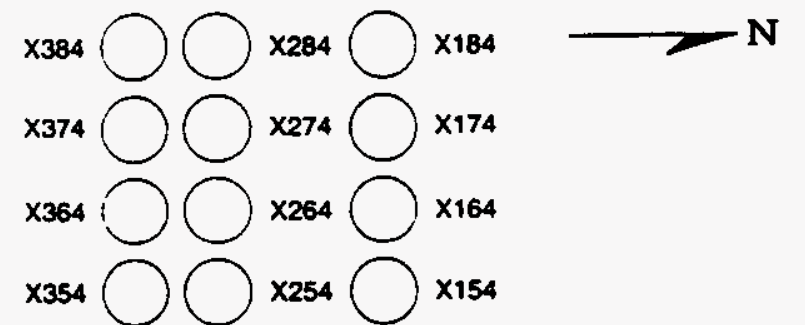

Tier 4

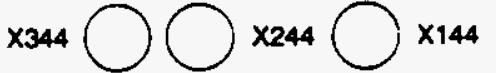

${ }^{3334} \bigcirc \bigcirc \times 234 \bigcirc \times 134$

$\times 324 \bigcirc \times 224 \bigcirc \times 124$

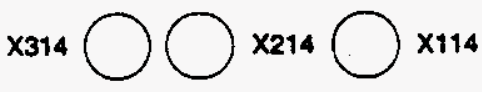

Row 325

x385 $\bigcirc \times 285 \bigcirc x 185$

$x 375 \bigcirc \times 275 \bigcirc \times 175$

$x 365 \bigcirc \times 265 \bigcirc \times 165$

$\times 355 \bigcirc \times 1255 \bigcirc \times 155$

$\times 345 \bigcirc \times 245 \bigcirc \times 145$

$x 335 \bigcirc \times 235 \bigcirc \times 135$

$x 325 \bigcirc \times x 225 \times 125$

$x 315 \bigcirc \bigcirc 215 \bigcirc \times 115$

Row 32

${ }^{x 386} \bigcirc \bigcirc \times 286 \bigcirc \times 186$

$x 376 \bigcirc \times 276 \bigcirc \times 176$

${ }^{x 366} \bigcirc \bigcirc \times 266 \bigcirc \times 166$

${ }^{x 356} \bigcirc \bigcirc \times 256 \bigcirc \times 156$

$\times 346 \bigcirc \times 246 \bigcirc \times 146$

x336 $\bigcirc \times 236 \bigcirc \times 136$

${ }^{3} 326 \bigcirc \times 226 \bigcirc \times 126$

x316 $\bigcirc \bigcirc 216 \bigcirc \times 116$

Tier 6

Note: Drum number indicates Test-Row-Bay-Tier.

Figure 4. Rack Storage Drum Location Diagram 


$$
\begin{aligned}
& A=\text { the burn area }\left(\mathrm{m}^{2}\right), \text { and } \\
& \Delta H_{c}=\text { the heat of combustion of the diesel fuel }(\mathrm{kJ} / \mathrm{kg}) .
\end{aligned}
$$

Burning properties for the fuel can be obtained from either fire tests or in the literature. In this case, properties for kerosene were used since it had similar burning properties as diesel fuel and the information was available in the literature. The maximum burning rate and heat of combustion were provided by Babrauskas [1 988 ] and were taken as $0.039 \mathrm{~kg} / \mathrm{m}^{2}-\mathrm{s}$ and 40 MJ/kg respectively. The ares of the diesel pool shown in Figure 3 is about $16.6 \mathrm{~m}^{2}\left(179 \mathrm{ft}^{2}\right)$. From Equation 1, this yielded a maximum heat release rate of approximately $25.9 \mathrm{MW}$.

The height of the flame was estimated in order to determine how many tiers of drums would be exposed to the flames. For this, a correlation for the visible flame height $\left(L_{f}\right)$ was used [Heskestad, 1988]:

$$
L_{1}=0.23 \dot{Q}^{2 / 5}-1.02 D \quad[\mathrm{~m}, \mathrm{kM}
$$

where $D$ is the equivalent diameter of the pool fire given by

$$
D=2 \sqrt{\frac{A}{\pi}}
$$

An equivalent diameter was used so that the height of the square base fire could be estimated by Equation 3 which was for a circular source fire. For a pool area of $16.6 \mathrm{~m}^{2}$, this yielded an equivalent diameter of about $4.6 \mathrm{~m}(15.1 \mathrm{ft})$. From Equation 2 , with a heat release rate of $25.9 \mathrm{MW}$, this yielded a visible flame height of about $8.7 \mathrm{~m}(28.6 \mathrm{ft})$. Since the height of the rack system is only $7.54 \mathrm{~m}(24.75 \mathrm{ft})$, the flame would be expected to directly expose all six tiers of the array. These predictions were made based on a planned pool area of 16.6 $\mathrm{m}^{2}\left(179 \mathrm{ft}^{2}\right)$. [Note: In the actual test, an $18.2 \mathrm{~m}^{2}\left(196 \mathrm{ft}^{2}\right)$.pool was actually used. This increase in pool size increases the flame height slightly but does not change the resulting damage.]

The burning duration of the fire is a function of the mass of fuel, the steady mass burning rate, and the area of the pool. The burning duration was estimated by

$$
t_{b}=\frac{m}{\dot{m}^{\prime \prime} A}
$$

where $t_{b} \quad$ = the burning duration (s), and

$m$ the total mass of fuel $(\mathrm{kg})$.

The total amount of diesel fuel was approximately $208 \mathrm{~L}(55 \mathrm{gal})$. Again using properties for kerosene, assuming a density of $0.825 \mathrm{~kg} / \mathrm{L}$ [Kanury, 1975], this yielded a total fuel mass of about $171.6 \mathrm{~kg}$. Using Equation 4 with a steady mass loss rate of $0.039 \mathrm{~kg} / \mathrm{m}^{2}-\mathrm{s}$ and a burn area of $16.6 \mathrm{~m}^{2}$, this would provide a burning duration of 265 seconds $1-4-5$ minutes). [Note: In the actual test, $284 \mathrm{~L}(75$ gall of fuel was used.) 


\subsubsection{Predicted Response of Rack Storage Drums}

The emissive power of the pool fire was estimated from the literature. The two principle correlations of emissive power available in the fiterature are by Mudan and Croce [1988j and Shokri and Beyler [1989]. The correlation of Mudan and Croce yielded an emissive power of $80 \mathrm{~kW} / \mathrm{m}^{2}$ for a $4.6 \mathrm{~m}$ diameter pool source. The Shokri and Beyler correlation yielded an emissive power of $53 \mathrm{~kW} / \mathrm{m}^{2}$ with data as high as $70 \mathrm{~kW} / \mathrm{m}^{2}$. Based on these results, a conservative estimate of $80 \mathrm{~kW} / \mathrm{m}^{2}$ was chosen for the emissive power. This indicates that any drum at the edge of the flame would be exposed to this flux and was expected to experience lid loss failure. Drums within the pool area would be within the flame and would be exposed to higher fluxes. These drums, within the flames, would experience lid loss. In Rows 2 and 3 (see Figure 4), drums in Bays 1-4 are fully engulfed by the flame. All six tiers of drums in these positions would be expected to experience lid loss failure. In the these rows, the first drum outside the pool fire (drums $\times 25_{-}$and $\times 35$ in Figure 4 ) would be directly exposed to the pool fire and expected to experience lid loss. In Row 1, all drums in Bays 1-4 would be expected to experience lid loss since they also were directly adjacent to the pool. While this conclusion for the drums directly exposed to the edige of the fire is unlikely to be realized on the top two tiers due to flame shape and drum obstruction effects, these effects could not be excluded analytically.

The separation distance from the flame required to prevent lid loss is governed by the minimum heat flux required to cause lid loss. As indicated previously, this heat flux was in the range of 45 to $75 \mathrm{~kW} / \mathrm{m}^{2}$. To maintain conservatism in the prediction, calculations were based on a required heat flux of $45 \mathrm{~kW} / \mathrm{m}^{2}$. The configuration factor required to yield an incident flux of $45 \mathrm{~kW} / \mathrm{m}^{2}$ was $45 / 80$ or 0.56 . Of course, the configuration factor is reduced as one moves away from the pool, and at some distance, lid loss will no longer be possible. It then remains to determine which drums outside the flame have configuration factors in excess of 0.56 , using the cylindrical flame model and a vertical target.

In Rows 2 and 3, drums $\times 26_{-} \times 27_{-}, \times 28_{-} \times 36_{-}, \times 37_{-}$, and $\times 38_{-}$(see Figure 4) would be substantially shielded from the flame by drums between the target and the assumed cylindrical flame. As such, these drums were not expected to experience lid loss due to flame radiation. Thus, in Rows 2 and 3, the only drums outside the pool which would experience lid loss are drums $\times 25_{-}$and $\times 35_{-}$.

In Row 1, for the Bay 5 drums, the configuration factors at the base level was approximately at the limiting configuration factor. As such, all tiers of that position were expected to be lost. Again, this appears to be somewhat conservative at the uppermost tiers. At Bay 6, at the midheight of the flame (around Tier 4), the configuration factor was greater than the critical value, but at the base of the bay the configuration factor was less than the critical value. As such, the middle four tiers only were expected to be lost. At Bay 7 , at the midheight of the flame, the configuration factor was less than the critical value. As such, all tiers in this bay were less than the critical value, and no lid losses in this position were expected due to flame radiation from the pool. Thus, in Row 1 all drums in Bays 1-5 were expected to experience lid loss failure and drums in Tiers 2-5 at Bay 6 would also experience lid loss due to flame radiation.

In the earlier work, it was shown that the radiant energy from a burning drum was not sufficient to cause lid loss failure of neighboring drums. The analysis included both lateral and 
vertical fire propagation. As such, no additional lid losses were expected due to exposure to burning drums.

As a result of the lid loss drums, the standard load contents would be expelled within the rack and onto the floor. As it was shown in the earlier testing, the average heat flux from drum content fires was on the order of $25 \mathrm{~kW} / \mathrm{m}^{2}$. Since this was less than the minimum heat flux required to cause lid loss, no additional lid loss failures would be expected due to drum content fires.

Based on a heat transfer analysis of the rack system in the earlier report, it was expected that the steel uprights would reach critical failure temperatures of $500 \cdot 600^{\circ} \mathrm{C}$ within approximately 100 seconds. Failure of the steel members would cause deformation of the rack. Since seismic bars were in place and based on the geometry of the racks with respect to one-another, it was not expected that the drums would fall out of the rack. As such, no additional lid losses were expected due to failure of the rack system.

The drums which would experience lid loss due to the pool fire are summarized in Figure 5 in which an $X$ is placed on these drums. The total number of drums conservatively predicted to experience lid loss are 48 within the pool area and 46 adjacent to the pool fire. These predictions treat the pool fire as a cylindrical radiator with an average flame emissive power of $80 \mathrm{~kW} / \mathrm{m}^{2}$ and a critical flux for lid loss of $45 \mathrm{~kW} / \mathrm{m}^{2}$.

If the emissive power used was $53 \mathrm{~kW} / \mathrm{m}^{2}$ as indicated by Shokri and Beyler and a critical heat flux for lid loss of $60 \mathrm{~kW} / \mathrm{m}^{2}$ (average of 45 and $75 \mathrm{~kW} / \mathrm{m}^{2}$ extremes) was used, then no drums outside the pool would experience lid loss. Since the accuracy of an average emissive power would be reduced as one approaches the pool edge, there are real changes in the appropriate emissive power depending on the height of the target. Based on these calculations, the number of drum lids lost outside the pool would be expected to be in the range of 0 to 46 .

\subsection{PALlet STORAGE TEST}

Figures. 6 and 7 provide diagrams of the proposed test arrangement for the pallet storage test configuration. The configuration consisted of three rows of pallets with each row being three tiers high ( $-3.1 \mathrm{~m}$ assuming $15 \mathrm{~cm}$ high pallets). Two rows used wooden pallets and one row used steel paliets. The rows of pallots were separated by $0.91 \mathrm{~m}(3.0 \mathrm{ft})$ aisles. Within a row, the pallets were positioned flush with one another. 
WHC-SD-WM-TRP-246 REV. 0

Tier 1

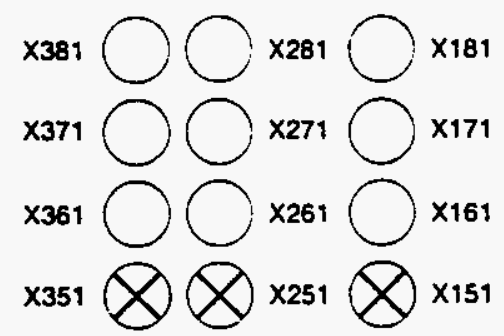

${ }^{\times 341} \otimes \otimes^{\times 241} \bigotimes \times 141$

${ }^{3331} \bigotimes \bigotimes^{\times 231} \bigotimes^{{ }^{131}}$

x321 $\bigotimes$ ×221

x311 $\bigotimes \times 211 \bigotimes \times 111$

Row $3 \quad 2 \quad 1$

x382 $\bigcirc \times 282 \bigcirc \times 182$

$\times 372 \bigcirc \times 272 \bigcirc \times 172$

${ }^{x 362} \bigcirc{ }^{x 262} \bigotimes \times 162$

${ }^{3352} \bigotimes \bigotimes^{\times 252} \otimes^{\times 152}$

Tier 2

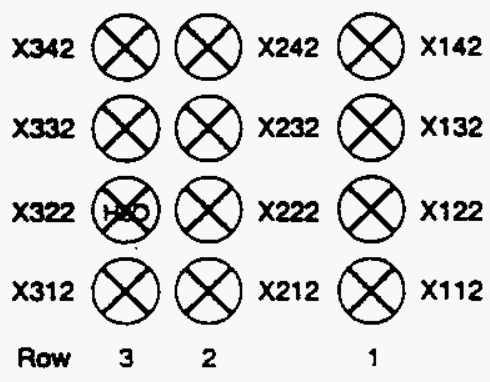

${ }^{3383} \bigcirc \bigcirc \times 283 \bigcirc \times 183$

$x 373 \bigcirc \times 273 \bigcirc \times 173$

${ }^{x 363} \bigcirc \bigcirc \times 263 \bigotimes \times 163$

${ }^{\times 353} \bigotimes \bigotimes^{\times 253} \otimes^{\times 153}$

Tier 3

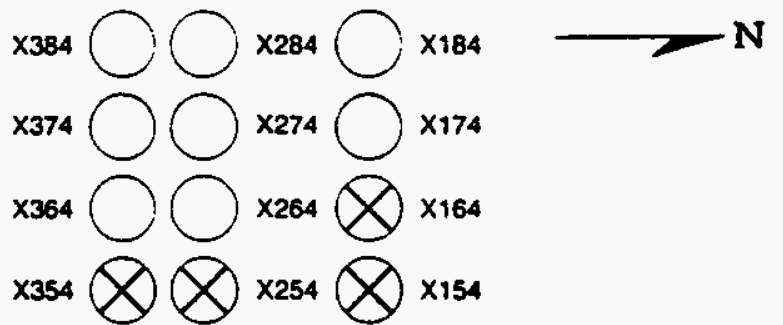

×344 $\bigotimes \times 244 \bigotimes \times 144$ ${ }^{\times 334} \bigotimes \bigotimes^{\times 234} \bigotimes$ ${ }^{x 324} \bigotimes_{\times 224} \bigotimes^{\times 124}$ x314 $\bigotimes \times 214 \bigotimes \times 114$ Row $3 \quad 2 \quad 1$

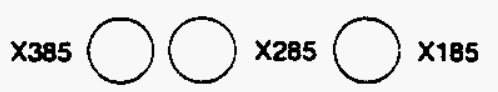
$x 375 \bigcirc \times 275 \bigcirc \times 175$ x365 $\bigcirc \times 265 \bigotimes \times 165$ x355 $\bigotimes \times \bigotimes^{x 255}$ ${ }^{345} \bigotimes \bigotimes^{\times 245} \bigotimes^{\times 145}$ x335 $\bigotimes^{\times 235} \bigotimes_{\text {xi35 }}$ ${ }^{3252} \bigotimes \bigotimes \times \bigotimes^{x 225}$ x315 $\bigotimes \times 215 \bigotimes \times 115$ Row 32

Tier 4

Tier 5

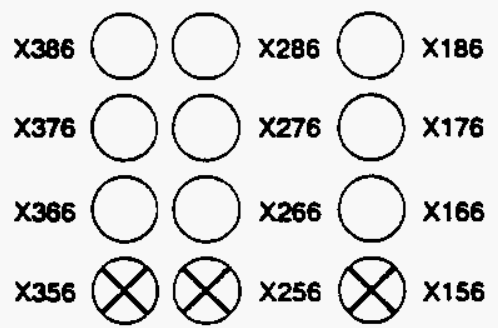
×346 $\otimes \times 246 \otimes \times 146$ Tier 6 ${ }^{\times 336} \bigotimes \bigotimes_{\times 236} \bigotimes^{\times 136}$ ${ }^{\times 326} \otimes \otimes_{\times 226} \otimes_{\times 126}$ ×316 $\bigotimes \times \bigotimes_{\text {×216 }} \otimes_{116}$

Note: Drum number indicates Test-Row-Bay-Tier.

$\bigotimes$ Potential Lid Loss

Figure 5. Predicted Rack Storage Lid Losses 
WHC-SD-WM-TRP-246 REV. 0

?
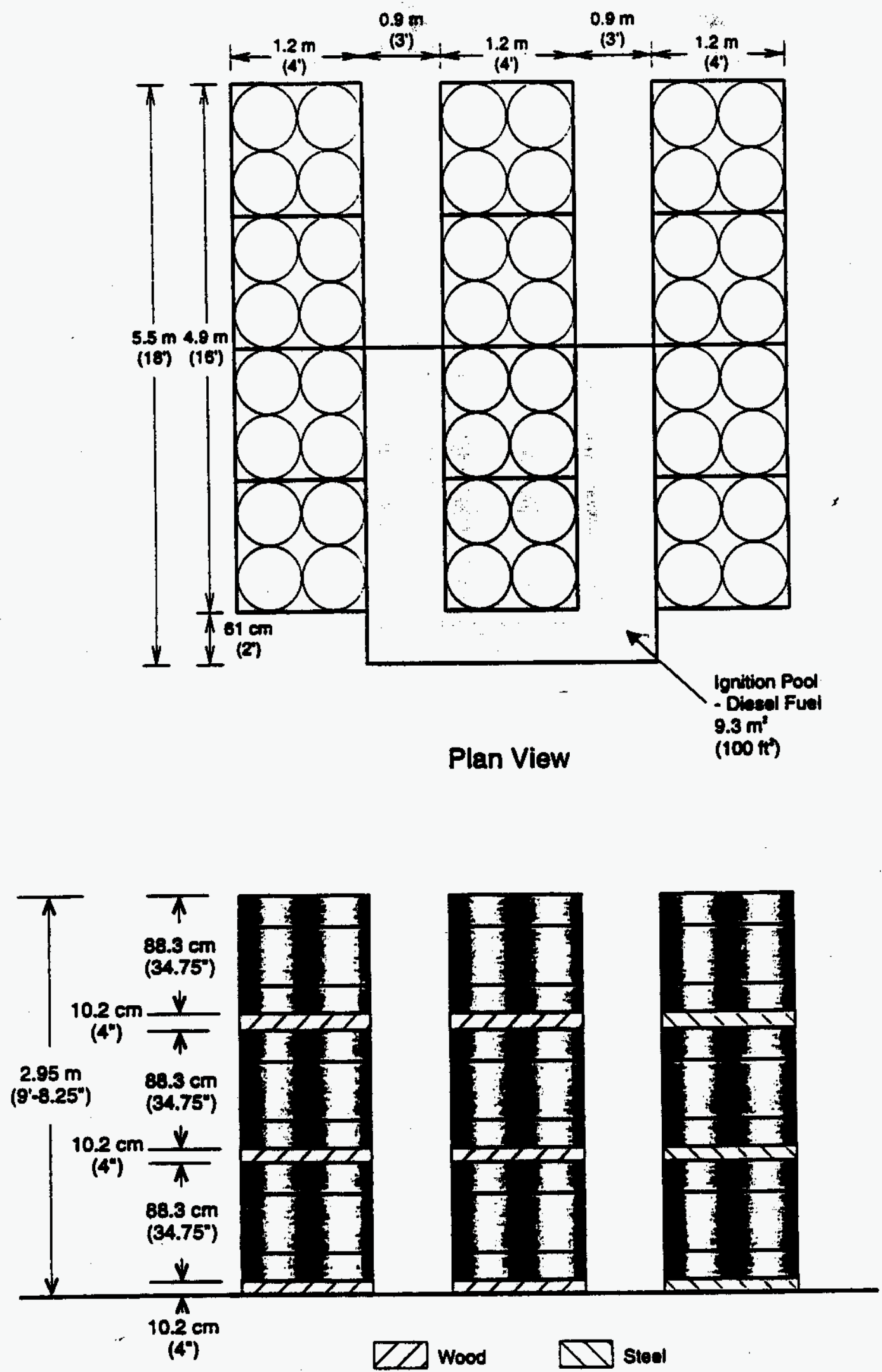

Side View

Figure 6. Planned Overall Rack Storage Experimental Setup 
WHC-SD-WM-TRP-246 REV. 0

Tier 1

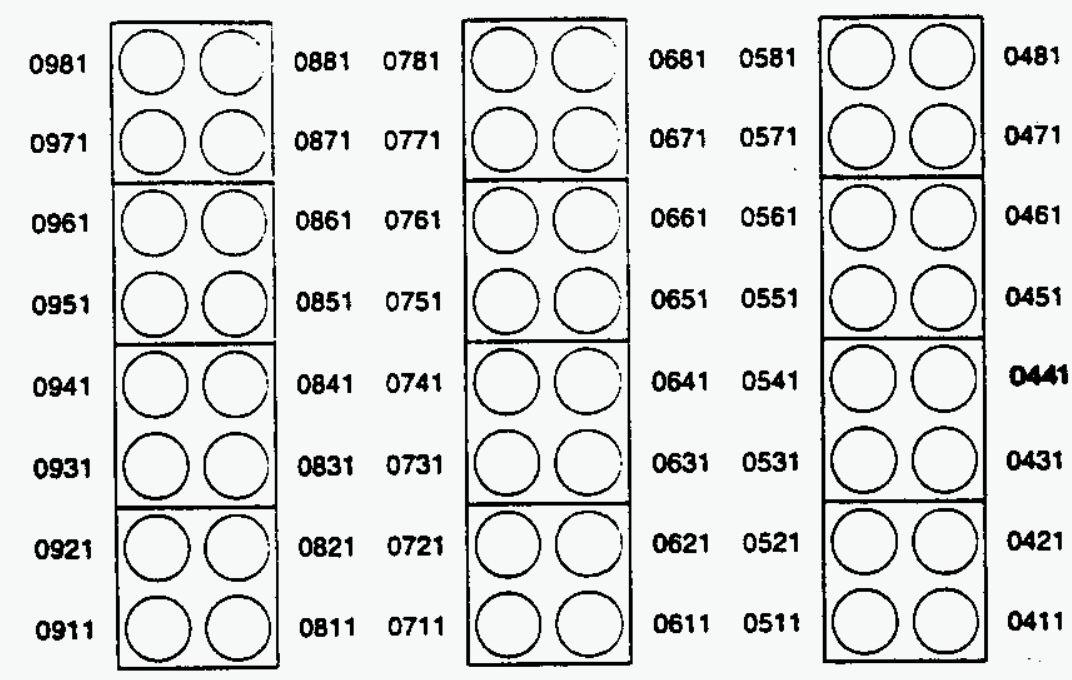

Tier 2

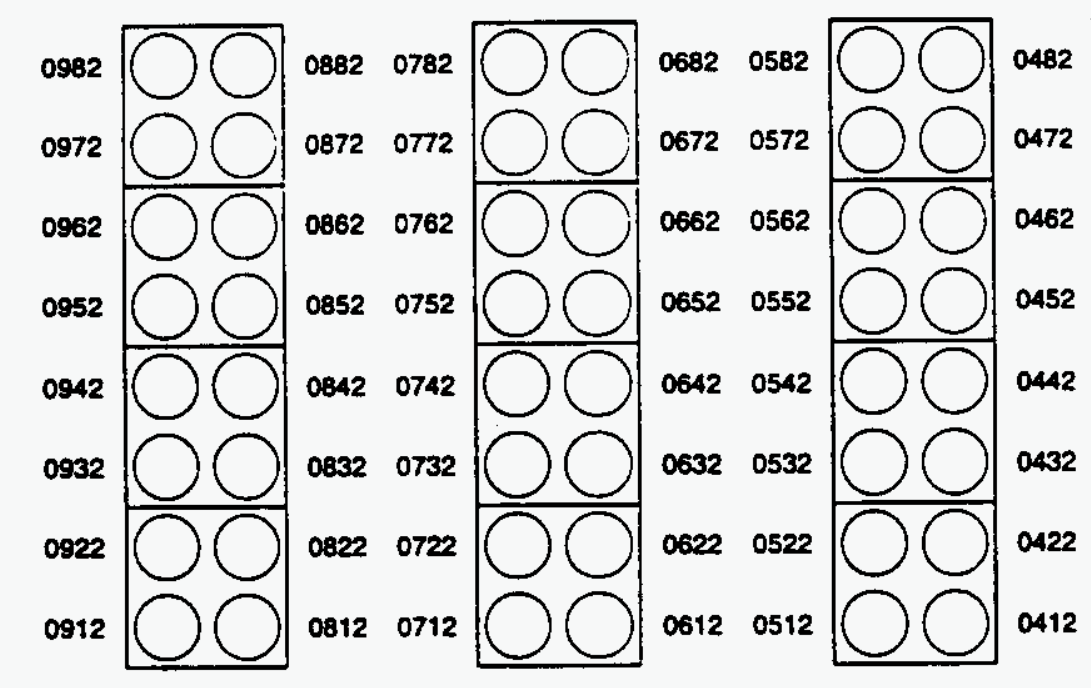

Tier 3

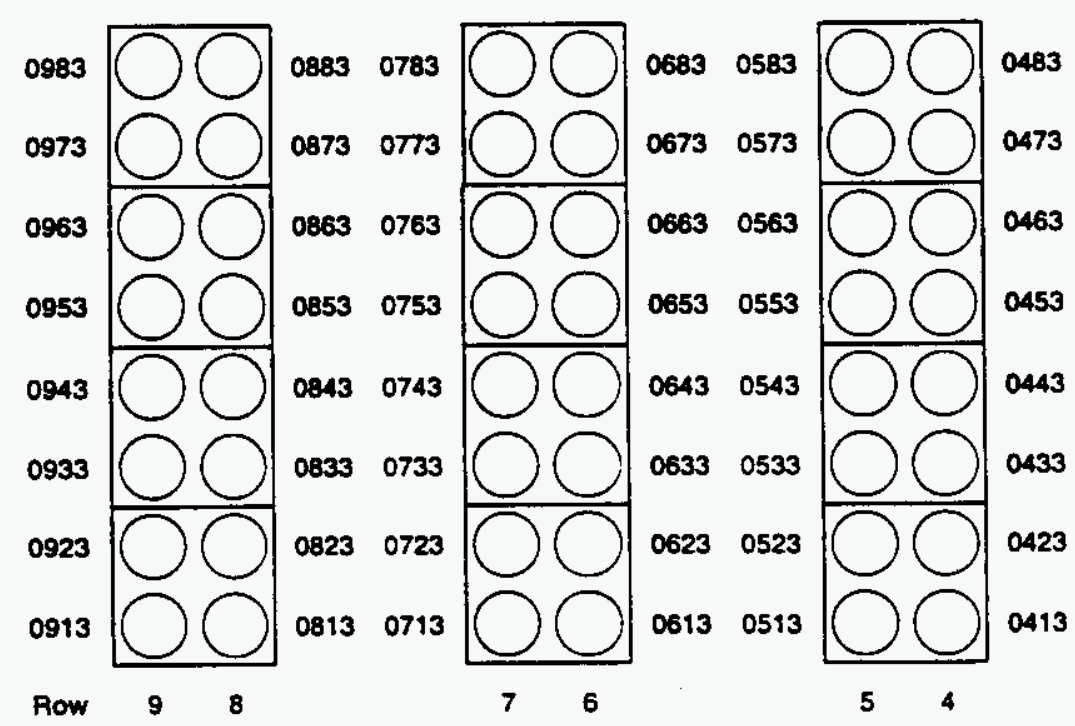

Note: Drum number indicates Test-Row-Bay-Tier.

Figure 7. Pallet Storage Drum Location Diagram 
A total of 144 drums were used in this test. The overall weight of each drum was $79.6 \mathrm{~kg}(175.6 \mathrm{lb})$, and it contained both combustible and noncombustible materials. Each pallet held four drums. The four drums were banded together by a single, horizontal, high tensile steel strap. The fire source consisted of approximately $135 \mathrm{~L}$ (35 gal) diesel fuel spilled into an area of about $9.3 \mathrm{~m}^{2}\left(100 \mathrm{ft}^{2}\right)$ as shown in Figure 5 . The methodology developed in earlier work was applied to this fire scenario in order to predict the number of drum failures and the extent of fire propagation.

\subsubsection{Description of Fire Exposure}

In order to determine the exposure to the drums, it was necessary to determine the burning behavior of the liquid pool fire. Based on the configuration of the fuel shown in Figure 6, estimates of the fire growth rate, maximum heat relesse rate, fleme height, and burning duration were calculated.

Typically, flammable/combustible liquid pool fires grow to their maximum intensity very quickly. Since the fire growth time would be small compared to the overall burning duration, it was assumed that the pool fire burned at its maximum heat release rate for the duration of the experiment. The maximum heat release rate for the diesel pool fire was estimated from Equation 1. For a pool area of $9.3 \mathrm{~m}^{2}$, this yielded a maximum heat release rate of $14.5 \mathrm{MW}$. The height of the flame was estimated from Equations 2 and 3 . The equivalent diameter of the pool was approximately $3.4 \mathrm{~m}$, and the visible flame height was $7.1 \mathrm{~m}$. Since the height of the pallet stacks is about $3.1 \mathrm{~m}$, the flame would expose all three tiers of drums. The burning duration of the fire was calculated using Equation 4. The total amount of diesel fuel was $135 \mathrm{~L}$. For a density of $0.825 \mathrm{~kg} / \mathrm{L}$, this yielded a total fuel mass of $111.4 \mathrm{~kg}$. This would provide a burning duration of 307 seconds ( -5 minutes). (Note: In the actual test, $142 \mathrm{~L}$ of fuel was used.] The predicted heat release and burn duration does not include a reduction in the burning rate due to shielding the pool surface by the drums.

\subsubsection{Predicted Response of Pallet Storage Drums}

The emissive power of the pool fire was estimated from the literature. The two principle correlations of emissive power available in the literature are by Mudan and Croce [1988] and Shokri and Beyler [1989]. The correlation of Mudan and Croce yielded an emissive power of $100 \mathrm{~kW} / \mathrm{m}^{2}$ for a $3.4 \mathrm{~m}$ diameter pool source. The Shokri and Beyler correlation yielded an emissive power of $55 \mathrm{~kW} / \mathrm{m}^{2}$ with data as high as $70 \mathrm{~kW} / \mathrm{m}^{2}$. Based on these results, a conservative estimate of $100 \mathrm{~kW} / \mathrm{m}^{2}$ was chosen for the emissive power. This indicates that any drum within the flame or at the edge of the flame would be exposed to this flux and was expected to experience lid loss failure. The drums on the first two pallet stacks in Rows 6 and 7 (drum nos. O6_ and 07., Bays 1.4 in Figure 7) would be completely engulfed in the flames. It would be expected that all of these drums would experience lid loss failure. The drums in Rows 5 and 8 (drum nos. 05 _ and 08 , Bays 1-4) and the drums in Bay 5 of Rows 6 and 7 (drum nos. 065 and 075 ) would be immediately adjacent to the pool fire and would be exposed to fluxes equivalent to the emissive power of the pool fire which exceeds the critical flux for lid loss failure. As such, these drums were expected to experience lid loss. 
The separation distance from the flame required to prevent lid loss is governed by the minimum heat flux required to cause lid loss. As indicated previously, this heat flux was in the range of 45 to $75 \mathrm{~kW} / \mathrm{m}^{2}$. To maintain conservatism in the prediction, calculations were based on a required heat flux of $45 \mathrm{~kW} / \mathrm{m}^{2}$. The configuration factor required to yield an incident flux of $45 \mathrm{~kW} / \mathrm{m}^{2}$ is $45 / 100$ or 0.45 . Of course, the configuration factor is reduced as one moves away from the pool and at some distance lid loss will no longer be possible. It then remains to determine which drums outside the flame have configuration factors in excess of 0.45 , using the cylindrical flame model and a vertical target.

Drums beyond Bay 5 in Rows 6 and 7 would be significantly shielded from the pool fire and were not expected to experience lid loss. Bays 5, 6, and 7 in Rows 5 and 8 were predicted to have configuration factors in excess of the critical values. However, this ignores the significant shielding provided by drums in Rows 6 and 7 in conjunction with the narrow aisle widths. Based on this shielding, only Bays 5 and 6 were expected to experience lid loss due to pool fire radiation.

The drums which were expected to experience lid loss due to pool fire radiation are all the drums within the pool as well as the following drums outside the pooi: 051,052 , 053_.054_,055_,056_065_075_.081_,082_083_,084_,085_, and 086_. Thus, 24 drums within the pool were expected to experience lid loss and 42 drums outside the pool were expected to experience lid loss due to pool fire radiation. These predictions treat the pool fire as a cylindrical radiator with an average flame emissive power of $100 \mathrm{~kW} / \mathrm{m}^{2}$ and a critical flux for lid loss of $45 \mathrm{~kW} / \mathrm{m}^{2}$.

If the emissive power used was $55 \mathrm{~kW} / \mathrm{m}^{2}$ as indicated by Shokri and Beyler and a critical heat flux for lid loss of $60 \mathrm{~kW} / \mathrm{m}^{2}$ (average of 45 and $75 \mathrm{~kW} / \mathrm{m}^{2}$ extremes) were used, then no drums outside the pool would experience lid loss. Since the accuracy of an average emissive power would be reduced as one approaches the pool edge, there are real changes in the appropriate emissive power depending on the height of the target. Based on these calculations, the number of drum lids lost outside the pool would be expected to be in the range of 0 to 52 .

When drums on the lower tiers experience lid loss failure, the force from the lid release could potentially tip over the pallet stacks above. A Drum Array Stability Model was developed in earlier work which calculated the minimum release pressure necessary to tip over the stacked drums. The model was based on the geometry and weight of the drums and pallets. No credit was taken for banding drums on the pallets. Since both steel and wooden pallets were used in the test, the minimum pressure required to topple the two types of pallets will vary. For the specific test arrangement shown in Figure 5, the mass of the drums was taken as $79.6 \mathrm{~kg}(175.6 \mathrm{lb})$ and the mass of the steel pallets was taken as $91 \mathrm{~kg}(200 \mathrm{lb})$. In the stability analysis, only the mass of the steel pallets were considered since these would be more difficult to topple than the wooden pallets. For a drum lid loss in the bottom tier, the minimum release pressure needed to topple the top two pallet stacks was approximately 20 psig. If the mass of the steel pallet was ignored, the minimum pressure was approximately 17 psig. This indicates that the critical pressure for the wooden pallet was between 17 and 20 psig. Based on the earlier drum response tests, failure pressures were measured to be in the range of 13 to 28 psig. As such, 20 psig is a reasonable failure pressure, and it would be expected that drums experiencing lid loss will topple the pallet stacks above. 
Since at least some drums were predicted to experience lid loss in the first three pallets of each row, all drums on the top two tiers of these pallets would be expected to topple to the floor. The drums in Rows 4 and 5 and the drums in Rows 8 and 9 would be expected to fall aivay from the fire. For the center rows, the rotation of the pallet stacks depends on which drum experiences lid loss first. Drums which fall to the floor were assumed to lose their lids. The total number of drums which would lose their lids due to falling on the floor is 72. This includes the top two tiers of drums in positions 1-6 for all of the rows. Of the 72 drums, 44 would have experienced lid loss anyway, due to radiation from the flame. Although it is assumed that all of the drums which experience lid loss due to radiation from the fire will fail at pressures above 20 psig, it is likely that some might fail at lower pressures. In these cases, the pallet stacks may not topple, and the failure mode of the drums would be that described earlier for the pool fire radiation.

As a result of the lid loss drums, the standard load contents would be expelled on top of other drums and onto the floor. As it was shown in the earlier testing, the average heat flux from drum content fires was on the order of $25 \mathrm{~kW} / \mathrm{m}^{2}$. Since this is less than the minimum heat flux required to cause lid loss, no additional lid loss failures would be expected due to drum content fires.

The drums which experience lid loss due to the pool fire are summarized in Figure 8 in which an $X$ is placed on these drums. The total number of drums conservatively predicted to experience lid loss are 66 due to radiation from the pool fire and an additional 28 due to drums falling to the floor. These predictions treat the pool fire as a cylindrical radiator with an average flame emissive power of $100 \mathrm{~kW} / \mathrm{m}^{2}$, and a critical flux for lid loss of $45 \mathrm{~kW} / \mathrm{m}^{2}$. It is further assumed that all drums experiencing lid loss due to radiation from the fire will cause the drums above to topple to the floor and lose their lids. 
WHC-SD-WM-TRP-246 REV. 0

Tier 1

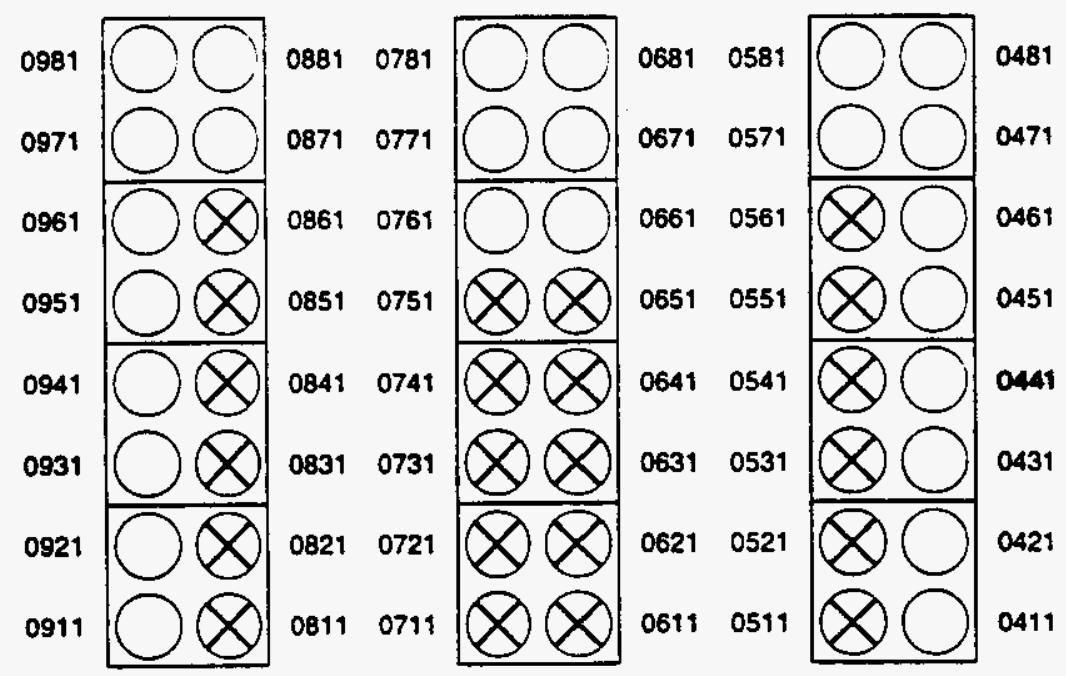

Tier 2

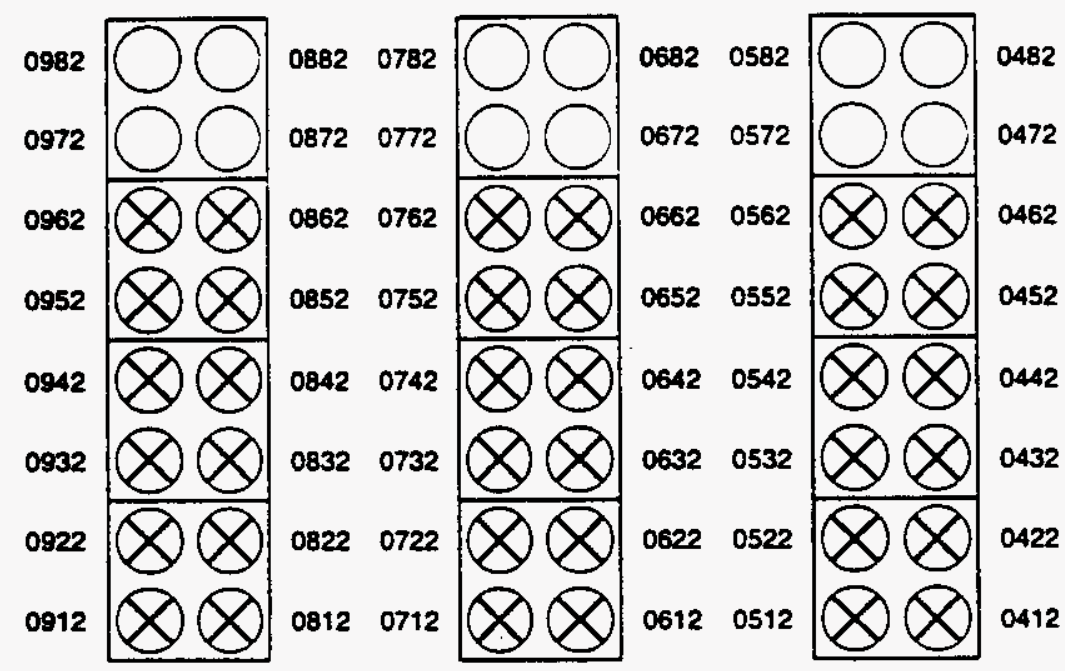

Tier 3

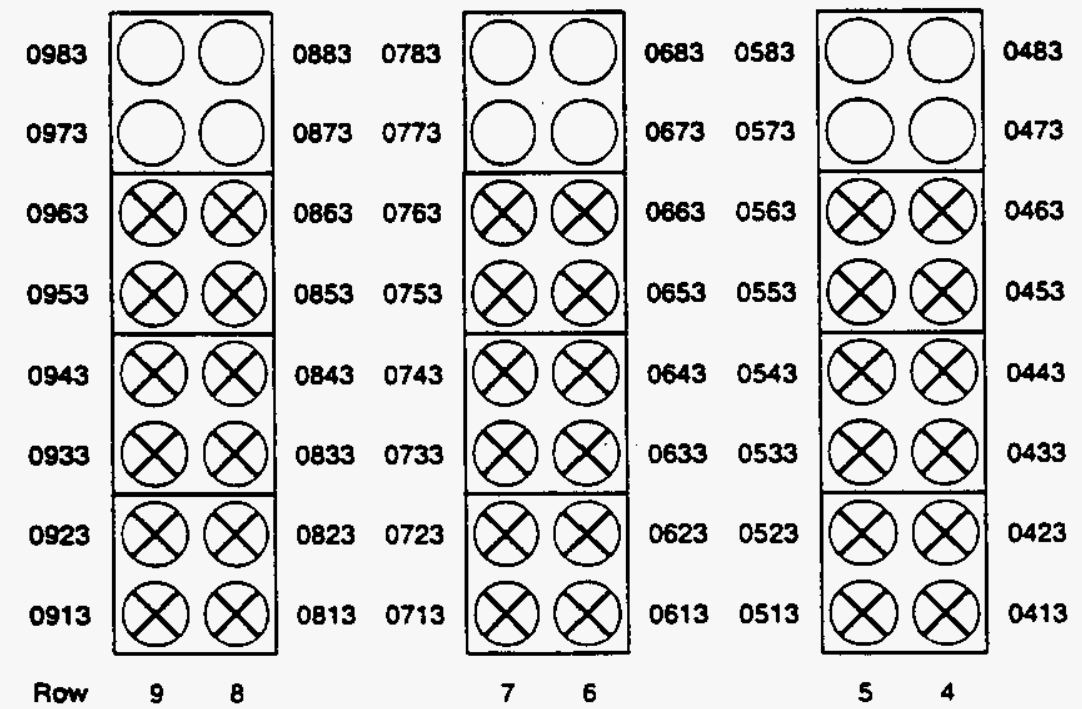

Note: Drum number indicates Test-Row-Bay-Tier.

$$
\bigotimes \text { Potential Lid Loss }
$$

Figure 8. Predicted Pallet Storage Lid Losses 


\subsection{EXPERIMENTAL - GENERAL}

In this work, one rack storage array test and one pallet array test were conducted. These configurations represented the two primary storage types under consideration. In actual practice, the rack storage configuration may vary in the number of tiers included, the nature of the structure as well as the nature of the drum supports. The pallet configuration represented storage configurations in which the drums are stacked in vertical arrays. In actual practice, the number of drum leveis within the array may vary, and the properties of the level separations may vary. In particular, current WHC practice and/or plans include pallets or plywood as level separations. The configurations which were used in these tests were reasonable representations of their respective storage configurations.

While the test setups used in the tests were intended to be representative of the general storage configurations in use, the initiating fire was designed to maximize the damage potential. Pool fires having a 1-2 m diameter yield the highest flame emissive power. Both smaller and larger diameters have lower emissive powers. As such, the pool fire initiating sources used for the tests were large enough to yield optically thick flames $(1 \mathrm{~m}$ or more) providing the maximum exposure to the drums. In addition, the quantity of fuel was designed to burn longer than any drum lid loss failure time in earlier testing. This assured that if a lid loss could occur, it would occur in the testing. This is consistent with a philosophy of "testing to failure." While this philosophy cannot be fully implemented in a single test situation, the use of a very severe (optically thick and long duration) initiating source avoids the concern that a longer duration or larger pool fire might have a more severe effect. Clearly, if a shorter fire duration is expected in practice, the data will be of value up to the pool fire burn time expected.

The size of the storage array was dictated by the need for an optically thick initiating fire exposure as well as the need for the storage array to be sufficiently large relative to the initiating source such that fire propagation remote from the initiating source would be geometrically possible in the test. Implied in the test array size criterion was that if the fire propagates to the end of the storage array, it would be expected to propagate indefinitely. While this can only be assured in an indefinitely long storage array, the array length had be long enough to approach this limit credibly, but practically.

While instrumenting every drum in detail was desirable, this was not an economically feasible option. The level of instrumentation used was sufficient to allow the fire propagation to be understood in terms of the modeling and testing performed in the earlier work. Extensive use of thermocouples was made, since these are relatively inexpensive, very diagnostic, and do not interfere with drum movement. Pressure transducers on the drums were used to a lesser extent due to cost, data value, and the interference of transducer tubing on drum movement. Heat flux transducers were used in sufficient numbers to yield a reasonable mapping of the initiating fire heat fluxes. A few drum calorimeters were used in the tests to provide total heat input to selected locations. Since these drums were filled with water, they could potentially interfere with the outcome of the test. As such, their use was limited in number and were used in locations which were unlikely to interfere with the test outcome while still providing needed data. 


\subsection{TEST FACILITY}

The tests were conducted at Southwest Research institute (SwRI) in San Antonio, Texas [Parker, 1995]. The tests were conducted inside SwRI's Sprinkler/Research Building, which was $12.2 \mathrm{~m}$ (40 ft) wide $\times 18.3 \mathrm{~m}(60 \mathrm{ft})$ long with a $9.1 \mathrm{~m}(30 \mathrm{ft})$ high ceiling. Use of this facility reduced the effects of wind, rain, etc. on the fire tests. Ventilation during the tests consisted of opening the two personnel doors located in the northeast and northwest corners of the building, six louvered windows ( $4 \mathrm{ft} \times 4 \mathrm{ft}$ ) located at ground level along the periphery of the building and two large wall openings located at the top of the north and west walls.

\subsection{INSTRUMENTATION}

During the fire tests, measurements were made for temperatures, pressure rise inside selected drums, and total heat flux of the fire. Specific locations of the instrumentation used in each test is provided in Sections 5.2 and 6.2.

\subsubsection{Temperatures}

Temperatures were measured using bare 24 gauge, type $K$ (chromel-alumel) thermocouples. The thermocouples were positioned at the following locations:

(1) Thermocouples Trees - either two (palletized storage) or three (rack storage) thermocouple trees were used to monitor the temperature at various heights above the floor of the pool fire.

(2) Drum wall temperatures - specific drums had thermocouples placed on the exterior surfaces. The thermocouples were positioned at one-fourth of the drum height up from the bottom and at one-fourth of the drum height down from the top. The thermocouples were welded to the exterior drum wall surface such that the two leads from the thermocouple wire were slightly flattened and welded approximately $3 \mathrm{~mm}(1 / 8 \mathrm{in}$.) apart to the cleaned drum surface. In instances where the thermocouple location was on a drum seam, the thermocouple was moved $25 \mathrm{~mm}(1 \mathrm{in.})$ to the left of the seam. Figure 9 provides a photograph showing the thermocouple placement on a test drum.

(3) Rack temperatures - Temperature measurements were also made at specific locations on the racks, containment pans, and support mesh during the rack storage test. These thermocouples were attached in a similar manner as those on the drum wall surfaces. 
WHC-SD-WM-TRP-246 REV. 0

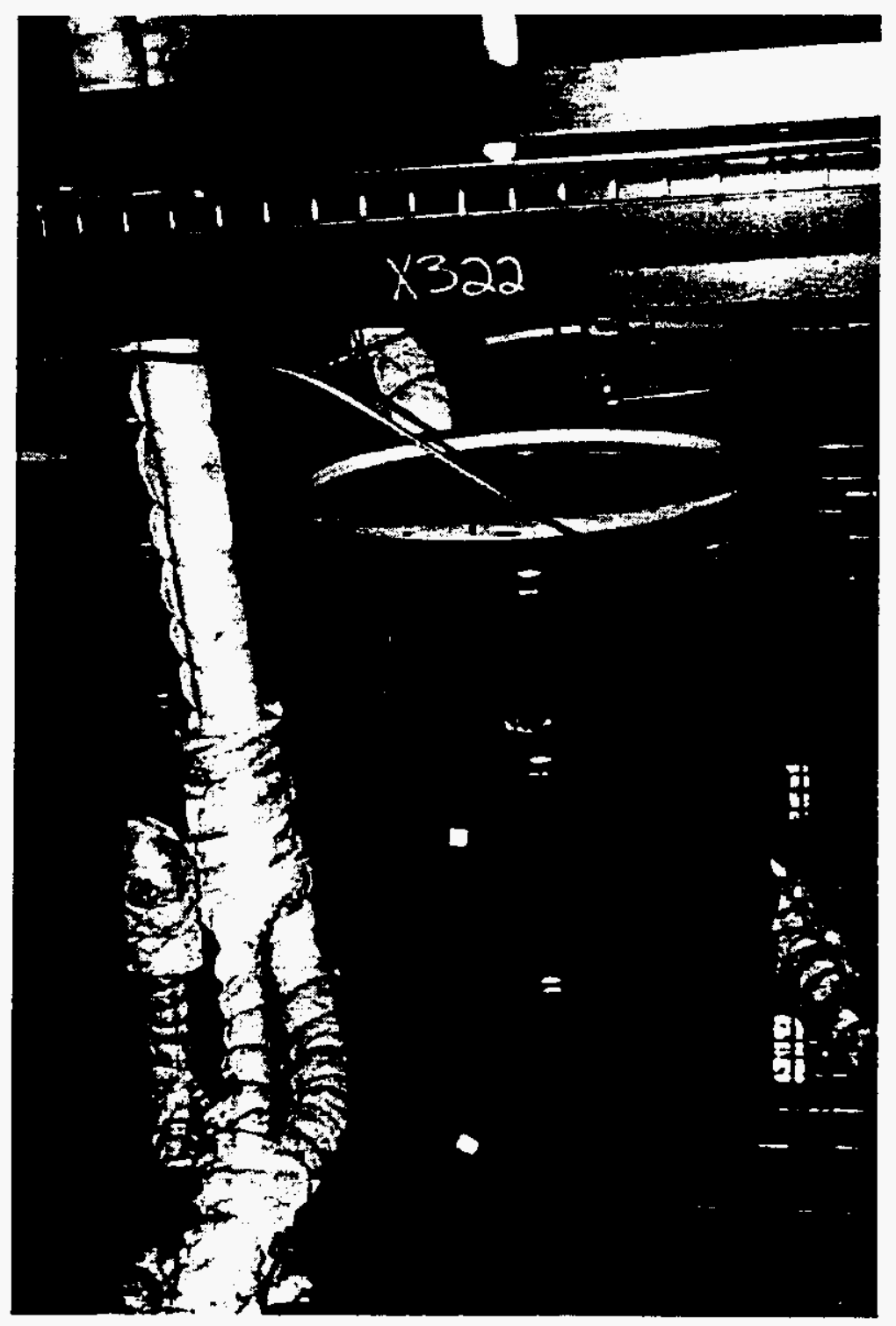

Figure 9. Drum with Temperature and Pressure Instrumentation 
Water temperatures - During the rack storage test, several water filled drums were used (see Section 4.5.1). The temperature of the water was measured at three locations inside the drum via inconel sheathed, type $K$, thermocouples.

\subsubsection{Pressure}

The internal pressure rise of selected drums was measured during the fire tests. The pressures were monitored using $0-350 \mathrm{kPag}$ (0-50 psig) transducers (Setra, Model No. 206). The pressure measurements were taken via a pressure tap placed in the drum wall approximately $25 \mathrm{~mm}$ (1 in.) below the top of the drum. Prior to each test, the pressure transducers were verified using a Beamex Pressure Calibrator, Model No. PG105. Prior to each test, the monitored drums were also checked for leaks prior to the test. The drums were pressurized to a pressure of $35 \mathrm{kPag}(5 \mathrm{psig})$ and held for five minutes to verify tightness of the drum. Figure 9 also shows the placement of the pressure tap on a test drum.

\subsubsection{Heat Flux}

Total heat flux measurements were made at specific locations in order to measure the local heat flux of the fire. The measurement were made using $0-200 \mathrm{~kW} / \mathrm{m}^{2}$ Medtherm total heat flux transducers, Model No. 64-20-5B-19. The heat flux transducers were verified for proper operation prior to each test.

\subsubsection{Mass Loss}

The total mass loss for each drum was measured. Prior to and after each test, each drum was weighed, and its mass loss was calculated.

\subsection{DATA ACQUISITION}

Two different data acquisition systems were used during these tests. The first system was dedicated to the pressure transducers. This system consisted of an IBM-based data acquisition system setup to scan and record the outputs of the pressure transducers every 20 milliseconds. The second system was dedicated to the recording of the thermocouples and the heat flux transducers. This system consisted of seven Helios A-D converters each with its own IBM-based computer. The Helios data acquisition system was capable of reading 20 channels per second, but actually required approximately 1.76 seconds to read and save 20 channels to the computer disk. In instances where more than 20 channels of instrumentation were connected to one Helios, the actual scan time was increased to accommodate the delay in scanning and saving the data.

The instrumentation was broken down into groups according to similar instrumentation or location on the test assembly, and each group was connected to one of seven Helioses. The Helioses were setup to scan as fast as possible, and the raw data were processed to provide temperatures and total heat flux measurements every second. The values for every second were calculated using an Excel spreadsheet program which calculated a straight line 
interpolation between each real data point. Post-test graphical analysis of the raw data and the processed data showed negligible differences between the two data sets.

\subsection{VISUAL DOCUMENTATION}

Documentation of the tests was provided by $35 \mathrm{~mm}$ photographs and video tape taken prior to each test, during each test, and after each test.

\subsection{ADDITIONAL DOCUMENTATION}

Data collection pertaining to drums and their combustible fuel content was recorded on the Drum Data Sheets. There is a data sheet for each drum used in each of the tests. As drums were removed from the test site, they were re-numbered (as needed) with the appropriate drum identification number. The drums were then weighed and inspected. A post-test video was taken of each drum and its contents by HAl personnel.

After the rack storage test, the rack system was assessed and documented, and deflections in the rack structure due to twisting, bending, or thermal expansion were measured with respect to the original position by HAl personnel.

\subsection{DRUMS}

A total of 144 drums were used in each test. The drums were new 17-H, 55-gallon (208-L) UN1A1 drums, painted black. The drums were identified as $1 A 2 / Y 1.5 / 100 / 95 / U S A / U L 3$. The drums were sealed using removable lids with styrenebutadiene sponge rubber gaskets and metal closure rings, rounded-section type. After loading, the closure rings were tightened per WHC specification [Carver, 1993] to $54.2 \mathrm{~J}(40.0 \mathrm{ft}-\mathrm{lb}$ ) torque. The lids were torqued to $54.2 \mathrm{~J}(40.0 \mathrm{ft}-\mathrm{lb})$, the bolt was backed off, and then retightened to $54.2 \mathrm{~J}(40.0 \mathrm{ft}-\mathrm{lb})$. This resulted in a separation between the ends of the closure ring of $12.7 \mathrm{~mm}(0.5 \mathrm{in.}$ ) or less. On all drums, the closure ring was oriented with the bolt on the opposite side of the drum as the seam. The drums, gaskets, and closure rings used in these tests were the same as those used in the earlier work.

In the rack storage test, 140 of the drums represented typical WHC loaded drums [Boothe, 1994] and contained the standard load which was developed in the earlier work [Rhodes, et al. 1995]. Four drums were filled with water to serve as water calorimeters. In the palletized storage test, all of the drums contained the standard load. As each drum was prepared, it was identified with a painted number according to the drum location diagram for that test. All preparation was documented on a Drum Data Sheet. An example of a Drum Data Sheet is provided in Figure 10. 
WHC-SD-WM-TRP-246 REV. 0

\section{Rack Storage Drum Data Sheet}

Drum No.

Description (Check):

Standard load

Instrumented for pressure

Instrumented for temperature

$\mathrm{H}_{2} \mathrm{O}$ filled drum

\begin{tabular}{|c|c|c|c|}
\hline Loading Starting at Bottom & Layer 1 & Layer 2 & Layer 3 \\
\hline 3 pieces of steel & & N/A & N/A \\
\hline $1 / 3$ of the brown paper & & & \\
\hline $1 / 3$ of the $10-$-mil plastic & & & \\
\hline $1 / 3$ of the bond paper & & & \\
\hline $1 / 3$ of the towels & & & \\
\hline $1 / 3$ of the bags & & & \\
\hline $1 / 3$ of the cut paper & & & \\
\hline $1 / 3$ of the fabric & & & \\
\hline $1 / 3$ of the rubber & & & \\
\hline & Go to Layer 2 & Go to Layer 3 & \\
\hline \hline
\end{tabular}

Drum Filled:

Date

Initial

Ring bolt placed opposite drum seam

Date

Initial and in down position

Lid sealed and torqued to $40 \mathrm{ft} / \mathrm{lb}$ :

Date

Initial

Leak tested:

N/A or Pressure (psig)

Pressure (psig)

Date

Time (min) $\frac{0}{5}$

Initial

Pre-test weight (lb)

Target weight $-175.6 \mathrm{lb}$

Post-test weight (lb)

Loss $=$

Figure 10. Drum Data Sheet 


\subsubsection{Water-filled Drums}

Each of the water-filled drums had a Sureflow Corporation air operated diaphragm submersible pump Model No. $1500 \mathrm{~F}$ placed inside the drum to mix the water, thus assuring a uniform temperature profile. The drums were filled with water to within $101.6 \mathrm{~mm}$ (4 in.) of the top and instrumented as described in Section 4.2.1. The actual placement of the drums in the rack storage test is provided in Section 5.0 .

\subsubsection{Standard Load Drums}

Each of the non-water filled drums was loaded with the standard combustible load of $25.9 \mathrm{~kg}(57.0 \mathrm{lb})$ and a noncombustible load of $31.7 \mathrm{~kg}(70 \mathrm{lb})$ for an overall loading of 57.6 $\mathrm{kg}(127.0 \mathrm{lb})$ in each drum. Based on these values and the breakdown of the materials used in the earlier work, the average contents of each drum are given in Table 1.

Table 1. Drum Loading Breakdown

\begin{tabular}{|c|c|c|}
\hline Material & Weight & Percent by Weight \\
\hline \hline Rubber & $2.7 \mathrm{~kg}(6.0 \mathrm{lb})$ & 4.7 percent \\
\hline Plastic & $13.4 \mathrm{~kg}(29.5 \mathrm{lb})$ & 23.2 percent \\
\hline Paper & $6.6 \mathrm{~kg}(14.5 \mathrm{lb})$ & 11.4 percent \\
\hline Cotton & $3.2 \mathrm{~kg}(7.0 \mathrm{lb})$ & 5.5 percent \\
\hline Steel & $31.7 \mathrm{~kg}(70.0 \mathrm{lb})$ & 55.2 percent \\
\hline Total & $57.6 \mathrm{~kg}(127.0 \mathrm{lb})$ & 100 percent \\
\hline
\end{tabular}

The combustible drum contents consisted of the following commercially available items which simulate the actual drum contents:

- Rubber - The rubber material was simulated using neoprene rubber sheets approximately $3.2 \mathrm{~mm}(0.125 \mathrm{in}$.) thick and approximately $152.4 \times 304.8 \mathrm{~mm}$ $(6$ in. $\times 12$ in.).

- Plastic - The plastic commodities consisted of 50 percent by weight 6-mil polyethylene plastic bags and 50 percent by weight 10 -mil polyethylene sheet.

- Paper - The paper consisted of $1 / 3$ by weight brown paper sheet $0.61-\mathrm{m}$ (24in.) wide rolls, $1 / 3$ by weight of $215.9 \times 279.4-\mathrm{mm}(8.5 \times 11$-in.) bond paper, and $1 / 3$ by weight cut-up $108.0 \times 139.7-\mathrm{mm}(4.25 \times 5.5-\mathrm{in}$.$) bond paper$ (simulating filter paper).

- Cotton - The cotton consisted of 57 percent by weight cotton fabric and 43 percent by weight cotton towels. 
Table 2 provides a breakdown of the combustible contents for the standard combustible load, and Table 3 provides the procedure for its loading into the drums. The loading procedure required that materials such as the brown paper, the plastic sheet, and the cotton fabric be unfolded when placed into the drums. This combustible loading required compression as it was placed into the drums, so that the entire load would be placed in the drum.

Table 2. Standard Combustible Loading

\begin{tabular}{|c|c|}
\hline \multicolumn{2}{|c|}{ Combustible Contents } \\
\hline Material & Standard Loading \\
\hline Plastic bags & $6.7 \mathrm{~kg}(14.8 \mathrm{lb})$ \\
\hline 10 -mil plastic sheet & $6.7 \mathrm{~kg}(14.8 \mathrm{lb})$ \\
\hline Rubber, $15 \times 30 \mathrm{~cm}(6 \times 12 \mathrm{in.})$ & $2.7 \mathrm{~kg}(6.0 \mathrm{lb})$ \\
\hline Brown paper & $2.2 \mathrm{~kg}(4.8 \mathrm{lb})$ \\
\hline Bond paper, $22 \times 28 \mathrm{~cm}(8.5 \times 11 \mathrm{in.})$ & $2.2 \mathrm{~kg}(4.8 \mathrm{lb})$ \\
\hline Cut paper & $2.2 \mathrm{~kg}(4.8 \mathrm{lb})$ \\
\hline Cotton towels & $1.4 \mathrm{~kg}(3.0 \mathrm{lb})$ \\
\hline Cotton fabric & $1.8 \mathrm{~kg}(4.0 \mathrm{lb})$ \\
\hline Total & $25.9 \mathrm{~kg}(57.0 \mathrm{lb})$ \\
\hline
\end{tabular}


Table 3. Standard Loading - Into Drum

\begin{tabular}{|c|c|}
\hline Order Starting at the Bottom & Material \\
\hline 1 & $1 / 3$ of the brown paper \\
\hline 2 & $1 / 3$ of the 10 -mil plastic \\
\hline 3 & $1 / 3$ of the bond paper \\
\hline 4 & $1 / 3$ of the towels \\
\hline 5 & $1 / 3$ of the bags \\
\hline 6 & $1 / 3$ of the cut paper \\
\hline 7 & $1 / 3$ of the fabric \\
\hline 8 & $1 / 3$ of the rubber \\
\hline 9 & Repeat Nos. $1-8$ \\
\hline 10 & Repeat Nos. $1-8$ \\
\hline
\end{tabular}

In addition to the standard combustible load, the noncombustible contents were composed of $50.8-\mathrm{mm}$ (2-in.) diameter round carbon steel bar stock. Three pieces, each $0.66 \mathrm{~m}$ (26 in.) long, were placed vertically and evenly spaced along the inside wall of the drum. The primary purpose of the steel was to simulate the typical weight of the noncombustible contents.

Each drum was loaded according to the Rack Storage Drum Data Sheets shown in Figure 10 as follows:

(1) Insert three steel bars vertically along the sides of the drums,

(2) Place one-third of the standard load into the drum in the order shown in Table 3.

(3) Place one-third of the standard load into the drum in the order shown in Table 3 , and

(4) Place final one-third of the standard load into the drum in the order shown in Table 3.

Upon completion of the loading, each drum was weighed to the nearest $0.09 \mathrm{~kg} 1 \pm 0.2$ (b) accuracy. The drum ID number and weight was then recorded on the Drum Data Sheet for each drum and all drums were loaded and positioned in the racks. The drums instrumented for internal pressure measurements were leak tested to ensure the integrity of the drum. The drums were pressurized to $34.5 \mathrm{kPa}(5 \mathrm{psig})$ for a period of 5 minutes. After 5 minutes, the internal pressure could not be below $31.0 \mathrm{kPa}$ (4.5 psig). 
WHC-SD-WM-TRP-246 REV. 0

\subsection{TEST PROCEDURES}

In general, the following procedures were followed:

(1) loading, weighing and identification of test drums;

(2) instrumenting test drums as required;

(3) placement of test drums into rack array and development of drum ID versus position diagram;

(4) instrumentation of racks, pans, etc.;

(5) instrumentation and/or connection of pressure transducers, heat flux transducers, and thermocouples;

(6) verification of instrumentation after connection to data acquisition system;

(7) creation of fuel spill;

(8) verification of documentation;

(9) start of test;

(10) extinguishment as required; and

(11) post-test damage assessments.

A Pre-test Check List for each test is included in Appendix $A$ and the Test Procedure Check List is included as Appendix B. All procedures were carried out by SwRI personnel and overseen by HAl test engineers. The test engineer or appointed designee verified and initialize all checklists and data sheets. Prior to the test being conducted, the HAl test supervisor verified that all items have been completed.

\subsection{TEST TERMINATION}

Data collection and video recording were continued after the completion of the 5minute diesel fuel fire during which time the extent of fire propagation was assessed. The test was terminated when the HAl representatives determined that further fire propagation was unlikely and further drum seal failures were improbable or unless a safety hazard was noted. Upon termination of the test, extinguishment of all burning items was performed. 


\subsection{RACK STORAGE TEST}

Figure 11 provides an overall view of the rack storage test arrangement configuration. The test configuration consisted of three racks, each measuring approximately $7.6 \mathrm{~m} \mathrm{(25 \textrm {ft } )}$ long $\times 0.9 \mathrm{~m}$ (34 in.) wide $\times 7.9 \mathrm{~m}(26 \mathrm{ft})$ high, with each rack having six tiers. Racks 2 and 3 (middle and left racks, respectively) were positioned $254 \mathrm{~mm}(10 \mathrm{in}$.) apart and bolted together using rack spacers. Rack 1 (right rack) was free standing and separated from the double rack by a $1.57 \mathrm{~m}(5.17 \mathrm{ft})$ aisle space. Rack 1 was attached to the structural steel of the test facility using $76.2 \times 76.2 \mathrm{~mm}(3 \times 3 \mathrm{in}$.) angle iron welded at the top tier of the rack system, running horizontally to a supporting column.

Figure 12 provides a diagram of the drum placement in each of the racks. Figure 13 provides a plan view of the drum placement along with the drum numbering system that was used to uniquely identify each drum.

The drums in Row 1 were positioned with the bolts facing north, the bolts on the drums in Row 2 faced south, and the bolts on the drums in Row 3 faced north.

Figures 14 and 15 provide photographs of the rack storage array prior to test.

\subsection{RACK SYSTEM}

The rack system was provided by Los Alamos Technical Associates, Inc. (LATA) and installed by a local contractor familiar with the rack design, construction, and erection process. The rack system included containment pans attached to the mesh screen flooring under each drum. Seismic event bars were also installed for drum containment. Figures 16 and 17 provide diagrams showing this arrangement. Figure 18 provides a diagram of side supports. The rack system was anchored to the concrete floor of the test facility using concrete anchors. The single rack was also secured to the structural steel of the building. Each beam pair of the rack system had a rated capacity of $2,272.3 \mathrm{~kg}(6,000 \mathrm{lb})$, and each upright had a rated capacity of $14,515.1 \mathrm{~kg}(32,000 \mathrm{lb})$. The containment pans measured 736.6 wide $x 711.2$ long and $50.8 \mathrm{~mm}$ deep ( 29 wide $x 28$ long and $2 \mathrm{in}$. deep) and were suspended by "S" hooks from the mesh screen. There was a $152.4 \mathrm{~mm}$ ( $6 \mathrm{in}$.) air space between adjacent pans.

\subsection{TEST INSTRUMENTATION}

Figure 19 provides a diagram showing an overall view of the instrumentation layout used in this test. Figures $20-22$ provide diagrams showing the thermocouple layout for the tree thermocouples. Figures 23 - 25 provide diagrams showing the thermocouple layout for the thermocouples mounted on the rack system, containment pans, and mesh. Figures 26 31 provide diagrams showing the instrumentation layout for each tier of the test array. 


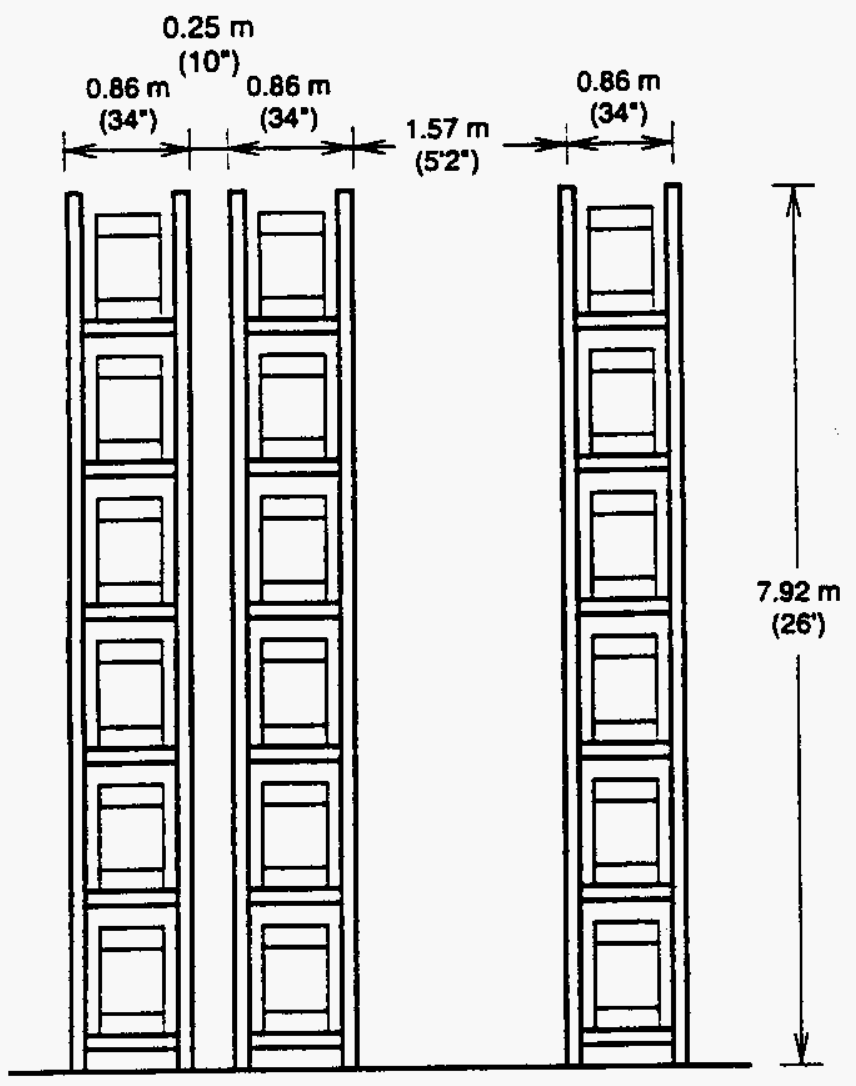

Side View

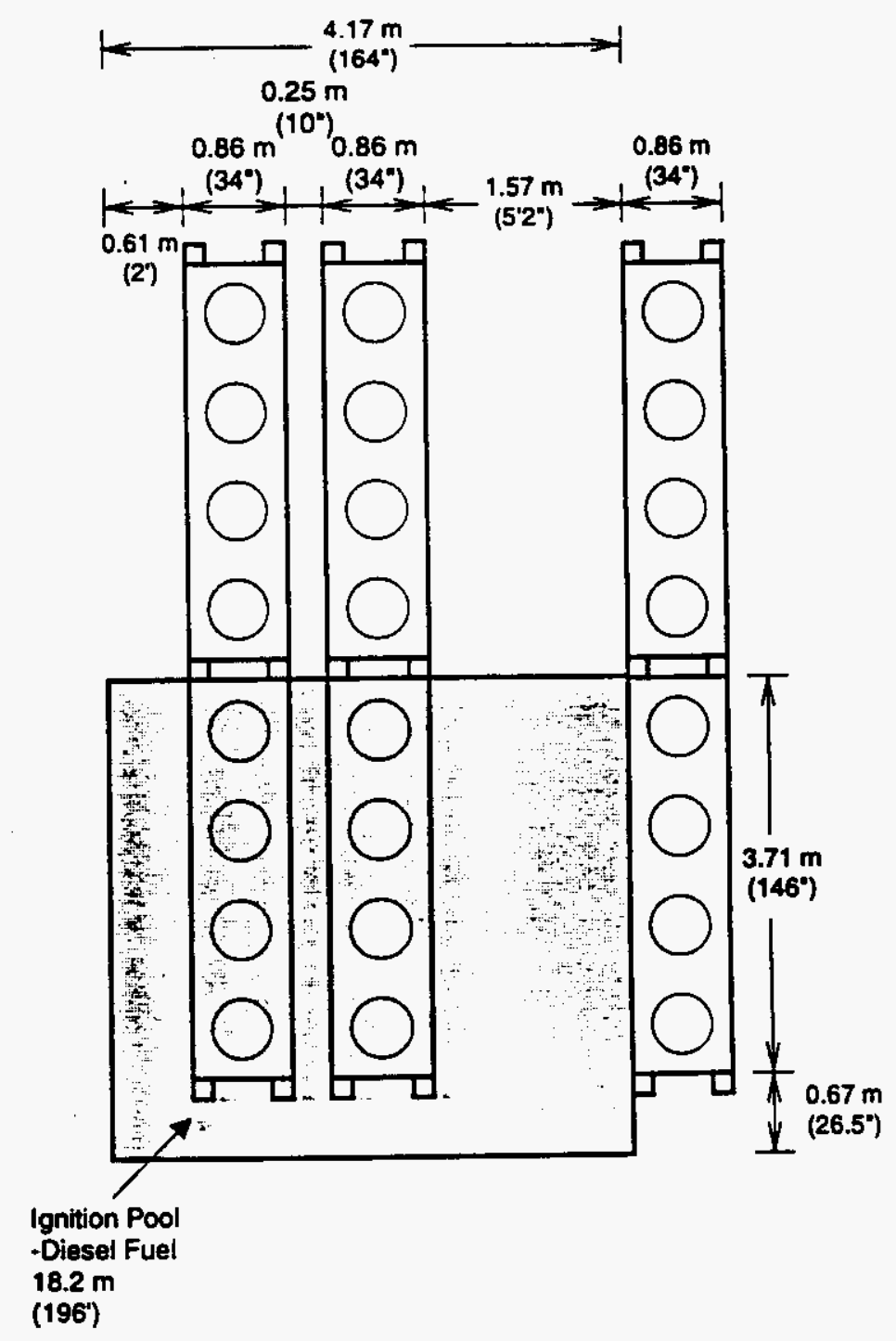

Plan View

Figure 11. Overall Rack Storage Experimental Setup 


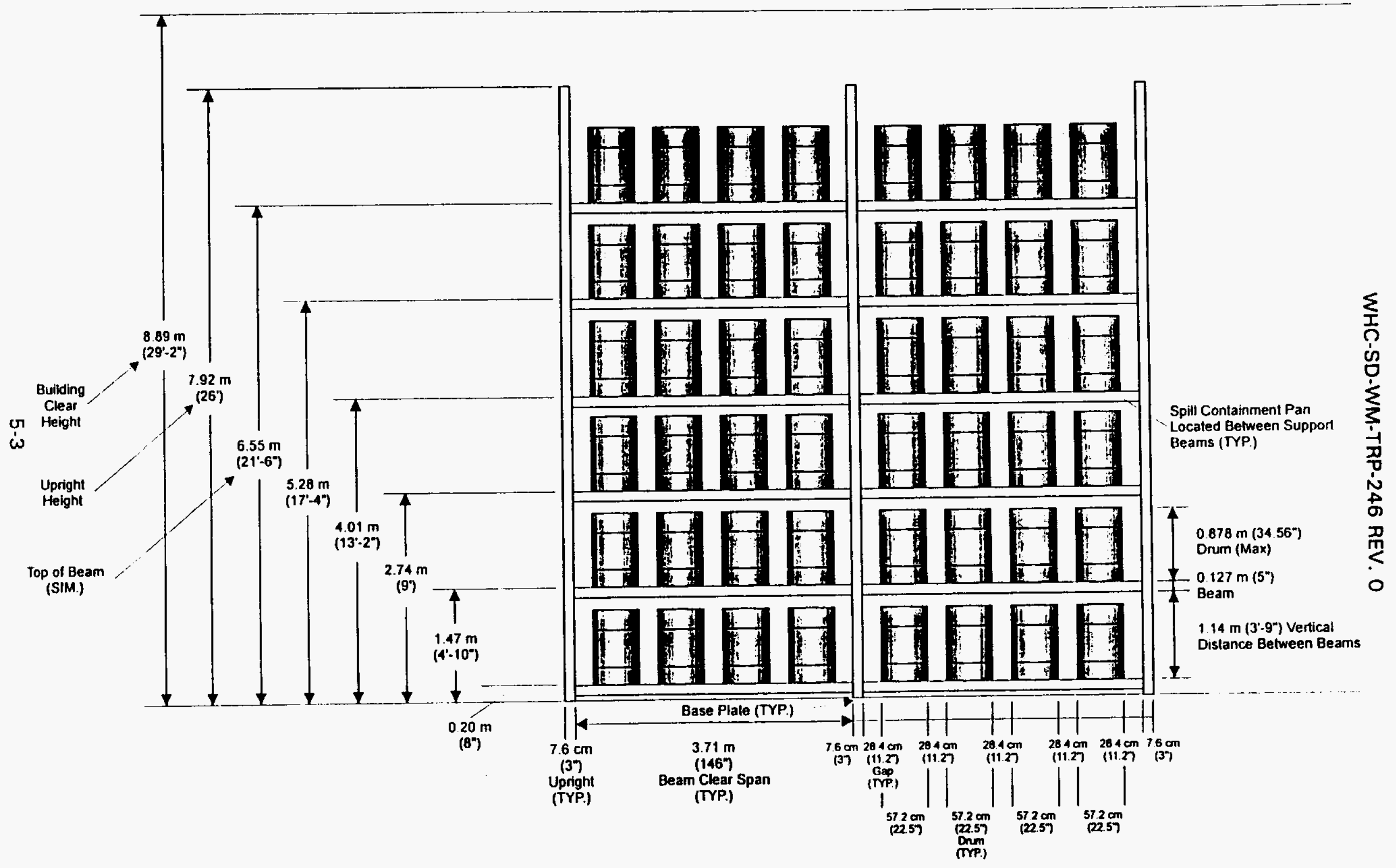

Figure 12. Face View of Rack Array 
Tier 1

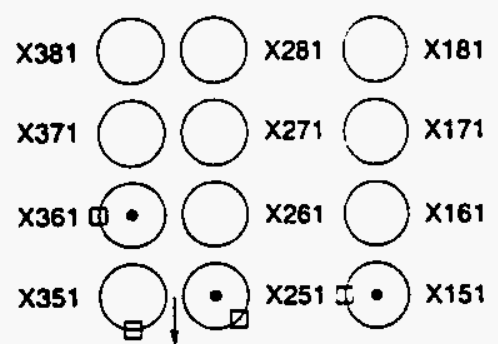

x341 $\bigcirc \times 241 \bigcirc \times 141$

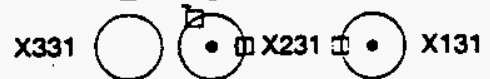
$\times 321$ 中. $\mathrm{H} 2 \mathrm{O} \times 221 \bigcirc \times 121$

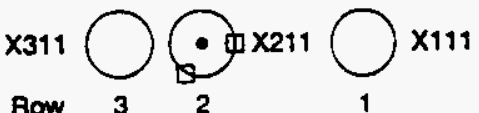
x382 $\bigcirc \times 282 \bigcirc \times 182$ $\times 372 \bigcirc \times 272 \bigcirc \times 172$ x362 $\bigcirc \times 262 \bigcirc \times 162$ x352 $\bigcirc \times 252 \curvearrowleft \times 152$

Tier 2
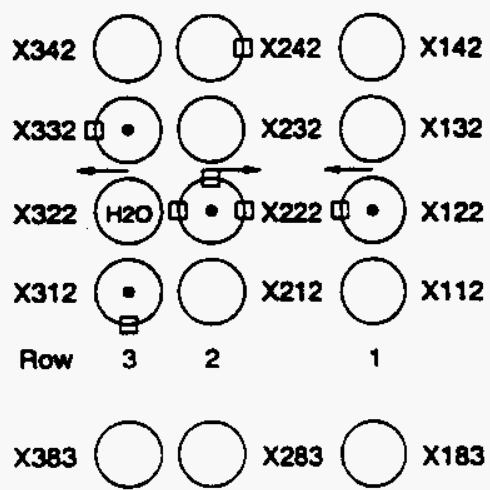

$\times 373 \bigcirc \times 273 \bigcirc \times 173$
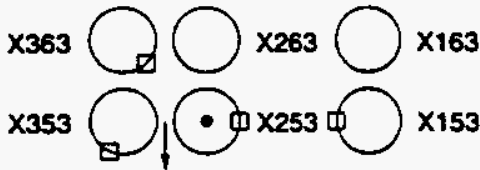

Tier 3

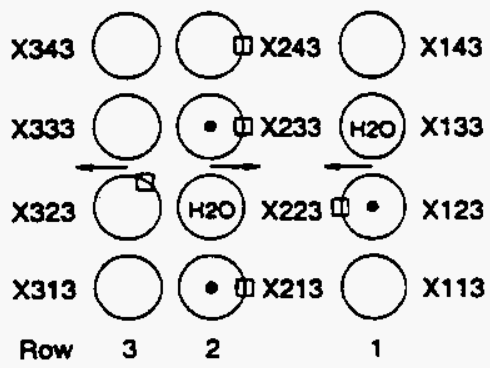

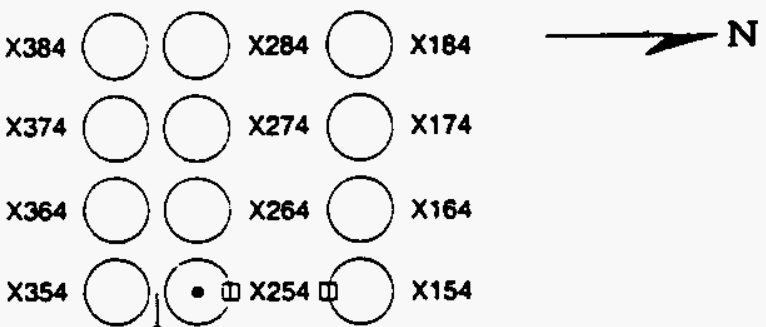

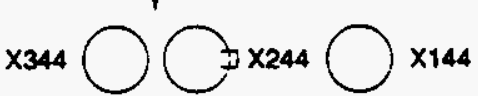

Tier 4

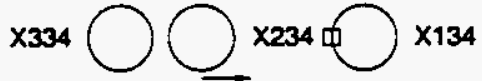

x324中 $\bigcirc \times 1224 \bigcirc \times 124$

$\times 314 \bigcirc \times 214 \bigcirc \times 114$

x385 $\bigcirc \times 285 \bigcirc \times 185$

x375 $\bigcirc \times 275 \bigcirc \times 175$

x365 $\bigcirc \times 265 \bigcirc \times 165$

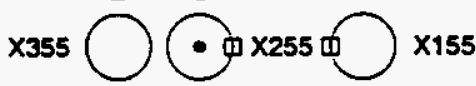

x345中 $\bigcirc 245 \bigcirc \times 145$

x335中 $\bigcirc 235 \bigcirc \times 135$

$\times 325 \bigcirc \times 225$ 中 125

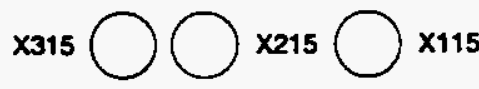

Row $3 \quad 2 \quad 1$

$\times 386 \bigcirc \times 286 \bigcirc \times 186$

$\times 376 \bigcirc \times 276 \bigcirc \times 176$

$\times 366 \bigcirc \times 266 \bigcirc \times 166$

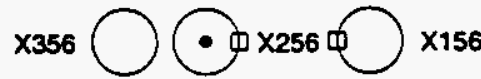

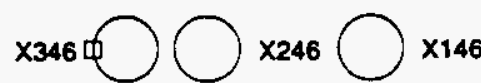

Tier 6

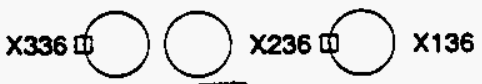

x326 $\bigcirc \times 226 \bigcirc \times 126$

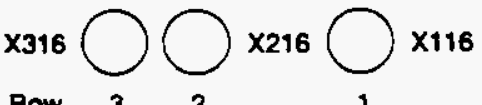

Tier 5

Note: Drum number indicates Test-Row-Bay-Tier

Figure 13. Rack Storage Drum Location Diagram 
WHC-SD-WM-TRP-246 REV. 0

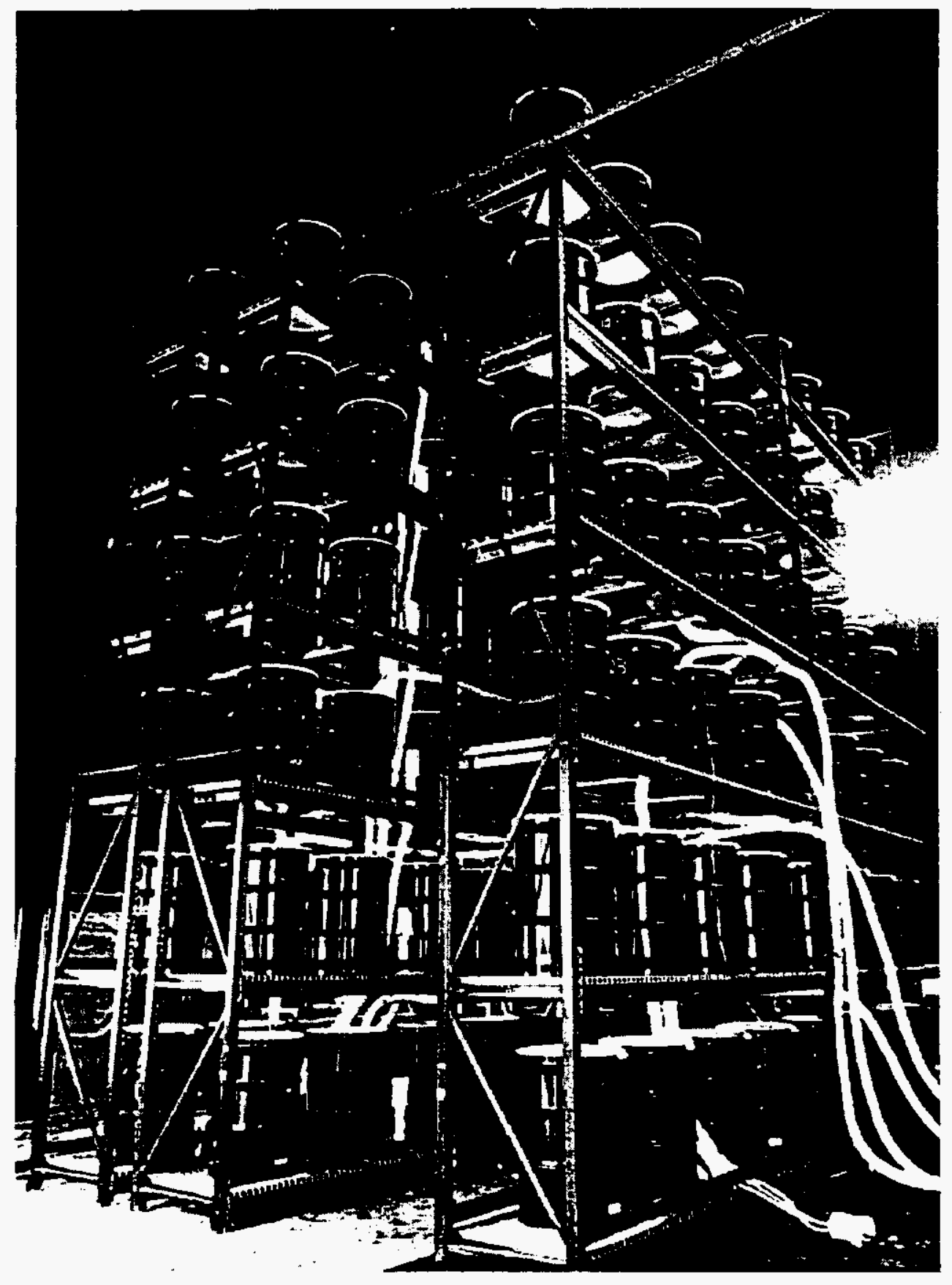

Figure 14. Pre-test Rack Storage Array 
WHC-SD-WM-TRP-246 REV. 0

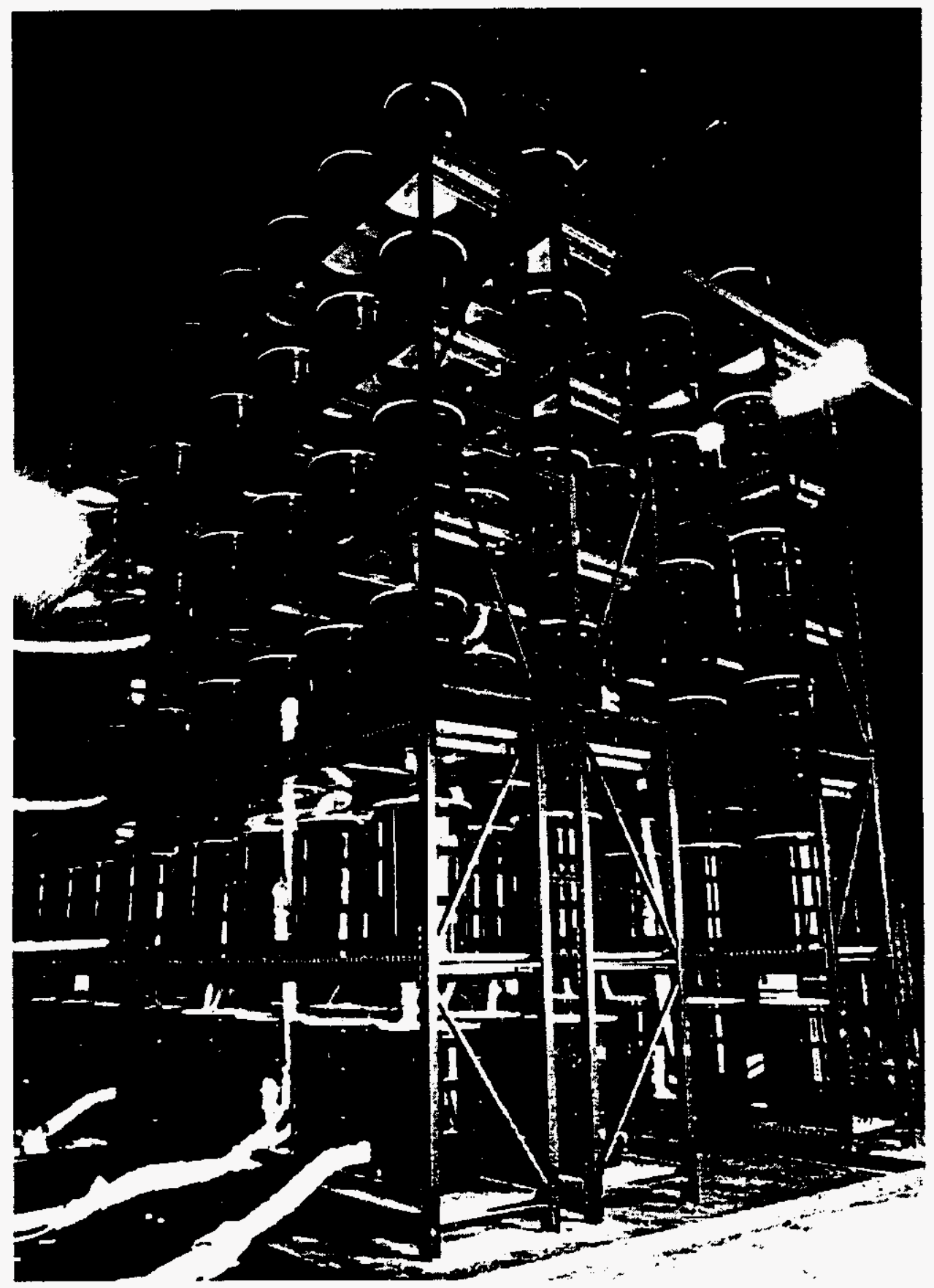

Figure 15. Pre-test Rack Storage Array, View Looking Northwest 


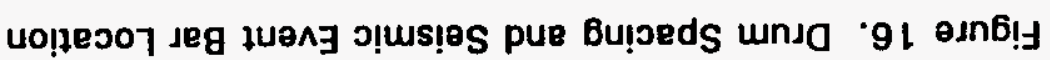

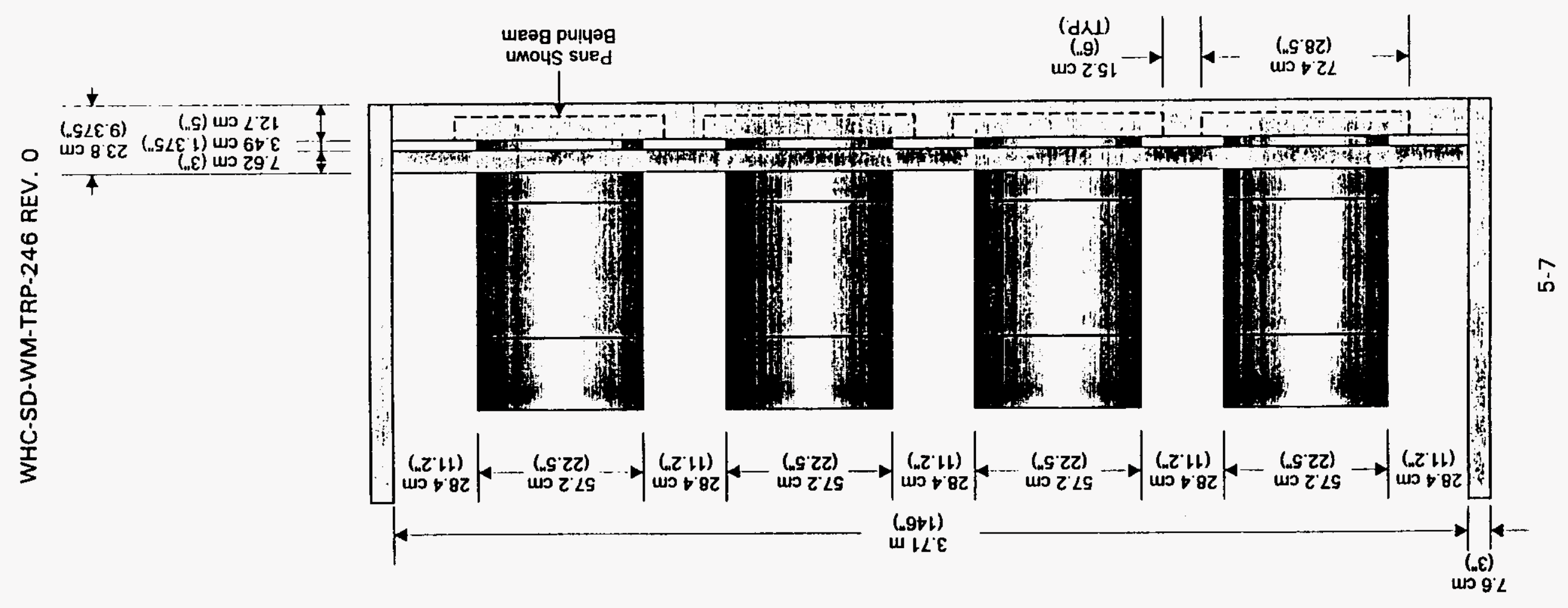



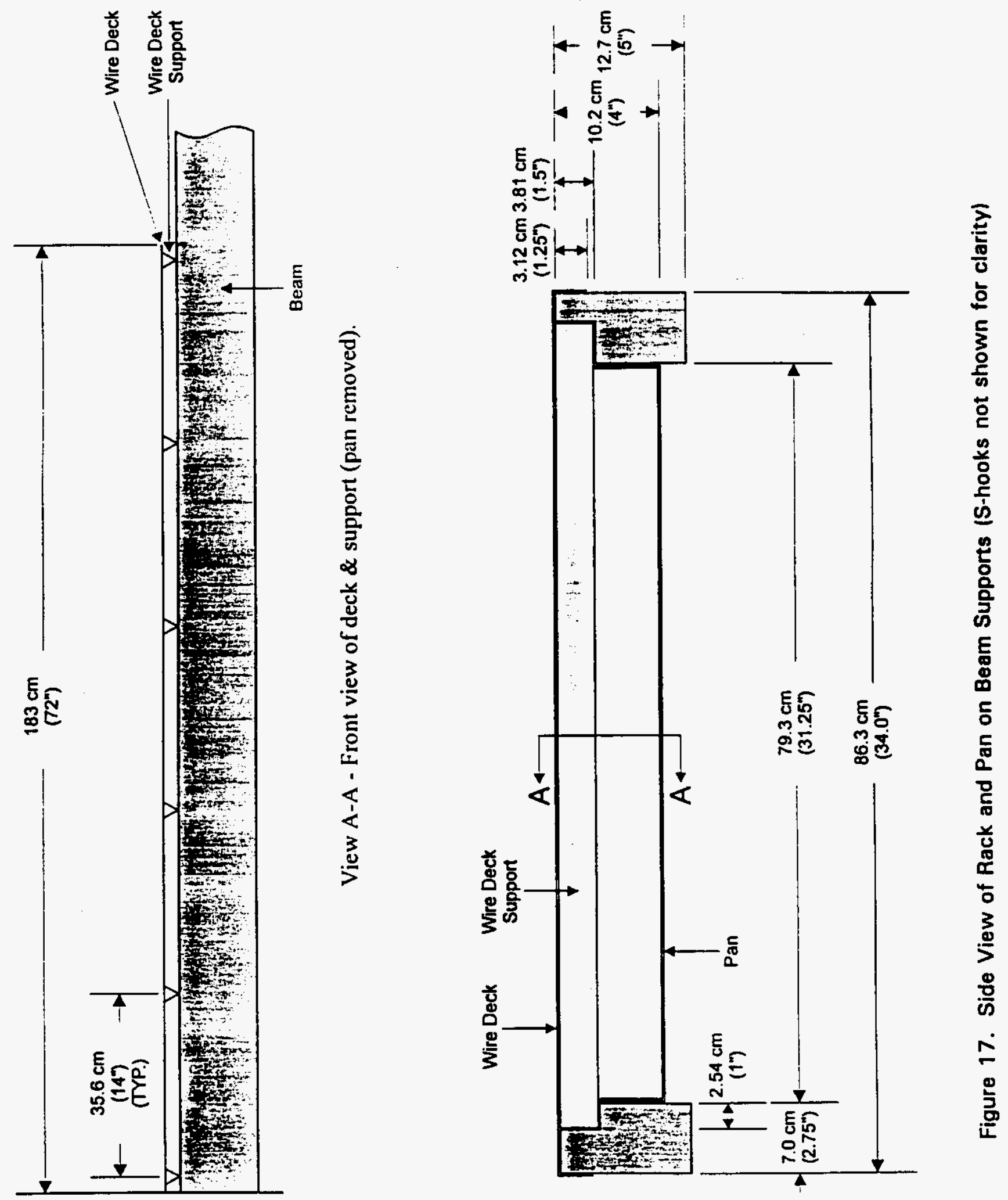
WHC-SD-WM-TRP-246 REV. 0

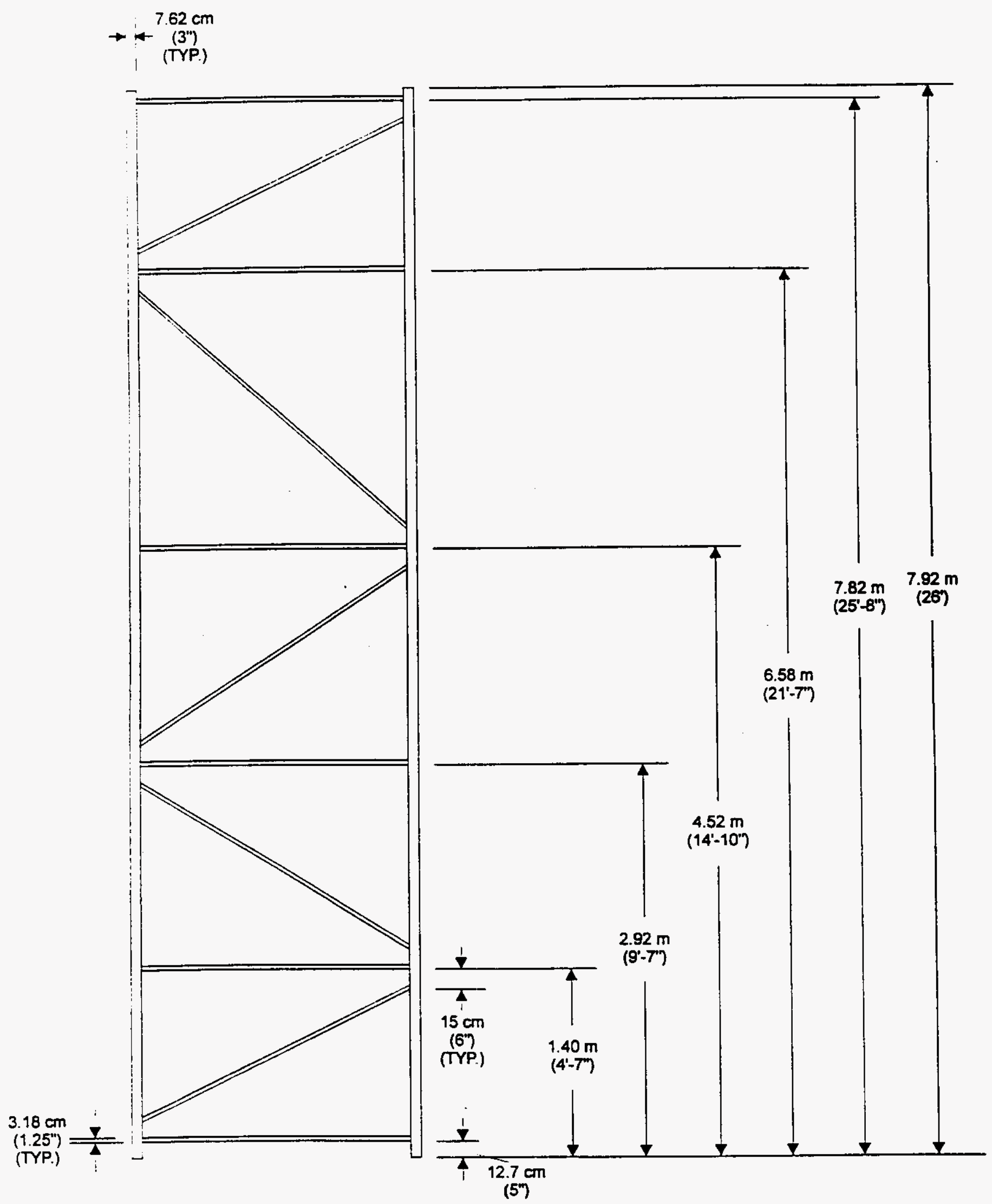

Figure 18. End View of a Rack 
WHC-SD-WM-TRP-246 REV. 0
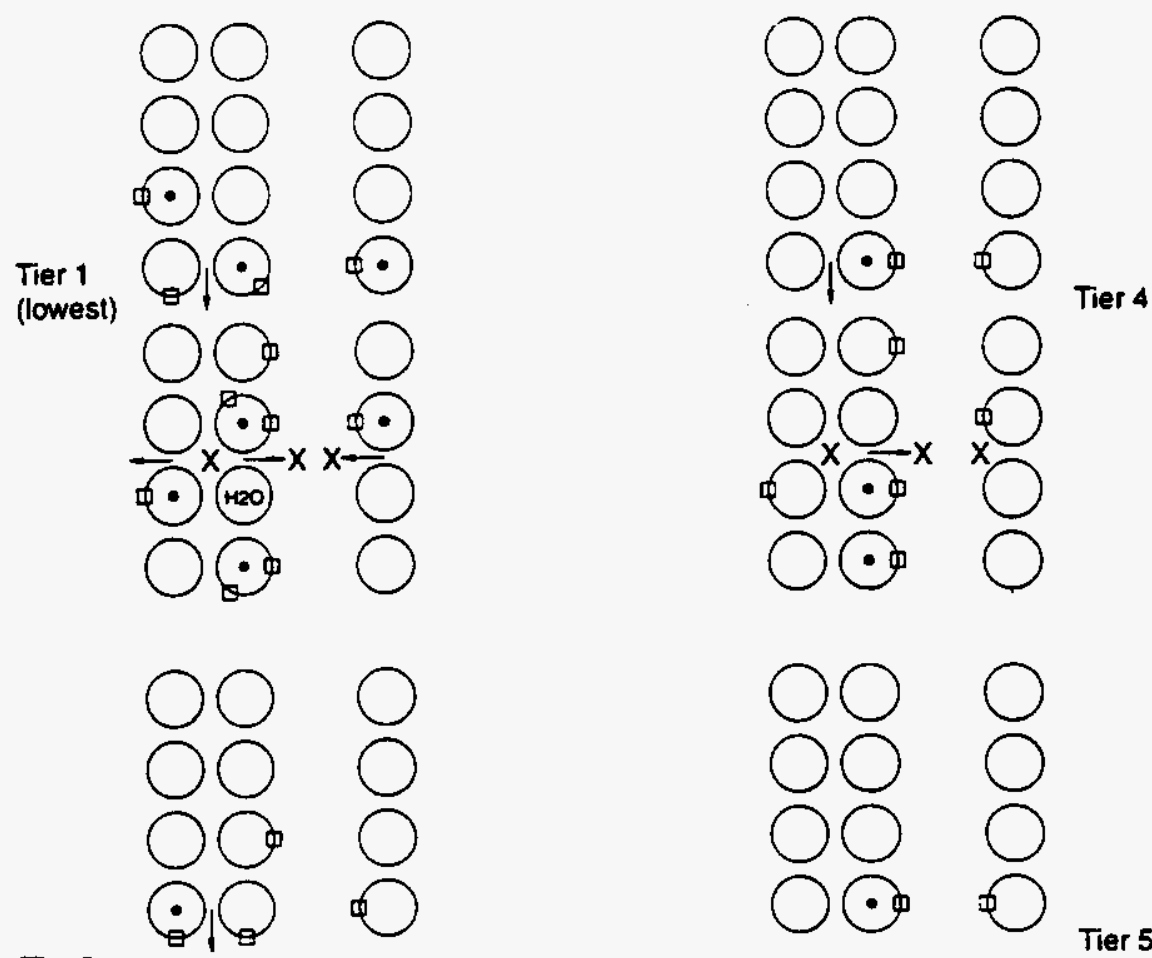

Tier 2
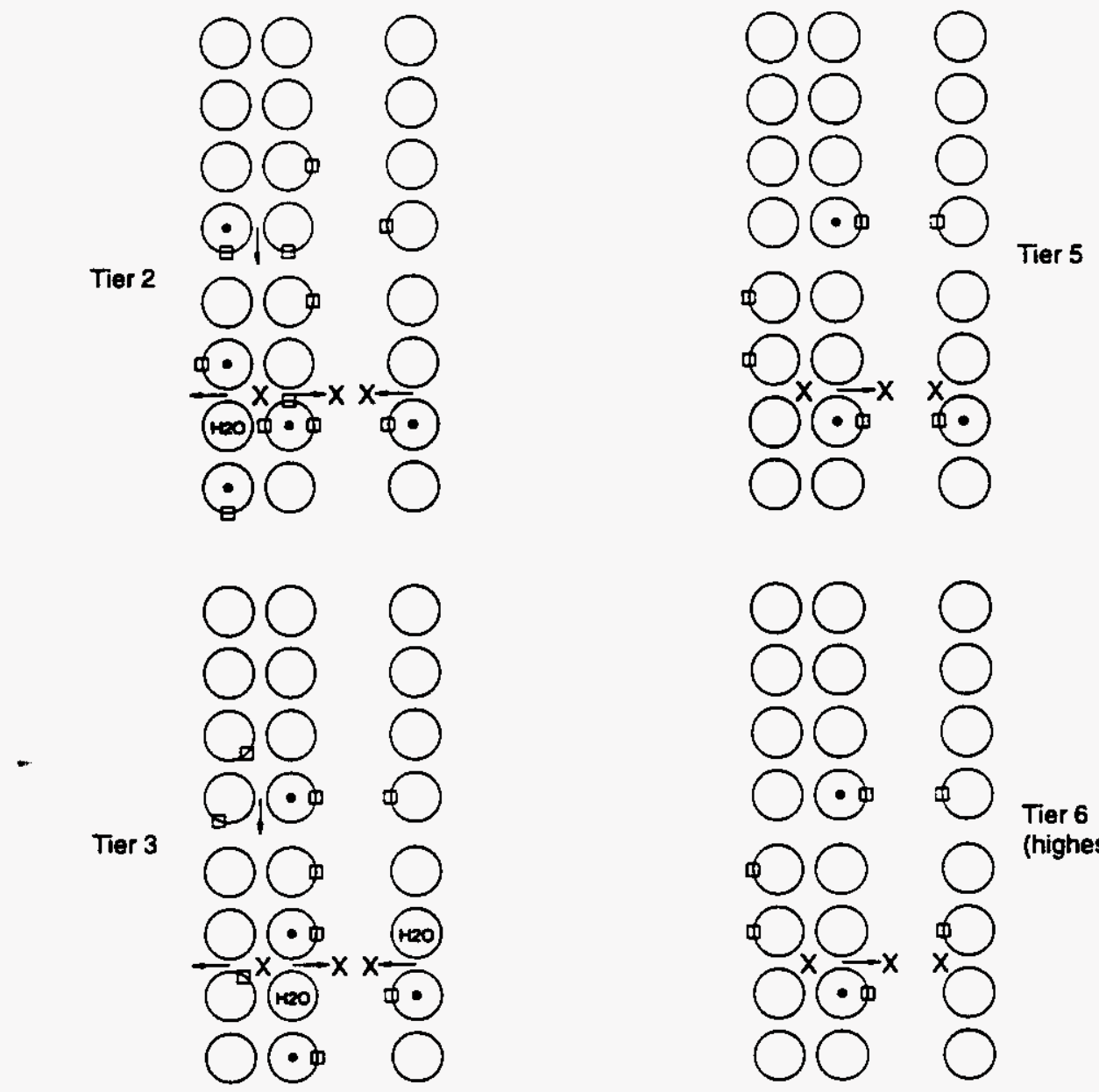

(highest)

Notes:

Except As Noted, All Drums Standard Load - w/Steel

- = Pressure $=24$ Locations

$\square=$ Drum Surface -50 Locations $=100 \mathrm{TC}$ 's

$\mathrm{H} 2 \mathrm{O}=$ Temps - In $\mathrm{H} 2 \mathrm{O}$ (3/Drum) $=12$

Temps On Racksi/Pans $=41$

$X=$ Temp in Fire $=$ TC Trees - Each 13 TC's X $3=39$ TC's Total

$\rightarrow=$ Heat Flux Gauges $=16$ Total

Total - 192 TC's

Figure 19. Rack Storage Experimental Setup and Instrumentation Layout 


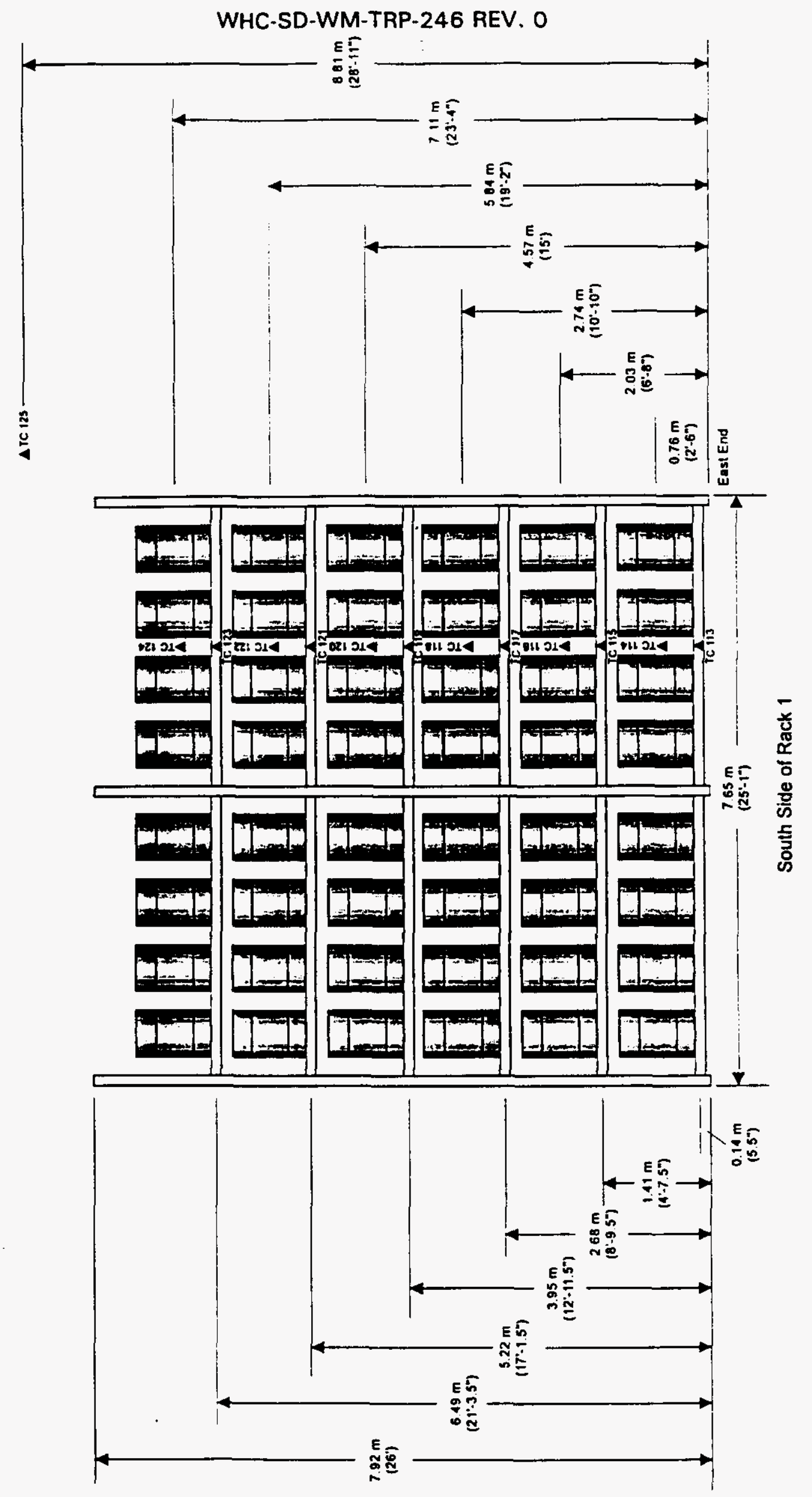


WHC-SD-WM-TRP-246 REV. 0

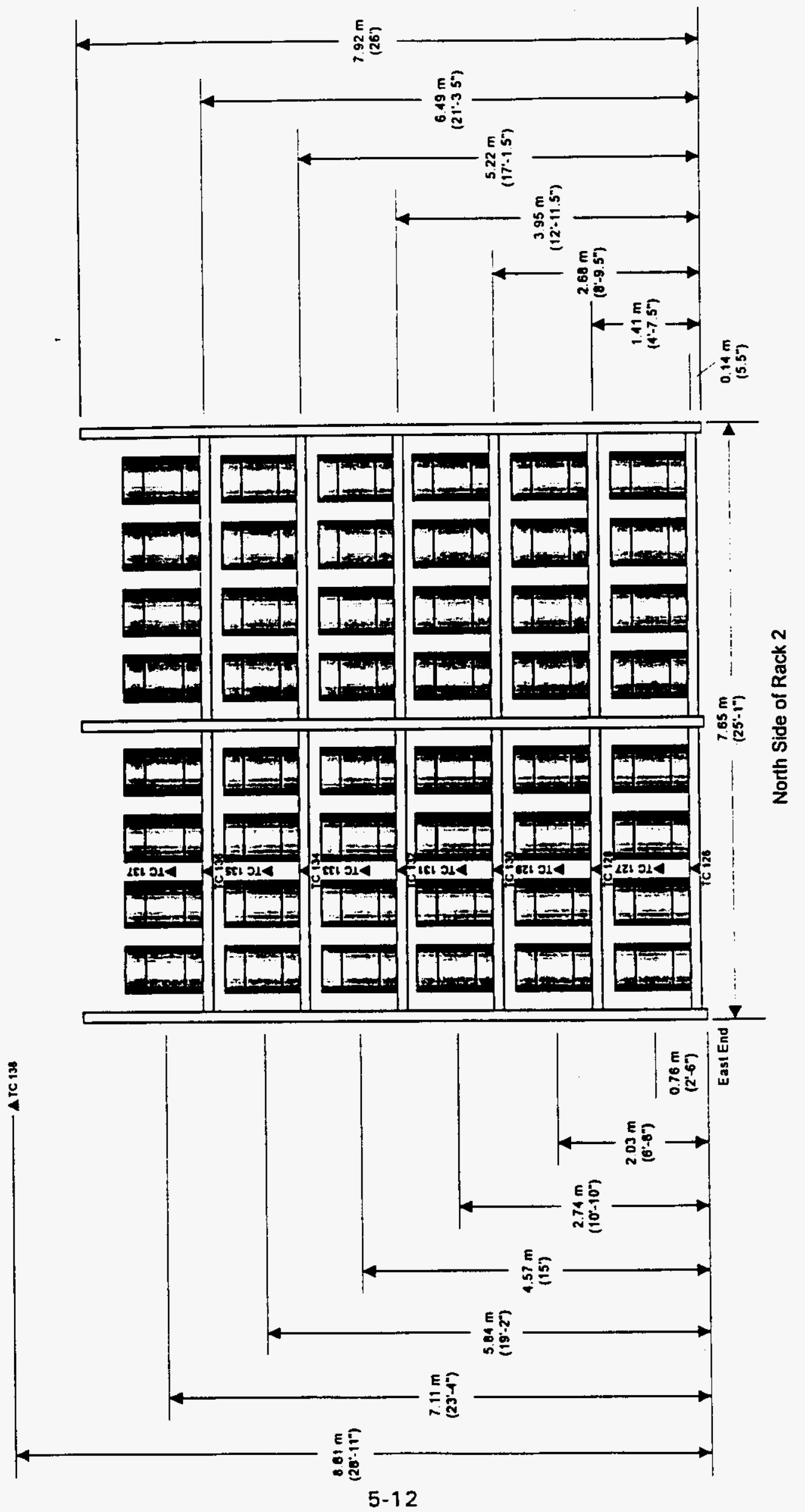


WHC-SD-WM-TRP-246 REV. 0
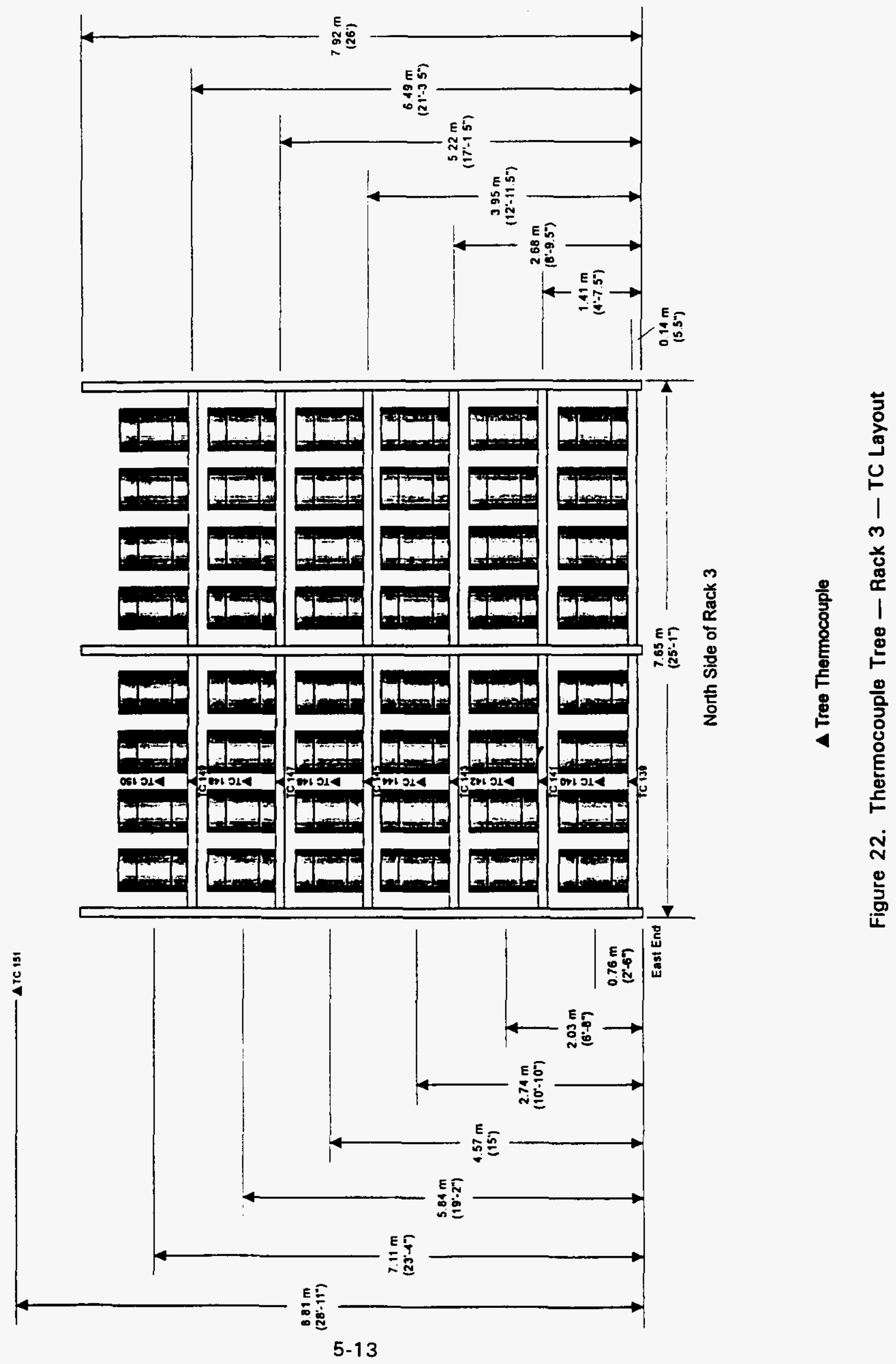


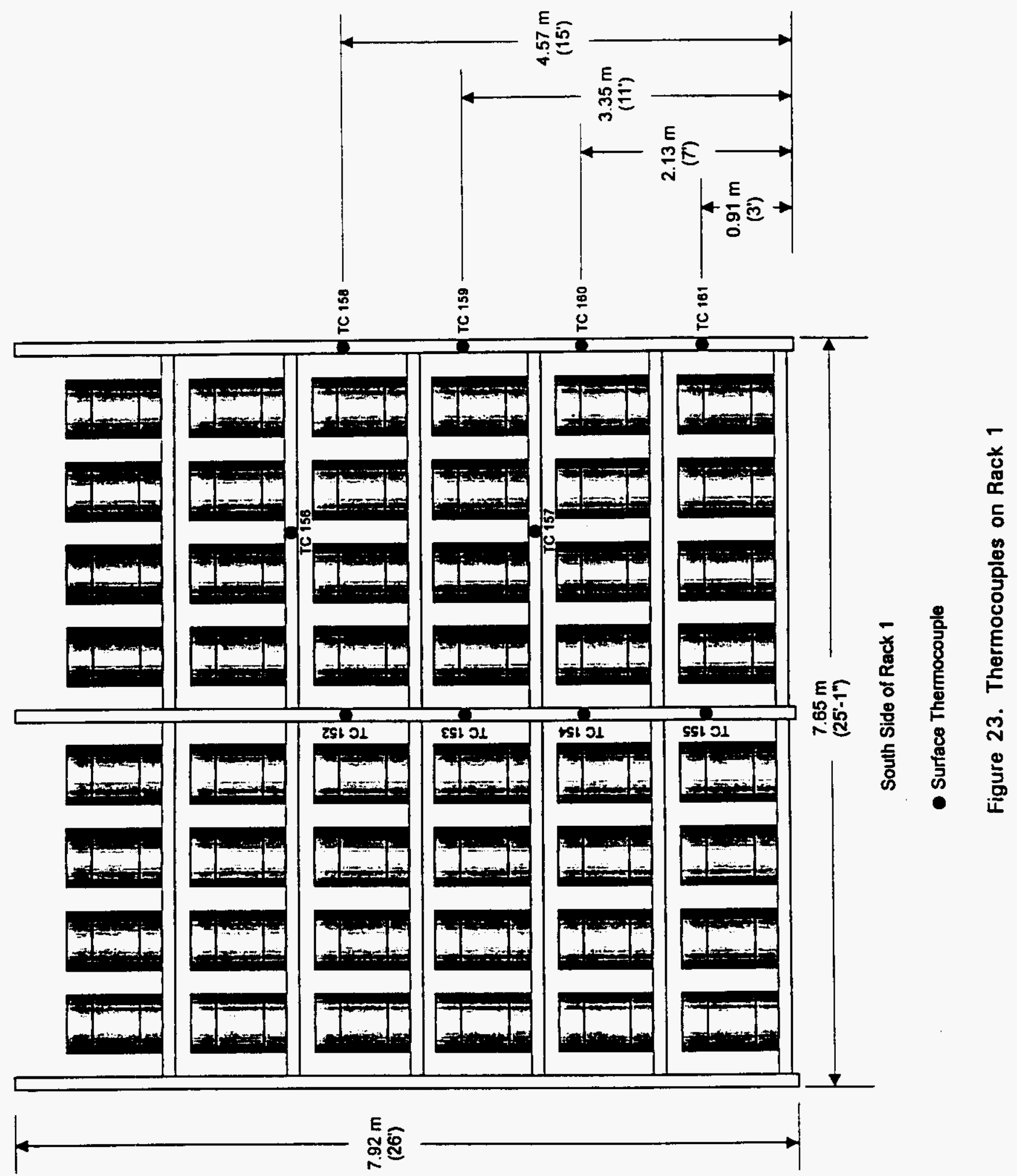




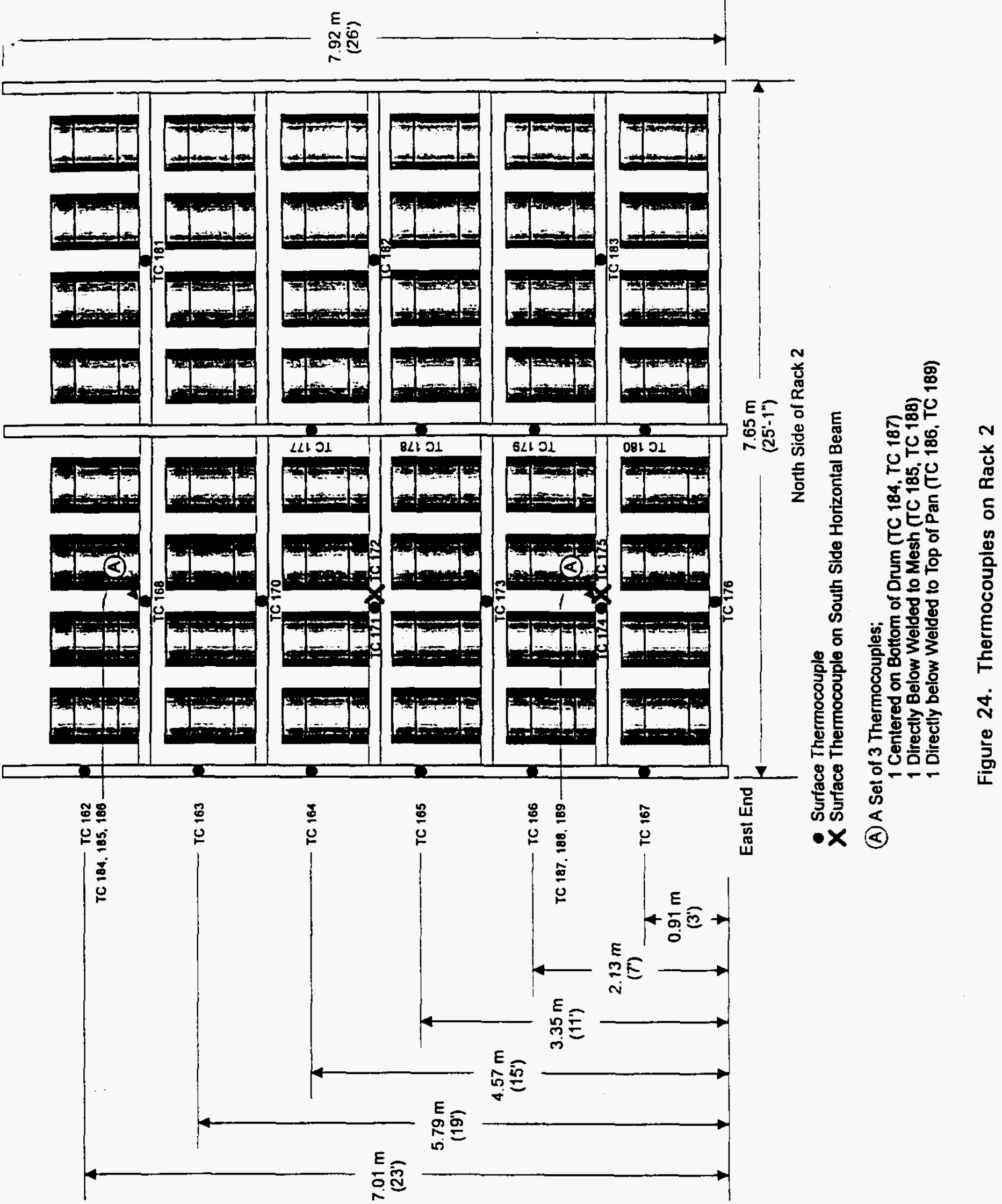




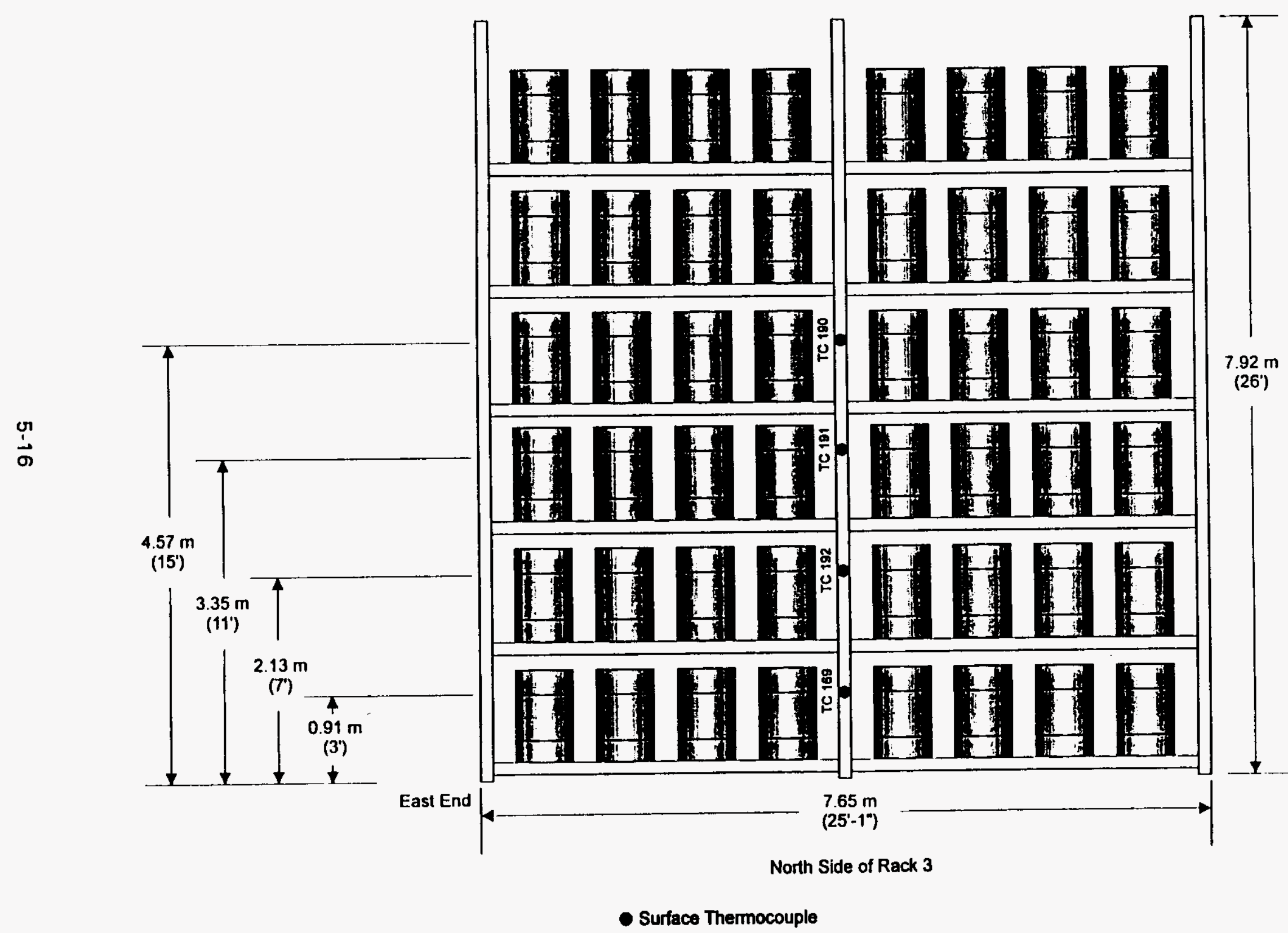

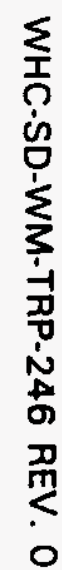

Figure 25. Thermocouples on Rack 3 

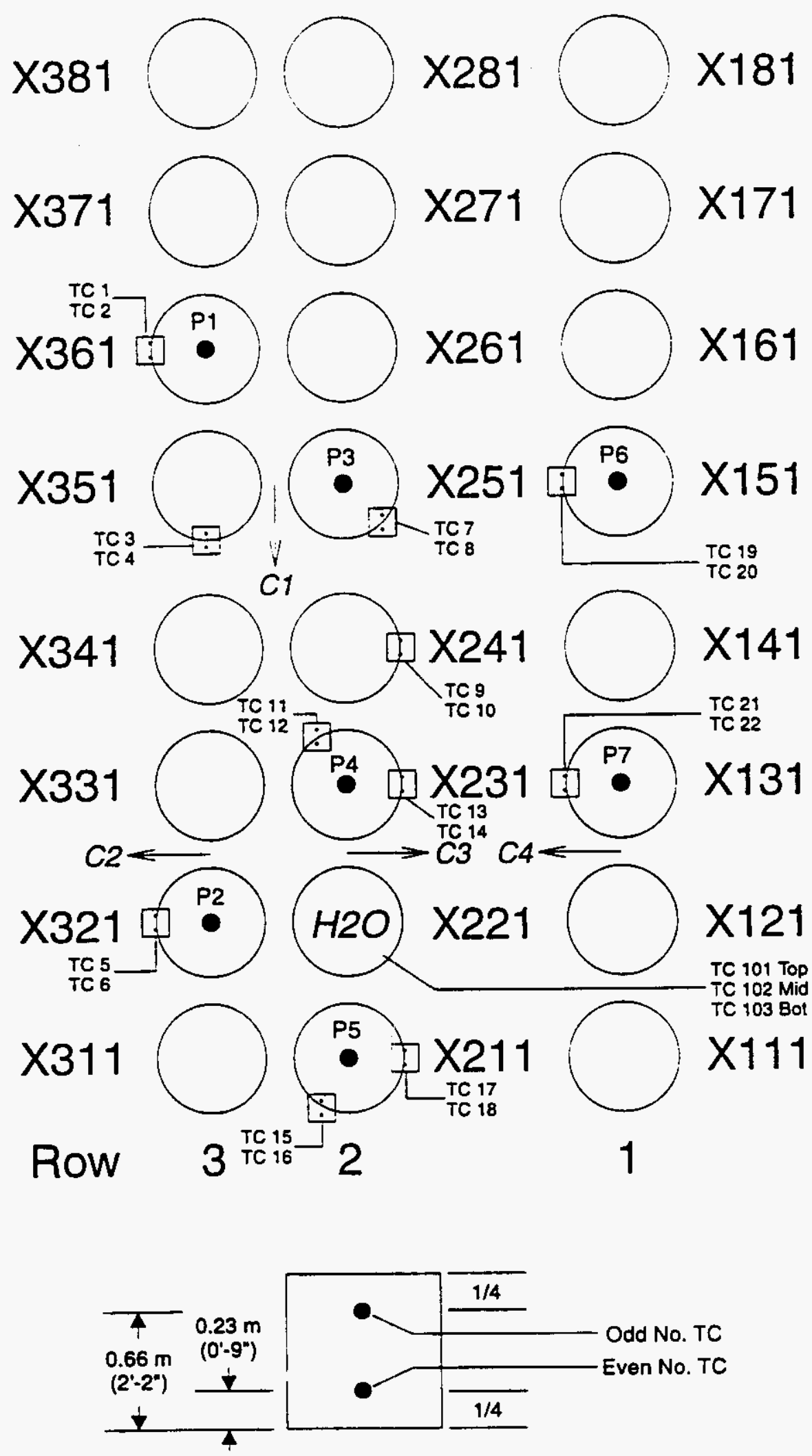

Figure 26. Instrumentation Layout - Tier 1 
WHC-SD-WM-TRP-246 REV. 0
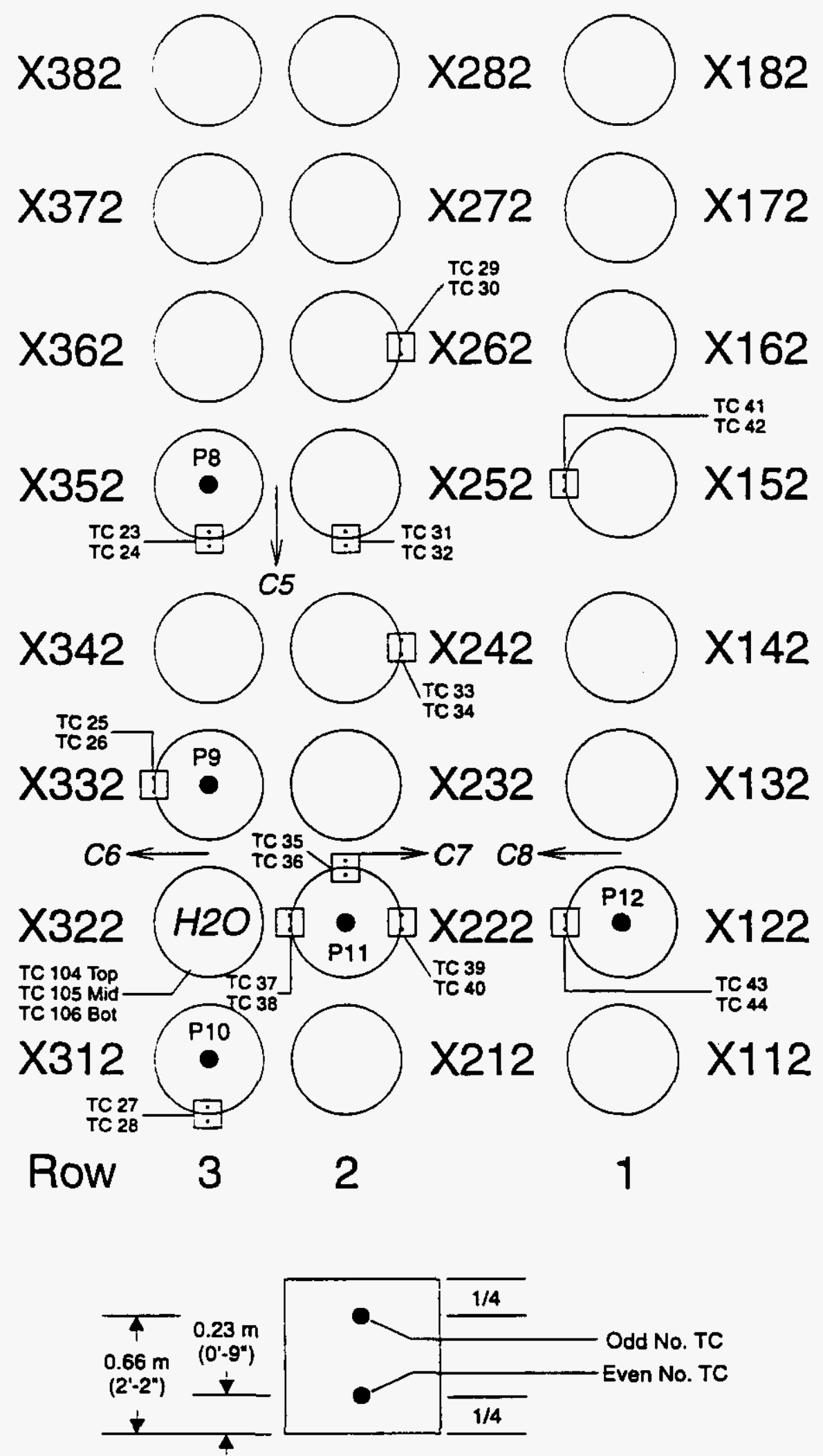

Figure 27. Instrumentation Layout - Tier 2 

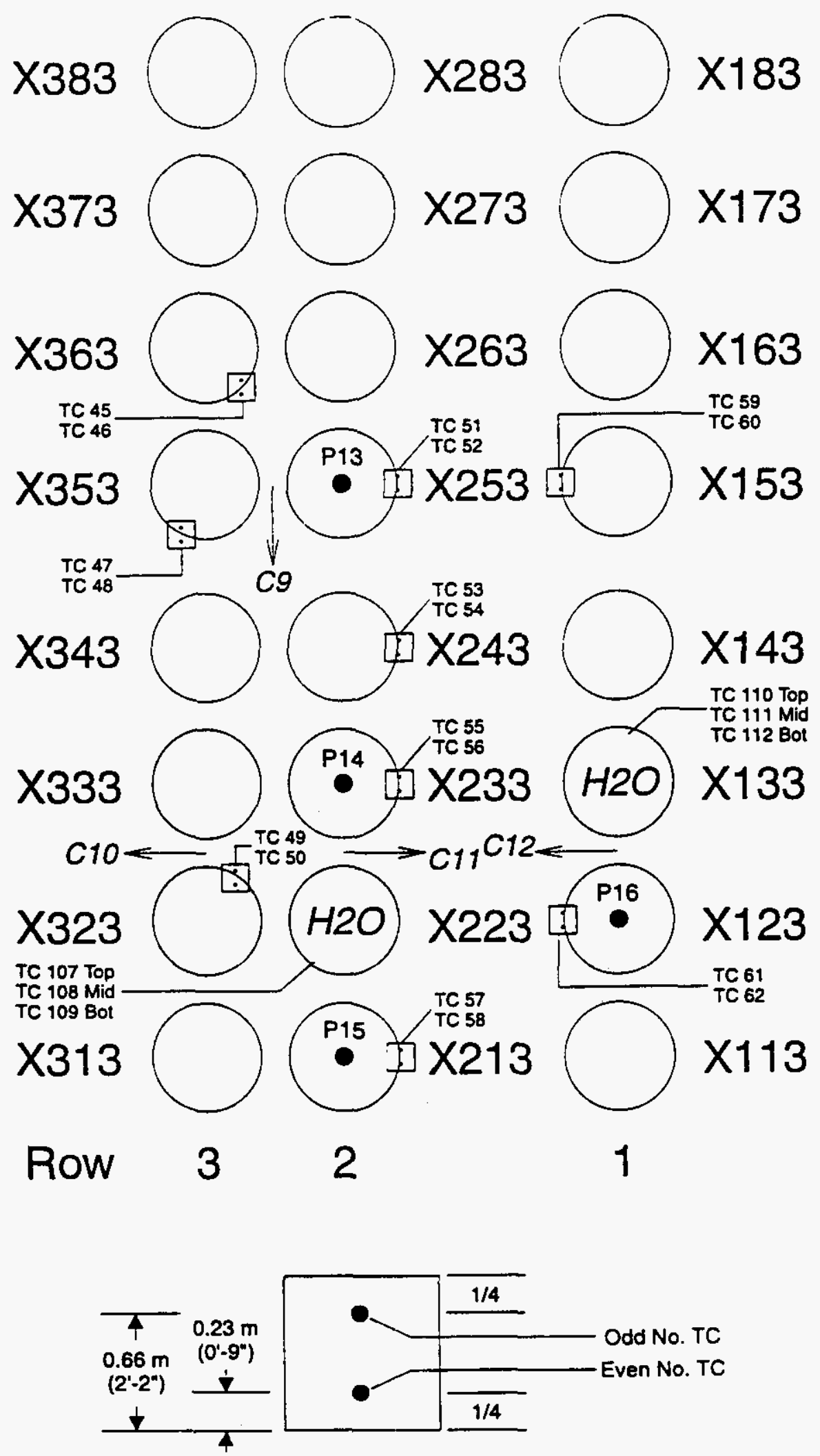

Figure 28. Instrumentation Layout - Tier 3 
WHC-SD-WM-TRP-246 REV. 0
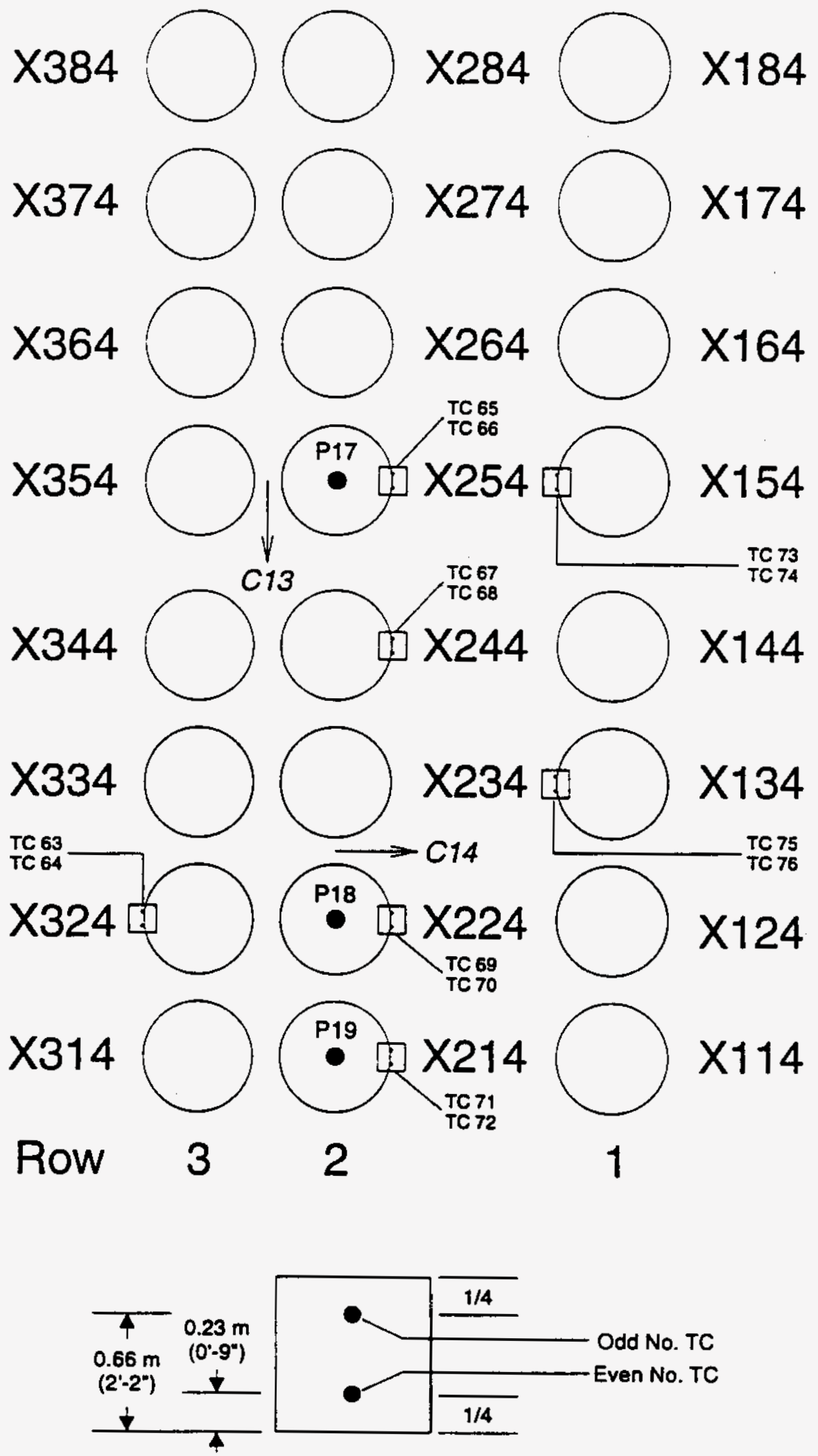

Figure 29. Instrumentation Layout - Tier 4 
WHC-SD-WM-TRP-246 REV. 0
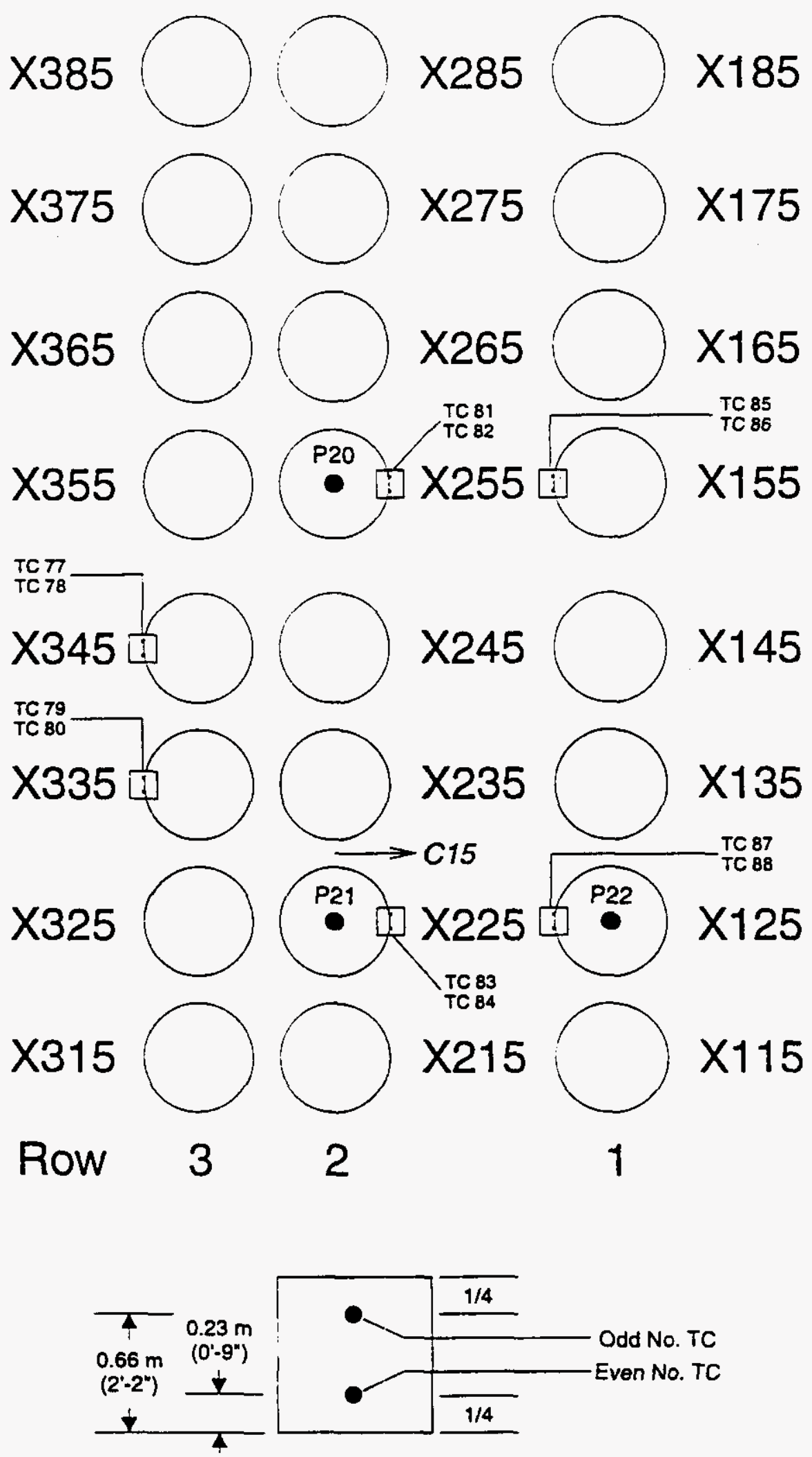

Figure 30. Instrumentation Layout - Tier 5 
WHC-SD-WM-TRP-246 REV. 0
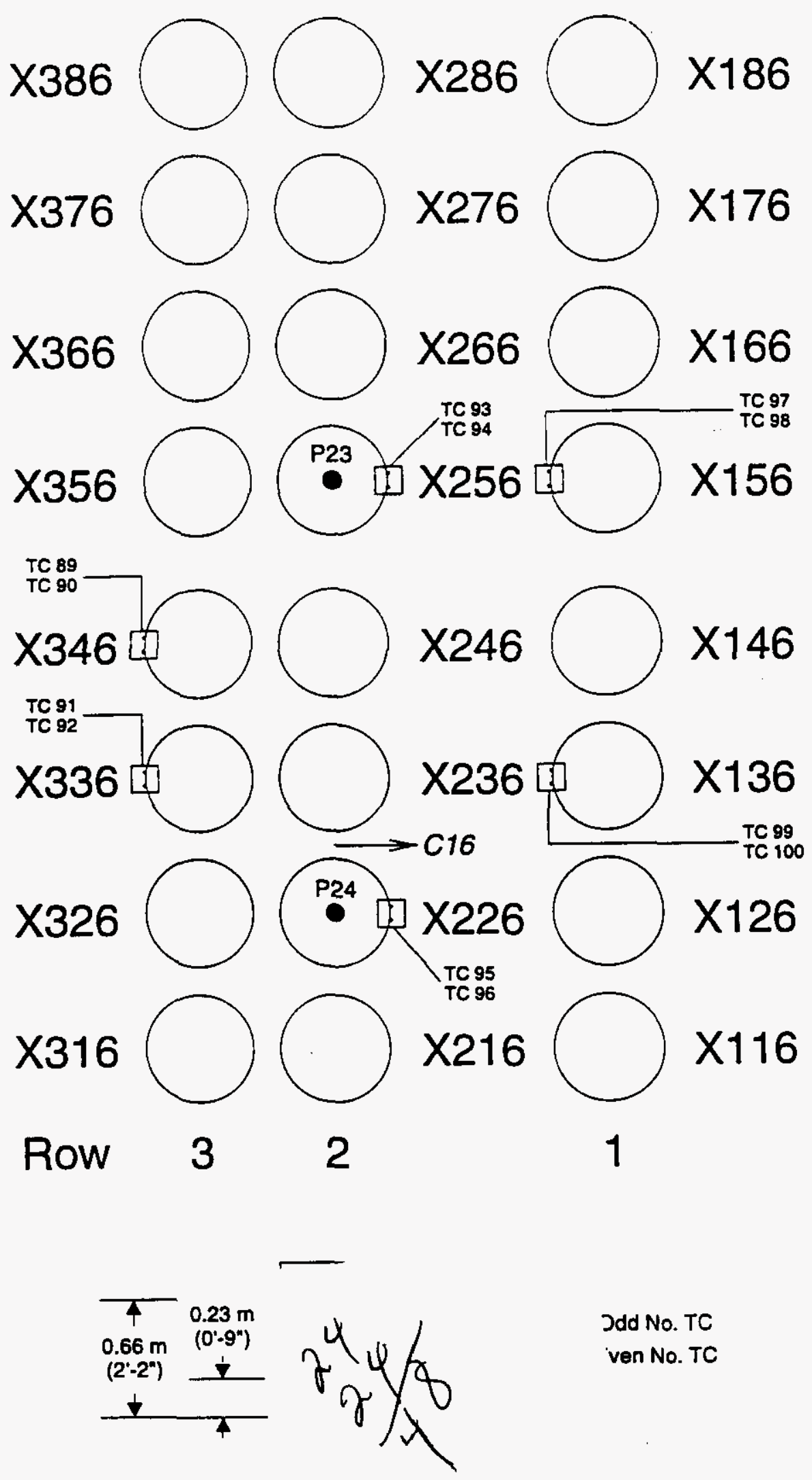

Jdd No. TC

ven No. TC

Figure 31. Instrumentation Layout - Tier 6 


\subsection{FIRE SOURCE}

The ignition source for the rack storage test was a $18.3 \mathrm{~m}^{2}\left(196.5 \mathrm{ft}^{2}\right)$ diesel fuel pool fire po:itioned under a portion of the rack array as shown in Figure 11. The fuel spill area was constructed of a watertight dam, $4.2 \mathrm{~m} \times 4.4 \mathrm{~m}(13.7 \mathrm{ft} \times 14.4 \mathrm{ft})$ utilizing $101.6 \times 101.6$ $\mathrm{mm}$ ( $3 \times 3$ in.) angle bolted to the concrete floor. Approximately a $25.4 \mathrm{~mm}$ ( 1 in.) deep layer of water was poured into the spill fire area and $265.0 \mathrm{~L}$ ( $70 \mathrm{gal}$ ) of diesel fuel was then spilled on top of the water. An additional $18.9 \mathrm{~L}(5 \mathrm{gal})$ of gasoline was poured over the diesel fuel to insure that the entire pool fire surface would ignite quickly and evenly. It was estimated that this quantity of fuel would provide a burn time of approximately five minutes. The 5minute burn time was chosen based on previous work conducted by $\mathrm{HAl}$, which showed that in engulfing fires, the drums experienced lid loss in less than 3 minutes. Additionally, the $265.0 \mathrm{~L}$ (70 gal) of fuel represented a conservatively high value for a credible fuel spill that could be experienced by WHC. 
Blank 


\subsection{PALLETIZED STORAGE TEST}

Figure 32 provides an overall view of the palletized storage test arrangement configuration. The test configuration consisted of three rows of palletized drums with each row being three tiers high. There were four drums per pallet and four pallets per row. The middle and left rows consisted of wooden pallets, and the right row consisted of steel pallets. The rows of pallets were separated by a $0.91 \mathrm{~m}(3 \mathrm{ft})$ aisle space. Within each row, the pallets were positioned flush with one another.

\subsection{PALLETS AND BANDING}

The steel pallets, similar to that in use at WHC, were provided by LATA. The pallets measured $1.2 \times 1.2 \mathrm{~m}(48.25 \times 48.25 \mathrm{in.})$ by $104.8 \mathrm{~mm}(4.1 \mathrm{in.})$ high and weighed approximately $29.0 \mathrm{~kg}(64.5 \mathrm{lb})$. All wood pallets were new and built by a local pallet company using hardwood. The wood pallets were approximately $1.2 \times 1.2 \mathrm{~m}$ (48 $\times 48$ in.) by $120.7 \mathrm{~mm}$ (4.75 in.) high and the average weight was $29.8 \mathrm{~kg}(65.8 \mathrm{lb})$. The weight of the wood pallets varied between 28 and $32 \mathrm{~kg}(62 \mathrm{lb}$ and $70 \mathrm{lb})$. Figures 33 and 34 provide construction details of the steel and wood pallets, respectively.

Four drums were loaded onto each pallet with the bolts facing into the center of the grouping, putting the seam at the corner of the pallet. A single horizontal band, located approximately $152.4 \mathrm{~mm}$ ( 6 in.) below the top of the drum, was used to secure the four drums together. The band was McMaster-Carr $6.4 \times 0.5 \mathrm{~mm}(0.25 \times 0.020$ in. $)$ thick high tensile steel strapping, Catalogue No. 2071T61. There was no specification for band tightness, except that the bands are secured "good and tight." Figure 35 provides a sketch of the pallet loading for each pallet. Figure 36 provides a plan view of the drum placement along with the drum numbering system that was used to uniquely identify each drum. Figures 37 and 38 provide photographs showing the pretest arrangement of the pallet array.

\subsection{TEST INSTRUMENTATION}

Figure 39 provides a diagram showing an overall view of the instrumentation layout used in this test. Figures $40-42$ provide diagrams showing the instrumentation layout for each tier of the test array.

\subsection{FIRE SOURCE}

The ignition source for the palletized storage test was a $9.3 \mathrm{~m}^{2}\left(100 \mathrm{ft}^{2}\right)$ diesel fuel pool fire positioned under a portion of the pallet array as shown in Figure 32 . The fuel spill area was constructed of a watertight dam $3.0 \times 3.0 \mathrm{~m}(10 \times 10 \mathrm{ft})$ utilizing $101.6 \times 101.6$ $\mathrm{mm}(3 \times 3 \mathrm{in.})$ angle bolted to the concrete floor. Approximately a $25.4 \mathrm{~mm}$ ( $1 \mathrm{in}$.) deep layer of water was poured into the spill fire area and $132.5 \mathrm{~L}$ (35 gal) of diesel fuel were then spilled on top of the water. An additional $9.5 \mathrm{~L}(2.5 \mathrm{gal})$ of gasoline were poured over the diesel fuel 
WHC-SD-WM-TRP-246 REV. 0

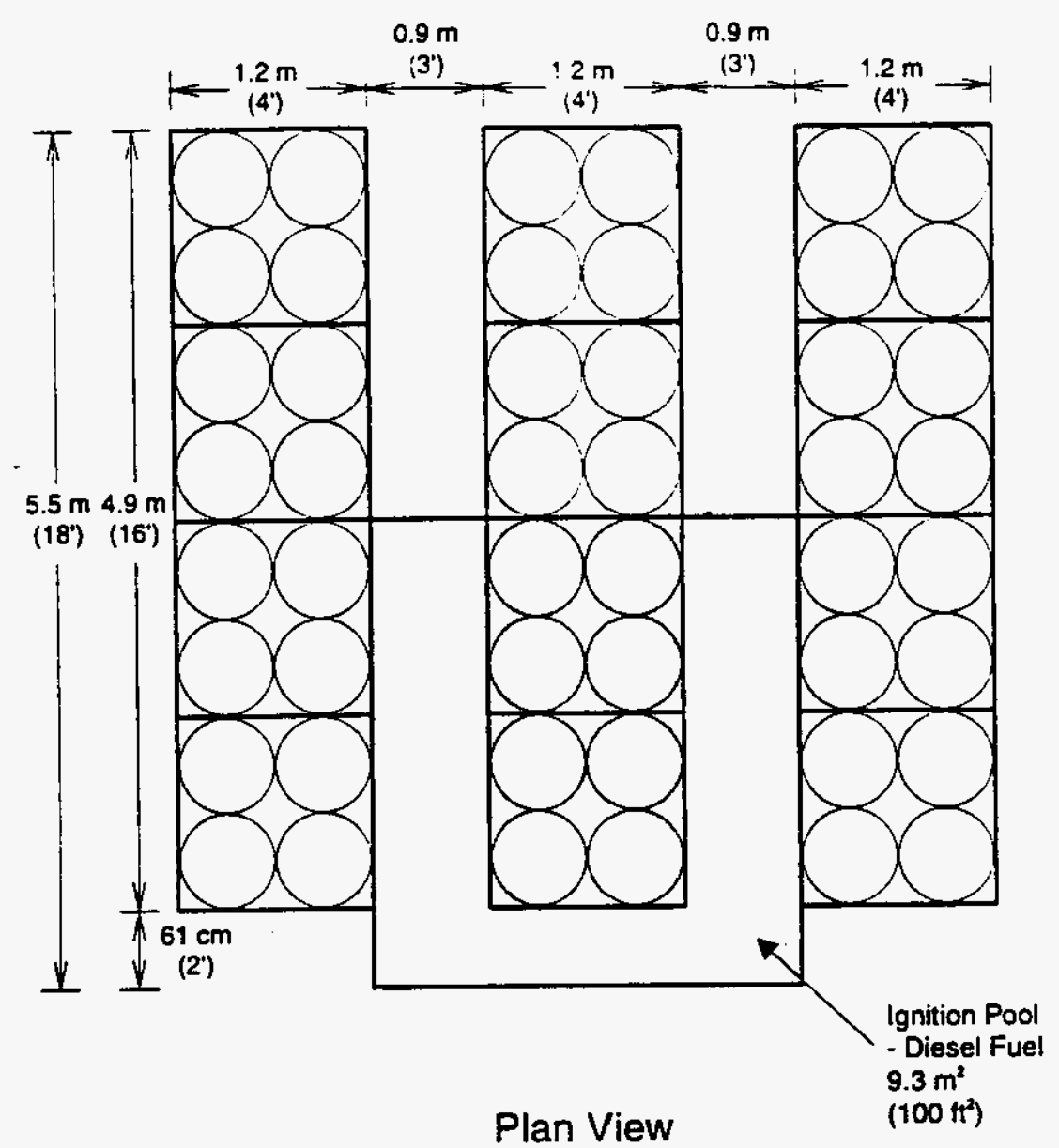

Plan View

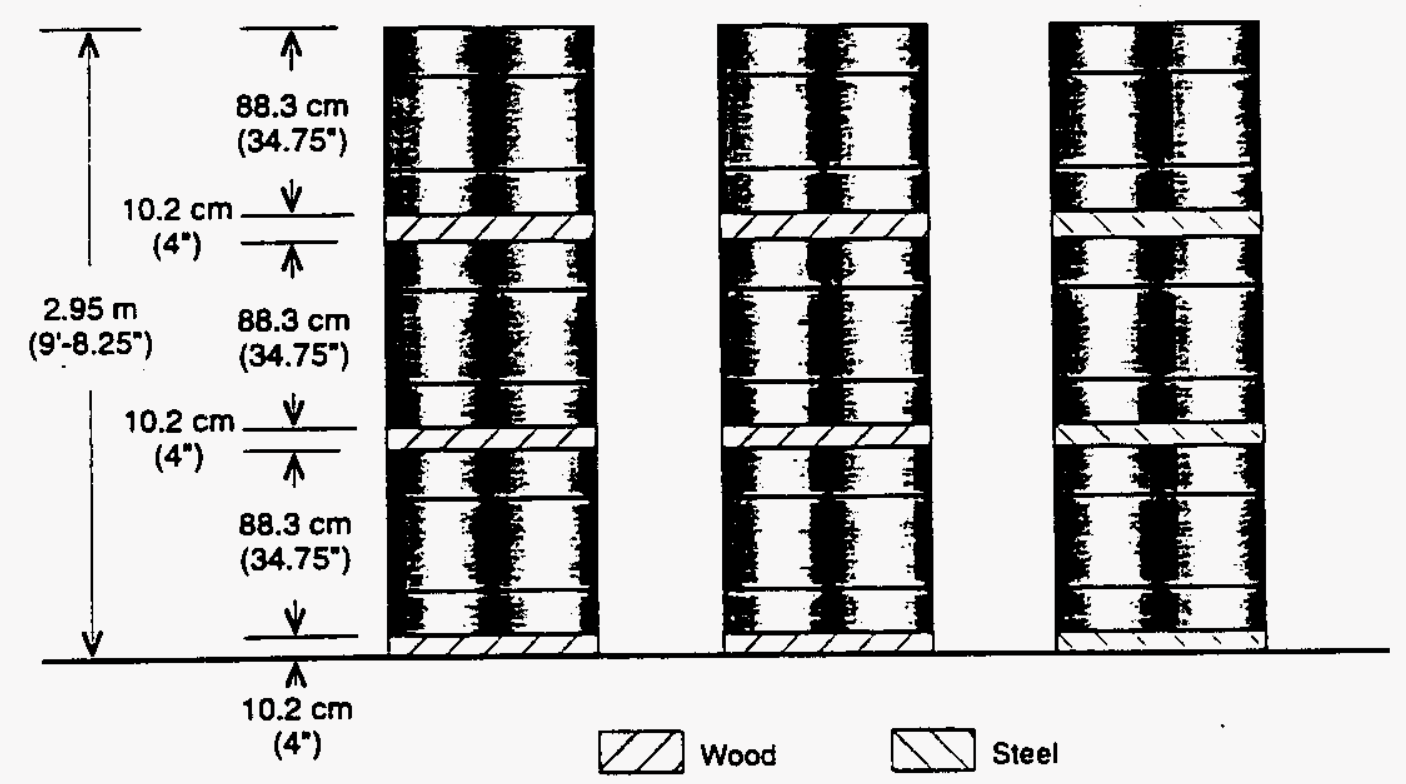

Side View

Figure 32. Overall Pallet Storage Setup 
WHC-SD-WM-TRP-246 REV. O

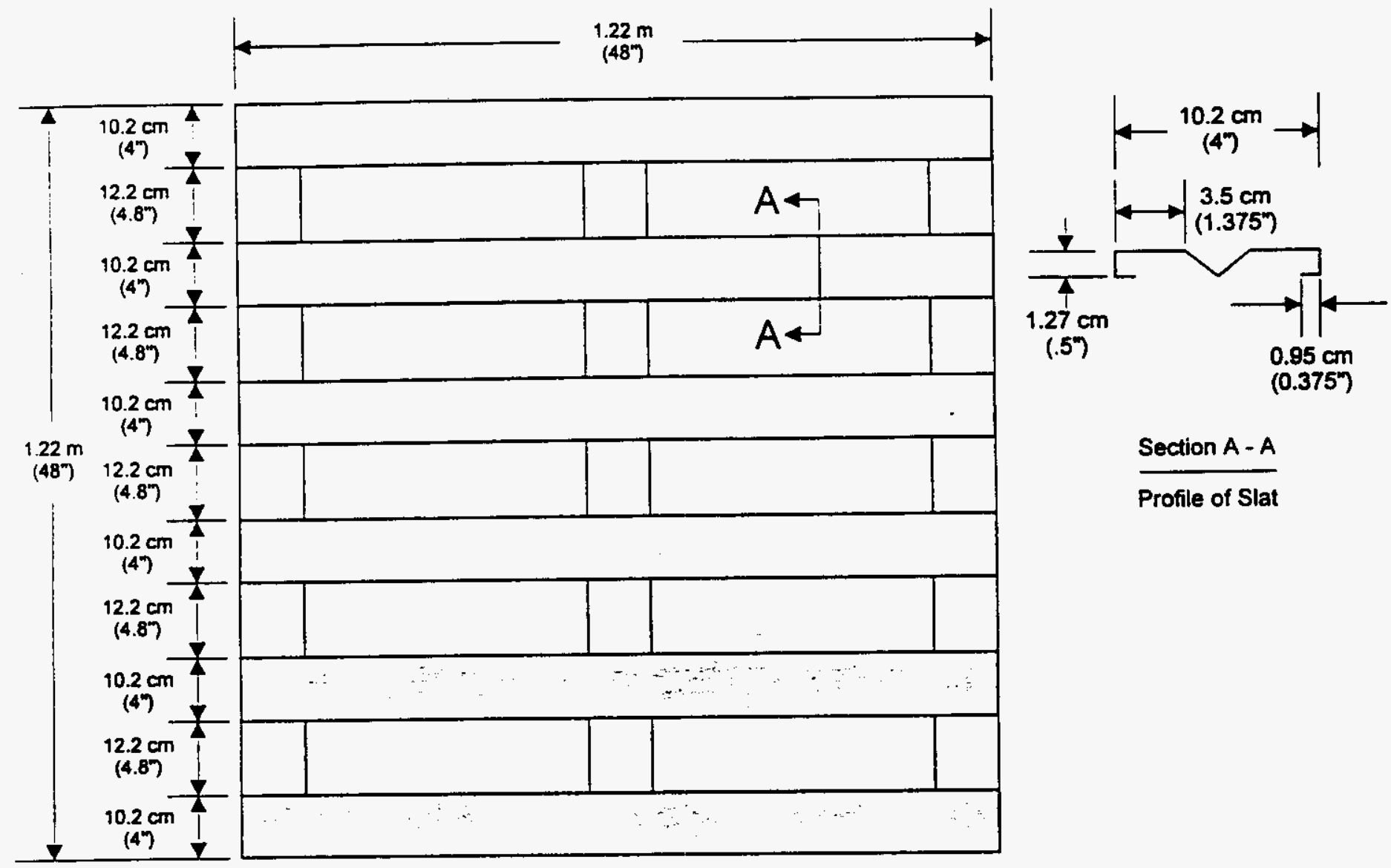

Top View

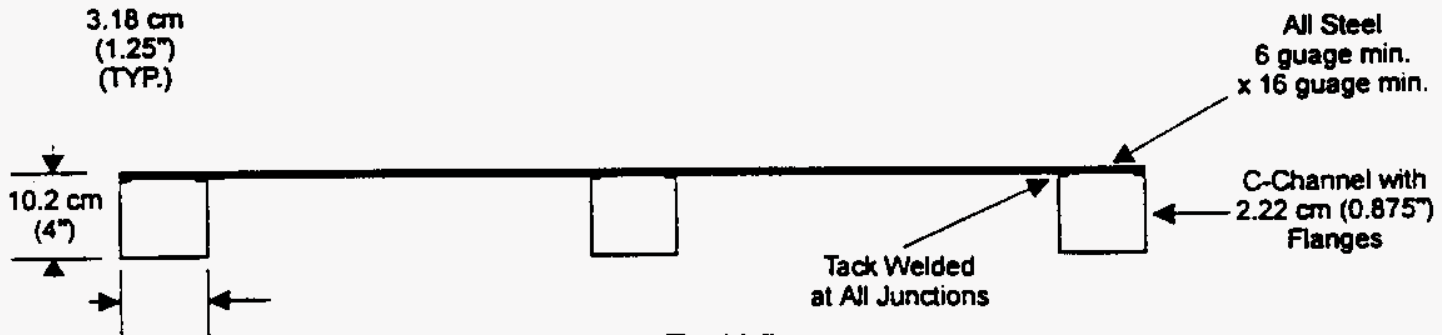

$10.2 \mathrm{~cm}$

(4)

End View

Figure 33. Steel Pallet Construction 
WHC-SD-WM-TRP-246 REV. 0

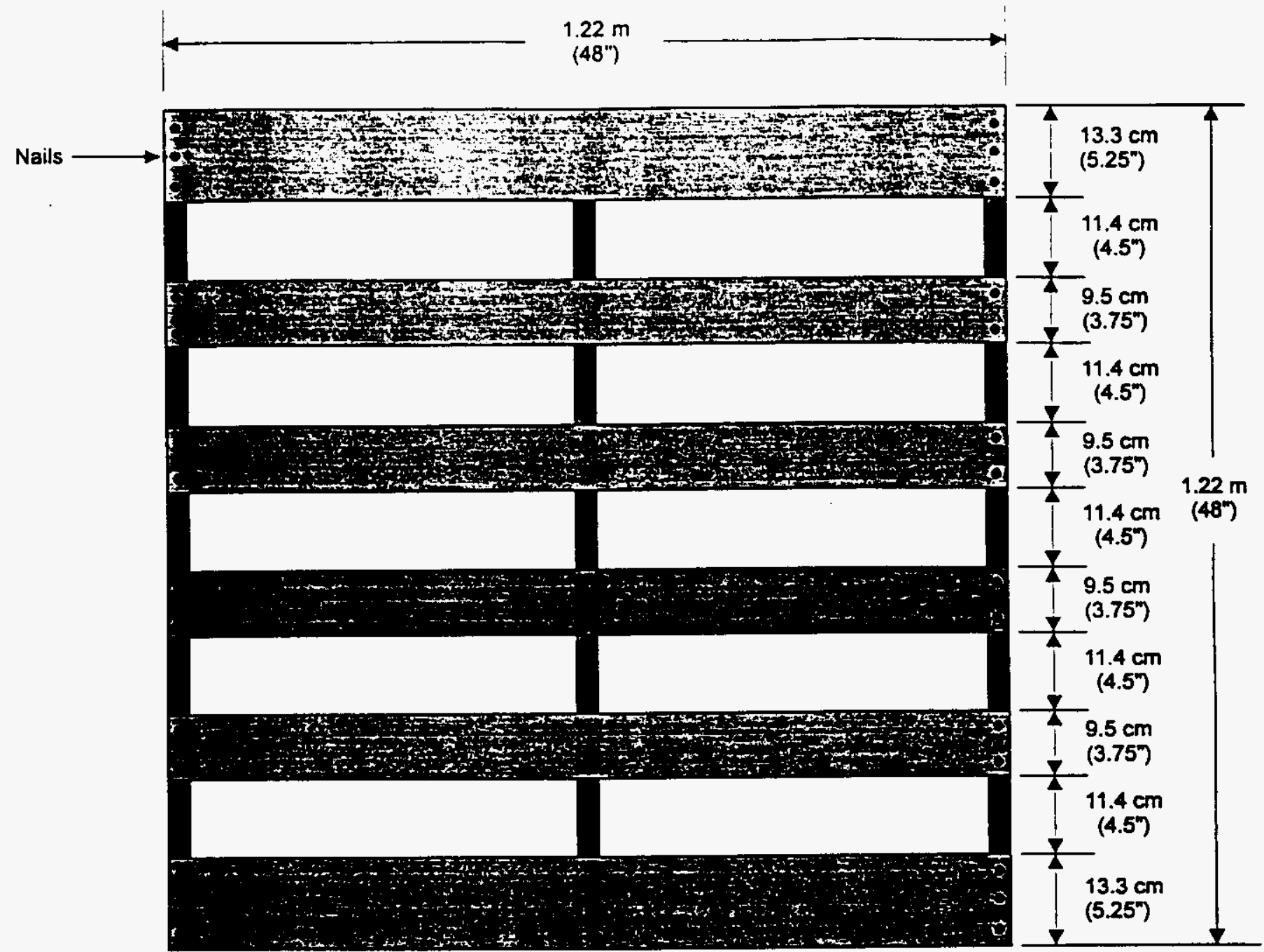

Top View $: \overparen{A}$

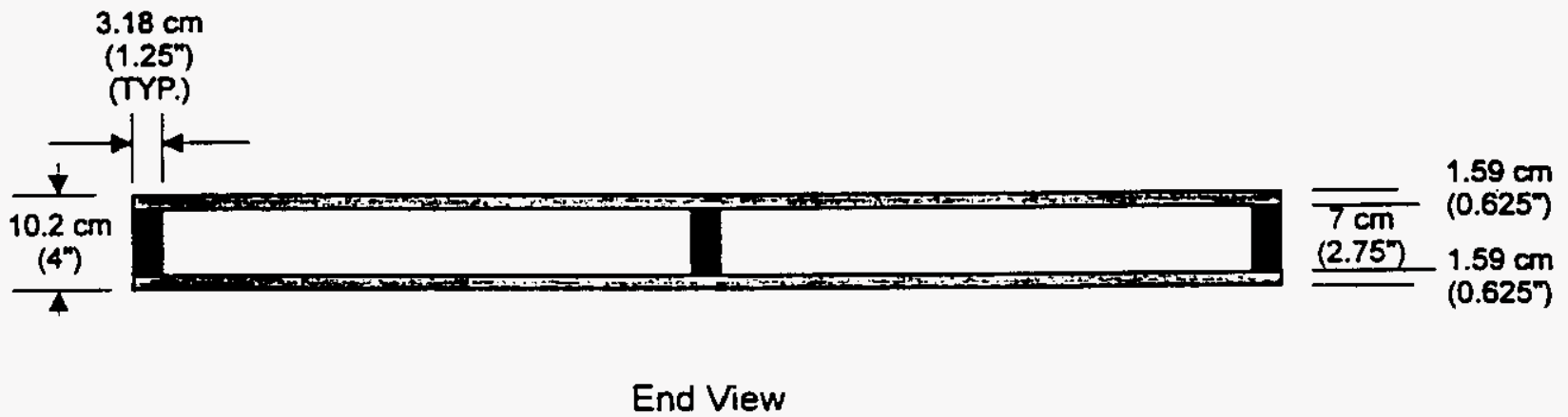

Note $\bar{A}$ Bottom is constructed similarly except with three $9.5 \mathrm{~cm}$ slats instead of four.

Figure 34. Wood Paliet Construction 
WHC-SD-WM-TRP-246 REV. 0
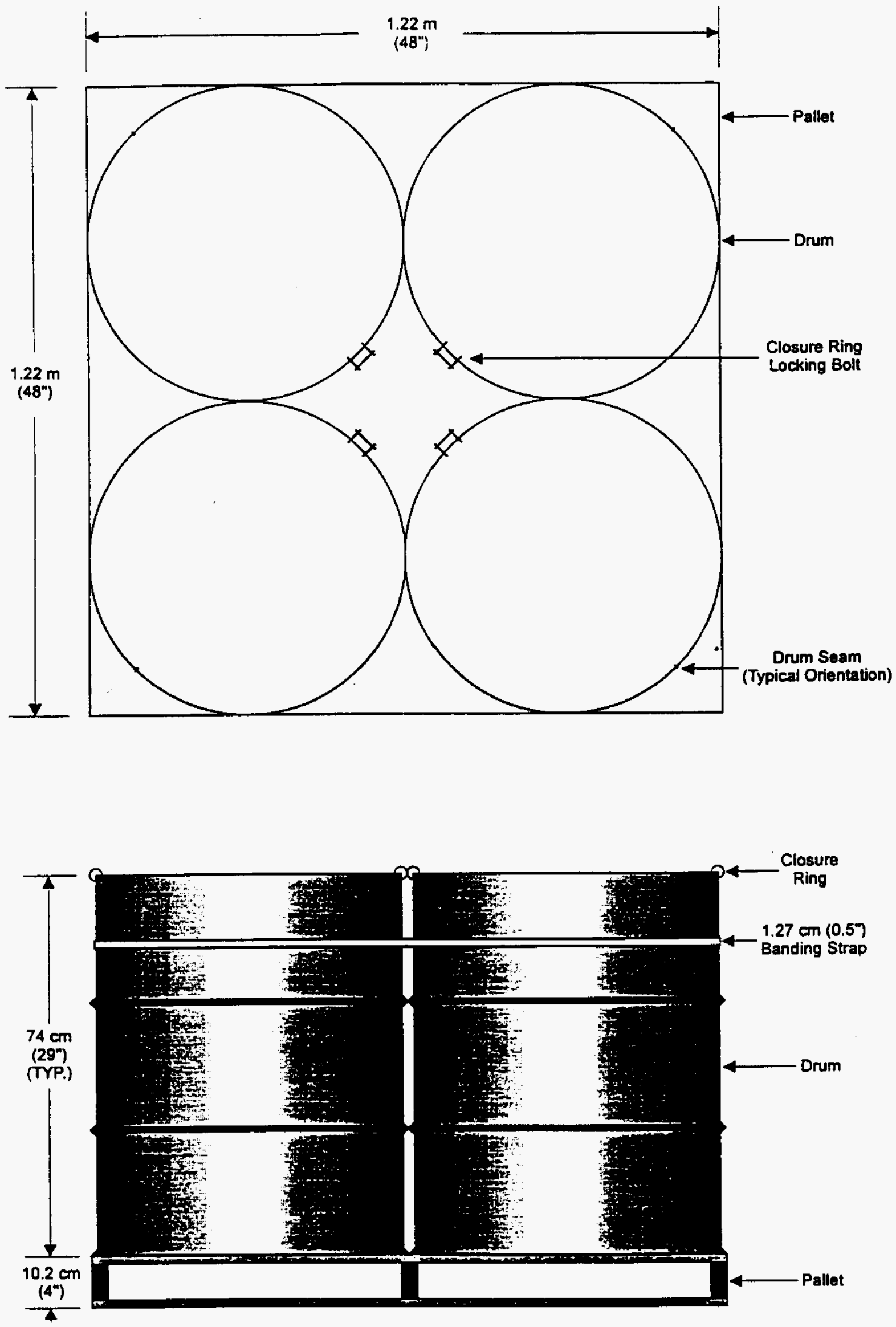

Figure 35. Drum Arrangement on Pallet 


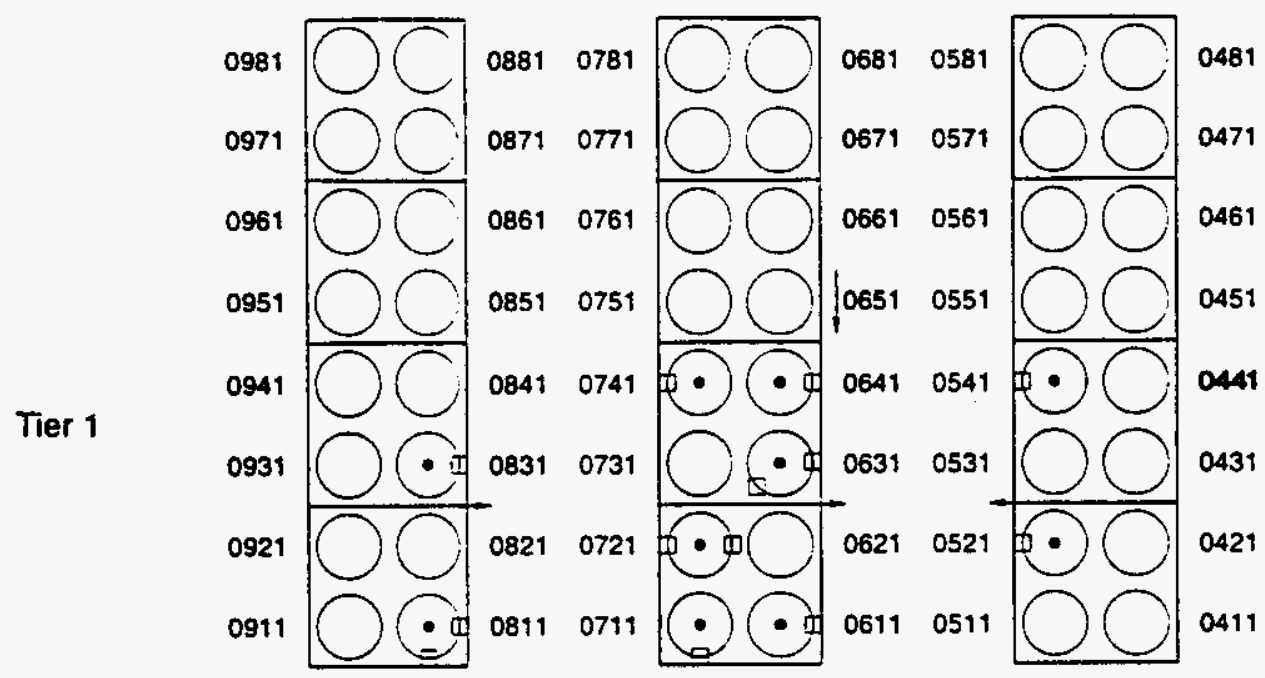

Tier 2

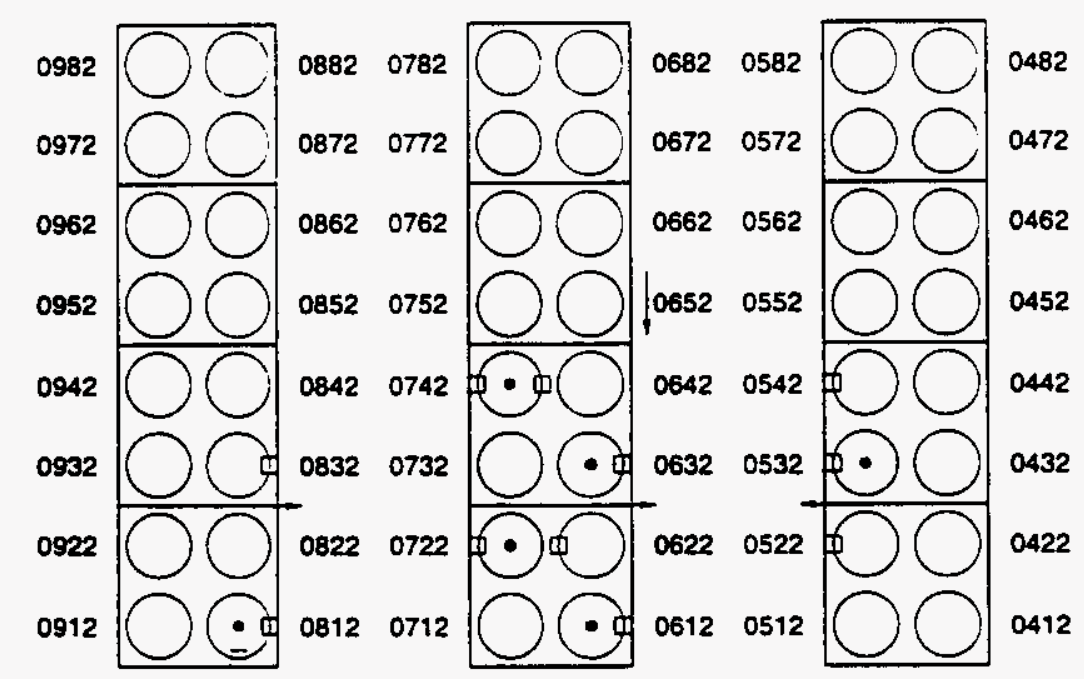

Tier 3

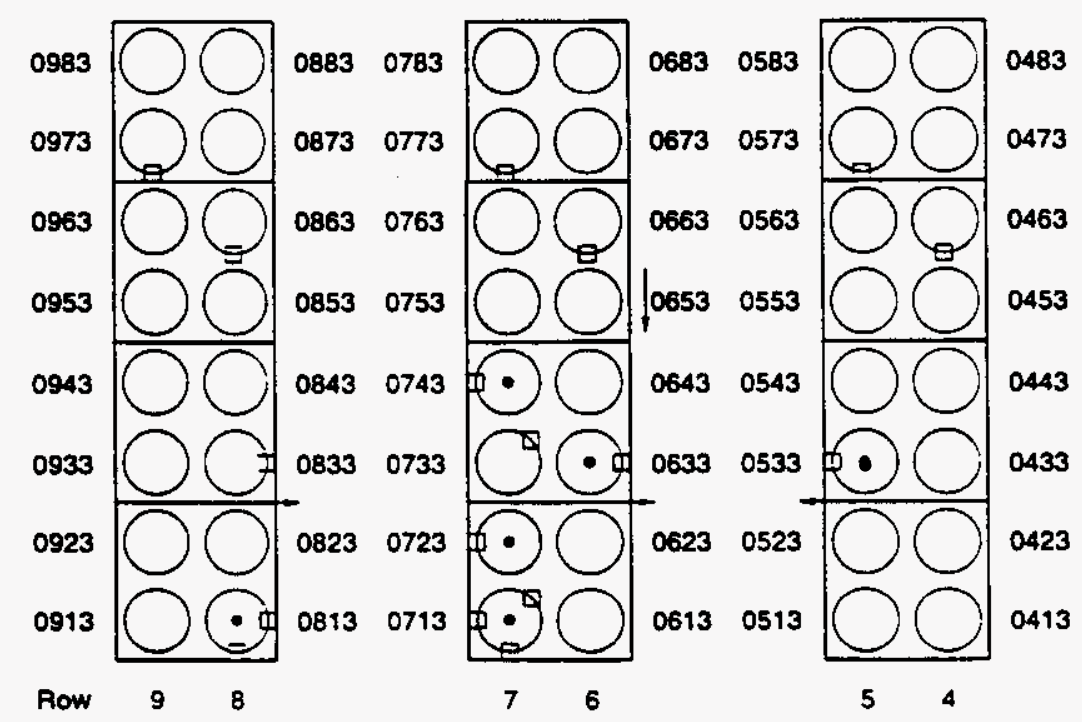

Note: Drum number indicates Test-Row-Bay-Tier

Figure 36. Pallet Storage Drum Location Diagram 
WHC-SD-WM-TRP-246 REV. 0

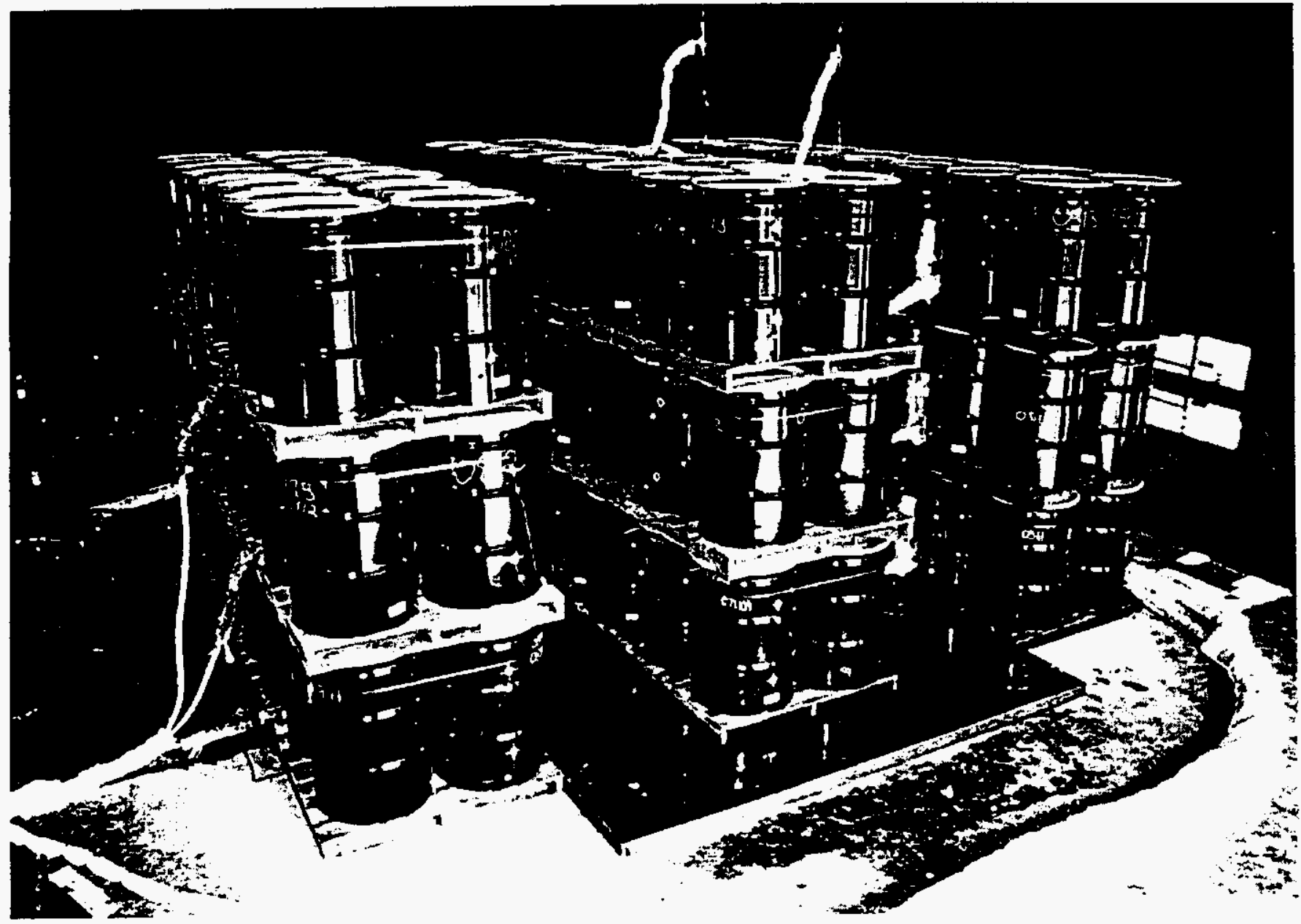

Figure 37. Pre-test Pallet Storage Array, View Looking Northwest 


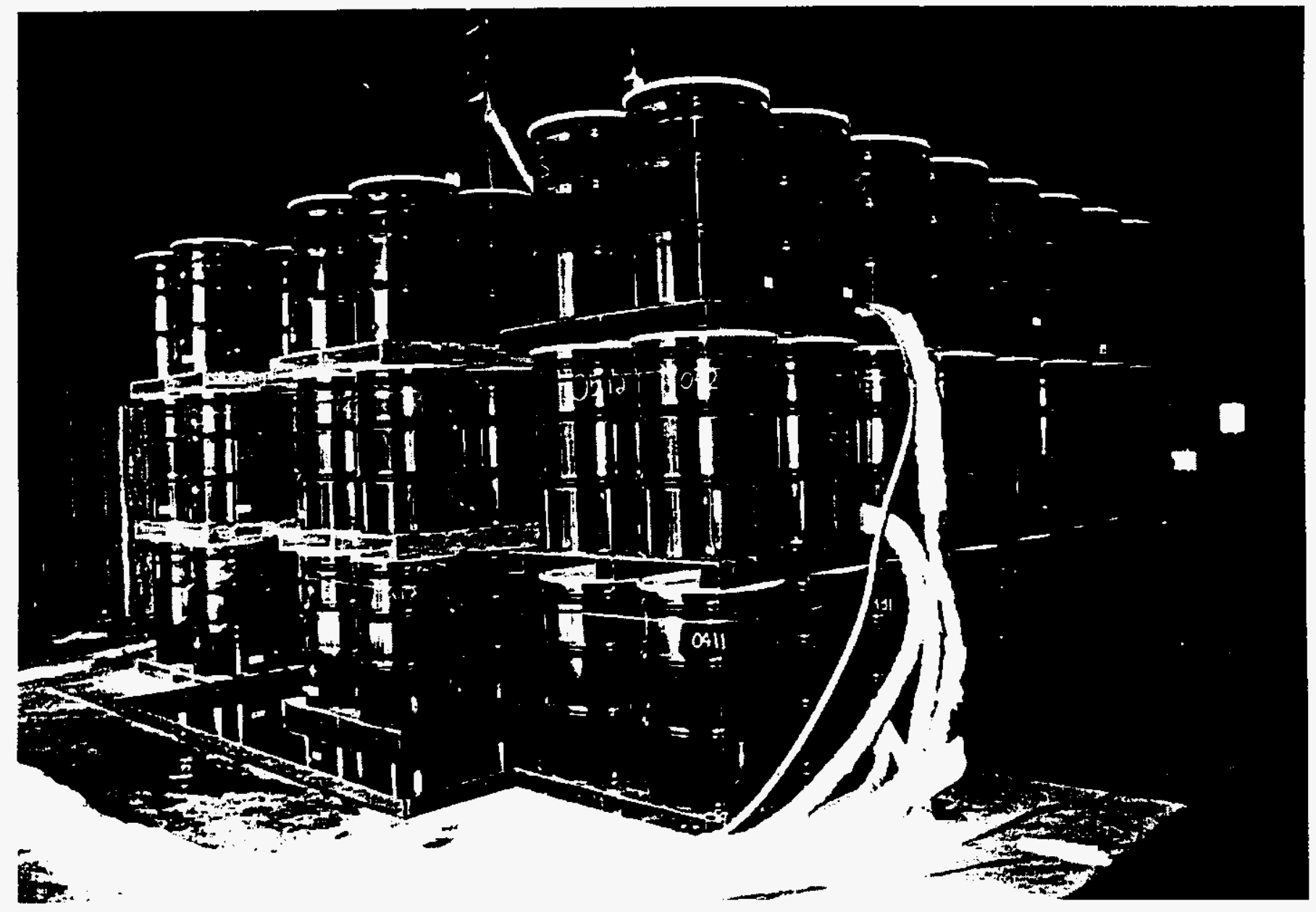

Figure 38. Pre-test Pallet Storage Array, View Looking Southwest 
WHC-SD-WM-TRP-246 REV. 0
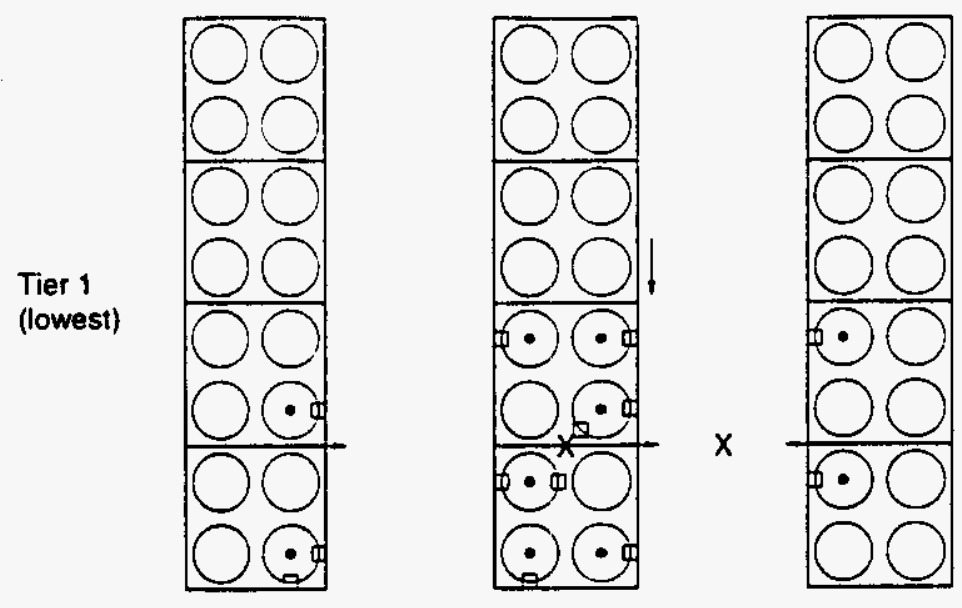

Tier 2
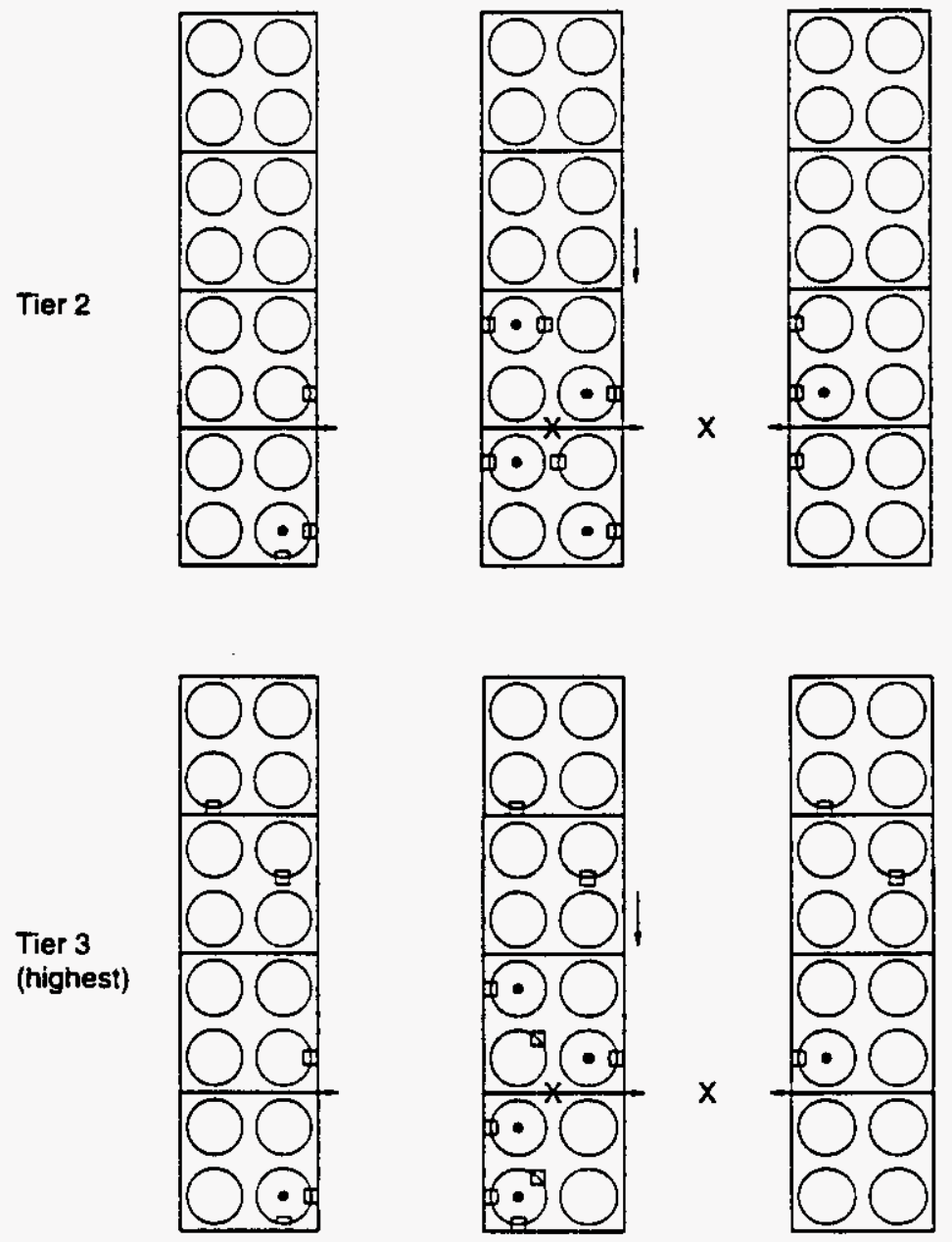

Notes:

All Drums Standard Load w/Steel

- = Pressure -22 Locations

$\square=$ Drum Surface -2 Each -42 Locations $=84$

$X=$ Temps in Fire - Two TC Trees - 15 TC's $X 2=30$ (every $61 \mathrm{~cm}$ (2') starting $61 \mathrm{~cm}$ above the floor) Total - 114 TC's

$=$ Heat Flux $=12$ Total

Figure 39. Pallet Storage Experimental Setup and Instrumentation Layout 
WHC-SD-WM-TRP-246 REV. 0

1

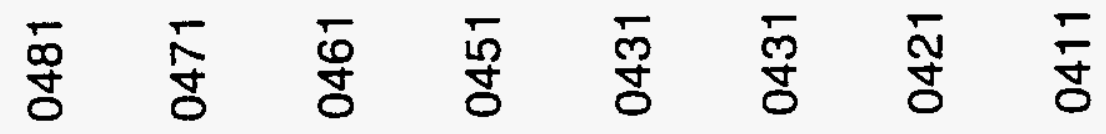

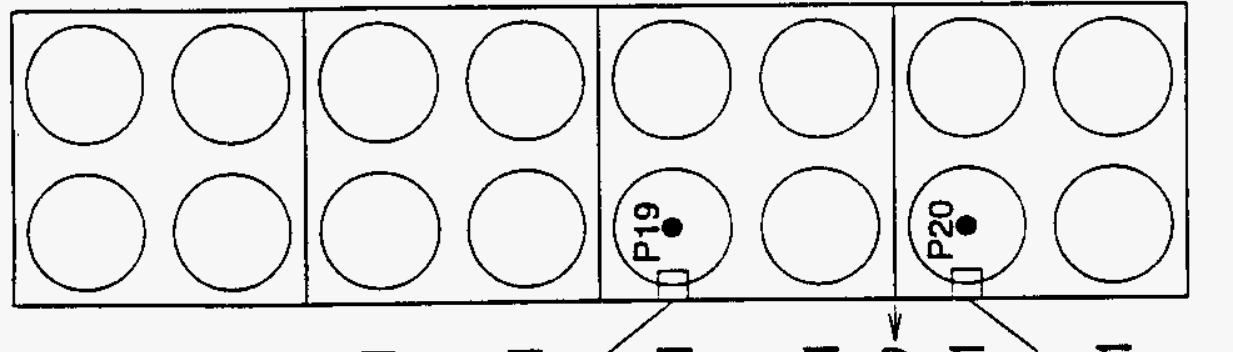

$\nabla$

น

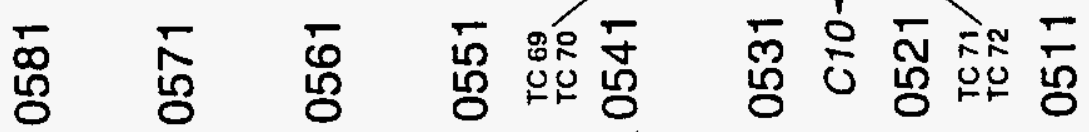

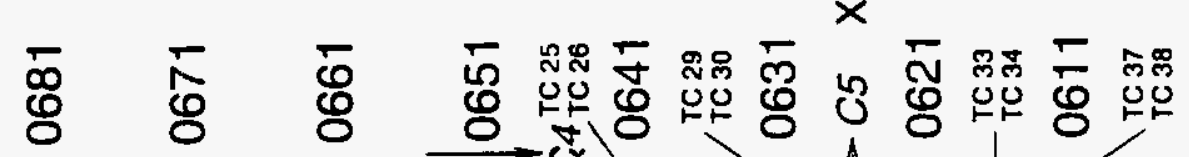

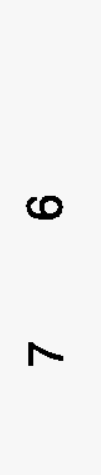

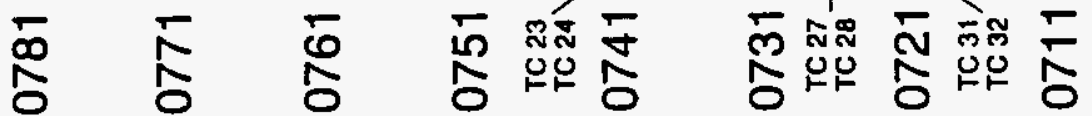

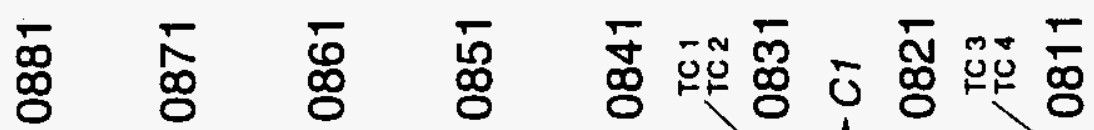

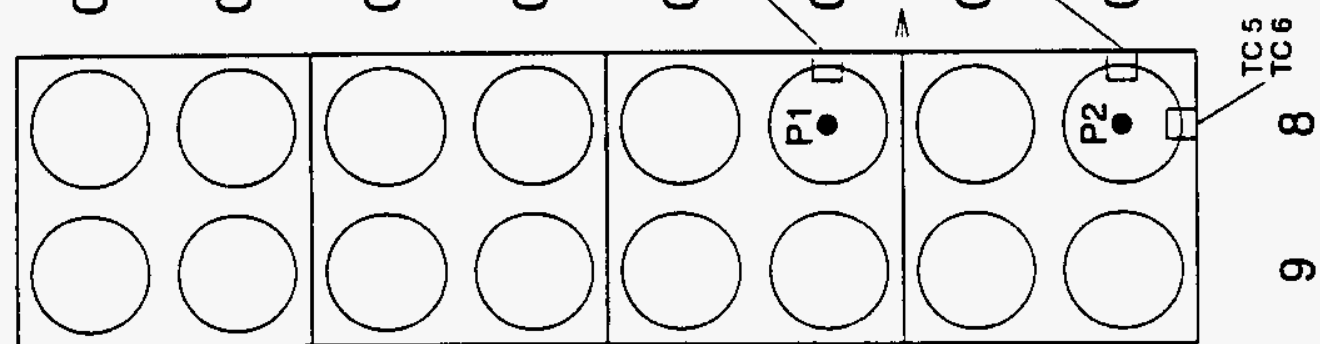

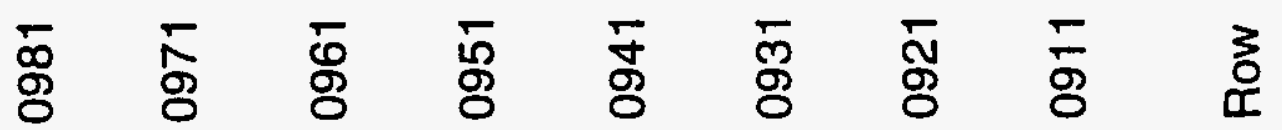

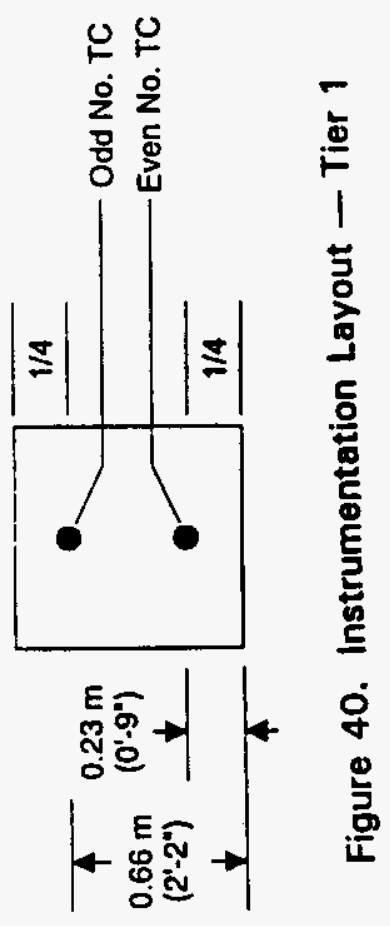




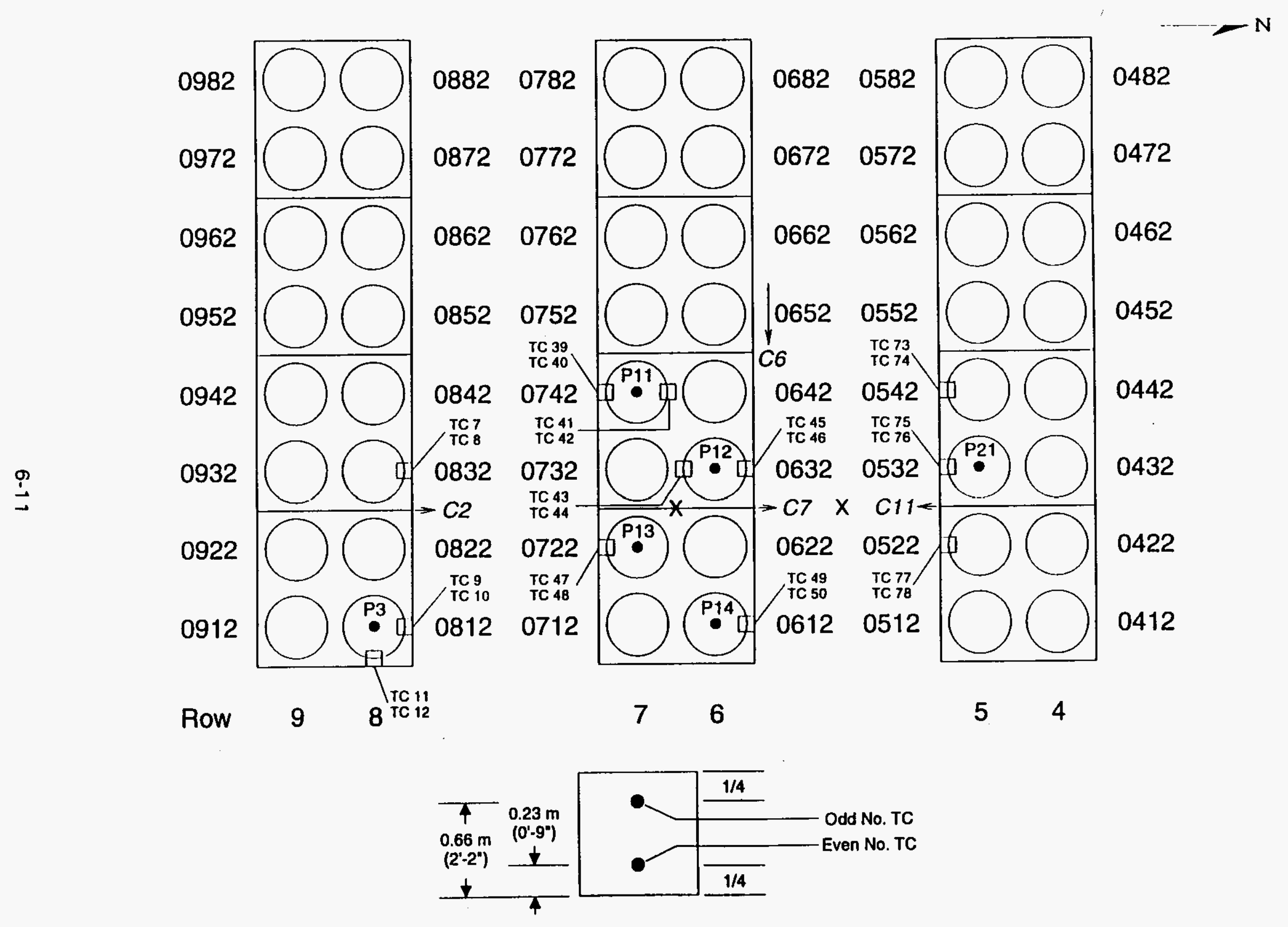

Figure 41. Instrumentation Layout - Tier 2 


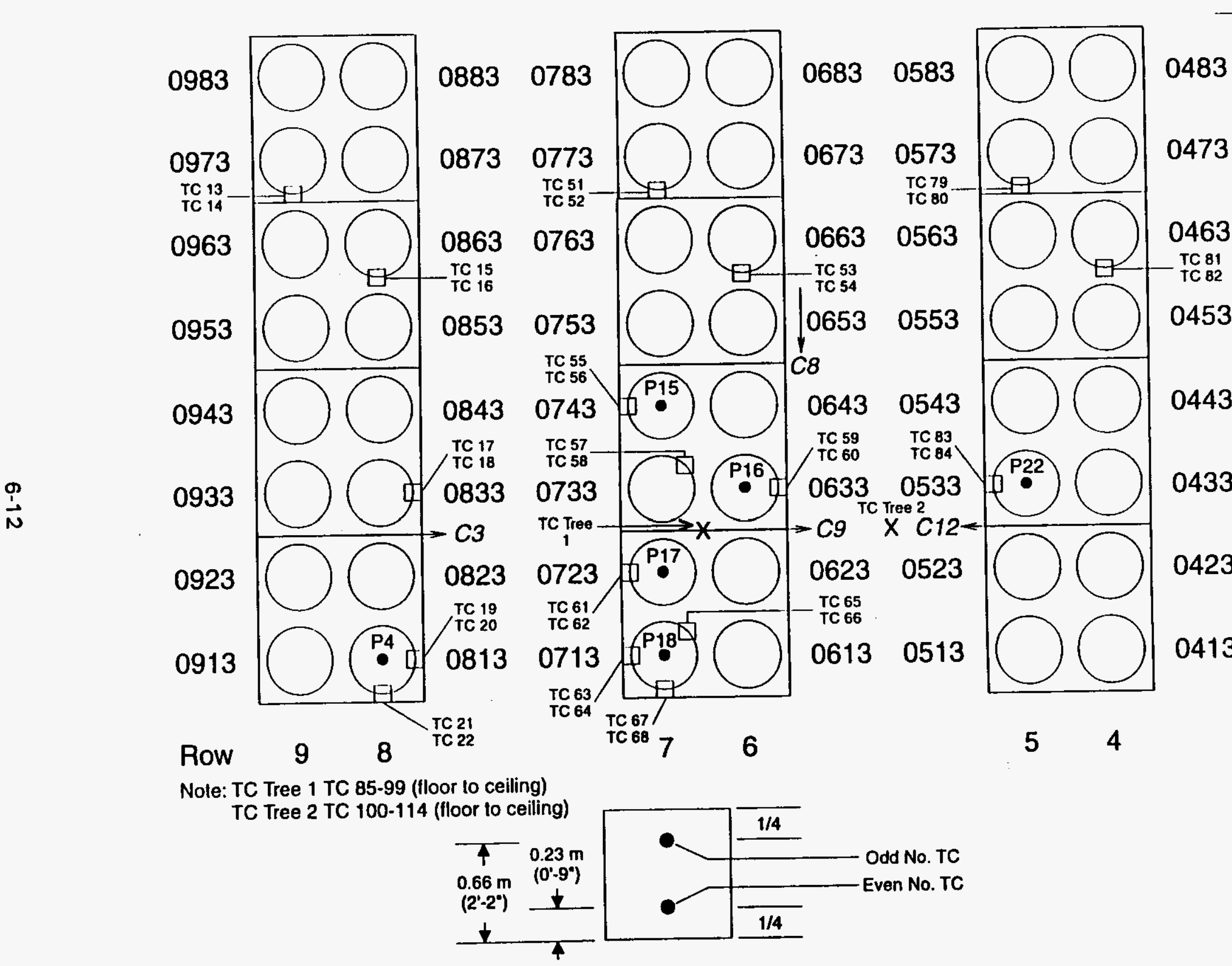

Figure 42. Instrumentation Layout - Tier 3 
to insure that the entire pool fire surface would ignite quickly and evenly. It was estimated that this quantity of fuel would provide a burn time of approximately five minutes. The 5minute burn time was chosen based on previous work conducted by $\mathrm{HAl}$, which showed that in the engulfing fires, the drums experienced lid loss in less than 3 minutes. Additionally, the $132.5 \mathrm{~L}$ (35 gal) of fuel represented a conservatively high value for a credible fuel spill that could be experienced by WHC. 
Blank 


\subsection{TEST RESULTS}

\subsection{RACK STORAGE TEST}

The rack storage test was conducted on June 6,1995 . The ambient temperature at the start of the test was $28^{\circ} \mathrm{C}\left(82^{\circ} \mathrm{F}\right)$. Data plots for the pressure, temperature, and heat flux data obtained during this test is provided in Appendix $C$.

\subsubsection{Visual Observations}

During the fire test, visual observations were made. A summary of the observations is provided in Appendix $C$. It should be noted that determinations of specific drums rupturing or exploding were difficult due to viewing angles and the engulfing pool fire itself.

The first drum exploded at 1:41 after ignition of the pool fire. Prior to this, flaming around the lids of several drums in the center of the pool fire was noted. During the test, the observers could hear the drums venting, and as the venting/pressure increased, the drum would explode. From the observations, it was estimated that 21 to 25 drums exploded during the test.

When several of the drums exploded, the lids would move upward and hit the underside of the containment pans. The force of the explosion and/or the lids would be sufficient to cause the containment pans to disengage from the " $S$ " hook supports and either completely or partially fall on top of the drum below it.

During the test, two lids were expelled from the racks. One lid fell into the center aisle between racks 2 and 3 at 2:46 into the test. Another lid was noted on the southside of rack 3 after the test. Also on the southside of Rack 3, two containment pans had fallen out of the rack onto the floor. During the test, no other lids were violently expelled from the racks.

Several times during the fire, airborne flaming contents could be observed in the facility. Generally, these were small pieces of materials, and no significant amount of expelled contents was noted either on the floor or on/in the racks after the test.

At approximately 3:30 into the test, significant movement and distortion of the vertical uprights of racks 2 and 3 was noted. This distortion continued throughout the remainder of the test. The outermost vertical upright (southeast side) of Rack 3 basically collapsed on itself along with the other east side vertical upright of Rack 3 also showing collapse but not as extensive as the outermost upright. The effect of this collapse was to shift and distort rack 3 toward the southeast corner of the test building. Rack 2 aiso showed coliapse of the east side uprights but not as extensive as that noted in Rack 3. The movement of Rack 3 also caused breakage of lower two horizontal ties between Rack 3 and Rack 2. 
At approximately 5:24 into the test period, the pool fire was extinguished. At this time, all of the drums that were in the pool fire area in Racks 2 and 3 were burning. Figure 43 provides a photograph at this time into the test. The drums were burning through openings in the lids created when they exploded. Several of the drums in Row 2, Tiers 1.6 and Bays $1-4$ were also burning around their lids. It appeared that these flames were approximately 76 $152 \mathrm{~mm}(3-6 \mathrm{in.})$ in length.

After extinguishment was started, two additional drums in the upper portion of the pool fire area exploded. The pool fire was extinguished at approximately 6:30 into the test period. Due to continued movement/collapse of the uprights of Rack 3, and with safety considerations in mind, the test was terminated at 7:18 into the test period. At this time, extinguishment of the burning drums in Racks 2 and 3 was begun. The drums were extinguished at 10:30 into the test period.

\subsubsection{Pressure Data}

Of the 24 drums instrumented with pressure transducers, 8 were positioned west of the pool fire in Racks 2 and $3(\times 151, \times 251, \times 361, \times 352, \times 253, \times 254, \times 255$, and X256) and 4 were positioned adjacent to the pool fire in Rack 1 (X131, X122, X123, and X125). All of these drums experienced negligible pressure increase with a peak pressure measured being $35.9 \mathrm{kPa}(5.20 \mathrm{psig})$ in Drum $\times 131$. The remaining 12 drums instrumented with pressure transducers were positioned inside the pool fire. The peak pressures measured ranged from $46.6 \mathrm{kPa}(6.76 \mathrm{psig})(\times 226)$ to $15.3 \mathrm{kPa}(16.72 \mathrm{psig})(\times 222)$ and averaged $68.3 \mathrm{kPa}(9.9$ psig). Table 4 shows the order each pressure transducer reached its peak pressure and the corresponding time.

Drum $\times 224$ reached a peak of approximately $46.6 \mathrm{kPa}(6.75 \mathrm{psig})$ and then experienced a relatively slow venting to a pressure of approximately $17.2 \mathrm{kPa}(2.5 \mathrm{psig})$. The pressure began to rise up to the peak of $49.3 \mathrm{kPa}(7.15 \mathrm{psig})$ at which time the drum may have exploded or vented, resulting in a total pressure drop. This double spike, also observed on Drums $X 233, X 225$, and $\times 226$ may be attributed to a pressure rise until venting occurred which relieved the pressure, followed by an increase in pressure which the venting action could not relieve thus causing the drum to explode.

\subsubsection{Total Heat Flux Data}

A total of 22 total heat flux transducers (calorimeters) with $180^{\circ}$ view angles were positioned at various locations throughout the rack system to define the heat flux environment imposed on the rack system and the drums from the pool fire. The first group of calorimeters (C1, C5, C9, and C13) were located outside the fire between Racks 2 and 3 looking east (into the fire) and measured peak total heat flux values between approximately 30 and $45 \mathrm{~kW} / \mathrm{m}^{2}$. The second group of calorimeters (C2, C6, and $\mathrm{C} 10)$ were located in the fire on Rack 3 looking south (out of the fire) and measured peak total heat flux values between approximately 160 and $200 \mathrm{~kW} / \mathrm{m}^{2}$. The third group of calorimeters $(C 3, C 7$, and $\mathrm{C} 11)$ were located in the fire on Rack 2 (Tiers 1, 2, and 3) looking north and measured peak total heat flux values of approximately $225 \mathrm{~kW} / \mathrm{m}^{2}$. Calorimeters $\mathrm{C} 14, \mathrm{C15}$, and $\mathrm{C} 16$ located on Tiers 4, 5, and 6 
WHC-SD-WM-TRP-246 REV. 0

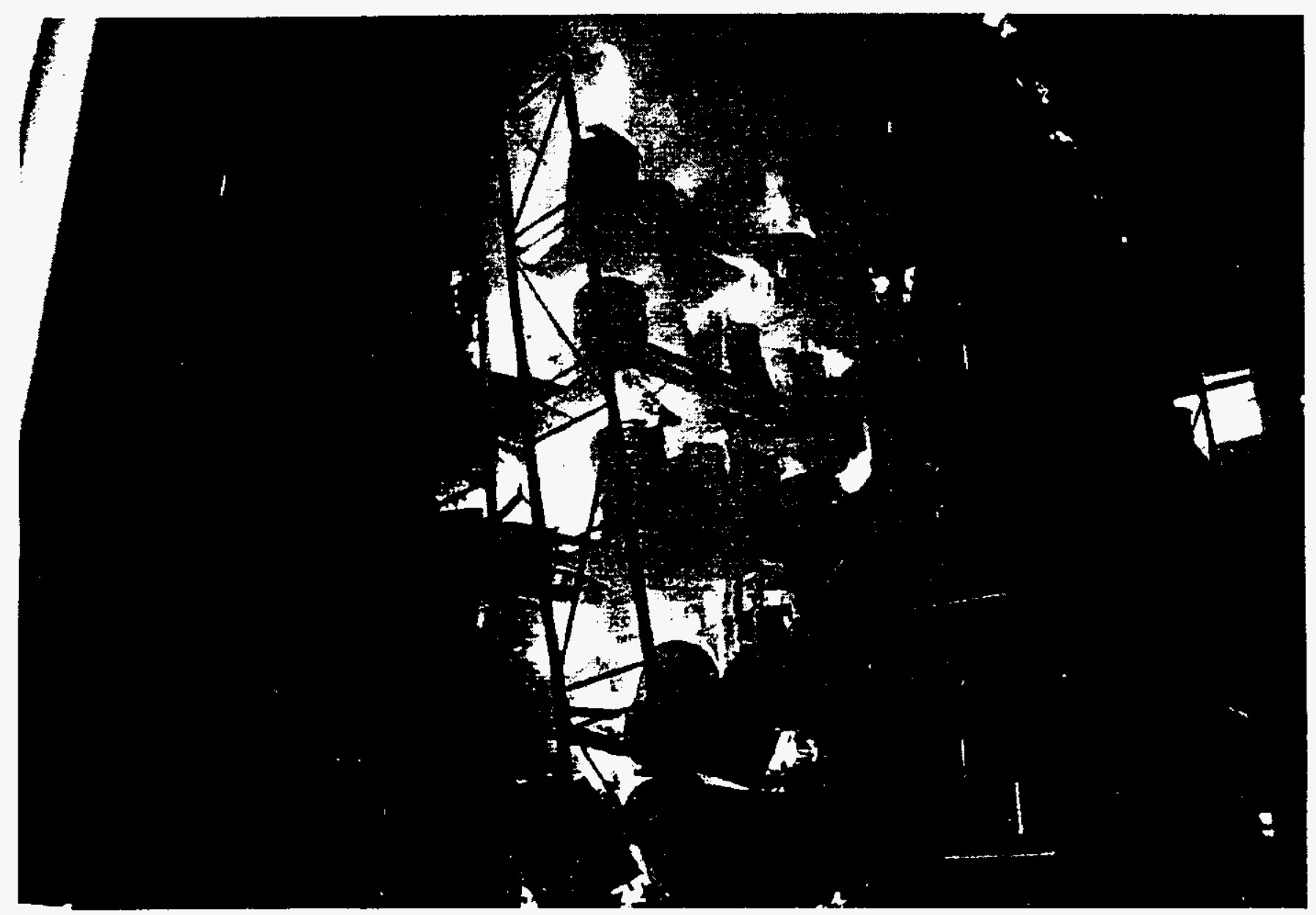

Figure 43. Rack Storage Test after Pool Fire Extinguishment 
looking in the same direction as calorimeters $\mathrm{C} 3, \mathrm{C7}$, and $\mathrm{C} 11$ measured peak total heat flux values of approximately $140 \mathrm{~kW} / \mathrm{m}^{2}$. The last group of calorimeters, C4, C8, and C12, were located outside the fire on Rack 1 looking south (into the fire) and measured peak total heat flux values of approximately 100 to $150 \mathrm{~kW} / \mathrm{m}^{2}$.

Table 4. Peak Pressures Measured For Each Drum

\begin{tabular}{|c|c|c|c|}
\hline Order & Drum No. & $\begin{array}{c}\text { Peak Pressure } \\
\text { psig } \\
\end{array}$ & $\begin{array}{c}\text { Time } \\
\text { (min:sec) }\end{array}$ \\
\hline 1 & $\times 222$ & 16.5 & $1: 41$ \\
\hline 2 & $\times 312$ & 11.0 & $1: 50$ \\
\hline 3 & $\times 211$ & 12.4 & $1: 51$ \\
\hline 4 & $\times 213$ & 11.0 & $1: 58$ \\
\hline 5 & $\times 321$ & 10.0 & 2:01 \\
\hline 6 & $\times 231$ & 8.7 & $2: 42$ \\
\hline 7 & $\times 214$ & 11.0 & $2: 43$ \\
\hline 8 & $\times 131$ & 5.2 & $2: 46$ \\
\hline 9 & $\times 122$ & 3.0 & $3: 27$ \\
\hline 10 & $\times 224$ & 7.1 & $3: 31$ \\
\hline 11 & $\times 225$ & 7.8 & $3: 58$ \\
\hline 12 & $\times 332$ & 8.4 & 4:08 \\
\hline 13 & $\times 233$ & 7.5 & $4: 33$ \\
\hline 14 & $\times 226$ & 6.7 & $4: 35$ \\
\hline 15 & $\times 123$ & 2.7 & $4: 56$ \\
\hline 16 & $\times 256$ & 3.8 & $4: 58$ \\
\hline 17 & $\times 251$ & 1.4 & $5: 29$ \\
\hline 18 & $\times 125$ & 2.4 & $5: 57$ \\
\hline 19 & $\times 361$ & 0.4 & $6: 27$ \\
\hline 20 & $\times 254$ & 1.3 & $8: 07$ \\
\hline 21 & $\times 255$ & 2.6 & $8: 14$ \\
\hline 22 & $\times 253$ & 0.9 & $8 ; 15$ \\
\hline 23 & $\times 352$ & 0.9 & $8: 16$ \\
\hline 24 & $\times 151$ & 1.4 & $17: 15$ \\
\hline
\end{tabular}

A typical hydrocarbon pool fire (one composed of diesel, gasoline, etc.) is characterized by total incident heat flux levels of approximately $200 \mathrm{~kW} / \mathrm{m}^{2}$ and temperatures ranging from 
approximately 800 to $1100^{\circ} \mathrm{C}\left(1470\right.$ to $\left.2000^{\circ} \mathrm{F}\right)$. Calorimeters C3, C7, and C11, which were totally immersed inside the fire, measured typical total heat flux values. The remaining calorimeters were located in positions facing the fire with obstructions in their view angle, (i.e. drums, rack members, etc.) or only intermittently was there flame filling the entire $180^{\circ}$ view angle resulting in some cases very low total heat flux levels.

\subsubsection{Drum Surface and Tree Temperatures}

The peak surface temperatures measured on the drums positioned in the west half of the three racks (Bays $5,6,7$, and 8 ) ranged in temperature from approximately 75 to $400^{\circ} \mathrm{C}$ $\left(167\right.$ to $\left.752^{\circ} \mathrm{F}\right)$. The drums in Bay 5, adjacent to the pool fire, ranged in peak temperature from 200 to $400^{\circ} \mathrm{C}\left(392\right.$ to $\left.752^{\circ} \mathrm{F}\right)$ with the remaining drums staying below $100^{\circ} \mathrm{C}\left(212^{\circ} \mathrm{F}\right)$. The four drums in Rack 1 adjacent to the fire (Bays 1, 2, 3, and 4) ranged from 450 to $600^{\circ} \mathrm{C}$ $\left(842\right.$ to $1112^{\circ} \mathrm{F}$ ) with little change in temperature as a function of tier level. The drums which were situated directly inside the pool fire ranged in peak temperature from $300^{\circ} \mathrm{C}\left(572^{\circ} \mathrm{F}\right)$ to peaks as high as $1300^{\circ} \mathrm{C}\left(2370^{\circ} \mathrm{F}\right)$. It is interesting to note that the drums in Bay 4 were approximately 100 to $1000^{\circ} \mathrm{C}\left(212\right.$ to $\left.1830^{\circ} \mathrm{F}\right)$ cooler as a result of intermittently being completely surrounded by a thick flame layer. The hottest location measured on the surface of a drum was in the $1 \mathrm{st}$ and 2 nd bays of Rack 3 . All peak surface temperatures measured on the drums in these locations were between 700 and $1300^{\circ} \mathrm{C}\left(1290\right.$ and $\left.2370^{\circ} \mathrm{F}\right)$. It should be noted that as Racks 3 and 2 began to collapse, some of the drum surface thermocouples may have been dislodged. It was not possible to make a good assessment of this due to safety concerns during post-test observations.

Three water filled drums were located in Racks 2 and 3 inside the fire, and the fourth drum was located in Rack 1 adjacent to the fire. The three water drums in the fire reached an internal temperature of $100^{\circ} \mathrm{C}\left(212^{\circ} \mathrm{F}\right)$ at approximately $400 \mathrm{sec}$ and remained at that level for the duration of the test. The internal temperatures in the water drum located in Rack 1 only rose approximately $10^{\circ} \mathrm{C}\left(50^{\circ} \mathrm{F}\right)$ above ambient. At the end of the fire test, all air operated pumps were operational and functioning properly.

Thermocouple (TC) Tree No. 1, 6 in. $(152.4 \mathrm{~mm})$ off Rack 1, measured peak temperatures up to approximately $800^{\circ} \mathrm{C}\left(1470^{\circ} \mathrm{F}\right)$ from Tiers 1 to 3 with averages between 500 and $600^{\circ} \mathrm{C}\left(930\right.$ and $\left.1110^{\circ} \mathrm{F}\right)$. The TC's measuring temperatures at Tiers 4,5 , and 6 measured peaks between 600 and $700^{\circ} \mathrm{C}\left(1110\right.$ and $\left.1290^{\circ} \mathrm{F}\right)$, and the averages dropped to approximately $400^{\circ} \mathrm{C}\left(750^{\circ} \mathrm{F}\right)$ with the hottest temperatures measured at the higher tiers. The ceiling TC peaked at approximately $800^{\circ} \mathrm{C}\left(1470^{\circ} \mathrm{F}\right)$ and averaged temperatures around 600 to $700^{\circ} \mathrm{C}\left(1110\right.$ to $\left.1290^{\circ} \mathrm{F}\right)$. TC Tree No. 2, $152.4 \mathrm{~mm}$ (6 in.) off Rack 2, measured peak temperatures of 1000 to $1100^{\circ} \mathrm{C}\left(1830\right.$ to $\left.2000^{\circ} \mathrm{F}\right)$ up to Tier 5 with averages around $1000^{\circ} \mathrm{C}\left(1830^{\circ} \mathrm{F}\right)$. The TC's around Tiers 4,5 , and 6 had some peaks around $1000^{\circ} \mathrm{C}$ $\left(1830^{\circ} \mathrm{F}\right)$, but the average dropped to approximately 700 to $800^{\circ} \mathrm{C}\left(1290\right.$ to $\left.1470^{\circ} \mathrm{F}\right)$. The ceiling TC measured a spike between 1300 to $1400^{\circ} \mathrm{C}\left(2370\right.$ to $\left.2550^{\circ} \mathrm{F}\right)$, but averaged approximately $800^{\circ} \mathrm{C}\left(1470^{\circ} \mathrm{F}\right)$.

Temperatures measured by TC Tree No. 3, between Racks 2 and 3, measured peak temperatures of $1000^{\circ} \mathrm{C}\left(1830^{\circ} \mathrm{F}\right)$ at Tier 1 and increased to approximately $1300^{\circ} \mathrm{C}\left(2370^{\circ} \mathrm{F}\right)$ at Tiers 2 through 6 and the ceiling TC, with averages between 800 and $900^{\circ} \mathrm{C}(1470$ and $1650^{\circ} \mathrm{F}$ ) for all tiers and the ceiling TC. The average temperatures in this TC tree were higher 
than measured by TC Trees 1 and 2 due to the large volume of flame inside the racks from both the pool fire and the burning drums and contents.

On Rack 1, TC's weided to the structural steel measured temperatures on the East end of the rack (TC's 158 through 161) of approximately 250 to $300^{\circ} \mathrm{C}\left(480\right.$ to $570^{\circ} \mathrm{F}$ ), on the middle support (TC's 156 and 157) they ranged from 500 to $650^{\circ} \mathrm{C}$ (930 to $1200^{\circ} \mathrm{F}$ ) (facing the fire), and at the center of the rack (TC's 152 through 155) they were approximately 250 to $300^{\circ} \mathrm{C}\left(480\right.$ to $\left.570^{\circ} \mathrm{F}\right)$.

Temperatures measured on the East end of Rack 2 (TC's 162 through 167) ranged from 700 to $1200^{\circ} \mathrm{C}\left(1290\right.$ to $\left.2190^{\circ} \mathrm{F}\right)$, as expected, since this end of the rack was immersed in the fire. TC's $167,0.89 \mathrm{~m}$ (35 in.) above the ground and TC $166,1.2 \mathrm{~m}(4 \mathrm{ft}$ ) above TC 167, measured steel temperatures of approximately 1100 to $1200^{\circ} \mathrm{C}\left(2000\right.$ to $\left.2190^{\circ} \mathrm{F}\right)$ which led to the structural collapse of the rack system. TC's 168, 170, 171, 173, 174, and 176, located at the middle of the East half of Rack 2, measured steel temperatures ranging from 800 to $1100^{\circ} \mathrm{C}\left(1470\right.$ to $\left.2000^{\circ} \mathrm{F}\right)$. TC's 177 through 180 , attached to the middle of the north side of Rack 2, measured temperatures of approximately 200 to $300^{\circ} \mathrm{C}\left(390\right.$ to $\left.570^{\circ} \mathrm{F}\right)$ and TC's 181 through 183, attached to the middle of the West half, North face of Rack 2. measured steel temperatures of 75 to $225^{\circ} \mathrm{C}\left(167\right.$ to $\left.437^{\circ} \mathrm{F}\right)$. TC's 172 and 175 , which were attached to the South side of Rack 2 (between Racks 2 and 3) in the middle of the East half, in the fire measured structural steel temperatures of approximately $1000^{\circ} \mathrm{C}\left(1830^{\circ} \mathrm{F}\right)$. Four TC's welded to the North side of Rack 3 (between Racks 2 and 3) at the middle of the rack system, measured steel temperatures ranging from 160 to $350^{\circ} \mathrm{C}\left(320\right.$ to $\left.660^{\circ} \mathrm{F}\right)$.

A group of three TC's were (1) Welded to the bottom of Drum X232 (TC 187), (2) directly below the bottom center of the drum on the mesh (TC 188), and (3) directly below the bottom center of the drum, weided to the top of the pan (TC 189). Another group of three TC's were welded below Drum X236 (TC's 184, 185, 186, respectively). The TC's welded to the bottom of the drum and the wire mesh measured temperatures of approximately $700^{\circ} \mathrm{C}$ $\left(1290^{\circ} \mathrm{F}\right)$ for Drum $X 236$ and $850^{\circ} \mathrm{C}\left(1650^{\circ} \mathrm{F}\right)$ for Drum $X 232$. The temperatures on the top of the pan were approximately $850^{\circ} \mathrm{C}\left(1650^{\circ} \mathrm{F}\right)$ under Drum $\times 236$, and the TC under Drum $\times 232$ malfunctioned, providing no temperature reading.

\subsubsection{Drum and Rack Inspection}

Post-test inspection of the drums showed that all drums in Racks 2 and 3 positioned in the pool fire (positions 1 through 4) experienced extensive burning of the contents and lid loss varying from a complete loss of lid (i.e., lid fallen to floor) to the lid being open (i.e., ruptured), but still attached to the drum. The remaining drums on the west half of Racks 2 and 3 and the entire Rack 1 were intact and showed no signs of lid loss or bulging. The lids in Rack 1 adjacent to the fire (Bays 1 through 4) remained on the drum without signs of rupture, but almost all experienced some degree of gasket burning around the bolt and/or on the fire side. The fire side of all drums had the paint burned off the drum, and Tiers 4 through 6 showed a heavy accumulation of soot. On the 3rd to 6 th tier, the underside of the pans (facing lower tier drum lids) experienced a heavy accumulation of soot. The drums on the top three tiers of Racks 2 and 3 had a heavy accumulation of soot on them and experienced a slight amount of heat damage. Post-test examination of the drums was performed and the results are provided in Appendix $D$. Appendix $D$ contains both the pre-test drum data sheets and the post-test drum data sheets for each test drum. Figure 44 provides a post-test damage 
sketch of the drums showing lid loss information and burn patterns. In this sketch, the term "lid loss" pertains to those drums which had the lid completely separated from the drum, i.e., lid was completely out of closing ring. The term, "lid rupture," pertains to those drums which had the lid partially removed from the metal closure ring. Overall, a total of 19 lids were separated from the metal closure ring, and 18 lids were partially separated from the closure ring.

Drum weight loss values are provided in Figure 45. The weight loss values for the drums in the immediate fire area (Rows 2 and 3, Bays 1-4, Tiers 1-6) are suspect for the following reasons:

- Water was used to either extinguish or partially extinguish these drums, and while free water was poured out of the drums prior to post-test weighing, the cotton and charred materials did retain some of the water; and

- Some of the drums continued to smolder after the test and could not be extinguished until they were removed from the test building. This delay allowed some drums to smolder for $12-16$ hours.

It is estimated that the drums in the immediate fire area lost 50-75 percent of their combustible contents.

The drums in Rack 1 showed very little weight loss, i.e., from $0-0.3 \mathrm{~kg}(0-0.6 \mathrm{lb})$. The drums in Racks 2 and 3, Bays 5-8, Tiers 1-6 also showed very little weight loss. The drums in the sixth tier, outside the immediate fire area showed the greatest weight loss 10 $0.5 \mathrm{~kg} 10-1.2 \mathrm{lb} /)$ when compared to drums in the same position but in other tiers. This is probably due to their exposure in the hot layer for a longer period of time than the drums in the lower tiers.

The seismic bar on Tier 1 was bowed outward approximately $101.6 \mathrm{~mm}$ (4 in.) due to thermal expansion from the fire. The remaining main beams and seismic bars in Rack 1 did not show any visible signs of heat distortion. The west half of Racks 2 and 3 experienced no heat damage, but Tiers 4,5 , and 6 were heavily covered with soot. The main beams and seismic bars were intact and straight.

The east half of Racks 2 and 3 were completely destroyed and were listing approximately 0.91 to $1.2 \mathrm{~m}(3$ to $4 \mathrm{ft})$ to the south at the top from their original positions. Figure 46 provides a diagram showing the distortion of the east end of Rack 3 . The interior of the rack system collapsed onto itself with the drums on the 1 st tier acting as columns and supporting the remaining tiers. The wire mesh that was used to support the drums in the 
WHC-SD-WM-TRP-246 REV. 0

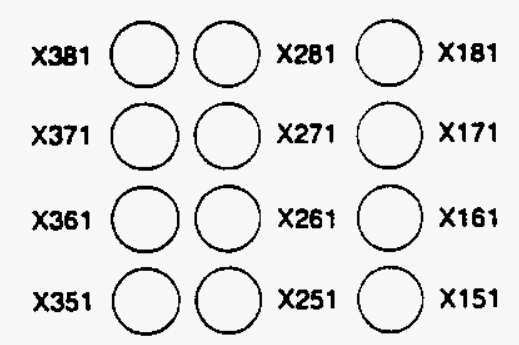

Tier 1

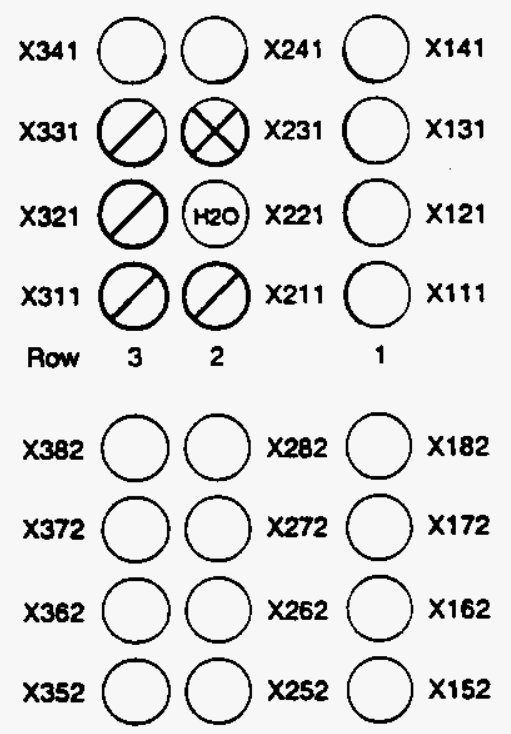

Tier 2
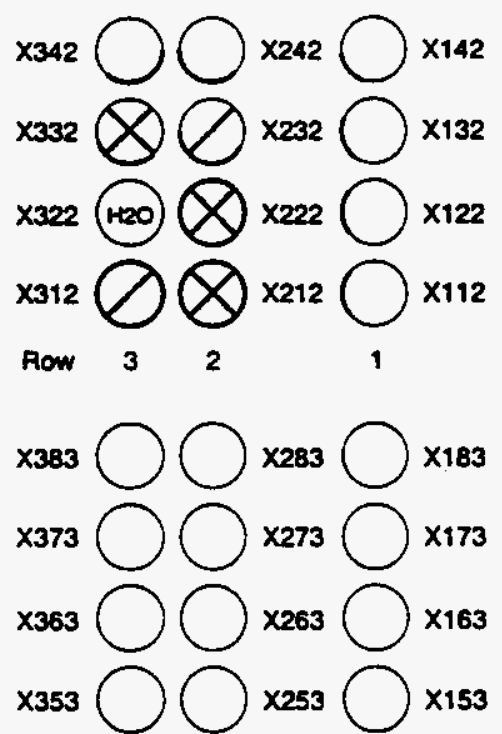

Tier 3

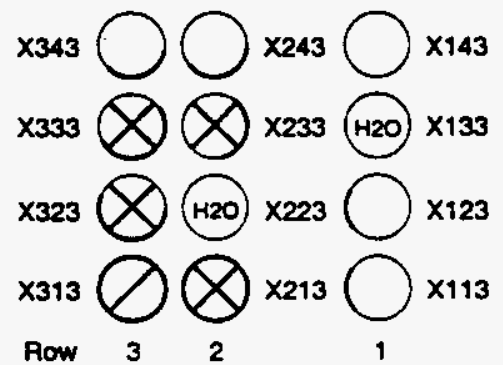

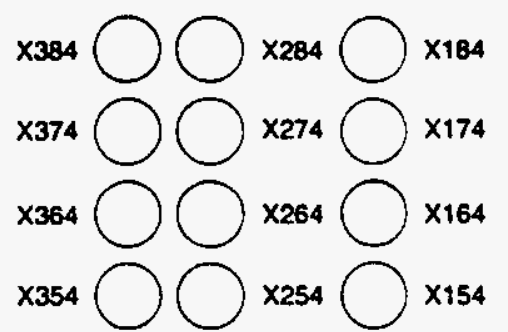

${ }^{x 344} \bigcirc \bigcirc \times 244 \bigcirc \times 144$

${ }^{\times 334} \bigcirc \times 134$

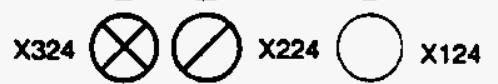

x314 $\bigotimes \times 214 \bigcirc \times 114$

now $3 \quad 2$,

x385 $\bigcirc \times 285 \bigcirc \times 185$

$x 375 \bigcirc \times 275 \bigcirc \times 175$

x365 $\bigcirc \times 265 \bigcirc \times 165$

x355 $\bigcirc \times 255 \bigcirc \times 155$

x345 $\bigcirc \bigcirc \times 245 \bigcirc \times 145$

${ }^{3335} \bigcirc{ }^{\times 235} \bigcirc \times 135$

${ }^{x 325} \bigotimes \bigcirc \times 225 \bigcirc \times 125$

${ }^{315} \bigcirc \bigcirc \times 215 \bigcirc \times 115$

Row 32

${ }^{3386} \bigcirc \bigcirc \times 286 \bigcirc \times 186$

$\times 376 \bigcirc \times 276 \bigcirc \times 176$

${ }_{x 366} \bigcirc \bigcirc \times 266 \bigcirc \times 166$

x356 $\bigcirc \times 256 \bigcirc \times 156$

x346 $\bigotimes \times 246 \bigcirc \times 146$

${ }^{x 336} \bigotimes \bigcirc_{\times 236} \bigcirc^{136}$

${ }^{326} \otimes \bigcirc_{\times 226} \bigcirc_{x 126}$

x316 $\bigcirc \times 216 \bigcirc \times 116$

Tier 4

Tier 5

Tier 6

Note: Drum number indicates Test-Row-Bay-Tier.

$\otimes$ Lid Loss $\oslash$ Rupture $\bigcirc$ Bum Pattern

Figure 44. Post-test Drum Assessment 
Tier 1

$$
\begin{aligned}
& x 381 \bigcirc 0 \times 2810 \times 181 \\
& x 371 \bigcirc \times 0710 \times 171 \\
& x 361000 \times 26100 \times 161 \\
& x 35100 \times 2510 \times 151
\end{aligned}
$$$$
x 34100.0 \times 2410 \times 141
$$$$
\times 331 \text { (42) } 8.92310 .1 \times 131
$$$$
\times 321 \text { (20) } 1020.2210121
$$$$
\times 3113.23 .7 \times 2110.9 \times 111
$$$$
\text { Row } 32
$$$$
x 38200 \times 2820 \times 182
$$$$
x 37200 \times 2720 \times 172
$$$$
x 362002620 \times 162
$$$$
x 352002520 \times 152
$$

Tier 2

Tier 3

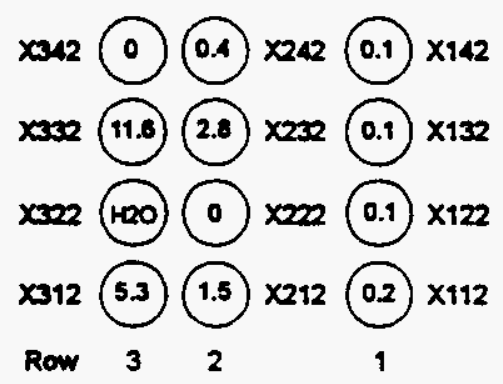

$x 38300 \times 2830 \times 183$

$x 37300 \times 2730 \times 173$

$x 36300 \times 2600103$

$x 353002530 \times 153$

$\times 3430.20 .1 \times 2430 \times 143$

$\times 333$ (13.8) $\times 233$ (12.9 $\times 133$

x323 12.0 $\times 223010123$

$\times 313$ 12.2 $13.0 \times 213 \bigcirc \times 113$

Row 321

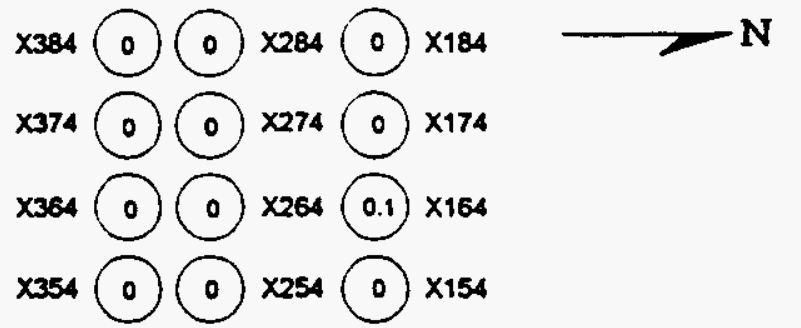

$\times 34400.7 \times 2440 \times 144$ $\times 3345.8010 .3 \times 2340 \times 134$ $\times 324$ (23.2) $02240 \times 124$ $\times 314$ (11.6) $\times 2140.1 \times 114$ Row 32

$x 3850 \times 2850 \times 185$ $x 37500 \times 275 \bigcirc \times 175$ $\times 365 \bigcirc \times 205010.165$ $x 355 \bigcirc \times 2550 \times 155$ $\times 345$ (18.7) $0.7 \times 2450.1 \times 145$ x335 $17.482 \times 2350 \times 135$ $\times 325$ (172) $32250 \times 125$ $x 315 \underset{\text { Row }}{12.7} \times \underbrace{0.5}_{2} \times 215 \underbrace{0}_{1} \times 115$ x386 0.1 $0.1 \times 2860.1 \times 186$ $\times 3760.402 \times 276010 \times 176$ $\times 3680.502030106$ $\times 3560.502500156$ $\times 346$ (14.6) $15.2 \times 2460146$ $\times 336$ 19.4 $0236013 \times 136$ $\times 326$ (18.5) $\times 2260126$ $\times 316$ (19.1) $5.6 \times 2160116$ Row 321
Tier 4

Tier 5

Tier 6

Note: Drum number indicates Test-Row-Bay-Tier.

Value $=$ Weight Loss $(\mathrm{kg})$

Figure 45. Weight Loss Data - Rack Storage Test 
WHC-SD-WM-TRP-246 REV. 0

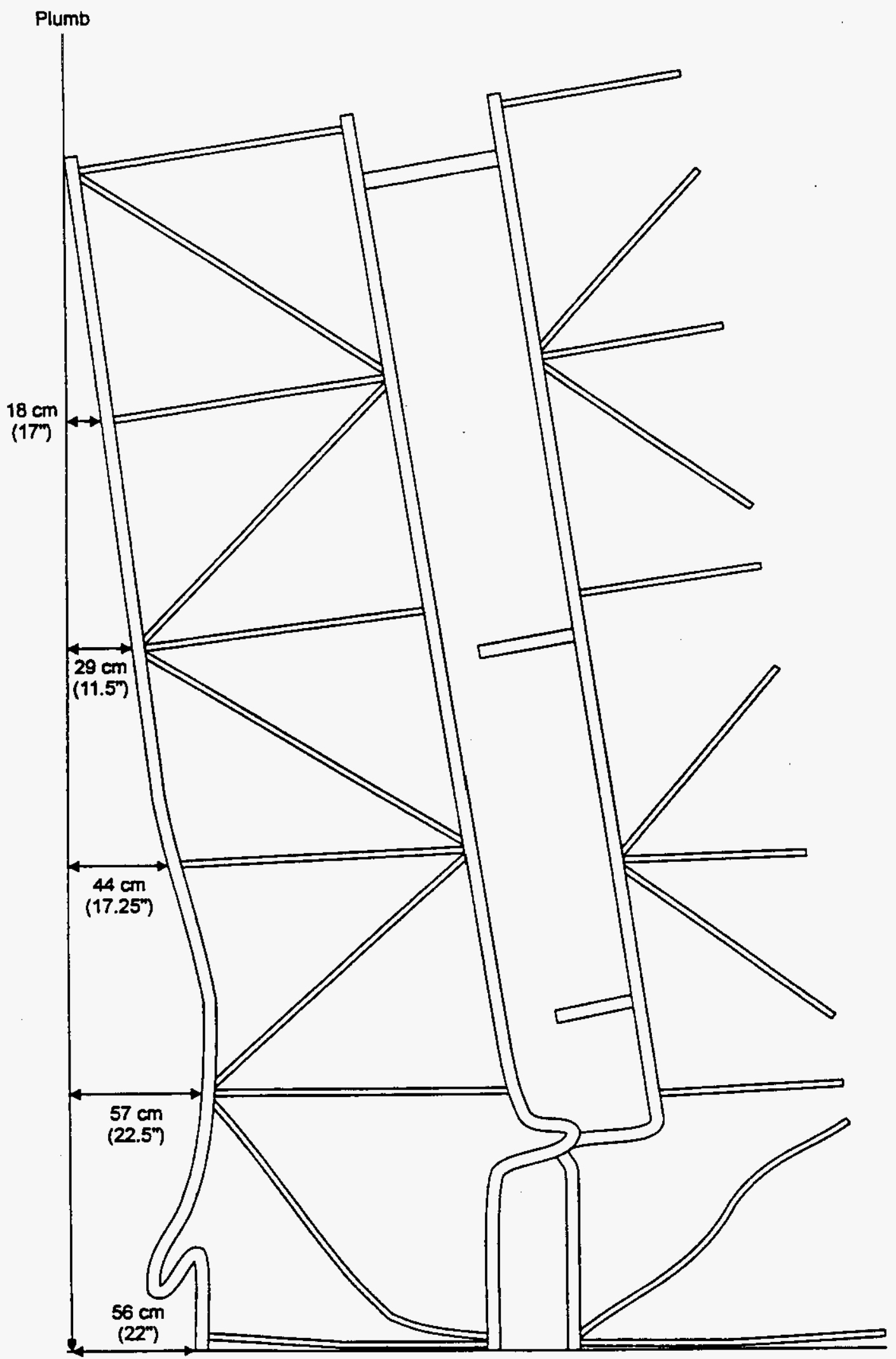

Figure 46. Post-test Damage, End of Rack 3 
racks, was distorted such that the weight of the drums caused the mesh to collapse. This movement allowed the drums to rest on top of each other with the mesh and the containment pans in between the drums. The distortion of the rack also caused the drums to slightly topple between the horizontal beams. No drums fell out of the rack, but drums in Bays 1, 2, and 3 had fallen over to some degree with Bay 1 being the most severe. The seismic bars and the horizontal members provided the support so that the drums did not fall from the rack. The drums in Bay 4 were only slightly tilted to the east end of the rack. Figures 47 and 48 provide post-test photographs of the rack storage array.

\subsection{PALLETIZED STORAGE TEST}

The palletized storage test was conducted on June 12, 1995. The ambient temperature at the start of the tests was $23^{\circ} \mathrm{C}\left(73^{\circ} \mathrm{F}\right)$. Data plots for the pressures, temperature, and heat flux data obtained during this test is provided in Appendix $E$.

\subsubsection{Visual Observations}

During the fire test, visual observations were made. A summary of the observations is provided in Appendix E. It should be noted that determinations of specific drums rupturing or exploding were difficult due to viewing angles and the engulfing pool fire itself.

In general, the first drum exploded at 2:07 after ignition of the pool fire. Prior to this, flaming around the lids of several drums in the pool fire area was noted. During the test, the observers could hear the drums venting and as the venting/pressure increased, the drum would explode. From the observations, it was estimated that $14-16$ drums exploded during the test.

During the test, no lids were completely expelled from the drums. Several times during the fire, a drum would explode, would expel burning pieces of drum contents, and these contents would become airborne. No large pieces of contents were expelled, and only several small pieces were observed on the floor, post-test.

At 5:46 into the test period, the extinguishment of the pool fire was initiated. The pool fire was extinguished at 5:57 into the test period. The drums in Rows $6,7,8$, and 9, Bays 1 4 , continued to burn after the pool fire was extinguished. The wood pallets were also burning in these areas. In Row 4, no burning of the drums was noted and in Row 5 several of the drums continued to burn around the lid area with small flames present.

The burning of the drums in Rows 6 -9. Bays $1-4$, gradually reduced in intensity as the drums cooled and the wood pallets completely burned and charred. At approximately 12 minutes, the drums in Rows 8 and 9. Bays 1-2, Tier 3, began to lean. The leaning was due to the disintegration of the wood pallet under these drums. The steel band did allow the drums to remain in place and they did not fall. 


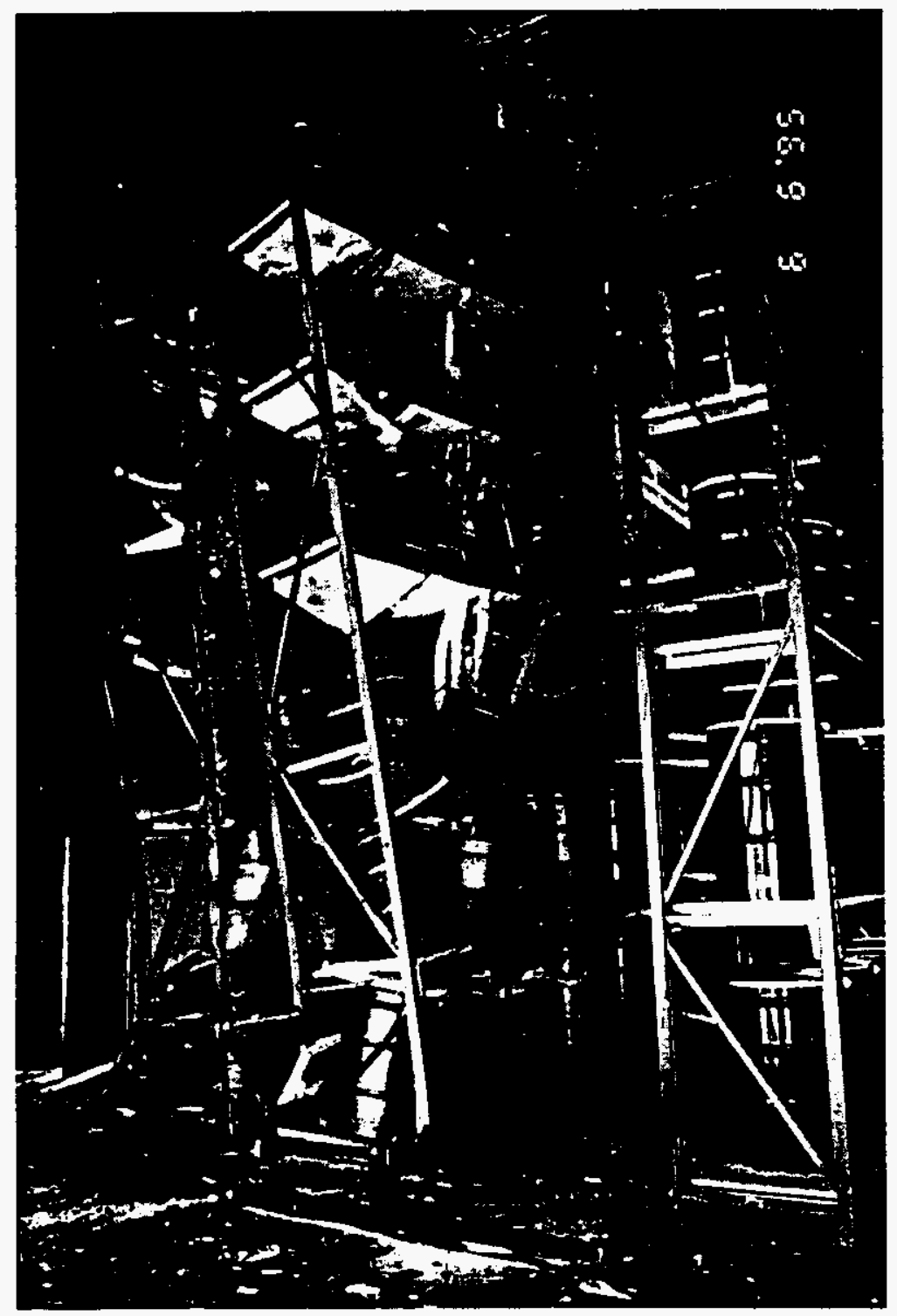

Figure 47. Post-test Photo - Rack Array 

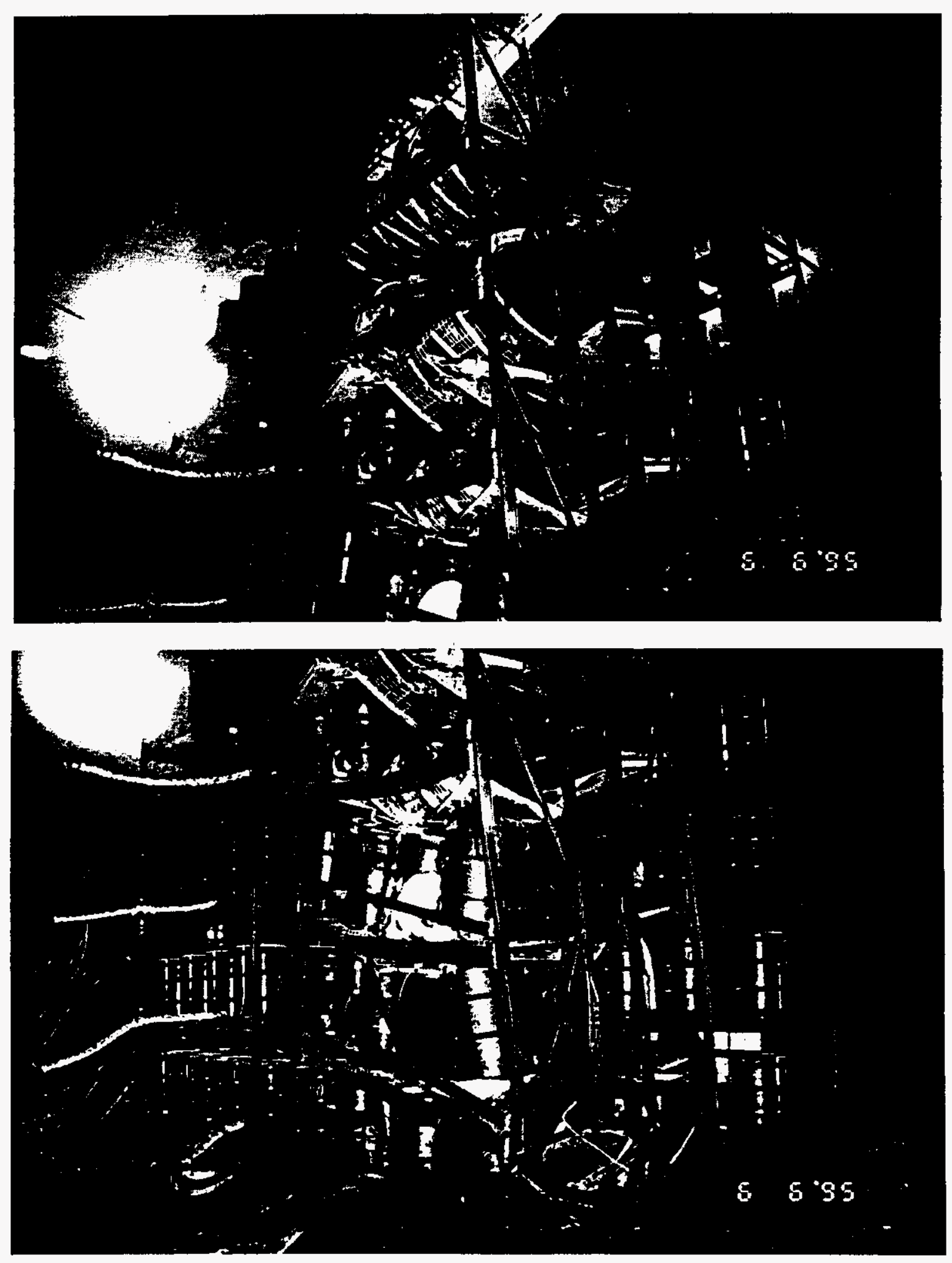

Figure 48. Post-test Photo - Rack 3 
The fire slowly progressed to the west through Rows $6,7,8$, and 9 . The progression of the fire was due to the wood pallets burning. As the pallet burned, it would heat the drum below it, cause burning around the lid, the pallet fire would intensify and heat the drum above, which in turn would begin to burn around its lid. During this progression of fire, no drums exploded however, venting was occurring.

The fire did progress through Rows 6 and 7, and it progressed through three of the pallets for Rows 8 and 9 . Figure 49 provides photographs showing the fire as is progressed through the pallet rows. The steel pallets did not exhibit any fire progression. Overall, it took approximately 70 minutes to progress through Rows 6 and 7 .

During the test, no drums fell to the floor or toppled from their initial positions.

\subsubsection{Pressure Data}

Of the 22 drums instrumented with pressure transducers, 16 showed a sudden pressure drop and the internal pressure rose slowly in the remaining 6 drums before venting or cooling resulted in a slow pressure decrease. Table 5 shows the order each pressure transducer reached a peak pressure, the time it occurred.

Drum 0743 peaked at a pressure of approximately $27.6 \mathrm{kPa}$ (4 psig) and then experienced a sharp pressure decrease due most likely to a quick venting, not an expiosion because of the relatively slow pressure drop off to ambient. Immediately after venting, the internal drum pressure began to rise over approximately the next $300 \mathrm{sec}$ to a peak pressure of approximately $43.1 \mathrm{kPa}(6.25 \mathrm{psig})$ and then slowly vented to ambient.

Several of the drums (0612 and 0633) had a double spike in the internal pressure/time plots. This may be attributed to a pressure rise until venting occurred which relieved the pressure, followed by an increase in pressure which the venting action could not relieve thus causing the drum to explode.

\subsubsection{Total Heat Flux Data}

A total of 12 total heat flux transducers (calorimeters) with $180^{\circ}$ view angles were positioned at various locations throughout the pallet system to define the heat flux environment imposed on the pallets and the drums in the pool fire.

The first group of calorimeters $\left(C 1, C_{2}\right.$, and $\left.C 3\right)$ were located outside the fire between Positions 2 and 3 of Row 8 looking north (into the fire) and measured peak total heat flux values between approximately 200 and $325 \mathrm{~kW} / \mathrm{m}^{2}$ with averages between 50 and 100 $\mathrm{kW} / \mathrm{m}^{2}$. The sharp peaks measured by $\mathrm{C} 2$ and $\mathrm{C} 3$ occurred at the same time possibly indicating that a nearby drum vented directly into the transducer' field of view, producing the extremely high instantaneous heat flux levels. The second group of calorimeters (C4, C6, and C8) were located on a vertical stand outside the fire next to Row 6, Position 5 drums looking east (into the fire) and measured peak total heat flux values between approximately 140 and 

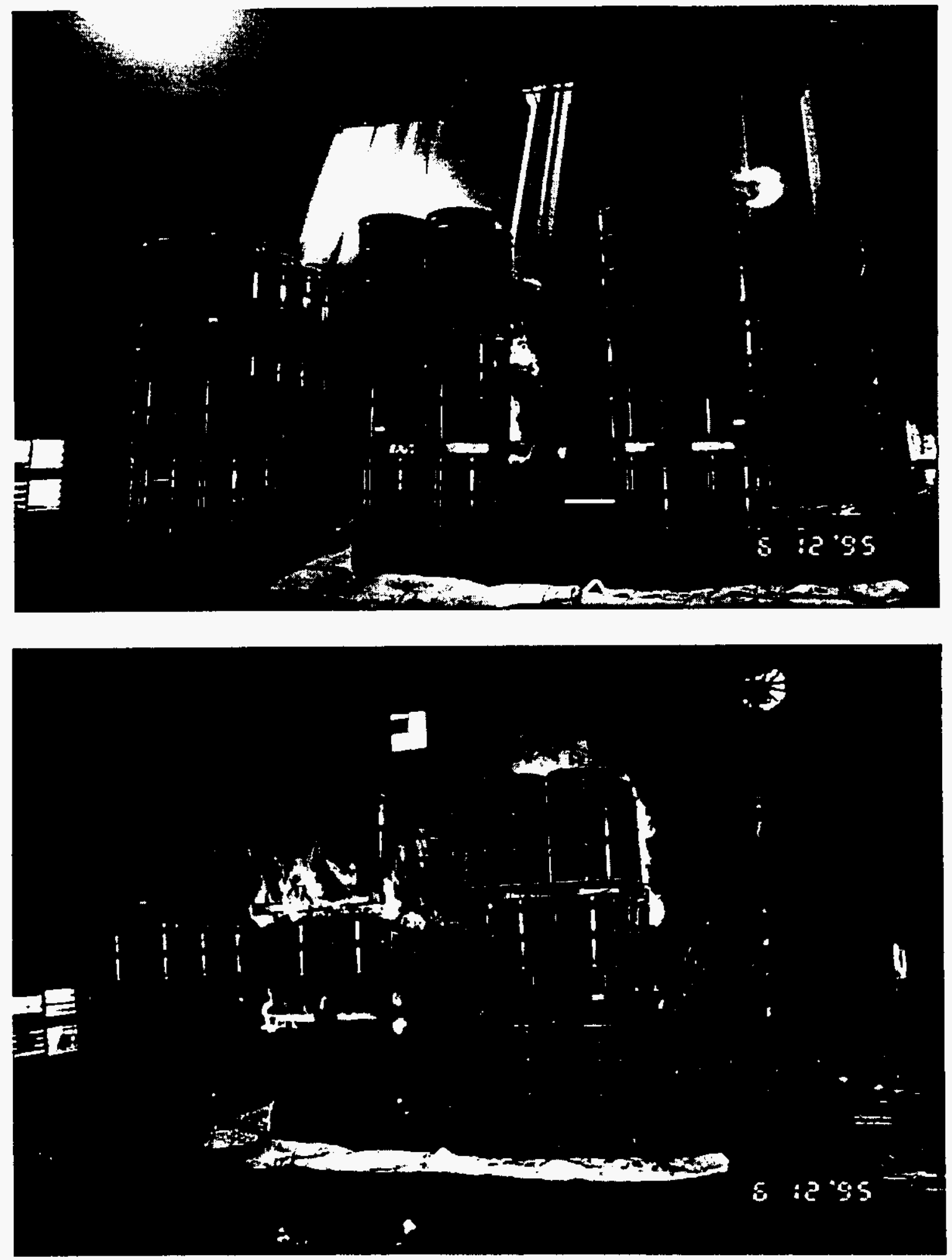

Figure 49. Burning Progressing through Pallet Array 
$225 \mathrm{~kW} / \mathrm{m}^{2}$ with averages around $100 \mathrm{~kW} / \mathrm{m}^{2}$. The third group of calorimeters $(\mathrm{C} 5, \mathrm{C} 7$, and C9) were located in the fire between Positions 2 and 3 of Row 6 looking north and measured peak total heat flux values of approximately $225 \mathrm{~kW} / \mathrm{m}^{2}$ with averages around 75 to 150 $\mathrm{kW} / \mathrm{m}^{2}$. Calorimeters $\mathrm{C} 10, \mathrm{C} 11$, and $\mathrm{C} 12$ were located outside the fire between Positions 2 and 3 of Row 5 looking south (into the fire) and measured peak total heat flux values of approximately 225 to $250 \mathrm{~kW} / \mathrm{m}^{2}$ and averages around 100 to $175 \mathrm{~kW} / \mathrm{m}^{2}$.

Table 5. Peak Pressures Measured by Each Drum

\begin{tabular}{|c|c|c|c|}
\hline Order & Drum No. & Peak Pressure (psig) & Time (min:sec) \\
\hline 1 & 0631 & 16.5 & $2: 27$ \\
\hline 2 & 0812 & 17.4 & $2: 32$ \\
\hline 3 & 0811 & 11.4 & $2: 32$ \\
\hline 4 & 0713 & 15.4 & $2: 34$ \\
\hline 5 & 0632 & 17.3 & $2: 35$ \\
\hline 6 & 0711 & 14.0 & $2: 37$ \\
\hline 7 & 0721 & 12.0 & $2: 40$ \\
\hline 8 & 0611 & 14.5 & $2: 43$ \\
\hline 9 & 0612 & 9.5 & $2: 47$ \\
\hline 10 & 0722 & 16.1 & $2: 49$ \\
\hline 11 & 0813 & 14.7 & $2: 54$ \\
\hline 12 & 0723 & 11.6 & 3:02 \\
\hline 13 & 0741 & 3.1 & $3: 12$ \\
\hline 14 & 0641 & 9.0 & $3: 14$ \\
\hline 15 & 0521 & 12.8 & $3: 17$ \\
\hline 16 & 0532 & 11.4 & $3: 32$ \\
\hline 17 & 0633 & 9.9 & 4:05 \\
\hline 18 & 0533 & 11.7 & $4: 14$ \\
\hline 19 & 0541 & 6.9 & $4: 44$ \\
\hline 20 & 0831 & 5.8 & $5: 36$ \\
\hline 21 & 0742 & 8.6 & $8: 38$ \\
\hline 22 & 0743 & 6.4 & 12:35 \\
\hline
\end{tabular}

As with the rack storage test, the calorimeters which were not positioned in the fire measured total heat flux levels lower than the typical pool fire value $200 \mathrm{~kW} / \mathrm{m}^{2}$ probably because the flames did not fill the entire $180^{\circ}$ view due to the turbulence of the fire. As the 
wood pallets burned, Calorimeters $\mathrm{C} 1, \mathrm{C} 2, \mathrm{C} 3, \mathrm{C} 5, \mathrm{C} 7$, and $\mathrm{C} 9$ were shifted, changing their view, and Calorimeter $\mathrm{C} 3$ fell into the pool fire at $663 \mathrm{sec}$ during the test. When the pool fire was initially extinguished at $346 \mathrm{sec}$, all transducers responded with a sharp drop off to 20 or $30 \mathrm{~kW} / \mathrm{m}^{2}$. Subsequent re-ignition of the pool fire can be seen as spikes on the graphs.

\subsubsection{Drum Surface and Tree Temperatures}

TC's weided to the surface of drums located directly in the pool fire measured peak surface temperatures between 800 and $1100^{\circ} \mathrm{C}\left(1470\right.$ to $\left.2000^{\circ} \mathrm{F}\right)$ with Tier 1 being the coolest at 700 to $800^{\circ} \mathrm{C}\left(1290\right.$ to $\left.1470^{\circ} \mathrm{F}\right)$ and Tier 2 the hottest at $1100^{\circ} \mathrm{C}\left(2000^{\circ} \mathrm{F}\right)$.

The drums located in Rows 6 and 7, Bay 4, were the coolest, approximately 500 to $700^{\circ} \mathrm{C}\left(930\right.$ to $\left.1290^{\circ} \mathrm{F}\right)$ because there was not the total immersion in the flame. The drums in the first four bays of Rows 4, 5, 8 and 9, adjacent to the fire measured temperatures of approximately 500 to $600^{\circ} \mathrm{C}\left(930\right.$ to $\left.1110^{\circ} \mathrm{F}\right)$. Some peaks were around $1000^{\circ} \mathrm{C}\left(1830^{\circ} \mathrm{F}\right)$ as the fire was deflected into these rows.

As the fire propagated back through Rows 6 through 9, the initial temperatures were relatively low, under $200^{\circ} \mathrm{C}\left(390^{\circ} \mathrm{F}\right)$, but as the flame front approached, the surface temperatures peaked at around $800^{\circ} \mathrm{C}\left(1470^{\circ} \mathrm{F}\right)$.

TC Tree No. 1 (located in the fire between Rows 5 and 6, Bays 2 and 3) measured air temperatures in the first $1.2 \mathrm{~m}(4 \mathrm{ft})$ around $1000^{\circ} \mathrm{C}\left(1830^{\circ} \mathrm{F}\right)$, and every $1.2 \mathrm{~m}(4 \mathrm{ft})$ increment decreased by approximately $100^{\circ} \mathrm{C}\left(212^{\circ} \mathrm{F}\right)$. Until about $4.9 \mathrm{~m}(16 \mathrm{ft})$ above the floor (TC's 19 and 92), the temperature dropped to approximately $500^{\circ} \mathrm{C}\left(930^{\circ} \mathrm{F}\right.$ ), and over the next $3.7 \mathrm{~m}(12 \mathrm{ft})$, the average air temperature dropped slowly to approximately 350 to $400^{\circ} \mathrm{C}\left(660\right.$ to $\left.750^{\circ} \mathrm{F}\right)$. The TC located $76.2 \mathrm{~mm}(3 \mathrm{in}$.) below the ceiling peaked at approximately $400^{\circ} \mathrm{C}$ with averages around $300^{\circ} \mathrm{C}$. Because the pallets disrupted the pool fire, flame heights of less than $3.7 \mathrm{~m}(12 \mathrm{ft})$ were observed.

TC Tree No. 2 (between Rows 6 and 7, Bays 2 and 3, in the pallet array) measured air temperatures which peaked around $1000^{\circ} \mathrm{C}\left(1830^{\circ} \mathrm{F}\right)$ up to the ceiling, but occurred after $1000 \mathrm{sec}$ when the pallet array was fully burning. In the first $1000 \mathrm{sec}$, TC Trees 1 and 2

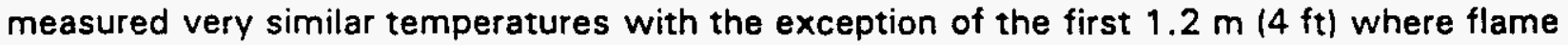
temperatures were measured by TC Tree No. 1. The peaks of $1000^{\circ} \mathrm{C}\left(1830^{\circ} \mathrm{F}\right)$ measured by TC Tree No. 2 lasted for approximately 500 sec.

\subsubsection{Post-Test Pallet Inspection}

Post-test inspection of the drums on the steel pallets (Rows 4 and 5) showed that only one drum (0521) experienced a lid loss. When the pallet above drum 0521 was lifted, the lid to drum 0521 was laying loose on top of the drum. Post-test examination of the drums showed that several failure mechanisms occurred. Figure 50 provides a photograph of Drum 0623 showing a ruptured lid. Figure 51 provides a photograph of Drum 0783 showing the bulging that occurred to many of the drums. This bulging may have allowed the drums to vent at the bottom seams of the drum. Figure 52 provides a photograph of Drum 0963 which shows that the bottom seam was ruptured allowing venting to occur. A majority of the drums adjacent to the fire showed signs of gasket burning around the bolt and/or on the fire side, and 


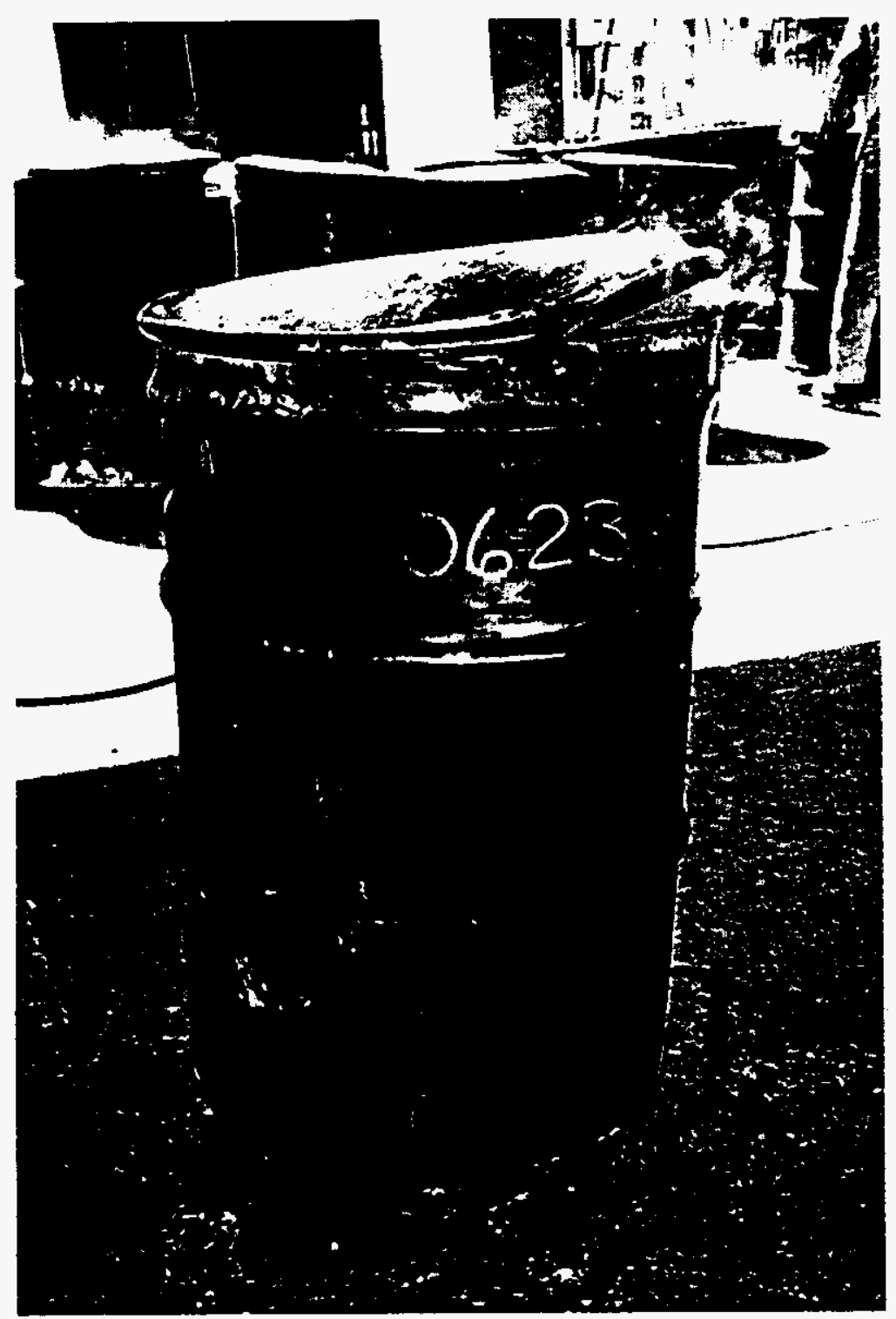

Figure 50. Photo - Lid Rupture 


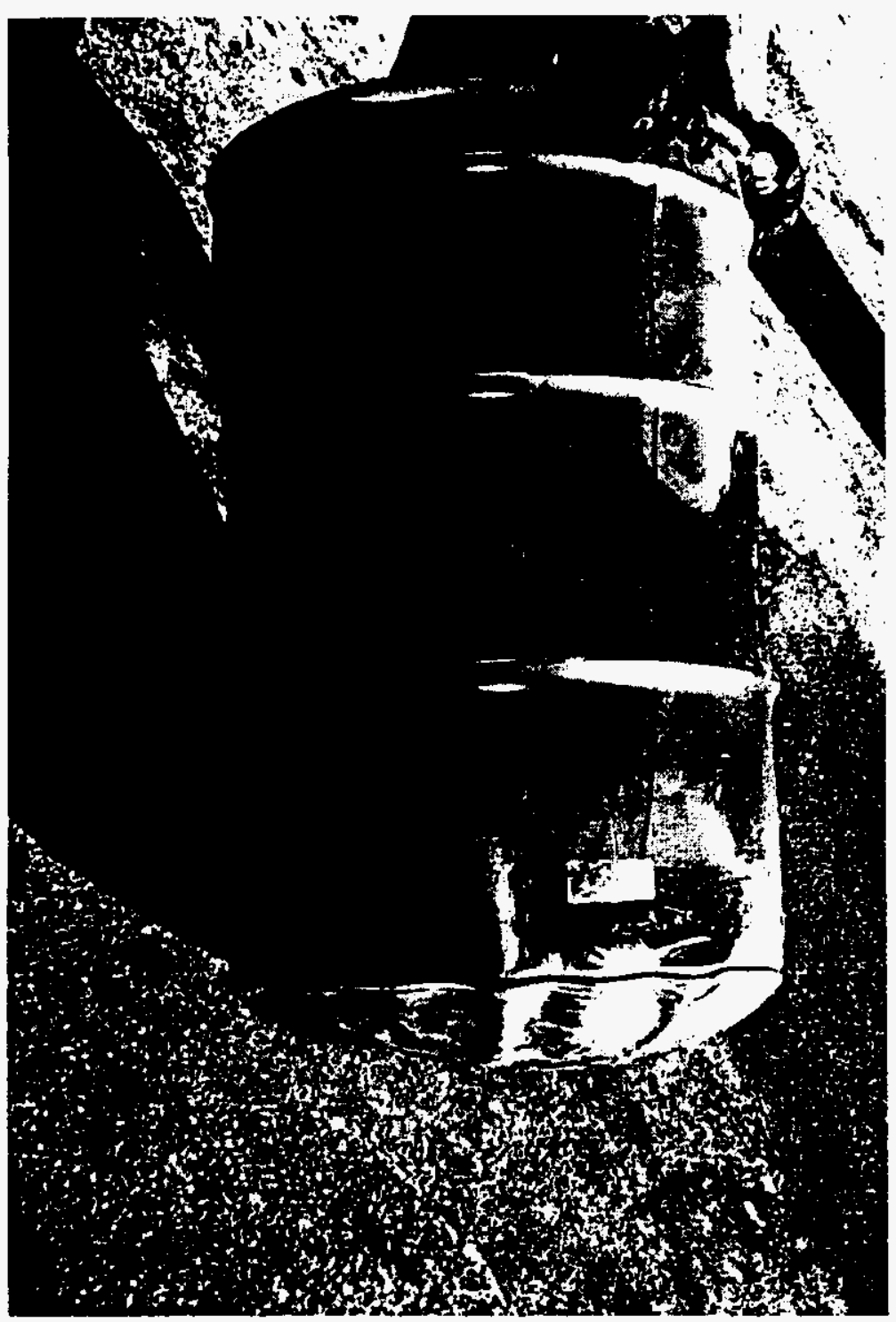

Figure 51. Photo - Bulging Drum 
WHC-SD-WM-TRP-246 REV. 0

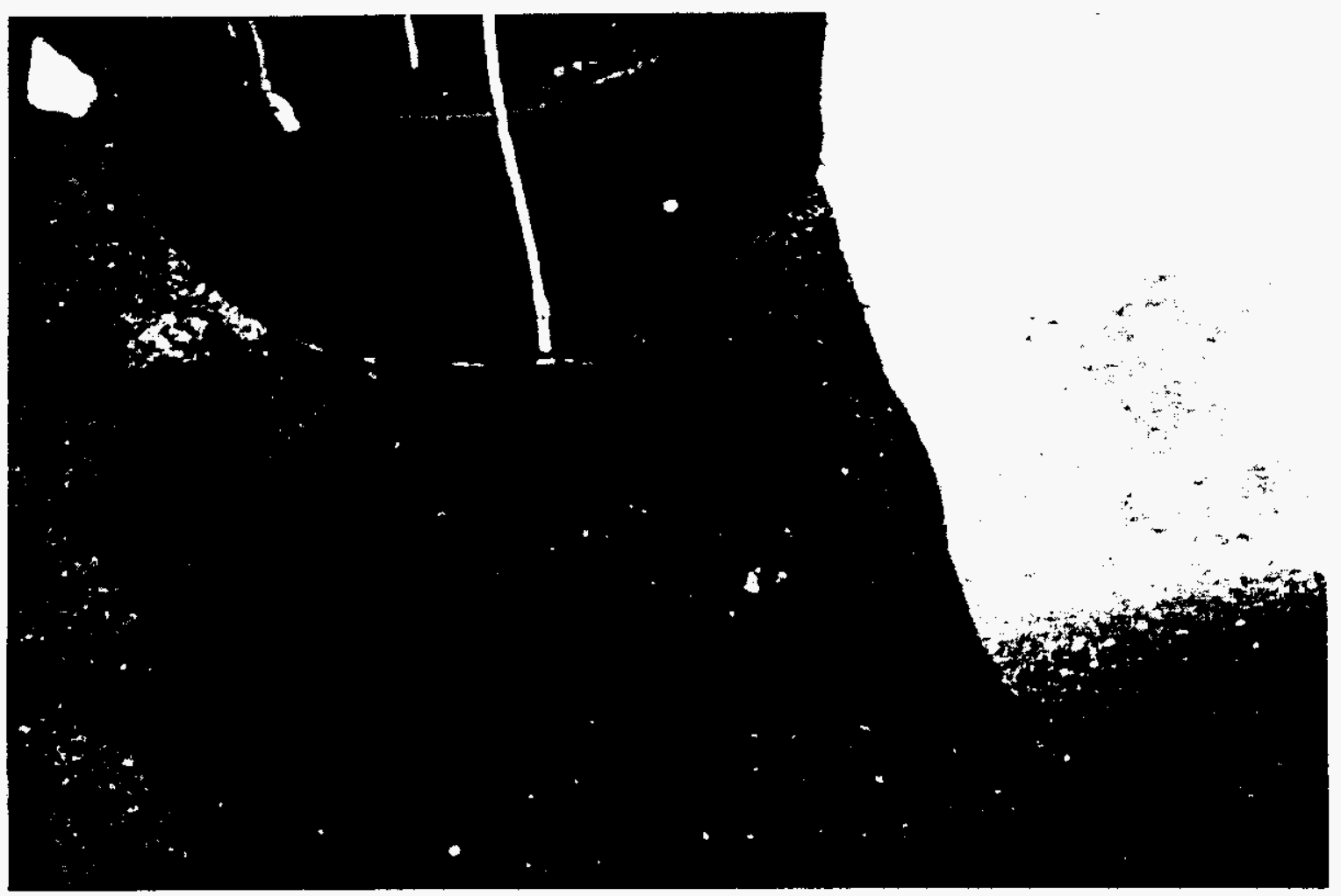

Figure 52. Photo - Seam Failure 
the fire side of all drums had the paint burned off the drums. Post-test examination of the drums was performed and the results are provided in Appendix $F$. Appendix $F$ contains both the pretest drum data sheets as well as the post-test drum data sheet for each test drum. Figure 53 provides a post-test damage sketch of the drums showing lid loss information and burn patterns. Overall, a total of 4 lids were separated from the metal closure ring, and 15 lids were partially separated from the closure ring.

Drum weight loss values are provided in Figure 54. The weight loss values for the drums in the immediate fire area (Rows 6 and 7, Bays 1-4, Tiers $1-3$ ) and the drums immediately adjacent (Rows 8 and 9, Bays 1-4, Tiers 1-3) are suspect for the following reasons:

- Water was used to either extinguish or partially extinguish some of these drums, and while free water was poured out of the drums prior to post-test weighing, the cotton and charred materials did retain some of the water; and

- Some of the drums continued to smoulder after the test and could not be extinguished until they were removed from the test building. This delay allowed some drums to smolder for 12-16 hours.

The drums in Row 4 showed very little weight loss, i.e., from $0-0.1 \mathrm{~kg}(0-0.2 \mathrm{lb})$. The drums in Row 5 also showed slightly higher weight loss, i.e., from $0-1.9 \mathrm{~kg}(0-4.2 \mathrm{lb})$. The drums in Rows 6, 7, 8, and 9 in Bays 5, 6, 7, and 8 show differing weight loss. The drums in Tier 3 generally show greater weight loss than the drums in Tier 2 which showed greater weight loss than the drums in Tier 1 . This is due to the fire spreading through the pallet array such that the higher tiers exhibited more flaming than in the lower tiers.

The steel pallets were not deformed in any way due to the heat from the fire. The drums positioned on the wood pallets inside the pool fire area experienced extensive burning of the contents and the lids were ruptured (i.e., not completely loose from the closure ring). The four wooden pallets (two in the flames and the two adjacent to the flames) were completely consumed and the drums on the upper tiers of these pallets had started to fall over (i.e., lean) during the test. Figures 55 and 56 provide post-test photographs of the rack storage array test. 
WHC-SD-WM-TRP-246 REV. 0

Tier 1

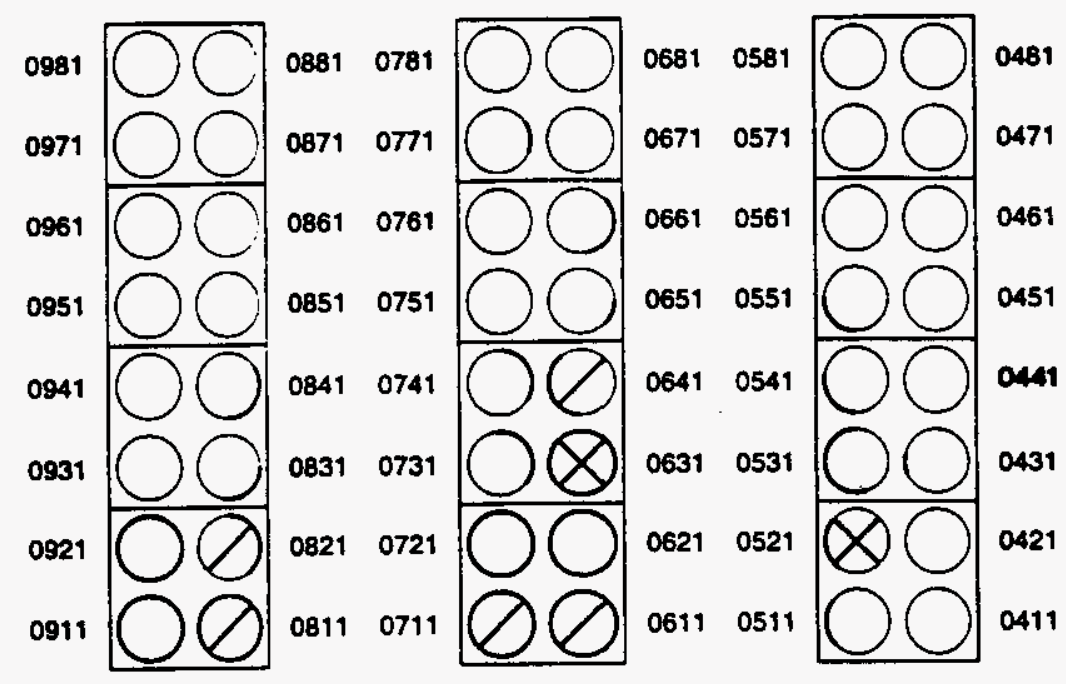

Tier 2

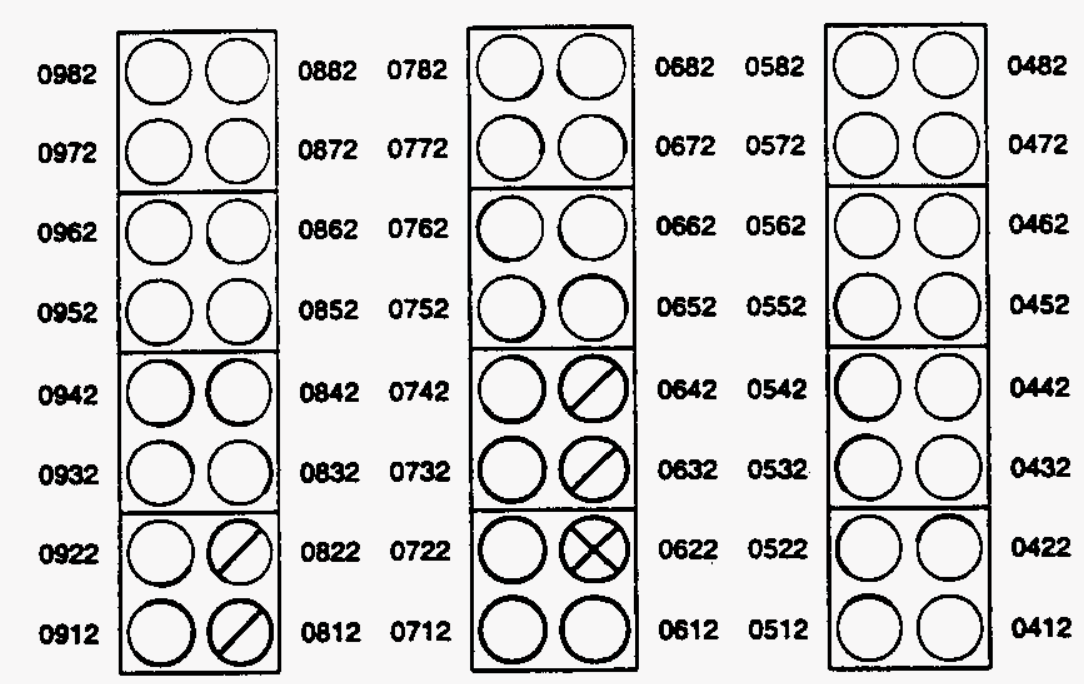

Tier 3

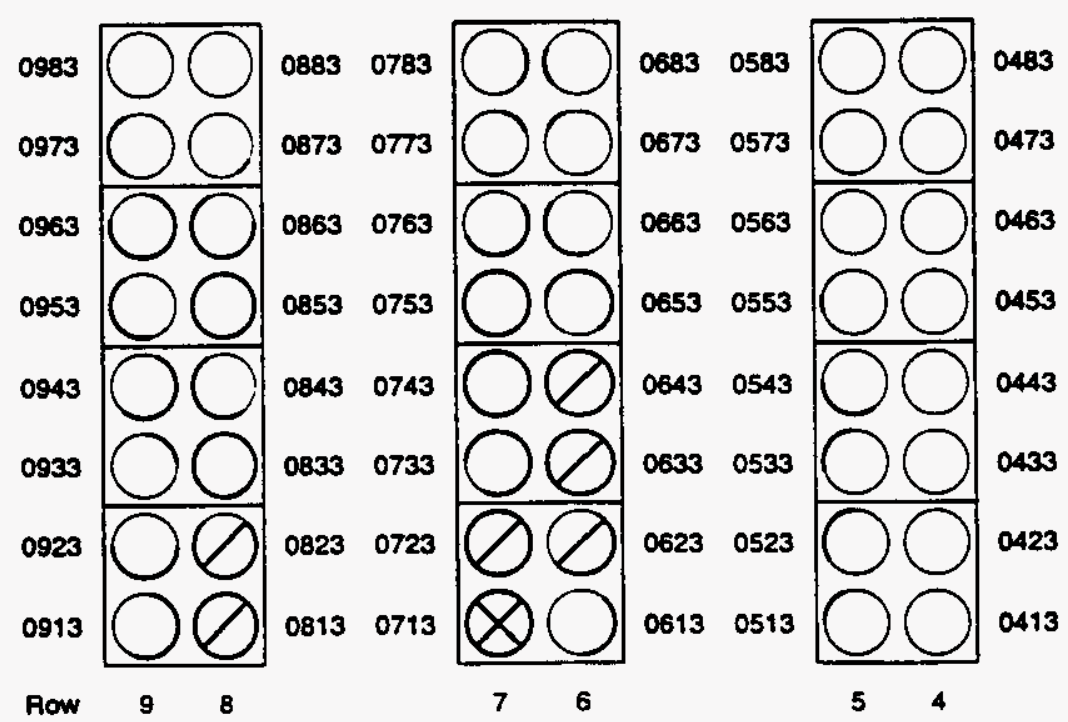

Note: Drum number indicates Test-Row-Bay-Tier.

$\otimes$ Lid Loss $\oslash$ Rupture $O$ Burn Pattem

Figure 53. Pallet Storage Drum Location Diagram 
WHC-SD-WM-TRP-246 REV. 0

Tier 1

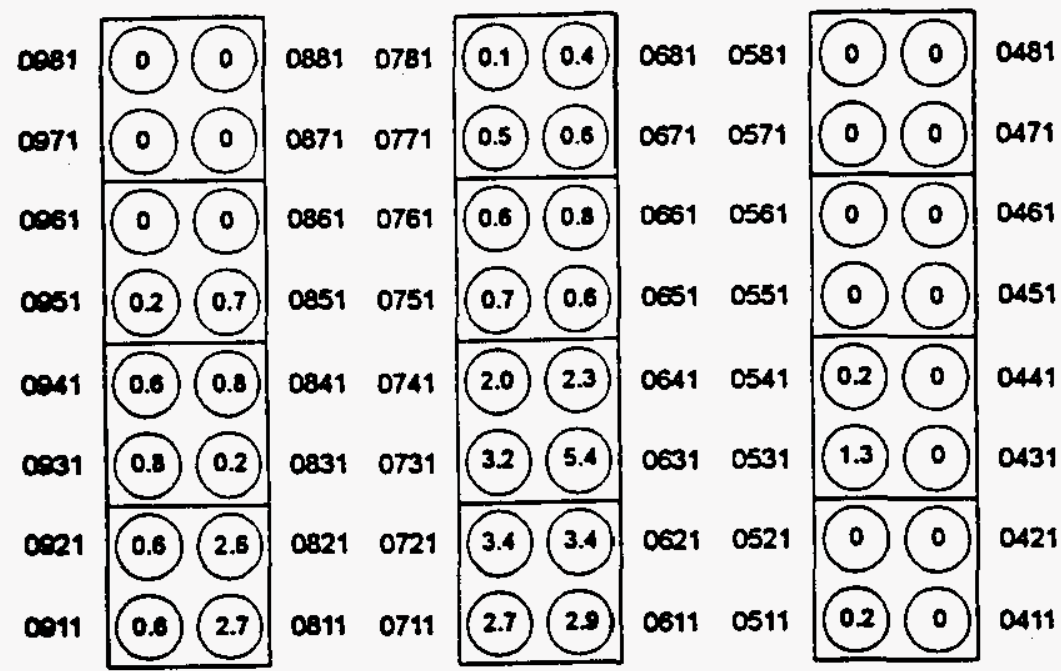

Tier 2

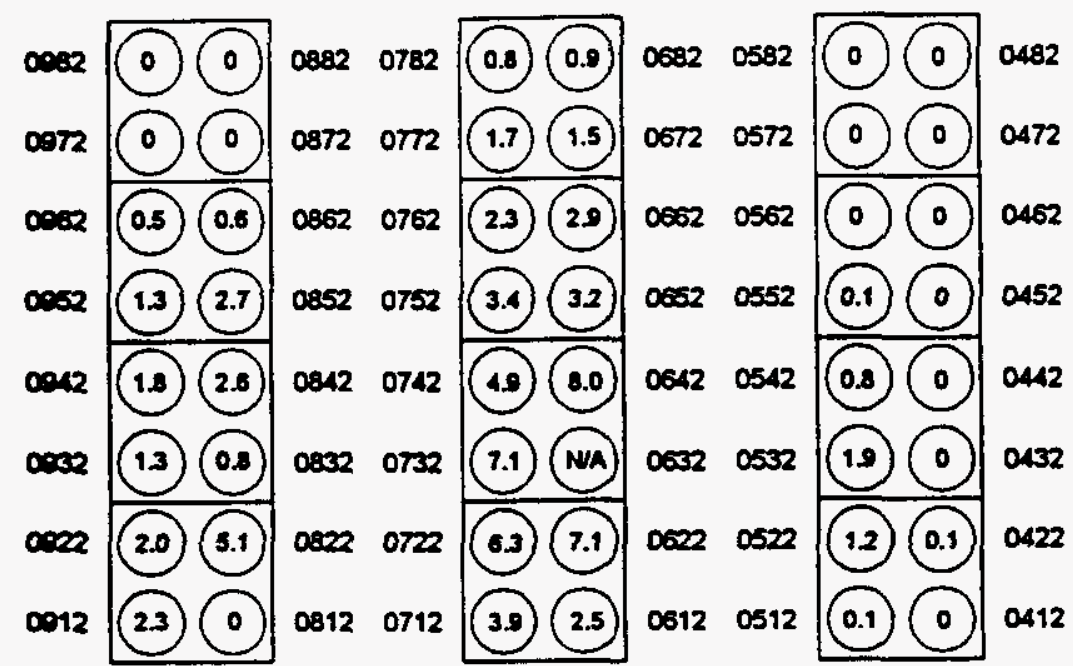

Tier 3

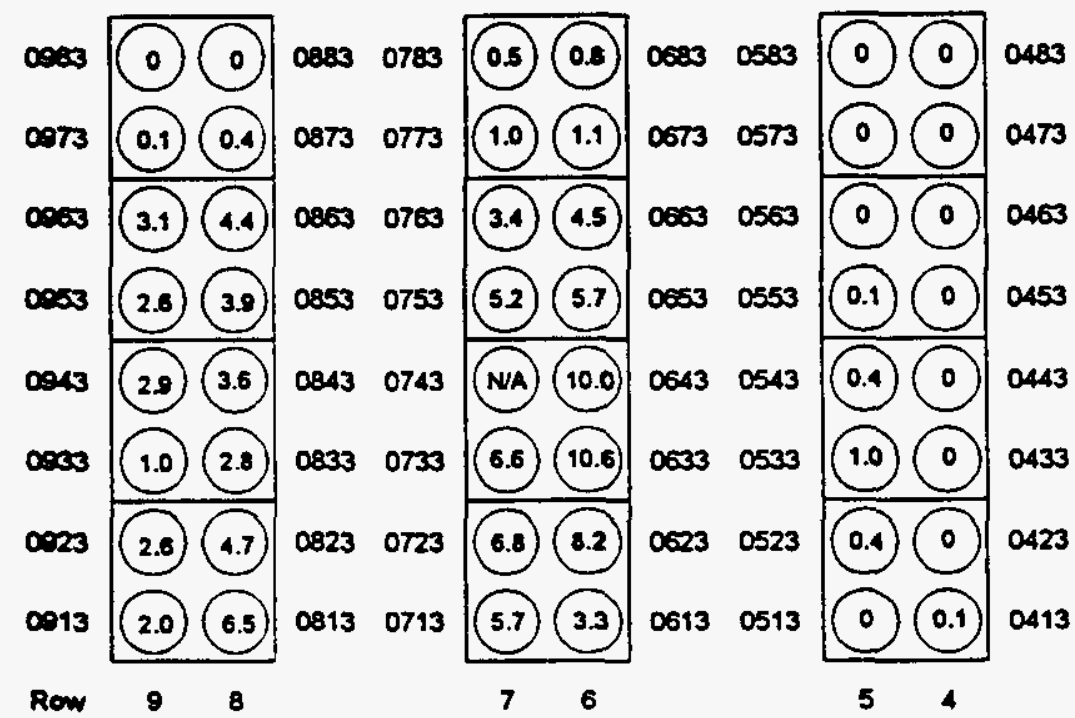

Note: Drum number indicates Test-Row-Bay-Tier.

$$
\text { Value }=\text { Weight Loss }(\mathrm{kg})
$$

Figure 54. Weight Loss Data - Pallet Array Test 


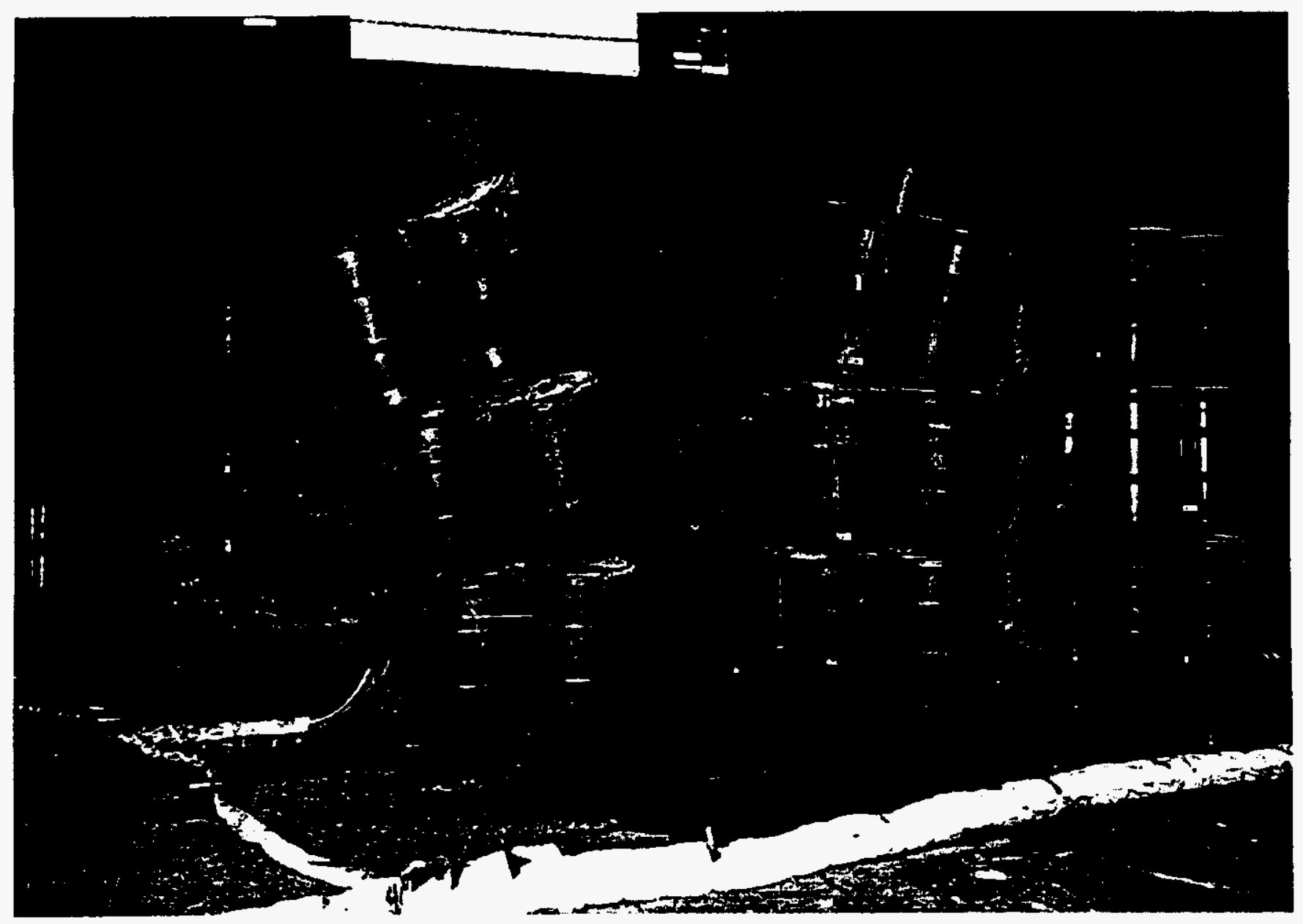

Figure 55. Photo of Post-test Pallet Array 
WHC-SD-WM-TRP-246 REV. 0

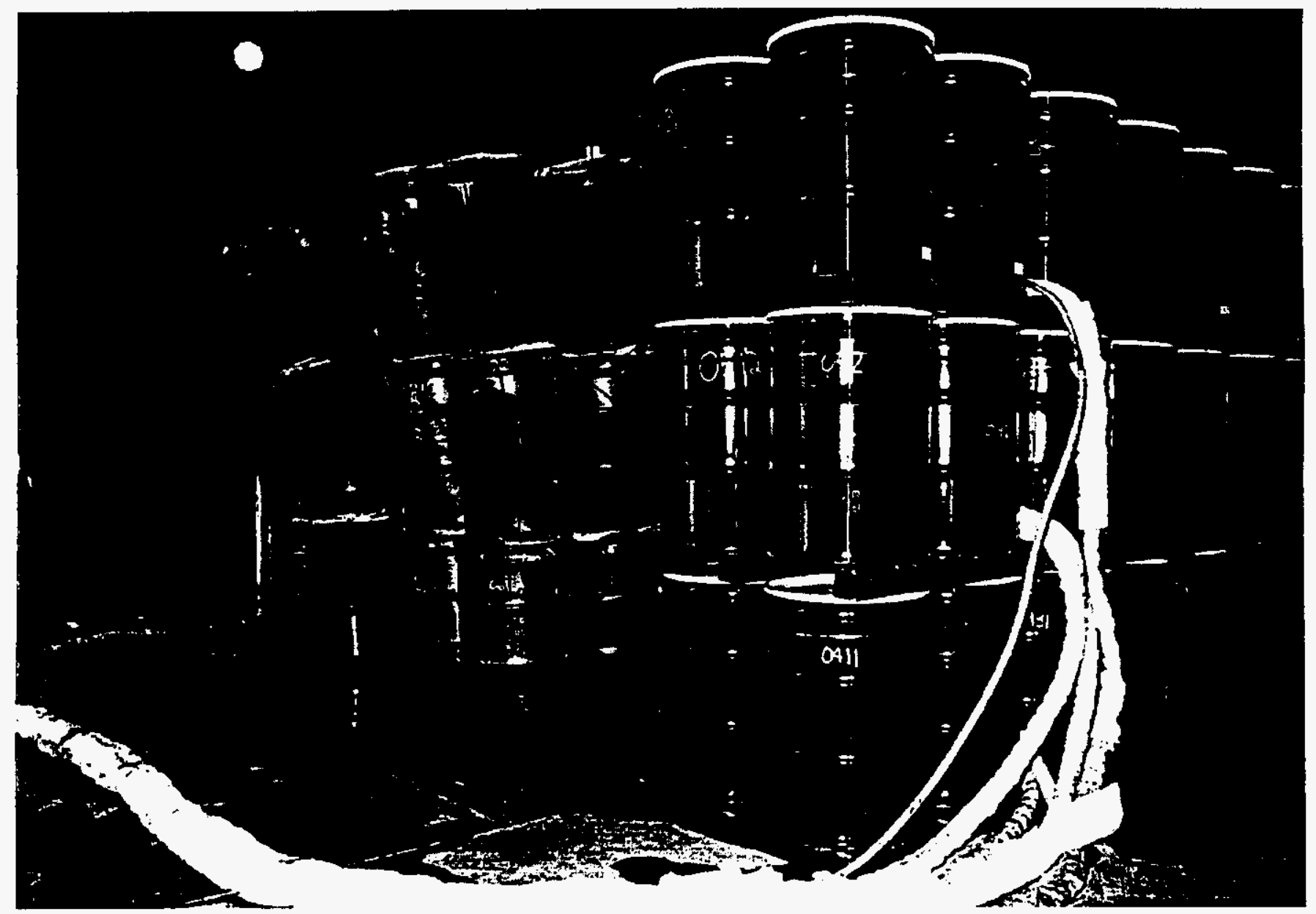

Figure 56. Photo of Post-test Pallet Array 
Beank 


\subsection{ANALYSIS OF RESULTS}

Based on the earlier HAl analytical and experimental results, the performance of solid waste drums during the current rack and paliet full-scale fire tests were predicted (see Section 3.0). The criteria used to characterize drum performance were developed from the earlier HAl individual drum fire tests. Pool fire heat transfer data was taken from the literature and analytical assessments were used to predict drum response and potential fire propagation.

It is believed that the models developed during earlier HAl work represent worst case conditions and serve as a conservative estimate of drum performance in an actual fire event. The purpose of the full-scale drum array fire tests is to demonstrate that the analytical and experimental methods developed in the earlier HAl work are indeed conservative. A comparison of the predictions presented in Section 3.0 and the actual full-scale rack and pallet test results will serve as validation of the drum response methodology developed in earlier $\mathrm{HAl}$ work.

\subsection{GENERAL}

In the earlier $\mathrm{HAl}$ individual drum fire tests, two failure modes were identified for the drums: (1) lid loss, and (2) lid seal failure. The most significant potential result of drum heating by an exposure fire is the loss of the lid. Lid loss results in the burning of drum contents and the distribution of hazardous materials which might be contained in the drum. Lid seal failure is a less severe drum failure mode. Lid seal failure results in loss of containment, but also results in drum pressure relief which can prevent lid loss. By preventing lid loss, lid seal failure prevents burning of the contents and thus markedly reduces material releases. During the earlier $\mathrm{HAl}$ individual drum testing, lid loss and lid seal failure were the only failure modes observed for the drums.

One of the primary concerns with a fire in a solid waste drum storage facility is the release and distribution of hazardous materials as airborne particulates due to burning drum contents. As such, the methods developed to predict lid loss are ultimately used to estimate the quantity of drum contents consumed in the fire. All of the previous HAl drum tests in which lid loss was not observed, lid seal failure occurred and resulted in minimal contents loss. For the current rack and pallet tests, a third failure mode was observed. In many cases, the drum lid was not completely blown from the drum but was partially held in place by the closure ring. Such an event is defined as a lid rupture failure and is different than lid loss and lid seal failure in several regards. Unlike lid loss failure, the contents are not significantly ejected from the drum. However, a lid rupture results in a partial separation of the lid and drum allowing sufficient air to support the burning of drum contents within the drum. This does not occur for drums experiencing lid seal failure. Although the burning rate of drum contents will differ for ruptured and lid loss drums, the quantity of hazardous material consumed is equivalent. As such, in evaluating the potential hazard of the full-scale fire tests, a drum rupture is treated as equivalent to drum lid loss. In the context of this report, both drum ruptures and lid loss failures are defined as lid failures.

Although the models developed during earlier $\mathrm{HAl}$ work were based on criteria established for lid loss failure, they are extended to include lid ruptures. Based on the similar heating conditions and locations of ruptured and lid loss drums during the current tests, it is 
not possible to distinguish between the two events. The basic mechanics of lid rupture and lid loss are the same. The primary difference is the severity of the event. In lid rupture, the forces on the lid are simply not sufficient to fully dislodge the lid from the lid clamp. In cases where lid loss drums will have significantly different consequences than ruptured drums, the impact of such an assumption shall be discussed.

\subsection{RACK STORAGE TEST}

Figure 57 indicates the predicted drum performance for the rack storage test. The methodology and models used for the predictions are discussed in Section 3.0. In determining the location of drums which would experience lid failure, conservative assumptions were made regarding the shape and height of the flame, emissive power of the flame, and the critical heat flux required for lid loss failure. The purpose for such conservatism is to ensure that the predicted performance of the drums does not underestimate the actual performance and the extent of fire propagation during the test. The models developed during earlier HAl work are intended to represent worst case conditions and serve as a conservative estimate of drum performance in an actual fire event.

For the rack storage test, the drum response models indicated that 90 drums (discounting the water filled drums) would experience lid failure. Of the 90 drums, 45 are within the pool fire and 45 are outside the pool. These predictions treat the pool fire as a cylindrical radiator with an average flame emissive power of $80 \mathrm{~kW} / \mathrm{m}^{2}$ and a critical heat flux of $45 \mathrm{~kW} / \mathrm{m}^{2}$ required for lid failure. As discussed in Section 3.0 , these represent conservative estimates and are intended to overpredict the number of lid failures. A more realistic estimate of lid failure is briefly discussed in Section 3.0 where the emissive power of the flame is taken as $53 \mathrm{~kW} / \mathrm{m}^{2}$ and a critical heat flux of $60 \mathrm{~kW} / \mathrm{m}^{2}$ is required for lid loss. With this, all drums within the flame are still predicted to experience lid failure; however, drums outside the flame are predicted to experience lid seal failure only. Depending on the degree of conservatism required, the predicted number of lid failure drums is $\mathbf{4 5}$ within the flame and anywhere from 0 to 45 outside the flame (see Figure 57).

Figure 58 indicates the actual drum performance for the rack storage test compared to the predicted drum performance. The failure mode of the drums were determined from post-test analysis of the drums. It is seen that nearly all of the drums within the flame experience lid failure whereas no drums outside the flame experience lid failure. The total number of actual lid failure drums is 37 . Of these, 18 drums ruptured as opposed to completely losing their lid (see Figure 58). There was no apparent pattern to the location of ruptured drums with respect to lid loss drums and they contributed equally to the severity of the fire. Again, in this analysis, both ruptured and lid loss drums are treated as lid failures. 


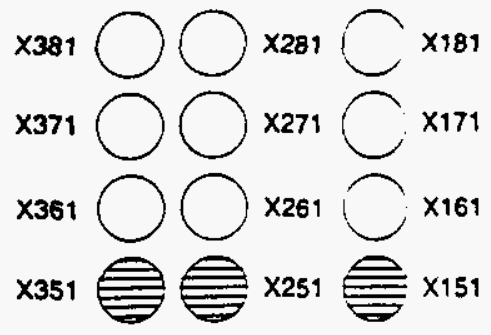

Tier 1
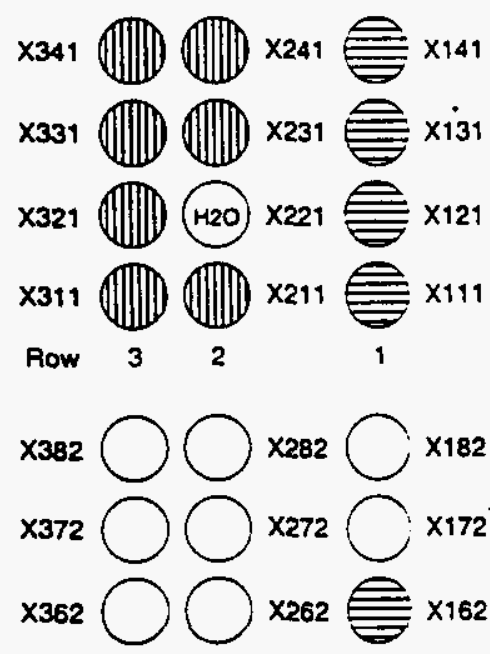

${ }^{x 352} \triangleq{ }_{x 252} \triangleq 152$

Tier 2

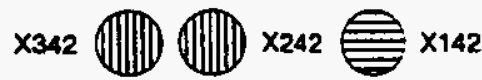

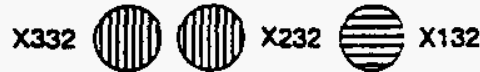

$\times 322$ H20 OIIIII $\times 222 \rightleftharpoons x_{122}$

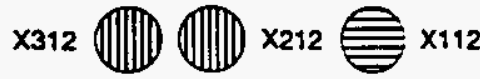

Row 322
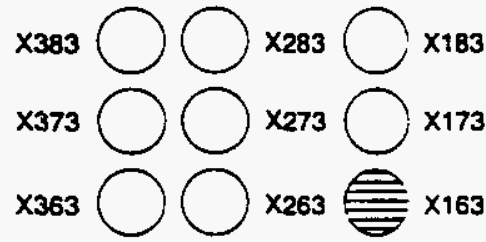

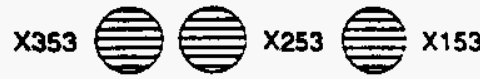

Tier 3

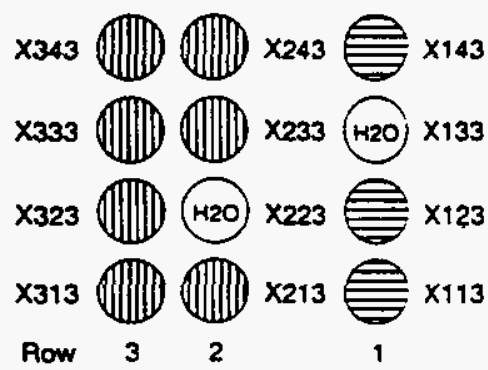

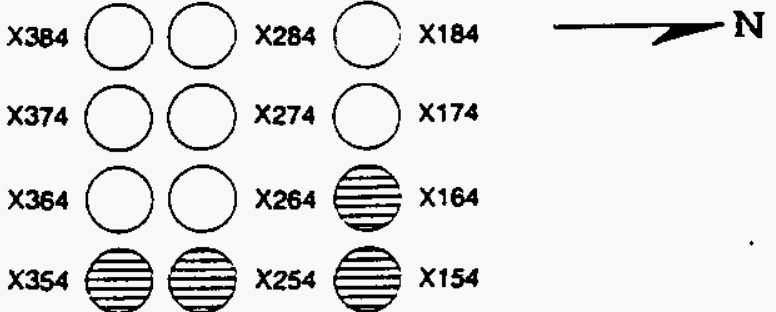

Tier 4

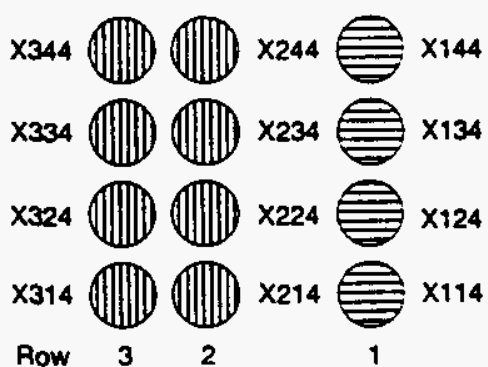

X385 $\bigcirc \times 285 \bigcirc \times 185$

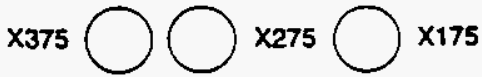

X365 $\bigcirc \times 265 \bigcirc \times 165$

$\times 355 \Leftrightarrow \times 255 \Leftrightarrow \times 155$

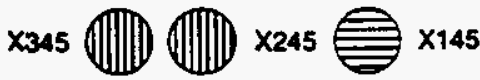

x335 (IIII) (IIIII) x235

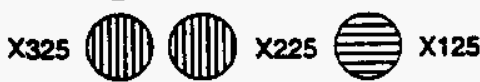

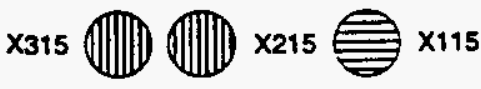

Row 32

x386 $\bigcirc \times 286 \bigcirc \times 186$

$\times 376 \bigcirc \times 276 \bigcirc \times 176$

x366 $\bigcirc \times 266 \bigcirc \times 166$

$\times 356 \triangleq \times 256 \triangleq 156$

$\times 346$ (IIIII) (IIII) $\times 246 \rightleftharpoons \times 146$

Tier 6

Tier 5

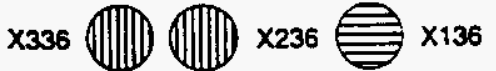

$\times 326$ (IIII) (III) $\times 226 \rightleftharpoons \times 126$

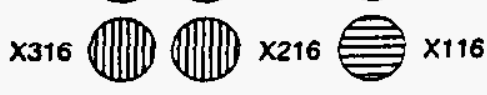

Row 321

Note: Drum number indicates Test-Row-Bay-Tier. (111) Predicted Lid Loss Failure $\vartheta$ Predicted Possible Lid Loss Failure

Figure 57. Predicted Drum Performance - Rack Storage Test 
WHC-SD-WM-TRP-246 REV. 0

Tier 1
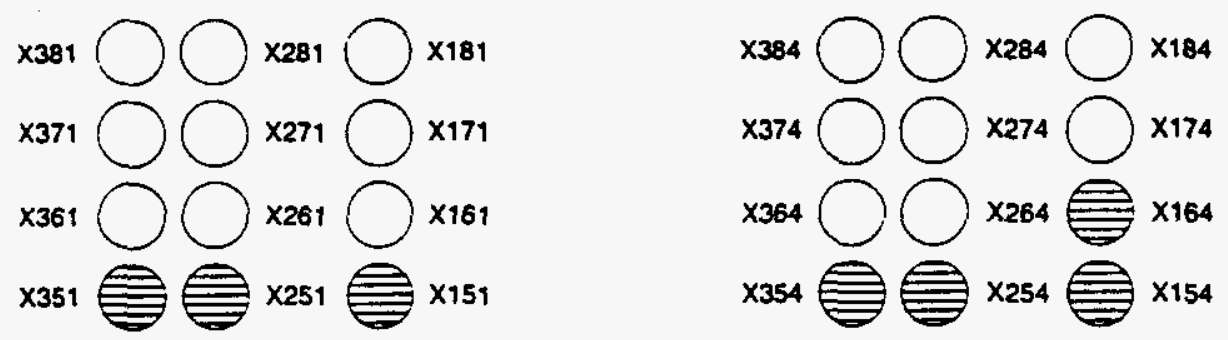

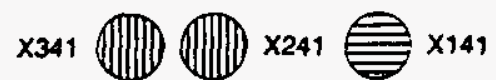

$\times 344$ (II) (III) X244

Tier 4

Tier 2

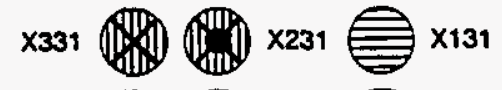

$\times 334$ (1010 $\times 234 \triangleq 134$

$\times 324$ (III) $\times 224 \Rightarrow \times 124$

$\times 314$ (III) $\times 214 \Leftrightarrow \times 114$

Row 325

x385 $\bigcirc \times 285 \bigcirc \times 185$

$\times 375 \bigcirc \times 275 \bigcirc \times 175$

$\times 365 \bigcirc \times 265 \bigcirc \times 165$

$\times 355 \Longleftrightarrow \times 255 \Leftrightarrow$ ×155

Tier 5

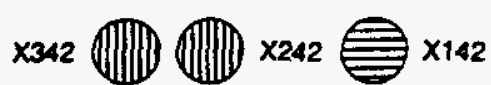

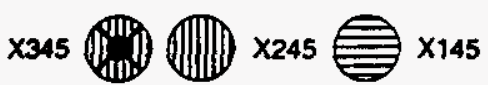

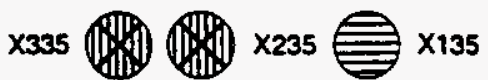

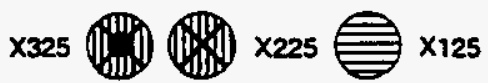

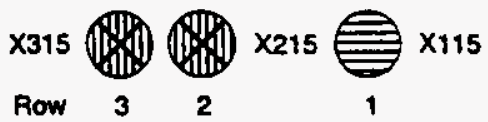

Row 32

$\times 383 \bigcirc \times 283 \bigcirc \times 183$

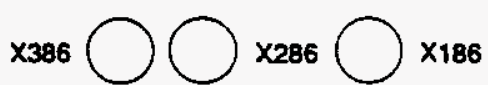

$\times 373 \bigcirc \times 273 \bigcirc \times 173$

X376 $\bigcirc \times 276 \bigcirc \times 176$

$\times 363 \bigcirc \times 263 \triangleq \times 163$

$\times 366 \bigcirc \times 266 \bigcirc \times 166$

x353 $\Longleftrightarrow$ X253 $\triangleq 153$

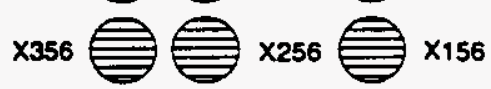

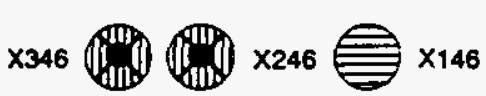

Tier 6

Tier 3

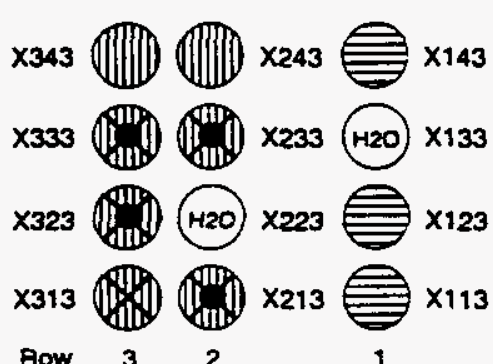

$\times 336$ (II)

$\times 326$ (III) $\times 226 \Rightarrow 126$

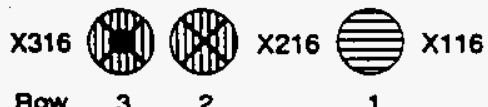

Note: Drum number indicates Test-Row-Bay-Tier.

(11) Predicted Lid Loss Failure $\quad 8$ Actual Lid Loss Failure

$\bigoplus$ Predicted Possible Lid Loss Failure $\bigotimes$ Actual Lid Rupture Failure

Figure 58. Actual and Predicted Drum Performance - Rack Storage Test 
Overall, the models used to predict drum performance were conservative for the rack storage test. It appears that the predictions based on a flame emissivity of $53 \mathrm{~kW} / \mathrm{m}^{2}$ and a critical heat flux of $60 \mathrm{~kW} / \mathrm{m}^{2}$ for lid failure better predict the actual drum performance. In order to better assess the validity of the models, it is necessary to investigate the experimental data for the drums and compare it to the criteria established during the earlier HAl individual drum testing. For the rack storage test, the time at which drums experienced lid failure was not easily determined by visual inspection. Rather, the specific time of lid failure could only be determined for those drums having pressure transducers due to the rapid decline in pressure at time of lid failure. Since it is necessary to know the drum wall temperature land if possible the heat flux and pressure) at the time of lid failure, only those drums instrumented with pressure transducers are assessed in this analysis:

\subsubsection{Drums Within The Flame}

Table 6 provides a summary of the experimental data for the pressure instrumented rack storage drums within the flame. Data for other drums is provided in Table 4 and Appendix $C$.

Table 6. Summary of Experimental Data for Pressure Instrumented Rack Storage Drums Within the Flame

\begin{tabular}{|c|c|c|c|c|c||}
\hline Drum No. & Lid Loss or & $\begin{array}{c}\text { Time of } \\
\text { Lid Faid Rupture } \\
\text { (s) }\end{array}$ & $\begin{array}{c}\text { Drum } \\
\text { Temp. at } \\
\text { Lid Failure } \\
\left({ }^{\circ} \mathrm{C}\right)\end{array}$ & $\begin{array}{c}\text { Pressure at } \\
\text { Lid Failure } \\
\text { (psig) }\end{array}$ & $\begin{array}{c}\text { Approx. Heat } \\
\text { Flux at Lid } \\
\text { Failure } \\
\text { (kW/m })^{*}\end{array}$ \\
\hline$\times 321$ & LID RUPTURE & 122 & 711 & 10.0 & 101 (cal 2) \\
\hline$\times 231$ & LID LOSS & 162 & 693 & 8.7 & 165 (cal 3) \\
\hline$\times 211$ & LID RUPTURE & 112 & 680 & 12.4 & NM \\
\hline$\times 332$ & LID LOSS & 249 & 400 & 8.4 & 66 (cal 6) \\
\hline$\times 312$ & LID RUPTURE & 110 & 701 & 11.0 & NM \\
\hline$\times 222$ & LID LOSS & 98 & 760 & 16.5 & 142 (cal 7) \\
\hline$\times 233$ & LID LOSS & 273 & 627 & 7.45 & 147 (cal 11) \\
\hline$\times 213$ & LID LOSS & 119 & 427 & 11.0 & NM \\
\hline$\times 224$ & LID RUPTURE & 211 & 592 & 7.1 & 75 (cal 14) \\
\hline$\times 214$ & LID LOSS & 163 & 483 & 11.0 & NM \\
\hline$\times 225$ & LID RUPTURE & 236 & 686 & 7.8 & 88 (cal 15) \\
\hline$\times 226$ & LID RUPTURE & 275 & 699 & 6.7 & 109 (cal 16) \\
\hline AVERAGE & & 178 & 622 & 9.8 & 112 \\
\hline
\end{tabular}

- See Figures 26-31 for calorimeter locations NM - not measured 
All of the drums within the flame which were instrumented with pressure transducers experienced lid failure. The lid failure times ranged from 98 to 275 seconds with an average lid failure time of 178 seconds. This is longer than the average lid failure time of 90 seconds observed during the HAl individual drum testing but is similar to prior testing as reviewed in the earlier HAl report [Rhodes et al., 1995]. In addition, during the individual drum testing, lid failure did not occur after 145 seconds for any of the fire exposures. The longest lid failure time for the rack storage test is nearly double this time. During the earlier HAl individual drum tests, the exposures were well controlled fires which reached their maximum intensity very quickly. As such, the incident heat flux to the drums remained approximately steady during the duration of the test. This is not always the case for the rack storage test.

Figures 59 and 60 show the approximate heat flux and temperature of drum no. $\times 226$ during the test. The heat flux measurement was taken adjacent to the drum with the transducer facing the center of the flame. As such, it does not necessarily represent the average heat flux over the surface of the drum. In fact, the back side of the drum is likely exposed to a muct lower incident heat flux since the flame is not optically thick in this area. It is seen in Figure 59 that the flame heat flux during the first 150 to 200 seconds of the test is approximately $20 \mathrm{~kW} / \mathrm{m}^{2}$. This is much lower than the minimum heat flux required for lid failure (i.e., $45-75 \mathrm{~kW} / \mathrm{m}^{2}$ ) and apparently is not high enough to cause lid seal failure in this case either. Once the flames begin impinging the drum, the flame heat flux rapidly increases to approximately $100 \mathrm{~kW} / \mathrm{m}^{2}$ which is well above the minimum heat flux required for lid failure. At this point, lid failure occurs within 100 seconds which is comparable to the individual drum test results. Figure 60 shows the same trend for the temperature where after 150 to 200 seconds into the test the temperature rapidly increases due to the increase in flame heat flux. Even on the lower tiers, this type of behavior is typical for the drums which have long lid loss times (i.e., X332, X233, X224, X225). As such, the lid failure times observed in the rack test are consistent with our understanding developed in earlier HAl work.

The maximum drum wall temperature is the primary criteria which was used to predict lid failure. Based on the earlier $\mathrm{HAl}$ individual drum testing, it is expected that drums which achieve temperatures in excess of $750^{\circ} \mathrm{C}$ will experience lid failure; drums in the range of 600 to $750^{\circ} \mathrm{C}$ will sometimes result in lid failure; and drum temperatures less than $600^{\circ} \mathrm{C}$ will result in lid seal failure only. In general, drum wall temperatures were in excess of $600^{\circ} \mathrm{C}$ at lid failure. In all cases, where the orum wall temperature exceeded $600^{\circ} \mathrm{C}$, lid failure occurred. There were no seal failures at temperatures greater than $600^{\circ} \mathrm{C}$. However, several of the drums (i.e., $\times 332, \times 213, \times 214$ ) had unusually low drum wall temperatures and still resulted in lid failure. A comparison of drum wall temperatures at lid and seal failure in the rack test and the prior individual drum tests is shown in Table 7. The most probable cause of the low measured temperatures at lid failure is the location of the thermocouple with respect to the edge of the flame. As seen in Figure 57, the drums which have low wall temperatures are at the edge of the fire. Due to the limited instrumentation, it is not possible to accurately determine the temperature distribution over the drum surface. Since the drum temperatures are much lower than observed in prior testing, it is expected that the surface temperature was higher at other locations on the drum. Such temperature variations were recorded for other drums having several thermocouple locations on the surface (i.e., X231, $X 222$; see Appendix $C$ for data). Without a better knowledge of the temperature distribution for these drums, further assessment of their performance is not possible, and no change in the predictive methods is indicated. 
WHC-SD-WM-TRP-246 REV. 0

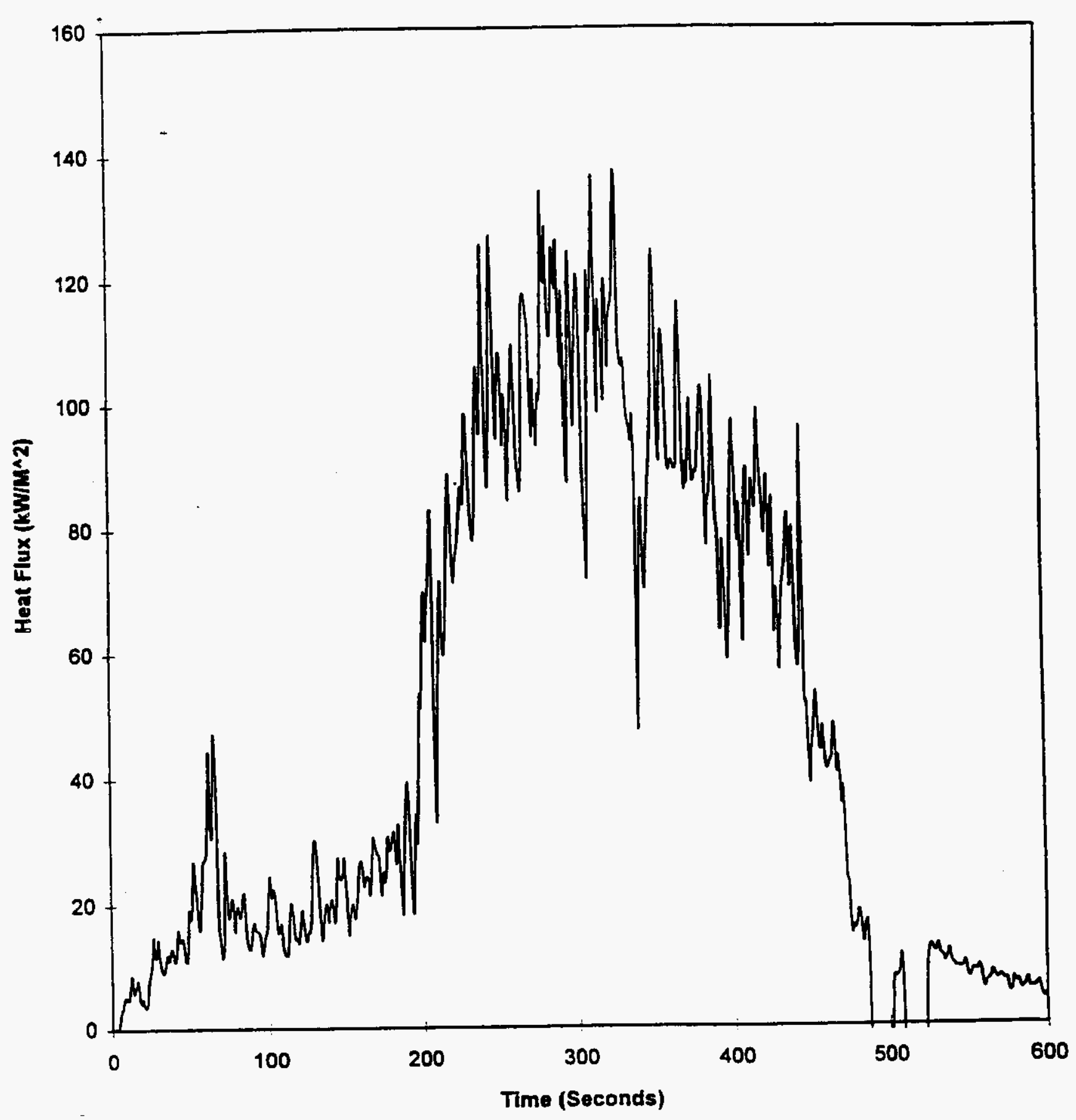

Figure 59. Approximate Heat Flux to Drum No. X226 (Cal\#16) 
WHC-SD-WM-TRP-246 REV. 0

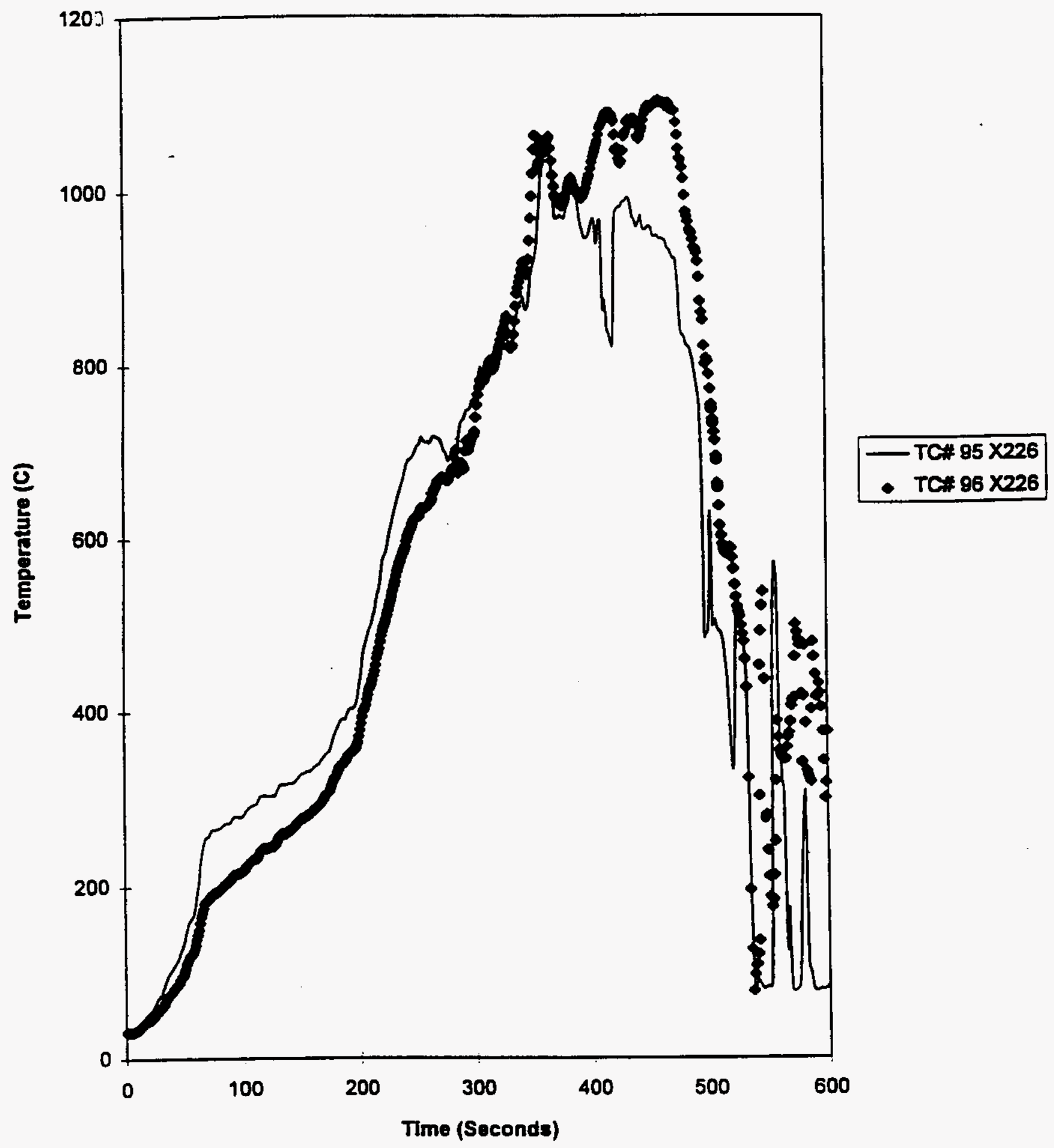

Figure 60. Drum Wall Temperature for Drum No. X226 
Tabie 7. Comparison of Drum Wall Temperatures at Lid and Seal Failure for the Rack Test with Prior Individual Drum Tests

\begin{tabular}{|c|c|c|c|c|}
\hline \multirow{2}{*}{$\begin{array}{c}\text { Temperature } \\
\text { Range }\left(^{\circ} \mathrm{C}\right)\end{array}$} & Rack & Individual & Rack & Individual \\
\cline { 2 - 5 } & 1 & 0 & 1 & 0 \\
\hline$\leq 400$ & 1 & 0 & 0 & 1 \\
\hline $401-450$ & 1 & 0 & 0 & 5 \\
\hline $451-500$ & 0 & 0 & 2 & 1 \\
\hline $501-550$ & 1 & 0 & 2 & 1 \\
\hline $551-600$ & 1 & 0 & 0 & 7 \\
\hline $601-650$ & 4 & 2 & 0 & 6 \\
\hline $651-700$ & 3 & 7 & 0 & 4 \\
\hline$>700$ & 12 & 9 & 5 & 25 \\
\hline Total & & & & \\
\hline
\end{tabular}

The incident heat flux to the drum is another criteria used to predict lid failure. Based on the earlier $\mathrm{HAl}$ individual drum testing, it is expected that drums exposed to radiant exposures less than $45 \mathrm{~kW} / \mathrm{m}^{2}$ will not experience lid failure; exposures in the range of 45 to $75 \mathrm{~kW} / \mathrm{m}^{2}$ will sometimes result in lid failure; and exposures greater than $75 \mathrm{~kW} / \mathrm{m}^{2}$ will experience lid failure. Heat flux measurements were obtained during the rack test using total heat flux transducers mounted between the drums and using water filled drum calorimeters. Neither of these methods provide exact measurements of the actual incident heat flux to the drum surface. However, the heat flux transducers can be used to estimate the maximum flame heat flux seen by a drum and the water filled drum calorimeters provide an estimate of the average heat input to a drum.

As seen in Table 6 , the heat flux measurements for the lid failure drums range from 66 to $165 \mathrm{~kW} / \mathrm{m}^{2}$, which are well within the range of heat fluxes expected to cause lid failure. Not all of the drums listed in Table 6 have heat flux data because the measurements are applicable only to drums adjacent to heat flux transducers. Since the transducers are located between drums and not on the drum surface, the actual incident heat flux to the drum surface will vary. The transducer measurements do, however, provide insight for the flame heat flux associated with different locations in the fire. As expected, points near or facing the center of the fire are typically exposed to higher heat fluxes than points near or facing the edge of the fire. As such, the measured heat flux for drum nos. $\times 231, \times 222$, and $\times 233$ are higher than for the other lid failure drums located further from the center of the pool.

From the rate of temperature rise for the water filled drums within the flame (i.e., $\times 221, \times 322, \times 2231$, an average heating rate is determined for the drums. Temperature and heating rate data for the water filled drums is included in Appendix $C$. The temperature of the drums steadily increase during the test yielding an average heat flux to the surface of the water filled drums of 90 to $100 \mathrm{~kW} / \mathrm{m}^{2}$. This is an average value over the entire drum wall. This corresponds well with the range of heat flux transducer measurements. The average 
heating rate of the water filled drums for the full-scale test is significantly higher than that measured during earlier $\mathrm{HAl}$ testing. During earlier HAl individual drum testing, the average heat flux for full flame engulfment tests was approximately $65 \mathrm{~kW} / \mathrm{m}^{2}$. This exposure resulted in lid failure for 7 of 8 drum tests. As such, it is not surprising that most drums within the flame for the full-scale test are also experienced lid failure.

During the earlier HAl testing, it was not possible to develop criteria for lid failure based on drum pressure data. It was found that lid failure pressures ranged from about 13 to 28 psig with an average value of approximately 20 psig. For the current rack storage test, 11 of the 12 drums which experienced lid failure and had pressure transducer measurements failed at a pressure less than $13 \mathrm{psig}$ (drum no. $\times 222$ failed at $16.5 \mathrm{psig}$ ) with an average value of 9.8 psig (see Table 6). During earlier HAl individual drum tests, it was observed that standard load drums failed at a lower pressure than other drum loadings. However, the current rack test lid failure pressures are even lower than that observed previously. Part of the reason may be that the fire exposure for the rack test is much more severe than that in the earlier HAl individual drum tests (as seen by the water calorimeter heat flux results). The lower pressures may also be responsible for the lid ruptures observed for many of the drums during the rack test where it was not during the individual drum tests. In any case, similar to the earlier $\mathrm{HAl}$ tests, systematic pressure trends are not evident for the rack storage test.

Although all of the drums within the flame were predicted to experience lid failure, 8 of the $\mathbf{4 5}$ standard load drums within the flame resulted in lid seal failure oniy. All of the 8 drums were located in drum Bay 4 near the edge of the pool. As noted earlier, drums near the edge of the pool are subjected to a less severe exposure than drums near the center. Although several other drums near the edge experienced lid failure, the flame impingement and heating rate may not have been uniform in this area. Of the 8 drums experiencing lid seal failure within the pool area, 4 were equipped with thermocouples. During the first 300 seconds of the test (the time in which lid failure was observed), the maximum temperature for these drums was typically 300 to $400^{\circ} \mathrm{C}$. This is well below the minimum temperature criteria established for lid failure (i.e., $600^{\circ} \mathrm{C}$ ). The lower temperatures indicate that the drums were not heated to the same extent as other drums experiencing lid loss failure. This is consistent with visual inspection of these drums. Based on the temperature data, it is not surprising that these drums resulted in lid seal failure only.

It was initially predicted that all drums within the pool area will result in lid failure. The lid failure times, drum temperatures, and heat flux data for drums within the flame generally agree with the criteria established during the earlier HAl drum testing. Approximately, 82 percent of the drums within the flame experienced lid failure. Although not all of the drums within the flame experienced lid failure, there is no predictable basis to distinguish between these few drums and the remaining drums which did result in lid failure. As such, modifications to the methodology are not necessary. The criteria and models used provide a reasonable and conservative estimate for the performance of drums within the flame.

\subsubsection{Drums Outside The Flame}

No drums outside the pool fire experienced lid loss failure. Table 8 provides a summary of the experimental data for the pressure instrumented rack storage drums outside the flame. Data for other drums is provided in Table 4 and Appendix $C$. 
Table 8. Summary of Experimental Data for Pressure Instrumented Rack Storage Drums Outside the Flame

\begin{tabular}{|c|c|c|c|c|c||}
\hline Drum No. & $\begin{array}{c}\text { Lid Seal } \\
\text { Failure }\end{array}$ & $\begin{array}{c}\text { Time of Lid } \\
\text { Seal Failure } \\
(\mathrm{s})\end{array}$ & $\begin{array}{c}\text { Max. Drum } \\
\text { Temperature } \\
\left({ }^{\circ} \mathrm{C}\right)\end{array}$ & $\begin{array}{c}\text { Max. } \\
\text { Pressure } \\
(\mathrm{psig})\end{array}$ & $\begin{array}{c}\text { Approx. } \\
\text { Heat Flux } \\
\left(\mathrm{kW} / \mathrm{m}^{2}\right)\end{array}$ \\
\hline \hline$\times 131$ & YES & 166 & 592 & 5.2 & 95 (cal 4) \\
\hline$\times 251$ & NO & -- & 254 & 1.4 & 23 (cal 1) \\
\hline$\times 361$ & NO & -- & 87 & 0.4 & NM \\
\hline$\times 122$ & YES & 207 & 574 & 3.0 & 68 (cal 8) \\
\hline$\times 352$ & NO & -- & 250 & 0.9 & 23 (cal 5) \\
\hline$\times 123$ & YES & 296 & 528 & 2.7 & 64 (cal 12) \\
\hline$\times 253$ & NO & -- & 145 & 0.9 & 28 (cal 9) \\
\hline$\times 254$ & NO & -- & 169 & 1.3 & 37 (cal 13) \\
\hline$\times 125$ & YES & 362 & 507 & 2.4 & NM \\
\hline$\times 255$ & NO & -- & 215 & 2.6 & NM \\
\hline$\times 256$ & YES & 298 & 339 & 3.8 & NM \\
\hline
\end{tabular}

NM - not measured

Drums outside the flame are subjected to a much less severe exposure than drums within the flame. Figure 61 shows Tier 1 heat flux measurements taken at the edge of the pool along the north edge. The heat flux along the north edge of the pool ranges from approximately 70 to $100 \mathrm{~kW} / \mathrm{m}^{2}$. This agrees well with the emissive power indicated by Mudan and Croce [1988] of $80 \mathrm{~kW} / \mathrm{m}^{2}$. In this region, the flame is optically thick and the heat flux transducer is located directly adjacent to the flame. Although this is higher than the critical heat flux criteria established for lid failure, no lid failure drums resulted. In the model used to predict lid failure, the critical average incident heat flux was determined based on flames impinging one-half the drum surface, which is a conservative estimate. For the rack test, drums along the edge of the pool were not exposed to direct flame contact. Air being entrained into the fire through the rack separated the flames from the drums. Since the drums are separated from the flame, view factors between the flame and the drums and the curvature of the drum surfaces will reduce the average heat flux to the drum surface. As such, the transducer measurements are higher than the average heat flux to the drum surface. Determining the average flame heat flux from the transducer data is not possible because small variations in the configuration of the drums and the separation from the flame significantly affect the radiation to the drums. In the drum response model, no credit is taken for a reduction in heat flux due to the configuration and geometry of the drums. This results in a conservative estimate of lid failure.

For the earlier HAl individual drum tests where the drums were exposed to direct flame contact over approximately one-fourth of the surface, transducer heat flux measurements were approximately $80 \mathrm{~kW} / \mathrm{m}^{2}$; whereas, the average heat flux to the surface was $45 \mathrm{~kW} / \mathrm{m}^{2}$ based 
WHC-SD-WM-TRP-246 REV. 0

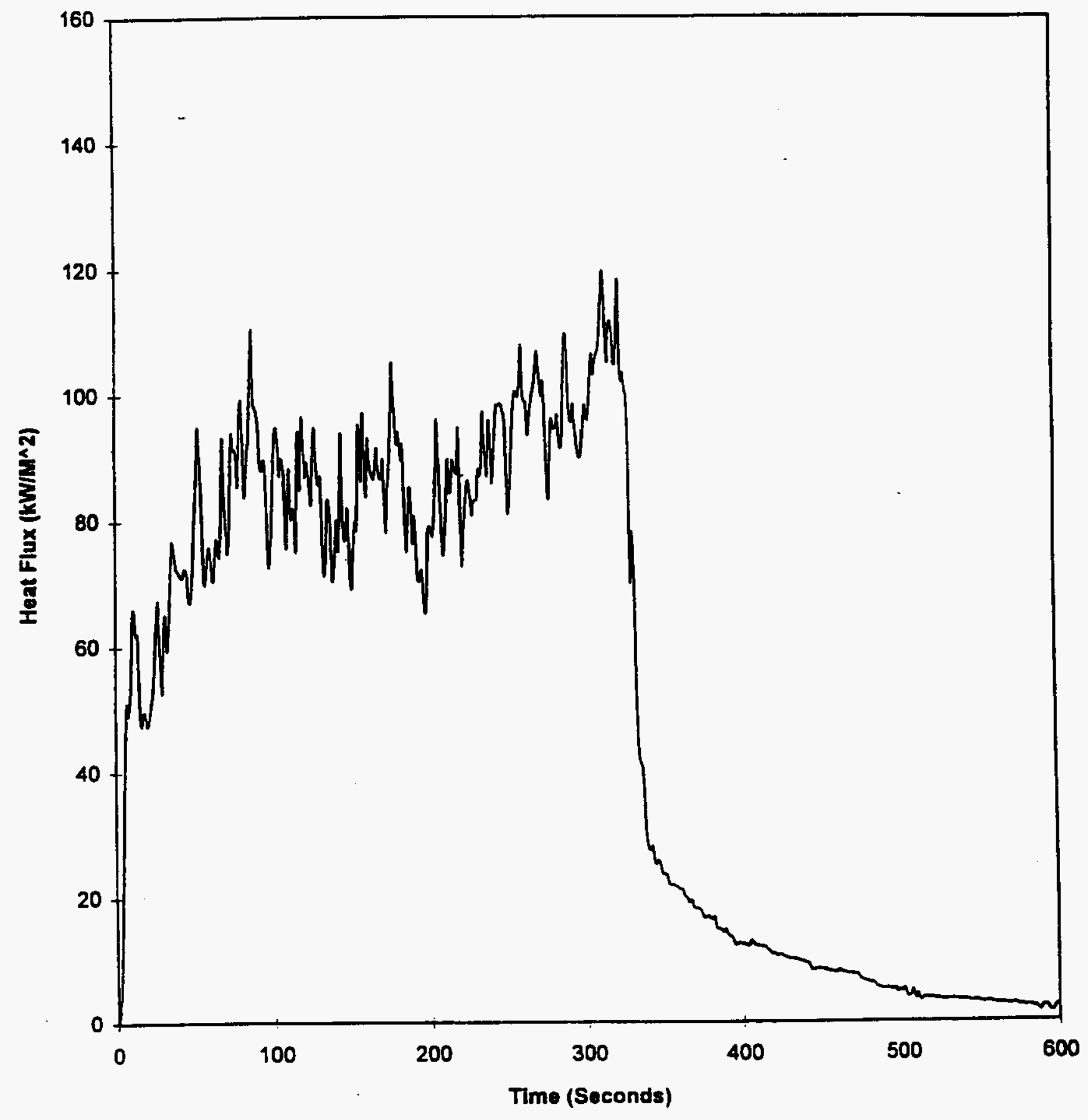

Figure 61. Rack Storage Test - Transducer Heat Flux Measurement along North Edge of Pool 
on water calorimeter results. Although the fire in the rack storage test was larger than this exposure, since there is no direct flame contact with the drums outside the pool, the average heat flux is expected to be less than $45 \mathrm{~kW} / \mathrm{m}^{2}$. The water calorimeter located along the north edge of the pool (i.e., $\times 133$ ) yields an average heat flux of approximately $15 \mathrm{~kW} / \mathrm{m}^{2}$ over onehalf the drum surface. Given the magnitude of the rack test pool fire, this value appears to be low. However, it illustrates that the heat input to a drum separated from the fire is much less than for drums in direct contact with the flame. As seen in Table 8, all of the pressure instrumented drums along the north edge of the pool experienced lid seal failure. The maximum wall temperature for these drums ranges from approximately 500 to $600^{\circ} \mathrm{C}$, which is less than the established critical value of $600^{\circ} \mathrm{C}$ required for lid failure.

The heat flux to drums along the west edge of the pool is less than that along the north edge. Figure 62 shows Tier 1 heat flux measurements taken along the west edge of the pool. The heat flux is lower because the flame is not optically thick in this area as it is in the aisle between Rows 1 and 2 . In addition, the transducer views the drums within the flame (i.e., $\times 341, \times 241$ ) which are initially cool. As these drums heat up, the re-radiation from the steel drum walls increase the measured heat flux. This is seen in Figure 62 where the heat flux steadily increases during the test. Again, the transducer measurement is higher than the average heat flux to the adjacent drum surfaces. Even so, the maximum heat flux is less than $25 \mathrm{~kW} / \mathrm{m}^{2}$ which is not sufficient to cause lid failure. The only drums to the west of the pool fire which experienced lid seal failure were on the sixth tier. Lid seal failure on the sixth tier is likely due to the hot gas layer descending and engulfing the drums. There was no significant damage to any other drums to the west of the pool. As seen in Table 8 , the temperatures for all of the drums to the west of the pool were less than $350^{\circ} \mathrm{C}$. This is less than the established critical value of $600^{\circ} \mathrm{C}$ required for lid failure.

Predictions of lid failure were based on critical drum wall temperatures as a result of heat exposures from the flame. The temperature of the drums outside the flame were estimated using conservative heat flux values based on literature data of pool fire emissive powers. Using an average value of $80 \mathrm{~kW} / \mathrm{m}^{2}$ for the emissive power of the flame, which is consistent with transducer measurements, overpredicts the number of lid failures. Using a flame emissive power of $53 \mathrm{~kW} / \mathrm{m}^{2}$, as suggested by Shokri and Beyler [1989], yields an accurate prediction of lid failure. The lower emissive power effectively accounts for the reduction in incident heat flux to the drums due to drum configuration and geometry. Whereas considerable calculations can be performed to estimate the heat flux to drums outside the pool, based on the individual drum tests and the rack storage test, it appears that if drums are not in direct contact with the flame, lid failure will not occur. For drums directly exposed to the flame, lid failure may not always occur but should be anticipated. This provides a simplified and representative estimate of drum performance during a fire event.

\subsubsection{Thermal Response of Rack Structure}

During the rack test, heat transfer to the steel columns and beams caused deformation of the rack structure. Temperature data for the rack is provided in Appendix $C$. As predicted, local rack temperatures exceeded 500 to $600^{\circ} \mathrm{C}$ within 100 seconds of the test. Although steel begins to lose structural integrity at these temperatures, the rack did not start to significantly deform until approximately 200 seconds into the test (see Section 7.1). This is likely due to the design of the rack in that steel members which were not heated to such temperatures continued to support the structure. Due to the seismic bars, no drums fell from 
WHC-SD-WM-TRP-246 REV. 0

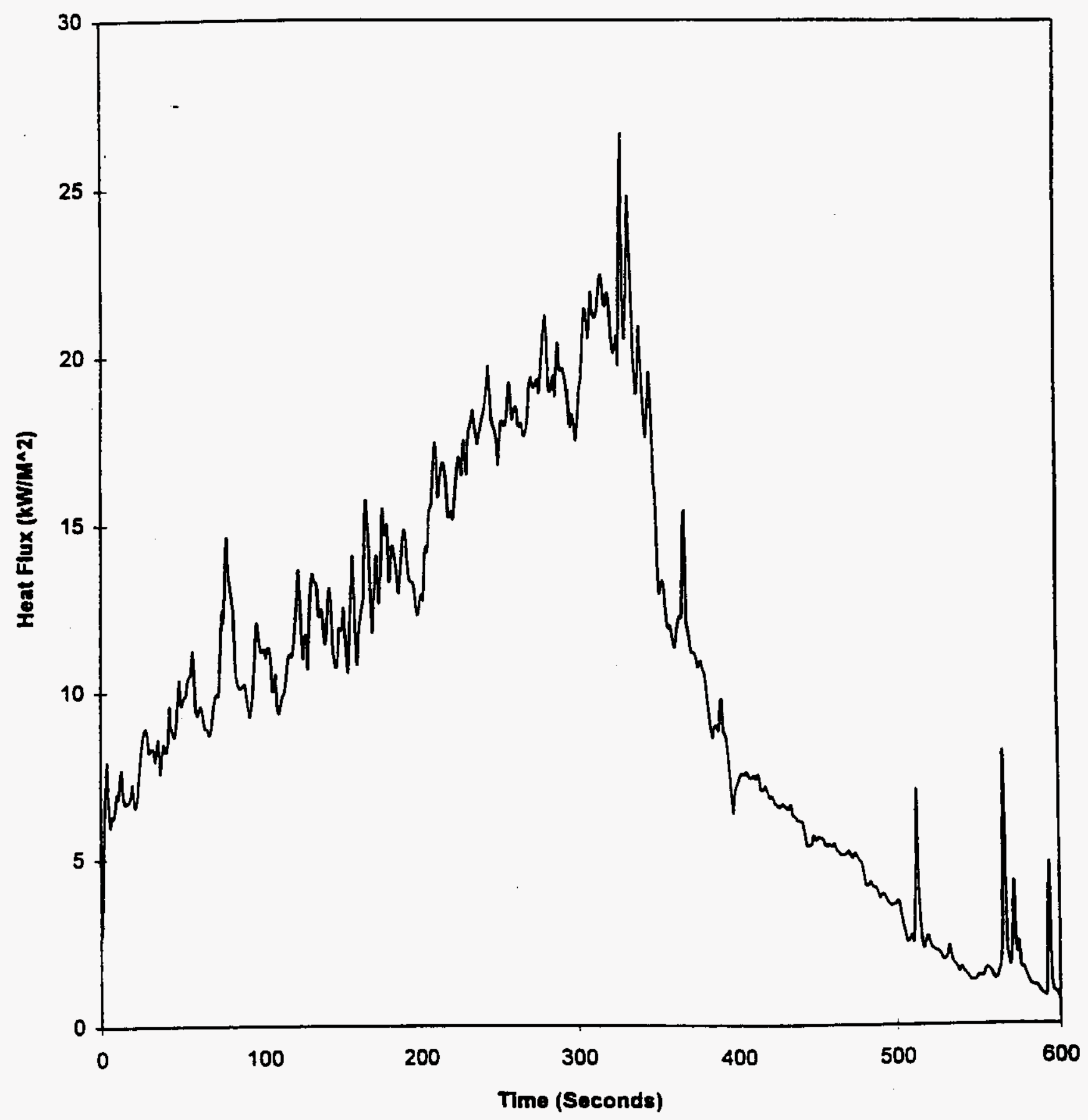

Figure 62. Rack Storage Test - Transducer Heat Flux Measurements along West Edge of Pool (Cal \#1) 
the rack during the test. If the fire had been allowed to continue, it is expected that the entire rack structure would have collapsed. This type of behavior can be expected for an unprotected rack within a pool fire for longer than several minutes.

\subsection{PALLET STORAGE TEST}

Figure 63 indicates the predicted drum performance for the pallet storage test. The methodology and models used for the predictions are discussed in Section 3.0. In determining the location of drums which would experience lid failure, conservative assumptions were made regarding the shape and height of the flame, emissive power of the flame, and the critical heat flux required for lid loss failure. The purpose for such conservatism is to ensure that the predicted performance of the drums does not underestimate the actual performance and the extent of fire propagation during the test. The models developed during earlier HAl testing are intended to represent worst case conditions and serve as a conservative estimate of drum performance in an actual fire event.

For the paliet storage test, the drum response models indicated that 94 drums would experience lid failure. Of the 94 drums, 68 are due to radiation from the pool fire and an additional 28 are due to drums falling to the floor. These predictions treat the pool fire as a cylindrical radiator with the visible flame height and an equivalent pool diameter with an average flame emissive power of $100 \mathrm{~kW} / \mathrm{m}^{2}$ and a critical heat flux of $45 \mathrm{~kW} / \mathrm{m}^{2}$ required for lid failure. It is further assumed that all drums experiencing lid loss due to radiation from the fire will cause the drums above to fall to the floor and experience lid failure. As discussed in Section 3.0, these represent conservative estimates and are intended to overpredict the number of lid failures.

Figure 64 indicates the actual drum performance for the pallet storage test compared so the predicted drum performance. The failure mode of the drums were determined from post-test analysis of the drums. It is seen that 11 of the 24 drums within the flame and 7 of the 30 drums adjacent to the pool experience lid failure. The total number of actual lid failure drums is 18. Of these, only 2 resulted in lid loss, whereas the remaining drums resulted in lid ruptures. For the paliet storage, there are potentially different consequences for lid loss and lid rupture drums. The stability model used to estimate the lid release pressure necessary to topple the pallet stacks above assumes that the lid is completely removed from the drum. It is not possible for a lid rupture to cause such an event. However, for the pallet test, there were no observed differences in the impact of lid loss and lid rupture drums on other drums in the array.

Overall, the models used to predict drum performance were conservative for the pallet storage test. In order to better assess the validity of the models, it is necessary to investigate the experimental data for the drums and compare it to the criteria established during the earlier $\mathrm{HAl}$ testing. For the pallet storage test, the time at which drums experienced lid failure was not easily determined by visual inspection. Rather, the specific time of lid failure could only be determined for those drums having pressure transducers due to the rapid decline in pressure at time of lid failure. Since it is necessary to know the drum wall temperature (and if possible the heat flux and pressure) at the time of lid failure, only those drums instrumented with pressure transducers are assessed in this analysis. 
WHC-SD-WM-TRP-246 REV. 0

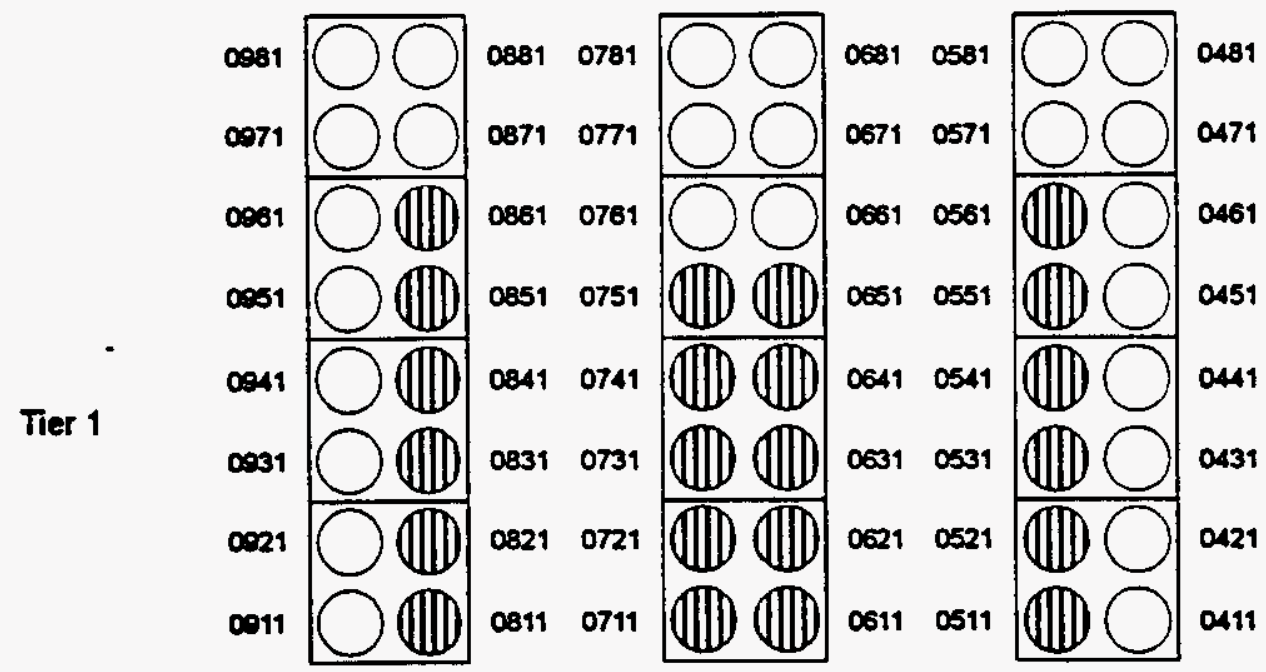

Tier 2

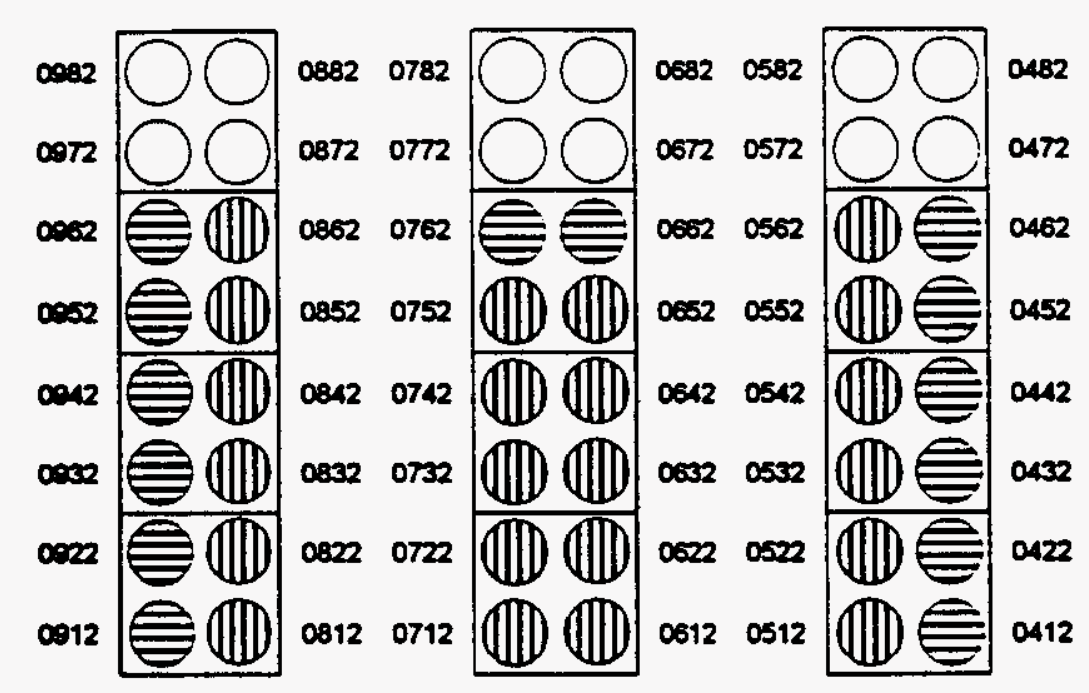

Tier 3

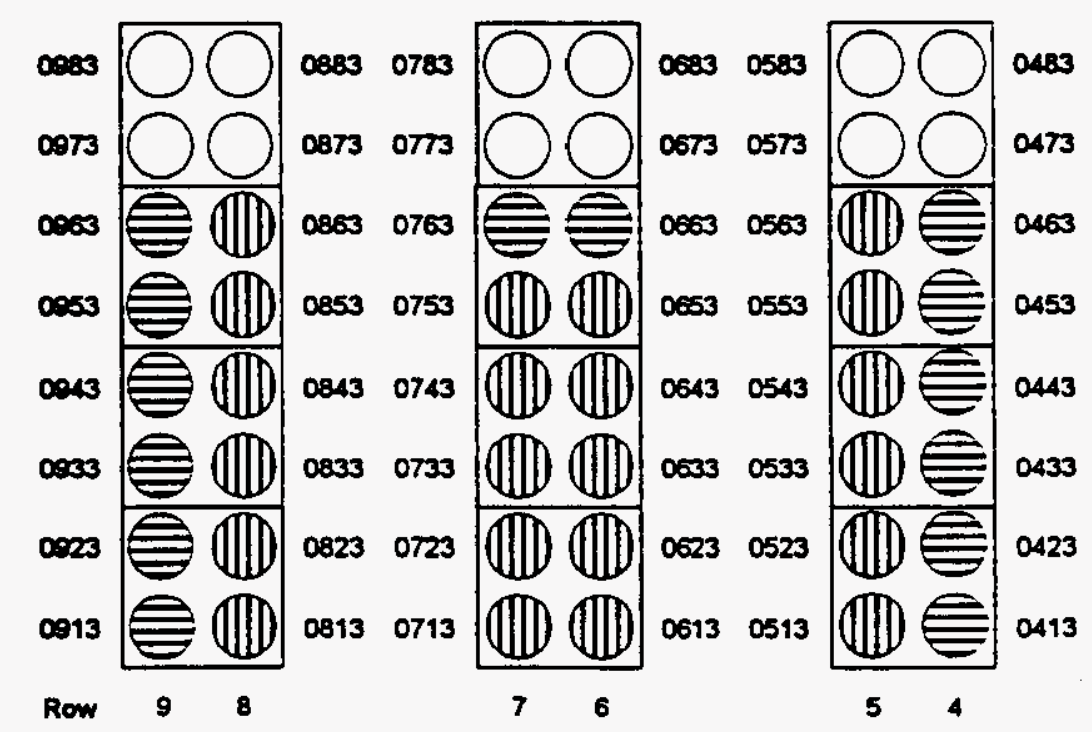

Note: Drum number indicates Test-Row-Bay-Tier.

(1) Predidod Lid Loses Dus to Flame Radiation F Predided Lid Loes Dus to Toppling

Figure 63. Predicted Drum Performance - Pallet Storage Test 
WHC-SD-WM-TRP-246 REV. 0

Tier 1

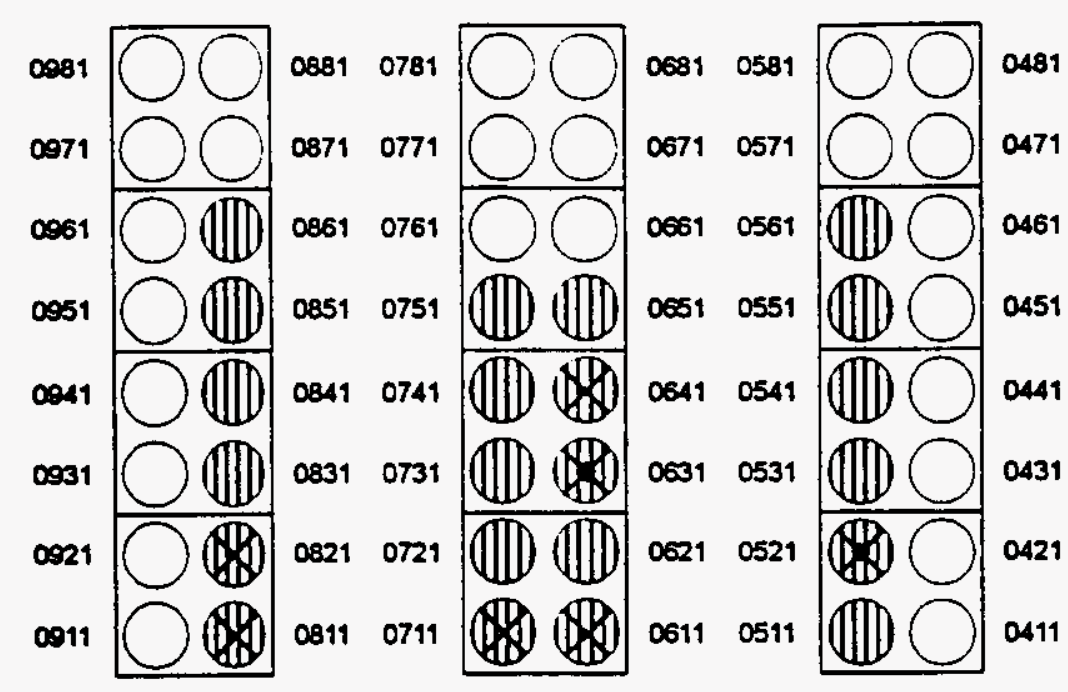

Tier 2

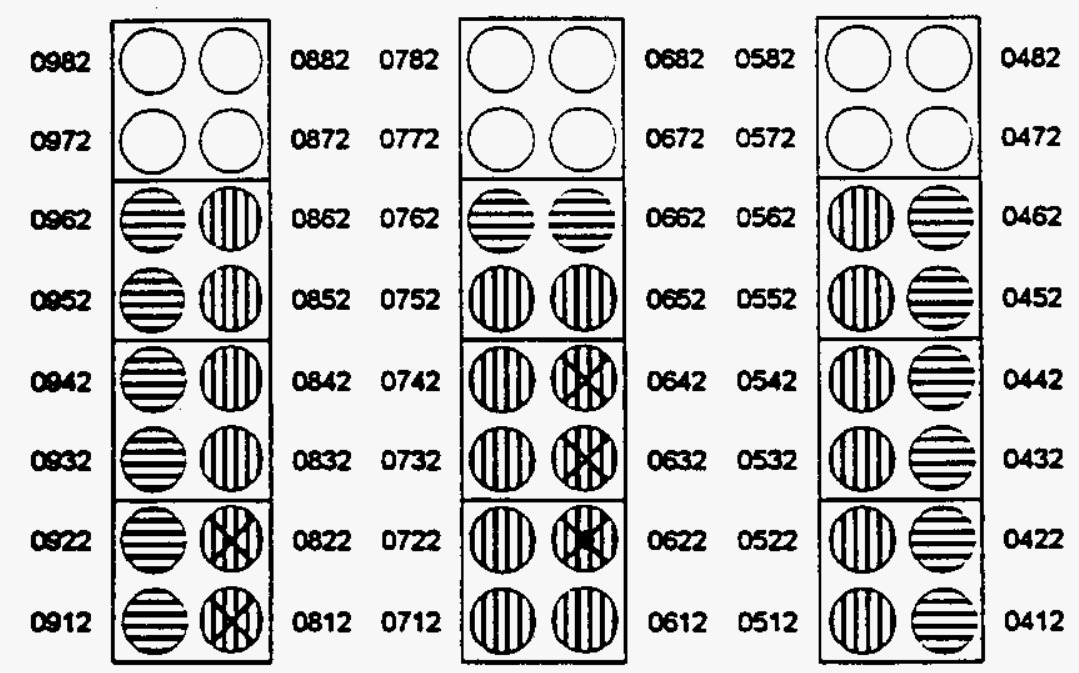

Tier 3

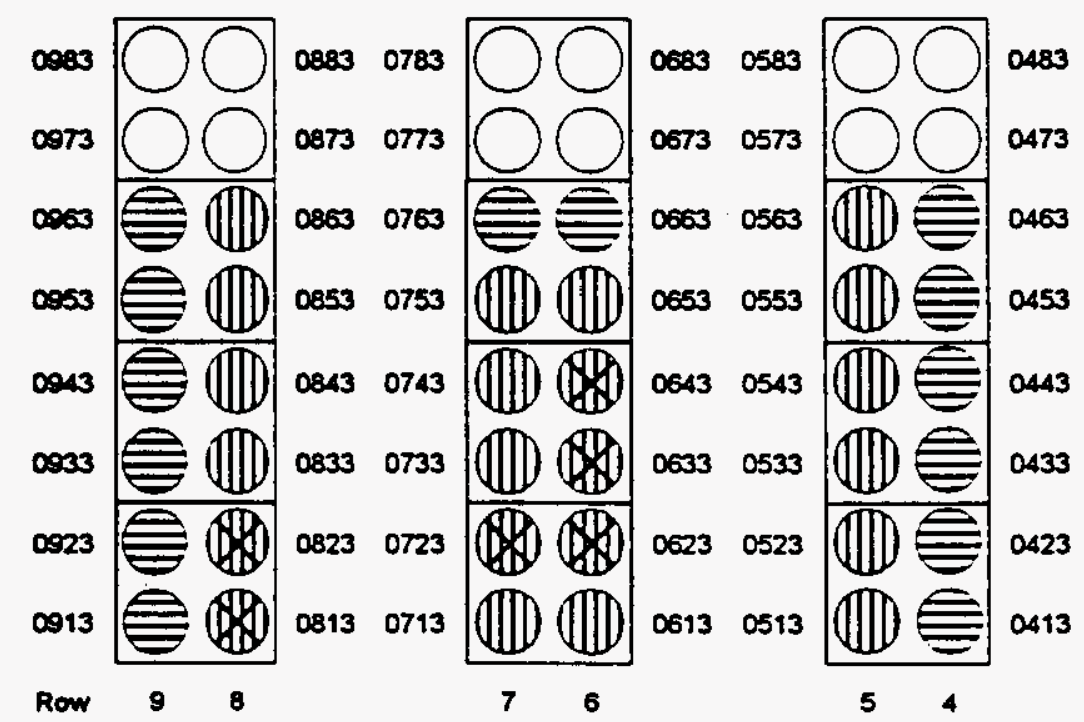

Note: Drum number indicates Test-Row-Bay-Tier.

8 Actual Lid Loss Falure

8 Actual Lto Rupture Faikure

(1) Predictod Lid Loss Dus io Ftame Radiation

OProdictod Lid Loss Dua 10 Toppling

Figure 64. Predicted and Actual Drum Performance - Pallet Storage Test 


\subsubsection{Drums Within The Flame}

Table 9 provides a summary of the experimental data for the pressure instrumented pallet storage drums within the flame. Data for other drums is provided in Table 5 and Appendix E.

Table 9. Summary of Experimental Data for Pressure Instrumented Pallet Storage Drums Within the Flame

\begin{tabular}{|c|c|c|c|c|c||}
\hline $\begin{array}{c}\text { Drum } \\
\text { No. }\end{array}$ & $\begin{array}{c}\text { Lid Loss, Lid } \\
\text { Rupture, or } \\
\text { Vent }\end{array}$ & $\begin{array}{c}\text { Time of } \\
\text { Failure } \\
(\mathrm{s})\end{array}$ & $\begin{array}{c}\text { Drum Temp. } \\
\text { at Failure } \\
\left({ }^{\circ} \mathrm{C}\right)\end{array}$ & $\begin{array}{c}\text { Pressure at } \\
\text { Failure } \\
(\mathrm{psig})\end{array}$ & $\begin{array}{c}\text { Approx. } \\
\text { Heat Flux } \\
\left(\mathrm{kW} / \mathrm{m}^{2}\right)\end{array}$ \\
\hline \hline 0711 & LID RUPTURE & 159 & 732 & 14.0 & $\mathrm{NM}$ \\
\hline 0721 & VENT & 160 & 744 & 12.0 & NM \\
\hline 0741 & VENT & 195 & 600 & 3.1 & $\mathrm{NM}$ \\
\hline 0611 & LID RUPTURE & 165 & 731 & 14.5 & $\mathrm{NM}$ \\
\hline 0631 & LID LOSS & 147 & 803 & 16.4 & 166 (cal 5) \\
\hline 0641 & LID RUPTURE & 197 & 699 & 8.9 & 77 (cal 4) \\
\hline 0722 & VENT & 169 & 932 & 16.4 & NM \\
\hline 0742 & VENT & 518 & 778 & 8.6 & NM \\
\hline 0612 & VENT & 172 & 937 & 9.5 & NM \\
\hline 0632 & LID RUPTURE & 156 & 876 & 17.3 & 135 (cal 7) \\
\hline 0713 & VENT & 154 & 908 & 15.4 & NM \\
\hline 0723 & LID RUPTURE & 185 & 759 & 11.6 & NM \\
\hline 0743 & VENT & 763 & 526 & 6.4 & NM \\
\hline 0633 & LID RUPTURE & 245 & 891 & 9.7 & 81 (cal 9) \\
\hline
\end{tabular}

- See Figures 40-42 for calorimeter locations

NM - not measured

Half of the drums within the flame which were instrumented with pressure transducers experienced lid failure whereas the other half vented due to lid seal degradation. The lid failure times ranged from 147 to 245 seconds with an average lid failure time of 179 seconds. This is longer than the average lid failure time of 90 seconds observed during the earlier $\mathrm{HAl}$ individual drum testing but is similar to the average lid failure time for the rack test $\left(\mathrm{t}_{\mathrm{avg}}=178\right.$ s, see Section 8.2.1) and to prior testing as reviewed in the eariier HAl report [Rhodes et al., 1995]. In general, the drums which vented due to lid seal degradation did so in the same time frame.

The maximum drum wall temperature is the primary criteria which was used to predict lid failure. Based on the earlier $\mathrm{HAl}$ drum testing, it is expected that drums which achieve 
temperatures in excess of $750^{\circ} \mathrm{C}$ will experience lid failure; drums in the range of 600 to $750^{\circ} \mathrm{C}$ will sometimes result in lid failure; and drum temperatures less than $600^{\circ} \mathrm{C}$ will result in lid seal failure oniy. Drum wall temperatures within the flame are generally higher than those for the rack storage test. Temperatures exceed approximately $700^{\circ} \mathrm{C}$ at lid failure for all of the drums within the flame instrumented with pressure transducers. In general, even the drums which experienced lid seal failure only had temperatures indicative of lid failure drums. As seen in Table 9, several of the drums which vented due to lid seal degradation failed at temperatures greater than $900^{\circ} \mathrm{C}$. It is not clear why these drums did not experience lid failure.

Table 10 shows a comparison of drum wall temperatures at lid and seal failure in the pallet test and the prior individual drum tests. The wall temperatures at lid failure in the pallet tests were generally higher than the previous individual drum tests. Again, it must be remembered that in the pallet tests only two thermocouples were used per drum so the average hot wall temperatures determined in the pallet tests must be regarded as crude estimates. No change in the predictive methods is indicated by these results.

Table 10. Comparison of Drum Wall Temperatures at Lid and Seal Failure for the Pallet Test with Prior Individual Drum Tests

\begin{tabular}{|c|c|c|c|c|}
\hline \multirow{2}{*}{$\begin{array}{c}\text { Temperature } \\
\text { Range }\left({ }^{\circ} \mathrm{C}\right)\end{array}$} & Pallet & Individual & Pallet & Individual \\
\cline { 2 - 5 } & 0 & 0 & 0 & 0 \\
\hline$\leq 400$ & 0 & 0 & 0 & 1 \\
\hline $401-450$ & 0 & 0 & 0 & 5 \\
\hline $451-500$ & 0 & 0 & 2 & 1 \\
\hline $501-550$ & 0 & 0 & 0 & 1 \\
\hline $551-600$ & 0 & 0 & 1 & 7 \\
\hline $601-650$ & 1 & 2 & 1 & 6 \\
\hline $651-700$ & 10 & 7 & 7 & 4 \\
\hline$>700$ & 11 & 9 & 11 & 25 \\
\hline \hline Total & & & & \\
\hline
\end{tabular}

The incident heat flux to the drum is another criteria used to predict lid failure. Based on the earlier HAl testing, it is expected that drums exposed to radiant exposures less than $45 \mathrm{~kW} / \mathrm{m}^{2}$ will not experience lid failure; exposures in the range of 45 to $75 \mathrm{~kW} / \mathrm{m}^{2}$ will sometimes result in lid failure; and exposures greater than $75 \mathrm{~kW} / \mathrm{m}^{2}$ will experience lid failure. Heat flux measurements were obtained during the pallet test using total heat flux transducers mounted between the drums. Water filled drum calorimeters were not used during the pallet storage test. The heat flux transducers do not provide exact measurements of the actual incident heat flux to the drum surface. However, they can be used to estimate the maximum flame heat flux seen by a drum. 
As seen in Table 9, the heat flux measurements for the lid failure drums range from 77 to $166 \mathrm{~kW} / \mathrm{m}^{2}$, which are comparable to the rack test results and well within the range of heat fluxes expected to cause lid failure. Not all of the drums tisted in Table 8 have heat flux data becatse the measurements are applicable only to drums adjacent to heat flux transducers.

During the earlier $\mathrm{HAl}$ individual drum testing, it was not possible to develop criteria for lid failure based on drum pressure data. It was found that lid failure pressures ranged from about 13 to 28 psig with an average value of approximately 20 psig. For the current pallet storage test, lid failure pressures ranged from 8.9 to 17.3 psig with an average value of 13.2 psig. This is slightly higher than the failure pressure for the rack test. This is possibiy due to the additional weight of the stacked drums and pallets on top of the drums. However, the failure pressures generally agree with the earlier HAl individual drum lid failure pressures for the standard load drums which were smaller than the lid failure pressures for lighter drum loadings. The failure pressures for drums which vented ranged from 3.1 to 16.4 psig. This is higher than venting drums for the rack test but is consistent with venting pressures observed for the earlier HAl individual drum tests.

Although all of the drums within the flame were predicted to experience lid failure, more than half resulted in only lid seal failure. Only 46 percent of the drums in the flame experienced lid failure. Based on the temperature, flame heat flux, and pressure data, it is not possible to predict that these drums will not result in lid failure. There does not appear to be any distinguishable differences between many of the lid failure and lid seal failure drums within the flame. It is not expected that the weight of the stacked drums had any significant effect on lid failure. The load pressure of a standard drum (weight of drum per unit area of drum base) is a mere fraction of a psi, and as such is insignificant relative to the drum release pressures observed. Many of the lid seal failure drums had temperatures and exposures exceeding the criteria for lid failure. Since the drums, contents, and loading procedures were the same for both the earlier HAl individual drum tests the current rack and pallet tests, this is a surprising result. It is believed that modifications to the methodology are not necessary. If less conservatism is desired, it is possible to use a probabilistic approach to predict drum performance. As noted earlier, only about half of the drums within the flame experienced lid failure. Such response could be taken as indicative of the pallet storage configuration. However, caution should be taken in basing the probabilistic drum performance on a single test. The criteria and models currently used provide a reasonable and conservative estimate for the performance of drums within the flame.

\subsubsection{Drums Outside The Flame}

Unlike for the rack test, several drums outside the pool area experienced lid failure. Table 11 provides a summary of the experimental data for the pressure instrumented pallet storage drums outside the flame. Data for other drums is provided in Table 5 and Appendix E.

The heat flux to drums outside the pool is very different for the pallet test than the rack test. Figure 65 and 66 show transducer heat flux measurements along the west and north edge of the pool respectively. As seen in Figure 65, the flame emissive power along the west 
WHC-SD-WM-TRP-246 REV. 0

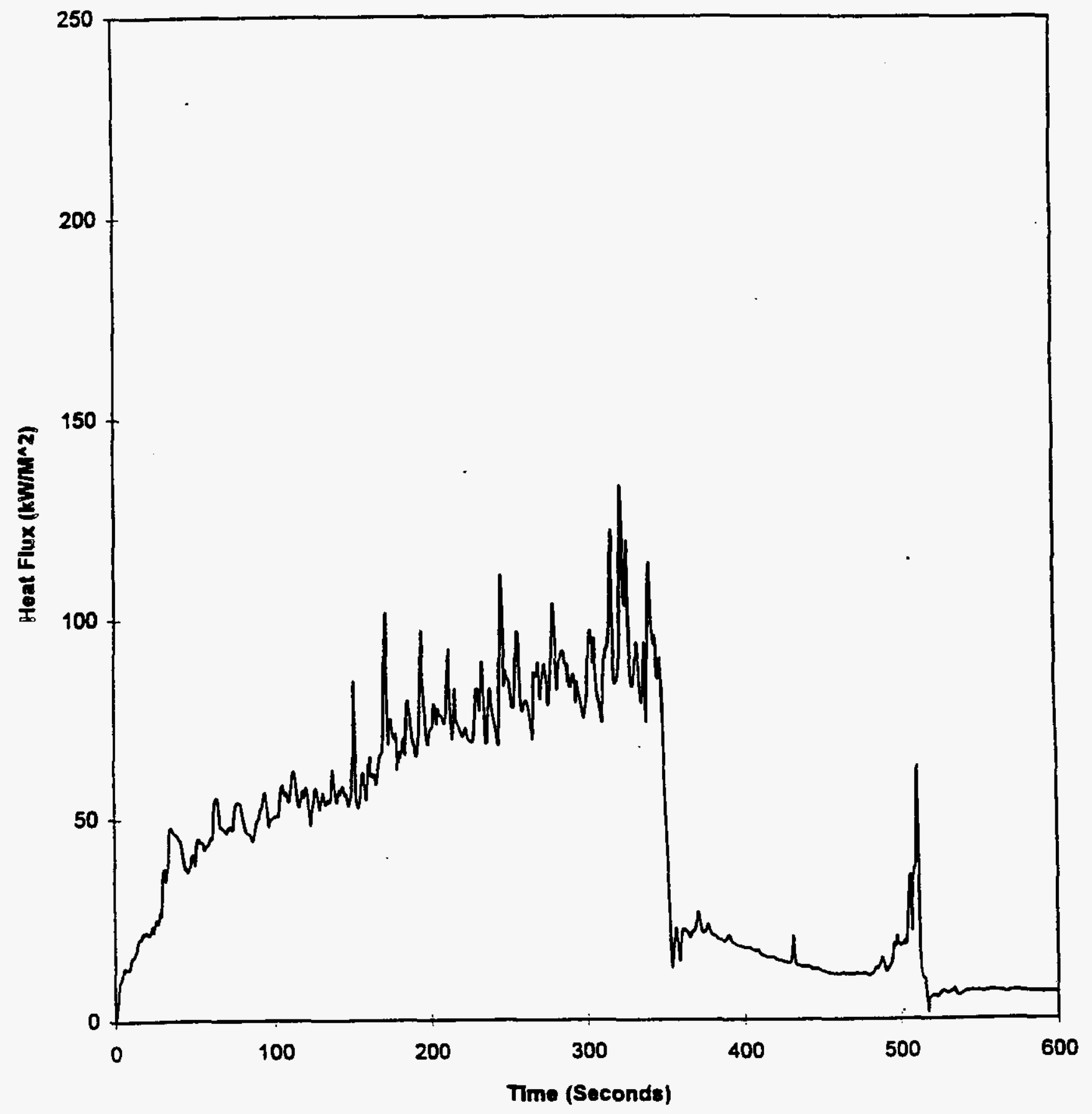

Figure 65. Pallet Storage Test - Transducer Heat Flux Measurement along West Edge of Pool (Cal \#4) 
WHC-SD-WM-TRP-246 REV. 0

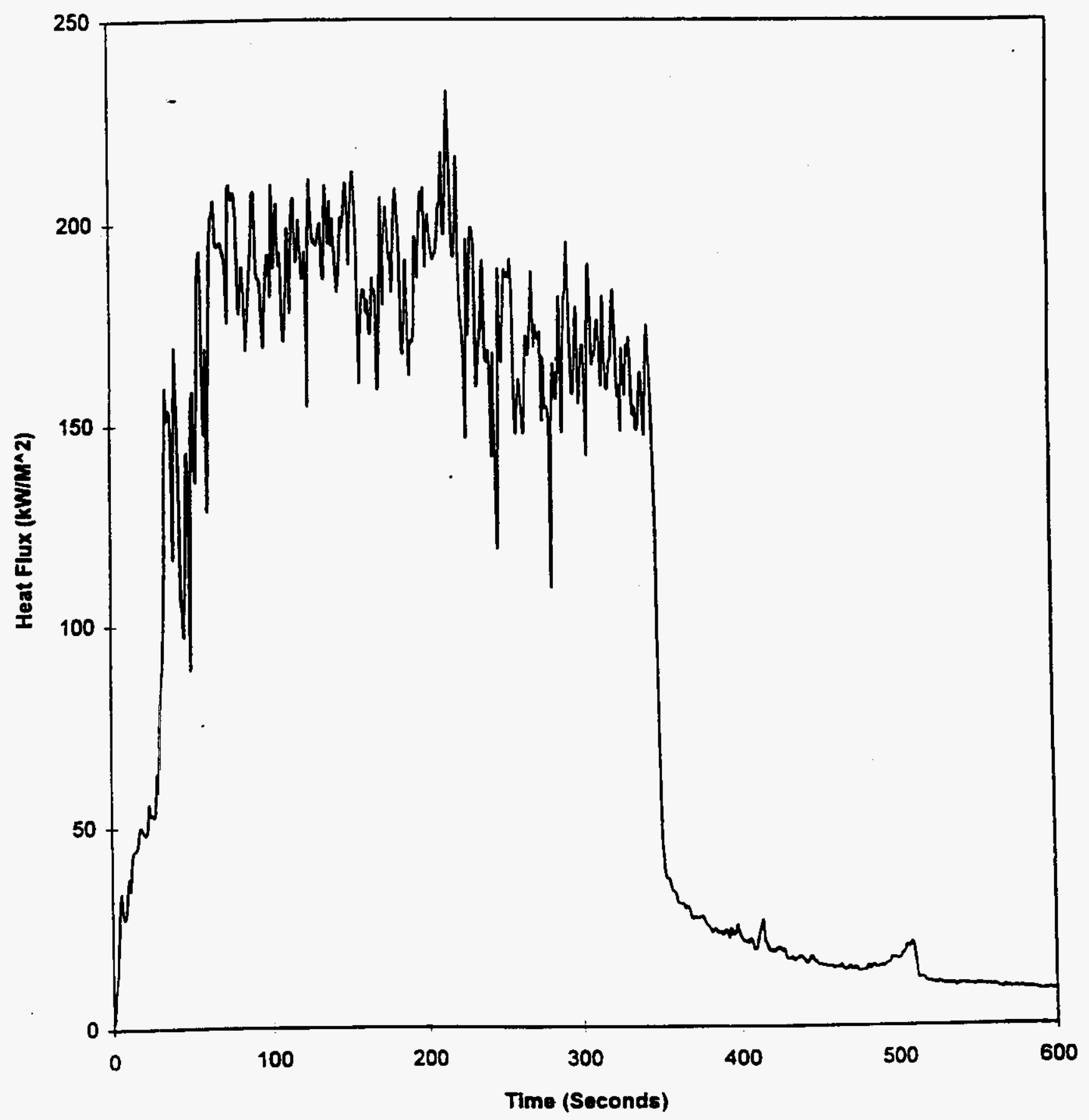

Figure 66. Pallet Storage Test - Transducer Heat Flux Measurement along North Edge of Pool (Cal \#10) 
edge becomes steady at approximately 80 to $90 \mathrm{~kW} / \mathrm{m}^{2}$. This is consistent with literature values and comparable to the rack test pool fire emissive power. However, as seen in Figure 66 , the flame heat flux along the north edge ranges from 150 to $200 \mathrm{~kW} / \mathrm{m}^{2}$. The difference in heat fluxes along the edge is due to the air entrainment into the fire. Along the west edge, air is free to enter the fire in the aisle. However, along the north edge, air can not be entrained through the drum stacks and therefore, the flame leans outward and impinges the drums. As such, uniike for the rack test, the drums along the north and south edge of the pool are subjected to direct flame exposure. As noted earlier, if the flame does not contact the drum, lid failure is not expected. In this case, the flame impinges the drums and causes lid failure for several of the drums.

Table 11. Summary of Experimental Data for Pressure Instrumented Pallet Storage Drums Outside the Flame

\begin{tabular}{|c|c|c|c|c|c||}
\hline $\begin{array}{c}\text { Drum } \\
\text { No. }\end{array}$ & $\begin{array}{c}\text { Lid Loss, Lid } \\
\text { Rupture, or } \\
\text { Vent }\end{array}$ & $\begin{array}{c}\text { Time of } \\
\text { Failure } \\
(\mathrm{s})\end{array}$ & $\begin{array}{c}\text { Drum Temp. } \\
\text { at Failure } \\
\left({ }^{\circ} \mathrm{C}\right)\end{array}$ & $\begin{array}{c}\text { Pressure at } \\
\text { Failure } \\
(\mathrm{psig})\end{array}$ & $\begin{array}{c}\text { Approx. Heat } \\
\text { Flux } \\
\left(\mathrm{kW} / \mathrm{m}^{2}\right)^{*}\end{array}$ \\
\hline \hline 0811 & LID RUPTURE & 156 & 857 & 11.3 & $\mathrm{NM}$ \\
\hline 0831 & VENT & 363 & 538 & 5.8 & 85 (cal 1) \\
\hline 0521 & LID LOSS & 203 & 811 & 12.8 & 200 (ca) 10) \\
\hline 0541 & VENT & 292 & 698 & 6.9 & $\mathrm{NM}$ \\
\hline 0812 & LID RUPTURE & 152 & 851 & 17.4 & $\mathrm{NM}$ \\
\hline 0532 & VENT & 212 & 918 & 11.4 & 176 (cal 11) \\
\hline 0813 & LID RUPTURE & 175 & 951 & 14.6 & $\mathrm{NM}$ \\
\hline 0533 & VENT & 254 & 920 & 11.6 & 124 (cal 12) \\
\hline
\end{tabular}

* See Figures 40.42 for calorimeter locations NM - not measured

Similar to drums within the flame, many of the drums adjacent to the pool which experienced lid seal failure had temperatures exceeding the criteria established for lid failure. Again, it is unclear why these drums did not result in lid failure. As seen in Figure 64, all of the drums adjacent to the flame are predicted to experience lid failure. Only 29 percent of these drums results in lid failure. As noted earlier for drums within the flame, a probabilistic approach could be used to predict drum performance. However, assuming that only one-third (i.e., approximately 29 percent) of the drums along the aisle experience lid failure based solely on a single test may not be appropriate. Given that some of these drums did experience lid failure and since many of them had temperature, heat flux, and pressure data consistent with lid failure drums, it is not analytically possible to discount the drums along the edge of the pool from experiencing lid failure. 
Drums in Bay 6 in Rows 5 and 8 were predicted to experience lid failure due to the radiant heat exposure from the flame. This was based on conservative estimates of incident heat fluxes and did not account for drum geometry and storage configuration (i.e., blockage from other drums). As suggested by the rack and pallet test results, since these drums are not in direct contact with the flame, they should not be expected to experience lid failure.

\subsubsection{Impact of Wood Pallets}

Following extinguishment of the pool fire, the wood pallets continued to burn and slowly propagate the fire along the rows. The flame took approximately one hour to spread to the end of the center row of pallets and caused additional lid seal failures along the way. Although this did not result in any additional lid failure drums, flame propagation from pallet to pallet was not expected. Prior testing of steel drum storage on wooden pallets [Snook, 1992] indicated that wooden pallets did not significantly ignite during the test and basically self extinguished once the ignition source was consumed. However, this test involved only non-combustible orum contents. For the current pallet test, drums experiencing lid seal failure often resulted in flaming jets which impinged upon the pallets. As the fire propagated from pallet to pallet, additional drums vented with jets which sustained the flame front on the pallet. This process continued until the fire reached the end of the pallet row.

Given the slow nature of the fire spread and minimal impact on the exposed drums, the wood pallets did not significantly affect the severity of this event. However, fire propagation should be expected if wood pallets are used. This will result in additional lid seal failure drums and may spread the fire to other areas not initially involved in the fire. Corrective actions such as providing barriers between wood pallets or using steel pallets would eliminate this problem.

\subsubsection{Assessment of Drum Stack Stability Model}

Based on the drum array stability model developed during earlier $\mathrm{HAl}$ testing, it was predicted that drums on lower tiers which experience lid failure would tip over the pallet stacks above. As a result, it was assumed that drums falling to the floor would experience lid failure. The model is based on the geometry and weight of the drums and pallets. Conservative assumptions are made regarding the force associated with lid loss and rotation of pallet stacks. It was estimated that the lid release pressure of a drum on the bottom tier required to topple the two pallet load above is approximately 17 to 20 psig. For a drum experiencing lid loss on the second tier, a lid release pressure of approximately 13 to 16 psig is required to topple the top tier. The model is based on the lid being completely removed from the drum. As such, lid ruptures can not topple the drums above.

There were two lid loss drums on the first tier for the pallet test (drum nos. 0631 and 05311 . The lid release pressure for 0631 was $16.4 \mathrm{psig}$ and for 0531 was $12.8 \mathrm{psig}$. Neither of the lid loss drums tipped the pallet stacks above. Although the lid release pressures are less than the 17 to 20 psig required, there was no rotation or significant displacement of the pallets above the lid loss drums observed. Since the ruptured drums can not topple the pallet stacks, they could not be used to assess the stability model. 
On the second tier, only a single drum experienced lid loss (drum no. 0622). This drum was not instrumented with a pressure transducer so pressure data is not available. Again, no rotation or significant displacement of the pallet above the lid loss drum was observed.

It is believed that the drum array stability model is overly conservative in estimating the release pressure required to topple stacked drums. There was no evidence that lid failures significantly affected the stability of drums on pallets above. Even though the drums were banded, the drums would not have fallen to the floor due to lid failures below. However, the banding did prevent several drums from falling due to wood pallets burning and losing their strength.

\subsection{REASSESSMENT OF THE MODEL PERFORMANCE}

The predictions of the results of the tests reported in Section 3 of this report were made prior to the conduct of the tests and were based on the modeling and individual drum tests reported in an earlier report [Rhodes et al., 1995]. The performance of the models in predicting the rack and pallets tests was reviewed in the previous sections (Sections 8.2 and 8.3, respectively). In the light of these comparisons with data, is appropriate to reassess the model with the view of enhancing the ability to predict drum array performance in the future.

Before addressing specific elements of the model, it is important to note that the performance of the model in predicting the results of these tests is both a function of the model and the model users. Inasmuch as the model users here are the model developers, one would assume that the results did not suffer from uninformed or inappropriate use of the model. Nonetheless, the model users here were motivated to provide conservative results. When an uncertainty arose, the more conservative choice was made. While this is an appropriate strategy for model use, it does not generally lead to the most realistic results possible. In the following discussion, changes in the use of the model which can reasonably used to improve the realism of the predicted results are discussed.

The primary cause for conservatism in the model use was the selection of the emissive power of the pool fire. This was not a subject investigated in the prior individual drum work [Rhodes et al., 1995], because such information is readily available in the literature. Unfortunately, the available sources of emissive power correlations are not in full agreement, and the differences can result in very different results in this application. Further, there are relatively little heat flux data to target very near pool fires. Most data are at least one pool radius away from the pool edge. As such, the reliability of the correlations in lowest in the area of most importance in this application. Coupled with the emissive power uncertainties is the range of drum wall temperatures found at lid failure in the individual drum tests. The results ranged from about $625^{\circ} \mathrm{C}$ to near $800^{\circ} \mathrm{C}$. Coupling the use of a conservative emissive power with a conservative drum wall temperature at lid failure resulted in much over predicted lid failures outside the pool area.

Using the Shokri/Beyler correlation and an average rather than conservative drum wall temperature in the rack predictions results in the correct prediction that no lid failures are expected. It is significant that in the rack tests the pool fire flame did not directly contact the drums. The air entrained between the drums in the rack caused the flame to neck as it normally does in an open pool fire such that a model for exposures to targets outside the flame is appropriate. Conversely, the flames in the pailet test did directly contact the drums adjacent 
to the pool fire. This results from the very tightly packed nature of the pallet array which did not allow significant air entrainment to the flame through the array. As a result, the flame did not "neck-in" (narrow) as it normally does in a free pool fire, and as a result, the flame contacted the drums adjacent to the pool. This results in a qualitatively different thermal exposure which did cause some lid failures. This condition is much more similar to a target within a pool fire than it is similar to a target outside the pool fire. Treating the drum as a target within this pool fire results in heat fluxes significantly above those required for lid loss. Thus, for this situation, the use of a correlation for targets outside the flame like the Shokri/Beyler or the Mudan/Croce correlations is not appropriate. The simple view that arises out of the testing and modeling is that for large scale pool fires, lid failures are to be expected where flame contact occurs and are not expected when there is no flame contact. We know that in open rack arrangements, drums outside the pool area will not experience direct pool flame contact whereas in the tight palletized storage configuration pool flame contact is expected. This simple approach is consistent with the use of the Shokri/Beyler emissive power correlation in conjunction with an average drum wall temperature criterion of $700^{\circ} \mathrm{C}$.

Table 12 shows a comparison of the original predictions of lid failure, predictions based on the revised predictive methods, and the results of the rack and pallet experiments. Clearly, based on the revisions to the predictive method discussed above, no change in the pretest predictions within the pool area have been made. In the rack test, the revised predictions provide a very good prediction of the actual results. Discrepancies result from eight drums within the pool fire area which experienced lid seal failure. These drums were the least severely exposed drums within the pool area as the flame leaned inward toward the pool center significantly during the test. Without the use of computation fluid mechanics models of pool fires, there is no hope of predicting these detailed features of the flame. While this is an active area of research, no reliable methods of analysis are currently available. The

Table 12. Comparison of Heat-induced Lid Failures in the Pretest Predictions, the Revised Predictive Method, and the Drum Array Test

\begin{tabular}{||l|c|c|c||}
\hline \multicolumn{4}{|c|}{ Rack Test } \\
\hline & Within Pool* & Outside Pool & Total \\
\hline \hline Pretest Predictions & 45 & 46 & 91 \\
\hline Revised Predictive Method & 45 & 0 & 45 \\
\hline Rack Test Results & 37 & 0 & 37 \\
\hline \multicolumn{3}{|c|}{ Pallet Test } & \\
\hline & Within Pool & Outside Pool & Total \\
\hline \hline Pretest Predictions & 24 & 42 & 66 \\
\hline Revised Predictive Method & 24 & 24 & 48 \\
\hline Pallet Test Results & 12 & 7 & 19 \\
\hline
\end{tabular}

* excludes three water-filled drums. 
computer models which currently exist are strictly research tools at this time and are not yet available or appropriate for use. In the pallet test, the revised predictions overestimate the total number of lid failures by a factor of 2-3 both within the pool area and outside the pool area. Within the pool only half the drums experienced lid failure and only 30 percent of the drums outside the pool exposed to flame contact experienced lid failure. There were clear asymmetries in the flame geometry and resulting heat fluxes. Such asymmetries cannot be predicted, and this leads to conservative predictions. Even without such considerations, most of the drums with direct flame contact would have been expected to experience lid loss based on drum wall temperatures. It is unclear why many of the drums experienced lid seal failure rather than lid failure.

The other area of major conservatism in the pretest predictions is in the area of array stability. The original predictions were based on a release pressure of 20 psig, which represented the full individual drum test series results. However, the drums with normal loading in the individual drum tests had release pressures systematically less than the overall average (13-16 psig). Thus, a lower release pressure was indicated by the individual drum test results for normally loaded drums. In the pallet tests, the release pressures ranged from 9-17 psig. The failure mode of the drum lids in the pallet test were typified by lid rupture. This, of course, violates one of the assumptions in the stability model, i.e. that the drum lid release is via simple rotation and lid deformation does not occur. Clearly, the lid deformation reduces the likelihood of loss of array stability. This limitation was recognized at the outset, but no practical means of including lid deformation was apparent at the time, and no solution is apparent even now. As such, we recognize the stability model to be very conservative, but the extent of conservatism is not known since no loss of stability was ever observed. Based on the evidence available at this time, normally loaded drums in pallet arrays with metal banding are not expected to topple. Further, the assumption that the drums would experience lid failure due to falling is also a conservative assumption which was made due to a lack of information to the contrary.

Other areas where the model was challenged was the flame propagation via vent jets and wooden pallets. The model simply did not include this phenomenon. Based on prior testing with noncombustible drum contents such behavior was not anticipated. The pallet test clearly showed this is a viable method for flame propagation, but that lid failures do not result. No modeling approach currently exists for this problem, and it is not at all clear that such a model is needed given the range of solutions available for the problem. The test further verifies that drum to drum fire propagation with lid failure does not occur as was predicted. This, in the end, is the single most important result and prediction.

For future applications, it would be most appropriate to make use of the revised methodology which makes use of the insights provided by the drum array testing. 
Blank 


\subsection{CONCLUSIONS AND RECOMMENDATIONS}

The two full-scale fire tests were successfully performed, and the results of the tests have been analyzed in detail. The two tests conducted were representative of a three tier, palletized storage configuration and a six tier, high rack storage configuration.

The high rack storage test resulted in extensive drum and rack damage within the confines of the pool fire. The rack suffered serious structural damage as expected and all drums within the pool fire vented. Lid failures were experienced on all tiers of the rack within the pool area. No lid failures were observed outside the pool fire area. No propagation to any drum outside the pool area was observed.

The palletized storage test resulted in extensive drum damage within the confines of the pool fire. Drums in the pallet stacks directly adjacent to the pool also experienced lid failures and some had jet flames from the vent. There was no loss of stability of the pallet arrays in the test. The major surprise of the test was the ability of fire to propagate along the pallet rows by a combination of pallet burning and vent flames. While the propagation was extremely slow, it did reach the end of the storage array, indicating that it could have continued indefinitely. Prior results from the literature indicated that the palletized storage of drums containing noncombustibles could not support this type of propagation. As such, it appears that a combination of pallet and vent flames from the drums is required for this propagation. No such propagation was observed for the metal pallet row.

The predictions of the behavior of the drum arrays was generally conservative relative to the actual test results. For drums within the flame, lid failure is expected to occur. Although this was not the case for all of the drums, there are no apparent criteria to distinguish between lid failure and seal failure drums. For the rack and pallet tests, there were significant differences for drums along the edge of the pool. For the rack test, spaces between drums and tiers allowed sufficient air to be entrained through the rack to the fire. This resulted in necking of the flame such that there was no significant flame impingement on the drums along the pool edge. For the pallet test, the closely spaced drums and stacked pallets inhibited the air entrainment to the fire. This resulted in the flame attaching itself to the drums along the edge of the pool. Whereas there were no lid failures for drums along the edge of the pool in the rack test, several lid failures occurred for the pallet test. It appears that if the flames do not impinge upon the drum, lid failure will not occur.

For drums outside the immediate flame region, two radiation models from the literature were used to predict drum performance. The less severe radiation model (i.e., Shokri and Beyler [1989]) provided better predictive results than the more severe Mudan and Croce [1988] model. The Shokri and Beyler model yielded no lid failures outside of the flame which is consistent with the experimental results.

The predictions for the palletized storage differed from the experiments in two important ways. First, the experiments had no loss of stability of the pallet arrays. The model indicated that some loss of stability was expected. Two factors are thought to contribute to the differences in expected and observed stability. The pressures measured at lid loss in these full scale tests were significantly less than that observed in the prior individual drum tests. No rationale for this difference has yet been identified though prior work did indicate a definite trend of failure pressure with drum contents. Further, the stability model as developed was 
known to be conservative in its assumptions. The second phenomenon which was not correctly predicted by the model was the propagation of the fire via the pallet/vented drum mechanism. This phenomenon had never before been observed and was not anticipated by the model. There are many ways of preventing this type of propagation, including the use of metal pallets.

Overall, the experiments validated the predictions of the model and indicated that propagation outside the area of the initiating fire are not expected under the conditions studied except by the wood pallet propagation mechanism. The propagation of the fire in the pallet rows via the pallet/vented drum mechanism is the primary weakness of the model which was uncovered by the full scale testing.

The experimental work has shown that the rack storage array as tested has serious structural stability problems. The steel uprights due to their design will not resist a worst case fire. The use of the tested wire mesh to support the drums is questionable due to its response under loaded fire conditions. For the rack storage array, it is recommended that future designs account for the structural stability problems as seen in the test. This may include but not be limited to other shapes or sizes for vertical uprights and support mesh, use of fireproofing materials, or other design fire protection features.

The palletized system, as was tested, showed that fire propagation through the length of the rows could be obtained when wood pallets are used. The steel pallets did not exhibit this propagation. It should be noted, however, that even though the propagation did occur, it was not rapid but rather a slow process. Manual intervention could have stopped the progression fairly easily. The use of noncombustible (i.e., steel) pallets is one alternative. Other less costly alternatives would be to cause a fire break either by using a single noncombustible tier of pallets at specific intervals such as every third pallet, or by placing a noncombustible material such a single layer of 5/8-in. thick, Type $X$ gypsum wallboard between some interval of pallets such as every third pallet. The gypsum wallboard should be $1.2 \mathrm{~m}(4 \mathrm{ft})$ wide (same width as pallets) and extend $\sim 0.3 \mathrm{~m}(\sim 1 \mathrm{ft}$ ) above the top of the highest drum.

Based on these tests, trash expulsion is negligible during a fire. Fire propagation via this mechanism was not observed and is not expected.

The behavior of the drum lids was significantly different than that noted in the earlier test work by HAl. In the earlier work, drum lids either remained on the drum or were violently expelled from the drum. In both of the array tests, no violent expulsion of the lids was noted. In the array tests, the lids showed the rupture failure mechanism which was not experienced in our earlier work. 
known to be conservative in its assumptions. The second phenomenon which was not correctly predicted by the model was the propagation of the fire via the pallet/vented drum mechanism. This phenomenon had never before been observed and was not anticipated by the model. There are many ways of preventing this type of propagation, including the use of metal pallets.

Overall, the experiments validated the predictions of the model and indicated that propagation outside the area of the initiating fire are not expected under the conditions studied except by the wood pallet propagation mechanism. The propagation of the fire in the pallet rows via the pallet/vented drum mechanism is the primary weakness of the model which was uncovered by the full scale testing.

The experimental work has shown that the rack storage array as tested has serious structural stability problems. The steel uprights due to their design will not resist a worst case fire. The use of the tested wire mesh to support the drums is questionable due to its response under loaded fire conditions. For the rack storage array, it is recommended that future designs account for the structural stability problems as seen in the test. This may include but not be limited to other shapes or sizes for vertical uprights and support mesh, use of fireproofing materials, or other design fire protection features.

The palletized system, as was tested, showed that fire propagation through the length of the rows could be obtained when wood pallets are used. The steel pallets did not exhibit this propagation. It should be noted, however, that even though the propagation did occur, it was not rapid but rather a slow process. Manual intervention could have stopped the progression fairly easily. The use of noncombustible (i.e., steel) pallets is one alternative. Other less costly alternatives would be to cause a fire break either by using a single noncombustible tier of pallets at specific intervals such as every third pallet, or by placing a noncombustible material such a single layer of 5/8-in. thick, Type $X$ gypsum waliboard between some interval of pallets such as every third pallet. The gypsum wallboard should be $1.2 \mathrm{~m}(4 \mathrm{ft}$ ) wide (same width as pallets) and extend $-0.3 \mathrm{~m}(\sim 1 \mathrm{ft}$ ) above the top of the highest drum.

Based on these tests, trash expulsion is negligible during a fire. Fire propagation via this mechanism was not observed and is not expected.

The behavior of the drum lids was significantly different than that noted in the earlier test work by HAl. In the earlier work, drum lids either remained on the drum or were violently expelled from the drum. In both of the array tests, no violent expulsion of the lids was noted. In the array tests, the lids showed the rupture failure mechanism which was not experienced in our earlier work. 
blank 


\section{*alls View/Frint Document Cover Sheet tow}

This document was retrieved from the Documentation and Records Manaqement (DRM) ISEARCH System. It is intended for Information only and may not be the most recent or updated version. Contact a Document Service Center (see Hanford Info for locations) if you need additional retrieval information.

Accession \#: D195061618

Document \#: SD-WM-TRP-246

Title/Desc:

SOLID WASTE DRUM ARRAY FIRE PERFORMANCE [SEC 2 OF 7]

Pages: 151 
THIS DOCUMENT WAS TOO LARGE TO SCAN AS A SINGLE

DOCUMENT. IT REQUIRED BREAKING INTO SMALLER SECTIONS.

DOCUMENT NUMBER: SD-WM-TRP-2HL

SECTION: $2 \quad$ OF 7

TITLE: Lolid wante Drum array fure Performance

AUTHOR: Louies RL

RECIPIENT:

DATE: $11 / 07 / 95$

KEYWORDS:

REFERENCES: EDT-612232 
Bank 


\subsection{REFERENCES}

Babrauskas, V. (1988), "Burning Rates," Section 2/Chapter 1, The SFPE Handbook of Fire Protection Engineering, National Fire Protection Association, P. DiNenno, Editor-inChief, Society of Fire Protection Engineers, Boston, MA.

Boothe, G.F. (1994), "Radiological and Chemical Source Terms for Solid Waste Operations, Complex," WHC-SD-W306-TI-001 REV 1, 1994.

Carver, M.R. (1993), "Drums, DOT UN1A2, Solid Material, 114/208/322 Liter Saivage, Galvanized, Steel, "HS-V-P0010, Impact Level 3-SQ, Westinghouse Hanford Company, January 20, 1993.

Heskestad, G., (1988), "Fire Plumes," Section 1/Chapter 6, The SFPE Handbook of Fire Protection Engineering, National Fire Protection Association, P. DiNenno, Editor-inChief, Society of Fire Protection Engineers, Boston, MA.

Kanury, A.M.(1975), Introduction to Combustion Phenomena, Gordon and Breach Science Publishers, New York..

Mudan, K.S., and P.A. Croce, (1988) "Fire Hazard Calculations for Large Open Hydrocarbon Fires," The SFPE Handbook of Fire Protection Engineering, National Fire Protection Association, P. DiNenno, Editor-in-Chief, Society of Fire Protection Engineers, Boston, MA.

Parker, A.J. (1995), "Rack and Palletized Storage Array Tests of Solid Waste Drums," Final Report, Volume I - Test Report, SwRI Project No. 01-7106-001, prepared for Los Alamos Technical Associates, Southwest Research Institute, San Antonio, TX, June 1995.

Rhodes, B.T., Beitel, J.J., Gottuk, D.T., Beyler, C.L., and Rosenbaum, E.R. (1995), "Analytical and Experimental Evaluation of Solid Waste Drum Fire Performance," Hughes Associates Inc., prepared for Westinghouse Hanford Company, April 27, 1995.

Shokri, M., and C.L. Beyler, (1989) "Radiation from Large Pool Fires," Journal of Fire Protection Engineering, Vol. 1, No. 4.

Snook, B. (1992), "Pallet Fire Test for Steel Drum Storage on Wooden Pallets," Martin Marietta, Portsmouth Gaseous Diffusion Plant, 1992. 
Bank 
WHC-SD-WM-TRP-246 REV. 0

Appendix A

Rack Storage and Palletized Storage

Pre-test Checklist 
WHC-SD-WM-TRP-246 REV. 0

Rack Storage Pre-test Checklist

Racks:

3 rows of 6 tiers installed per test plan

Bottom rack units anchored to floor

Single rack secured to ceiling

Back-to-back rows fastened together

All hardware secure

Containment pans and mesh installed

Structures plumb and level

Seismic event bars installed

Verify dimensions of rack storage system

Drums:

144 drums labeled per Drum Location Diagram (DDS)

All drums identified correctly on DDS (initialize description)

All (except $\mathrm{H}_{2} \mathrm{O}$ filled drums) Joaded with standard load and steel

Drums sealed, torqued, and documented on DDS

Leak test drums with pressure measurements and document of DDS

All drums weighed and recorded on DDS

Thermocouples installed on appropriate drums

Photograph typical setup of each drum type

Videotape typical setup of each drum type

Drums positioned per Drum Location Diagram

_ Verify correct spacing between drums as installed in racks

Instrumentation:

Check each pressure transducer calibration

Catalog all heat flux transducer, certified calibration sheets

- Verify correct calibrations for instruments in data acquisition

Ambient pressure measurements verified

Verify operation and location of thermocouples through computer (apply heat)

Verify operation and location of heat flux transducers through computer

Verify operation of $\mathrm{H}_{2} \mathrm{O}$ calorimeter pumps

Fuel:

$14.7 \mathrm{~m}^{2}\left(162 \mathrm{ft}^{2}\right)$ pool area fabricated per test plan

Pool area filled with $-2.5 \mathrm{~cm}$ ( 1 in.) of water and inspected for leaks

$208 \mathrm{~L}(55 \mathrm{gal})$ of diesel fuel staged

$8 \mathrm{~L}(2 \mathrm{gal})$ of gasoline staged

Foam extinguishing system in place and tested 


\section{Rack Storage Pre-test Checklist (Continued)}

Data Acquisition:

Data acquisition system ready

Pre-test video and still photographs complete

Video cameras positioned per test plan and operable

Camera 1 tape set

Camera 2 tape set

Photographer positioned and ready

Remote video monitors setup

Remote monitors setup for data acquisition if required

Observation structure in place under overhead door

Tape recorders loaded and ready 
Pallet Storage Pre-test Checklist

Pallets:

Banded together

Properiy oriented per test plan

Drums:

144 drums labeled per Drum Location Diagram (DDS)

All drums identified correctly on DDS (QA: initialize description)

Drums sealed, torqued, and documented on DDS

Leak test drums with pressure measurements and document of DDS

All drums weighed and recorded on DDS

Thermocouples installed on appropriate drums

Photograph typical setup of each drum type

Videotape typical setup of each drum type

Drums positioned per Drum Location Diagram

Instrumentation:

Check each pressure transducer calibration

Catalog all heat flux transducer, certified calibration sheets

Verify correct calibrations for instruments in data acquisition

Heat flux transducers positioned per test plan

Thermocouples positions per test plan

Ambient pressure measurements verified

Verify operation and location of thermocouples through computer (apply heat)

Verify operation and location of heat flux transducers through computer

Fuel:

$9.3 \mathrm{~m}^{2}\left(100 \mathrm{ft}^{2}\right)$ pool area fabricated per test plan

Pool area filled with $-2.5 \mathrm{~cm}$ ( 1 in.) of water and inspected for leaks

$135 \mathrm{~L}(35 \mathrm{gal})$ of diesel fuel staged

$8 \mathrm{~L}$ ( $2 \mathrm{gal})$ of gasoline staged

Foam extinguishing system in place and tested 


\section{Pallet Storage Pre-test Checklist (Continued)}

Data Acquisition:

Data acquisition system ready

Pre-test video and still photographs complete

Video cameras positioned per test plan and operable

Camera 1 tape set

Camera 2 tape set

Still photography camera positioned and ready

Remote video monitors setup if required

Remote monitors setup for data acquisition if required

Observation structure in place under overhead door

Tape recorders loaded and ready 
blank 
WHC-SD-WM-TRP-246 REV. 0

Appendix B

Rack Storage and Palletized Storage

Test Procedure Checklist 


\section{Rack Storage and Pallet Storage}

Test Procedure Checklist

Pre-test checklist complete

Verify all vents in building open

All observers in designated locations

Firefighters suited and standing by

Foam extinguishing system charged

Start tape recorders

Start video tapes

Start data acquisition and synchronize with video

Pour diesel fuel

Pour gasoline

Ignite fuel (data acquisition time recorded)

Test termination criteria 


\title{
Solid Waste Drum Array Fire Performance
}

\author{
Volume II \\ Appendix C. Rack Storage Test Data \\ Appendix D. Rack Storage Drum Data Sheets
}

\author{
Prepared for \\ Westinghouse Hanford Company \\ Richland, WA 99352
}

Prepared by

Chris F. Haecker

Los Alamos Technical Associates, Inc.

Kennewick, WA 99306

Jesșe J. Beitel, Daniel T. Gottuk, Brian T. Rhodes, and Craig L. Beyler Hughes Associates, Inc.

Baltimore, MD 21227 
Blante 
WHC-SD-WM-TRP-246 REV. 0

Appendix C

Rack Storage Test Data

C-1 
Beante 


\section{RACK STORAGE TEST \\ INTERNAL DRUM PRESSURES}

(TIER \#1)

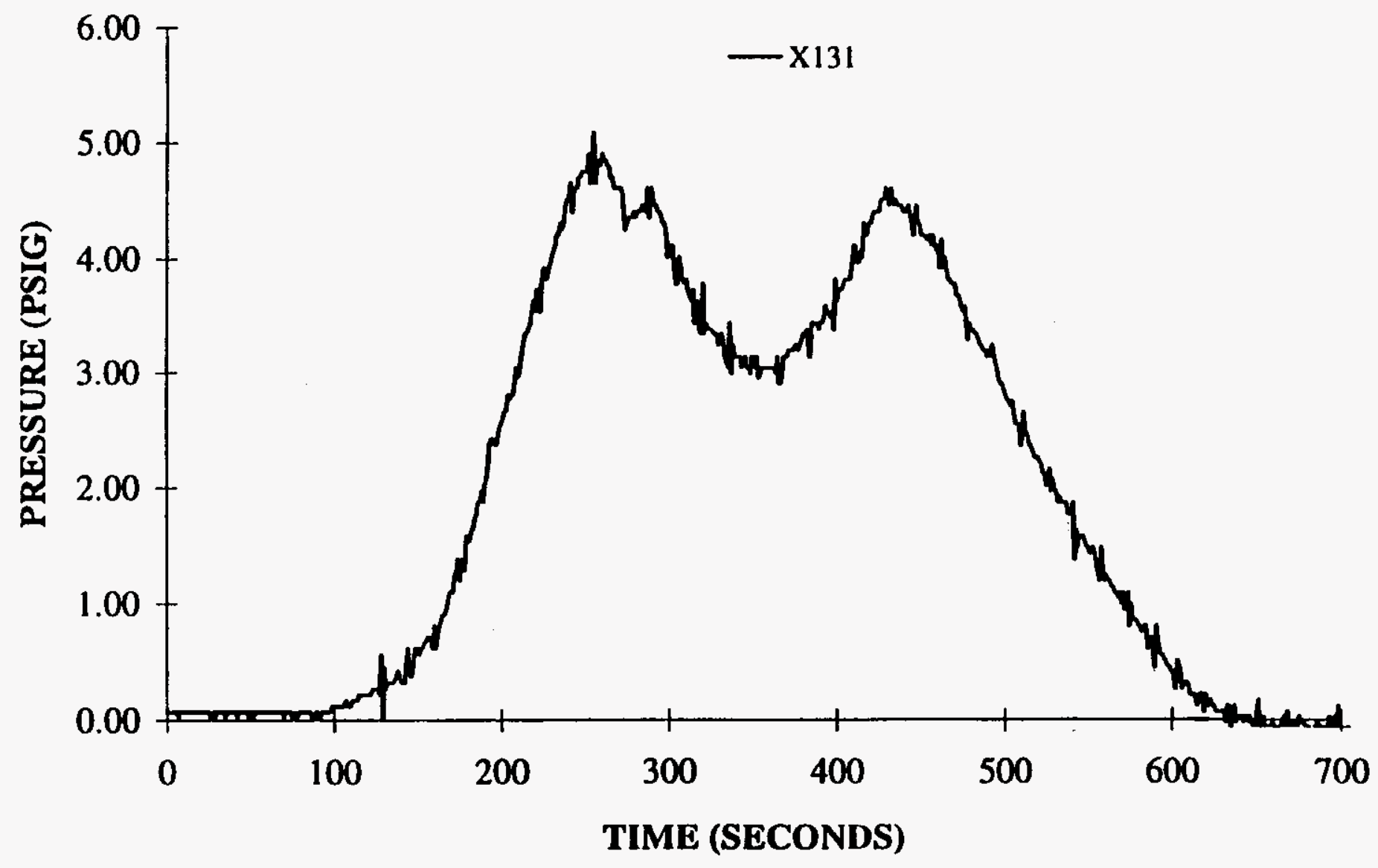


CLIENT: LATA

PROJECT NO.: SwRI 01-7106

DATE: 6 JUNE 1995

TEST ID: P24.PRN

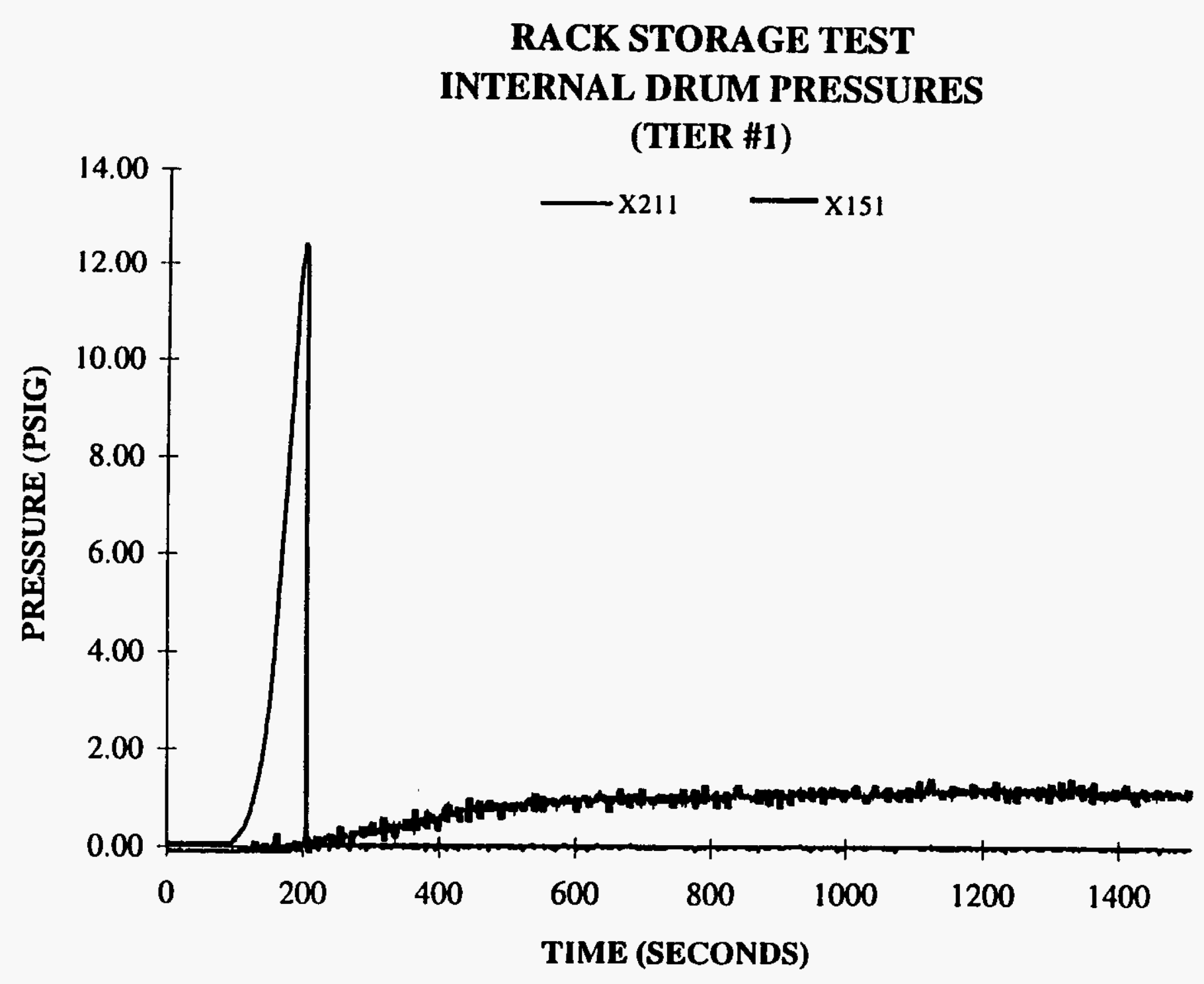


CLIENT: LATA

PROJECT NO.: SwRI 01-7106

DATL: 6 JUNE 1995

TEST ID: P24.PRN

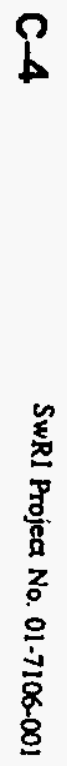

\section{RACK STORAGE TEST INTERNAL DRUM PRESSURES}

(TIER \#1)

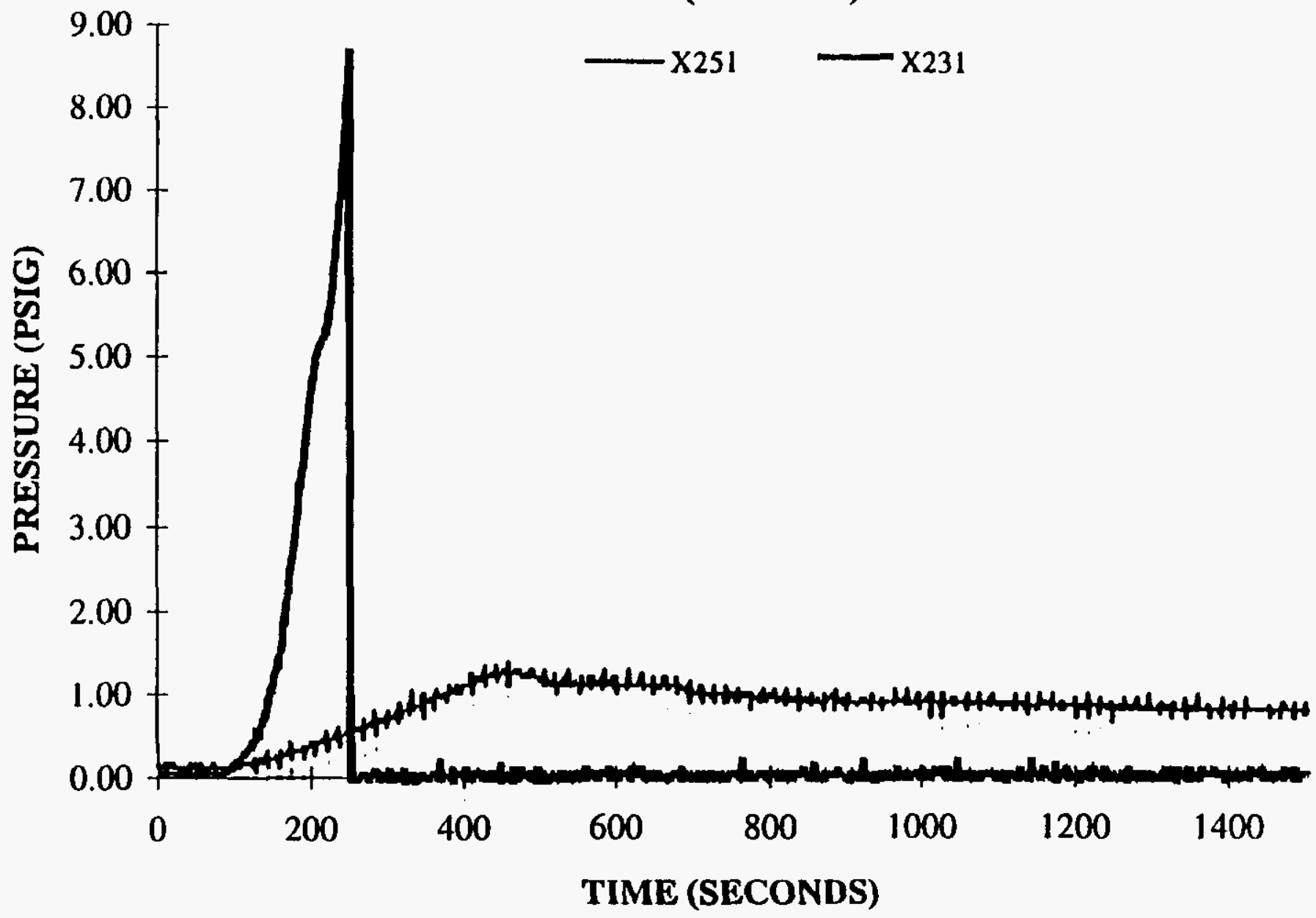


CLIENT: LATA

PROJECT NO.: SwRI 01-7106

DATE: 6 JUNE 1995

TEST ID: P24.PRN

\section{RACK STORAGE TEST \\ INTERNAL DRUM PRESSURES}

(TIER \#1)

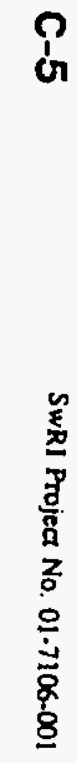

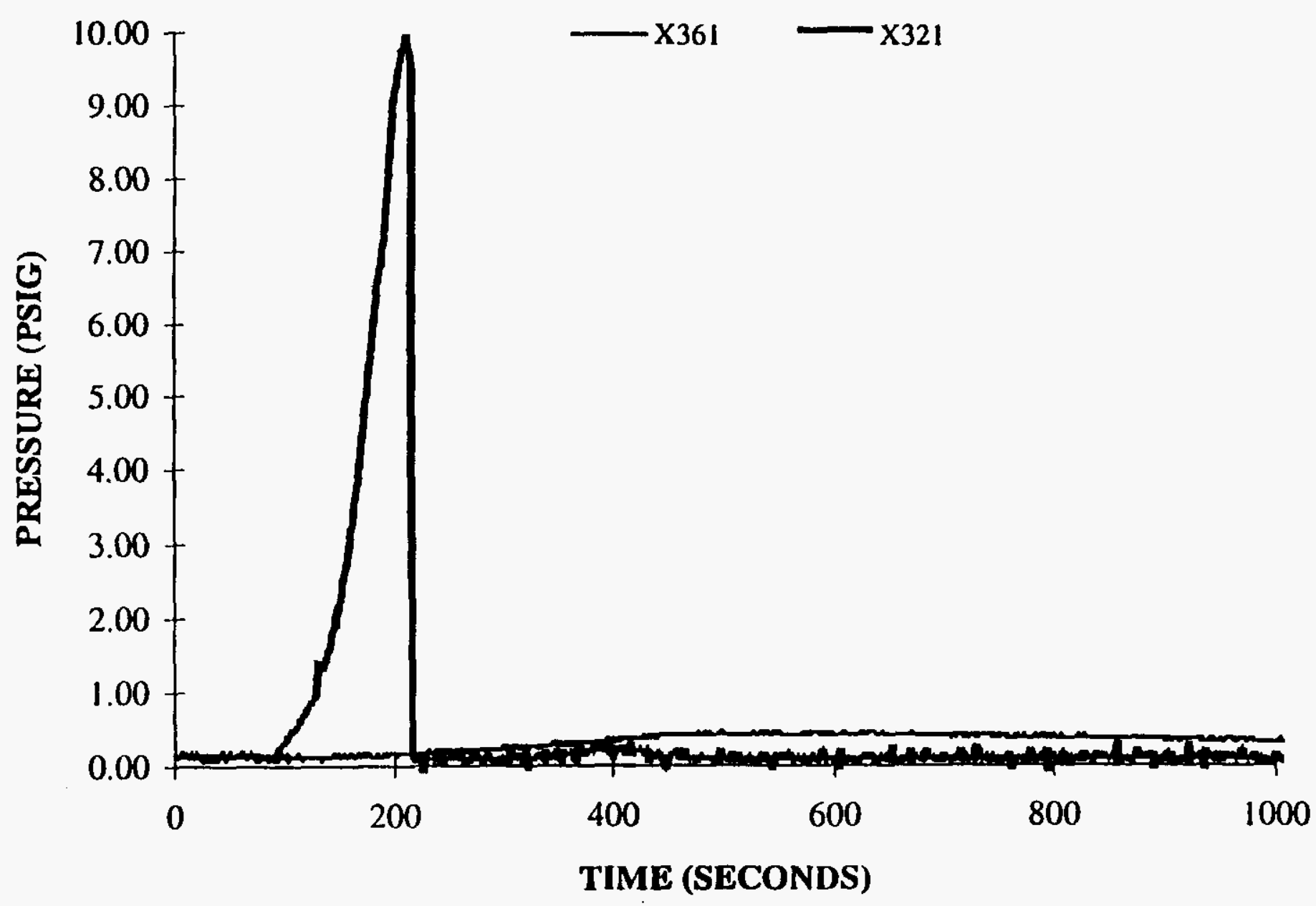




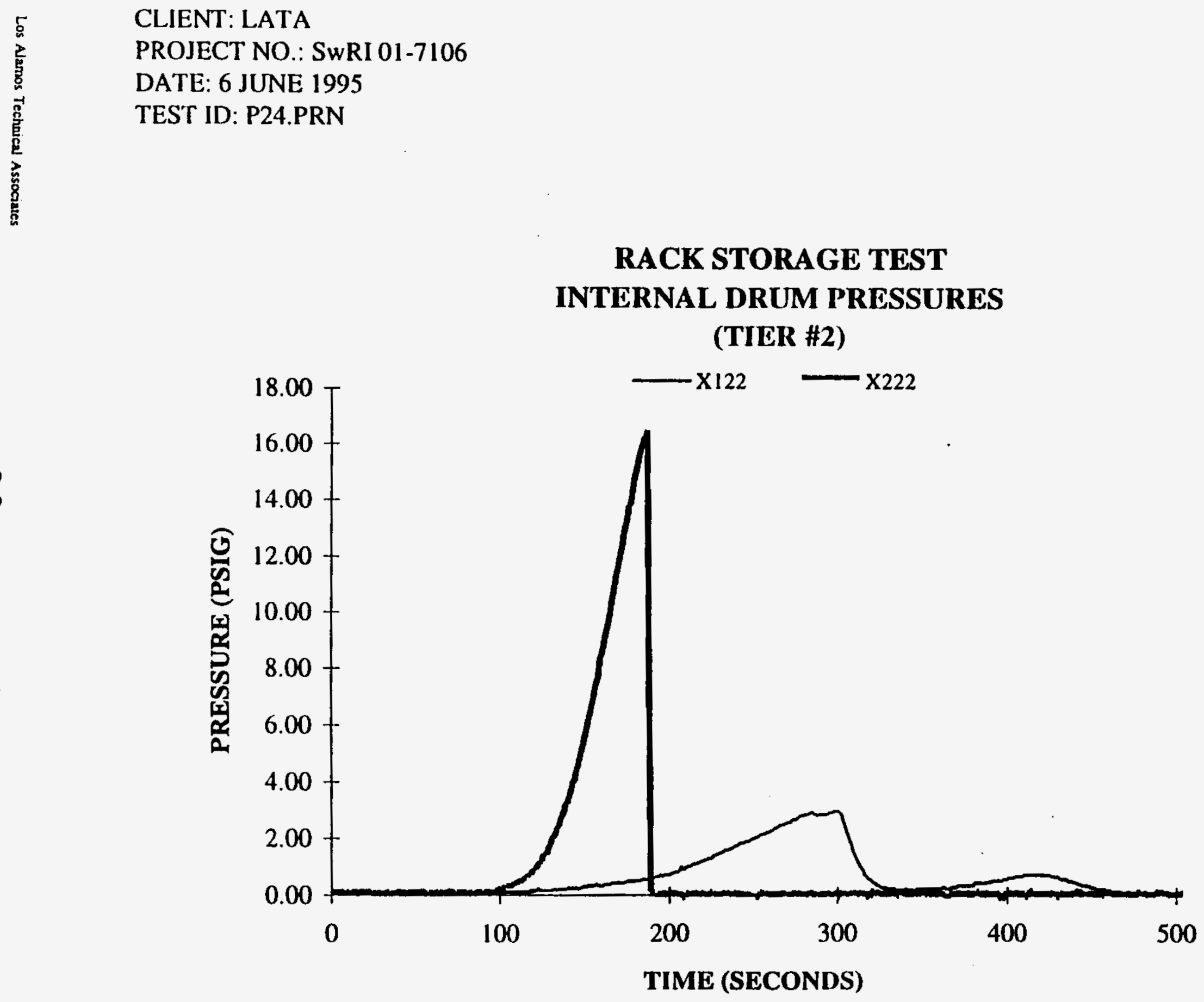


CLIENT: LATA

PROJECT NO.: SwRI 01-7106

DATE: 6 JUNE 1995

TEST ID: P24.PRN

\section{RACK STORAGE TEST \\ INTERNAL DRUM PRESSURES}

(TIER \#2)
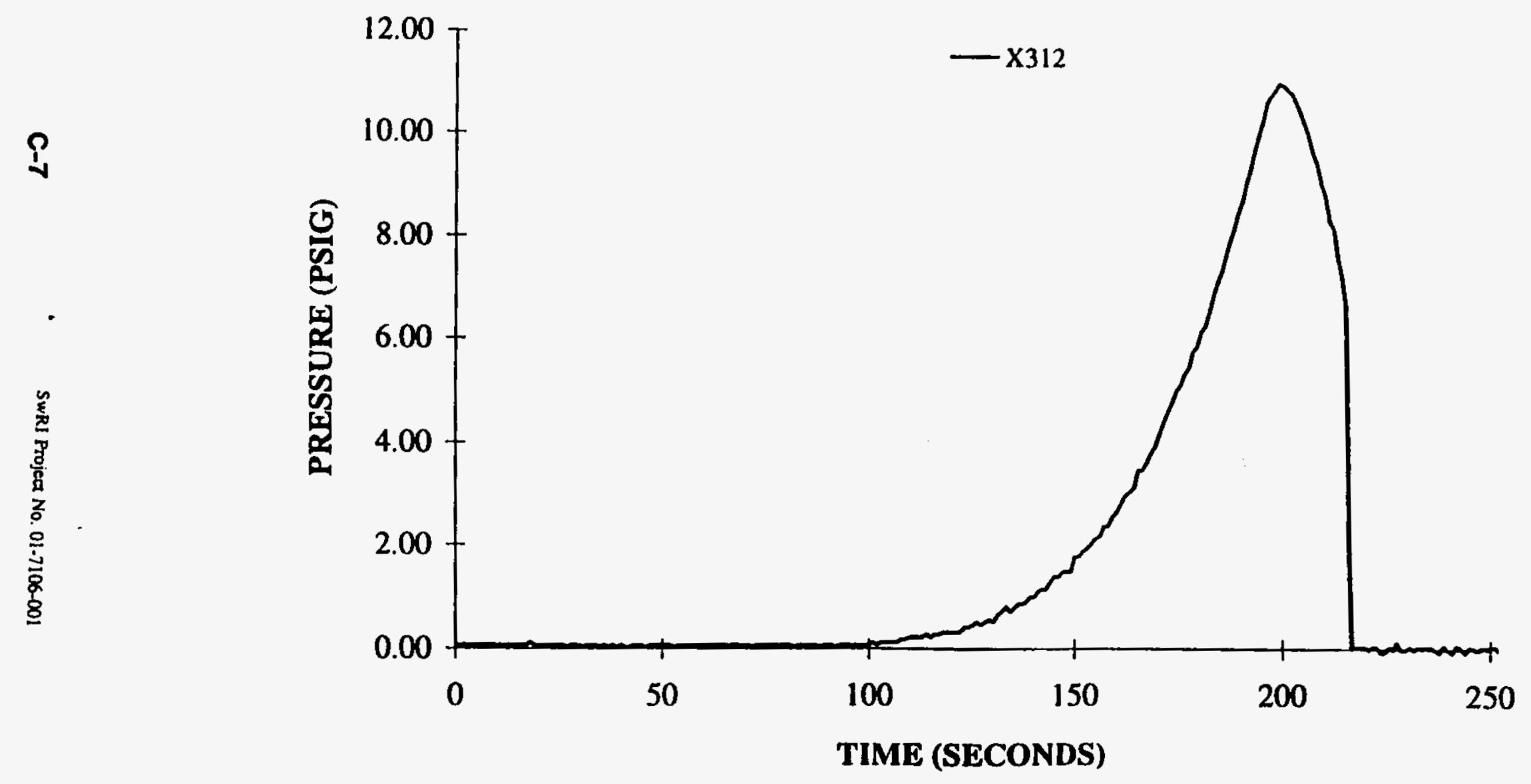
WHC-SD-WM-TRP-246 REV. 0

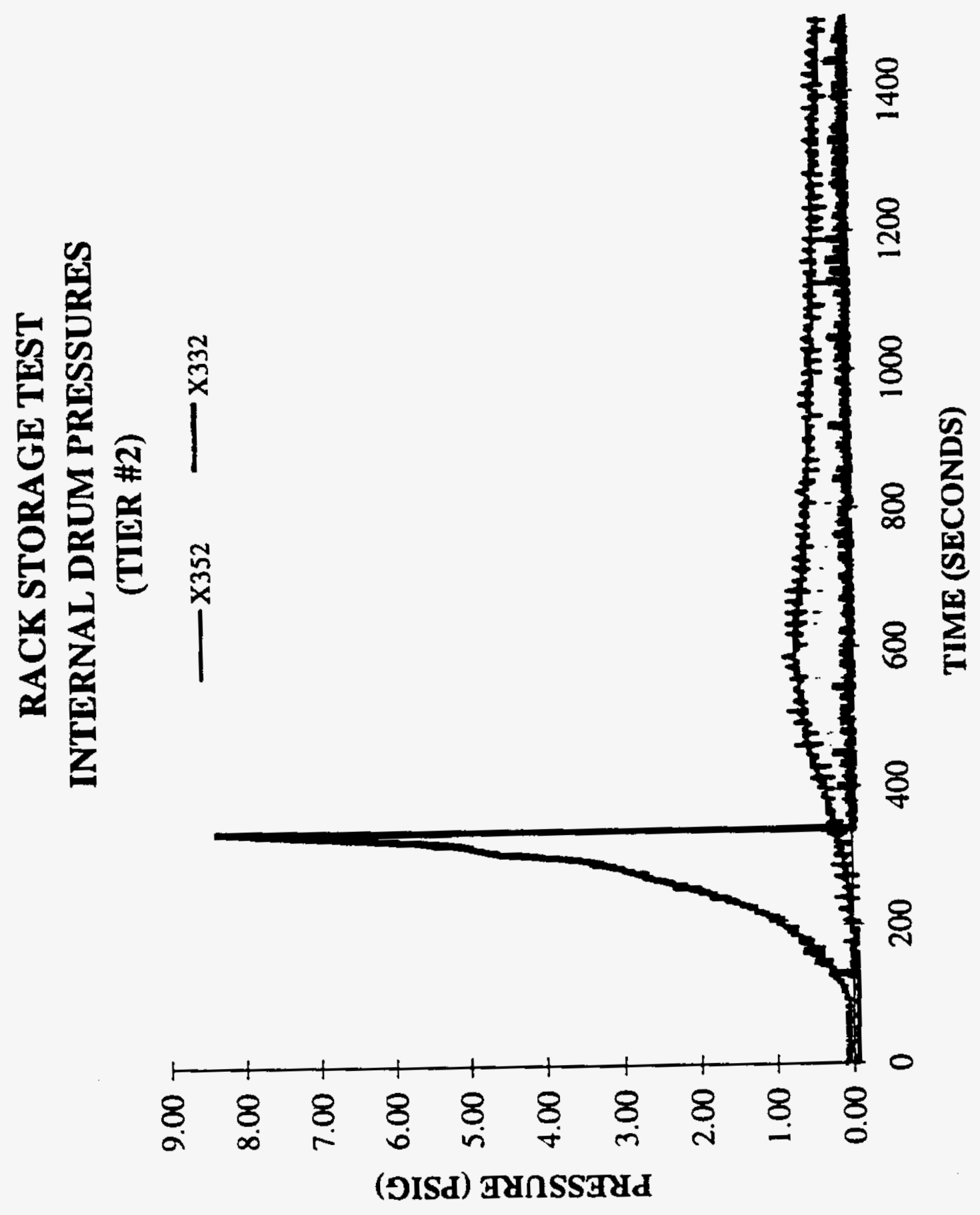


WHC-SD-WM-TRP-246 REV. 0

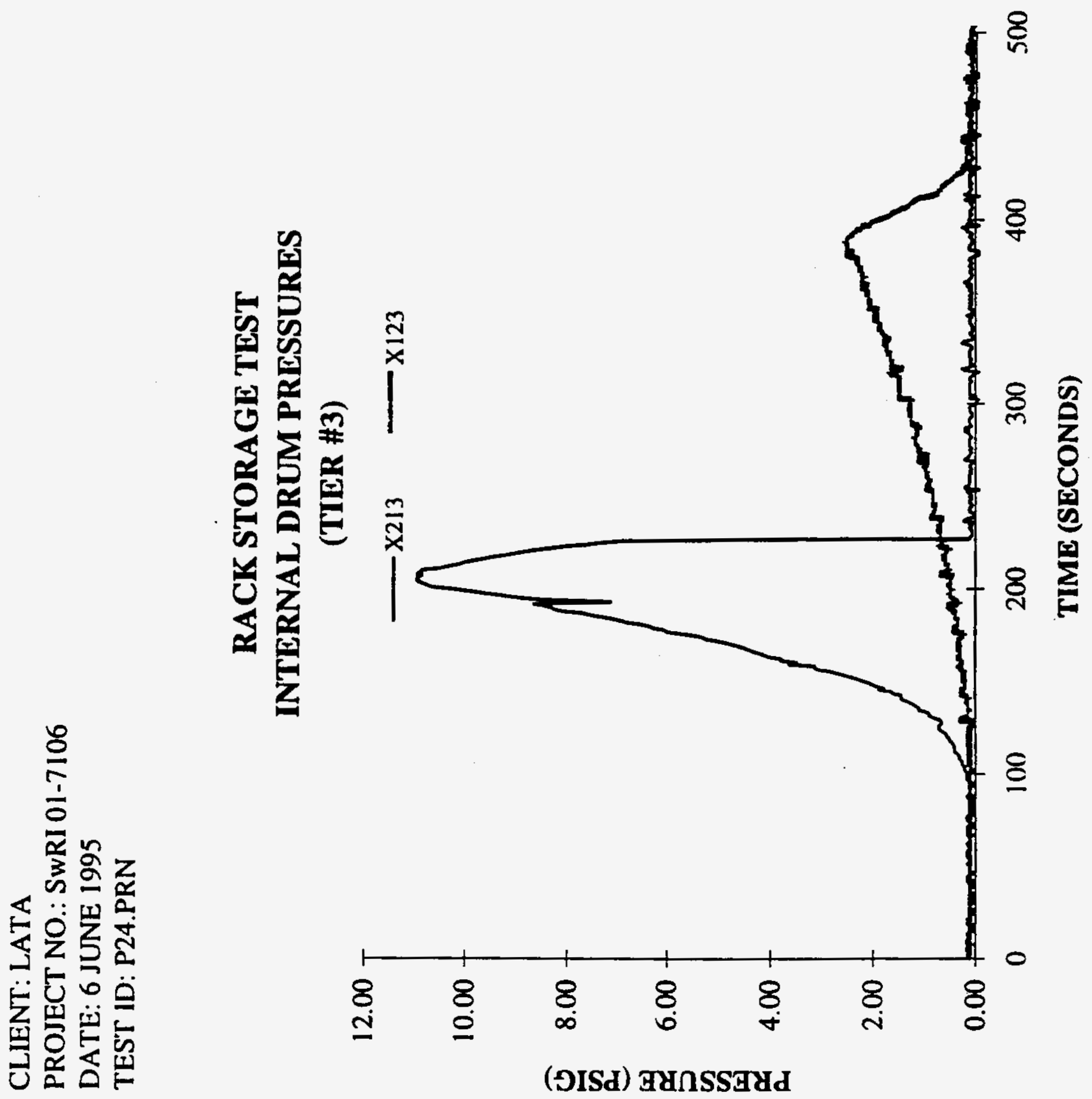




\section{CLIENT: LATA}

PROJECT NO.: SwRI 01-7106

DATE: 6 JUNE 1995

TEST ID: P24.PRN

?

\section{RACK STORAGE TEST \\ INTERNAL DRUM PRESSURES}

(TIER \#3)

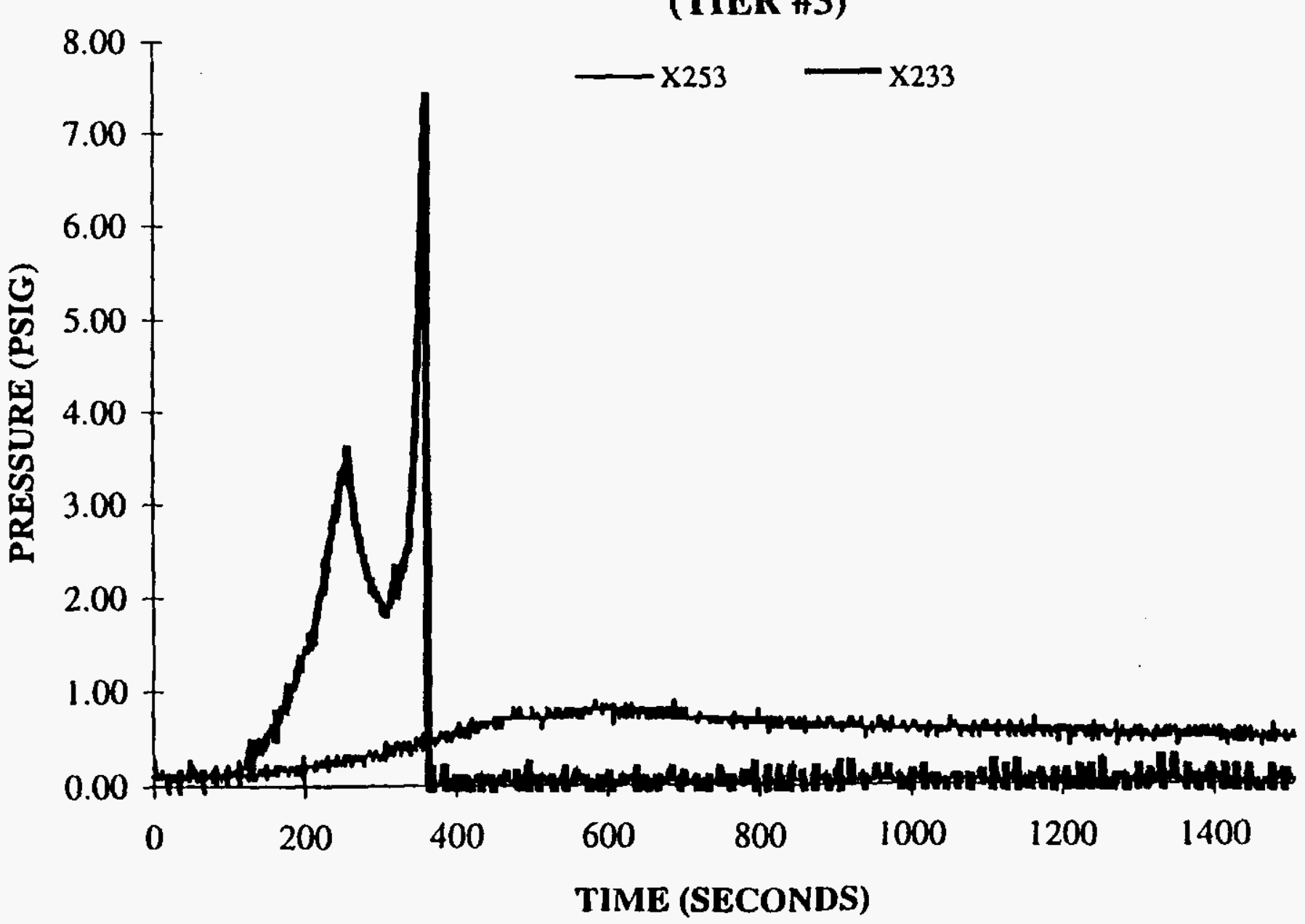



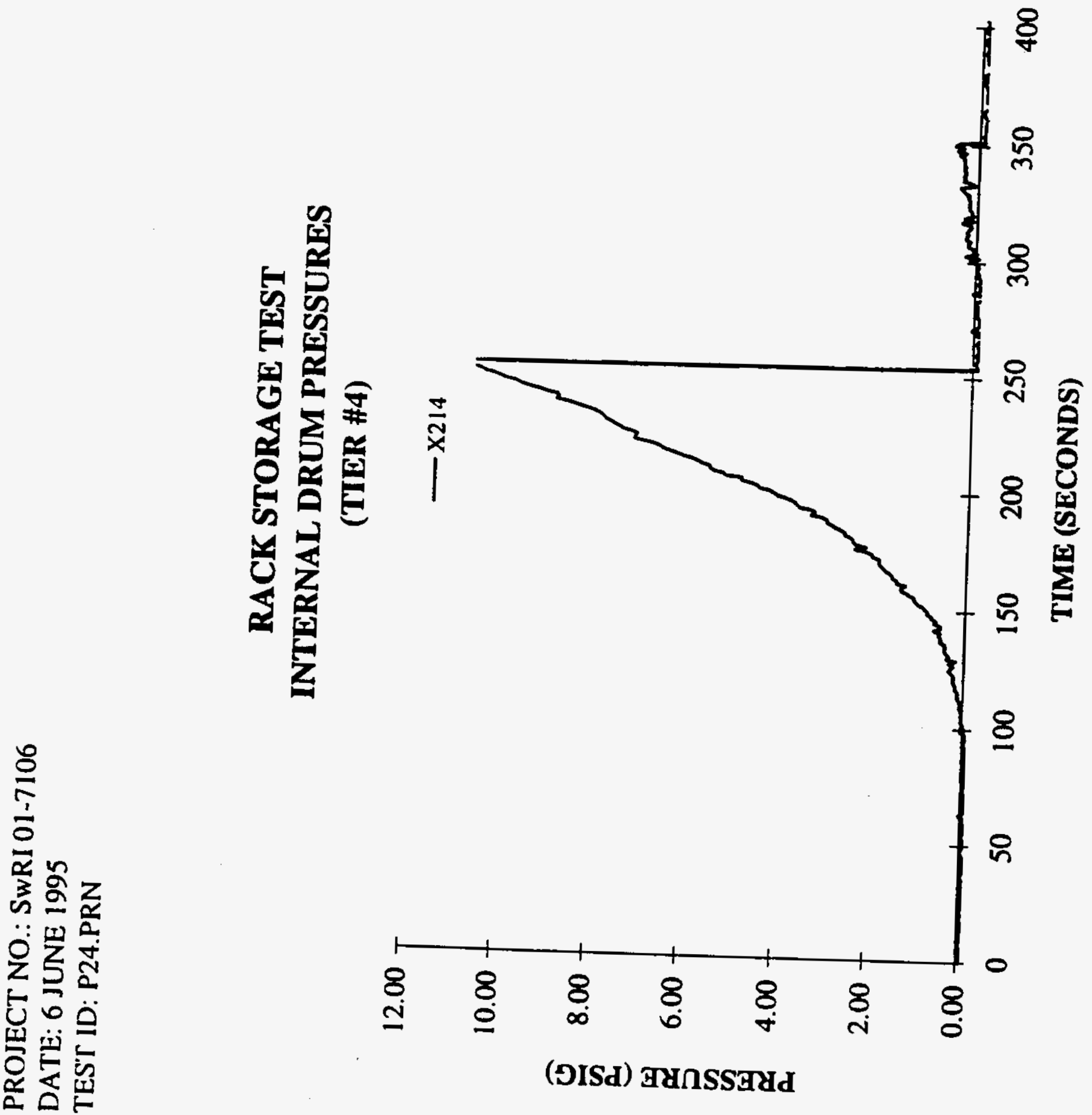


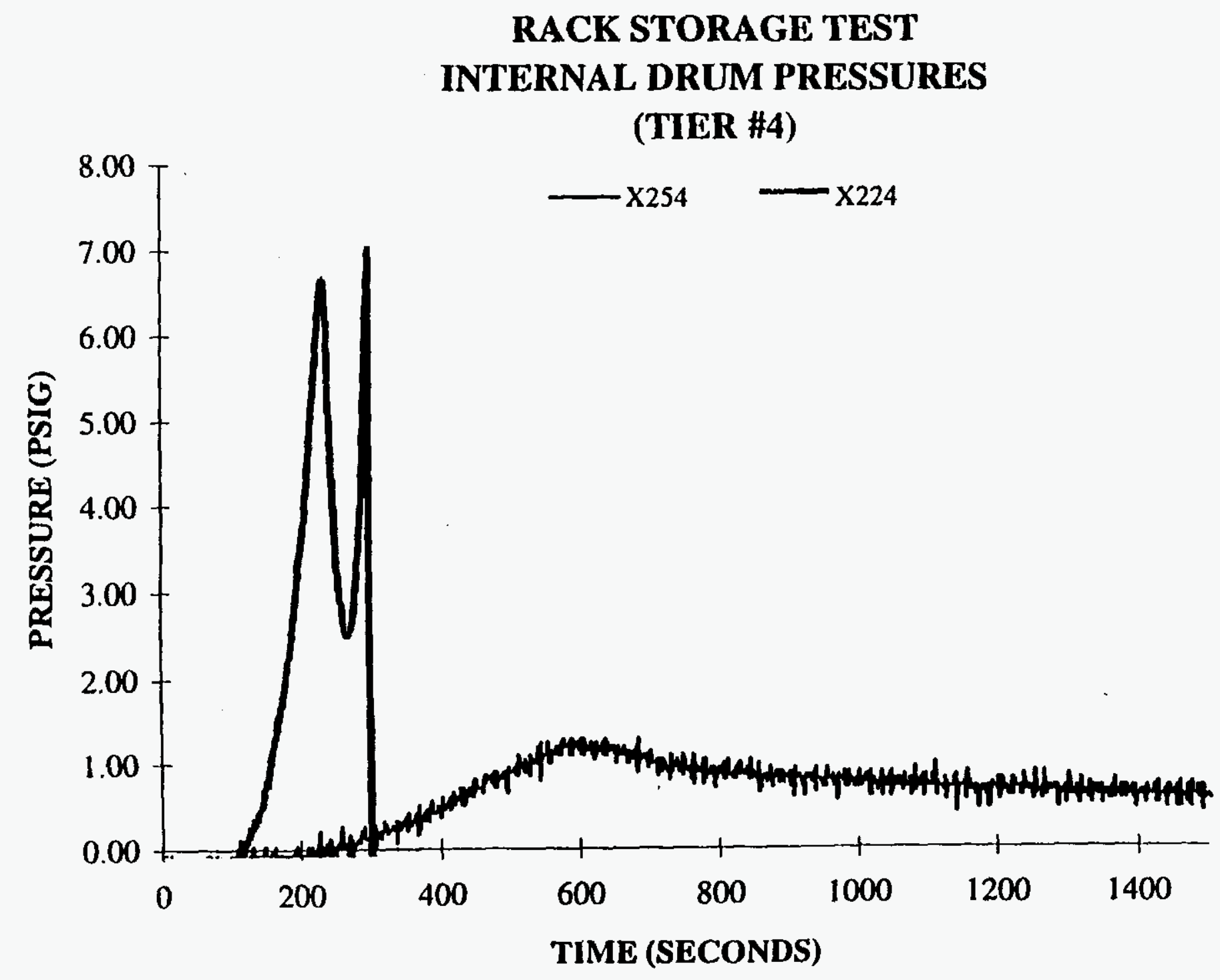




\section{CLIENT: LATA}

PROJECT NO.: SwRI 01-7106

DATE: 6 JUNE 1995

TEST ID: P24.PRN

\section{RACK STORAGE TEST \\ INTERNAL DRUM PRESSURES}

(TIER \#5)

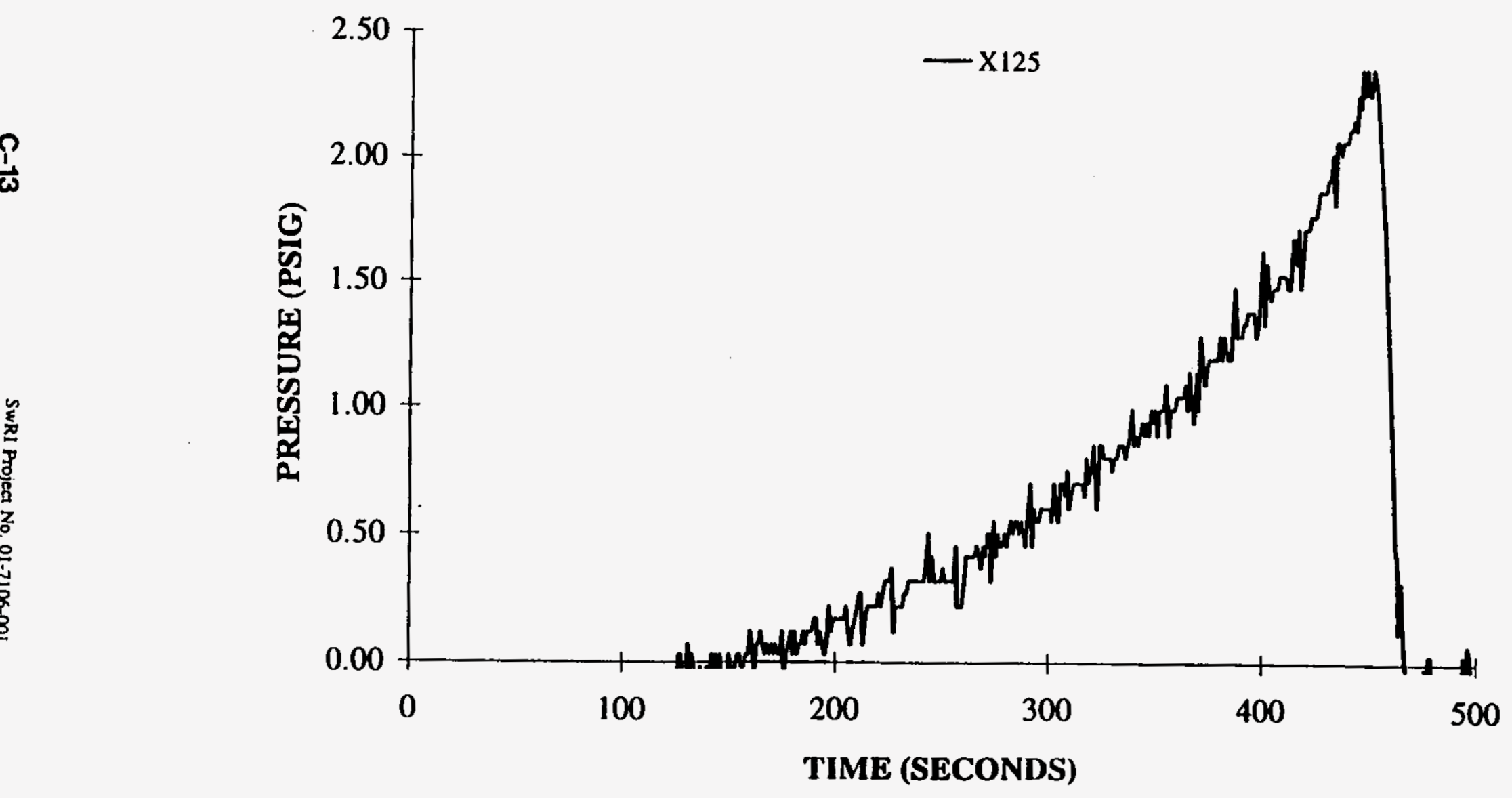

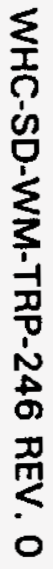

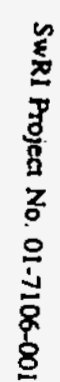

$\stackrel{?}{\omega}$ 


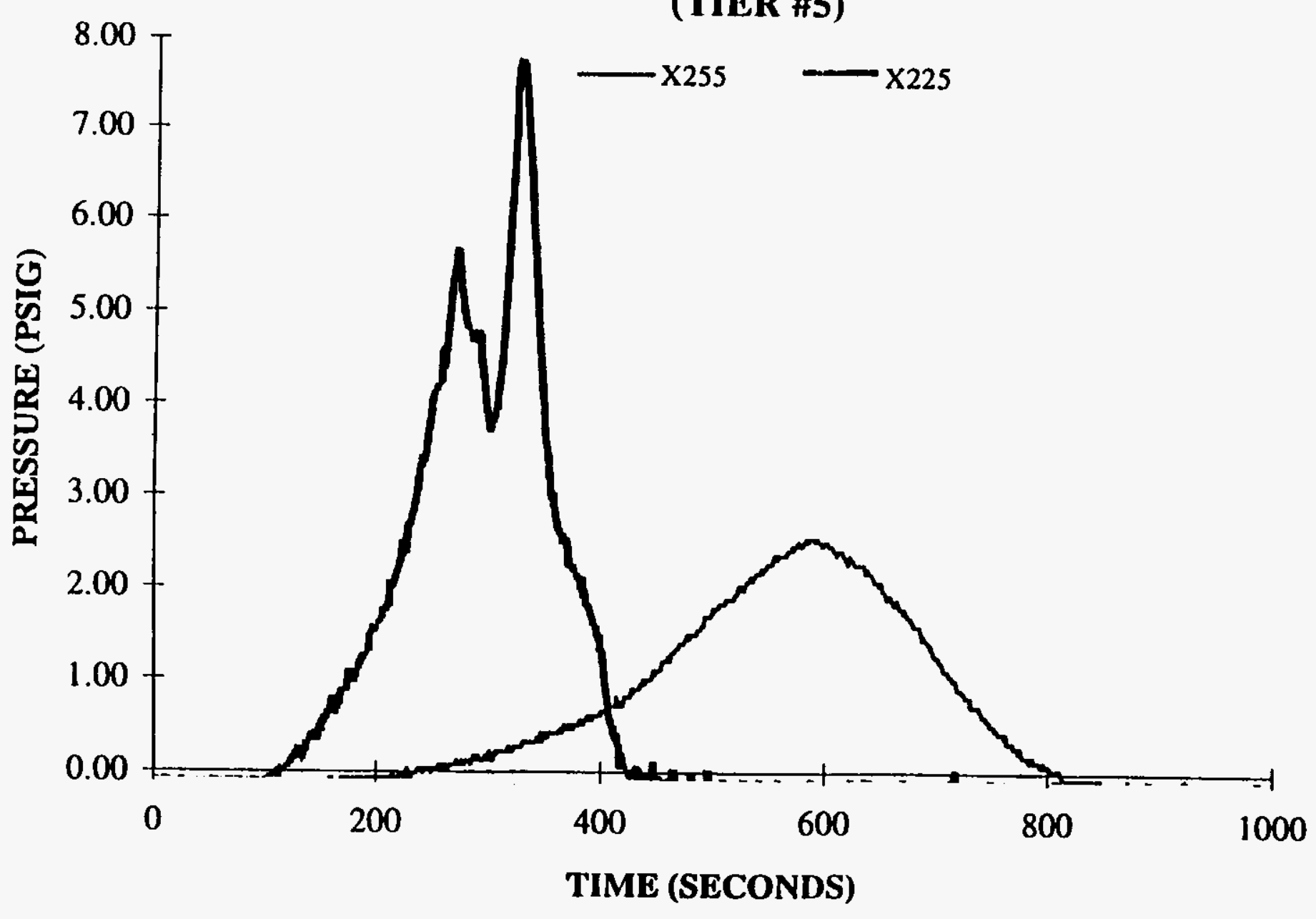


PROJECT NO.: SwRI 01-7106

DATE: 6 JUNE 1995

TEST ID: P24.PRN

\section{RACK STORAGE TEST \\ INTERNAL DRUM PRESSURES}

(TIER \#6)

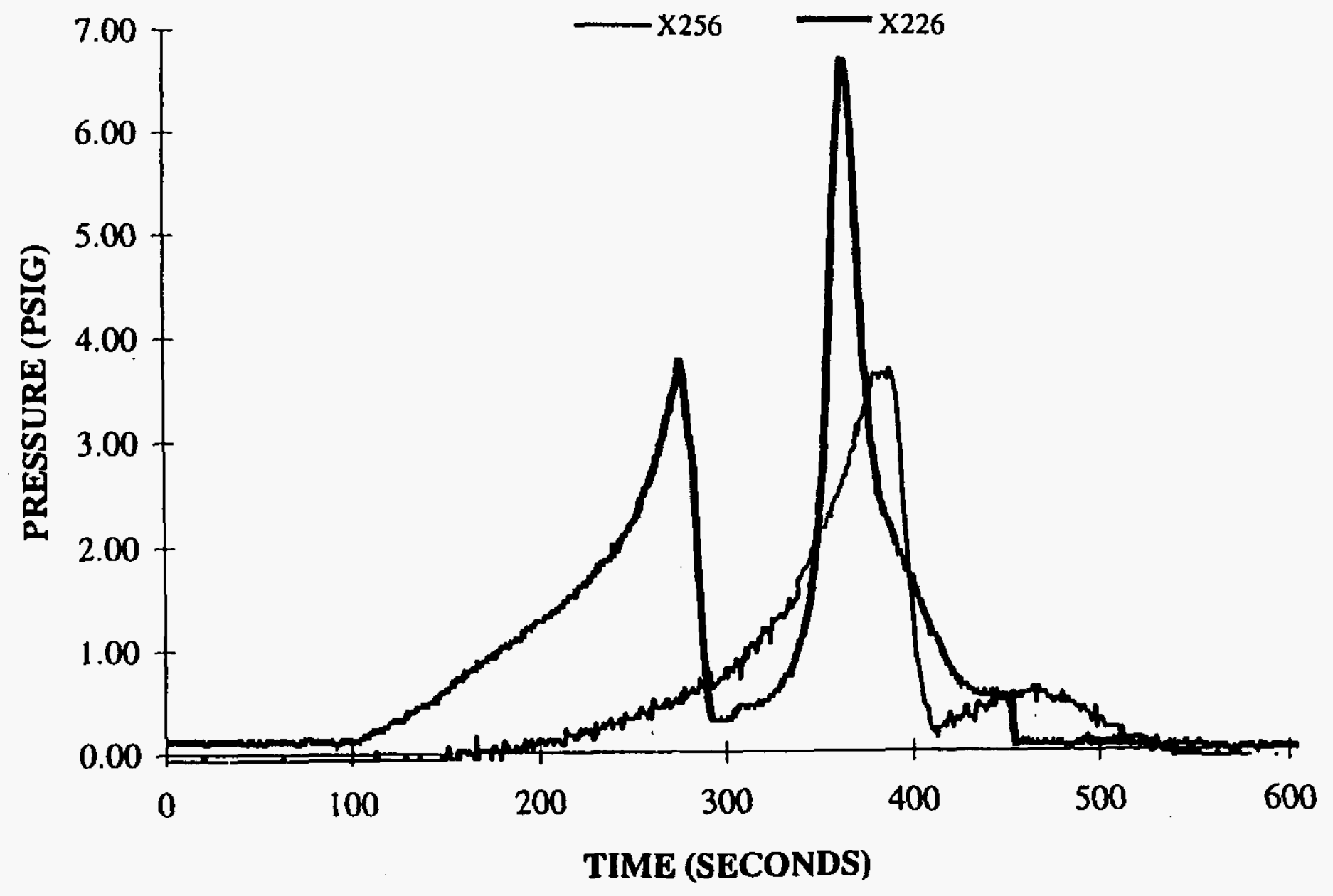


CLIENT: LATA

PROJECT NO.: SwRI 01-7106

DATE: 6 JUNE 1995

TEST ID: 157LR6.DAT

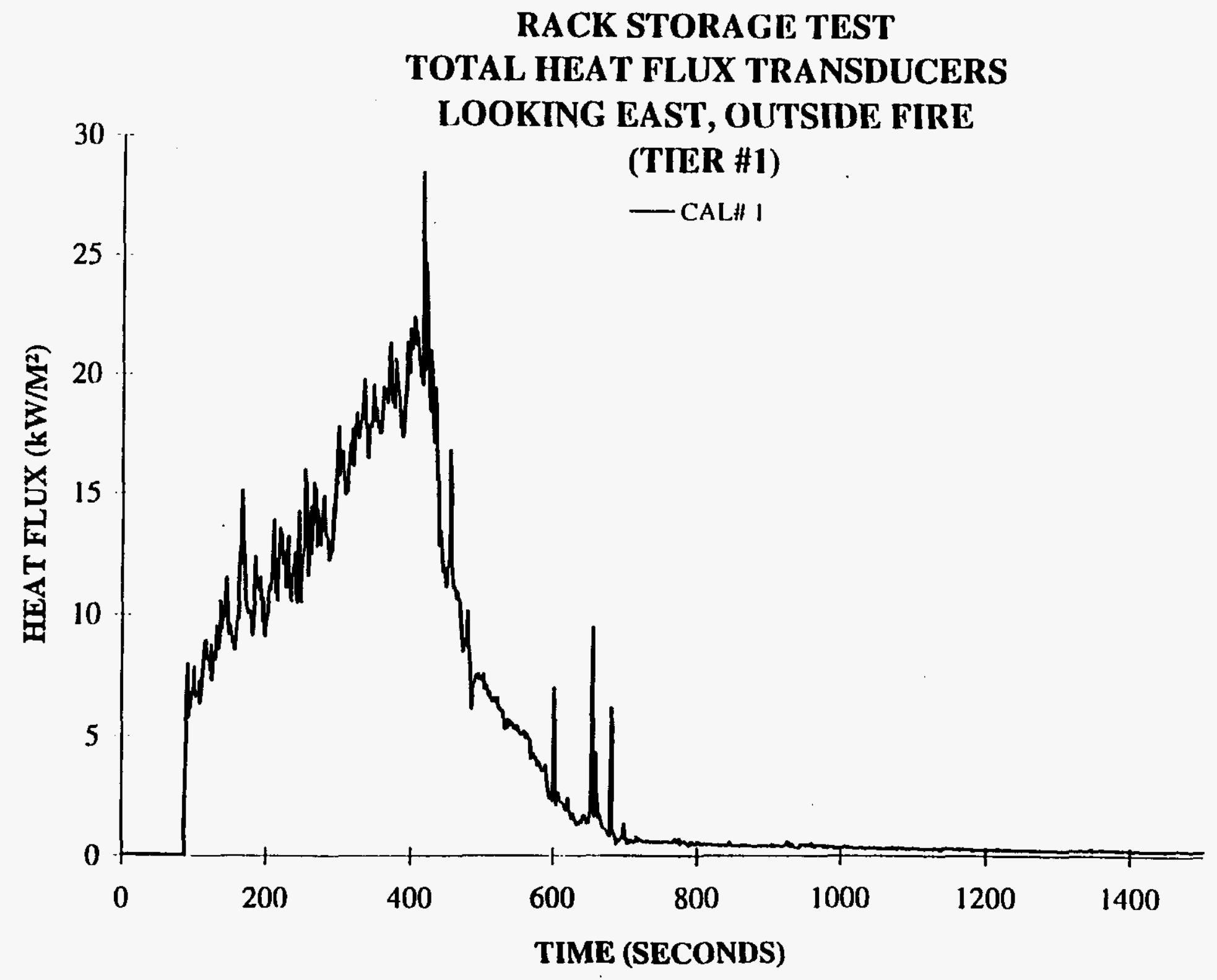




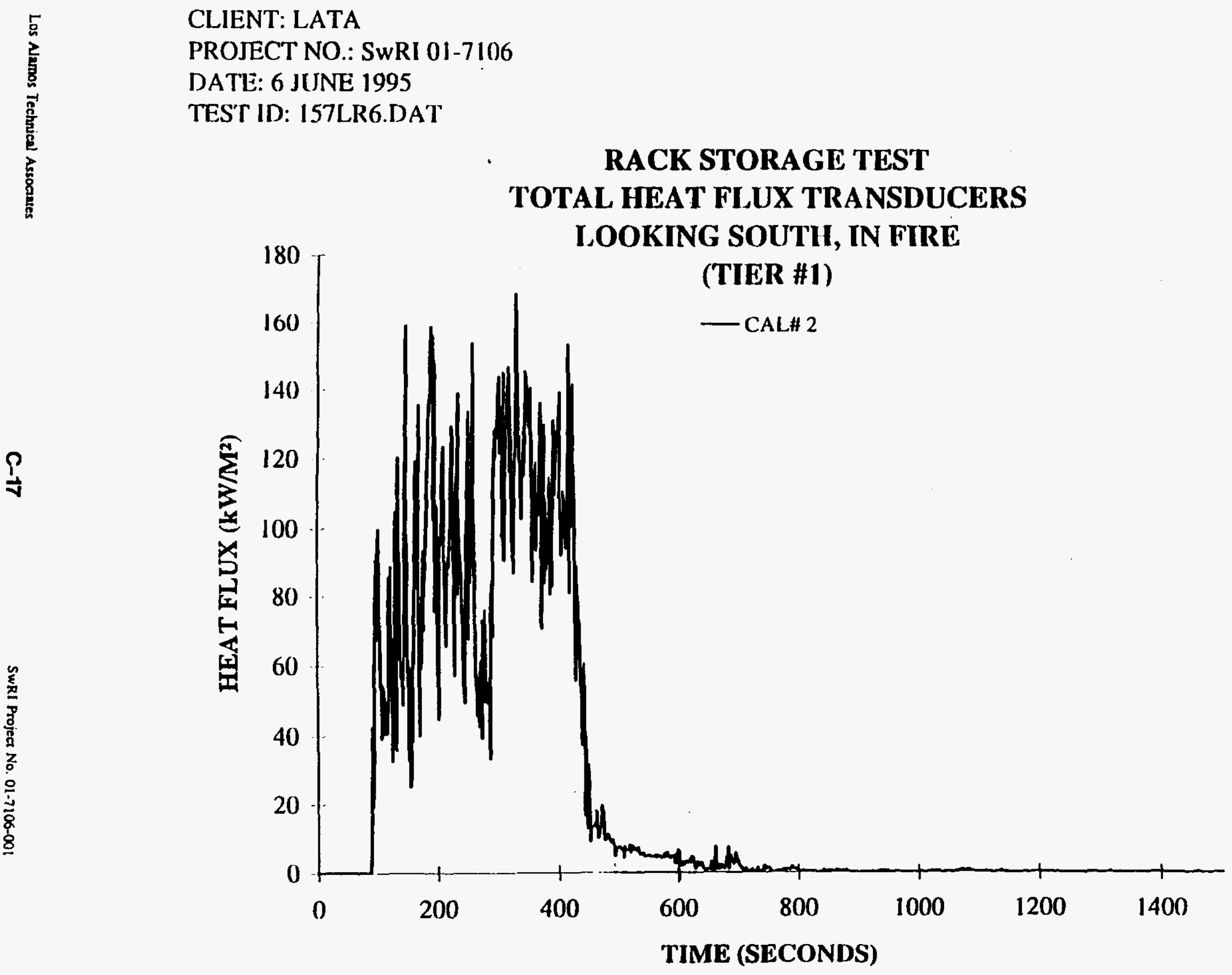




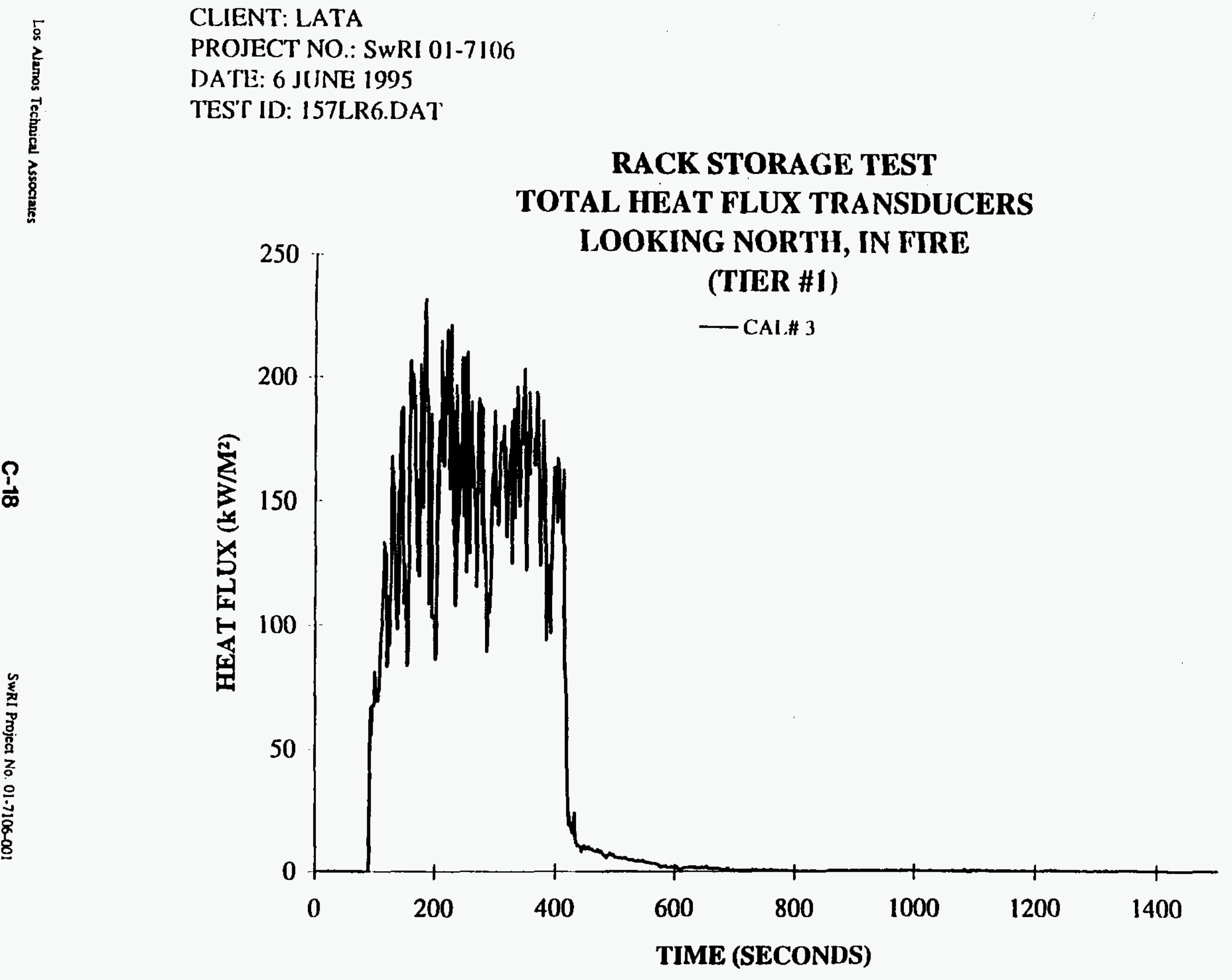




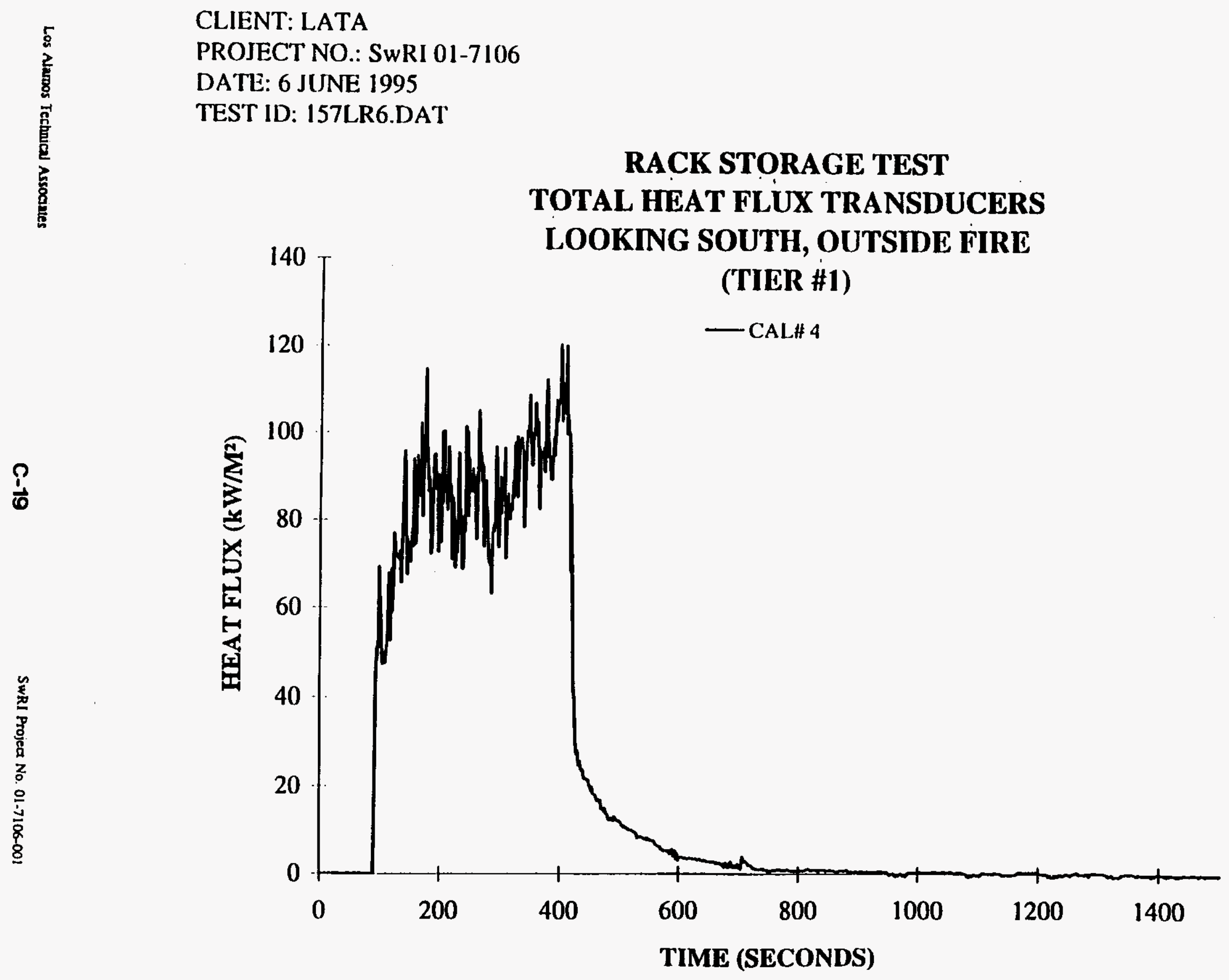




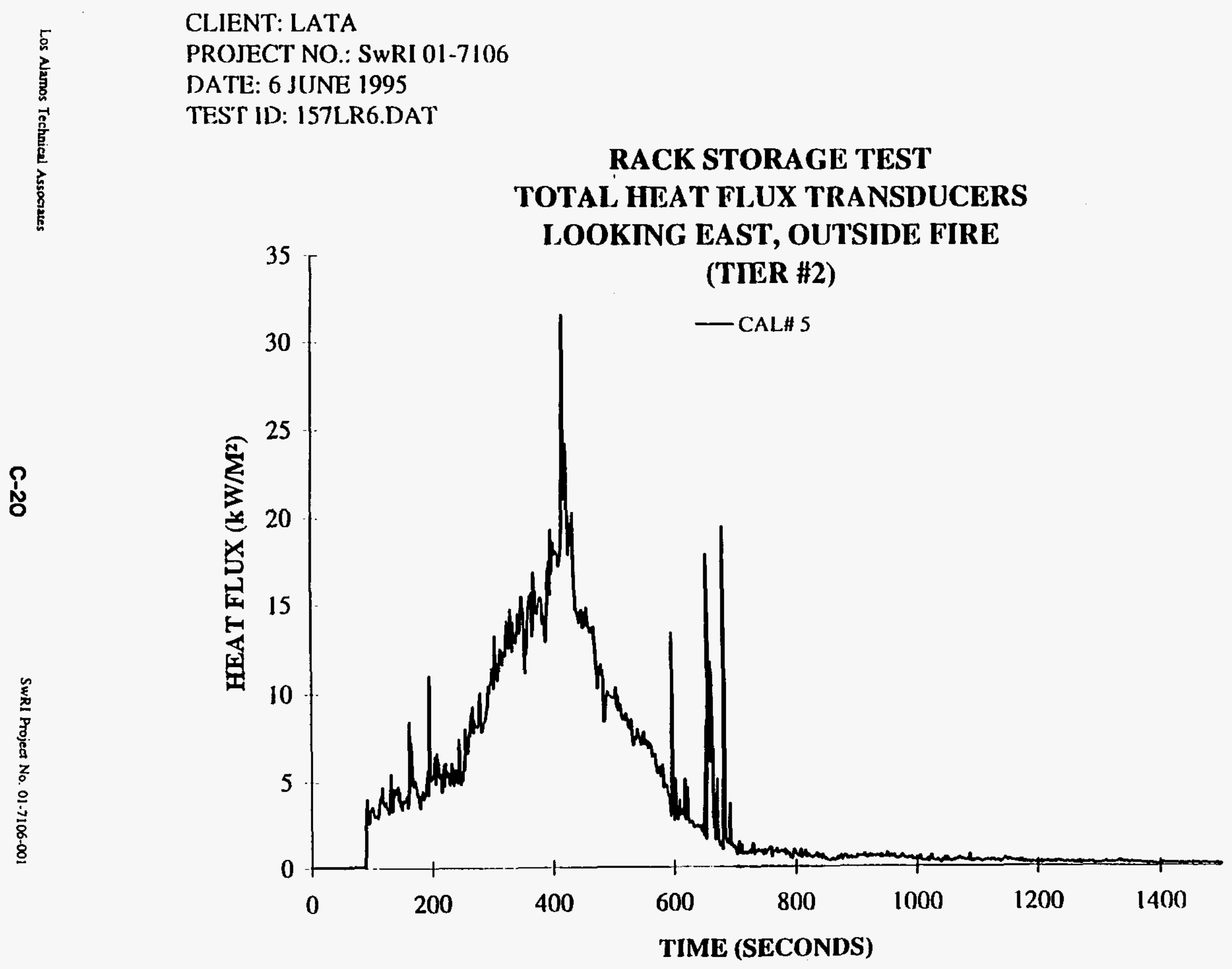

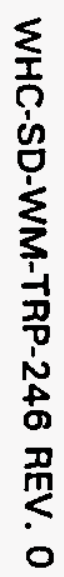


CLIENT: LATA

PROJECT NO.: SWRI 01-7106

DATE: 6 JUNE 1995

TEST ID: 157LR6.DAT

$\stackrel{8}{\mathcal{N}}$

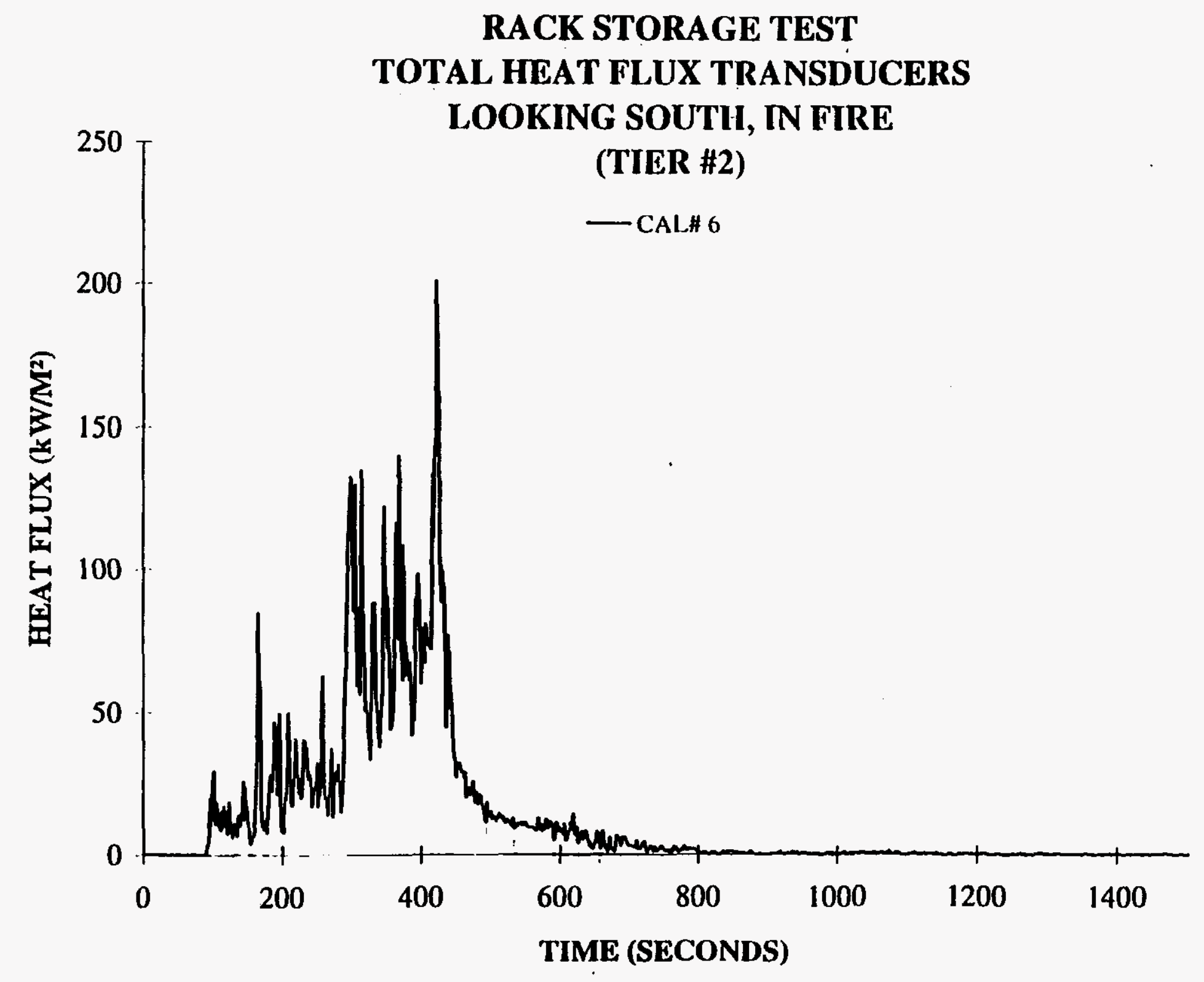


TEST ID: 157LR6.DAT

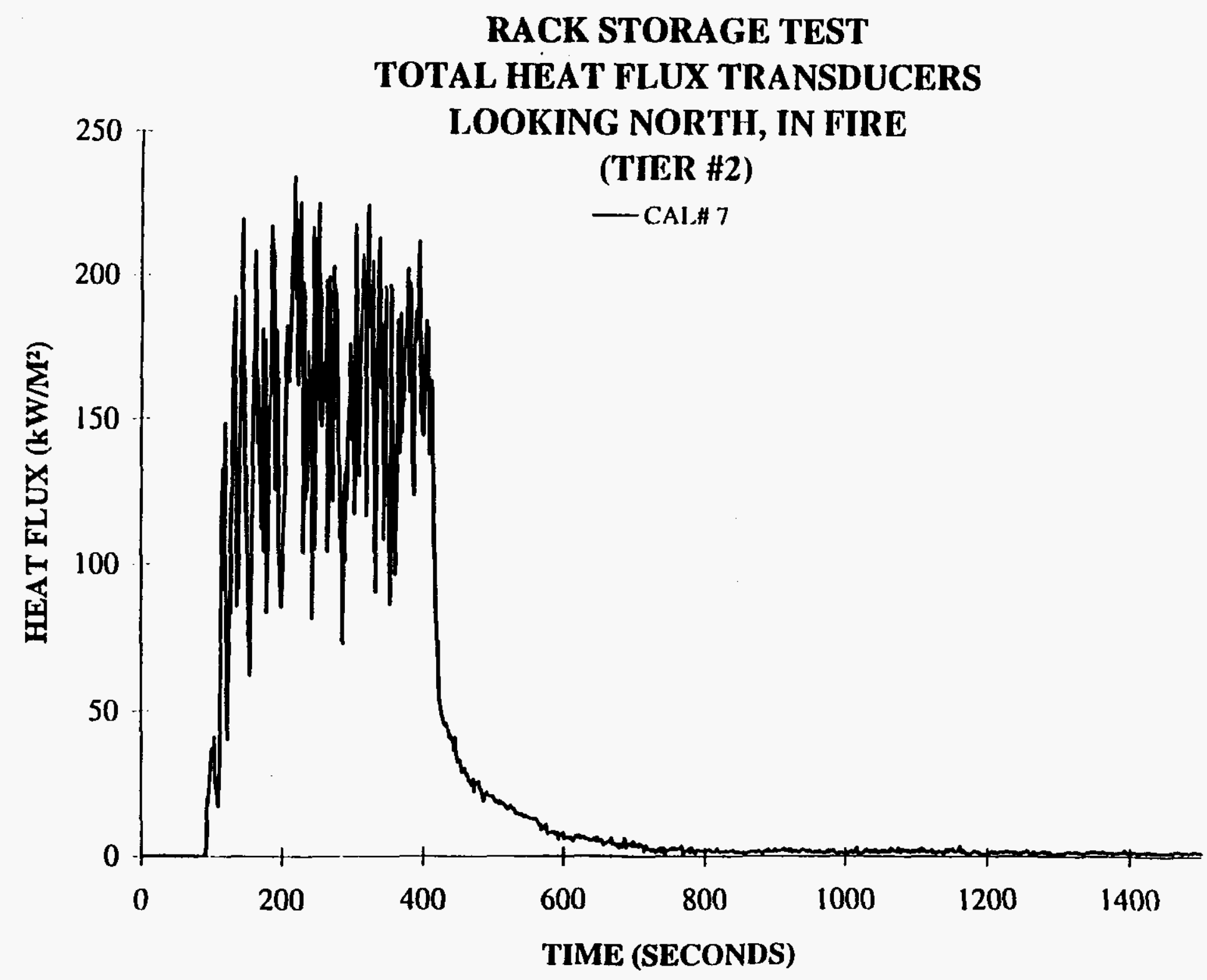

$\stackrel{p}{N}$ 
CLIENT: LATA

PROJECT NO.: SwRI 01-7106

DATE: 6 JUNE 1995

TES"T ID: I57LR6.DAT

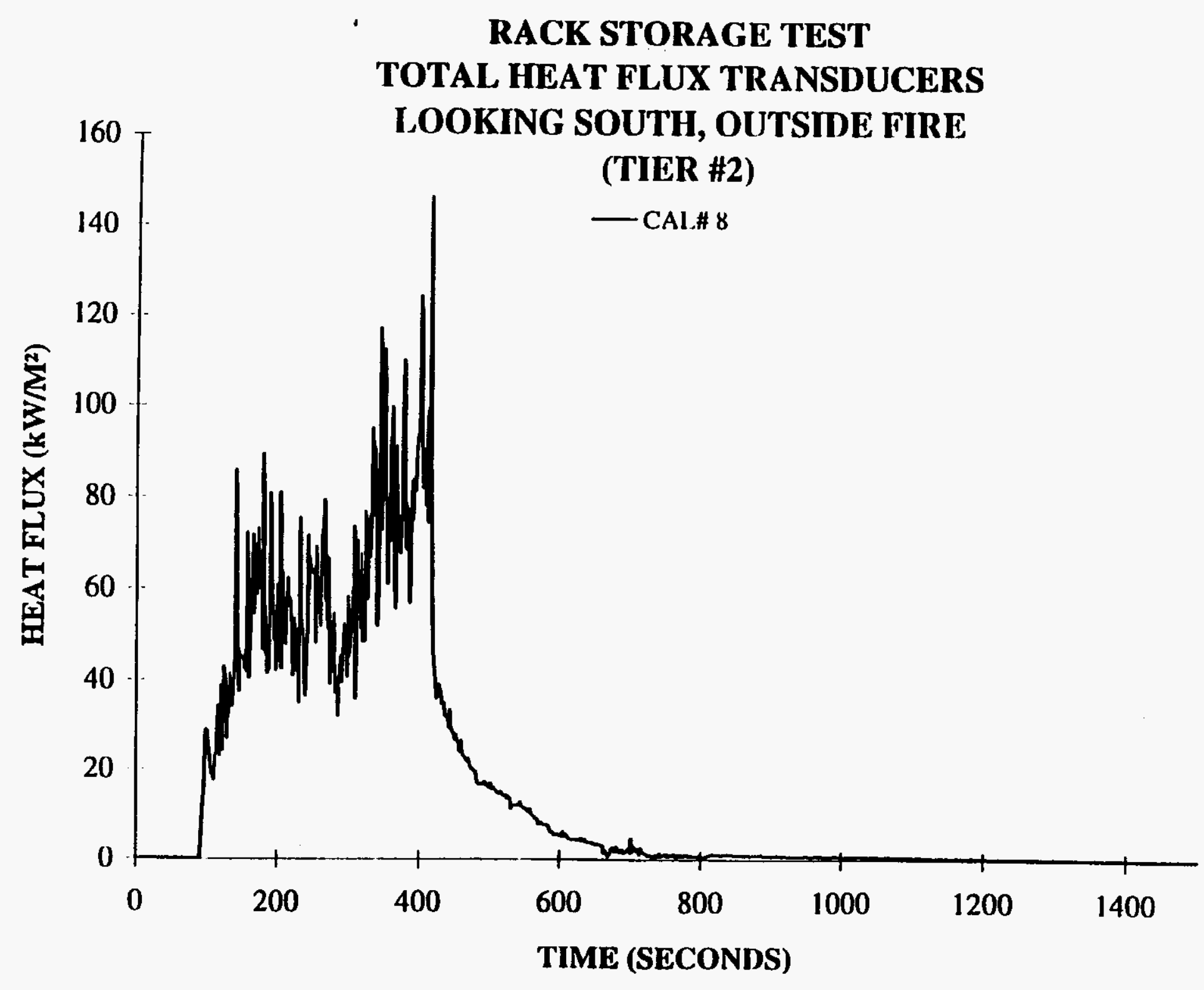

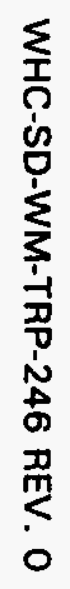




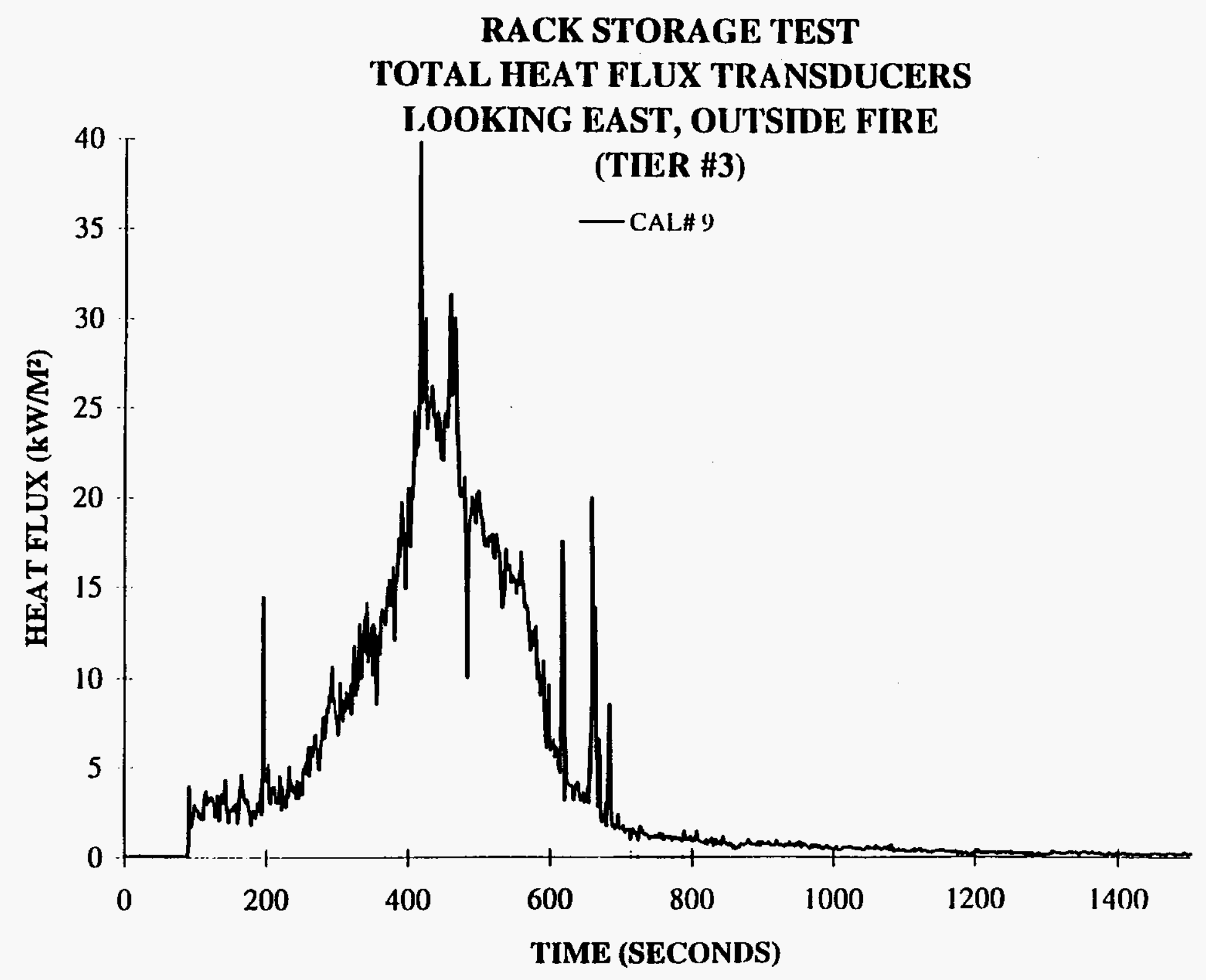




\section{CLIENT: LATA}

PROJECT NO.: SwRI 01-7106

DATE: 6 JUNE 1995

TEST ID: 157LR6.DAT

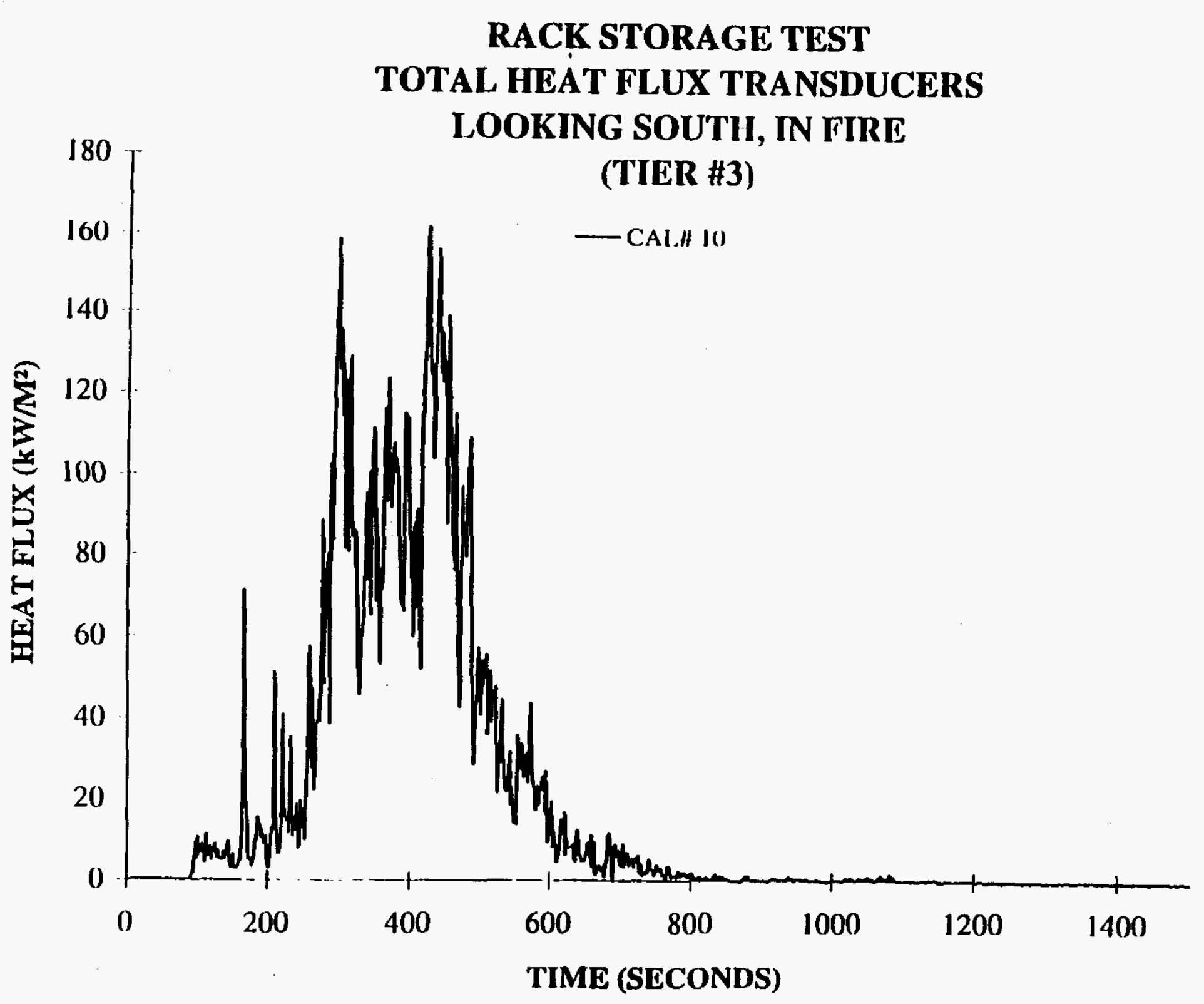


CLIENT: LATA

PROJECT NO.: SwRI 01-7106

DATE: 6 JUNE 1995

TEST ID: 157LR6.DAT

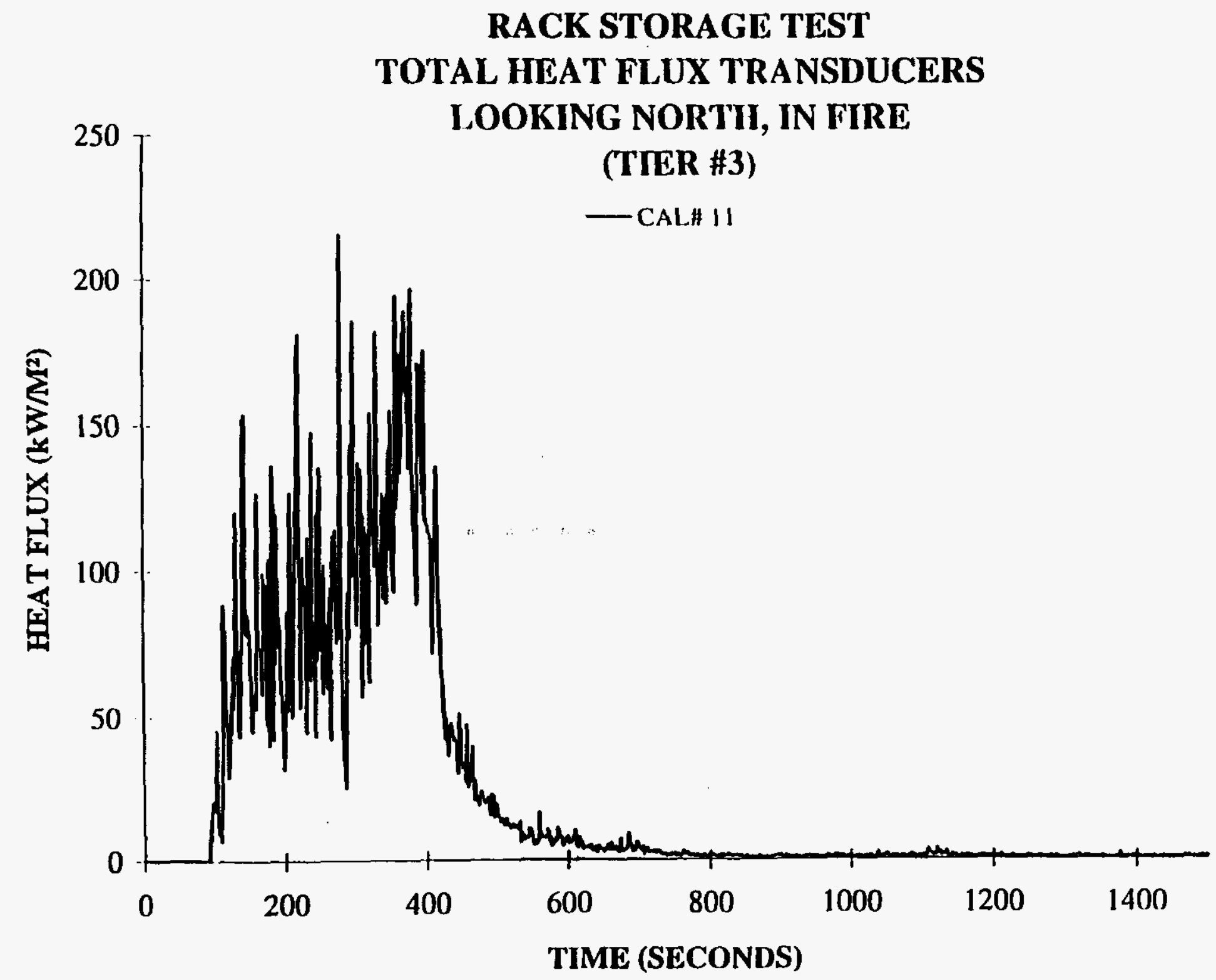

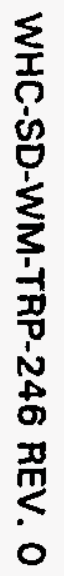

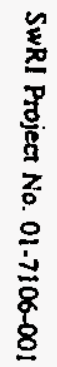

ก̊

TIME (SECONDS) 
PROJECT NO.: SwRI 01-7106

DATE: 6 JUNE 1995

TEST ID: I57LR6.DAT

กู

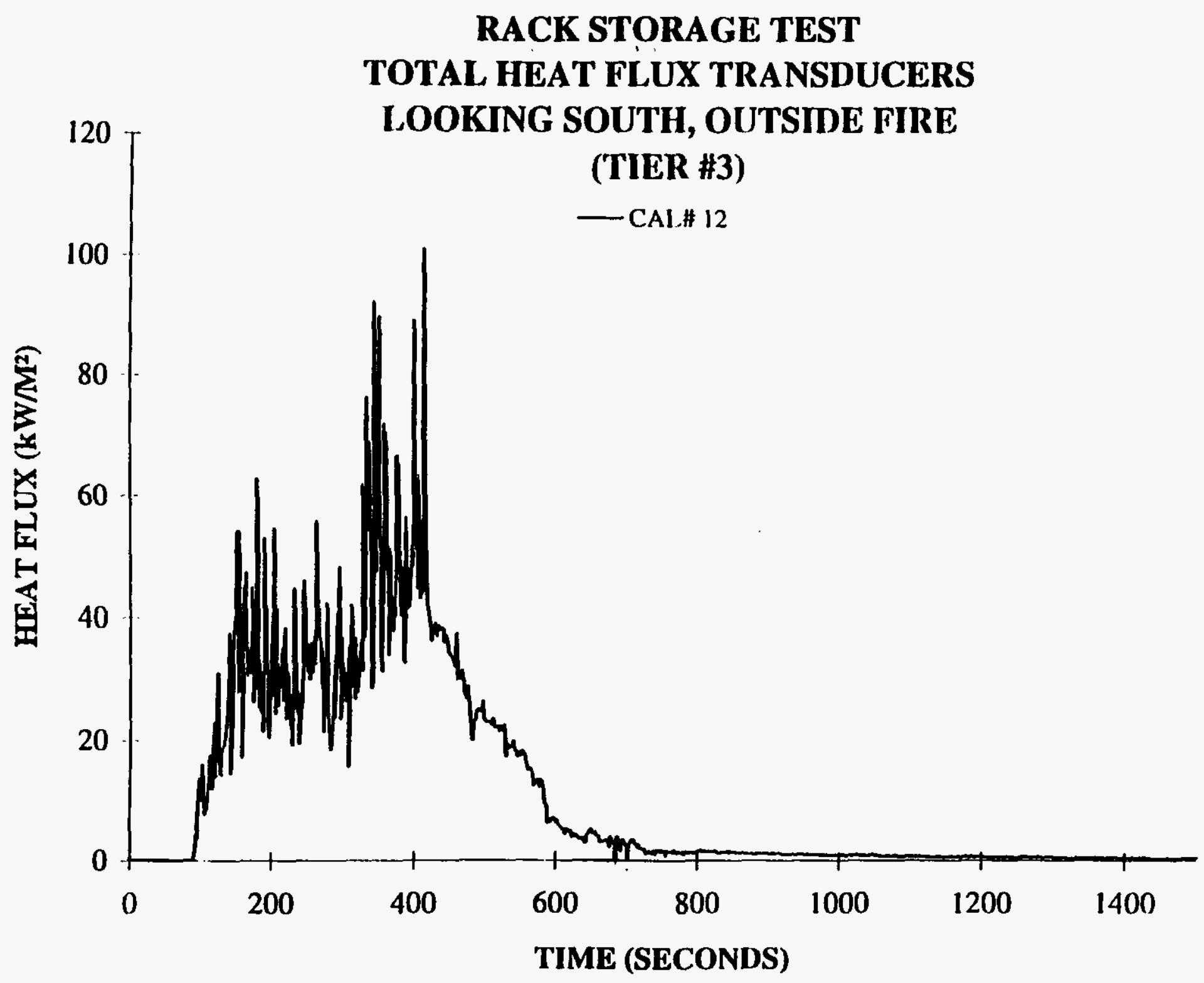


CLIENT: LATA

PROJECT NO.: SwRI 01-7106

DATE: 6 JUNE 1995

TEST ID: 157LR6.DAT

RACK STORAGE TEST

TOTAL HEAT FLUX TRANSDUCERS

I,OOKING EAST, OUTSIDE FIRE

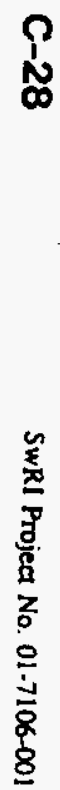

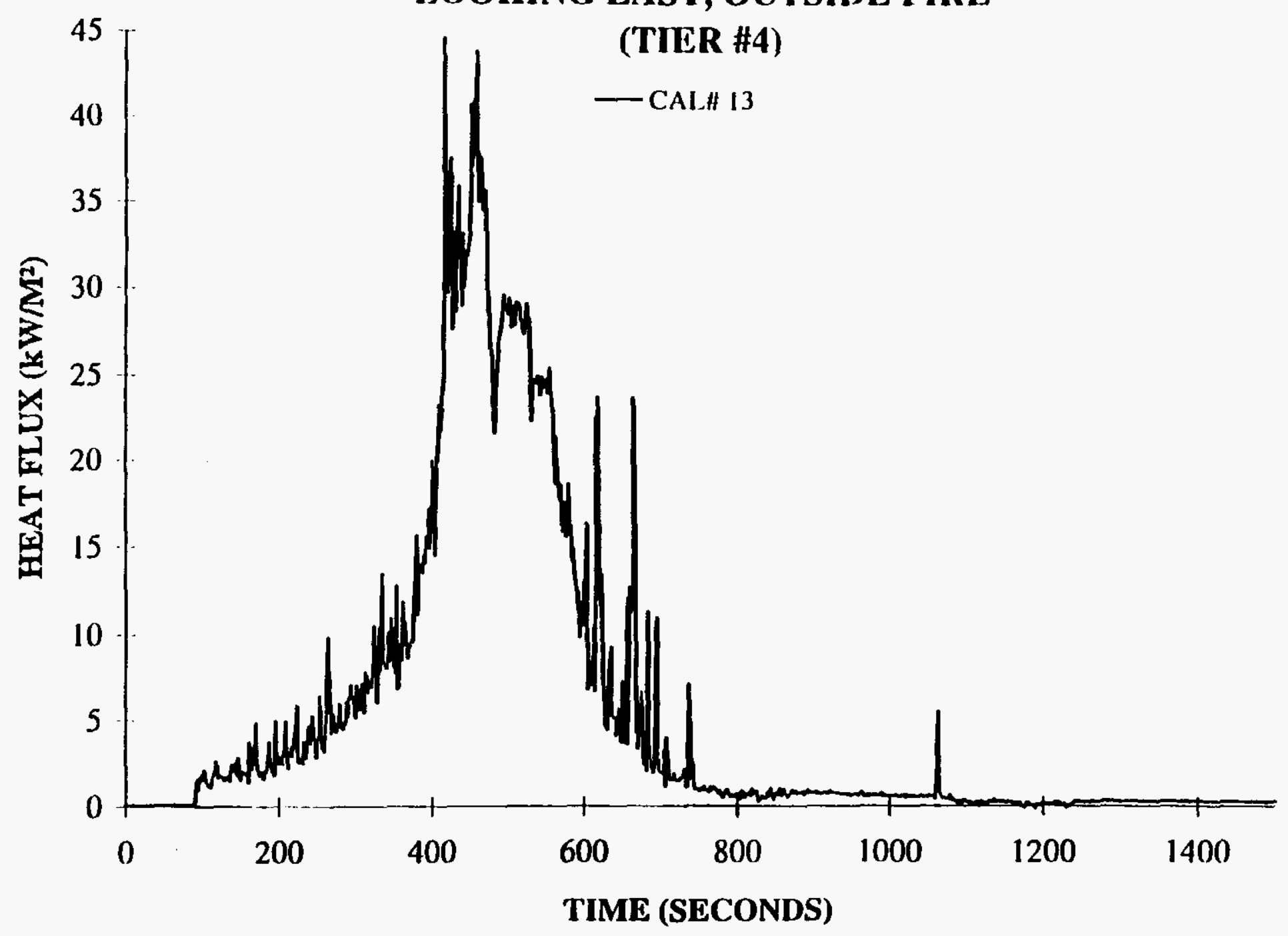

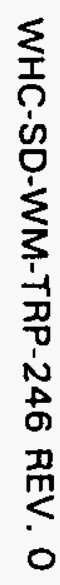

TIME (SECONDS) 


\section{RACK STORAGE TEST \\ TOTAL HEAT FLUX TRANSDUCERS \\ LOOKING NORTH, IN FIRE}

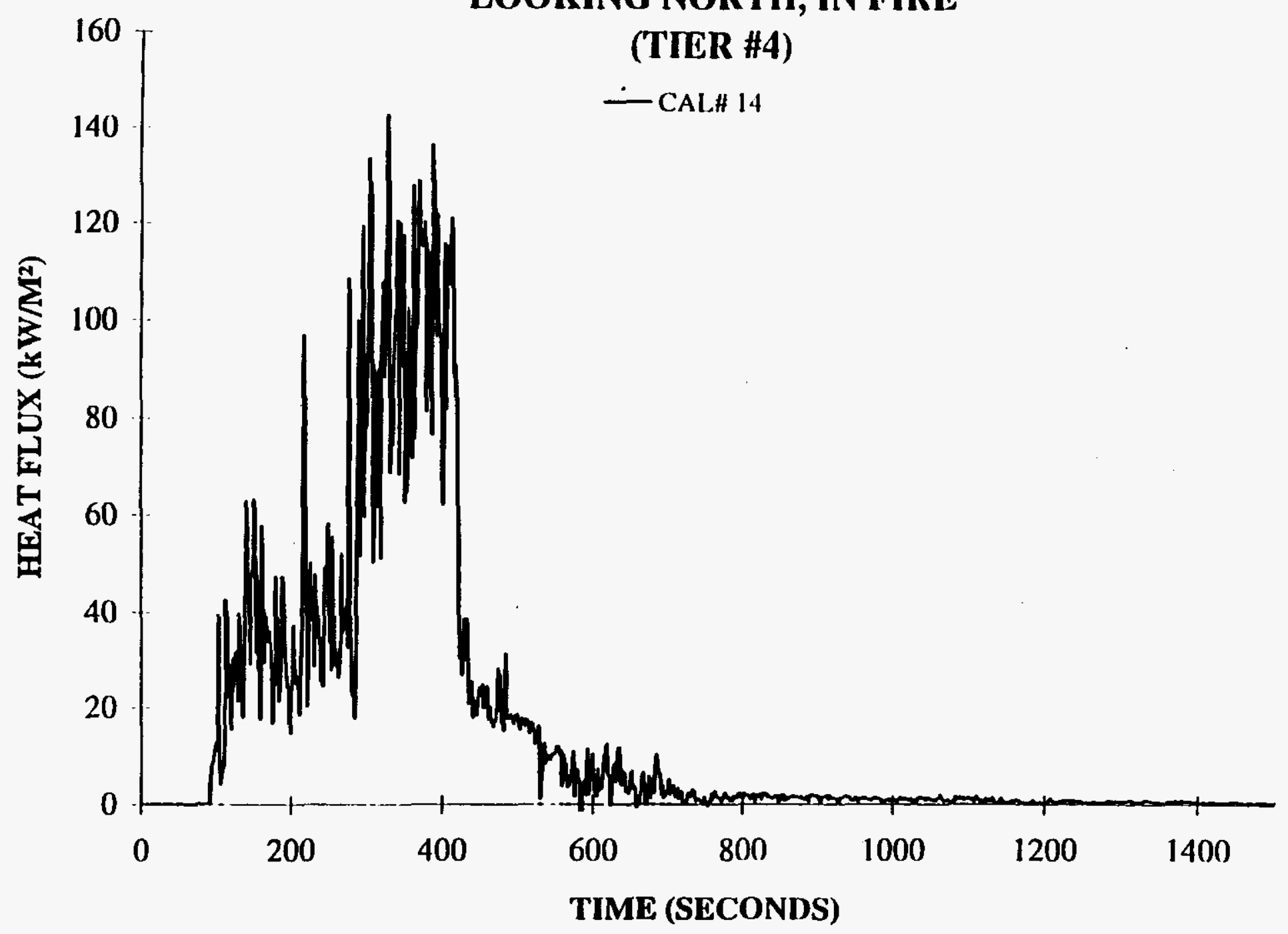


TEST ID: 157LR6.DAT

\section{RACK STORAGE TEST TOTAL HEAT FLUX TRANSDUCERS} I,OOKING NORTH, IN FIRE

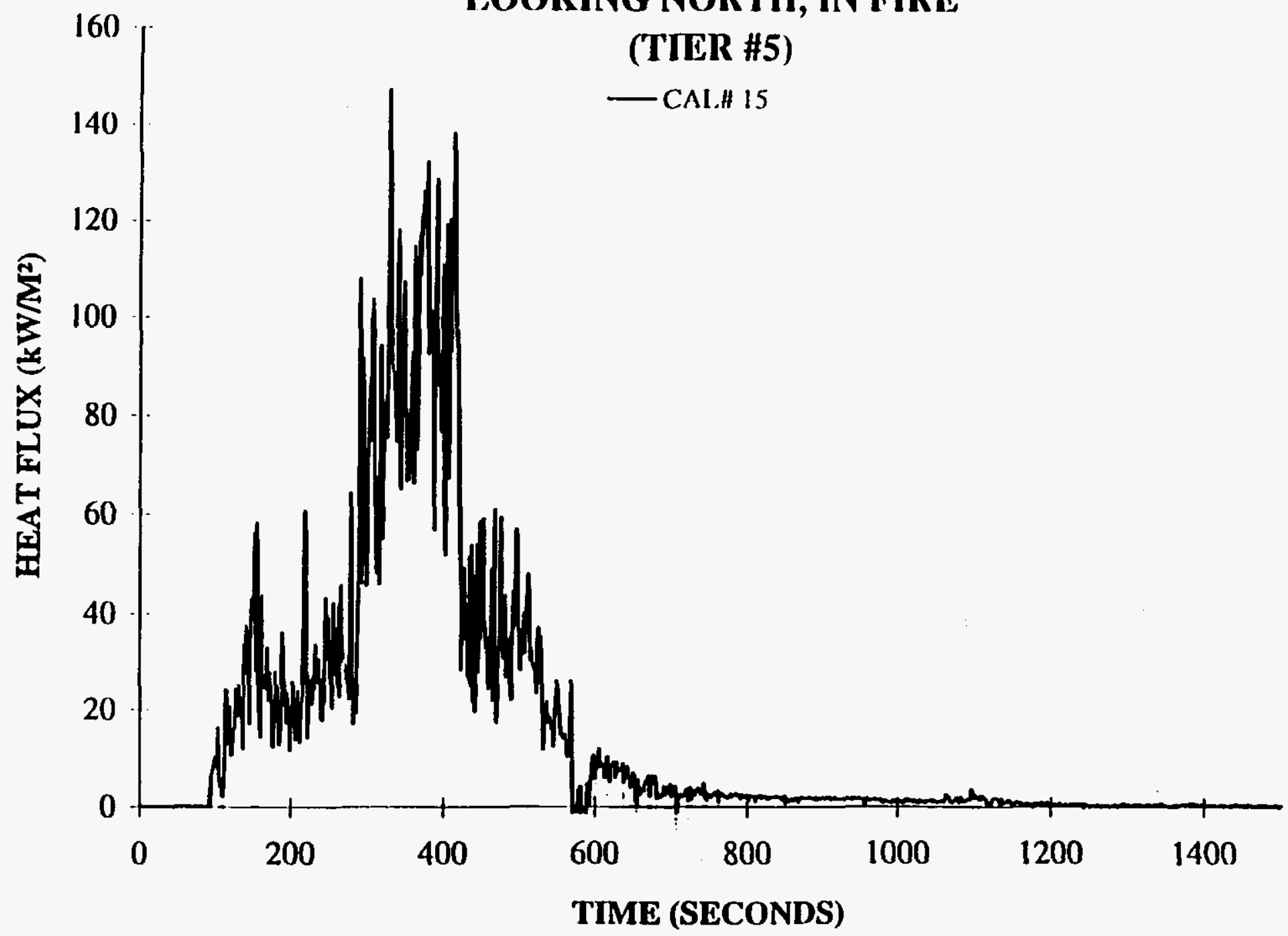



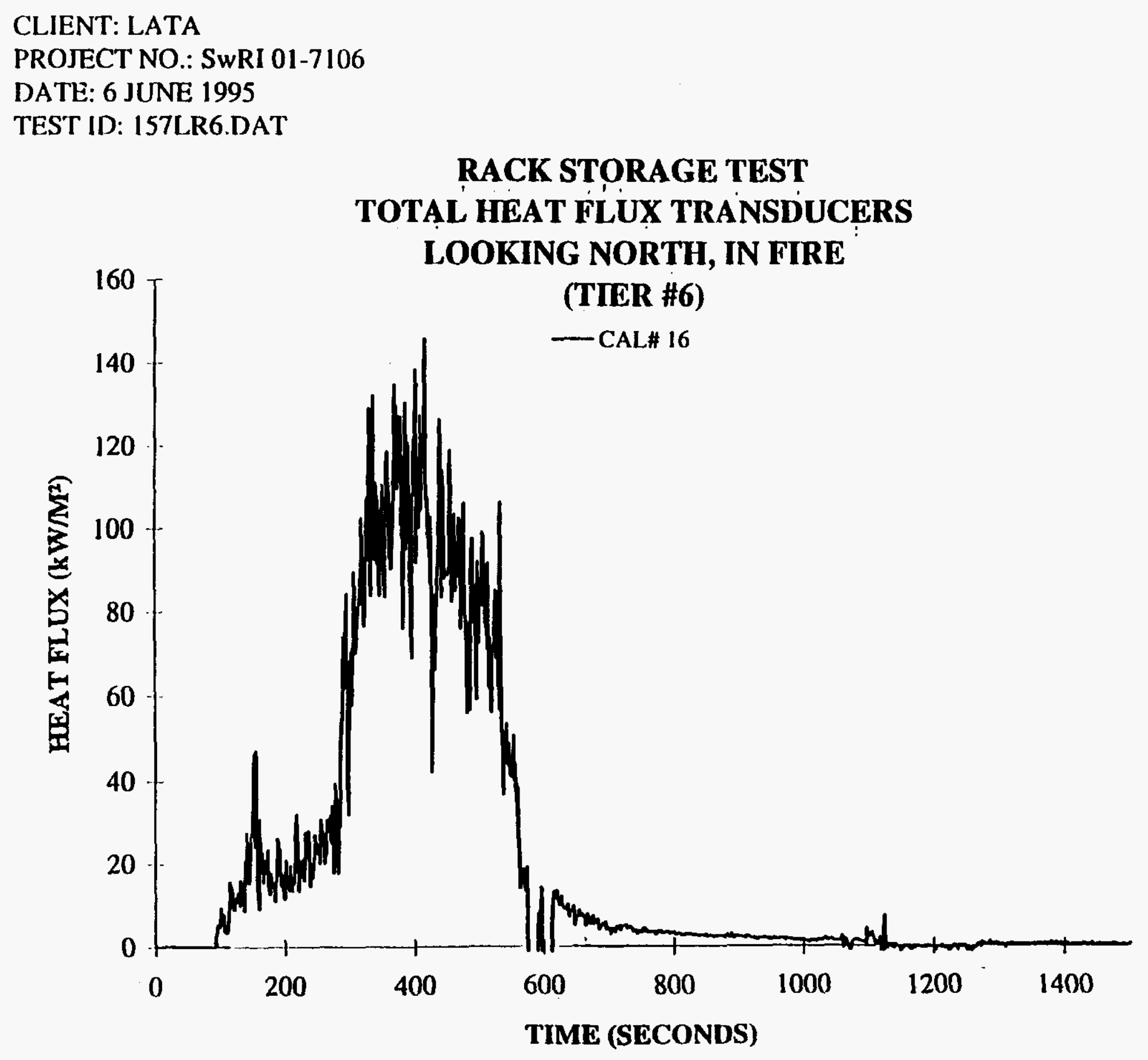


\section{RACK STORAGE TEST}

\section{DRUM SURFACE TC'S}

$\stackrel{\rho}{\omega}$

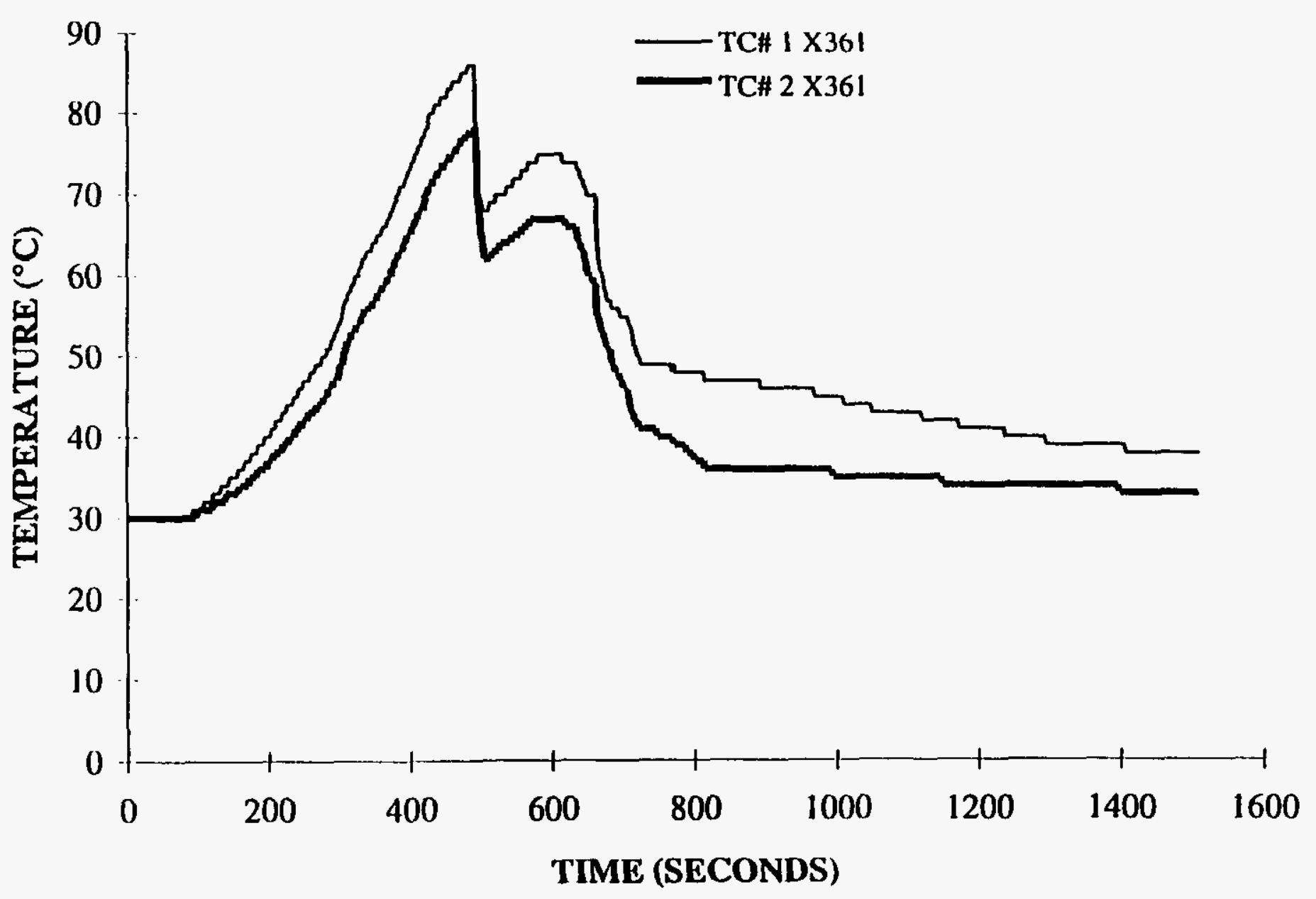




\section{CLIENT: LATA}

PROJECT NO.: SwRI 01-7106

DATE: 6 JUNE 1995

TEST ID: 157LR3.DAT

RACK STORAGE TEST

DRUM SURFACE TC'S

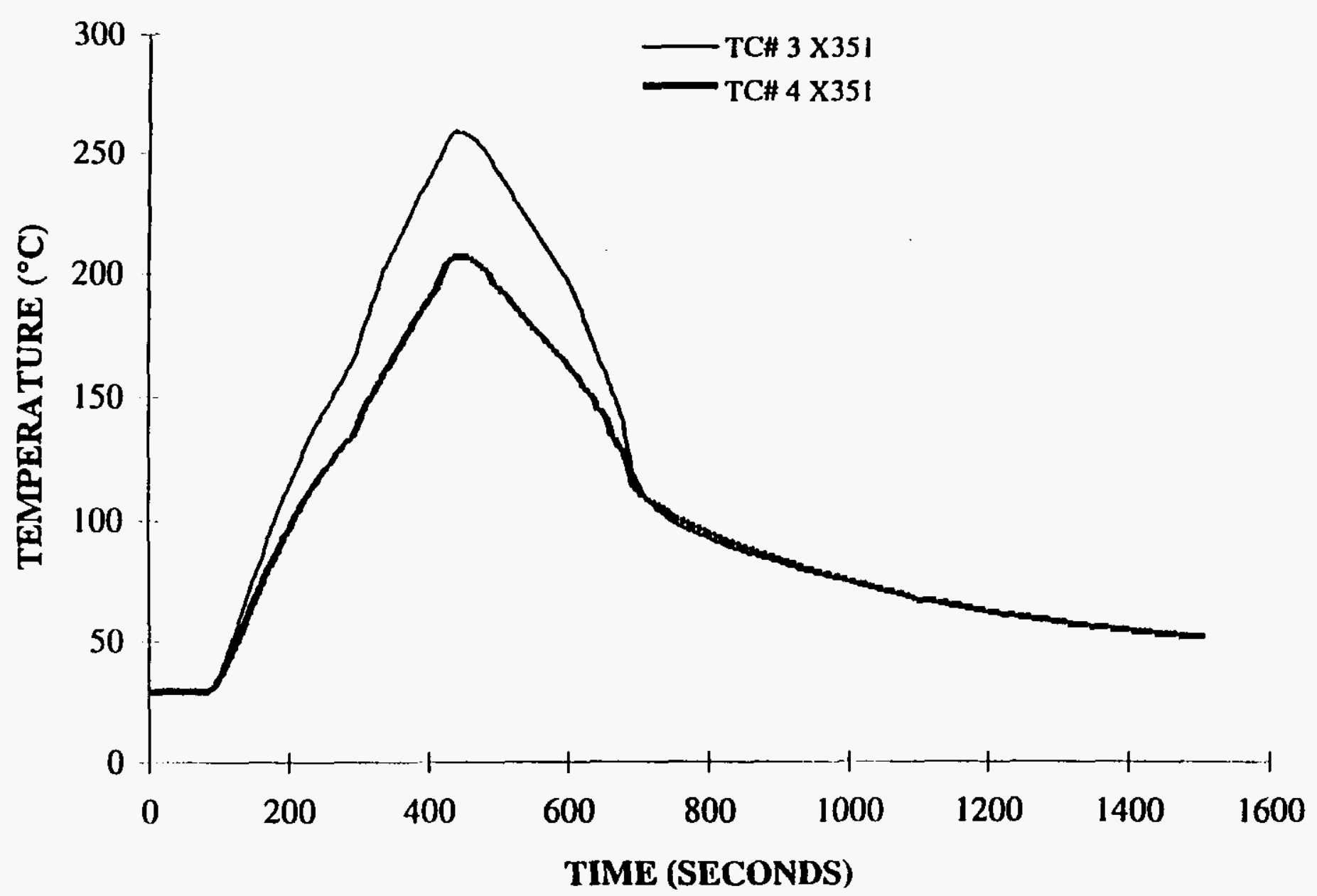


CLIENT: LATA

PROJECT NO.: SwRI 01-7106

DATE: 6 JUNE 1995

TEST ID: 157LRI.DAT

RACK STORAGE TEST

DRUM SURFACE TC'S

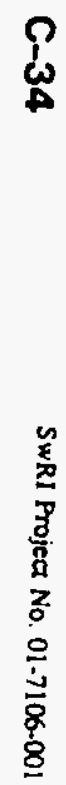

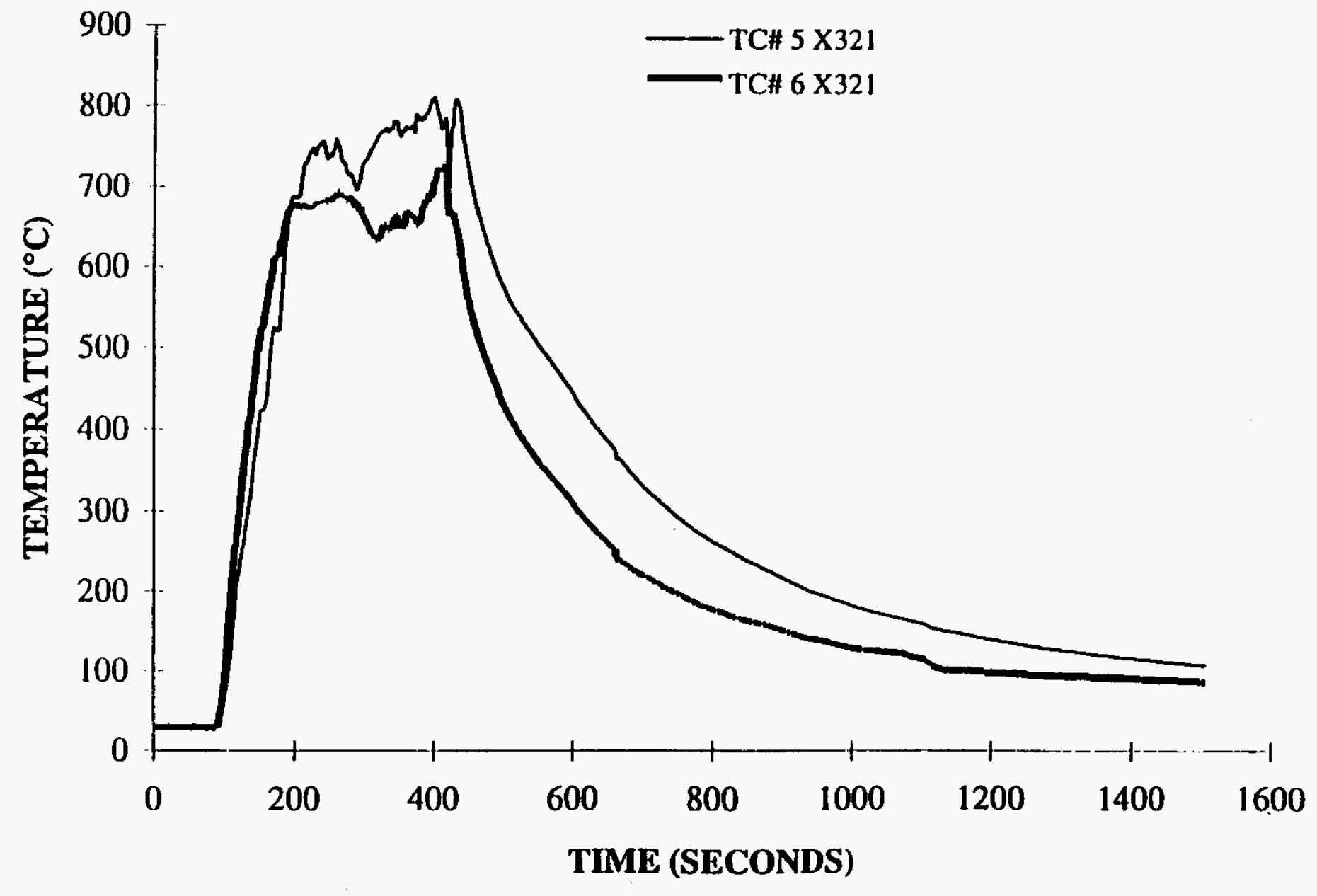


CLIENT: LATA

PROJECT NO.: SwRI 01-7106

DATE: 6 JUNE 1995

TEST ID: 157LRI.DAT

\section{RACK STORAGE TEST}

\section{DRUM SURFACE TC'S}

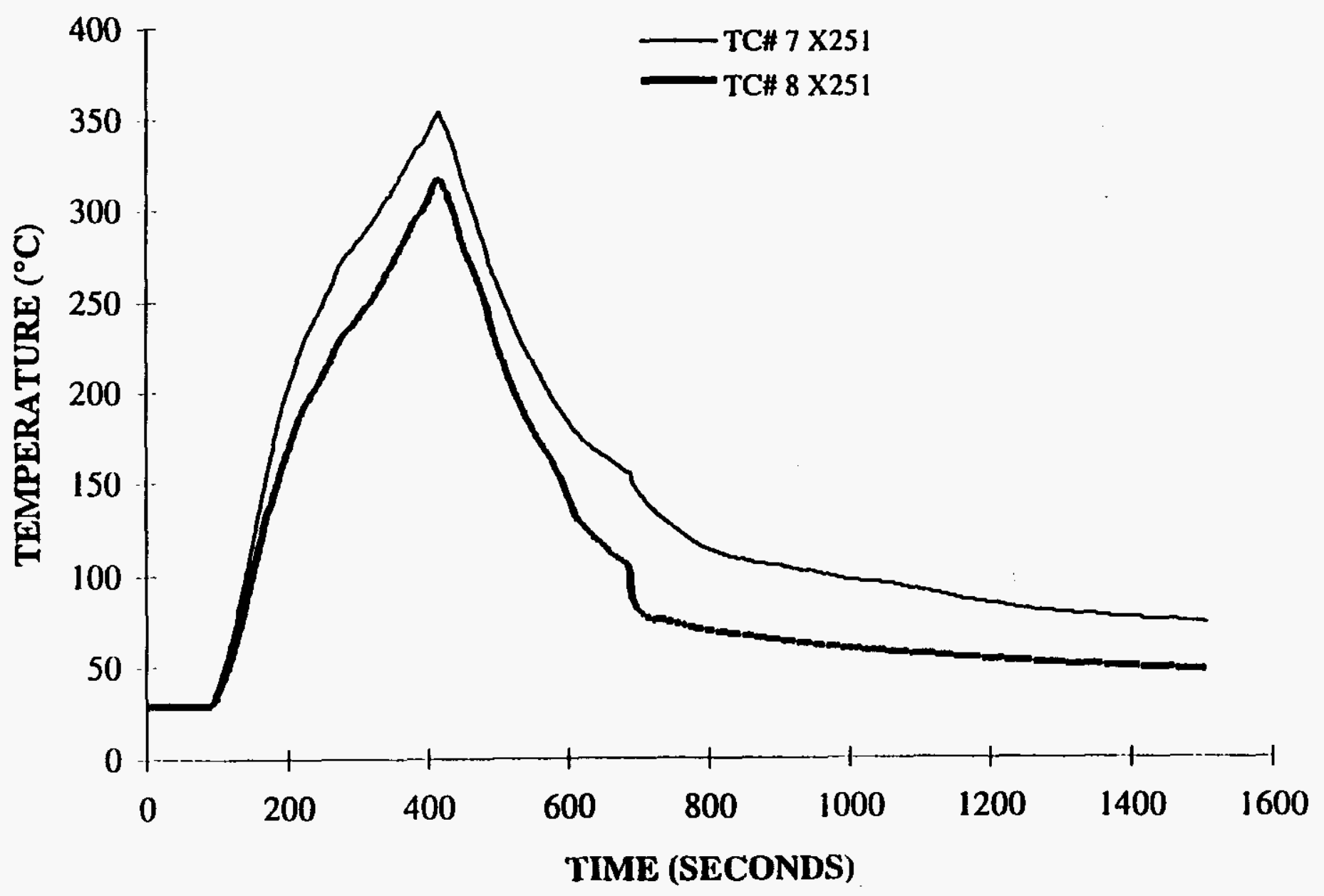


RACK STORAGE TEST DRUM SURFACE TC'S

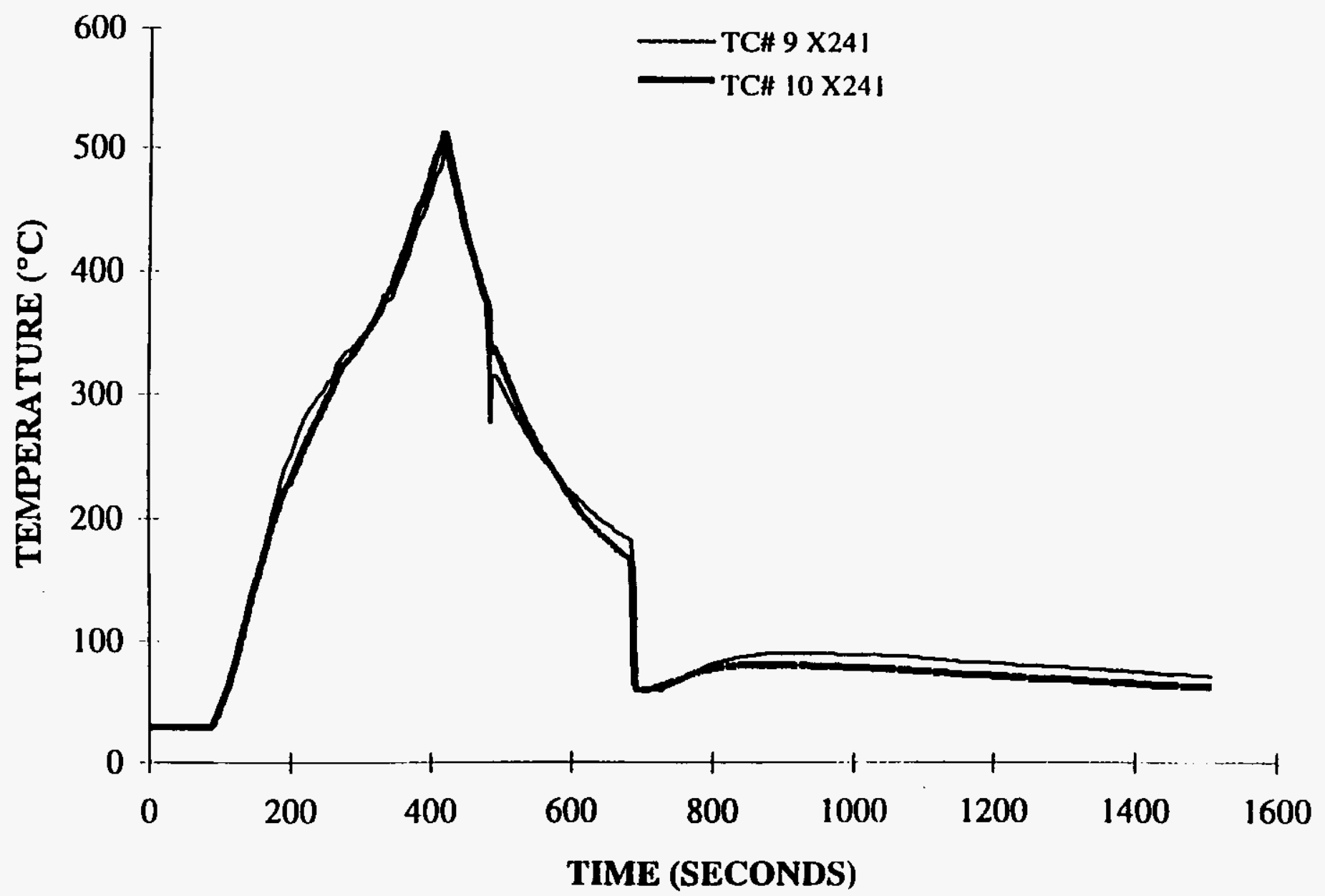


CLIENT: LATA

PROJECT NO.: SwRI 01-7106

DATE: 6 JUNE 1995

TEST ID: 157LR3.DAT

\section{RACK STORAGE TEST}

\section{DRUM SURFACE TC'S}

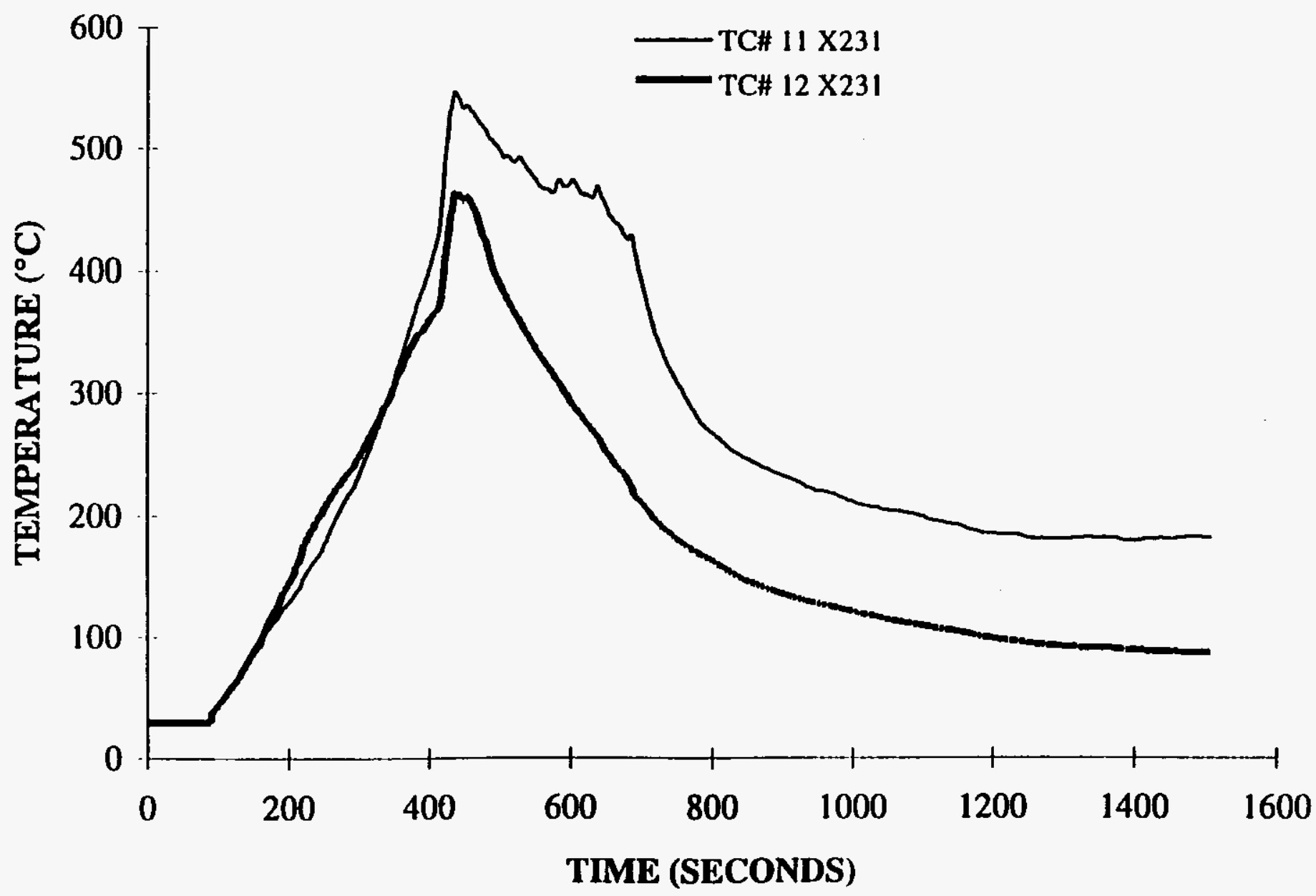


CLIENT: LATA

PROJECT NO.: SwRI 01-7106

DATE: 6 JUNE 1995

TES'T ID: 157LRI.DAT

RACK STORAGE TEST

DRUM SURFACE TC'S

$\stackrel{\wp}{\infty}$

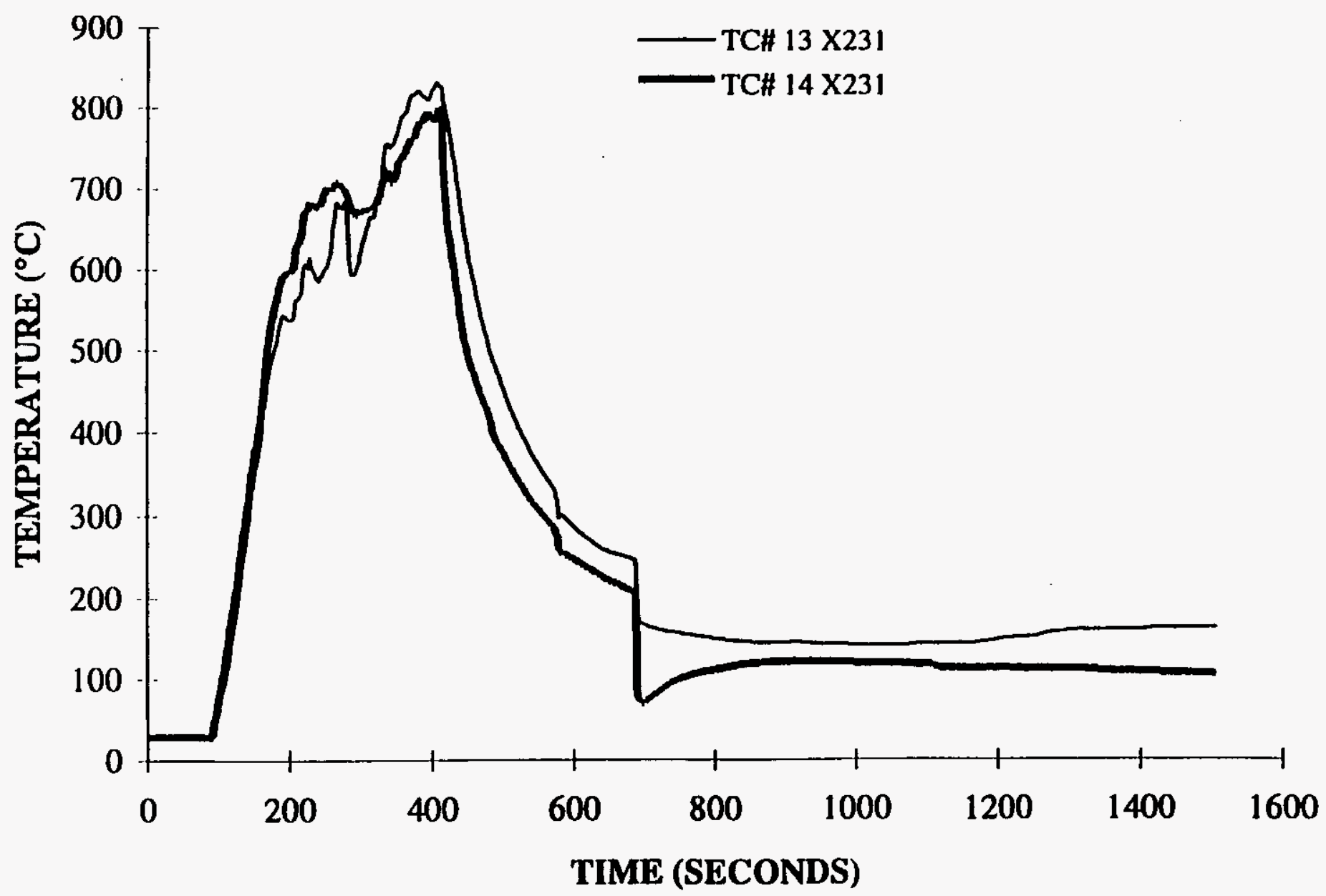


CLIENT: LATA

PROJECT NO.: SwRI 01-7106

DATE: 6 JUNE 1995

TEST ID: 157LR3.DAT

RACK STORAGE TEST

DRUM SURFACE TC'S

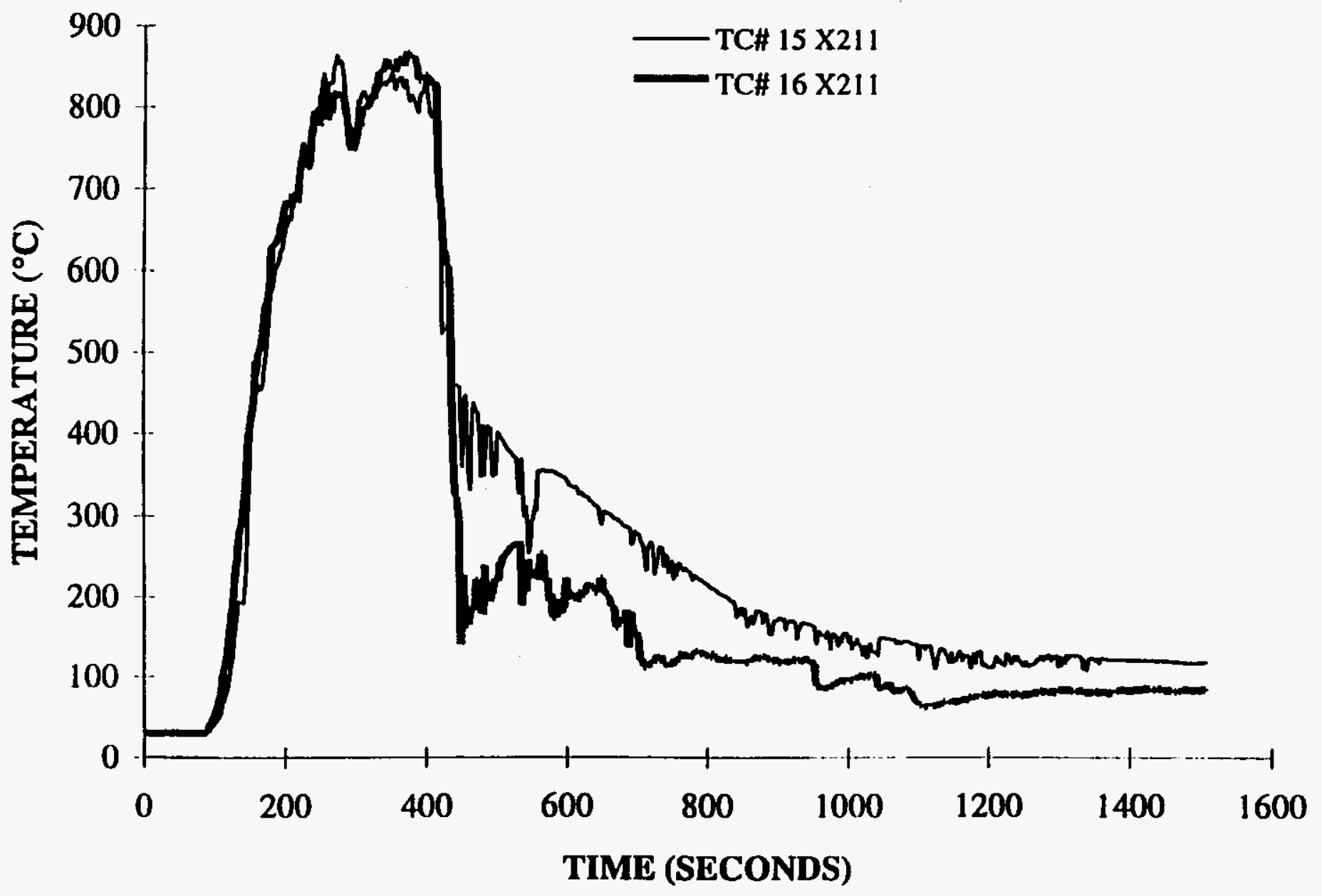




\section{RACK STORAGE TEST}

\section{DRUM SURFACE TC'S}

f

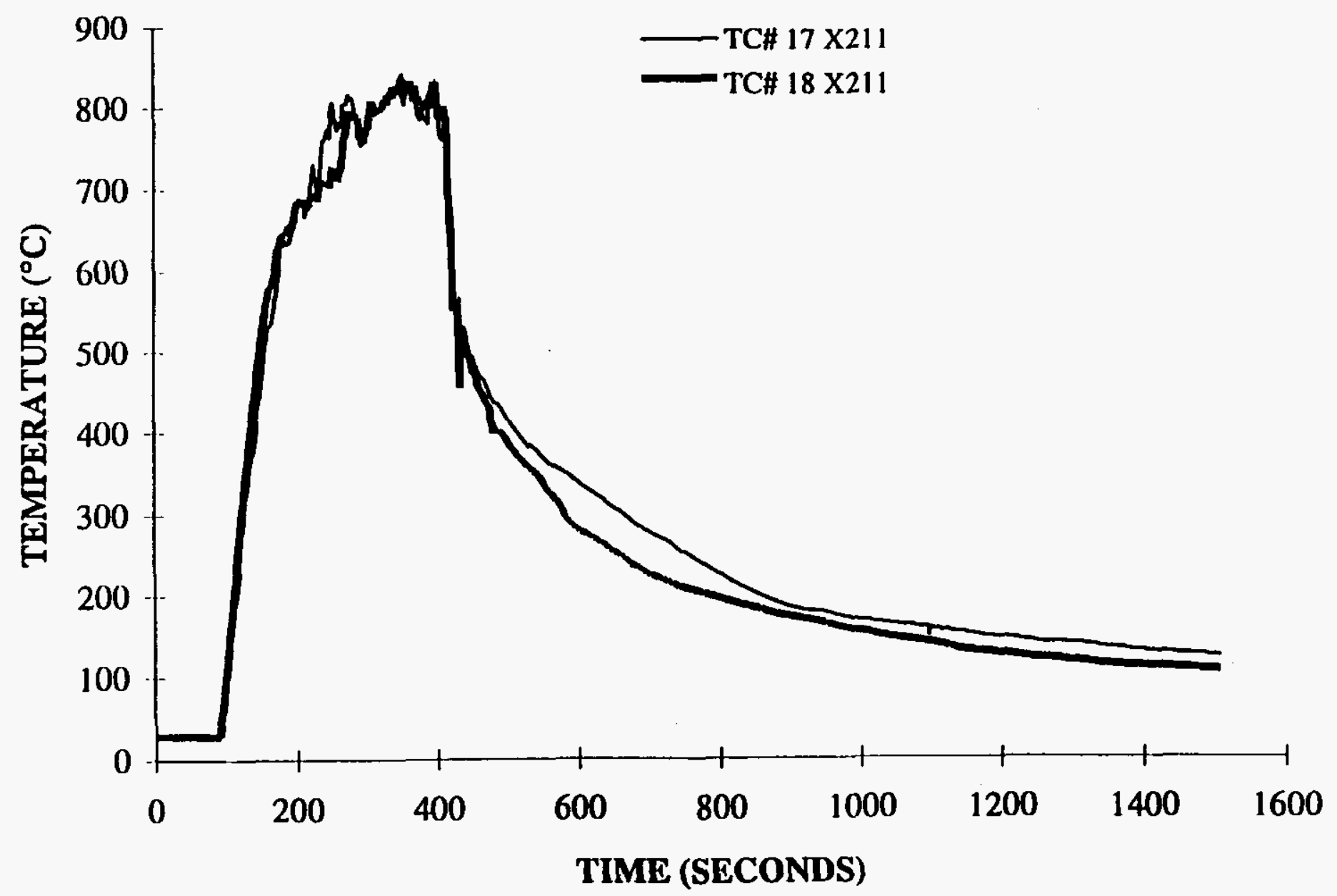


CLIENT: LATA

PROJECT NO.: SwRI 01-7106

DATE: 6 JUNE 1995

TEST ID: 157LRI.DAT

\section{RACK STORAGE TEST}

DRUM SURFACE TC'S

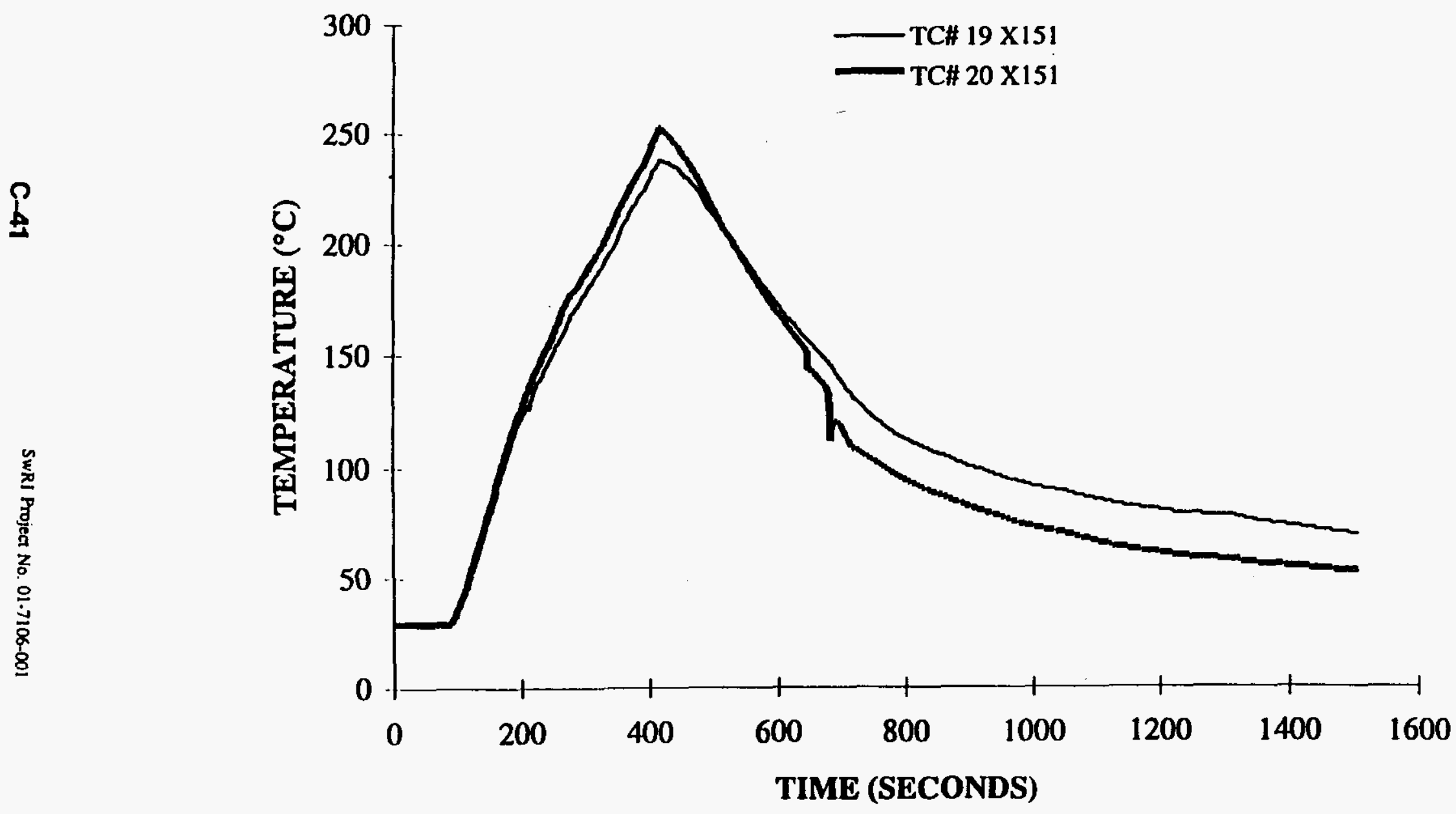


CLIENT: LATA

PROJECT NO.: SwRI 01-7106

DATE: 6 JUNE 1995

TEST ID: 157LRI.DAT

RACK STORAGE TEST

DRUM SURFACE TC'S

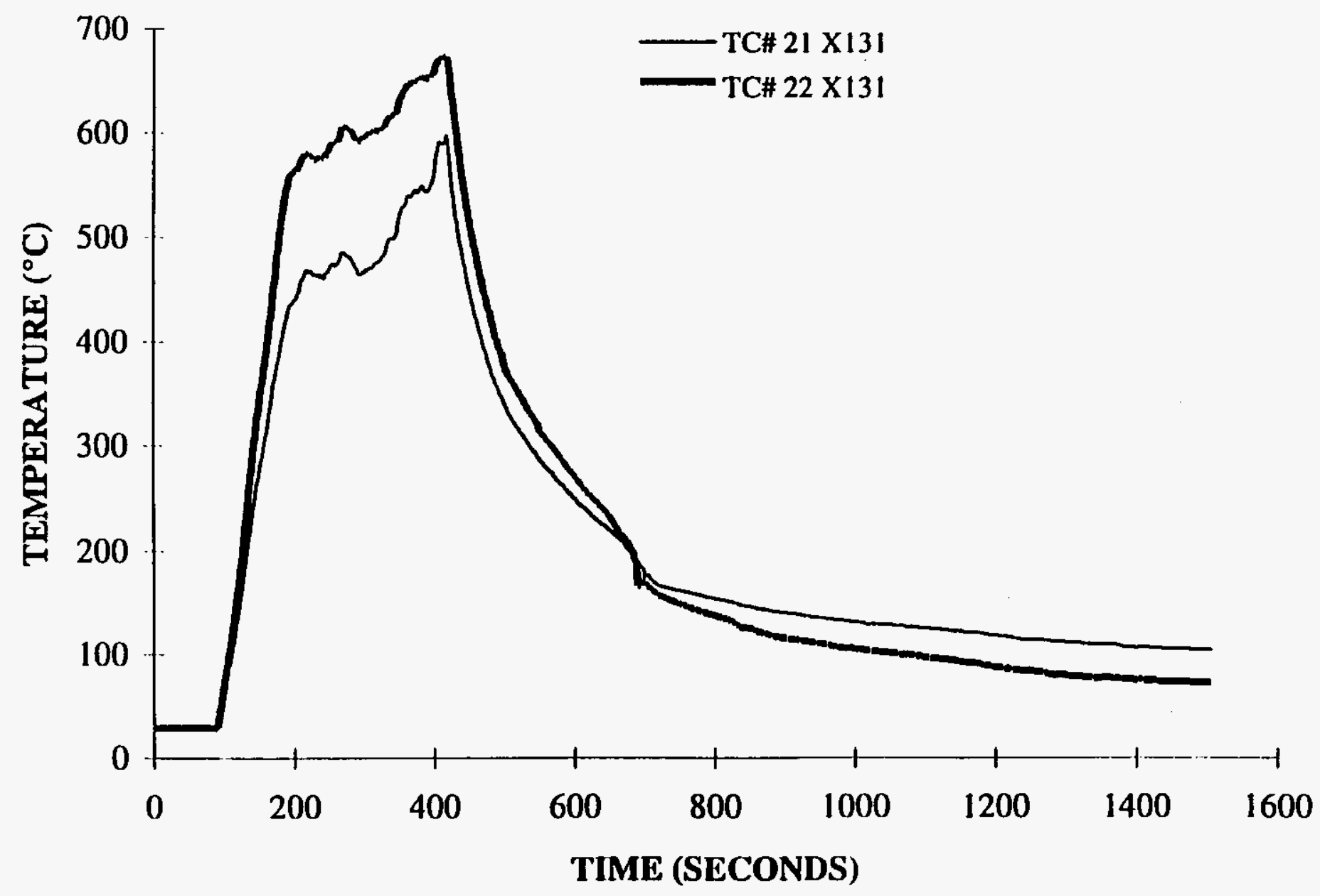


CLIENT: LATA

PROJECT NO.: SwRI 01-7106

DATE: 6 JUNE 1995

TEST ID: 157LRI.DAT

\section{RACK STORAGE TEST}

DRUM SURFACE TC'S

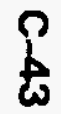

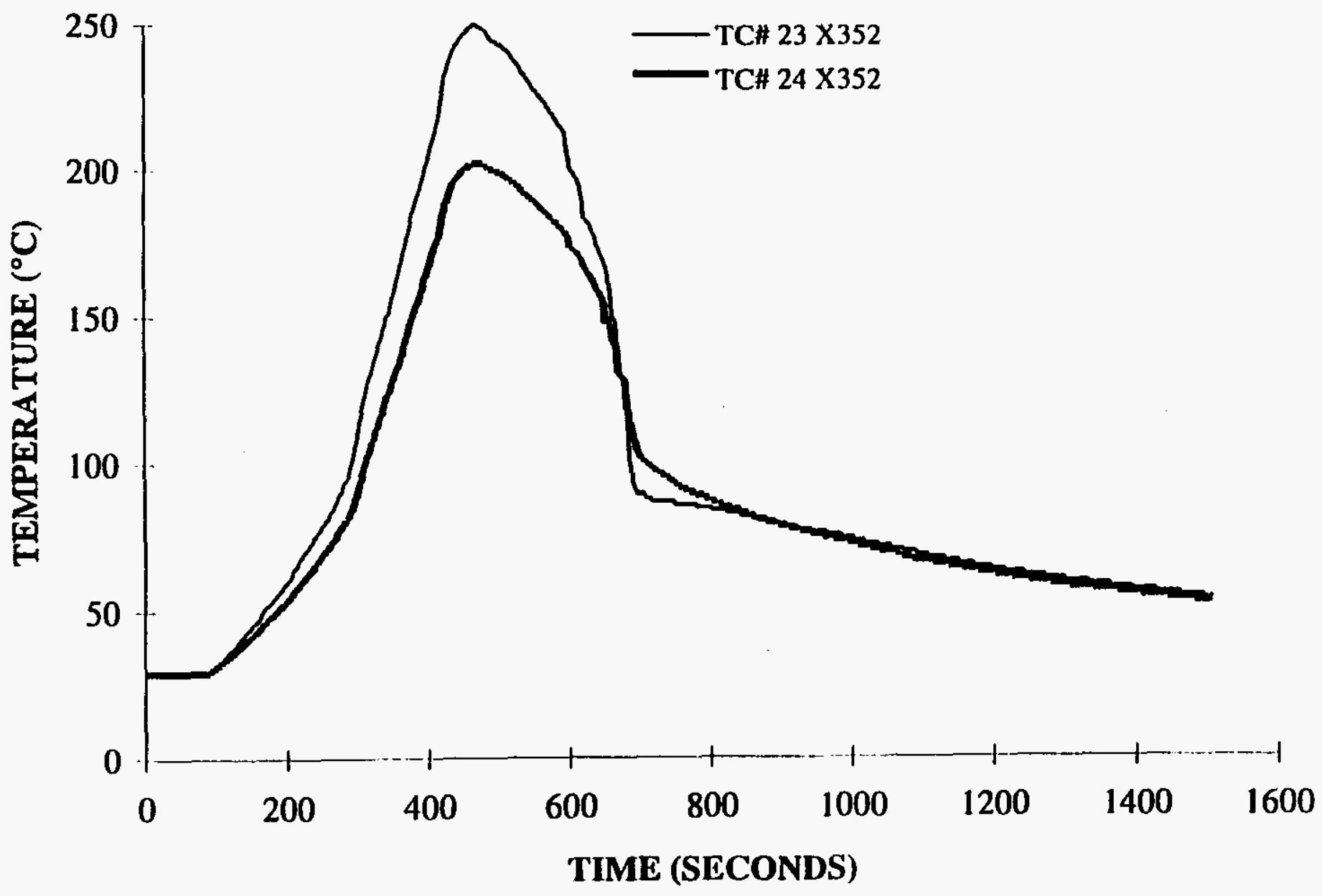


CLIENT: LATA

PROJECT NO.: SwRI 01-7106

DATE: 6 JUNE 1995

TEST ID: 157LR I.DAT

\section{RACK STORAGE TEST}

DRUM SURFACE TC'S

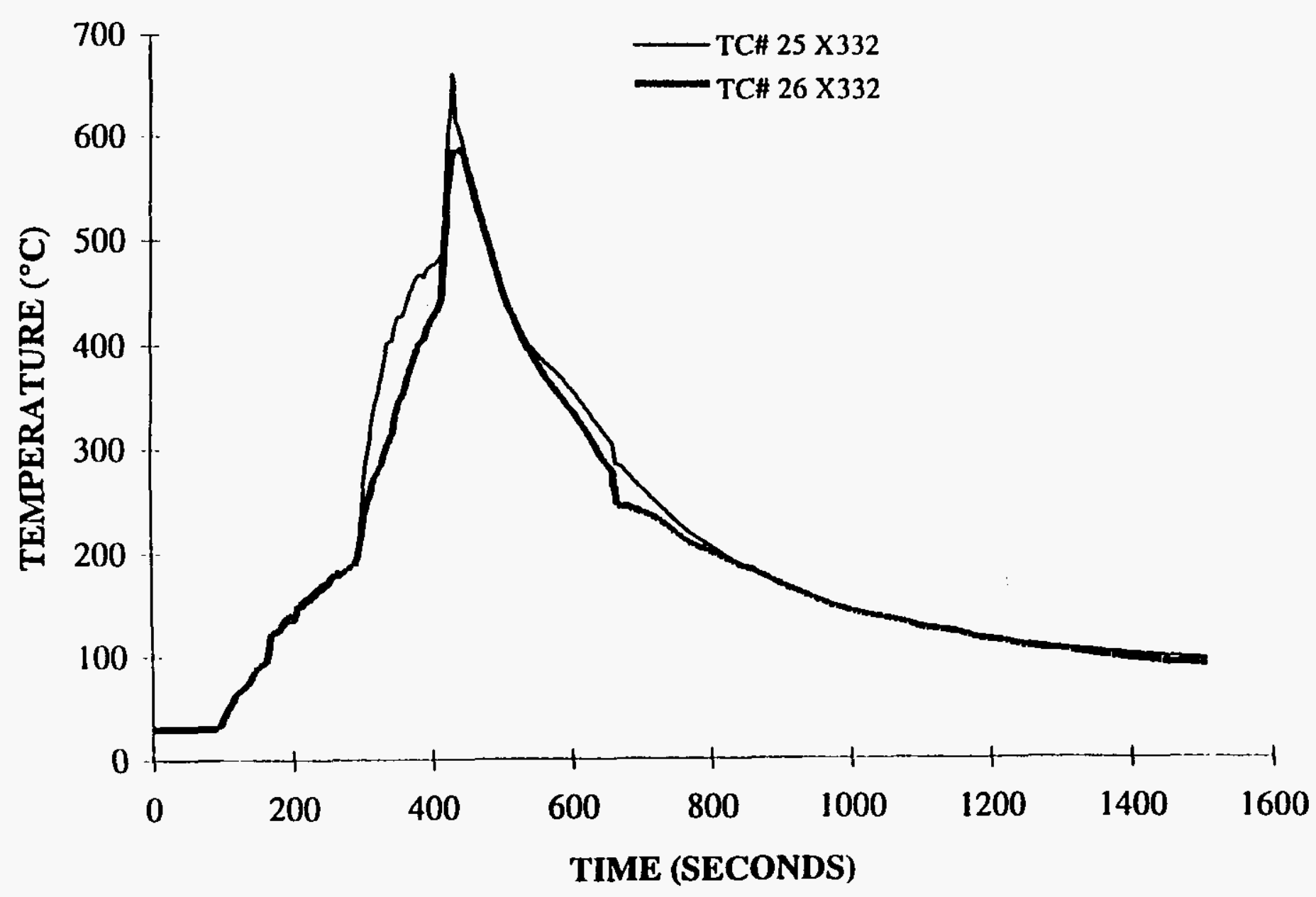

TIME (SECONDS) 
CLIENT: LATA

PROJECT NO.: SwRI 01-7106

DATE: 6 JUNE 1995

TEST ID: 157LRI.DAT

\section{RACK STORAGE TEST} DRUM SURFACE TC'S

\&

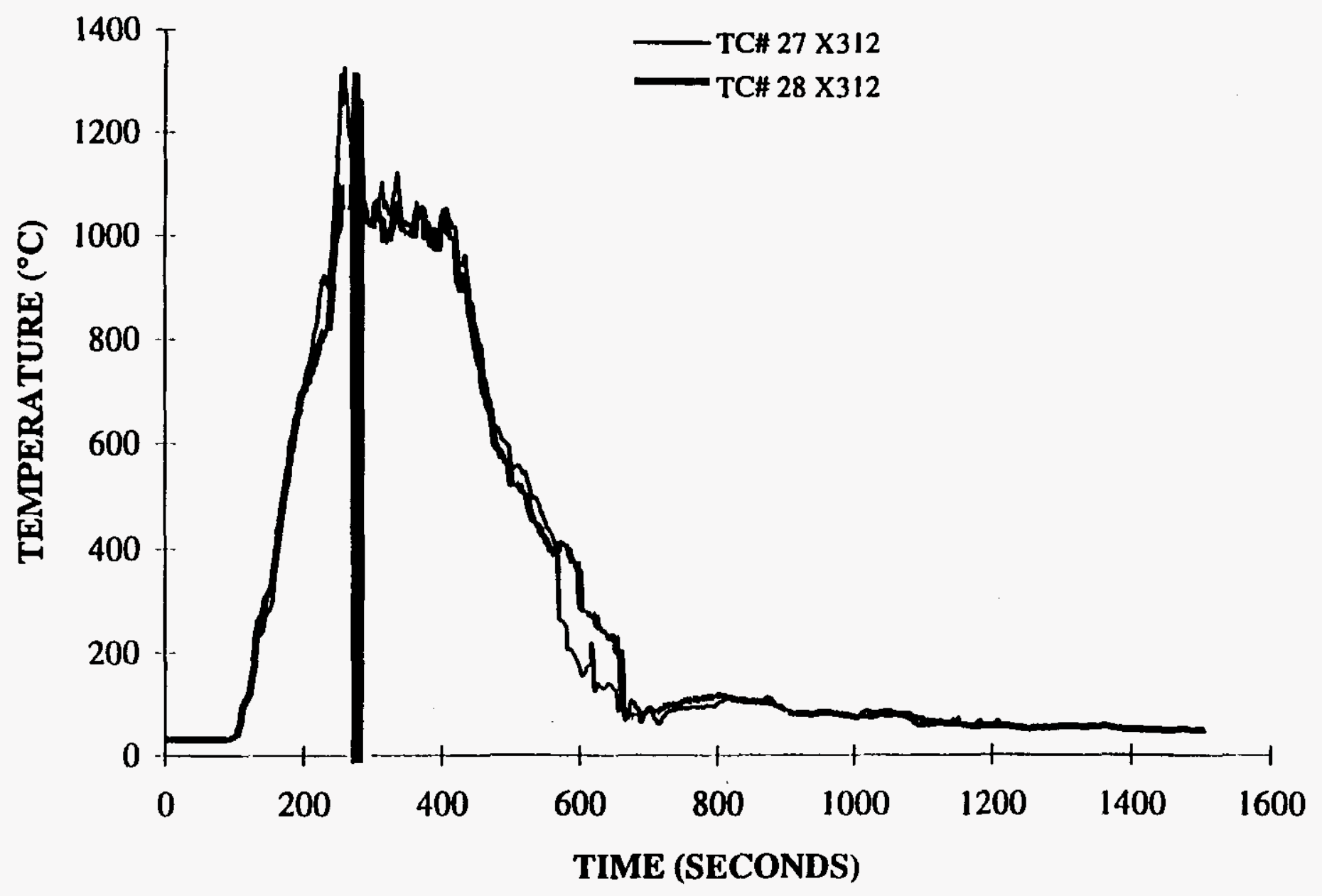




\section{CLIENT: LATA}

PROJECT NO.: SwRI 01-7106

DATE: 6 JUNE 1995

TEST ID: 157LR3.DAT

\section{RACK STORAGE TEST}

\section{DRUM SURFACE TC'S}

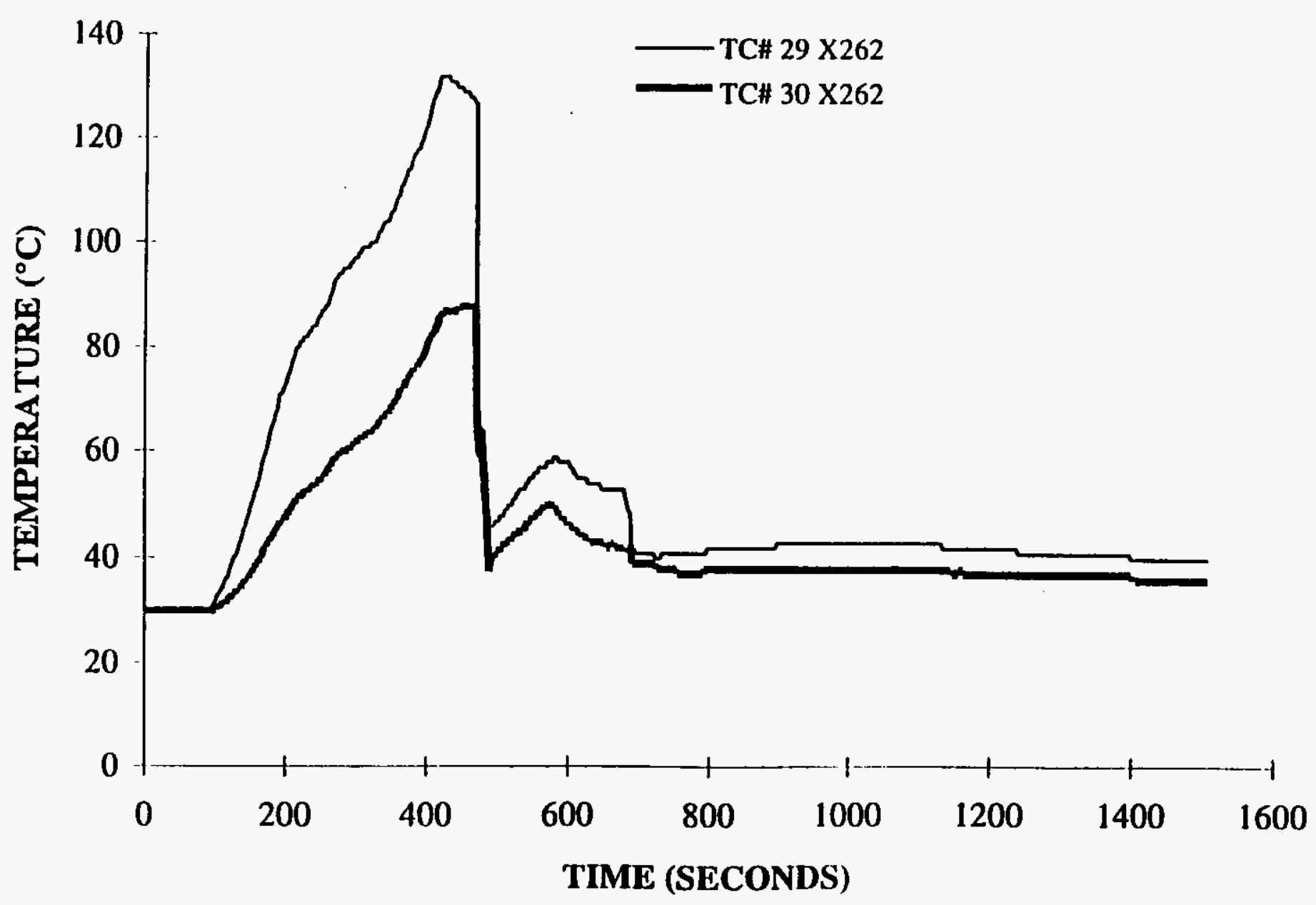

f 
TEST ID: 157LR3.DAT

\section{RACK STORAGE TEST}

\section{DRUM SURFACE TC'S}

$?$

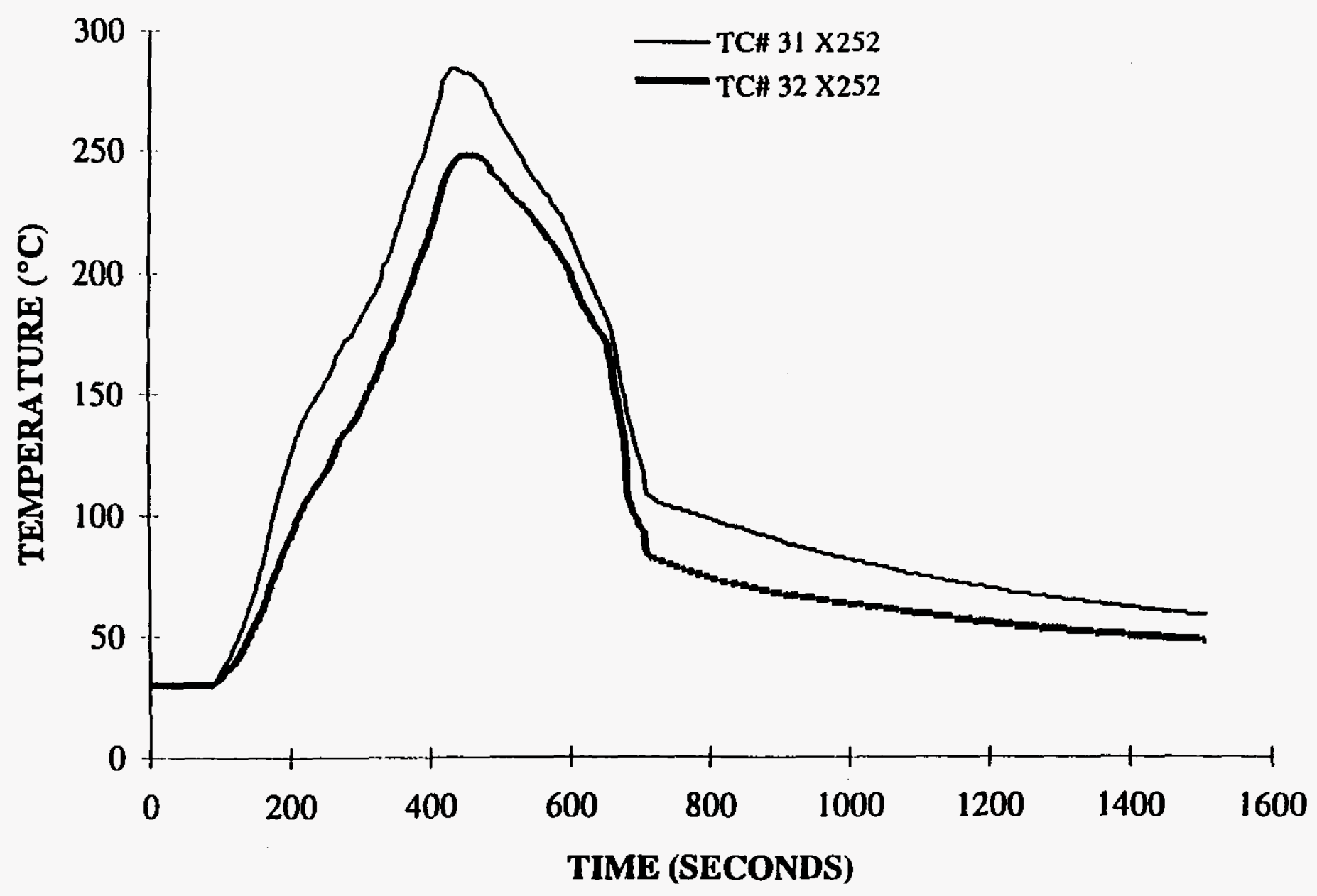


CLIENT: LATA

PROJECT NO.: SwRI 01-7106

DATE: 6 JUNE 1995

TEST ID: 157LR3.DAT

\section{RACK STORAGE TEST}

DRUM SURFACE TC'S

\&

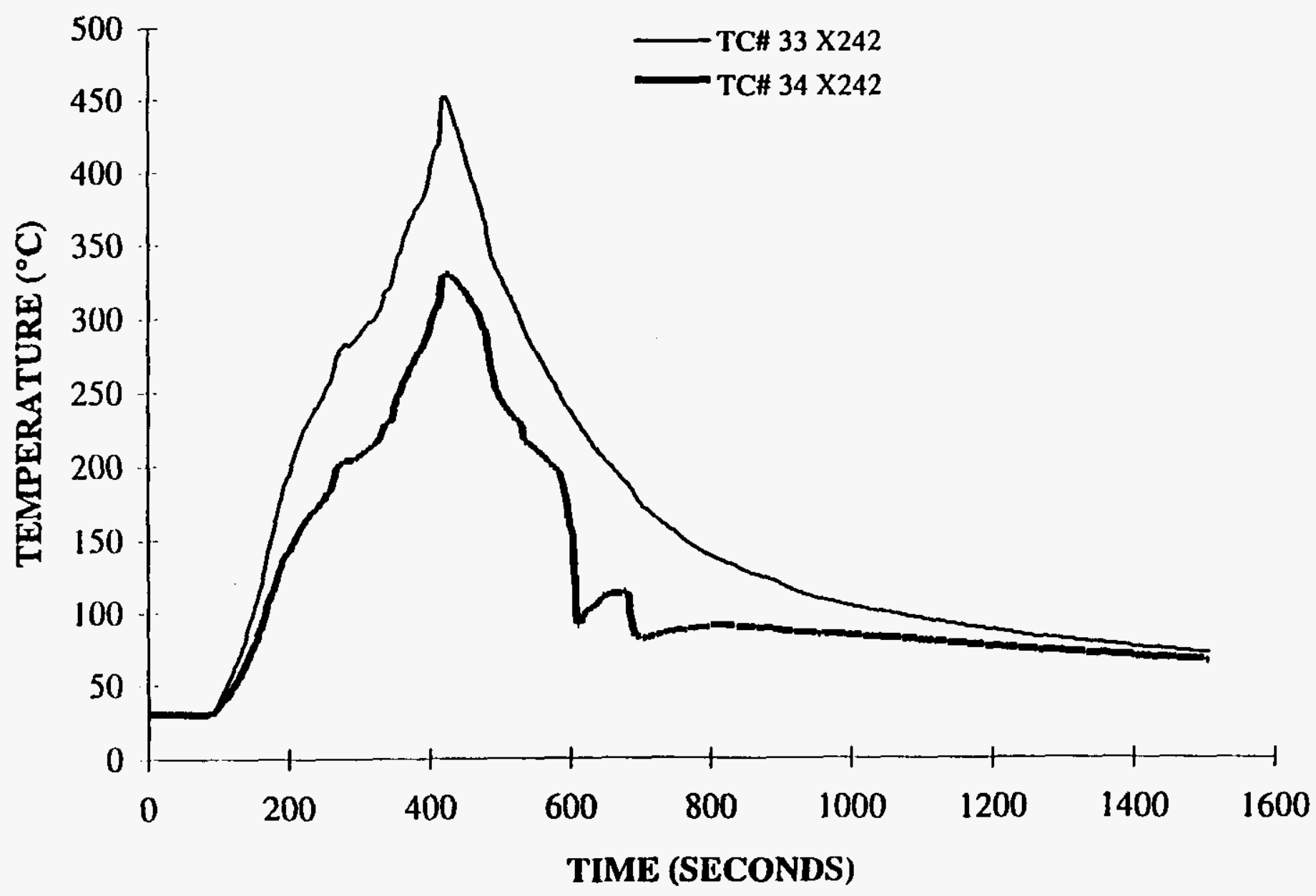


CLIENT: LATA

PROJECT NO.: SwRI 01-7106

DATE: 6 JUNE 1995

TEST ID: 157LR3.DAT

\section{RACK STORAGE TEST}

DRUM SURFACE TC'S

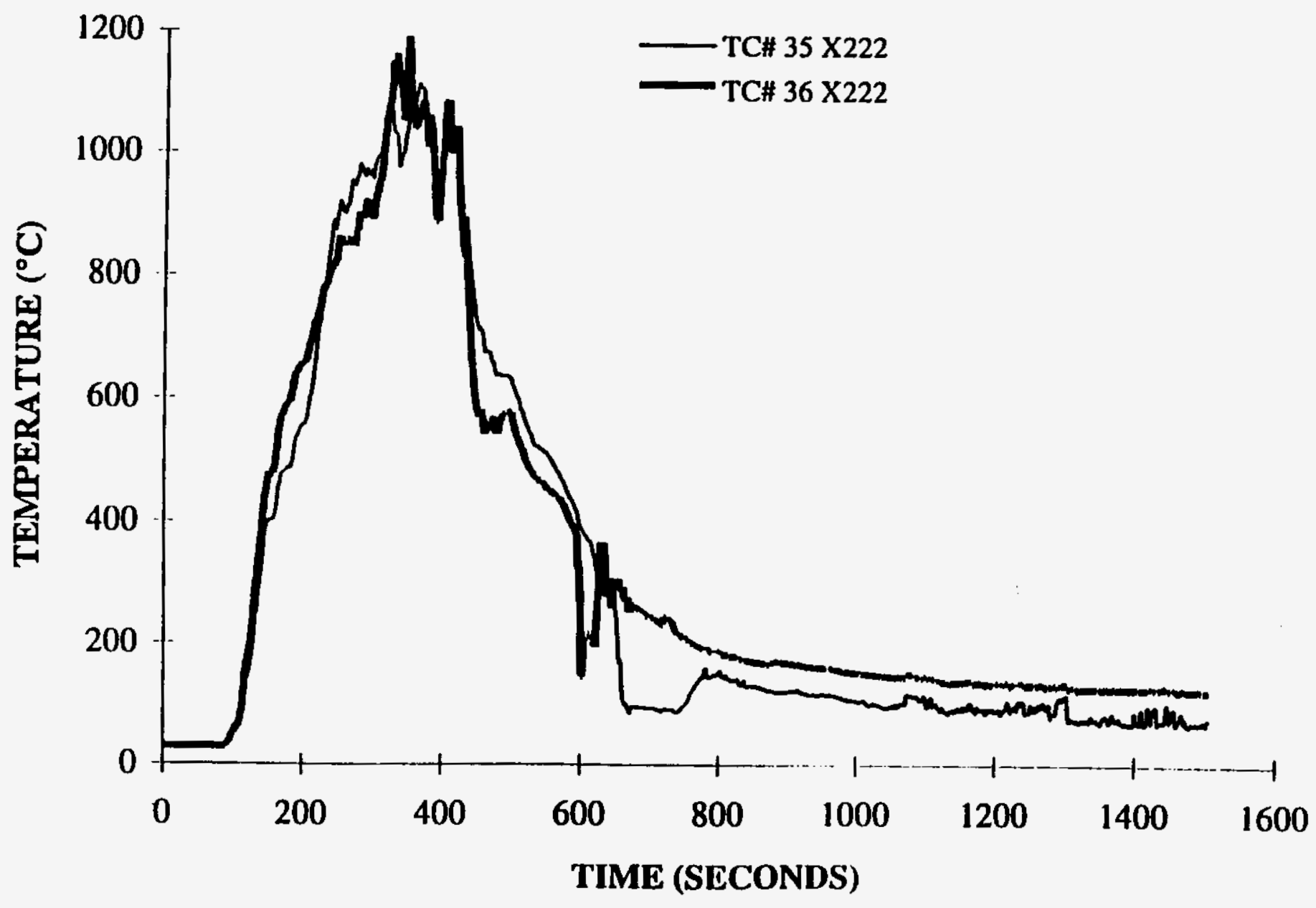


CLIENT: LATA

PROJECT NO.: SwRI 01-7106

DATE: 6 JUNE 1995

TEST ID: 157LR3.DAT

\section{RACK STORAGE TEST}

DRUM SURFACE TC'S

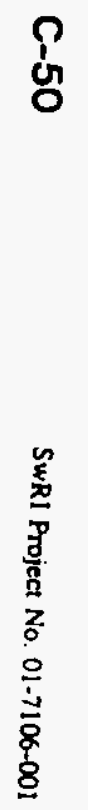

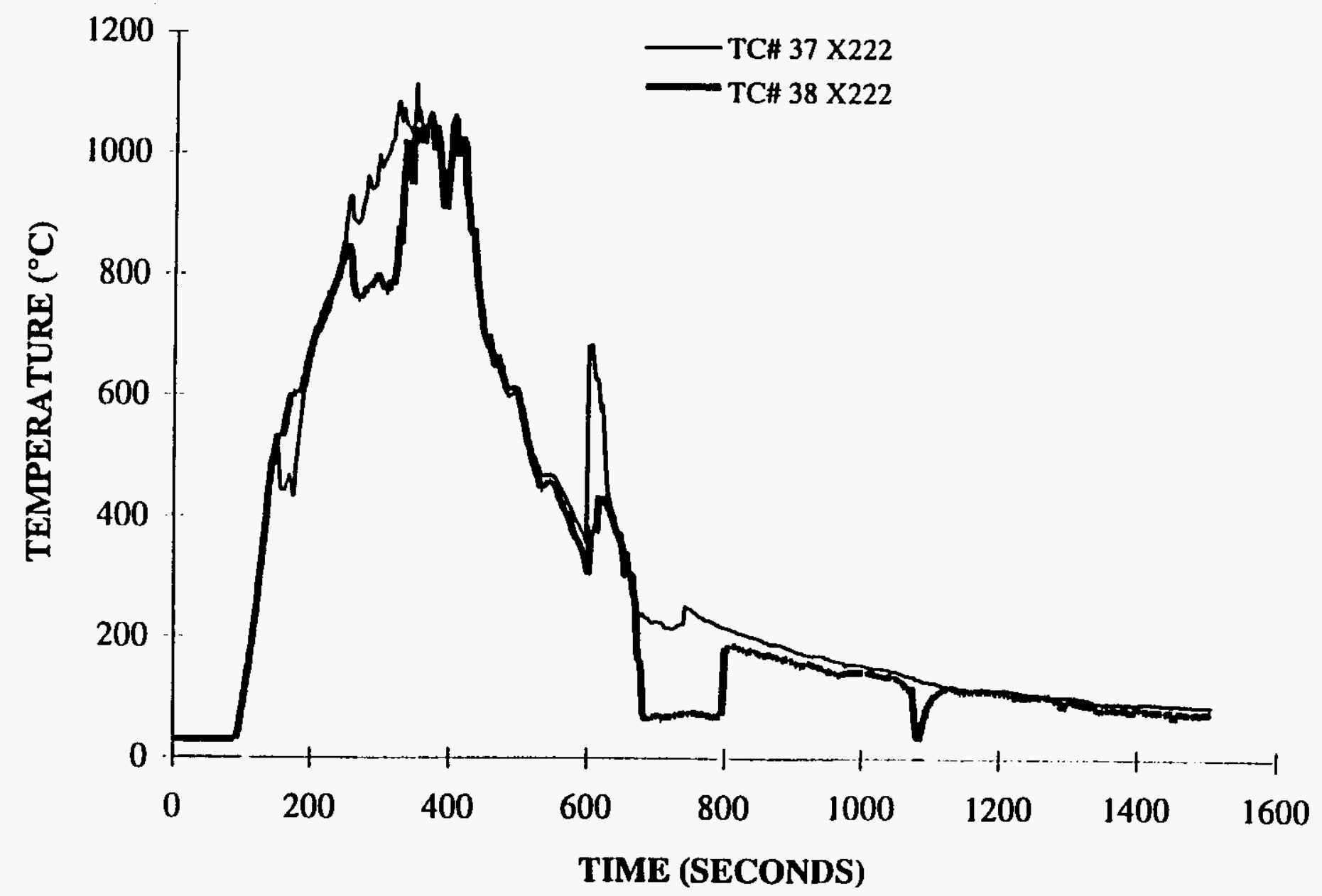

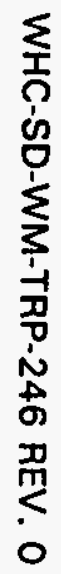

TIME (SECONDS) 
CLIENT: LATA

PROJECT NO.: SwRI 01-7106

DATE: 6 JUNE 1995

TEST ID: 157LRI.DAT

\section{RACK STORAGE TEST}

DRUM SURFACE TC'S

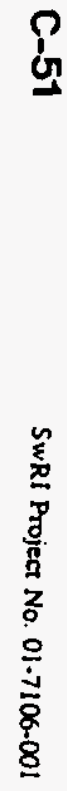

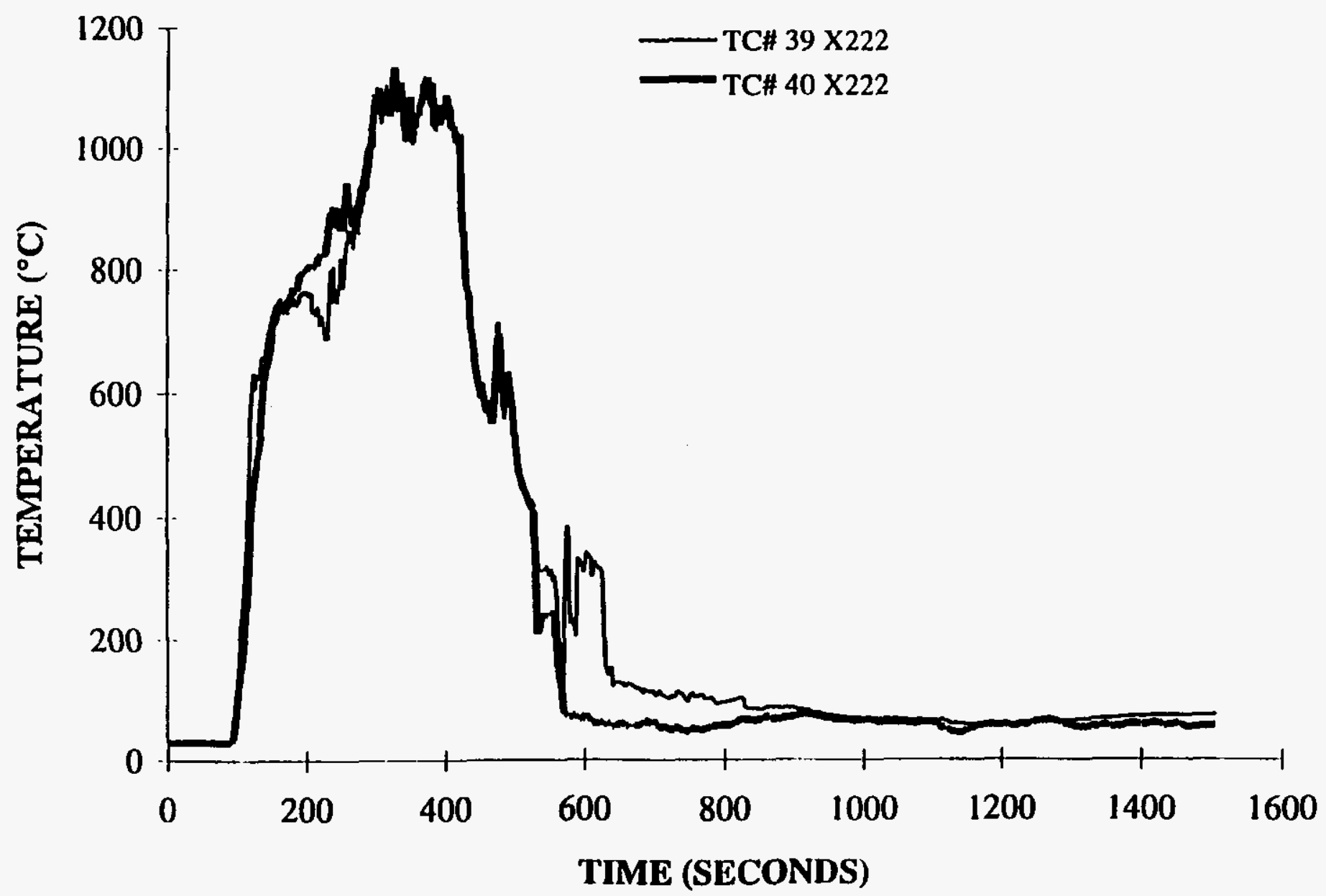


CLIENT: LATA

PROJECT NO.: SwRI 01-7106

DATE: 6 JUNE 1995

TEST ID: 157LR3.DAT

RACK STORAGE TEST

DRUM SURFACE TC'S

en

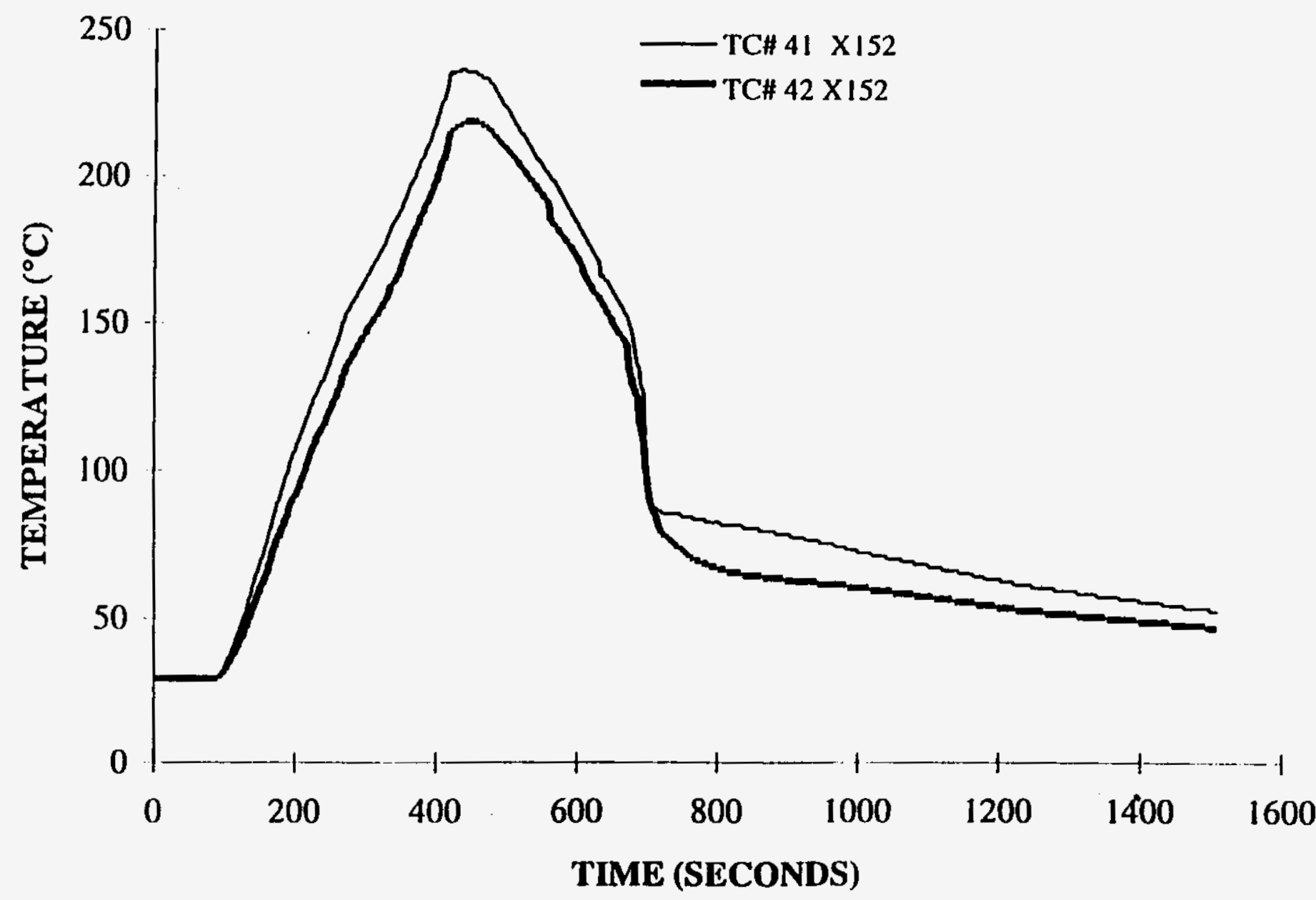

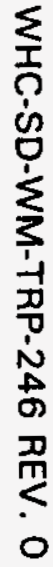

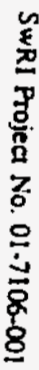

TIME (SECONDS) 


\section{CLIENT: LATA}

PROJECT NO.: SwRI 0]-7106

DATE: 6 JUNE 1995

TEST ID: 157LR2.DAT

\section{RACK STORAGE TEST}

DRUM SURFACE TC'S

@

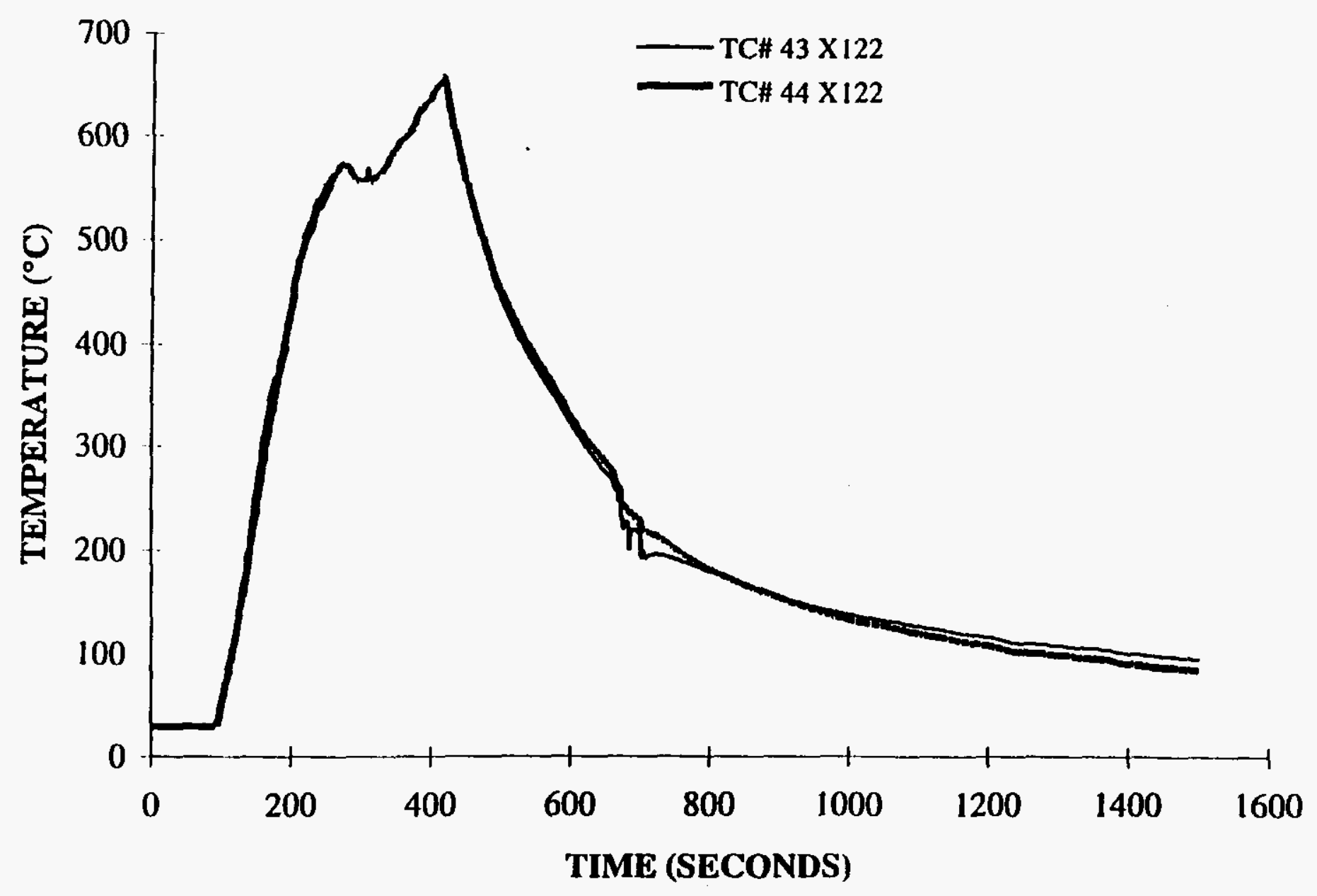


CLIENT: LATA

PROJECT NO.: SwRI 01-7106

DATE: 6 JUNE 1995

TEST ID: 157LRI.DAT

\section{RACK STORAGE TEST DRUM SURFACE TC'S}

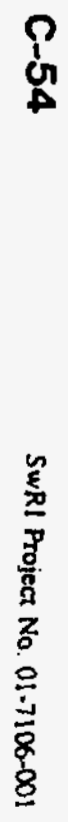

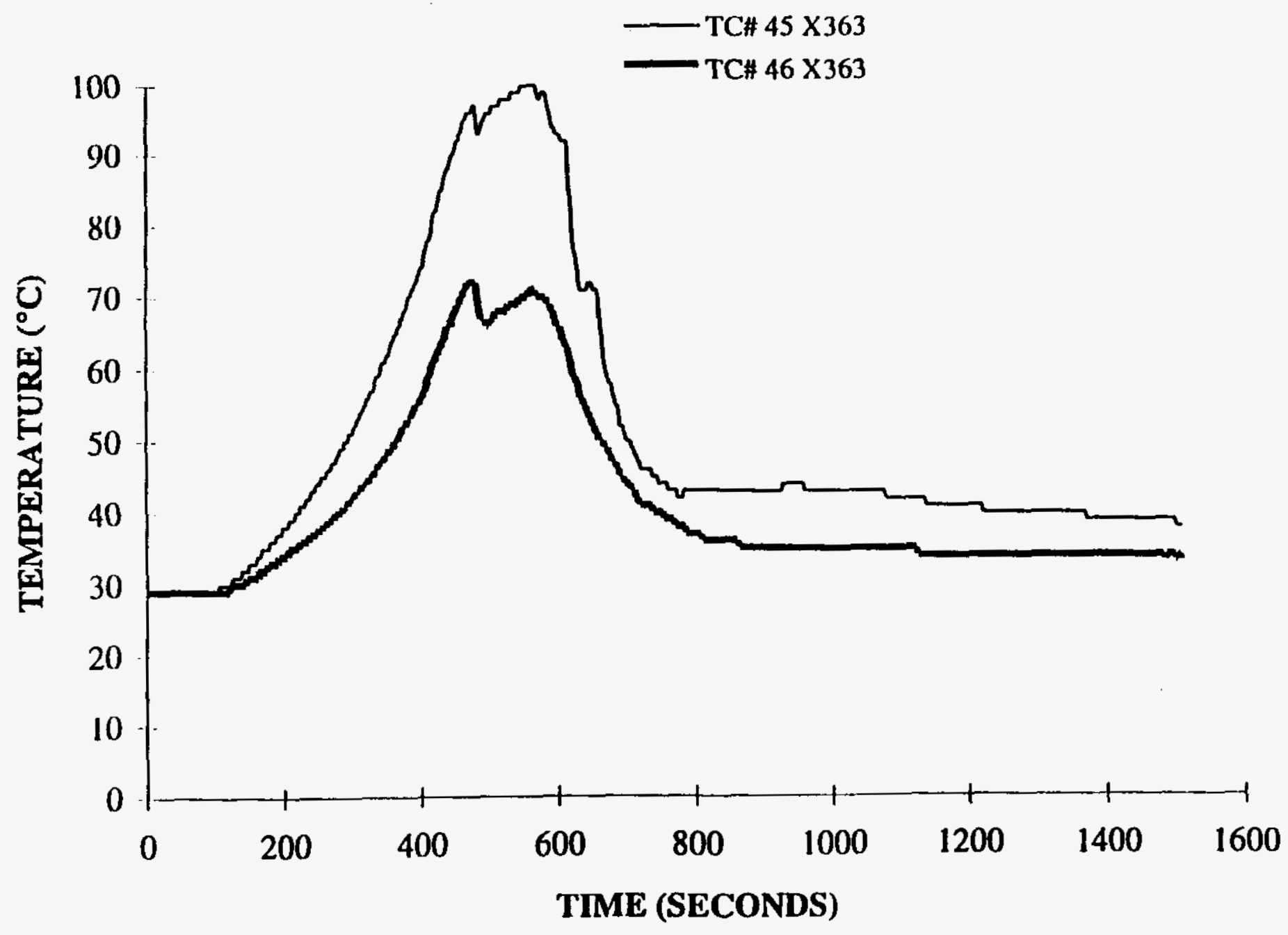


CLIENT: LATA

PROJECT NO.: SwRI 01-7106

DATE: 6 JUNE 1995

TEST ID: 157LR3.DAT

\section{RACK STORAGE TEST DRUM SURFACE TC'S}

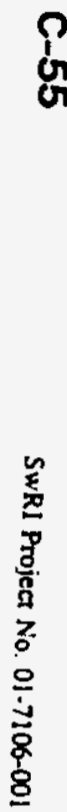

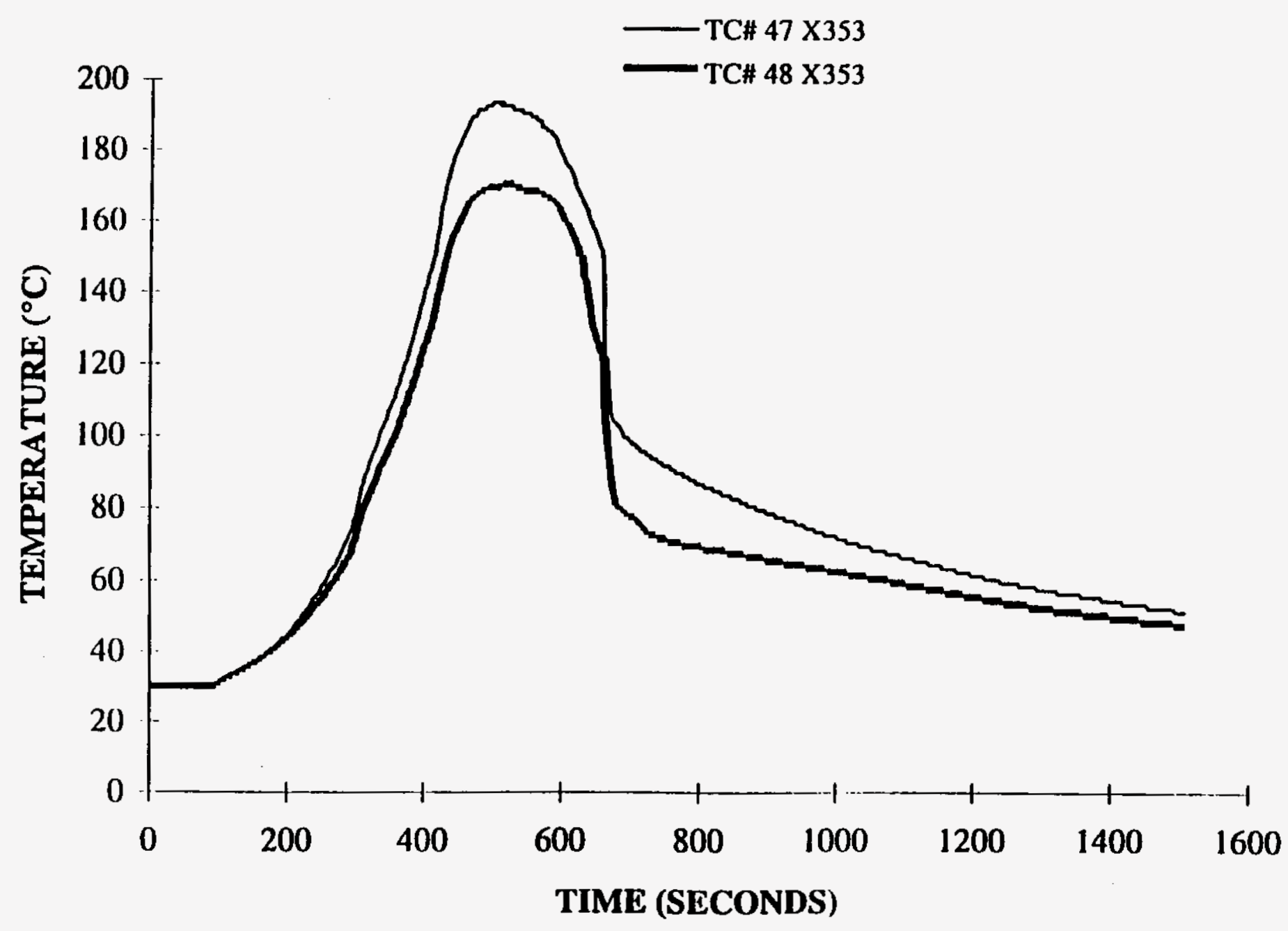


CLIENT: LATA

PROJECT NO.: SwRI 01-7106

DATE: 6 JUNE 1995

TEST ID: 157LR3.DAT

RACK STORAGE TEST

DRUM SURFACE TC'S

\&

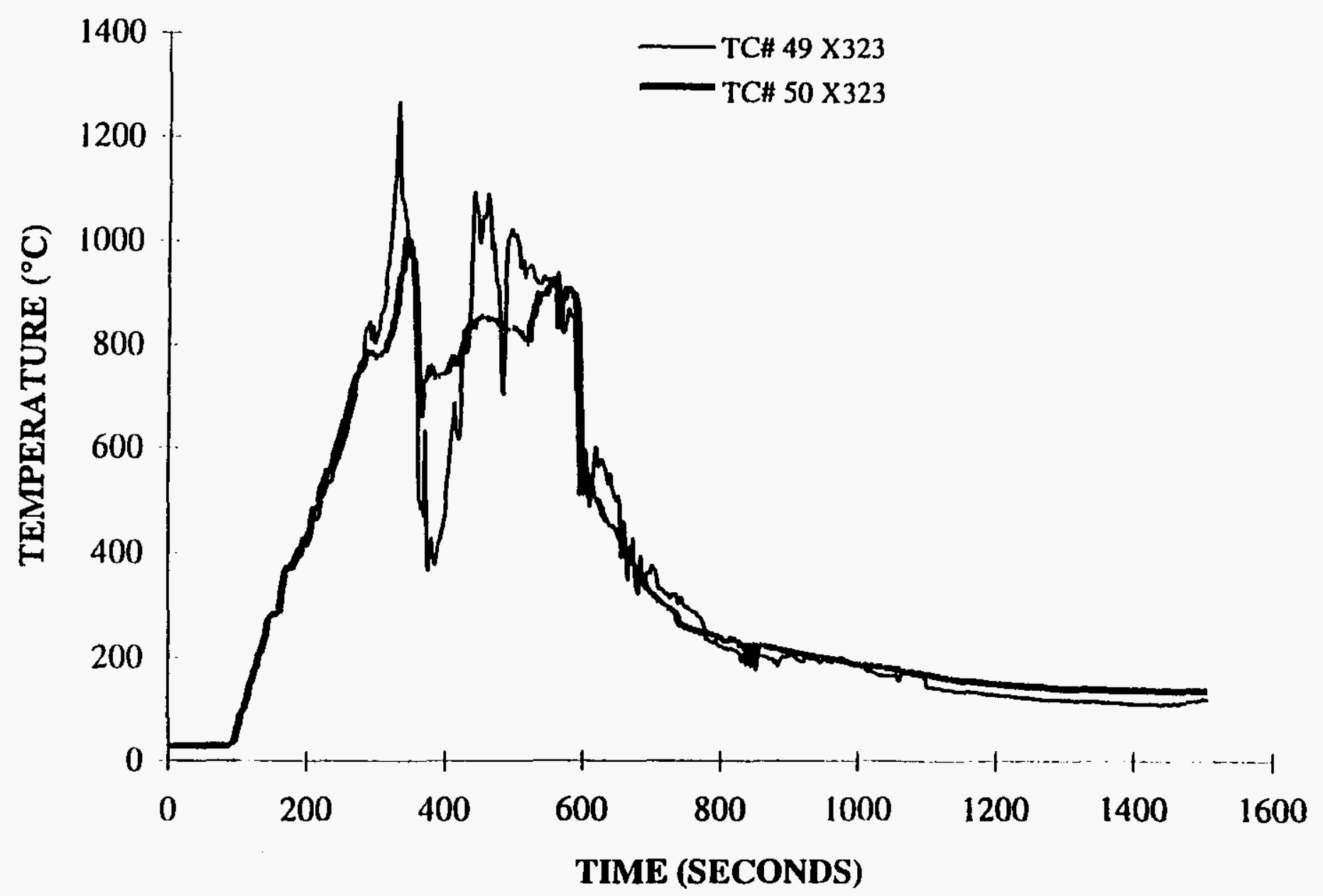

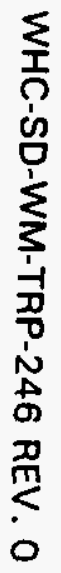

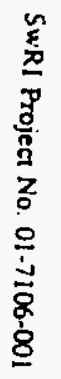

TIME (SECONDS) 
CLIENT: LATA

PROJECT NO.: SwRI 01-7106

DATE: 6 JUNE 1995

TEST ID: 157LR2.DAT

\section{RACK STORAGE TEST DRUM SURFACE TC'S}

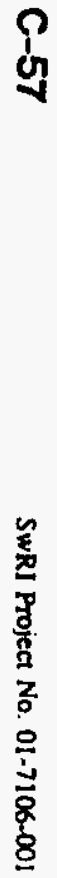

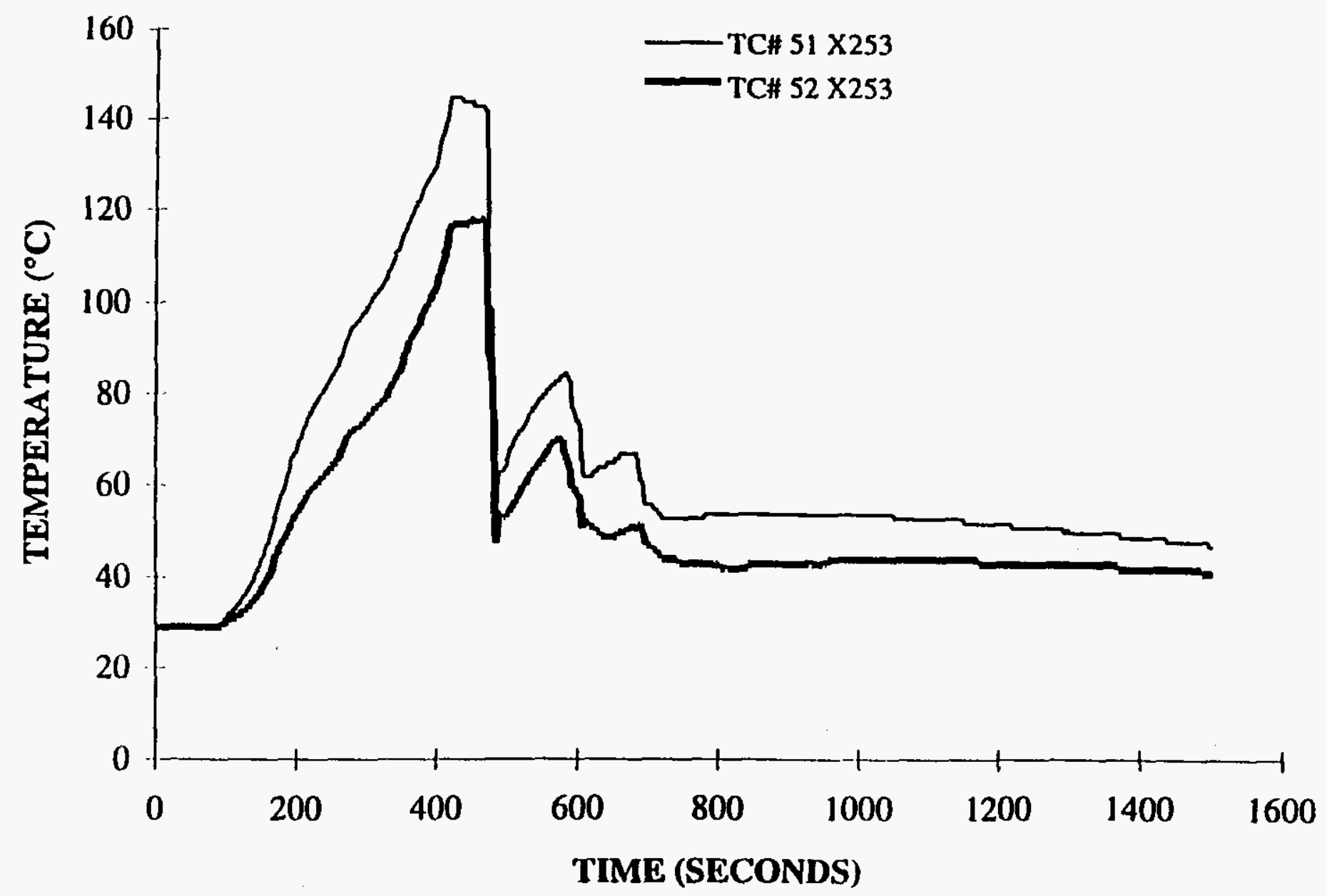


WHC-SD-WM-TRP-246 REV. O

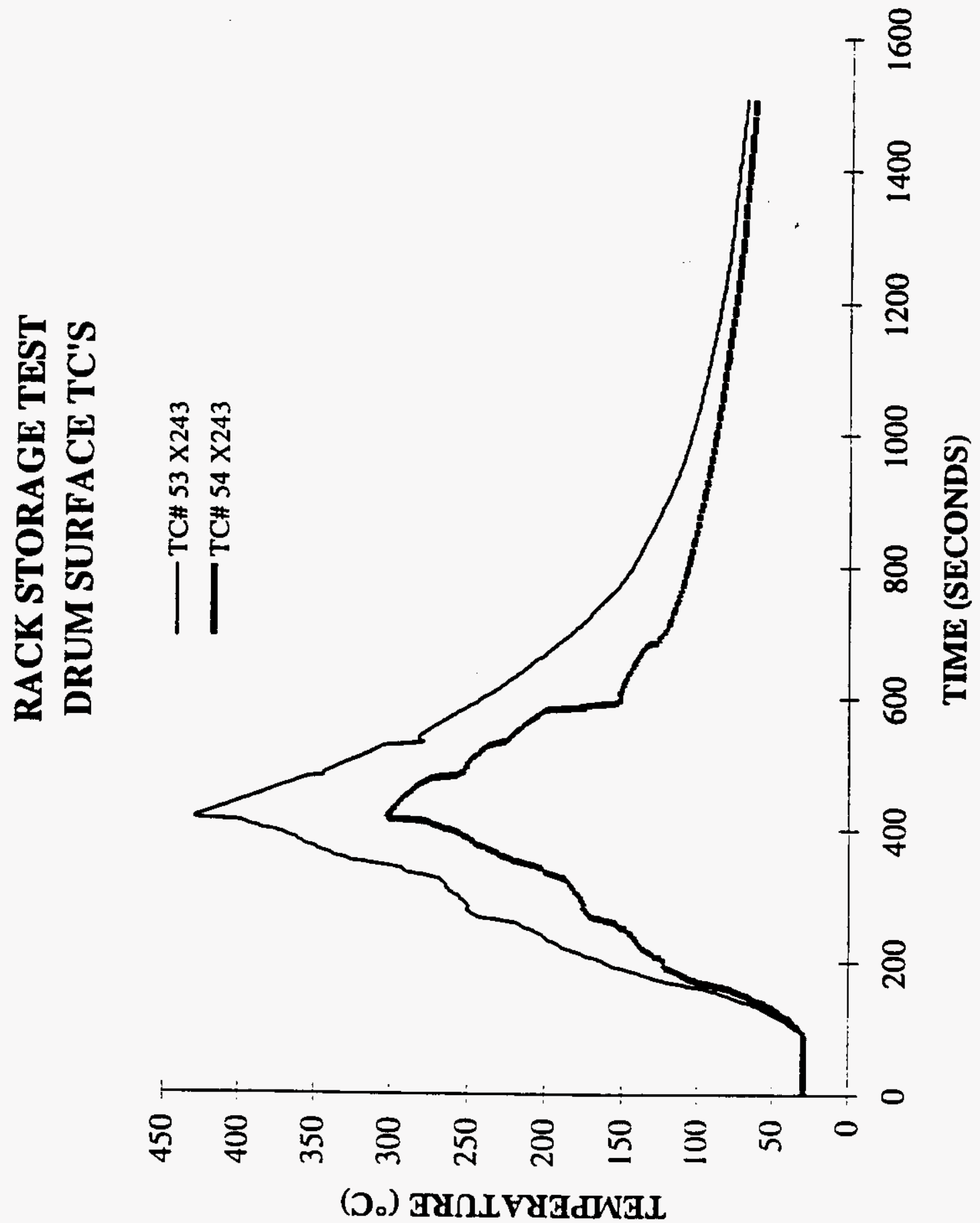


CLIENT: LATA

PROJECT NO.: SwRI 01-7106

DATE: 6 JUNE 1995

TEST ID: 157LR2.DAT

RACK STORAGE TEST

DRUM SURFACE TC'S

\&

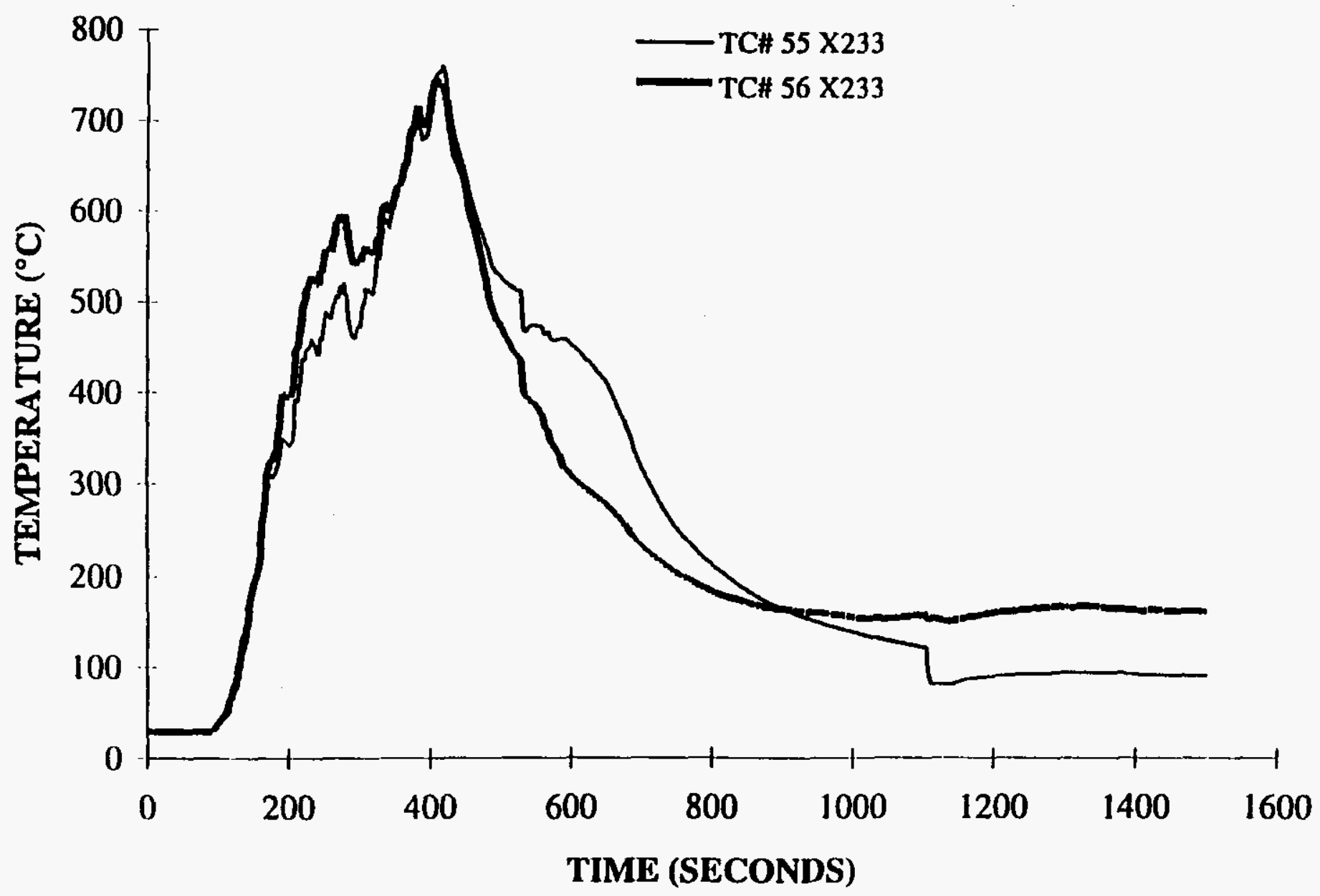

\o⿱ 
CLIENT: LATA

PROJECT NO.: SwRI 01-7106

DATE: 6 JUNE 1995

TEST ID: 157LR2.DAT

\section{RACK STORAGE TEST \\ DRUM SURFACE TC'S}

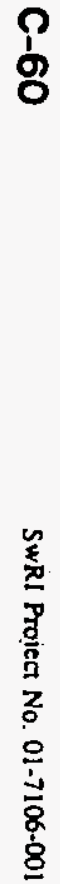

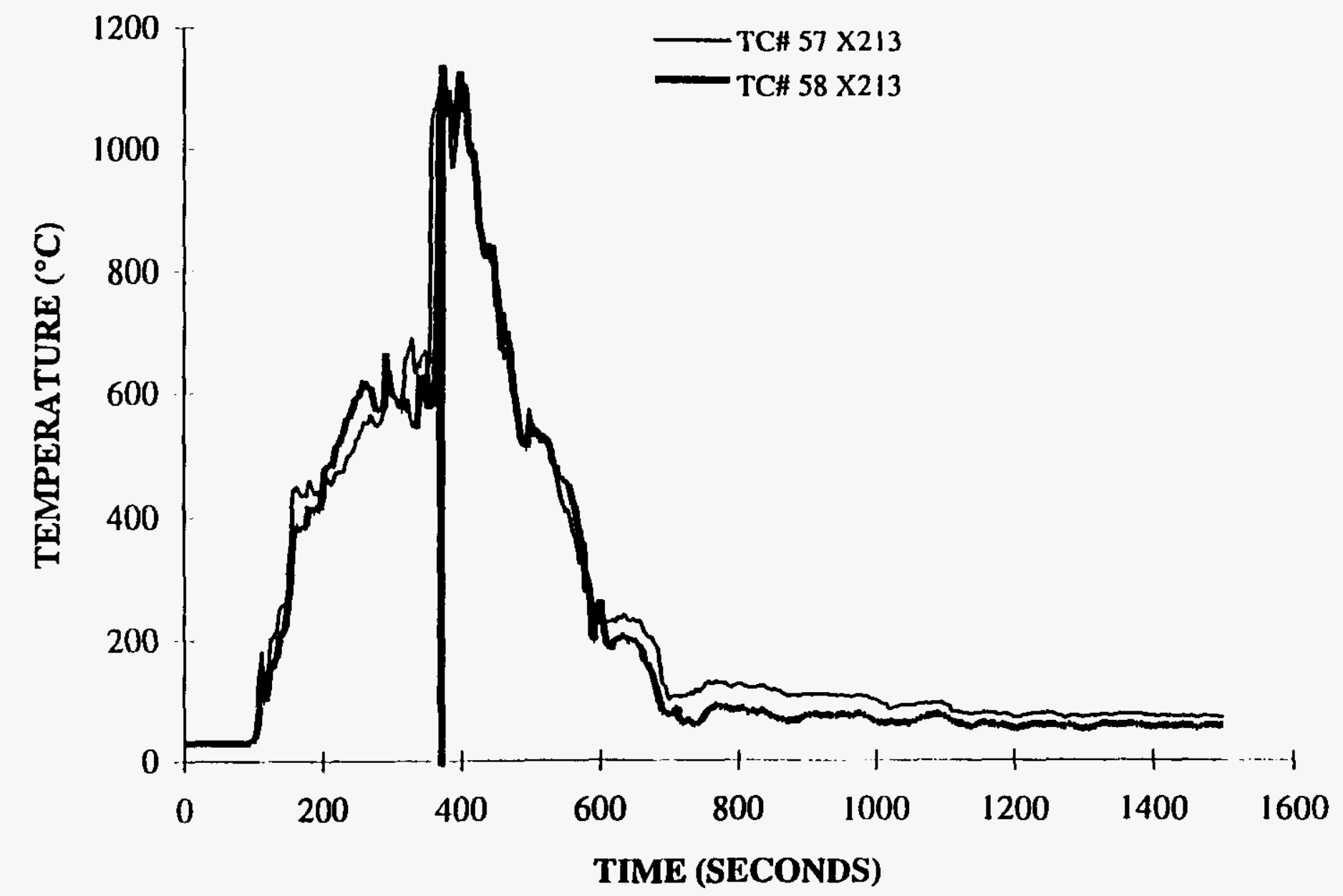

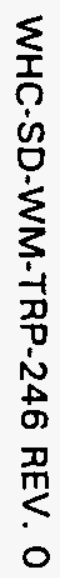

8 
CLIENT: LATA

PROJECT NO.: SwRI 01-7106

DATE: 6 JUNE 1995

TEST ID: 157LRI.DAT

\section{RACK STORAGE TEST}

DRUM SURFACE TC'S

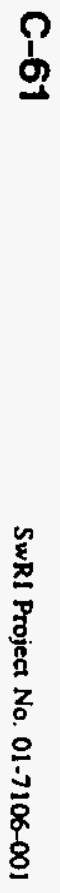

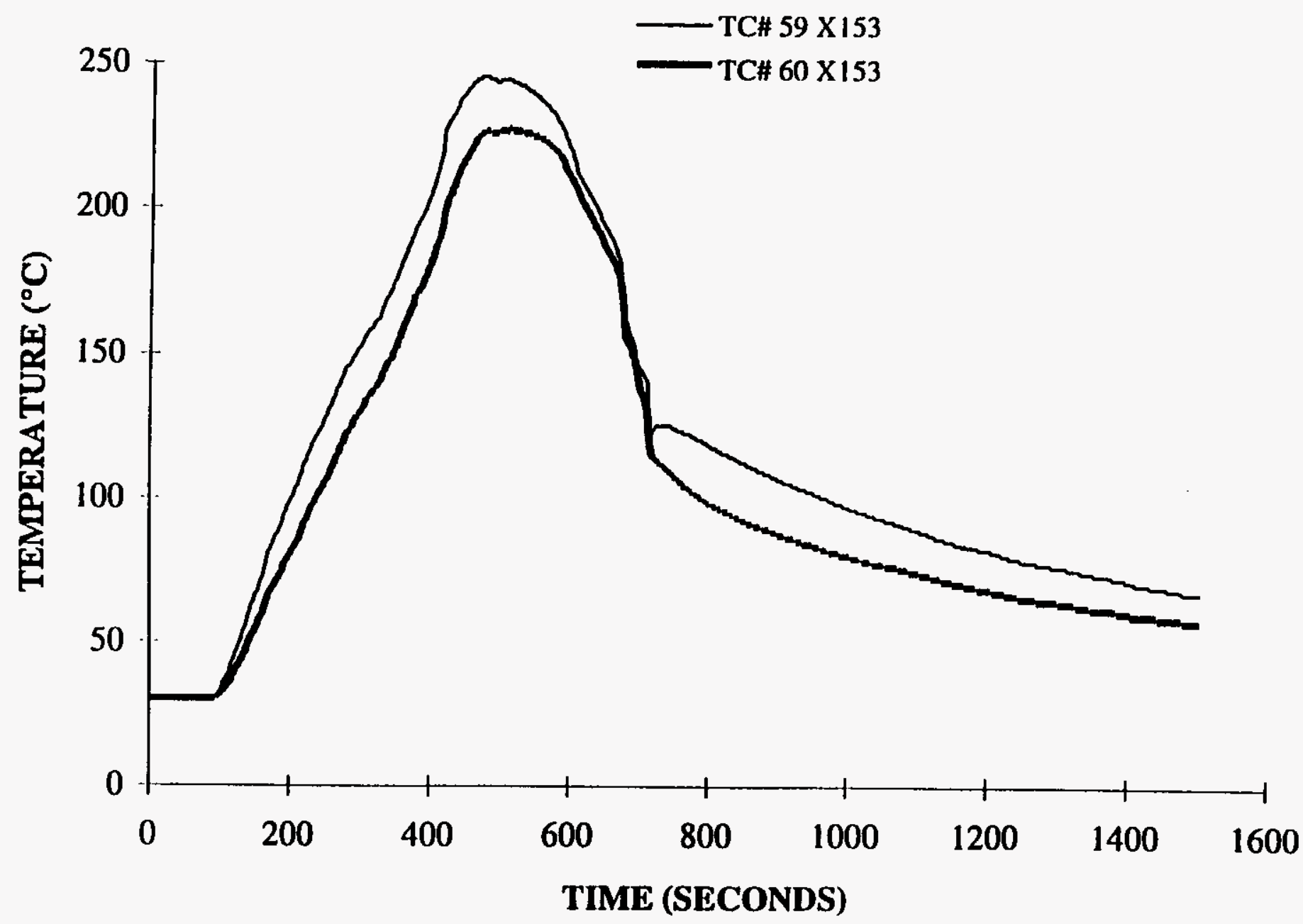


CLIENT: LATA

PROJECT NO.: SWRI 01-7106

DATE: 6 JUNE 1995

TEST ID: 157LR2.DAT

\section{RACK STORAGE TEST DRUM SURFACE TC'S}

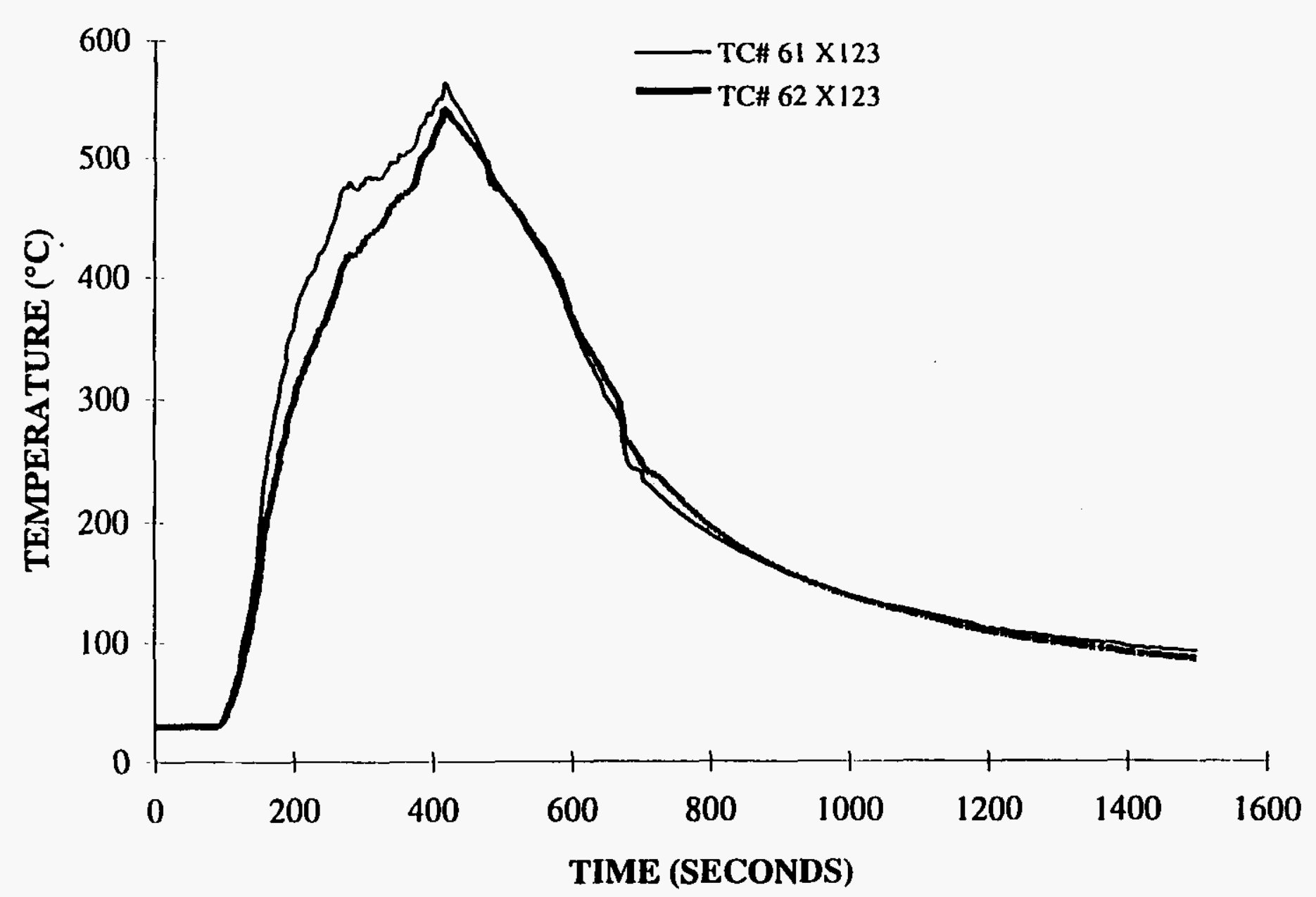


CLIENT: LATA

PROJECT NO.: SwRI 01-7106

DATE: 6 JUNE 1995

TEST ID: 157LR4.DAT

RACK STORAGE TEST

DRUM SURFACE TC'S

\&

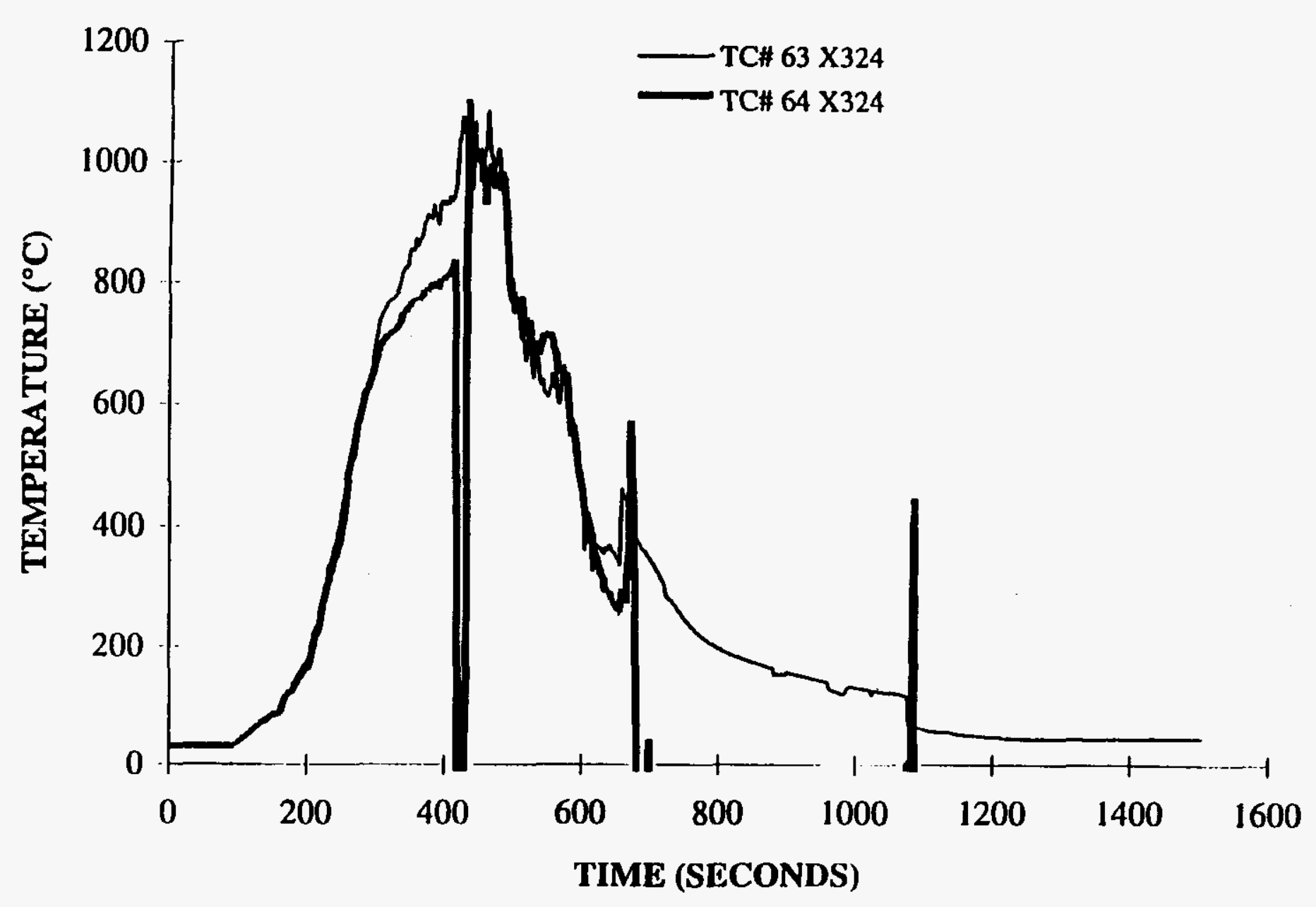

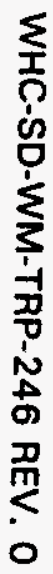

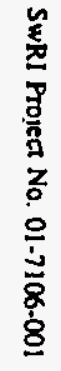


CLIENT: LATA

PROJECT NO.: SwRI 01-7106

DATE: 6 JUNE 1995

TEST ID: 157LR2.DAT

RACK STORAGE TEST

DRUM SURFACE TC'S

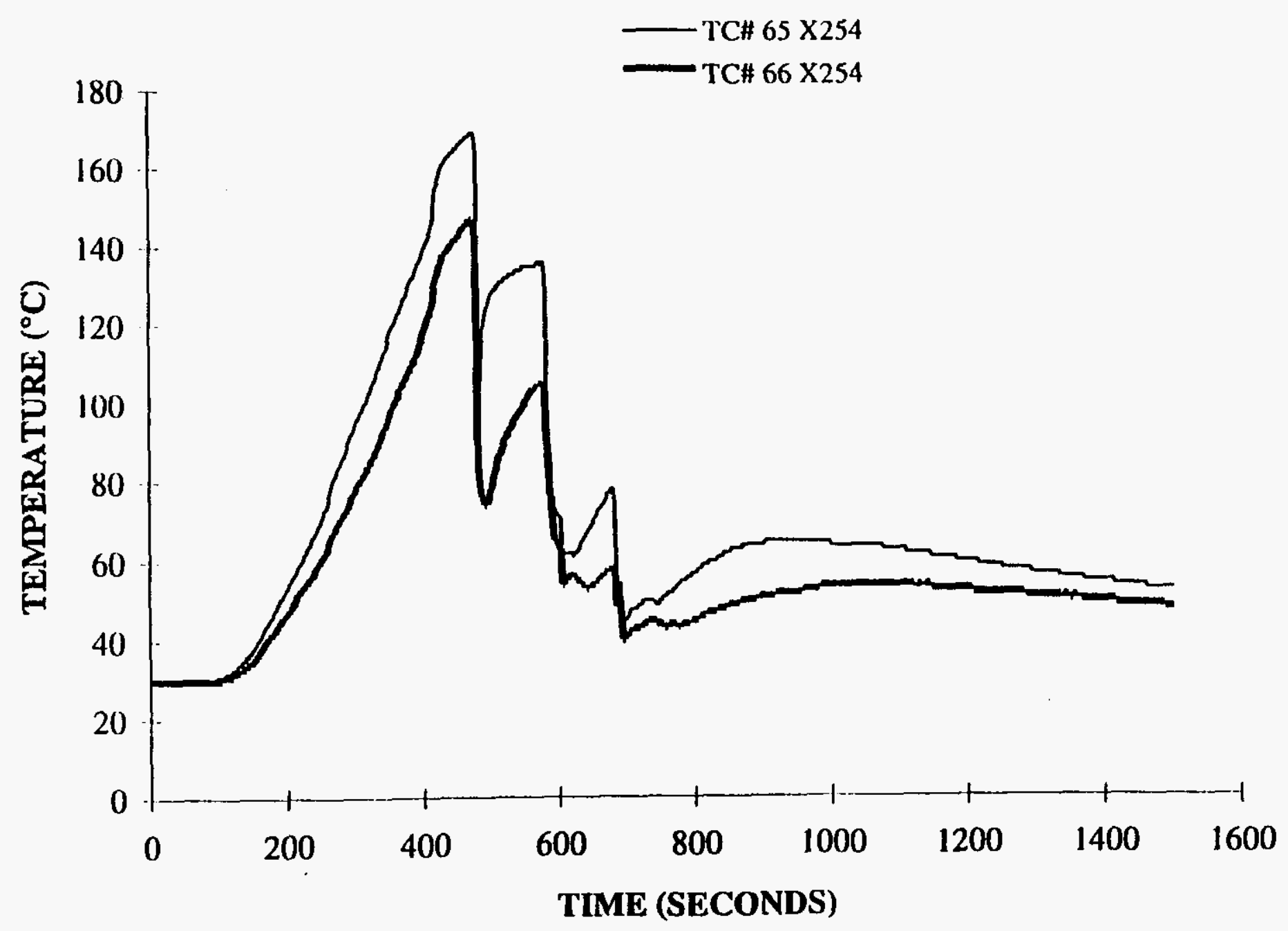

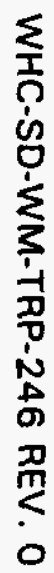

$\stackrel{8}{8}$

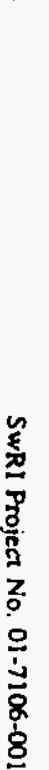


CLIENT: LATA

PROJECT NO.: SwRI 01-7106

DATE: 6 JUNE 1995

TEST ID: 157LR4.DAT

\section{RACK STORAGE TEST \\ DRUM SURFACE TC'S}

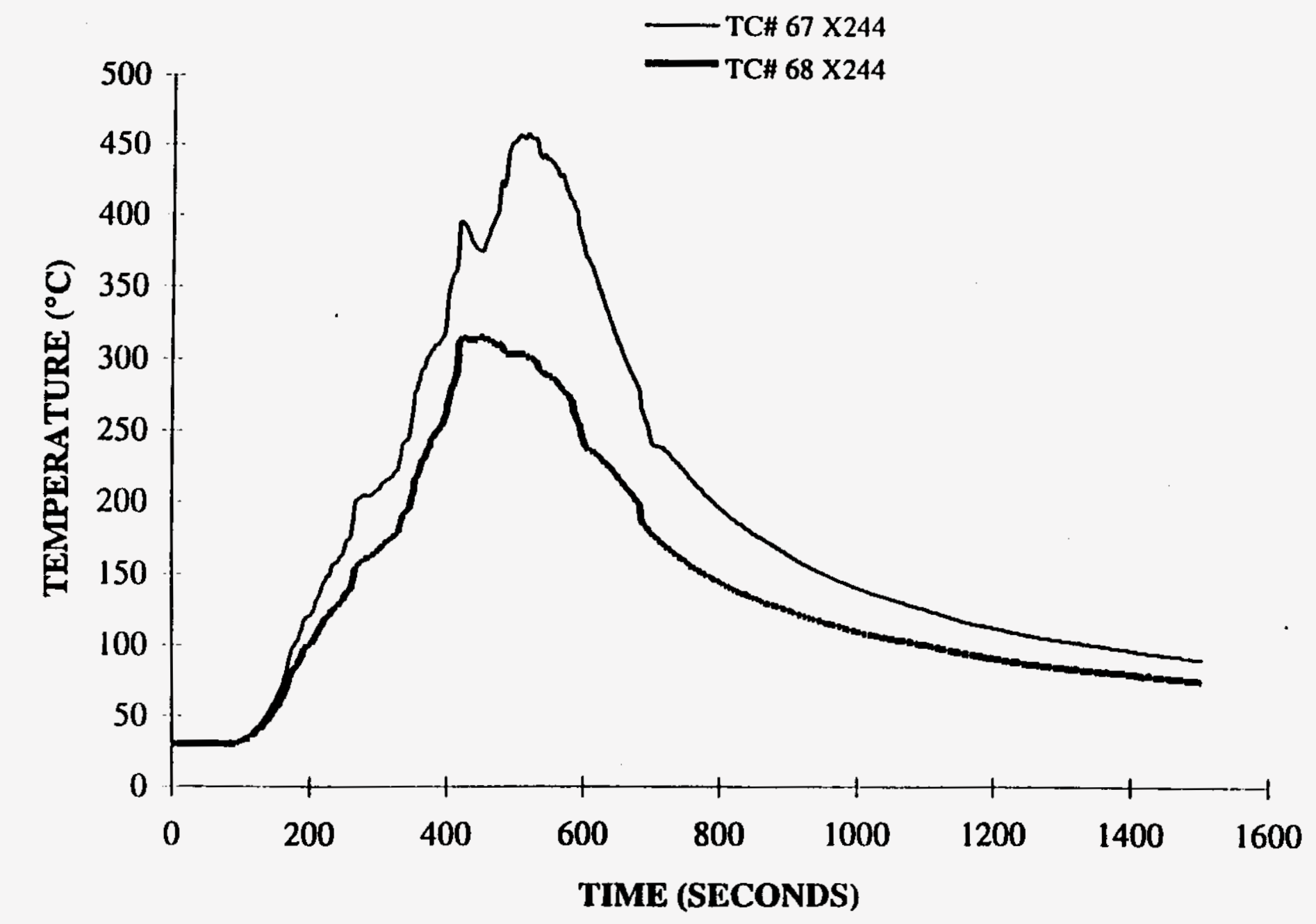

\& 
CLIENT: LATA

PROJECT NO.: SwRI 01-7106

DATE: 6 JUNE 1995

TES'T ID: 157LR2.DAT

\section{RACK STORAGE TEST DRUM SURFACE TC'S}

ร

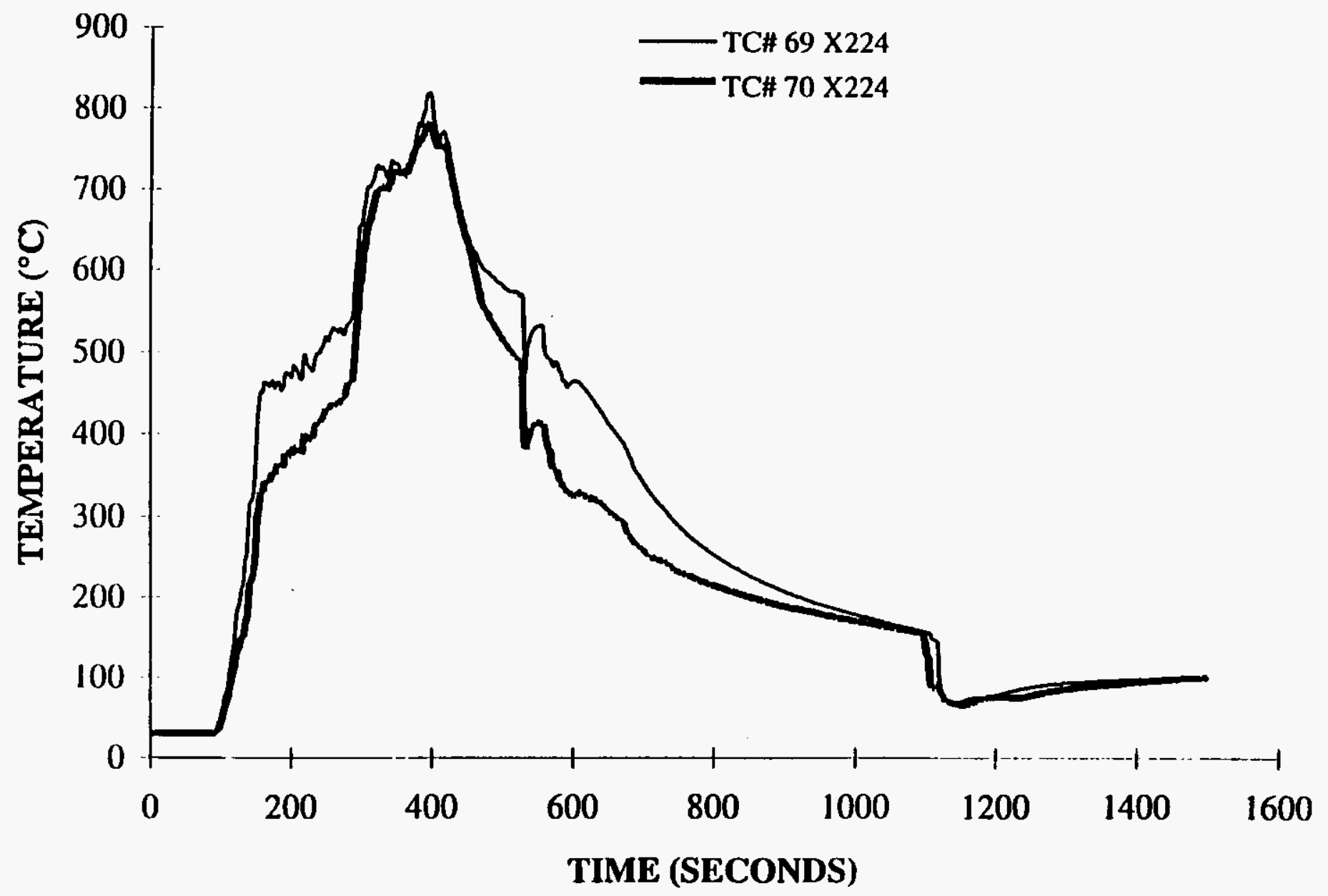


CLIENT: LATA

PROJECT NO.: SwRI 01-7106

DATE: 6 JUNE 1995

TEST ID: 157LR2.DAT

RACK STORAGE TEST

DRUM SURFACE TC'S

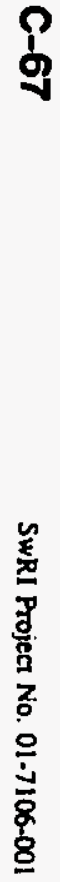

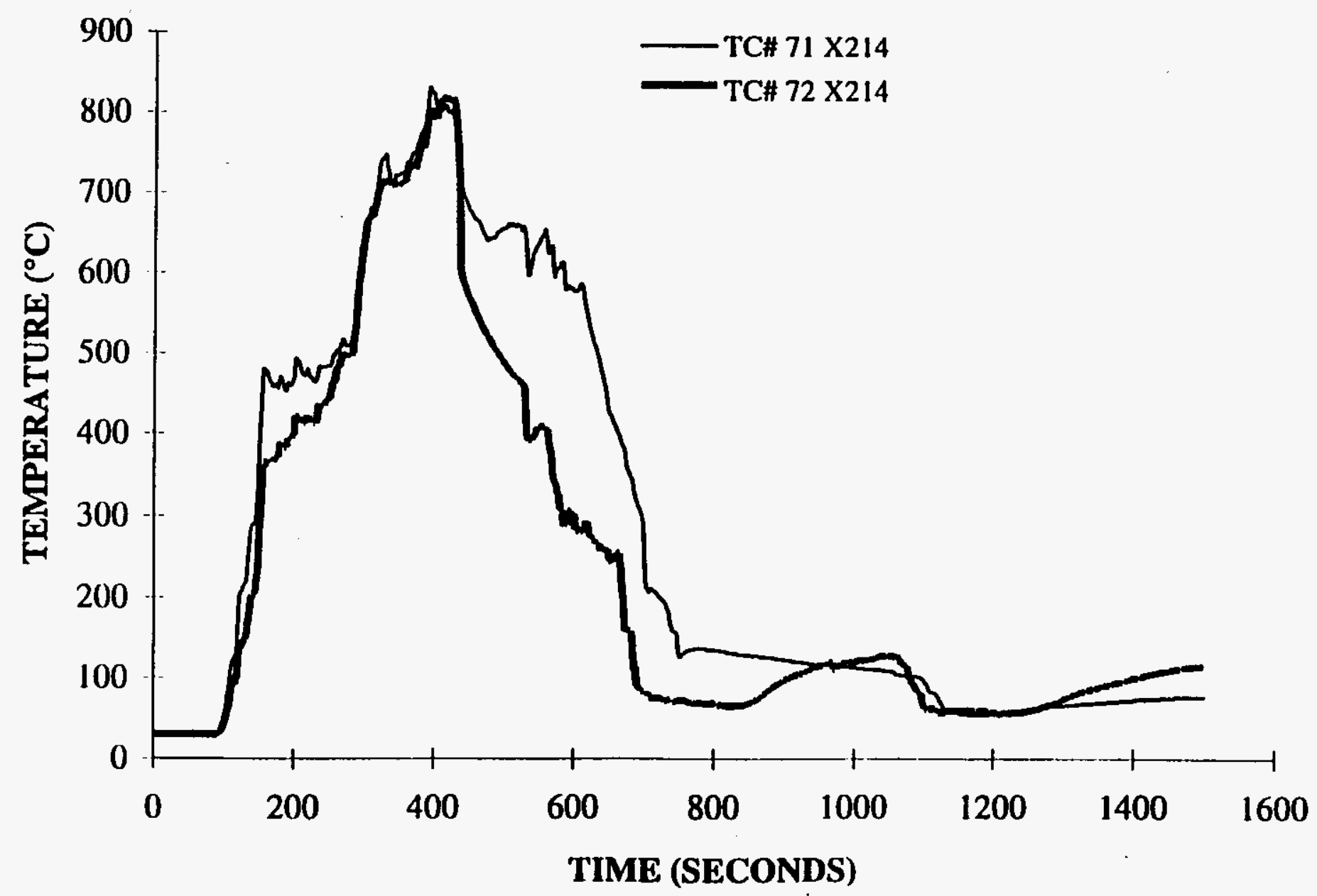

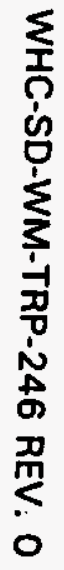


CLIENT: LATA

PROJECT NO.: SwRI 01-7106

DATE: 6 JUNE 1995

TEST ID: 157LR4.DAT

\section{RACK STORAGE TEST DRUM SURFACE TC'S}

尽

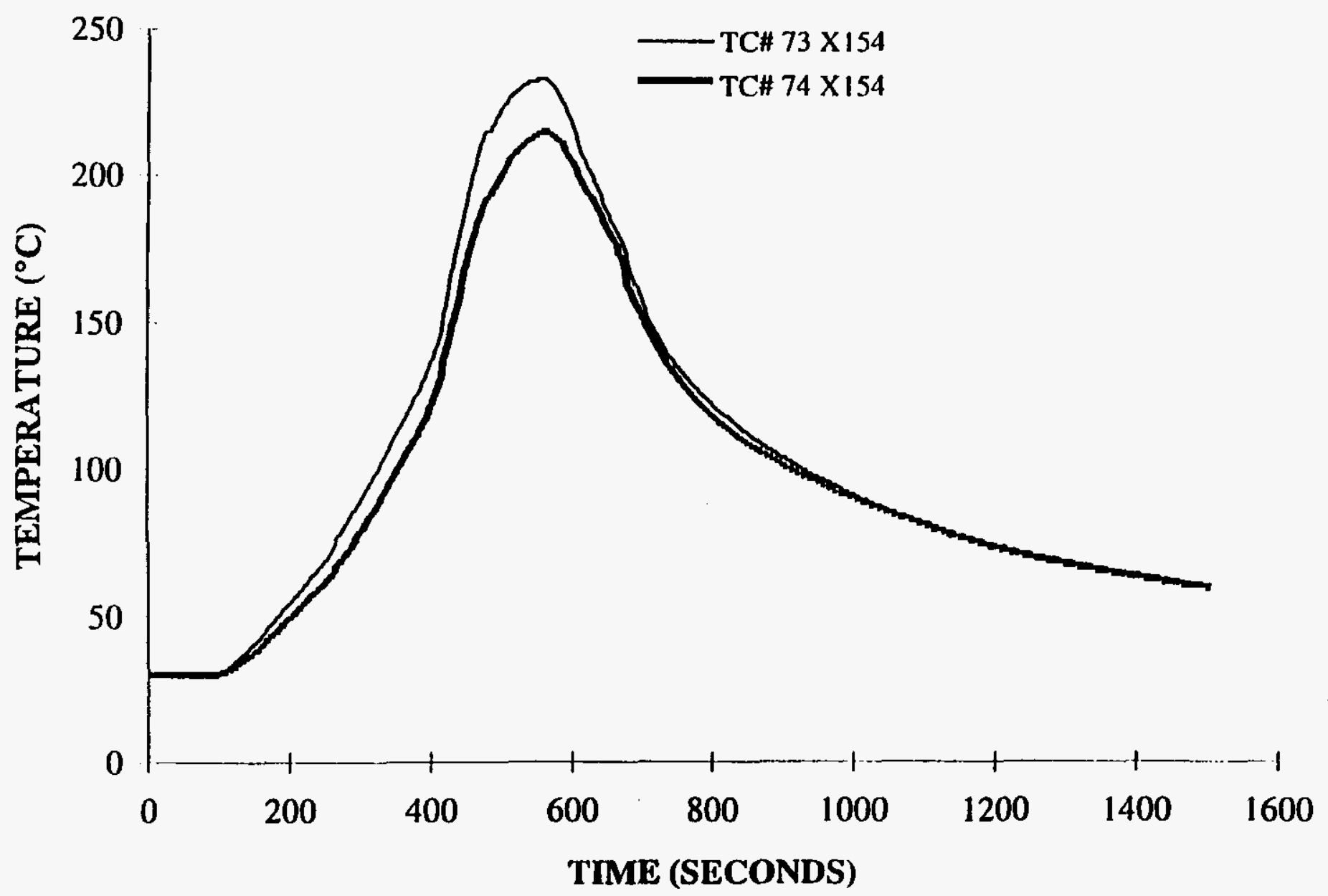


CLIENT: LATA

PROJECT NO.: SWRI 01-7106

DATE: 6 JUNE 1995

TEST ID: 157LR4.DAT

RACK STORAGE TEST

\section{DRUM SURFACE TC'S}

?

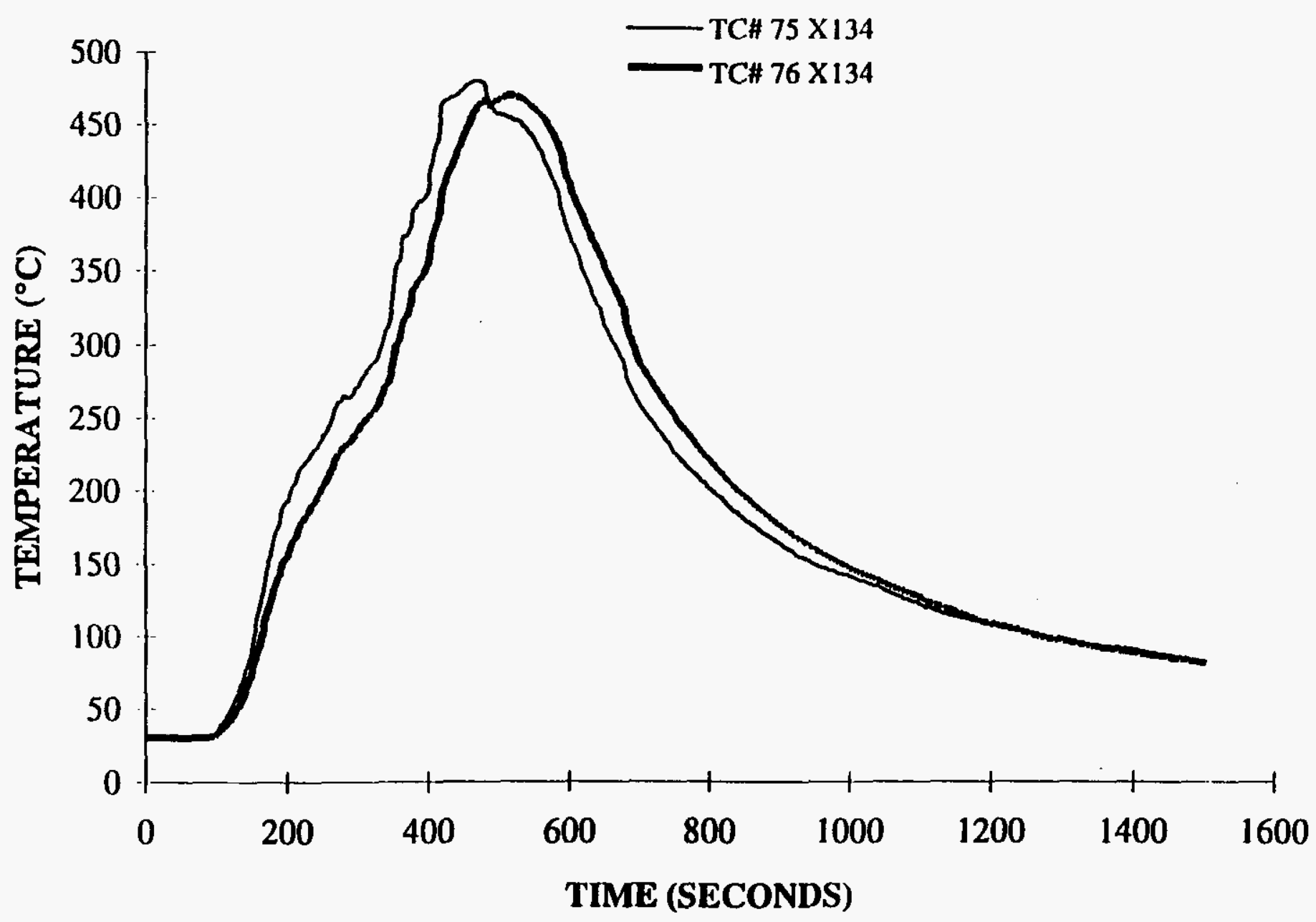

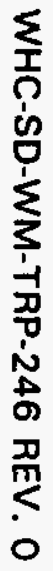

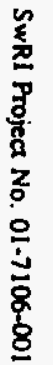

TIME (SECONDS) 
CLIENT: LATA

PROJECT NO.: SwRI 01-7106

DATE: 6 JUNE 1995

TEST ID: 157LR4.DAT

\section{RACK STORAGE TEST \\ DRUM SURFACE TC'S}

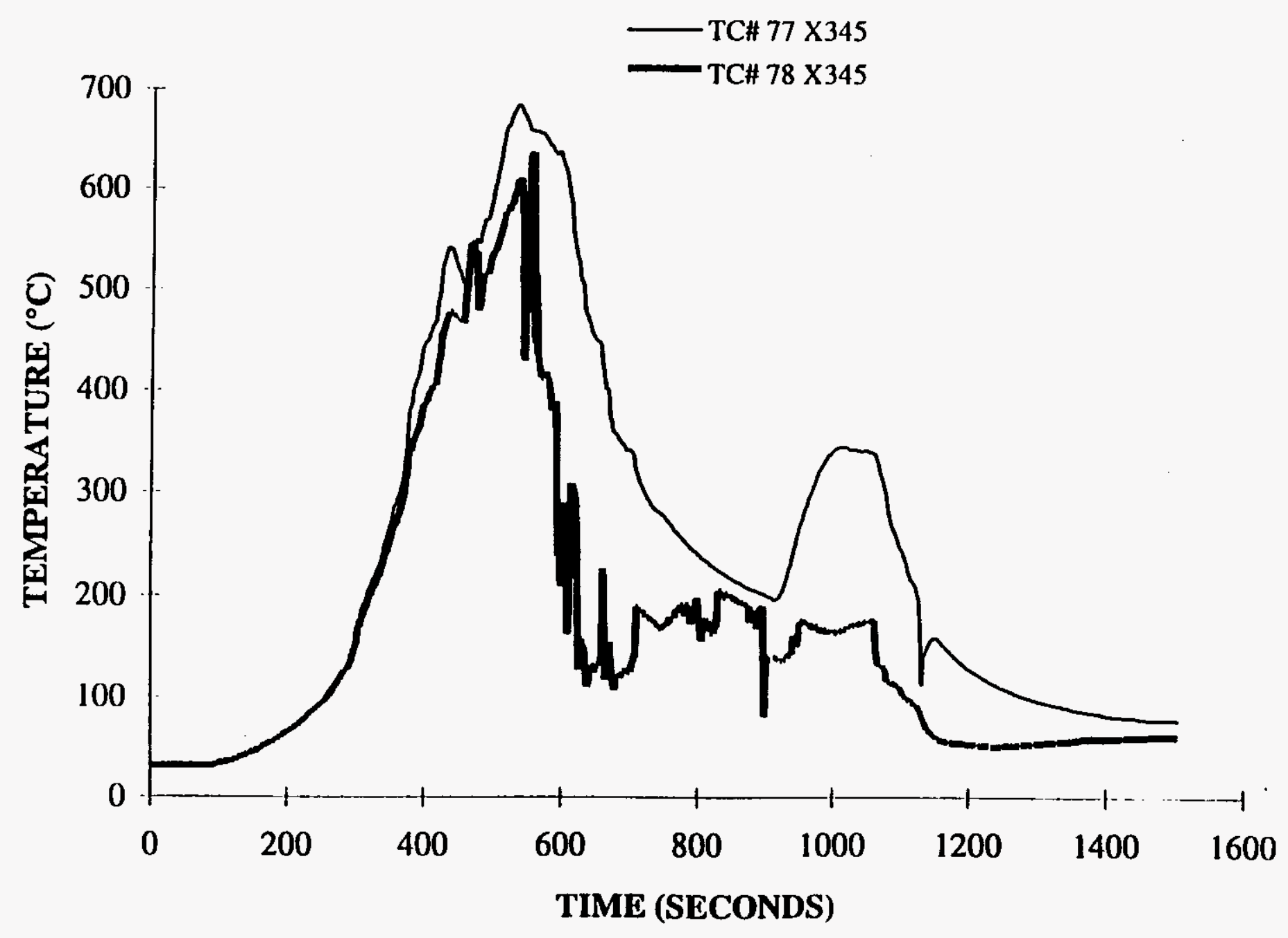


WHC-SD-WM-TRP-246 REV. 0

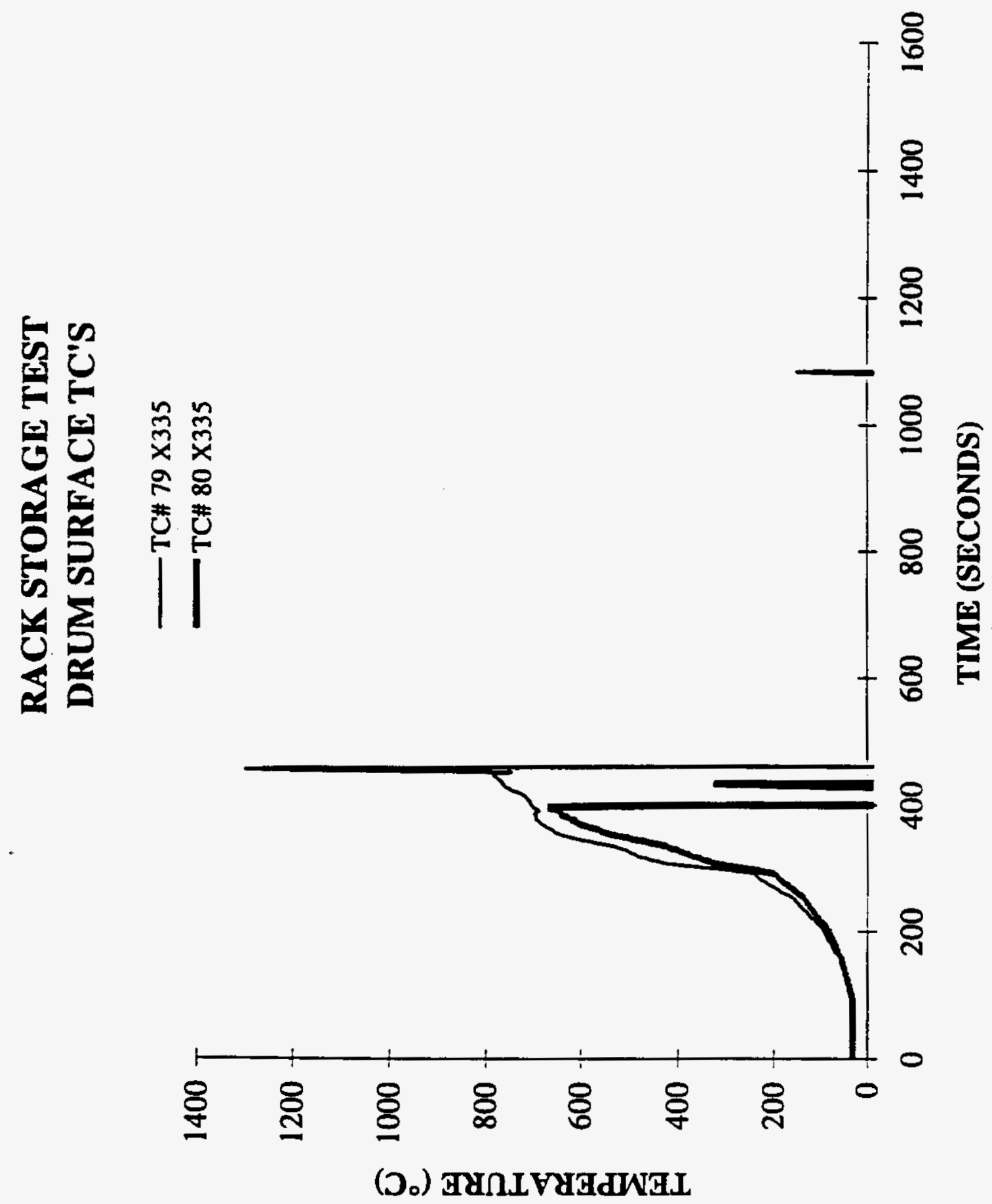


CLIENT: LATA

PROJECT NO.: SwRI 01-7106

DATE: 6 JUNE 1995

TEST ID: 157LR4.DAT

\section{RACK STORAGE TEST \\ DRUM SURFACE TC'S}

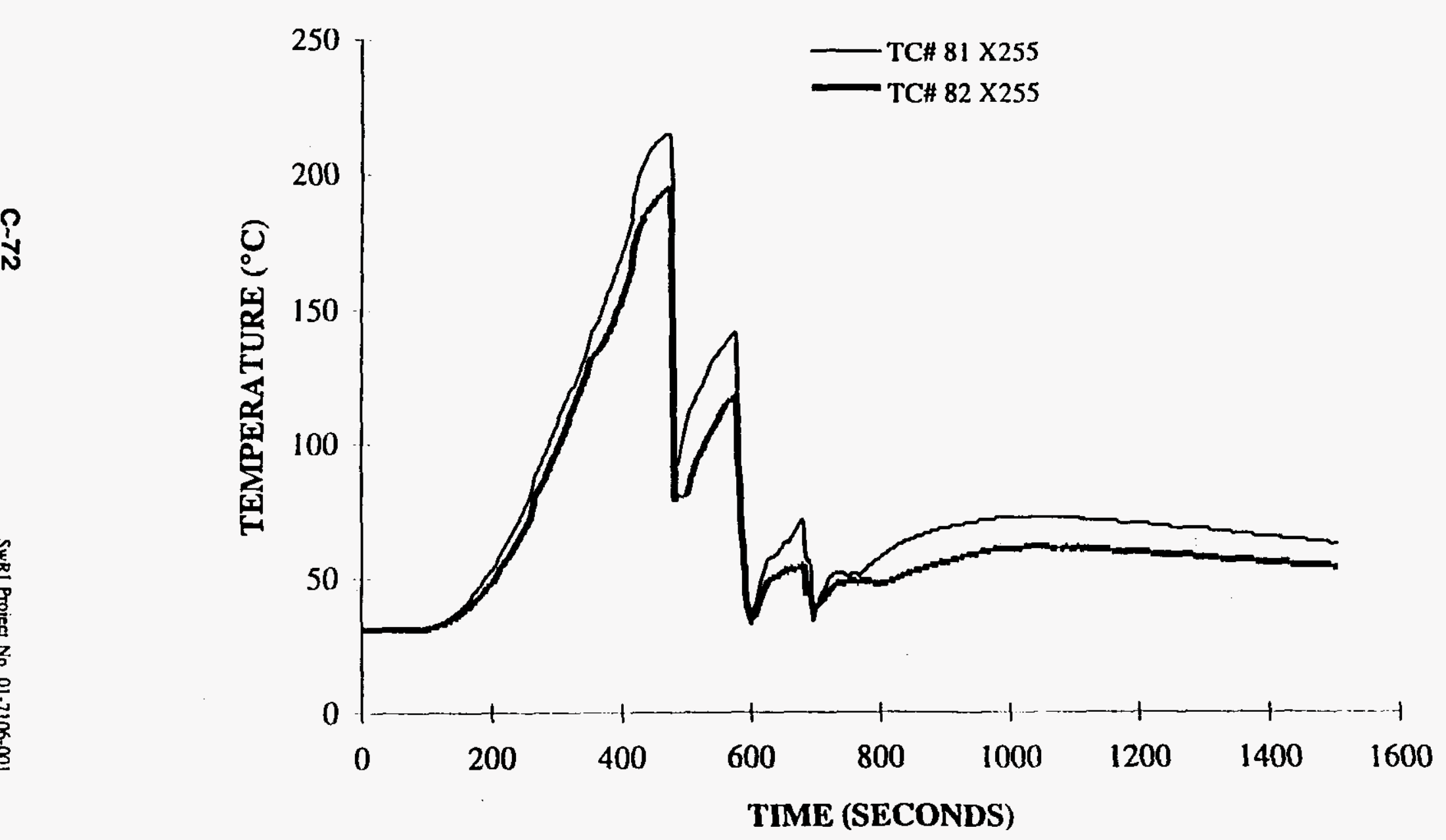


CLIENT: LATA

PROJECT NO.: SwRI 01-7106

DATE: 6 JUNE 1995

TEST ID: 157LR2.DAT

\section{RACK STORAGE TEST}

DRUM SURFACE TC'S

$\frac{\rho}{\omega}$

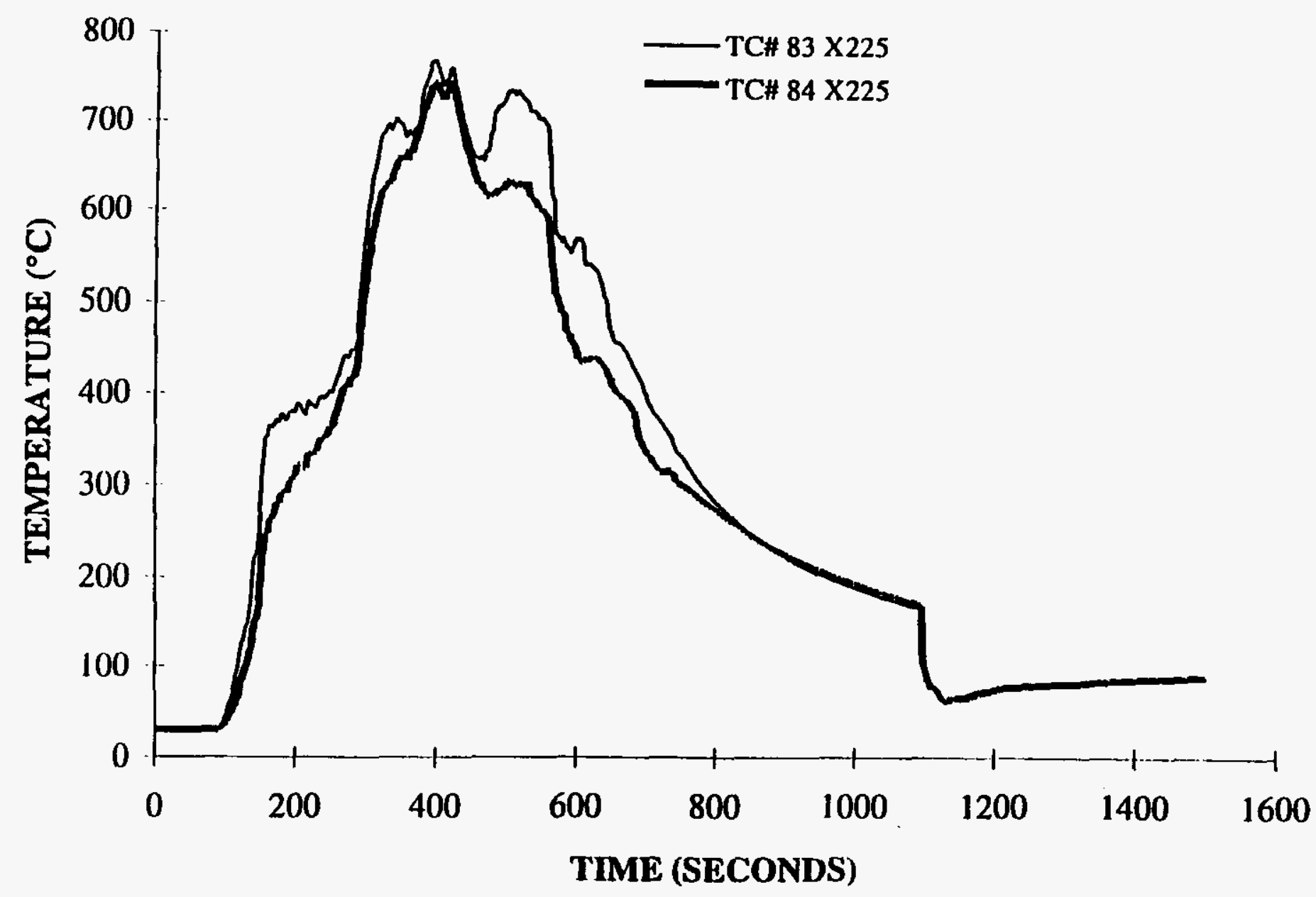


CLIENT: LATA

PROJECT NO.: SWRI 01-7106

DATE: 6 JUNE 1995

TEST ID: 157LR4.DAT

\section{RACK STORAGE TEST}

DRUM SURFACE TC'S

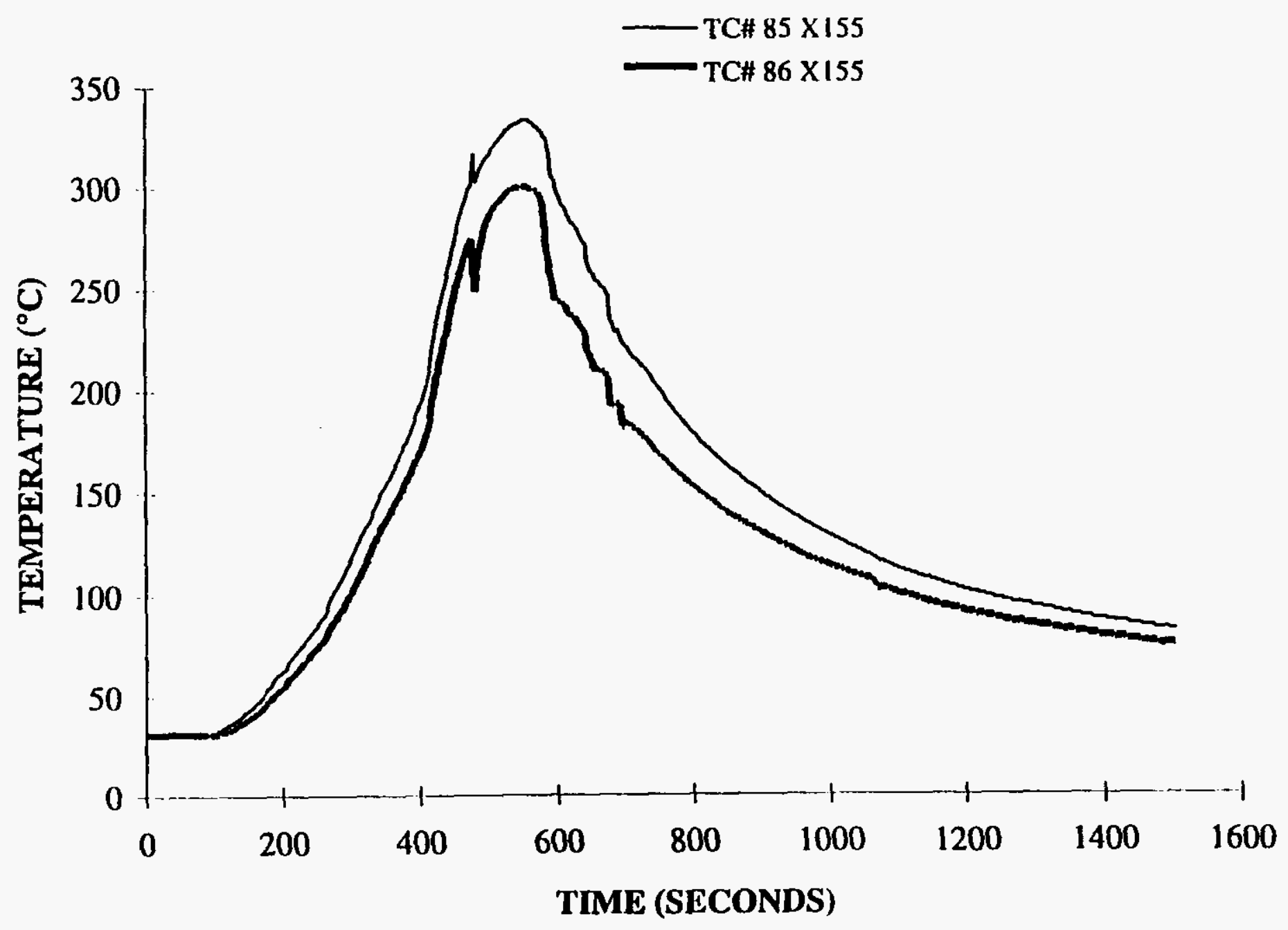


CLIENT: LATA

PROJECT NO.: SwRI 01-7106

DATE: 6 JUNE 1995

TEST ID: I57LR4.DAT

\section{RACK STORAGE TEST}

DRUM SURFACE TC'S

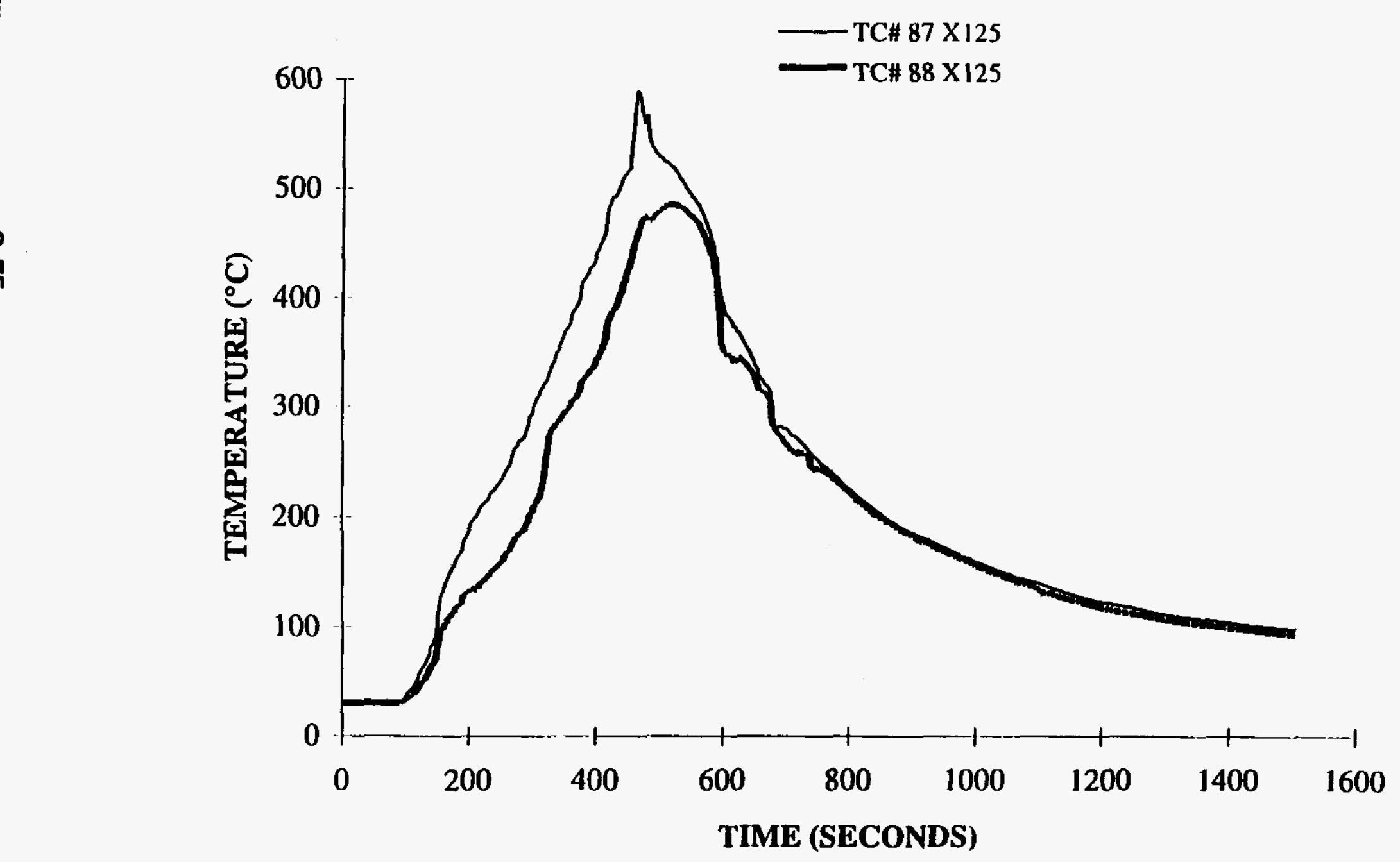

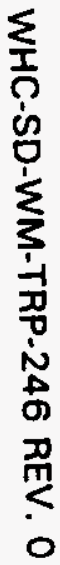

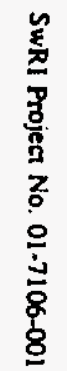

ง 
CLIENT: LATA

PROJECT NO.: SwRI 01-7106

DATE: 6 JUNE 1995

TEST ID: 157LR4.DAT

\section{RACK STORAGE TEST DRUM SURFACE TC'S}

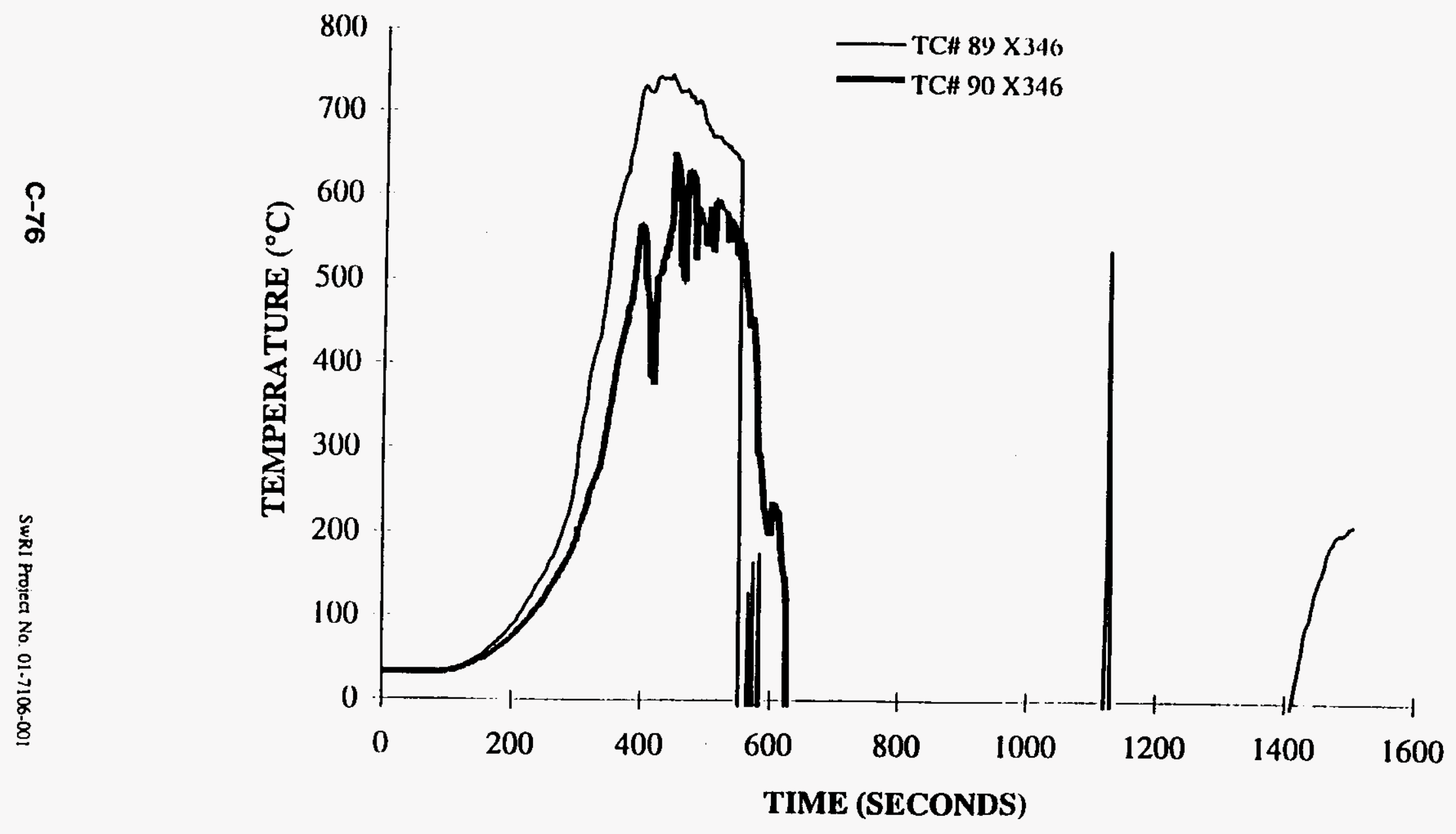


CLIENT: LATA

PROJECT NO.: SwRI 01-7106

DATE: 6 JUNE 1995

TEST ID: 157LR4.DAT

\section{RACK STORAGE TEST DRUM SURFACE TC'S}

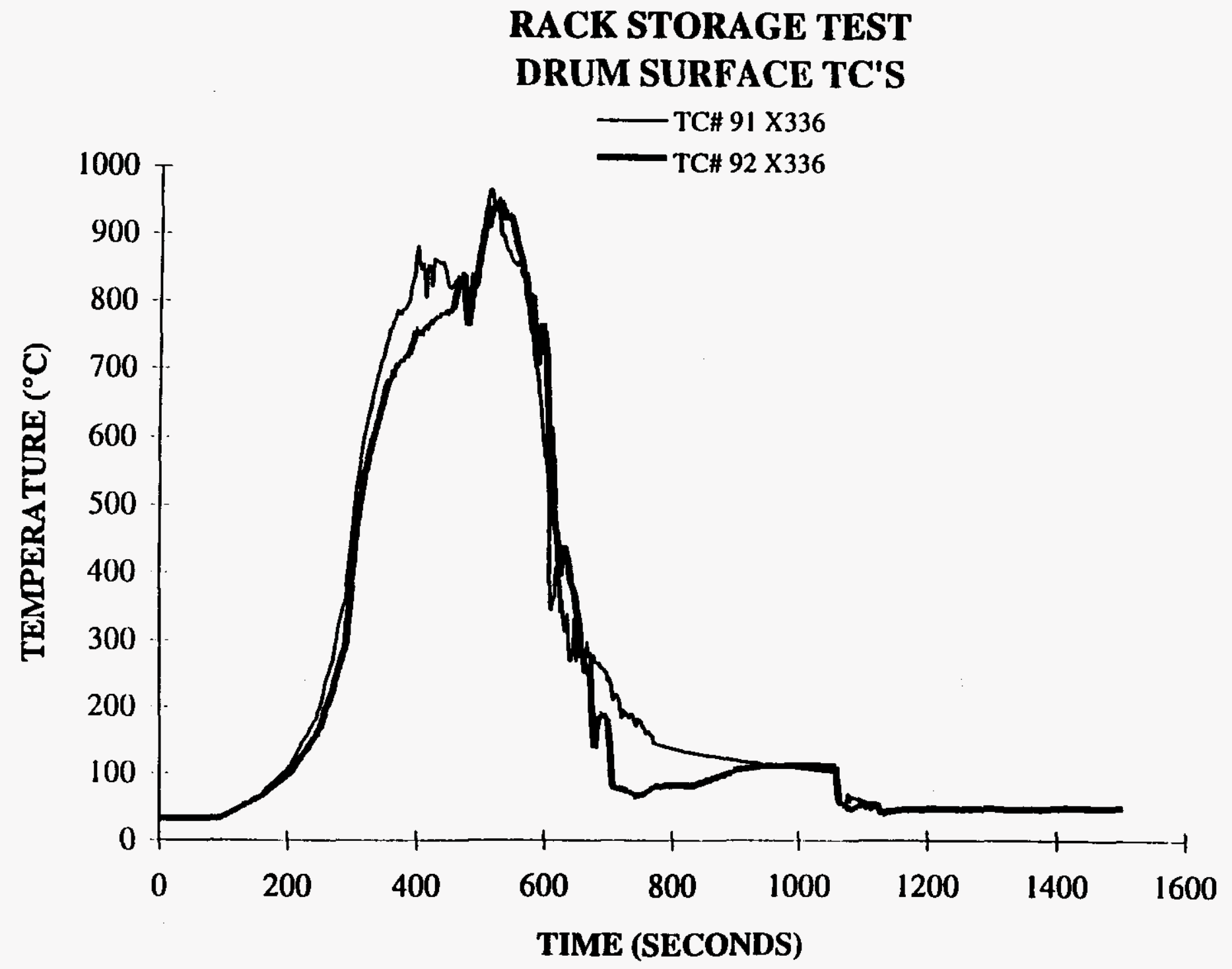


CLIENT: LATA

PROJECT NO.: SwRI 0I-7106

DATE: 6 JUNE 1995

TEST ID: 157LR4.DAT

\section{RACK STORAGE TEST DRUM SURFACE TC'S}

$\underset{\infty}{\stackrel{8}{0}}$

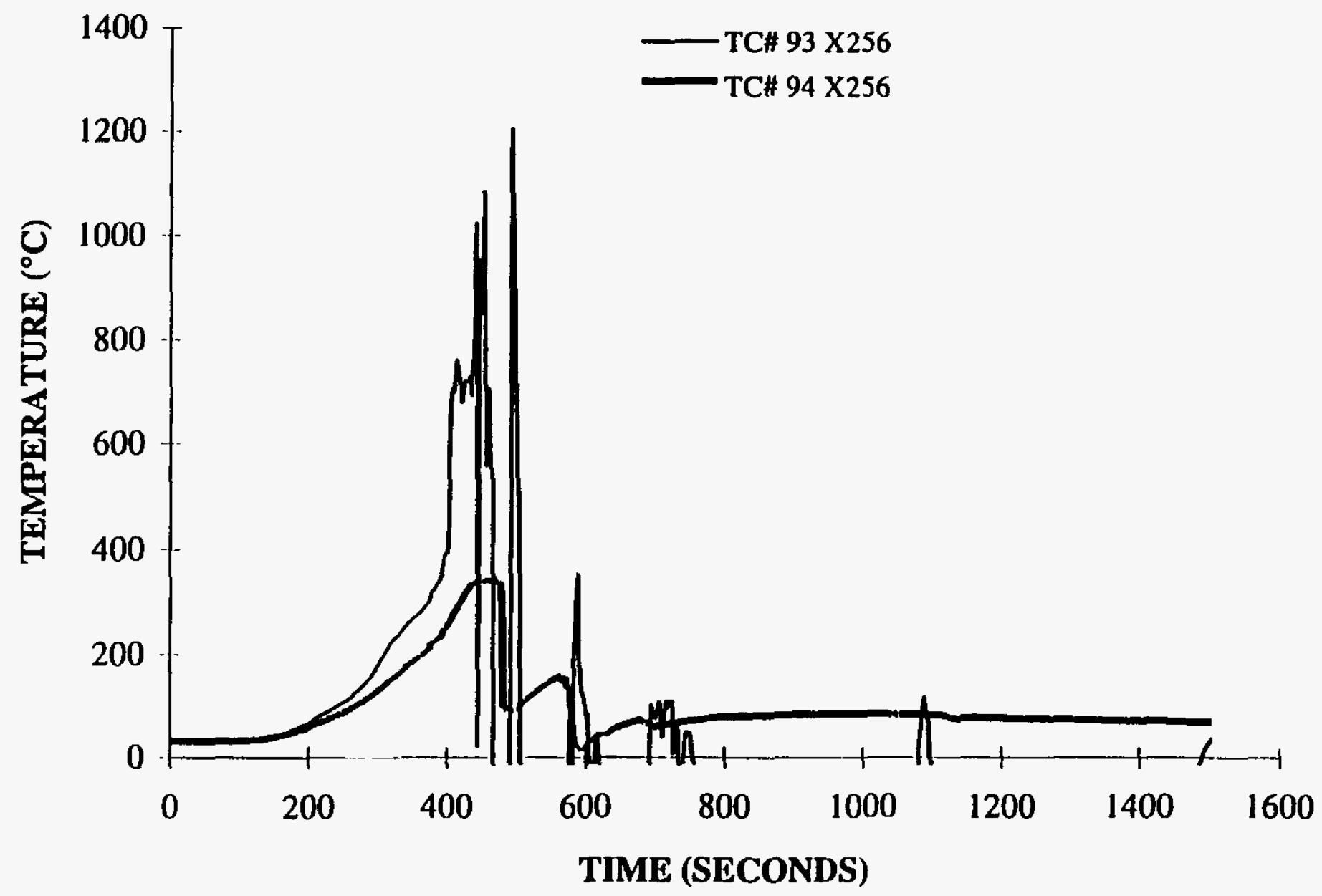


Blank 
CLIENT: LATA

PROJECT NO.: SwRI 01-7106

DATE: 6 JUNE 1995

TEST ID: 157LR2.DAT

RACK STORAGE TEST DRUM SURFACE TC'S

$\stackrel{?}{0}$

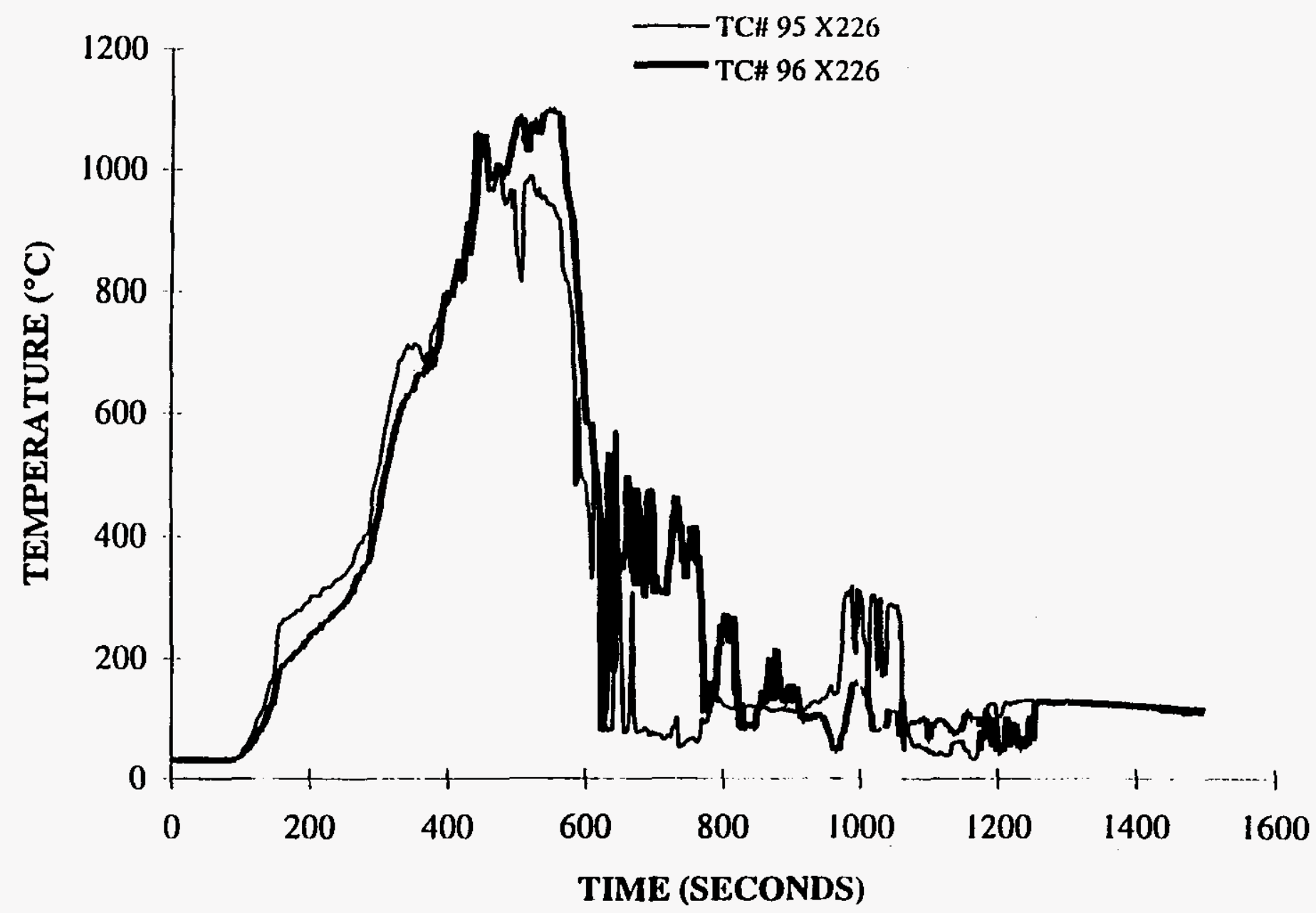

TIME (SECONDS) 
CLIENT: LATA

PROJECT NO.: SwRI 01-7106

DATE: 6 JUNE 1995

TEST ID: 157LR4.DAT

\section{RACK STORAGE TEST \\ DRUM SURFACE TC'S}

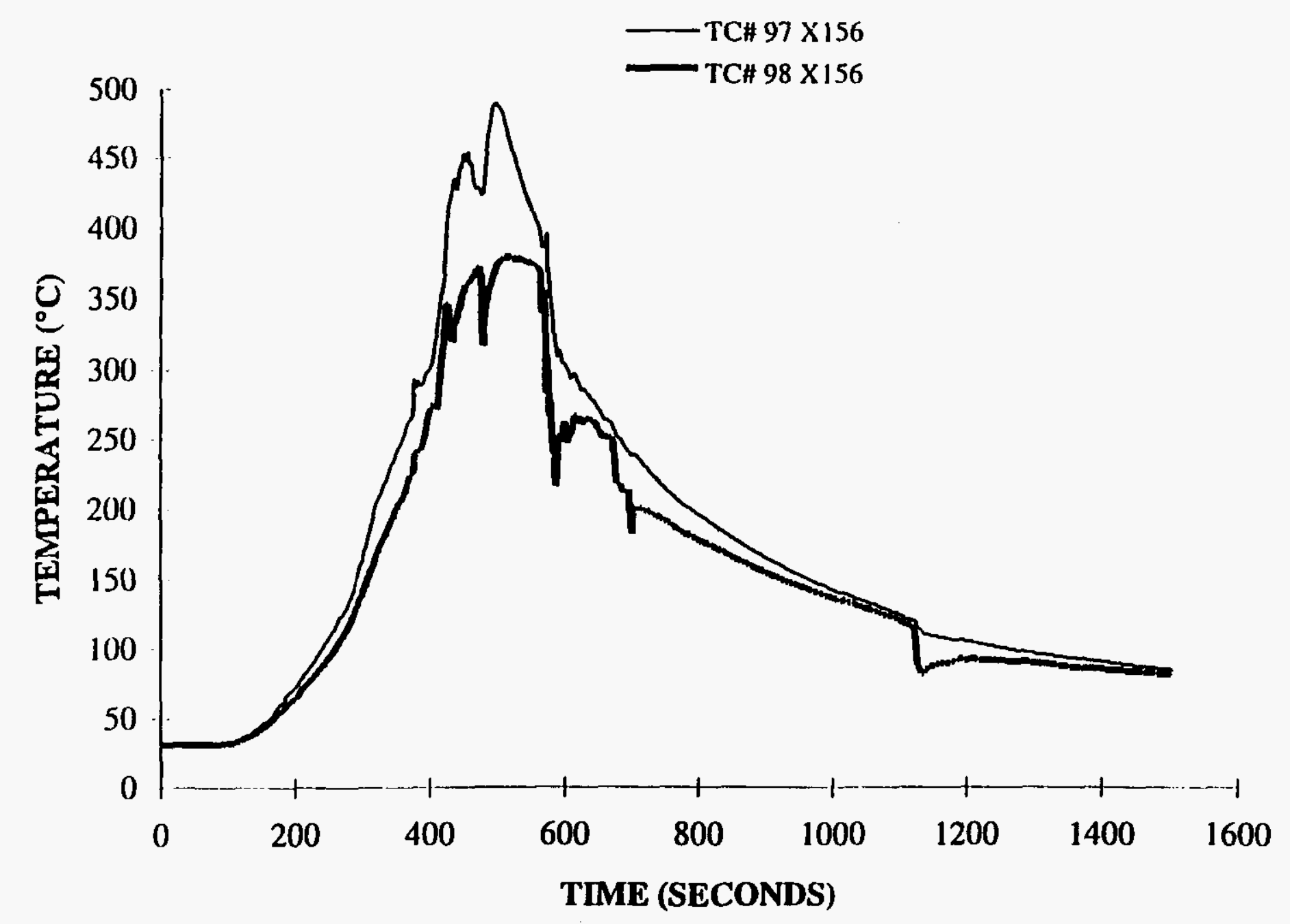

@̊

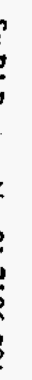


CLIENT: LATA

PROJECT NO.: SwRI 01-7106

DATE: 6 JUNE 1995

TEST ID: 157LR4.DAT

\section{RACK STORAGE TEST \\ DRUM SURFACE TC'S}

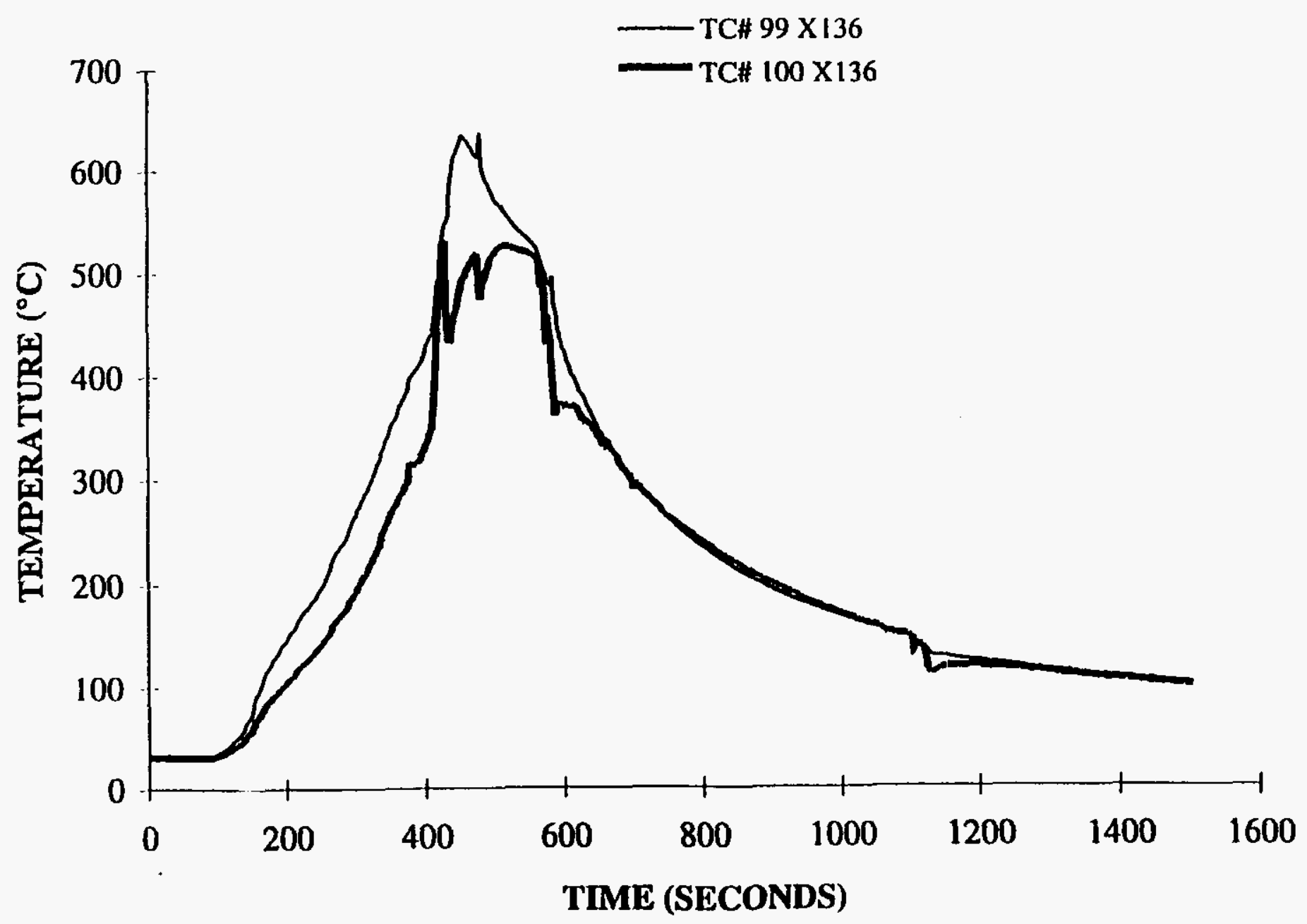


CLIENT: LATA

PROJECT NO.: SWRI 01-7106

DATE: 6 JUNE 1995

TEST ID: 157LR5.DAT

\section{RACK STORAGE TEST \\ DRUM SURFACE TC'S}

-TC\# $101 \times 221$

TC\# 102 X221

@

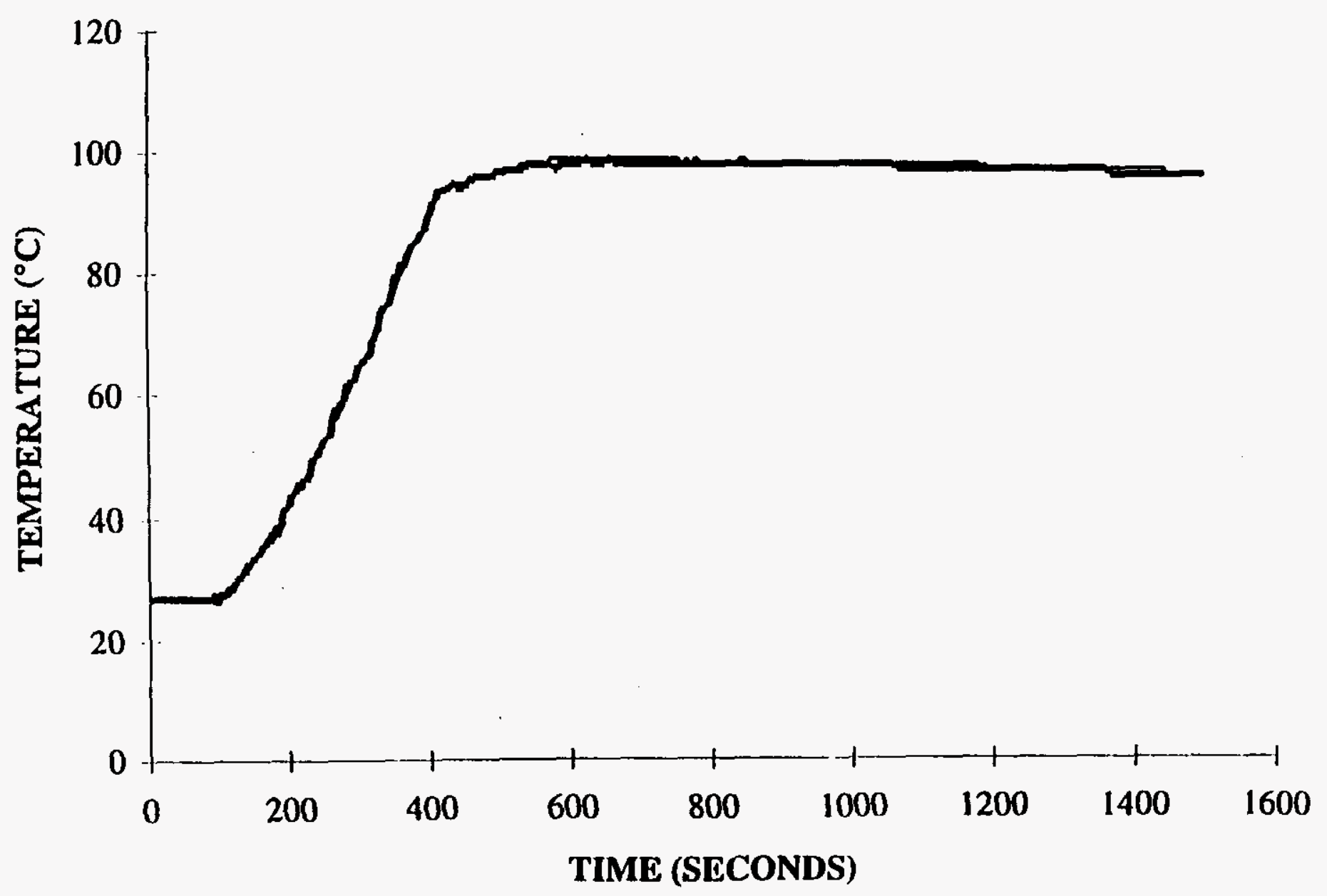


CLIENT: LATA

PROJECT NO.: SwRI 01-7106

DATE: 6 JUNE 1995

TEST ID: 157LR4.DAT

\section{RACK STORAGE TEST \\ DRUM SURFACE TC'S}

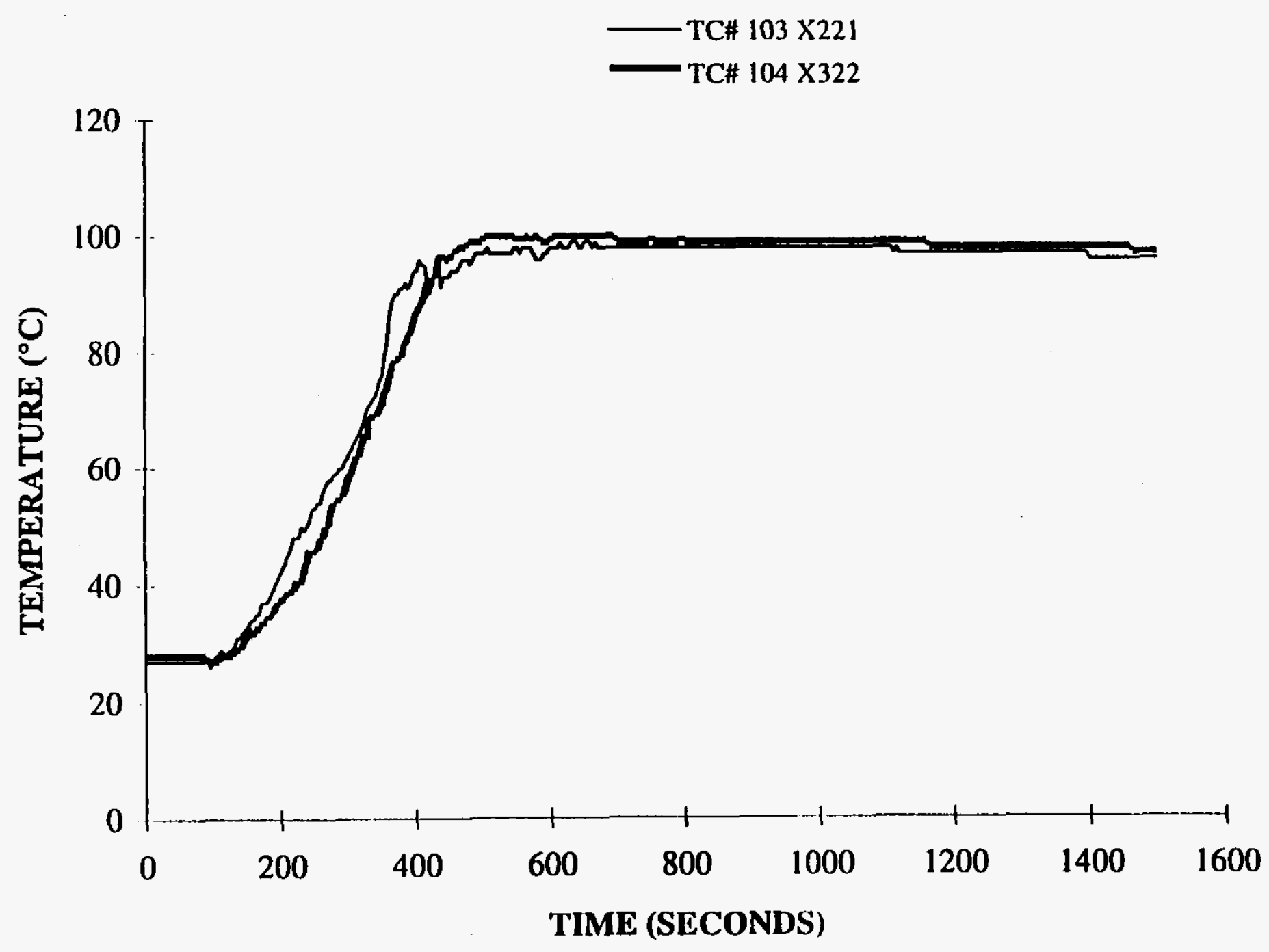


CLIENT: LATA

PROJECT NO.: SwRI 01-7106

DATE: 6 JUNE 1995

TEST ID: 157LR5.DAT

RACK STORAGE TEST

\section{DRUM SURFACE TC'S}

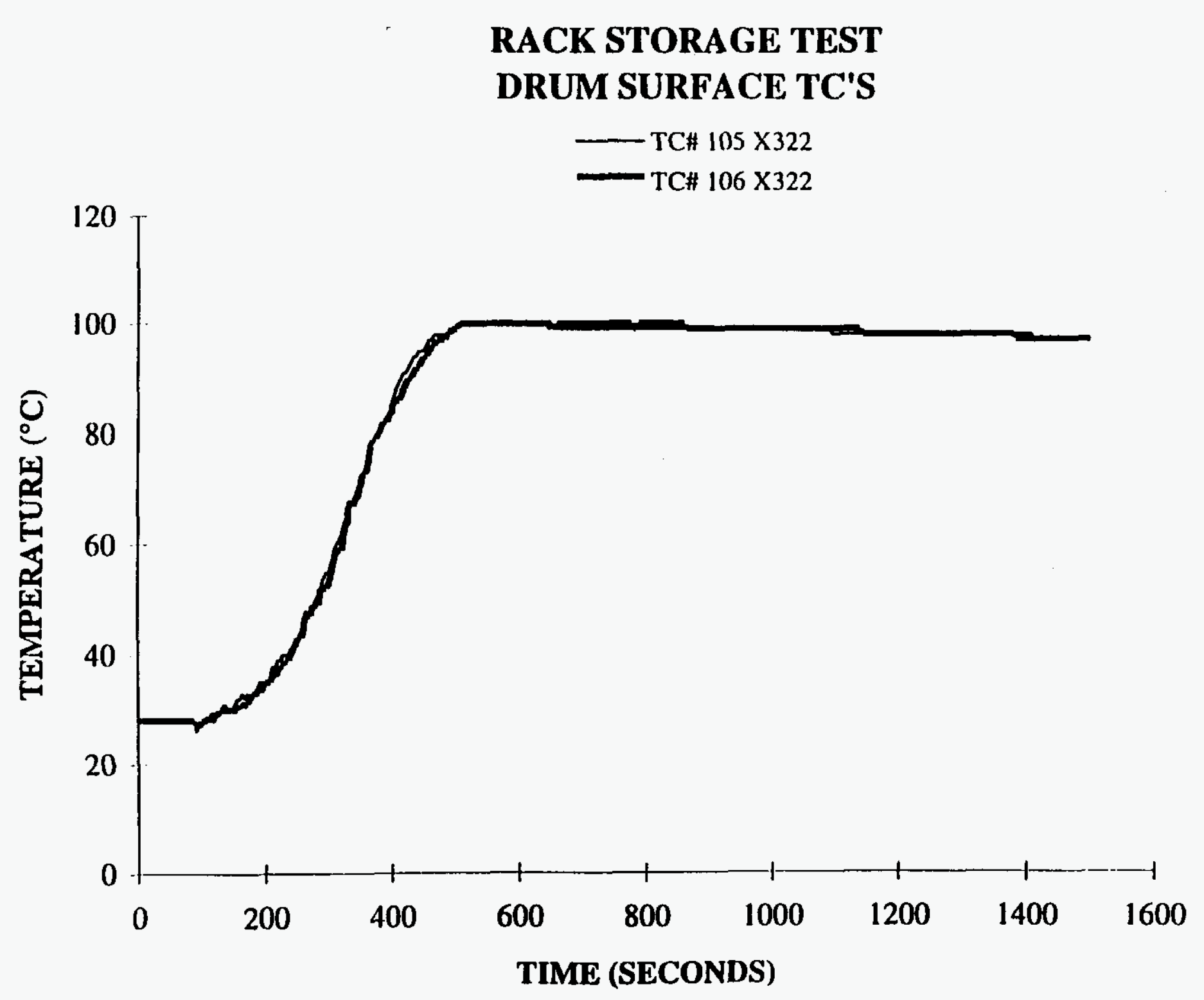


CLIENT: LATA

PROJECT NO.: SwRI 01-7106

DATE: 6 JUNE 1995

TEST ID: 157LR5.DAT

\section{RACK STORAGE TEST} DRUM SURFACE TC'S

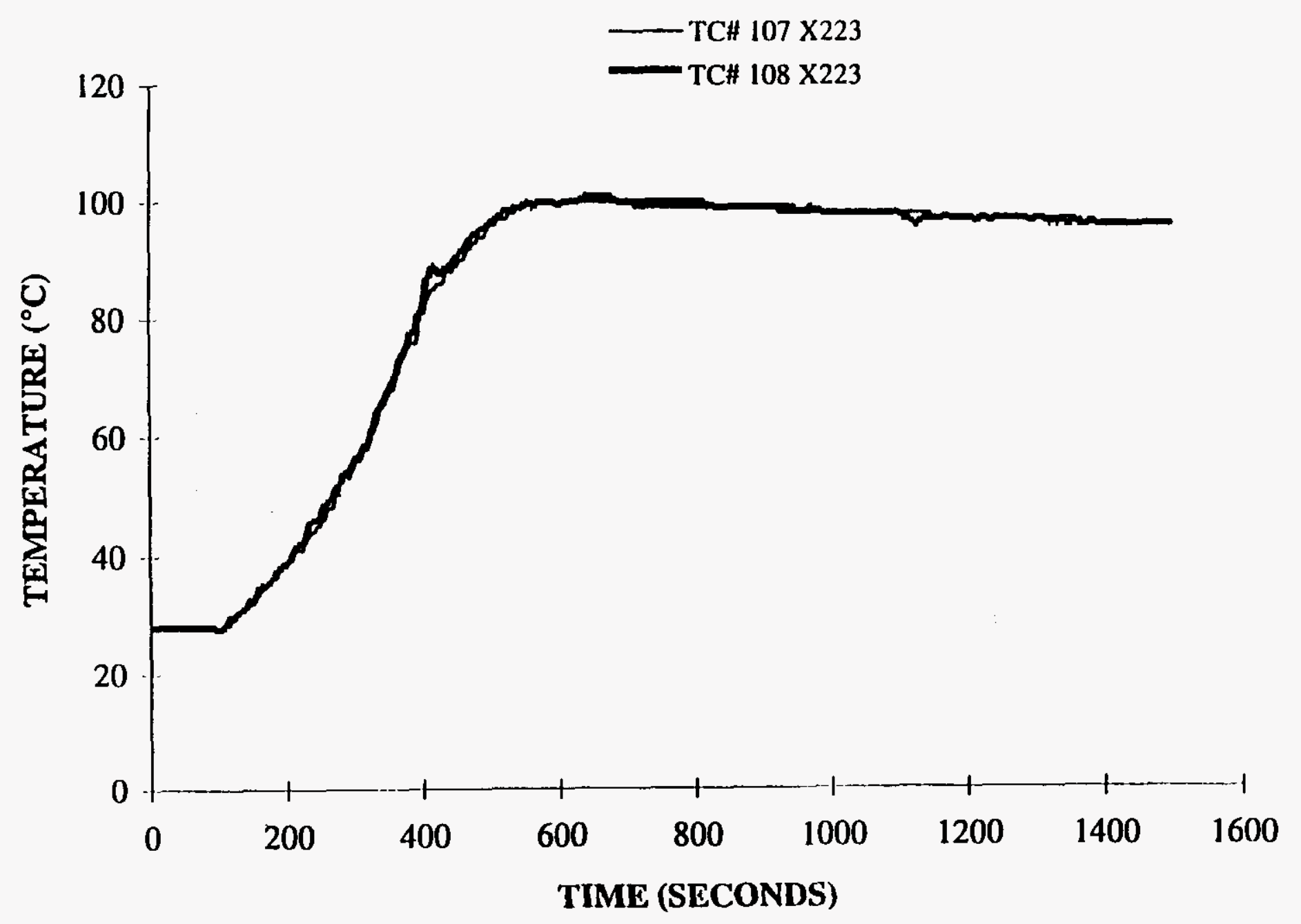

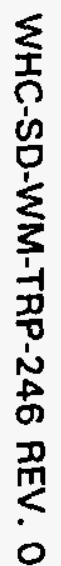


CLIENT: LATA

PROJECT NO.: SwRI 01-7106

DATE: 6 JUNE 1995

TEST ID: 157LR5.DAT

\section{RACK STORAGE TEST} DRUM SURFACE TC'S

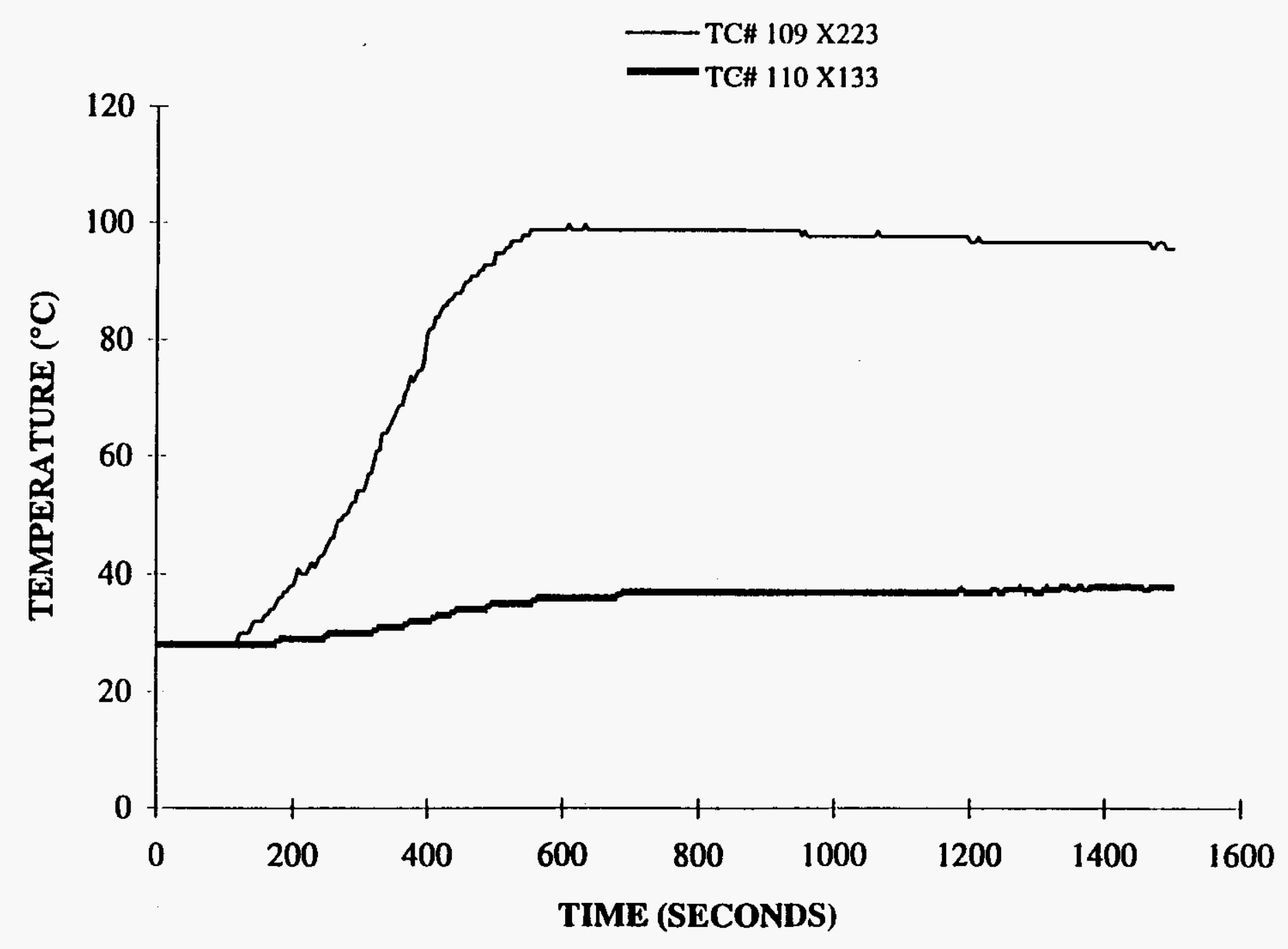


CLIENT: LATA

PROJECT NO.: SwRI 01-7106

DATE: 6 JUNE 1995

TEST ID: 157LR5.DAT

$\stackrel{?}{\infty}$

RACK STORAGE TEST

DRUM SURFACE TC'S

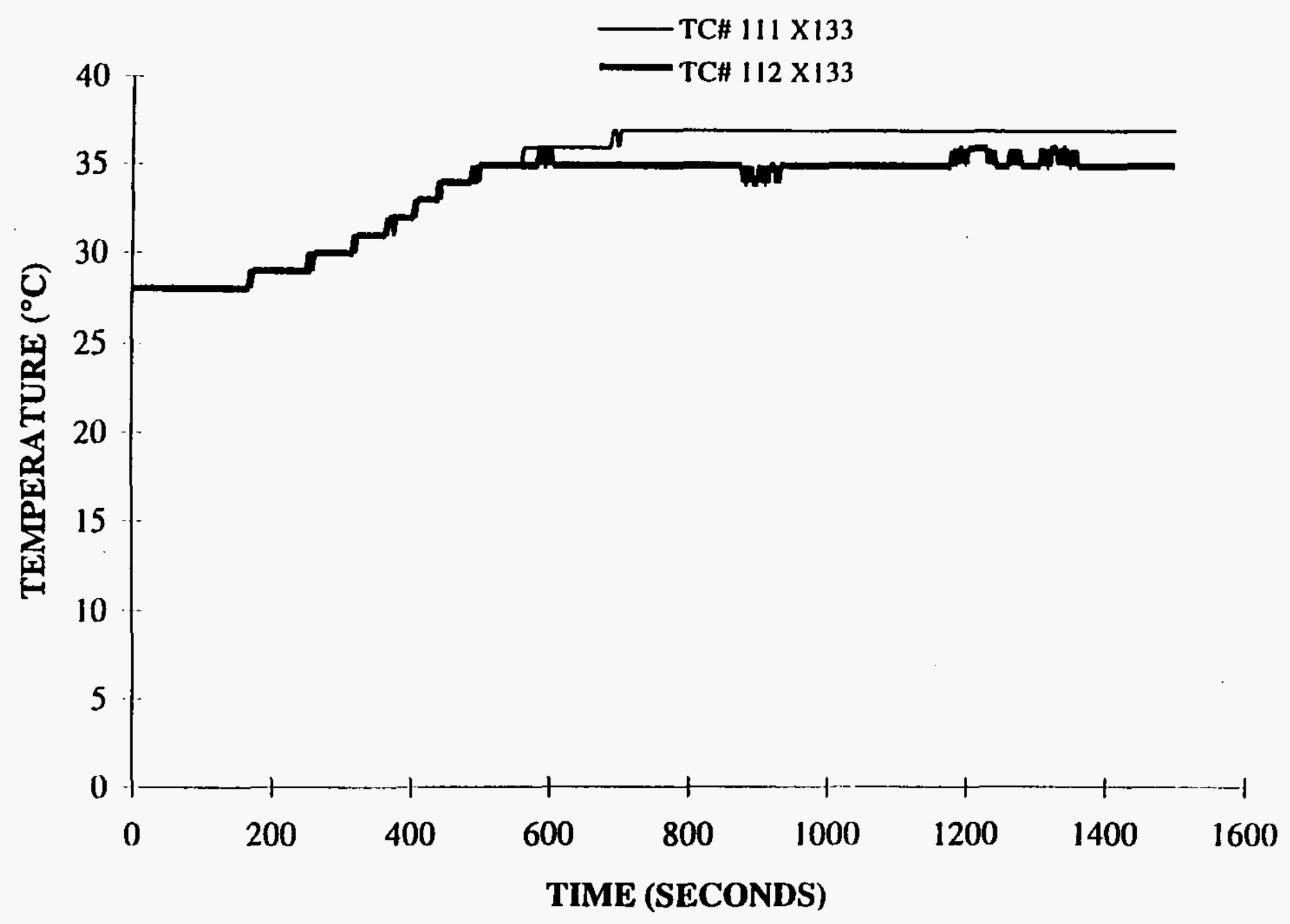

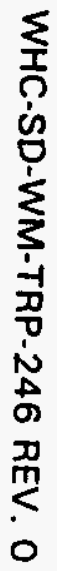




\section{CLIENT: LATA}

PROJECT NO.: SwRI 01-7106

DATE: 6 JUNE 1995

TEST 1D: 157LR7.DAT

RACK STORAGE TEST

TC TREE \#1 (6" OFF RACK No. 1)

?̊

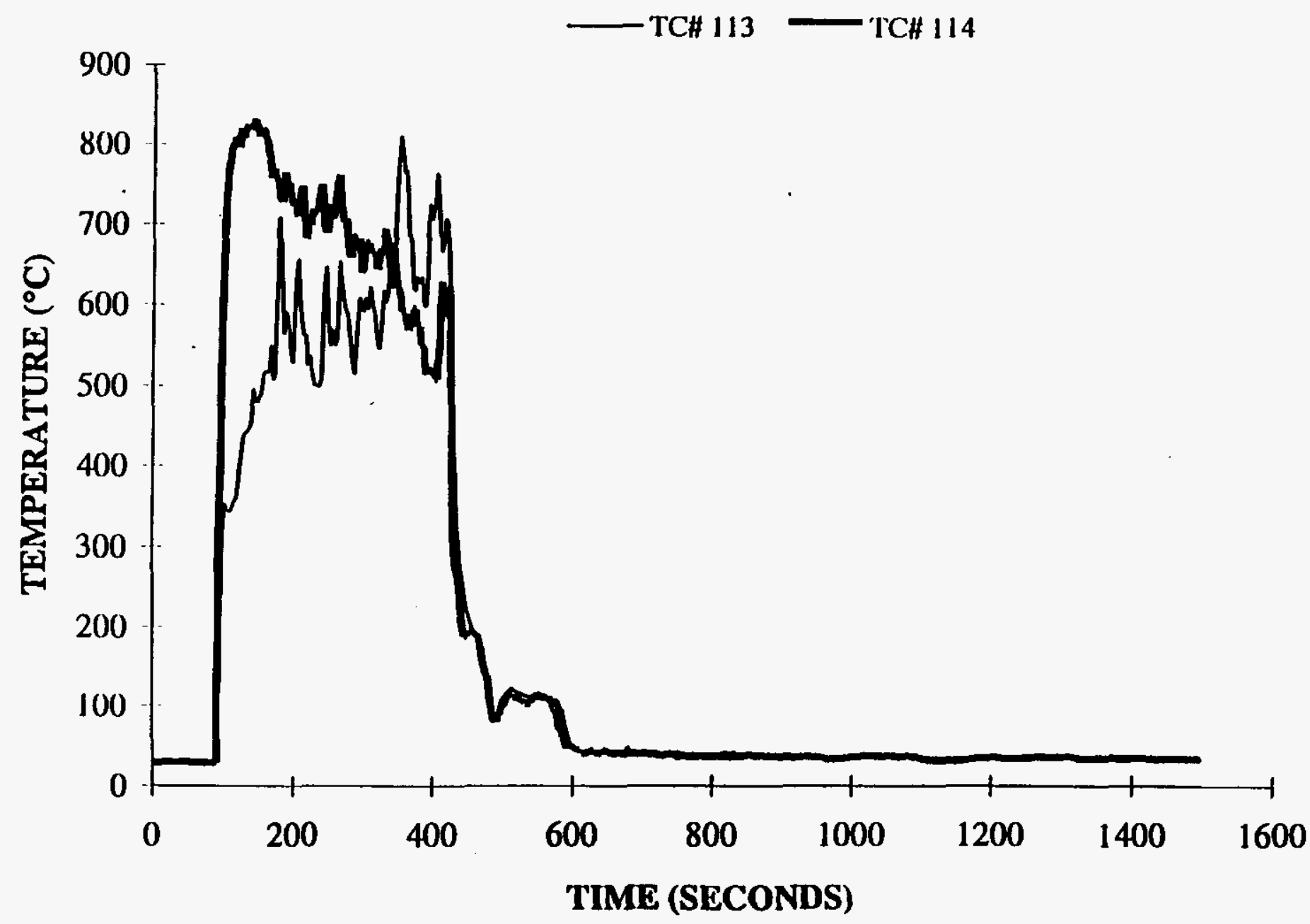


CLIENT: LATA

PROJECT NO.: SWRI 01-7106

DATE: 6 JUNE 1995

TEST ID: 157LR7.DAT

\section{RACK STORAGE TEST \\ TC TREE \#1 (6" OFF RACK No. 1)}

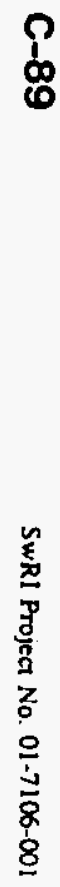

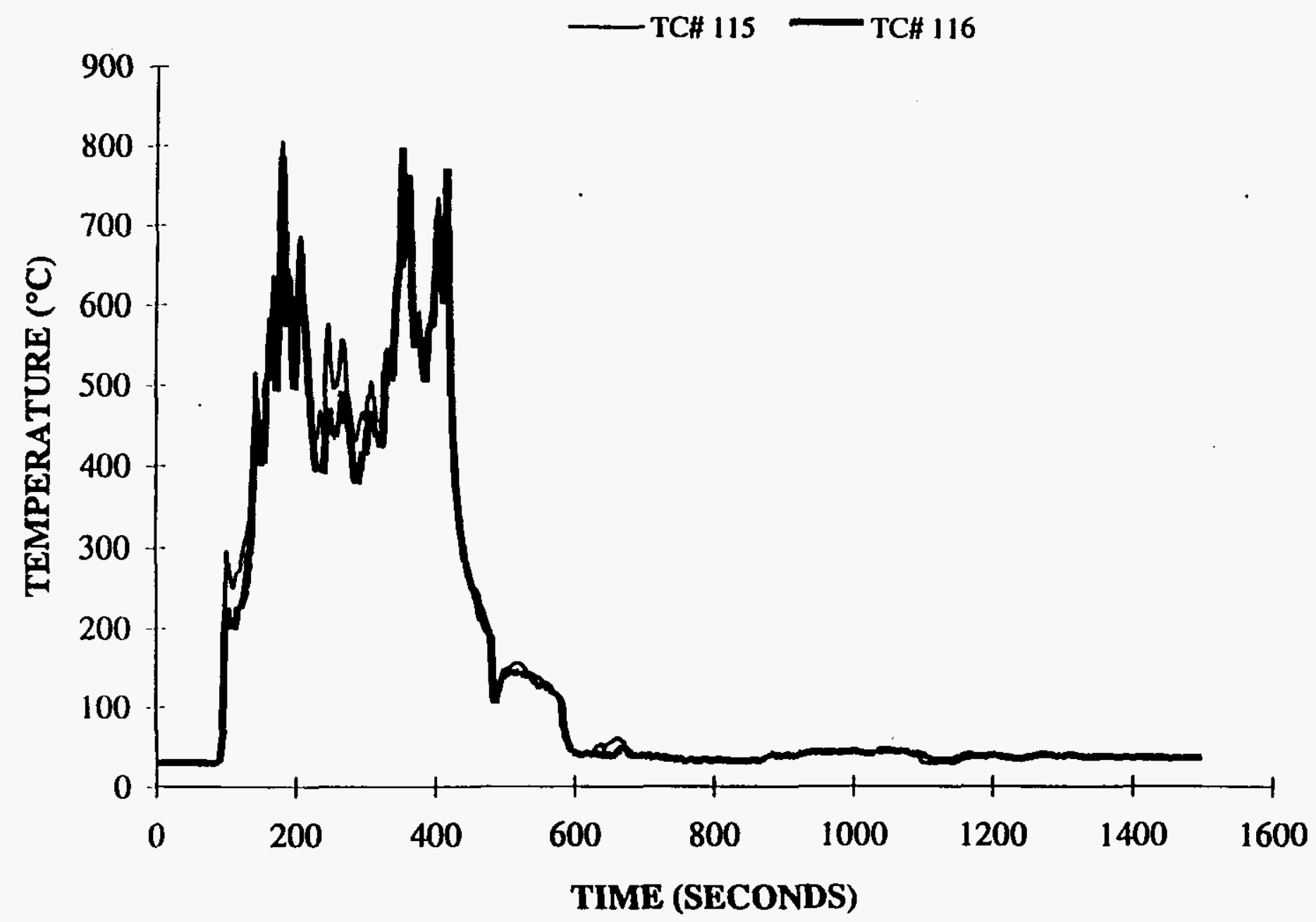


CLIENT: LATA

PROJECT NO.: SwRI 01-7106

DATE: 6 JUNE 1995

TEST ID: 157LR7.DAT

\section{RACK STORAGE TEST \\ TC TREE \#1 (6" OFF RACK No. 1)}

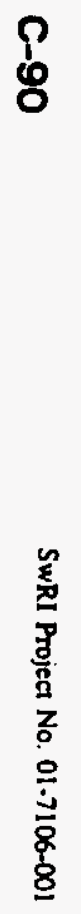

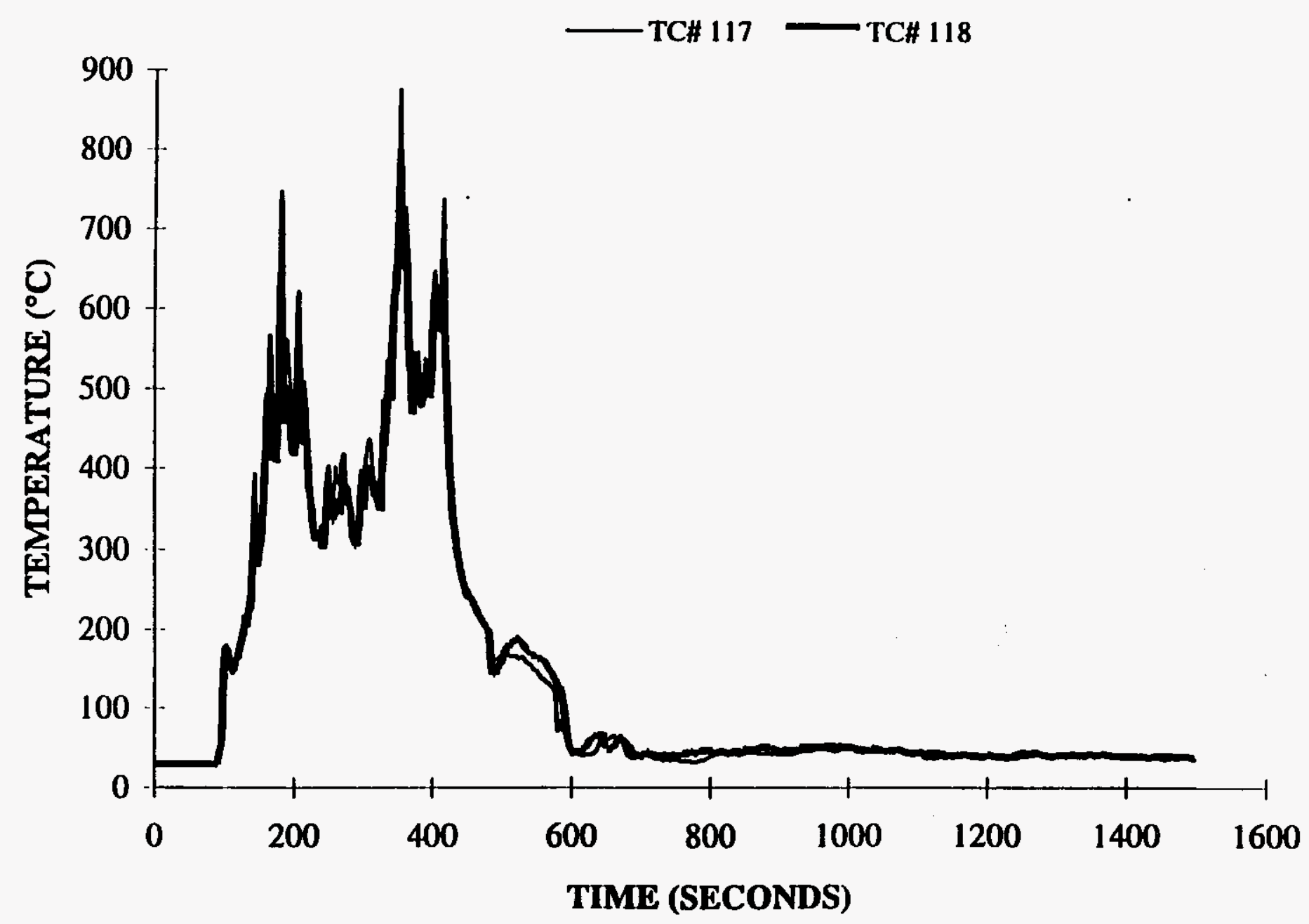


CLIENT: LATA

PROJECT NO.: SwRI 01-7106

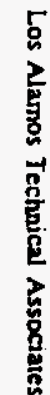

DATE: 6 JUNE 1995

TEST ID: 157LR7.DAT

\section{RACK STORAGE TEST \\ TC TREE \#1 (6" OFF RACK No. 1)}

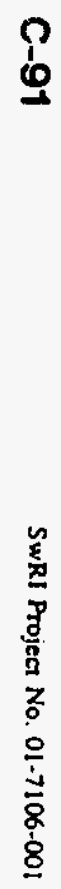

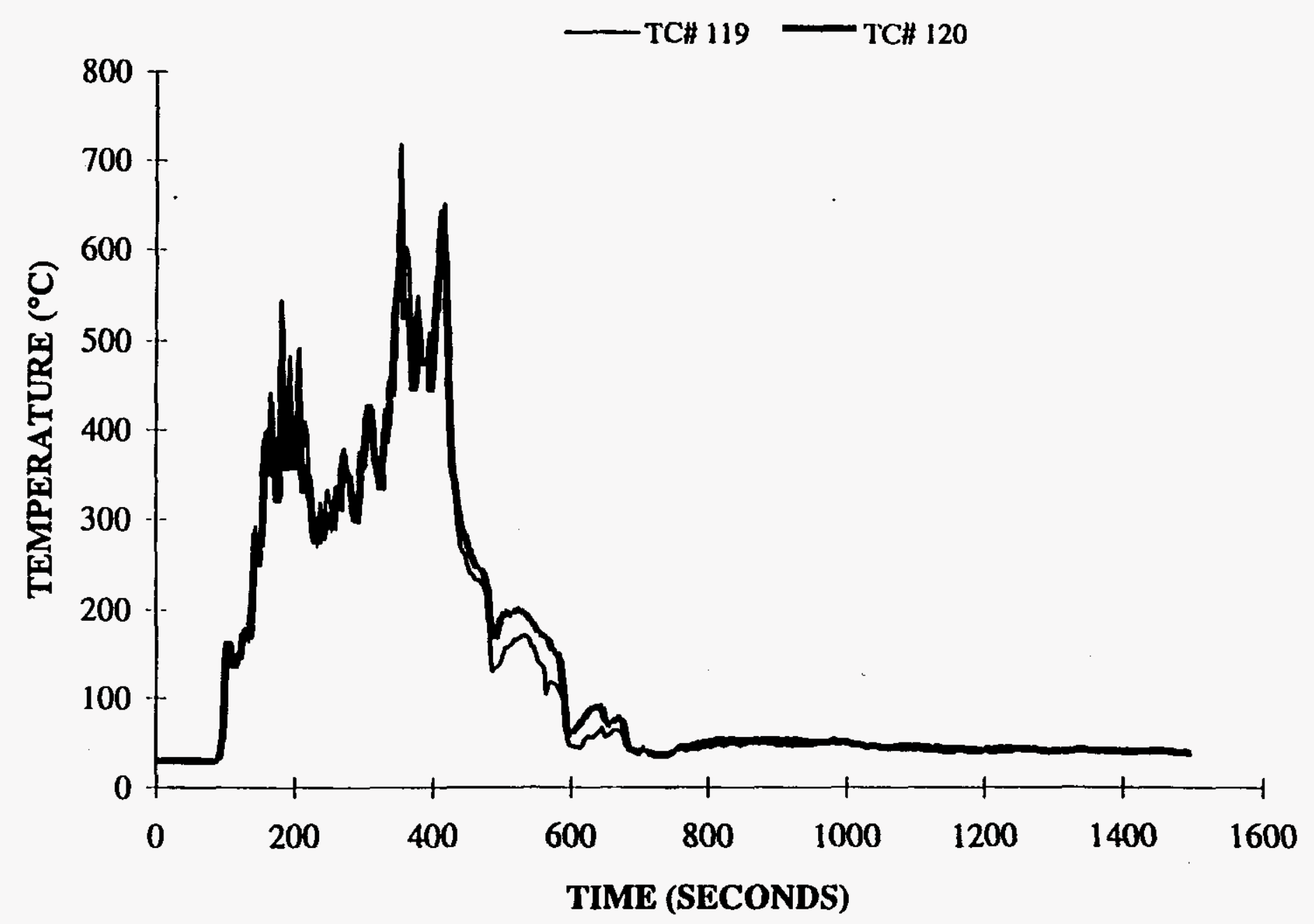
TIME (SECONDS) 
CLIENT: LATA

PROJECT NO.: SwRI 01-7106

DATE: 6 JUNE 1995

TEST ID: 157LR7.DAT

\section{RACK STORAGE TEST \\ TC TREE \#1 (6" OFF RACK No. 1)}

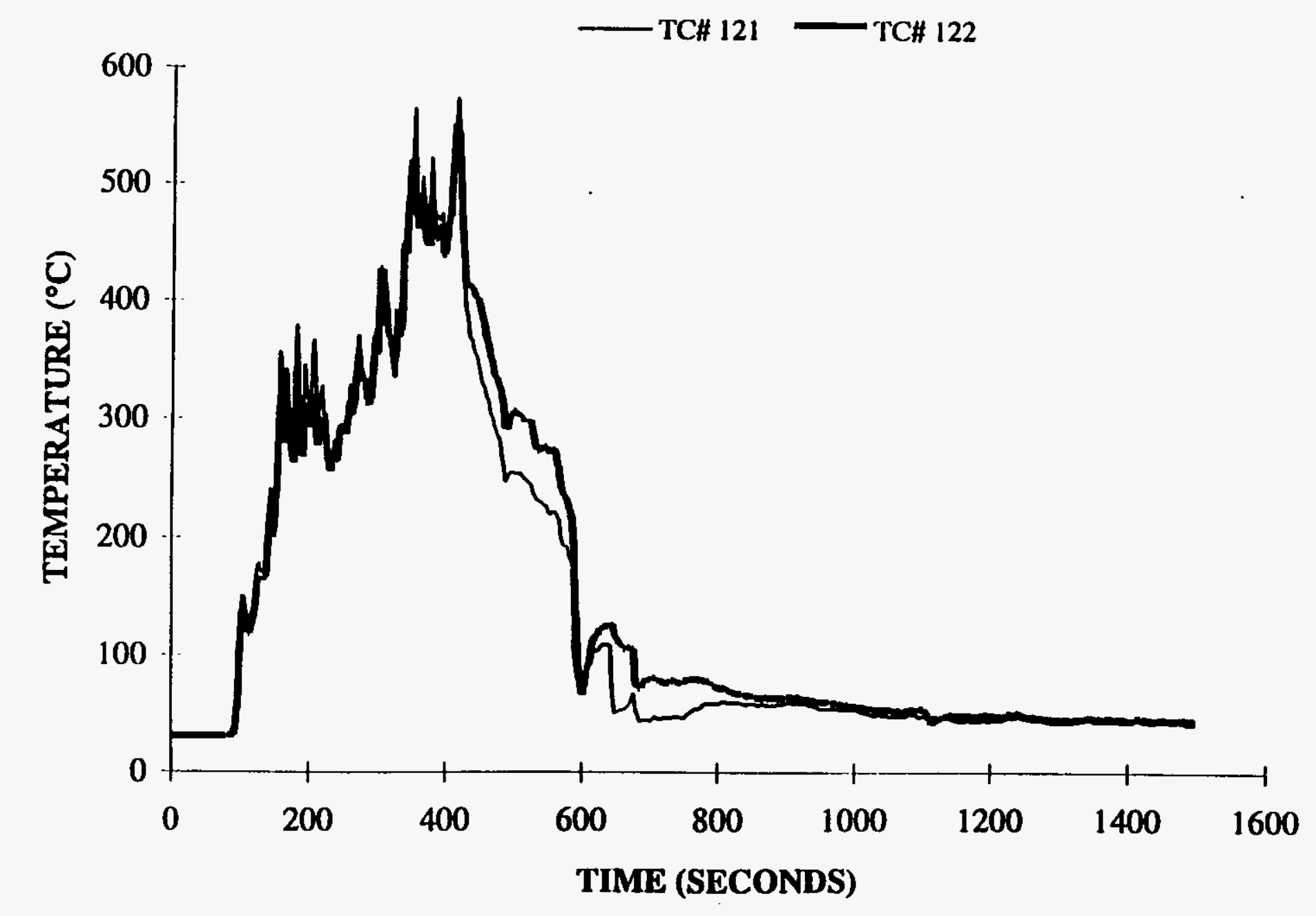


CLIENT: LATA

PROJECT NO.: SwRI 01-7106

DATE: 6 JUNE 1995

TEST ID: 157LR7.DAT

RACK STORAGE TEST

TC TREE \#1 (6" OFF RACK No. 1)

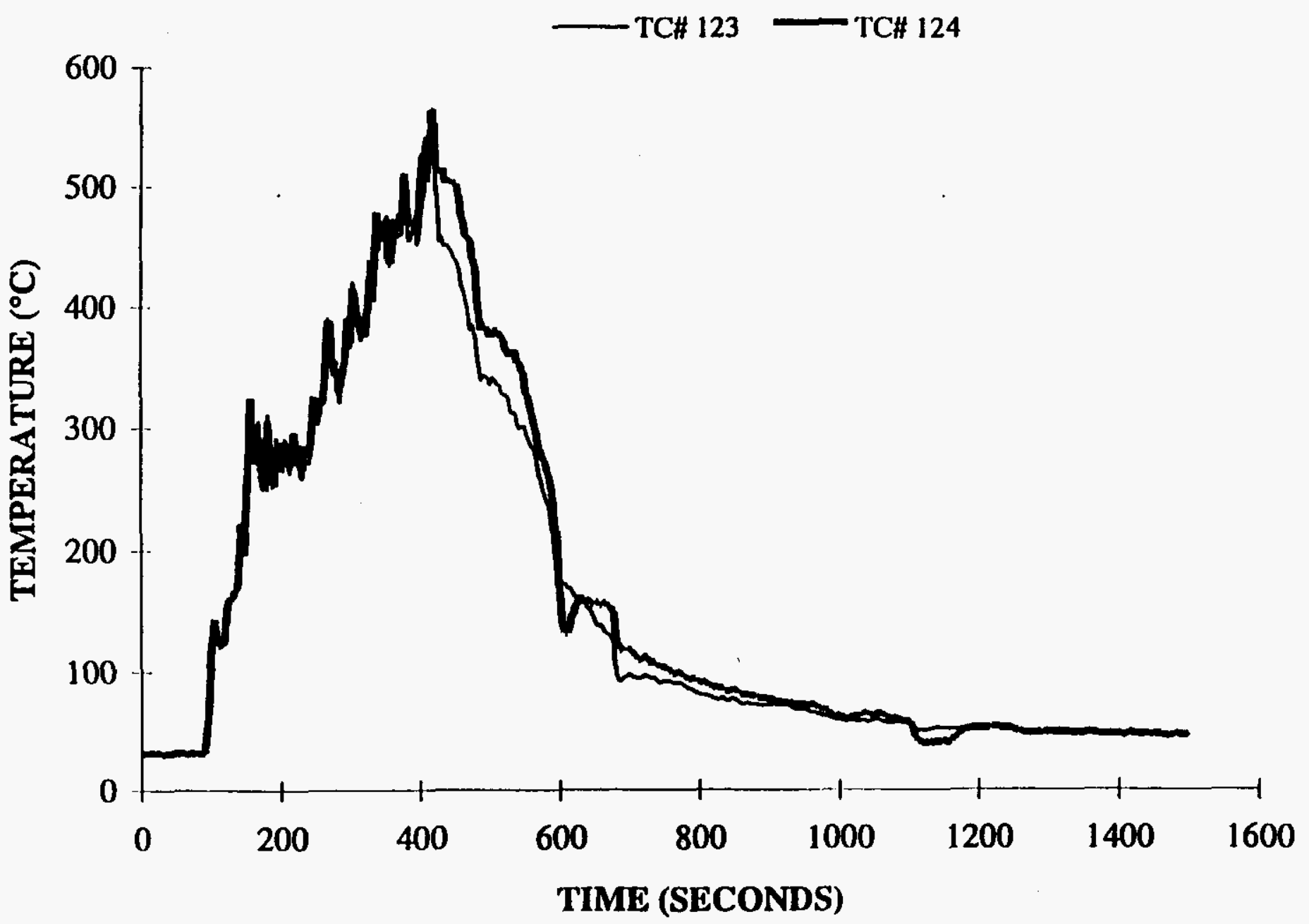

$\stackrel{?}{\vdots}$ 
CLIENT: LATA

PROJECT NO.: SwRI 01-7106

DATE: 6 JUNE 1995

TEST ID: 157LR7.DAT

\section{RACK STORAGE TEST \\ TC TREE \#1 (6" OFF RACK No. 1)}

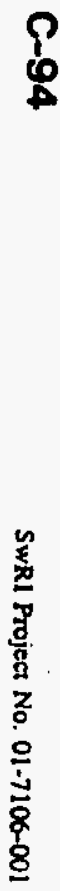

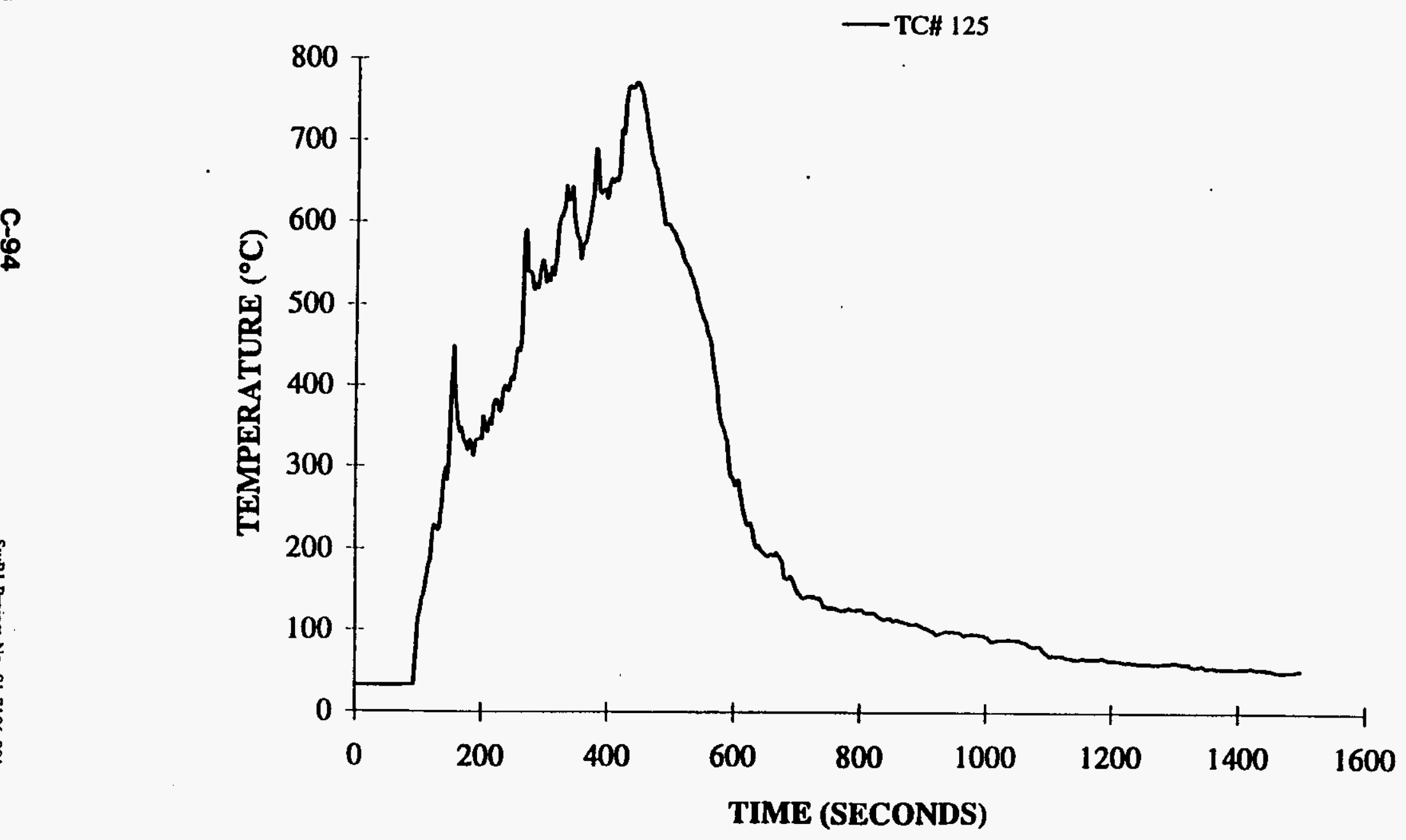

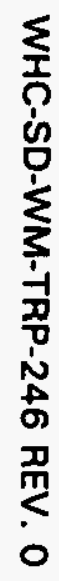


CLIENT: LATA

PROJECT NO.: SWRI 01-7106

DATE: 6 JUNE 1995

TEST ID: 157LR7.DAT

\section{RACK STORAGE TEST \\ TC TREE \#2 (6" OFF RACK No. 2)}

@

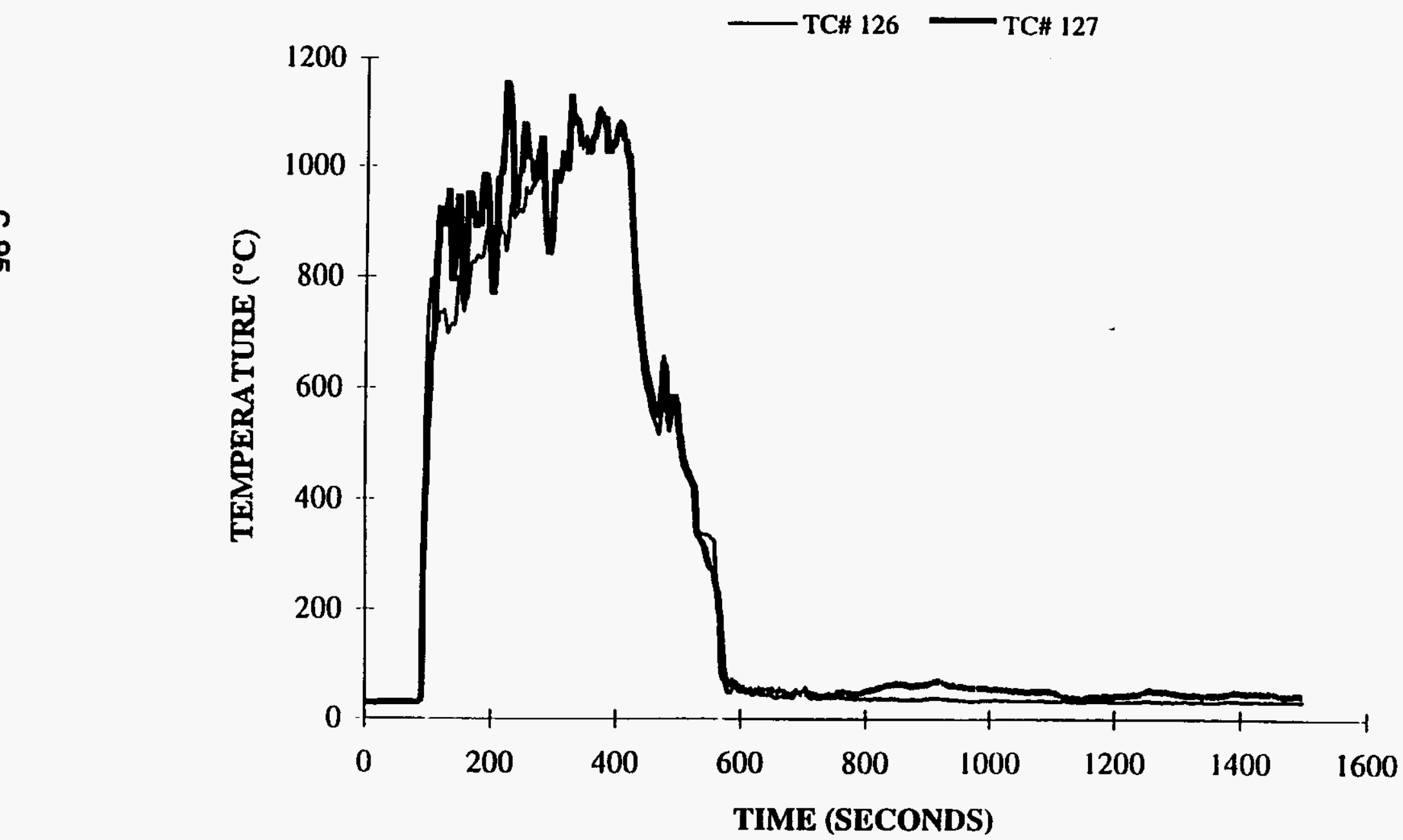

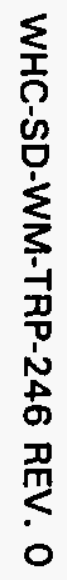

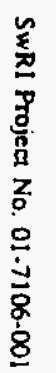


CLIENT: LATA

PROJECT NO.: SwRI 01-7106

TEST ID: 157LR7.DAT

RACK STORAGE TEST

TC TREE \#2 (6" OFF RACK No. 2)

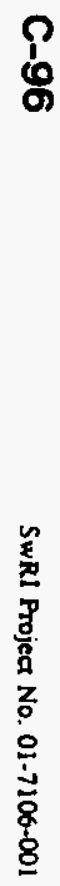

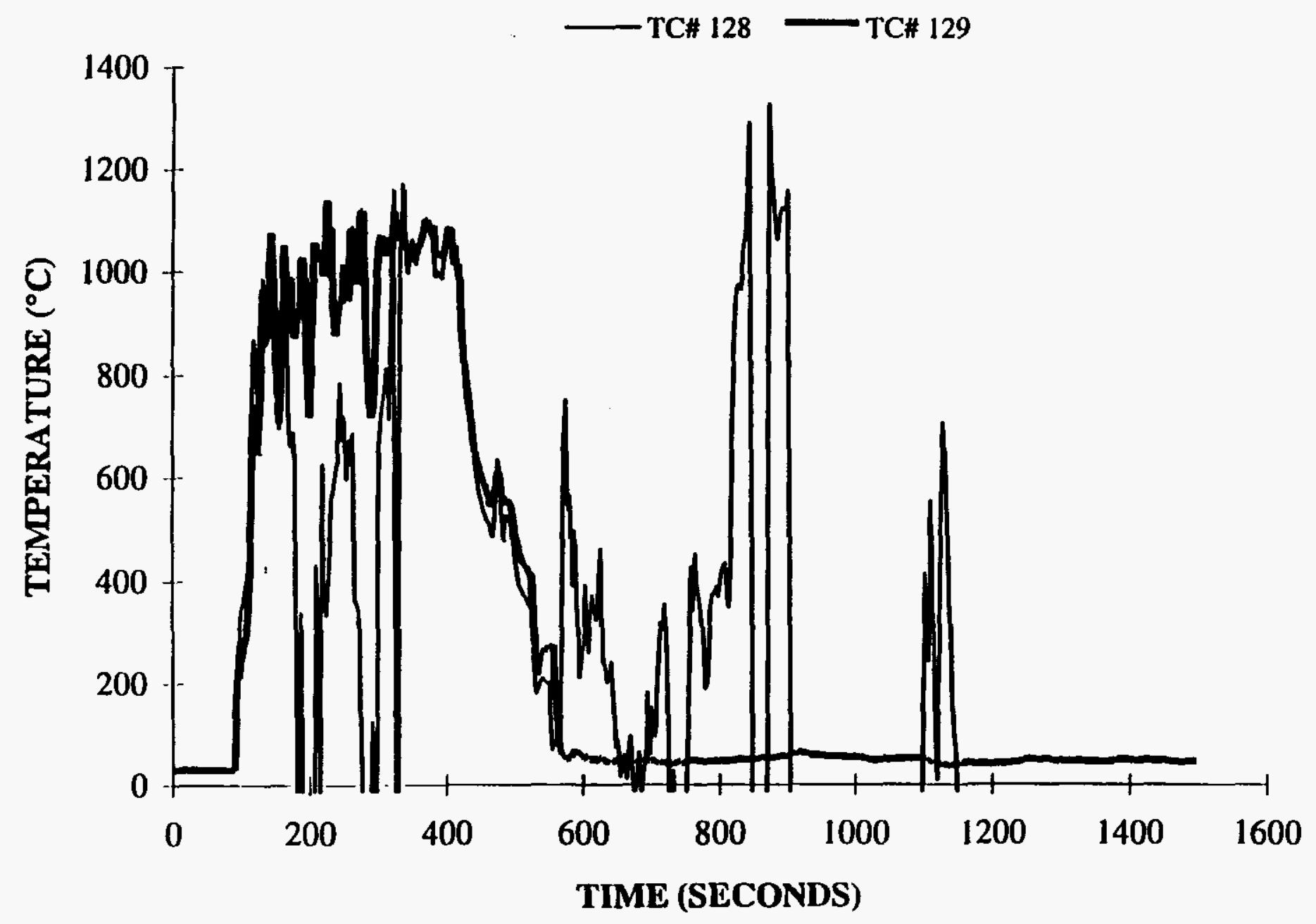

TIME (SECONDS) 
PROJECT NO.: SwRI 01-7106

DATE: 6 JUNE 1995

TEST ID: 157LR7.DAT

\section{RACK STORAGE TEST \\ TC TREE \#2 (6" OFF RACK No. 2)}

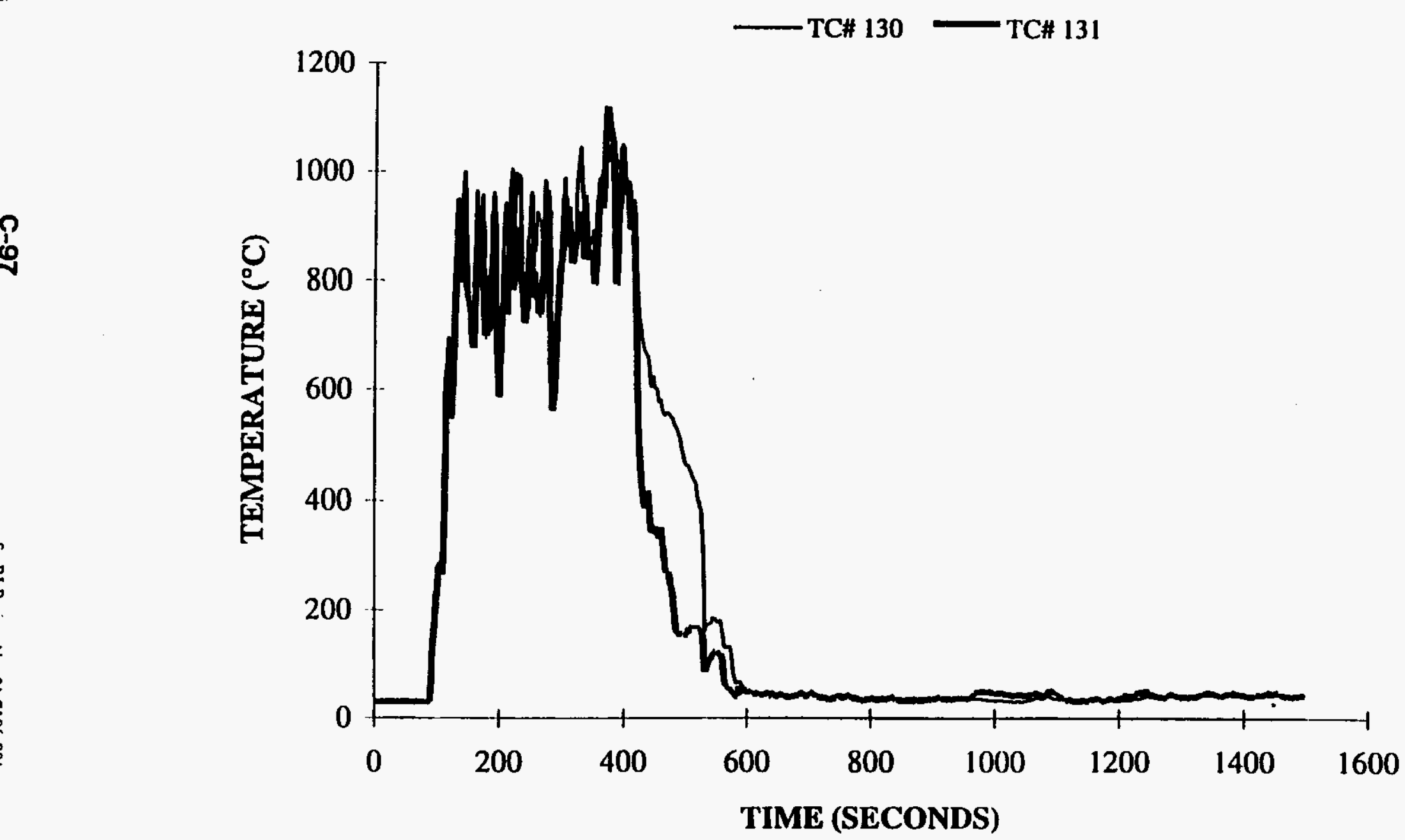


CLIENT: LATA

PROJECT NO.: SwRI 01-7106

DATE: 6 JUNE 1995

TEST ID: 157LR7.DAT
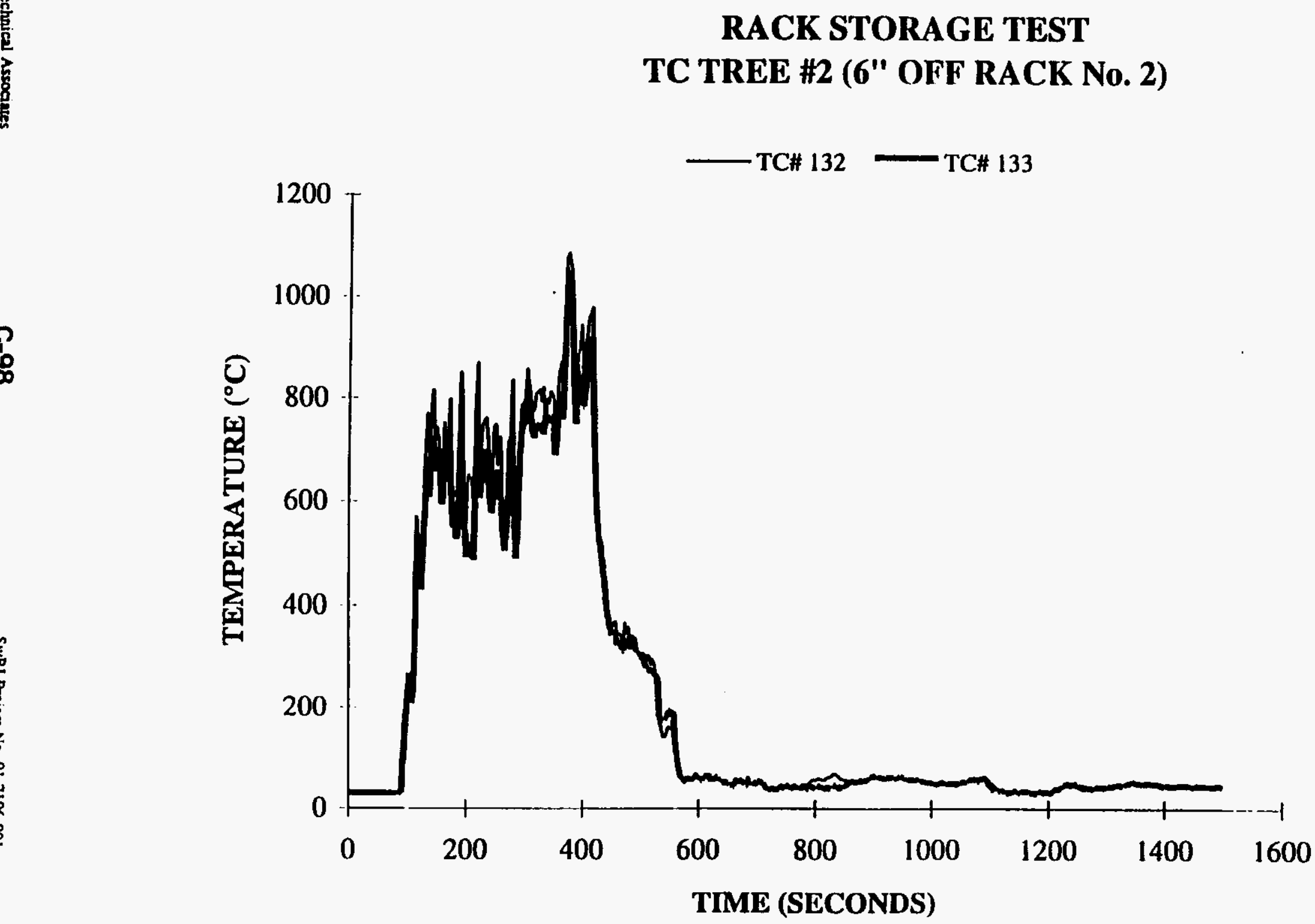

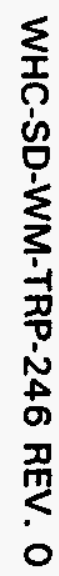

$\stackrel{8}{0}$ 
CLIENT: LATA

PROJECT NO.: SwRI 01-7106

DATE: 6 JUNE 1995

TEST ID: 157LR7.DAT

\section{RACK STORAGE TEST \\ TC TREE \#2 (6" OFF RACK No. 2)}

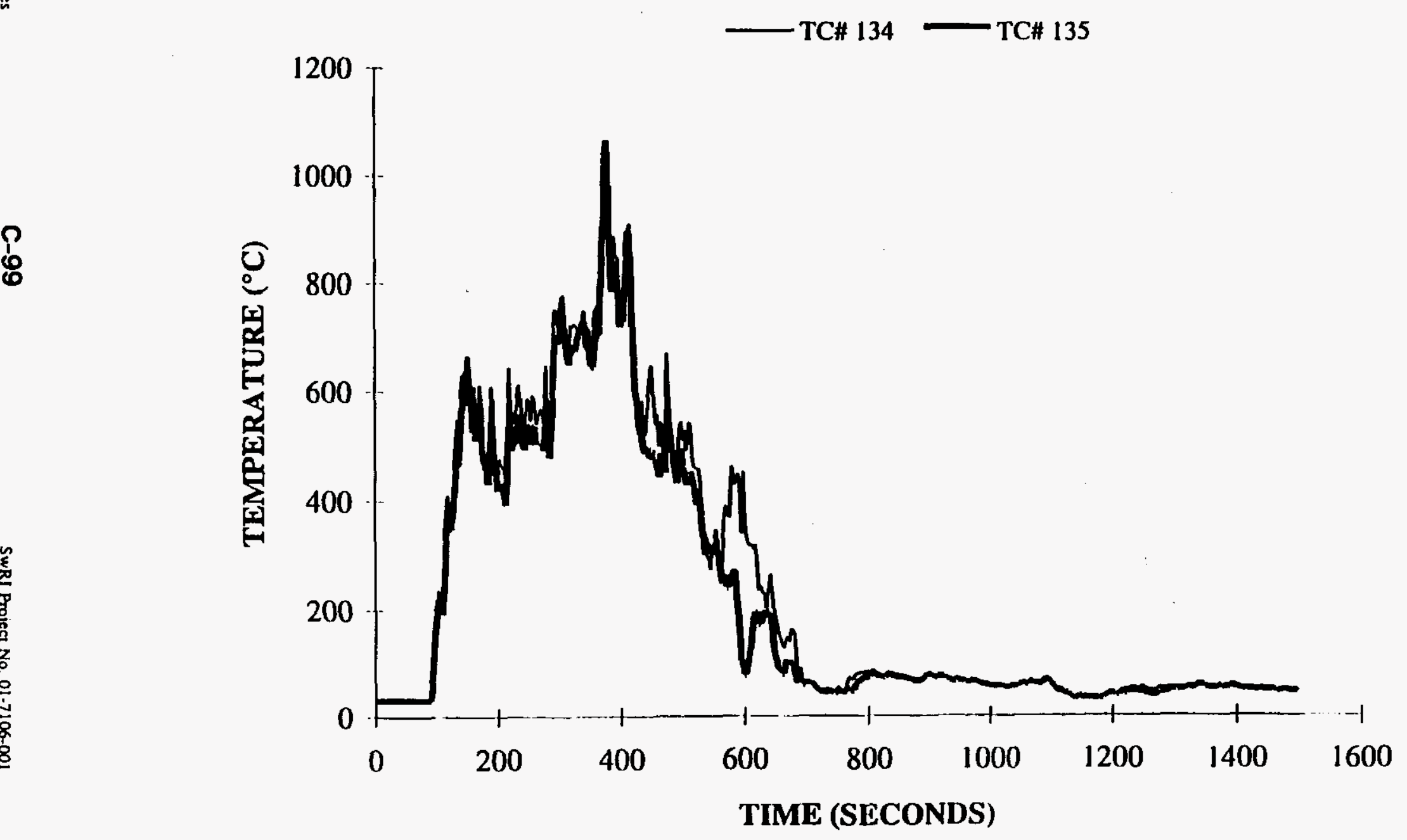

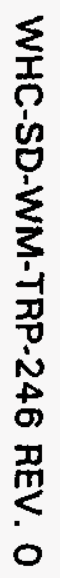

$\stackrel{8}{8}$ 
CLIENT: LATA

PROJECT NO.: SwRI 01-7106

DATE: 6 JUNE 1995

TEST ID: 157LR7.DAT

RACK STORAGE TEST

TC TREE \#2 (6" OFF RACK No. 2)

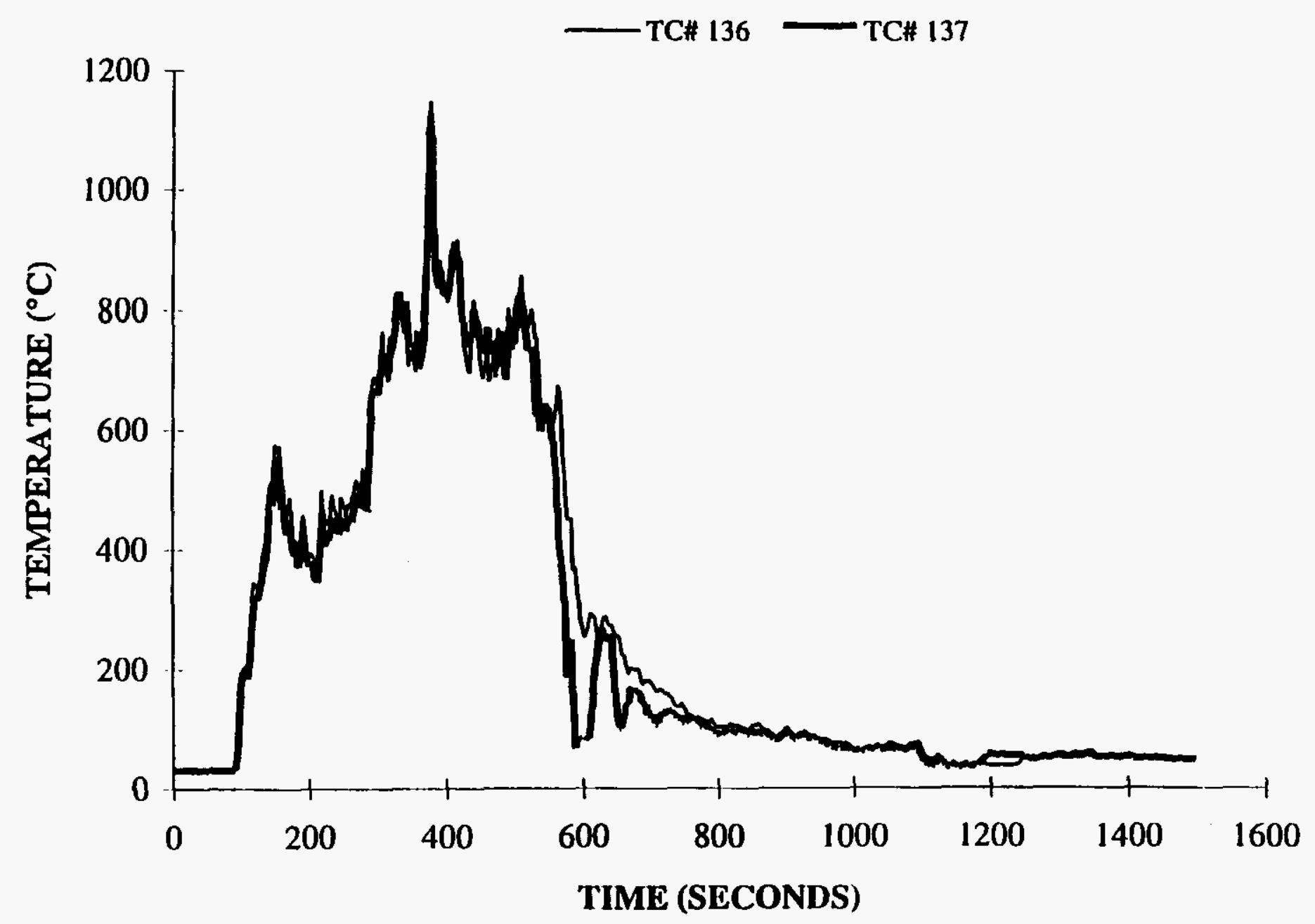


CLIENT: LATA

PROJECT NO.: SwRI 01-7106

DATE: 6 JUNE 1995

TEST ID: 157LR7.DAT

\section{RACK STORAGE TEST \\ TC TREE \#2 (6" OFF RACK No. 2)}

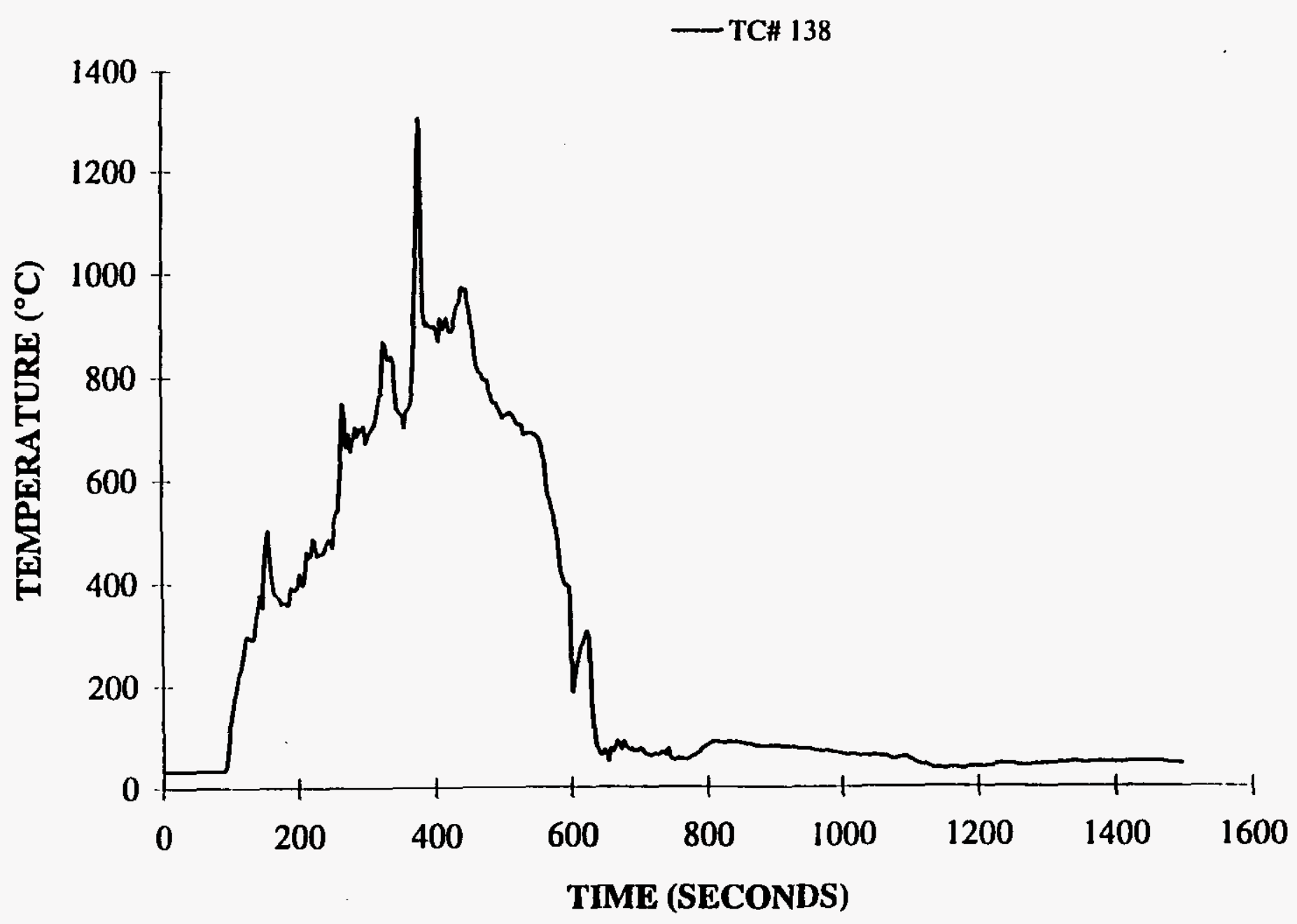


CLIENT: LATA

PROJECT NO.: SwRI 01-7106

DATE: 6 JUNE 1995

TEST ID: 157LR7.DAT

RACK STORAGE TEST

TC TREE \# 3 (BETWEEN RACKS 2\&3)

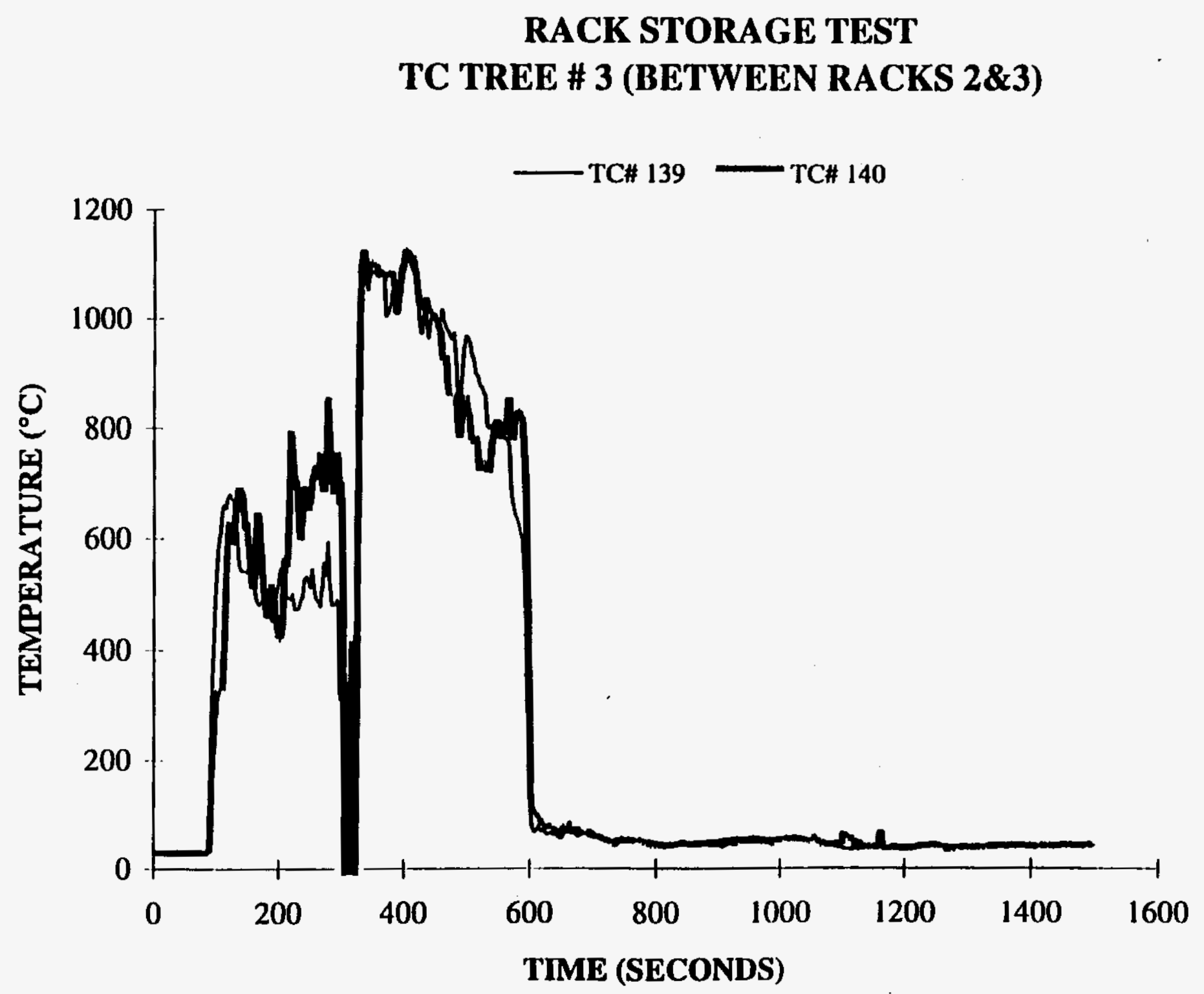

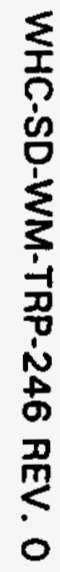

疍

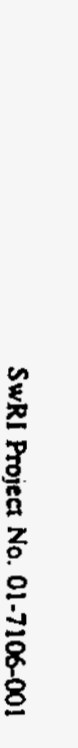


CLIENT: LATA

PROJECT NO.: SwRI 01-7106

DATE: 6 JUNE 1995

TEST ID: 157LR7.DAT

\section{RACK STORAGE TEST \\ TC TREE \# 3 (BETWEEN RACKS 2\&3)}

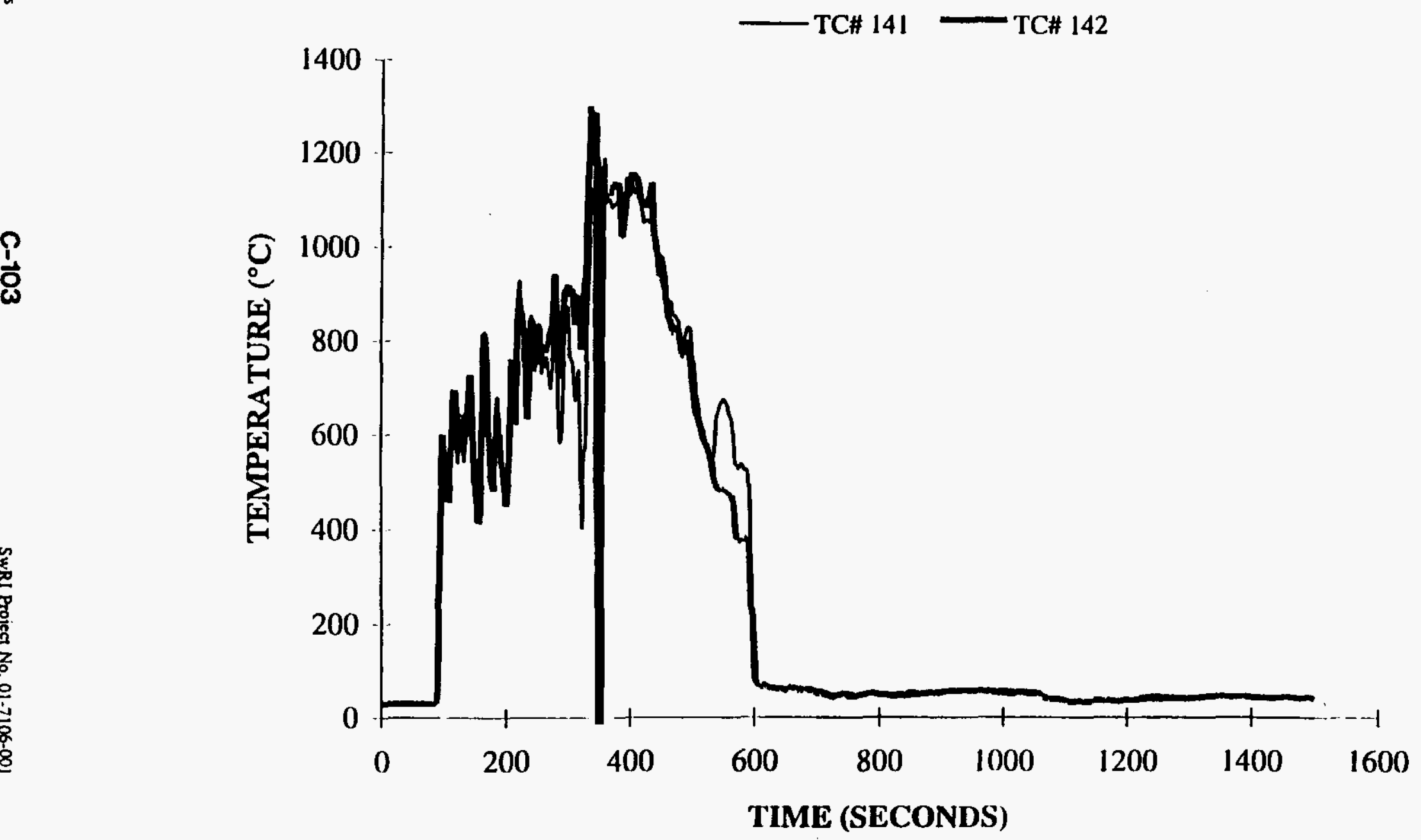


CLIENT: LATA

PROJECT NO.: SwRI 01-7106

DATE: 6 JUNE 1995

TEST ID: 157LR7.DAT

\section{RACK STORAGE TEST \\ TC TREE \# 3 (BETWEEN RACKS 2\&3)}

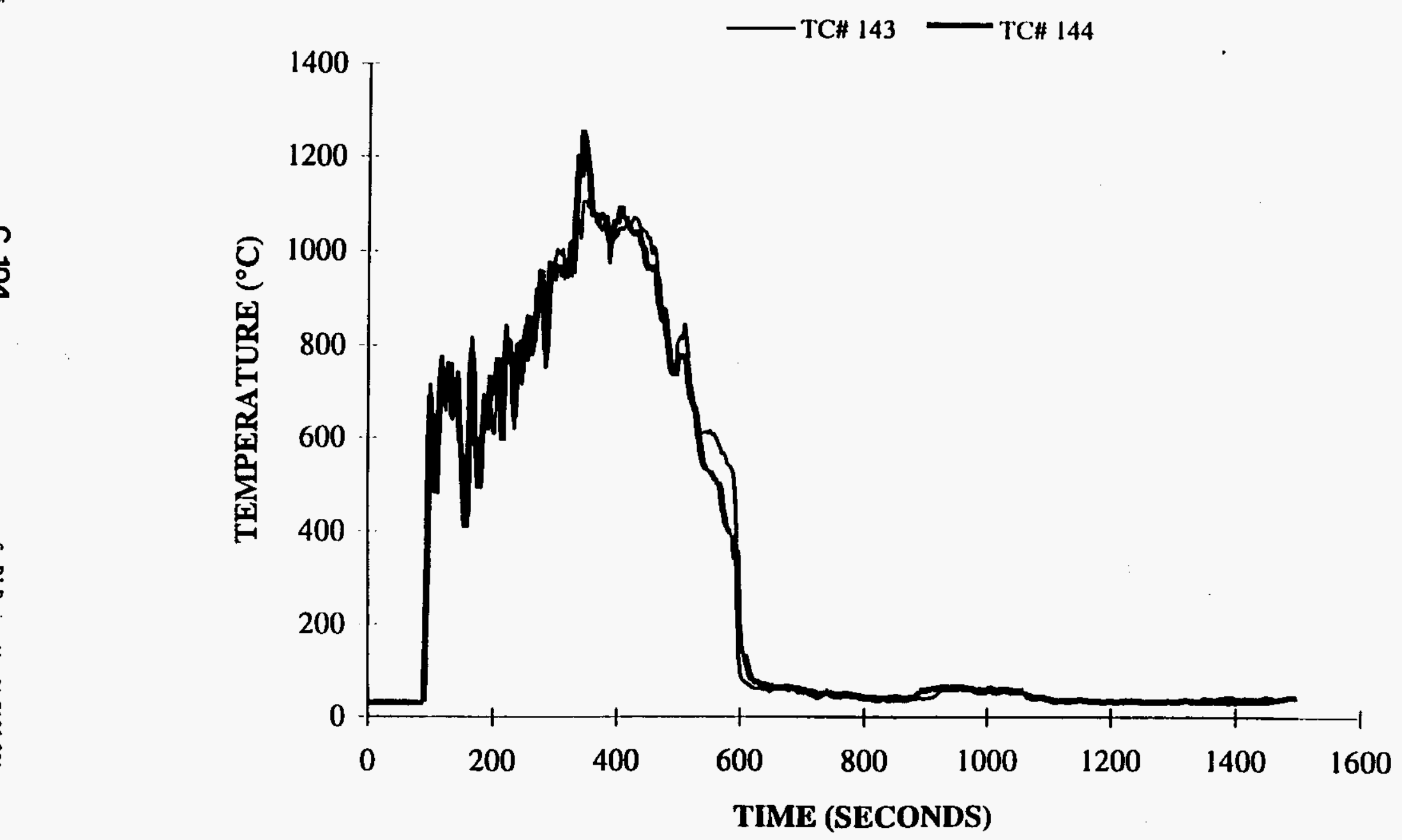




\section{CLIENT: LATA}

PROJECT NO.: SwRI 01-7106

DATE: 6 JUNE 1995

TEST ID: 157LR7.DAT

\section{RACK STORAGE TEST \\ TC TREE \# 3 (BETWEEN RACKS 2\&3)}

웅

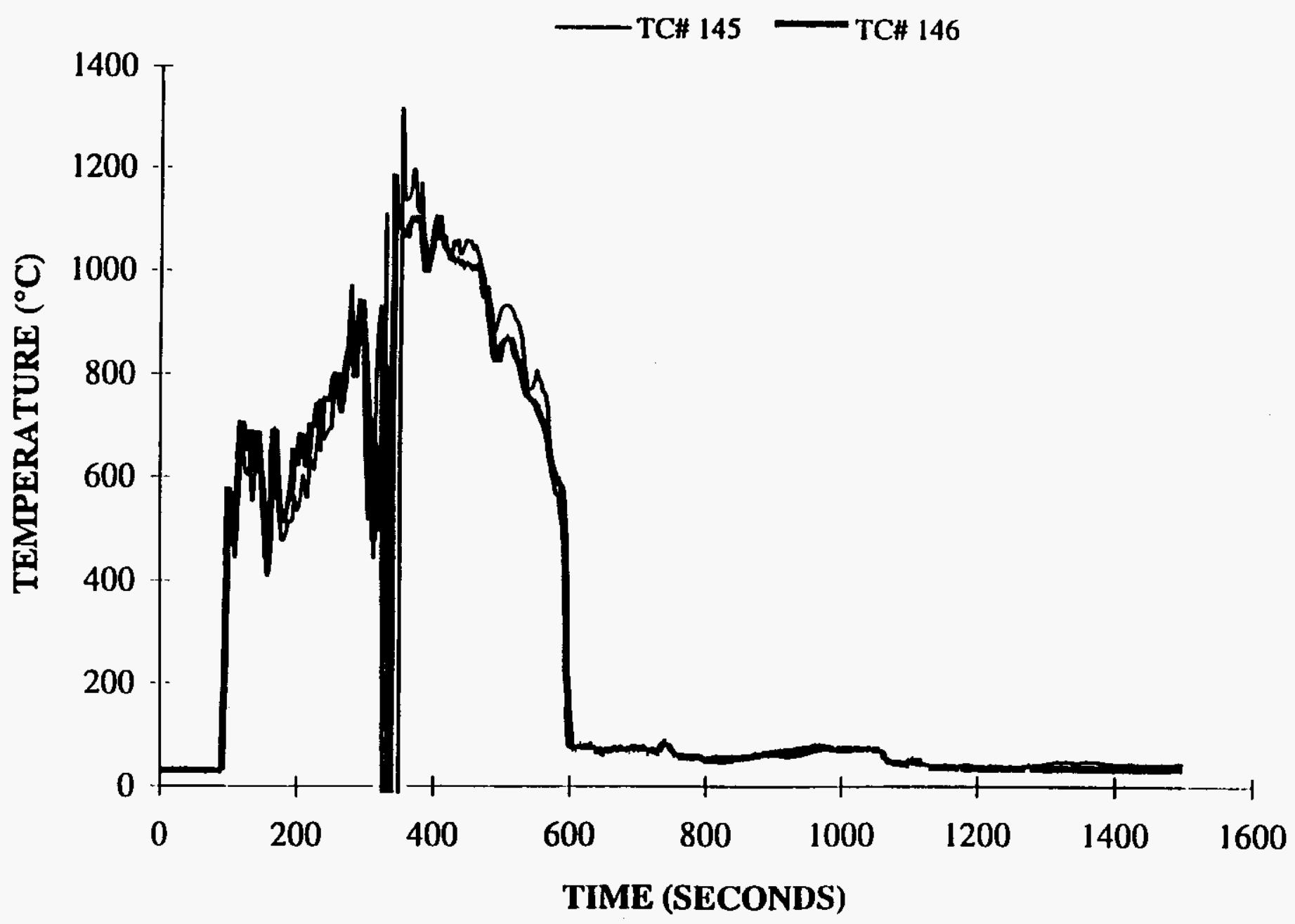

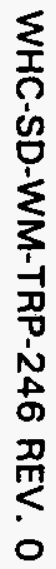


CLIENT: LATA

PROJECT NO.: SwRI 01-7106

DATE: 6 JUNE 1995

TEST ID: 157LR7.DAT

\section{RACK STORAGE TEST \\ TC TREE \# 3 (BETWEEN RACKS 2\&3)}

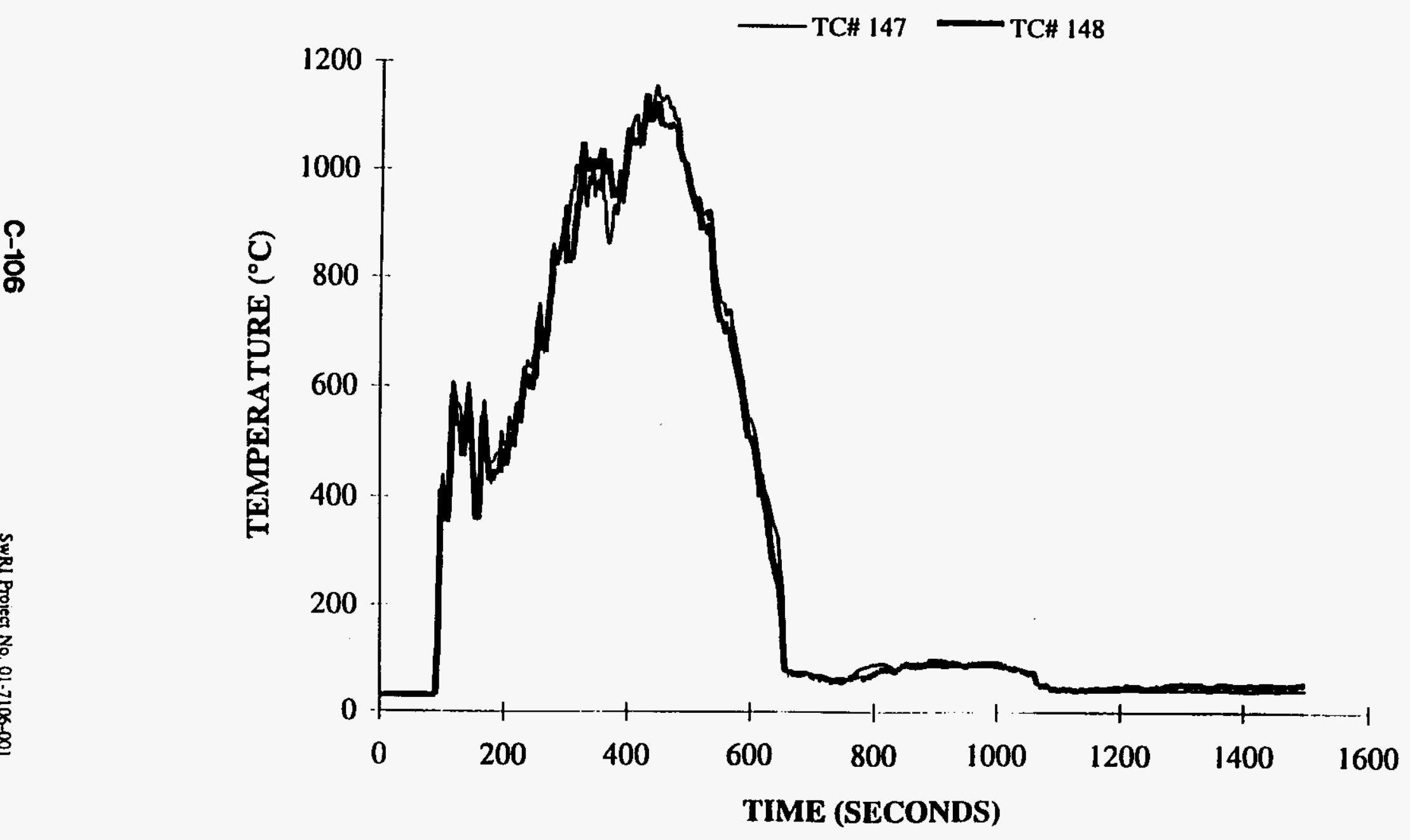

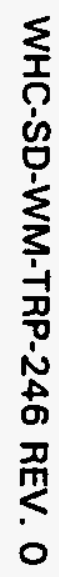

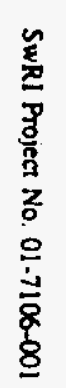

? 
CLIENT: LATA

PROJECT NO.: SwRI 01-7106

DATE: 6 JUNE 1995

TEST ID: 157LR7.DAT

RACK STORAGE TEST

TC TREE \# 3 (BETWEEN RACKS 2\&3)

?

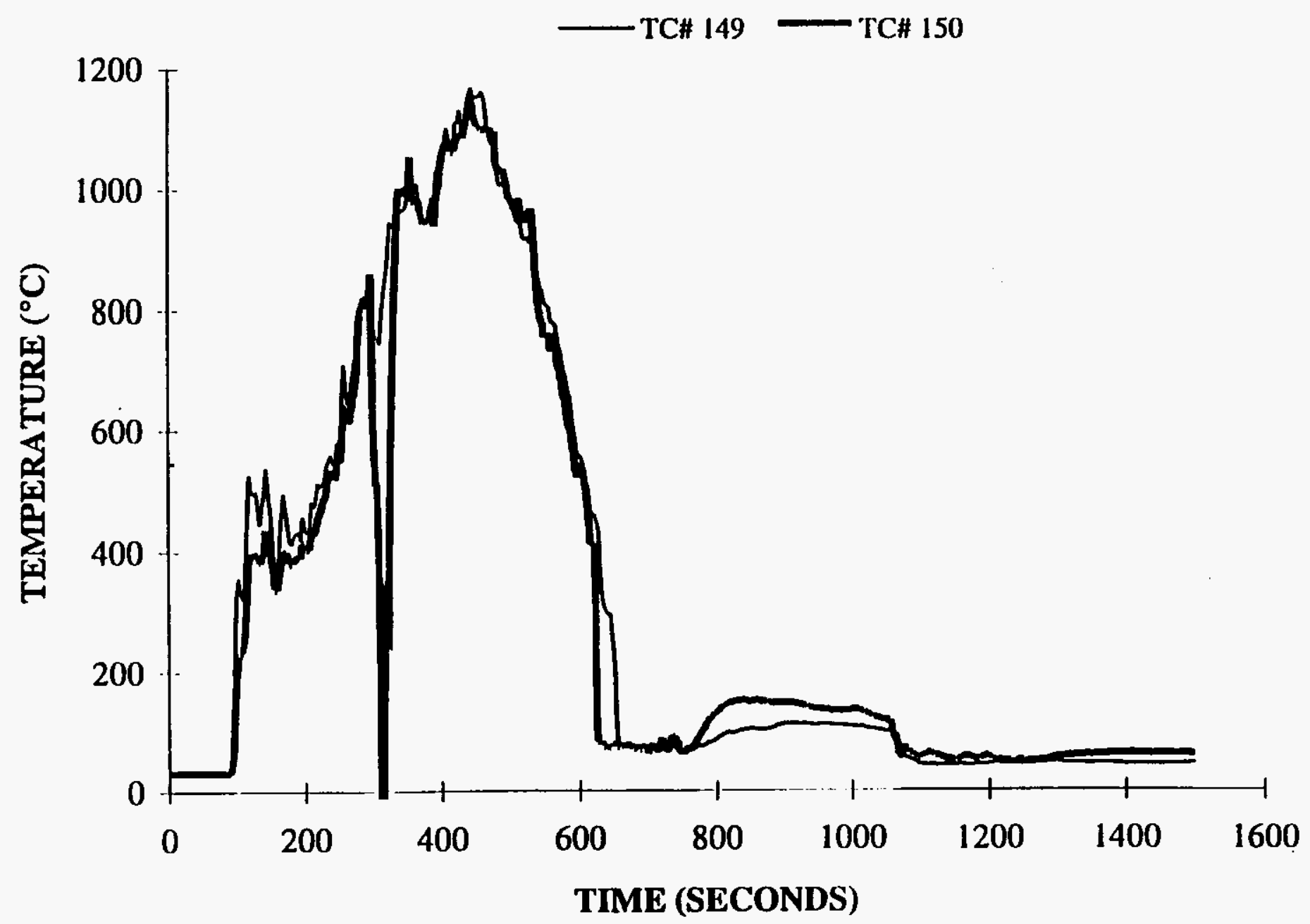

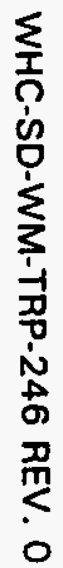


CLIENT: LATA

PROJECT NO.: SwRI 01-7106

DATE: 6 JUNE 1995

TEST ID: 157LR7.DAT

\section{RACK STORAGE TEST}

TC TREE \# 3 (BETWEEN RACKS 2\&3)

$\stackrel{?}{\stackrel{\infty}{\circ}}$

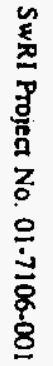

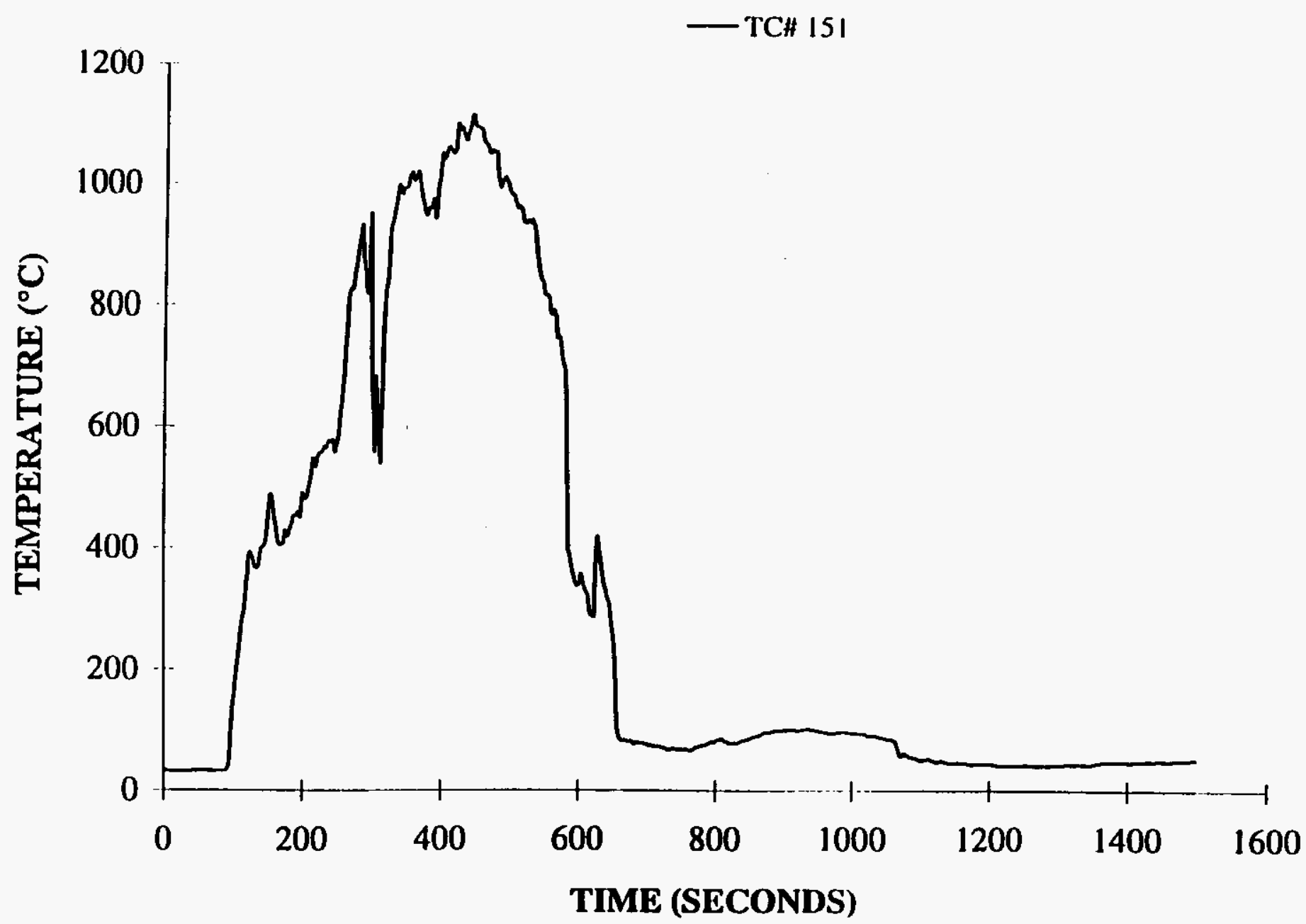


CLIENT: LATA

PROJECT NO.: SwRI 01-7106

DATE: 6 JUNE 1995

TEST ID: 157LR5.DAT

\section{RACK STORAGE TEST \\ MIDDLE BEAM, SOUTH SIDE \\ OF RACK 1}

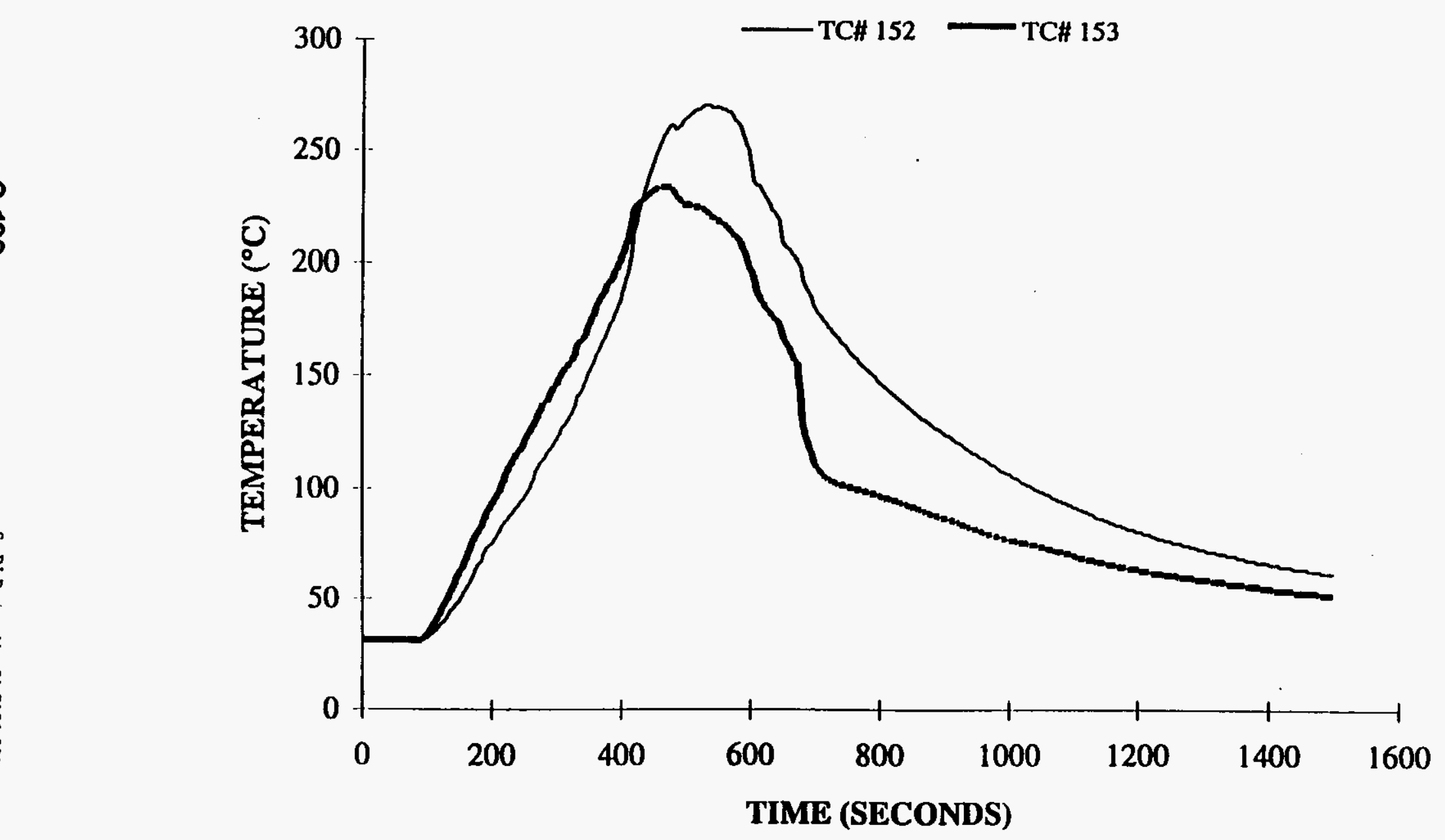

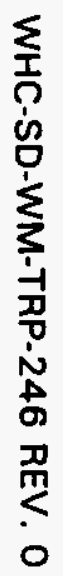


CLIENT: LATA

PROJECT NO.: SwRI 01-7106

DATE: 6 JUNE 1995

TEST ID: 157LR5.DAT

\section{RACK STORAGE TEST \\ MIDDLE BEAM, SOUTH SIDE \\ OF RACK 1}

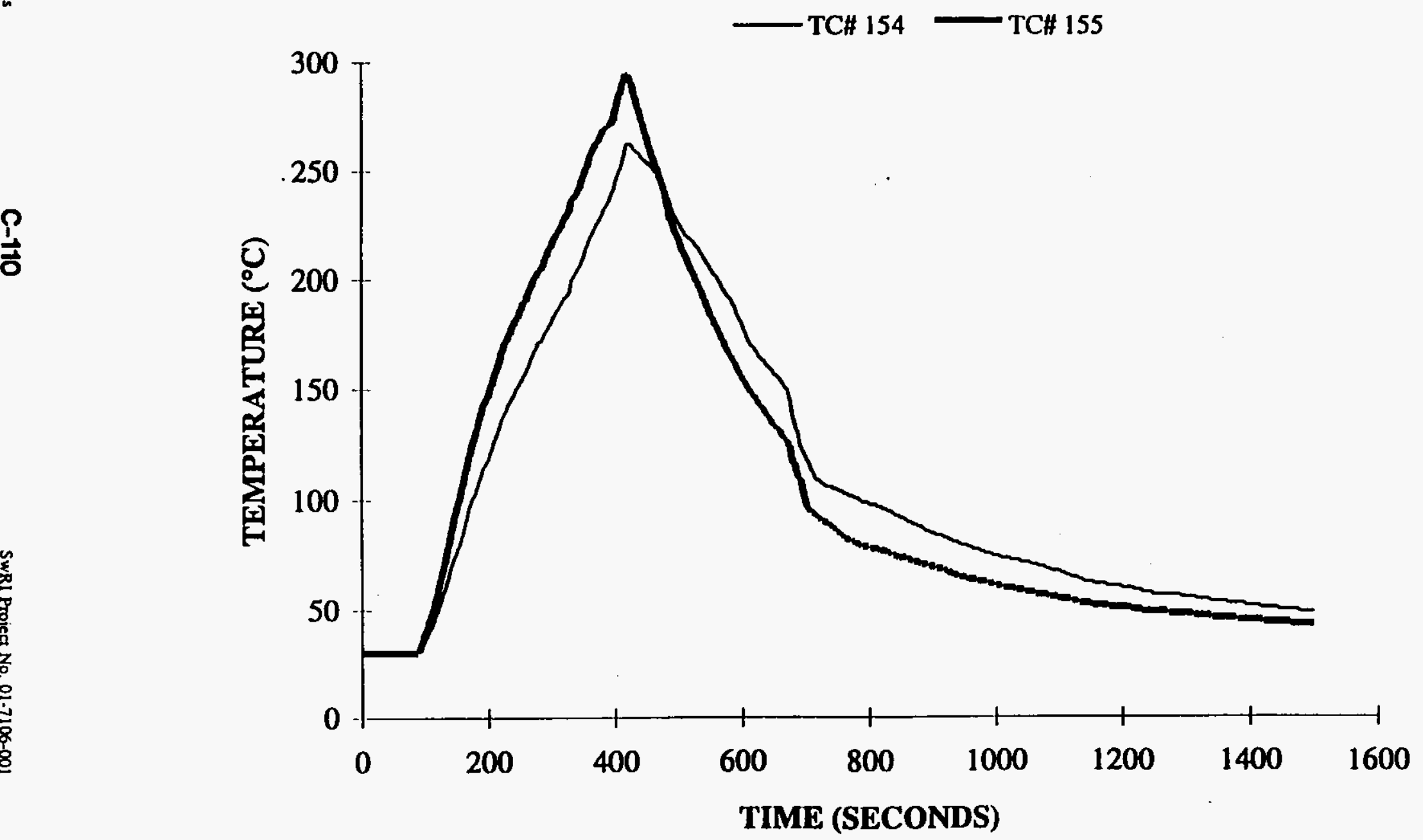


CLIENT: LATA

PROJECT NO.: SwRI 01-7106

DATE: 6 JUNE 1995

TEST ID: 157LR5.DAT

\section{RACK STORAGE TEST \\ EAST SIDE, SOUTH SIDE OF \\ RACK 1}

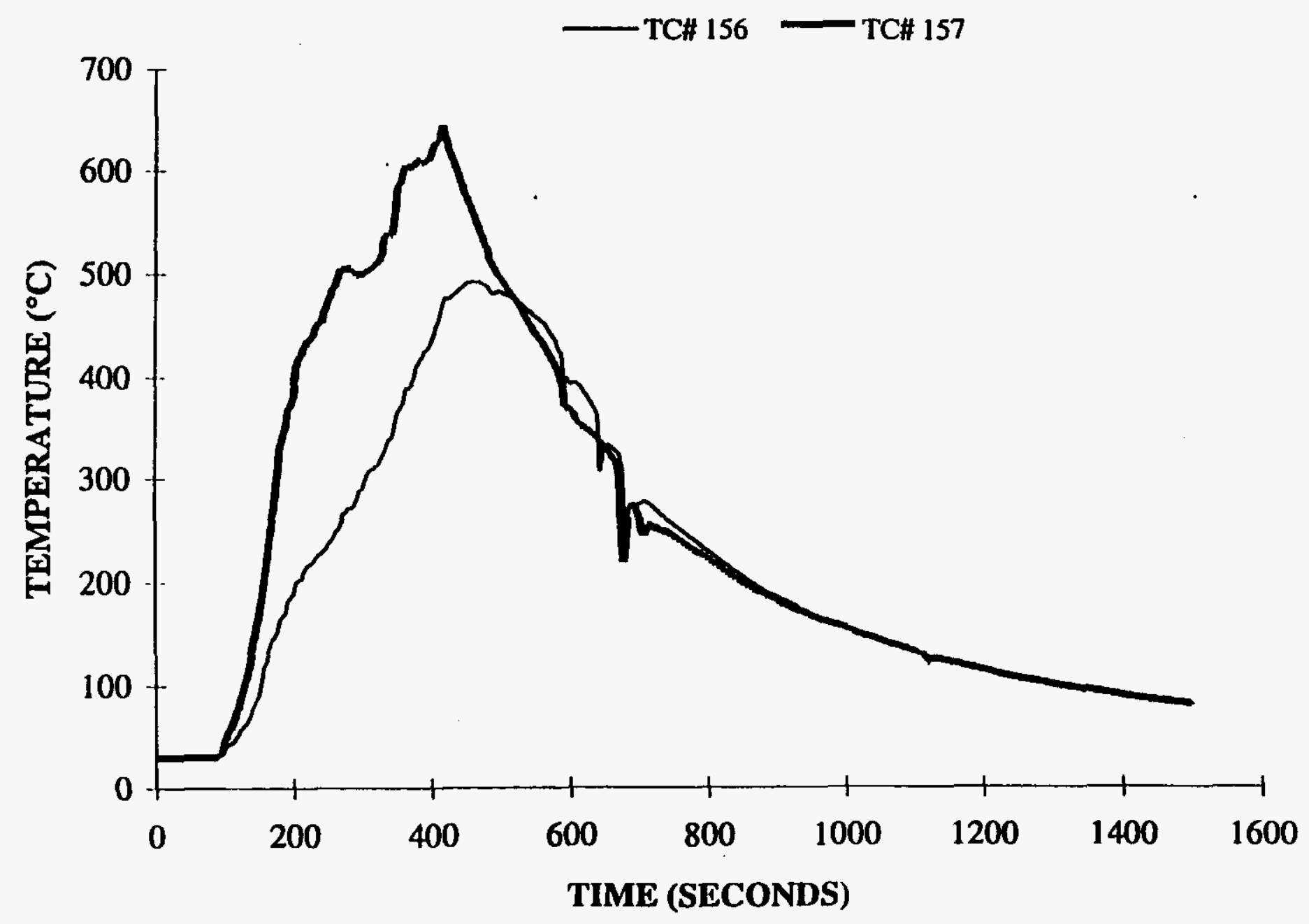




\section{CLIENT: LATA}

PROJECT NO.: SwRI 01-7106

DATE: 6 JUNE 1995

TEST ID: 157LR5.DAT

\section{RACK STORAGE TEST EAST END OF RACK 1}

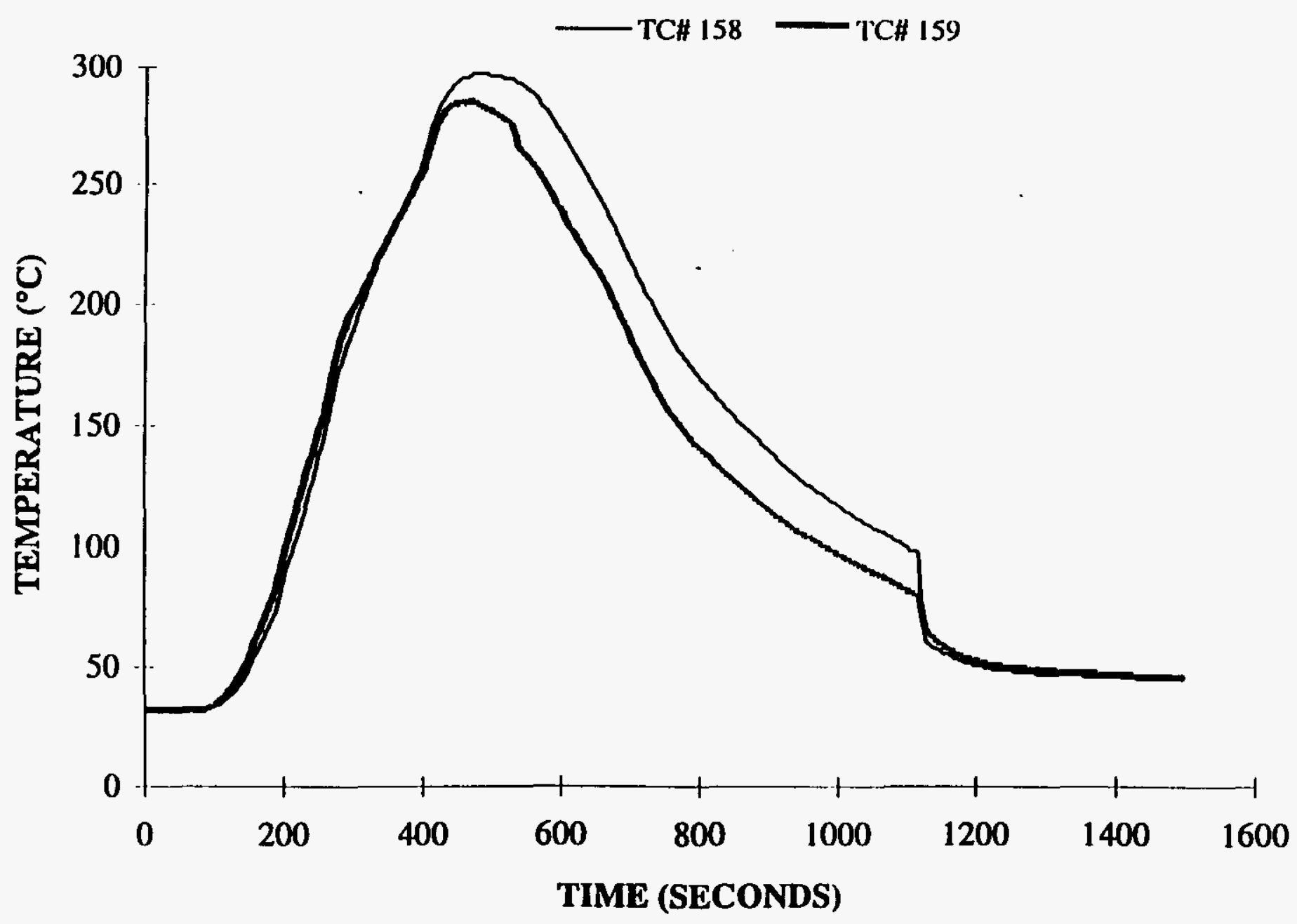

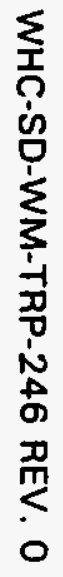


CLIENT: LATA

PROJECT NO.: SwRI 01-7106

DATE: 6 JUNE 1995

TEST ID: 157LR5.DAT

\section{RACK STORAGE TEST}

EAST END OF RACK 1

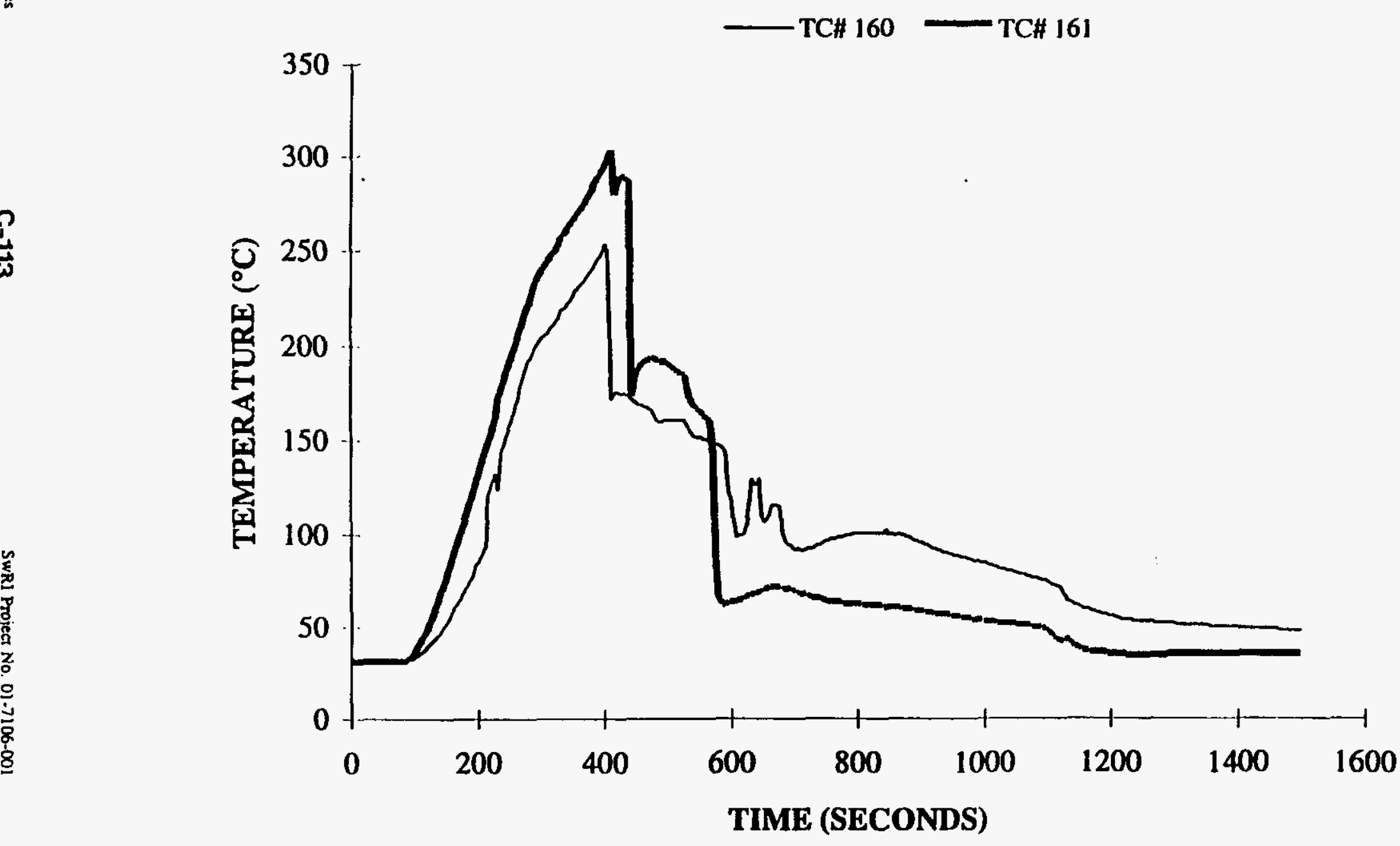

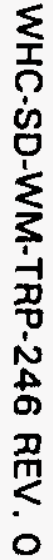

$\frac{\rho}{\vec{\omega}}$ 


\section{CLIENT: LATA}

PROJECT NO.: SwRI 01-7106

DATE: 6 JUNE 1995

TEST ID: 157LR5.DAT

\section{RACK STORAGE TEST}

EAST END OF RACK 2

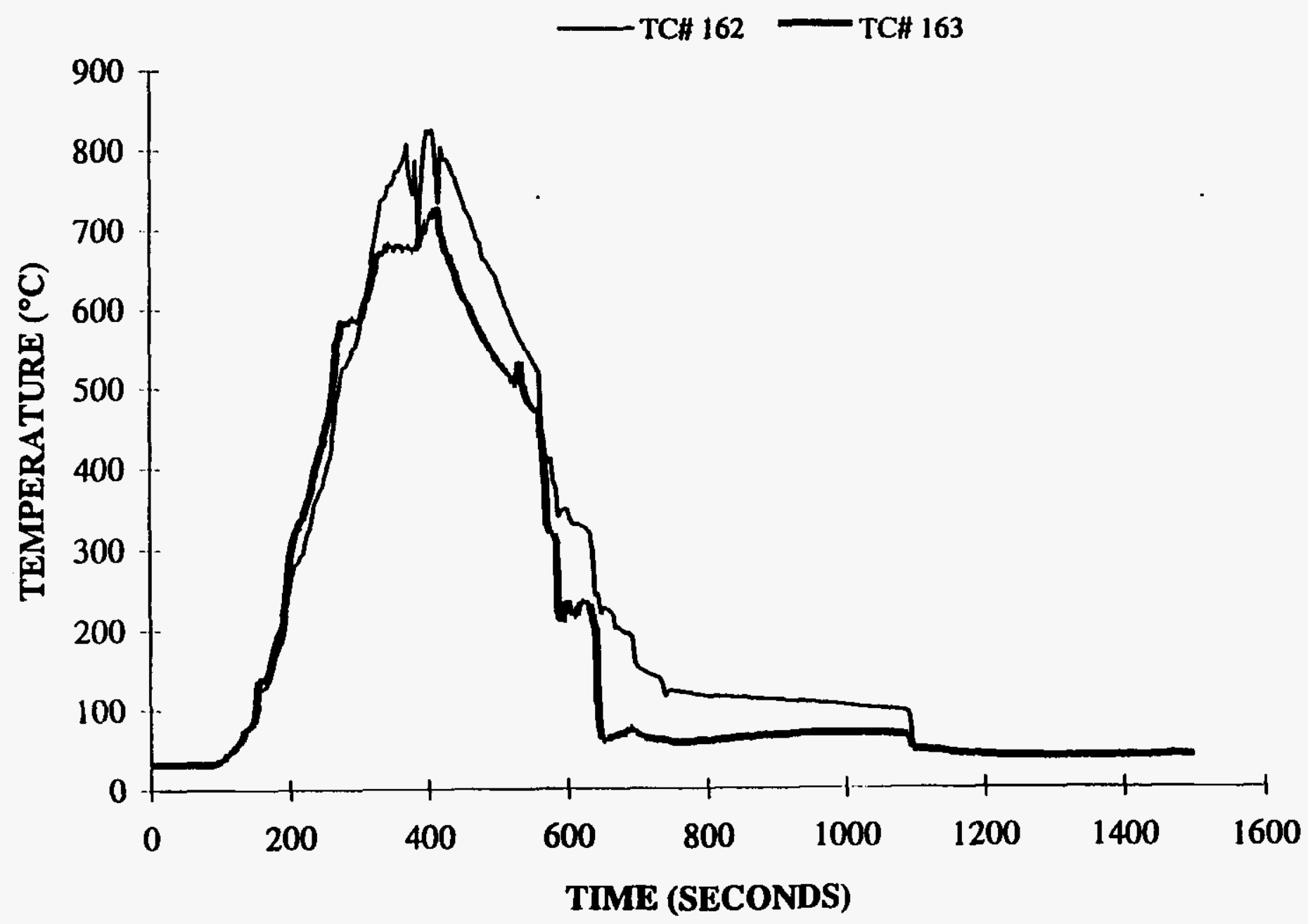

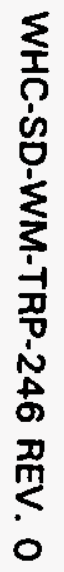

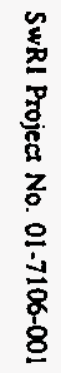

$\frac{\rho}{\frac{1}{5}}$ 
CLIENT: LATA

PROJECT NO.: SwRI 01-7106

DATE: 6 JUNE 1995

TEST ID: 157LR5.DAT

\section{RACK STORAGE TEST}

EAST END OF RACK 2

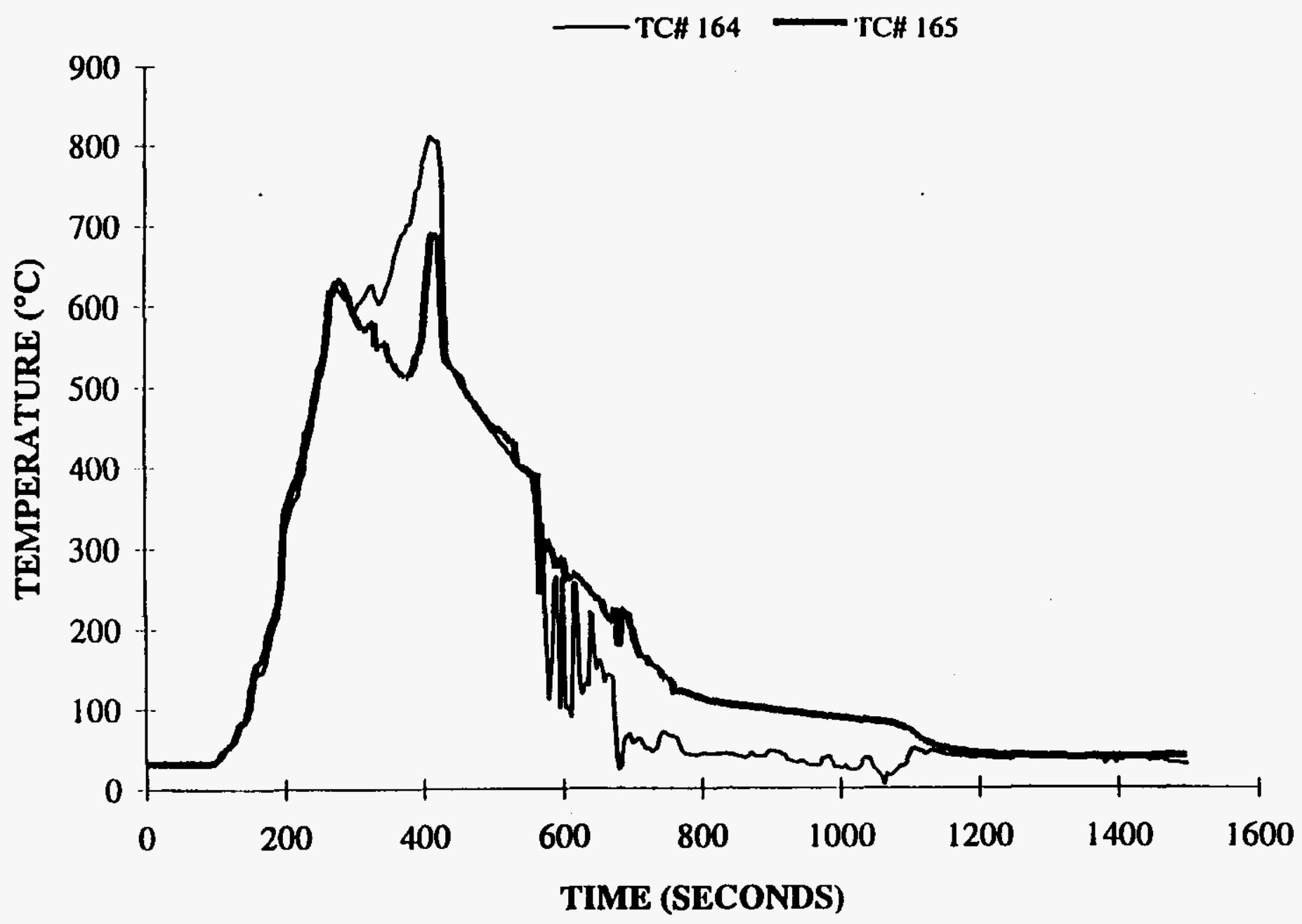

ज 
CLIENT: LATA

PROJECT NO.: SwRI 01-7106

DATE: 6 JUNE 1995

TEST ID: 157LR5.DAT

\section{RACK STORAGE TEST EAST END OF RACK 2}

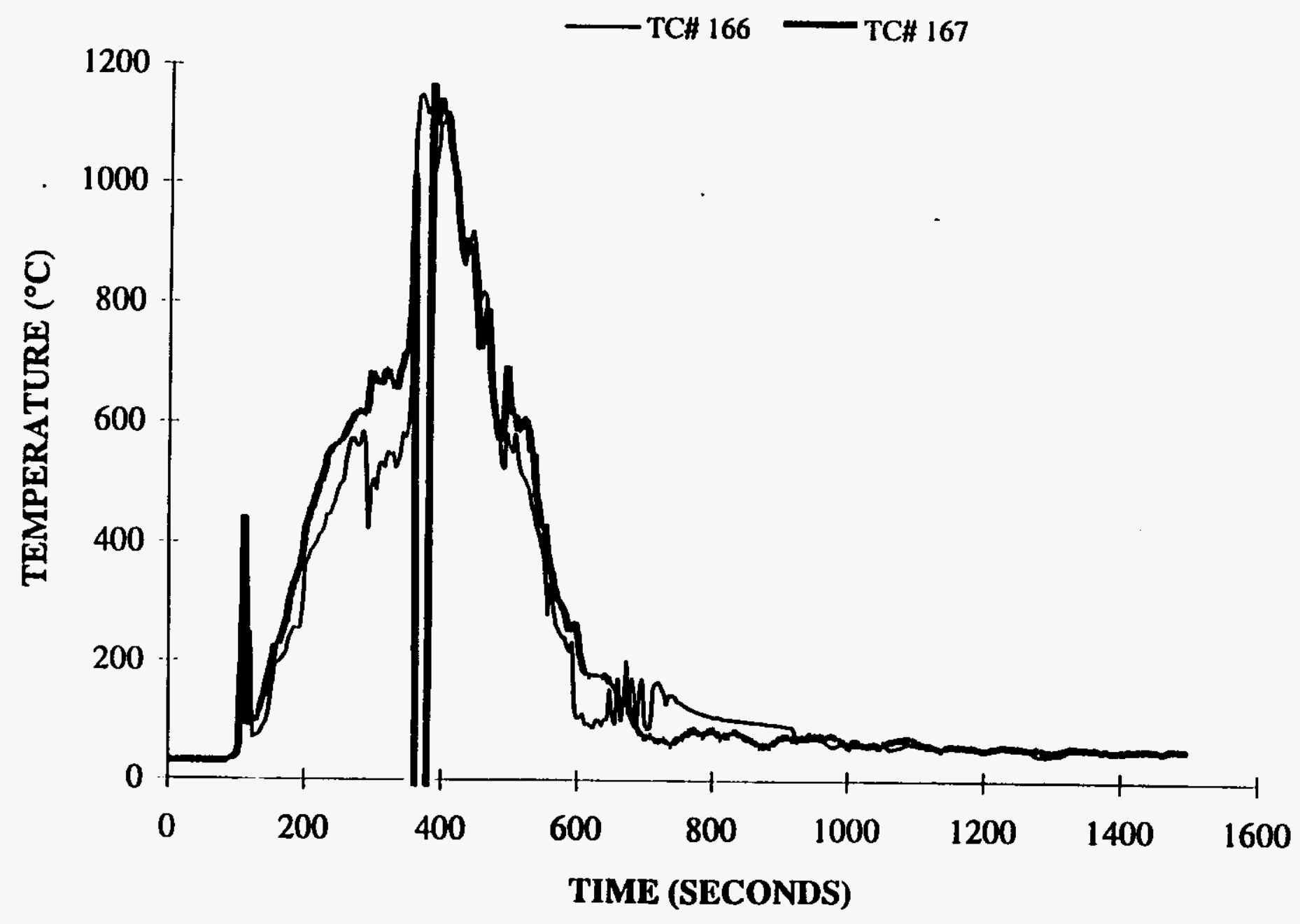

$\stackrel{?}{\frac{?}{5}}$ 
CLIENT: LATA

PROJECT NO.: SwRI 01-7106

DATE: 6 JUNE 1995

TEST ID: 157LR5.DAT

\section{RACK STORAGE TEST \\ EAST SIDE, NORTH FACE OF \\ RACK 2}

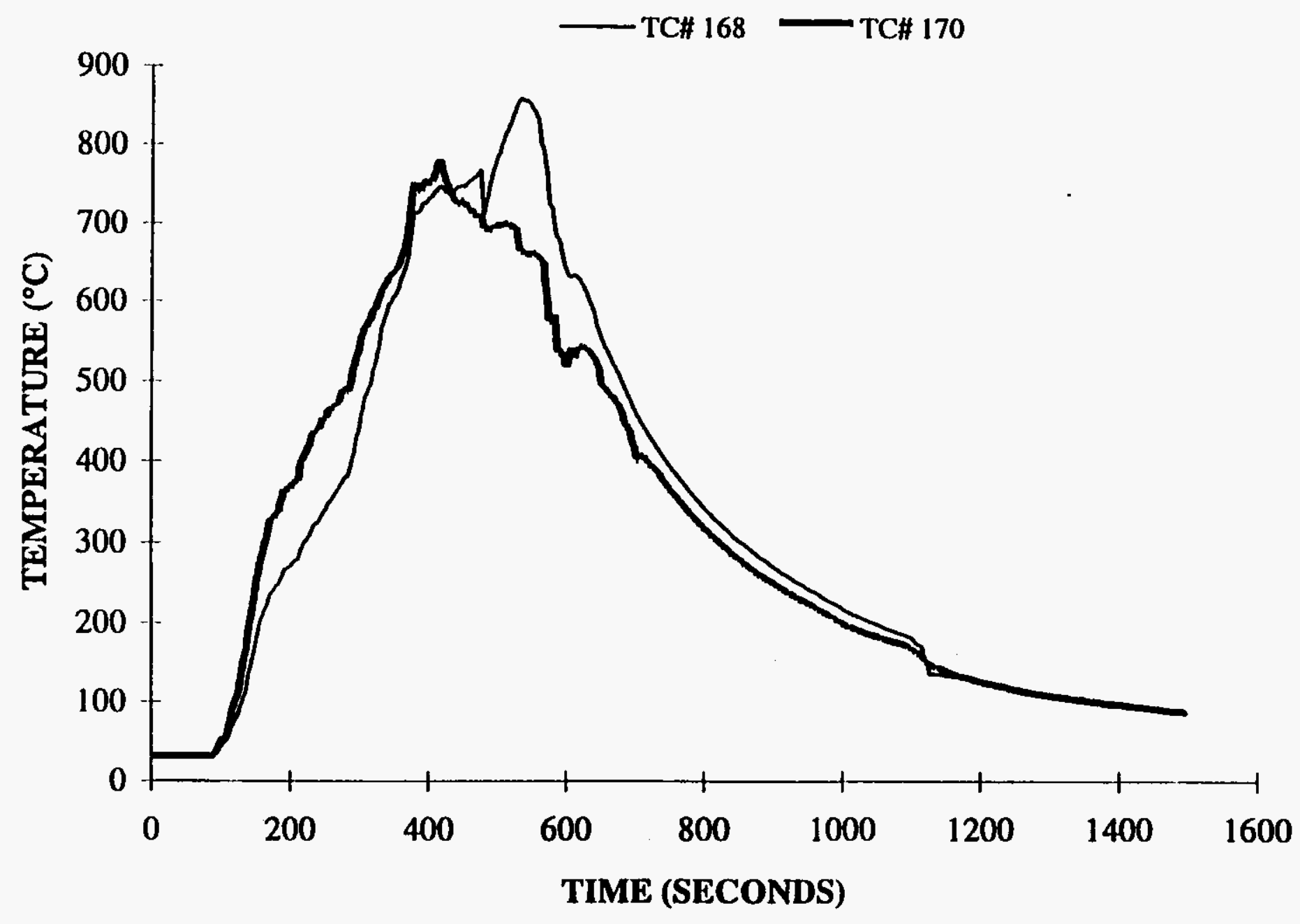


CLIENT: LATA

PROJECT NO.: SwRI 01-7106

DATE: 6 JUNE 1995

TEST ID: 157LR5.DAT

\section{RACK STORAGE TEST \\ EAST SIDE, NORTH FACE OF}

RACK 2

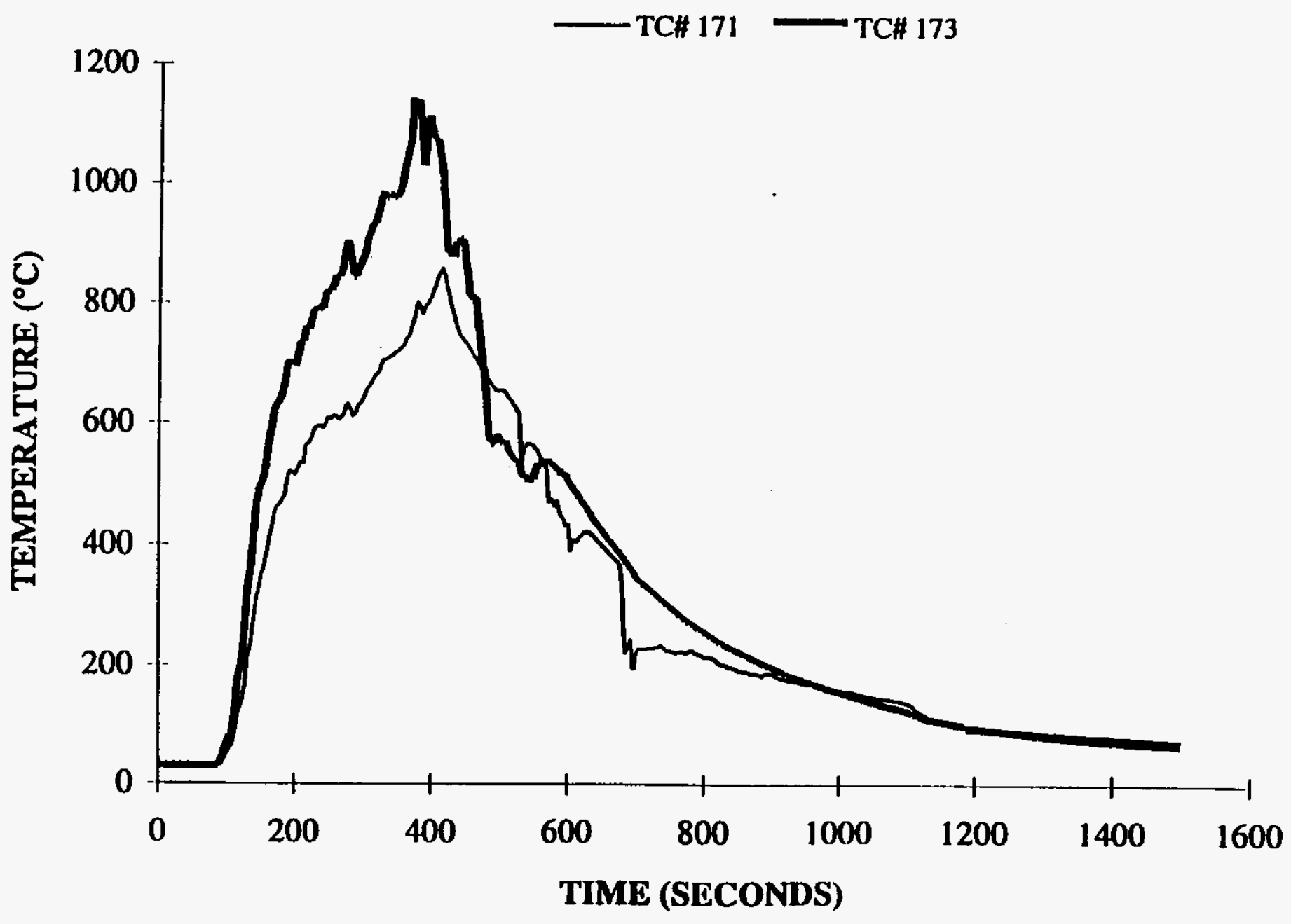


CLIENT: LATA

PROJECT NO.: SwRI 01-7106

DATE: 6 JUNE 1995

TEST ID: 157LR5.DAT

\section{RACK STORAGE TEST \\ EAST SIDE, NORTH FACE OF}

RACK 2

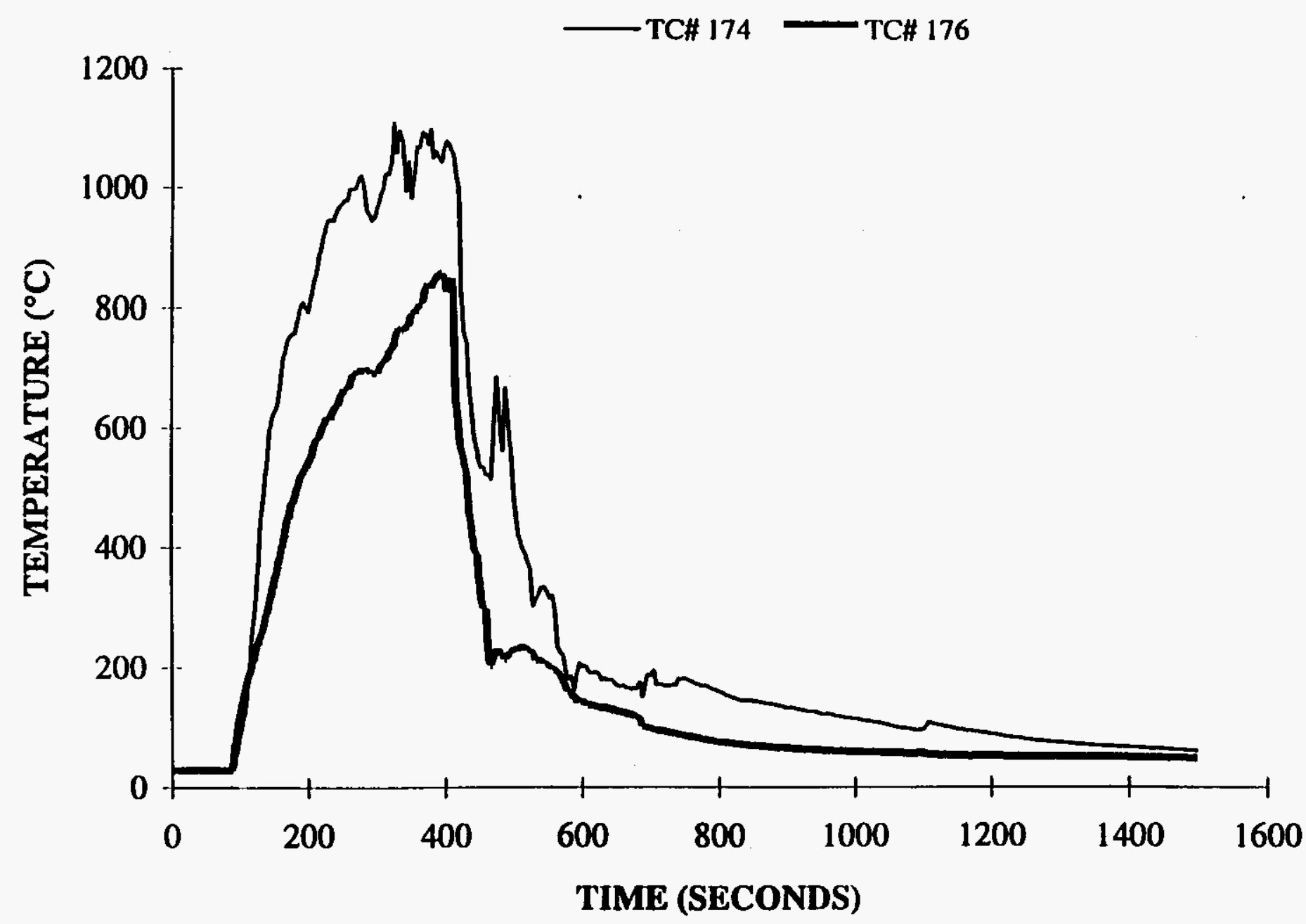




\section{CLIENT: LATA}

PROJECT NO.: SwRI 01-7106

DATE: 6 JUNE 1995

TEST ID: 157LR5.DAT

\section{RACK STORAGE TEST \\ EAST SIDE, SOUTH FACE OF \\ RACK 2}

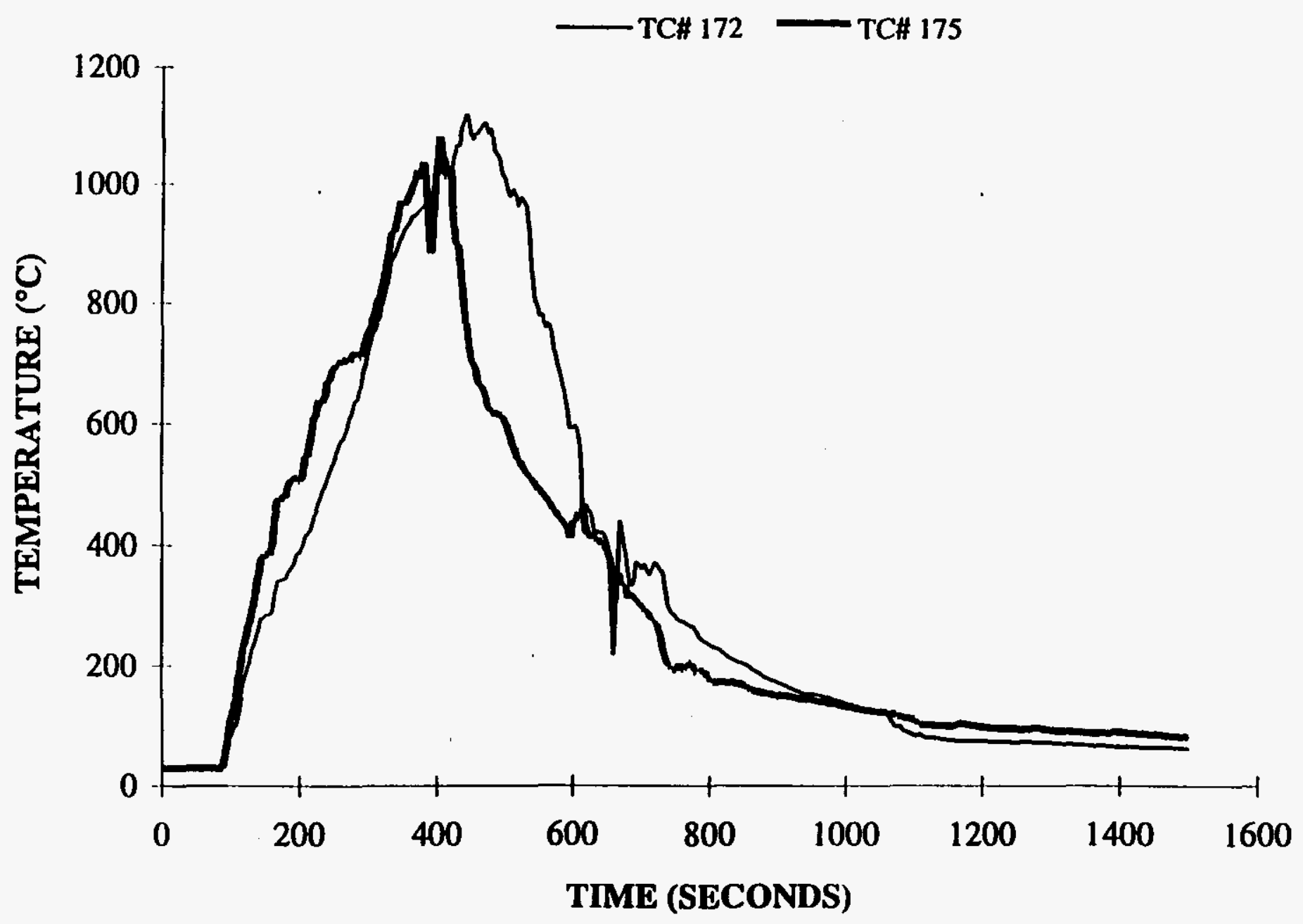


CLIENT: LATA

PROJECT NO.: SwRI 01-7106

DATE: 6 JUNE 1995

TEST ID: 157LR5.DAT

\section{RACK STORAGE TEST \\ MIDDLE BEAM, NORTH SIDE}

OF RACK 2

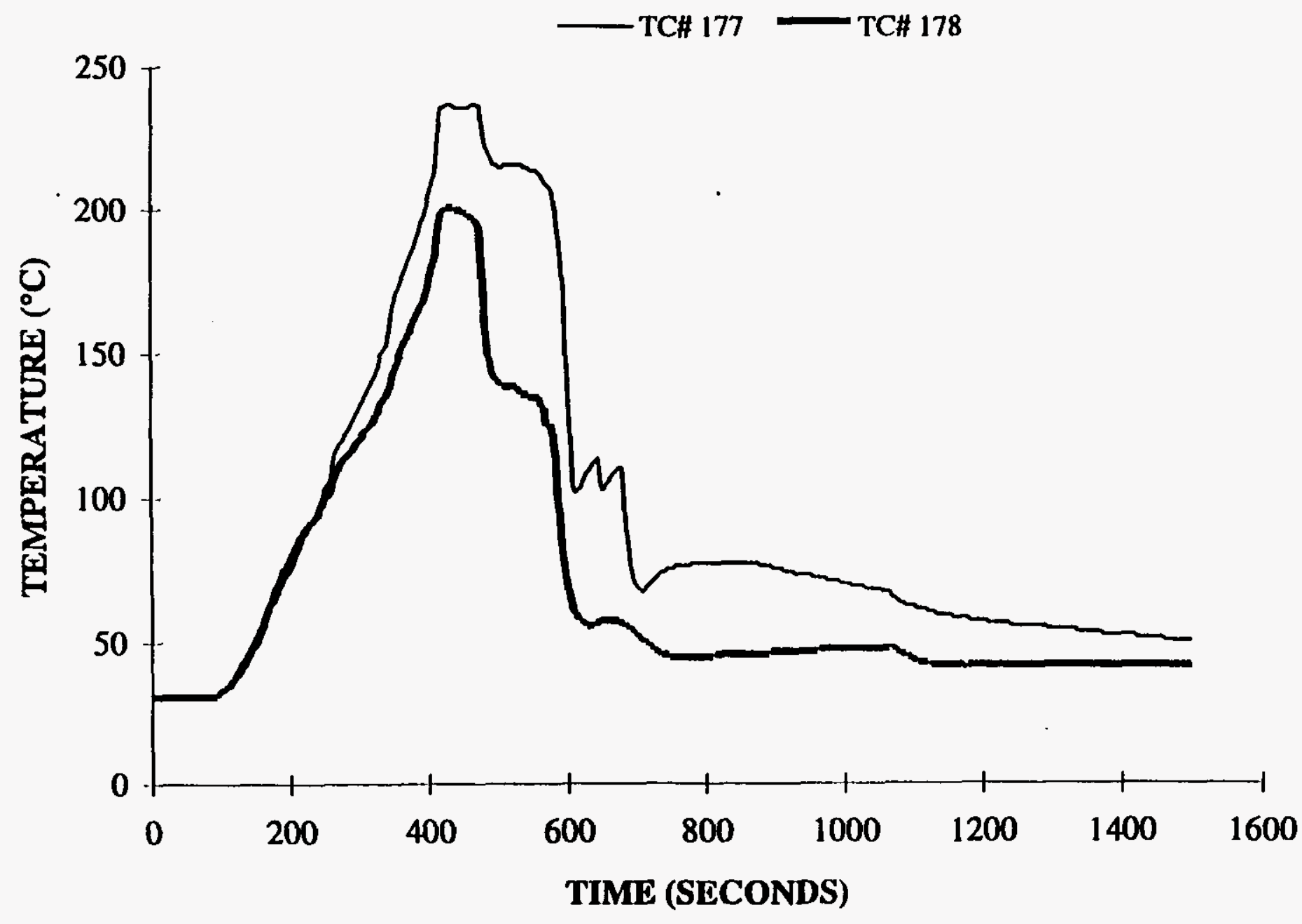

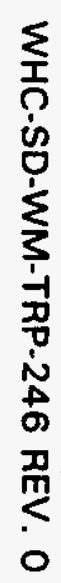


CLIENT: LATA

PROJECT NO.: SwRI 01-7106

DATE: 6 JUNE 1995

TEST ID: 157LR5.DAT

\section{RACK STORAGE TEST \\ MIDDLE BEAM, NORTH SIDE}

OF RACK 2

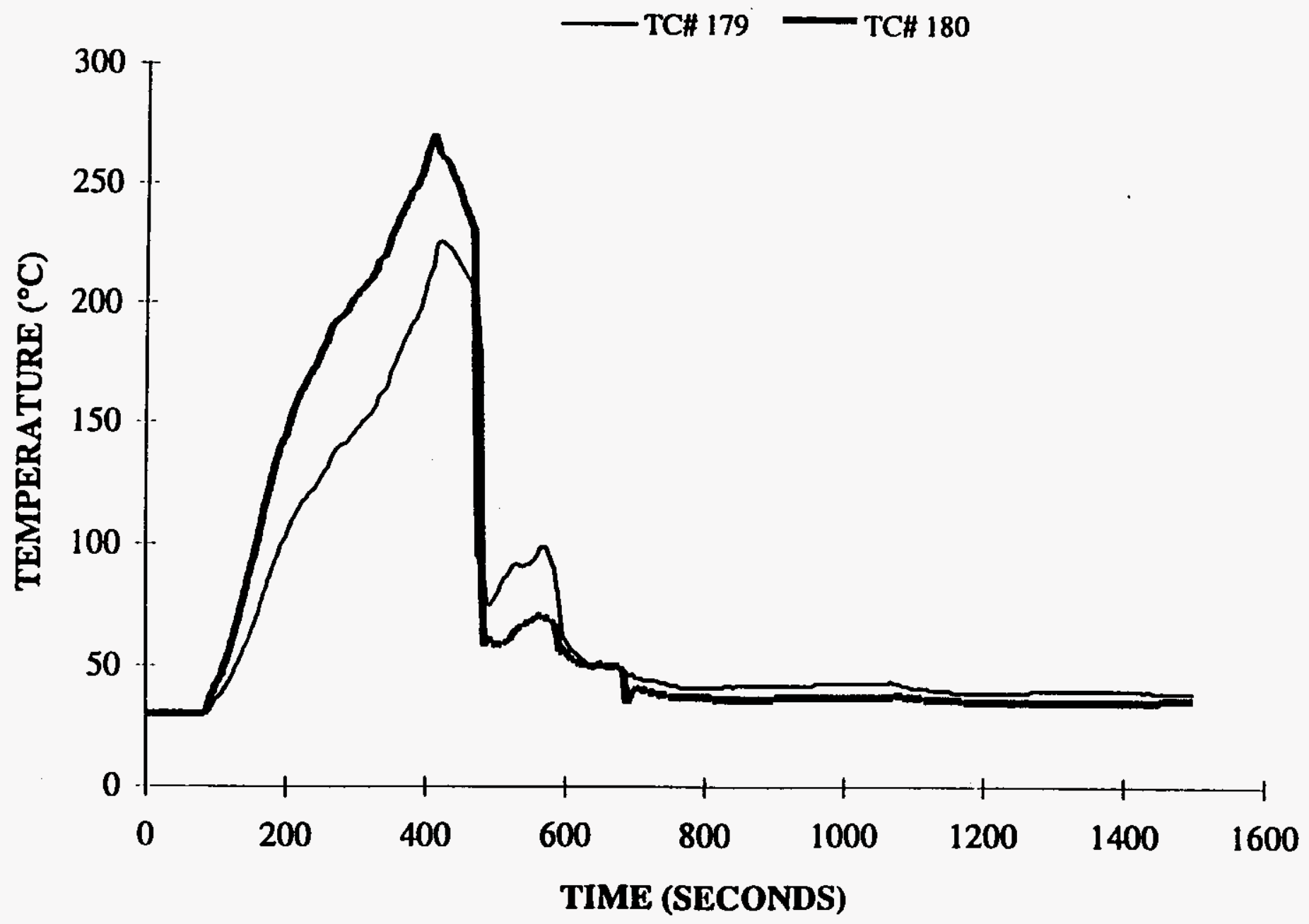


CLIENT: LATA

PROJECT NO.: SwRI 01-7106

DATE: 6 JUNE 1995

TEST ID: 157LR5.DAT

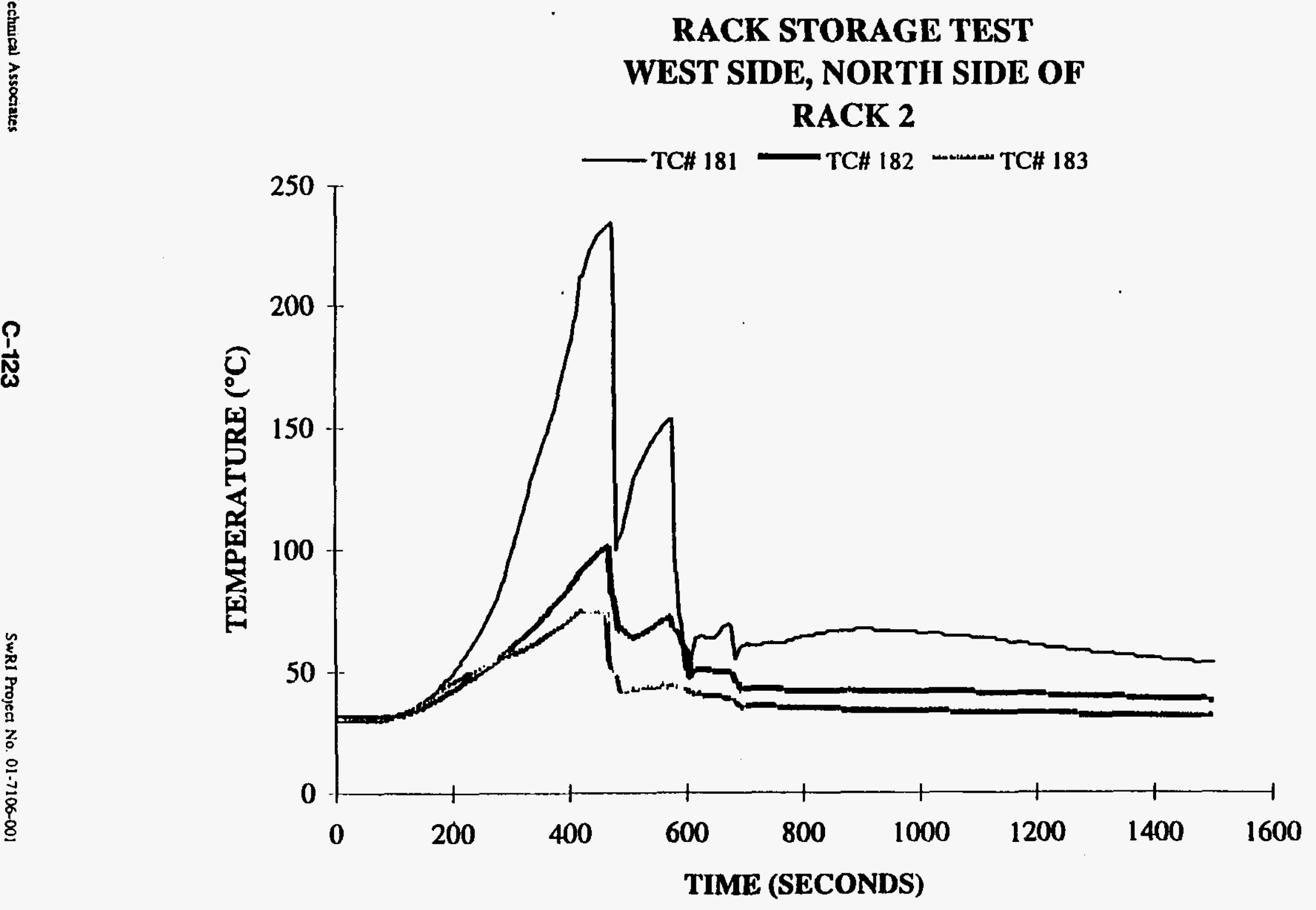


CLIENT: LATA

PROJECT NO.: SwRI 01-7106

DATE: 6 JUNE 1995

TEST ID: 157LR5.DAT

RACK STORAGE TEST

AT DRUM X236

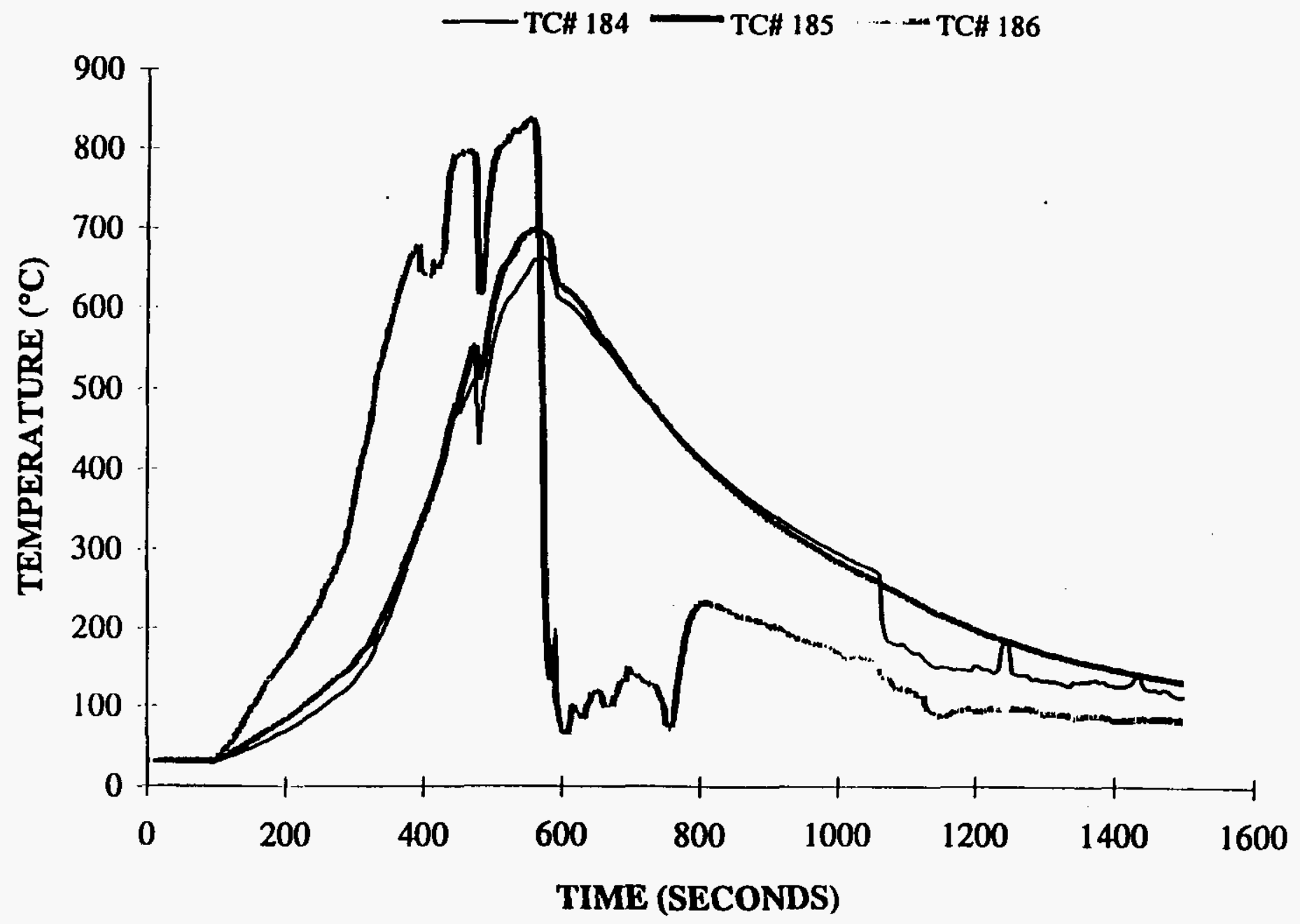

TIME (SECONDS) 
CLIENT: LATA

PROJECT NO.: SwRI 01-7106

DATE: 6 JUNE 1995

TEST ID: 157LR5.DAT

\section{RACK STORAGE TEST \\ AT DRUM X232}

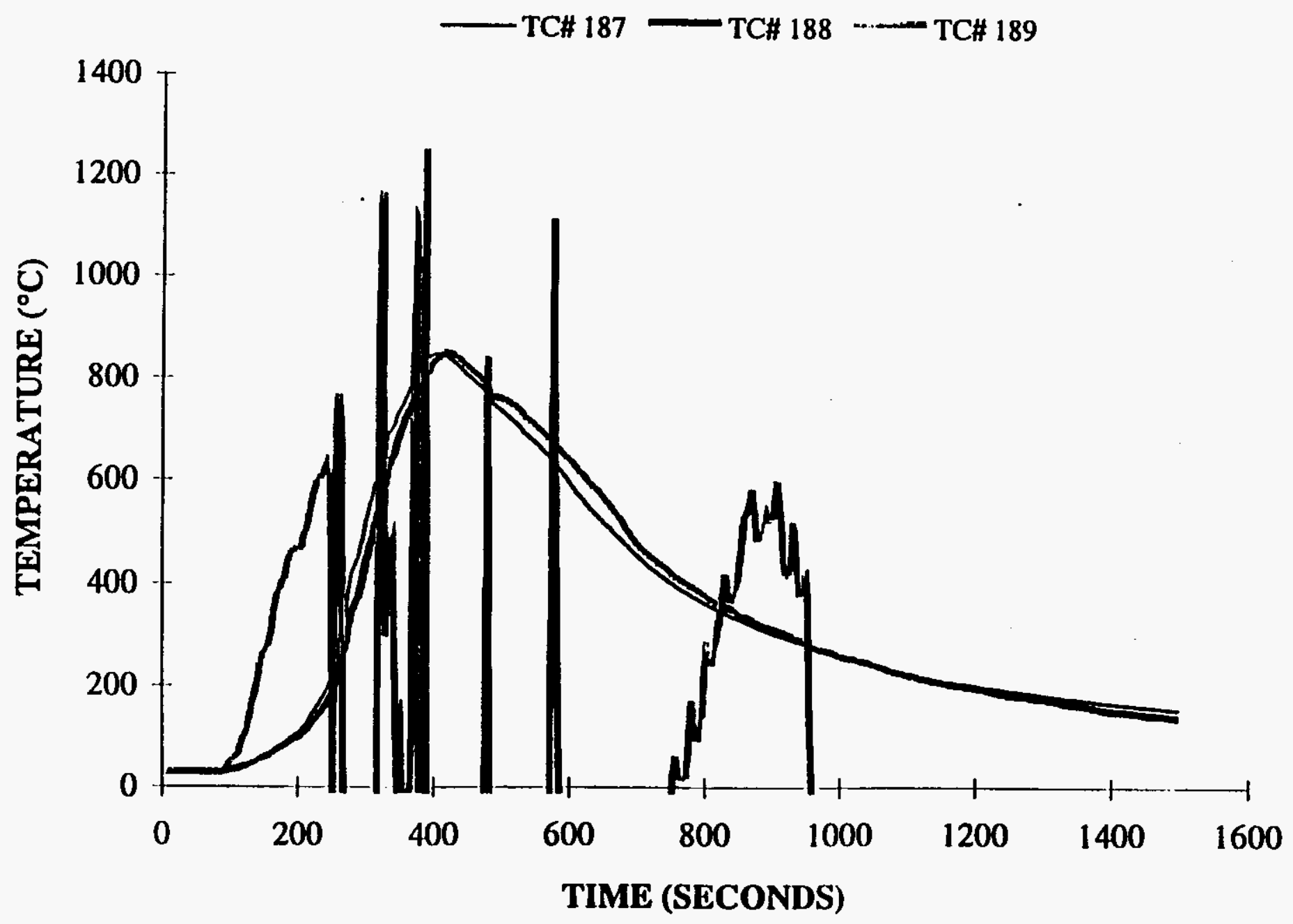


CLIENT: LATA

PROJECT NO.: SwRI 01-7106

DATE: 6 JUNE 1995

TEST ID: 157LR5.DAT

\section{RACK STORAGE TEST \\ MIDDLE BEAM, NORTH SIDE \\ OF RACK 3}

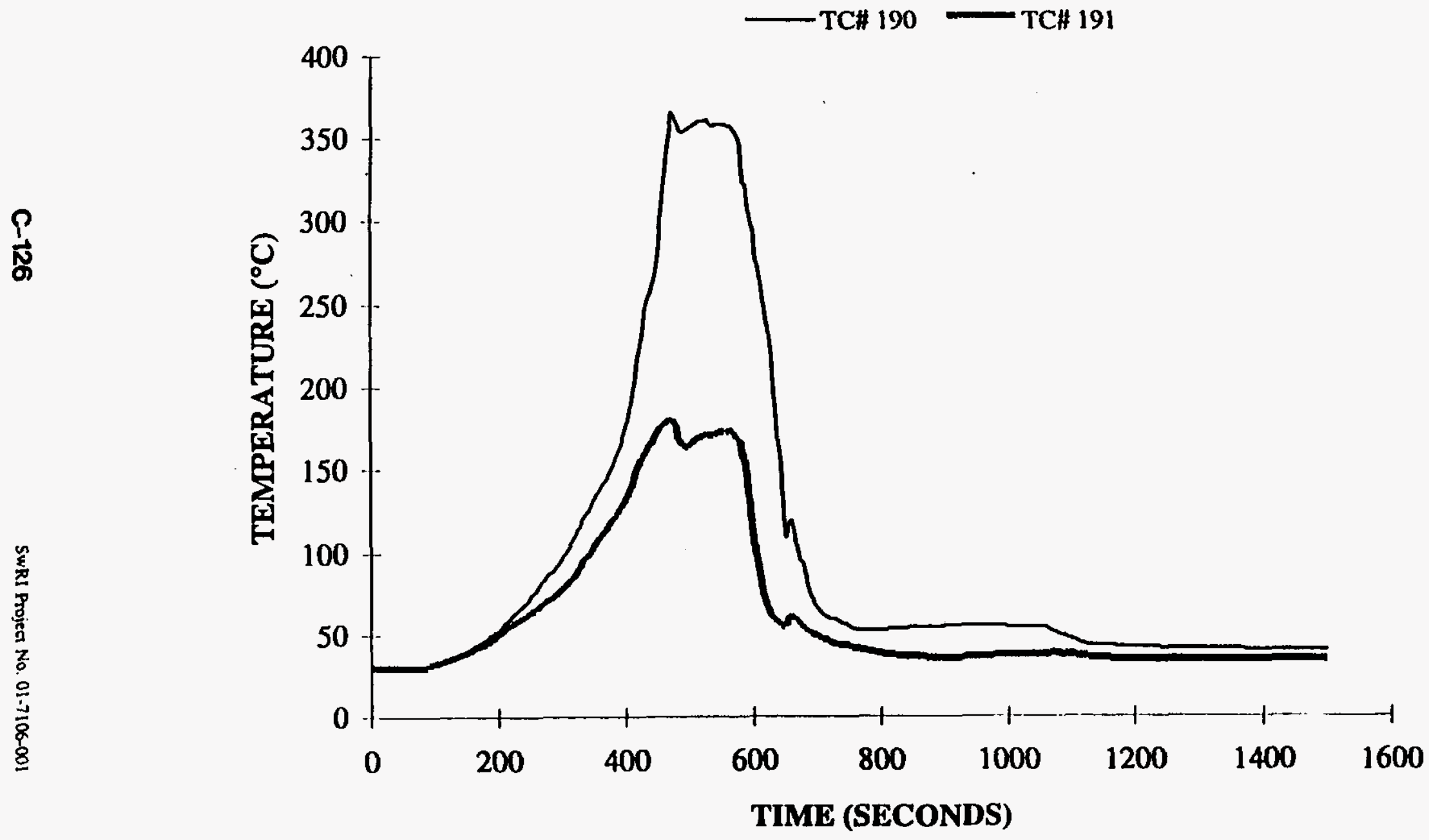


CLIENT: LATA

PROJECT NO.: SwRI 01-7106

DATE: 6 JUNE 1995

TEST ID: 157LR5.DAT

\section{RACK STORAGE TEST \\ MIDDLE BEAM, NORTH SIDE \\ OF RACK 3}

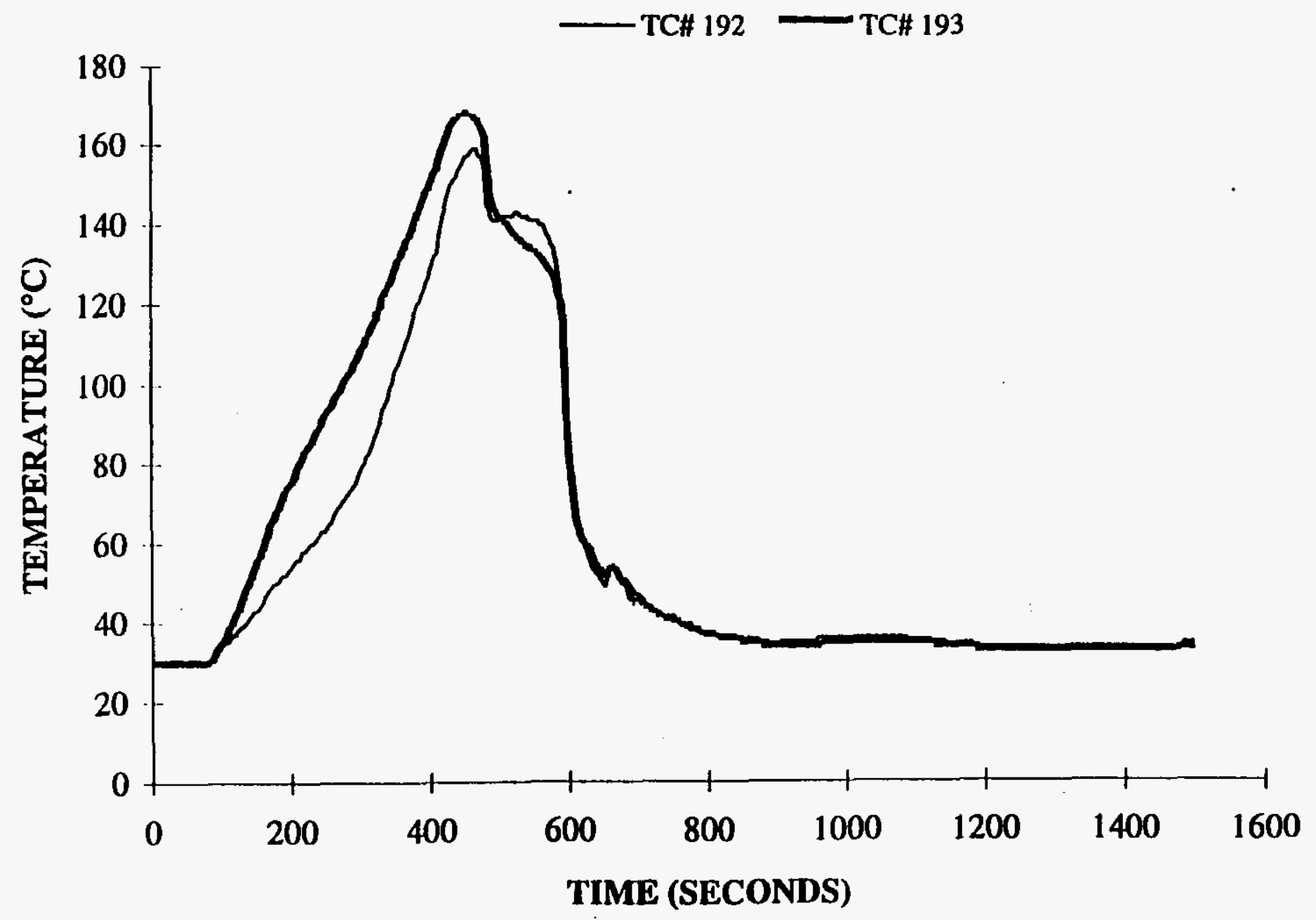

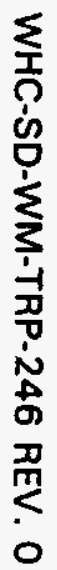


WHC-SD-WM-TRP-246 REV. 0

$\overline{-} \mid$
.0
0
0
1

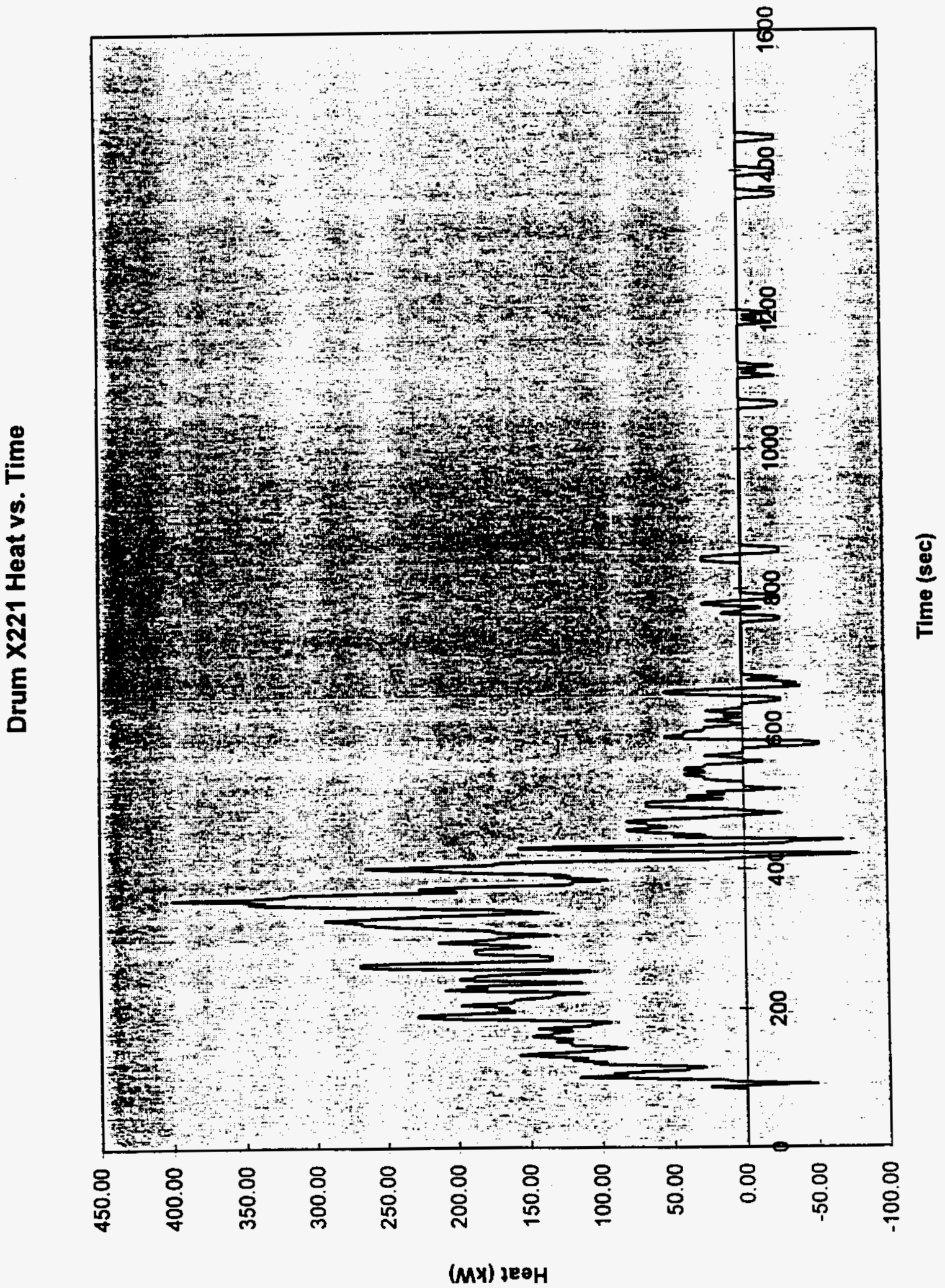



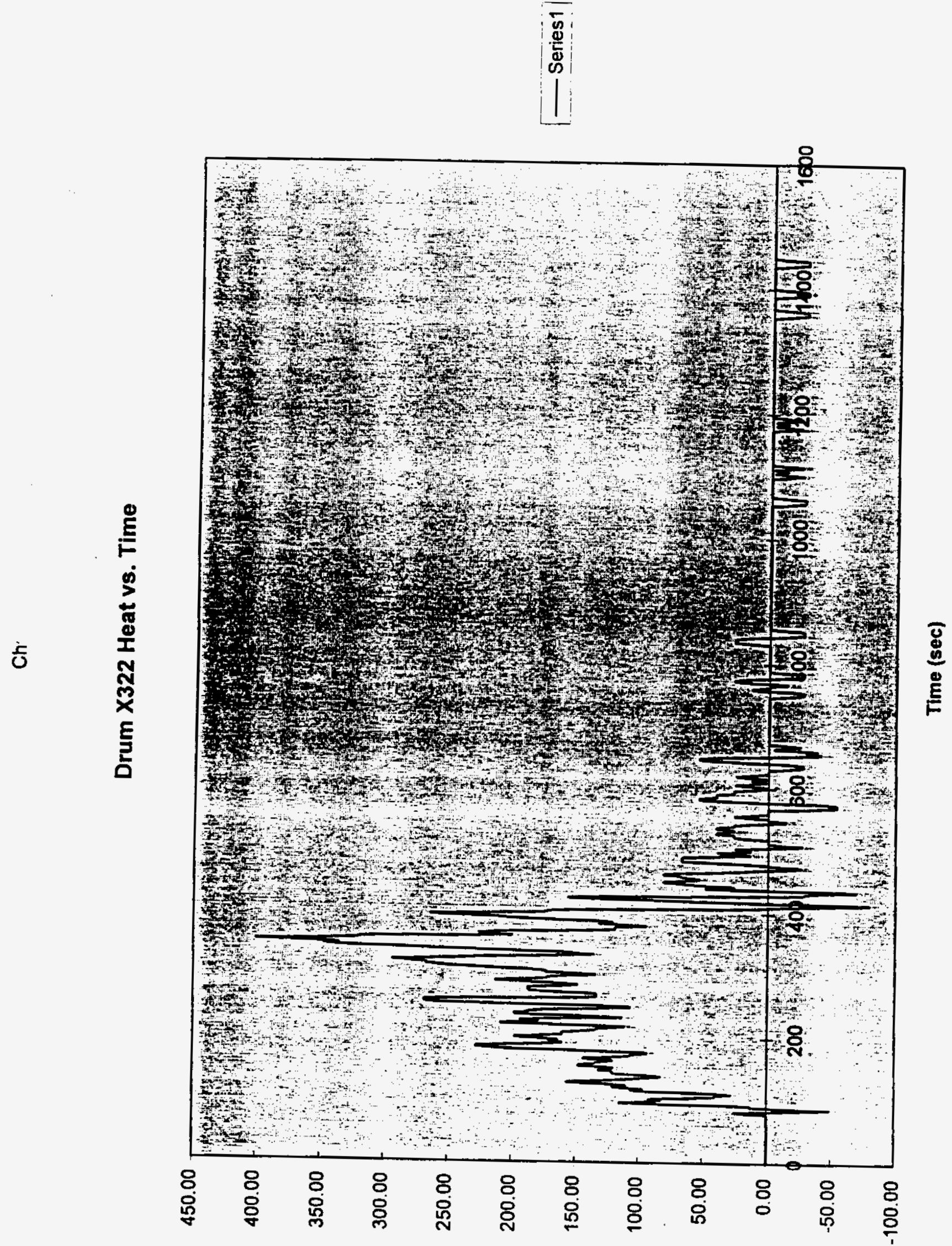

(Мי) зеЕн 
OEL-ว

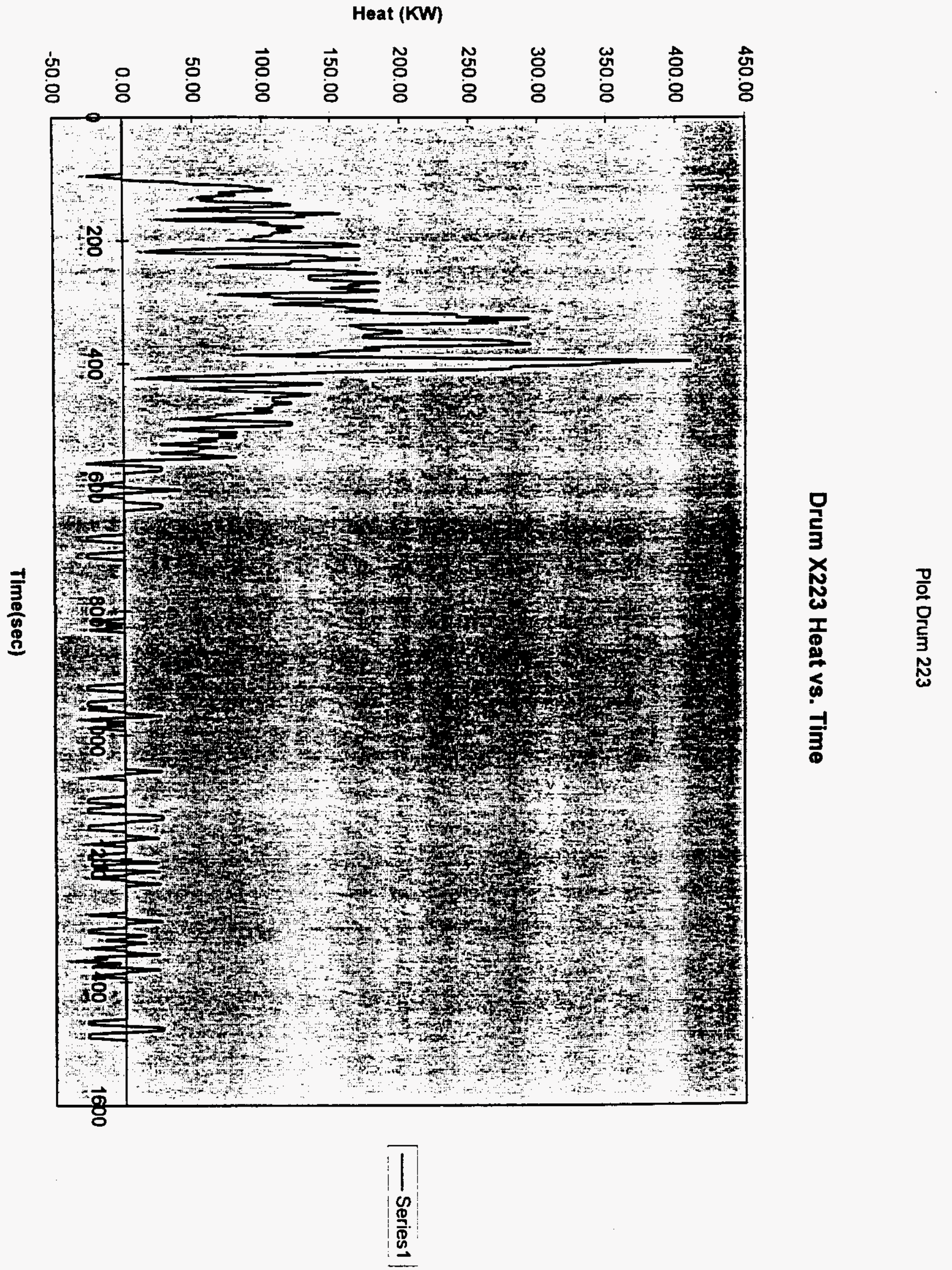


WHC-SD-WM-TRP-246 REV. 0

\section{Appendix D}

Rack Storage Drum Data Sheets

D-1 
WHC-SD-WM-TRP-246 REV. 0

Rack Storage Drum Data Sheet

Drum No. $\quad X 111$

Description (check):

Standard load

Instrumented for pressure

Instrumented for temperature

$\mathrm{H}_{2} \mathrm{O}$ filled drum

\begin{tabular}{|c|c|c|c|}
\hline Loading Starting at Bottom & Layer 1 & Layer 2 & Layer 3 \\
\hline 3 pieces of steel & $\sim$ & $N / A$ & $N / A$ \\
\hline $1 / 3$ of the brown paper & $\checkmark$ & $\nu$ & $\checkmark$ \\
\hline $1 / 3$ of the 10 -mil plastic & $\checkmark$ & $C$ & $\checkmark$ \\
\hline $1 / 3$ of the bond paper & 2 & r & \\
\hline $1 / 3$ of the towels & 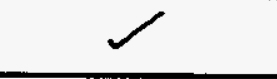 & & $\mathcal{L}$ \\
\hline $1 / 3$ of the bags & 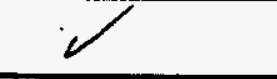 & $\checkmark$ & 2 \\
\hline $1 / 3$ of the cut paper & $\checkmark$ & $\sim$ & $\checkmark$ \\
\hline $1 / 3$ of the fabric & & $\checkmark$ & $\checkmark$ \\
\hline $1 / 3$ of the rubber & & $\nu$ & - \\
\hline & Go to Layer 2 & Go to Layer 3 & \\
\hline
\end{tabular}

Drum Filled:

Date

$5-1-95$

Initial AP

Lid sealed and torqued: $\quad$ Date $5-1-95$ Initial AP

Leak tested:

16

Pre-test weight $(\mathrm{kg})$

Post-test weight $(\mathrm{kg})$

Loss $=$
N/A or Pressure (psig)

Pressure (psig)

Date

175.3 


\section{Rack Storage Drum Data Sheet}

Drum No. $\quad \underline{x 111} u t=175.01 \mathrm{~b}$

\section{Post-test Assessment}

Position:

original:

tipped:

toppled

Lid on drum: $\quad \times$ Yes No

Gasket missing (cm) 35 "

Contents expelled: _ues $\underline{x}$ No

Seal failure: $\quad$ Yes $\quad x$ Not visible

Burn pattern:

$24 "$ at mid $l t$.

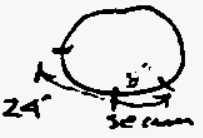

Photo taken:

Yes $\stackrel{\infty}{\longrightarrow}$ No

Leak tested: $\quad$ N/AO Or Pressure (psig)

Pressure (psig)

Date

\begin{tabular}{lll}
- & Time $(\min )$ & 0 \\
\hline & Time $(\min )$
\end{tabular}

$\overline{\text { Initial }}$

Interior assessment:

$\frac{7-3 \text { "han ou fire side - plastic melted on fan inde }}{\text { but litte to no chan }}$

$\therefore$ Dees 43

Photo taken:

$\underline{X}$ Yes

Comments: 
Drum No. $\quad x 121$

Description (check):

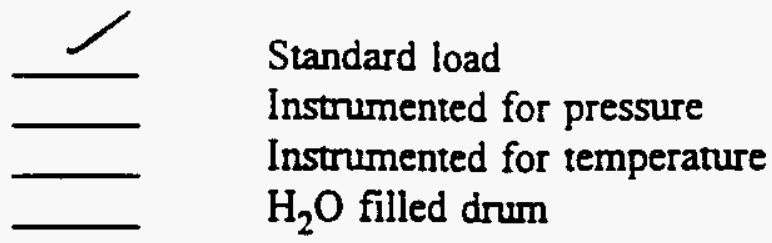

\begin{tabular}{|c|c|c|c|}
\hline Loading Starting at Bottom & Layer 1 & Layer 2 & Layer 3 \\
\hline 3 pieces of steel & 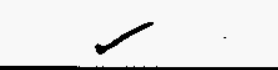 & $N / A$ & $N / A$ \\
\hline $1 / 3$ of the brown paper & $\checkmark$ & $c$ & $r$ \\
\hline $1 / 3$ of the $10-\mathrm{mil}$ plastic & $\checkmark$ & 0, & $\checkmark$ \\
\hline $1 / 3$ of the bond paper & $\checkmark$ & $t^{\prime}$ & $\int$ \\
\hline $1 / 3$ of the towels & $\checkmark$ & $\checkmark$ & $\mathcal{V}$ \\
\hline $1 / 3$ of the bags & $V$ & $\checkmark$ & $\checkmark$ \\
\hline $1 / 3$ of the cut paper & $V$ & $\checkmark$ & U \\
\hline $1 / 3$ of the fabric & $\checkmark$ & 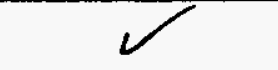 & $\checkmark$ \\
\hline $1 / 3$ of the rubber & $\checkmark$ & $\checkmark$ & $\checkmark$ \\
\hline & Go to Layer 2 & Go to Layer 3 & \\
\hline
\end{tabular}

Drum Filled:

Lid sealed and torqued:

Leak tested:

ib

Pre-test weight $(\mathrm{kg})$

Post-test weight $(\mathrm{kg})$

Loss $=$
Date $5-1-95$ Initial AP

Initial $A P$

N/A or Pressure (psig)

Pressure (psig)

Date

\begin{tabular}{lll} 
& Time (min) & 0 \\
\hline Initial & Time (min)
\end{tabular}


*ak RMIS View/Frint Document Cover Sheet tow

This document was retrieved from the Documentation and Records Manaqement (DRM) ISEARCH System. It is intended for Information only and may not be the most recent or updated version. Contact a Document Service Center (see Hanford Info for locations) if you need additional retrieval information.

Accession \#: D195061638

Document \#: SD-WM-TRP-246

Title/Desc:

SOLID WASTE DRUM ARRAY FIRE PERFORMANCE [SEC 3 OF 7]

Pages: 151 
THIS DOCUMENT WAS TOO LARGE TO SCAN AS A SINGLE DOCUMENT. IT REQUIRED BREAKING INTO SMALLER SECTIONS.

DOCUMENT NUMBER: SD-WM.TRP. 246

SECTION: $\quad 3 \quad$ OF 7

TITLE: shlid waste Drum array fine Performance

AUTHOR: Lowies PL

RECIPIENT:

DATE: $11 / 07 / 95$

KEYWORDS:

REFERENCES: EDT-612232 
Blanter 


\section{Rack Storage Drum Data Sheet}

Drum No. $\underline{x 121}-175.1$ ib.

\section{Post-tert Assessment}

Position: __ original; __ tipped; __ toppled

Lid on drum: $\quad X$ Yes $\quad$ No

Gasket missing (cm) ___ 37 " on bum one

Contents expelled: _ـ Yes $X$ No

Seal failure: $\quad$ Yes $\underset{\text { Not visible }}{ }$

Burn pattern: -
26 "at mid Lt. centered m seam

Photo taken:

$$
\text { Yes } \underset{x}{\sim} \text { No }
$$

Leak tested:

N/A or Pressure (psig)

Pressure (psig)

Date \begin{tabular}{lll} 
& Time (min) & 0 \\
\hline & Time (min)
\end{tabular}

Interior assessment:

2 "chan on burn area, barely discolored on opposite

ince 35

Photo taken:

$\underline{X}$ Yes No

Comments: 
WHC-SD-WM-TRP-246 REV. 0

\section{Rack Storage Drum Data Sheet}

Drum No. $x_{1} 3(P)$

Description (Check):

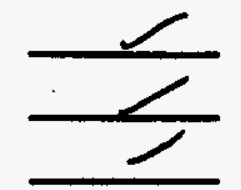

Standard load

Instrumented for Pressure

Instrumented for temperature

$\mathrm{H}_{2} \mathrm{O}$ filled dnum

\begin{tabular}{|c|c|c|c|}
\hline Loading Starting at Bottom & Layer 1 & Layer 2 & Layer 3 \\
\hline 3 pieces of stcel & & & \\
\hline $1 / 3$ of the brown paper & & & \\
\hline $1 / 3$ of the 10-mil plastic & & & \\
\hline $1 / 3$ of the bond paper & & & \\
\hline $1 / 3$ of the towels & & & \\
\hline $1 / 3$ of the bags & & & \\
\hline $1 / 3$ of the cut paper & & & \\
\hline $1 / 3$ of the fabric & & & \\
\hline $1 / 3$ of the rubber & Go to Layer 2 & Go to Layer 3 & \\
\hline
\end{tabular}

Drum Filled:

Ring bolt placed opposite drum seam and in down position

Lid sealed and torqued to $40 \mathrm{ft} / \mathrm{lb}$ :

Leak tested:

Pre-test weight (lb)

Post-test weight (lb)
Date 5.2.95 Initial L.K

Date 5.2 .95 Initial L./L
N/A or Pressure (psig)

Pressure (psig)

Date

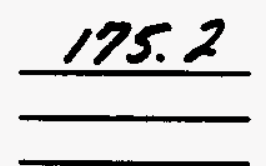

Time (min) 0 Time (min) 5

Loss = 


\section{Rack Storage Drum Data Sheet}

Drum No. $\quad X / 31(p)$ et. 174.9 ih

\section{Post-test Assessment}

Position: original; tipped; toppled

Lid on drum: $\quad \quad \quad X$ Yes No

Gasket missing (cm) __ $29^{\prime \prime}$

Contents expeiled: _ Yes $\backslash$ No

Seal failure: $\quad$ Yes $X$ Not visible

Burn pattern: $24^{\circ}$

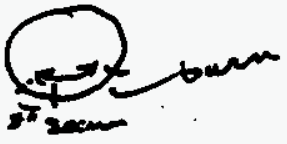

Photo taken:

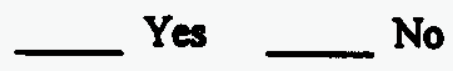

Leak tested:

N/A or Pressure (psig)

Time $(\min ) \quad \frac{0}{5}$
Time $(\min )$

Pressure (psig)

Date

Initial

Interior assessment:

2" at bum area other side unchaned.

Photo taken:

Yes

Comments: 


\section{Rack Storage Drum Data Sheet}

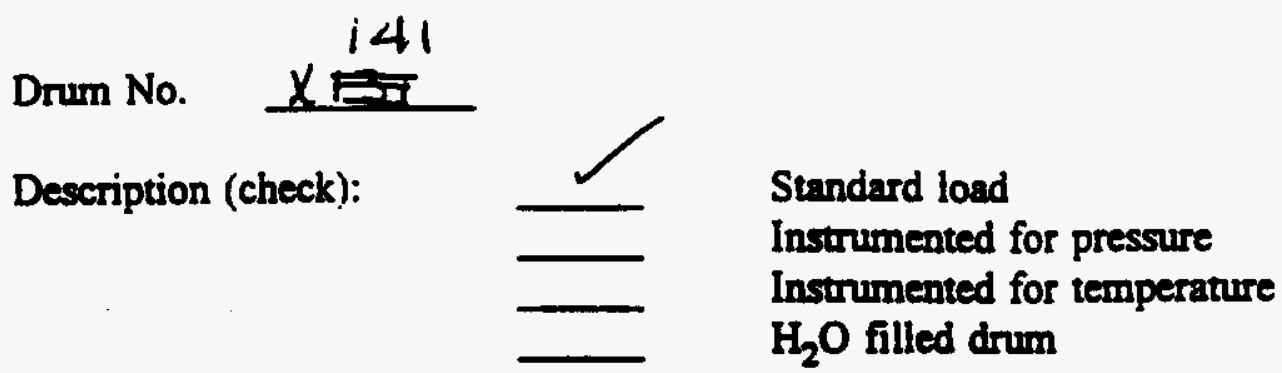

\begin{tabular}{|c|c|c|c|}
\hline Loading Starting at Bottom & Layer 1 & Layer 2 & Layer 3 \\
\hline 3 pieces of steel & & \\
\hline $1 / 3$ of the brown paper & & \\
\hline $1 / 3$ of the 10 -mil plastic & & \\
\hline $1 / 3$ of the bond paper & & & \\
\hline $1 / 3$ of the toweis & & & \\
\hline $1 / 3$ of the bags & & \\
\hline $1 / 3$ of the cut paper & & \\
\hline $1 / 3$ of the fabric & & Go to Layer 3 & \\
\hline $1 / 3$ of the rubber & & \\
\hline
\end{tabular}

Drum Filled:

Date $5-1-55$ Initial th

Lid sealed and torqued: Date $5-1-95$ Initial AP

Leak tested:

Pre-test weight (1/8)
Post-test weight (kg)

Loss $=$

Post-test weight (kg)
N/A or Pressure (psig) Pressure (psig)

Date

\section{2}


WHC-SD-WM-TRP-246 REV. 0

Rack Storage Drum Data Sheet

Drum No. $\underline{x / 41} \quad 1755 \mathrm{ib}$.

Post-test Assessment

Position: original: tipped; toppled

Lid on drum: $\quad X$ Yes No

Gasket missing (cm) _ 22" at bum area - rest of gas bet like arew Contents expelled: $\longrightarrow$ Yes $X$ No

Seal failure: : Yes $X$ Not visible

Burn pattern: $17 \%$

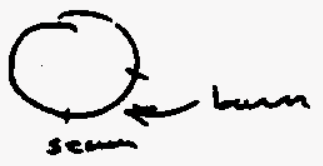

Photo taken: Y Yes $\underset{\text { No }}{\longrightarrow}$

Leak tested: N/A or Pressure (psig) Pressure (psig) Date

\begin{tabular}{lll}
- & Time (min) & 0 \\
\hline & Time (min)
\end{tabular}

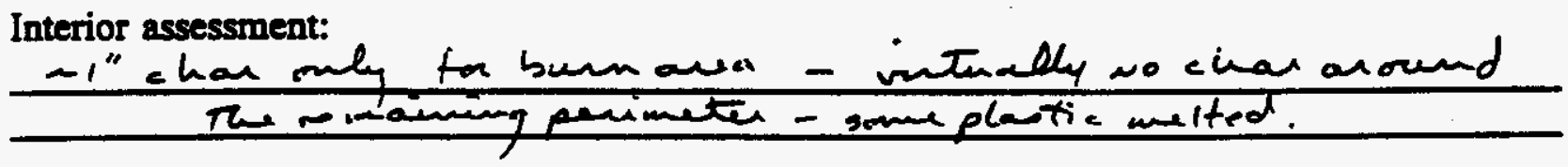

$v:$ ise i3

Photo taken:

$x$ Yes

Comments: 


\section{Rack Storage Drum Data Sheet}

Drum No. $X_{1} 51(P)$

Description (Check):

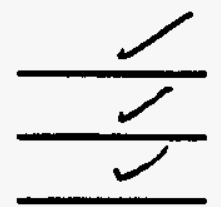

Standard load

Instrumented for Pressure

Instrumented for temperature

$\mathrm{H}_{2} \mathrm{O}$ filled drum

\begin{tabular}{|c|c|c|c|}
\hline Loading Starting at Bottom & Layer 1 & Layer 2 & Layer 3 \\
\hline 3 pieces of steel & & & \\
\hline $1 / 3$ of the brown paper & & & \\
\hline $1 / 3$ of the 10-mil plastic & & & \\
\hline $1 / 3$ of the bond paper & & & \\
\hline $1 / 3$ of the toweis & & & \\
\hline $1 / 3$ of the bags & & & \\
\hline $1 / 3$ of the cut paper & & & \\
\hline $1 / 3$ of the fabric & & & \\
\hline $1 / 3$ of the rubber & Go to Layer 2 & Go to Layer 3 & \\
\hline & & & \\
\hline
\end{tabular}

Drum Filled:

Date 5.2 .95 Initial L.R

Ring bolt placed opposite drum seam Date $5-2-95$ Initial L.K

and in down position

Lid sealed and torqued to $40 \mathrm{fthb}$ Date $5=2.95$

Leak tested:

Pre-test weight (lb)

Post-test weight (lb)
N/A or Pressure (psig)

Pressure (psig)

Date

\begin{tabular}{lll}
- & Time $(\min )$ & 0 \\
\hline & Time $(\min )$ & 5 \\
\hline
\end{tabular}

Initial

$126.6 \quad$ Target weight $-175.6 \mathrm{lb}$ 


\section{Rack Storage Drum Data Sheet}

Drum No. $\quad x \mid 51(P) \quad 176.61 b$.

\section{Post-test Assessment}

Position:

original:

tipped; toppled

Lid on drum: $\quad x$ Yes No

Gasket missing (cm) $D$ compreshed thand for 28 "

Contents expelled: _ Yes $X$ No

Seal failure: $\quad$ Yes $X$ Not visible

Burn pattern:

none

Photo taken: _ Yes $X$ No

Leak tested: $\quad$ T/A or Pressure (psig)

Pressure (psig)

Date

\begin{tabular}{lll}
- & Time (min) & 0 \\
\hline
\end{tabular}

Interior assessment:

I"chan is" span to right d seam

ivideo bes

Proto taken:

$\underline{x}$ Yes

No

Comments:

 
Rack Storage Drum Data Sheet

Drum No. $\times \stackrel{1621}{\times}$

Description (check):

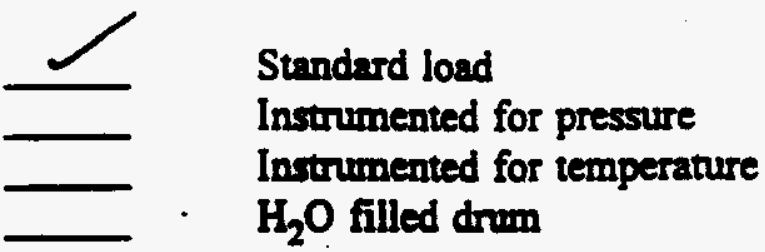

\begin{tabular}{|c|c|c|c|}
\hline Loading Starting at Bottom & Layer 1 & Layer 2 & Layer 3 \\
\hline 3 pieces of steel & & $N / A$ & $N / A$ \\
\hline \multicolumn{4}{|l|}{$1 / 3$ of the brown paper } \\
\hline \multicolumn{4}{|l|}{$1 / 3$ of the 10 -mil plastic } \\
\hline \multicolumn{4}{|l|}{$1 / 3$ of the bond paper } \\
\hline \multicolumn{4}{|l|}{$1 / 3$ of the towels } \\
\hline $1 / 3$ of the bags & 1 & 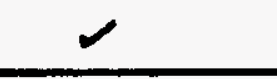 & \\
\hline \multicolumn{4}{|l|}{$1 / 3$ of the cur paper } \\
\hline \multicolumn{4}{|l|}{$1 / 3$ of the fabric } \\
\hline \multicolumn{4}{|l|}{$1 / 3$ of the rubber } \\
\hline & Go to Layer 2 & Go to Layer 3 & \\
\hline
\end{tabular}

Drum Filled:

Date $5-1-95$ Initial $A P$

Lid sealed and torqued: Date $5-1-95$ Initial AP

Leak tested:

N/A or Pressure (psig)

Pressure (psig)

Date

$-\quad$ Time (min) $\frac{0}{5}$

ib

Pre-test weight (kg)

Post-test weight (kg)

1753

Loss $=$ 
WHC-SD-WM-TRP-246 REV. 0

Rack Storage Drum Data Sheet

Drum No. $\quad x / 61 \quad \underline{t}=175.713$.

Post-test Assessment

Position:

original;

tipped; toppled

Lid on drum: $\quad X$ Yes No

Gasket missing (cm) $O$ like vew

Contents expelled: __ Yes $x$ No

Seal failure: $\quad$ Yes $x$ Not visible

Bum pattern: Nowe

Photo taken: $\quad$ Yes $\underline{x}$ No

Leak tested: N/A or Pressure (psig) Pressure (psig)

Date

$\square \quad$\begin{tabular}{ll} 
Time $(\min )$ & 0 \\
\hline & Time $(\min )$
\end{tabular}

Interior assessment:

like new

Initial

xidec 59

Xivoto taken:

$X$ Yes _ No

Comments:

iveny dean 
WHC-SD-WM-TRP-246 REV. 0

Rack Storage Drum Data Sheet

Drum No. $\quad x \mapsto \neg 1$

Description (check):

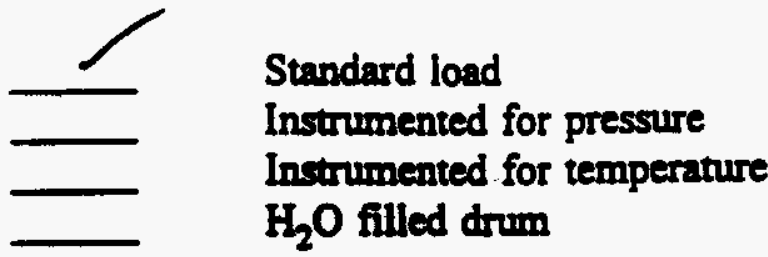

\begin{tabular}{|c|c|c|c|}
\hline Loading Starting at Bottom & Layer 1 & Layer 2 & Layer 3 \\
\hline 3 pieces of steel & $\checkmark$ & $N / A$ & $N / A$ \\
\hline \multicolumn{4}{|l|}{$1 / 3$ of the brown paper } \\
\hline \multicolumn{4}{|l|}{$1 / 3$ of the 10 -mil plastic } \\
\hline \multicolumn{4}{|l|}{$1 / 3$ of the bond paper } \\
\hline \multicolumn{4}{|l|}{$1 / 3$ of the towels } \\
\hline \multicolumn{4}{|l|}{$1 / 3$ of the bags } \\
\hline \multicolumn{4}{|l|}{$1 / 3$ of the cut paper } \\
\hline $1 / 3$ of the fabric & $r$ & $r$ & $\checkmark$ \\
\hline \multicolumn{4}{|l|}{$1 / 3$ of the rubber } \\
\hline & Go to Layer 2 & Go to Layer 3 & \\
\hline
\end{tabular}

Drum Filled:

Lid sealed and torqued:

Leak tested:

Pre-test weight (fb

Post-test weight $(\mathrm{kg})$
Date $5-1.95$

Date $5-1-95$

N/A or Pressure (psig)

Pressure (psig)

Date

Initial

Initial AP

$$
\text { Loss }=
$$

075.7 
WHC-SD-WM-TRP-246 REV. 0

\section{Rack Storage Drum Data Sheet}

Drum No. $\quad x 171, u t=176.416$

Post-test Assessment

Position: ___ original: ___ tipped; __ toppled

Lid on drum: $\quad x$ Yes No

Gasket missing (cm) 0 gas but like weser

Contents expelled: —ues $x$ No

Seal failure: $\quad$ Yes $\quad X$ Not visible

Burn pattern:

NoNe

Photo taken:

Yes

Leak tested:

N/A or Pressure (psig)

Pressure (psig)

Date

$\square \quad$ Time $(\min ) \quad \frac{0}{5}$

Interior assessment:

No chan

vioeo $=8$

Photo taken:

$\longrightarrow$ Yes

Comments:

very dean 
WHC-SD-WM-TRP-246 REV. 0

Rack Storage Drum Data Sheet

Drum No. $\times 181$

Description (check):

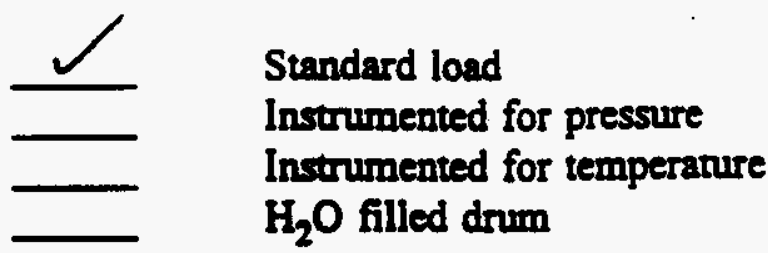

\begin{tabular}{|c|c|c|c|}
\hline Loading Starting at Bottom & Layer 1 & Layer 2 & Layer 3 \\
\hline 3 pieces of steel & & \\
\hline $1 / 3$ of the brown paper & & & \\
\hline $1 / 3$ of the 10 -mil plastic & & & \\
\hline $1 / 3$ of the bond paper & & & \\
\hline $1 / 3$ of the towels & & & \\
\hline $1 / 3$ of the bags & & & \\
\hline $1 / 3$ of the cut paper & & & \\
\hline $1 / 3$ of the fabric & & & \\
\hline $1 / 3$ of the rubber & & & \\
\hline & Go to Layer 2 & Go to Layer 3 & \\
\hline
\end{tabular}

Drum Filled:

Date

$5-1-95$

Initial AP

Lid sealed and torqued:

Date $5-1-55$

Initial AP

Leak tested:

Pre-test weight (kst)

Post-test weight (kg)
N/A or Pressure (psig)

Pressure (psig)

Date

175.6

Loss = 
WHC-SD-WM-TRP-246 REV. 0

\section{Rack Storage Drum Data Sheet}

Drum No. $\quad$ wis 1 it. $=175.6$

Post-test Assessment

Position:

original: ___ tipped:

toppled

Lid on drum: $\quad X$ Yes No

Gasket missing (cm) O feels like war

Contents expelled: _ Yes $X$ No

Seal failure: $\quad$ Yes $X$ Not visible

Burn pattern:

\section{NONe}

Photo taken: $\quad$ Yes $\varnothing$ No

Leak tested: $\quad$ N/A or Pressure (psig) Pressure (psig)

Date

\begin{tabular}{lll} 
Time (min) & 0 \\
\hline & Time (min)
\end{tabular}

Interior assessment:

filly intact - wo chaning

upec il

thoto taken:

$x$ Yes No

Comments: 


\section{Reck Storage Drum Data Sheet}

Drum No. $X 211(P)$

Description (Check):

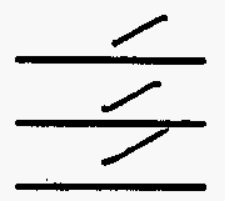

Standard load

Instrumented for Pressure

Instrumented for temperature

$\mathrm{H}_{2} \mathrm{O}$ filled drum

\begin{tabular}{|c|c|c|c|}
\hline Loading Sturting at Bottom & Layer 1 & Layer 2 & Layer 3 \\
\hline 3 pieces of steel & & & \\
\hline $1 / 3$ of the brown paper & & & \\
\hline $1 / 3$ of the 10-mil plastic & & & \\
\hline $1 / 3$ of the bond paper & & & \\
\hline $1 / 3$ of the towels & & & \\
\hline $1 / 3$ of the bags & & & \\
\hline $1 / 3$ of the cut paper & & & \\
\hline $1 / 3$ of the fabric & Go to Layer 2 & Go to Layer 3 & \\
\hline $1 / 3$ of the rubber & & & \\
\hline
\end{tabular}

Drum Filled: Ring bolt placed opposite drum seam
and in down position

Lid sealed and torqued to $40 \mathrm{ft} / \mathrm{lb}$ :

Leak tested:

Pre-test weight (lb)

Post-test weight (lb)
Date $5-2-95$ Initial $P K$

Date s-2.95 Initial R.K

Date 5-2.95 Initial R.K

N/A or Pressure (psig)

Pressure (psig)

Date

$\overline{\text { Initial }}$

Time (min) 0

Time $(\min )-5$

Target weight $-175.6 \mathrm{lb}$ 
WHC-SD-WM-TRP-246 REV. 0

\section{Rack Storage Drum Data Sheet}

Drum No. $\quad \underline{x(1(P)} 168.8 \mathrm{Ib}$.

Post-tert Assessment

Position: __ original; __ tipped; __ toppled

Lid on drum: $\quad X$ Yes No cop was wot meanened

Gasket missing (cm) At ll

Contents expelled: _u Yes $x$ No

Seal failure: $\quad X$ Yes Not visible

Burn pattern:

All acound

Photo taken: $\quad$ Yes $\leq$ No

Leak tested: $\quad$ or Pressure (psig)

Pressure (psig)

Date

\begin{tabular}{lll} 
Time (min) & 0 \\
\hline & Time (min)
\end{tabular}

Interior assessment:

$1 z^{\prime \prime}$ folerued -outente

pideo 91

photo taken:

$x$ Yes

No

Comments: 
WHC-SD-WM-TRP-246 REV. 0

\section{Rack Storage Drum Data Sheet}

Drum No. $\times 231(p)$

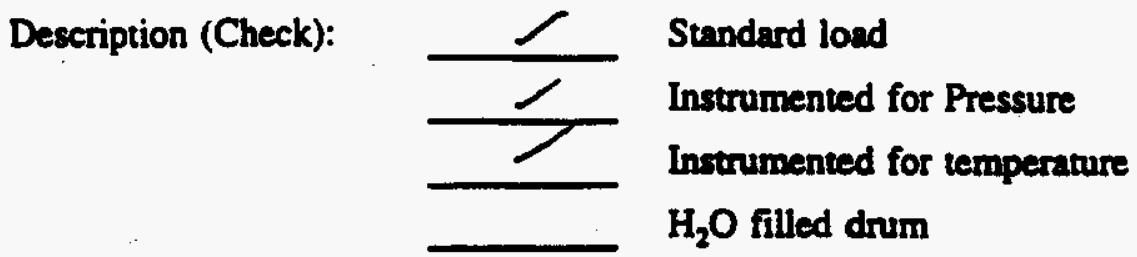

\begin{tabular}{|c|c|c|c|}
\hline Loading Starting at Bottom & Layer 1 & Layer 2 & Layer 3 \\
\hline 3 pieces of steel & & N/A & N/A \\
\hline $1 / 3$ of the brown paper & & $\checkmark$ & \\
\hline $1 / 3$ of the 10 -mil plastic & r & & \\
\hline $1 / 3$ of the bond paper & & & \\
\hline $1 / 3$ of the towels & & & \\
\hline $1 / 3$ of the bags & & - & \\
\hline $1 / 3$ of the cut paper & & & \\
\hline $1 / 3$ of the fabric & & & \\
\hline $1 / 3$ of the nubber & & & \\
\hline & Go to Layer 2 & Go to Layer 3 & \\
\hline
\end{tabular}

Drum Filled:

Ring bolt placed opposite drum seam and in down position

Lid sealed and torqued to $40 \mathrm{ft} / \mathrm{lb}$ :

Leak tested:

Pre-test weight (lb)

Post-test weight (lb)
Date $5.2 .95 \quad$ Initial LL

Date 5.2 .85 Initial 4.2
N/A or Pressure (psig)

Pressure (psig)

Date

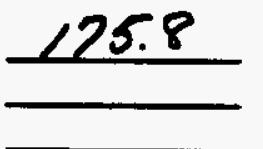

Time (min) 0

Time (min) 
WHC-SD-WM-TRP-246 REV. 0

Rack Storage Drum Data Sheet

Drum No. $\quad \underline{231} 156.0$

Post-test Assessment

Position: __ original; ___ _ _ _ _ _

Lid on drum: $\quad$ Yes $x$ No

Gasket missing (cm)

Contents expelled: __ Yes _ No

Seal failure: $\quad X$ Yes $\quad$ Not visible

Burm partern: $46^{4}$ at mind lt.

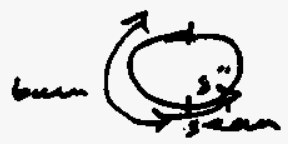

Photo taken:

\begin{tabular}{llll}
\multicolumn{1}{c}{ Yes No } & \\
$\begin{array}{l}\text { N/Abr Pressure (psig) } \\
\text { Pressure (psig) } \\
\text { Date }\end{array}$ & $\overline{\text { Time (min) }}$ & 0 \\
\hline
\end{tabular}

Interior assessment:

26 "frention to top of contents ichaund)

$v, 0<0130$

Photo taken:

$\infty$ Yes No

Comments: 


\section{Rack Storage Drum Data Sheet}

Drum No. $\times 241$

Description (Check):

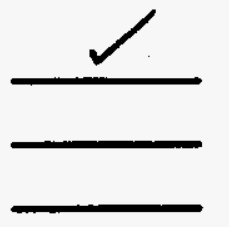

Standard load

Instrumented for Pressure

Instrumented for temperature

$\mathrm{H}_{2} \mathrm{O}$ filled drum

\begin{tabular}{|c|c|c|c|}
\hline Loading Starting at Bottom & Layer 1 & Layer 2 & Layer 3 \\
\hline 3 pieces of steel & & & \\
\hline $1 / 3$ of the brown paper & & & \\
\hline $1 / 3$ of the 10-mil plastic & & & \\
\hline $1 / 3$ of the bond paper & & & \\
\hline $1 / 3$ of the towels & & & \\
\hline $1 / 3$ of the bags & & & \\
\hline $1 / 3$ of the cut paper & & & \\
\hline $1 / 3$ of the fabric & & & \\
\hline $1 / 3$ of the rubber & Go to Layer 2 & Go to Layer 3 & \\
\hline
\end{tabular}

Drum Filled:

Ring bolt placed opposite drum seam and in down position

Lid sealed and torqued to $40 \mathrm{ft} / \mathrm{b}$ :

Leak tested:

Pre-test weight (lb)

Post-test weight (lb)
Date $5-2.85 \quad$ Initial $N=$

Date $5.9 .95 \quad$ Initial $\$$

Date 5.2 .95 Initial L.K

N/A or Pressure (psig)

Pressure (psig)

Date

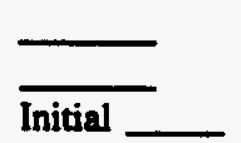

Time (min) 0

Time (min) 5

$175.5 \quad$ Target weight $-175.6 \mathrm{lb}$ 
WHC-SD-WM-TRP-246 REV. 0

Rack Storage Drum Data Sheet

Drum No. $\times 24 ! 175.616$.

Post-test Assessment

Position: original; tipped; toppled

Lid on drum: $\quad x$ Yes No nolge $14^{\text {* }}$ left $f$ ivear

Gasket missing (cm) ___ $39^{\prime \prime}$

Contents expelled: —Yes $\$ No

Seal failure: $\quad$ Yes $X$ Not visible

Burn pattern:
$17^{4}$ at mid lat.
sean $\tau_{\text {legt }}$

Photo taken:

Yes $x$ No

Leak tested:

Agressure (psig)

Pressure (psig)

Date
Time (min) $\quad 0$

Time (min) 5

Initial

Interior assessment:

top a hauned up to $7^{4}$ bum side

videc i29

photo taken:

$x$ Yes

No

Comments: 


\section{Rack Storage Drum Data Sheet}

Drum No. $\times 251$ (P)

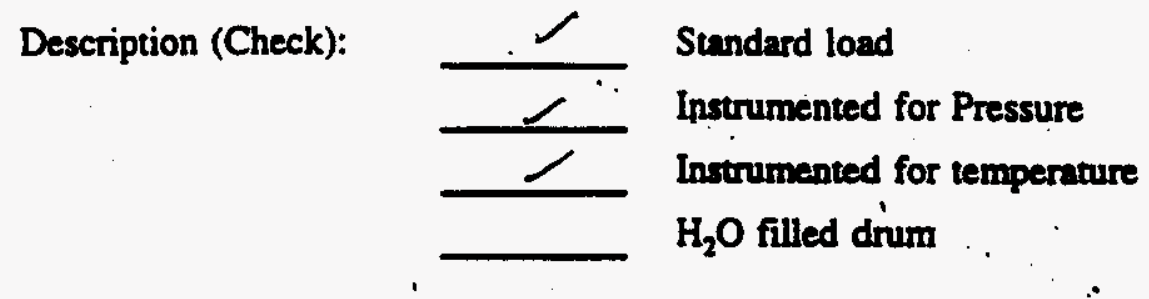

\begin{tabular}{|c|c|c|c|}
\hline Loading Starting at Bottom & Layer 1 & Layer 2 & Layer 3 \\
\hline 3 pieces of steel & & & \\
\hline $1 / 3$ of the brown paper & & & \\
\hline $1 / 3$ of the 10-mil plastic & & & \\
\hline $1 / 3$ of the bond paper & & & \\
\hline $1 / 3$ of the towels & & & \\
\hline $1 / 3$ of the bags & & & \\
\hline $1 / 3$ of the cut paper & & & \\
\hline $1 / 3$ of the fabric & & & \\
\hline $1 / 3$ of the rubber & Go to Layer 2 & Go to Layer 3 & \\
\hline
\end{tabular}

Drum Filled:

Ring boilt placed opposite drum seam and in down position

Lid sealed and torqued to $40 \mathrm{ft} / \mathrm{lb}$ :

Leak tested:

Pre-test weight (lb)

Post-test weight (lb)

Loss $=$
Date 5.2 .95 Initial $L . R$

Date 5.2 .95

Initial $P . R$

Date 5.2.95 Initial LR

N/A or Pressure (psig)

Pressure (psig)

Date

Initial

Time (min) 0

Time (min) $\frac{0}{5}$

$176.7 \quad$ Target weight $-175.6 \mathrm{lb}$ 


\section{Rack Storage Drum Data Sheet}

Drum No. $\times 25 /(P)$ at. $177.21 \mathrm{~s}$

Post-test Assessment

Position: __ original; __ tipped; __ toppled

Lid on drum: $\quad \not \subset$ Yes No

Gasket missing (cm) 0 62" compuesed s hand, gasket is buown Contents expelled: _u Yes $x$ No

Seal failure: $\quad$ Y Yes _ Not visible

Burn pattern:

NONe

Photo taken:

Yes $\chi x$ No

Leak tested:

(VIA)or Pressure (psig)

Pressure (psig)

Date

\begin{tabular}{lll} 
Tnitial & Time (min) & 0 \\
\hline
\end{tabular}

Interior assessment:

-36" seav toleft melted plastic $r$ i $/ 2$ " of than

Soec is

Photo taken:

$x$ Yes

Comments:

beern macks on lid. 


\section{Rack Storage Drum Data Sheet}

Drum No. $\times 261$

Description (Check):

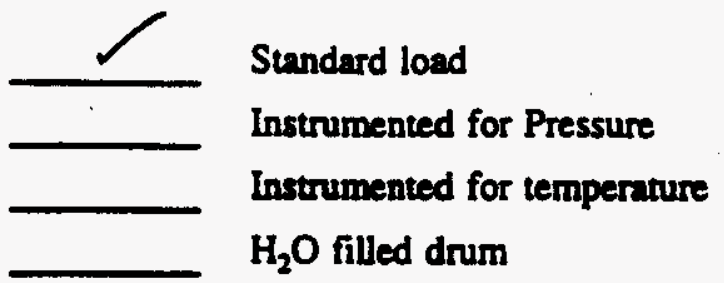

\begin{tabular}{|c|c|c|c|}
\hline Loading Starting at Bottom & Layer 1 & Layer 2 & Layer 3 \\
\hline 3 pieces of steel & $L$ & N/A & N/A \\
\hline $1 / 3$ of the brown paper & $\sim$ & & \\
\hline $1 / 3$ of the 10-mil plastic & & & \\
\hline $1 / 3$ of the bond paper & & & \\
\hline $1 / 3$ of the towels & - & & \\
\hline $1 / 3$ of the bags & & & \\
\hline $1 / 3$ of the cut paper & $\checkmark$ & & \\
\hline $1 / 3$ of the fabric & - & & \\
\hline $1 / 3$ of the rubber & & & \\
\hline
\end{tabular}

Drum Filled:

Ring bolt placed opposite drum seam and in down position

Lid sealed and torqued to $40 \mathrm{ft} / \mathrm{lb}$ :

Leak tested:

Pre-test weight (lb)

Post-test weight (lb)
Date $5-1.95$ Initial A.S.

Date 5-1.95 Initial A.S.

Date $s-1-9 s \quad$ Initial $\boldsymbol{Q} . \boldsymbol{s}$
N/A or Pressure (psig)

Pressure (psig)

Date

176.4
Time (min) $\quad 0$

Time (min) 5 
WHC-SD-WM-TRP-246 REV. 0

Rack Storage Drum Data Sheet

Drum No. $\times 261 \ldots$ N. $^{\circ}=176.516$.

Post-test Assessment

Position: original; tipped; toppled

Lid on drum: $\quad \underline{x}$ Yes No

Gasket missing (cm) O like wew

Contents expelled: —u Yes $x$ No

Seal failure: $\quad$ Yes $\quad X$ Not visible

Burn pattern: vone

Photo taken: Yes $x$ No

Leak tested: $\quad$ NID or Pressure (psig) Pressure (psig)

Date $\begin{array}{ll}\text { Time }(\min ) & 0 \\ \text { Time (min) } & \end{array}$

Initial

Interior assessment:

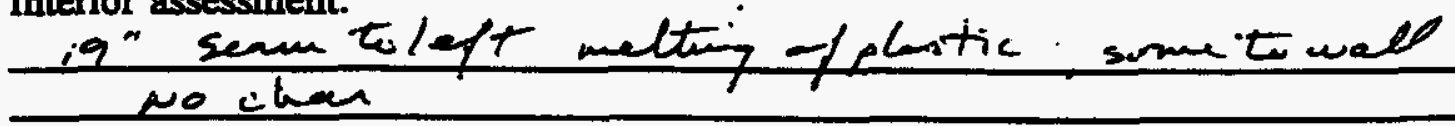

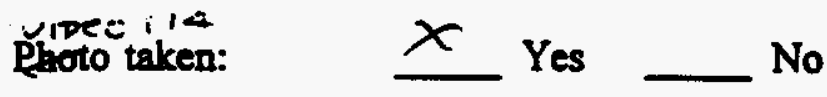

Comments: 


\section{Rack Storage Drum Data Sheet}

Drum No. $\times 271$

Description (Check):

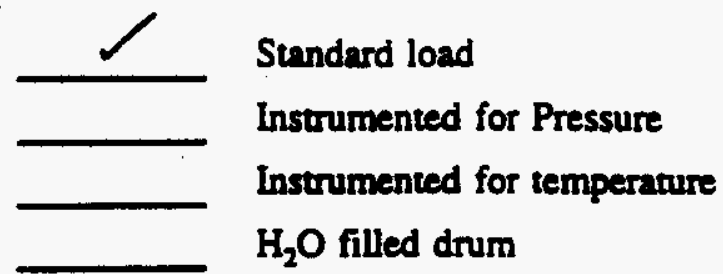

\begin{tabular}{|c|c|c|c|}
\hline Loading Starting at Bottom & Layer 1 & Layer 2 & Layer 3 \\
\hline 3 pieces of steel & 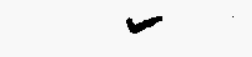 & $\mathbf{N} / \mathbf{A}$ & $\mathbf{N} / \mathbf{A}$ \\
\hline $1 / 3$ of the brown paper & $r$ & $r$ & 5 \\
\hline $1 / 3$ of the 10 -mil plastic & $س$ & 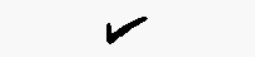 & $\boldsymbol{V}$ \\
\hline $1 / 3$ of the bond paper & $レ$ & $\checkmark$ & 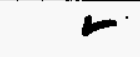 \\
\hline $1 / 3$ of the towels & $w$ & 一 & レ \\
\hline $1 / 3$ of the bags & $r$ & $L$ & \\
\hline $1 / 3$ of the cut paper & 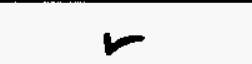 & $\boldsymbol{V}$ & $V$ \\
\hline $1 / 3$ of the fabric & $\sim$ & $\longleftarrow$ & - \\
\hline $1 / 3$ of the nubber & 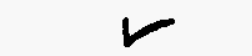 & 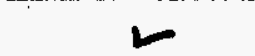 & - \\
\hline & Go to Layer 2 & Go to Layer 3 & \\
\hline
\end{tabular}

Drum Filled:

Ring bolt placed opposite drum seam Date $5-1.95$ Initial A.S . and in down position

Lid sealed and torqued to $40 \mathrm{ft} / \mathrm{lb}:$ Date $5 \cdot 1.95$ Initial A.5.

Leak tested:

Pre-test weight (lb)

Post-test weight (lb)

Loss =
N/A or Pressure (psig)

Pressure (psig)

Date

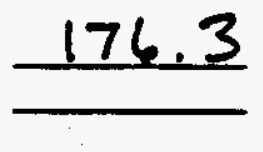

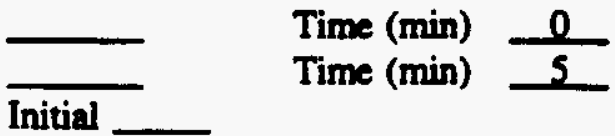

Target weight $-175.6 \mathrm{lb}$ 
WHC-SD-WM-TRP-246 REV. 0

Rack Storage Drum Data Sheet

Drum No. $\times 271 \quad 175.416$.

Post-test Assessment

Position:

original: tipped; toppled

Lid on drum: $\quad X$ Yes No

Gasket missing (cm) $O$ rike wew

Contents expelled: $\quad$ Yes $\underline{X}$ No

Seal failure: $\quad$ Yes $\underline{x}$ Not visible

Burn pattern:

none

Photo taken:

Yes

Leak tested:

NTh or Pressure (psig)

Pressure (psig)

Date

$\longrightarrow \quad$ Time $(\min ) \quad \frac{0}{5}$

Interior assessment:

rey slight melting in me spet $\sim 7$ " to left of sing seam

inseo 116

Phow taken:

$\checkmark$ Yes

Comments: 


\section{Rack Storage Drum Data Sheet}

Drum No. $\times 281$

Description (Check):

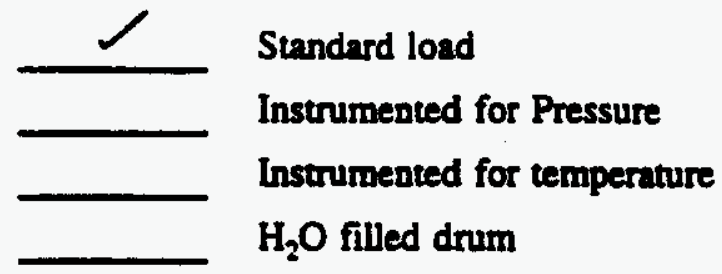

\begin{tabular}{|c|c|c|c|}
\hline Loading Starting at Bottom & Layer 1 & Layer 2 & Layer 3 \\
\hline 3 pieces of steel & & N/A & \\
\hline $1 / 3$ of the brown paper & & & \\
\hline $1 / 3$ of the 10-mil plastic & & & \\
\hline $1 / 3$ of the bond paper & & & \\
\hline $1 / 3$ of the towels & & & \\
\hline $1 / 3$ of the bags & & & \\
\hline $1 / 3$ of the cut paper & $\checkmark$ & & \\
\hline $1 / 3$ of the fabric & $\checkmark$ & & \\
\hline $1 / 3$ of the rubber & Go to Layer 2 & Go to Layer 3 & \\
\hline
\end{tabular}

Drum Filled:

Date S-1.95 Initial A.S.

Ring bolt placed opposite drum seam Date $5-1.95$ Initial $\mathrm{A}-\mathrm{S}$. and in down position

Lid sealed and torqued to $40 \mathrm{ft} / \mathrm{b}: \quad$ Date $5-1.95$ Initial $A .5$.

Leak tested:

Pre-test weight (lb)

Post-test 'weight (lb)

Loss $=$
N/A or Pressure (psig)

Pressure (psig)

Date

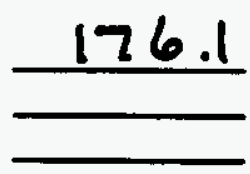

Time (min) 0

Time (min) 5

Initial

Target weight $-175.6 \mathrm{lb}$ 
WHC-SD-WM-TRP-246 REV. 0

Rack Storage Drum Data Sheet

Drum No. $\times 281$ w.t. $176: 4$ in.

Post-test Assessment

Position: __ original; ___ tipped; __ _ toppled

Lid on drum: $\quad x$ Yes No

Gasket missing (cm) O like weer

Contents expelled: _u Yes $X$ No

Seal failure: $\quad$ Yes $X$ Not visible

Burn pattern:

Nente

Photo taken:

Yes $\not 2$ No

Leak tested:

N/A. ơ Pressure (psig)

Pressure (psig)

Date

$\longrightarrow \quad$ Time (min) $\frac{0}{5}$

Interior assessment:

NO me ltin.

ve chas?

pirover : “7

$x$ Yes _ No

Comments: 
WHC-SD-WM-TRP-246 REV. 0

\section{Rack Storage Drum Data Sheet}

Drum No. $\times 311$

Description (Check):

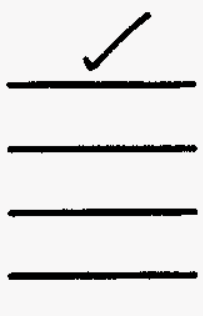

Standard load

Instrumented for Pressure

Instrumented for temperature

$\mathrm{H}_{2} \mathrm{O}$ filled drum

\begin{tabular}{|c|c|c|c|}
\hline Loading Starting at Bottom & Layer 1 & Layer 2 & Layer 3 \\
\hline 3 pieces of steel & & & \\
\hline $1 / 3$ of the brown paper & & & \\
\hline $1 / 3$ of the 10-mil piastic & & & \\
\hline $1 / 3$ of the bond paper & & & \\
\hline $1 / 3$ of the towels & & & \\
\hline $1 / 3$ of the bags & & & \\
\hline $1 / 3$ of the cut paper & & & \\
\hline $1 / 3$ of the fabric & & & \\
\hline $1 / 3$ of the rubber & Go to Layer 2 & Go to Layer 3 & \\
\hline & & & \\
\hline
\end{tabular}

Drum Filled:

Date $5-7.95$

Initial

Ring bolt placed opposite dnum seam and in down position

Date $5 \cdot 7 \cdot 95$

Initial

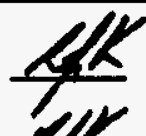

Lid sealed and torqued to $40 \mathrm{ft} / \mathrm{b}: \quad$ Date $s=2.95$ Initial $\angle L$

Leak tested:

N/A or Pressure (psig)

Pressure (psig)

Date

\begin{tabular}{|c|c|c|}
\hline & Time (min) & 0 \\
\hline & Time (min) & 5 \\
\hline
\end{tabular}

Pre-test weight (lb)

Post-test weight (lb)

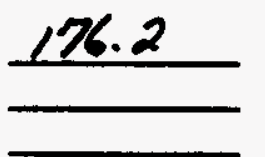

Target weight $-175.6 \mathrm{lb}$ 


\section{WHC-SD-WM-TRP-246 REV. 0 \\ Rack Storage Drum Data Sheet}

Drum No. $\quad \underline{x \cdot 311 \quad 167.116}$

\section{Post-test Assessment}

Position: original: tipped; toppled

Lid on drum: $\quad X$ Yes No $18^{\prime \prime} \times 26^{\prime \prime}$ Gep ceround boit

Gasket missing (cm) All (Iid siffited on drem)

Contents expelled: _ Yes No

Seal failure: $\quad X$ Yes Not visible

Burn pattern: Woround

Photo taken:

$$
\text { Yes } x \text { No }
$$

Leak tested: Nrisor Pressure (psig)

Pressure (psig)

Date

$-\quad$ Time (min) $\quad \frac{0}{5}$

Interior assessment:

13" from top to tep fichaned contents

videc is 3

Photo taken:

$Y$ Yes

Comments: 


\section{Rack Storage Drum Data Sheet}

Drum No. $\times 321(P)$

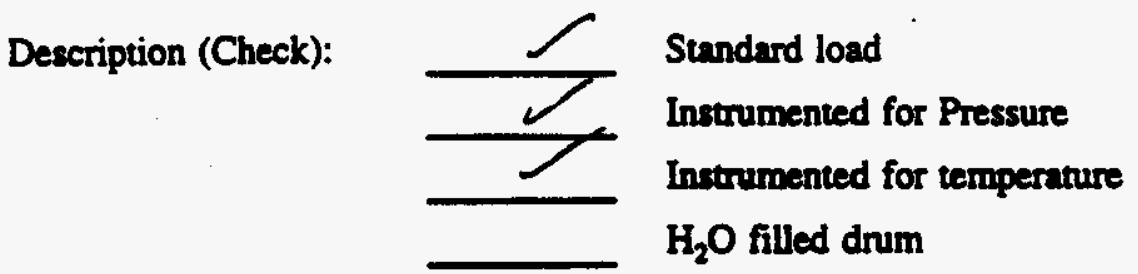

\begin{tabular}{|c|c|c|c|}
\hline Loading Starting at Bottom & Layer 1 & Layer 2 & Layer 3 \\
\hline 3 pieces of steel & & & \\
\hline $1 / 3$ of the brown paper & & & \\
\hline $1 / 3$ of the 10-mil plastic & & & \\
\hline $1 / 3$ of the bond paper & & & \\
\hline $1 / 3$ of the towels & & & \\
\hline $1 / 3$ of the bags & & & \\
\hline $1 / 3$ of the cut paper & & & \\
\hline $1 / 3$ of the fabric & & & \\
\hline $1 / 3$ of the rubber & Go to Layer 2 & Go to Layer 3 & \\
\hline
\end{tabular}

Drum Filled:

Ring boit placed opposite drum seam and in down position

Lid sealed and torqued to $40 \mathrm{f} / \mathrm{hb}$ :

Leak tested:

Pre-test weight (lb)

Post-test weight (lb)
Date $5-2.95 \quad$ Initial

Date $5.2 .95 \quad$ Initial L.K

Date $5.2 \cdot 95$ Initial $1 K$
N/A or Pressure (psig)

Pressure (psig)

Date

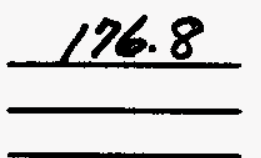

Time ( $\mathrm{min}) \quad 0$

Time (min) 5
Initial

Target weight $-175.6 \mathrm{lb}$ 
WHC-SD-WM-TRP-246 REV. 0

\section{Rack Storage Drum Data Sheet}

Drum No. $\times 321(p), 70.4 \mathrm{is}$

Post-tert Assessment

Position: original: tipped; toppled

Lid on drum: $\quad x$ Yes No $1 / 8^{\prime \prime}-48$ "gap (lid,lifted m dum)

Gasket missing (cm) AlI

Contents expeiled: _ـ Yes _ No

Seal failure: $\quad \quad X$ Yes $\quad$ Not visible

Burn pattern:

all around

Photo taken:

$$
\text { Y Yes } x \text { No }
$$

Leak tested:

$\overparen{\text { HA }}$ or Pressure (psig)

Pressure (psig)

Date

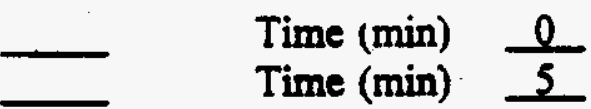

Interior assessment: 14 " top of dum te top fol haukd contents

widec is 4

Phototaken:

$x$ Yes _ No

Com̄inents: 
WHC-SD-WM-TRP-246 REV. 0

\section{Reck Storage Drum Data Sheet}

Drum No. $\times 331$

Description (Check):

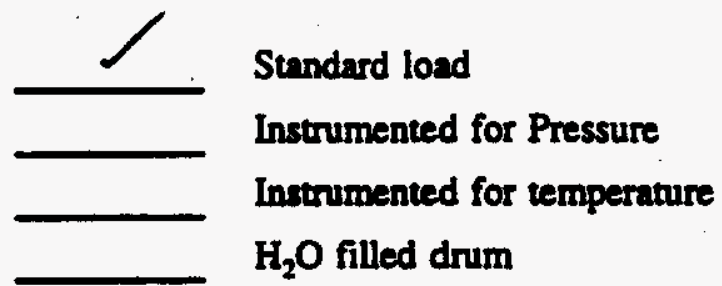

\begin{tabular}{|c|c|c|c|}
\hline Loading Starting at Bottom & Layer 1 & Layer 2 & Layer 3 \\
\hline 3 pieces of steel & & N/A & N/A \\
\hline $1 / 3$ of the brown paper & $\checkmark$ & & \\
\hline $1 / 3$ of the 10-mil plastic & - & & \\
\hline $1 / 3$ of the bond paper & $\checkmark$ & & \\
\hline $1 / 3$ of the towels & $\checkmark$ & $\checkmark$ & \\
\hline $1 / 3$ of the bags & $\checkmark$ & $\checkmark$ & \\
\hline $1 / 3$ of the cut paper & $\checkmark$ & $\checkmark$ & \\
\hline $1 / 3$ of the fabric & $\checkmark$ & $\checkmark$ & \\
\hline $1 / 3$ of the rubber & $\checkmark$ & $\checkmark$ & \\
\hline & Go to Layer 2 & Go to Layer 3 & \\
\hline
\end{tabular}

Drum Filled:

Date $5-1-95$ Initial A.S.

Ring bolt placed opposite drum seam Date 51.95 Initial A.S. and in down position

Lid sealed and torqued to $40 \mathrm{ft} / \mathrm{b}$ : Date $5-1.95$ Initial A.S.

Leak tested:

Post-test weight (lb)
N/A or Pressure (psig)

Pressure (psig)

Date

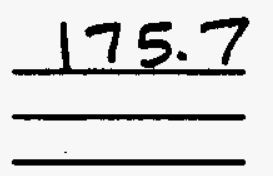

Time $(\min )$

Time (min) 5

Initial

Target weight $-175.6 \mathrm{lb}$ 


\section{WHC-SD-WM-TRP-246 REV. 0}

\section{Rack Storage Drum Data Sheet}

Drum No. $\times 331 \quad 106.4 \mathrm{ib}$.

Post-test Assessment

Position: original: tipped; toppled

Lid on drum: $\times$ Yes No $11 / 4$ "by 15 "gat

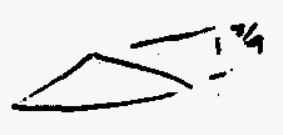

Gasket missing (cm)

Contents expelled: __ Yes __ No

Seal failure: $\quad x$ Yes Not visible

Burn pattern:

45 at mid lit

Photo taken:

Yes $\leq$ No

Leak tested:

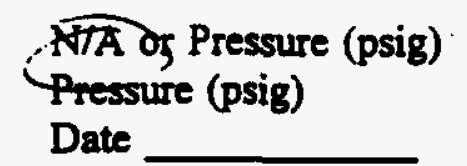

$=\quad$\begin{tabular}{ll} 
Time (min) & 0 \\
\hline
\end{tabular}

Date

Initial

Interior assessment:

$12^{2}$ tef fodum te top of banned eontents No

Comments: 


\section{Rack Storage Drum Data Sheet}

Drum No. $\times 341$

Description (Check):

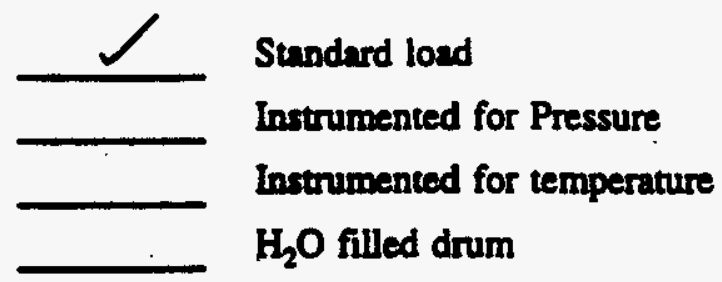

\begin{tabular}{|c|c|c|c|}
\hline Loading Starting at Bottom & Layer 1 & Layer 2 & Layer 3 \\
\hline 3 pieces of steel & - & N/A & N/A \\
\hline $1 / 3$ of the brown paper & $\boldsymbol{r}$ & レ & - \\
\hline $1 / 3$ of the 10 -mil plastic & 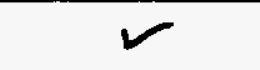 & $\checkmark$ & $\checkmark$ \\
\hline $1 / 3$ of the bond paper & ש & r & - \\
\hline $1 / 3$ of the toweis & $\boldsymbol{r}$ & - & ש \\
\hline $1 / 3$ of the bags & $\boldsymbol{\nu}$ & L & $\longleftarrow$ \\
\hline $1 / 3$ of the cut paper & レ & 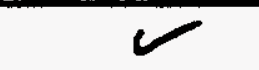 & L \\
\hline $1 / 3$ of the fabric & ᄂ & r & $\infty$ \\
\hline $1 / 3$ of the rubber & $r$ & $V$ & $\boldsymbol{V}$ \\
\hline & Go to Layer 2 & Go to Layer 3 & \\
\hline
\end{tabular}

Drum Filled:

Ring bolt placed opposite drum seam and in down position

Lid sealed and torqued to $40 \mathrm{f} / \mathrm{b}$ :

Leak tested:

Pre-test weight (lb)

Post-test weight (lb)

Loss =

Date 51.95 Initial A.S,

Date 5.1 .95 Initial A.S.

Date $5-1.45$ Initial A.S.

N/A or Pressure (psig)

Pressure (psig)

Date

175.2 Target weight $-175.6 \mathrm{lb}$ 
WHC-SD-WM-TRP-246 REV. 0

Rack Storage Drum Data Sheet

Drum No. $\times 341$

Post-tert Assessment

Position: ___ original; ___ tipped; ___ toppled

Lid on drum: $\quad X$ Yes _ No

Gacket missing (cm) _

Contents expelled: __ Yes $x$ No

Seal failure: $\quad$ Y_ Yes $\quad$ Not visible

Bum pattern:

Photo taken: $\quad$ Yes $\longrightarrow$ No

Leak tested: N/A or Pressure (psig)

Pressure (psig)

Date

$\begin{array}{ll}\text { Time (min) } & 0 \\ \text { Time (min) } & 5\end{array}$

Initial

Interior assessment:

$4-\zeta^{4}$ chai all around

Ptoro taken:

$b$ Yes No

Comments: 


\section{Rack Storage Drum Data Sheet}

Drum No. $\times 351$

Description (Check):

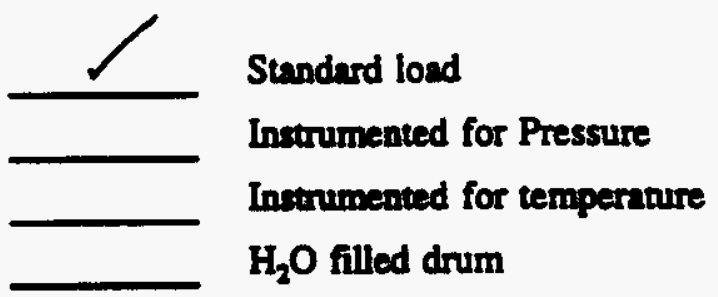

\begin{tabular}{|c|c|c|c|}
\hline Loading Starting at Bottom & Layer 1 & Layer 2 & Layer 3 \\
\hline 3 pieces of steel & & & \\
\hline $1 / 3$ of the brown paper & & & \\
\hline $1 / 3$ of the 10-mil plastic & & & \\
\hline $1 / 3$ of the bond paper & & & \\
\hline $1 / 3$ of the towels & & & \\
\hline $1 / 3$ of the bags & & & \\
\hline $1 / 3$ of the cut paper & & & \\
\hline $1 / 3$ of the fabric & Go to Layer 2 & Go to Layer 3 & \\
\hline $1 / 3$ of the rubber & & \\
\hline
\end{tabular}

Drum Filled:

Date 5.2 .85

Initial $\mathscr{P L}$

Ring bolt placed opposite drum seam Date 5.2 .95
and in down position

Lid sealed and torqued to $40 \mathrm{ft} / \mathrm{b}:$ Date 5.2 .95 Initial

Leak tested:

N/A or Pressure (psig)

Pressure (psig)

Date

\begin{tabular}{ll}
- Time (min) & 0 \\
\hline & Time (min)
\end{tabular}

Pre-test weight (lb)

$195.7 \quad$ Target weight $-175.6 \mathrm{lb}$

Post-test weight (lb)

Loss = 
WHC-SD-WM-TRP-246 REV. 0

Rack Storage Drum Data Sheet

Drum No. $\times 351$ at. 176.216.

Post-test Assessment

Position: __ original; __ tipped; __ toppled

Lid on drum: $\quad X$ Yes $\quad$ No

Gasket missing (cm) D compressed g hand $25 "$

Contents expelled: _ـ Yes $X$ No

Seal failure: $\quad$ Yes $X$ Not visible

Burn pattern:

None.

Photo taken:

Yes $x$ No

Leak tested:

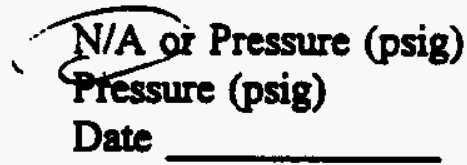

$\longrightarrow \quad$\begin{tabular}{ll} 
Time (min) & 0 \\
\hline
\end{tabular}$\quad$ Time (min)

Interior assessment:

$\frac{34^{\prime \prime} \text { from seam te right meltip plastic, }-12^{\text {"metted to well }}}{\text { Nie char }}$

Photo taken:

$\Longleftarrow$ Yes _ No

Comments: 


\section{Rack Storage Drum Data Sheet}

Drum No. $\times 3601(p)$

Description (Check):

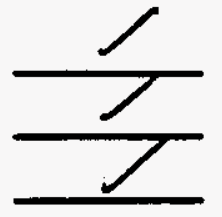

Standard load

Instrumented for Pressure

Instrumented for temperature

$\mathrm{H}_{2} \mathrm{O}$ filled drum

\begin{tabular}{|c|c|c|c|}
\hline Loading Starting at Bottom & Layer 1 & Layer 2 & Layer 3 \\
\hline 3 pieces of steel & & N/A & N/A \\
\hline $1 / 3$ of the brown paper & 7 & & \\
\hline $1 / 3$ of the 10 -mil plastic & 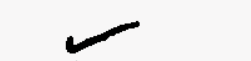 & & \\
\hline $1 / 3$ of the bond paper & $\swarrow$ & & \\
\hline $1 / 3$ of the towels & & & \\
\hline $1 / 3$ of the bags & & & $\sim$ \\
\hline $1 / 3$ of the cut paper & 1 & & \\
\hline $1 / 3$ of the fabric & $\nearrow$ & J & $r$ \\
\hline $1 / 3$ of the rubber & 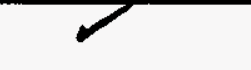 & $\gamma$ & $\nearrow$ \\
\hline & Go to Layer 2 & Go to Layer 3 & \\
\hline
\end{tabular}

Drum Filled:

Ring bolt placed opposite drum seam and in down position

Lid sealed and torqued to $40 \mathrm{ft} / \mathrm{b}$ :

Leak tested:

Pre-test weight (lb)

Post-test weight (lb)

Date $5.2 .95 \quad$ Initial Lee

Date 5.2 .95 Initial \&.K

Date 5.2 .95 Initial 2.

N/A or Pressure (psig)

Pressure (psig)

Date

175.9
Time (min) $\frac{0}{5}$

Initial

Target weight $\sim 175.6 \mathrm{lb}$ 


\section{Rack Storage Drum Data Sheet}

Drum No. $\quad \times 36 /(p) 176 .+16$

Post-test Assessment

Position:

original: ___ tipped; ___ toppled

Lid on drum: $\quad x$ Yes No

Gasket missing (cm) $O$ like wew

Contents expelled: —u Yes $x$ No

Seal failure: $\quad$ Yes $X$ Not visible

Burn pattern:

NoNe

Photo taken: $\quad$ Yes $X$ No

Leak tested:

(N/A

Date

$-\quad$\begin{tabular}{ll} 
Time (min) & 0 \\
\hline & Time (min)
\end{tabular}

Interior assessment:

bouly wetted plastic from sean to st. F26"l aly mants

thoec taken: $\quad X$ Yes No

Comments: 


\section{Rack Storage Drum Data Sheet}

Drum No. $\times 371$

Description (Check)

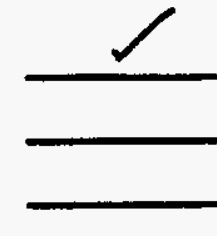

Standard load Instrumented for Pressure Instramented for temperature $\mathrm{H}_{2} \mathrm{O}$ filled drum

\begin{tabular}{|c|c|c|c|}
\hline Loading Starting at Bottom & Layer l & Layer 2 & Layer 3 \\
\hline 3 pieces of steel & & N/A & \\
\hline $1 / 3$ of the brown paper & & & \\
\hline $1 / 3$ of the 10-mil plastic & & & \\
\hline $1 / 3$ of the bond paper & & & \\
\hline $1 / 3$ of the towels & & & \\
\hline $1 / 3$ of the bags & & & \\
\hline $1 / 3$ of the cut paper & & & \\
\hline $1 / 3$ of the fabric & & & \\
\hline $1 / 3$ of the rubber & Go to Layer 2 & Go to Layer 3 & \\
\hline
\end{tabular}

Drum Filled:

Ring bolt placed opposite drum seam and in down position

Lid sealed and torqued to $40 \mathrm{ft} / \mathrm{b}$ :

Leak tested:

Pre-test weight (lb)

Post-test weight (lb)
Date 5.1 .95 Initial A.S.

Date 5.1 .95 Initial A.S.
N/A or Pressure (psig)

Pressure (psig)

Date

175.8
Time (min) $\frac{0}{5}$

Time (min)

Initial

Target weight $-175.6 \mathrm{lb}$ 
WHC-SD-WM-TRP-246 REV. 0

\section{Rack Storage Drum Data Sheet}

Drum No. $\times 371 \quad 176.2 \mathrm{~b}$.

Post-test Assessment

Position: __ original: ___ tipped; __ toppled

Lid on drum: $\quad X$ Yes No

Gasket missing (cm) $D$ like wew

Contents expelled: _ Yes $X$ No

Seal failure: $\quad$ Yes $X$ Not visible

Burn pattern:

NONZ

Photo taken:

Yes $x$ No

Leak tested:

A or Pressure (psig)

Pressure (psig)

Date

$=\quad$\begin{tabular}{ll} 
Time (min) & 0 \\
\hline & Time (min)
\end{tabular}

Interior assessment:

$\frac{\text { No neter }}{\text { No deal }}$

$v i b e c$ : $t 0$

Fioto taken:

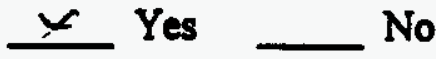

Comments: 


\section{Rack Storage Drum Data Sheet}

Drum No. $\times 381$

Description (Check):

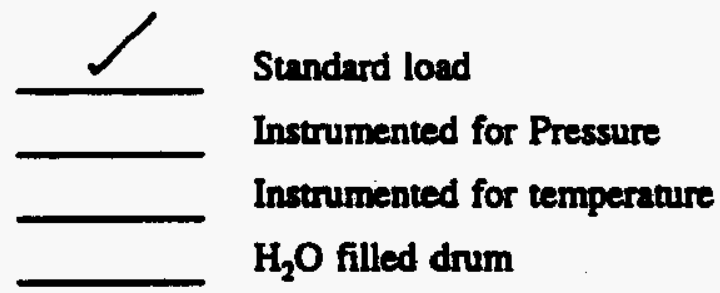

\begin{tabular}{|c|c|c|c|}
\hline Loading Starting at Bottom & Layer 1 & Layer 2 & Layer 3 \\
\hline 3 pieces of steel & & & \\
\hline $1 / 3$ of the brown paper & & & \\
\hline $1 / 3$ of the 10-mil plastic & & & \\
\hline $1 / 3$ of the bond paper & & & \\
\hline $1 / 3$ of the towels & & & \\
\hline $1 / 3$ of the bags & $\checkmark$ & & \\
\hline $1 / 3$ of the cut paper & & & \\
\hline $1 / 3$ of the fabric & & & \\
\hline $1 / 3$ of the rubber & Go to Layer 2 & Go to Layer 3 & \\
\hline
\end{tabular}

Drum Filled:

Date 5-1-9.5 Initial A.S.

Ring bolt placed opposite drum seam Date $5 \cdot 1 \cdot 95$ Initial A.S.

and in down position

Lid sealed and torqued to $40 \mathrm{fthb}$ : Date $5 . / .95$ Initial $Q . S$.

Leak tested:

N/A or Pressure (psig)

Pressure (psig)

Date

\begin{tabular}{ll} 
Time $(\min )$ & $\frac{0}{5}$ \\
\hline Initial & Time $(\min )$
\end{tabular}

Pre-test weight (lb)

$176.1 \quad$ Target weight $\sim 175.6 \mathrm{lb}$

Post-test weight (lb)

Loss = 
WHC-SD-WM-TRP-246 REV. 0

Rack Storage Drum Data Sheet

Drum No. X381 wat. $=176.3 \mathrm{H}$.

Post-tert Assessment

Position: __ original; ___ tipped; __ toppled

Lid on drum: $\quad X$ Yes $\quad$ No

Gasket missing (cm) $D$ smewhat compressed, li he vew

Contents expelled: _ـ Yes $\underline{x}$ No

Seal failure: $\quad$ Yes $X$ Not visible

Burn pattern:

NONF

Photo taken: $\quad$ Yes $\searrow$ No

Leak tested:

Pressure (psig)

Date

\begin{tabular}{ll} 
Time (min) & 0 \\
\hline
\end{tabular}

Initial

Interior assessment:

No ne chan

t.0ee 109

Photo taken:

$x$ Yes

No

Comments:

like vew 
Drum No. $\quad \times 112$

Description (check):

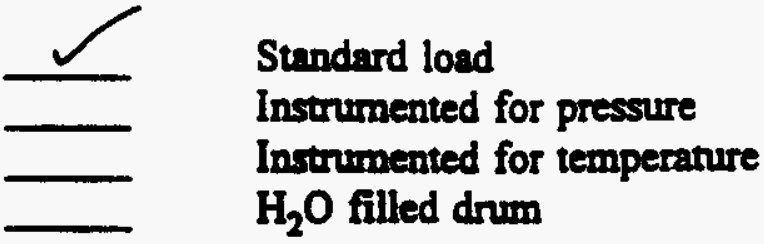

\begin{tabular}{|c|c|c|c|}
\hline Loading Sturting at Bottom & Layer 1 & Layer 2 & Layer 3 \\
\hline 3 pieces of steel & & & \\
\hline $1 / 3$ of the brown paper & & & \\
\hline $1 / 3$ of the 10-mil plastic & & $\checkmark$ & \\
\hline $1 / 3$ of the bond paper & $\checkmark$ & $\checkmark$ & \\
\hline $1 / 3$ of the towels & $\checkmark$ & $\checkmark$ & \\
\hline $1 / 3$ of the bags & $\checkmark$ & \\
\hline $1 / 3$ of the cur paper & $\checkmark$ & \\
\hline $1 / 3$ of the fabric & $\checkmark$ & \\
\hline $1 / 3$ of the rubber & $\checkmark$ & Go to Layer 3 & \\
\hline & Go to Layer 2 & & \\
\hline
\end{tabular}

Drum Filled:

Lid sealed and torqued:

Leak tested:

Pre-test weight (1)

Post-test weight (kg)

Loss $=$
Date $5-1-95$ Initial AP

Initial $A P$
N/A or Pressure (psig)

Pressure (psig)

Date

175.3

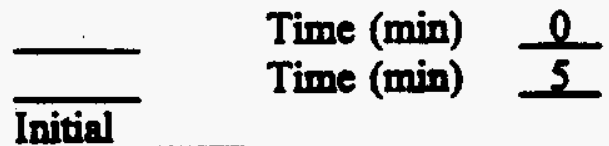

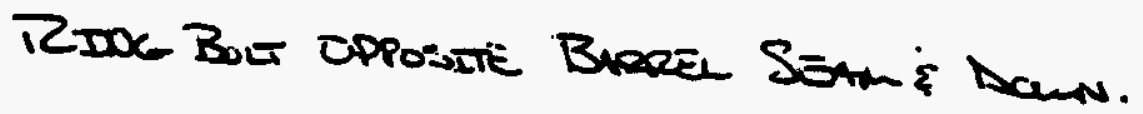


WHC-SD-WM-TRP-246 REV. 0

Rack Storage Drum Data Sheet

Drum No. $x \perp 12 \quad \omega t=174.9 \mathscr{R}_{1}$.

Post-test Assessment

Position: original:

tipped; toppled

Lid on drum:

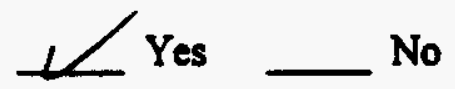

Gasket missing (cm) $29.5^{\prime \prime}$

Contents expelled: Yes No

Seal failure: Yes Not visible

Burn pattern:

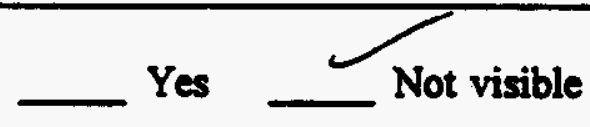

Photo taken: Yes $\underline{x}$ No

Leak tested:
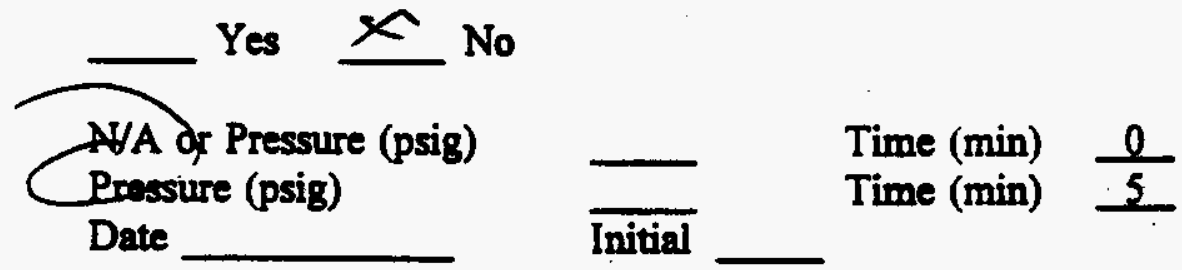

Interior assessment:

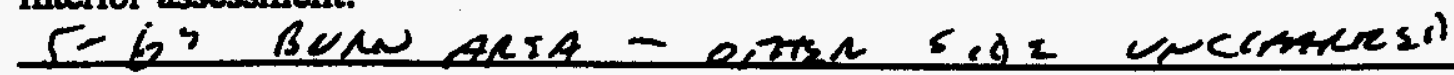

Photo taken:

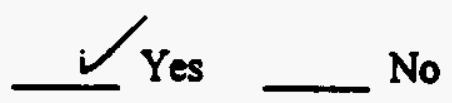

Comments: 
WHC-SD-WM-TRP-246 REV. 0

\section{Reck Storage Drum Data Sheet}

Drum No. $\times 122(8)$

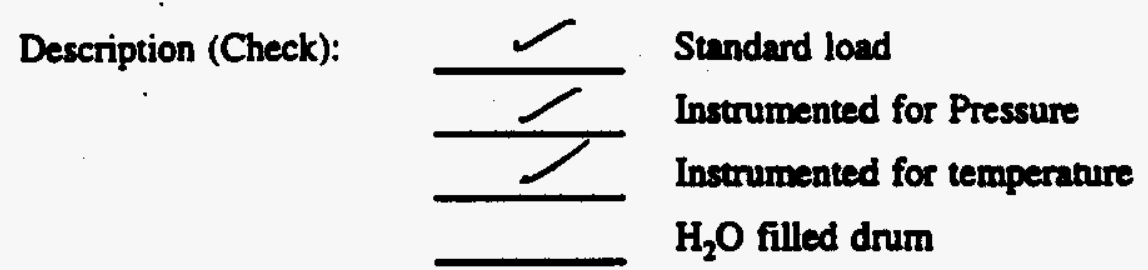

\begin{tabular}{|c|c|c|c|}
\hline Lading Staring at Bottom & Layer 1 & Layer 2 . & Layer 3 \\
\hline 3 pieces of steel & & N/A & $\mathbf{N} / \mathbf{A}$ \\
\hline \multicolumn{4}{|l|}{$1 / 3$ of the brown paper } \\
\hline \multicolumn{4}{|l|}{$1 / 3$ of the 10 -mil plastic } \\
\hline \multicolumn{4}{|l|}{$1 / 3$ of the bond paper } \\
\hline $1 / 3$ of the towels & $v$ & & \\
\hline \multicolumn{4}{|l|}{$1 / 3$ of the bags } \\
\hline \multicolumn{4}{|l|}{$1 / 3$ of the cut paper } \\
\hline \multicolumn{4}{|l|}{$1 / 3$ of the fabric } \\
\hline \multicolumn{4}{|l|}{$1 / 3$ of the rubber } \\
\hline & Go to Layer 2 & Go to Layer 3 & \\
\hline
\end{tabular}

Drum Filled:

Date $5=3-95$ Initial $\underline{R}$

Ring bolt placed opposite drum seam Date $5=3-85$ Initial L.R and in down position

Lid sealed and torqued to $40 \mathrm{fthb}$ : Date $5=3.85$ Initial L.K

Leak tested:

Pre-test weight (lb)

Post-test weight (lb)
N/A or Pressure (psig)

Pressure (psig)

Date

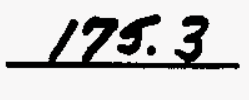

Time (min)

Initial 
WHC-SD-WM-TRP-246 REV. 0

\section{Rack Storage Drum Data Sheet}

Drum No. $\times 1.22$ wt. i75. 1 ib.

\section{Post-test Assessment}

Position: __ original; __ tipped; __ toppled

Lid on drum: $\quad X$ Yes No

Gasket missing (cm) _33" othen sich is compressed and stiff

Contents expelled: $\longrightarrow$ Yes $X$ No

Seal failure: $\quad$ Yes $X$ Not visible

Burn pattern:

21 " centened on sean

Photo taken:

Leak tested:

N/A Ot Pressure (psig)

Pressure (psig)

Date

\section{Yes $\underline{x}$ No}

$=\quad$ Time $(\min ) \quad \frac{0}{5}$

Interior assessment:

-3" at bumarea -o Then side not chaned

:0e041

Photo taken:

$X$ Yes №

Comments: 
WHC-SD-WM-TRP-246 REV. 0

\section{Rack Storage Drum Data Sheet}

Drum No. $\times 132$

Description (Check):

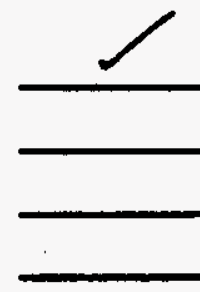

Standard load

Instrumented for Pressure

Instrumented for temperature

$\mathrm{H}_{2} \mathrm{O}$ filled drum

\begin{tabular}{|c|c|c|c|}
\hline Loading Starting at Bottom & Layer 1 & Layer 2 & Layer 3 \\
\hline 3 pieces of steel & & & \\
\hline $1 / 3$ of the brown paper & & & \\
\hline $1 / 3$ of the 10-mil plastic & & & \\
\hline $1 / 3$ of the bond paper & & & \\
\hline $1 / 3$ of the towels & & & \\
\hline $1 / 3$ of the bags & & & \\
\hline $1 / 3$ of the cut paper & & & \\
\hline $1 / 3$ of the fabric & & Go to Layer 3 & \\
\hline $1 / 3$ of the rubber & & Go to Layer 2 & Go \\
\hline
\end{tabular}

Drum Filled:

Ring bolt placed opposite drum seam and in down position

Lid sealed and torqued to $40 \mathrm{ft} / \mathrm{b}$ :

Leak tested:

Prie-test weight (lb)

Post-test weight (lb)
Date 5.145 Initial $Q P$

Date 5-145 Initial

Date $5-1-95$ Initial AP
N/A or Pressure (psig)

Pressure (psig)

Date

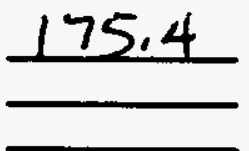

Time (min) 0

Time (min) 5
Initial

Target weight $-175.6 \mathrm{lb}$ 


\section{Rack Storage Drum Data Sheet}

Drum No. $\times 132 \quad 175.216$

Post-test Assessment

Position: __ original; __ tipped; __ toppled

Lid on drum: $\quad X$ Yes No

Gasket missing (cm) __ 30" centared on baun area

Contents expeiled: _ Yes $\not \subset$ No

Seal failure: $\quad$ Yes $\quad X$ Not visible

Burn pattern:

$17^{\prime \prime}$

Photo taken:

Yes $\underline{x}$ No

Leak tested:

ST/A Dr Pressure (psig)

Pressure (psig)

Date

Time (min) $\frac{0}{5}$

Initial

Time (min) 5

Interior assessment:

3"char at boun area - nothing m back part.

vipeo 34

Photo taken:

$\varnothing$ Yes _ No

Comments: 
WHC-SD-WM-TRP-246 REV. 0

Rack Storage Drum Data Sheet

Drum No. $\times 142$

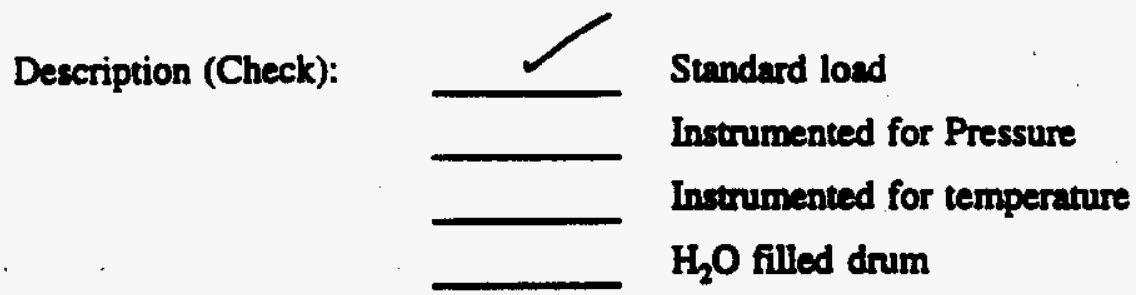

\begin{tabular}{|c|c|c|c|}
\hline Loading Starting at Bottom & Layer 1 & Layer 2 & Layer 3 \\
\hline 3 pieces of steel & & $\mathbf{N} / \mathbf{A}$ & $\mathbf{N} / \mathbf{A}$ \\
\hline \multicolumn{4}{|l|}{$1 / 3$ of the brown paper } \\
\hline \multicolumn{4}{|l|}{$1 / 3$ of the 10 -mil plastic } \\
\hline \multicolumn{4}{|l|}{$1 / 3$ of the bond paper } \\
\hline \multicolumn{4}{|l|}{$1 / 3$ of the towels } \\
\hline \multicolumn{4}{|l|}{$1 / 3$ of the bags } \\
\hline \multicolumn{4}{|l|}{$1 / 3$ of the cut paper } \\
\hline \multicolumn{4}{|l|}{$1 / 3$ of the fabric } \\
\hline \multicolumn{4}{|l|}{$1 / 3$ of the rubber } \\
\hline & Go to Layer 2 & Go to Layer 3 & \\
\hline
\end{tabular}

\section{Drum Filled:}

Ring bolt placed opposite drum seam and in down position

Lid sealed and torqued to $40 \mathrm{ft} / \mathrm{b}: \quad$ Date $5-1-95$ Initial $A P$
Date $5-1-95$ Initial AP

Date $-5-1-95$ Initial AP
Leak tested:

Pre-test weight (lb)

Post-test weight (lb)
N/A or Pressure (psig)

Pressure (psig)

Date

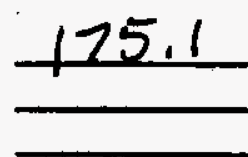


WHC-SD-WM-TRP-246 REV. 0

Rack Storage Drum Data Sheet

Drum No. $\quad y / 42$ T. $175.6 \mathrm{lb}$

\section{Post-test Assessment}

Position:

original: tipped; toppled

Lid on drum: $\quad$ Yes _ No

Gasket missing (cm) ___ is" on baun area - other gabket is acmpreased

Contents expelled: $\quad$ Yes $\longrightarrow$ No

Seal failure: $\quad$ Yes $X$ Not visible no buiging $f$ lid

Burn pattern:

$-10$

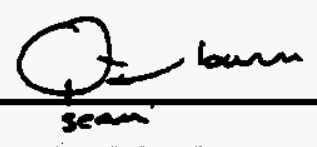

Photo taken:

Yes $\searrow$ No

Leak tested:

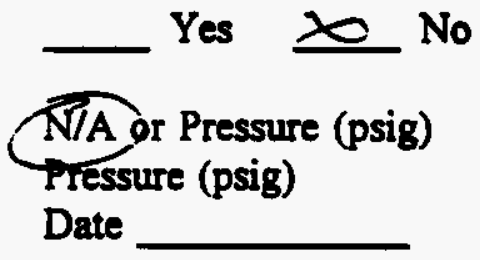

\begin{tabular}{lll} 
Initial & Time (min) & 0 \\
\hline & Time (min)
\end{tabular}

Interior assessment:

${ }_{2}$ " chan at bum area

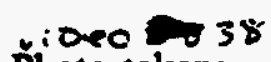

Phote taken:

$X$ Yes No

Comments: 
WHC-SD-WM-TRP-246 REV. 0

\section{Reck Storage Drum Data Sheet}

Drum No. $\times 152$

Description (Check):

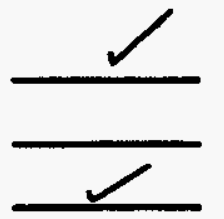

Standard load

Instrumented for Pressure

Instrumented for temperature

$\mathrm{H}_{2} \mathrm{O}$ filled drum

\begin{tabular}{|c|c|c|c|}
\hline Loading Starting at Bottom & Layer 1 & Layer 2 & Layer 3 \\
\hline 3 pieces of steel & & & \\
\hline $1 / 3$ of the brown paper & & & \\
\hline $1 / 3$ of the 10-mil plastic & & & \\
\hline $1 / 3$ of the bond paper & & & \\
\hline $1 / 3$ of the towels & & & \\
\hline $1 / 3$ of the bags & & & \\
\hline $1 / 3$ of the cut paper & & & \\
\hline $1 / 3$ of the fabric & & & \\
\hline $1 / 3$ of the rubber & Go to Layer 2 & Go to Layer 3 & \\
\hline
\end{tabular}

Drum Filled:

Date $5-1-95$ Initial DL

Ring bolt placed opposite drum seam Date $5-1-35$ Initial AP and in down position

Lid sealed and torqued to $40 \mathrm{ftllb}$ : Date $5-1-95$ Initial $\underline{A P}$

Leak tested:

Pre-test weight (lb)

Post-test weight (lb)
N/A or Pressure (psig) Pressure (psig)

Date

176.1

Time (min) 0

Time (min)

Initial

Target weight $-175.6 \mathrm{lb}$ 
WHC-SD-WM-TRP-246 REV. 0

Rack Storage Drum Data Sheet

Drum No. $\quad \times 152 \quad$ LT. $=2$

$176 . \mathrm{i}$

Post-test Assessment

Position: ___ original; ___ tipped; ___ toppled

Lid on drum: $\quad \quad X$ Yes _ No

Gasket missing (cm) $O \sim 13 "$ compresand and hand

Contents expelled: __ Yes $X$ No

Seal failure: $\quad$ Yes $X$ Not visible

Bum pattern:

NONe

Photo taken:

Yes $x$ No

Leak tested:

NAtor Pressure (psig)

Pressure (psig)

Date

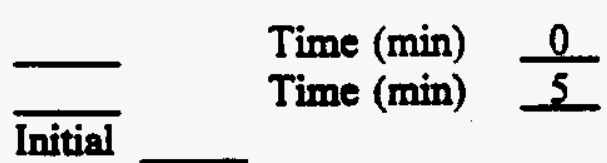

Interior assessment:

$\frac{\text { some plostic melted to right of seam }}{\text { nochan }}$

$\because p e 06 !$

"jpeo 61
phote taken:

Yes _ No

Comments: 
WHC.SD-WM-TRP-246 REV. 0

Rack Storage Drum Date Sheet

Drum No. $\times 142$

Description (Check):

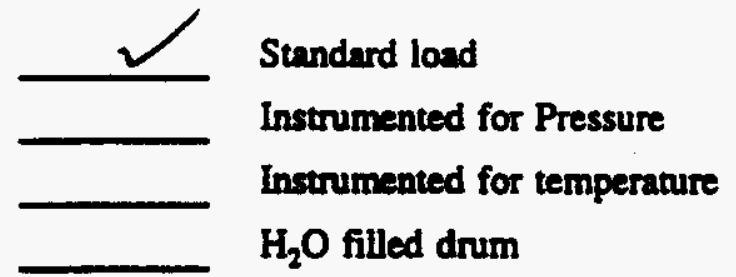

\begin{tabular}{|c|c|c|c|}
\hline Lading Starting at Bottom & Layer 1 & Layer 2 & Layer 3 \\
\hline 3 pieces of steel & & & \\
\hline $1 / 3$ of the brown paper & & & \\
\hline $1 / 3$ of the 10-mil plastic & & & \\
\hline $1 / 3$ of the bond paper & & & \\
\hline $1 / 3$ of the towels & & & \\
\hline $1 / 3$ of the bags & & & \\
\hline $1 / 3$ of the cut paper & & & \\
\hline $1 / 3$ of the fabric & & & \\
\hline $1 / 3$ of the rubber & Go to Layer 2 & Go to Layer 3 & \\
\hline
\end{tabular}

Drum Filled:

Date $5-1-95$ Initial $A P$

Ring bolt placed opposite drum seam Date $5-1-95$ Initial AP and in down position

Lid sealed and torqued to $40 \mathrm{fthb}$ : Date $5-1-95$ Initial AP

Leak tested:

Pre-test weight (lb)

Post-test weight (lb)
N/A or Pressure (psig)

Pressure (psig)

Date

$175 \cdot 2$
Time (min) 0

Time (min) 5

\section{Loss $=$}


WHC-SD-WM-TRP-246 REV. 0

Rack Storage Drum Data Sheet

Drum No. $\quad x i 62 \quad 175.216$.

\section{Post-test Assessment}

Position: __ original: __ tipped; __ toppled

Lid on drum: $\quad \quad \quad X$ Yes $\quad$ No

Gasket missing (cm) 2

Contents expelled: —Yes $x$ No

Seal failure: $\quad$ Yes $\longrightarrow$ Not visible

Burn pattern:

none

Photo taken:

Yes $X$ No

Leak tested:

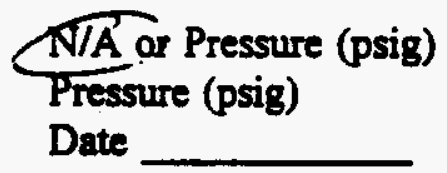

Interior assessment:

som plastic metted to wall al in 19" trom sem to rightside. wo chan

vioeo 62

Photo taken:

$x$ Yes No

Comments: 
WHC-SD-WM-TRP-246 REV. 0

Rack Storage Drum Data Sheet

Drum No. $\times 172$

Description (Check):

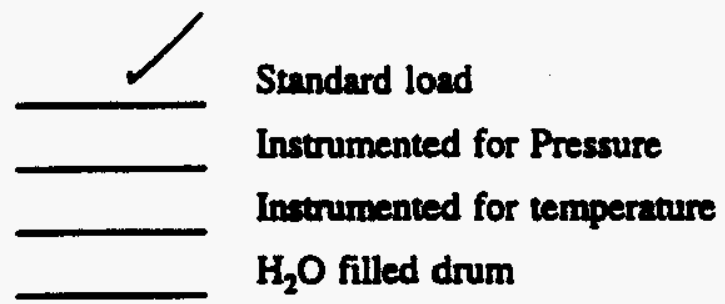

\begin{tabular}{|c|c|c|c|}
\hline Loading Staning at Bottom & Layer 1 & Layer 2 & Layer 3 \\
\hline 3 pieces of steel & & & \\
\hline $1 / 3$ of the brown paper & & & \\
\hline $1 / 3$ of the 10-mil plastic & & & \\
\hline $1 / 3$ of the bond paper & & & \\
\hline $1 / 3$ of the towels & & & \\
\hline $1 / 3$ of the bags & & & \\
\hline $1 / 3$ of the cut paper & & & \\
\hline $1 / 3$ of the fabric & & Go to Layer 3 & \\
\hline $1 / 3$ of the rubber & & Gayer 2 & Go to \\
\hline
\end{tabular}

Drum Filled:

Date $5-1-95$ Initial $4 P$

Ring bolt placed opposite drum seam Date $5-1-45$ Initial AP and in down position

Lid sealed and torqued to $40 \mathrm{ft} / \mathrm{b}: \quad$ Date $5-1-95$ Initial AP

Leak tested:

Pre-test weight (lb)

Post-test weight (lb)
N/A or Pressure (psig)

Pressure (psig)

Date

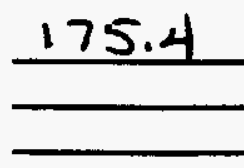

Time (min)

Time (min) $\frac{5}{5}$ 


\section{WHC-SD-WM-TRP-246 REV. 0}

\section{Rack Storage Drum Data Sheet}

Drum No. $\quad \underline{x / 72}-2+.=175.4 \mathrm{~b}$.

\section{Post-test Assessment}

Position: original; tipped; toppled

Lid on drum: $\quad \underline{x}$ Yes No

Gasket missing (cm) 0

Contents expelled: _ Yes $x$ No

Seal failure: $\quad$ Yes $X$ Not visible

Burn pattern:

None

Photo taken:

Yes $x$ No

Leak tested:

Interior assessment:

bavely meted $\sim 4$ " sean of elastic $\sim q^{\prime \prime} t$ right of ream

Photo taken:

$x$ Yes No

Comments: 


\section{Rack Storage Drum Data Sheet}

Drum No. $\times 183$

Description (Check):

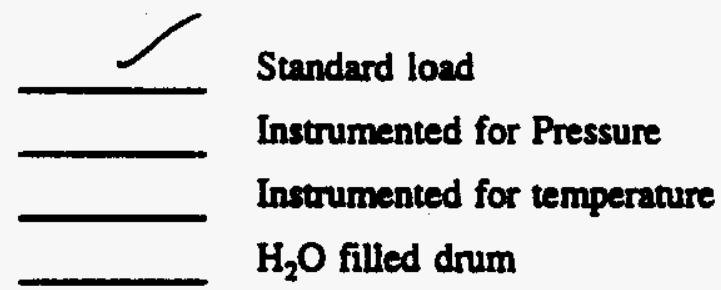

\begin{tabular}{|c|c|c|c|}
\hline Loading Starting at Bottom & Layer 1 & Layer 2 & Layer 3 \\
\hline 3 pieces of steel & & N/A & \\
\hline $1 / 3$ of the brown paper & & & \\
\hline $1 / 3$ of the 10-mil plastic & & & \\
\hline $1 / 3$ of the bond paper & & & \\
\hline $1 / 3$ of the towels & & & \\
\hline $1 / 3$ of the bags & & & \\
\hline $1 / 3$ of the cut paper & & & \\
\hline $1 / 3$ of the fabric & & & \\
\hline $1 / 3$ of the rubber & Go to Layer 2 & Go to Layer 3 & \\
\hline & & & \\
\hline
\end{tabular}

Drum Filled:

Date $5-195$ Initial $A P$

Ring bolt placed opposite drum seam Date $5-1-95$ Initial AP and in down position

Lid sealed and torqued to $40 \mathrm{fthb}$ Date $5-1-95$ Initial

Leak tested:

Post-test weight (lb)
N/A or Pressure (psig)

Pressure (psig)

Date

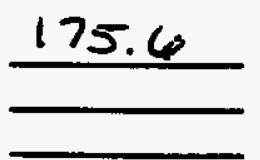

Time (min) 0

Time (min) 5

Initial

Target weight $-175.6 \mathrm{lb}$ 
WHC-SD-WM-TRP-246 REV. 0

Rack Storage Drum Data Sheet

Drum No. $\quad \underline{182 \quad u t .=175.8}$

Post-test Assessment

Position: __ original: __ tipped; __ toppled

Lid on drum: $\quad X$ Yes No

Gasket missing (cm)

Contents expelled: _ Yes $\measuredangle$ No

Seal failure: $\quad$ Yes $\quad$ Not visible

Burn pattern:

NONC

Photo taken:

Yes $X$ No

Leak tested:

TJA) Pressure (psig)

Pressure (psig)

Date

$\longrightarrow \quad$\begin{tabular}{ll} 
Time (min) & 0 \\
\hline
\end{tabular}

Initial

Interior assessment:

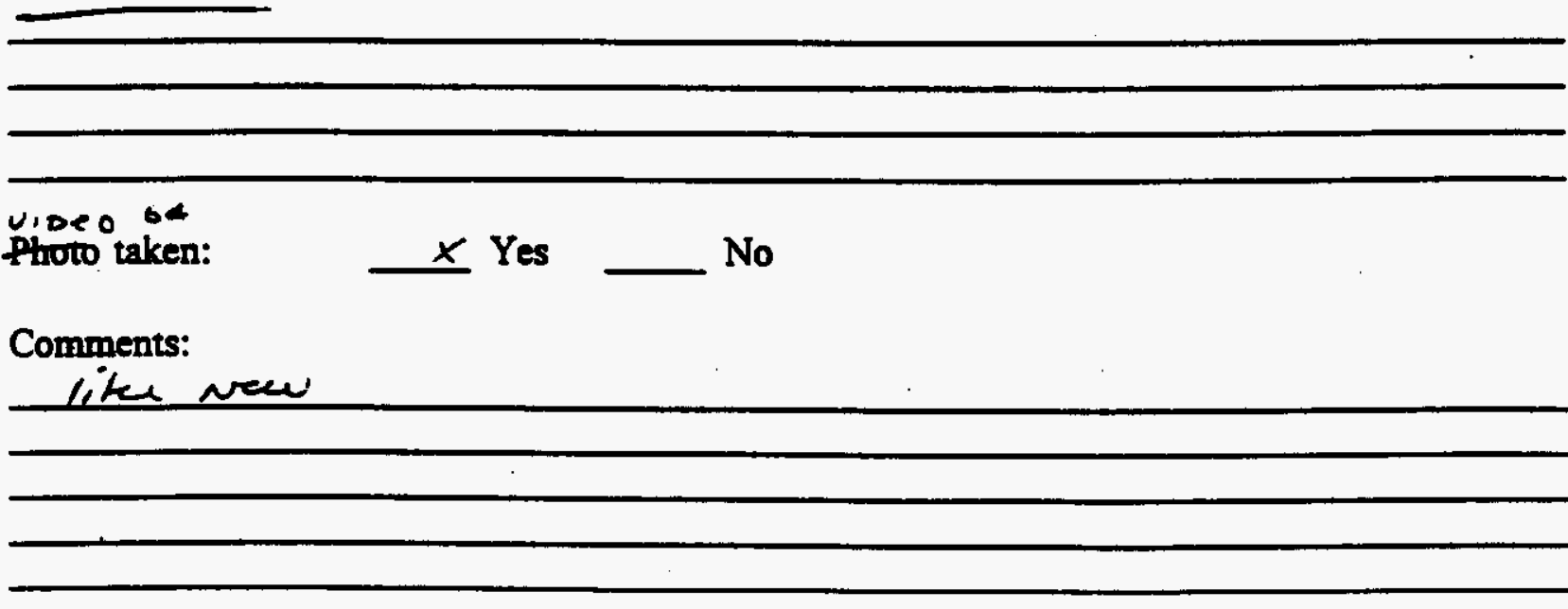




\section{Rack Storage Drum Data Sheet}

Drum No. $X 212$

Description (Check):

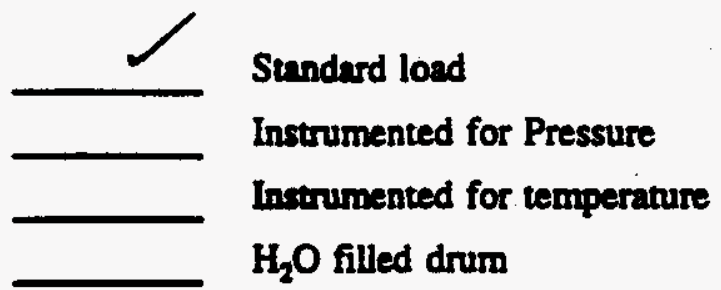

\begin{tabular}{|c|c|c|c|}
\hline Loading Starting at Bottom & Layer 1 & Layer 2 & Layer 3 \\
\hline 3 pieces of steel & & N/A & \\
\hline $1 / 3$ of the brown paper & & & \\
\hline $1 / 3$ of the 10-mil plastic & & & \\
\hline $1 / 3$ of the bond paper & & & \\
\hline $1 / 3$ of the towels & & & \\
\hline $1 / 3$ of the bags & & & \\
\hline $1 / 3$ of the cut paper & & & \\
\hline $1 / 3$ of the fabric & & & \\
\hline $1 / 3$ of the rubber & Go to Layer 2 & Go to Layer 3 & \\
\hline & & & \\
\hline
\end{tabular}

Drum Filled:

Date $5-1-95$ Initial $Q P$

Ring boit placed opposite drum seam Date $5-1-95$ Initial pP and in down position

Lid sealed and torqued to $40 \mathrm{ftlb}$ : Date $5-1-95$ Initial AP

Leak tested:

Pre-test weight (lb)

Post-test weight (lb)
N/A or Pressure (psig)

Pressure (psig)

Date

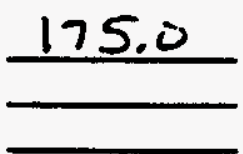

Time (min) 0

Time (min) 5

Target weight $-175.6 \mathrm{lb}$ 
WHC-SD-WM-TRP-246 REV. 0

Rack Storage Drum Data Sheet

Drum No. $\times 212 \quad \omega t=171.7 \mathrm{lb}$.

Post-test Assessment

Position: __ original; __ tipped; __ toppled

Lid on drum: $\quad$ Yes $X$ No

Gasket missing (cm) all

Contents expelled: __ Yes _ No

Seal failure: $\quad X$ Yes $\quad$ Not visible

Burn pattern:

allaround

Photo taken: $\quad$ Yes $\quad$ No

Leak tested: - NTA or Pressure (psig)

Pressure (psig)

Date __ Initial

$\begin{array}{ll}\text { Time (min) } & 0 \\ \text { Time }(\min ) & \underline{5}\end{array}$

Interior assessment:

-24 " from Top of dur $t$ top $f$ reminimg chaned centents

V. Dea 16

photo taken:

$\underset{X}{X}$ Yes No

Comments: 
WHC-SD-WM-TRP-246 REV. 0

\section{Rack Storage Drum Data Sheet}

Drum No. $\times 222(P)$

Description (Check):

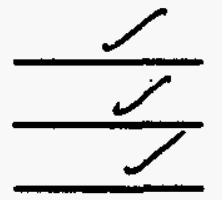

Standard load

Instrumented for Pressure

Instrumented for temperature

$\mathrm{H}_{2} \mathrm{O}$ filled drum

\begin{tabular}{|c|c|c|c|}
\hline Loading Starting at Bottom & Layer 1 & Layer 2 & Layer 3 \\
\hline 3 pieces of steel & & & \\
\hline $1 / 3$ of the brown paper & & & \\
\hline $1 / 3$ of the 10 -mil plastic & & & \\
\hline $1 / 3$ of the bond paper & & & \\
\hline $1 / 3$ of the towels & & & \\
\hline $1 / 3$ of the bags & & & \\
\hline $1 / 3$ of the cut paper & & & \\
\hline $1 / 3$ of the fabric & Go to Layer 2 & Go to Layer 3 & \\
\hline $1 / 3$ of the rubber & & \\
\hline
\end{tabular}

Drum Filled:

Date 5.3.95 Initial Le

Ring bolt placed opposite drum seam Date $5-3-75$ Initial LCK and in down position

Lid sealed and torqued to $40 \mathrm{ft} / \mathrm{b}: \quad$ Date $5.3 \cdot 75$ Initial LK

Leak tested:

Pre-test weight (lb)

Post-test weight (lb)

Loss =
N/A or Pressure (psig)

Pressure (psig)

Date

Initial

$175.8 \quad$ Target weight $-175.6 \mathrm{lb}$
Time (min) $\frac{0}{5}$
Time $(\min )$ 
WHC-SD-WM-TRP-246 REV. 0

Rack Storage Drum Data Sheet

Drum No. $\quad \mathbf{2 2 2}-\pi .=185.616$ Ne iid

Post-test Assessment

Position: __ original; ___ tipped; __ toppled

Lid on drum: _ Yes $X$ No missing lidering

Gasket missing (cm)

Contents expelled: __ Yes _ No

Seal failure: $\quad \longrightarrow$ Yes Not visible

Burn pattern:

NoTruanabl - allaround

Photo taken:

Yes _ No

Leak tested:

W1A or Pressure (psig)

Pressure (psig)

Date

Initial

Time $(\min ) \quad \frac{0}{5}$
Time (min)

Interior assessment: $14^{\circ}$ of remaining contents chaured m eutside but intact

Vioer II

Photo taken:

$x$ Yes

No

Comments: 
WHC-SD-WM-TRP-246 REV. 0

\section{Rack Storage Drum Data Sheet}

Dnum No. $x 232$

Description (Check):

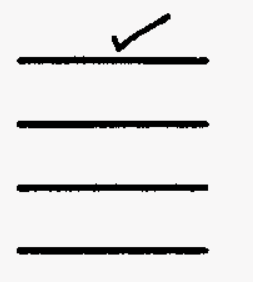

Standard load

Instrumented for Pressure

Instrumented for temperature

$\mathrm{H}_{2} \mathrm{O}$ filled drum

\begin{tabular}{|c|c|c|c|}
\hline Lading Starting at Bottom & Layer 1 & Layer 2 & Layer 3 \\
\hline 3 pieces of steel & & N/A & \\
\hline $1 / 3$ of the brown paper & & & \\
\hline $1 / 3$ of the 10-mil plastic & & & \\
\hline $1 / 3$ of the bond paper & & & \\
\hline $1 / 3$ of the towels & & & \\
\hline $1 / 3$ of the bags & & & \\
\hline $1 / 3$ of the cut paper & & & \\
\hline $1 / 3$ of the fabric & & & \\
\hline $1 / 3$ of the rubber & Go to Layer 2 & Go to Layer 3 & \\
\hline
\end{tabular}

Drum Filled:

Ring bolt placed opposite dnum seam and in down position

Lid sealed and torqued to $40 \mathrm{ft} / \mathrm{b}$ :

Leak tested:

Pre-test weight (lb)

Post-lest weight (lb)

Loss $=$
Date $5-1.95$ Initial Q.S.

Date $5-1.95$ Initial A.S.

Date $5 \cdot 1.95$ Initial A.S.
N/A or Pressure (psig)

Pressure (psig)

Date

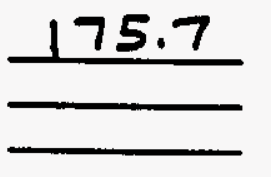

Time (min) 0

Time (min)
Initial

Target weight - $175.6 \mathrm{lb}$ 
WHC-SD-WM-TRP-246 REV. 0

Rack Storage Drum Data Sheet

Drum No. $\times 232$ it. $169.5 \mathrm{lb}$

Post-test Assessment

Position: ___ origina; ___ __tipped; __ toppled

Lid on drum: $X$ Yes N_ No $" 4 " x "$ jap is" to left fream

Gasket missing (cm) AII

Contents expelled: __ Yes $X$ No

Seal failure: $\quad \times$ Yes N Not visible

Bum pattem:

Photo taken:

Yes

Leak tested: $\quad$ N/A or Pressure (psig)

Pressure (psig)

Date

\begin{tabular}{lll} 
& Time $(\min )$ & 0 \\
\hline & Time $(\min )$
\end{tabular}

Interior assessment:

-14" top fdum to top of chanud contents

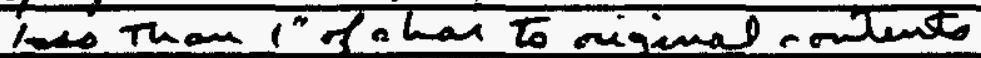

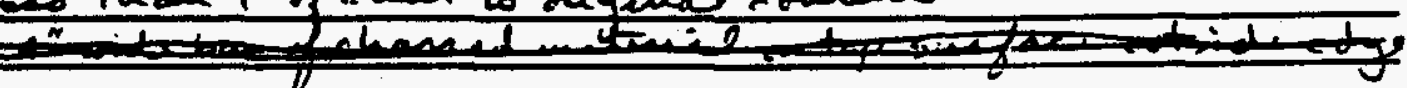

vioeo 17

thoto taken:

$\triangle$ Yes No

Comments: 
WHC-SD-WM-TRP-246 REV. 0

\section{Rack Storage Drum Dato Sheet}

Drum No. $\times 242$

Description (Check):

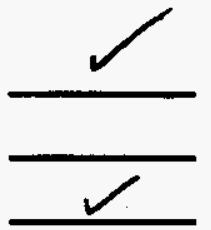

Standard load

Instrumented for Pressure

Instrumented for temperature

$\mathrm{H}_{2} \mathrm{O}$ filled drum

\begin{tabular}{|c|c|c|c|}
\hline Loading Starting at Bottom & Layer 1 & Layer 2 & Layer 3 \\
\hline 3 pieces of steel & & & \\
\hline $1 / 3$ of the brown paper & & & \\
\hline $1 / 3$ of the 10-mil plastic & & & \\
\hline $1 / 3$ of the bond paper & & & \\
\hline $1 / 3$ of the towels & & & \\
\hline $1 / 3$ of the bags & & & \\
\hline $1 / 3$ of the cut paper & & & \\
\hline $1 / 3$ of the fabric & Go to Layer 2 & Go to Layer 3 & \\
\hline $1 / 3$ of the rubber & & & \\
\hline & & & \\
\hline
\end{tabular}

Drum Filled:

Ring bolt placed opposite drum seam and in down position

Lid sealed and torqued to $40 \mathrm{f} / \mathrm{bb}$ : Date $5 \cdot 1.95$ Initial A.S.

\section{Lenk tested:}

Pre-test weight (lb)

Post-test weight (lb)
Date $5-1.95$ Initial A.S.

Date $5-1.95$ Initial $A . S$.

N/A or Pressure (psig)

Pressure (psig)

Date

$\overline{\text { Initial }}$

$175.7 \quad$ Target weight $-175.6 \mathrm{lb}$ 
WHC-SD-WM-TRP-246 REV. 0

\section{Rack Storage Drum Data Sheet}

Drum No. $\times 242$ its 174.9 is

Post-iest Assessment

Position: $\quad$ _ original: __ tipped; __ toppled

Lid on drum: $\quad X$ Yes No

Gasket missing (cm) __ 12.5" on bumed side

Contents expelled: . __ Yes X No

Seal failure: $\quad$ Yes $X$ Not visible

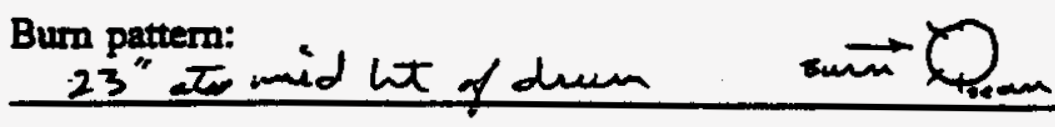

Photo taken:

Yes — No

Leak tested:

N/A ot Pressure (psig)

Time (min) $\quad 0$

Pressure (psig)

Date

\begin{tabular}{lll} 
Time $(\min )$ & 0 \\
\hline & Time $(\min )$
\end{tabular}

Interior assessment:

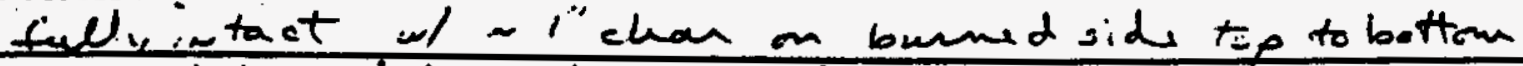

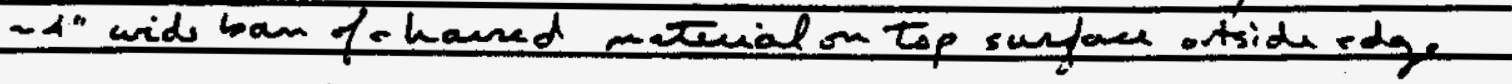

videc is

Photo taken:

$\chi^{\prime}$ Yes

No

Comments: 
WHC-SD-WM-TRP-246 REV. 0

\section{Rack Storage Drum Data Sheet}

Drum No. $\times 252$

Description (Check):

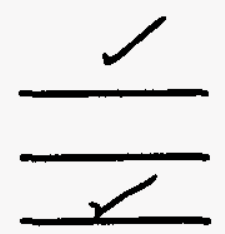

Standard load

Instrumented for Pressure

Instrumented for temperature

$\mathrm{H}_{2} \mathrm{O}$ filled drum

\begin{tabular}{|c|c|c|c|}
\hline Loading Starting at Bottom & Layer 1 & Layer 2 & Layer 3 \\
\hline 3 pieces of steel & & & \\
\hline $1 / 3$ of the brown paper & & & \\
\hline $1 / 3$ of the 10-mil plastic & & & \\
\hline $1 / 3$ of the bond paper & & & \\
\hline $1 / 3$ of the towels & & & \\
\hline $1 / 3$ of the bags & & & \\
\hline $1 / 3$ of the cut paper & & & \\
\hline $1 / 3$ of the fabric & & & \\
\hline $1 / 3$ of the rubber & Go to Layer 2 & Go to Layer 3 & \\
\hline
\end{tabular}

Drum Filled:

Date 5.1 .95 Initial A.S.

Ring bolt placed opposite drum seam Date $S-1-95$ Initial $A . S$. and in down position

Lid sealed and torqued to $40 \mathrm{fthb}$ : Date $\$ \cdot 1.95$ Initial A.S.

Leak tested:

Pre-test weight (lb)

Post-test weight (lb)
N/A or Pressure (psig) Pressure (psig)

Date

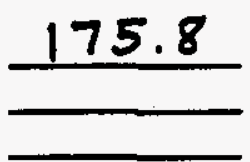

$\longrightarrow \quad$ Time (min) $\frac{0}{5}$

Initial

Target weight $-175.6 \mathrm{lb}$ 
WHC-SD-WM-TRP-246 REV. 0

Rack Storage Drum Data Sheet

Drum No. $\times 252176.216$.

Post-test Assessment

Position: __ original; ___ tipped; __ toppled

Lid on drum: $\quad \quad X$ Yes _ No

Gasket missing (cm) $O$ compremed thand 228 "

Contents expelled: __ Yes $X$ No

Seal failure: $\quad$ Y Yes $x$ Not visible

Burn pattern:

nONe

Photo taken:

Yes No

Leak tested:

N/A gे Pressure (psig)

Pressure (psig)

Date

$\begin{array}{ll}\text { Time }(\min ) & \frac{0}{5} \\ \text { Time }(\min ) & \end{array}$

$\overline{\text { Initial }}$

Interior assessment:

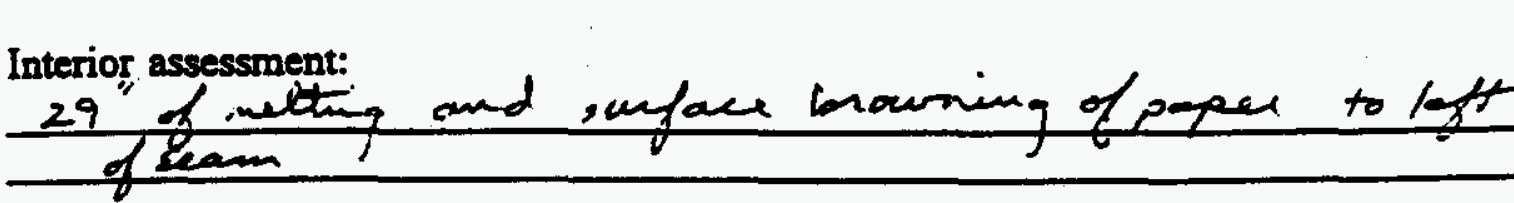

i) 8 eec 82

Photo taken:

$X$ Yes

Comments: 


\section{Rack Storage Drum Data Sheet}

Drum No. $\times 262$

Description (Check):

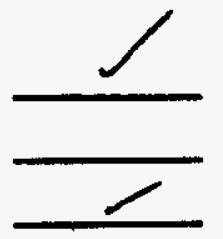

Standard load

Instrumented for Pressure

Instrumented for temperature

$\mathrm{H}_{2} \mathrm{O}$ filled drum

\begin{tabular}{|c|c|c|c|}
\hline Loading Starting at Bottom & Layer 1 & Layer 2 & Layer 3 \\
\hline 3 pieces of steel & $V$ & $\mathbf{N} / \mathbf{A}$ & $\mathbf{N} / \mathbf{A}$ \\
\hline $1 / 3$ of the brown paper & $\checkmark$ & $\checkmark$ & $\checkmark$ \\
\hline $1 / 3$ of the 10 -mil plastic & & $\checkmark$ & $V$ \\
\hline $1 / 3$ of the bond paper & & $\checkmark$ & $V$ \\
\hline $1 / 3$ of the towels & & $\checkmark$ & $V$ \\
\hline $1 / 3$ of the bags & & $\checkmark$ & $\checkmark$ \\
\hline $1 / 3$ of the cut paper & & $\checkmark$ & $r$ \\
\hline $1 / 3$ of the fabric & & 7 & $r$ \\
\hline $1 / 3$ of the rubber & & $\checkmark$ & $\checkmark$ \\
\hline & Go to Layer 2 & Go to Layer 3 & \\
\hline
\end{tabular}

Drum Filled:

Date 5.1 .95 Initial A.S.

Ring bolt placed opposite drum seam Date $5 \cdot 1 \cdot 95$ Initial $A . S$. and in down position

Lid sealed and torqued to $40 \mathrm{ft} / \mathrm{b}: \quad$ Date $5-1.95$ Initial A.S.

Leak tested:

Pre-test weight (lb)

Post-test weight (lb)
N/A or Pressure (psig)

Pressure (psig)

Date

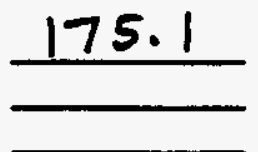

$\begin{array}{ll}\text { Time }(\min ) & 0 \\ \text { Time }(\min ) & \end{array}$

Target weight $-175.6 \mathrm{lb}$ 
WHC-SD-WM-TRP-246 REV. 0

\section{Rack Storage Drum Data Sheet}

Drum No. $\times 262$ wt. $=175.7$

\section{Post-test Assessment}

Position: __ original; ___ tipped; __ toppled

Lid on drum: $\quad X$ Yes No

Gasket missing (cm) D semi hand for $\sim 6$ "

Contents expelled: _ Yes $x$ No

Seal failure: $\quad$ Y Yes $X$ Not visible

Burn pattern:

NONR

Photo taken:

Y Yes $x$ No

Leak tested:

NT/Aुor'Pressure (psig)

Time (min) $\frac{0}{5}$

Pressure (psig)

Date

T Time (min) $\frac{0}{5}$

Interior assessment:

som meltin of plastic for $\sim 10^{\circ}$ /ft of sean

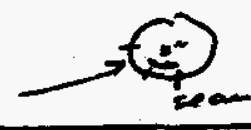

videc का

Photo taken:

$\stackrel{x}{ }$ Yes

Comments: 
WHC-SD-WM-TRP-246 REV. 0

\section{Reck Storage Drum Data Sheet}

Drum No. $\times 272$

Description (Check):

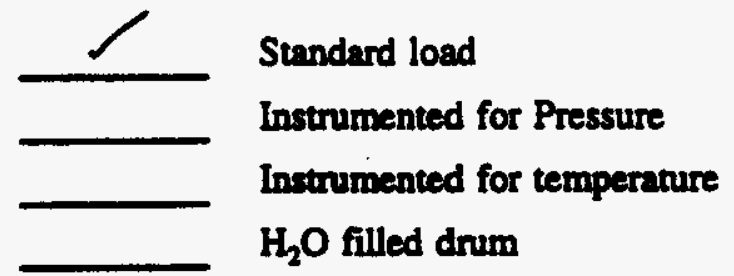

\begin{tabular}{|c|c|c|c|}
\hline Losding Starting at Bottom & Layer 1 & Layer 2 & Layer 3 \\
\hline 3 pieces of steel & $V$ & $\mathbf{N} / \mathbf{A}$ & $\mathbf{N} / \mathbf{A}$ \\
\hline $1 / 3$ of the brown paper & $\checkmark$ & $\checkmark$ & $\checkmark$ \\
\hline $1 / 3$ of the 10 -mil plastic & $\checkmark$ & $\gamma$ & $\boldsymbol{r}$ \\
\hline $1 / 3$ of the bond paper & $\checkmark$ & $\checkmark$ & 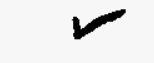 \\
\hline $1 / 3$ of the towels & $\checkmark$ & $\mathscr{L}$ & $\mathscr{V}$ \\
\hline . $\quad 1 / 3$ of the bags & & $\checkmark$ & $\checkmark$ \\
\hline $1 / 3$ of the cut paper & r & $r$ & 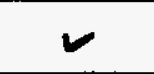 \\
\hline $1 / 3$ of the fabric & $V$ & $\checkmark$ & - \\
\hline $1 / 3$ of the rubber & & $\checkmark$ & $V$ \\
\hline & Go to Layer 2 & Go to Layer 3 & \\
\hline
\end{tabular}

Drum Filled:

Date $5-1.95$ Initial $A-S$.

Ring bolt placed opposite drum seam Date $5-1-95$ Initial A.S. and in down position

Lid sealed and torqued to $40 \mathrm{f} / 1 \mathrm{~b}: \quad$ Date $5-1-95$ Initial A.S.

Leak tested:

Pre-test weight (lb)

Post-test weight (lb)
N/A or Pressure (psig)

Pressure (psig)

Date

175.0
Time (min) 0

Initial

Time (min) 
WHC-SD-WM-TRP-246 REV. 0

\section{Rack Storage Drum Data Sheet}

Drum No. $\times 272$. At. $=175.7 \mathrm{16}$

\section{Post-test Assessment}

Position: original; tipped; toppled

Lid on drum: $\quad X$ Yes No

Gasket missing (cm) 0 almoot i. ihe wrew

Contents expelled: __ Yes $x$ No

Seal failure: $\quad$ Yes $\quad x$ Not visible

Burn pattern: NONe

Photo taken: $\quad$ Yes $X$ No

Leak tested: AfA or Pressure (psig) Pressure (psig)

Date \begin{tabular}{lll}
- & Time (min) & 0 \\
\hline & Time (min)
\end{tabular} Initial

Interior assessment:

bauly metted plastic $10 "$ to left of rem

$\therefore$ roto 3

Bhote taken:

$x$ Yes No

Comments: 
WHC-SD-WM-TRP-246 REV. 0

\section{Rack Storage Drum Data Sheet}

Drum No. $x-282$

Description (Check):

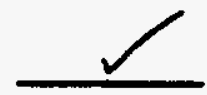

Standard load

Instrumented for Pressure

Instrumented for temperanure

$\mathrm{H}_{2} \mathrm{O}$ filled drum

\begin{tabular}{|c|c|c|c|}
\hline Loading Starting at Bottom & Layer 1 & Layer 2 & Layer 3 \\
\hline 3 pieces of steel & $\boldsymbol{r}$ & $\mathbf{N} / \mathbf{A}$ & $\mathbf{N} / \mathbf{A}$ \\
\hline $1 / 3$ of the brown paper & $r$ & & $V$ \\
\hline $1 / 3$ of the 10 -mil plastic & $\checkmark$ & ح & V \\
\hline $1 / 3$ of the bond paper & 2 & $レ$ & $\sim$ \\
\hline $1 / 3$ of the towels & $\checkmark$ & $\checkmark$ & $\sim$ \\
\hline $1 / 3$ of the bags & & $\checkmark$ & 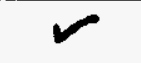 \\
\hline $1 / 3$ of the cut paper & $r$ & $\sim$ & 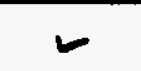 \\
\hline $1 / 3$ of the fabric & $V$ & $\checkmark$ & $レ$ \\
\hline $1 / 3$ of the rubber & $V$ & $\nu$ & $V$ \\
\hline & Go to Layer 2 & Go to Layer 3 & \\
\hline
\end{tabular}

Drum Filled:

Date $5 \cdot 1 \cdot 95$ Initial $A \cdot S$

Ring bolt placed opposite drum seam Date $5-1-95$ Initial $\mathbf{A . S}$.

and in down position

Lid sealed and torqued to $40 \mathrm{ftlb}$ : Date $5 \cdot 1 \cdot 95$ Initial A.S.

Leak tested:

Post-test weight (lb)

Loss $=$
N/A or Pressure (psig)

Pressure (psig)

Date

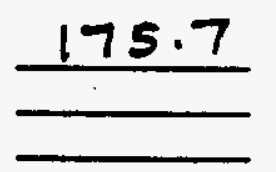

Time (min) 0

Time (min) 5

Target weight $-175.6 \mathrm{lb}$ 
WHC-SD-WM-TRP-246 REV. 0

\section{Rack Storage Drum Data Sheet}

Drum No. $\times 2 \$ 2 \quad \omega_{1}=176.1 \mathrm{ib}$

\section{Post-test Asressment}

Position: ___ original; ___ _ _ _ _ _

Lid on drum: $\quad x^{*}$ Yes No

Gasket missing (cm) O like wew

Contents expelled: _ـ Yes $x$ No

Seal failure: $\quad$ _ $\quad$ Yes $\times$ Not visible

Bum pattern:

None

Photo taken: $\quad$ Yes $X$ No

Leak tested: $\quad$ N/A or Pressure (psig)

Pressure (psig)

Date

\begin{tabular}{lll} 
& Time $(\min )$ & 0 \\
\hline & Time $(\min )$
\end{tabular}

Interior assessment:

vo c hal - No metaing

Video 12

pheto taken:

$x^{\circ}$ Yes

Comments: 


\section{Rack Storage Drum Data Sheet}

Drum No. $x 312(P)$

Description (Check):

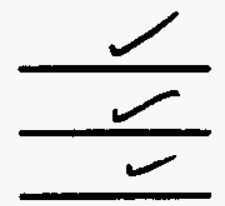

Standard load

Instrumented for Pressure

Instrumented for temperature

$\mathrm{H}_{2} \mathrm{O}$ filled drum

\begin{tabular}{|c|c|c|c|}
\hline Loading Starting at Bottom & Layer 1 & Layer 2 & Layer 3 \\
\hline 3 pieces of steel & & N/A & \\
\hline $1 / 3$ of the brown paper & & & \\
\hline $1 / 3$ of the 10-mil plastic & & & \\
\hline $1 / 3$ of the bond paper & & & \\
\hline $1 / 3$ of the toweis & & & \\
\hline $1 / 3$ of the bags & & & \\
\hline $1 / 3$ of the cut paper & & & \\
\hline $1 / 3$ of the fabric & & & \\
\hline $1 / 3$ of the rubber & Go to Layer 2 & Go to Layer 3 & \\
\hline
\end{tabular}

Drum Filled:

Date 5.1 .95 Initial A.S.

Ring bolt placed opposite drum seam Date $5-1.95$ Initial $A . S$. and in down position

Lid sealed and torqued to $40 \mathrm{fthb}$ Date $5 \cdot 1.95$ Initial A.S.

Leak tested:

Pre-test weight (lb)

Post-test weight (lb)
N/A or Pressure (psig)

Pressure (psig)

Date
$\longrightarrow$
Initial

Time (min) 0

Time (min) $\frac{1}{5}$

\section{$175.5 \quad$ Target weight $-175.6 \mathrm{lb}$}


WHC-SD-WM-TRP-246 REV. 0

\section{Rack Storage Drum Data Sheet}

Drum No. $\quad \times 312 \quad 163.9$

Post-test Assessment

Position: original: ___ tipped; toppled

Lid on drum: $\quad X$ Yes $\quad$ No Gep was wet measened

Gasket missing (cm) $\mathrm{Al}^{\prime}$

Contents expelled: __ Yes $X$ No

Seal failure: $\quad X$ Yes $\quad$ Not visible

Burn pattern:

All around.

Photo taken:

$X$ Yes No

Leak tested: $\quad$ N/P or Pressure (psig)

Pressure (psig)

Date

Initial

Interior assessment:

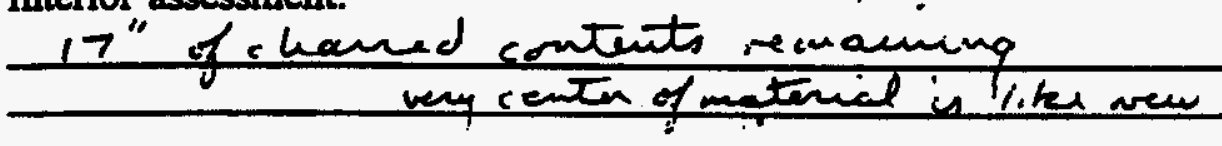

$\therefore$ Deo \& 9

Photo taken: $\quad X$ Yes N No

Comments: 


\section{Rack Storage Drum Data Sheet}

Drum No. $\times 332(p)$

\begin{tabular}{|c|c|c|c|}
\hline Description (Check): & $\begin{array}{l}\text { Standard load } \\
\text { Instrumented for Presst } \\
\text { Instrumented for tempe } \\
\mathrm{H}_{2} \mathrm{O} \text { filled drum }\end{array}$ & re & \\
\hline Loading Starting at Bottom & Layer 1 & Layer 2 & Layer 3 \\
\hline 3 pieces of steel & $\checkmark$ & N/A & $\mathbf{N} / \mathbf{A}$ \\
\hline $1 / 3$ of the brown paper & $\checkmark$ & $\checkmark$ & \\
\hline $1 / 3$ of the 10 -mil plastic & $r$ & $\checkmark$ & $\mathscr{L}$ \\
\hline $1 / 3$ of the bond paper & & r & $\longleftarrow$ \\
\hline $1 / 3$ of the towels & & $\checkmark$ & $V$ \\
\hline $1 / 3$ of the bags & & $\sim$ & - \\
\hline $1 / 3$ of the cut paper & & 7 & - \\
\hline $1 / 3$ of the fabric & & $\checkmark$ & $\leftarrow$ \\
\hline $1 / 3$ of the rubber & & $r$ & r \\
\hline & Go to Layer 2 & Go to Layer 3 & \\
\hline
\end{tabular}

Drum Filled:

Date $5-1.95$ Initial A.S.

Ring bolt placed opposite drum seam Date $5 \cdot 1-95$ Initial $A \cdot S$. and in down position

Lid sealed and torqued to $40 \mathrm{fthb}$ Date $5-1.95$ Initial A.S.

Leak tested:

Pre-test weight (lb)

Post-test weight (lb)
N/A or Pressure (psig)

Pressure (psig)

Date

\begin{tabular}{|c|c|c|}
\hline & Time (min) & 0 \\
\hline 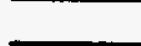 & Time (min) & 5 \\
\hline
\end{tabular}

\section{$175.8 \quad$ Target weight $-175.6 \mathrm{lb}$}




\section{WHC-SD-WM-TRP-246 REV. 0 \\ Rack Storage Drum Data Sheet}

Drum No. $\times 332$ 150.316. was inet

Post-test Assessment

Position: __ original; __ tipped; __ toppled

Lid on drum: $\quad$ Yes $X$ No

Gasket missing (cm) A-H

Contents expelled: _ Yes — No

Seal failure: $\quad X$ Yes $\perp$ Not visible

Burn pattern:
24 " at mid lt. seam $t$ right

Photo taken:

Yes

Leak tested:

N/A or Pressure (psig)

Pressure (psig)

Date

$\longrightarrow \quad$\begin{tabular}{ll} 
Time (min) & 0 \\
\hline & Time (min)
\end{tabular}

Initial

Interior assessment:

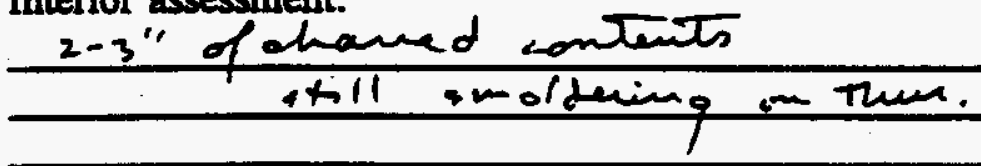

woec 124

Photo taken:

$x$ Yes

Comments: 


\section{Rack Storage Drum Data Sheet}

Drum No. $\times 342$

Description (Check):

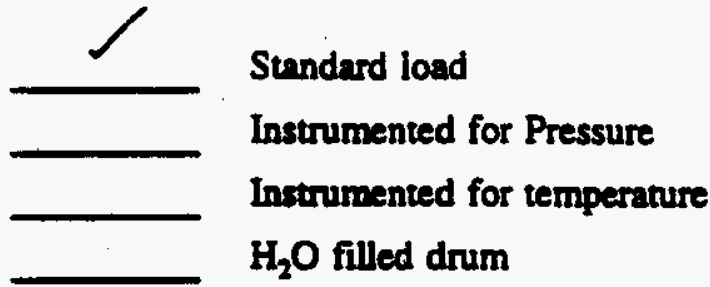

\begin{tabular}{|c|c|c|c|}
\hline Loading Starting at Bottom & Layer 1 & Layer 2 & Layer 3 \\
\hline 3 pieces of steel & N/A & \\
\hline $1 / 3$ of the brown paper & & & \\
\hline $1 / 3$ of the 10-mil plastic & & & \\
\hline $1 / 3$ of the bond paper & & & \\
\hline $1 / 3$ of the rowels & & & \\
\hline $1 / 3$ of the bags & & & \\
\hline $1 / 3$ of the cut paper & & & \\
\hline $1 / 3$ of the fabric & & & \\
\hline $1 / 3$ of the rubber & Go to Layer 2 & Go to Layer 3 & \\
\hline & & & \\
\hline
\end{tabular}

\section{Drum Filled:}

Ring bolt placed opposite drum seam and in down position

Lid sealed and torqued to $40 \mathrm{ft} / \mathrm{tb}:$ Date $5-1-95$ Initial AP

Leak tested:

Pre-test weight (lb)

Post-test weight (lb)
Date $5-1 \cdot 95$ Initial $A \cdot S$.

Date 5.1 .95 Initial A.S.
N/A or Pressure (psig)

Pressure (psig)

Date

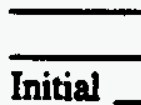

Time (min) 0

Time (min) 5 


\section{WHC-SD-WM-TRP-246 REV. 0}

\section{Rack Storage Drum Data Sheet}

Drum No. $\times 342 \quad 176.316$.

Post-test Assessment

Position: __ original; ___ tipped; __ toppled

Lid on drum: $\quad x$ Yes No

Gasket missing (cm) _ 43 "

Contents expelled: _ Yes $x$ No

Seal failure: $\quad$ Yes $\quad X$ Not visible

Burn pattern: aid le stuts i6" right feeam

Photo taken: $\quad$ Yes $x$ No

Leak tested: $\quad$ NTADor Pressure (psig) Pressure (psig)

Date

$\longrightarrow \quad$\begin{tabular}{cc} 
Time (min) & 0 \\
\hline & Time (min)
\end{tabular}

Interior assessment:

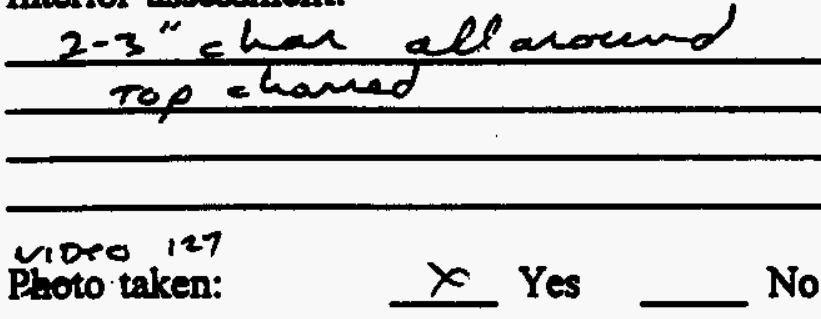

Comments: 


\section{Rack Storage Drum Data Sheet}

Drum No. $x 452$

Description (Check):

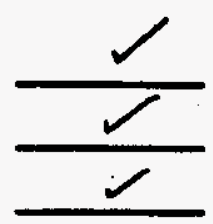

Standard load

Instrumented for Pressure

Instrumented for cemperature

$\mathrm{H}_{2} \mathrm{O}$ filled drum

\begin{tabular}{|c|c|c|c|}
\hline Loading Starting at Bottom & Layer 1 & Layer 2 & Layer 3 \\
\hline 3 pieces of steel & & N/A & \\
\hline $1 / 3$ of the brown paper & & & \\
\hline $1 / 3$ of the 10-mil plastic & & & \\
\hline $1 / 3$ of the bond paper & & & \\
\hline $1 / 3$ of the towels & & & \\
\hline $1 / 3$ of the bags & & & \\
\hline $1 / 3$ of the cut paper & & & \\
\hline $1 / 3$ of the fabric & & & \\
\hline $1 / 3$ of the rubber & Go to Layer 2 & Go to Layer 3 & \\
\hline
\end{tabular}

Drum Filled:

Date $5-1-95$ Initial $A P$

Ring bolt placed opposite drum seam Date $5-(-9.5$ Initial AP and in down position

Lid sealed and torqued to $40 \mathrm{ft} / \mathrm{lb}$ : Date $\$ .1 .95$ Initial $A S$

Leak tested:

Pre-test weight (lb)

Post-test weight (lb)
N/A or Pressure (psig) Pressure (psig)

Date

175.8
Time (min) 0

Time (min) 5

Initial

Target weight $-175.6 \mathrm{lb}$ 
WHC-SD-WM-TRP-246 REV. 0

Rack Storage Drum Data Sheet

Drum No. $\quad \leq 352(P) \quad 176.6 \mathrm{it}$

Post-tert Assessment

Position: __ original; ___ tipped; __ toppled

Lid on drum: $\quad X$ Yes No

Gasket missing (cm) 0 compresard - fairly hand all the way anound.

Contents expelled: _ Yes $x$ No

Seal failure: $\quad$ Yes $x$ Not visible

Burn pqttern:

Photo taken: $\quad$ Yes $\perp$ No

Leak tested:

Pressure (psig)

Date

Interior assessment:

24 "plastic unthd - no char et if seam

Time $(\min )$
Time $(\min )$

Time $(\min ) \quad 5$

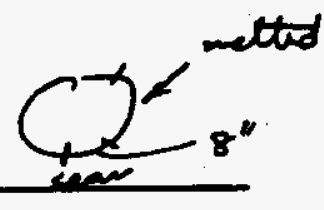

No

Comments: 


\section{Rack Storage Drum Data Sheet}

Drum No. $\times 362$

Description (Check):

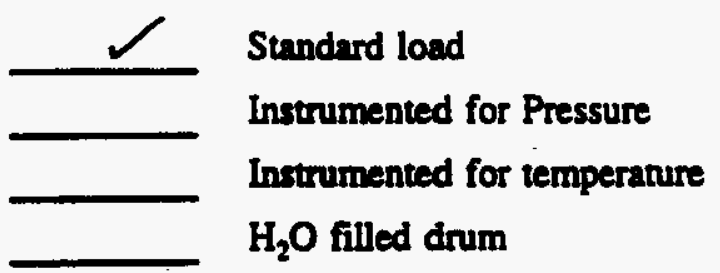

\begin{tabular}{|c|c|c|c|}
\hline Loading Starting at Bottom & Layer 1 & Layer 2 & Layer 3 \\
\hline 3 pieces of steel & & N/A & N/A \\
\hline $1 / 3$ of the brown paper & & & \\
\hline $1 / 3$ of the 10-mil plastic & & & \\
\hline $1 / 3$ of the bond paper & & & \\
\hline $1 / 3$ of the towels & & & \\
\hline $1 / 3$ of the bags & & & \\
\hline $1 / 3$ of the cut paper & & & \\
\hline $1 / 3$ of the fabric & & & \\
\hline $1 / 3$ of the rubber & Go to Layer 2 & Go to Layer 3 & \\
\hline
\end{tabular}

Drum Filled:

Ring bolt placed opposite drum seam and in down position

Lid sealed and torqued to $40 \mathrm{ft} / \mathrm{b}$ :

Leak tested:

Pre-test weight (lb)

Post-test weight (lb)

Loss $=$
Date $\$-1.95$ Initial $A . S$.

Date $s-1.95$ Initial $A . S$. Date 5-1-95 Initial AP
N/A or Pressure (psig)

Pressure (psig)

Date

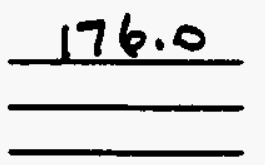

Time (min) 0 Time (min) 5 


\section{WHC-SD-WM-TRP-246 REV. 0}

\section{Rack Storage Drum Data Sheet}

Drum No. $\quad \times 362 \quad 176.21 \mathrm{~b}$

Post-test Assessment

Position: __ original; ___ tipped; ___ toppled

Lid on drum: $\quad X$ Yes No

Gasket missing (cm) $O$ like vew, true what compresured $\sim 18$ "

Contents expelled: —u Yes $x$ No

Seal failure:

Yes $X$ Not visible

Burn pattern:

NONE

Photo taken:

—ues $x$ No

Leak tested:

NIA Pressure (psig)

Pressure (psig)

Date

- Time $(\min ) \quad \frac{0}{5}$

Interior assessment:

20 " seam to right slight meltury

บTDeo 1: 7

Photo taken:

$\infty$ Yes

Comments: 
WHC-SD-WM-TRP-246 REV. 0

\section{Rack Storage Drum Data Sheet}

Drum No. X 372

Description (Check):

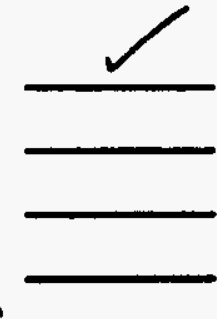

Standard load

Instrumented for Pressure

Instrumented for temperature

$\mathrm{H}_{2} \mathrm{O}$ filled drum

\begin{tabular}{|c|c|c|c|}
\hline Loding Starting at Bottom & Layer 1 & Layer 2 & Layer 3 \\
\hline 3 pieces of steel & $\sim$ & $\mathbf{N} / \mathbf{A}$ & $\mathbf{N} / \mathbf{A}$ \\
\hline $1 / 3$ of the brown paper & $w$ & $r$ & 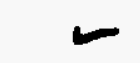 \\
\hline $1 / 3$ of the 10 -mil plastic & 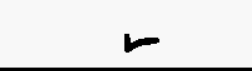 & - & - \\
\hline $1 / 3$ of the bond paper & $\checkmark$ & - & - \\
\hline $1 / 3$ of the towels & - & $r$ & $\sim$ \\
\hline $1 / 3$ of the bags & 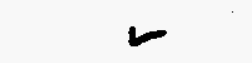 & - & - \\
\hline $1 / 3$ of the cut paper & $r$ & $\sim$ & - \\
\hline $1 / 3$ of the fabric & w & - & 一 \\
\hline $1 / 3$ of the rubber & $\sim$ & 2 & $r$ \\
\hline & Go to Layer 2 & Go to Layer 3 & \\
\hline
\end{tabular}

Drum Filled:

Ring bolt placed opposite drum seam and in down position

Lid sealed and torqued to $40 \mathrm{ft} / \mathrm{b}$ :

Leak tested:

Pre-test weight (lb)

Post-test weight (lb)
Date $\$-1.95$ Initial A.S.

Date $5 \cdot 1.95$ Initial A.S.
Date S-1.95 Initial A.S.

N/A or Pressure (psig)

Pressure (psig)

Date

\begin{tabular}{ll} 
Time $(\min ) \frac{0}{5}$ \\
\hline Initial
\end{tabular}

Target weight $-175.6 \mathrm{lb}$ 
WHC-SD-WM-TRP-246 REV. 0

Rack Storage Drum Data Sheet

Drum No. $\times 372$ ut. $=176.0$

\section{Post-test Assessment}

Position: __ original; __ tipped; __ toppled

Lid on drum: $\quad X$ Yes No

Gasket missing $(\mathrm{cm}) \underline{O}$ like vew, slightly crmpreased

Contents expelled: — Yes $X$ No

Seal failure: $\quad$ Y Yes $X$ Not visible

Burn pattern:

none

Photo taken:

Yes $\searrow$ No

Leak tested:

FTE or Pressure (psig) Pressure (psig)

Date
Time (min) $\quad 0$

Time $(\min ) \frac{0}{5}$

Interior assessment:

no chan

No buning

vioec 111

Photo taken:

$\varnothing$ Yes

Comments: 
WHC-SD-WM-TRP-246 REV. 0

\section{Rack Storage Drum Data Sheet}

Drum No. $\times 382$

Description (Check):

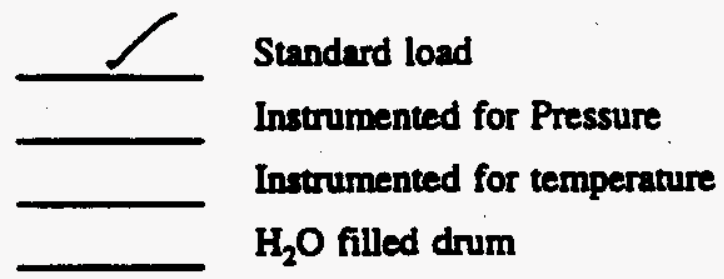

\begin{tabular}{|c|c|c|c|}
\hline Loading Starting at Bottom & Layer 1 & Layer 2 & Layer 3 \\
\hline 3 pieces of steel & $V$ & $\mathbf{N} / \mathbf{A}$ & $\mathbf{N} / \mathbf{A}$ \\
\hline $1 / 3$ of the brown paper & $r$ & $レ$ & $\checkmark$ \\
\hline $1 / 3$ of the 10 -mil plastic & & $r$ & $\checkmark$ \\
\hline $1 / 3$ of the bond paper & & $\checkmark$ & $\checkmark$ \\
\hline $1 / 3$ of the towels & $\sim$ & $\checkmark$ & $\checkmark$ \\
\hline $1 / 3$ of the bags & $\checkmark$ & $\checkmark$ & $\checkmark$ \\
\hline $1 / 3$ of the cut paper & 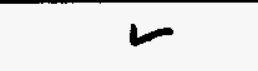 & $\checkmark$ & $\checkmark$ \\
\hline $1 / 3$ of the fabric & ש & $V$ & $\nu$ \\
\hline $1 / 3$ of the rubber & $\nabla$ & 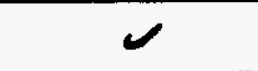 & $V$ \\
\hline & Go to Layer 2 & Go to Layer 3 & \\
\hline
\end{tabular}

Drum Filled:

Date S-1.95 Initial A.S.

Ring bolt placed opposite drum seam Date $5-1-95$ Initial A.S. and in down position

Lid sealed and torqued to $40 \mathrm{f} / \mathrm{bb}:$ Date $5 \cdot 1.95$ Initial

Leak tested:

(lb)

Post-test weight (lb) Loss $=$
N/A or Pressure (psig) Pressure (psig)

Date

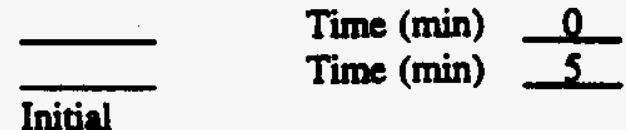

$176.3 \quad$ Target weight $-175.6 \mathrm{lb}$ 
WHC-SD-WM-TRP-246 REV. 0

Rack Storage Drum Data Sheet

Drum No. $\times 382,176.61 \mathrm{~h}$

Post-test Assessment

Position: __ original; __ tipped; __ toppled

Lid on drum: $\quad X$ Yes No

Gasket missing (cm) $O$ like wew

Contents expelled: —ues $\simeq$ No

Seal failure: $\quad$ Yes $X$ Not visible

Burn pattern:

none

Photo taken:

Yes $\varnothing$ No

Leak tested:

(NTA or Pressure (psig)

Pressure (psig)

Date

$\square \quad$ Time (min) $\frac{0}{5}$

Interior assessment:

No uelting

broto taken:

$\times$ Yes

Comments: 
WHC-SD-WM-TRP-246 REV. 0

Rack Storage Drum Data Sheet

Drum No. $\times 113$

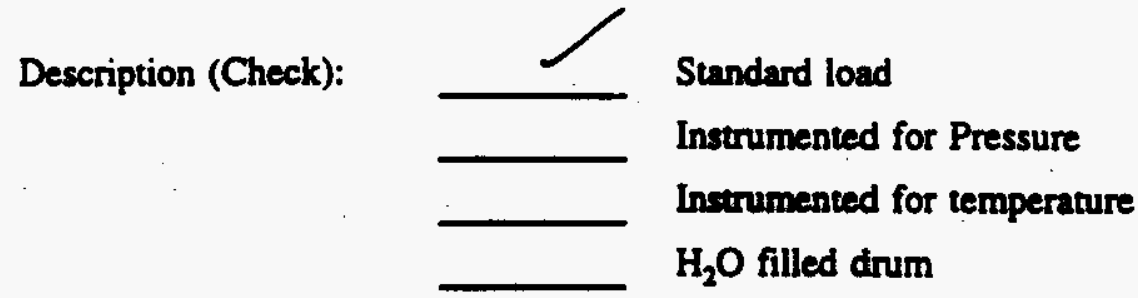

\begin{tabular}{|c|c|c|c|}
\hline Loading Starting at Bottom & Layer 1 & Layer 2 & Layer 3 \\
\hline 3 pieces of steel & & N/A & N/A \\
\hline \multicolumn{4}{|l|}{$1 / 3$ of the brown paper } \\
\hline \multicolumn{4}{|l|}{$1 / 3$ of the 10 -mil plastic } \\
\hline \multicolumn{4}{|l|}{$1 / 3$ of the bond paper } \\
\hline \multicolumn{4}{|l|}{$1 / 3$ of the towels } \\
\hline \multicolumn{4}{|l|}{$1 / 3$ of the bags } \\
\hline \multicolumn{4}{|l|}{$1 / 3$ of the cut paper } \\
\hline \multicolumn{4}{|l|}{$1 / 3$ of the fabric } \\
\hline \multicolumn{4}{|l|}{$1 / 3$ of the nubber } \\
\hline & Go to Layer 2 & Go to Layer 3 & \\
\hline
\end{tabular}

\section{Drum Filled:}

Ring bolt placed opposite drum seam and in down position

Lid sealed and torqued to $40 \mathrm{ft} / \mathrm{b}$ :

Lenk tested:

Pre-test weight (lb)

Post-test weight (lb)
Date $5-1.95$ Initial AP

Date $5-1-95$ Initial AP

Date $5-1-95$ Initial AP
N/A or Pressure (psig)

Pressure (psig)

Date

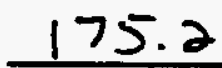

Time (min) 0

Time (min) 5 
WHC-SD-WM-TRP-246 REV. 0

\section{Rack Storage Drum Data Sheet}

Drum No. $\quad x 113$ wt. $=175.2$

\section{Post-test Assessment}

Position:

original;

tipped; toppled

Lid on drum: $\quad x$ Yes No

Gasket missing (cm) _ $25^{\prime \prime}$

Contents expelled: _ـ Yes $X$ No

Seal failure: $\quad$ Y $\quad$ Yes $\quad x$ Not visible

Burn pattern:

$-12^{\prime \prime}$ centund on sear only top i/s of demm

Photo taken:

Yes $x$ No

Leak tested:

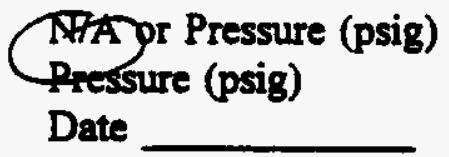

\begin{tabular}{lll}
- & Time (min) & 0 \\
\hline & Time $(\min )$
\end{tabular}

$\overline{\text { Initial }}$

Interior assessment:

$1-2^{4}$ ofthan in burned holf

Videe 42

Photo taken:

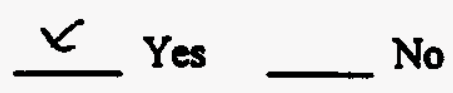

Comments: 
WHC-SD-WM-TRP-246 REV. 0

Rack Storage Drum Data Sheet

Drum No. $\times 123(p)$

Description (Check):

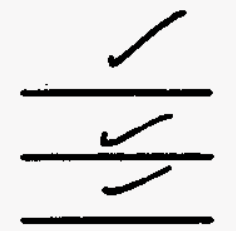

Standard load

Instrumented for Pressure

Instrumented for temperature

$\mathrm{H}_{2} \mathrm{O}$ filled drum

\begin{tabular}{|c|c|c|c|}
\hline Lading Starting at Bottom & Layer 1 & Layer 2 & Layer 3 \\
\hline 3 pieces of steel & & N/A & $\mathbf{N} / \mathbf{A}$ \\
\hline \multicolumn{4}{|l|}{$1 / 3$ of the brown paper } \\
\hline \multicolumn{4}{|l|}{$1 / 3$ of the 10 -mil plastic } \\
\hline \multicolumn{4}{|l|}{$1 / 3$ of the bond paper } \\
\hline \multicolumn{4}{|l|}{$1 / 3$ of the towels } \\
\hline \multicolumn{4}{|l|}{$1 / 3$ of the bags } \\
\hline \multicolumn{4}{|l|}{$1 / 3$ of the cut paper } \\
\hline \multicolumn{4}{|l|}{$1 / 3$ of the fabric } \\
\hline \multicolumn{4}{|l|}{$1 / 3$ of the rubber } \\
\hline & Go to Layer 2 & Go to Layer 3 & \\
\hline
\end{tabular}

Drum Filled: Ring bolt placed opposite drum seam
and in down position

Lid seaied and torqued to $40 \mathrm{ft} / \mathrm{b}$ :

Leak tested:

Pre-test weight (lb)

Post-test weight (lb)
Date $5-1-95$ Initial IfP

Date $5-1-95$ Initial aP
N/A or Pressure (psig)

Pressure (psig)

Date

175.6
Time (min) 0 Time (min) 5

\section{Loss $=$}




\section{WHC-SD-WM-TRP-246 REV. 0}

\section{Rack Storage Drum Data Sheet}

Drum No. $\quad x_{i 23(\beta)}+176.1$

\section{Post-iest Assessment}

Position: original: tipped: toppled

Lid on drum: $\quad X$ Yes No

Gasket missing (cm) $21^{\prime \prime}$

Contents expelled: Yes $X$ No

Seal failure: Yes $X$ Not visible

Burn pattern:

i4" at ind Lt. to $2 / 3$ of drum centend on seam

Photo taken: Yes $\searrow$ No

Leak tested:

1FA ot Pressure (psig)

Pressure (psig)

Date
Time (min) 0

Time (min) 5

Initial

Interior assessment:

i" at barn area

V. Dee 44

Photo taken:

Yes

No

Comments: 


\section{Rack Storage Drum Data Sheet}

Drum No. $\times 143$

Description (Check):

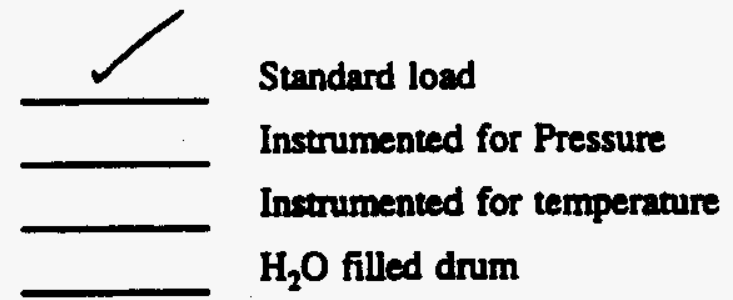

\begin{tabular}{|c|c|c|c|}
\hline Loading Starting at Bottom & Layer 1 & Layer 2 & Layer 3 \\
\hline 3 pieces of steel & & & \\
\hline $1 / 3$ of the brown paper & & & \\
\hline $1 / 3$ of the 10-mil piastic & & & \\
\hline $1 / 3$ of the bond paper & & & \\
\hline $1 / 3$ of the towels & & & \\
\hline $1 / 3$ of the bags & & & \\
\hline $1 / 3$ of the cut paper & & & \\
\hline $1 / 3$ of the fabric & & Go to Layer 3 & \\
\hline $1 / 3$ of the rubber & & & \\
\hline
\end{tabular}

Drum Filled:

Date $5-1-95 \quad$ Initial $4 /$

Ring bolt placed opposite drum seam Date $5-1-95$ Initial AP and in down position

Lid sealed and torqued to $40 \mathrm{ft} / \mathrm{lb}:$ Date $5-1-75$ Initial AP

Leak tested:

Pre-test weight (lb)

Post-test weight (lb)
N/A or Pressure (psig) Pressure (psig)

Date

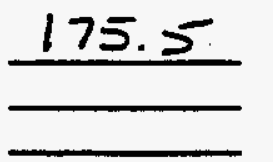

Time (min) 0 Time (min) 5 
WHC-SD-WM-TRP-246 REV. 0

Rack Storage Drum Data Sheet

Drum No. $\quad \underline{\times 143} \quad 175.416$

Post-test Assessment

Position: __ original; __ tipped; __ toppled

Lid on drum: $\quad x$ Yes No

Gasket missing (cm) $Q-12$ "is dauk brown

Contents expelied: __ Yes $x$ No

Seal failure: $\quad$ Yes $X$ Not visible

Burm pattern:

mone

Photo taken: — Yes $\searrow$ No

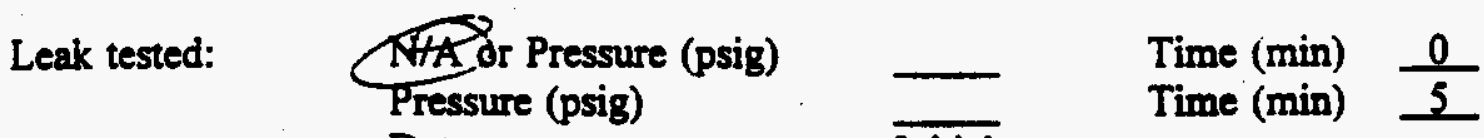

Date

Initial

Interior assessment:

-2"p(wic drelted ( - 12" ppan) tt suam vatually ne charring

woec 45

Photo taken:

$\infty$ Yes

Comments: 
WHC-SD-WM-TRP-246 REV. 0

Rack Storage Drum Data Sheet

Drum No. $\times 153$

Description (Check):

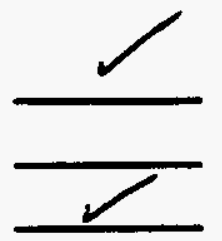

Standard load

Instrumented for Pressure

Instrumented for temperature

$\mathrm{H}_{2} \mathrm{O}$ filled drum

\begin{tabular}{|c|c|c|c|}
\hline Loading Starting at Bottom & Layer 1 & Layer 2 & Layer 3 \\
\hline 3 pieces of steel & & $\mathbf{N} / \mathbf{A}$ & N/A \\
\hline \multicolumn{4}{|l|}{$1 / 3$ of the brown paper } \\
\hline \multicolumn{4}{|l|}{$1 / 3$ of the 10 -mil plastic } \\
\hline \multicolumn{4}{|l|}{$1 / 3$ of the bond paper } \\
\hline \multicolumn{4}{|l|}{$1 / 3$ of the towels } \\
\hline \multicolumn{4}{|l|}{$1 / 3$ of the bags } \\
\hline \multicolumn{4}{|l|}{$1 / 3$ of the cut paper } \\
\hline \multicolumn{4}{|l|}{$1 / 3$ of the fabric } \\
\hline \multicolumn{4}{|l|}{$1 / 3$ of the rubber } \\
\hline & Go to Layer 2 & Go to Layer 3 & \\
\hline
\end{tabular}

Drum Filled:

Date $5-1-95$ Initial $A P$

Ring bolt placed opposite drum seam Date $54-95$ Initial AP

and in down position

Lid sealed and torqued to $40 \mathrm{ft} / \mathrm{b}:$ Date $5-1-9.5$ Initial AP

Leak tested:

N/A or Pressure (psig)

Pressure (psig)

Date

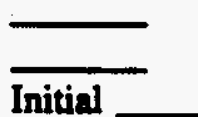

Time (min) 0

Time (min) 5

Pre-test weight (lb)

$175.8 \quad$ Target weight $-175.6 \mathrm{lb}$

Post-test weight (lb)

Loss $=$ 
WHC-SD-WM-TRP-246 REV. 0

Rack Storage Drum Data Sheet

Drum No. $\times 153-176.1$ ib.

Post-test Assessment

Position: ___ original; ___ tipped; __ toppled

Lid on drum: $\quad \underline{X}$ Yes No

Gasket missing (cm) O $211^{\prime \prime}$ compressed and hand

Contents expeiled: _ـ Yes $x$ No

Seal failure: $\quad$ Yes $X$ Not visible

Burn pattern:

none

Photo taken: _u Yes $\underline{x}$ No

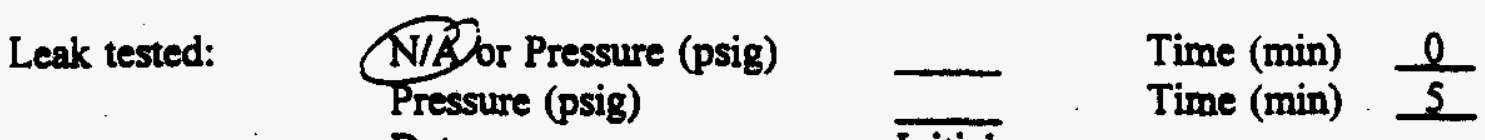

Date

Initial

Interior assessment:

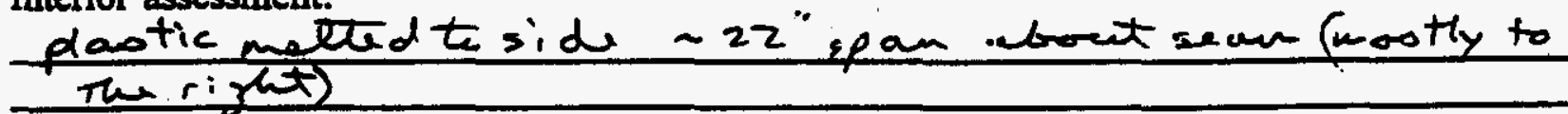
no chan

Wideo \&5

Photo taken:

$X$ Yes

Comments: 


\section{Rack Storage Drum Data Sheet}

Drum No. $x<63$

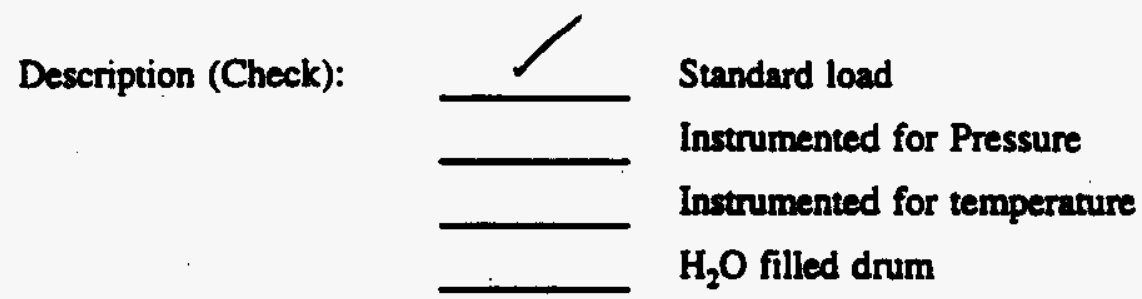

\begin{tabular}{|c|c|c|c|}
\hline Laading Starting at Bottom & Layer 1 & Layer 2 & Layer 3 \\
\hline 3 pieces of steel & & & \\
\hline $1 / 3$ of the brown paper & & & \\
\hline $1 / 3$ of the 10-mil plastic & & & \\
\hline $1 / 3$ of the bond paper & & & \\
\hline $1 / 3$ of the towels & & & \\
\hline $1 / 3$ of the bags & & & \\
\hline $1 / 3$ of the cut paper & & & \\
\hline $1 / 3$ of the fabric & & & \\
\hline $1 / 3$ of the rubber & Go to Layer 2 & Go to Layer 3 & \\
\hline & & & \\
\hline & & & \\
\hline
\end{tabular}

Drum Filled:

Ring bolt placed opposite drum seam and in down position

Lid sealed and torqued to $40 \mathrm{ft} / \mathrm{lb}$ :

Leak tested:

Pre-test weight (lb)

Post-test weight (lb)
Date $5-1-95$ Initial $A P$

Date $5-1.95$ Initial AP

Date $5-1-55$ Initial AP
N/A or Pressure (psig)

Pressure (psig)

Date

175 12.4
Time (min) 0

Time (min) 5 
WHC-SD-WM-TRP-246 REV. 0

Rack Storage Drum Data Sheet

Drum No. $\times 163$ wt. $=175.7$ ib

Post-test Assessment

Position: __ original; ___ tipped; __ toppled

Lid on drum: $\quad X$ Yes No

Gasket missing (cm) $O$ houd + confucesed $\sim 11 "$ spom

Contents expelled: __ Yes $X$ No

Seal failure: $\quad$ Y Yes $X$ Not visible

Bum pattern:

none

Photo taken: $\quad$ Yes $\chi 0$ No

Leak tested: $\quad \begin{array}{lll}\text { N/A or Pressure (psig) } & \text { Time (min) } & 0 \\ \text { Pressure (psig) } & - \text { Time (min) } & -\end{array}$

Interior assessment:

$\frac{\text { plestic weted to well }-15^{\prime \prime} \text { sear and to the right }}{\text { No chan }}$

LCDCO 6C

Phote.taken:

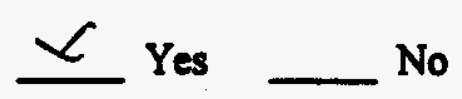

Comments: 
WHC-SD-WM-TRP-246 REV. 0

\section{Rack Storage Drum Data Sheet}

Drum No. $\times 173$

Description (Check):

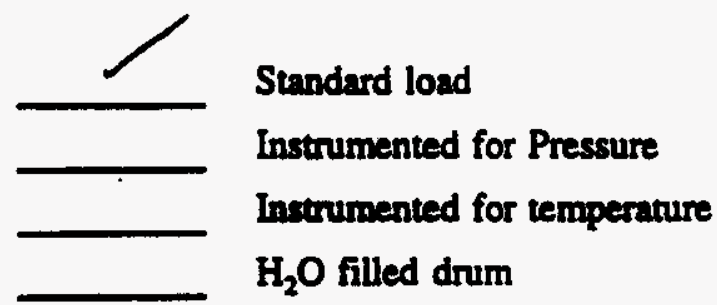

\begin{tabular}{|c|c|c|c|}
\hline Loading Starting at Bottom & Layer 1 & Layer 2 & Layer 3 \\
\hline 3 pieces of steel & & & \\
\hline $1 / 3$ of the brown paper & & & \\
\hline $1 / 3$ of the 10-mil plastic & & & \\
\hline $1 / 3$ of the bond paper & & & \\
\hline $1 / 3$ of the toweis & & & \\
\hline $1 / 3$ of the bags & & & \\
\hline $1 / 3$ of the cut paper & & & \\
\hline $1 / 3$ of the fabric & & Go to Layer 3 & \\
\hline $1 / 3$ of the rubber & Go to Layer 2 & G & \\
\hline & & & \\
\hline
\end{tabular}

Drum Filled:

Ring bolt placed opposite drum seam and in down position

Lid sealed and torqued to $40 \mathrm{ft} / \mathrm{b}$ :

Leak tested:

Pre-test weight (lb)

Post-test weight (Ib)
Date $5-1-95$ Initial $A P$

Date $5-1-15$ Initial Af
N/A or Pressure (psig)

Pressure (psig)

Date

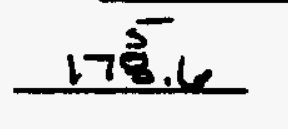

Time (min) 0

Initial

Target weight $-175.6 \mathrm{lb}$ 
WHC-SD-WM-TRP-246 REV. 0

Rack Storage Drum Data Sheet

Drum No. $\times 173$ wt. $=175.5 \mathrm{~b}$.

Post-test Assessment

Position: __ original; __ tipped; _

Lid on drum: $\quad \underline{X}$ Yes No

Gasket missing (cm) '

Contents expelled: —u Yes $x$ No

Seal failure: $\quad$ Yes $x$ Not visible

Burn pattern:

none

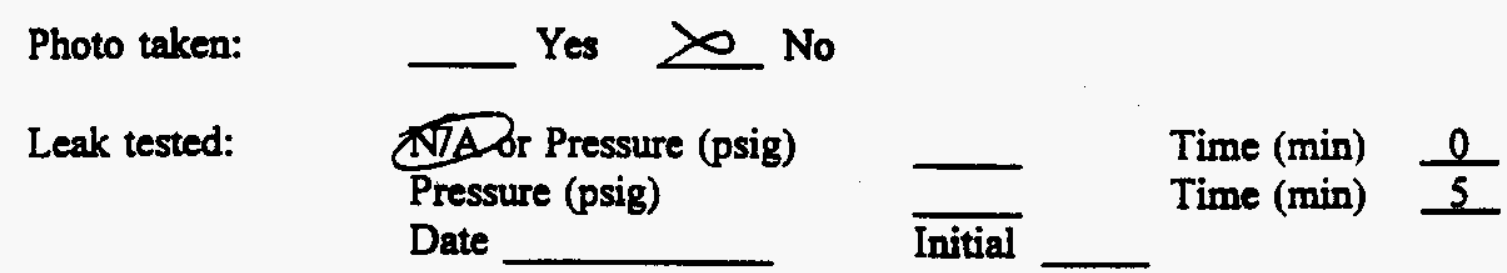

Interior assessment:

plastic bavely welted at seam

Y.pro 67

Photo taken:

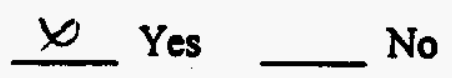

Comments: 


\section{Rack Storage Drum Data Sheet}

Drum No. $\times 183$

Description (Check):

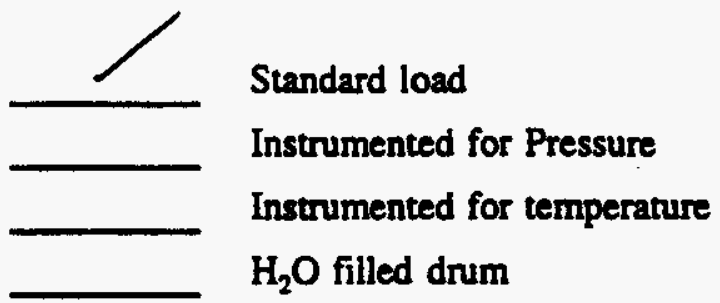

\begin{tabular}{|c|c|c|c|}
\hline Loading Starting at Bottom & Layer 1 & Layer 2 & Layer 3 \\
\hline 3 pieces of steel & & N/A & N/A \\
\hline \multicolumn{4}{|l|}{$1 / 3$ of the brown paper } \\
\hline \multicolumn{4}{|l|}{$1 / 3$ of the 10 -mil plastic } \\
\hline \multicolumn{4}{|l|}{$1 / 3$ of the bond paper } \\
\hline \multicolumn{4}{|l|}{$1 / 3$ of the towels } \\
\hline \multicolumn{4}{|l|}{$1 / 3$ of the bags } \\
\hline \multicolumn{4}{|l|}{$1 / 3$ of the cut paper } \\
\hline \multicolumn{4}{|l|}{$1 / 3$ of the fabric } \\
\hline \multicolumn{4}{|l|}{$1 / 3$ of the rubber } \\
\hline & Go to Layer 2 & Go to Layer 3 & \\
\hline
\end{tabular}

Drum Filled:

Date $5-1-95$ Initial $A P$

Ring bolt placed opposite drum seam Date $5-1-35$ Initial $A P$ and in down position

Lid seaied and torqued to $40 \mathrm{ft} / \mathrm{lb}$ :

Date

Initial

Leak tested:

Pre-test weight (lb)

Post-test weight (lb)
N/A or Pressure (psig) Pressure (psig)

Date

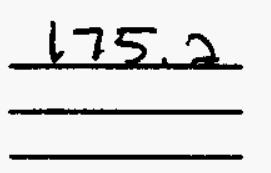

Time (min) 0 Time (min) 5 
WHC-SD-WM-TRP-246 REV. 0

\section{Rack Storage Drum Data Sheet}

Drum No. $\quad x 183 \ldots t .=175.616$.

\section{Post-test Assessment}

Position: original; tipped; toppled

Lid on drum: $\quad X$ Yes No

Gasket missing (cm) 0

Contents expelled: $\quad$ Yes $X$ No

Seal failure: $\quad$ Yes $X$ Not visible

Burn pattern:

NONe

Photo taken:

Yes $\stackrel{x}{\longrightarrow}$ No

Leak tested:

NTA ot Pressure (psig)

Pressure (psig)

Date

nitial

Time (min) 0

Time (min) 5

Interior assessment:

plastic barely nelted at seam

rioec 68

$X$ Yes No

Comments: 


\section{Rack Storage Drum Data Sheet}

Drum No. $\times 213(p)$

Description (Check):

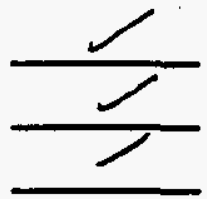

Standard load

Instrumented for Pressure

Instrumented for temperature

$\mathrm{H}_{2} \mathrm{O}$ filled drum

\begin{tabular}{|c|c|c|c|}
\hline Loading Starting at Bottom & Layer 1 & Layer 2 & Layer 3 \\
\hline 3 pieces of steel & & & \\
\hline $1 / 3$ of the brown paper & & & \\
\hline $1 / 3$ of the 10-mil plastic & & \\
\hline $1 / 3$ of the bond paper & & & \\
\hline $1 / 3$ of the towels & & \\
\hline $1 / 3$ of the bags & & & \\
\hline $1 / 3$ of the cut paper & Go to Layer 2 & Go to Layer 3 & \\
\hline $1 / 3$ of the fabric & & \\
\hline $1 / 3$ of the rubber & & & \\
\hline & & & \\
\hline
\end{tabular}

Drum Filled:

Date 5.3 .95 Initial $2 . K$

Ring bolt placed opposite drum seam Date $5 \cdot 3-95$ Initial R.R and in down position

Lid sealed and torqued to $40 \mathrm{ft} / \mathrm{lb}$ :

Date 5.3.95 Initial R.R

Leak tested:

N/A or Pressure (psig) Pressure (psig)

Date

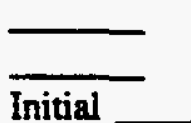

Time $(\min )$

Time (min) 5

Pre-test weight (lb)

Post-test weight (lb)

176.2 Target weight $-175.6 \mathrm{lb}$

Loss $=$ 
WHC-SD-WM-TRP-246 REV. 0

Rack Storage Drum Data Sheet

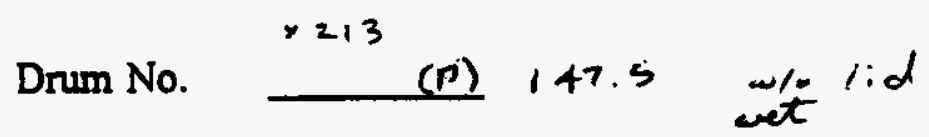

Post-test Assessment

Position: ___ original; ___ tipped; ___ toppled

Lid on drum: $\quad \quad$ Yes $X$ No

Gasket missing (cm)

Contents expelled: __ Yes _ No

Seal failure: $\quad X$ Yes Not visible

Burn pattern:

all anound

Photo taken: $\quad$ Yes $x$ No

Leak tested: N/A or Pressure (psig)

Pressure (psig)

Date

Initial

$\begin{array}{ll}\text { Time (min) } & 0 \\ \text { Time (min) } & \end{array}$

Interior assessment:

$\sim 3^{4}$ chand a ontensts

$\therefore$ is<o is9

photortaken:

$x$ Yes

Comments: 


\section{Rack Storage Drum Data Sheet}

Drum No. $\times 233(P)$

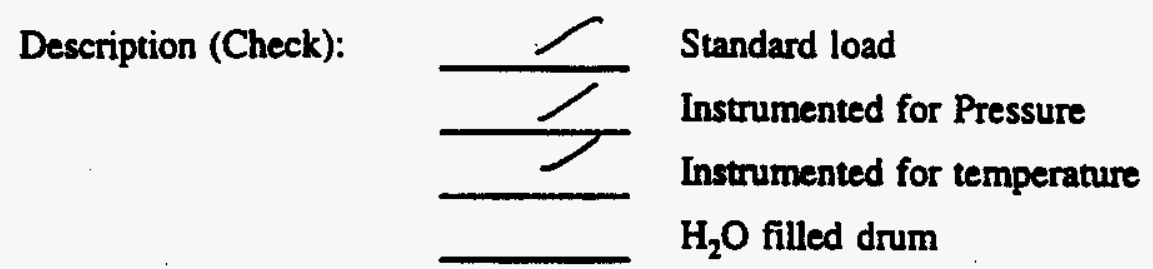

\begin{tabular}{|c|c|c|c|}
\hline Loading Starting at Bottom & Layer 1 & Layer 2 & Layer 3 \\
\hline 3 pieces of stcel & & N/A & $\mathbf{N} / \mathbf{A}$ \\
\hline \multicolumn{4}{|l|}{$1 / 3$ of the brown paper } \\
\hline \multicolumn{4}{|l|}{$1 / 3$ of the 10 -mil plastic } \\
\hline \multicolumn{4}{|l|}{$1 / 3$ of the bond paper } \\
\hline \multicolumn{4}{|l|}{$1 / 3$ of the towels } \\
\hline \multicolumn{4}{|l|}{$1 / 3$ of the bags } \\
\hline \multicolumn{4}{|l|}{$1 / 3$ of the cut paper } \\
\hline \multicolumn{4}{|l|}{$1 / 3$ of the fabric } \\
\hline \multicolumn{4}{|l|}{$1 / 3$ of the rubber } \\
\hline & Go to Layer 2 & Go to Layer 3 & \\
\hline
\end{tabular}

Drum Filled:

Ring bolt placed opposite drum seam and in down position

Lid sealed and torqued to $40 \mathrm{ft} / \mathrm{lb}$ :

Leak tested:

Pre-test weight (lb)

Post-test weight (lb)

Loss $=$
Date $5=3.85 \cdots$ Initial $E K$

Date 5-3-85 Initial R.R

Date 5.3 .95 Initial R.R
N/A or Pressure (psig)

Pressure (psig)

Date

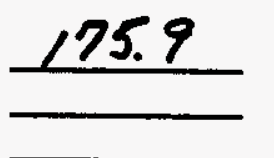

Time (min) 0

Time (min) 5
Initial

Target weight $-175.6 \mathrm{lb}$ 
WHC-SD-WM-TRP-246 REV. 0

\section{Rack Storage Drum Data Sheet}

Drum No. $\quad \underline{233(P)}: T=147.4 \quad$ stil smoldeing

Post-test Assessment

Position: __ original; __ tipped; __ toppled

Lid on drum: $\quad$ Yes $\quad X$ No

Gasket missing (cm) AHI

Contents expelled: _ـ Yes _ No

Seal failure: $\quad \underline{x}$ Yes Not visible

Burn pattern:

$\sim 28^{4}$ ad $h t$.

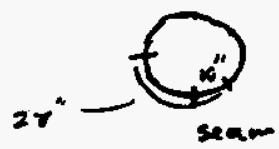

Photo taken:

Yes ㄴ No

Leak tested:

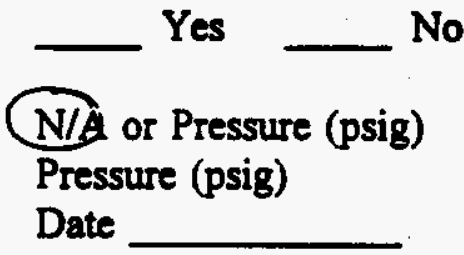

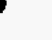




\section{Rack Storage Drum Data Sheet}

Drum No. $\times 243$

Description (Check):

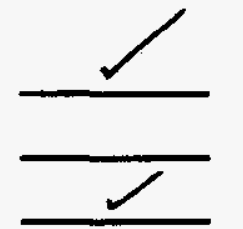

Standard load

Instrumented for Pressure

Instrumented for temperature

$\mathrm{H}_{2} \mathrm{O}$ filled drum

\begin{tabular}{|c|c|c|c|}
\hline Loading Starting at Bottom & Layer 1 & Layer 2 & Layer 3 \\
\hline 3 pieces of steel & & & \\
\hline $1 / 3$ of the brown paper & & & \\
\hline $1 / 3$ of the $10-$ mil plastic & & & \\
\hline $1 / 3$ of the bond paper & & & \\
\hline $1 / 3$ of the towels & & & \\
\hline $1 / 3$ of the bags & & & \\
\hline $1 / 3$ of the cut paper & & & \\
\hline $1 / 3$ of the fabric & & & \\
\hline $1 / 3$ of the rubber & Go to Layer 2 & Go to Layer 3 & \\
\hline & & \\
\hline
\end{tabular}

Drum Filled:

Date $5-1-95 \cdots$ Initial off

Ring bolt placed opposite drum seam Date $5-1-95$ Initial $A P$ and in down position

Lid sealed and torqued to $40 \mathrm{ft} / \mathrm{b}: \quad$ Date 5.1 .95 Initial A.S.

Leak tested:

Pre-test weight (lb)

Post-test weight (lb)
N/A or Pressure (psig)

Pressure (psig)

Date

175.5
Time (min) $\frac{0}{5}$

Time (min) 5

Initial

Target weight $-175.6 \mathrm{lb}$ 
WHC-SD-WM-TRP-246 REV. 0

Rack Storage Drum Data Sheet

Drum No. $\times 243: t=175.316$

Post-test Assessment

Position: original: tipped; toppled

Lid on drum:

$X$ Yes No

Gasket missing (cm) $52.5^{\prime \prime}$

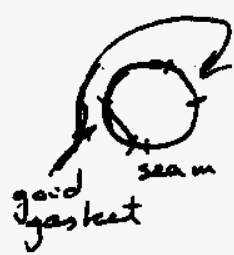

Contents expelled: Yes $X$ No

Seal failure:

Yes $\quad x$ Not visible

Burn pattern:

$.21^{\circ}+$ t d l t

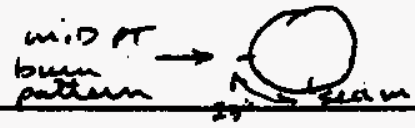

Photo taken:

Yes $x$ No

Leak tested:

X/A Dr Pressure (psig) Pressure (psig)

Date

\begin{tabular}{ll} 
Time $(\min )$ & 0 \\
\hline & Time $(\min )$
\end{tabular}

Interior assessment:

vipec 21

Diototaken:

$X$ Yes

Comments: 


\section{Rack Storage Drum Data Sheet}

Drum No. $x \geq 53(P)$

\begin{tabular}{|} 
Description (Check): \\
\hline Loading Starting at Bottom
\end{tabular}

Drum Filled:

Ring bolt placed opposite drum seam and in down position

Lid seaied and torqued to $40 \mathrm{ft} / \mathrm{lb}$ :

Leak tested:

Pre-test weight (lb)

Post-test weight (lb)
Date 53.95 Initial 10 C

Date 5.3.95 Initial L.K
N/A or Pressure (psig)

Pressure (psig)

Date

175.0
Time (min)

Time (min) 5

Initial

Target weight $\sim 175.6 \mathrm{lb}$ 
WHC-SD-WM-TRP-246 REV. 0

Rack Storage Drum Data Sheet

Drum No. $\times 253(P) \quad 175.4 \mathrm{ib}$.

Post-test Assessment

Position: ___ original; ___ tipped; ___ toppled

Lid on drum: $\quad \underline{x}$ Yes No

Gasket missing (cm) O compressed o hand fon 15"

Contents expelled: _ues $\not$ No

Seal failure: _ Yes $X$ Not visible

Burn pattern:

NONe

Photo taken:

Yes $\infty$ No

Leak tested:

N/A'orPressure (psig)

Pressure (psig)

Date

$\begin{array}{ll}\text { Time }(\mathrm{min}) & 0 \\ \text { Time }(\mathrm{min}) & \underline{5}\end{array}$

$\overline{\text { Initial }}$

Interior assessment:

31 "plastic inelted very, light inowing of top layen of eloth "Tsem

UIDeC 34

Photo taken:

$x$ Yes

Comments: 
WHC-SD-WM-TRP-246 REV. 0

\section{Rack Storage Drum Data Sheet}

Drum No. $\times 26,3$

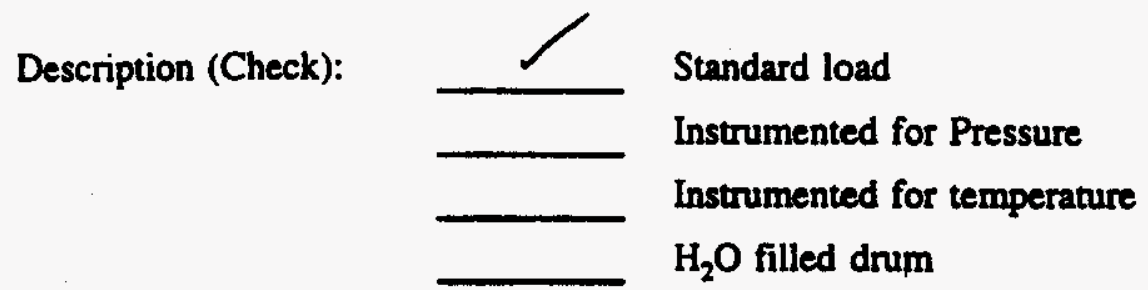

\begin{tabular}{|c|c|c|c|}
\hline Loading Starting at Bottom & Layer 1 & Layer 2 & Layer 3 \\
\hline 3 pieces of steel & & N/A & $\mathbf{N} / \mathbf{A}$ \\
\hline \multicolumn{4}{|l|}{$1 / 3$ of the brown paper } \\
\hline \multicolumn{4}{|l|}{$1 / 3$ of the 10 -mil plastic } \\
\hline \multicolumn{4}{|l|}{$1 / 3$ of the bond paper } \\
\hline \multicolumn{4}{|l|}{$1 / 3$ of the towels } \\
\hline \multicolumn{4}{|l|}{$1 / 3$ of the bags } \\
\hline $1 / 3$ of the cut paper & & & س \\
\hline $1 / 3$ of the fabric & & & $\longleftarrow$ \\
\hline \multicolumn{4}{|l|}{$1 / 3$ of the rubber } \\
\hline & Go to Layer 2 & Go to Layer 3 & \\
\hline
\end{tabular}

Drum Filled:

Ring bolt placed opposite drum seam and in down position

Lid sealed and torqued to $40 \mathrm{ft} / \mathrm{b}$ :

Leak tested:

Pre-test weight (lb)

Post-test weight (lb)
Date $5-1-95$ Initial AP

Date $5-1-95$ Initial $A P$

Date $5-1-95$ Initial $A S$.
N/A or Pressure (psig)

Pressure (psig)

Date

175.6
Time (min)

Initial

Loss $=$ 
WHC-SD-WM-TRP-246 REV. 0

Rack Storage Drum Data Sheet

Drum No. $\quad \underline{263} \omega t=176,1 \mathrm{~b}$

Post-test Assessment

Position: __ original; ___ tipped; __ toppled

Lid on drum: $\quad X$ Yes No

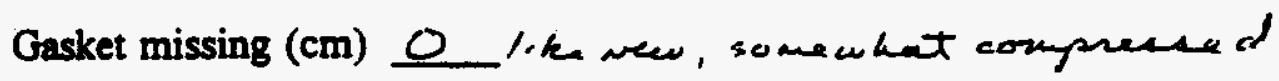

Contents expelied: __ Yes $x$ No

Seal failure: $\quad$ Yes $X$ Not visible

Burn pattern:

None

Photo taken:

Yes $X$

Leak tested:

(N/A or Pressure (psig)

Pressure (psig)

Date

Time (min) $\quad 0$

Time (min) 5

Interior assessment:

bouly meloted plasthe fror seam to $12^{*}$ to left

v:Dewi";

Pheto taken:

$x$ Yes

No

Comments: 
WHC-SD-WM-TRP-246 REV. 0

\section{Rack Storage Drum Data Sheet}

Drum No. $\times 273$

Description (Check):

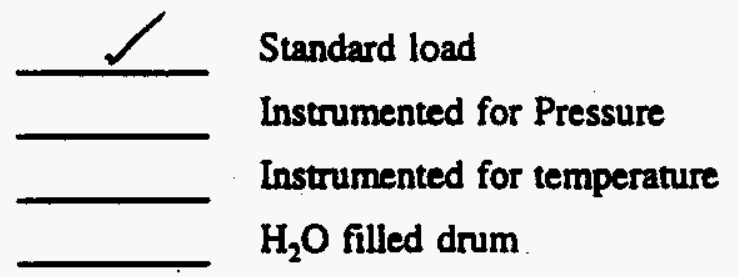

\begin{tabular}{|c|c|c|c|}
\hline Loading Starting at Bottom & Layer 1 & Layer 2 & Layer 3 \\
\hline 3 pieces of steel & & & \\
\hline $1 / 3$ of the brown paper & & & \\
\hline $1 / 3$ of the 10-mil plastic & & & \\
\hline $1 / 3$ of the bond paper & & & \\
\hline $1 / 3$ of the toweis & & & \\
\hline $1 / 3$ of the bags & & & \\
\hline $1 / 3$ of the cut paper & & & \\
\hline $1 / 3$ of the fabric & & Go to Layer 3 & \\
\hline $1 / 3$ of the rubber & Go to Layer 2 & G & \\
\hline
\end{tabular}

Drum Filled:

Date $5-1-95$ Initial kP

Ring bolt placed opposite drum seam Date $5-95$ Initial AP and in down position

Lid sealed and torqued to $40 \mathrm{ft} / \mathrm{lb}: \quad$ Date 5.1 .95 Initial A.S.

Leak tested:

Pre-test weight (lb)

Post-test weight (lb)
N/A or Pressure (psig)

Pressure (psig)

Date

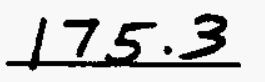

Time (min) $\frac{0}{5}$
Time (min)

Initial

Target weight $-175.6 \mathrm{lb}$

\footnotetext{
Loss =
} 
WHC-SD-WM-TRP-246 REV. 0

Rack Storage Drum Data Sheet

Drum No. $\times 273$ at. $=175.8 \mathrm{ib}$.

Post-test Assessment

Position: __ original; ___ tipped; __ toppled

Lid on drim: $\quad \quad x$ Yes No

Gasket missing (cm) O like New

Contents expelled: _ Yes $\varnothing$ No

Seal failure: $\quad$ Yes $X$ Not visible

Bum pattern:

wone

Photo taken:

Yes $⿻$ No

Leak tested:

N7/A or Pressure (psig)

Pressure (psig)

Date

\begin{tabular}{lll} 
& Time (min) & 0 \\
\hline & Time (min)
\end{tabular}

Interior assessment:

barely imall spot of plastic $10^{\circ}$ ift of ream

i. oee 77

pinoto taken:

$\underline{y}$ Yes No

Comments: 
WHC-SD-WM-TRP-246 REV. 0

\section{Rack Storage Drum Data Sheet}

Drum No. $\times 283$

Description (Check):

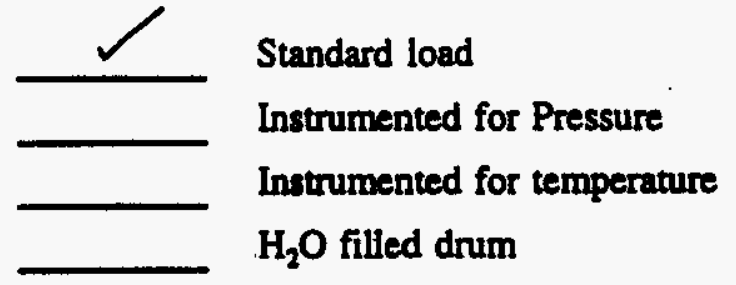

\begin{tabular}{|c|c|c|c|}
\hline Loading Starting at Bottom & Layer 1 & Layer 2 & Layer 3 \\
\hline 3 pieces of steel & & N/A & \\
\hline $1 / 3$ of the brown paper & & & \\
\hline $1 / 3$ of the 10-mil plastic & & & \\
\hline $1 / 3$ of the bond paper & & & \\
\hline $1 / 3$ of the towels & & & \\
\hline $1 / 3$ of the bags & $\checkmark$ & & \\
\hline $1 / 3$ of the cut paper & & & \\
\hline $1 / 3$ of the fabric & & & \\
\hline $1 / 3$ of the rubber & Go to Layer 2 & Go to Layer 3 & \\
\hline & & & \\
\hline
\end{tabular}

Drum Filled:

Date $5-1-95$ Initial $A P$

Ring bolt placed opposite drum seam Date $5-1-75$ Initial AP and in down position

Lid seaied and torqued to $40 \mathrm{ft} / \mathrm{b}$ :

Date $5 \cdot 1 \cdot 95$ Initial $A 5$

Leak tested:

N/A or Pressure (psig)

Pressure (psig)

Date

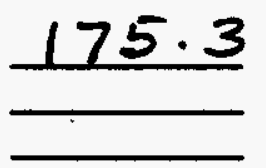

Time (min)

Time (min)

Pre-test weight (lb)

Post-test weight (lb)

Loss $=$ 
WHC-SD-WM-TRP-246 REV. 0

\section{Rack Storage Drum Data Sheet}

Drum No. $\times 283 \quad w t .=175.7 \mathrm{lb}$.

\section{Post-test Assessment}

Position: __ original; ___ tipped; __ toppled

Lid on drum: $\quad x$ Yes No

Gasket missing (cm) $O$ like wew, some what compressed.

Contents expelled: __ Yes $x$ No

Seal failure: $\quad$ Yes $X$ Not visible

Burn pattern:

None

Photo taken:

Yes $X$ No

Leak tested:

NT/Agr Pressure (psig)

Pressure (psig)

Date

$\begin{array}{lll}\text { Time (min) } & \frac{0}{5}\end{array}$

$\overline{\text { Initial }}$

Interior assessment:

Interior assessment:
no Chou, no melting

$v$ ipevis

pheto taken:

X Yes

No

Comments: 
WHC-SD-WM-TRP-246 REV. 0

Rack Storage Drum Data Sheet

Drum No. $\times 313$

Description (Check):

Standard load

Instrumented for Pressure

Instrumented for temperature

$\mathrm{H}_{2} \mathrm{O}$ filled drum

\begin{tabular}{|c|c|c|c|}
\hline Loading Starting at Bottom & Layer 1 & Layer 2 & Layer 3 \\
\hline 3 pieces of steel & & & \\
\hline $1 / 3$ of the brown paper & & & \\
\hline $1 / 3$ of the 10-mil plastic & & & \\
\hline $1 / 3$ of the bond paper & & & \\
\hline $1 / 3$ of the towels & & & \\
\hline $1 / 3$ of the bags & & & \\
\hline $1 / 3$ of the cut paper & & & \\
\hline $1 / 3$ of the fabric & Go to Layer 2 & Go to Layer 3 & \\
\hline $1 / 3$ of the rubber & & & \\
\hline & & & \\
\hline
\end{tabular}

Drum Filled:

Ring bolt placed opposite drum seam and in down position

Lid sealed and torqued to $40 \mathrm{ft} / \mathrm{b}: \quad$ Date $5 \cdot 1.95$ Initial $A .5$.

Leak tested:

Pre-test weight (lb)

Post-test weight (lb)

Loss $=$
N/A or Pressure (psig)

Pressure (psig)

Date

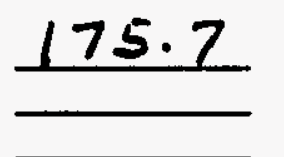

$\begin{array}{ll}-\quad \text { Time }(\min ) & \frac{0}{5}\end{array}$

Initial

Target weight $-175.6 \mathrm{lb}$ 
WHC-SD-WM-TRP-246 REV. 0

Rack Storage Drum Data Sheet

Drum No. $\times 3 / 3$. wt. 148.8

Post-test Assessment

Position: __ original; ___ tipped; __ toppled

Lid on drum: L/Yes No $\quad 21$ " under ringi $\sim 1 / 4 "$ jap anound

Gasket missing (cm)

Contents expelled: _ Yes $X$ No

Seal failure: $\quad X$ Yes $\quad$ Not visible

Burn pattern:
All wound

Photo taken: $\quad$ Yes $x$ No

Leak tested: NN/A Dressure (psig) Pressure (psig)

Date

\begin{tabular}{lll} 
Time (min) & 0 \\
\hline
\end{tabular}

$\overline{\text { Initial }}$

Interior assessment:

$14^{\circ}$ of chened critent

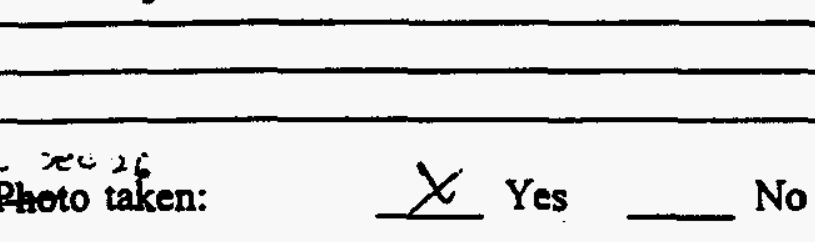

Comments: 
WHC-SD-WM-TRP-246 REV. 0

Rack Storage Drum Data Sheet

Drum No. $\times 32.3$

Description (Check):

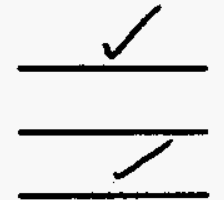

Standard load

Instrumented for Pressure

Instrumented for temperature

$\mathrm{H}_{2} \mathrm{O}$ filled drum

\begin{tabular}{|c|c|c|c|}
\hline Loading Starting at Bottom & Layer 1 & Layer 2 & Layer 3 \\
\hline 3 pieces of steel & & & \\
\hline $1 / 3$ of the brown paper & & & \\
\hline $1 / 3$ of the 10-mil plastic & & & \\
\hline $1 / 3$ of the bond paper & & & \\
\hline $1 / 3$ of the towels & & & \\
\hline $1 / 3$ of the bags & & & \\
\hline $1 / 3$ of the cut paper & & & \\
\hline $1 / 3$ of the fabric & & & \\
\hline $1 / 3$ of the rubber & Go to Layer 2 & Go to Layer 3 & \\
\hline
\end{tabular}

Drum Filled:

Date $5-1-95$ Initial $A P$

Ring bolt placed opposite drum seam Date $5-1-95$ Initial AP

and in down position

Lid sealed and torqued to $40 \mathrm{ft} / \mathrm{b}: \quad$ Date $5 \cdot 1.95$ Initial A.S.

Leak tested:

Pre-test weight (lb)

Post-test weight (lb)
N/A or Pressure (psig)

Pressure (psig)

Date

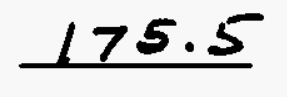

Time (min)

Time $(\min )$

Initial

Target weight - $175.6 \mathrm{lb}$ 
WHC-SD-WM-TRP-246 REV. 0

Rack Storage Drum Data Sheet

Drum No. $\quad \underline{3.23}$ ut $=1470$

Post-test Assessment

Position: __ original; ___ tipped; ___ toppled

Lid on drum: $\quad$ Yes $X$ No

Gasket missing (cm) All

Contents expeiled: _ Yes _ No

Seal failure: $\quad X$ Yes Not visible

Burn pattern:

$39^{\prime \prime}$

Photo taken: $\quad$ Yes $X$ No

Leak tested:

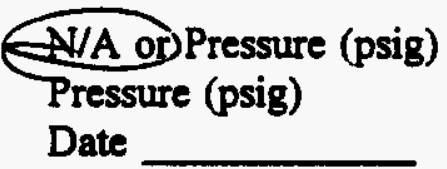

Pressure (psig)

Date

\begin{tabular}{lll}
$-\quad$ Time $(\min )$ & $\frac{0}{5}$ \\
\hline
\end{tabular}

Interior assessment:

-9" chaned isutents

Proc $\div 5$

Phote taken:

$X$ Yes No

Comments: 
WHC-SD-WM-TRP-246 REV. 0

Rack Storage Drum Data Sheet

Drum No. $\times 333$

Description (Check):

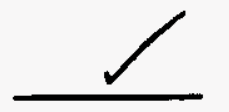

Standard load

Instrumented for Pressure

Instrumented for temperature

$\mathrm{H}_{2} \mathrm{O}$ filled drum

\begin{tabular}{|c|c|c|c|}
\hline Loading Starting at Bottom & Layer 1 & Layer 2 & Layer 3 \\
\hline 3 pieces of steel & & N/A & $\mathbf{N} / \mathbf{A}$ \\
\hline \multicolumn{4}{|l|}{$1 / 3$ of the brown paper } \\
\hline \multicolumn{4}{|l|}{$1 / 3$ of the 10 -mil plastic } \\
\hline \multicolumn{4}{|l|}{$1 / 3$ of the bond paper } \\
\hline \multicolumn{4}{|l|}{$1 / 3$ of the towels } \\
\hline \multicolumn{4}{|l|}{$1 / 3$ of the bags } \\
\hline \multicolumn{4}{|l|}{$1 / 3$ of the cut paper } \\
\hline $1 / 3$ of the fabric & & $\swarrow$ & $\sim$ \\
\hline \multicolumn{4}{|l|}{$1 / 3$ of the rubber } \\
\hline & Go to Layer 2 & Go to Layer 3 & \\
\hline
\end{tabular}

Drum Filled:

Date $5-1-95$ Initial AP

Ring bolt placed opposite drum seam Date $5-1-95$ Initial $A P$

and in down position

Lid sealed and torqued to $40 \mathrm{ftlb}: \quad$ Dates-1.95 Initial A.S.

Leak tested:

-test weight (lb)

Post-test weight (lb)
N/A or Pressure (psig)

Pressure (psig)

Date

175.2
Time (min) 0

Time (min) 5

Initial

Target weight $-175.6 \mathrm{lb}$ 


\section{WHC-SD-WM-TRP-246 REV. 0 \\ Rack Storage Drum Data Sheet}

Drum No. $\times 333 \quad$ it. $=17.8$ ib has $\mu_{2} \mathrm{O}$

Post-test Assessment

Position: __ original; ___tipped; ___ toppled

Lid on drum: $\quad$ Yes $X$ No

Gasket missing (cm) All

Contents expelled: __ Yes _ No

Seal failure: $\quad \times$ Yes $\quad$ Not visible

Burn pattern:

$33^{\prime c h e n t "}$

Photo taken:

Yes $\underline{x}$ No

Leak tested:

ATA-Aressure (psig)

Pressure (psig)

Date \begin{tabular}{lll}
$-\quad$ Time $(\min )$ & 0 \\
\hline
\end{tabular}

Initial

Interior assessment:

-3" chaned contents

Les i.4

phote-taken:

$X$ Yes

Comments: 
WHC-SD-WM-TRP-246 REV. 0

Rack Storage Drum Data Sheet

Drum No. $\times 343$

Description (Check):

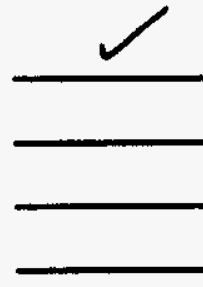

Standard load

Instrumented for Pressure

Instrumented for temperature

$\mathrm{H}_{2} \mathrm{O}$ filled drum

\begin{tabular}{|c|c|c|c|}
\hline Loading Starting at Bottom & Layer 1 & Layer 2 & Layer 3 \\
\hline 3 pieces of steel & & N/A & N/A \\
\hline $1 / 3$ of the brown paper & & & \\
\hline $1 / 3$ of the 10-mil plastic & & & \\
\hline $1 / 3$ of the bond paper & $\checkmark$ & & \\
\hline $1 / 3$ of the towels & $\checkmark$ & \\
\hline $1 / 3$ of the bags & $\checkmark$ & \\
\hline $1 / 3$ of the cut paper & $\checkmark$ & $\checkmark$ & \\
\hline $1 / 3$ of the fabric & $\checkmark$ & $\checkmark$ & \\
\hline $1 / 3$ of the rubber & Go to Layer 2 & Go to Layer 3 & \\
\hline
\end{tabular}

Drum Filled:

Ring bolt placed opposite drum seam and in down position

Lid sealed and torqued to $40 \mathrm{ft} / \mathrm{lb}$ :

Leak tested:

Pre-test weight (lb)

Post-test weight (lb)

Date $5-1.95$ Initial A.S.

Date 5.1 .95 Initial $\Lambda . S$

Date $5-1.95$ Initial A.S.

N/A or Pressure (psig) Pressure (psig)

Date

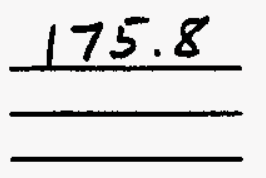

Time (min) 0

Time (min) 5

Loss = 
WHC-SD-WM-TRP-246 REV. 0

\section{Rack Storage Drum Data Sheet}

Drum No. $\times 343$ U. $175,2,16$

Post-test Assessment

Position:

original:

tipped;

toppled

Lid on drim: $\quad \underline{X}$ Yes $\quad$ No

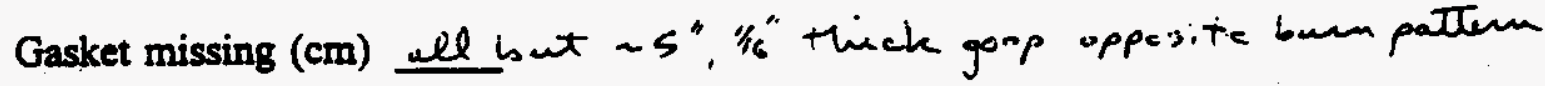

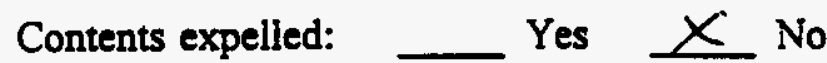

Seal failure:

Yes $X$ Not visible

Burn pattern:

Photo taken:

Yes $\longrightarrow$ No

Leak tested:

NTA Pressure (psig)

Pressure (psig)

Date

\begin{tabular}{lll} 
& Time (min) & 0 \\
\hline Initial & Time (min)
\end{tabular}

Interior assessment:

-1" depth chan an burned side vove in otherside

Teo 23

Puoto taken:

$x$ Yes

No

Comments: 
WHC-SD-WM-TRP-246 REV. 0

Rack Storage Drum Data Sheet

Drum No. $\times 353$

Description (Check):

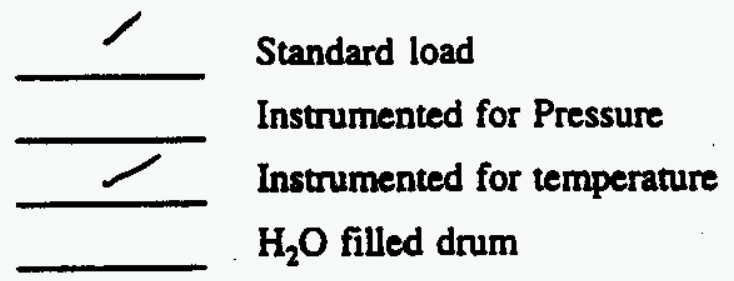

\begin{tabular}{|c|c|c|c|}
\hline Loading Starting at Bottom & Layer 1 & Layer 2 & Layer 3 \\
\hline 3 pieces of steel & $\boldsymbol{\sim}$ & N/A & N/A \\
\hline $1 / 3$ of the brown paper & $\checkmark$ & $\checkmark$ & $\checkmark$ \\
\hline $1 / 3$ of the 10 -mil plastic & $\checkmark$ & $\checkmark$ & $\checkmark$ \\
\hline $1 / 3$ of the bond paper & $r$ & $\checkmark$ & $\checkmark$ \\
\hline $1 / 3$ of the towels & $\checkmark$ & 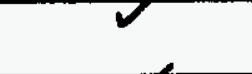 & $V$ \\
\hline $1 / 3$ of the bags & 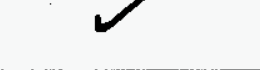 & $\checkmark$ & $\checkmark$ \\
\hline $1 / 3$ of the cut paper & $\checkmark$ & $V$ & $\checkmark$ \\
\hline $1 / 3$ of the fabric & $\checkmark$ & $\checkmark$ & $\checkmark$ \\
\hline $1 / 3$ of the rubber & $\checkmark$ & $\checkmark$ & 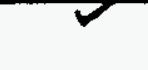 \\
\hline & Go to Layer 2 & Go to Layer 3 & \\
\hline
\end{tabular}

Drum Filled:

Date $5-1 \cdot 95$ Initial A.S.

Ring bolt placed opposite drum seam Date $5-1.95$ Initial A.S. and in down position

Lid sealed and torqued to $40 \mathrm{f} / \mathrm{bb}: \quad$ Date 5.1 .95 Initial A.S.

Leak tested:

Pre-test weight (lb)

Post-test weight (lb)
N/A or Pressure (psig)

Pressure (psig)

Date

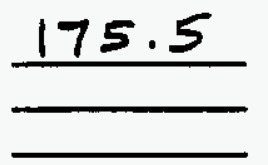

Time (min) 0

Time (min)

Target weight $\sim 175.6 \mathrm{lb}$ 
WHC-SD-WM-TRP-246 REV. 0

Rack Storage Drum Data Sheet

Drum No. $\quad x_{353}$ ut. $=176.216$.

Post-test Assessment

Position: __ original; __ tipped; __ toppled

Lid on drum: $\quad x$ Yes No

Gasket missing (cm) O 15" had o compuessed

Contents expeiled: _ Yes $\quad X$ No

Seal failure: $\quad$ Yes $\quad X$ Not visible

Burn pattern:

None

Photo taken:

Yes $\underline{x}$ No

Leak tested:

NTANor Pressure (psig)

Pressure (psig)

Date

$\longrightarrow \quad$\begin{tabular}{ll} 
Time (min) & 0 \\
\hline & Time (min)
\end{tabular}

Initial

Interior assessment: $27 "$ Interior assessment:
no chaning rifht melting fplastic - some to wall

f

以代仍

Photo taken:

$x$ Yes

Comments: 
WHC-SD-WM-TRP-246 REV. 0

Reck Storage Drum Data Sheet

Drum No. $\times 363$

Description (Check):

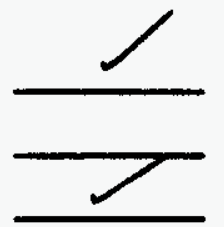

Standard load

Instrumented for Pressure

Instrumented for temperature

$\mathrm{H}_{2} \mathrm{O}$ filled drum

\begin{tabular}{|c|c|c|c|}
\hline Loading Starting at Bottom & Layer 1 & Layer 2 & Layer 3 \\
\hline 3 pieces of steel & $\checkmark$ & N/A & N/A \\
\hline $1 / 3$ of the brown paper & $\checkmark$ & $\boldsymbol{v}$ & $\checkmark$ \\
\hline $1 / 3$ of the 10 -mil plastic & $\checkmark$ & レ & $r$ \\
\hline $1 / 3$ of the bond paper & $\checkmark$ & $r$ & $\nabla$ \\
\hline $1 / 3$ of the toweis & 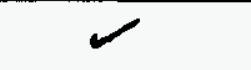 & $\checkmark$ & $\boldsymbol{V}$ \\
\hline $1 / 3$ of the bags & $\checkmark$ & $\checkmark$ & $\checkmark$ \\
\hline $1 / 3$ of the cut paper & $\checkmark$ & $J$ & $r$ \\
\hline $1 / 3$ of the fabric & $\checkmark$ & $\checkmark$ & $\checkmark$ \\
\hline \multirow[t]{2}{*}{$1 / 3$ of the rubber } & $\checkmark$ & $\checkmark$ & $\nabla$ \\
\hline & Go to Layer 2 & Go to Layer 3 & \\
\hline
\end{tabular}

Drum Filled:

Date $5-1.95$ Initial A.S.

Ring bolt placed opposite drum seam Date $5 \cdot 1.95$ Initial $A . S$. and in down position

Lid sealed and torqued to $40 \mathrm{ft} / \mathrm{b}: \quad$ Date $5-1.95$ Initial

Leak tested:

Pre-test weight (lb)

Post-test weight (lb)
N/A or Pressure (psig)

Pressure (psig)

Date

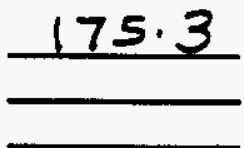

Time $(\min ) \frac{0}{5}$

Initial

Target weight $-175.6 \mathrm{lb}$ 
WHC-SD-WM-TRP-246 REV. 0

Rack Storage Drum Data Sheet

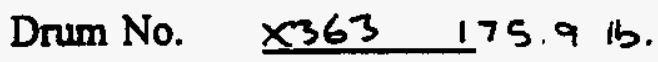

Post-test Assessment

Position:

original; ___ tipped; toppled

Lid on drum: $\quad X$ Yes No

Gasket missing (cm) 2 like weer

Contents expeiled: _u Yes $X$ No

Seal failure: _ _ Yes $x$ Not visible

Burn pattern:

nove

Photo taken:

Y.Pes $x$ No

Leak tested:

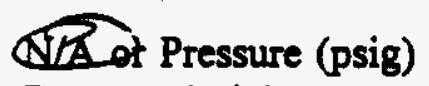
Pressure (psig)

Date

Time (min) 0 Time (min) 5

Interior assessment:

$\frac{13 \text { " seam to right slightmethy }}{\text { Nochas }}$

Pecco cś

$x$ Yes

Comments: 
WHC-SD-WM-TRP-246 REV. 0

\section{Rack Storage Drum Data Sheet}

Drum No. $\times 373$

Description (Check):

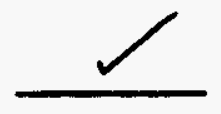

Standard load

Instrumented for Pressure

Instrumented for temperature

$\mathrm{H}_{2} \mathrm{O}$ filled drum

\begin{tabular}{|c|c|c|c|}
\hline Loading Starting at Bottom & Layer 1 & Layer 2 & Layer 3 \\
\hline 3 pieces of steel & & N/A & \\
\hline $1 / 3$ of the brown paper & & & \\
\hline $1 / 3$ of the 10-mil plastic & & & \\
\hline $1 / 3$ of the bond paper & & & \\
\hline $1 / 3$ of the toweis & & & \\
\hline $1 / 3$ of the bags & & & \\
\hline $1 / 3$ of the cut paper & & & \\
\hline $1 / 3$ of the fabric & & & \\
\hline $1 / 3$ of the rubber & Go to Layer 2 & Go to Layer 3 & \\
\hline & & & \\
\hline
\end{tabular}

Drum Filled:

Ring bolt placed opposite drum seam and in down position

Lid sealed and torqued to $40 \mathrm{ftlb}$ : Date $5-1.95$ Initial A. S

Leak tested:

Pre-test weight (lb)

Post-test weight (lb)

Date $5-1.95$ Initial A.S.

Date $5-1.95$ Initial A.S.

N/A or Pressure (psig)

Pressure (psig)

Date

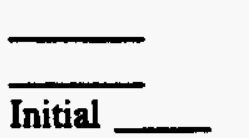

Time (min) $\frac{0}{5}$
Time (min)

$176.0 \quad$ Target weight $\sim 175.6 \mathrm{lb}$ 
WHC-SD-WM-TRP-246 REV. 0

\section{Rack Storage Drum Data Sheet}

Drum No. $\times 373 \quad T=176.3 \mathrm{~h}$.

Post-test Assessment

Position: __ original; ___ tipped; __ toppled

Lid on drum: $\quad y$ Yes No

Gasket missing (cm) D like vew', i lightly cmpressed

Contents expelled: — Yes $\varnothing$ No

Seal failure: $\quad$ Yes $\quad x$ Not visible

Burn pattern:

wove - sooty

Photo taken:

Yes $\perp \in$ No

Leak tested:

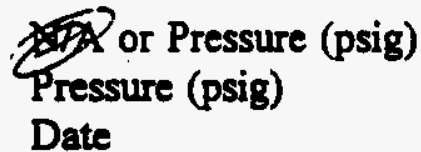

Date

$-\quad$ Time (min) $\quad \frac{0}{5}$

Interior assessment:

No retting

No chan

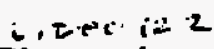

ploto taken:

$\underline{x}$ Yes No

Comments: 
WHC-SD-WM-TRP-246 REV. 0

\section{Rack Storage Drum Data Sheet}

Drum No. $\times 383$

Description (Check):

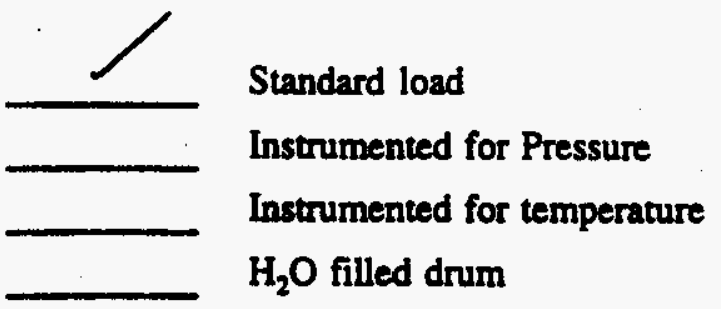

\begin{tabular}{|c|c|c|c|}
\hline Loading Starting at Bottom & Layer 1 & Layer 2 & Layer 3 \\
\hline 3 pieces of steel & & N/A & \\
\hline $1 / 3$ of the brown paper & & & \\
\hline $1 / 3$ of the 10-mil plastic & & & \\
\hline $1 / 3$ of the bond paper & & & \\
\hline $1 / 3$ of the towels & & & \\
\hline $1 / 3$ of the bags & & & \\
\hline $1 / 3$ of the cut paper & & & \\
\hline $1 / 3$ of the fabric & & & \\
\hline $1 / 3$ of the rubber & Go to Layer 2 & Go to Layer 3 & \\
\hline
\end{tabular}

Drum Filled:

Date $5-1.95$ Initial A.S.

Ring bolt placed opposite drum seam and in down position

Lid seaied and torqued to $40 \mathrm{ft} / \mathrm{lb}$ :

Leak tested:

Pre-test weight (lb)

Post-test weight (lb)
Date 5-1.95 Initial A.S.

Date $5 \cdot 1.95$ Initial A.S.
N/A or Pressure (psig)

Pressure (psig)

Date

175.5
Time (min) 0

Time (min) 
WHC-SD-WM-TRP-246 REV. 0

\section{Rack Storage Drum Data Sheet}

Drum No. $\lcm{353} \omega t=, 75.7 \mathrm{~b}$

\section{Post-test Assessment}

Position: original: ___ tipped; ___ toppled

Lid on drum: $\quad x$ Yes $\quad$ No

Gasket missing (cm) $O$ failly hand $\sim / 2$ "

Contents expelled: _ Yes $\varnothing$ No

Seal failure: $\quad$ Yes $\quad x$ Not visible

Burm pattern: nove - slialutty iodty

Photo taken:

Yes _ No

Leak tested:

N/A or Pressure (psig)

Pressure (psig)

Date

\begin{tabular}{lll} 
& Time (min) & $\frac{0}{5}$ \\
\hline Initial & Time (min)
\end{tabular}

Interior assessment:

no mellin

viped 120

photo taken:

120 Yes _ No

Comments:

like ven arept in soot 
WHC-SD-WM-TRP-246 REV. 0

Rack Storage Drum Data Sheet

Drum No. $\times 114$

Description (Check):

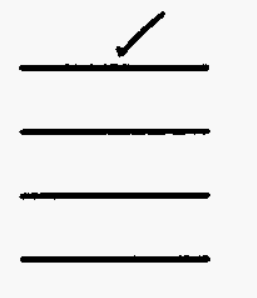

Standard load

Instrumented for Pressure

Instrumented for temperature

$\mathrm{H}_{2} \mathrm{O}$ filled drum

\begin{tabular}{|c|c|c|c|}
\hline Loading Starting at Bottom & Layer 1 & Layer 2 & Layer 3 \\
\hline 3 pieces of steel & N/A & \\
\hline $1 / 3$ of the brown paper & & & \\
\hline $1 / 3$ of the 10-mil plastic & & & \\
\hline $1 / 3$ of the bond paper & & & \\
\hline $1 / 3$ of the towels & & & \\
\hline $1 / 3$ of the bags & & & \\
\hline $1 / 3$ of the cut paper & & & \\
\hline $1 / 3$ of the fabric & & Go to Layer 3 & \\
\hline $1 / 3$ of the rubber & & & \\
\hline & & & \\
\hline
\end{tabular}

Drum Filled:

Date $5=2-95$ Initial L.K

Ring bolt placed opposite drum seam Date $5 \cdot 2.95$ Initial R.K

and in down position

Lid sealed and torqued to $40 \mathrm{f} / 1 \mathrm{~b}: \quad$ Date 5.2 .95 Initial $1 K$

Leak tested:

N/A or Pressure (psig)

Pressure (psig)

Date

$\longrightarrow \quad$ Time $(\min ) \frac{0}{5}$

Pre-test weight (lb)

$\angle 75.9 \quad$ Target weight $-175.6 \mathrm{lb}$

Post-test weight (lb)

Loss = 
WHC-SD-WM-TRP-246 REV. 0

Rack Storage Drum Data Sheet

Drum No. $\quad \underline{x 14} \quad 175.715$

\section{Post-test Assessment}

Position: original: ___ tipped; ___ toppled

Lid on drum: $\quad X$ Yes No

Gasket missing (cm) $28^{\circ}$.

Contents expelled: —ues $x$ No

Seal failure: $\quad$ Yes $\quad \chi$ Not visible

Burn pattern:

$9^{\prime \prime}$ top $2 / 3$ of drum at seam

Photo taken:

Yes $\not \underline{ }$ No

Leak tested:

Pressure (psig)

Date

Initial

Time (min) 0 Time (min) 5

Interior assessment:

2-3" d chour t weted plastic seam holf of drum backe half has ineled plastic but little chan

Visec 49

Photo taken:

$\triangle$ Yes No

Comments: 
WHC-SD-WM-TRP-246 REV. 0

\section{Rack Storage Drum Data Sheet}

Drum No. $\times 124$

Description (Check):

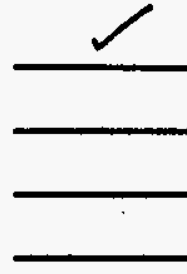

Standard load

Instrumented for Pressure

Instrumented for temperature

$\mathrm{H}_{2} \mathrm{O}$ filled drum

\begin{tabular}{|c|c|c|c|}
\hline Loading Starting at Bottom & Layer 1 & Layer 2 & Layer 3 \\
\hline 3 pieces of steel & & $\mathbf{N} / \mathbf{A}$ & N/A \\
\hline \multicolumn{4}{|l|}{$1 / 3$ of the brown paper } \\
\hline \multicolumn{4}{|l|}{$1 / 3$ of the 10 -mil plastic } \\
\hline \multicolumn{4}{|l|}{$1 / 3$ of the bond paper } \\
\hline \multicolumn{4}{|l|}{$1 / 3$ of the towels } \\
\hline \multicolumn{4}{|l|}{$1 / 3$ of the bags } \\
\hline \multicolumn{4}{|l|}{$1 / 3$ of the cut paper } \\
\hline \multicolumn{4}{|l|}{$1 / 3$ of the fabric } \\
\hline \multicolumn{4}{|l|}{$1 / 3$ of the rubber } \\
\hline & Go to Layer 2 & Go to Layer 3 & \\
\hline
\end{tabular}

Drum Filled:

Ring bolt placed opposite drum seam and in down position

Lid sealed and torqued to $40 \mathrm{ft} / \mathrm{b}$ :

Leak tested:

Pre-test weight (lb)

Post-test weight (lb)
Date 5.2.85 Initial R.K

Date 5.7 .95 Initial R.K

Date 5.2 .95 Initial R.K

N/A or Pressure (psig)

Pressure (psig)

Date

\begin{tabular}{|c|c|c|}
\hline & Time (min) & 0 \\
\hline . & Time (min) & 5 \\
\hline
\end{tabular}


WHC-SD-WM-TRP-246 REV. 0

Rack Storage Drum Data Sheet

Drum No. $\quad \underline{x 124} u t .=176.5 \mathrm{ib}$

Post-test Assessment

Position:

original;

tipped; toppled

Lid on drum: $\quad \underline{X}$ Yes No

Gasket missing (cm) _ـ $26^{\prime \prime}$ at seam.

Contents expelled: $\quad$ Yes $\underline{x}$ No

Seal failure: $\quad$ Yes $X$ Not visible

Burn pattern:

Nove - some discolcratein to right $f$ seom

Photo taken: Yes $\not \subset$ No

Leak tested:

Pror Pressure (psig)

Pressure (psig)

Date \begin{tabular}{lll}
- & Time $(\min )$ & 0 \\
\hline
\end{tabular}$\quad$ Time $(\min ) \quad$

Interior assessment:

-3 " chan at seam 26 "span

snec 47

Fhoto taken:

$\varnothing$ Yes

Comments: 
WHC-SD-WM-TRP-246 REV. 0

\section{Rack Storage Drum Data Sheet}

Drum No. $\times 13+$

Description (Check):

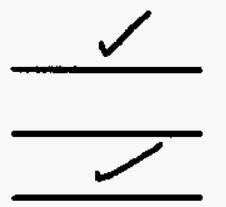

Standard load

Instrumented for Pressure

Instrumented for temperature

$\mathrm{H}_{2} \mathrm{O}$ filled drum

\begin{tabular}{|c|c|c|c|}
\hline Loading Starting at Bottom & Layer 1 & Layer 2 & Layer 3 \\
\hline 3 pieces of steel & & N/A & N/A \\
\hline $1 / 3$ of the brown paper & & & \\
\hline $1 / 3$ of the 10-mil plastic & & & \\
\hline $1 / 3$ of the bond paper & & & \\
\hline $1 / 3$ of the towels & & & \\
\hline $1 / 3$ of the bags & & & \\
\hline $1 / 3$ of the cut paper & & & \\
\hline $1 / 3$ of the fabric & & & \\
\hline $1 / 3$ of the rubber & & - & \\
\hline & Go to Layer 2 & Go to Layer 3 & \\
\hline
\end{tabular}

Drum Filled:

Date 5.2 .95 Initial R.K

Ring bolt placed opposite drum seam Date 5.2 .95 Initial L.K and in down position

Lid sealed and torqued to $40 \mathrm{ftllb}: \quad$ Date 5.2 .95 Initial R.K.

Leak tested:

Pre-test weight (lb)

Post-test weight (lb)

Loss =
N/A or Pressure (psig)

Pressure (psig)

Date

$\begin{array}{ll}-\quad \text { Time (min) } & \frac{0}{5}\end{array}$


WHC-SD-WM-TRP-246 REV. 0

\section{Rack Storage Drum Data Sheet}

Drum No. $\times 134-T$ T. $i 76.5 \mathrm{ib}$.

Post-test Assessment

Position: __ original; __ tipped; __ toppled

Lid on drum: $\quad X$ Yes No

Gasket missing (cm) _ـ $20^{\circ}$ at ream

Contents expelled: _ues $x$ No

Seal failure: $\quad$ Yes $\ldots$

Burn pattern: - discolnation 12 " at sean to $2 / 3$ of dum
NONe. -

Photo taken: —ues $\searrow$ No

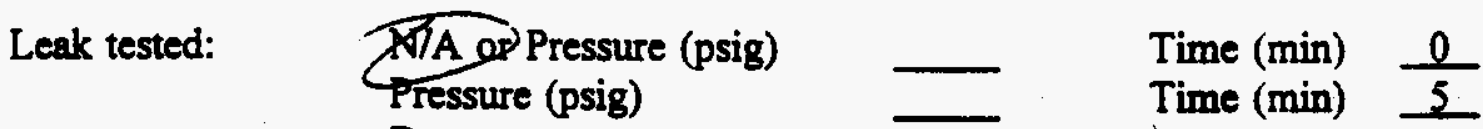

Date

$\overline{\text { Initial }}$

Interior assessment:

$\frac{3^{\prime \prime} \text { mett of plastic } \sim 1-i \text { f char at seam, othen side }}{\text { is like arew }}$

Phote taken:

$X$ Yes

Comments: 
WHC-SD-WM-TRP-246 REV. 0

\section{Rack Storage Drum Data Sheet}

Drum No. $\times 144$

Description (Check):

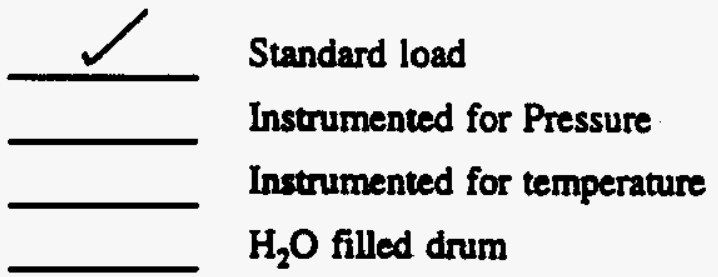

\begin{tabular}{|c|c|c|c|}
\hline Loading Starting at Bottom & Layer 1 .... . Layer 2 & Layer 3 \\
\hline 3 pieces of steel & N/A & \\
\hline $1 / 3$ of the brown paper & & & \\
\hline $1 / 3$ of the 10 -mil plastic & & & \\
\hline $1 / 3$ of the bond paper & & & \\
\hline $1 / 3$ of the towels & & & \\
\hline $1 / 3$ of the bags & & & \\
\hline $1 / 3$ of the cut paper & & & \\
\hline $1 / 3$ of the fabric & Go to Layer 2 & Go to Layer 3 & \\
\hline $1 / 3$ of the rubber & & \\
\hline
\end{tabular}

Drum Filled:

Date $5.2 \cdot 95^{\cdots}$ Initial L.K

Ring bolt placed opposite drum seam Date 5.7 .95 Initial L.K and in down position

Lid sealed and torqued to $40 \mathrm{ft} / \mathrm{bb}: \quad$ Date 5.7 .95 Initial

Leak tested:

Pre-test weight (lb)

Post-test weight (lb)
N/A or Pressure (psig)

Pressure (psig)

Date

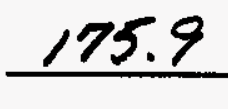

Time (min) 0

Time (min)

Initial

Target weight $-175.6 \mathrm{lb}$ 


\section{WHC-SD-WM-TRP-246 REV. 0}

Rack Storage Drum Data Sheet

Drum No. $\times 134 \quad \omega t .=176.1$

\section{Post-test Assessment}

Position: ___ original; ___ tipped; ___ toppled

Lid on drum: $\quad \quad \quad X$ Yes $\quad$ No

Gasket missing (cm) __ 6 " to right of seam

Contents expelled: __ Yes $x$ No

Seal failure: $\quad$ Yes $X$ Not visible

Burn pattern:

nonve sooty on front hal

Photo taken:

Yes X No

Leak tested:

N/A or Pressure (psig)

Pressure (psig)

Date

$\longrightarrow \quad$ Time (min) $\quad \frac{0}{5}$

Initial

Interior assessment:

$\sim 3 "$ mett of plastic rohan to irght of seam, $\sim 6$ "span

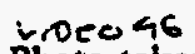

photo taken:

$\underline{\chi}$ Yes No

Comments: 


\section{Rack Storage Drum Data Sheet}

Drum No. $x 154$

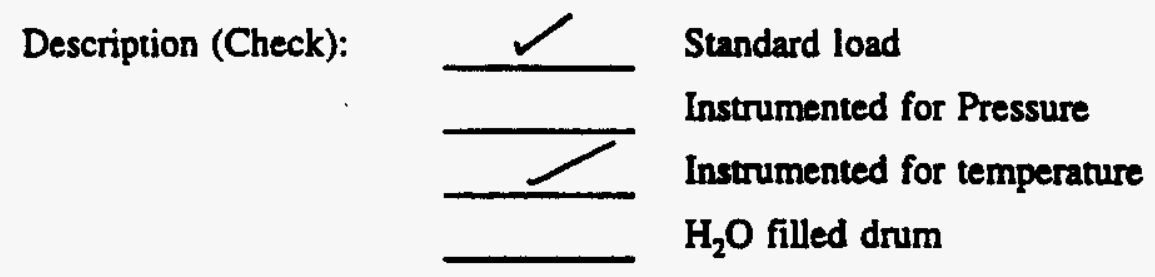

\begin{tabular}{|c|c|c|c|}
\hline Loading Starting at Bottom & Layer 1 & Layer 2 & Layer 3 \\
\hline 3 pieces of steel & & & \\
\hline $1 / 3$ of the brown paper & & & \\
\hline $1 / 3$ of the 10-mil plastic & & & \\
\hline $1 / 3$ of the bond paper & & & \\
\hline $1 / 3$ of the towels & & & \\
\hline $1 / 3$ of the bags & & & \\
\hline $1 / 3$ of the cut paper & & & \\
\hline $1 / 3$ of the fabric & Go to Layer 2 & Go to Layer 3 & \\
\hline $1 / 3$ of the rubber & & \\
\hline
\end{tabular}

Drum Filled:

Date $5.2 .95 \cdots \cdots$ Initial L.K

Ring bolt placed opposite drum seam Date $5=2.95$ Initial 12 and in down position

Lid sealed and torqued to $40 \mathrm{ftllb}: \quad$ Date 5.2 .95 Initial $\underline{R K}$

Leak tested:

Pre-test weight (lb)

Post-test weight (lb)
N/A or Pressure (psig)

Pressure (psig)

Date

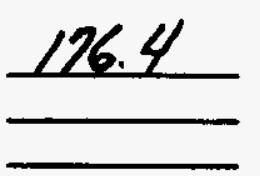

Time (min)

Time (min) 5

Target weight $-175.6 \mathrm{lb}$ 
WHC-SD-WM-TRP-246 REV. 0

Rack Storage Drum Data Sheet

Drum No. $\times 154$ int $=176.41 \mathrm{~b}$

Post-test Assessment

Position: ___ original; ___ tipped; __ toppled

Lid on drum: $\quad X$ Yes $\quad$ No

Gasket missing (cm) 0 compressed and hand 220 "

Contents expelled: _u Yes $x$ No

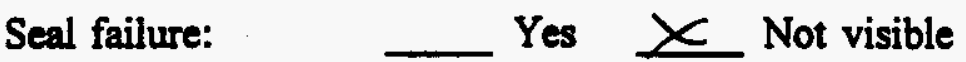

Burn pattern:

NoNe - sooty on sean haly

Photo taken:

Yes $x$ No

Leak tested:

NIA or Pressure (psig)

Pressure (psig)

Date

\begin{tabular}{lll} 
& Time $(\min )$ & 0 \\
\hline & Time (min)
\end{tabular}

Interior assessment:

plestic melted to side $\sim 20 "$ about seam

Video 71

Pheto taken:

$\nsim$ Yes No

Comments: 
Drum No. $\times 164$

Description (Check):

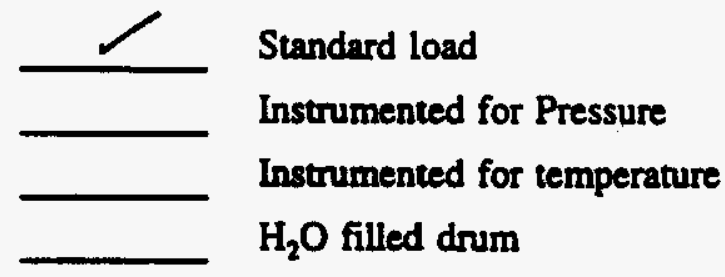

\begin{tabular}{|c|c|c|c|}
\hline Loading Starting at Bottom & Layer 1 & Layer 2 & Layer 3 \\
\hline 3 pieces of steel & & $\mathbf{N} / \mathbf{A}$ & N/A \\
\hline \multicolumn{4}{|l|}{$1 / 3$ of the brown paper } \\
\hline \multicolumn{4}{|l|}{$1 / 3$ of the $10-$ mil plastic } \\
\hline \multicolumn{4}{|l|}{$1 / 3$ of the bond paper } \\
\hline \multicolumn{4}{|l|}{$1 / 3$ of the towels } \\
\hline \multicolumn{4}{|l|}{$1 / 3$ of the bags } \\
\hline \multicolumn{4}{|l|}{$1 / 3$ of the cut paper } \\
\hline \multicolumn{4}{|l|}{$1 / 3$ of the fabric } \\
\hline \multicolumn{4}{|l|}{$1 / 3$ of the rubber } \\
\hline & Go to Layer 2 & Go to Layer 3 & \\
\hline
\end{tabular}

Drum Filled:

Ring bolt placed opposite drum seam and in down position

Lid sealed and torqued to $40 \mathrm{ft} / \mathrm{lb}$ :

Leak tested:

Pre-test weight (lb)

Post-test weight (lb)
Date 5.2 .95 Initial R.K

Date 5.2 .95 Initial R.K

Date 5.2 .95 Initial K.K
N/A or Pressure (psig)

Pressure (psig)

Date

176.5
Time (min) 0

Time (min) 5
Initial

Target weight $-175.6 \mathrm{lb}$ 
WHC-SD-WM-TRP-246 REV. 0

Rack Storage Drum Data Sheet

Drum No. Xita int. $=176.316$.

Post-test Assessment

Position: original; tipped; toppled

Lid on drum: $\quad x$ Yes No

Gasket missing (cm) $2-12$ " sorme what hand

Contents expelled: —Yes $X$

Seal failure: $\quad$ Yes $\chi$ Not visible

Burn pattern:

nous - sooty anound dunm

Photo taken: $\quad$ Yes $\longrightarrow$ No

Leak tested: $\quad$ Adar Pressure (psig) Pressure (psig)

Date

\begin{tabular}{lll}
$-\quad$ Time $(\min )$ & 0 \\
\hline & Time (min)
\end{tabular}

Interior assessment:

plastic metted $\tau_{\text {e side }}$ iz" fram seam to right

$\therefore 8<073$

Photo taken:

$\not$ Yes No

Comments: 
WHC-SD-WM-TRP-246 REV. 0

\section{Rack Storage Drum Data Sheet}

Drum No. $\times 174$

Description (Check):

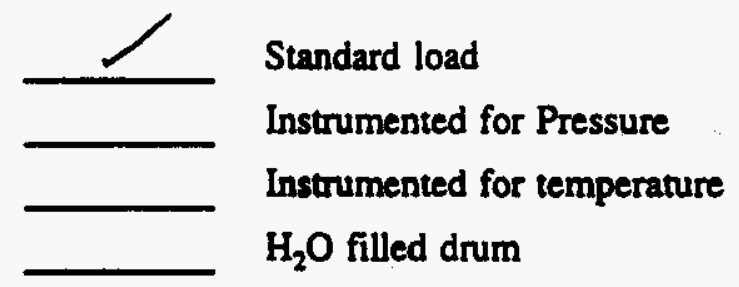

\begin{tabular}{|c|c|c|c|}
\hline Loading Starting at Bottom & Layer 1 & Layer 2 & Layer 3 \\
\hline 3 pieces of steel & & N/A & $\mathbf{N} / \mathbf{A}$ \\
\hline \multicolumn{4}{|l|}{$1 / 3$ of the brown paper } \\
\hline \multicolumn{4}{|l|}{$1 / 3$ of the 10 -mil plastic } \\
\hline \multicolumn{4}{|l|}{$1 / 3$ of the bond paper } \\
\hline \multicolumn{4}{|l|}{$1 / 3$ of the towels } \\
\hline \multicolumn{4}{|l|}{$1 / 3$ of the bags } \\
\hline \multicolumn{4}{|l|}{$1 / 3$ of the cut paper } \\
\hline \multicolumn{4}{|l|}{$1 / 3$ of the fabric } \\
\hline \multicolumn{4}{|l|}{$1 / 3$ of the rubber } \\
\hline & Go to Layer 2 & Go to Layer 3 & \\
\hline
\end{tabular}

Drum Filled:

Date 5-2.95 Initial L.K

Ring bolt placed opposite drum seam Date $5=2.85$ Initial te. and in down position

Lid sealed and torqued to $40 \mathrm{ft} / \mathrm{b}: \quad$ Date 5.2 .95 Initial R.K

Leak tested:

Pre-test weight (lb)

Post-test weight (lb)

Loss $=$
N/A or Pressure (psig)

Pressure (psig)

Date

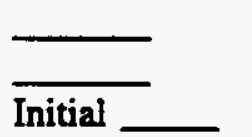

Time (min) 0

Time (min) 5

M6 $8 \quad$ Target weight $-175.6 \mathrm{lb}$ 
WHC-SD-WM-TRP-246 REV. 0

\section{Rack Storage Drum Data Sheet}

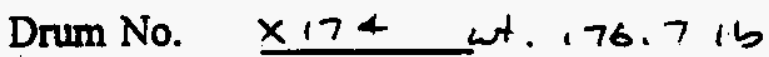

\section{Post-test Assessment}

Position: __ original; __ tipped; __ toppled

Lid on drum: . $\quad x$ Yes No

Gasket missing (cm) 0

Contents expelied: _u Yes $\underline{x}$ No

Seal failure: $\quad \longrightarrow$ Yes $\quad x$ Not visible

Burn pattern: None - soaty

Photo taken: Yes es No

Leak tested: Cor Pressure (psig) Pressure (psig) Date

Initial $\begin{array}{ll}\text { Time (min) } & 0 \\ \text { Time (min) }\end{array}$

Interior assessment:

some platic melted at seam inot to dum no char

ritoce To

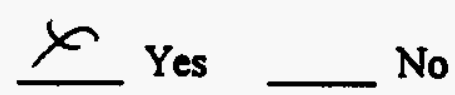

Comments: 
WHC-SD-WM-TRP-246 REV. 0

\section{Rack Storage Drum Data Sheet}

Drum No. $\times 184$

Description (Check):

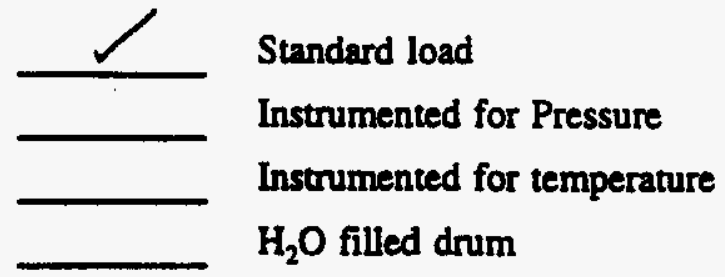

\begin{tabular}{|c|c|c|c|}
\hline Loading Starting at Bottom & Layer 1 & Layer 2 & Layer 3 \\
\hline 3 pieces of steel & & N/A & $\mathbf{N} / \mathbf{A}$ \\
\hline \multicolumn{4}{|l|}{$1 / 3$ of the brown paper } \\
\hline \multicolumn{4}{|l|}{$1 / 3$ of the 10 -mil plastic } \\
\hline \multicolumn{4}{|l|}{$1 / 3$ of the bond paper } \\
\hline \multicolumn{4}{|l|}{$1 / 3$ of the towels } \\
\hline \multicolumn{4}{|l|}{$1 / 3$ of the bags } \\
\hline \multicolumn{4}{|l|}{$1 / 3$ of the cut paper } \\
\hline \multicolumn{4}{|l|}{$1 / 3$ of the fabric } \\
\hline \multicolumn{4}{|l|}{$1 / 3$ of the rubber } \\
\hline & Go to Layer 2 & Go to Layer 3 & \\
\hline
\end{tabular}

Drum Filled:

Date $5-2-95$ Initial

Ring bolt placed opposite drum seam Date $5=2.95$ Initial Le. and in down position

Lid sealed and torqued to $40 \mathrm{ftlb}: \quad$ Date $5=2-95$ Initial $L . K$

Leak tested:

Pre-test weight (lb)

Post-test weight (lb)
N/A or Pressure (psig)

Pressure (psig)

Date

175.8
Time (min) $\frac{0}{5}$

Initial

Target weight $\sim 175.6 \mathrm{lb}$ 


\section{*alls View/Frint Document Cover Sheet tow}

This document was retrieved from the Documentation and Records Manaqement (DRM) ISEARCH System. It is intended for Information only and may not be the most recent or updated version. Contact a Document Service Center (see Hanford Info for locations) if you need additional retrieval information.

Accession \#: D195061639

Document \#: SD-WM-TRP-246

Title/Desc:

SOLID WASTE DRUM ARRAY FIRE PERFORMANCE [SEC 4 OF 7]

Pages: 151 
THIS DOCUMENT WAS TOO LARGE TO SCAN AS A SINGLE

DOCUMENT. IT REQUIRED BREAKING INTO SMALLER SECTIONS.

DOCUMENT NUMBER: SD-WM.TRP.2HL

SECTION: $\quad 4 \quad$ OF 7

TITLE: Solid waote Drum array fire Performance

AUTHOR: LoUwi RL

RECIPIENT:

DATE: $11 / 07 / 95$

KEYWORDS:

REFERENCES: $E D T-612232$ 
Blank 
WHC-SD-WM-TRP-246 REV. 0

Rack Storage Drum Data Sheet

Drum No. $x_{i 54}, 75.8 \mathrm{bl}$.

Post-test Assessment

Position: __ original; __ t _ tipped; __ toppled

Lid on drum: $\quad \longrightarrow$ Yes No

Gasket missing (cm) $O \sim 11$ " hand o coupressed.

Contents expelled: __ Yes $x$ No

Seal failure: $\quad$ Yes $\quad$ Not visible

Burn pattern:

None-sooky seam to rt. side

Photo taken:

Yes $\chi$ No

Leak tested:

Nagr Pressure (psig)

Pressure (psig)

Date

Initial

$\begin{array}{ll}\text { Time (min) } & 0 \\ \text { Time (min) } & \end{array}$

Interior assessment:

some plastic bancly welted

UDDeo 72

Photo taken:

$\underline{X}$ Yes

Comments: 


\section{Rack Storage Drum Data Sheet}

Drum No. $\times 214(p)$

Description (Check):

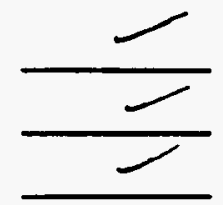

Standard load

Instrumented for Pressure

Instrumented for temperature

$\mathrm{H}_{2} \mathrm{O}$ filled drum

\begin{tabular}{|c|c|c|c|}
\hline Loading Starting at Bottom & Layer 1 & Layer 2 & Layer 3 \\
\hline 3 pieces of steel & & & \\
\hline $1 / 3$ of the brown paper & & & \\
\hline $1 / 3$ of the 10 -mil plastic & & & \\
\hline $1 / 3$ of the bond paper & & & \\
\hline $1 / 3$ of the towels & & & \\
\hline $1 / 3$ of the bags & & & \\
\hline $1 / 3$ of the cut paper & & & \\
\hline $1 / 3$ of the fabric & & & \\
\hline $1 / 3$ of the rubber & Go to Layer 2 & Go to Layer 3 & \\
\hline
\end{tabular}

Drum Filled:

Ring bolt placed opposite drum seam and in down position

Lid sealed and torqued to $40 \mathrm{ft} / \mathrm{lb}$ :

Leak tested:

Pre-test weight (lb)

Post-test weight (lb)

Date 5.2.95 Initial RK

Date 5.2 .95 Initial $/ . /$

Date 5.2 .95 Initial R.K

N/A or Pressure (psig)

Pressure (psig)

Date

$\overline{\text { Initial }}$

$175.6 \quad$ Target weight $-175.6 \mathrm{lb}$
Time (min) 0

Time (min) 5 


\section{Rack Storage Drum Data Sheet}

Drum No. $\times 214(p) \quad \omega t .=145.3 \mathrm{lb}$.

Post-test Assessment

Position: original; tipped; toppled

Lid on drum: $\quad$ Yes $\quad X$ No

Gasket missing (cm) All

Contents expelled: __ Yes — No

Seal failure: $\quad$ Yes _ Not visible

Burn pattern:

all the way

around

Photo taken:

Yes

Leak tested:

N/A or Pressure (psig)

Pressure (psig)

Date

$\longrightarrow \quad$\begin{tabular}{ll} 
Time (min) & 0 \\
\hline & Time (min)
\end{tabular}

$\overline{\text { Initial }}$

Interior assessment:

26 " trom tep te bol top of rewining coutents

Vider 4

Photo taken:

Yes _ No

Comments: 


\section{Rack Storage Drum Data Sheet}

Drum No. $\times 224(P)$

Description (Check):

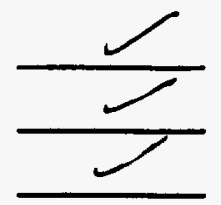

Standard load

Instrumented for Pressure

Instrumented for temperature

$\mathrm{H}_{2} \mathrm{O}$ filled drum

\begin{tabular}{|c|c|c|c|}
\hline Loading Starting at Bottom & Layer 1 & Layer 2 & Layer 3 \\
\hline 3 pieces of steel & & & \\
\hline $1 / 3$ of the brown paper & & & \\
\hline $1 / 3$ of the $10-$ mil plastic & & & \\
\hline $1 / 3$ of the bond paper & & & \\
\hline $1 / 3$ of the towels & & & \\
\hline $1 / 3$ of the bags & & & \\
\hline $1 / 3$ of the cut paper & & Go to Layer 3 & \\
\hline $1 / 3$ of the fabric & & & \\
\hline $1 / 3$ of the rubber & & Gayer 2 & Go to \\
\hline
\end{tabular}

Drum Filled:

Date $5 \cdot 2 \cdot 95$ Initial EL

Ring bolt placed opposite drum seam Date 5.2 .95 Initial $2 K$ and in down position

Lid sealed and torqued to $40 \mathrm{ft} / \mathrm{lb}: \quad$ Date $5.7 .95 \quad$ Initial $\leq \mathcal{K}$

Leak tested:

Pre-test weight (lb)

Post-test weight (lb)

Loss =
N/A or Pressure (psig)

Pressure (psig)

Date

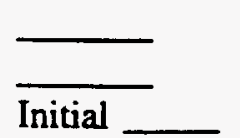

Time (min) $\quad 0$

Time $(\min )$ 
WHC-SD-WM-TRP-246 REV. 0

Rack Storage Drum Data Sheet

Drum No. $\times 224(P) \quad \omega t=.04 .6$

$\underline{\text { Post-test Assessment }}$

Position: original: tipped; toppled

Lid on drum: $\quad X$ Yes No $\quad$ Ngap 13 " ing

Gasket missing $(\mathrm{cm}) \leq$ Ul

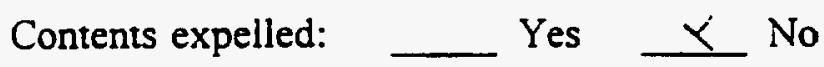

Seal failure: $\quad x$ Yes $\quad$ Not visible

Burn pattern:

All an ound

Photo taken:

Yes №

Leak tested:

N/Aor Pressure (psig)

Pressure (psig)

Date

\begin{tabular}{lll} 
& Time (min) & 0 \\
\hline Initial & Time (min) &
\end{tabular}

Interior assessment:

$17 \% 2$ " from top to remaining cortents

V. $2 \cos 3$

Photo taken:

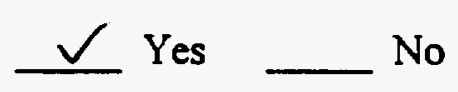

Comments: 
WHC-SD-WM-TRP-246 REV. 0

\section{Rack Storage Drum Data Sheet}

Drum No. $X 234$

Description (Check):

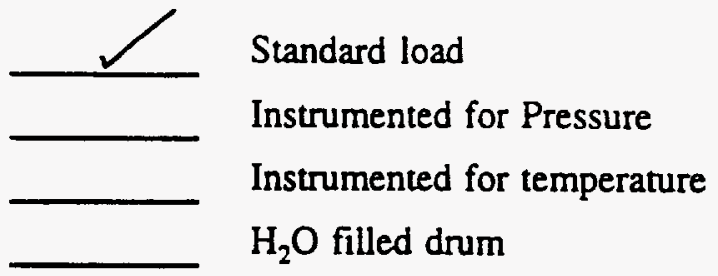

\begin{tabular}{|c|c|c|c|}
\hline Loading Starting at Bottom & Layer 1 & Layer 2 & Layer 3 \\
\hline 3 pieces of steel & N/A \\
\hline $1 / 3$ of the brown paper & & & \\
\hline $1 / 3$ of the 10 -mil plastic & & & \\
\hline $1 / 3$ of the bond paper & & & \\
\hline $1 / 3$ of the towels & & & \\
\hline $1 / 3$ of the bags & & & \\
\hline $1 / 3$ of the cut paper & & & \\
\hline $1 / 3$ of the fabric & Go to Layer 2 & Go to Layer 3 & \\
\hline $1 / 3$ of the rubber & & \\
\hline
\end{tabular}

Drum Filled:

Date 5.2 .95 Initial $A P$

Ring bolt placed opposite drum seam Date $5-2-45$ Initial and in down position

Lid sealed and torqued to $40 \mathrm{ft} / \mathrm{lb}:$ Date $5-2-45$ Initial $\not \subset \varnothing$

Leak tested:

Pre-test weight (lb)

Post-test weight (lb)

Loss $=$
N/A or Pressure (psig)

Pressure (psig)

Date

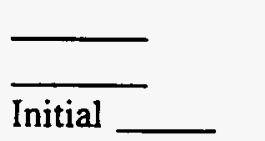

$176.1 \quad$ Target weight $-175.6 \mathrm{lb}$
Time (min) 0

Time $(\min ) \quad 5$ 
WHC-SD-WM-TRP-246 REV. 0

Rack Storage Drum Data Sheet

Drum No. $\times 234 \quad \omega t=153.416$

Post-test Assessment

Position: original: tipped; toppled

Lid on drum: $\quad$ Yes $X$ No

Gasket missing (cm)

Contents expelled: _ Yes _ No

Seal failure: $\quad X$ Yes $\quad$ Not visible

Burn pattern:

$X$ Yes $\quad$ Not visible

729" clean

Photo taken: Yes — No

Leak tested:

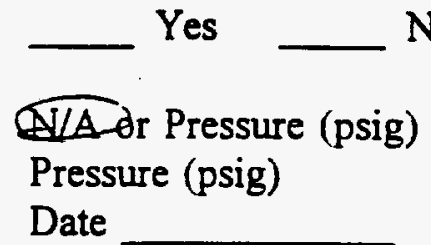
No 


\section{Rack Storage Drum Data Sheet}

Drum No. $\times 244$

Description (Check):

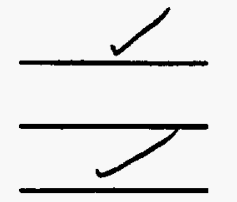

Standard load

Instrumented for Pressure

Instrumented for temperature

$\mathrm{H}_{2} \mathrm{O}$ filled drum

\begin{tabular}{|c|c|c|c|}
\hline Loading Starting at Bottom & Layer 1 & Layer 2 & Layer 3 \\
\hline 3 pieces of steel & & N/A \\
\hline $1 / 3$ of the brown paper & & & \\
\hline $1 / 3$ of the 10 -mil plastic & & & \\
\hline $1 / 3$ of the bond paper & & & \\
\hline $1 / 3$ of the towels & & & \\
\hline $1 / 3$ of the bags & & & \\
\hline $1 / 3$ of the cut paper & & & \\
\hline $1 / 3$ of the fabric & Go to Layer 2 & Go to Layer 3 & \\
\hline $1 / 3$ of the rubber & & \\
\hline
\end{tabular}

Drum Filled:

Ring bolt placed opposite drum seam and in down position

Lid sealed and torqued to $40 \mathrm{ft} / \mathrm{lb}$ :

Leak tested:

Pre-test weight (lb)

Post-test weight (lb)

$$
\text { Loss = }
$$

Date $5-2.95$ Initial AP

Date $5-2-9.5$ Initial AP

Date $5-2-55$ Initial AP
N/A or Pressure (psig)

Pressure (psig)

Date

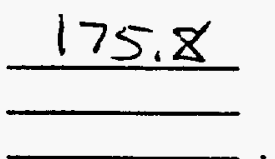

Time (min)

Time $(\mathrm{min}) \quad 5$
Initial

Target weight $-175.6 \mathrm{lb}$ 


\section{Rack Storage Drum Data Sheet}

Drum No. $\quad \geq 244 \quad$.t. $=174.1 \mathrm{ibs}$

Post-test Assessment

Position: __ original: ___ _ _ _ _ _ _

Lid on drum: $\quad X$ Yes No

Gasket missing (cm) Atl

Contents expelled: __ Yes $\longrightarrow$ No

Seal failure: $\quad$ Yes $x$ Not visible? slight bulge in lid but wo Burn pattern: $-1 / 2$ to $1=f+$ of seam

Photo taken: _ Yes _ No

Leak tested: $\quad$ N/A

Pressure (psig)

Date

\begin{tabular}{lll} 
& Time $(\min )$ & 0 \\
\hline & Time $(\min )$
\end{tabular}

$\overline{\text { Initial }}$

Interior assessment:

"1"Chas on unburnedside upto 2 "on beunedside

vioeo $=0$

Photo taken:

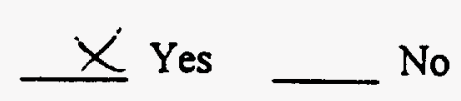

Comments:

some discolcring around top vecur lid probably vented. 


\section{Rack Storage Drum Data Sheet}

Drum No. $\times 254(p)$

Description (Check):

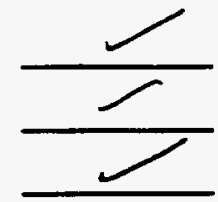

Standard load

Instrumented for Pressure

Instrumented for temperature

$\mathrm{H}_{2} \mathrm{O}$ filled drum

\begin{tabular}{|c|c|c|c|}
\hline Loading Starting at Bottom & Layer 1 & Layer 2 & Layer 3 \\
\hline 3 pieces of steel & & & \\
\hline $1 / 3$ of the brown paper & & & \\
\hline $1 / 3$ of the 10 -mil plastic & & & \\
\hline $1 / 3$ of the bond paper & & & \\
\hline $1 / 3$ of the towels & & & \\
\hline $1 / 3$ of the bags & Go to Layer 2 & Go to Layer 3 & \\
\hline $1 / 3$ of the cut paper & & \\
\hline $1 / 3$ of the fabric & & \\
\hline $1 / 3$ of the rubber & & \\
\hline
\end{tabular}

Drum Filled:

Date $\underline{5.3 .95}$ Initial R.K

Ring bolt placed opposite drum seam Date 5.3.95 Initial RK and in down position

Lid sealed and torqued to $40 \mathrm{ft} / \mathrm{b}$ :

Date $5 \cdot 3 \cdot 95$ Initial

Leak tested:

N/A or Pressure (psig)

Pressure (psig)

Date

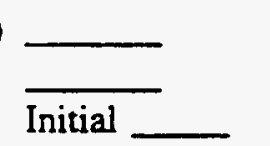

Time $(\min ) \quad 0$

Time $(\min ) \quad 5$

Pre-test weight (lb)

176.4

Target weight $\sim 175.6 \mathrm{lb}$

Post-test weight (lb)

Loss $=$ 


\section{Rack Storage Drum Data Sheet}

Drum No. $\times 254(p) \quad .76 .916$

Post-test Assessment

Position: original: ___ tipped: toppled

Lid on drum: $\quad \longrightarrow$ Yes No

Gasket missing (cm) O 47 " compresaed t hard (pan tially brouned) Contents expelled: _ Yes $x$ No

Seal failure: $\quad$ Yes $\quad X$ Not visible

Burn pattern:

Nove - soot all around

Photo taken: $\quad$ Yes $\simeq$ No

Leak tested:

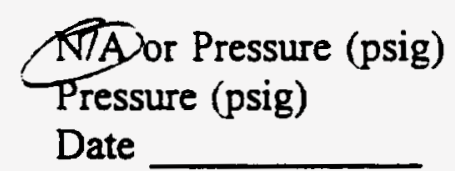

\begin{tabular}{lll} 
& Time $(\min )$ & 0 \\
\hline Initial & Time $(\min )$
\end{tabular}

Interior assessment:

$\frac{35^{\prime} \text { melted plastic lett of seam }}{24^{\prime \prime} \text { fo ieft of sean is }-8^{2} \text { span of " chand contento }}$

VIDec' $\$ 6$

Pheto taken:

$\longrightarrow$ Yes No

Comments: 
WHC-SD-WM-TRP-246 REV. 0

\section{Rack Storage Drum Data Sheet}

Drum No. $\times 264$

Description (Check):

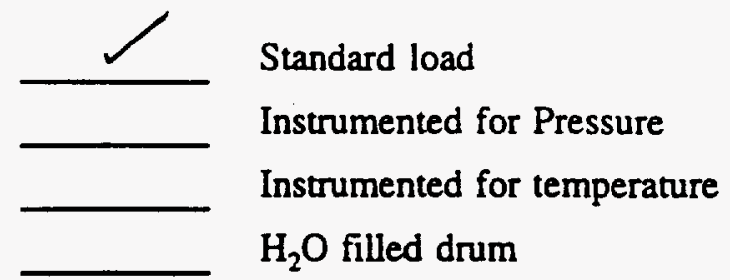

\begin{tabular}{|c|c|c|c|}
\hline Loading Starting at Bottom & Layer 1 & Layer 2 & Layer 3 \\
\hline 3 pieces of steel & N/A & \\
\hline $1 / 3$ of the brown paper & & & \\
\hline $1 / 3$ of the 10 -mil plastic & & & \\
\hline $1 / 3$ of the bond paper & & & \\
\hline $1 / 3$ of the towels & & & \\
\hline $1 / 3$ of the bags & & & \\
\hline $1 / 3$ of the cut paper & & Go to Layer 3 & \\
\hline $1 / 3$ of the fabric & Go to Layer 2 & & \\
\hline $1 / 3$ of the rubber & & \\
\hline
\end{tabular}

Drum Filled:

Ring bolt placed opposite drum seam and in down position

Lid sealed and torqued to $40 \mathrm{ft} / \mathrm{b}$ :

Leak tested:

Pre-test weight (lb)

Post-test weight (lb)
Date 5.2 .95 Initial $4 P$

Date $5-2.95 \quad$ Initial AP
N/A or Pressure (psig)

Pressure (psig)

Date

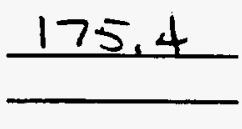

Time (min) $\quad 0$

Time (min) 5

$$
\text { Loss }=
$$




\section{Rack Storage Drum Data Sheet}

Drum No. $\quad \times 264 \quad \ldots t=175.7$ ib.

Post-test Assessment

Position: ___ original; ___ tipped: ___ toppled

Lid on drum: $\quad x$ Yes $\quad$ No

Gasket missing (cm) $O$ compresed t hand all around.

Contents expelled: $\quad$ Yes $\quad$ No

Seal failure: $\quad$ Yes $\quad \longrightarrow$ Not visible

Burn pattern:

Noxe - soety all around

Photo taken: $\quad$ Yes $\varnothing$ No

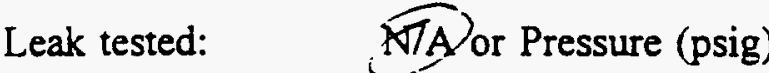

Pressure (psig)

Date

Initial

$\begin{array}{ll}\text { Time (min) } & 0 \\ \text { Time (min) } & \end{array}$

Interior assessment:

37" inctied soound Not to ide

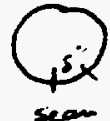

i.jee 13

photo taken:

$\underset{1}{x}$ Yes No

Comments: 
WHC-SD-WM-TRP-246 REV. 0

\section{Rack Storage Drum Data Sheet}

Drum No. $\times 274$

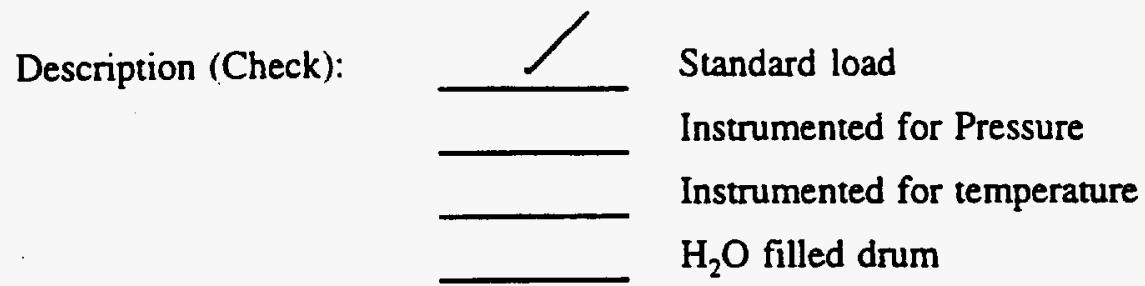

\begin{tabular}{|c|c|c|c|}
\hline Loading Starting at Bottom & Layer 1 & Layer 2 & Layer 3 \\
\hline 3 pieces of steel & N/A & N/A \\
\hline $1 / 3$ of the brown paper & & & \\
\hline $1 / 3$ of the 10 -mil plastic & & & \\
\hline $1 / 3$ of the bond paper & & & \\
\hline $1 / 3$ of the towels & & & \\
\hline $1 / 3$ of the bags & & & \\
\hline $1 / 3$ of the cut paper & & & \\
\hline $1 / 3$ of the fabric & Go to Layer 2 & Go to Layer 3 & \\
\hline $1 / 3$ of the rubber & & \\
\hline
\end{tabular}

Drum Filled:

Date $5 \cdot 2 \cdot 95$ Initial At

Ring bolt placed opposite drum seam Date $5-7-75$ Initial AP and in down position

Lid sealed and torqued to $40 \mathrm{ft} / \mathrm{lb}: \quad$ Date $5-2-75$ Initial AP

Leak tested:

Pre-test weight (lb)

Post-test weight (lb)
N/A or Pressure (psig)

Pressure (psig)

Date

176.4

Time (min)

Time (min) 5

Target weight $-175.6 \mathrm{lb}$ 


\section{Rack Storage Drum Data Sheet}

Drum No. $\times 274 \quad \omega T=i 76.516$.

Post-test Assessment

Position: ___ original: ___ tipped; ___ toppled

Lid on drum: $\quad X$ Yes $\quad$ No

Gasket missing (cm) $12-34^{\circ}$ tainl hand

Contents expelled: $\quad$ Yes $\longrightarrow$ No

Seal failure: $\quad$ Y $\quad$ Yes $\quad X$ Not visible

Burn pattern:

NONe - sooty all ancund

Photo taken:

Yes _ No

Leak tested:

N/A or Pressure (psig)

Pressure (psig)

Date

$\begin{array}{lll} & \text { Time (min) } & 0 \\ - & \text { Time (min) }\end{array}$

Interior assessment:

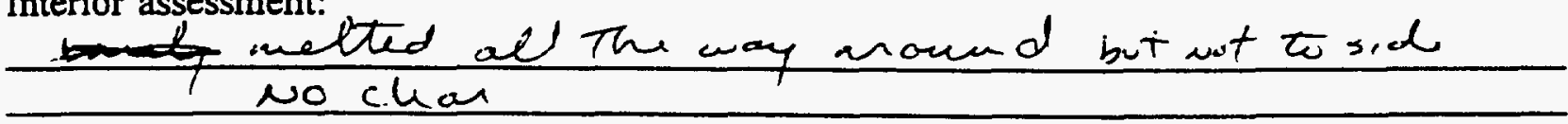

Photo taken:

$\times$ Yes

No

Comments: 
WHC-SD-WM-TRP-246 REV. 0

\section{Rack Storage Drum Data Sheet}

Drum No. $\times 284$

Description (Check):

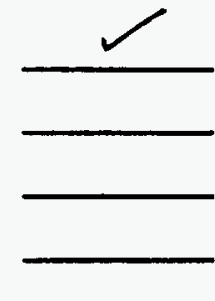

Standard load

Instrumented for Pressure

Instrumented for temperature

$\mathrm{H}_{2} \mathrm{O}$ filled drum

\begin{tabular}{|c|c|c|c|}
\hline Loading Starting at Bottom & Layer 1 & Layer 2 & Layer 3 \\
\hline 3 pieces of steel & & N/A & N/A \\
\hline \multicolumn{4}{|l|}{$1 / 3$ of the brown paper } \\
\hline \multicolumn{4}{|l|}{$1 / 3$ of the 10 -mil plastic } \\
\hline \multicolumn{4}{|l|}{$1 / 3$ of the bond paper } \\
\hline \multicolumn{4}{|l|}{$1 / 3$ of the towels } \\
\hline \multicolumn{4}{|l|}{$1 / 3$ of the bags } \\
\hline \multicolumn{4}{|l|}{$1 / 3$ of the cut paper } \\
\hline \multicolumn{4}{|l|}{$1 / 3$ of the fabric } \\
\hline \multicolumn{4}{|l|}{$1 / 3$ of the rubber } \\
\hline & Go to Layer 2 & Go to Layer 3 & \\
\hline
\end{tabular}

Drum Filled:

Date $5 \cdot 2 \cdot 95$ Initial $A P$

Ring bolt placed opposite drum seam Date $5-2-25$ Initial AP and in down position

Lid sealed and torqued to $40 \mathrm{ft} / \mathrm{lb}:$ Date $5-2-95$ Initial $A P$

Leak tested:

Pre-test weight (lb)

Post-test weight (lb)
N/A or Pressure (psig)

Pressure (psig)

Date

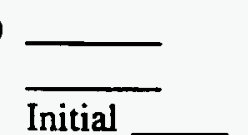

$\underline{175.9} \quad$ Target weight $-175.6 \mathrm{lb}$
Time $(\min ) \quad 0$

Time $(\min ) \quad 5$ 
WHC-SD-WM-TRP-246 REV. 0

Rack Storage Drum Data Sheet

Drum No. $\times 284 \quad 176.1$ ib.

Post-test Assessment

Position: __ original; ___ tipped; __ toppled

Lid on drum: $\quad \quad \quad x$ Yes

Gasket missing $(\mathrm{cm}) \mathcal{O}$ like weer, compressed sightly in cheas.

Contents expelled: _ Yes $x$ No

Seal failure: $\quad$ Yes $X$ Not visible

Burn pattern:

Photo taken: $\quad$ Yes $X$ No

Leak tested:

Pressure (psig)

Date

\begin{tabular}{lll} 
& Time $(\min )$ & 0 \\
\hline & Time $(\min )$ & $\underline{5}$
\end{tabular}

$\overline{\text { Initial }}$

Interior assessment:

slight meltu, of plastic all The way around net to drum

V.Dec 96

Photo taken:

$\varnothing$ Yes _ No

Comments: 


\section{Rack Storage Drum Data Sheet}

Drum No. $\times 3 / 4$

Description (Check):

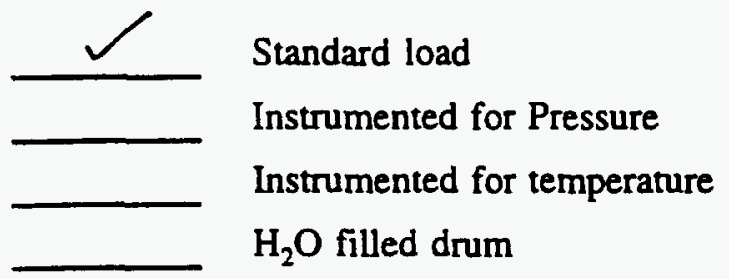

\begin{tabular}{|c|c|c|c|}
\hline Loading Starting at Bottom & Layer 1 & Layer 2 & Layer 3 \\
\hline 3 pieces of steel & $\sim$ & N/A & N/A \\
\hline $1 / 3$ of the brown paper & & $\gamma$ & $\checkmark$ \\
\hline $1 / 3$ of the 10 -mil plastic & $\nearrow$ & $>$ & $\checkmark$ \\
\hline $1 / 3$ of the bond paper & $\checkmark$ & $\gamma$ & 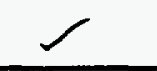 \\
\hline $1 / 3$ of the towels & $\gamma$ & $\checkmark$ & $\checkmark$ \\
\hline $1 / 3$ of the bags & 7 & $\checkmark$ & $\gamma$ \\
\hline $1 / 3$ of the cut paper & 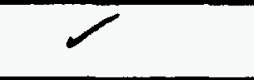 & $>$ & $r$ \\
\hline $1 / 3$ of the fabric & r & $\checkmark$ & - \\
\hline $1 / 3$ of the rubber & $\checkmark$ & $\zeta$ & $\checkmark$ \\
\hline & Go to Layer 2 & Go to Layer 3 & \\
\hline
\end{tabular}

Drum Filled:

Date $5 \cdot 2 \cdot 95$ Initial $A \varnothing$

Ring bolt placed opposite drum seam Date $5 \cdot 7-95$ Initial PP and in down position

Lid sealed and torqued to $40 \mathrm{ft} / \mathrm{b}$ : Date $5-2-35$ Initial AP

Leak tested:

Pre-test weight (lb)

Post-test weight (lb)
N/A or Pressure (psig)

Pressure (psig)

Date

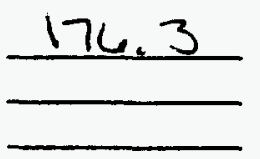

Time (min) $\quad 0$

Time (min) 5

$$
\text { Loss }=
$$


WHC-SD-WM-TRP-246 REV. 0

Rack Storage Drum Data Sheet

Drum No. $\times 314=14.0$

Post-test Assessment

Position: __ original; __ _ tipped: __ toppled

Lid on drum: $\quad$ Yes $\quad X$ No

Gasket missing (cm) All

Contents expelled: __ Yes _ No

Seal failure: $\quad X^{\prime}$ Yes $\quad$ Not visible

Burn pattern:

All around

Photo taken:

Yes _ No

Leak tested:

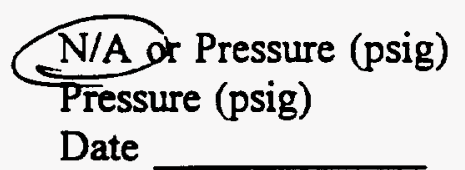

Date

Initial

$\begin{array}{ll}\text { Time (min) } & 0 \\ \text { Time (min) } & -5\end{array}$

Interior assessment:

6" ff chaned contents

i:Deo. 3

Ptroto taken:

$X^{Y}$ Yes

No

Comments: 


\section{Rack Storage Drum Data Sheet}

Drum No. $\times 224$

Description (Check):

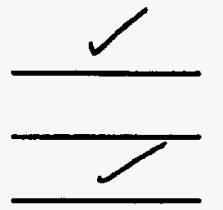

Standard load

Instrumented for Pressure

Instrumented for temperature

$\mathrm{H}_{2} \mathrm{O}$ filled drum

\begin{tabular}{|c|c|c|c|}
\hline Loading Starting at Bottom & Layer 1 & Layer 2 & Layer 3 \\
\hline 3 pieces of steel & & N/A & N/A \\
\hline $1 / 3$ of the brown paper & & & \\
\hline $1 / 3$ of the 10 -mil plastic & & & \\
\hline $1 / 3$ of the bond paper & & & \\
\hline $1 / 3$ of the towels & & & \\
\hline $1 / 3$ of the bags & & & \\
\hline $1 / 3$ of the cut paper & & & \\
\hline $1 / 3$ of the fabric & & & \\
\hline $1 / 3$ of the rubber & Go to Layer 2 & Go to Layer 3 & \\
\hline
\end{tabular}

Drum Filled:

Ring bolt placed opposite drum seam and in down position

Lid sealed and torqued to $40 \mathrm{ft} / \mathrm{b}$ :

Leak tested:

Pre-test weight (lb)

Post-test weight (lb)
Date $5 \cdot 2-95$ Initial AP

Date $5.2-45$ Initial AP
N/A or Pressure (psig)

Pressure (psig)

Date

176.1

Time (min) $\quad 0$ Time (min) 5

$$
\text { Loss = }
$$


WHC-SD-WM-TRP-246 REV. 0

\section{Rack Storage Drum Data Sheet}

Drum No. $\quad \times 324-\omega t .=125.0 \mathrm{lb} . \quad$ we i id

\section{Post-test Assessment}

Position:

original;

tipped;

toppled

Lid on drum: $\quad$ Yes $X$ No missing

Gasket missing (cm)

Contents expelled: __ Yes _ No

Seal failure: $\quad \longrightarrow$ Yes $\quad$ Not visible

Burn pattern:

el anound

Photo taken: Yes — No

Leak tested:

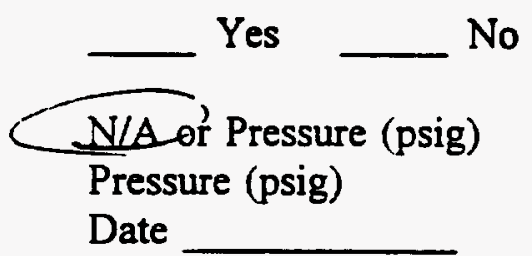

\begin{tabular}{lll} 
& Time $(\min )$ & 0 \\
\hline Initial & Time $(\min )$
\end{tabular}

Interior assessment:

$\checkmark 2 "$ chaned matenal in Lettem

vope 15
Photo taken:

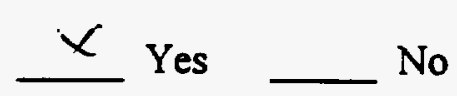

Comments:

Drum elougated OIz6" 
WHC-SD-WM-TRP-246 REV. O

\section{Rack Storage Drum Data Sheet}

Drum No. $\times 334$

Description (Check):

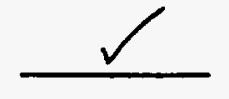

Standard load

Instrumented for Pressure

Instrumented for temperature

$\mathrm{H}_{2} \mathrm{O}$ filled drum

\begin{tabular}{|c|c|c|c|}
\hline Loading Starting at Bottom & Layer 1 & Layer 2 & Layer 3 \\
\hline 3 pieces of steel & & N/A & N/A \\
\hline \multicolumn{4}{|l|}{$1 / 3$ of the brown paper } \\
\hline $1 / 3$ of the 10 -mil plastic & $r$ & & \\
\hline \multicolumn{4}{|l|}{$1 / 3$ of the bond paper } \\
\hline \multicolumn{4}{|l|}{$1 / 3$ of the towels } \\
\hline \multicolumn{4}{|l|}{$1 / 3$ of the bags } \\
\hline \multicolumn{4}{|l|}{$1 / 3$ of the cut paper } \\
\hline \multicolumn{4}{|l|}{$1 / 3$ of the fabric } \\
\hline \multicolumn{4}{|l|}{$1 / 3$ of the rubber } \\
\hline & Go to Layer 2 & Go to Layer 3 & \\
\hline
\end{tabular}

Drum Filled:

Date 5.2 .95 Initial AP

Ring bolt placed opposite drum seam Date $5-2-45$ Initial $A ?$ and in down position

Lid sealed and torqued to $40 \mathrm{ft} / \mathrm{b}: \quad$ Date $5-2-95$ Initial $A P$

Leak tested:

Pre-test weight (lb)

Post-test weight (lb)
N/A or Pressure (psig)

Pressure (psig)

Date

175.9
Time $(\min ) \quad \frac{0}{5}$

Initial

Target weight $\sim 175.6 \mathrm{lb}$ 
WHC-SD-WM-TRP-246 REV. 0

Rack Storage Drum Data Sheet

Drum No. $\times 334$ int. $=103.216$

Post-test Assessment

Position:

original: tipped: toppled

Lid on drum:

$X$ Yes N No $1 " \times 11$ "gap 28 "to right of

Gasket missing (cm) All

Contents expelled: _u Yes $\not \subset$ No

Seal failure:

$X$ Yes Not visible

Burn pattern:

Allaround

Photo taken: $\quad$ Yes _ No

Leak tested:

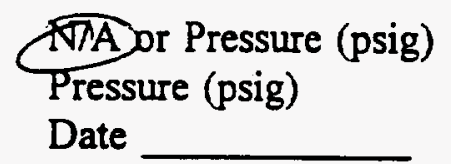

\begin{tabular}{lll}
- & Time $(\min )$ & 0 \\
\hline & Time $(\min )$
\end{tabular}

$\overline{\text { Initial }}$

Interior assessment:

16 " top of dum to top of contente 2 " char layer

Yidec i4
Photo taken:

$X$ Yes

Comments: 
WHC-SD-WM-TRP-246 REV. 0

Rack Storage Drum Data Sheet

Drum No. $\times 344$

Description (Check):

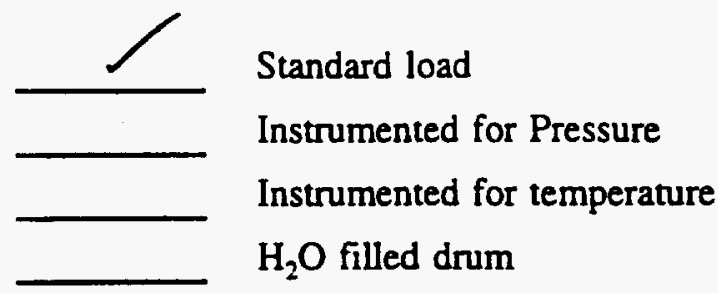

\begin{tabular}{|c|c|c|c|}
\hline Loading Starting at Bottom & Layer 1 & Layer 2 & Layer 3 \\
\hline 3 pieces of steel & & N/A & \\
\hline $1 / 3$ of the brown paper & & & \\
\hline $1 / 3$ of the 10 -mil plastic & & & \\
\hline $1 / 3$ of the bond paper & & & \\
\hline $1 / 3$ of the towels & & & \\
\hline $1 / 3$ of the bags & & & \\
\hline $1 / 3$ of the cut paper & & & \\
\hline $1 / 3$ of the fabric & Go to Layer 2 & Go to Layer 3 & \\
\hline $1 / 3$ of the rubber & & & \\
\hline
\end{tabular}

Drum Filled:

Ring bolt placed opposite drum seam and in down position

Lid sealed and torqued to $40 \mathrm{ft} / \mathrm{lb}$ :

Leak tested:

Pre-test weight (lb)

Post-test weight (lb)
Date $5=2.95 \quad$ Initial $\cong \mathbb{K}$

Date $5.2 .95 \quad$ Initial $\% \mathbb{R}$

Date 5.2 .95 Initial LLK

N/A or Pressure (psig)

Pressure (psig)

Date

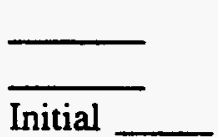

Time (min)

Time (min)

Loss = 
WHC-SD-WM-TRP-246 REV. 0

Rack Storage Drum Data Sheet

Drum No. $\times 344$ wit. 172.016

Post-test Assessment

Position: __ original; ___ tipped; __ toppled

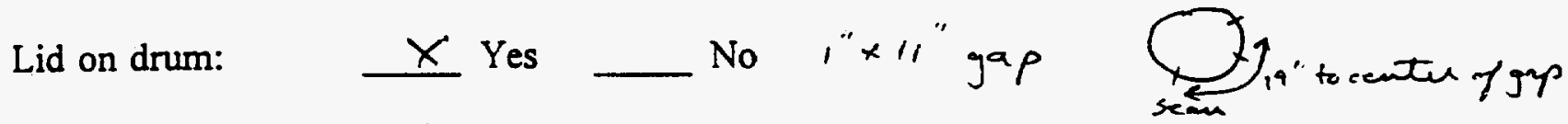

Gasket missing (cm) All

Contents expelled: _ـ Yes $\quad$ No

Seal failure: $\quad \quad X$ Yes $\quad$ Not visible

Burn pattern: "45" at mid ht.

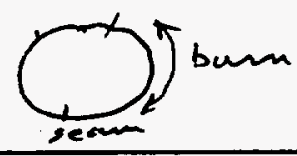

Photo taken:

Yes $x$ No

Leak tested:

A or Pressure (psig)

Pressure (psig)

Date

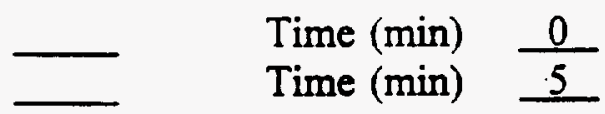

$\overline{\text { Initial }}$

Interior assessment:

9 "fru tep to Cp of chaned matinal

pito täken:

$\not \subset$ Yes No

Comments: 
WHC-SD-WM-TRP-246 REV. 0

\section{Rack Storage Drum Data Sheet}

Drum No. $\times 354$

Description (Check):

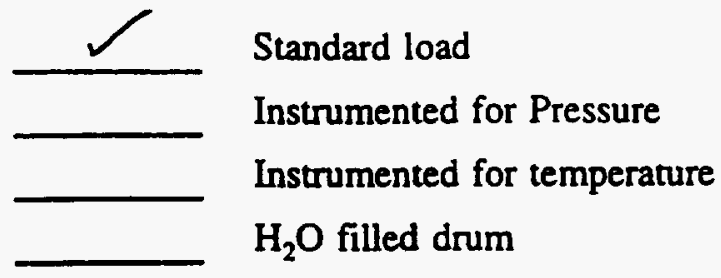

\begin{tabular}{|c|c|c|c|}
\hline Loading Starting at Bottom & Layer 1 & Layer 2 & Layer 3 \\
\hline 3 pieces of steel & N/A \\
\hline $1 / 3$ of the brown paper & & & \\
\hline $1 / 3$ of the 10 -mil plastic & & & \\
\hline $1 / 3$ of the bond paper & & & \\
\hline $1 / 3$ of the towels & & & \\
\hline $1 / 3$ of the bags & & & \\
\hline $1 / 3$ of the cut paper & & & \\
\hline $1 / 3$ of the fabric & Go to Layer 2 & Go to Layer 3 & \\
\hline $1 / 3$ of the rubber & & \\
\hline
\end{tabular}

Drum Filled:

Date $5 \cdot 2.95$ Initial

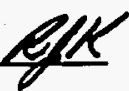

Ring bolt placed opposite drum seam

Date 5.2 .95

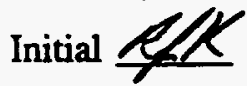

and in down position

Lid sealed and torqued to $40 \mathrm{ft} / \mathrm{b}: \quad$ Date 5.2 .95 Initial $\mathrm{CLK}$

Leak tested:

Pre-test weight (lb)

Post-test weight (lb)
N/A or Pressure (psig)

Pressure (psig)

Date

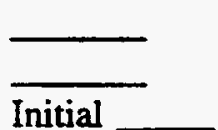

175.6
Time (min)

Time $(\min ) \underline{5}$

Target weight $\sim 175.6 \mathrm{lb}$ 
WHC-SD-WM-TRP-246 REV. 0

Rack Storage Drum Data Sheet

Drum No. $\times 354 \quad u t .=176.216$

Post-test Assessment

Position: ___ original: ___ tipped; ___ toppled

Lid on drum: $\quad \quad \quad x$ Yes No

Gasket missing (cm) _L_ 2" $20 "$ beoum + bunned, nost compressed thand

Contents expelled: _ Yes $\underline{x}$ No

$\checkmark$

Seal failure: $\quad$ Yes $\quad$ Y Not visible

Burn pattern:

Nore - seoty

Photo taken:

Yes No

Leak tested:

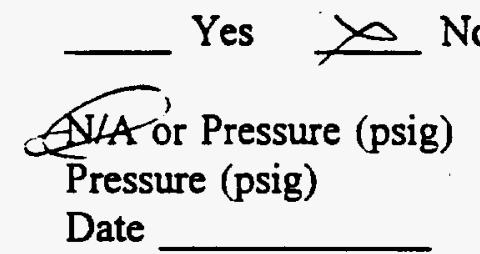

\begin{tabular}{lll} 
& Time (min) & 0 \\
\hline Initial & Time (min)
\end{tabular}

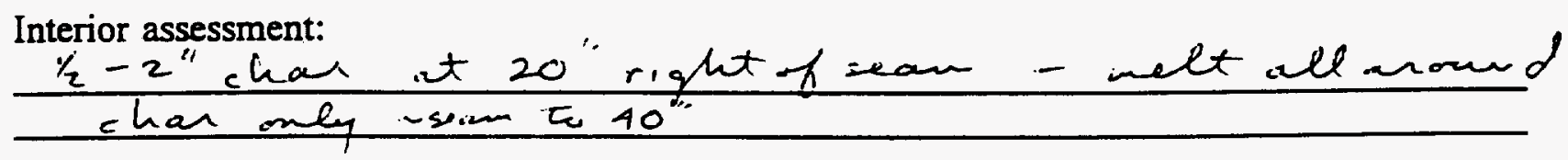

visec is

Photo taken:

$\leftarrow$ Yes No

Comments: 
WHC-SD-WM-TRP-246 REV. $O$

\section{Rack Storage Drum Data Sheet}

Drum No. $\times 364$

Description (Check):

$$
\begin{array}{ll}
\multicolumn{1}{c}{\text { Standard load }} \\
\text { Instrumented for Pressure } \\
\text { Instrumented for temperature } \\
\mathrm{H}_{2} \mathrm{O} \text { filled drum }
\end{array}
$$

\begin{tabular}{|c|c|c|c|}
\hline Loading Starting at Bottom & Layer 1 & Layer 2 & Layer 3 \\
\hline 3 pieces of steel & & N/A \\
\hline $1 / 3$ of the brown paper & & & \\
\hline $1 / 3$ of the 10-mil plastic & & & \\
\hline $1 / 3$ of the bond paper & & & \\
\hline $1 / 3$ of the towels & & & \\
\hline $1 / 3$ of the bags & & & \\
\hline $1 / 3$ of the cut paper & & & \\
\hline $1 / 3$ of the fabric & & & \\
\hline $1 / 3$ of the rubber & Go to Layer 2 & Go to Layer 3 & \\
\hline
\end{tabular}

\section{Drum Filled:}

Ring bolt placed opposite drum seam and in down position

Lid sealed and torqued to $40 \mathrm{ft} / \mathrm{b}$ :

Leak tested:

Pre-test weight (lb)

Post-lest weight (lb)
Date $5.2-95^{\circ}$ Initial

Date 5.2.95

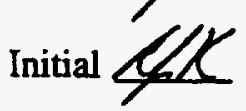

Date $5.2 .95 \quad$ Initial $\ell / K$

N/A or Pressure (psig)

Pressure (psig)

Date

Initial

176.1 Target weight $-175.6 \mathrm{lb}$
Time (min) $\quad 0$

Time (min)

\section{Loss $=$}


WHC-SD-WM-TRP-246 REV. 0

Rack Storage Drum Data Sheet

Drum No. $\times 364$.

Post-test Assessment

Position: __ original; ___ tipped; ___ toppled

Lid on drum: $\quad \quad \chi$ Yes $\quad$ No

Gasket missing (cm) $O$ i4" hand a compressed

Contents expelled: $\quad$ Yes $\longrightarrow$ No

Seal failure: $\quad$ Yes $\quad X$ Not visible

Burn pattern:

nove - seoly

Photo taken:

- Yes to No

Leak tested: Pressure (psig)

Date

Initial

$\begin{array}{ll}\text { Time }(\min ) & 0 \\ \text { Time }(\min ) & \end{array}$

Interior assessment:
iny ileght mething all wound

V:Deo, is

Photo taken:

$\underline{x}$ Yes

Comments: 
WHC-SD-WM-TRP-246 REV. O

\section{Rack Storage Drum Data Sheet}

Drum No. $\times 374$

Description (Check):

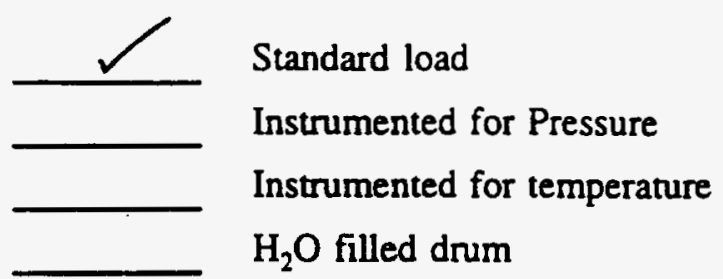

\begin{tabular}{|c|c|c|c|}
\hline Loading Starting at Bottom & Layer 1 & Layer 2 & Layer 3 \\
\hline 3 pieces of steel & & N/A \\
\hline $1 / 3$ of the brown paper & & & \\
\hline $1 / 3$ of the 10 -mil plastic & & & \\
\hline $1 / 3$ of the bond paper & & & \\
\hline $1 / 3$ of the towels & & & \\
\hline $1 / 3$ of the bags & & & \\
\hline $1 / 3$ of the cut paper & Go to Layer 2 & Go to Layer 3 & \\
\hline $1 / 3$ of the fabric & & & \\
\hline $1 / 3$ of the rubber & & & \\
\hline
\end{tabular}

Drum Filled:

Ring bolt placed opposite drum seam and in down position

Lid sealed and torqued to $40 \mathrm{ft} / \mathrm{lb}$ :

Leak tested:

Pre-test weight (lb)

Post-test weight (lb)
Date $5-2.95$ Initial QNK

Date $5.7 .95 \quad$ Initial $\frac{\mathbb{L}}{7}$

Date $5.2 .95 \quad$ Initial $L / K$

N/A or Pressure (psig)

Pressure (psig)

Date

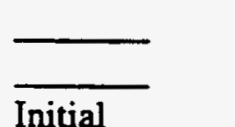

Time (min) $\frac{0}{5}$

Time (min)

$175.9 \quad$ Target weight $-175.6 \mathrm{lb}$ 
WHC-SD-WM-TRP-246 REV. 0

Rack Storage Drum Data Sheet

Drum No. $\quad \lcm{374} i 76.2 \quad 16$

Post-test Assessment

Position: __ original; ___ tipped; __ toppled

Lid on drum: $\quad x$ Yes No

Gasket missing (cm) I 46 " hand a compressed.

Contents expelled: _ues $\underset{x}{ }$ No

Seal failure: $\quad$ Yes $\quad x$ Not visible

Burn pattern:

none - sooty

Photo taken: _ Yes $\underline{x}$ No

\begin{tabular}{llll} 
Leak tested: & Time (min) & 0 \\
\hline Pressure (psig) & Time (min) & $\frac{5}{5}$
\end{tabular}

Date

$\overline{\text { Initial }}$

Interior assessment:

Interior assessment:
barely inelted right half of hur (from sean)
No tha

نlDes ¿1

Photo taken:

Yes — No

Comments: 
WHC-SD-WM-TRP-246 REV. 0

\section{Rack Storage Drum Data Sheet}

Drum No. $\times 384$

Description (Check):

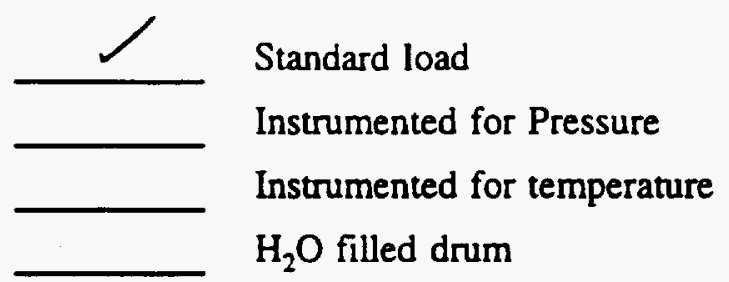

\begin{tabular}{|c|c|c|c|}
\hline Loading Starting at Bottom & Layer 1 & Layer 2 & Layer 3 \\
\hline 3 pieces of steel & & & \\
\hline $1 / 3$ of the brown paper & & & \\
\hline $1 / 3$ of the 10 -mil plastic & & & \\
\hline $1 / 3$ of the bond paper & & & \\
\hline $1 / 3$ of the towels & & & \\
\hline $1 / 3$ of the bags & & Go to Layer 3 & \\
\hline $1 / 3$ of the cut paper & & & \\
\hline $1 / 3$ of the fabric & Go to Layer 2 & G & \\
\hline $1 / 3$ of the rubber & & \\
\hline
\end{tabular}

Drum Filled:

Date $5-7.95 \quad$ Initial $/ 4$

Ring bolt placed opposite drum seam Date 5.2 .95 Initial and in down position

Lid sealed and torqued to $40 \mathrm{ft} / \mathrm{lb}: \quad$ Date 5.2 .95 Initial

Leak tested:

Pre-test weight (lb)

Post-test weight (lb)
N/A or Pressure (psig)

Pressure (psig)

Date

176.1
Time (min)

Time (min) 5

Initial

Target weight $-175.6 \mathrm{lb}$ 
WHC-SD-WM-TRP-246 REV. 0

Rack Storage Drum Data Sheet

Drum No. $\times 384.2 t .=176.31 \mathrm{~h}$.

$\underline{\text { Post-test Assessment }}$

Position: ___ original; ___ tipped; __ toppled

Lid on drum: $\quad$ Yes

Gasket missing (cm) O compreased, hand all but 12 "

Contents expelled: _— Yes $\rightleftharpoons$ No

Seal failure: $\quad$ Yes $\quad \chi$ Not visible

Burn pattern:

None - sooty all anowd

Photo taken:

- Yes $\cong$ No

Leak tested:

Dor Pressure (psig)

Pressure (psig)

Date

Time (min) 0

Time (min)

Initial

Interior assessment:

Interior assessment:
bacely melted all acound not to wael
wo chan

video

Photo taken:

119 Yes

No

Comments: 
WHC-SD-WM-TRP-246 REV. 0

\section{Rack Storage Drum Data Sheet}

Drum No. $\times 115$

Description (Check):

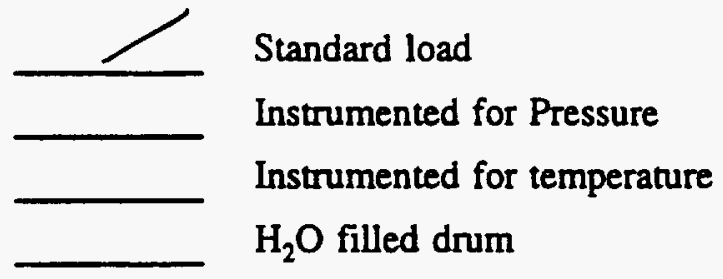

\begin{tabular}{|c|c|c|c|}
\hline Loading Starting at Bottom & Layer 1 & Layer 2 & Layer 3 \\
\hline 3 pieces of steel & & N/A & N/A \\
\hline \multicolumn{4}{|l|}{$1 / 3$ of the brown paper } \\
\hline \multicolumn{4}{|l|}{$1 / 3$ of the 10 -mil plastic } \\
\hline \multicolumn{4}{|l|}{$1 / 3$ of the bond paper } \\
\hline \multicolumn{4}{|l|}{$1 / 3$ of the towels } \\
\hline \multicolumn{4}{|l|}{$1 / 3$ of the bags } \\
\hline \multicolumn{4}{|l|}{$1 / 3$ of the cut paper } \\
\hline \multicolumn{4}{|l|}{$1 / 3$ of the fabric } \\
\hline \multicolumn{4}{|l|}{$1 / 3$ of the rubber } \\
\hline & Go to Layer 2 & Go to Layer 3 & \\
\hline
\end{tabular}

Drum Filled:

Ring bolt placed opposite drum seam and in down position

Lid sealed and torqued to $40 \mathrm{ftlb}$ :

Leak tested:

Pre-test weight (lb)

Post-test weight (lb)

Loss $=$
Date 5-3.95 Initial L.K

Date 5.3-95 Initial RK

Date 5-3.95 Initial R.K

N/A or Pressure (psig)

Pressure (psig)

Date

\begin{tabular}{lll}
- & Time $(\min )$ & 0 \\
\hline & Time $(\min )$
\end{tabular}

$176.2 \quad$ Target weight $-175.6 \mathrm{lb}$ 
WHC-SD-WM-TRP-246 REV. U

\section{Rack Storage Drum Data Sheet}

Drum No. $\times 115 \quad \ldots t=176.1$ ib.

Post-test Assessment

Position: __ original: ___ tipped: __ toppled

Lid on drum: $\quad \longrightarrow$ Yes No

Gasket missing $(\dot{\mathrm{cm}}) \_37^{\prime \prime}$

Contents expelled: __ Yes $X$ No

Seal failure: $\quad$ Yes $\quad x$ Not visible

Burn pattern:

$=12^{\circ}$ to $10 p+$ of seam

Photo taken:

Yes $\$ No

Leak tested:

Pressure (psig)

Date

Initial

Time (min)

Time (min) 5

Interior assessment:

$i-2 "$ sher seam hal $-1 / 2-i "$ on back holf

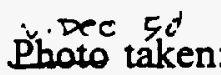

$X ?$ Yes No

Comments: 


\section{Rack Storage Drum Data Sheet}

Drum No. $x 125(p)$

Description (Check):

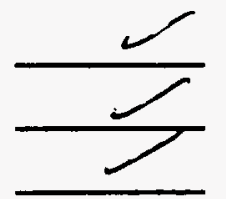

Standard load

Instrumented for Pressure

Instrumented for temperature

$\mathrm{H}_{2} \mathrm{O}$ filled drum

\begin{tabular}{|c|c|c|c|}
\hline Loading Starting at Bottom & Layer 1 & Layer 2 & Layer 3 \\
\hline 3 pieces of steel & & N/A & \\
\hline $1 / 3$ of the brown paper & & & \\
\hline $1 / 3$ of the 10 -mil plastic & & & \\
\hline $1 / 3$ of the bond paper & & & \\
\hline $1 / 3$ of the towels & & & \\
\hline $1 / 3$ of the bags & & & \\
\hline $1 / 3$ of the cut paper & & & \\
\hline $1 / 3$ of the fabric & Go to Layer 2 & Go to Layer 3 & \\
\hline $1 / 3$ of the rubber & & \\
\hline
\end{tabular}

Drum Filled:

Ring bolt placed opposite drum seam and in down position

Lid sealed and torqued to $40 \mathrm{ft} / \mathrm{lb}$ :

Leak tested:

Pre-test weight (lb)

Post-test weight (lb)
Date 5.2 .85 Initial

Date $5 \cdot 2 \cdot 95$ Initial
Date $=2-95 \quad$ Initial $L \cdot K$

N/A or Pressure (psig)

Pressure (psig)

Date

\begin{tabular}{lll} 
& Time $(\min )$ & 0 \\
\hline & Time $(\min )$
\end{tabular}

Target weight $\sim 175.6 \mathrm{lb}$ 
WHC-SD-WM-TRP-246 REV. 0

Rack Storage Drum Data Sheet

Drum No. $\frac{x, 25}{(P)}-t .=176.0$

Post-test Assessment

Position: __ original: __ tipped; __ toppled

Lid on drum: $\quad \quad \quad X$ Yes No

Gasket missing (cm) _ 35 "

Contents expelled: $\_$Yes $\longrightarrow$ No

Seal failure: $\quad$ Yes $\quad \times$ Not visible

Burn pattern:

-6" ot seam top $1 / 5$ of drum

Photo taken:

Yes $\perp$ No

Leak tested:

77 or Pressure (psig)

Pressure (psig)

Date

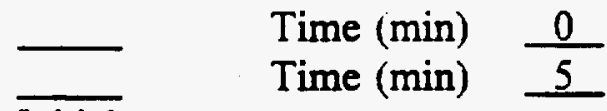

$\overline{\text { Initial }}$

Interior assessment:

2-3"char seam side, -1" on far side

pec 5 ín

$\times$ Yes

No

Comments: 
WHC-SD-WM-TRP-246 REV. U

\section{Rack Storage Drum Data Sheet}

Drum No. $\times 135$

Description (Check):

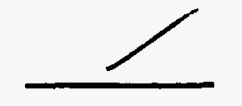

Standard load

Instrumented for Pressure

Instrumented for temperature

$\mathrm{H}_{2} \mathrm{O}$ filled drum

\begin{tabular}{|c|c|c|c|}
\hline Loading Starting at Bottom & Layer 1 & Layer 2 & Layer 3 \\
\hline 3 pieces of steel & & N/A \\
\hline $1 / 3$ of the brown paper & & & \\
\hline $1 / 3$ of the 10-mil plastic & & & \\
\hline $1 / 3$ of the bond paper & & & \\
\hline $1 / 3$ of the towels & & & \\
\hline $1 / 3$ of the bags & & & \\
\hline $1 / 3$ of the cut paper & & & \\
\hline $1 / 3$ of the fabric & Go to Layer 2 & Go to Layer 3 & \\
\hline $1 / 3$ of the rubber & & \\
\hline
\end{tabular}

Drum Filled:

Date $5 \cdot 3-95$ Initial $\underline{\ell . K}$

Ring bolt placed opposite drum seam Date 5.3.95 Initial and in down position

Lid sealed and torqued to $40 \mathrm{ft} / \mathrm{lb}: \quad$ Date $5=3.85$ Initial $2 K$

Leak tested:

Pre-test weight (lb)

Post-test weight (lb)
N/A or Pressure (psig)

Pressure (psig)

Date

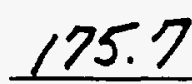

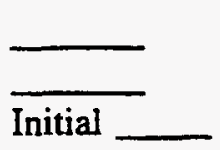

Target weight $\sim 175.6 \mathrm{lb}$

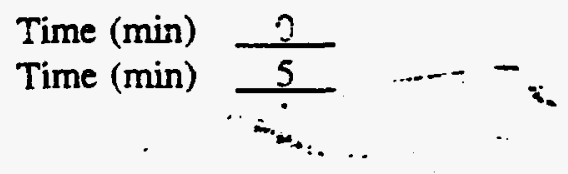

Time (min) $\frac{0}{5} \quad \ldots$

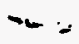


WHC-SD-WM-TRP-246 REV. 0

Rack Storage Drum Data Sheet

Drum No. $\times 135 \quad \omega T=.76 .616$

Post-test Assessment

Position: __ original: ___ tipped; ___ toppled

Lid on drum: $\quad X$ Yes No

Gasket missing (cm) __ $29^{\prime \prime}$

Contents expelled: _u Yes $\underline{X}$ No

Seal failure: $\quad$ Yes $\quad x$ Not visible

Burn pattern:

$\sim 4^{*}$ top is of drum at seam

Photo taken:

Yes $x$ No

Leak tested:

NA⿵⺆ Pressure (psig)

Pressure (psig)

Date
Time $(\min ) \quad \frac{0}{5}$

$\overline{\text { Initial }}$

Interior assessment:

3-4" "han depth seam side, 1-2" melted plasti- buck hay.

V.peo52

Photo taken:

$\underline{x}$ Yes

No

Comments: 
WHC-SD-WM-TRP-246 REV. 0

\section{Rack Storage Drum Data Sheet}

Drum No. $\times 145$

Description (Check):

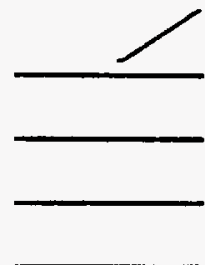

Standard load

Instrumented for Pressure

Instrumented for temperature

$\mathrm{H}_{2} \mathrm{O}$ filled drum

\begin{tabular}{|c|c|c|c|}
\hline Loading Starting at Bottom & Layer 1 & Layer 2 & Layer 3 \\
\hline 3 pieces of steel & & N/A & \\
\hline $1 / 3$ of the brown paper & & & \\
\hline $1 / 3$ of the 10 -mil plastic & & & \\
\hline $1 / 3$ of the bond paper & & & \\
\hline $1 / 3$ of the towels & & & \\
\hline $1 / 3$ of the bags & & & \\
\hline $1 / 3$ of the cut paper & & & \\
\hline $1 / 3$ of the fabric & & & \\
\hline $1 / 3$ of the rubber & Go to Layer 2 & Go to Layer 3 & \\
\hline
\end{tabular}

Drum Filled:

Ring bolt placed opposite drum seam and in down position

Lid sealed and torqued to $40 \mathrm{ft} / \mathrm{lb}$ :

Leak tested:

Pre-test weight (lb)

Post-test weight (lb)

Loss =
Date 5-3.95 Initial $\mathrm{eK}$

Date 5.3.95 Initial R.K

Date 5.3.95 Initial R.K
N/A or Pressure (psig)

Pressure (psig)

Date

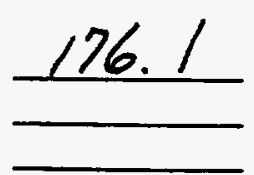

Time (min) 0

Time $(\min ) \quad 5$
Initial

Target weight $-175.6 \mathrm{lb}$ 
WHC-SD-WM-TRP-246 REV. 0

Rack Storage Drum Data Sheet

Drum No. $\quad \times 145 \quad$ of $=175.9 \mathrm{~b}$

Post-test Assessment

Position: __ original; ___ tipped; __ toppled

Lid on drum: $\quad \quad \quad X$ Yes $\quad$ No

Gasket missing (cm)

Contents expelled: _ Yes $\quad x^{\prime}$ No

Seal failure: $\quad$ Yes $\quad x$ Not visible

Burn pattern:

$9^{\prime \prime}$ to right of seam top $3 / 3$ of dum

Photo taken: $\quad$ Yes $\not C$ No

Leak tested: $\quad$ HiA ot Pressure (psig)

Pressure (psig)

Date

$\begin{array}{ll}\text { Time }(\min ) & 0 \\ \text { Time }(\min ) & -5\end{array}$

Initial

Interior assessment:

-1 "char -ic" span around seam other peremetes cloan

\begin{tabular}{l}
\hline \\
\hline pioce 53 \\
Photo taken: \\
Comments:
\end{tabular}


WHC-SD-WM-TRP-246 REV. 0

Rack Storage Drum Data Sheet

Drum No. $\times 155$

Description (Check):

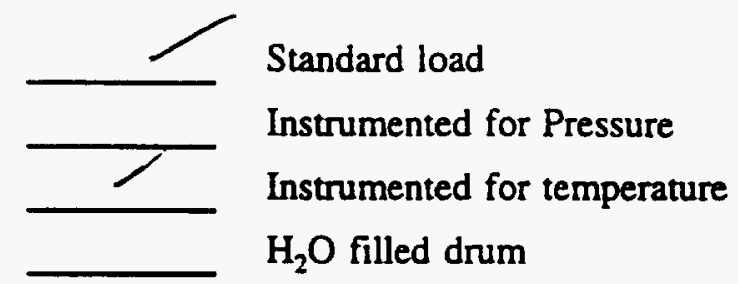

\begin{tabular}{|c|c|c|c|}
\hline Loading Starting at Bottom & Layer 1 & Layer 2 & Layer 3 \\
\hline 3 pieces of steel & & N/A & N/A \\
\hline $1 / 3$ of the brown paper & & & \\
\hline $1 / 3$ of the 10 -mil plastic & & $r$ & \\
\hline $1 / 3$ of the bond paper & & & \\
\hline $1 / 3$ of the towels & & & \\
\hline $1 / 3$ of the bags & & & \\
\hline $1 / 3$ of the cut paper & & & \\
\hline $1 / 3$ of the fabric & & & \\
\hline $1 / 3$ of the rubber & & $r$ & \\
\hline & Go to Layer 2 & Go to Layer 3 & \\
\hline
\end{tabular}

Drum Filled:

Date $5-3.95$ Initial

Ring bolt placed opposite drum seam Date $5-3-95$ Initial and in down position

Lid sealed and torqued to $40 \mathrm{ft} / \mathrm{lb}: \quad$ Date $5 \cdot 3.95$ Initial $1 . \times$

Leak tested:

N/A or Pressure (psig)

Pressure (psig)

Date __nitial

Time (min)

Time $(\mathrm{min}) 5$

Pre-test weight (lb)

Post-test weight (lb)

$176.3 \quad$ Target weight $-175.6 \mathrm{lb}$

Loss $=$ 


\section{WHC-SD-WM-TRP-246 REV. O}

Rack Storage Drum Data Sheet

Drum No. $\times 155$ ut. 176.316

Post-test Assessment

Position:

original:

tipped; toppled

Lid on drum: $\quad \longrightarrow$ Yes No

Gasket missing (cm) $2-10^{\prime \prime}$ broum

Contents expelled: _ Yes $X$ No

Seal failure: $\quad$ Yes $X$ Not visible

Burn pattern:

NOT visible - very socty on seam half

Photo taken:

Yes $\underline{x}$ No

Leak tested:

NTA or Pressure (psig)

Pressure (psig)

Date
Time (min) $\quad 0$

Time (min)

Interior assessment:

plaotic malted $/ 2$ drum about serm

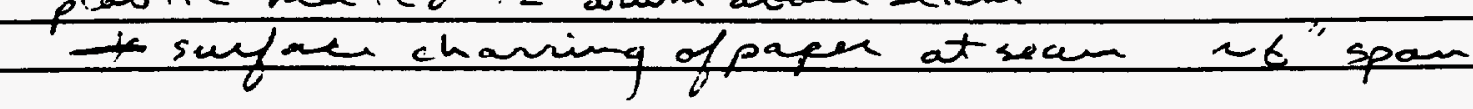

‥tec 69

Photo taken:

$\mathcal{L}$ Yes $\quad$ No

Comments: 


\section{Rack Storage Drum Data Sheet}

Drum No. $\times 165$

Description (Check):

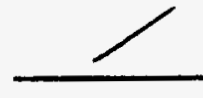

Standard load

Instrumented for Pressure

Instrumented for temperature

$\mathrm{H}_{2} \mathrm{O}$ filled drum

\begin{tabular}{|c|c|c|c|}
\hline Loading Starting at Bottom & Layer 1 & Layer 2 & Layer 3 \\
\hline 3 pieces of steel & & N/A & N/A \\
\hline $1 / 3$ of the brown paper & & & \\
\hline \multicolumn{4}{|l|}{$1 / 3$ of the 10 -mil plastic } \\
\hline $1 / 3$ of the bond paper & & & $\omega^{2}$ \\
\hline \multicolumn{4}{|l|}{$1 / 3$ of the towels } \\
\hline \multicolumn{4}{|l|}{$1 / 3$ of the bags } \\
\hline \multicolumn{4}{|l|}{$1 / 3$ of the cut paper } \\
\hline \multicolumn{4}{|l|}{$1 / 3$ of the fabric } \\
\hline \multicolumn{4}{|l|}{$1 / 3$ of the rubber } \\
\hline & Go to Layer 2 & Go to Layer 3 & \\
\hline
\end{tabular}

Drum Filled:

Date 5-3-95. Initial R.K

Ring bolt placed opposite drum seam Date 5.3.95 Initial L.K

and in down position

Lid sealed and torqued to $40 \mathrm{ft} / \mathrm{lb}$ : Date $5 \cdot 3-95$ Initial

Leak tested:

Pre-test weight (lb)

Post-test weight (lb)

Loss $=$
N/A or Pressure (psig)

Pressure (psig)

Date

175.7

Initial

Target weight $\sim 175.6 \mathrm{lb}$ 


\section{Rack Storage Drum Data Sheet}

Drum No. $\times 165 \quad$ at. $=175.4 \mathrm{ib}$.

Post-test Assessment

Position: __ original: __ tipped: __ toppled

Lid on drum: $\quad \quad \quad X$ Yes

Gasket missing (cm) $O$ hand all the way around

Contents expelled: _ Yes $\simeq$ No

Seal failure: $\quad$ Yes $\quad \chi$ Not visible

Burn pattern:

Noare - soot all arcund

Photo taken: _ Yes — No

Leak tested:

Pressure (psig)

Date

\begin{tabular}{lll}
- & Time (min) & 0 \\
\hline & Time (min)
\end{tabular}

$\overline{\text { Initial }}$

Interior assessment:

flosfic matted atl the war around prowesing of papen a falioic at seam

viveo 76

Photo taken:

$\chi^{-}$Yes No

Comments: 


\section{Rack Storage Drum Data Sheet}

Drum No. $\times 172$

Description (Check):

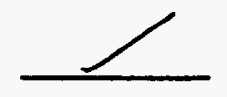

Standard load

Instrumented for Pressure

Instrumented for temperature

$\mathrm{H}_{2} \mathrm{O}$ filled drum

\begin{tabular}{|c|c|c|c|}
\hline Loading Starting at Bottom & Layer 1 & Layer 2 & Layer 3 \\
\hline 3 pieces of steel & & N/A & N/A \\
\hline $1 / 3$ of the brown paper & & & \\
\hline \multicolumn{4}{|l|}{$1 / 3$ of the 10 -mil plastic } \\
\hline $1 / 3$ of the bond paper & & & \\
\hline $1 / 3$ of the towels & & & \\
\hline \multicolumn{4}{|l|}{$1 / 3$ of the bags } \\
\hline \multicolumn{4}{|l|}{$1 / 3$ of the cut paper } \\
\hline \multicolumn{4}{|l|}{$1 / 3$ of the fabric } \\
\hline \multirow[t]{2}{*}{$1 / 3$ of the rubber } & & & \\
\hline & Go to Layer 2 & Go to Layer 3 & \\
\hline
\end{tabular}

Drum Filled:

Date 5-3.95 Initial L.K

Ring bolt placed opposite drum seam Date $5-3.95$ Initial P.K and in down position

Lid sealed and torqued to $40 \mathrm{ft} / \mathrm{b}$ : $\quad$ Date $5-3-95$ Initial

Leak tested:

Pre-test weight (lb)

Post-test weight (lb)
N/A or Pressure (psig)

Pressure (psig)

Date

176.1

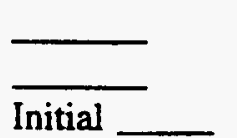

Time (min)

Time (min)

Target weight $\sim 175.6 \mathrm{lb}$ 


\section{WHC-SD-WM-TRP-246 REV. 0 \\ Rack Storage Drum Data Sheet}

Drum No. $\quad \underline{x, 75} \quad 176.2$

Post-test Assessment

Position: __ original: __ tipped; __ toppled

Lid on drum: $\quad X$ Yes No

Gasket missing $(\mathrm{cm}) Q$ most compressed and hand

Contents expelled: _ـ Yes $x$ No

Seal failure: $\quad$ Yes $\rightleftharpoons$ Not visible

Burn pattern:

None.

Photo taken: $\quad$ Yes $\underset{x}{ }$ No

Leak tested: $\begin{array}{llll}\widehat{\text { N/A or Pressure (psig) }} & & \text { Time (min) } & 0 \\ \begin{array}{l}\text { Pressure (psig) } \\ \text { Date }\end{array} & \overline{\text { Time (min) }} & \frac{5}{5}\end{array}$

Interior assessment:
plastic ilted all the way around peumeter - to wall aboct the seam no chou

Photo taken:

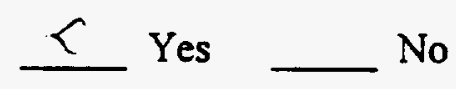

\section{Comments:}




\section{Rack Storage Drum Data Sheet}

Drum No. $\times 185$

Description (Check):

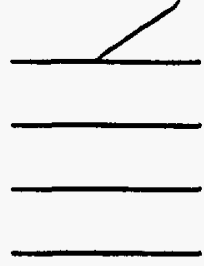

Standard load

Instrumented for Pressure

Instrumented for temperature

$\mathrm{H}_{2} \mathrm{O}$ filled drum

\begin{tabular}{|c|c|c|c|}
\hline Loading Starting at Bottom & Layer 1 & Layer 2 & Layer 3 \\
\hline 3 pieces of steel & & N/A & N/A \\
\hline $1 / 3$ of the brown paper & & & \\
\hline $1 / 3$ of the $10-\mathrm{mil}$ plastic & & & \\
\hline $1 / 3$ of the bond paper & & & \\
\hline $1 / 3$ of the towels & & & \\
\hline $1 / 3$ of the bags & & & \\
\hline $1 / 3$ of the cut paper & & 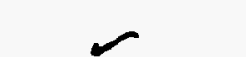 & \\
\hline $1 / 3$ of the fabric & & & \\
\hline $1 / 3$ of the nubber & 2 & & \\
\hline & Go to Layer 2 & Go to Layer 3 & \\
\hline
\end{tabular}

Drum Filled:

Ring bolt placed opposite drum seam and in down position

Lid sealed and torqued to $40 \mathrm{ft} / \mathrm{lb}$ :

Leak tested:

Pre-test weight (lb)

Post-test weight (lb)
Date 5.3.95 Initial R.K

Date $5-3.95 \quad$ Initial L.R

Date 5.3.95 Initial L.K

N/A or Pressure (psig)

Pressure (psig)

Date

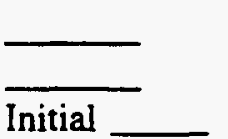

Time (min)

Time (min) 5

\section{$1760 \quad$ Target weight $\sim 175.6 \mathrm{lb}$}


WHC-SD-WM-TRP-246 REV. 0

Rack Storage Drum Data Sheet

Drum No. $\quad \underline{x 185} 175.9$

Post-test Assessment

Position: __ original: ___ tipped; __ toppled

Lid on drum: $\quad \quad \quad X$ Yes No

Gasket missing (cm) $O$ hand all the way around

Contents expelled: _ Yes $x$ No

Seal failure: $\quad$ Yes $\quad X$ Not visible

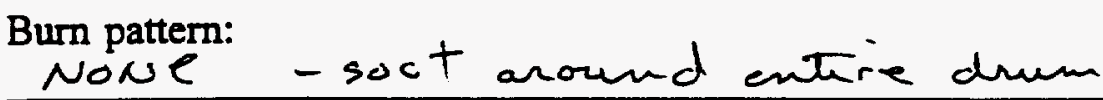

Photo taken: $\quad$ Yes $\quad x$ No

Leak tested: $\quad$ NT or Pressure (psig)

Pressure (psig)

Date

$\begin{array}{ll}\text { Time }(\min ) & 0 \\ \text { Time }(\min ) & \end{array}$

Initial

Interior assessment: melted ull the way around, to wall at seam

$\because 00075$

Photo taken:

$x$ Yes

Comments: 


\section{Rack Storage Drun Data Sheet}

Drum No. $\times 215$

Description (Check):

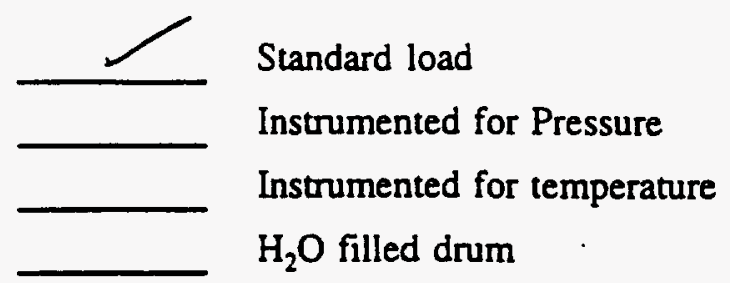

\begin{tabular}{|c|c|c|c|}
\hline Loading Starting at Bottom & Layer 1 & Layer 2 & Layer 3 \\
\hline 3 pieces of steel & & N/A & N/A \\
\hline $1 / 3$ of the brown paper & & & \\
\hline $1 / 3$ of the $10-$ mil plastic & & & \\
\hline $1 / 3$ of the bond paper & & & \\
\hline $1 / 3$ of the toweis & & & \\
\hline $1 / 3$ of the bags & & & \\
\hline $1 / 3$ of the cut paper & & & \\
\hline $1 / 3$ of the fabric & & & \\
\hline $1 / 3$ of the rubber & Go to Layer 2 & Go to Layer 3 & \\
\hline \multicolumn{2}{|l}{} \\
\hline \hline
\end{tabular}

Drum Filled:

Ring bolt placed opposite drum seam and in down position

Lid sealed and torqued to $40 \mathrm{ft} / \mathrm{b}$ :

Leak tested:

Pre-test weight (lb)

Post-test weight (lb)
Date 5.2-95 Initial L.R

Date $5=7.95 \quad$ Initial Let

Date $6.2 .95 \quad$ Initial Le

N/A or Pressure (psig)

Pressure (psig)

Date

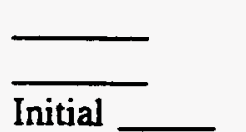

Time (min)

Time $(\mathrm{min}) \quad 5$

175.8

Target weight $\sim 175.6 \mathrm{lb}$ 
WHC-SD-WM-TRP-246 REV. 0

Rack Storage Drum Data Sheet

Drum No. $\quad x 215 \quad$ it. 174,7 B

Post-test Assessment

Position: __ original: __ tipped; __ toppled

Lid on drum: $\quad X$ Yes No $6^{\circ} \times 30^{\circ}$ gap at boit

Gasket missing $(\mathrm{cm}) \mathrm{All}$

Contents expelled: _ Yes $\_$No

Seal failure: $\quad X$ Yes $\quad$ Not visible

Burn pattern:

appeans to be bunned onmore on bott half of drum

Photo taken: $\quad$ Yes $\underline{x}$ No

$\begin{array}{lllll}\text { Leak tested: } & \begin{array}{l}\text { N/A or Pressure (psig) } \\ \text { Pressure (psig) } \\ \text { Date }\end{array} & \overline{\text { Time (min) }} & 0 \\ & & \overline{\text { Initial }} & \text { Time (min) } & -\end{array}$

Interior assessment:

"24" fra top f dum to top of chaned contento

videc 29

Photo taken:

X Yes — No

Comments: 


\section{Rack Storage Drum Data Sheet}

Drum No. $\times 225(r)$

Description (Check):

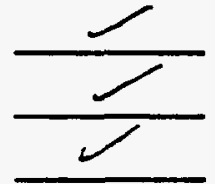

Standard load

Instrumented for Pressure

Instrumented for temperature

$\mathrm{H}_{2} \mathrm{O}$ filled drum

\begin{tabular}{|c|c|c|c|}
\hline Loading Starting at Bottom & Layer 1 & Layer 2 & Layer 3 \\
\hline 3 pieces of steel & & & \\
\hline $1 / 3$ of the brown paper & & & \\
\hline $1 / 3$ of the 10-mil plastic & & & \\
\hline $1 / 3$ of the bond paper & & & \\
\hline $1 / 3$ of the towels & & & \\
\hline $1 / 3$ of the bags & & & \\
\hline $1 / 3$ of the cut paper & & & \\
\hline $1 / 3$ of the fabric & & & \\
\hline $1 / 3$ of the rubber & Go to Layer 2 & Go to Layer 3 & \\
\hline
\end{tabular}

\section{Drum Filled:}

Ring bolt placed opposite drum seam and in down position

Lid sealed and torqued to $40 \mathrm{ft} / \mathrm{b}$ :

Leak tested:

Pre-test weight (lb)

Post-test weight (lb)
Date 5.2.95 Initial L.K

Date 5.7 .85 Initial

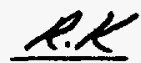

Date 5-2.95 Initial N.S

N/A or Pressure (psig)

Pressure (psig)

Date

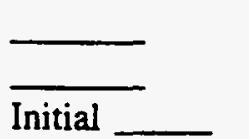

Time (min)

Time $(\min ) \quad 5$

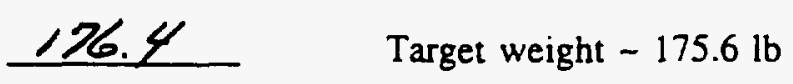


WHC-SD-WM-TRP-246 REV. 0

Rack Storage Drum Data Sheet

Drum No. $\times 225(P) \quad 168.0$

Post-test Assessment

Position: __ original: ___ tipped; __ toppled

Lid on drum: $\quad \quad \quad$ Yes No $3 / 4 " \times 9 "$ at boit

Gasket missing (cm)

Contents expelled: Yes $X$ No

Seal failure: $\quad \underline{X}$ Yes Not visible

Burn pattern:

Difficult To manom

$12^{*}$ are clean

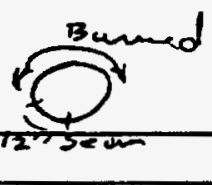

Photo taken:

Yes $\varnothing$ No

Leak tested: $\quad$ N/A ODPressure (psig) Pressure (psig)

Date

\begin{tabular}{ll} 
Time $(\min )$ & 0 \\
Time $(\min )$ & \multicolumn{5}{c}{}
\end{tabular}

$\overline{\text { Initial }}$

Interior assessment:

16 "from top to top of choned contents

videe $=2$

Photo taken:

$\underline{X}$ Yes

Comments: 


\section{Rack Storage Drum Data Sheet}

Drum No. $\times 235$

Description (Check):

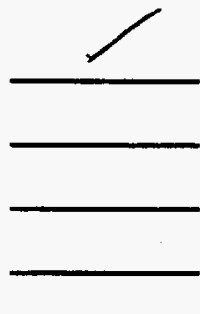

Standard load

Instrumented for Pressure

Instrumented for temperature

$\mathrm{H}_{2} \mathrm{O}$ filled drum

\begin{tabular}{|c|c|c|c|}
\hline Loading Starting at Bottom & Layer 1 & Layer 2 & Layer 3 \\
\hline 3 pieces of steel & & N/A \\
\hline $1 / 3$ of the brown paper & & & \\
\hline $1 / 3$ of the 10-mil plastic & & & \\
\hline $1 / 3$ of the bond paper & & & \\
\hline $1 / 3$ of the towels & & & \\
\hline $1 / 3$ of the bags & & & \\
\hline $1 / 3$ of the cut paper & & & \\
\hline $1 / 3$ of the fabric & Go to Layer 2 & Go to Layer 3 & \\
\hline $1 / 3$ of the rubber & & & \\
\hline
\end{tabular}

Drum Filled:

Ring bolt placed opposite drum seam and in down position

Lid sealed and torqued to $40 \mathrm{ft} / \mathrm{lb}$ :

Leak tested:

Pre-test weight (lb)

Post-test weight (lb)
Date 5-2.95 Initial L.R

Date $5=295$ Initial $h . K$

Date $=2.95$ Initial

N/A or Pressure (psig) Pressure (psig)

Date

Initial

Time (min) 0

Time (min) 5

$175.8 \quad$ Target weight $\sim 175.6 \mathrm{lb}$ 


\section{Rack Storage Drum Data Sheet}

Drum No. $\not 235$ sot. $=158.8$ ib wet inside

\section{Post-test Assessment}

Position: original; tipped; toppled

Lid on drum: $\quad \frac{1}{2}$ Yes No

Gasket missing (cm) all

Contents expelled: __ Yes _ No

Seal failure: $\quad \not \subset$ Yes Not visible

Burn pattern:

$$
40 \text { ind wit }
$$

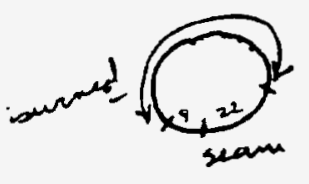

Photo taken:

$$
\text { Yes _ No }
$$

Leak tested:
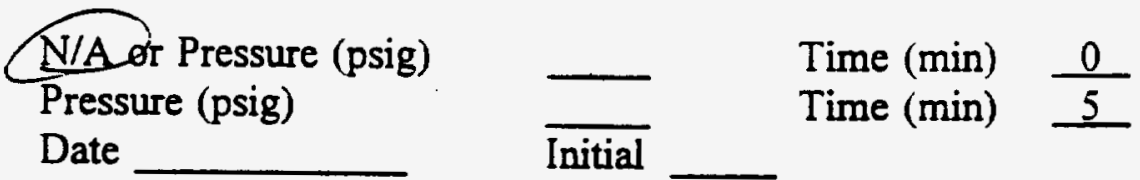

Interior assessment:
combustible
contents
yone exrept $\approx 6$ " lt

Photo taken:

Yes

No

Comments: 


\section{Rack Storage Drum Data Sheet}

Drum No. $\times 245$

Description (Check):

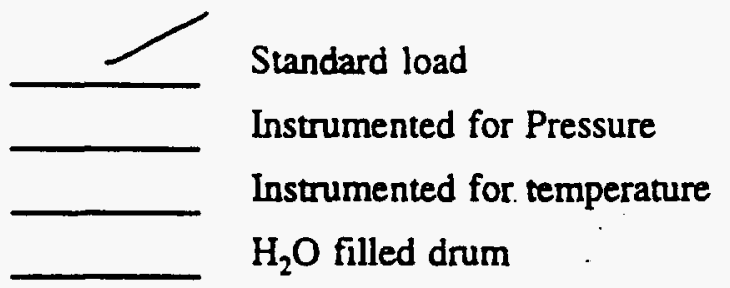

\begin{tabular}{|c|c|c|c|}
\hline Loading Starting at Bottom & Layer 1 & Layer 2 & Layer 3 \\
\hline 3 pieces of steel & & N/A & N/A \\
\hline \multicolumn{4}{|l|}{$1 / 3$ of the brown paper } \\
\hline \multicolumn{4}{|l|}{$1 / 3$ of the 10 -mil plastic } \\
\hline \multicolumn{4}{|l|}{$1 / 3$ of the bond paper } \\
\hline \multicolumn{4}{|l|}{$1 / 3$ of the towels } \\
\hline \multicolumn{4}{|l|}{$1 / 3$ of the bags } \\
\hline \multicolumn{4}{|l|}{$1 / 3$ of the cut paper } \\
\hline \multicolumn{4}{|l|}{$1 / 3$ of the fabric } \\
\hline \multicolumn{4}{|l|}{$1 / 3$ of the rubber } \\
\hline & Go to Layer 2 & Go to Layer 3 & \\
\hline
\end{tabular}

Drum Filled:

Ring bolt placed opposite drum seam and in down position

Lid sealed and torqued to $40 \mathrm{ft} / \mathrm{b}$ :

Leak tested:

Pre-test weight (lb)

Post-test weight (lb)
Date 5.2.95 Initial LL

Date 5.2 .95 Initial L.L
N/A or Pressure (psig)

Pressure (psig)

Date

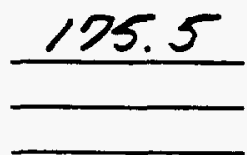

Time (min) 0 Time (min)

Initial

Target weight $\sim 175.6 \mathrm{lb}$ 
WHC-SD-WM-TRP-246 REV. 0

\section{Rack Storage Drum Data Sheet}

Drum No. $\times 245$ t. $173.9 \mathrm{in}$.

Post-test Assessment

Position:

original:

tipped;

toppled

Lid on drum: $\quad X$ Yes No

Gasket missing (cm) $\mathrm{All}$

Contents expelled: _— Yes $\chi$ No

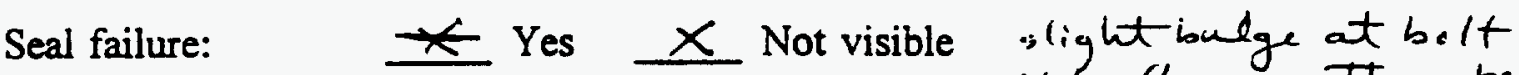
possible flame pattem below bolt

Burn pattern:

$\frac{\text { difficult to measme }}{\text { ellaround }}$

Photo taken:

Yes $x$ No

Leak tested:

N/AOr Pressure (psig)

Pressure (psig)

Date

$\begin{array}{ll}\text { Time }(\min ) & \frac{0}{5} \\ \text { Time }(\min ) & \end{array}$

Initial

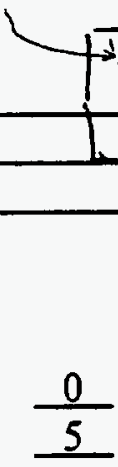

Interior assessment:

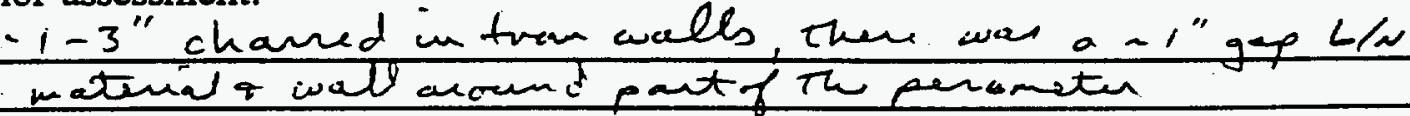

YDec 30

Phote taken:

$X$ Yes

Comments: 


\section{Rack Storage Drum Data Sheet}

Drum No. $\times 255(\%)$

Description (Check):

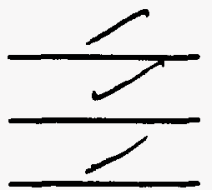

Standard load

Instrumented for Pressure

Instrumented for temperature

$\mathrm{H}_{2} \mathrm{O}$ filled drum

\begin{tabular}{|c|c|c|c|}
\hline Loading Starting at Bottom & Layer 1 & Layer 2 & Layer 3 \\
\hline 3 pieces of steel & & N/A \\
\hline $1 / 3$ of the brown paper & & & \\
\hline $1 / 3$ of the 10 -mil plastic & & & \\
\hline $1 / 3$ of the bond paper & & & \\
\hline $1 / 3$ of the towels & & & \\
\hline $1 / 3$ of the bags & & & \\
\hline $1 / 3$ of the cut paper & & & \\
\hline $1 / 3$ of the fabric & & & \\
\hline $1 / 3$ of the rubber & Go to Layer 2 & Go to Layer 3 & \\
\hline
\end{tabular}

Drum Filled:

Ring bolt placed opposite drum seam and in down position

Lid sealed and torqued to $40 \mathrm{ft} / \mathrm{b}$ :

Leak tested:

Pre-test weight (lb)

Post-test weight (lb)

Date 5.2-95 Initial R.K

Date $5.2 .95 \quad$ Initial R./

Date 5.2.95 Initial T.R

N/A or Pressure (psig)

Pressure (psig)

Date

176.2 \begin{tabular}{ll} 
Time (min) & 0 \\
Time $(\min )$ & 5 \\
\hline
\end{tabular}

Initial

Target weight $\sim 175.6 \mathrm{lb}$ 


\section{Rack Storage Drum Data Sheet}

Drum No. $\quad \underline{\times 255} \quad 176.7 \mathrm{lb}$

$\underline{\text { Post-test Assessment }}$

Position: original; tipped; toppled

Lid on drum:

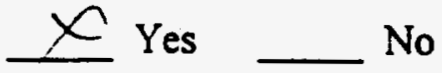

Gasket missing (cm) $O{ }^{12}$ "is burned $r$ ondy a slight icresing (<1/is")

Contents expelled: _ Yes $x$ No

Seal failure: $\quad$ Yes $\quad X$ Not visible

Burn pattern:

wene - 14" at aid Lt centund $26^{\circ}$ 1ft of seam

Photo taken:

Yes $\searrow$ No

Leak tested:

N/A or Pressure (psig)

Pressure (psig)

Date

\begin{tabular}{lll}
- & Time (min) & 0 \\
\hline
\end{tabular}$\quad$ Time (min)

Interior assessment:

28 "frm iean te left $2 z^{\prime \prime}$ chas plastic all around

videc i

Photo taken:

$X$ Yes

No

Comments: 


\section{Rack Storage Drum Data Sheet}

Drum No. $\times 265$

Description (Check):

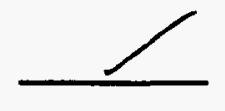

Standard load

Instrumented for Pressure

Instrumented for temperature

$\mathrm{H}_{2} \mathrm{O}$ filled drum

\begin{tabular}{|c|c|c|c|}
\hline Loading Starting at Bottom & Layer 1 & Layer 2 & Layer 3 \\
\hline 3 pieces of steel & & N/A & \\
\hline $1 / 3$ of the brown paper & & & \\
\hline $1 / 3$ of the 10 -mil plastic & & & \\
\hline $1 / 3$ of the bond paper & & & \\
\hline $1 / 3$ of the towels & & & \\
\hline $1 / 3$ of the bags & & & \\
\hline $1 / 3$ of the cut paper & & & \\
\hline $1 / 3$ of the fabric & & & \\
\hline $1 / 3$ of the rubber & Go to Layer 2 & Go to Layer 3 & \\
\hline
\end{tabular}

\section{Drum Filled:}

Ring bolt placed opposite drum seam and in down position

Lid sealed and torqued to $40 \mathrm{ft} / \mathrm{lb}$ :

Leak tested:

Pre-test weight (lb)

Post-test weight (lb)
Date $5.2 .95 \cdots$ Initial L.R

Date $5.2 .95 \quad$ Initial L.K

Date $5.2-95$ Initial

N/A or Pressure (psig)

Pressure (psig)

Date

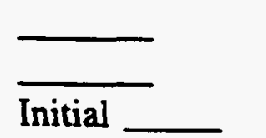

Time (min) $\frac{0}{5}$

Time (min) 5 
WHC-SD-WM-TRP-246 REV. 0

Rack Storage Drum Data Sheet

Drum No. $\quad x .265 \quad$ ats $=176.3$ ib

Post-test Assessment

Position: __ original: ___ tipped; __ toppled

Lid on drum: $\quad \quad \quad \quad$ Yes No

Gasket missing (cm) 12 haud \& compressed 50 " around.

Contents expelled: _— Yes $\searrow$ No

Seal failure: $\quad$ Yes $\quad$ Y Not visible

Burn pattern:

NONe - soly

Photo taken: __ Yes $\quad$ No

Leak tested: N/Äor Pressure (psig)

Pressure (psig)

Date

Time (min) $\quad 0$

Time (min) 5

Interior assessment:

melted all anownd to dum inspot - vo char itaibl

Vioecioc

Photo taken:

$\underset{7}{Y}$ Yes No

Comments: 


\section{Rack Storage Drum Data Sheet}

Drum No. $x 275$

Description (Check):

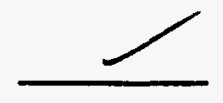

Standard load

Instrumented for Pressure

Instrumented for temperature

$\mathrm{H}_{2} \mathrm{O}$ filled drum

\begin{tabular}{|c|c|c|c|}
\hline Loading Starting at Bottom & Layer 1 & Layer 2 & Layer 3 \\
\hline 3 pieces of steel & & & \\
\hline $1 / 3$ of the brown paper & & & \\
\hline $1 / 3$ of the 10-mil plastic & & & \\
\hline $1 / 3$ of the bond paper & & & \\
\hline $1 / 3$ of the towels & & & \\
\hline $1 / 3$ of the bags & & & \\
\hline $1 / 3$ of the cut paper & & Go to Layer 3 & \\
\hline $1 / 3$ of the fabric & & & \\
\hline $1 / 3$ of the rubber & Go to Layer 2 & Go & \\
\hline
\end{tabular}

\section{Drum Filled:}

Ring bolt placed opposite drum seam and in down position

Lid sealed and torqued to $40 \mathrm{ft} / \mathrm{lb}$ :

Leak tested:

Pre-test weight (lb)

Post-test weight (lb)
Date S-2-95 Initial L.R

Date 5.2.95 Initial L.K

Date 5.2-95 Initial R./
N/A or Pressure (psig)

Pressure (psig)

Date

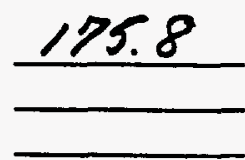

Time (min) $\quad 0$

Time (min) 


\section{Rack Storage Drum Data Sheet}

Drum No. $\quad \underline{275} w T=176.1 \mathrm{lb}$

Post-test Assessment

Position: original: tipped; toppled

Lid on drum: $\quad \times$ Yes $\quad$ No

Gasket missing (cm) O compressed, hand all around

Contents expelled: _ Yes $\underset{x}{\longrightarrow}$ No

Seal failure: $\quad$ Yes $\simeq$ Not visible

Burn pattern:

None - sooty

Photo taken:

Leak tested:

$$
\text { Yes } \chi \text { No }
$$

N/A or Pressure (psig) Pressure (psig)

Date

Initial

$\begin{array}{ll}\text { Time (min) } & 0 \\ \text { Time (min) } & \end{array}$

Interior assessment: metted oll around - to hum ondy sightly in itew places
No chou

fipec icke

$x$ Yes

Comments: 


\section{Rack Storage Drum Data Sheet}

\section{Drum No. $\times 285$}

Description (Check):

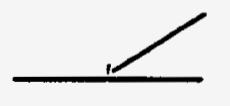

Standard load

Instrumented for Pressure

Instrumented for temperature

$\mathrm{H}_{2} \mathrm{O}$ filled drum

\begin{tabular}{|c|c|c|c|}
\hline Loading Starting at Bottom & Layer 1 & Layer 2 & Layer 3 \\
\hline 3 pieces of steel & & N/A & N/A \\
\hline $1 / 3$ of the brown paper & & & - \\
\hline $1 / 3$ of the 10 -mil plastic & & & \\
\hline \multicolumn{4}{|l|}{$1 / 3$ of the bond paper } \\
\hline $1 / 3$ of the towels & & & \\
\hline \multicolumn{4}{|l|}{$1 / 3$ of the bags } \\
\hline \multicolumn{4}{|l|}{$1 / 3$ of the cut paper } \\
\hline \multicolumn{4}{|l|}{$1 / 3$ of the fabric } \\
\hline \multicolumn{4}{|l|}{$1 / 3$ of the rubber } \\
\hline & Go to Layer 2 & Go to Layer 3 & \\
\hline
\end{tabular}

Drum Filled:

Date $5=2.95$ Initial L.K

Ring bolt placed opposite drum seam Date $5 \cdot 2 \cdot 95$ Initial R.K and in down position

Lid sealed and torqued to $40 \mathrm{ft} / \mathrm{b}: \quad$ Date 5.2 .95 Initial

Leak tested:

Post-test weight (lb)

Loss $=$
N/A or Pressure (psig)

Pressure (psig)

Date

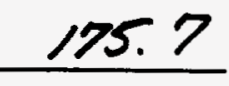

Time (min) 0

Time (min)

Initial

Target weight $-175.6 \mathrm{lb}$ 
WHC-SD-WM-TRP-246 REV. 0

Rack Storage Drum Data Sheet

Drum No. $\times 285$ ete 176.1 ib

Post-test Assessment

Position: ___ original; ___ tipped; ___ toppled

Lid on drum: $\quad x$ Yes No

Gasket missing (cm) O 19" compressed thand

Contents expelled: _ـ Yes $\longrightarrow$ No

Seal failure: $\quad$ Y Yes $\quad$ Not visible

Burn pattern: nONe

Photo taken: $\quad$ Yes $\$ No

Leak tested:

ATAjor Pressure (psig)

Pressure (psig)

Date

$\begin{array}{ll}\text { Time }(\min ) & 0 \\ \text { Time }(\min ) & \end{array}$

$\overline{\text { Initial }}$

Interior assessment:

melting ofplastic allaround - not to drum

ipeo ics

Photo taken:

$\not$ Yes _ No

Comments: 


\section{Rack Storage Drum Data Sheet}

Drum No. $\times 315$

Description (Check):

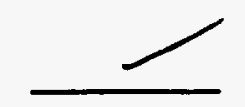

Standard load

Instrumented for Pressure

Instrumented for temperature

$\mathrm{H}_{2} \mathrm{O}$ filled drum

\begin{tabular}{|c|c|c|c|}
\hline Loading Starting at Bottom & Layer 1 & Layer 2 & Layer 3 \\
\hline 3 pieces of steel & $\checkmark$ & N/A & N/A \\
\hline $1 / 3$ of the brown paper & 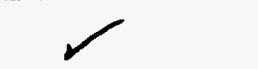 & 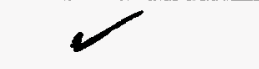 & $r$ \\
\hline $1 / 3$ of the 10 -mil plastic & r & $/$ & \\
\hline $1 / 3$ of the bond paper & $\checkmark$ & $\checkmark$ & r \\
\hline $1 / 3$ of the towels & $\checkmark$ & 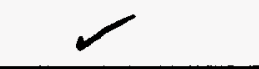 & 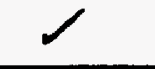 \\
\hline $1 / 3$ of the bags & $r$ & $\checkmark$ & \\
\hline $1 / 3$ of the cut paper & $\zeta$ & l & \\
\hline $1 / 3$ of the fabric & $\checkmark$ & r & - \\
\hline $1 / 3$ of the rubber & 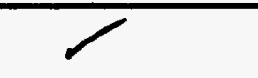 & $\checkmark$ & 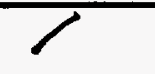 \\
\hline & Go to Layer 2 & Go to Layer 3 & \\
\hline
\end{tabular}

Drum Filled:

Date 5-2.95 Initial R.K

Ring bolt placed opposite drum seam Date $\underline{5.2 .95}$ Initial $\underline{\text { C.K }}$ and in down position

Lid sealed and torqued to $40 \mathrm{ft} / \mathrm{lb}: \quad$ Date $5.2-95 \quad$ Initial $\ell . K$

Leak tested:

Pre-test weight (lb)

Post-test weight (lb)
N/A or Pressure (psig)

Pressure (psig)

Date

$\overline{\text { Initial }}$

Target weight $-175.6 \mathrm{lb}$
Time (min)

Time (min) 5 
WHC-SD-WM-TRP-246 REV. 0

\section{Rack Storage Drum Data Sheet}

Drum No. $\times 315 \quad \omega t .=148.1 \mathrm{ib}$

Post-test Assessment

Position: original; tipped; toppled

Lid on drum:

$$
X \text { Yes No } 22^{\prime \prime \prime} \times 17^{\prime \prime} \mathrm{JP}
$$

$-12^{\prime \prime}$ Rt of seam

Gasket missing (cm) All Contents expelled: __ Yes _ No

Seal failure: $\quad \rtimes$ Yes $\quad$ Not visible

Burn pattern:

All around

Photo taken:

Yes — No

Leak tested:

N/A or Pressure (psig)

Pressure (psig)

Date 


\section{Rack Storage Drum Data Sheet}

Drum No. $\triangle 325$

Description (Check):

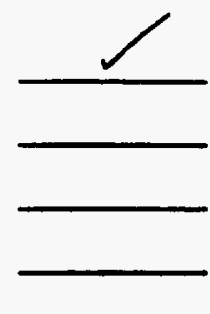

Standard load

Instrumented for Pressure

Instrumented for temperature

$\mathrm{H}_{2} \mathrm{O}$ filled drum

\begin{tabular}{|c|c|c|c|}
\hline Loading Starting at Bottom & Layer 1 & Layer 2 & Layer 3 \\
\hline 3 pieces of steel & & N/A & N/A \\
\hline \multicolumn{4}{|l|}{$1 / 3$ of the brown paper } \\
\hline \multicolumn{4}{|l|}{$1 / 3$ of the 10 -mil plastic } \\
\hline \multicolumn{4}{|l|}{$1 / 3$ of the bond paper } \\
\hline \multicolumn{4}{|l|}{$1 / 3$ of the towels } \\
\hline \multicolumn{4}{|l|}{$1 / 3$ of the bags } \\
\hline \multicolumn{4}{|l|}{$1 / 3$ of the cut paper } \\
\hline \multicolumn{4}{|l|}{$1 / 3$ of the fabric } \\
\hline \multicolumn{4}{|l|}{$1 / 3$ of the rubber } \\
\hline & Go to Layer 2 & Go to Layer 3 & \\
\hline
\end{tabular}

Drum Filled:

Date $\{-2.95 \quad$ Initial R.K

Ring bolt placed opposite drum seam Date $5=2.95$ Initial

and in down position

Lid sealed and torqued to $40 \mathrm{ft} / \mathrm{lb}: \quad$ Date 5.2 .95 Initial

Leak tested:

Pre-test weight (lb)

Post-test weight (lb)
N/A or Pressure (psig)

Pressure (psig)

Date

176.7
Time (min) 0

Time (min) 5

Initial

Target weight $\sim 175.6 \mathrm{lb}$ 
WHC-SD-WM-TRP-246 REV. 0

Rack Storage Drum Data Sheet

Drum No. $\times 325 \quad .4 t=138.81 \mathrm{~b}$

Post-test Assessment

Position:

original: tipped; toppled

Lid on drum: _ Yes $X$ No

Gasket missing (cm)

Contents expelled: __ Yes _ No

Seal failure: $\quad X$ Yes Not visible

Burn pattern:

39" bumed trinid Lt centued in bolt

Photo taken:

Yes _ No

Leak tested:

N/ADor Pressure (psig) Pressure (psig)

Date

\begin{tabular}{lll}
- & Time $(\min )$ & 0 \\
\hline
\end{tabular}$\quad$ Time (min)

\section{$\overline{\text { Initial }}$}

Interior assessment:

$\rightarrow$ " dpth of chaned inaterial

VIDrO 7

Photo taken:

Yes

No

Comments: 


\section{Rack Storage Drum Data Sheet}

Drum No. $\times 335$

Description (Check):

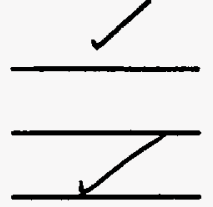

Standard load

Instrumented for Pressure

Instrumented for temperature

$\mathrm{H}_{2} \mathrm{O}$ filled drum

\begin{tabular}{|c|c|c|c|}
\hline Loading Starting at Bottom & Layer 1 & Layer 2 & Layer 3 \\
\hline 3 pieces of steel & & & \\
\hline $1 / 3$ of the brown paper & & & \\
\hline $1 / 3$ of the 10 -mil plastic & & & \\
\hline $1 / 3$ of the bond paper & & & \\
\hline $1 / 3$ of the towels & & C & \\
\hline $1 / 3$ of the bags & & Go to Layer 3 & \\
\hline $1 / 3$ of the cut paper & & & \\
\hline $1 / 3$ of the fabric & Go to Layer 2 & Go & \\
\hline $1 / 3$ of the rubber & & \\
\hline
\end{tabular}

Drum Filled:

Ring boit placed opposite drum seam and in down position

Lid sealed and torqued to $40 \mathrm{ft} / \mathrm{lb}$ :

Leak tested:

Pre-test weight (lb)

Post-test weight (lb)
Date 5.2.95 Initial L.R

Date $5=2-95 \quad$ Initial L.K

Date 5.2-95 Initial L.K

N/A or Pressure (psig)

Pressure (psig)

Date

$\overline{\text { Initial }}$

Time (min)

Time (min) 5

$176.4 \quad$ Target weight $-175.6 \mathrm{lb}$ 
WHC-SD-WM-TRP-246 REV. 0

Rack Storage Drum Data Sheet

Drum No. $\times 335 \quad \omega T=138.0$

Post-test Assessment

Position: original: tipped: toppled

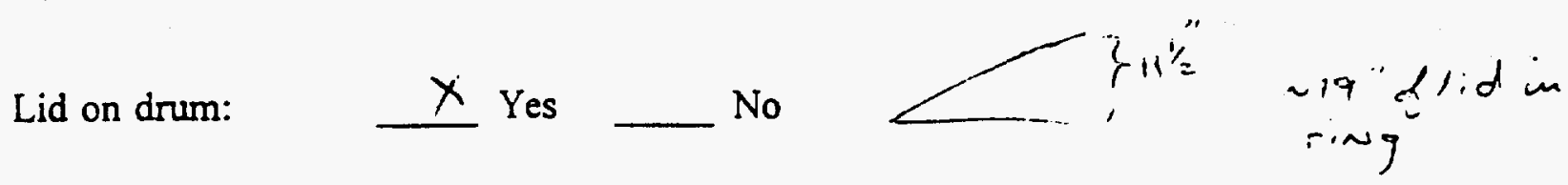

Gasket missing (cm)

Contents expelled: __ Yes _ No

Seal failure: $\quad X$ Yes Not visible

Burn pattern:

Difficult to measure - oll wound

Photo taken:

Yes — No

Leak tested:

N/A or Pressure (psig)

Pressure (psig)

Date

\begin{tabular}{lll} 
Time (min) & 0 \\
\hline Time (min) &
\end{tabular}

Interior assessment:

"3" of conterts remainim, (sharred)

VIDeo s

Photo taken:

Yes

No

\section{Comments:}




\section{Rack Storage Drum Data Sheet}

Drum No. $\times 345$

Description (Check):

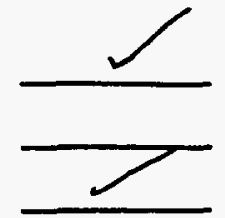

Standard load

Instrumented for Pressure

Instrumented for temperature

$\mathrm{H}_{2} \mathrm{O}$ filled drum

\begin{tabular}{|c|c|c|c|}
\hline Loading Starting at Bottom & Layer 1 & Layer 2 & Layer 3 \\
\hline 3 pieces of steel & & N/A & \\
\hline $1 / 3$ of the brown paper & & & \\
\hline $1 / 3$ of the 10 -mil plastic & & & \\
\hline $1 / 3$ of the bond paper & & & \\
\hline $1 / 3$ of the towels & & & \\
\hline $1 / 3$ of the bags & & & \\
\hline $1 / 3$ of the cut paper & & & \\
\hline $1 / 3$ of the fabric & & Go to Layer 3 & \\
\hline $1 / 3$ of the rubber & & & \\
\hline
\end{tabular}

Drum Filled:

Date $5.2 .95:$ Initial L.K

Ring bolt placed opposite drum seam Date $5=2.95$ Initial $C .4$ and in down position

Lid sealed and torqued to $40 \mathrm{ft} / 1 \mathrm{~b}: \quad$ Date $\underline{5-7.95}$ Initial

Leak tested:

Pre-test weight (lb)

Post-test weight (lb)
N/A or Pressure (psig)

Pressure (psig)

Date

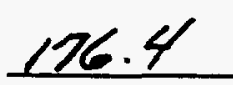

Time (min) $\quad 0$

Time $(\mathrm{min}) \quad 5$

Initial

Target weight - $175.6 \mathrm{lb}$

\section{Loss $=$}




\section{Rack Storage Drum Data Sheet}

Drum No. $\times 345$ wt $=135.1$

Post-test Assessment

Position: original; tipped; toppled

Lid on drum: $\quad$ Yes $X$ No

Gasket missing (cm) w

Contents expelled: __ Yes _ No

Seal failure: $\quad$ Y $\quad$ Yes _ Not visible

Burn pattern:

$$
22 " \text { t ind lt. 9"1-ftof sean }
$$

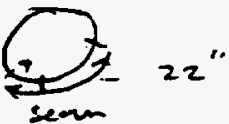

Photo taken: _ Yes _ No

Leak tested: \$N/A or Pressure (psig)

Pressure (psig)

Date $\overline{\text { Initial }}$

Time (min) $\quad \underline{0}$

Time (min) 5

Interior assessment:

-3" of contents

proeo :

$\underset{X}{X}$ Yes No

Comments: 


\section{Rack Storage Drum Data Sheet}

Drum No. $\times 355$

Description (Check):

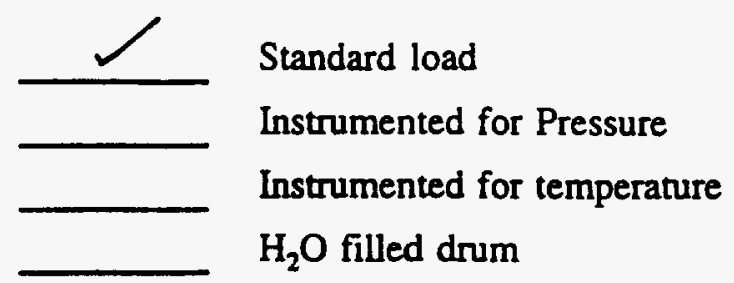

\begin{tabular}{|c|c|c|c|}
\hline Loading Starting at Bottom & Layer 1 & Layer 2 & Layer 3 \\
\hline 3 pieces of steel & & N/A & N/A \\
\hline $1 / 3$ of the brown paper & & & \\
\hline $1 / 3$ of the 10 -mil plastic & & & \\
\hline $1 / 3$ of the bond paper & & & \\
\hline $1 / 3$ of the towels & & & \\
\hline $1 / 3$ of the bags & & & \\
\hline $1 / 3$ of the cut paper & & & \\
\hline $1 / 3$ of the fabric & Go to Layer 2 & Go to Layer 3 & \\
\hline $1 / 3$ of the rubber & &
\end{tabular}

Drum Filled:

Ring bolt placed opposite drum seam and in down position

Lid sealed and torqued to $40 \mathrm{ft} / \mathrm{lb}$ :

Leak tested:

Pre-test weight (lb)

Post-test weight (lb)
Date $5-2.95$ Initial R.K

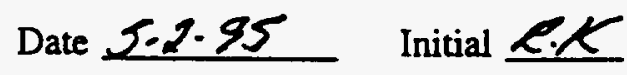

Date $5.7 .95 \quad$ Initial $\ell K$
N/A or Pressure (psig)

Pressure (psig)

Date

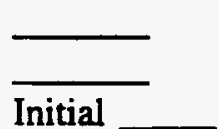

Time (min) 0 Time (min) 5 
WHC-SD-WM-TRP-246 REV. 0

Rack Storage Drum Data Sheet

Drum No. $x 355 \quad \omega t .=176.6$ ib

Post-test Assessment

Position: ___ original: ___ tipped; __ toppied

Lid on drum: $\quad \underline{x}$ Yes No

Gasket missing (cm) 20 29", rest is hand 9 compreased.

Contents expelled: —u Yes $x$ No

Seal failure: $\quad$ Yes $\quad x$ Not visible

Burn pattern:

$\frac{\text { sooty } 16^{\prime \prime} \text { mid lt }- \text { entend } 24^{\circ} \text { E right }}{\text { of stam }}$

Photo taken:

Yes $x$ No

Leak tested:

N/A Dr Pressure (psig)

Pressure (psig)

Date
Time $(\min ) \quad \underline{0}$

Time (min) 5

Interior assessment:

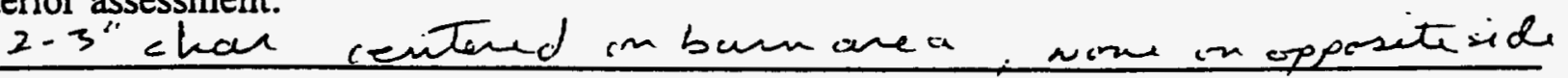
$\overline{\text { Initial }}$

isec 125

Dhoto taken:

$x$ Yes

No

Comments: 


\section{Rack Storage Drum Data Sheet}

Drum No. $\not 365$

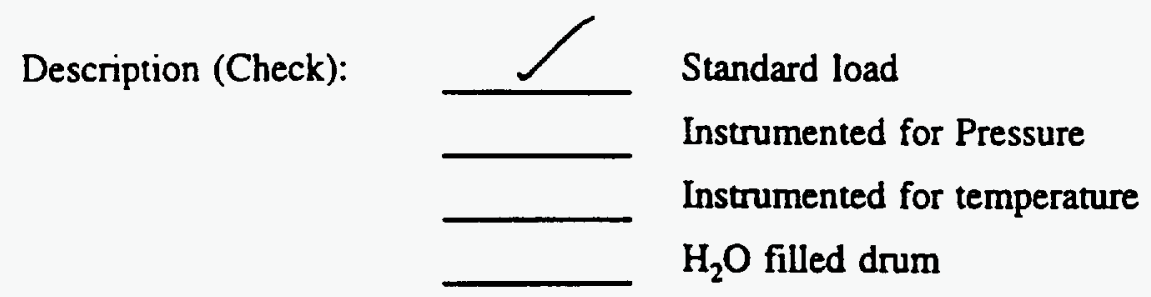

\begin{tabular}{|c|c|c|c|}
\hline Loading Starting at Bottom & Layer 1 & Layer 2 & Layer 3 \\
\hline 3 pieces of steel & & N/A & N/A \\
\hline $1 / 3$ of the brown paper & & & \\
\hline $1 / 3$ of the $10-$ mil plastic & & & \\
\hline $1 / 3$ of the bond paper & & & \\
\hline $1 / 3$ of the towels & & & \\
\hline $1 / 3$ of the bags & & & \\
\hline $1 / 3$ of the cut paper & & $\checkmark$ & \\
\hline $1 / 3$ of the fabric & Go to Layer 2 & Go to Layer 3 & \\
\hline $1 / 3$ of the rubber & & & \\
\hline &
\end{tabular}

Drum Filled:

Date $5 \cdot 2 \cdot 95$ Initial R.K

Ring bolt placed opposite drum seam Date $5-2.95$ Initial $\underline{L . K}$ and in down position

Lid sealed and torqued to $40 \mathrm{ft} / \mathrm{b}: \quad$ Date 5.2 .85 Initial $\underline{\text { P.K }}$

Leak tested:

Pre-test weight (lb)

Post-test weight (lb)
N/A or Pressure (psig)

Pressure (psig)

Date

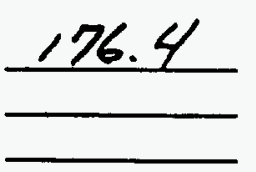

Time (min) $\quad 0$

Time (min) 5

Target weight $\sim 175.6 \mathrm{lb}$ 
WHC-SD-WM-TRP-246 REV. 0

Rack Storage Drum Data Sheet

Drum No. $\quad \times 365 \quad \omega t=176.71 \mathrm{~b}$

Post-test Assessment

Position: __ original; __ tipped; __ toppled

Lid on drum: $\quad x$ Yes No

Gasket missing (cm) 12 compressed o hand all wionend

Contents expelled: _ Yes $x$ No

Seal failure: $\quad$ Yes $X$ Not visible

Burn pattern:

wone-sooty

Photo taken:

Yes $\searrow$ No

Leak tested: Pressure (psig)

Date $\begin{array}{ll}\text { Time }(\min ) & 0 \\ \text { Time }(\min ) & \underline{5}\end{array}$ $\overline{\text { Initial }}$

Interior assessment:

meltin all mound some towall no ellan

video 126

Photo taken:

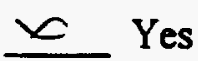

No

Comments: 


\section{Rack Storage Drum Data Sheet}

Drum No. $\times 375$

Description (Check):

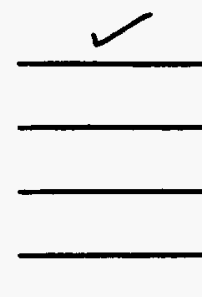

Standard load

Instrumented for Pressure

Instrumented for temperature

$\mathrm{H}_{2} \mathrm{O}$ filled drum

\begin{tabular}{|c|c|c|c|}
\hline Loading Starting at Bottom & Layer 1 & Layer 2 & Layer 3 \\
\hline 3 pieces of steel & & N/A \\
\hline $1 / 3$ of the brown paper & & & \\
\hline $1 / 3$ of the 10 -mil plastic & & & \\
\hline $1 / 3$ of the bond paper & & & \\
\hline $1 / 3$ of the towels & & & \\
\hline $1 / 3$ of the bags & & & \\
\hline $1 / 3$ of the cut paper & & Go to Layer 3 & \\
\hline $1 / 3$ of the fabric & Go to Layer 2 & - & \\
\hline $1 / 3$ of the rubber & & \\
\hline
\end{tabular}

Drum Filled:

Date $5-2.95$ Initial L.K

Ring bolt placed opposite drum seam Date S.2.95 Initial L.K and in down position

Lid sealed and torqued to $40 \mathrm{ftlb}$ : Date 5.2 .95 Initial L.K

Leak tested:

Pre-test weight (lb)

Post-test weight (lb)
N/A or Pressure (psig)

Pressure (psig)

Date

176.3
Time (min)

Time $(\min ) \quad 5$

Initial

Target weight $-175.6 \mathrm{lb}$ 


\section{Rack Storage Drum Data Sheet}

Drum No. $\times 375$.ut. $=176.916$

\section{Post-test Assessment}

Position: original; tipped; toppled

Lid on drum: $\quad \quad \quad \quad$ Yes

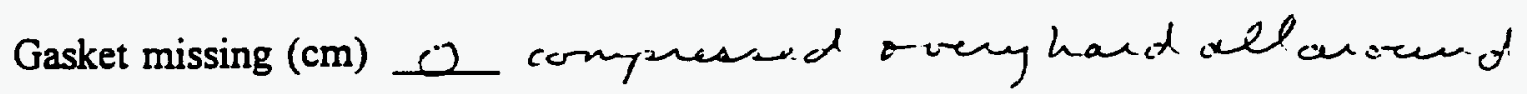

Contents expelled: __ Yes $\subseteq$ No

Seal failure: $\quad$ Yes $\quad X$ Not visible

Burn pattern:

None - sooty

Photo taken: Yes $x$ No

Leak tested: $\quad$ (N/A Pressure (psig)

Date

\begin{tabular}{lll} 
& Time (min) & 0 \\
\hline & Time $(\min )$
\end{tabular}

Interior assessment;
meltug all around - N tew spots to the wall
wo dha

voc: ics

Photo taken:

$x$ Yes $\quad$ No

Comments: 


\section{Rack Storage Drum Data Sheet}

Drum No. $\times 385$

Description (Check):

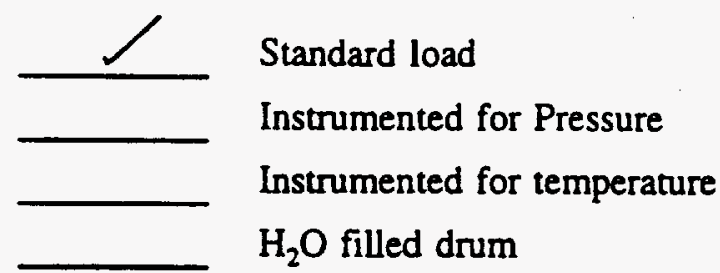

\begin{tabular}{|c|c|c|c|}
\hline Loading Starting at Bottom & Layer 1 & Layer 2 & Layer 3 \\
\hline 3 pieces of steel & & & \\
\hline $1 / 3$ of the brown paper & & & \\
\hline $1 / 3$ of the 10-mil plastic & & & \\
\hline $1 / 3$ of the bond paper & & & \\
\hline $1 / 3$ of the towels & & & \\
\hline $1 / 3$ of the bags & & & \\
\hline $1 / 3$ of the cut paper & & & \\
\hline $1 / 3$ of the fabric & Go to Layer 2 & Go to Layer 3 & \\
\hline $1 / 3$ of the rubber & & & \\
\hline
\end{tabular}

Drum Filled:

Ring bolt placed opposite drum seam and in down position

Lid sealed and torqued to $40 \mathrm{ft} / \mathrm{lb}$ :

Leak tested:

Pre-test weight (lb)

Post-test weight (lb)
Date $5.2 .95 \quad$ Initial L.K

Date 5.2 .95 Initial K.K

Date 5.2 .85 Initial L.K

N/A or Pressure (psig)

Pressure (psig)

Date

Initial

Time (min)

Time (min) 5

\section{$176.2 \quad$ Target weight $-175.6 \mathrm{lb}$}

Loss $=$ 
WHC-SD-WM-TRP-246 REV. 0

\section{Rack Storage Drum Data Sheet}

Drum No. $\quad \underline{385} \omega t .=176.6 \mathrm{ib}$.

Post-test Assessment

Position:

original: ___tipped; toppled

Lid on drum: $\quad \underline{x}$ Yes No

Gasket missing (cm) 0 compressed t hat all acound

Contents expelled: _ Yes $x$ No

Seal failure: $\quad$ Yes $\quad x$ Not visible

Burn pattern:

none - sooty

Photo taken: $\quad$ Yes $\quad$ No

Leak tested: $\quad$ NTA Or Pressure (psig) Pressure (psig)

Date

\begin{tabular}{lll} 
& Time (min) & 0 \\
\hline Initial & Time (min)
\end{tabular}

Interior assessment

melted all around sme to wall No chan

Hioto taken:

$x$ Yes No

Comments: 


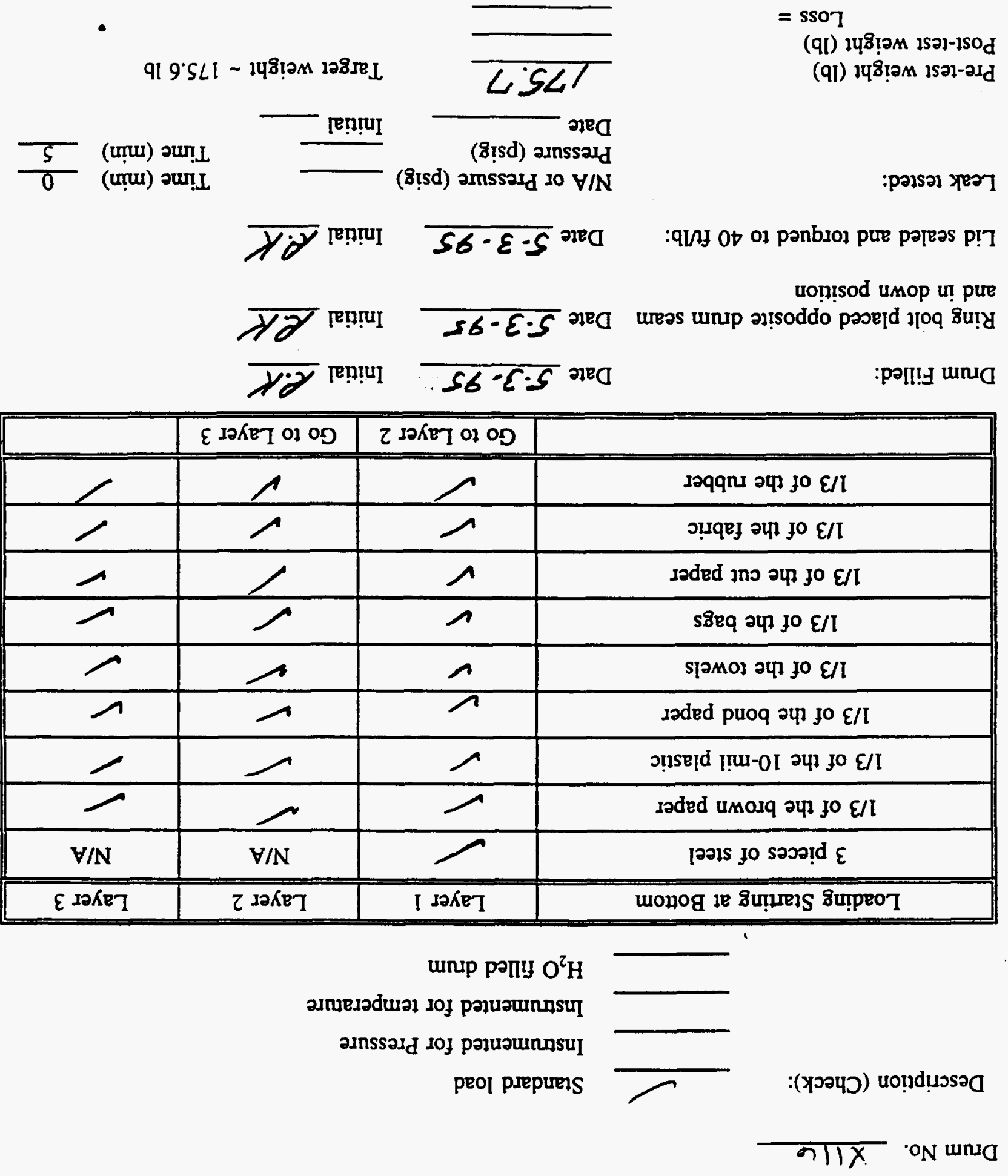




\section{Rack Storage Drum Data Sheet}

Drum'No. $\quad$ $116 \quad w T=.75 .2 \mathrm{ib}$

\section{Post-test Assessment}

Position:

original:

tipped:

toppled

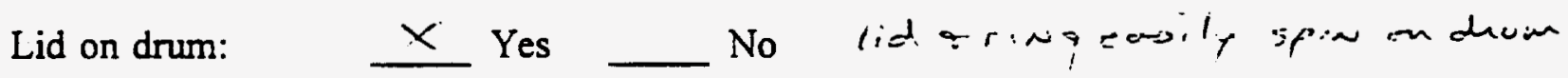

Gasket missing (cm) A1!

Contents expelled: _— Yes $\stackrel{x}{\longrightarrow}$ No

Seal failure: $\quad$ Yes $\quad$ Not visible

Burn pattern:

i" tep halfoflucm at seam

Photo taken:

Yes $\chi$ No

Leak tested:

Yes $\chi$ No

NIA Or Pressure (psig)

Pressure (psig)

Date

$\overline{\text { Initial }}$

$\begin{array}{ll}\text { Time }(\min ) & \frac{0}{5} \\ \text { Time }(\min ) & \end{array}$

Time (min)

Interior assessment:

chan contents 7 belore tep

"char seam side ind around - Top w all chandd.

$$
\text { (wote cthen dwions wl chan ins ide top is not). }
$$

T. T, eo 54

Photo taken:

$x$ Yes

No

Comments: 
WHC-SD-WM-TRP-246 REV. 0

\section{Rack Storage Drum Data Sheet}

Drum No. $\times 126$

Description (Check):

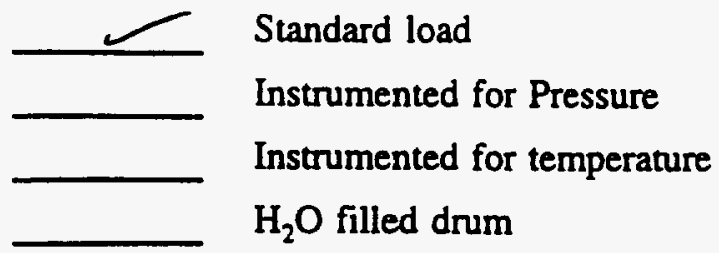

\begin{tabular}{|c|c|c|c|}
\hline Loading Starting at Bottom & Layer 1 & Layer 2 & Layer 3 \\
\hline 3 pieces of steel & & & \\
\hline $1 / 3$ of the brown paper & & & \\
\hline $1 / 3$ of the 10 -mil plastic & & & \\
\hline $1 / 3$ of the bond paper & & & \\
\hline $1 / 3$ of the towels & & & \\
\hline $1 / 3$ of the bags & & & \\
\hline $1 / 3$ of the cut paper & & Go to Layer 3 & \\
\hline $1 / 3$ of the fabric & Go to Layer 2 & G & \\
\hline $1 / 3$ of the rubber & & \\
\hline
\end{tabular}

Drum Filled:

Ring bolt placed opposite drum seam and in down position

Lid sealed and torqued to $40 \mathrm{ft} / \mathrm{b}$ :

Leak tested:

Pre-test weight (lb)

Post-test weight (lb)

Loss $=$
Date 5.3 .95 Initial P.F

Date 5.3 .95 Initial P.K

\section{Date 5.3.95 Initial P.K}

N/A or Pressure (psig)

Pressure (psig)

Date

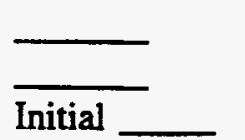

Time (min) 0

Time (min) 5

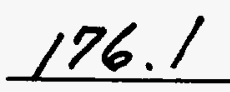

Target weight $-175.6 \mathrm{lb}$ 


\section{Rack Storage Drum Data Sheet}

Drun No. $\times 126$ ut. $175.6 \mathrm{lb}$

\section{Post-test Assessment}

Position:

original: tipped; toppled

Lid on drum: $\quad X$ Yes No ring r lid ectate irealy

Gasket missing $(\mathrm{cm})$

Contents expelled: _ Yes $\longrightarrow$ No

Seal failure:

Yes $X$ Not visible

Burn pattern:

$6 \times 9^{\prime \prime}$ at seam tip of chure.

Photo taken:

$$
\text { Yes } \quad x \text { No }
$$

Leak tested:

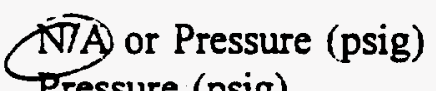

Pressure (psig)

Date \begin{tabular}{ll} 
Time (min) & 0 \\
Time (min) & \multicolumn{5}{c}{}
\end{tabular}

$\overline{\text { Initial }}$

Interior assessment:

Tos in chaned

i" chan all around

voeo 2,5

photo taken:

$x$ Yes

No

Comments: 


\section{Rack Storage Drum Data Sheet}

Drum No. $\times 136$

Description (Check):

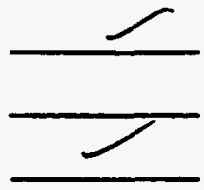

Standard load

Instrumented for Pressure

Instrumented for temperature

$\mathrm{H}_{2} \mathrm{O}$ filled drum

\begin{tabular}{|c|c|c|c|}
\hline Loading Starting at Bottom & Layer 1 & Layer 2 & Layer 3 \\
\hline 3 pieces of steel & & & \\
\hline $1 / 3$ of the brown paper & & & \\
\hline $1 / 3$ of the 10 -mil plastic & & & \\
\hline $1 / 3$ of the bond paper & & & \\
\hline $1 / 3$ of the towels & & & \\
\hline $1 / 3$ of the bags & & & \\
\hline $1 / 3$ of the cut paper & Go to Layer 2 & Go to Layer 3 & \\
\hline $1 / 3$ of the fabric & & & \\
\hline $1 / 3$ of the rubber & & & \\
\hline
\end{tabular}

Drum Filled:

Ring bolt placed opposite drum seam and in down position

Lid sealed and torqued to $40 \mathrm{ft} / \mathrm{lb}$ :

Leak tested:

Pre-test weight (lb)

Post-test weight (lb)
Date $5.3 \cdot 95$ Initial P.K

Date 5.3.85 Initial L.K
N/A or Pressure (psig)

Pressure (psig)

Date

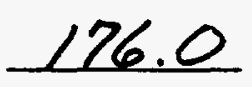

\begin{tabular}{ll} 
Time (min) & 0 \\
Time (min) & 5 \\
\hline
\end{tabular}

Initial

Target weight $\sim 175.6 \mathrm{lb}$ 


\section{Rack Storage Drum Data Sheet}

Drum No. $\quad x, 36-t=175.516$

\section{Post-test Assessment}

Position:

original:

tipped: toppled

Lid on drum: $\quad X$ Yes No

Gasket missing (cm) All

Contents expelled: _ Yes $\_$No

Seal failure: $\quad$ Yes $\quad x$ Not visible

Burn pattern:

Burn pattern: $t$ seam top of duim

Photo taken: $\quad x$ Yes No

Leak tested:

Pressure (psig)

Date

$\begin{array}{ll}\text { Time }(\min ) & 0 \\ \text { Time }(\min ) & \end{array}$

Initial

Interior assessment:

Top chared

ichan all arcund

$\because$ Dec sta

Dhotaken:

$ð$ Yes

Comments: 


\section{Rack Storage Drum Data Sheet}

Drum No. $\times 146$

Description (Check):

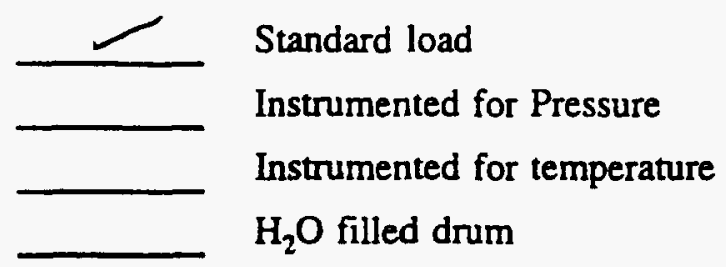

\begin{tabular}{|c|c|c|c|}
\hline Loading Starting at Bottom & Layer 1 & Layer 2 & Layer 3 \\
\hline 3 pieces of steel & & N/A & \\
\hline $1 / 3$ of the brown paper & & & \\
\hline $1 / 3$ of the 10-mil plastic & & & \\
\hline $1 / 3$ of the bond paper & & & \\
\hline $1 / 3$ of the towels & & & \\
\hline $1 / 3$ of the bags & & & \\
\hline $1 / 3$ of the cut paper & & & \\
\hline $1 / 3$ of the fabric & Go to Layer 2 & Go to Layer 3 & \\
\hline $1 / 3$ of the rubber & & & \\
\hline
\end{tabular}

Drum Filled:

Ring bolt placed opposite drum seam and in down position

Lid sealed and torqued to $40 \mathrm{ft} / \mathrm{lb}$ :

Leak tested:

Pre-test weight (lb)

Post-test weight (lb)
Date 5-3.95 Initial L.K

Date 5.3-95 Initial L.K

Date $5.3-95 \quad$ Initial L.K

N/A or Pressure (psig)

Pressure (psig)

Date

\begin{tabular}{lll} 
& Time (min) & 0 \\
\hline & Time (min)
\end{tabular}

Target weight $\sim 175.6 \mathrm{lb}$

175.9

Initial 
WHC-SD-WM-TRP-246 REV. 0

Rack Storage Drum Data Sheet

Drum No. $\times 146$ wt. $=175.3 \mathrm{lb}$

$\underline{\text { Post-test Assessment }}$

Position: __ original; ___ tipped; __ toppled

Lid on drum: $\quad X$ Yes No

Gasket missing (cm) All

Contents expeiled: _ Yes $x$ No

Seal failure: $\quad$ Yes $\quad x^{\prime}$ Not visible

Bum pattern:

$43 \times 6 "$ atsean top of duin 7 anound lid seam side

Photo taken:

Yes $\underline{x}$ No

Leak tested: $\quad$ N/A or Pressure (psig)

Pressure (psig)

Date

\begin{tabular}{lll}
- & Time $(\min )$ & 0 \\
\hline
\end{tabular}$\quad$ Time $(\min ) \quad \underline{5}$

Interior assessment:

top is chand $t \sim 1$ around peimeter

Biroc 47

$\underline{x}$ Yes

Comments: 


\section{Rack Storage Drum Data Sheet}

Drum No. $\times 154$

Description (Check):

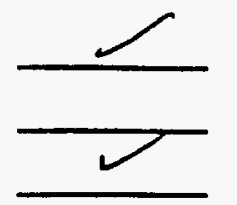

Standard load

Instrumented for Pressure

Instrumented for temperature

$\mathrm{H}_{2} \mathrm{O}$ filled drum

\begin{tabular}{|c|c|c|c|}
\hline Loading Starting at Bottom & Layer 1 & Layer 2 & Layer 3 \\
\hline 3 pieces of steel & N/A \\
\hline $1 / 3$ of the brown paper & & & \\
\hline $1 / 3$ of the 10 -mil plastic & & & \\
\hline $1 / 3$ of the bond paper & & & \\
\hline $1 / 3$ of the towels & & & \\
\hline $1 / 3$ of the bags & & & \\
\hline $1 / 3$ of the cut paper & Go to Layer 2 & Go to Layer 3 & \\
\hline $1 / 3$ of the fabric & & & \\
\hline $1 / 3$ of the rubber & & & \\
\hline
\end{tabular}

Drum Filled:

Ring bolt placed opposite drum seam and in down position

Lid sealed and torqued to $40 \mathrm{ft} / \mathrm{lb}$ :

Leak tested:

Pre-test weight (lb)

Post-test weight (lb)
Date $5 \cdot 3 \cdot 95 \quad$ Initial

Date $5-3-95$

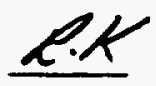

Initial R.R

Date 5-3.95 Initial R.K

N/A or Pressure (psig)

Pressure (psig)

Date

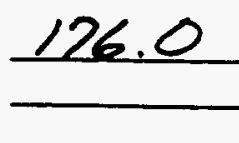

Time (min) 0

Time (min) 5

Target weight $-175.6 \mathrm{lb}$ 


\section{Rack Storage Drum Data Sheet}

Drum No. $\times 156 \quad 175.7$ ih.

\section{Post-test Assessment}

Position: original: tipped: toppled

Lid on drum: $\quad \underset{\text { Yes }}{\longrightarrow}$ No

Gasket missing (cm)

Contents expelled: _ Yes $\longrightarrow$ No

Seal failure: $\quad$ Yes $\quad \chi$ Not visible

Burn pattern:
Nove - hand to tell
$300+2,1$
The way
actoren of

Photo taken:

Yes $X$ No

Leak tested:

N/A Of Pressure (psig)

Pressure (psig)

Date
Time (min) $\frac{0}{5}$
Time (min)

Initial

Interior assessment:

top chaned

$1-2 \therefore$ chan it zear

inol on opposite side $\sim 5$ pionticielted

$\tau<$ inam

videc $>5$

Photo taken:

$x$ Yes

No

Comments: 
WHC-SD-WM-TRP-246 REV. 0

\section{Rack Storage Drum Data Sheet}

Drum No. $\times 166$

Description (Check):

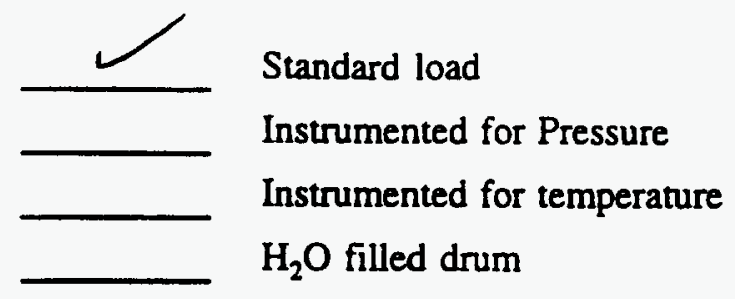

\begin{tabular}{|c|c|c|c|}
\hline Loading Starting at Bottom & Layer 1 & Layer 2 & Layer 3 \\
\hline 3 pieces of steel & & & \\
\hline $1 / 3$ of the brown paper & & & \\
\hline $1 / 3$ of the $10-$ mil plastic & & & \\
\hline $1 / 3$ of the bond paper & & & \\
\hline $1 / 3$ of the towels & & & \\
\hline $1 / 3$ of the bags & & & \\
\hline $1 / 3$ of the cut paper & & & \\
\hline $1 / 3$ of the fabric & & & \\
\hline $1 / 3$ of the rubber & Go to Layer 2 & Go to Layer 3 & \\
\hline
\end{tabular}

Drum Filled:

Date 5.3 .95 Initial e.t.

Ring bolt placed opposite drum seam Date 5.3 .95 Initial A.X and in down position

Lid sealed and torqued to $40 \mathrm{ft} / \mathrm{b}$ :

Date $5.3 \cdot 95$ Initial P.R

Leak tested:

Pre-test weight (lb)

Post-test weight (lb)
N/A or Pressure (psig)

Pressure (psig)

Date

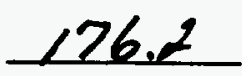

Time (min)

Time $(\min ) 5$

Loss = 


\section{Rack Storage Drum Data Sheet}

Drum No. $\quad x \in 6 \quad$ wet, $175 \leq 16$

\section{$\underline{\text { Post-test Assessment }}$}

Position: original: tipped; toppled

Lid on drum: $\quad X$ Yes No idd ring tum tireely

Gasket missing (cm) A+1

Contents expelled: __ Yes $x$ No

Seal failure: $\quad$ Yes $\quad x$ Not visible

Burn pattern:

hand to Tell-mainly sooty - possible bumat
top wear lid

Photo taken:

$$
\text { Yes } \underline{x} \text { No }
$$

Leak tested:

NTAOr Pressure (psig)

Pressure (psig)

Date

\begin{tabular}{lll} 
& Time $(\min )$ & 0 \\
\hline Initial & Time $(\min )$ & $\underline{5}$
\end{tabular}

Interior assessment:

top chanced

-2-3" char weloround roli wean seam

Piboec taken:

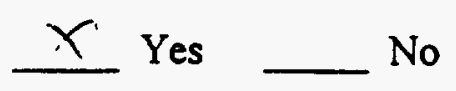

Comments: 


\section{Rack Storage Drum Data Sheet}

Drum No. $\times 176$

Description (Check):

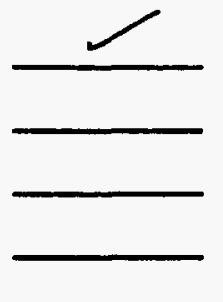

Standard load

Instrumented for Pressure

Instrumented for temperature

$\mathrm{H}_{2} \mathrm{O}$ filled drum

\begin{tabular}{|c|c|c|c|}
\hline Loading Starting at Bottom & Layer 1 & Layer 2 & Layer 3 \\
\hline 3 pieces of steel & & N/A \\
\hline $1 / 3$ of the brown paper & & & \\
\hline $1 / 3$ of the $10-$ mil plastic & & & \\
\hline $1 / 3$ of the bond paper & & & \\
\hline $1 / 3$ of the towels & & & \\
\hline $1 / 3$ of the bags & & & \\
\hline $1 / 3$ of the cut paper & & & \\
\hline $1 / 3$ of the fabric & & & \\
\hline $1 / 3$ of the rubber & Go to Layer 2 & Go to Layer 3 & \\
\hline
\end{tabular}

Drum Filled:

Date 5.3 .95 Initial $R \cdot K$

Ring bolt placed opposite drum seam Date $5 \cdot 3-95$ Initial and in down position

Lid sealed and torqued to $40 \mathrm{ft} / \mathrm{b}$ : $\quad$ Date 5.3 .95 Initial

Leak tested:

Pre-test weight (lb)

Post-test weight (lb)
N/A or Pressure (psig)

Pressure (psig)

Date

175.3
Time (min)

Time $(\mathrm{min})$

Initial

Target weight $\sim 175.6 \mathrm{lb}$ 


\section{Rack Storage Drum Data Sheet}

Drum No. $\quad x_{17}-175.51 b$.

Post-test Assessment

Position: __ originai: ___ tipped; __ toppled

Lid on drum: $\quad \quad \quad X$ Yes $\quad$ No

Gasket missing (cm) All

Contents expelled: _ Yes $\chi$ No

Seal failure: $\quad$ Yes $\quad x$ Not visible

Burn pattern:

NoNe soot all around -gasket isaking at

Photo taken:

Leak tested:

$$
\begin{aligned}
& \text { Yes } \longrightarrow \text { No } \\
& \begin{array}{l}
\text { Pressure (psig) } \\
\text { Date }
\end{array}
\end{aligned}
$$

Interior assessment:

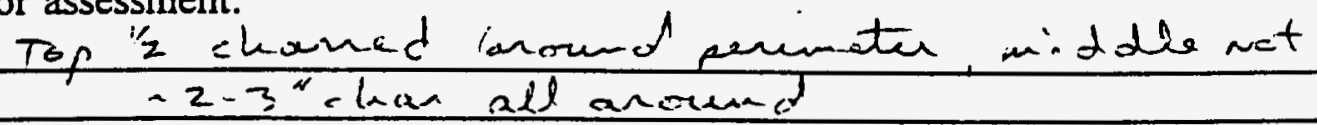

ineo 79
photo taken:

$X$ Yes

Comments: 


\section{Rack Storage Drum Data Sheet}

Drum No. $\times 1 \$ 6$

Description (Check):

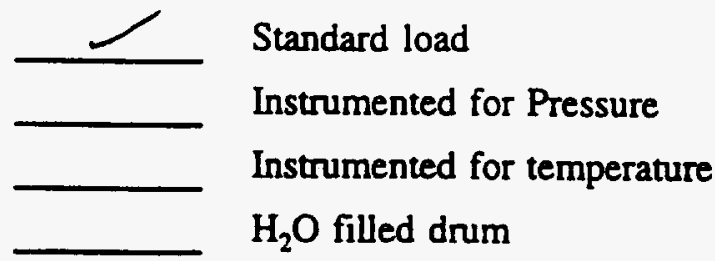

\begin{tabular}{|c|c|c|c|}
\hline Loading Starting at Bottom & Layer 1 & Layer 2 & Layer 3 \\
\hline 3 pieces of steel & & N/A \\
\hline $1 / 3$ of the brown paper & & & \\
\hline $1 / 3$ of the 10 -mil plastic & & & \\
\hline $1 / 3$ of the bond paper & & & \\
\hline $1 / 3$ of the towels & & & \\
\hline $1 / 3$ of the bags & & & \\
\hline $1 / 3$ of the cut paper & & Go to Layer 3 & \\
\hline $1 / 3$ of the fabric & Go to Layer 2 & G & \\
\hline $1 / 3$ of the rubber & & & \\
\hline
\end{tabular}

Drum Filled:

Date 5.3.95 Initial L.K

Ring bolt placed opposite drum seam Date 5-3-95 Initial L.K and in down position

Lid sealed and torqued to $40 \mathrm{ft} / \mathrm{b}: \quad$ Date 5.3 .95 Initial L.K

Leak tested:

Pre-test weight (lb)

Post-test weight (lb)

Loss =
N/A or Pressure (psig)

Pressure (psig)

Date
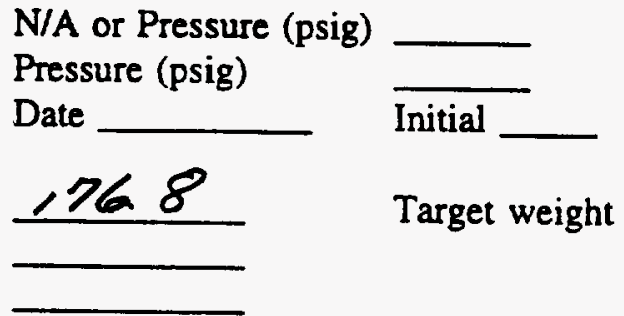

Time (min) 0

Time (min)

Target weight $-175.6 \mathrm{lb}$ 


\section{Rack Storage Drum Data Sheet}

Drum No. $\times 156 \quad 176.6$ ib.

Post-test Assessment

Position:

original; tipped; toppled

Lid on drum: $\quad \longrightarrow$ Yes No

Gasket missing (cm) All

Contents expelled: __ Yes $x$ No

Seal failure: $\quad$ Yes $\quad X$ Not visible

Burn pattern:

none soit allaround
gas het leakiry at seam

Photo taken: $\therefore$ Yes $\rightleftharpoons$ No

Leak tested:

NIAEr Pressure (psig)
Pressure (psig)
Date

\begin{tabular}{lll} 
& Time $(\min )$ & 0 \\
\hline & Time $(\min )$
\end{tabular}

Interior assessment:

in not chaned exiept at edge

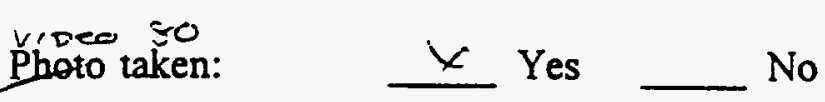

Comments: 


\section{Rack Storage Drum Data Sheet}

Drum No. $\times 216$

Description (Check):

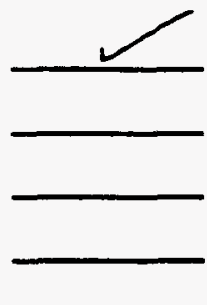

Standard load

Instrumented for Pressure

Instrumented for temperature

$\mathrm{H}_{2} \mathrm{O}$ filled drum

\begin{tabular}{|c|c|c|c|}
\hline Loading Starting at Bottom & Layer 1 & Layer 2 & Layer 3 \\
\hline 3 pieces of steel & & N/A & N/A \\
\hline \multicolumn{4}{|l|}{$1 / 3$ of the brown paper } \\
\hline \multicolumn{4}{|l|}{$1 / 3$ of the 10 -mil plastic } \\
\hline \multicolumn{4}{|l|}{$1 / 3$ of the bond paper } \\
\hline \multicolumn{4}{|l|}{$1 / 3$ of the towels } \\
\hline \multicolumn{4}{|l|}{$1 / 3$ of the bags } \\
\hline \multicolumn{4}{|l|}{$1 / 3$ of the cut paper } \\
\hline \multicolumn{4}{|l|}{$1 / 3$ of the fabric } \\
\hline \multicolumn{4}{|l|}{$1 / 3$ of the rubber } \\
\hline & Go to Layer 2 & Go to Layer 3 & \\
\hline
\end{tabular}

Drum Filled:

Date $5.3 .95 \quad$ Initial L.K

Ring bolt placed opposite drum seam Date 5.3.95 Initial L.K and in down position

Lid sealed and torqued to $40 \mathrm{ft} / \mathrm{lb}: \quad$ Date $\underline{5.3 .95}$ Initial L.K

Leak tested:

Pre-test weight (lb)

Post-test weight (lb)
N/A or Pressure (psig)

Pressure (psig)

Date

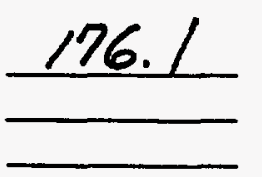

Time (min)

Time (min) 5 


\section{Rack Storage Drum Data Sheet}

Drum No. $\quad \times 216$ wt. $=103.316$

Post-test Assessment

Position: original: tipped: toppled

Lid on drum: $\quad X$ Yes 느 No $\begin{gathered}\sim 1 / 2 \text { off } \\ \sim 18^{\prime \prime} \text { flid in ring }\end{gathered}$

Gasket missing (cm)

Contents expelled: _ Yes

Seal failure: $\quad X$ Yes Not visible

Burn pattern:

allaround

Photo taken: Yes $\searrow$ No

Leak tested:

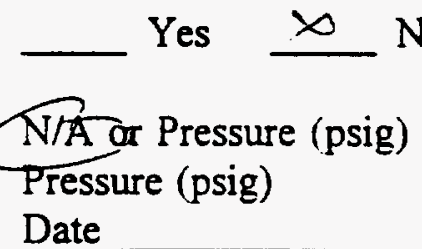

Date $\overline{\text { Initial }}$

Time $(\min ) \quad \underline{0}$ Time (min) 5

Interior assessment:

-6 " chared a critents

ii:

Photo taken:

$x \quad$ Yes No

Comments: 


\section{Rack Storage Drum Data Sheet}

Drum No. $\times 226(P)$

Description (Check):

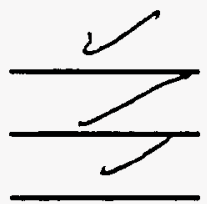

Standard load

Instrumented for Pressure

Instrumented for temperature

$\mathrm{H}_{2} \mathrm{O}$ filled drum

\begin{tabular}{|c|c|c|c|}
\hline Loading Starting at Bottom & Layer 1 & Layer 2 & Layer 3 \\
\hline 3 pieces of steel & & & \\
\hline $1 / 3$ of the brown paper & & & \\
\hline $1 / 3$ of the 10 -mil plastic & & & \\
\hline $1 / 3$ of the bond paper & & & \\
\hline $1 / 3$ of the towels & & & \\
\hline $1 / 3$ of the bags & & Go to Layer 3 & \\
\hline $1 / 3$ of the cut paper & Go to Layer 2 & Go & \\
\hline $1 / 3$ of the fabric & & \\
\hline $1 / 3$ of the rubber & & \\
\hline
\end{tabular}

Drum Filled:

Ring bolt placed opposite drum seam and in down position

Lid sealed and torqued to $40 \mathrm{ft} / \mathrm{lb}$ :

Leak tested:

Pre-test weight (lb)

Post-test weight (lb)
Date $5-2.95 \quad$ Initial R.K

Date $5-2.95$ Initial R.K

Date 5.2.95 Initial R.K

N/A or Pressure (psig)

Pressure (psig)

Date

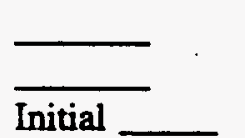

Time (min) $\frac{0}{5}$

Time (min)

Target weight $\sim 175.6 \mathrm{lb}$ 


\section{Rack Storage Drum Data Sheet}

Drum No. $\quad \underline{x_{22}}(p) \quad \omega t .=166.6$

\section{Post-test Assessment}

Position: __ original: __ tipped: __ toppled

Lid on drum: $\quad x$ Yes No $"{ }^{\prime \prime} \times 6$ " Gap atbolt

Gasket missing (cm) A-1I

Contents expelled: __ Yes $\underline{x}$ No

Seal failure: $\quad \quad \quad X$ Yes $\quad$ Not visible

Burn pattern:

allaround

Photo taken:

$$
\text { Yes } \underset{x}{ } \text { No }
$$

Leak tested:

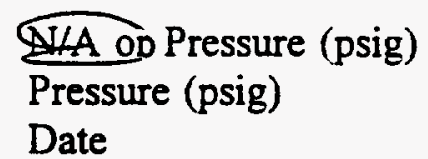

$\longrightarrow \quad$\begin{tabular}{lll}
- & Time (min) & 0 \\
\hline
\end{tabular}$\quad$ Time (min)

Interior assessment:
-16 " fram top

$$
\text { of hum te tep }
$$
of charred coutents

Comments: 
WHC-SD-WM-TRP-246 REV. 0

\section{Rack Storage Drum Data Sheet}

Drum No. $\times 236$

Description (Check):

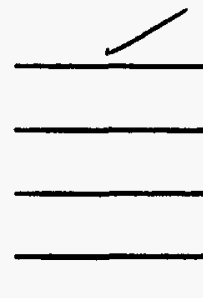

Standard load

Instrumented for Pressure

Instrumented for temperature

$\mathrm{H}_{2} \mathrm{O}$ filled drum

\begin{tabular}{|c|c|c|c|}
\hline Loading Starting at Bottom & Layer 1 & Layer 2 & Layer 3 \\
\hline 3 pieces of steel & & N/A & N/A \\
\hline \multicolumn{4}{|l|}{$1 / 3$ of the brown paper } \\
\hline \multicolumn{4}{|l|}{$1 / 3$ of the $10-$ mil plastic } \\
\hline \multicolumn{4}{|l|}{$1 / 3$ of the bond paper } \\
\hline $1 / 3$ of the towels & $\alpha$ & & \\
\hline \multicolumn{4}{|l|}{$1 / 3$ of the bags } \\
\hline $1 / 3$ of the cut paper & $r$ & & \\
\hline \multicolumn{4}{|l|}{$1 / 3$ of the fabric } \\
\hline \multicolumn{4}{|l|}{$1 / 3$ of the rubber } \\
\hline & Go to Layer 2 & Go to Layer 3 & \\
\hline
\end{tabular}

Drum Filled:

Ring bolt placed opposite drum seam and in down position

Lid sealed and torqued to $40 \mathrm{ft} / \mathrm{b}$ :

Leak tested:

Pre-test weight (lb)

Post-test weight (lb)
Date 5.3 .95 Initial R.R

Date 5.3 .85 Initial R.K
Date 5.3 .95 Initial R.K

N/A or Pressure (psig)

Pressure (psig)

Date

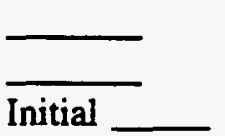

Time (min) $\frac{0}{5}$

Time (min) 5

\section{Loss $=$}


WHC-SD-WM-TRP-246 REV. 0

\section{Rack Storage Drum Data Sheet}

Drum No. $\quad x 236 \quad \omega t=195.0$

Post-test Assessment

Position:

original: tipped: toppled

Lid on drum:

$$
X \text { Yes No } 1{ }^{1 / 2}{ }^{\prime \prime} \times 22^{\prime \prime} \text { gap }
$$

Gasket missing (cm)

Contents expelled: __ Yes $\times$ No

Seal failure: $\quad \underline{X}$ Yes $\quad$ Not visible

Burn pattern:

Difficut to Asseas

Photo taken:

Yes $\quad$ No

Leak tested:

N/A or Pressure (psig) Pressure (psig)

Date

\begin{tabular}{lll} 
& Time (min) & 0 \\
\hline Initial & Time (min)
\end{tabular}

Interior assessment:

$20 "$ from tep to emaining contents - allchaned

video

Ptoto taken:

$X$ Yes

Comments: 


\section{Rack Storage Drum Data Sheet}

Drum No. $\times 246$

Description (Check):

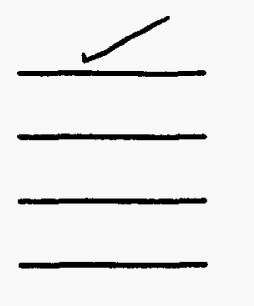

Standard load

Instrumented for Pressure

Instrumented for temperature

$\mathrm{H}_{2} \mathrm{O}$ filled drum

\begin{tabular}{|c|c|c|c|}
\hline Loading Starting at Bottom & Layer 1 & Layer 2 & Layer 3 \\
\hline 3 pieces of steel & & N/A & N/A \\
\hline \multicolumn{4}{|l|}{$1 / 3$ of the brown paper } \\
\hline $1 / 3$ of the 10 -mil plastic & & & \\
\hline \multicolumn{4}{|l|}{$1 / 3$ of the bond paper } \\
\hline \multicolumn{4}{|l|}{$1 / 3$ of the towels } \\
\hline \multicolumn{4}{|l|}{$1 / 3$ of the bags } \\
\hline \multicolumn{4}{|l|}{$1 / 3$ of the cut paper } \\
\hline \multicolumn{4}{|l|}{$1 / 3$ of the fabric } \\
\hline \multicolumn{4}{|l|}{$1 / 3$ of the rubber } \\
\hline & Go to Layer 2 & Go to Layer 3 & \\
\hline
\end{tabular}

Drum Filled:

Ring bolt placed opposite drum seam and in down position

Lid sealed and torqued to $40 \mathrm{ft} / \mathrm{lb}$ :

Leak tested:

Pre-test weight (lb)

Post-test weight (lb)
Date 5.3 .95 Initial R.K

Date 5.3 .95 Initial Let

Date 5.3 .95 Initial Le

N/A or Pressure (psig)

Pressure (psig)

Date

\begin{tabular}{ll} 
Time $(\min )$ & 0 \\
\hline & Time $(\min )$
\end{tabular}

Target weight $\sim 175.6 \mathrm{lb}$ 


\section{Rack Storage Drum Data Sheet}

Drum No. $\lcm{246 \quad \omega t}=142.216$.

Post-test Assessment

Position: original; tipped; toppled

Lid on drum: $\quad$ Yes $X$ No

Gasket missing (cm)

Contents expelled: __ Yes _ No

Seal failure: $\quad \square$ Yes $\quad$ Not visible

Burn pattern:

$$
\sim 32^{\circ} \text { at ind it. }
$$

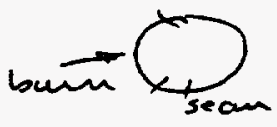

Photo taken: Yes _ No

Leak tested:

N/A or Pressure (psig)

Pressure (psig)

Date

\begin{tabular}{lll} 
& Time (min) & 0 \\
\hline Initial & Time (min)
\end{tabular}

Interior assessment;
3 " of chened

vedec 32

Photo taken:

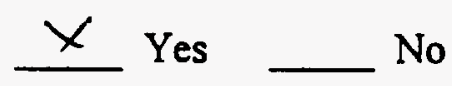

Comments: 


\section{Rack Storage Drum Data Sheet}

Drum No. $\times 256(P)$

Description (Check):

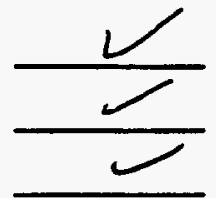

Standard load

Instrumented for Pressure

Instrumented for temperature

$\mathrm{H}_{2} \mathrm{O}$ filled drum

\begin{tabular}{|c|c|c|c|}
\hline Loading Starting at Bottom & Layer 1 & Layer 2 & Layer 3 \\
\hline 3 pieces of steel & & & \\
\hline $1 / 3$ of the brown paper & & & \\
\hline $1 / 3$ of the 10 -mil plastic & & & \\
\hline $1 / 3$ of the bond paper & & & \\
\hline $1 / 3$ of the towels & & & \\
\hline $1 / 3$ of the bags & & & \\
\hline $1 / 3$ of the cut paper & Go to Layer 2 & Go to Layer 3 & \\
\hline $1 / 3$ of the fabric & & \\
\hline $1 / 3$ of the rubber & & & \\
\hline
\end{tabular}

Drum Filled:

Ring bolt placed opposite drum seam and in down position

Lid sealed and torqued to $40 \mathrm{ft} / \mathrm{lb}$ :

Leak tested:

Pre-test weight (lb)

Post-test weight (lb)
Date $5.2-95$ Initial R.K

Date 5.2 .95 Initial P.K

Date $5 \cdot 2-95 \quad$ Initial LK

N/A or Pressure (psig)

Pressure (psig)

Date

$\overline{\text { Initial }}$

Time (min)

Time (min) 5

\subsection{Target weight $-175.6 \mathrm{lb}$}


WHC-SD-WM-TRP-246 REV. 0

\section{Rack Storage Drum Data Sheet}

Drum No. $\times 256 \quad 176.31 \mathrm{~b}$

Post-test Assessment

Position: __ original: __ tipped; __ toppled

Lid on drum: $\quad X$ Yes No

Gasket missing (cm)

Contents expelled: _ Yes $x$ No

Seal failure: $\quad$ Yes $\triangle$ Not visible

Burn pattern:
39 at inid lit.

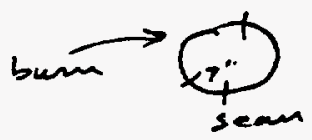

Photo taken: $\quad$ Yes $x$ No

Leak tested: $\quad$ N/AOr Pressure (psig)

Date

\begin{tabular}{lll} 
& Time (min) & 0 \\
\hline & Time (min)
\end{tabular}

Interior assessment:

top chaved, 4-5" chas all around

Uivec 99

photo taken:

$\underline{x^{\prime}}$ Yes

No

Comments: 


\section{Rack Storage Drum Data Sheet}

Drum No. $\times 264$

Description (Check):

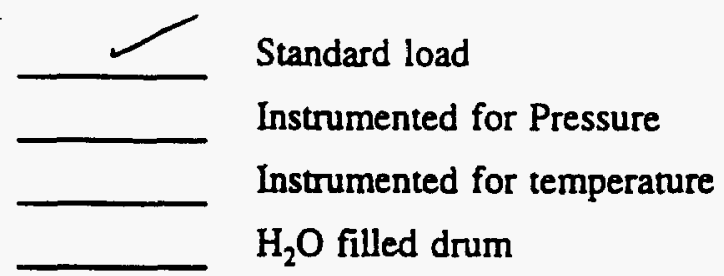

\begin{tabular}{|c|c|c|c|}
\hline Loading Starting at Bottom & Layer 1 & Layer 2 & Layer 3 \\
\hline 3 pieces of steel & & N/A & N/A \\
\hline $1 / 3$ of the brown paper & & & \\
\hline $1 / 3$ of the 10 -mil plastic & & & \\
\hline $1 / 3$ of the bond paper & & & \\
\hline $1 / 3$ of the towels & & & \\
\hline $1 / 3$ of the bags & & & \\
\hline $1 / 3$ of the cut paper & & & \\
\hline $1 / 3$ of the fabric & & $\checkmark$ & \\
\hline $1 / 3$ of the rubber & & & \\
\hline & Go to Layer 2 & Go to Layer 3 & \\
\hline
\end{tabular}

Drum Filled:

Ring bolt placed opposite drum seam and in down position

Lid sealed and torqued to $40 \mathrm{ft} / \mathrm{lb}$ :

Leak tested:

Pre-test weight (lb)

Post-test weight (lb)
Date 5.3 .95 Initial LK

Date 5.3 .95 Initial R.K

Date $5.3 .95 \quad$ Initial $\underline{R}$.

N/A or Pressure (psig)

Pressure (psig)

Date

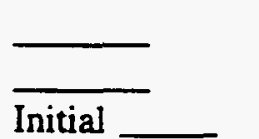

Time (min)

Time (min)

Target weight $-175.6 \mathrm{lb}$ 
WHC-SD-WM-TRP-246 REV. 0

\section{Rack Storage Drum Data Sheet}

Drum No. $\times 266 \quad$ wt. $=175.2$ ib.

Post-test Assessment

Position:

original: tipped; toppled

Lid on drum: $\quad x$ Yes No

Gasket missing (cm)

Contents expelled: $\quad$ Yes $\_$No

Seal failure: $\quad$ Y Yes $\quad x$ Not visible

Burn pattern:

top $2 / 3 \quad-26^{\prime \prime}$ wide centered on bott

Photo taken:

$<$ Yes

Leak tested:

WIA br Pressure (psig)

Pressure (psig)

Date

\begin{tabular}{lll}
- & Time $(\min )$ & 0 \\
\hline & Time $(\min )$
\end{tabular}

Interior assessment:

top chaned

i-6" allaround chomed

i'oec:ic4

Photo taken:

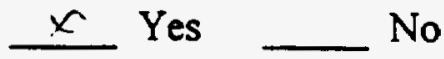

Comments: 


\section{Rack Storage Drum Data Sheet}

Drum No. $\times 276$

Description (Check):

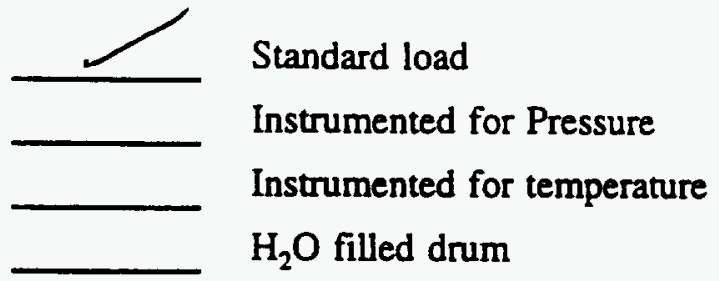

\begin{tabular}{|c|c|c|c|}
\hline Loading Starting at Bottom & Layer 1 & Layer 2 & Layer 3 \\
\hline 3 pieces of steel & & N/A & N/A \\
\hline \multicolumn{4}{|l|}{$1 / 3$ of the brown paper } \\
\hline \multicolumn{4}{|l|}{$1 / 3$ of the 10 -mil plastic } \\
\hline \multicolumn{4}{|l|}{$1 / 3$ of the bond paper } \\
\hline \multicolumn{4}{|l|}{$1 / 3$ of the towels } \\
\hline \multicolumn{4}{|l|}{$1 / 3$ of the bags } \\
\hline \multicolumn{4}{|l|}{$1 / 3$ of the cut paper } \\
\hline \multicolumn{4}{|l|}{$1 / 3$ of the fabric } \\
\hline \multicolumn{4}{|l|}{$1 / 3$ of the rubber } \\
\hline & Go to Layer 2 & Go to Layer 3 & \\
\hline
\end{tabular}

Drum Filled:

Date 5.3 .95 Initial R.K

Ring bolt placed opposite drum seam Date 5.3-95 Initial R.K and in down position

Lid sealed and torqued to $40 \mathrm{ft} / \mathrm{b}: \quad$ Date $5.3-95$ Initial R.K

Leak tested:

Pre-test weight (lb)

Post-test weight (lb)
N/A or Pressure (psig)

Pressure (psig)

Date

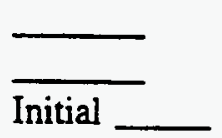

Time (min) $\frac{0}{5}$

Time (min) 5
Target weight $\sim 175.6 \mathrm{lb}$ 


\section{Rack Storage Drum Data Sheet}

Drum No. $\times 276 \quad 775.4$ ib

$\underline{\text { Post-test Assessment }}$

Position: original: tipped; toppled

Lid on drum: $\quad \longrightarrow$ Yes No

Gasket missing (cm) All

Contents expelled: _ Yes $X$ No

Seal failure: $\quad$ Yes $\quad x$ Not visible

Burn pattern: top half $\sim 16$ "wide below bitt

Photo taken: $\quad \chi$ Yes No

Leak tested: $\quad$ N $\quad$ A Pressure (psig)

Date

\begin{tabular}{lll}
- & Time $(\min )$ & 0 \\
\hline & Time $(\min )$
\end{tabular}

Interior assessment:

top chand. 4-5" of chan allaround

$\because 720 \times-101$

Photo taken:

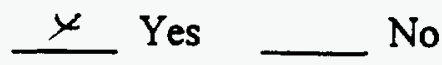

Comments: 


\section{Rack Storage Drum Data Sheet}

Drum No. $\times 286$

Description (Check):

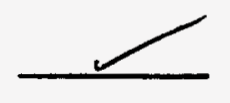

Standard load

Instrumented for Pressure

Instrumented for temperature

$\mathrm{H}_{2} \mathrm{O}$ filled drum

\begin{tabular}{|c|c|c|c|}
\hline Loading Starting at Bottom & Layer 1 & Layer 2 & Layer 3 \\
\hline 3 pieces of steel & & & \\
\hline $1 / 3$ of the brown paper & & & \\
\hline $1 / 3$ of the 10-mil plastic & & & \\
\hline $1 / 3$ of the bond paper & & & \\
\hline $1 / 3$ of the towels & & & \\
\hline $1 / 3$ of the bags & & & \\
\hline $1 / 3$ of the cut paper & Go to Layer 2 & Go to Layer 3 & \\
\hline $1 / 3$ of the fabric & & & \\
\hline $1 / 3$ of the rubber & & & \\
\hline
\end{tabular}

Drum Filled:

Ring bolt placed opposite drum seam and in down position

Lid sealed and torqued to $40 \mathrm{ft} / \mathrm{lb}$ :

Leak tested:

Pre-test weight (lb)

Post-test weight (lb)
Date 5-3-95 Initial R.K

Date 5.3.95 Initial R.K

Date 5.3 .95 Initial $\underline{R K}$
N/A or Pressure (psig)

Pressure (psig)

Date

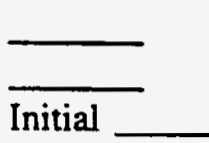

Time (min) $\frac{0}{5}$

Time (min) 


\section{Rack Storage Drum Data Sheet}

Drum No. $\times 236-175.016$.

Post-test Assessment

Position: __ original; ___ tipped: __ toppled

Lid on drum: $\quad X$ Yes No bolto bid rotite treetin

Gasket missing (cm) Ail

Contents expelled: _ـ Yes $X$ No

Seal failure: $\quad$ Yes $\quad X^{\prime}$ Not visible

Burn pattern:

only below rim - inall 4 " $\times 4 "$ spot belou bott

Photo taken:

Yes $x$ No

Leak tested:
NA or Pressure (psig) Pressure (psig)
Date

$\begin{array}{ll}\text { Time }(\min ) & 0 \\ \text { Time }(\min ) & \end{array}$

Initial

Interior assessment:

Top charre o

$2-4 "$ of chan all nownd.

pisec 103

photo taken:

$\varnothing$ Yes

Comments: 


\section{Rack Storage Drum Data Sheet}

Drum No. X316

Description (Check):

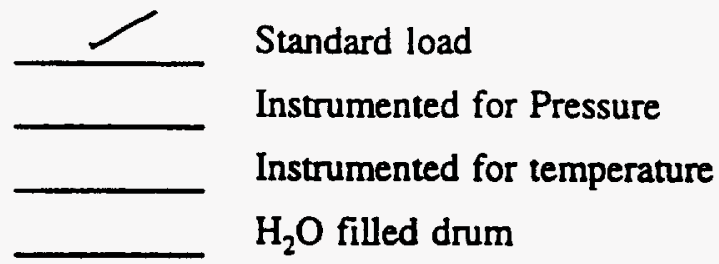

\begin{tabular}{|c|c|c|c|}
\hline Loading Starting at Bottom & Layer 1 & Layer 2 & Layer 3 \\
\hline 3 pieces of steel & & & \\
\hline $1 / 3$ of the brown paper & & & \\
\hline $1 / 3$ of the 10 -mil plastic & & & \\
\hline $1 / 3$ of the bond paper & & & \\
\hline $1 / 3$ of the towels & & & \\
\hline $1 / 3$ of the bags & & Go to Layer 3 & \\
\hline $1 / 3$ of the cut paper & & & \\
\hline $1 / 3$ of the fabric & & & \\
\hline $1 / 3$ of the rubber & Go to Layer 2 & Go & \\
\hline
\end{tabular}

Drum Filled:

Date 5.3 .95 Initial $2 K$

Ring bolt placed opposite drum seam

Date 5.3 .95 Initial $2 K$ and in down position

Lid sealed and torqued to $40 \mathrm{ft} / \mathrm{lb}$ :

Date 5.3 .95 Initial R.K

Leak tested:

N/A or Pressure (psig)

Pressure (psig)

Date

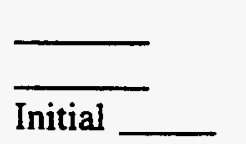

Time (min)

Time (min)

Pre-test weight (lb)

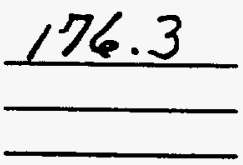

Target weight $-175.6 \mathrm{lb}$

Post-test weight (lb)

$$
\text { Loss = }
$$


WHC-SD-WM-TRP-246 REV. 0

Rack Storage Drum Data Sheet

Drum No. $\quad \begin{array}{r}316 \\ x\end{array}$

Post-test Assessment

Position: ___ original; ___ tipped; ___ toppled

Lid on drum: $\quad$ Yes $\times$ No

Gasket missing (cm) AtI

Contents expelled: __ Yes _ _ No

Seal failure: $\quad \quad X$ Yes _ Not visible

Burn pattern:
all around

Photo taken: $\quad$ Yes $\$ No

Leak tested: $\quad$ NFA or Pressure (psig)

Pressure (psig)

Date

\begin{tabular}{lll} 
& Time $(\min )$ & 0 \\
\hline & Time $(\min )$
\end{tabular}

$\overline{\text { Initial }}$

Interior assessment:

$-5^{*}$ chaned centents

viono it

Phototaken:

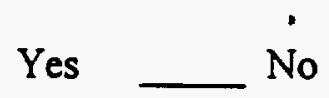

Comments: 


\section{Rack Storage Drum Data Sheet}

Drum No. $\times 32 c$

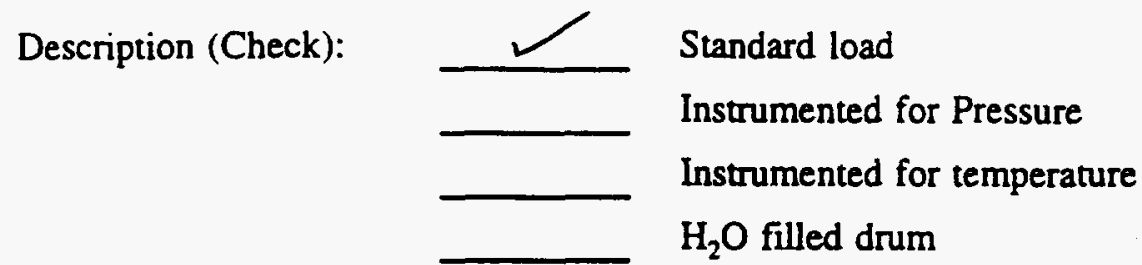

\begin{tabular}{|c|c|c|c|}
\hline Loading Starting at Bottom & Layer 1 & Layer 2 & Layer 3 \\
\hline 3 pieces of steel & & & \\
\hline $1 / 3$ of the brown paper & & & \\
\hline $1 / 3$ of the 10-mil plastic & & & \\
\hline $1 / 3$ of the bond paper & & & \\
\hline $1 / 3$ of the towels & & & \\
\hline $1 / 3$ of the bags & & & \\
\hline $1 / 3$ of the cut paper & & & \\
\hline $1 / 3$ of the fabric & & & \\
\hline $1 / 3$ of the rubber & Go to Layer 2 & Go to Layer 3 & \\
\hline
\end{tabular}

Drum Filled:

Ring bolt placed opposite drum seam and in down position

Lid sealed and torqued to $40 \mathrm{ft} / \mathrm{lb}$ :

Leak tested:

Pre-test weight (lb)

Post-test weight (lb)
Date 5.3 .95 Initial $\underline{\text { L.K }}$

Date 5-3-95 Initial $2 K$
N/A or Pressure (psig)

Pressure (psig)

Date

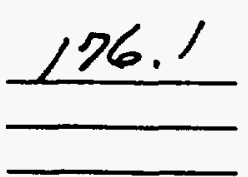

Time $(\min ) \frac{0}{5}$
Time $(\min )$

Initial

Target weight $-175.6 \mathrm{lb}$ 


\section{Rack Storage Drum Data Sheet}

Drum No. $\quad x 326 \quad$ wit. $=135.310$

Post-test Assessment

Position: __ original: __ tipped: __ toppled

Lid on drum: $\quad$ Yes $X$ No not withedrum

Gasket missing (cm)

Contents expelled: _ Yes

Seal failure: $\quad \quad \quad$ Yes $\quad$ Not visible

Burn pattern:

$43^{4}$ at mid lt. centend in sein

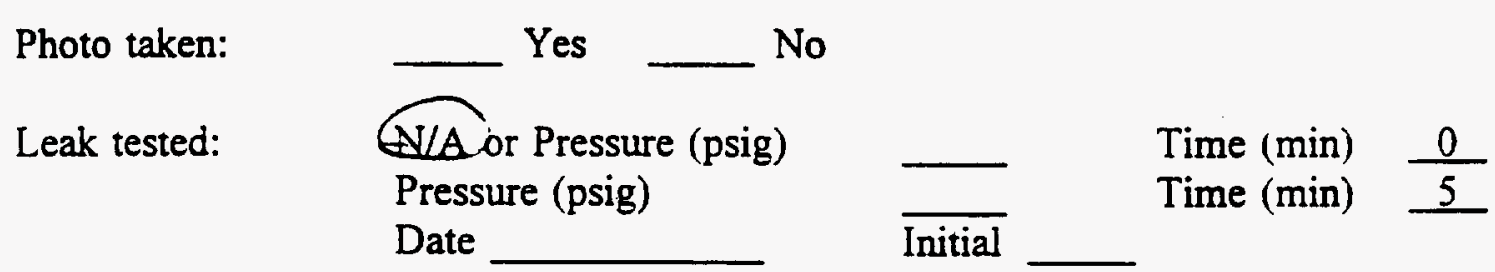

Interior assessment:

$-5 \%$ coutents

Tre 7

photo taken:

Yes No

Comments: 
WHC-SD-WM-TRP-246 REV. 0

\section{Rack Storage Drum Data Sheet}

Drum No. $\times 336$

Description (Check):

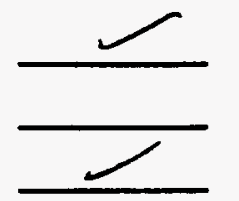

Standard load

Instrumented for Pressure

Instrumented for temperature

$\mathrm{H}_{2} \mathrm{O}$ filled drum

\begin{tabular}{|c|c|c|c|}
\hline Loading Starting at Bottom & Layer 1 & Layer 2 & Layer 3 \\
\hline 3 pieces of steel & & N/A & N/A \\
\hline \multicolumn{4}{|l|}{$1 / 3$ of the brown paper } \\
\hline \multicolumn{4}{|l|}{$1 / 3$ of the 10 -mil plastic } \\
\hline \multicolumn{4}{|l|}{$1 / 3$ of the bond paper } \\
\hline \multicolumn{4}{|l|}{$1 / 3$ of the towels } \\
\hline \multicolumn{4}{|l|}{$1 / 3$ of the bags } \\
\hline \multicolumn{4}{|l|}{$1 / 3$ of the cut paper } \\
\hline \multicolumn{4}{|l|}{$1 / 3$ of the fabric } \\
\hline \multicolumn{4}{|l|}{$1 / 3$ of the rubber } \\
\hline & Go to Layer 2 & Go to Layer 3 & \\
\hline
\end{tabular}

Drum Filled:

Date 5.3-95 Initial P.K

Ring bolt placed opposite drum seam

Date $5-3-95$

Initial and in down position

Lid sealed and torqued to $40 \mathrm{ft} / \mathrm{lb}$ :

Date 5-3-95 Initial Re.

Leak tested:

N/A or Pressure (psig)

Pressure (psig)

Date

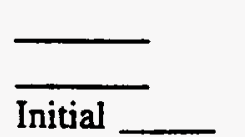

Time (min) $\quad 0$

Time (min)

Pre-test weight (lb)

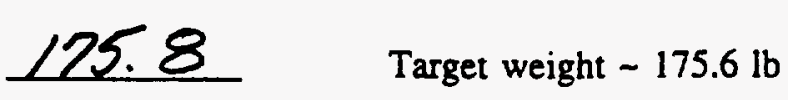

Post-test weight (lb) 


\section{Rack Storage Drum Data Sheet}

Drum No. $\times 336$ wt. 133, 1 ib. we lid

Post-test Assessment

Position: original: tipped; toppled

Lid on drum: Yes $X$ No missing

Gasket missing (cm)

Contents expelled: __ Yes _ No

Seal failure: $\quad x$ Yes $\quad$ Not visible

Burn pattern:

not meas meable - all around

Photo taken:

Yes $\longrightarrow$ No

Leak tested:

N/A or Pressure (psig)

Pressure (psig)

Date

\begin{tabular}{lll}
- & Time (min) & 0 \\
\hline & Time $(\mathrm{min})$ & $\underline{5}$
\end{tabular}

$\overline{\text { Initial }}$

Interior assessment:

-3 " of - haved ervtents

$\therefore 00 \% 12$

Photo taken:

$X$ Yes

No

Comments: 


\section{Rack Storage Drum Data Sheet}

Drum No. $\times 3+6$

Description (Check):

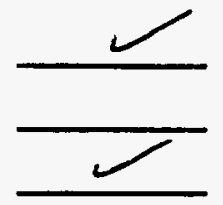

Standard load

Instrumented for Pressure

Instrumented for temperature

$\mathrm{H}_{2} \mathrm{O}$ filled drum

\begin{tabular}{|c|c|c|c|}
\hline Loading Starting at Bottom & Layer 1 & Layer 2 & Layer 3 \\
\hline 3 pieces of steel & N/A & \\
\hline $1 / 3$ of the brown paper & & & \\
\hline $1 / 3$ of the 10 -mil plastic & & & \\
\hline $1 / 3$ of the bond paper & & & \\
\hline $1 / 3$ of the towels & & & \\
\hline $1 / 3$ of the bags & & & \\
\hline $1 / 3$ of the cut paper & Go to Layer 2 & Go to Layer 3 & \\
\hline $1 / 3$ of the fabric & & & \\
\hline $1 / 3$ of the rubber & & & \\
\hline
\end{tabular}

Drum Filled:

Date $5-3-95$ Initial L.K

Ring bolt placed opposite drum seam Date 5.3 .95 Initial R.K and in down position

Lid sealed and torqued to $40 \mathrm{ft} / \mathrm{b}: \quad$ Date $5.3 .95 \quad$ Initial R.R

Leak tested:

N/A or Pressure (psig)

Pressure (psig)

Date

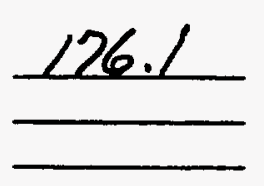

Time (min) $\quad 0$

Time (min) 5

Pre-test weight (lb)

Post-test weight (lb)

\begin{tabular}{lll}
$\longrightarrow$ & Time $(\min )$ & 0 \\
\hline Initial & Time $(\min )$ & 5
\end{tabular}

Target weight $\sim 175.6 \mathrm{lb}$ 
WHC-SD-WM-TRP-246 REV. 0

Rack Storage Drum Data Sheet

Drum No. $\times 346 \quad 143.9$

Post-test Assessment

Position: __ original: __ tipped; __ toppled

Lid on drum: $\quad$ Yes $\quad x$ No

Gasket missing (cm) All

Contents expelled: _ Yes _ No

Seal failure: $\quad X$ Yes Not visible

Burn pattern:

pifficut to ineasme

Photo taken: _ Yes _ No

Leak tested: Nor Pressure (psig)

Pressure (psig)

Date

\begin{tabular}{lll} 
& Time $(\min )$ & 0 \\
\hline & Time $(\min )$
\end{tabular}

Interior assessment:

4" of remouning contento

u. tec 5

phato taken:

$X$ Yes

Comments: 


\section{Rack Storage Drum Data Sheet}

Drum No. $\times 356(p)$

Description (Check):

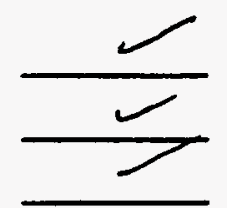

Standard load

Instrumented for Pressure

Instrumented for temperature

$\mathrm{H}_{2} \mathrm{O}$ filled drum

\begin{tabular}{|c|c|c|c|}
\hline Loading Starting at Bottom & Layer 1 & Layer 2 & Layer 3 \\
\hline 3 pieces of steel & & N/A & \\
\hline $1 / 3$ of the brown paper & & & \\
\hline $1 / 3$ of the 10-mil plastic & & & \\
\hline $1 / 3$ of the bond paper & & & \\
\hline $1 / 3$ of the towels & & & \\
\hline $1 / 3$ of the bags & & & \\
\hline $1 / 3$ of the cut paper & & & \\
\hline $1 / 3$ of the fabric & Go to Layer 2 & Go to Layer 3 & \\
\hline $1 / 3$ of the rubber & & & \\
\hline
\end{tabular}

\section{Drum Filled:}

Ring bolt placed opposite drum seam and in down position

Lid sealed and torqued to $40 \mathrm{ft} / \mathrm{b}$ :

Leak tested:

Pre-test weight (lb)

Post-test weight (lb)
Date $5=3 \cdot 95 \quad$ Initial

Date $5.3 .95 \quad$ Initial
N/A or Pressure (psig)

Pressure (psig)

Date

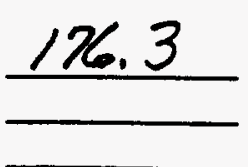

$\begin{array}{ll}\text { Time }(\min ) & 0 \\ \text { Time }(\min ) & 5\end{array}$

Initial

Target weight $\sim 175.6 \mathrm{lb}$ 
WHC-SD-WM-TRP-246 REV. 0

\section{Rack Storage Drum Data Sheet}

Drum No. $\quad \underline{356} \omega t_{1}=175.116$.

$\underline{\text { Post-test Assessment }}$

Position:

original;

tipped; toppled

Lid on drum: $\quad x$ Yes No l.d r ring retate frealy.

Gasket missing (cm) AII

Contents expelled: _ Yes $\not \subset$ No

Seal failure: $\quad$ Yes $x$ Not visible

Burn pattern:

ill around - mainly in bott half

Photo taken:

Yes $\underset{X}{X}$

Leak tested:

NT/A)or Pressure (psig)

Pressure (psig)

Date

\begin{tabular}{|c|c|}
\hline & Time (min) \\
\hline & Time $(\mathrm{min})$ \\
\hline
\end{tabular}

Interior assessment:
top chaned - upto $\delta^{\prime \prime}$ in of a han oll unound

V.rec is

Photo taken:

$x$ Yes

Comments: 


\section{Rack Storage Drum Data Sheet}

Drum No. $\times 3 i 6$

Description (Check):

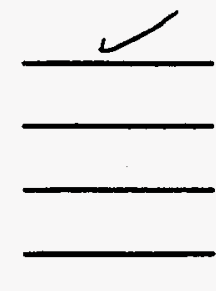

Standard load

Instrumented for Pressure

Instrumented for temperature

$\mathrm{H}_{2} \mathrm{O}$ filled drum

\begin{tabular}{|c|c|c|c|}
\hline Loading Starting at Bottom & Layer 1 & Layer 2 & Layer 3 \\
\hline 3 pieces of steel & & N/A & N/A \\
\hline $1 / 3$ of the brown paper & & & \\
\hline $1 / 3$ of the 10-mil plastic & & & \\
\hline $1 / 3$ of the bond paper & & & \\
\hline $1 / 3$ of the towels & & & \\
\hline $1 / 3$ of the bags & & & \\
\hline $1 / 3$ of the cut paper & & & \\
\hline $1 / 3$ of the fabric & & & \\
\hline $1 / 3$ of the rubber & Go to Layer 2 & Go to Layer 3 & \\
\hline
\end{tabular}

Drum Filled:

Date 5.3.95 Initial R.K

Ring bolt placed opposite drum seam Date $\underline{5-3.95}$ Initial

and in down position

Lid sealed and torqued to $40 \mathrm{ft} / \mathrm{lb}: \quad$ Date 5.3 .95 Initial R.K

Leak tested:

Pre-test weight (lb)

Post-test weight (lb)
N/A or Pressure (psig)

Pressure (psig)

Date

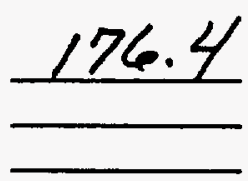

Time (min)

Time (min) 5

Initial

Target weight $-175.6 \mathrm{lb}$ 
WHC-SD-WM-TRP-246 REV. 0

Rack Storage Drum Data Sheet

Drum No. $\quad \times 366175.416$.

Post-test Assessment

Position: ___ original: ___ tipped; __ toppled

Lid on drum: $\quad$ Yes _ No ring ollid rotate treely

Gasket missing (cm) A1l

Contents expelled: __ Yes $\searrow$ No

Seal failure: $\quad$ Yes $\quad$ Not visible

Burn pattern:

woumd rim, top $2 / 3 \sim 15 "$, belm bolt

Photo taken:

Yes $\quad x$ No

Leak tested:

NTA or Pressure (psig)

Pressure (psig)

- Date

\begin{tabular}{lll}
- & Time $(\min )$ & 0 \\
\hline & Time $(\min )$
\end{tabular}

Interior assessment:

chamed m top

$2-5$ " char around drum

roco is z

photo taken:

$\times$ Yes

Comments: 


\section{Rack Storage Drum Data Sheet}

Drum No. $\times 376$

Description (Check):

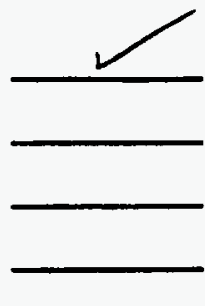

Standard load

Instrumented for Pressure

Instrumented for temperature

$\mathrm{H}_{2} \mathrm{O}$ filled drum

\begin{tabular}{|c|c|c|c|}
\hline Loading Starting at Bottom & Layer 1 & Layer 2 & Layer 3 \\
\hline 3 pieces of steel & N/A & \\
\hline $1 / 3$ of the brown paper & & & \\
\hline $1 / 3$ of the 10 -mil plastic & & & \\
\hline $1 / 3$ of the bond paper & & & \\
\hline $1 / 3$ of the towels & & & \\
\hline $1 / 3$ of the bags & & & \\
\hline $1 / 3$ of the cut paper & & Go to Layer 3 & \\
\hline $1 / 3$ of the fabric & & & \\
\hline $1 / 3$ of the rubber & Go to Layer 2 & G & \\
\hline
\end{tabular}

Drum Filled:

Date 5.3.95 Initial L.K

Ring bolt placed opposite drum seam Date $5-3-95$ Initial L.K and in down position

Lid sealed and torqued to $40 \mathrm{ft} / \mathrm{b}: \quad$ Date $5.3-95$ Initial C.K

Leak tested:

Pre-test weight (lb)

Post-test weight (lb)
N/A or Pressure (psig)

Pressure (psig)

Date

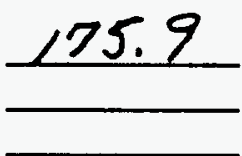

Time (min) $\quad 0$

Time (min) 5

Initial

Target weight $-175.6 \mathrm{lb}$ 


\section{WHC-SD-WM-TRP-246 REV. 0}

\section{Rack Storage Drum Data Sheet}

Drum No. $\quad \times 376,75.116$.

\section{Post-test Assessment}

Position: __ original: ___ tipped: ___ toppled

Lid on drum: $\quad \quad \quad$ Yes $\quad$ No

Gasket missing (cm) AlI

Contents expelled: _ Yes $\longrightarrow$ No

Seal failure: $\quad$ Yes $\not$ Not visible

Burn pattern: arownd rim \& top $2 / 3-14^{\prime \prime}$ wide below bolt.

Photo taken: _ Yes _ No

\begin{tabular}{llll} 
Leak tested: & N/A or Pressure (psig) \\
Pressure (psig) & $\longrightarrow$ & Time (min) & 0 \\
\hline
\end{tabular}

Date

Initial

Interior assessment:

top chaned

ip to $-s^{\prime \prime}$ char all around - showed on vadeo

UIDeO is!

Photo taken:

$x$ Yes

Comments: 
WHC-SD-WM-TRP-246 REV. 0

\section{Rack Storage Drum Data Sheet}

Drum No. $\times 386$

Description (Check):

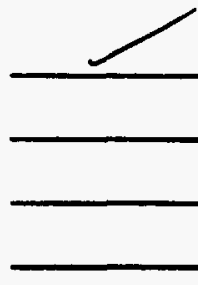

Standard load

Instrumented for Pressure

Instrumented for temperature

$\mathrm{H}_{2} \mathrm{O}$ filled drum

\begin{tabular}{|c|c|c|c|}
\hline Loading Starting at Bottom & Layer 1 & Layer 2 & Layer 3 \\
\hline 3 pieces of steel & & & \\
\hline $1 / 3$ of the brown paper & & & \\
\hline $1 / 3$ of the 10-mil plastic & & & \\
\hline $1 / 3$ of the bond paper & & & \\
\hline $1 / 3$ of the towels & & & \\
\hline $1 / 3$ of the bags & & & \\
\hline $1 / 3$ of the cut paper & & & \\
\hline $1 / 3$ of the fabric & & Go to Layer 3 & \\
\hline $1 / 3$ of the rubber & & & \\
\hline
\end{tabular}

Drum Filled:

Date $5=3.95$ Initial L.K

Ring bolt placed opposite drum seam Date $5 \cdot 3-95$ Initial L.K

and in down position

Lid sealed and torqued to $40 \mathrm{ft} / \mathrm{b}: \quad$ Date 5.3 .95 Initial R.K

Leak tested:

Pre-test weight (lb)

Post-test weight (lb)

Loss $=$
N/A or Pressure (psig)

Pressure (psig)

Date

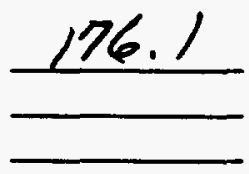

Time $(\min ) \quad 0$

Time (min) 5

Initial

Target weight $-175.6 \mathrm{lb}$ 


\section{Rack Storage Drum Data Sheet}

Drum No. $\quad \times 386-175.516$

Post-test Assessment

Position: original; tipped; toppled

Lid on drum: $\quad$ Yes No

Gasket missing (cm) All

Contents expelled: _ Yes $x$ No

Seal failure: $\quad$ Yes $\quad \chi$ Not visible

Burn pattern:

hand totel iovt defiartelf $\sim 12^{\prime \prime} \times 12^{\prime \prime}$ spot below bolt

Photo taken: Yes $\varnothing$ No

Leak tested:

NT Pressure (psig) Pressure (psig)

Date $\begin{array}{ll}\text { Time (min) } & 0 \\ \text { Time (min) } & \end{array}$

\begin{tabular}{lll} 
& Time (min) & 0 \\
\hline Initial & Time (min)
\end{tabular}

Interior assessment:

tof charn of

5-6" char all oround

vinec 36

Photo taken:

$x$ Yes No

Comments: 


\section{Rack Storage Drum Data Sheet}

Drum No. $\quad \underline{x 133}\left(\mathrm{H}_{2} \mathrm{O}\right)$

\section{Post-test Assessment}

Position: original: tipped; toppled

Lid on drum: Yes — No

Gasket missing (cm)

Contents expeiled: Yes No

Seal failure: $\quad$ Y Yes $\quad$ Not visible

Burn pattern:

Nove - sootyon seam half

Photo taken:

Yes

Leak tested:

N/A or Pressure (psig)

Pressure (psig)

Date

\begin{tabular}{lll}
- & Time (min) & 0 \\
\hline & Time (min)
\end{tabular}

Interior assessment:

Photo taken:

Yes

No

Comments: 
WHC-SD-WM-TRP-246 REV. 0

\section{Rack Storage Drum Data Sheet}

Drum No. $\times 221\left(\mathrm{H}_{2} \mathrm{O}\right)$

Post-test Assessment

Position: __ original: ___ tipped: __ toppled

Lid on drum: $\quad$ Yes

Gasket missing (cm)

Contents expelled:

Yes — No

Seal failure:

Yes

Not visible

Burn pattern:

Photo taken:

Yes

No

Leak tested:

N/A or Pressure (psig)

Pressure (psig)

Date

\begin{tabular}{lll} 
& Time (min) & 0 \\
\hline Initial & Time (min)
\end{tabular}

Interior assessment:

Photo taken:

Yes

No

Comments: 
WHC-SD-WM-TRP-246 REV. 0

\section{Rack Storage Drum Data Sheet}

Drum No. $\times 223\left(\mathrm{H}_{2} \mathrm{O}\right)$

Post-test Assessment

Position: __ original: __ tipped: __ toppled

Lid on drum: $\quad$ Yes $\quad$ No

Gasket missing (cm)

Contents expelled: __ Yes _ No

Seal failure: $\quad$ Yes _ N Not visible

Burn pattern:

Photo taken: $\quad$ Yes _ No

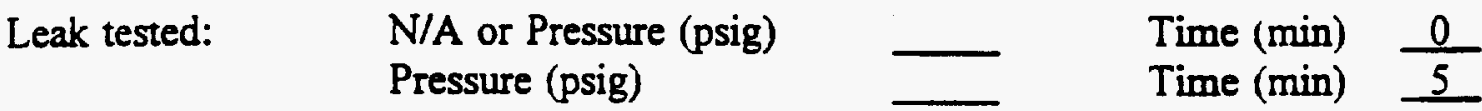

Date

$\overline{\text { Initial }}$

Interior assessment:

Photo taken: $\quad$ Yes _ No

Comments: 
WHC-SD-WM-TRP-246 REV. 0

\section{Rack Storage Drum Data Sheet}

Drum No. $\times 322\left(\mathrm{H}_{2} \mathrm{O}\right)$

$\underline{\text { Post-test Assessment }}$

Position: __ original: ___ tipped: __

Lid on drum: $\quad$ Yes $\quad$ No

Gasket missing (cm)

Contents expelled: _ Yes _ No

Seal failure: $\quad$ Yes $\quad$ Not visible

Burn pattern:

All around, very sooty

Photo taken:

Yes

Leak tested: $\quad$ N/A or Pressure (psig)

Pressure (psig)

Date

Time (min) $\quad \frac{0}{5}$

Time (min) 5

Interior assessment:

Photo taken: $\quad$ Yes $\quad$ No

Comments: 
Blank 
WHC-SD-WM-TRP-246 REV. 0

\title{
Solid Waste Drum Array Fire Performance
}

\author{
Volume III \\ Appendix E. Palletized Storage Test Data \\ Appendix F. Palletized Storage Drum Data Sheets
}

\author{
Prepared for \\ Westinghouse Hanford Company \\ Richland, WA 99352 \\ Prepared by \\ Chris F. Haecker \\ Los Alamos Technical Associates, Inc. \\ Kennewick, WA 99306 \\ Jesse J. Beitel, Daniel T. Gottuk, Brian T. Rhodes, and Craig L. Beyler \\ Hughes Associates, Inc. \\ Baltimore, MD 21227
}


blante 
WHC-SD-WM-TRP-246 REV. O

Appendix $E$

Palletized Storage Test Data 
CLIENT: LATA

PROJECT NO.: SwRI 01-7106

DATE: 6 JUNE 1995

TEST ID: P22.PRN

\section{PALLET STORAGE TEST INTERNAL DRUM PRESSURES}

(TIER \#1)

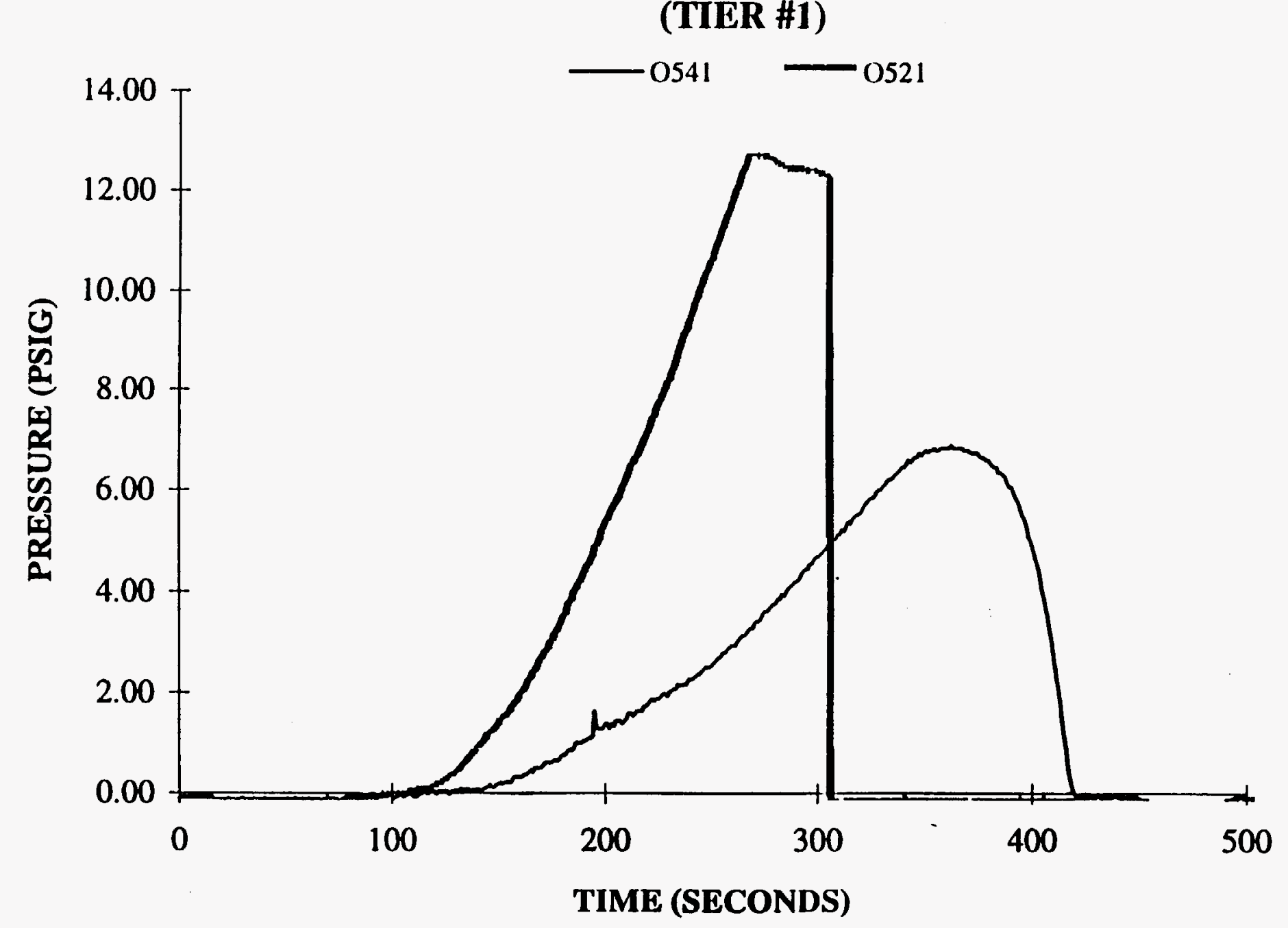

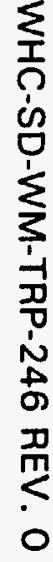

䍐 
CLIENT: LATA

PROJECT NO.: SwRI 01-7106

DATE: 6 JUNE 1995

TEST ID: P22.PRN

PALLET STORAGE TEST

INTERNAL DRUM PRESSURES

(TIER \#1)

䍐

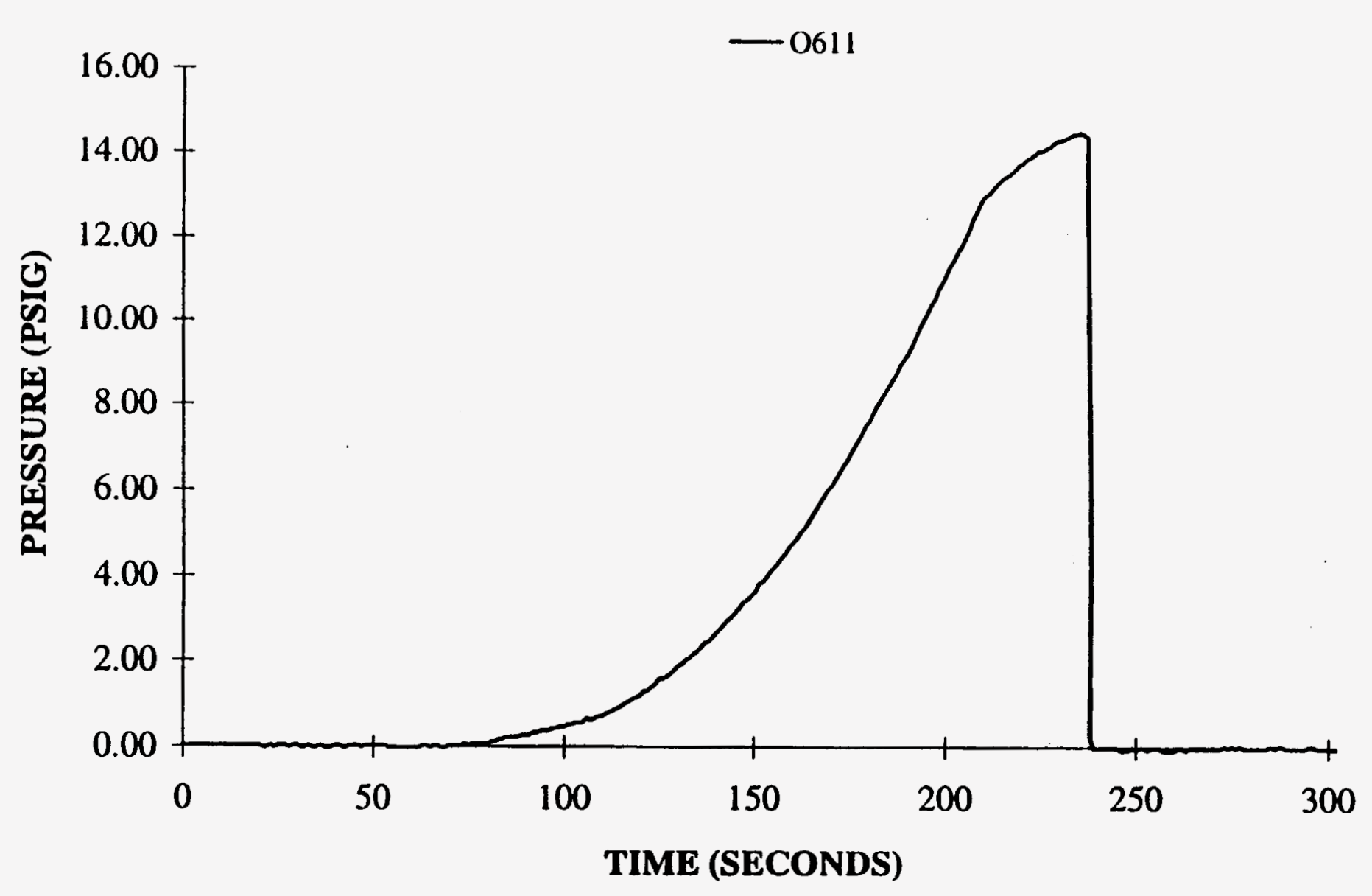

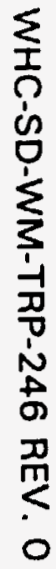

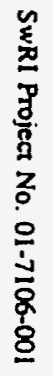

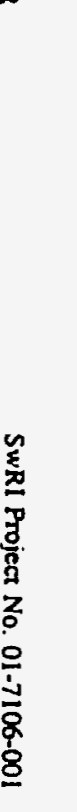


CLIENT: LATA

PROJECT NO.: SwRI 01-7106

DATE: 6 JUNE 1995

TEST ID: P22.PRN

\section{PALLET STORAGE TEST \\ INTERNAL DRUM PRESSURES}

(TIER \#1)

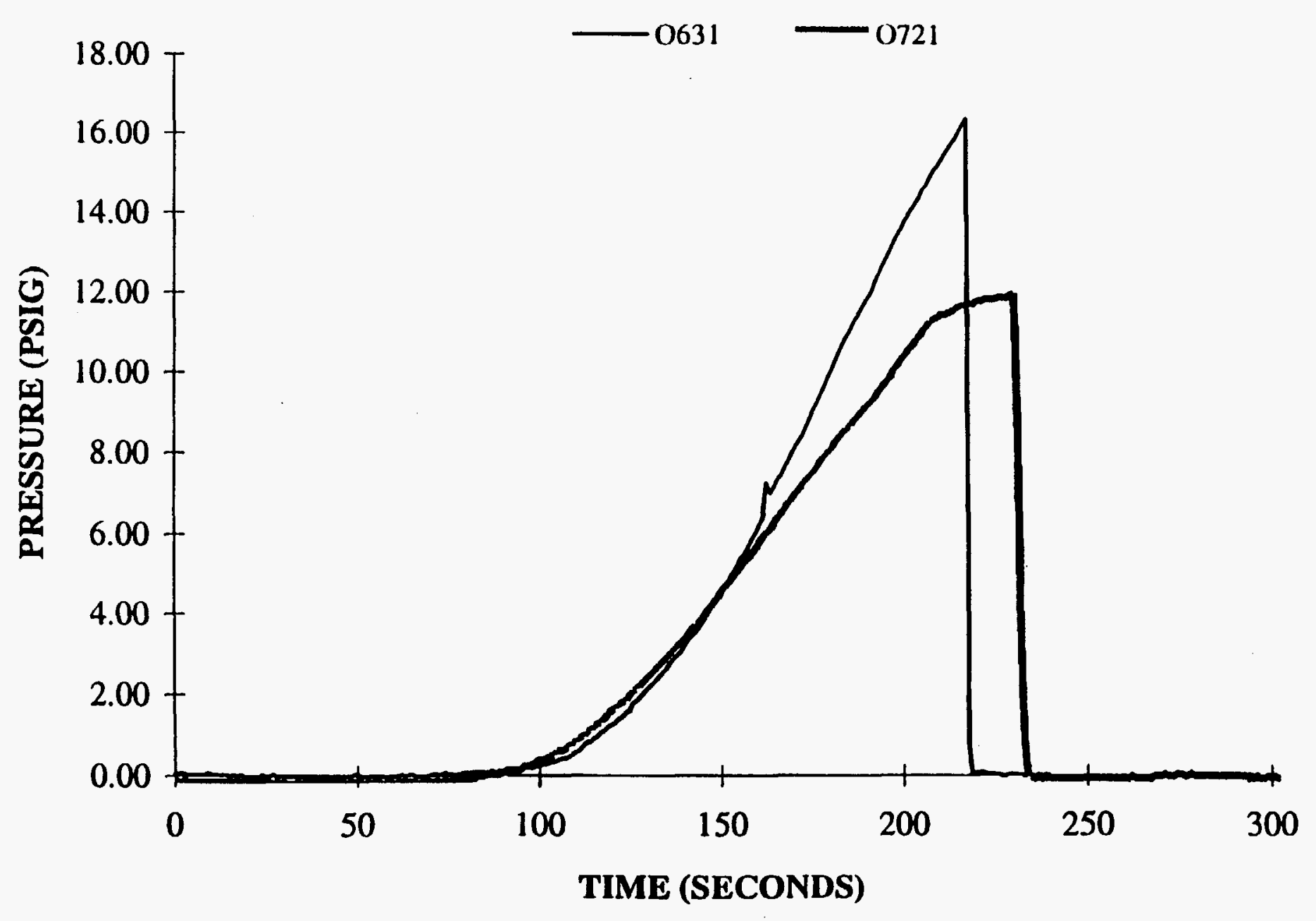

m

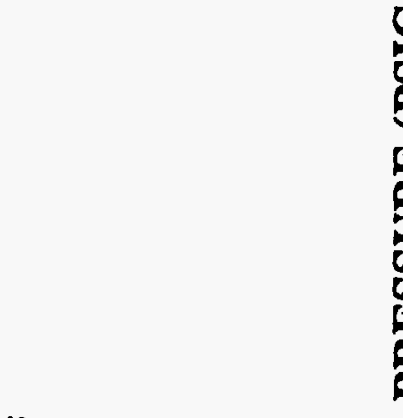


CLIENT: LATA

PROJECT NO.: SwRI 01-7106

DATE: 6 JUNE 1995

TEST ID: P22.PRN

\section{PALLET STORAGE TEST INTERNAL DRUM PRESSURES}

(TIER \#1)

ơ

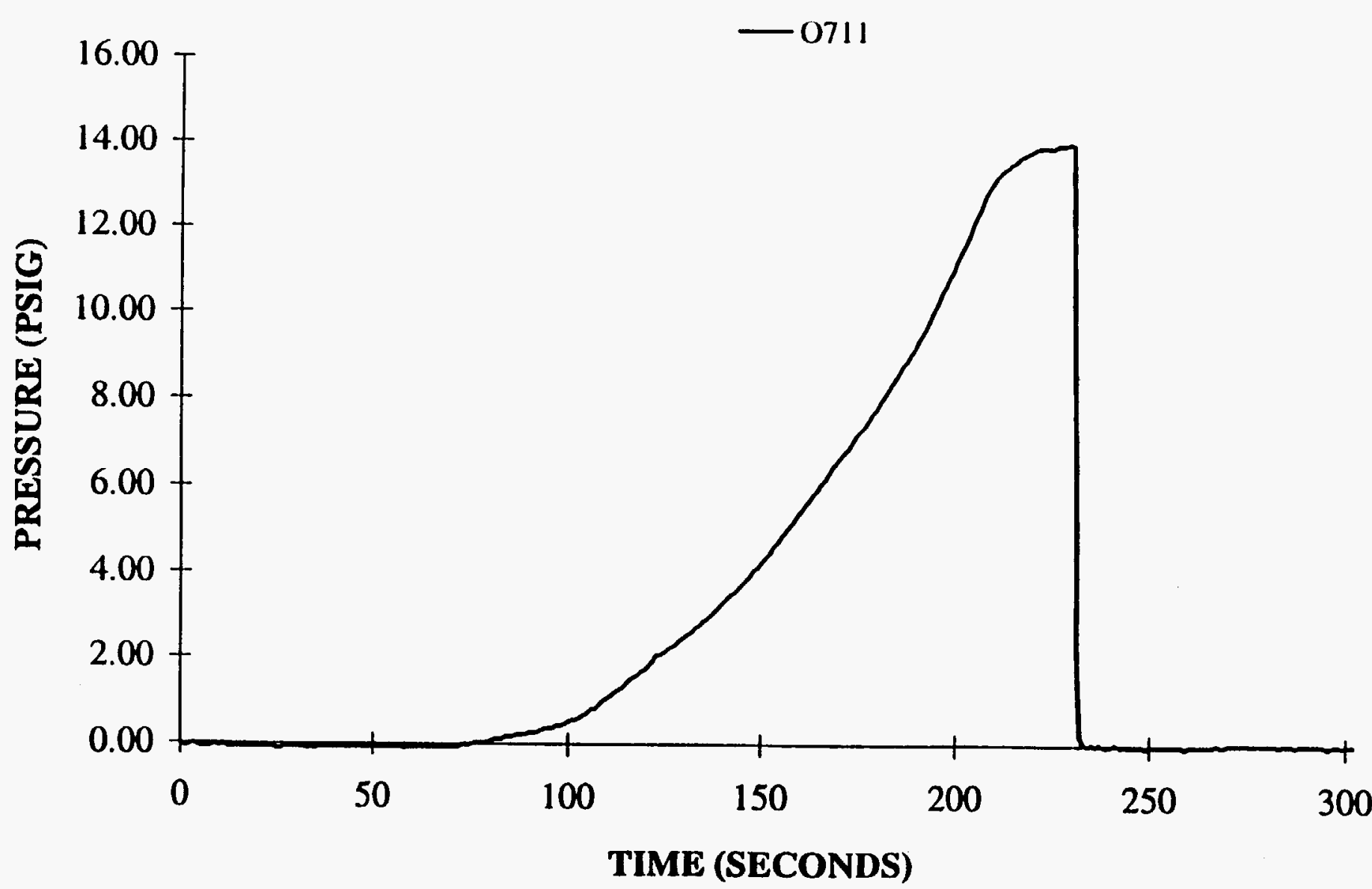

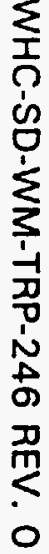


CLIENT: LATA

DATE: 6 JUNE 1995

TEST ID: P22.PRN

m

PALLET STORAGE TEST INTERNAL DRUM PRESSURES

(TIER \#1)

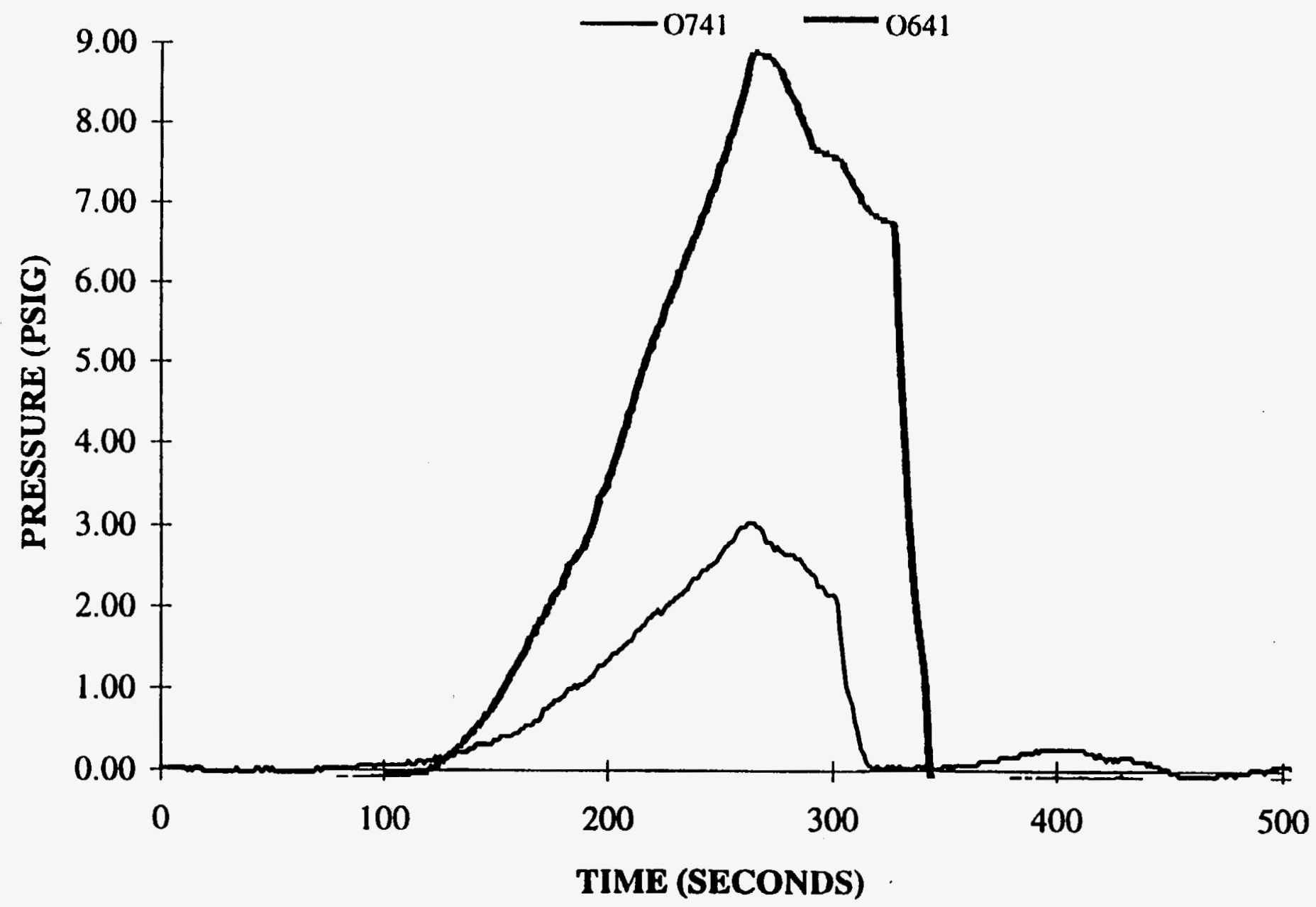


CLIENT: LATA

PROJECT NO.: SwRI 01-7106

DATE: 6 JUNE 1995

TEST ID: P22.PRN

\section{PALLET STORAGE TEST \\ INTERNAL DRUM PRESSURES}

(TIER \#1)

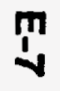

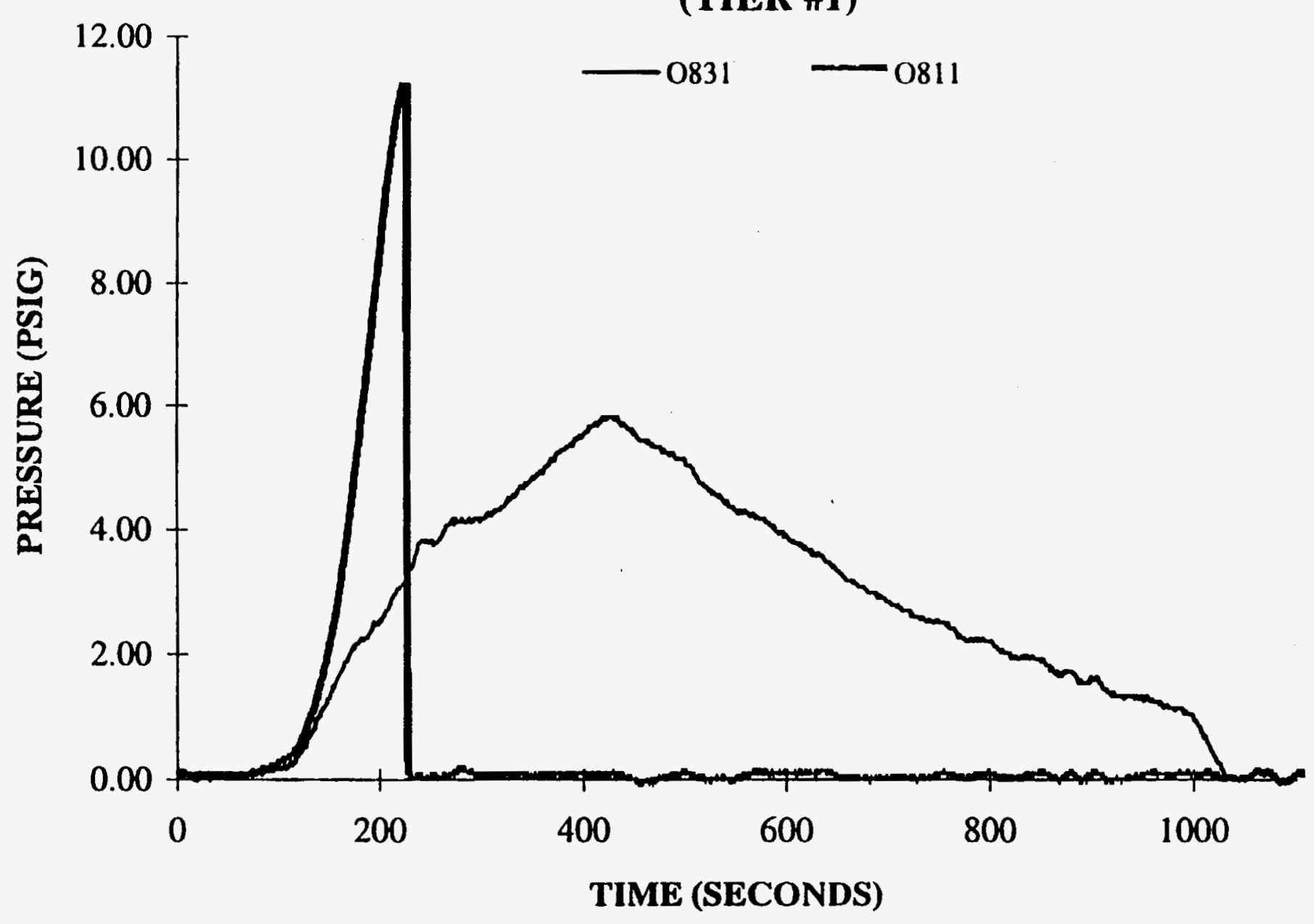


CLIENT: LATA

PROJECT NO.: SwRI 01-7106

TEST ID: P22.PRN

\section{PALLET STORAGE TEST INTERNAL DRUM PRESSURES}

(TIER \#2)

$\underset{\infty}{m}$

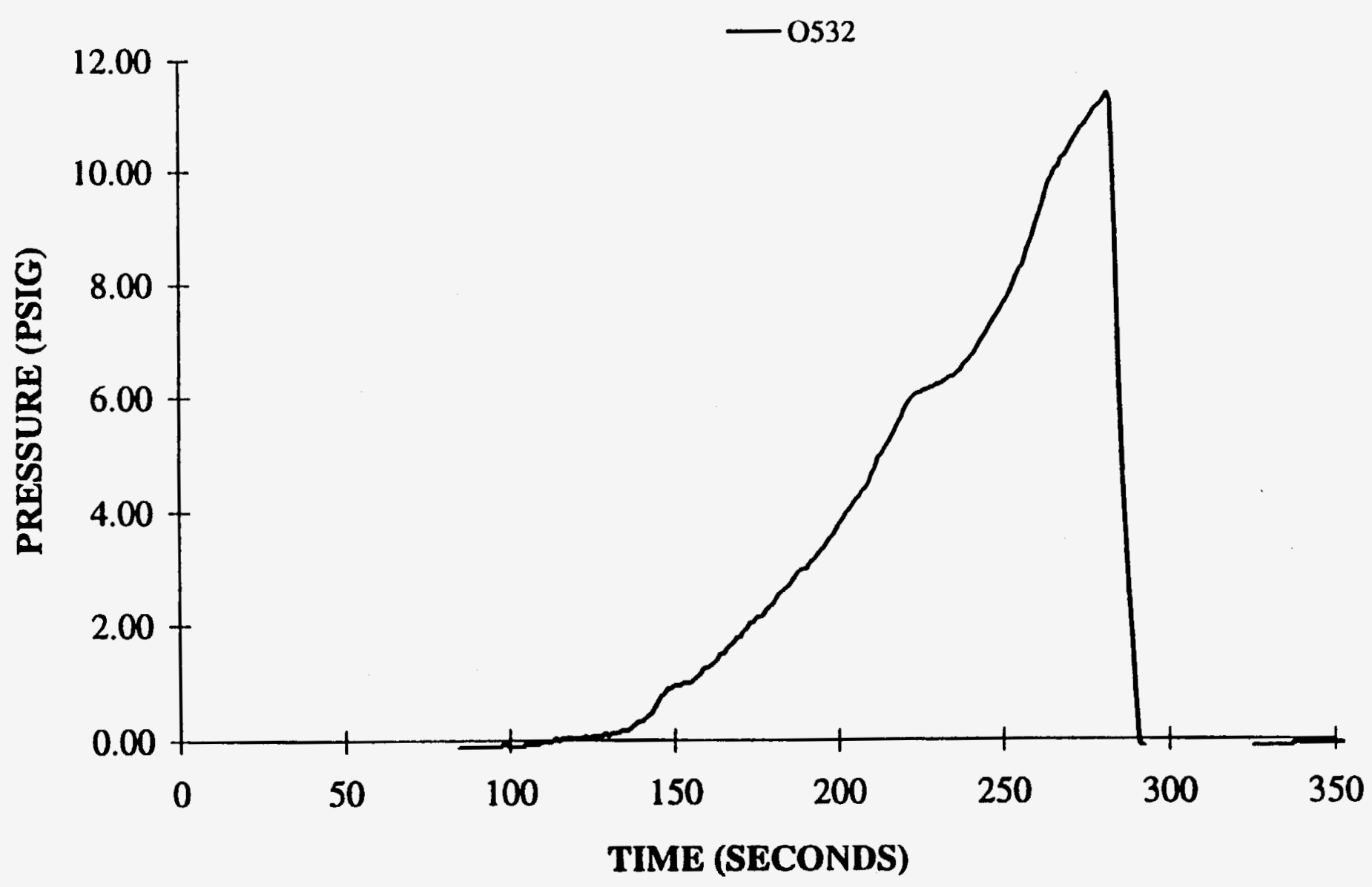


CLIENT: LATA

PROJECT NO.: SwRI 01-7106

DATE: 6 JUNE 1995

TEST ID: P22.PRN

\section{PALLET STORAGE TEST \\ INTERNAL DRUM PRESSURES}

(TIER \#2)

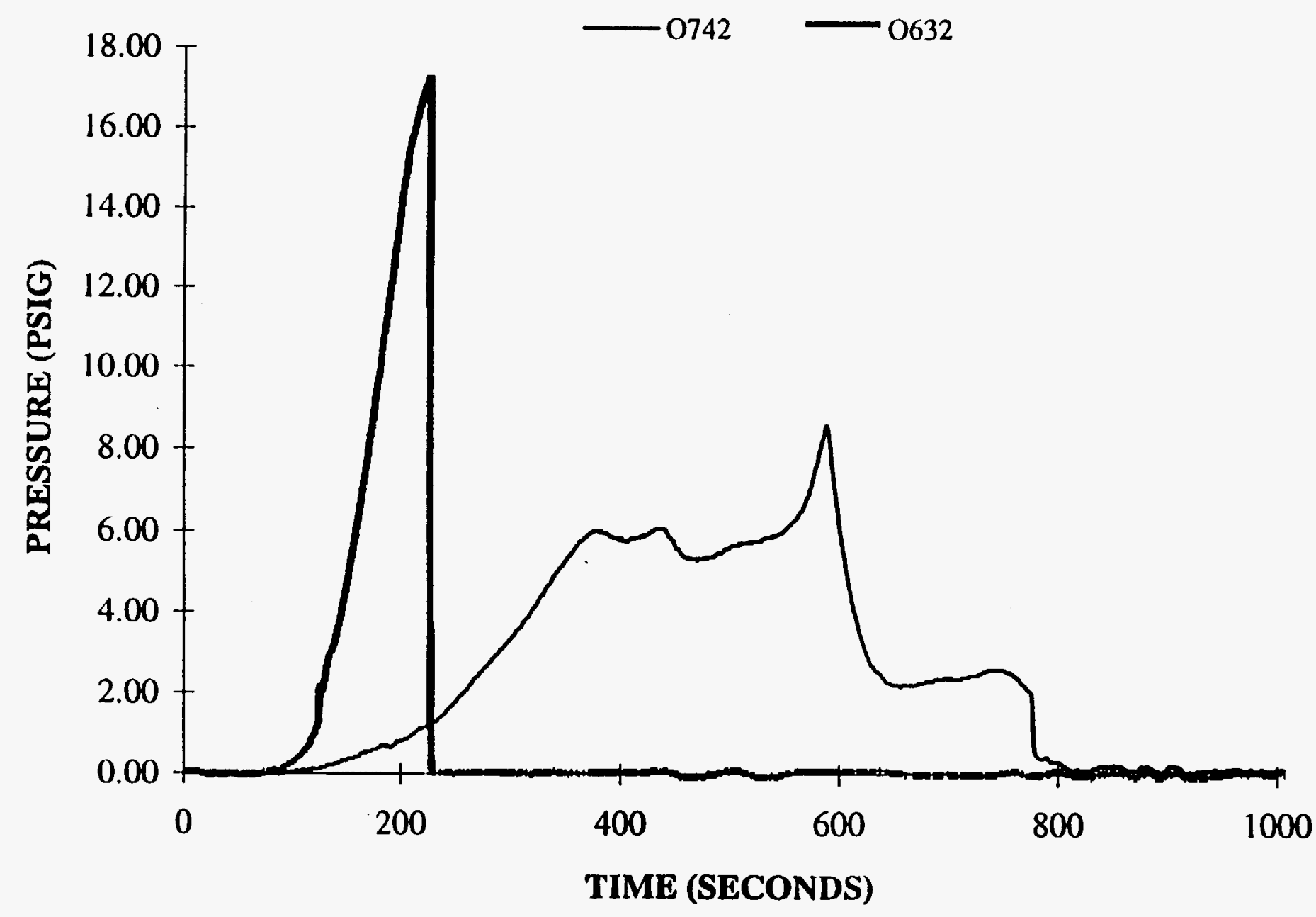

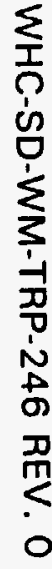


CLIENT: LATA

PROJECT NO.: SwRI 01-7106

DATE: 6 JUNE 1995

TEST ID: P22.PRN

\section{PALLET STORAGE TEST \\ INTERNAL DRUM PRESSURES}

(TIER \#2)

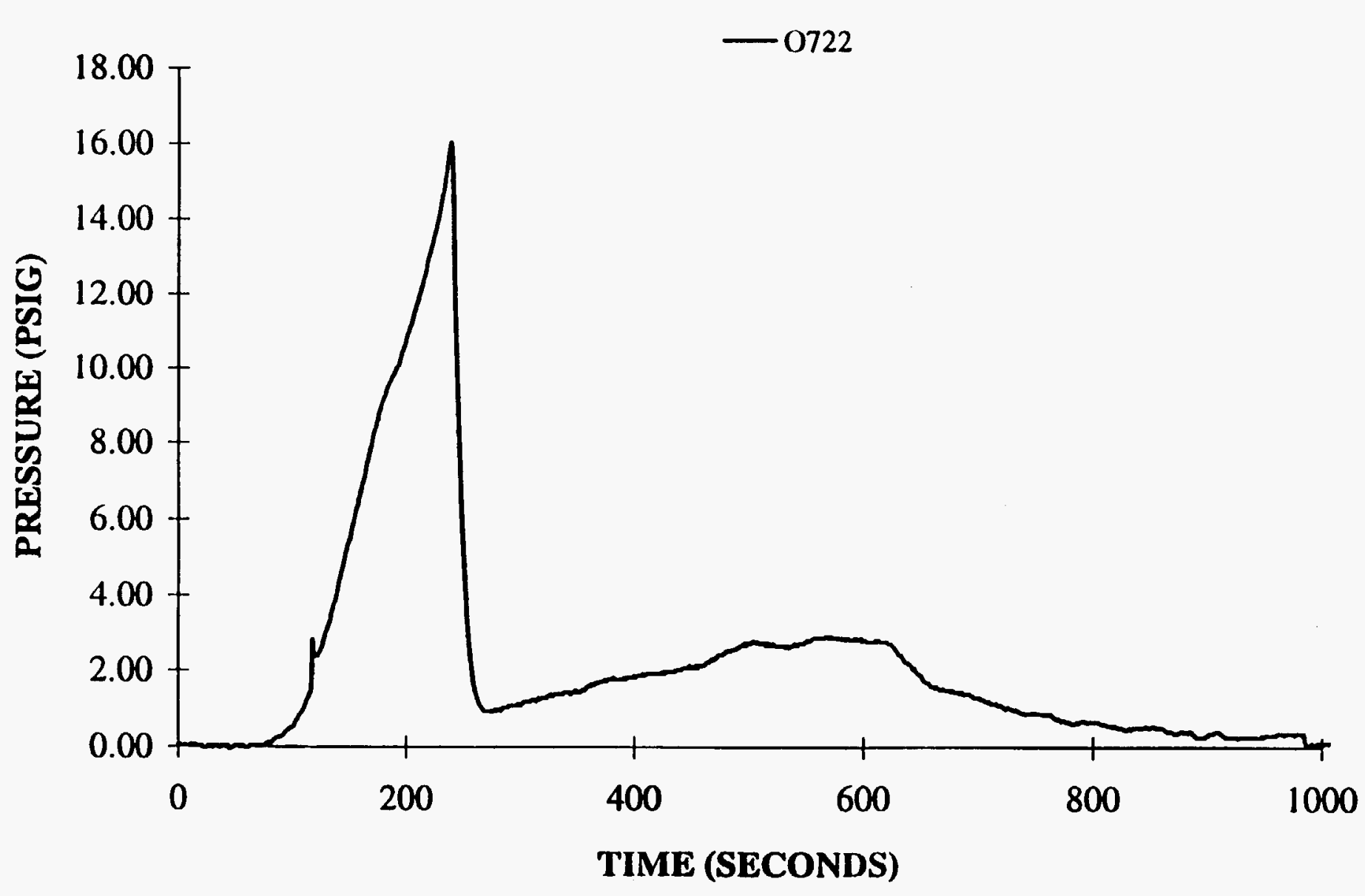


CLIENT: LATA

PROJECT NO.: SwRI 01-7106

DATE: 6 JUNE 1995

TEST ID: P22.PRN

PALLET STORAGE TEST

INTERNAL DRUM PRESSURES

(TIER \#2)

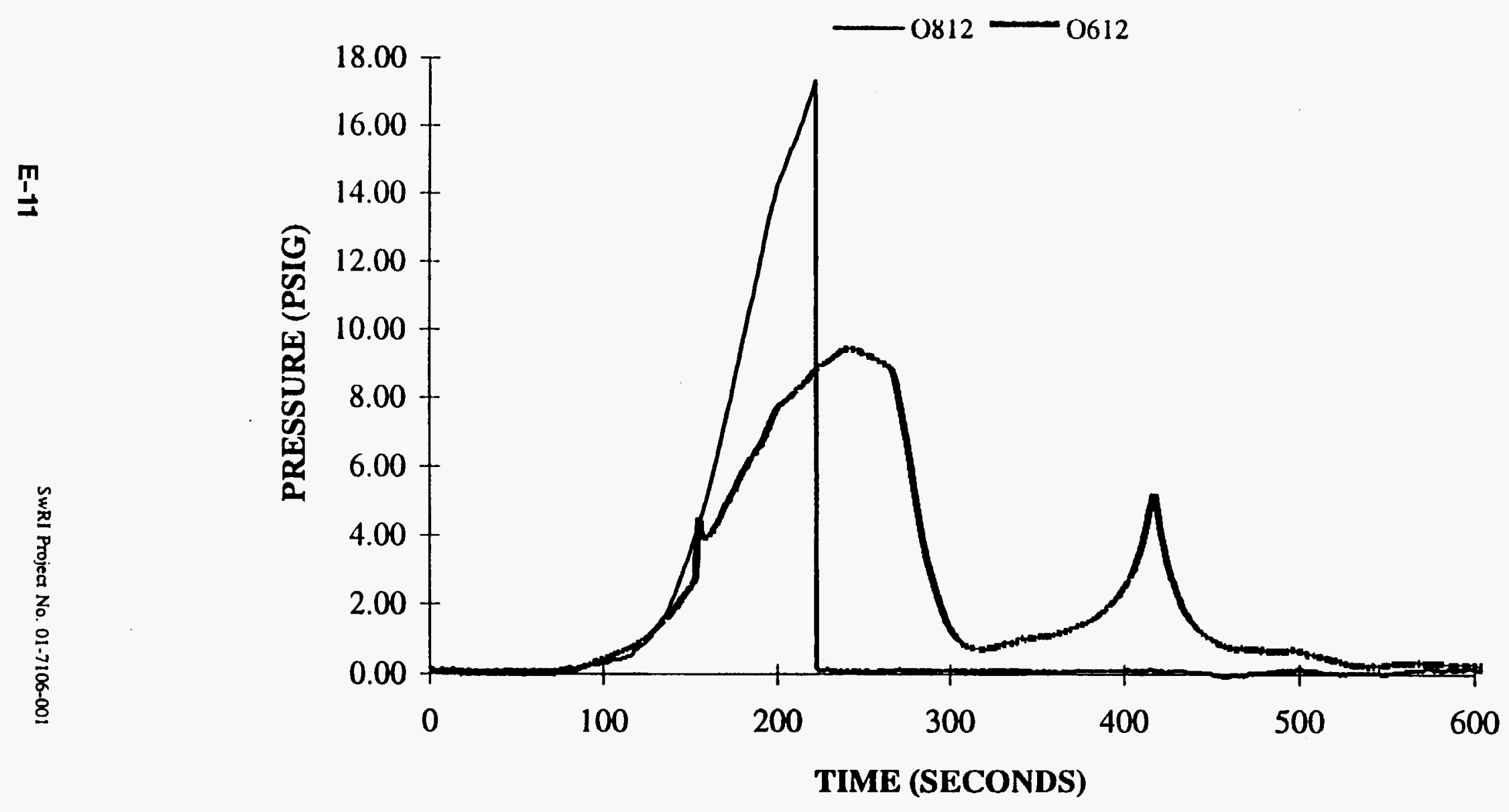

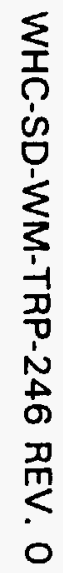


CLIENT: LATA

PROJECT NO.: SwRI 01-7106

DATE: 6 JUNE 1995

TEST ID: P22.PRN

\section{PALLET STORAGE TEST INTERNAL DRUM PRESSURES}

(TIER \#3)

$\stackrel{m}{\mathfrak{N}}$

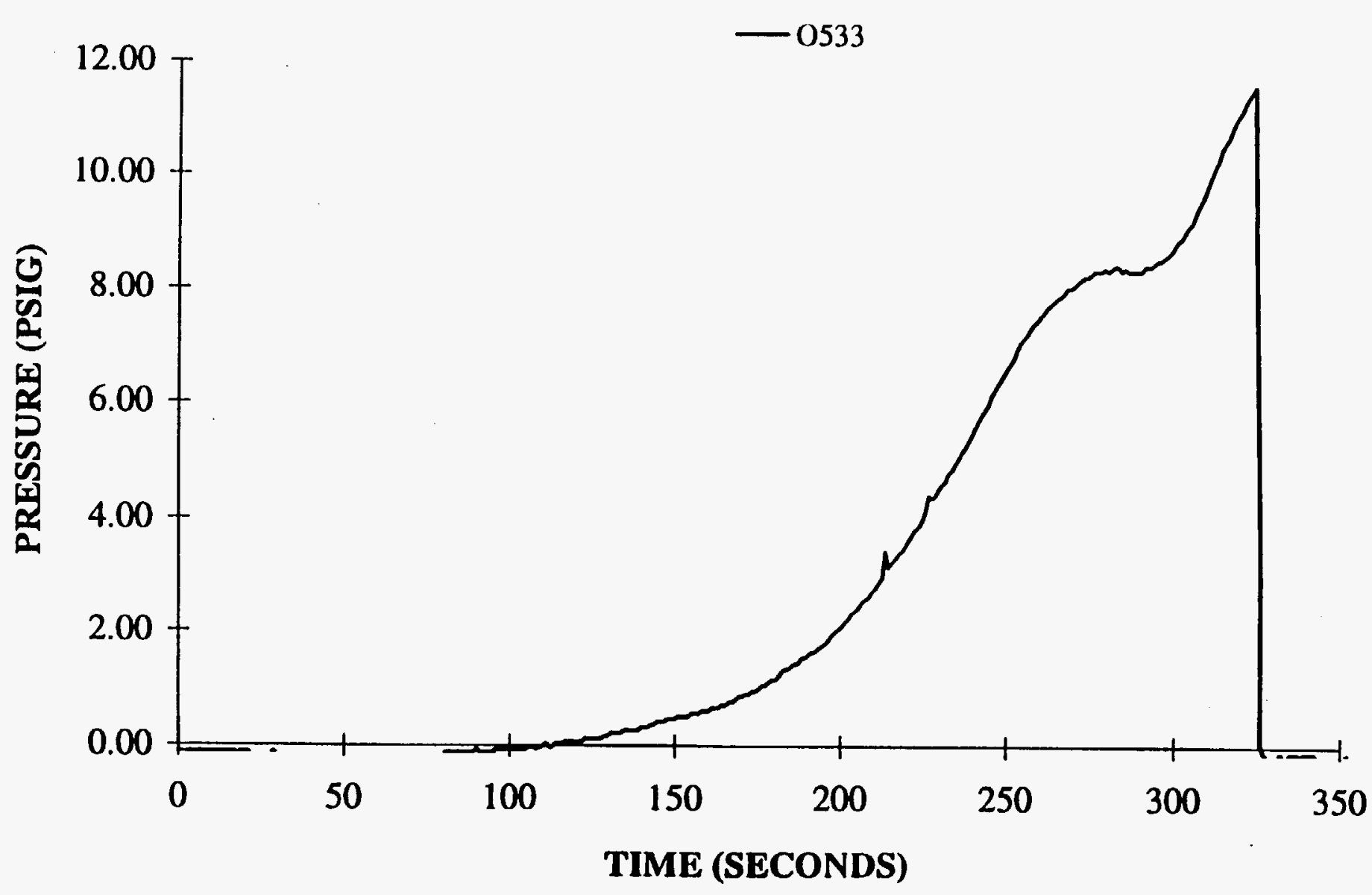

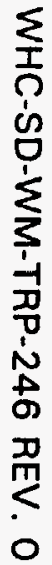


*ak RMIS View/Frint Document Cover Sheet tow

This document was retrieved from the Documentation and Records Manaqement (DRM) ISEARCH System. It is intended for Information only and may not be the most recent or updated version. Contact a Document Service Center (see Hanford Info for locations) if you need additional retrieval information.

Accession \#: D195061640

Document \#: SD-WM-TRP-246

Title/Desc:

SOLID WASTE DRUM ARRAY FIRE PERFORMANCE [SEC 5 OF 7]

Pages: 151 
THIS DOCUMENT WAS TOO LARGE TO SCAN AS A SINGLE

DOCUMENT. IT REQUIRED BREAKING INTO SMALLER SECTIONS.

DOCUMENT NUMBER: SD-WM.TRP.2HL

SECTION: $5 \quad$ OF 7

TITLE: Allid wante Drum array firie Performance

AUTHOR: LovUS RL

RECIPIENT:

DATE: $11 / 07 / 95$

KEYWORDS:

REFERENCES: EDT-612232 
blank 
CLIENT: LATA

PROJECT NO.: SwRI 01-7106

DATE: 6 JUNE 1995

TEST ID: P22.PRN

\section{PALLET STORAGE TEST INTERNAL DRUM PRESSURES}

(TIER \#3)

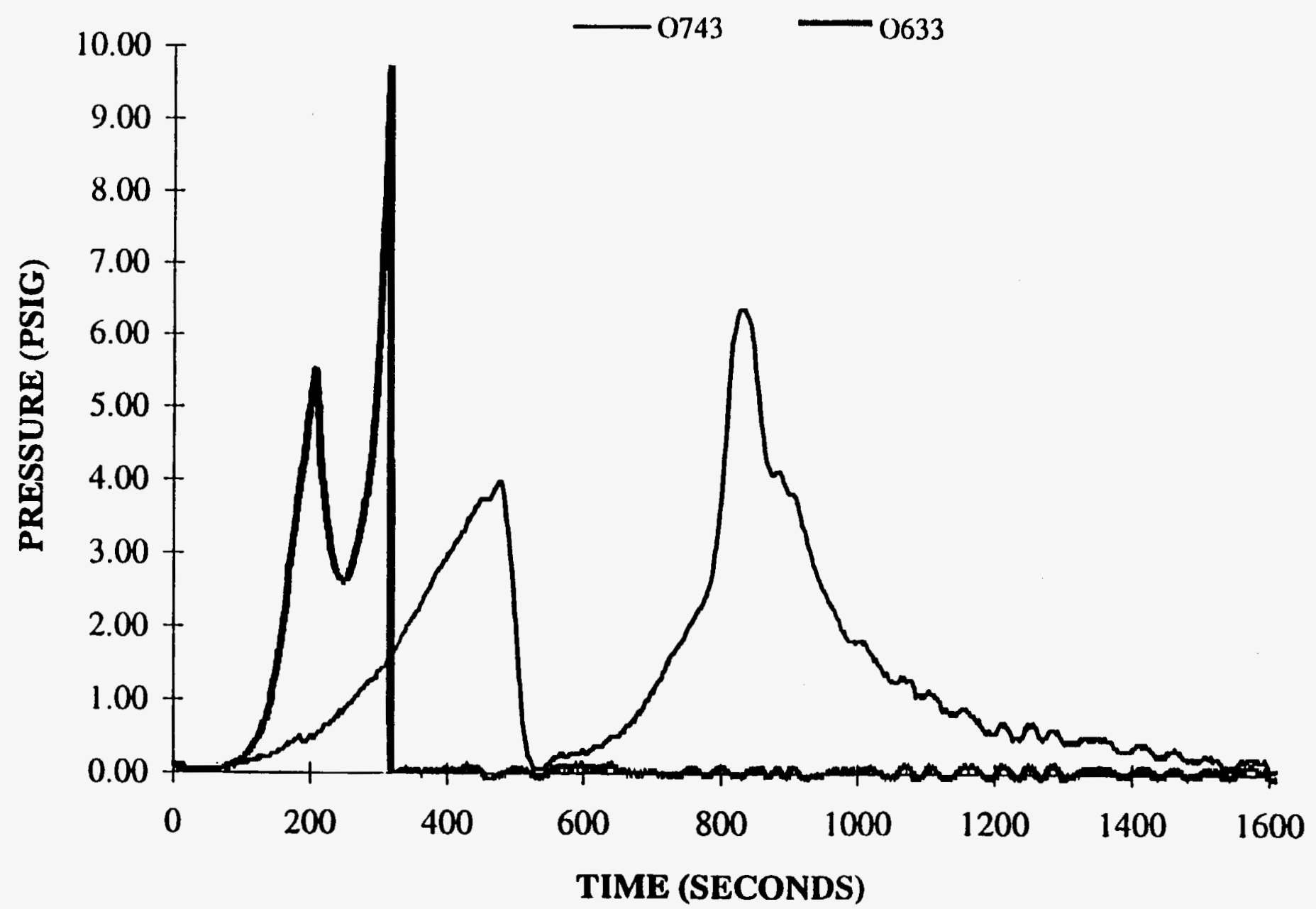

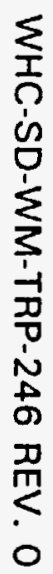


CLIENT: LATA

PROJECT NO.: SwRI 01-7106

DATE: 6 JUNE 1995

TEST ID: P22.PRN

\section{PALLET STORAGE TEST INTERNAL DRUM PRESSURES \\ (TIER \#3)}

$\prod_{i}^{i}$

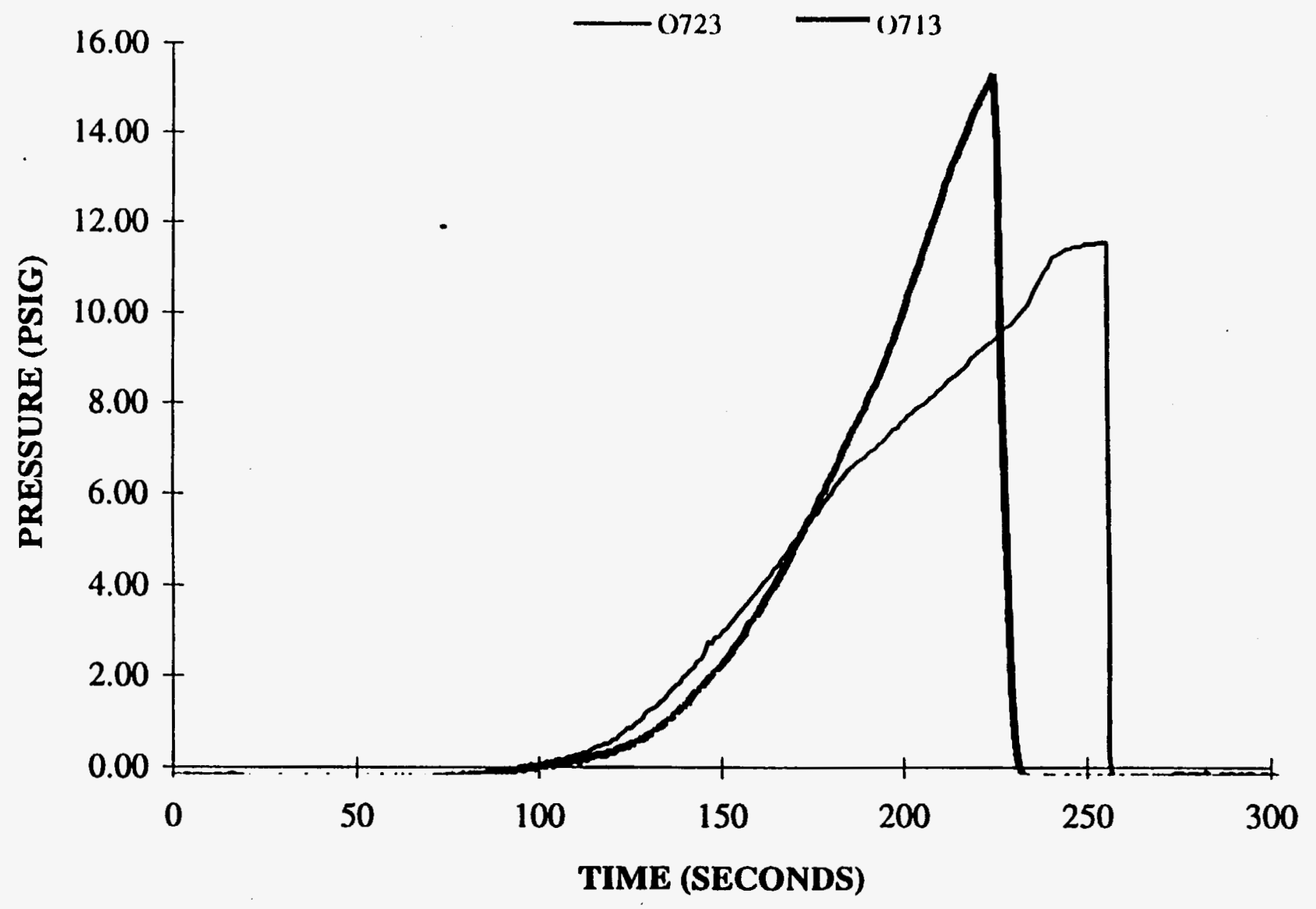

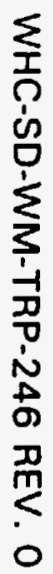

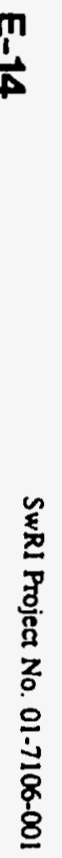

. 


\section{CLIENT: LATA}

PROJECT NO.: SwRI 01-7106

DATE: 6 JUNE 1995

TEST ID: P22.PRN

\section{PALLET STORAGE TEST \\ INTERNAL DRUM PRESSURES \\ (TIER \#3)}

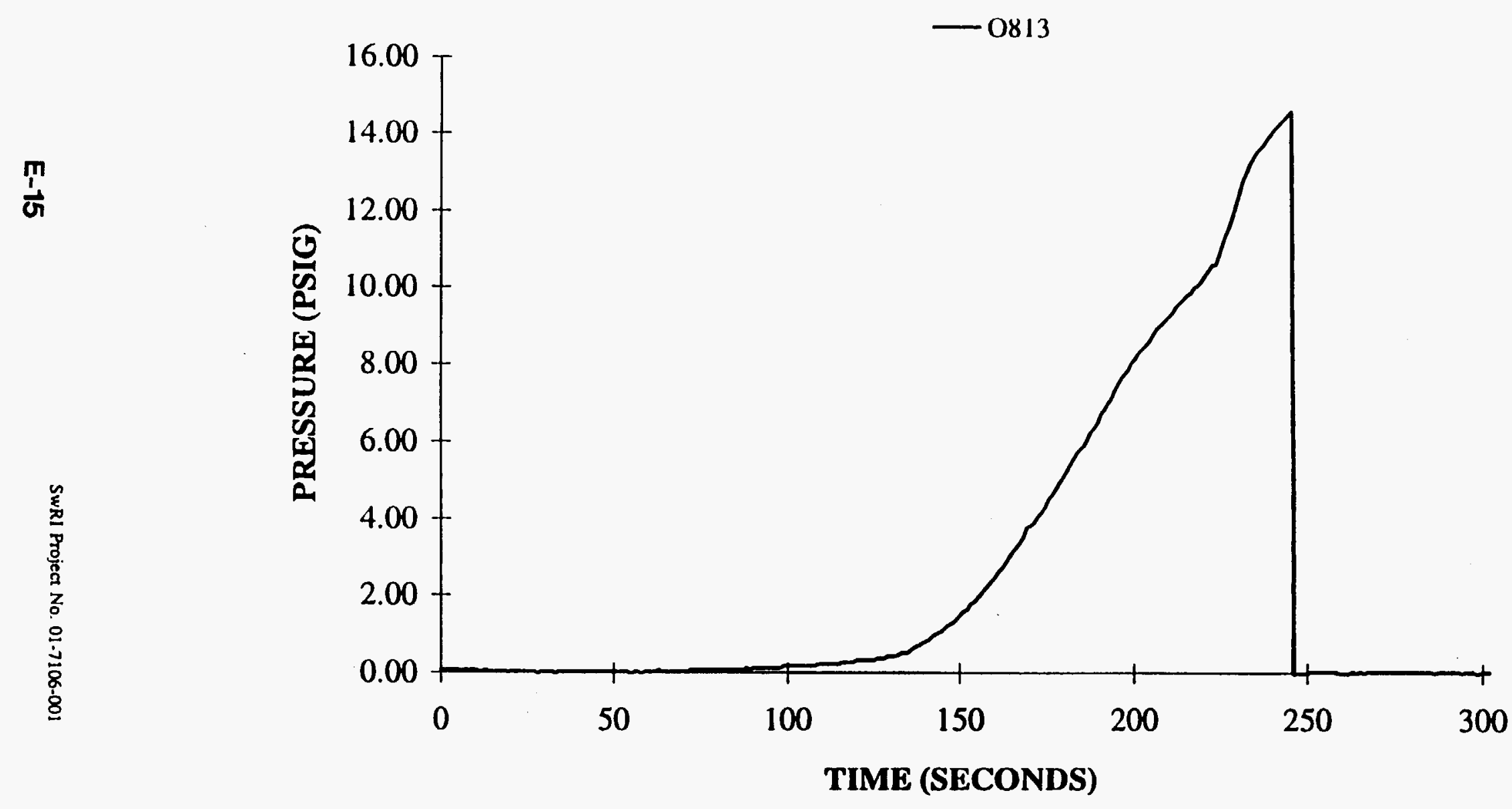


CLIENT: LATA

PROJECT NO.: SwRI 01-7106

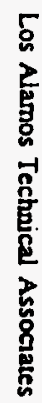

DATE: 6 JUNE 1995

TEST ID: 163LR6.DAT

कั

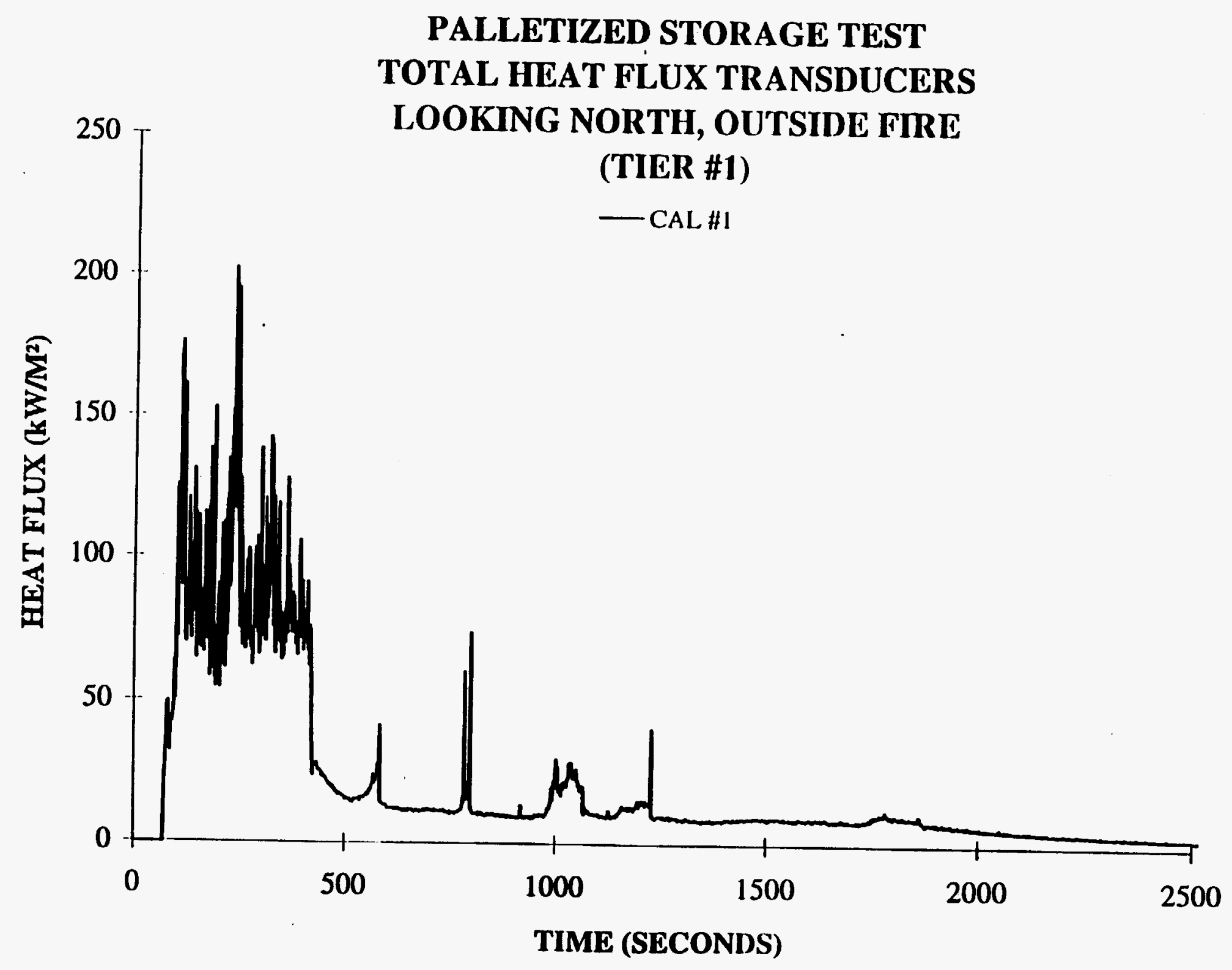

TIME (SECONDS) 


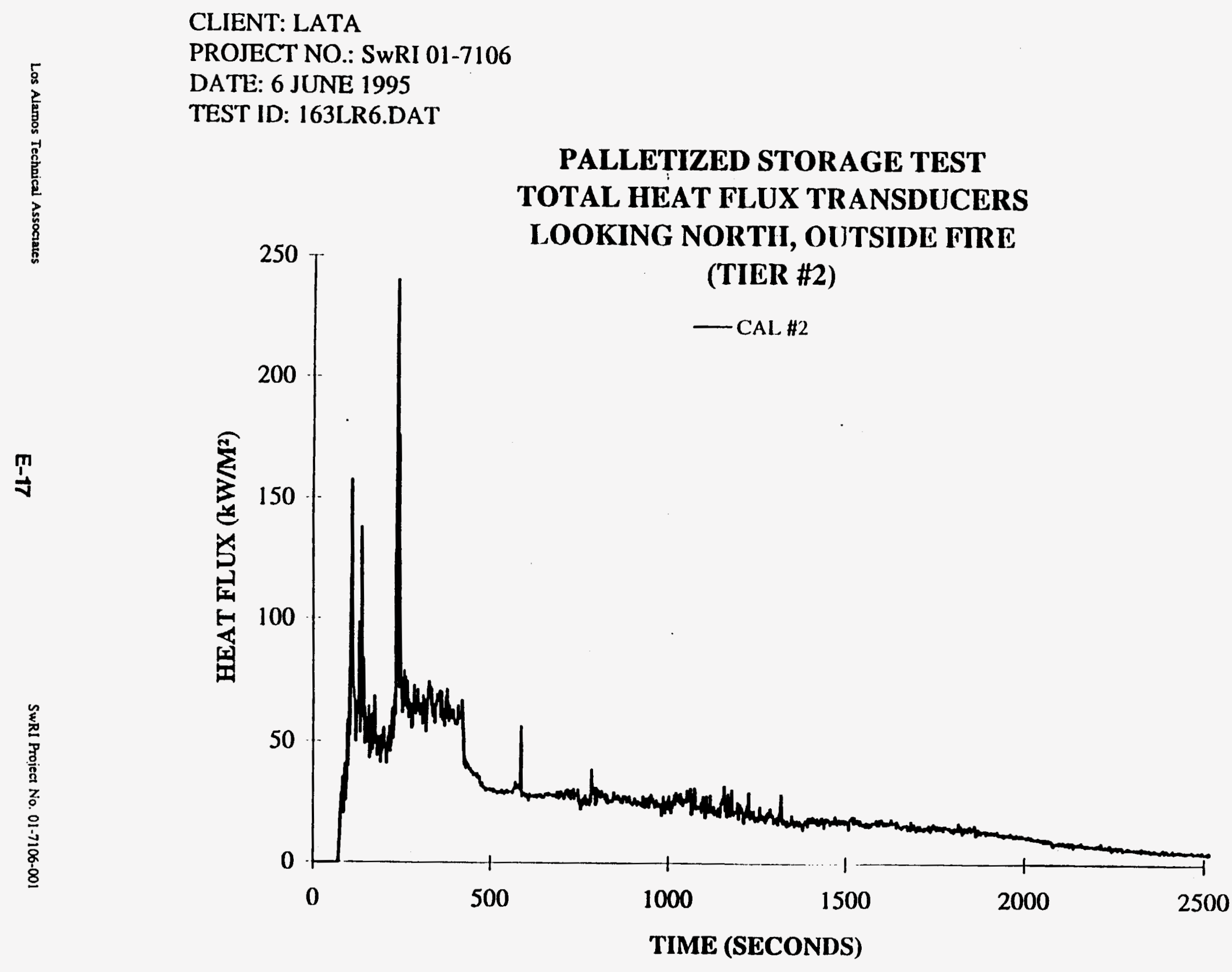

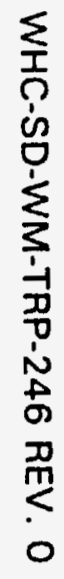


CLIENT: LATA

PROJECT NO.: SwRI 01-7106

DATE: 6 JUNE 1995

TEST ID: 163LR6.DAT

$\underset{\infty}{\infty}$

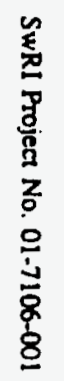

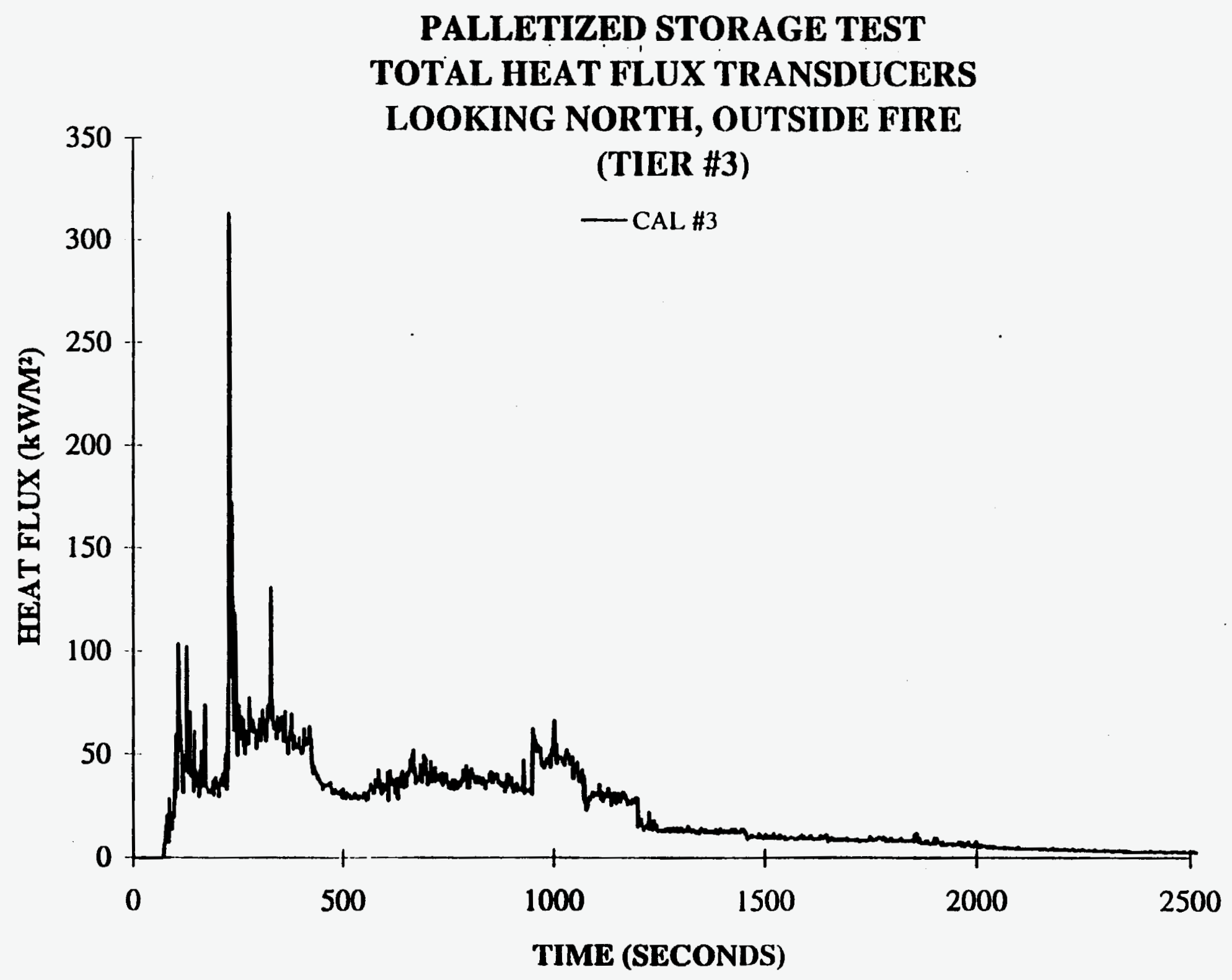




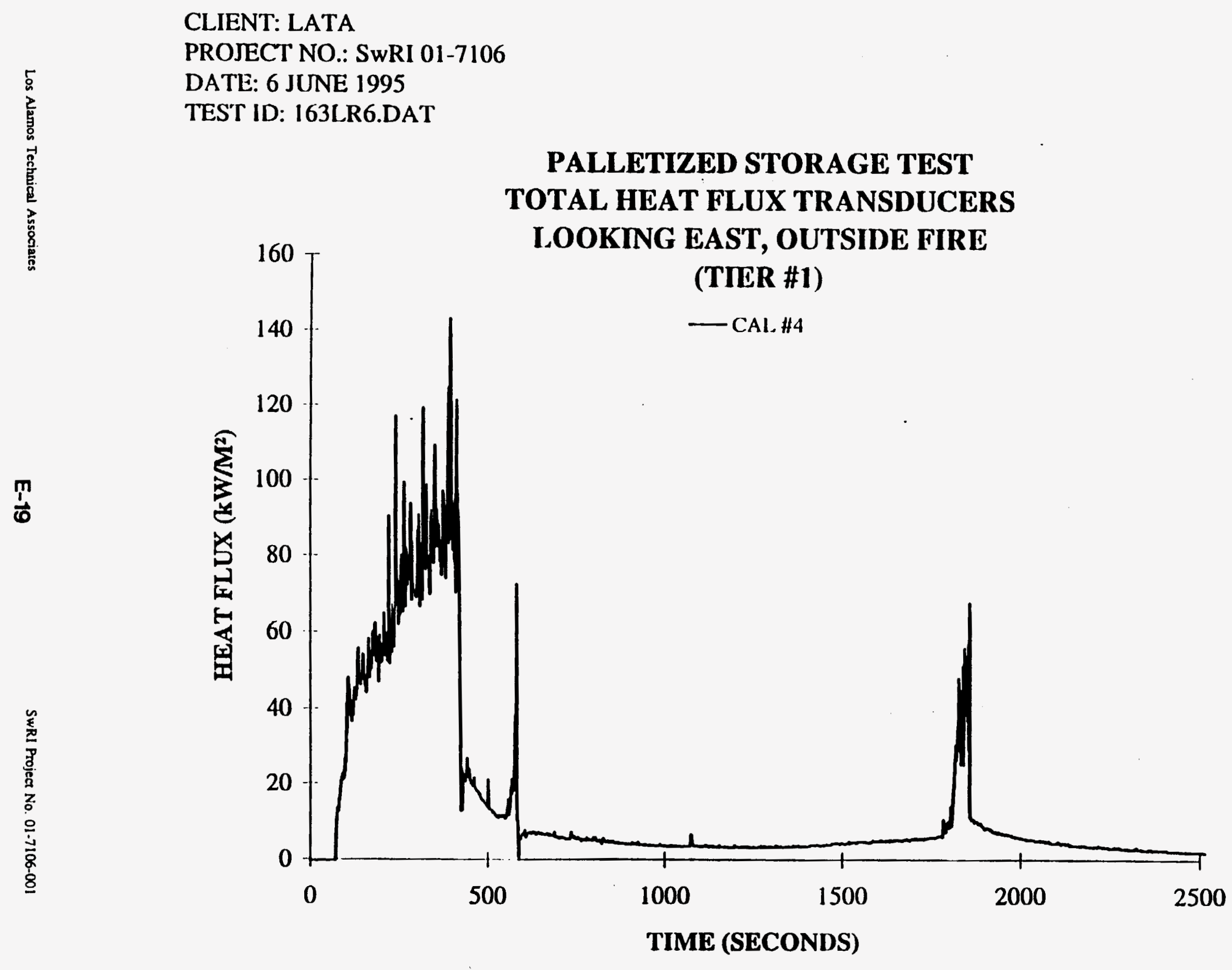


CLIENT: LATA

PROJECT NO.: SwRI 01-7106

DATE: 6 JUNE 1995

TEST ID: 163LR6.DAT

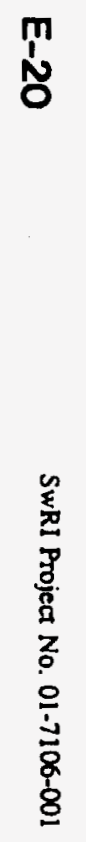

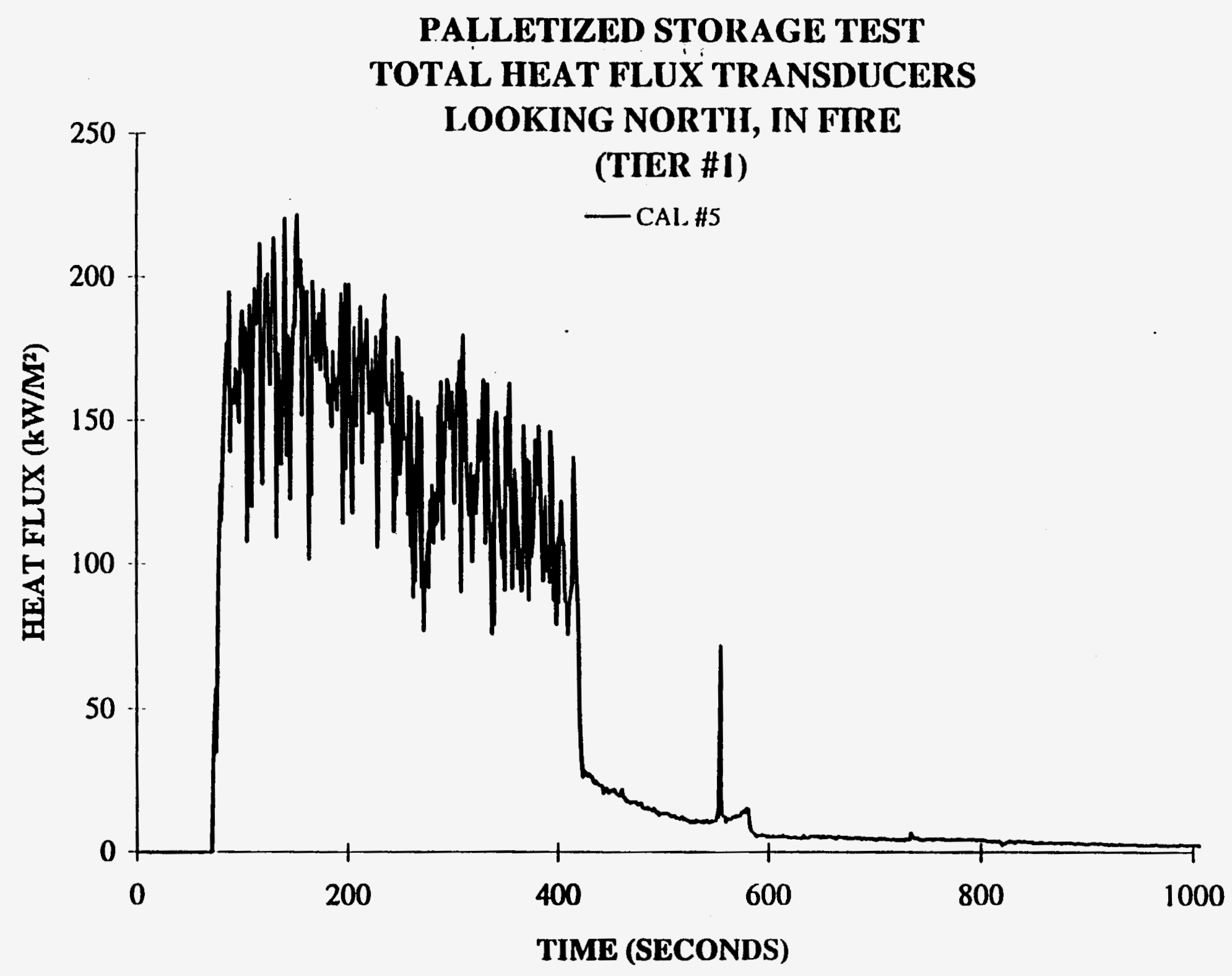

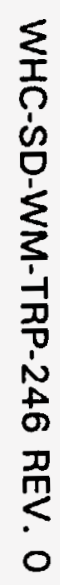

TIME (SECONDS) 


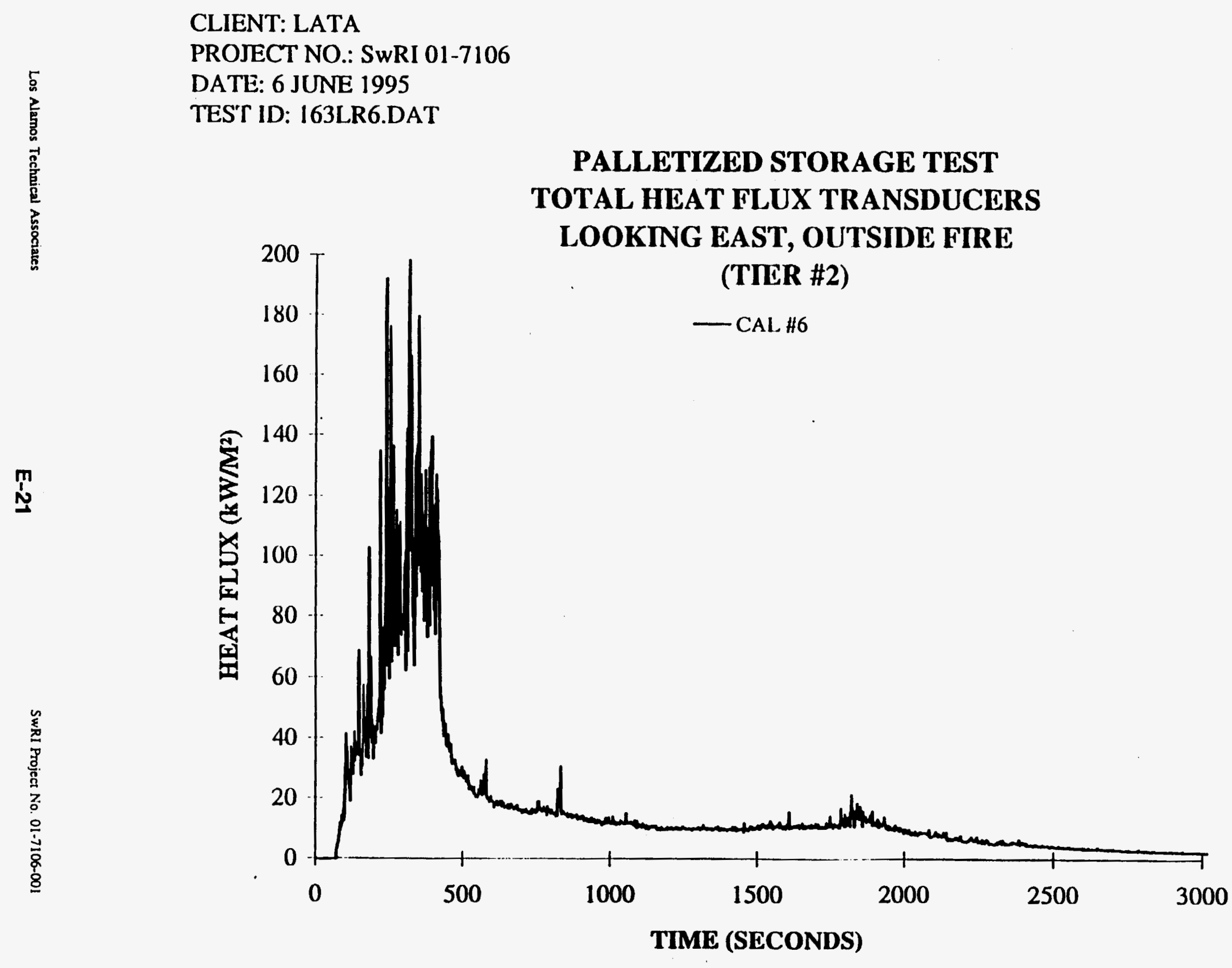




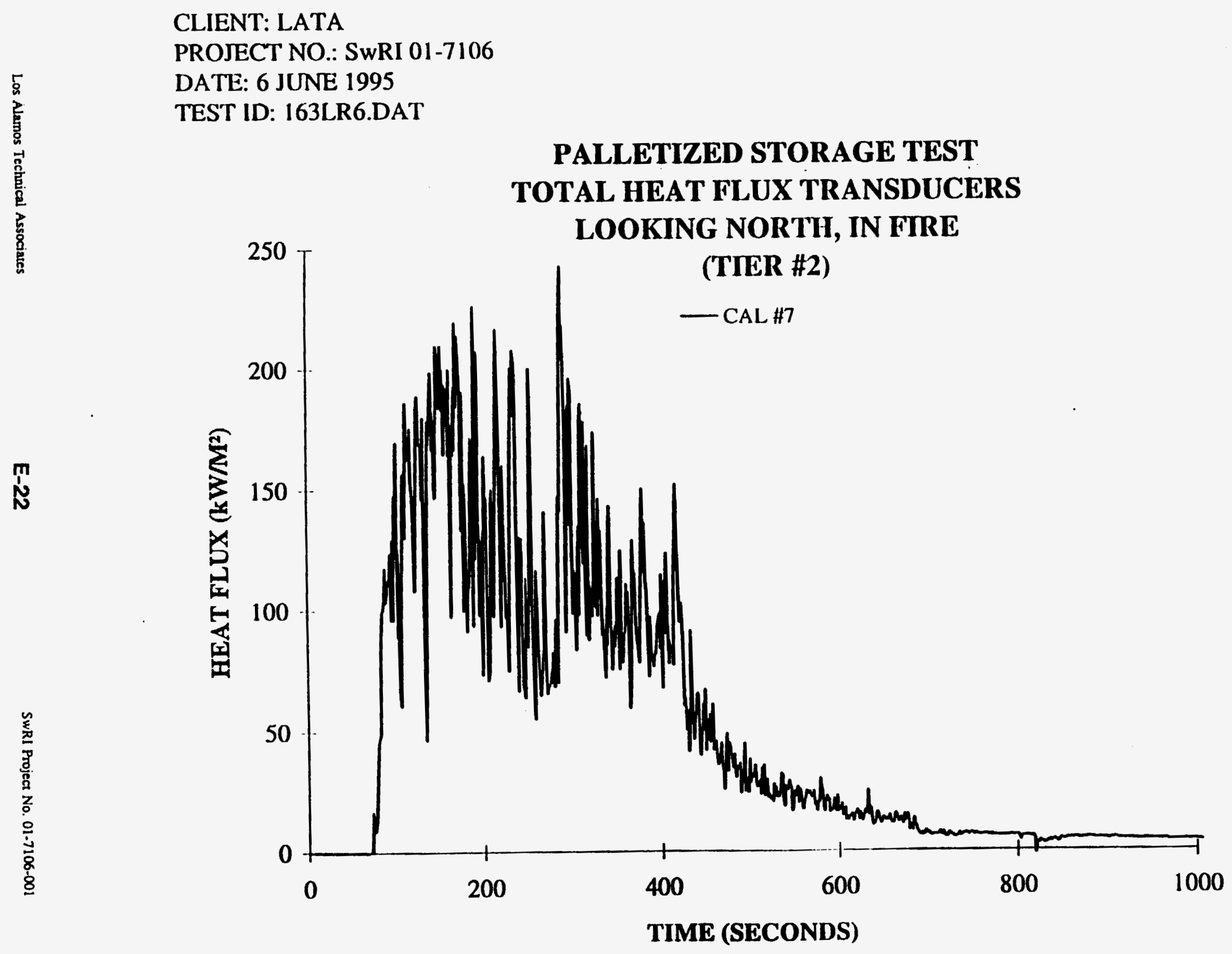


CLIENT: LATA

PROJECT NO.: SwRI 01-7106

DATE: 6 JUNE 1995

TEST ID: 163LR6.DAT

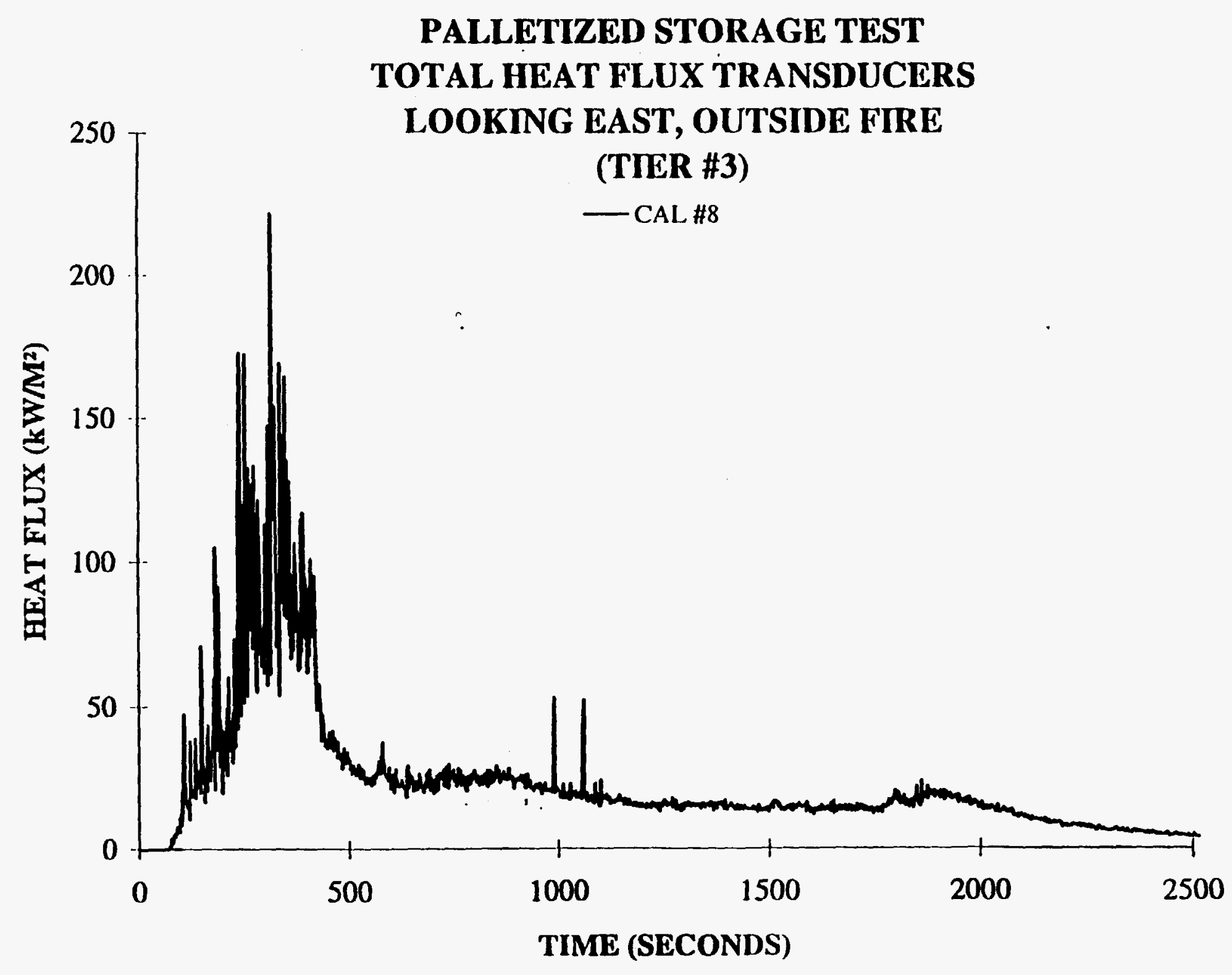




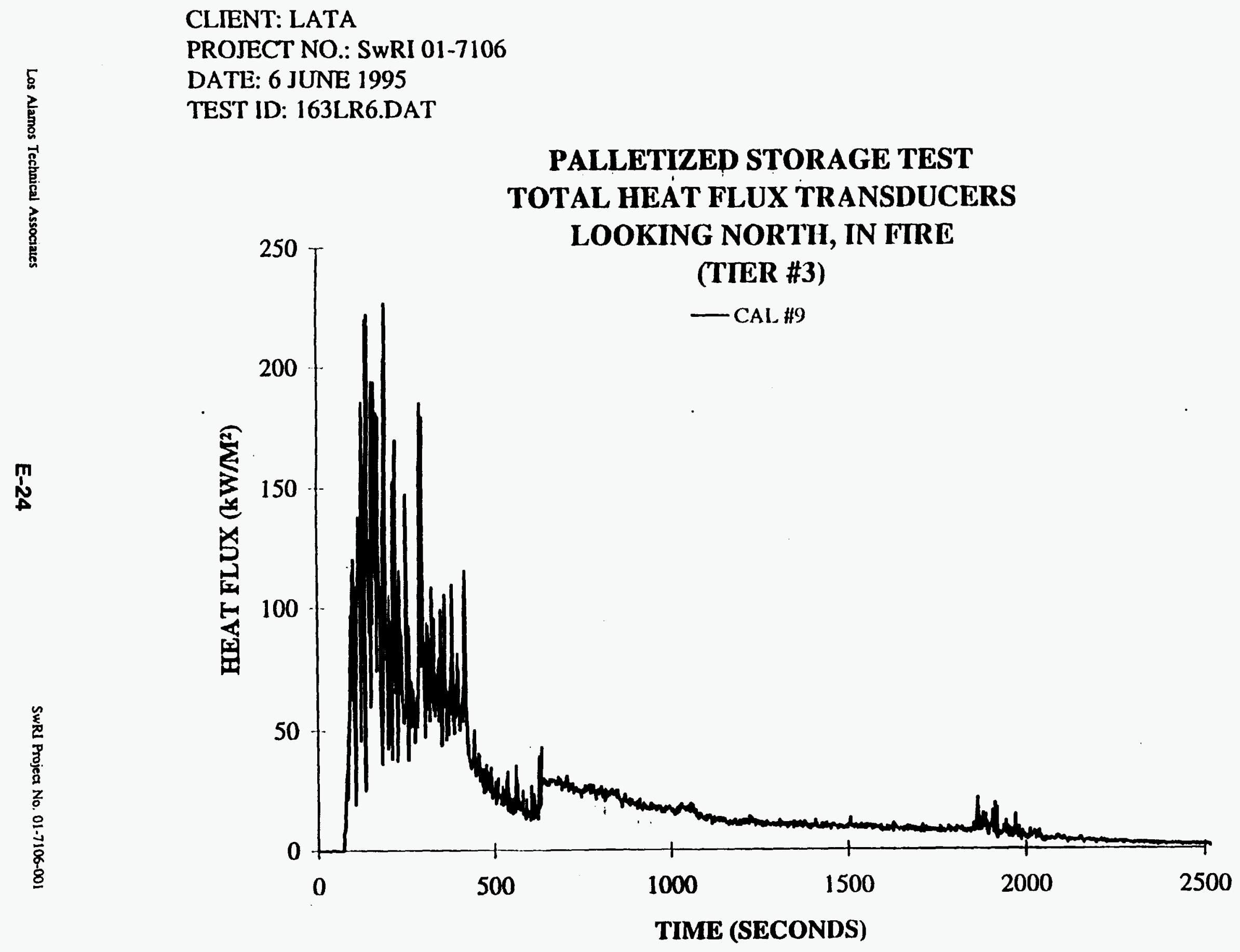

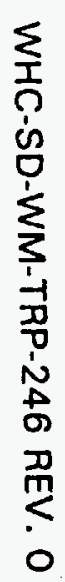




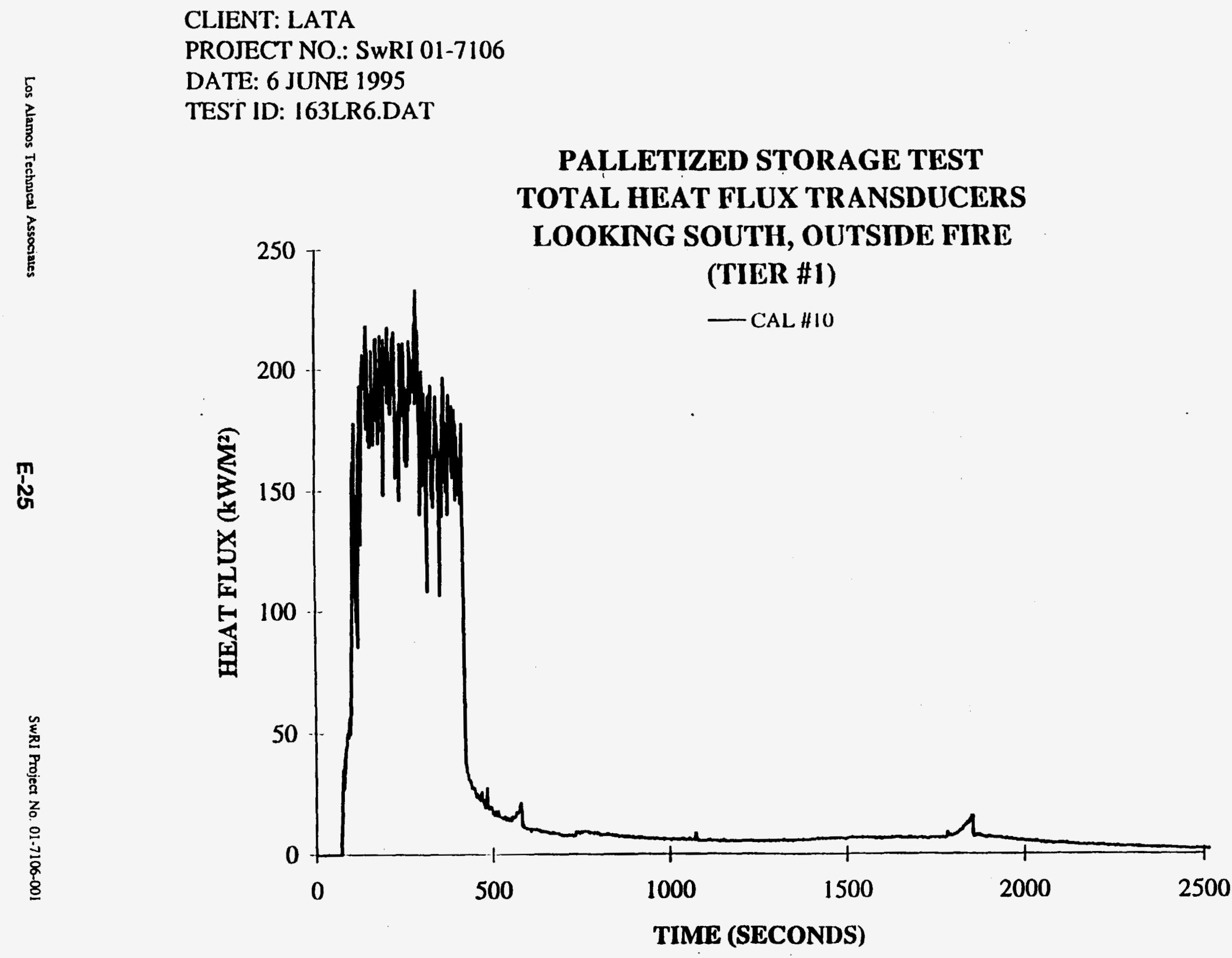




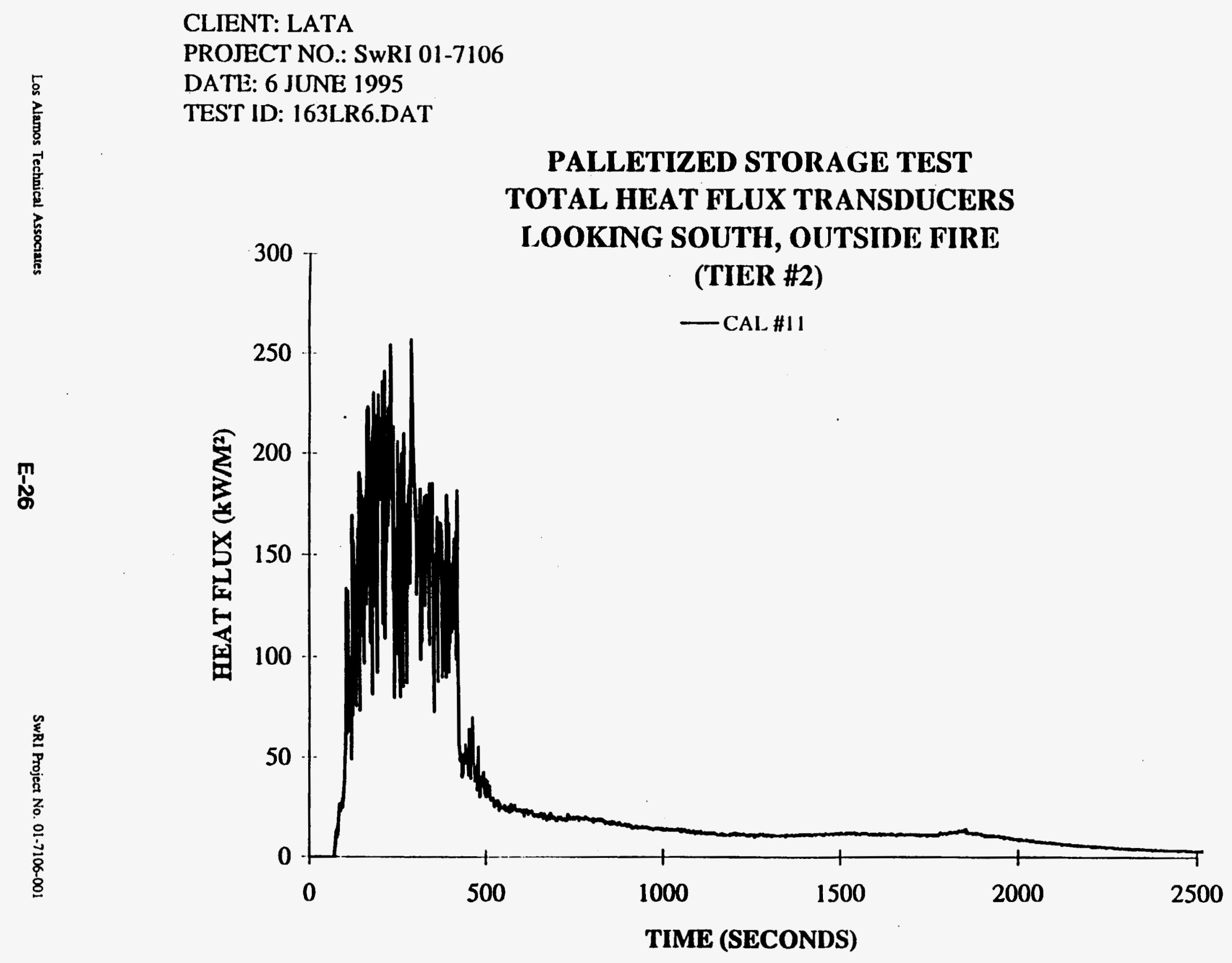




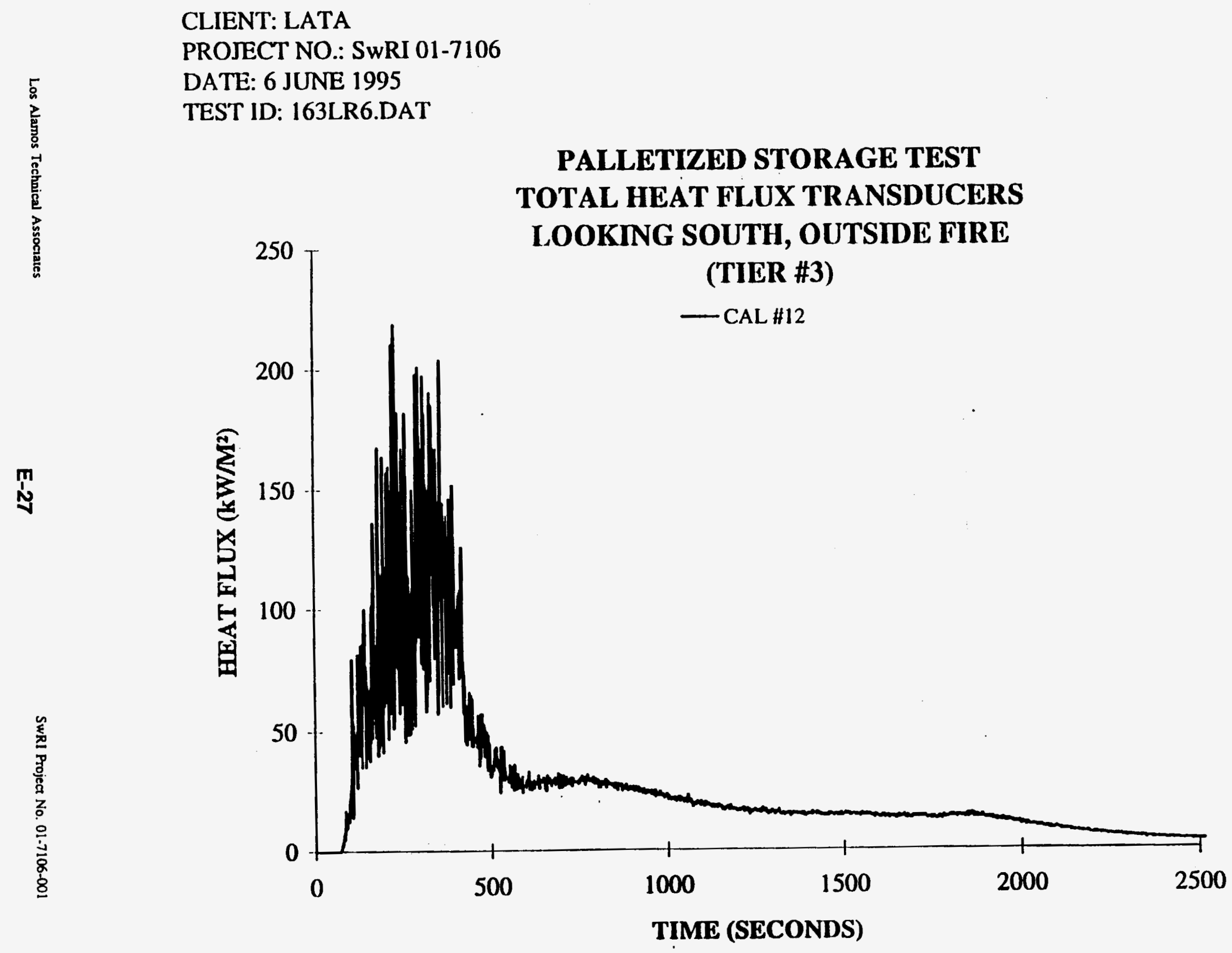


CLIENT: LATA

PROJECT NO.: SwRI 01-7106

DATE: 6 JUNE 1995

TEST ID: 163LP1.DAT

\section{PALLETIZED STORAGE TEST DRUM SURFACE TC'S}

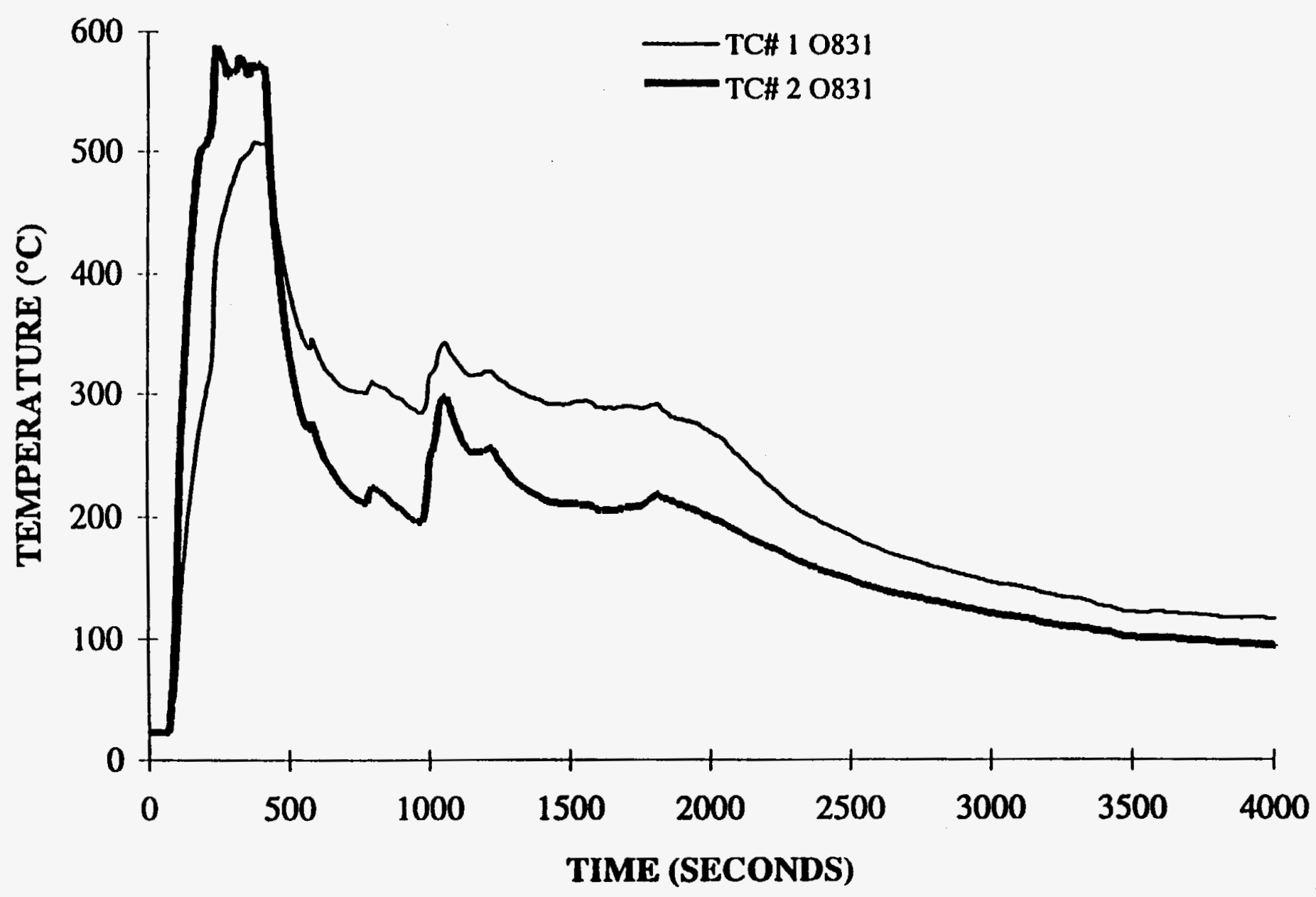


CLIENT: LATA

PROJECT NO.: SwRI 01-7106

DATE: 6 JUNE 1995

TEST ID: 163LP1.DAT

\section{PALLETIZED STORAGE TEST DRUM SURFACE TC'S}

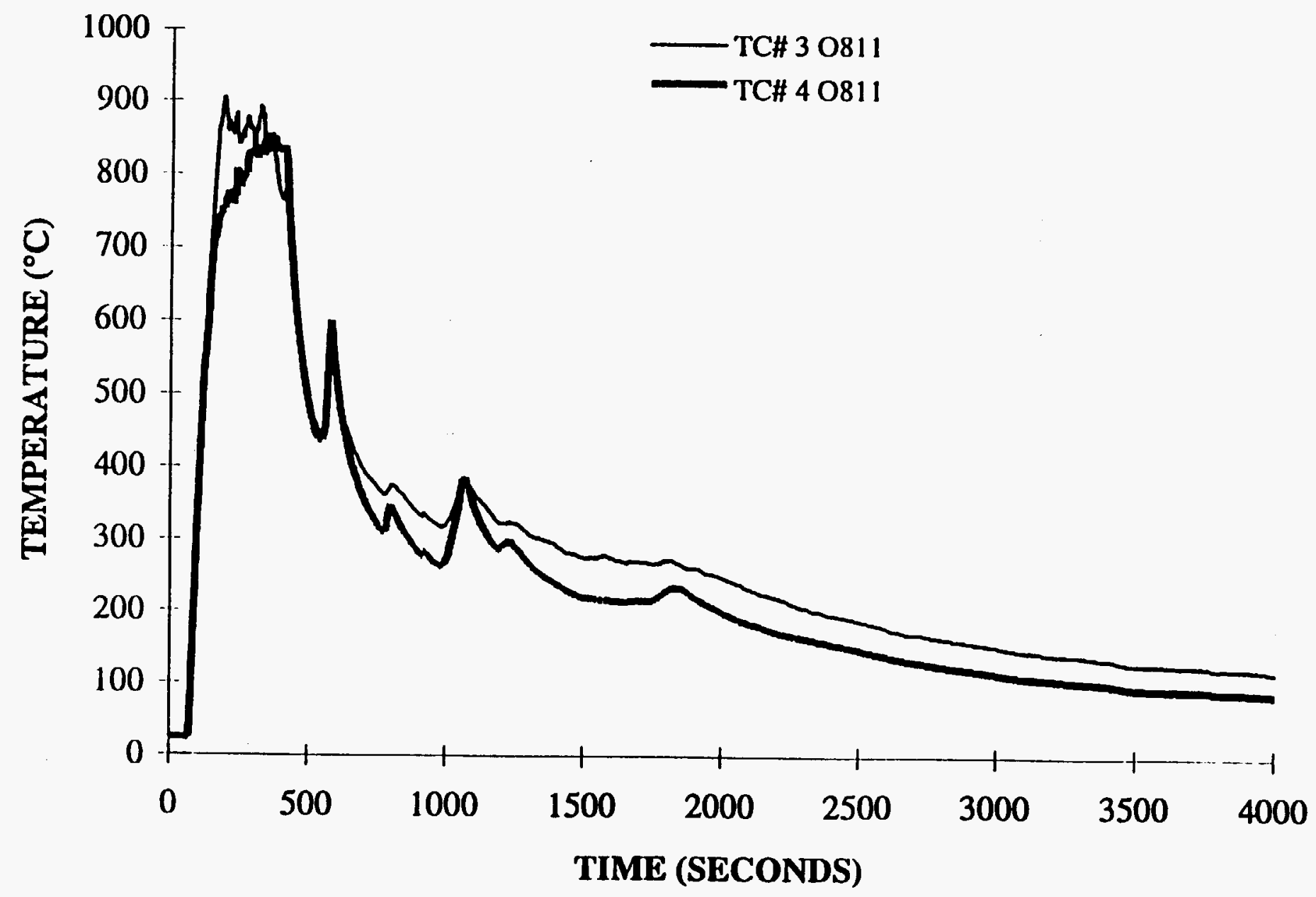


CLIENT: LATA

PROJECT NO.: SwRI 01-7106

DATE: 6 JUNE 1995

TEST ID: 163LP1.DAT

\section{PALlETIZED STORAGE TEST DRUM SURFACE TC'S}

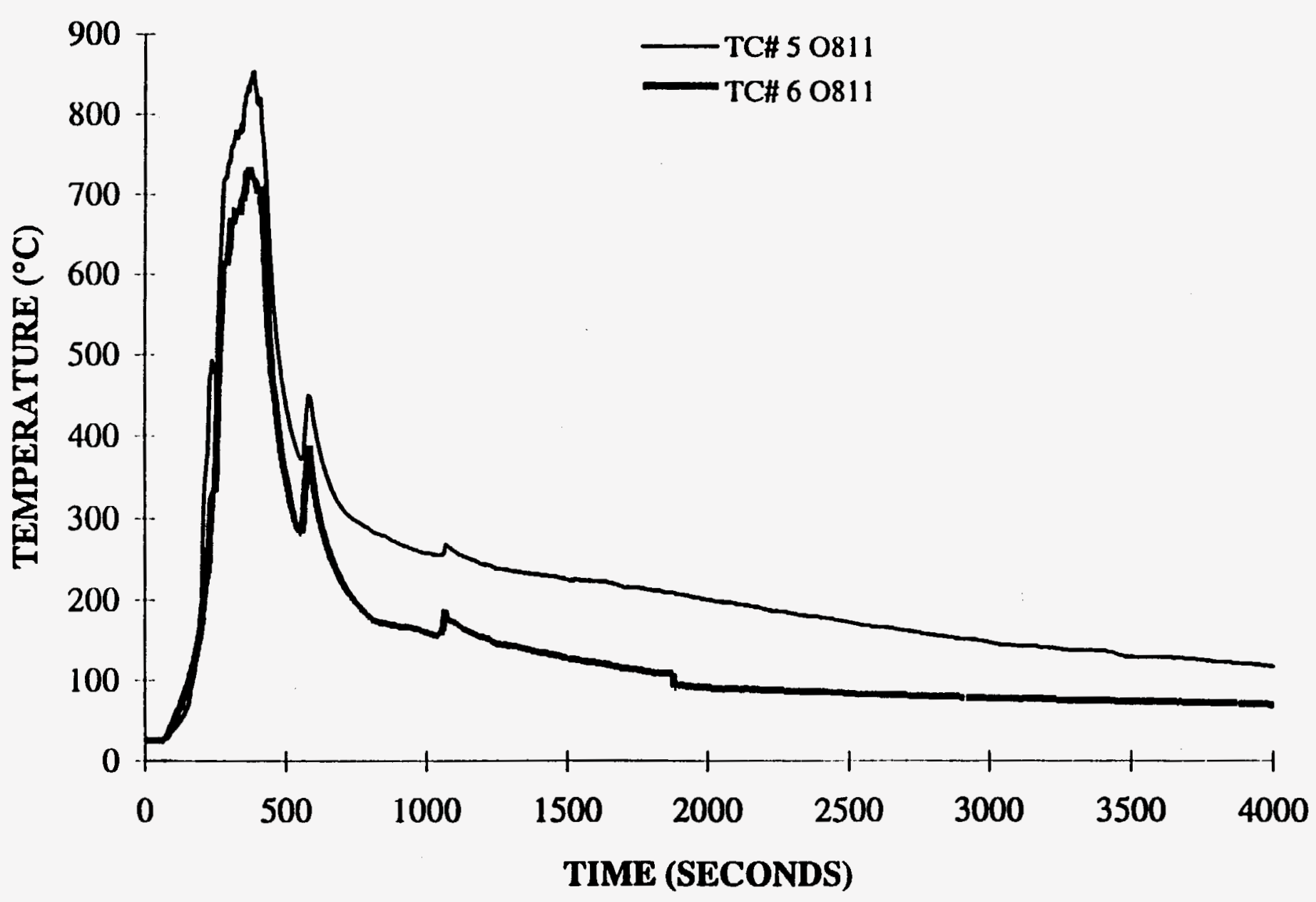




\section{CLIENT: LATA}

PROJECT NO.: SwRI 01-7106

DATE: 6 JUNE 1995

TEST ID: 163LP1.DAT

\section{PALLETIZED STORAGE TEST DRUM SURFACE TC'S}

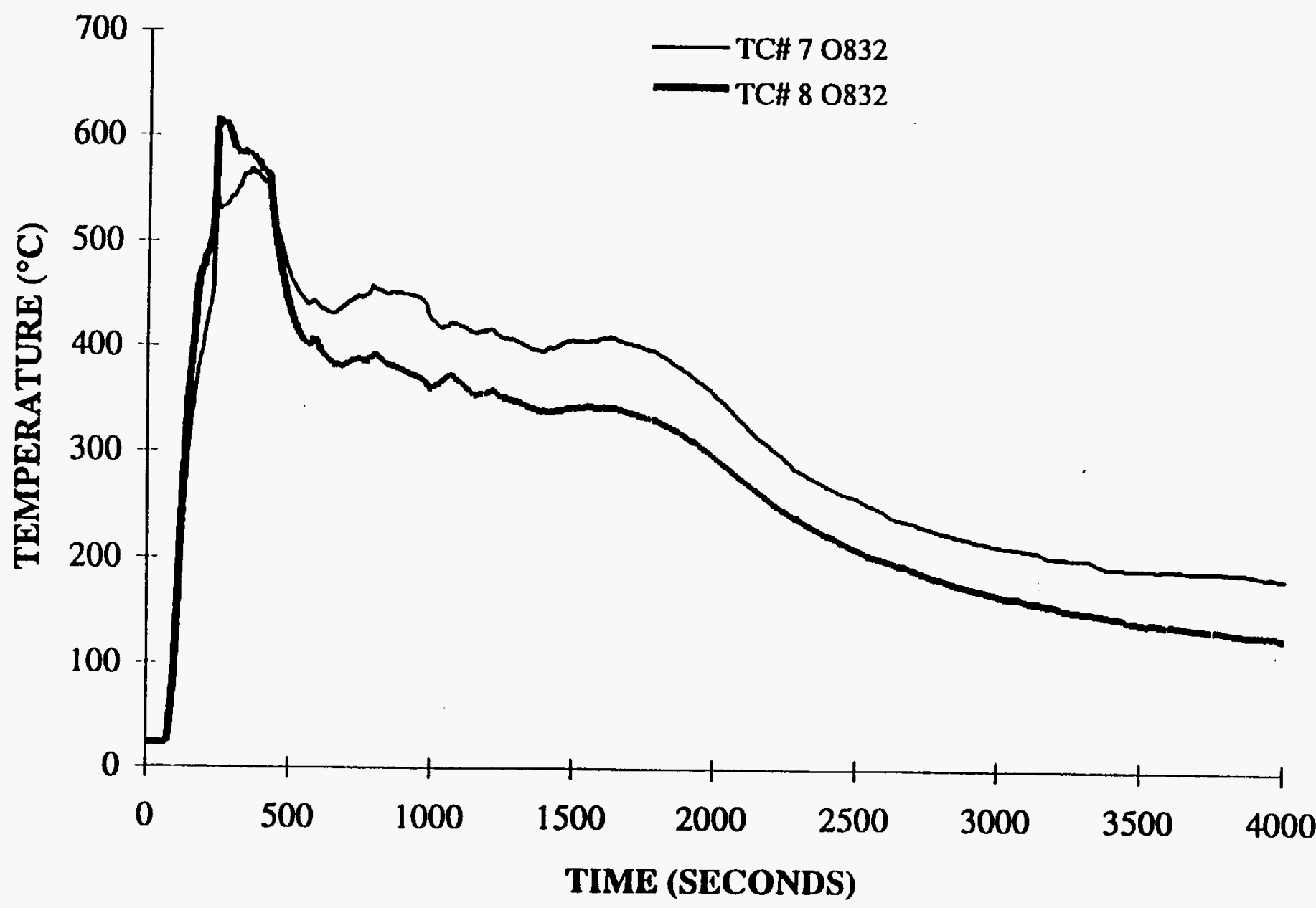




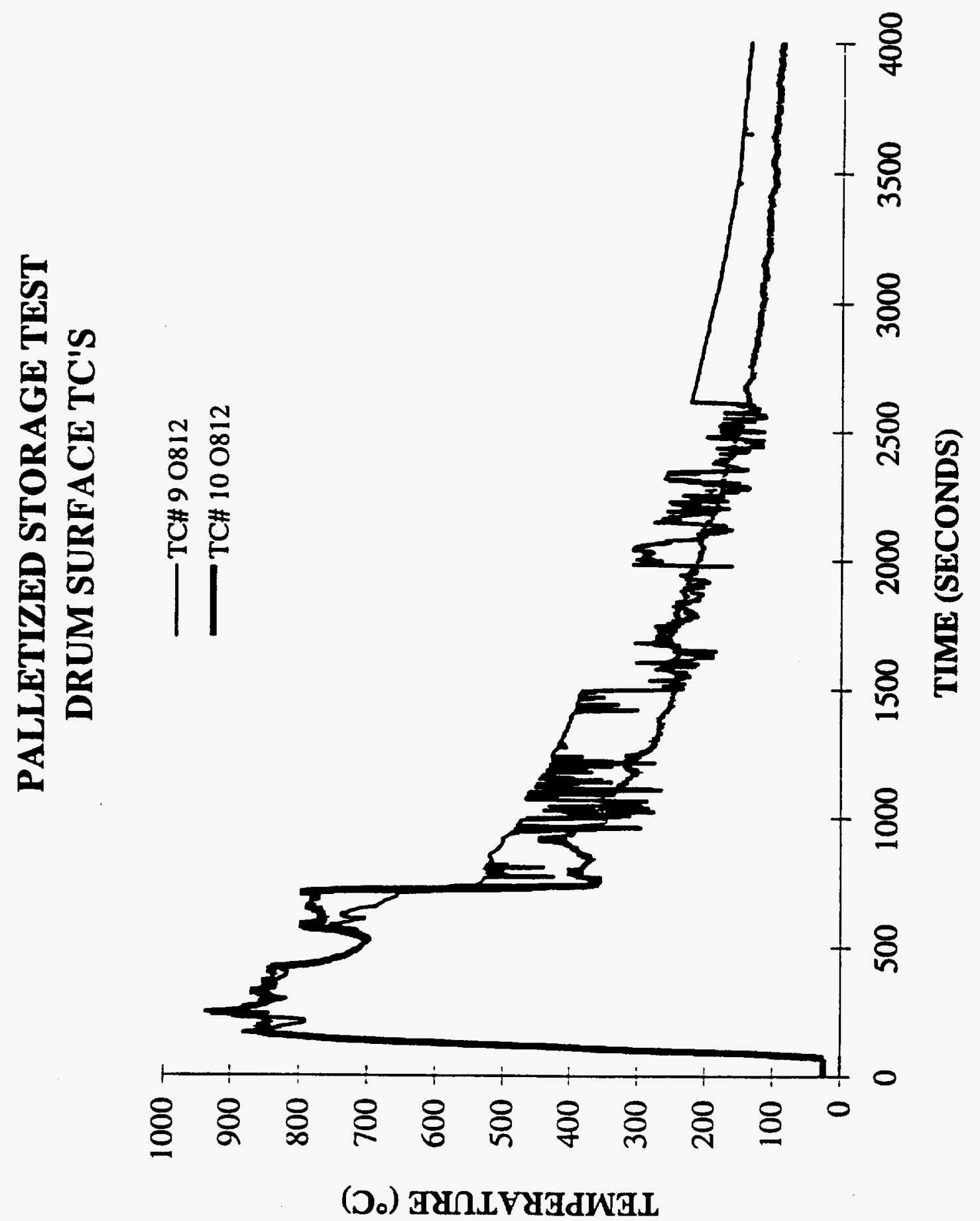


CLIENT: LATA

PROJECT NO.: SwRI 01-7106

DATE: 6 JUNE 1995

TEST ID: 163LP1.DAT

\section{PALLETIZED STORAGE TEST DRUM SURFACE TC'S}

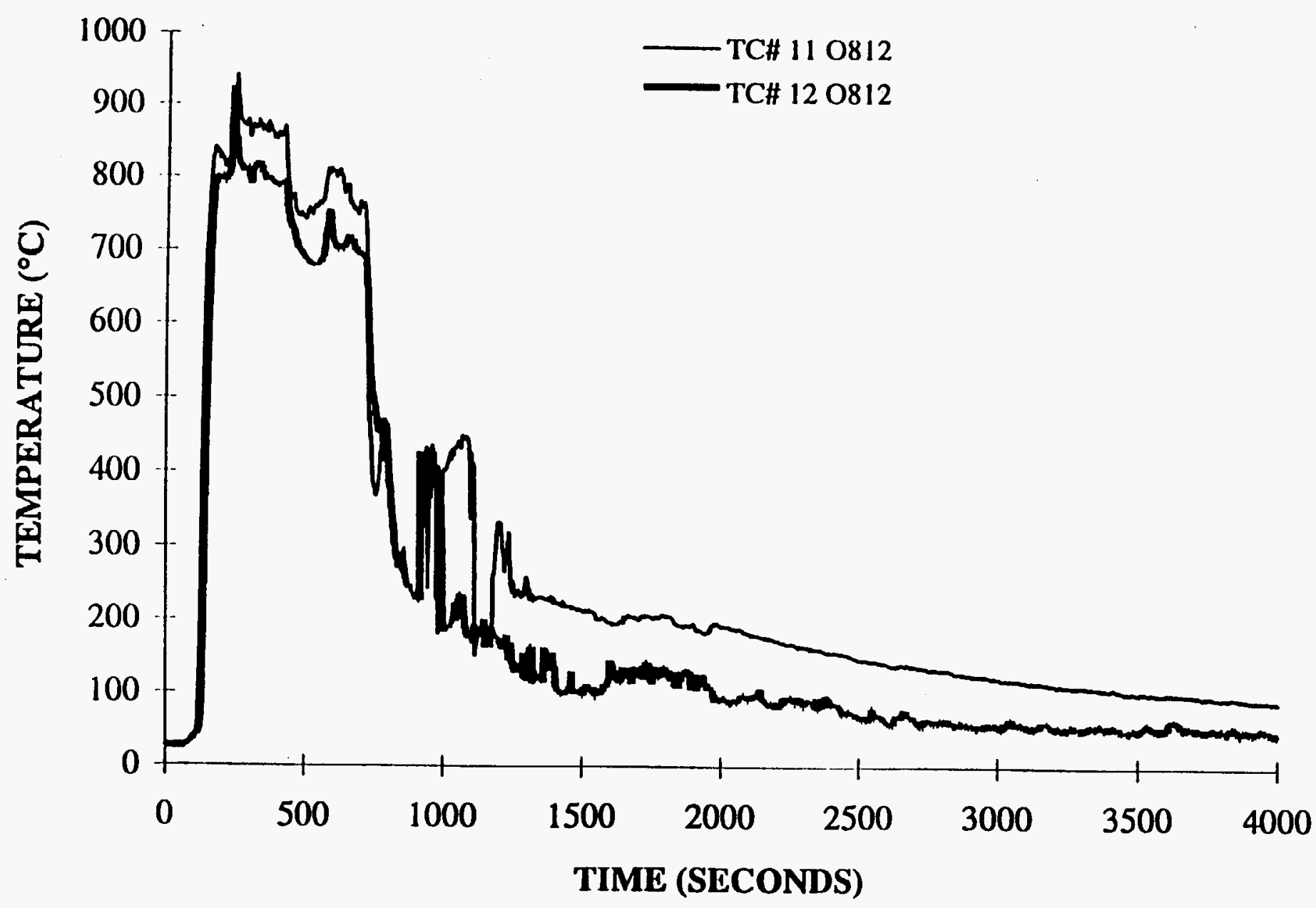


CLIENT: LATA

PROJECT NO.: SwRI 01-7106

DATE: 6 JUNE 1995

TEST ID: 163LP1.DAT

\section{PALLETIZED STORAGE TEST DRUM SURFACE TC'S}

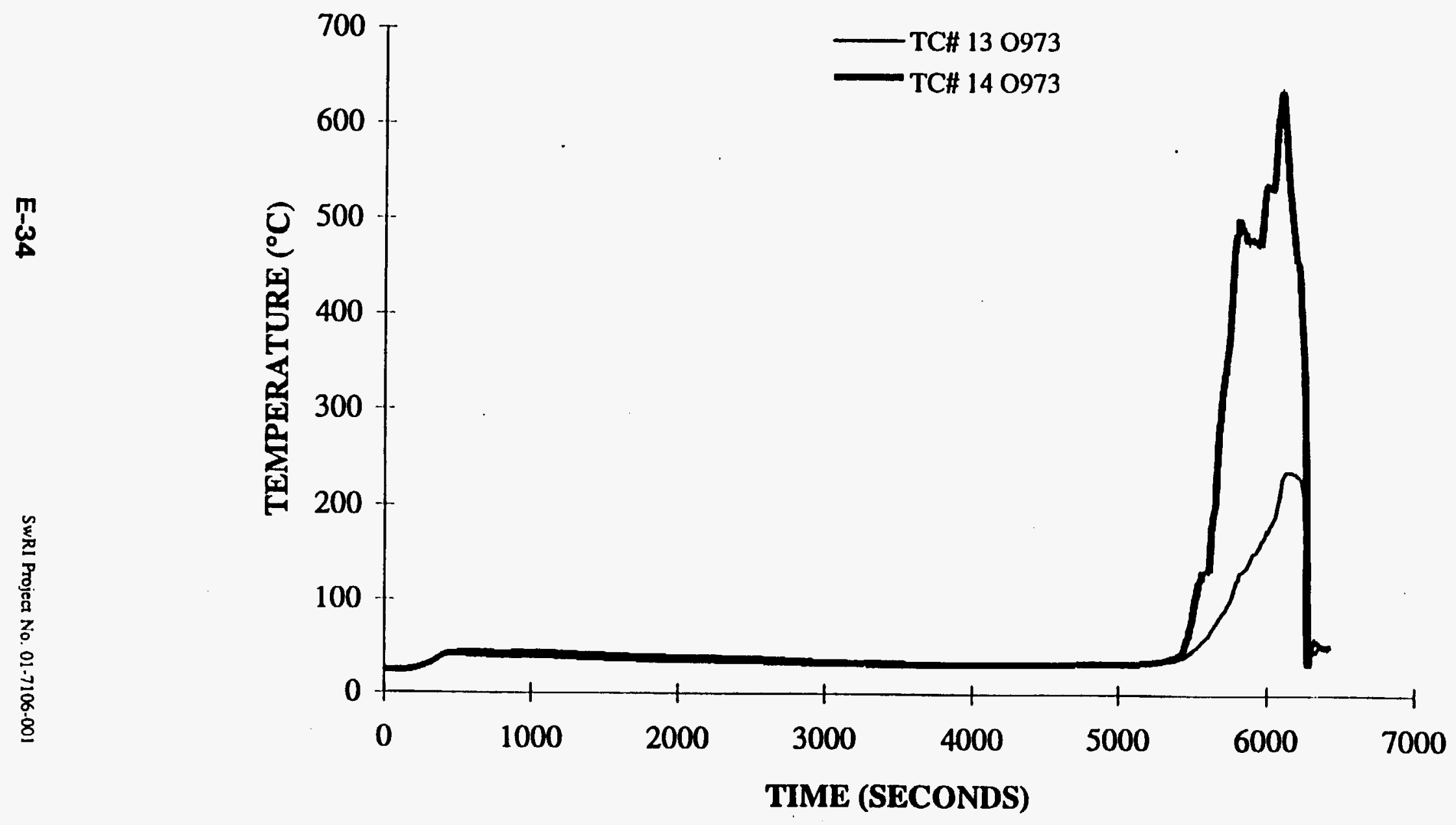


CLIENT: LATA

PROJECT NO.: SwRI 01-7106

DATE: 6 JUNE 1995

TEST ID: 163LP1.DAT

\section{PALLETIZED STORAGE TEST DRUM SURFACE TC'S}

$\underset{\substack{\omega \\ \omega}}{\omega}$

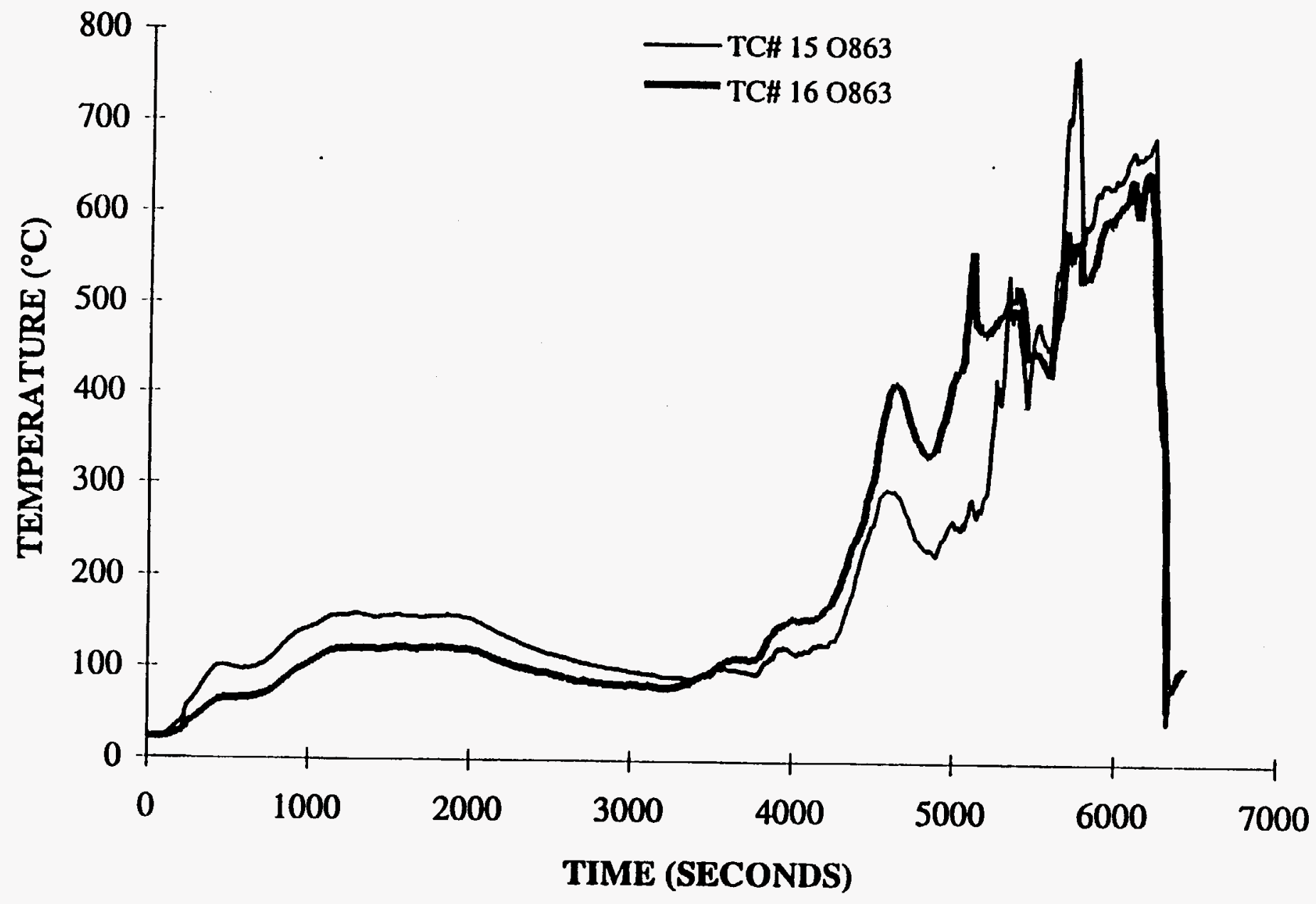


CLIENT: LATA

PROJECT NO.: SwRI 01-7106

DATE: 6 JUNE 1995

TEST ID: 163LP1.DAT

\section{PALLETIZED STORAGE TEST DRUM SURFACE TC'S}

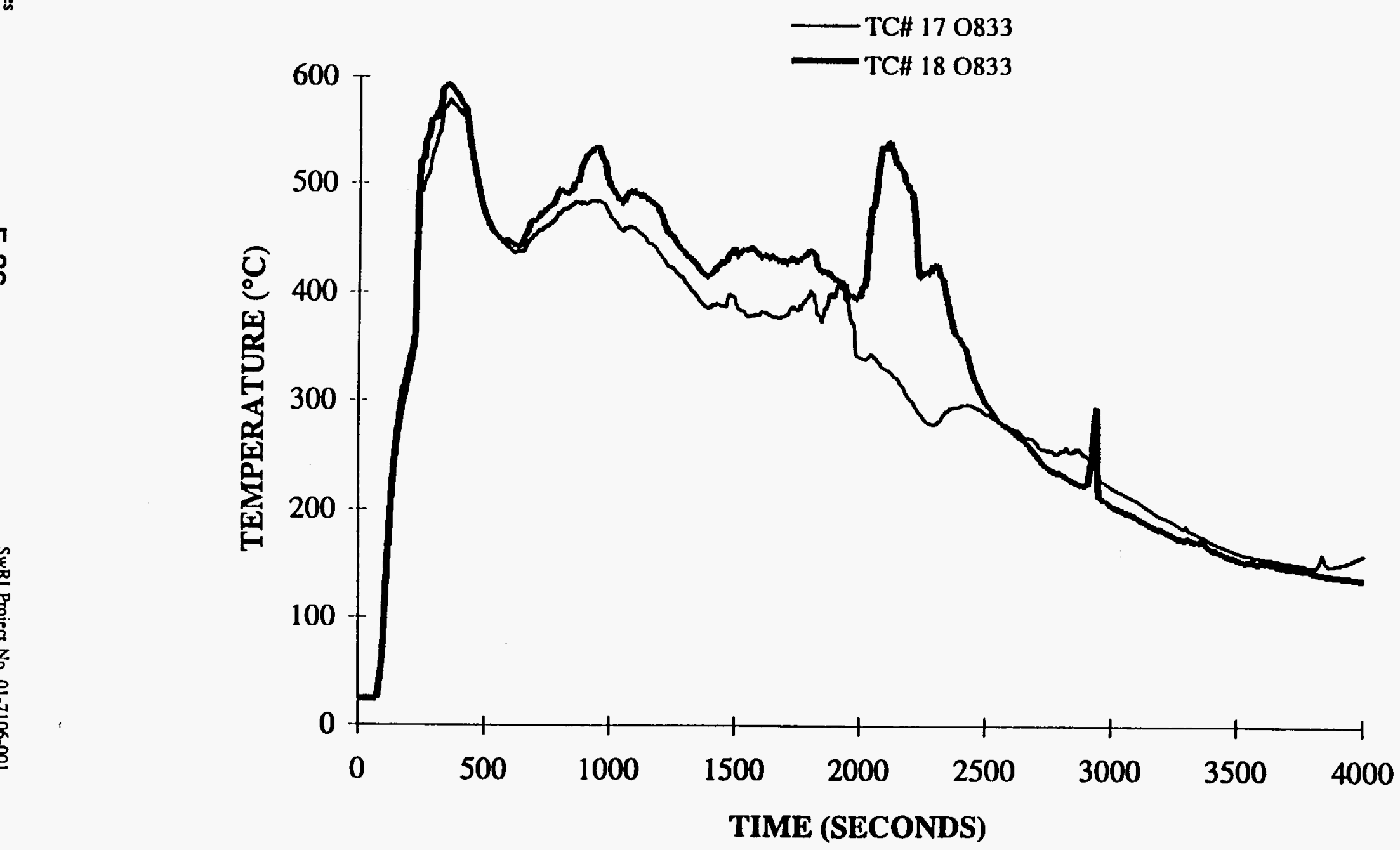

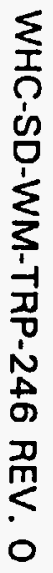

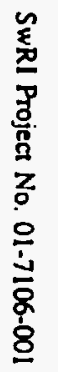

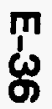


CLIENT: LATA

PROJECT NO.: SwRI 01-7106

DATE: 6 JUNE 1995

TEST ID: 163LP2.DAT

\section{PALLETIZED STORAGE TEST DRUM SURFACE TC'S}

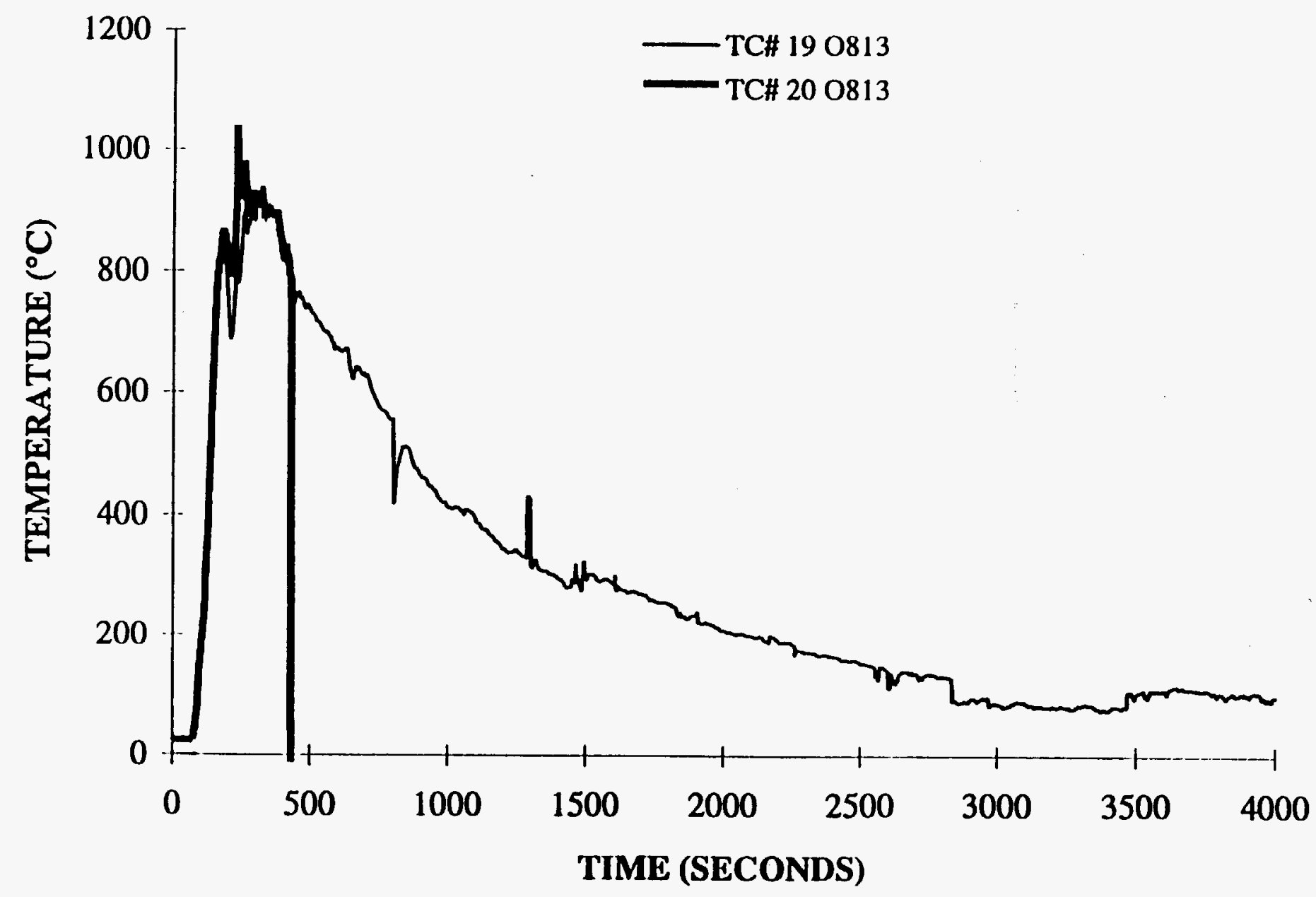


CLIENT: LATA

PROJECT NO.: SwRI 01-7106

DATE: 6 JUNE 1995

TEST ID: 163LP2.DAT

\section{PALLETIZED STORAGE TEST \\ DRUM SURFACE TC'S}

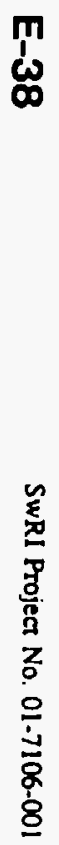

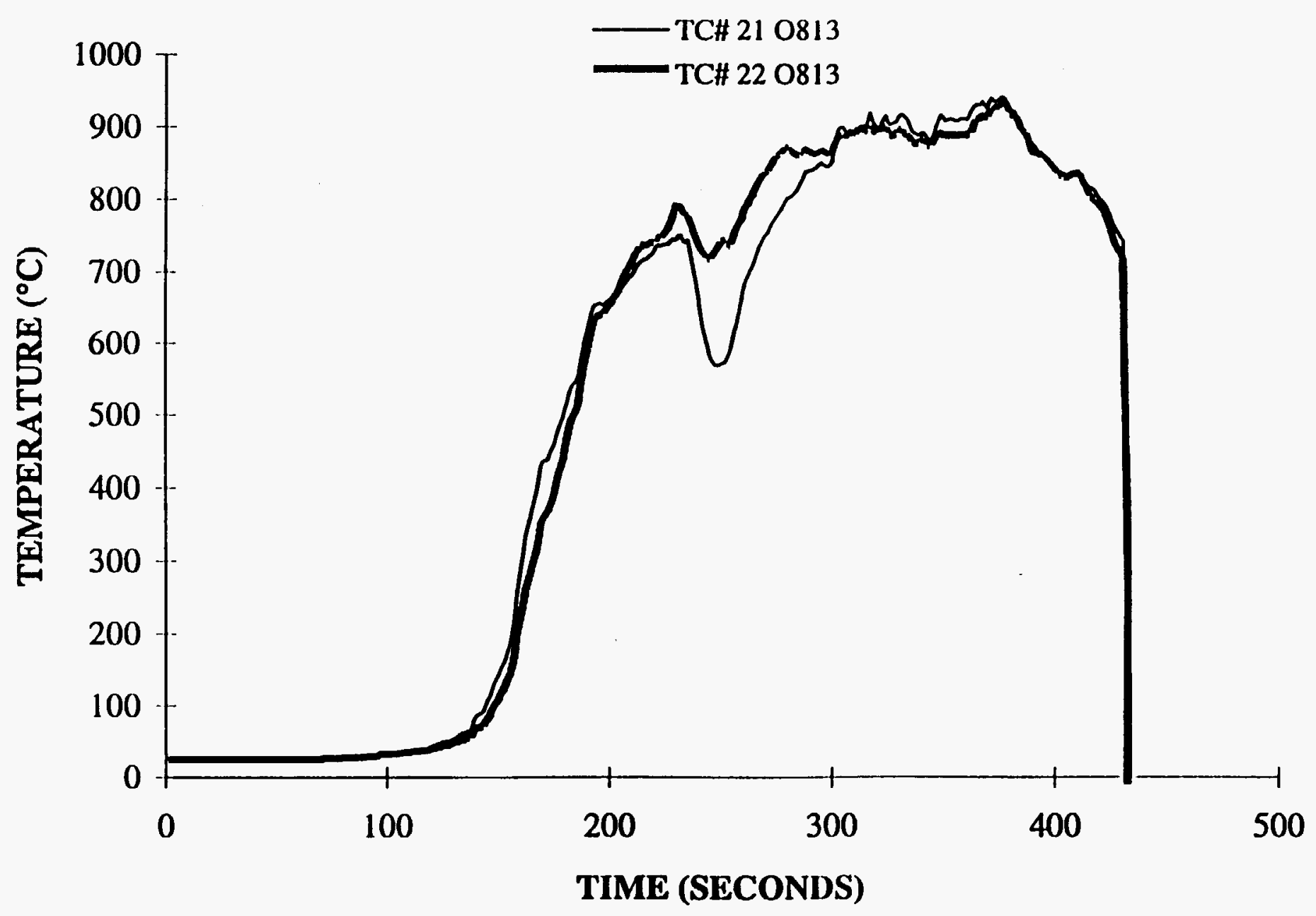


CLIENT: LATA

PROJECT NO.: SwRI 01-7106

DATE: 6 JUNE 1995

TEST ID: 163LP2.DAT

\section{PALLETIZED STORAGE TEST DRUM SURFACE TC'S}

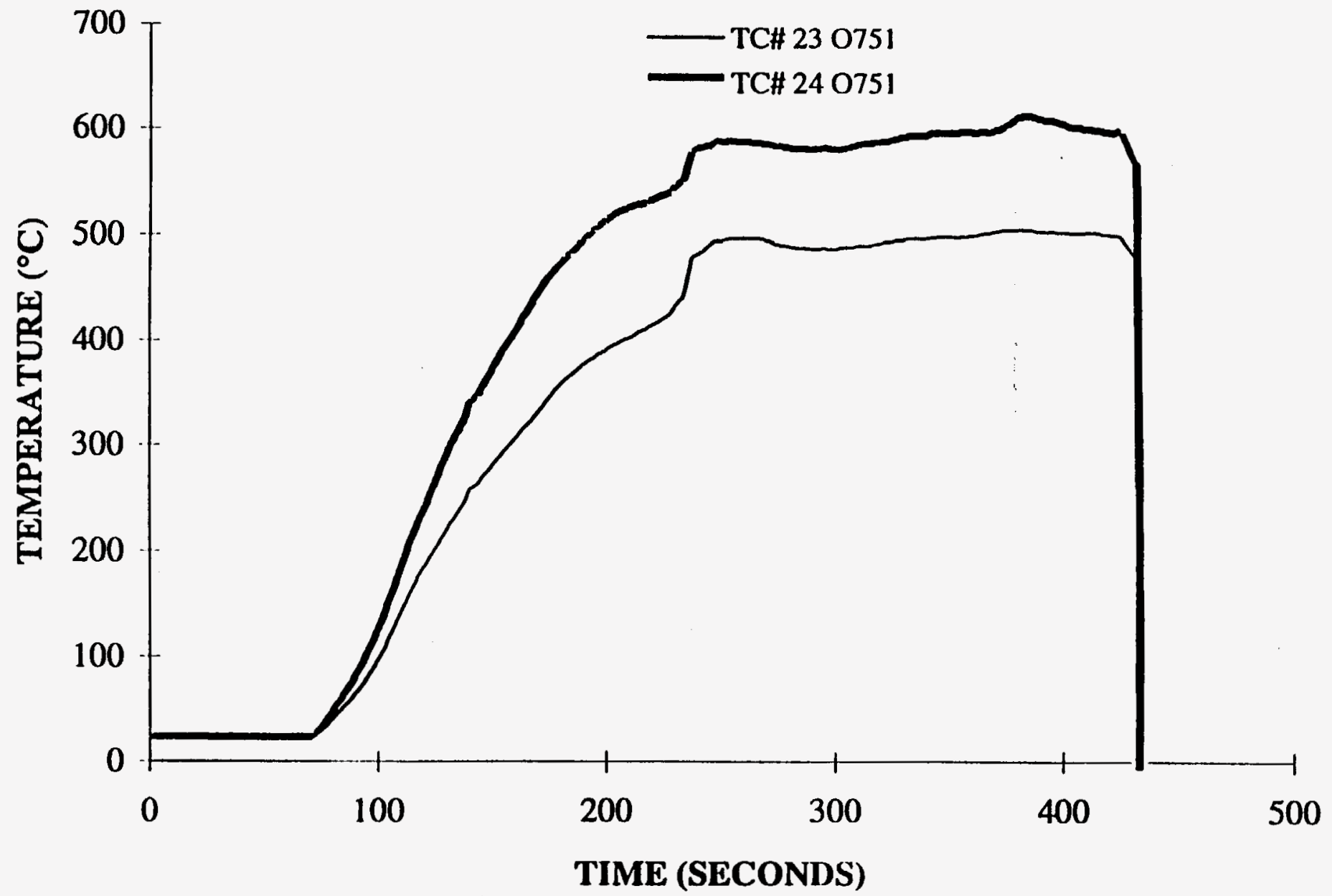




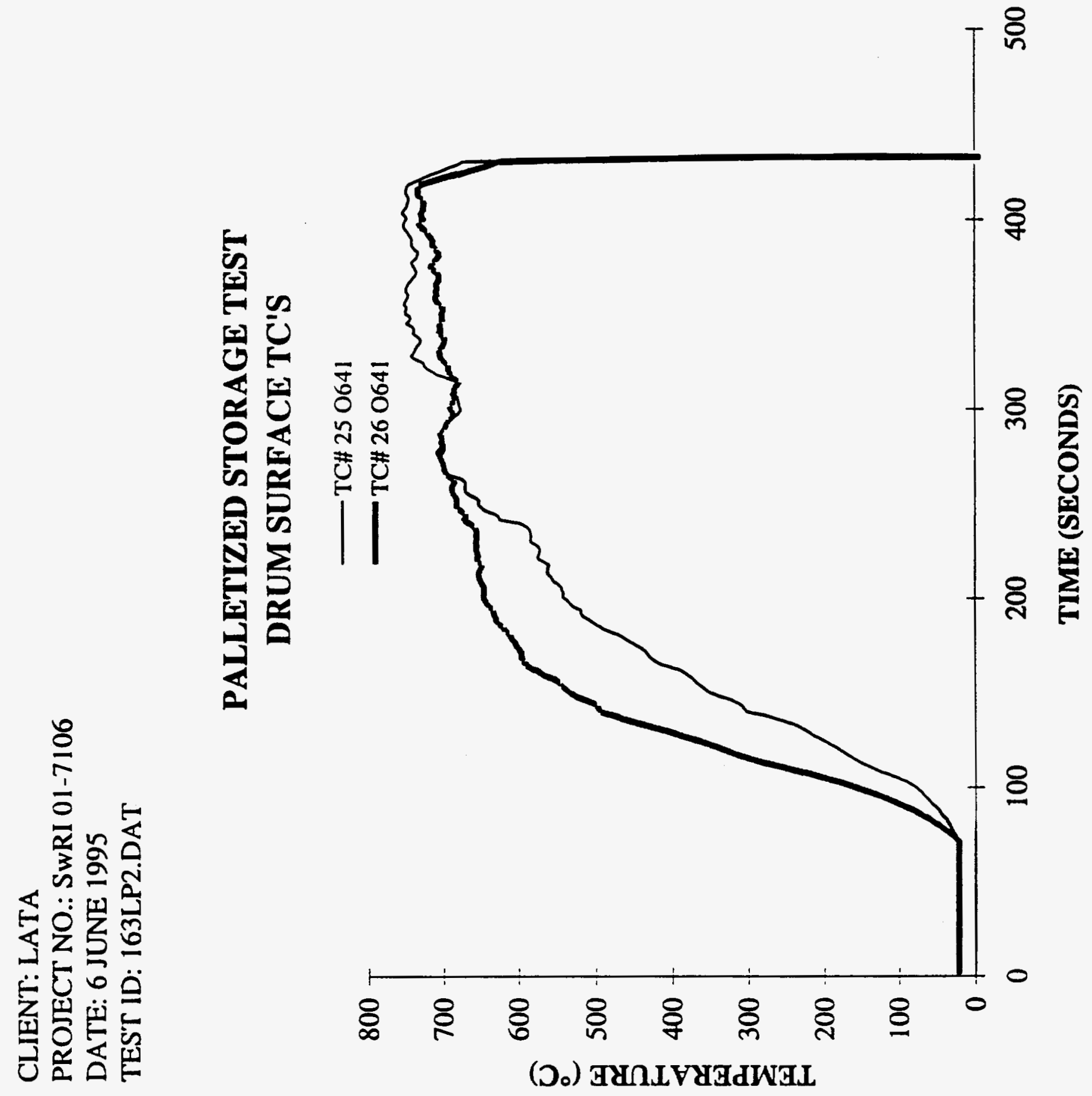


CLIENT: LATA

PROJECT NO.: SwRI 01-7106

DATE: 6 JUNE 1995

TEST ID: 163LP2.DAT

\section{PALLETIZED STORAGE TEST DRUM SURFACE TC'S}

$\stackrel{m}{a}$

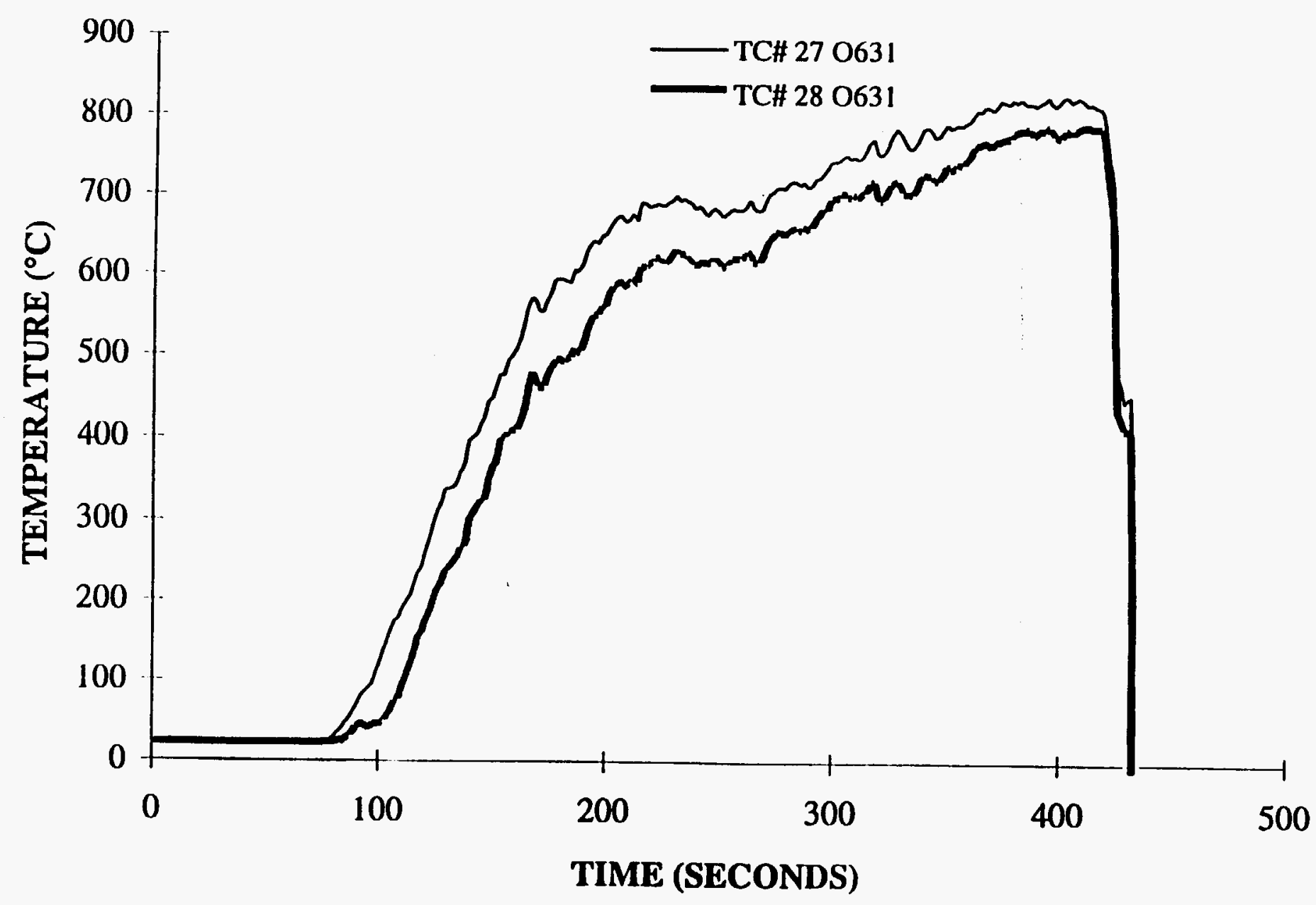


CLIENT: LATA

PROJECT NO.: SwRI 01-7106

DATE: 6 JUNE 1995

TEST ID: 163LP2.DAT

\section{PALLETIZED STORAGE TEST DRUM SURFACE TC'S}

ग0

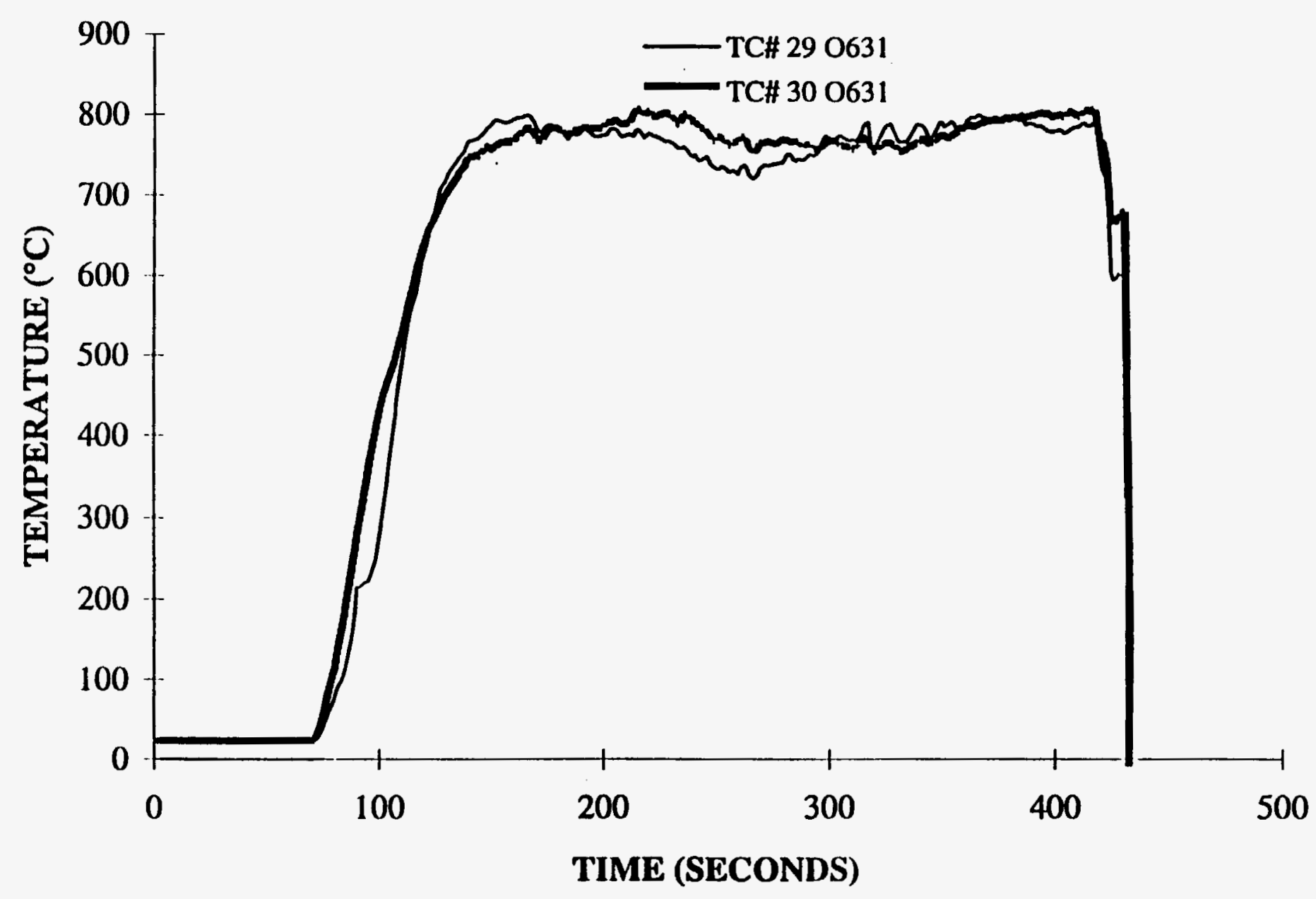

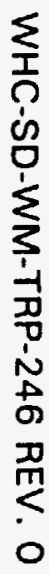

TIME (SECONDS) 
CLIENT: LATA

PROJECT NO.: SwRI 01-7106

DATE: 6 JUNE 1995

TES'T ID: 163LP2.DAT

\section{PALLETIZED STORAGE TEST DRUM SURFACE TC'S}

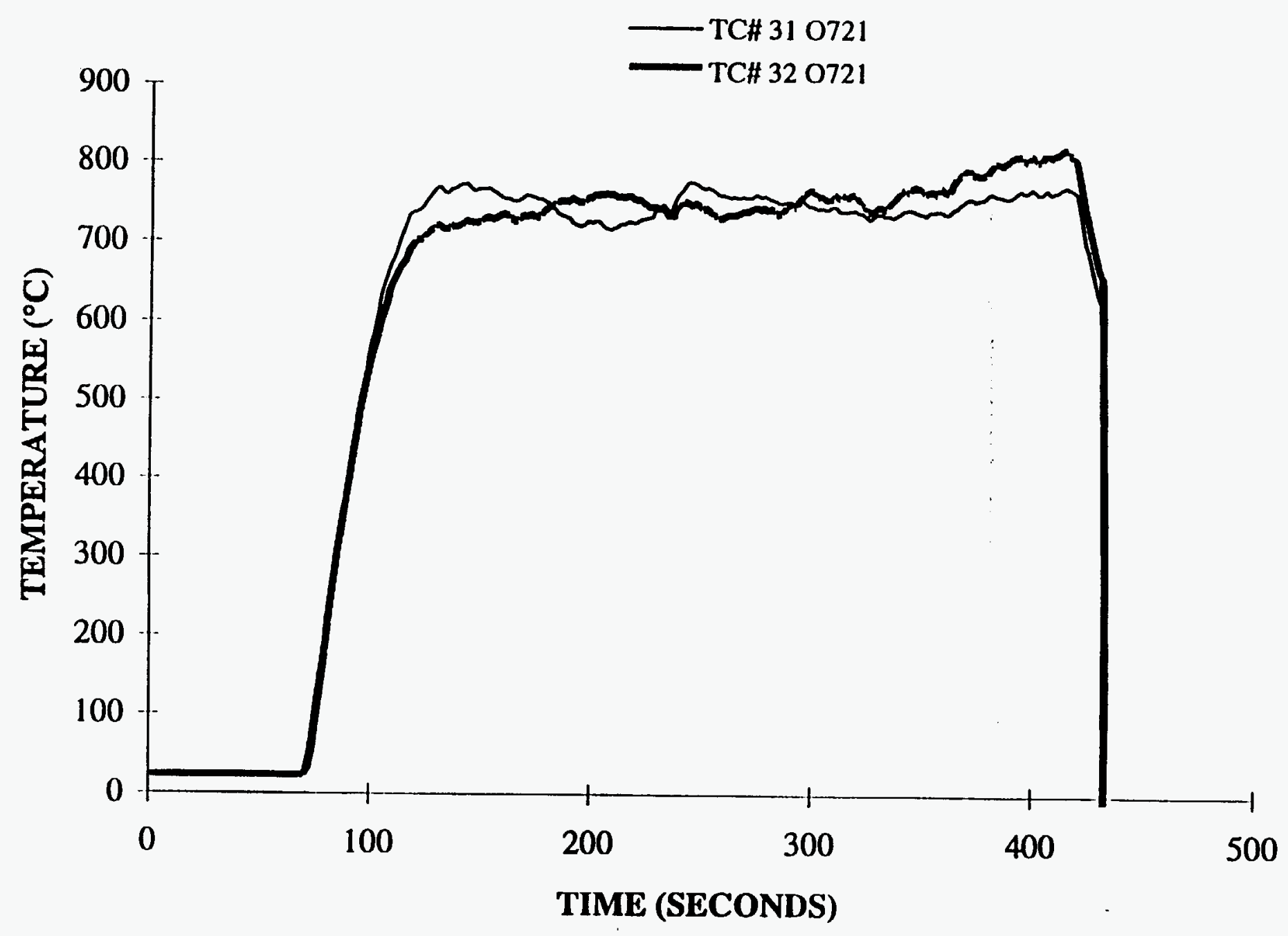

$m$
$\stackrel{1}{\omega}$

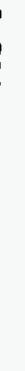

TIME (SECONDS) 


\section{CLIENT: LATA}

PROJECT NO.: SwRI 01-7106

DATE: 6 JUNE 1995

TEST ID: 163LP2.DAT

\section{PALLETIZED STORAGE TEST DRUM SURFACE TC'S}

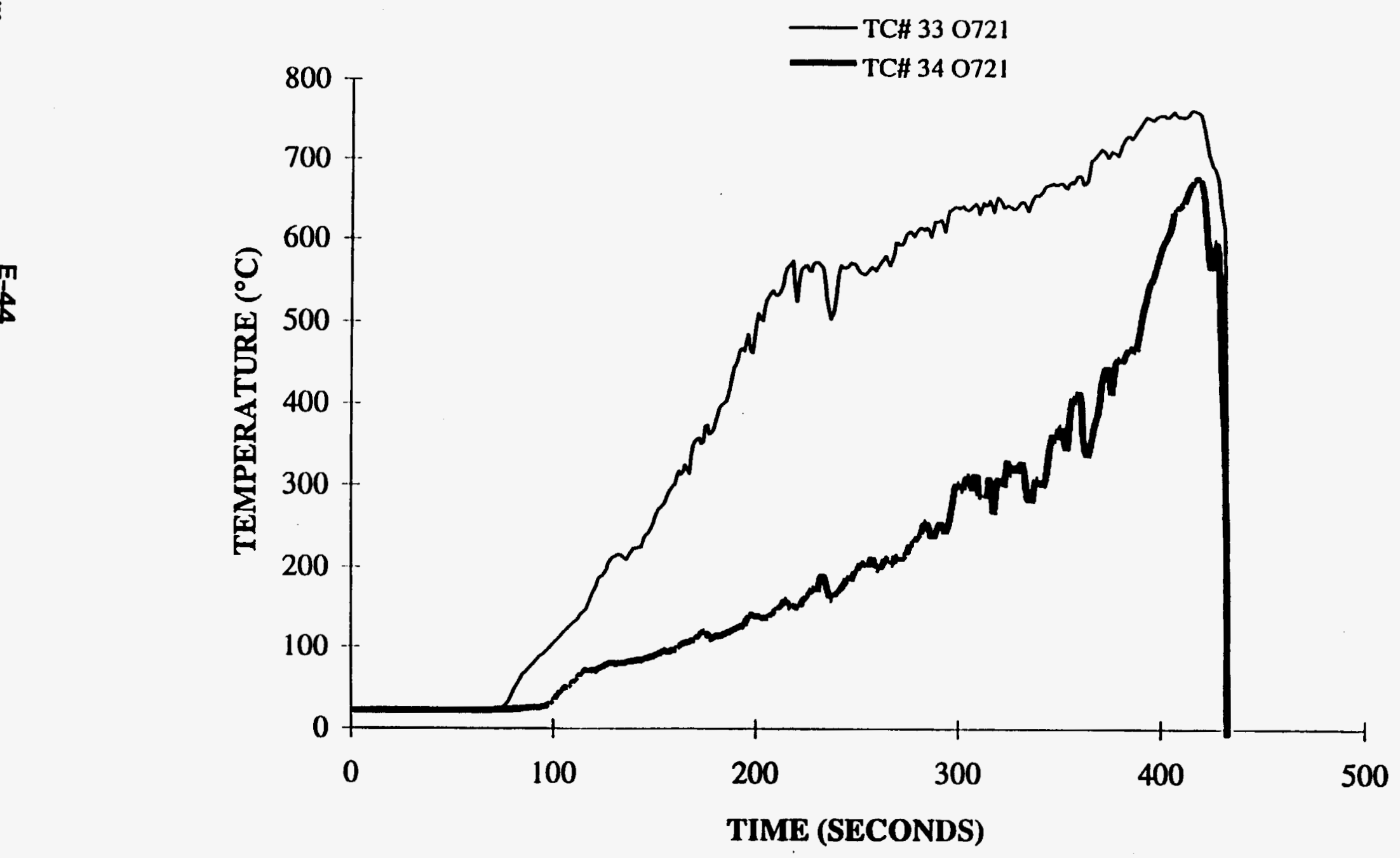

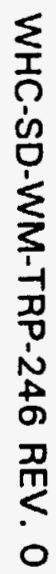

m 


\section{CLIENT: LATA}

PROJECT NO.: SwRI 01-7106

DATE: 6 JUNE 1995

TEST ID: 163LP2.DAT

\section{PALLETIZED STORAGE TEST DRUM SURFACE TC'S}

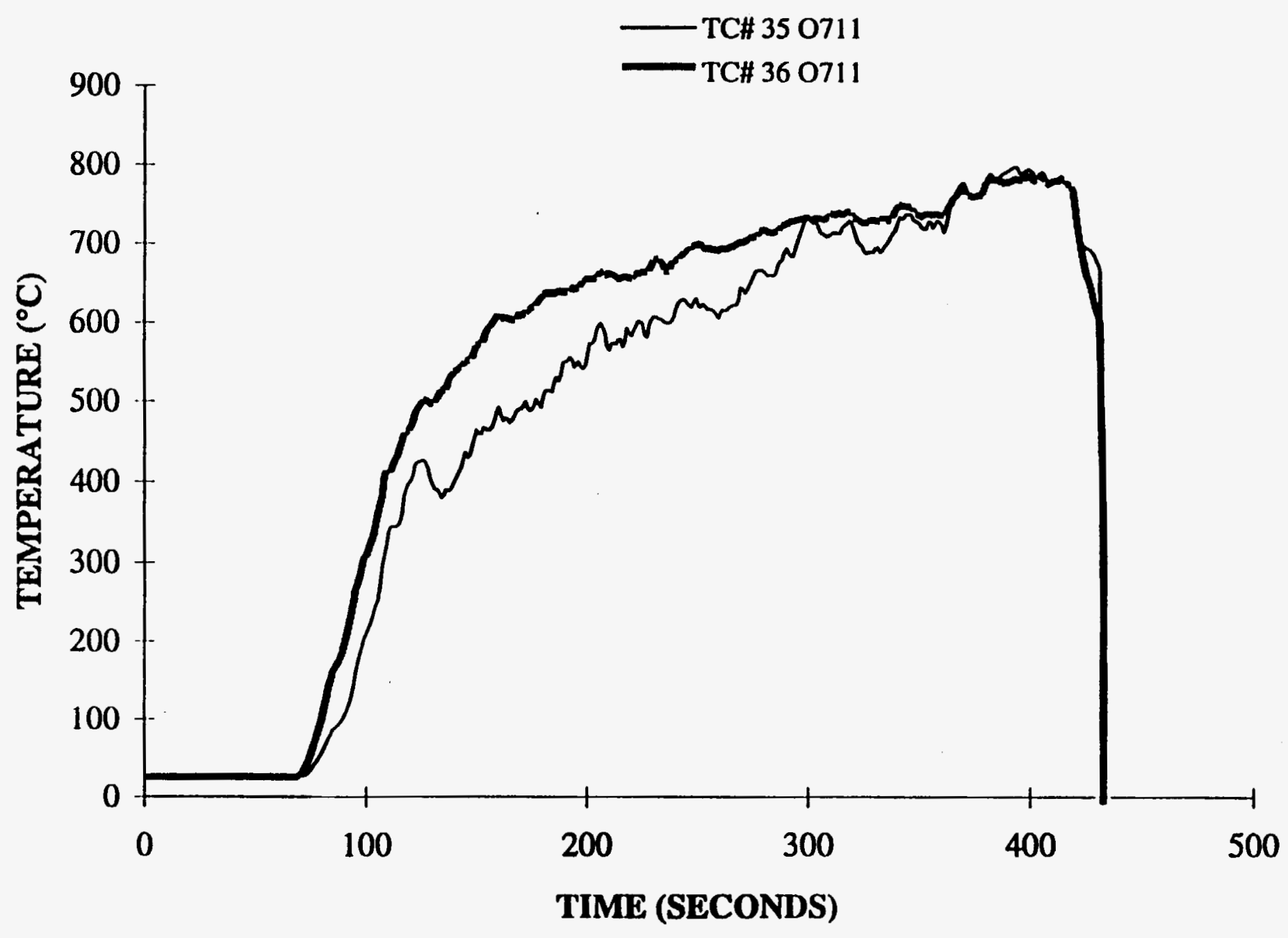


CLIENT: LATA

PROJECT NO.: SwRI 01-7106

DATE: 6 JUNE 1995

TEST ID: 163LP2.DAT

\section{PALLETIZED STORAGE TEST DRUM SURFACE TC'S}

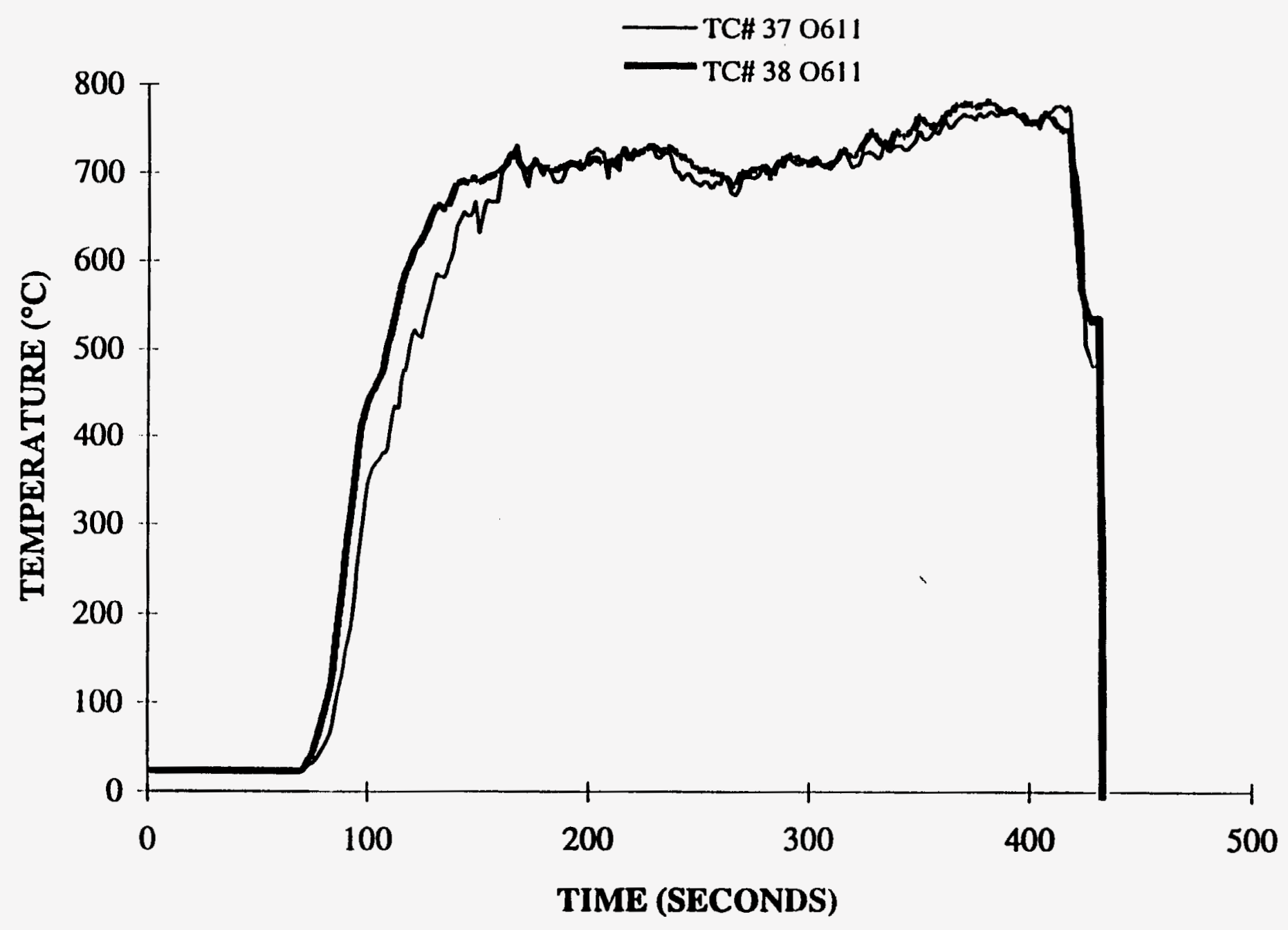


CLIENT: LATA

PROJECT NO.: SwRI 01-7106

DATE: 6 JUNE 1995

TEST ID: 163LPI.DAT

\section{PALLETIZED STORAGE TEST DRUM SURFACE TC'S}

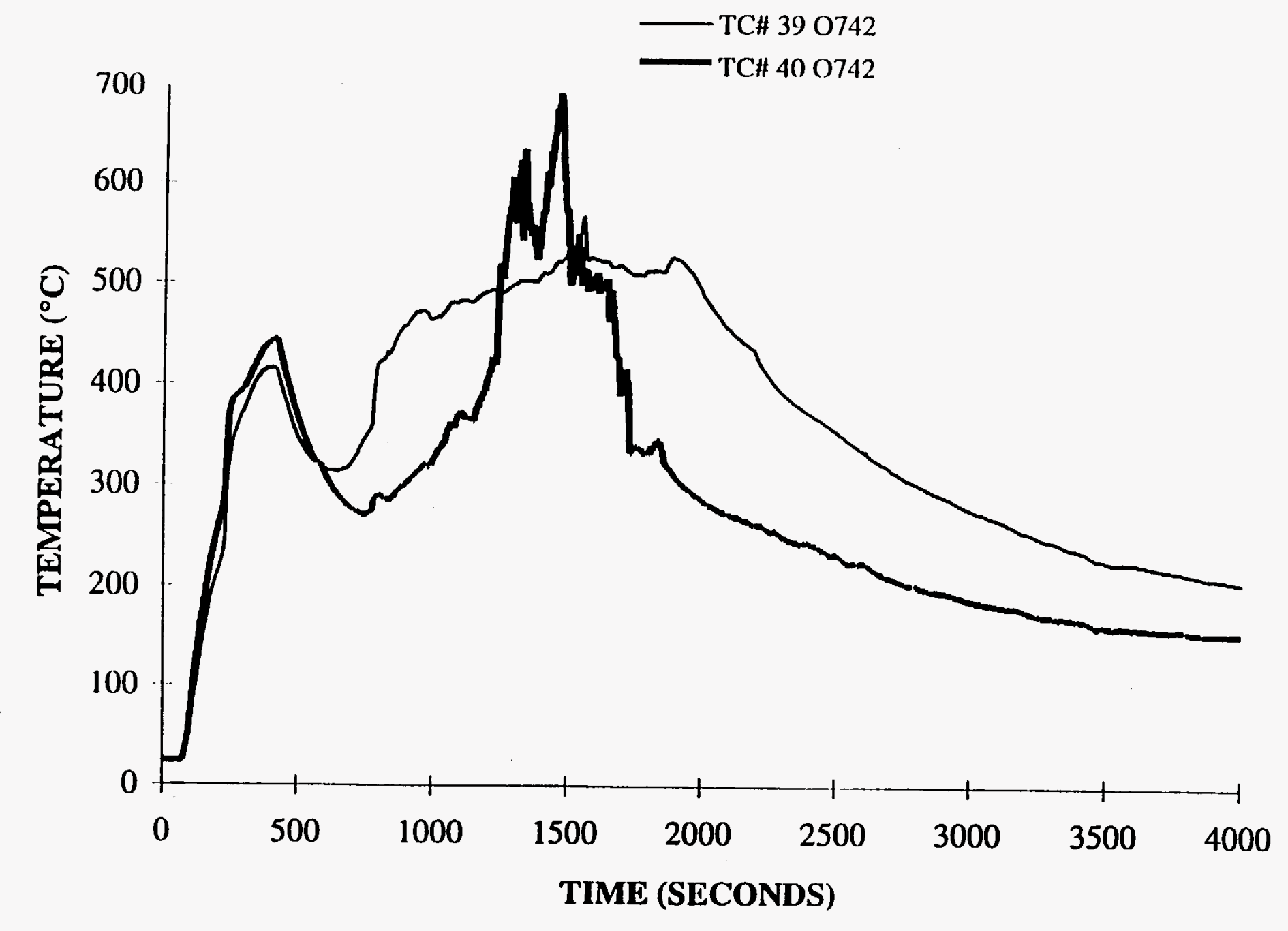

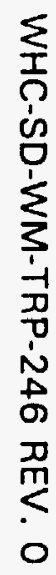


CLIENT: LATA

PROJECT NO.: SwRI 01-7106

DATE: 6 JUNE 1995

TEST ID: 163LP3.DAT

\section{PALLETIZED STORAGE TEST DRUM SURFACE TC'S}

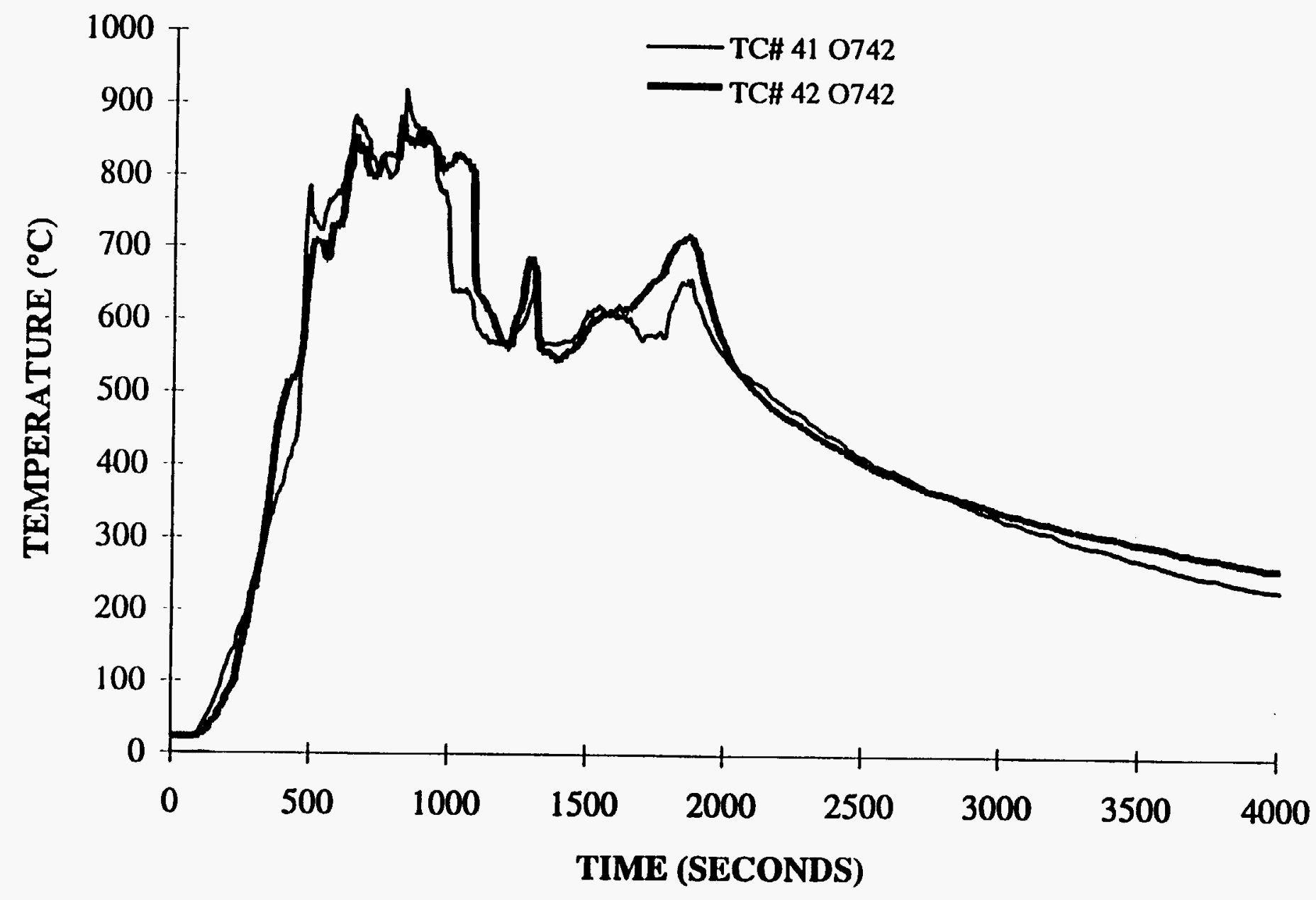


CLIENT: LATA

PROJECT NO.: SwRI 01-7106

DATE: 6 JUNE 1995

TEST ID: 163LP3.DAT

PALLETIZED STORAGE TEST

DRUM SURFACE TC'S

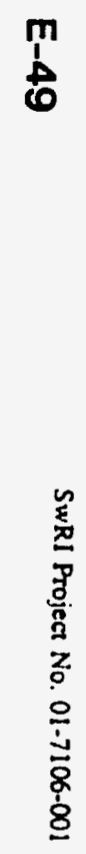

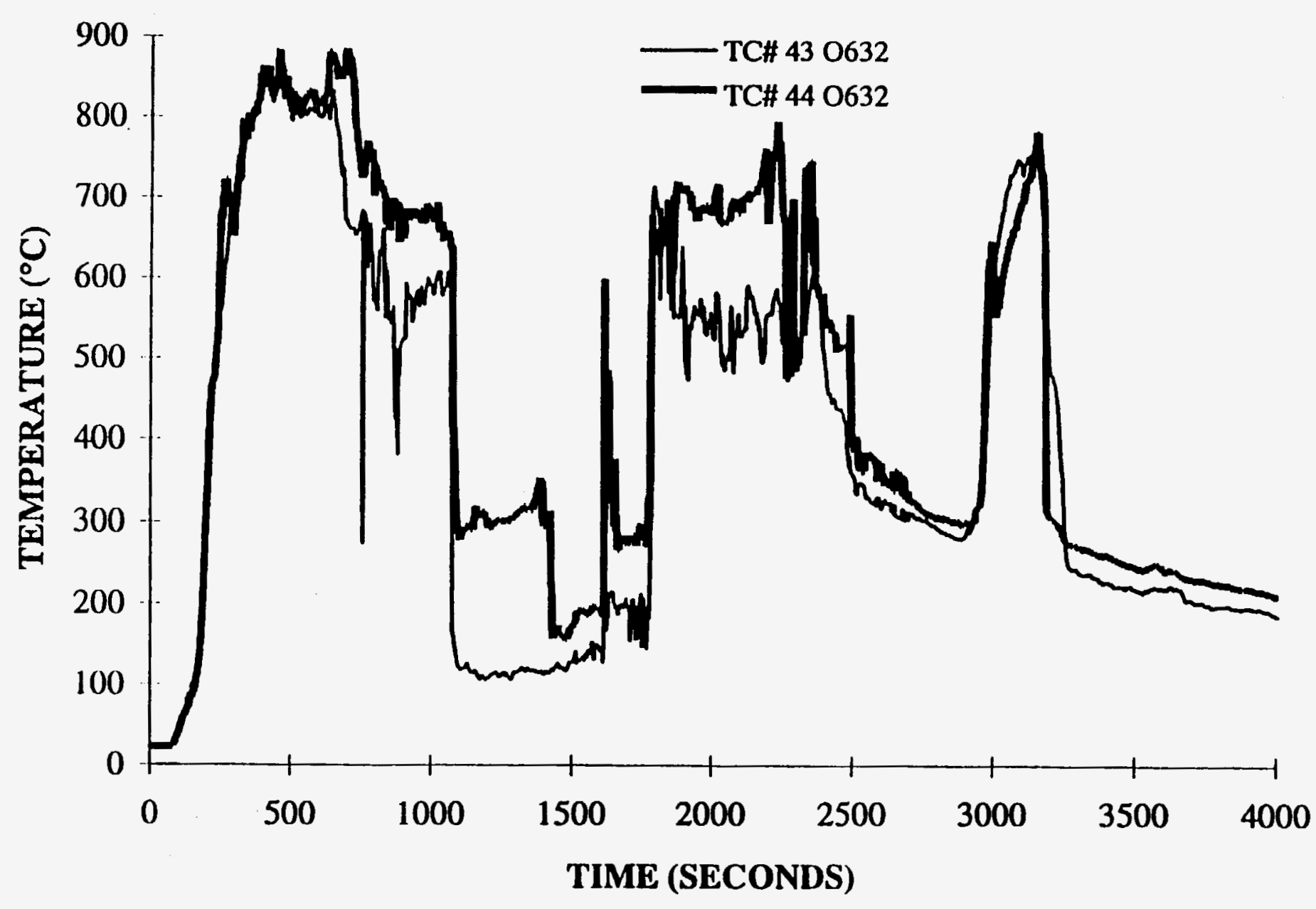

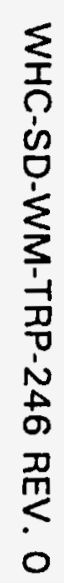

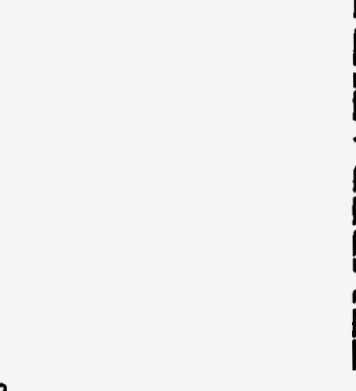


CLIENT: LATA

PROJECT NO.: SwRI 01-7106

DATE: 6 JUNE 1995

TEST ID: 163LP3.DAT

\section{PALLETIZED STORAGE TEST DRUM SURFACE TC'S}

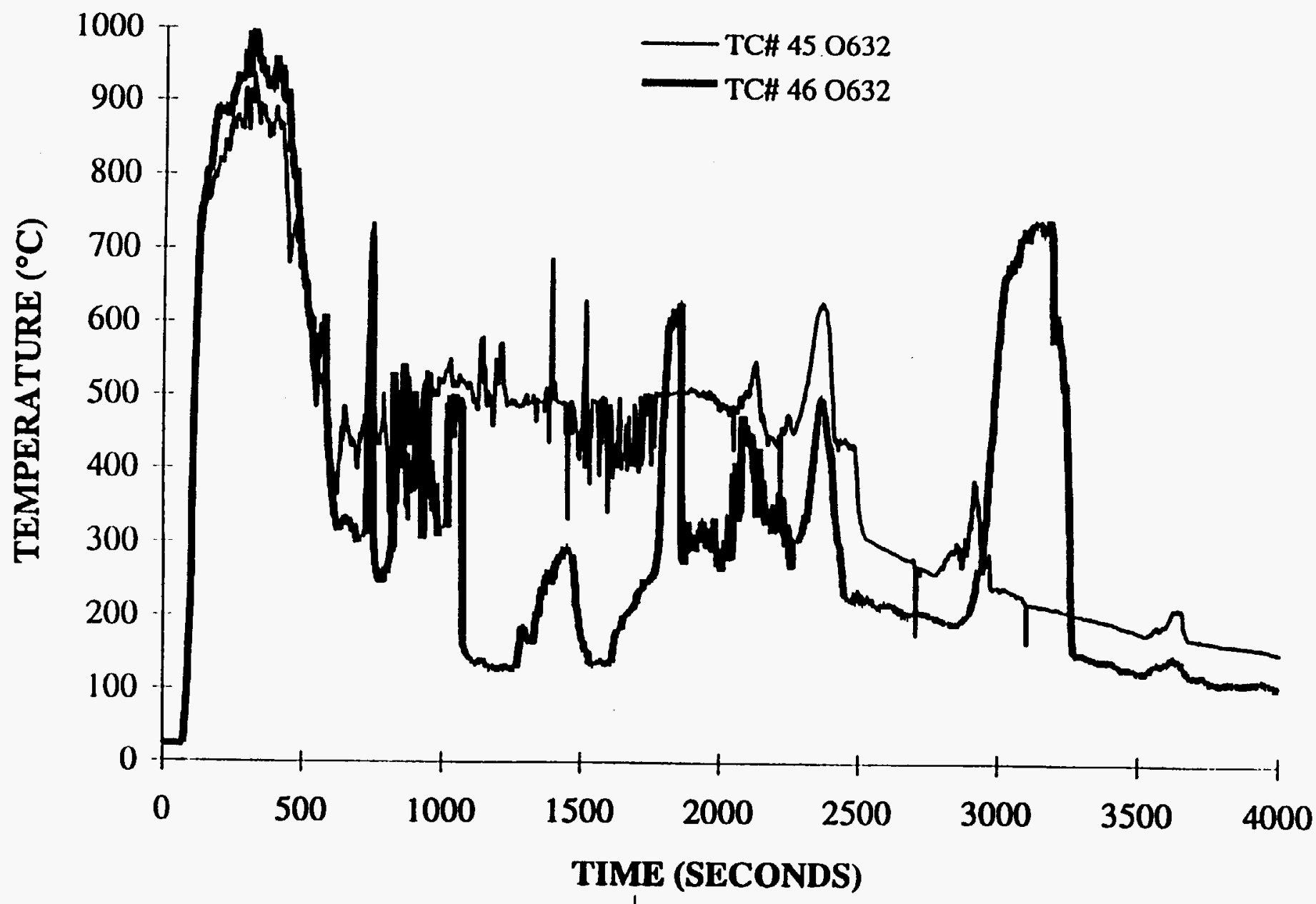

m 
WHC-SD-WM-TRP-246 REV. 0
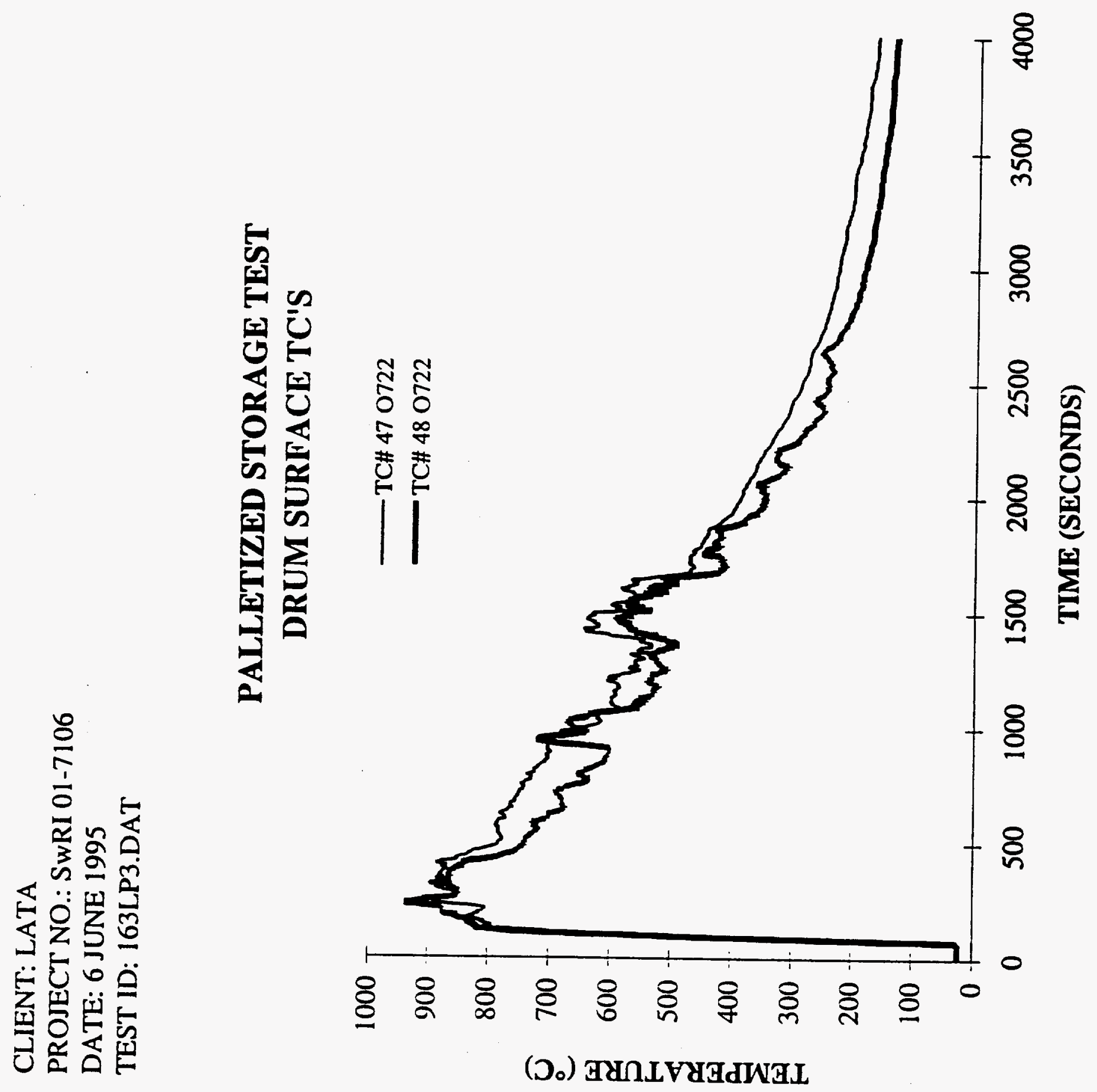

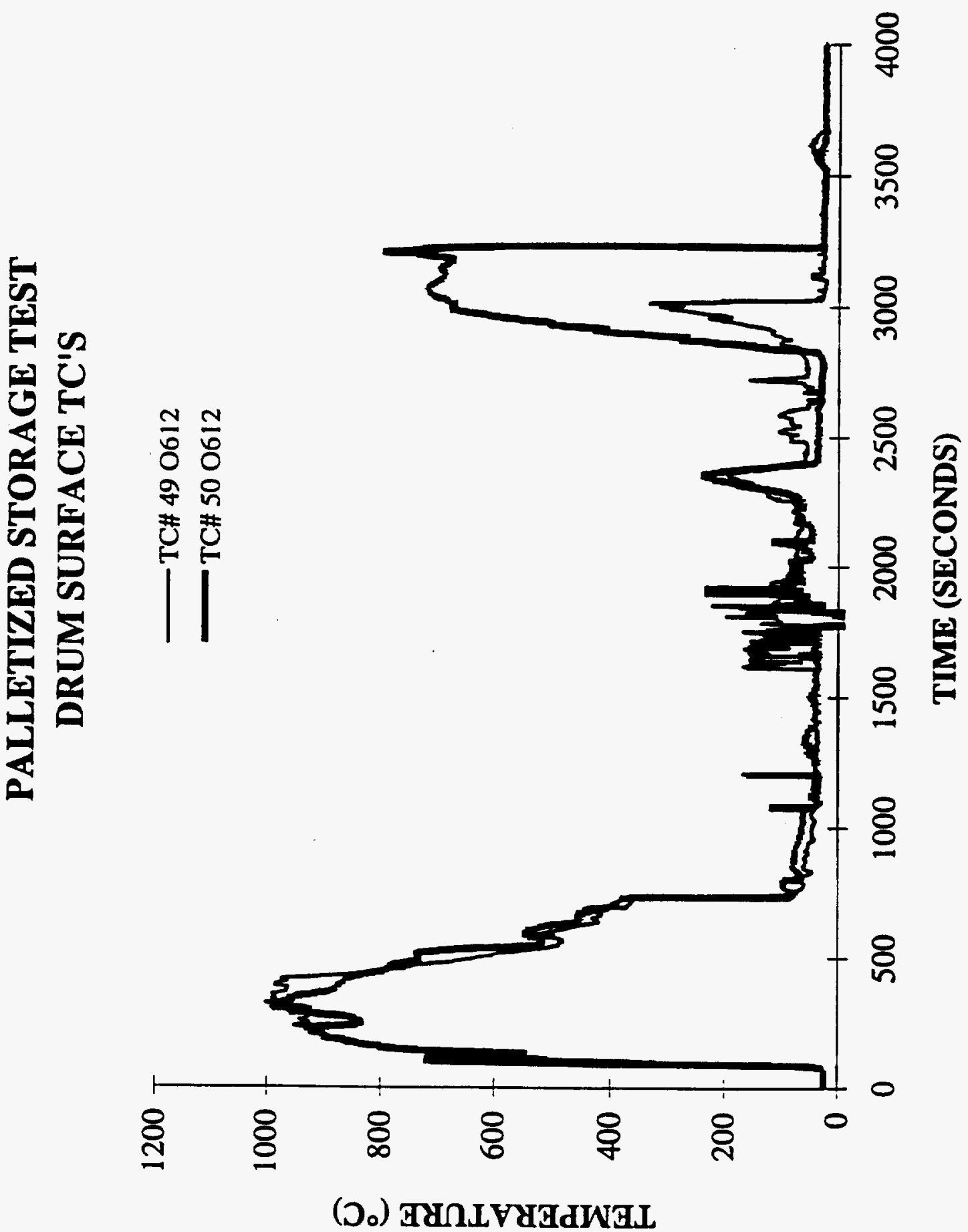
CLIENT: LATA

PROJECT NO.: SwRI 01-7106

DATE: 6 JUNE 1995

TEST ID: 163LP3.DAT

\section{PALLETIZED STORAGE TEST DRUM SURFACE TC'S}

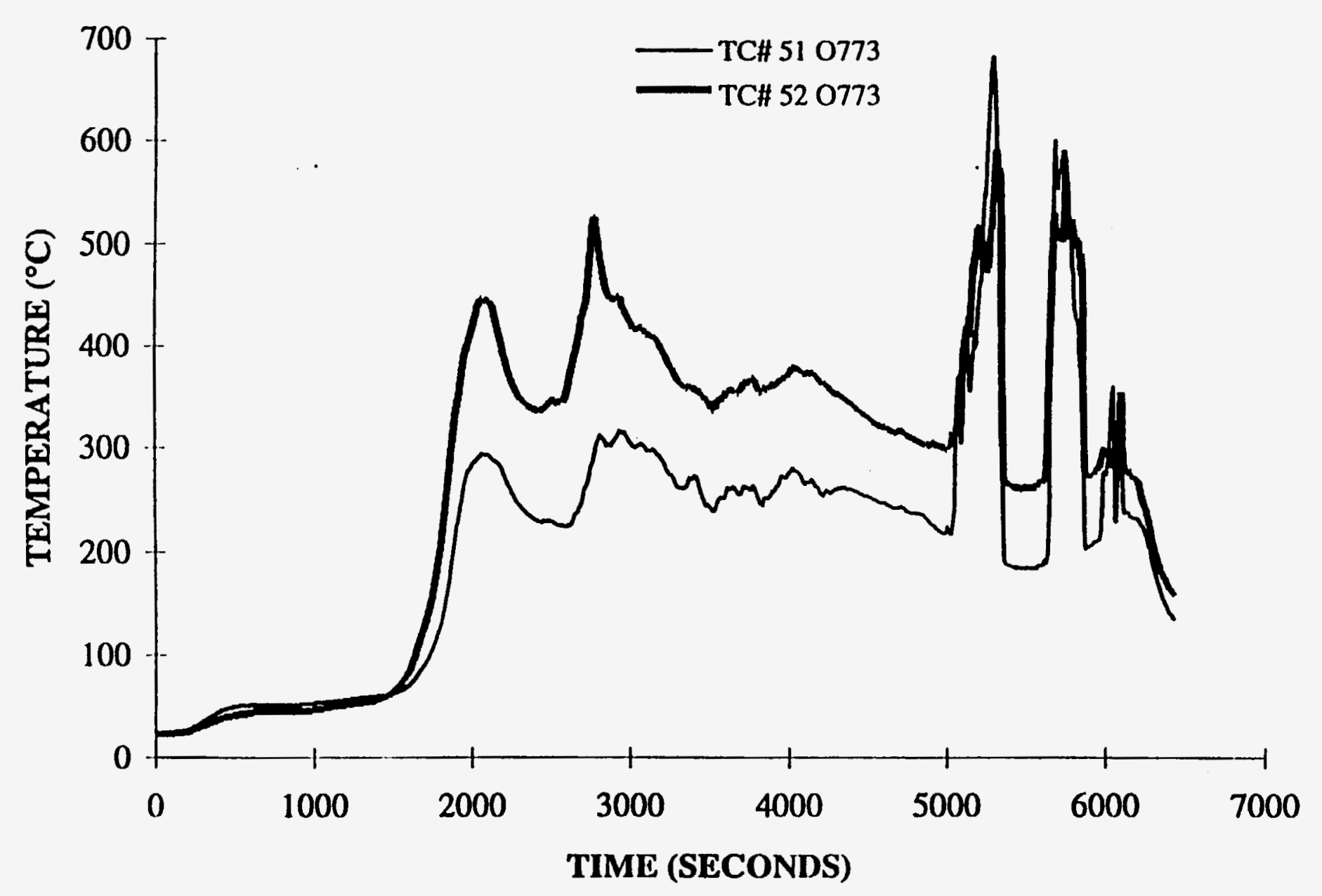

息

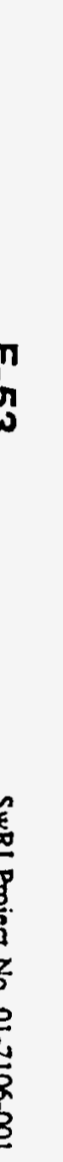


CLIENT: LATA

PROJECT NO.: SwRI 01-7106

DATE: 6 JUNE 1995

TEST ID: 163LP3.DAT

\section{PALLETIZED STORAGE TEST DRUM SURFACE TC'S}

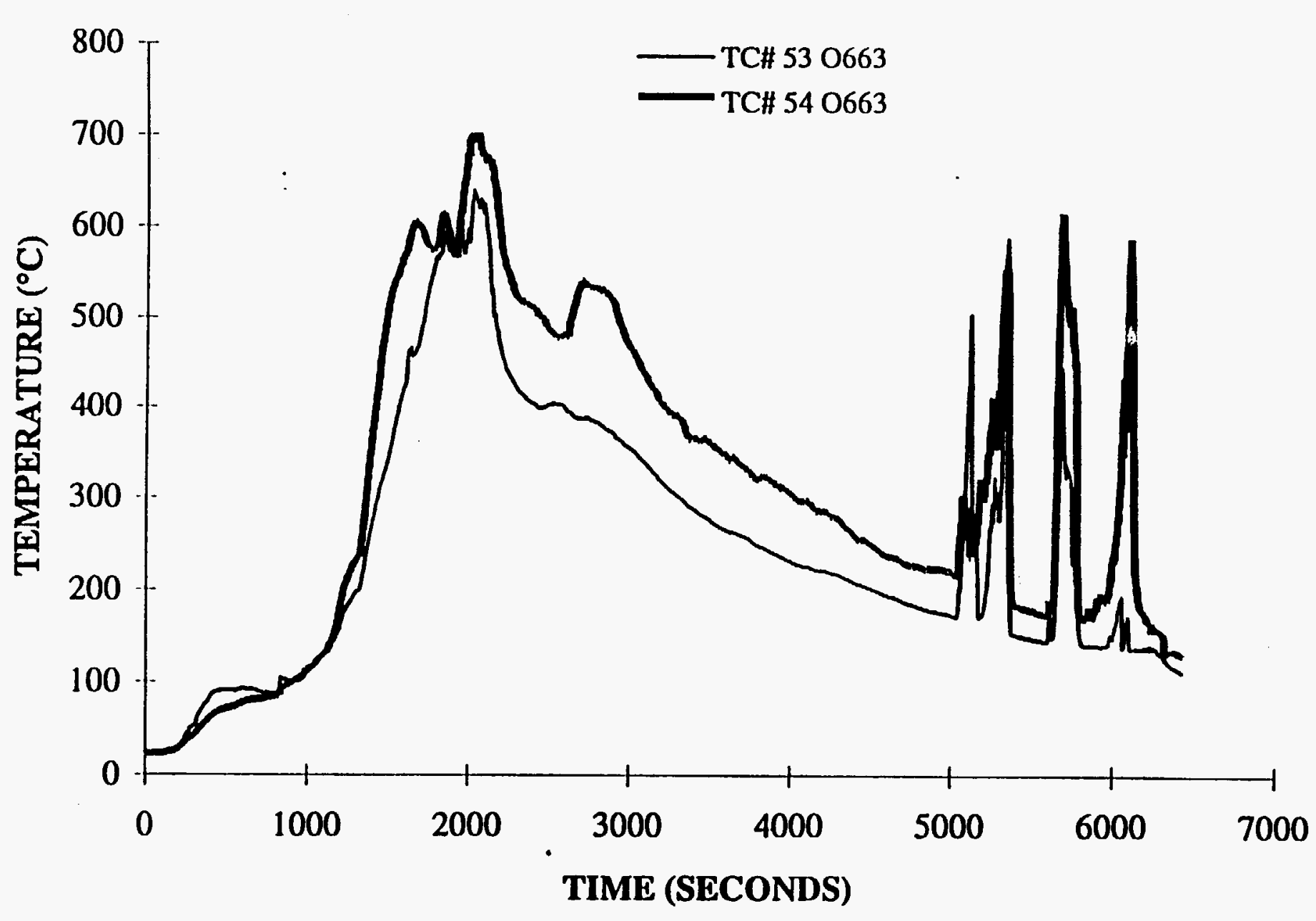


CLIENT: LATA

PROJECT NO.: SwRI 01-7106

DATE: 6 JUNE 1995

TEST ID: 163LP3.DAT

\section{PALLETIZED STORAGE TEST DRUM SURFACE TC'S}

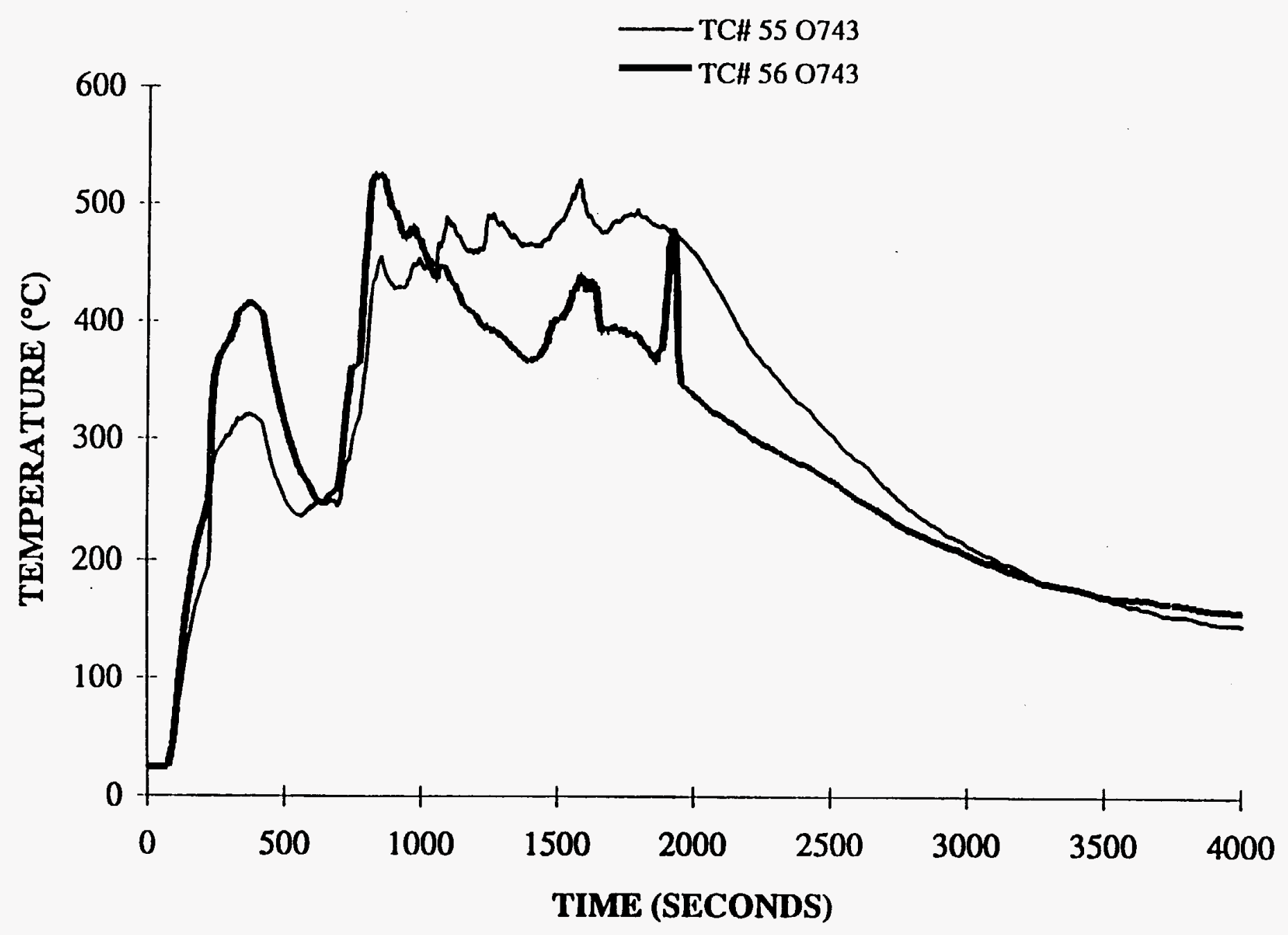


TEST ID: 163LP3.DAT

\section{PALLETIZED STORAGE TEST DRUM SURFACE TC'S}

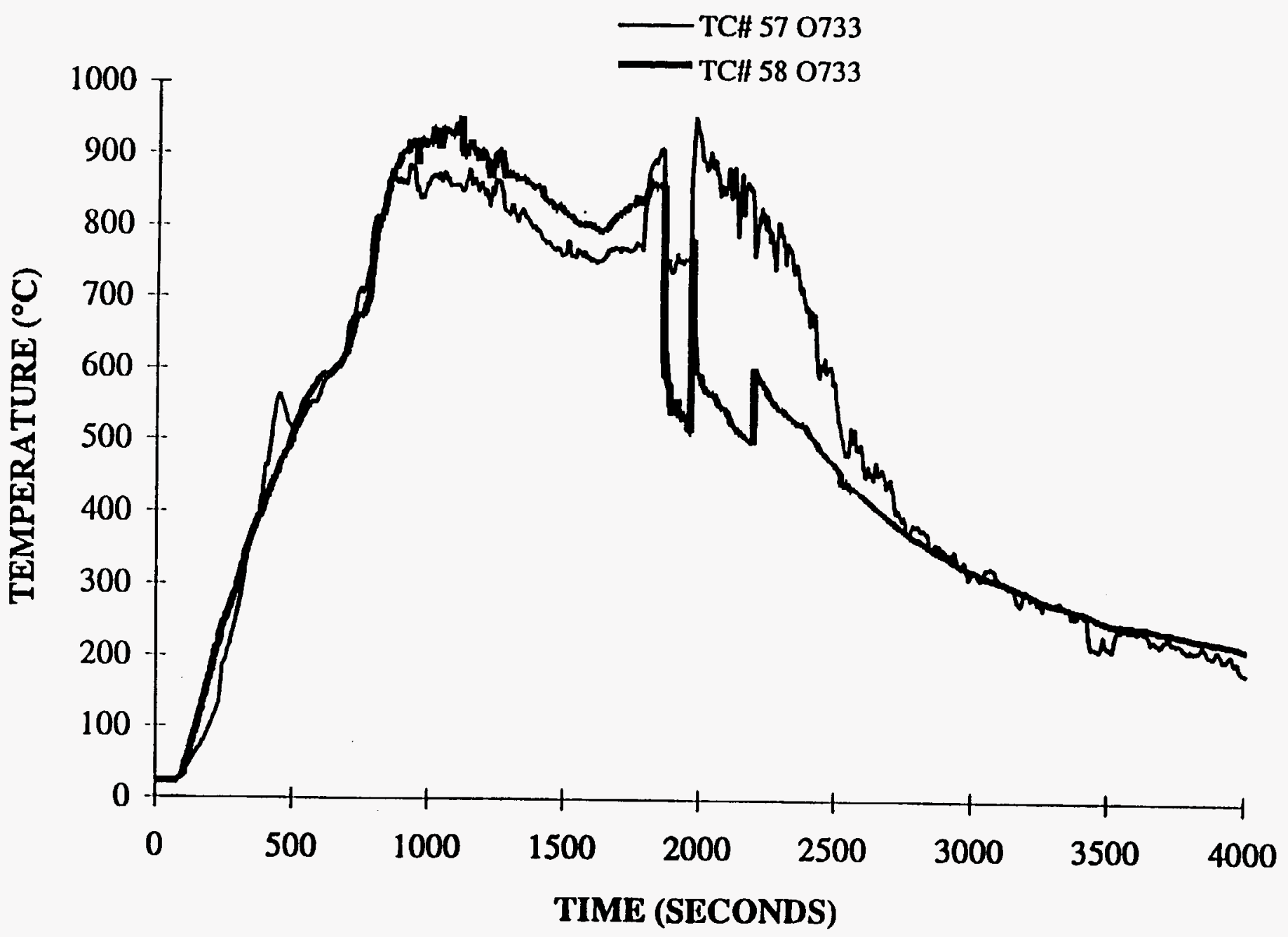


CLIENT: LATA

PROJECT NO.: SwRI 01-7106

TEST ID: 163LP4.DAT

\section{PALLETIZED STORAGE TEST} DRUM SURFACE TC'S

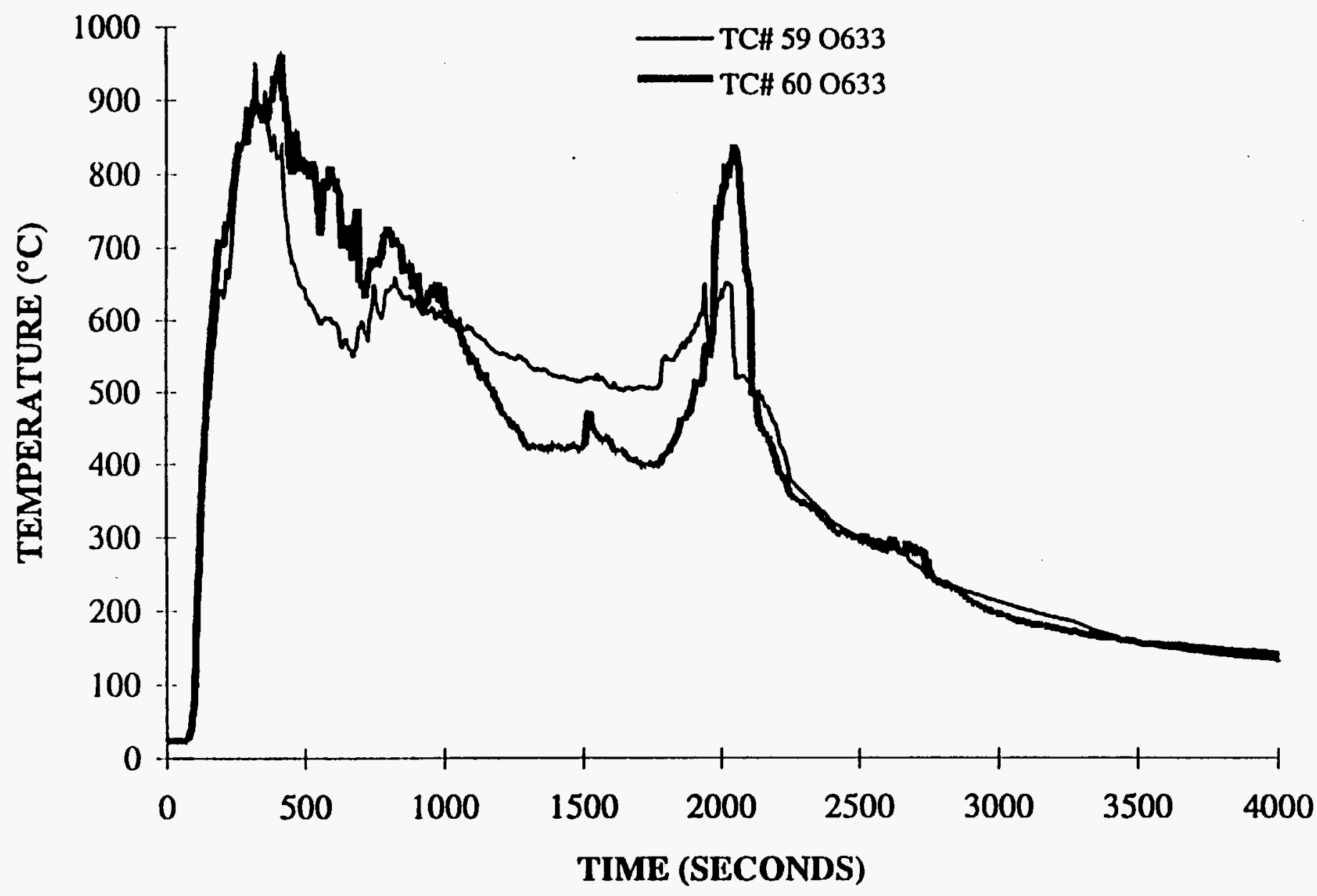


CLIENT: LATA

PROJECT NO.: SwRI 01-7106

DATE: 6 JUNE 1995

TEST ID: 163LP4.DAT

\section{PALLETIZED STORAGE TEST DRUM SURFACE TC'S}

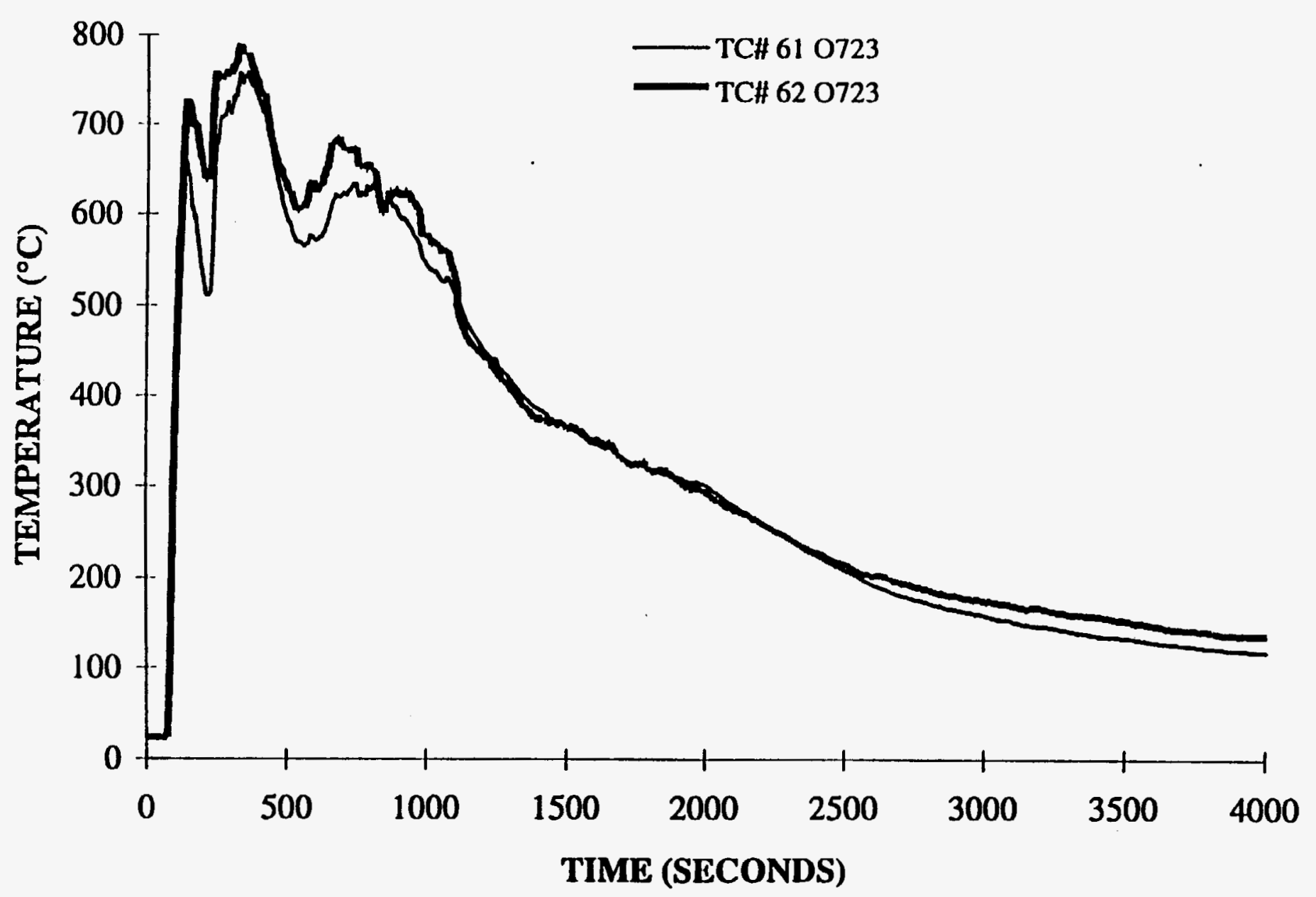



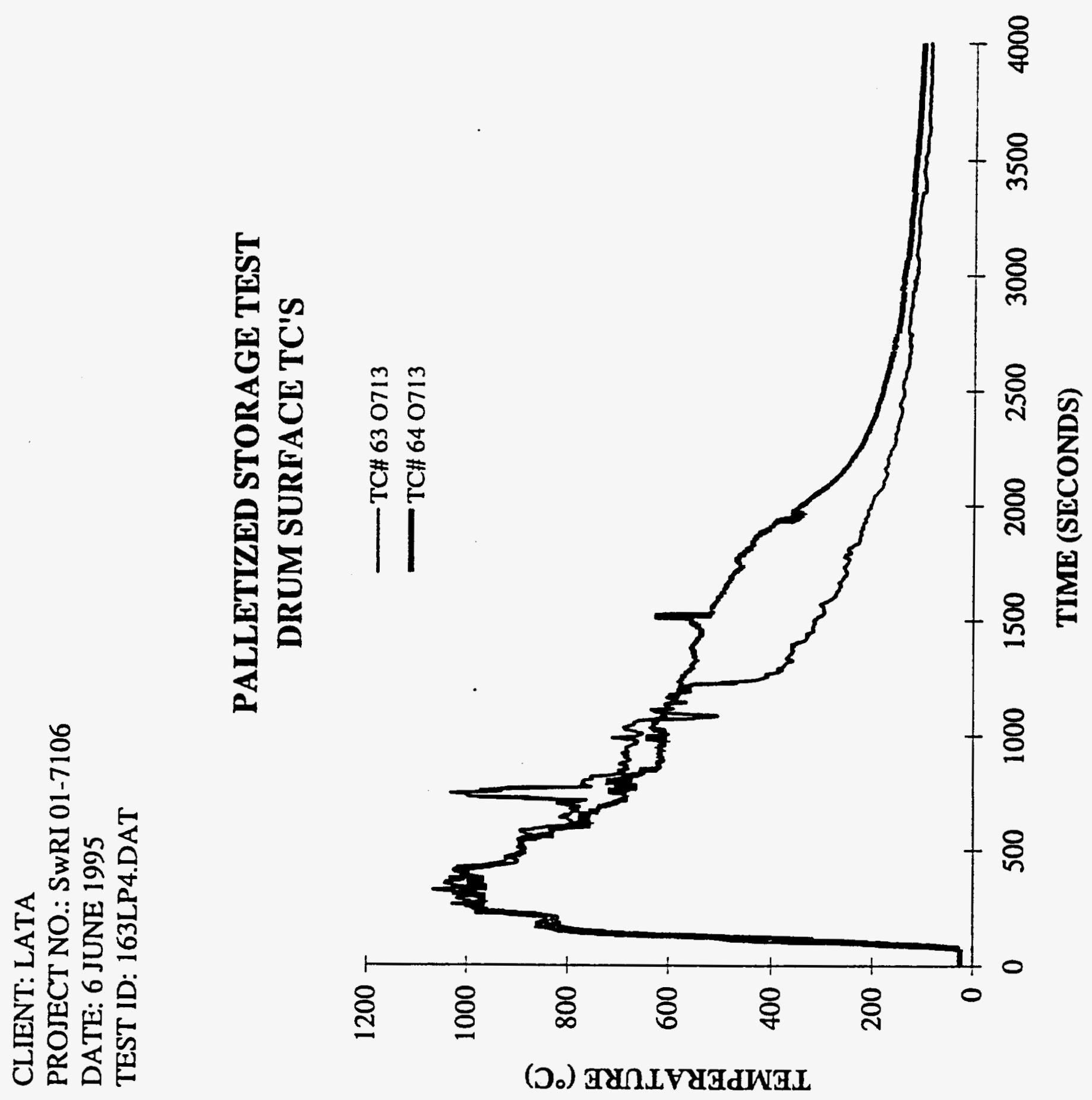
CLIENT: LATA

PROJECT NO.: SwRI 01-7106

DATE: 6 JUNE 1995

TEST ID: 163LP4.DAT

PALLETIZED STORAGE TEST DRUM SURFACE TC'S

m

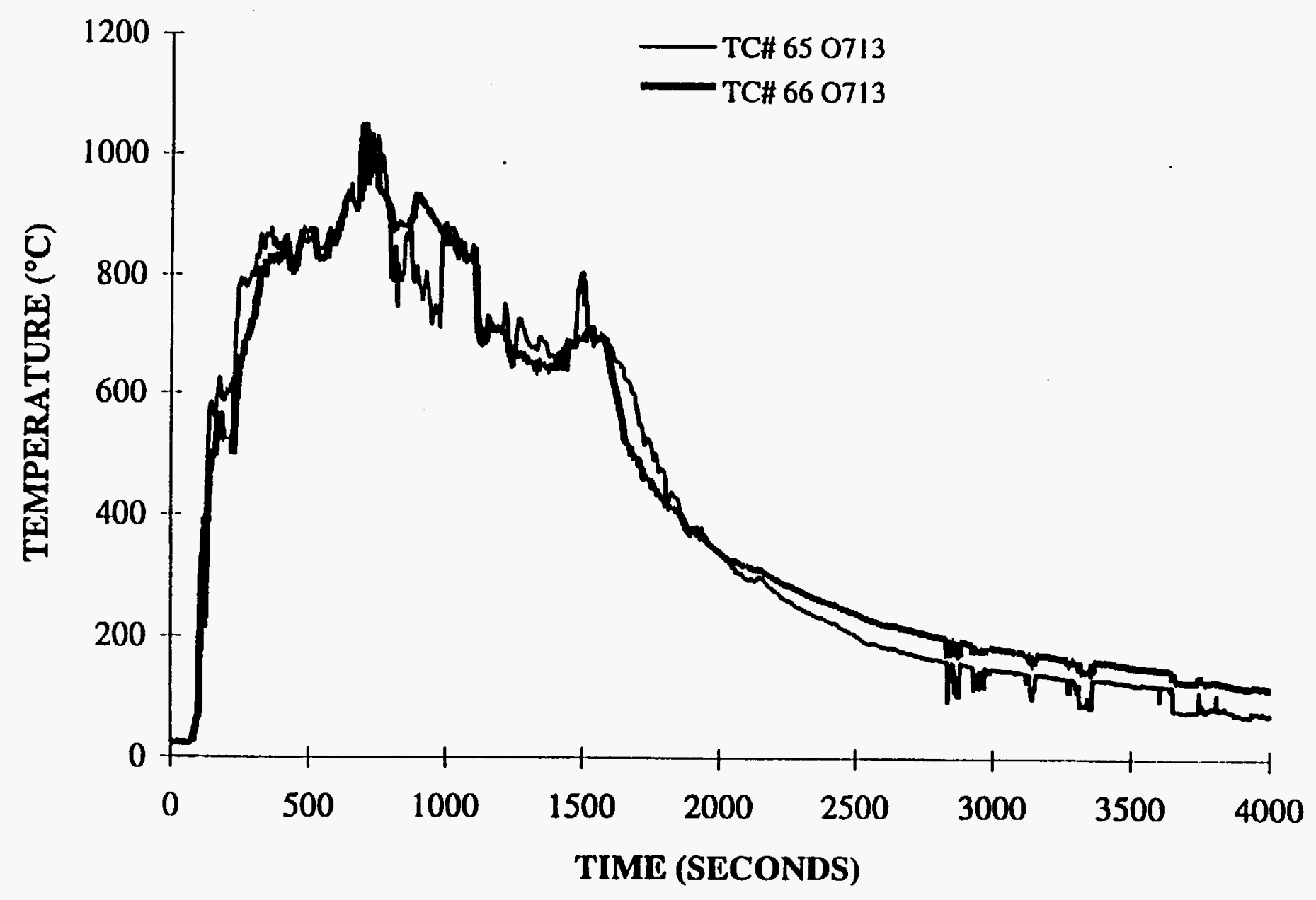


CLIENT: LATA

PROJECT NO.: SwRI 01-7106

DATE: 6 JUNE 1995

TEST ID: 163LP4.DAT

\section{PALLETIZED STORAGE TEST DRUM SURFACE TC'S}

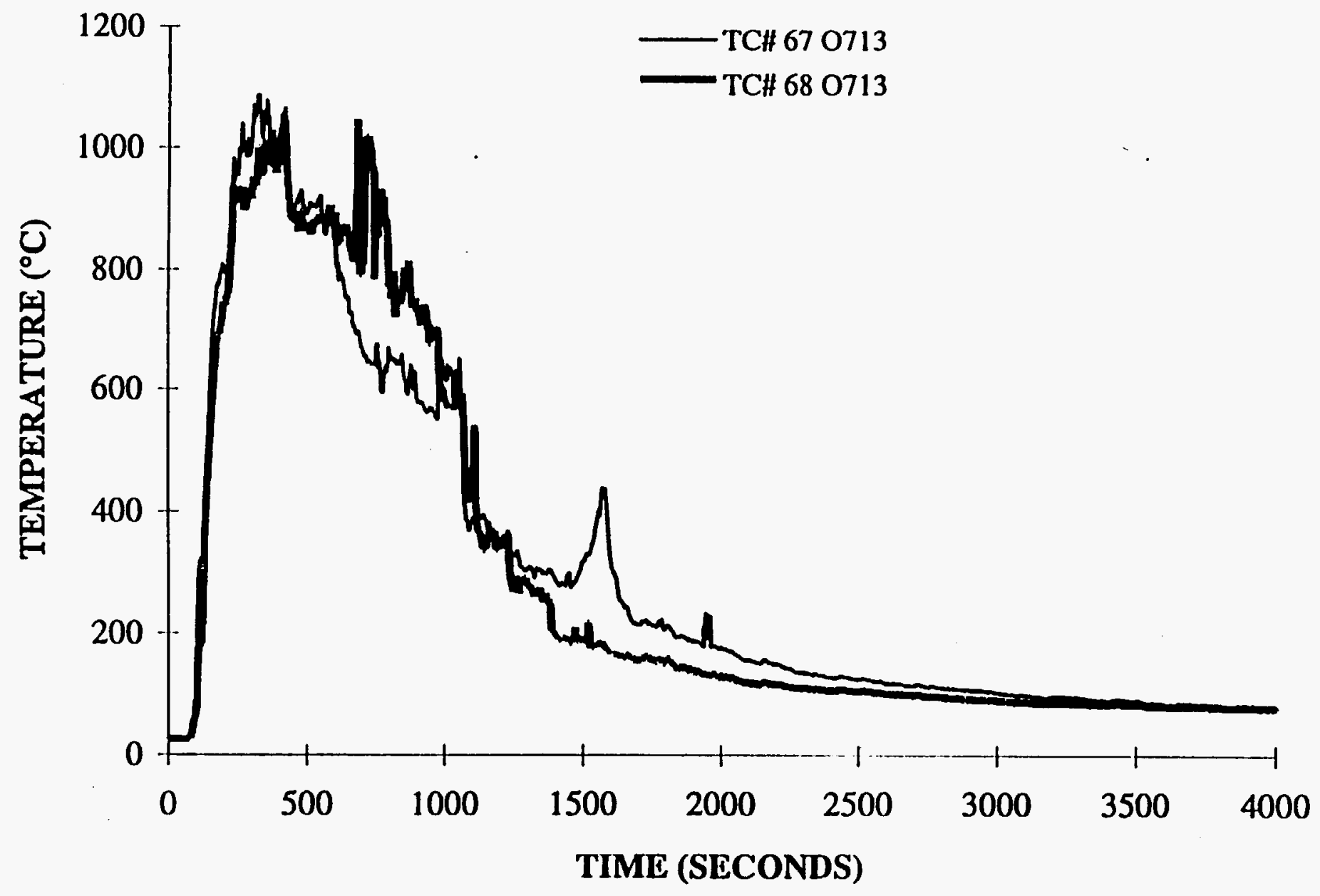


CLIENT: LATA

PROJECT NO.: SwRI 01-7106

DATE: 6 JUNE 1995

TEST ID: 163LP4.DAT

\section{PALLETIZED STORAGE TEST DRUM SURFACE TC'S}

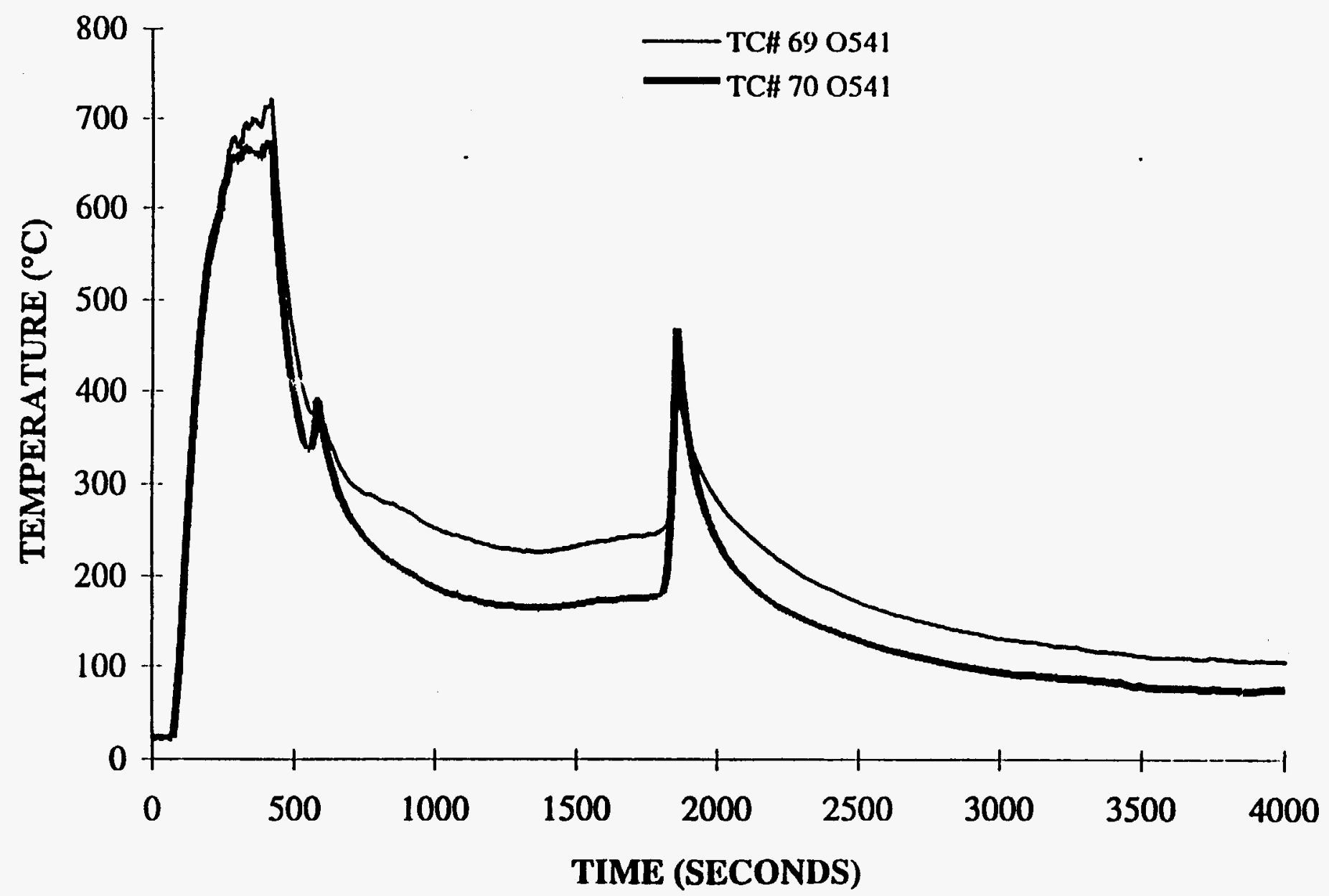




\section{CLIENT: LATA}

\section{PROJECT NO.: SwRI 01-7106}

DATE: 6 JUNE 1995

TEST ID: 163LP4.DAT

\section{PALLETIZED STORAGE TEST DRUM SURFACE TC'S}

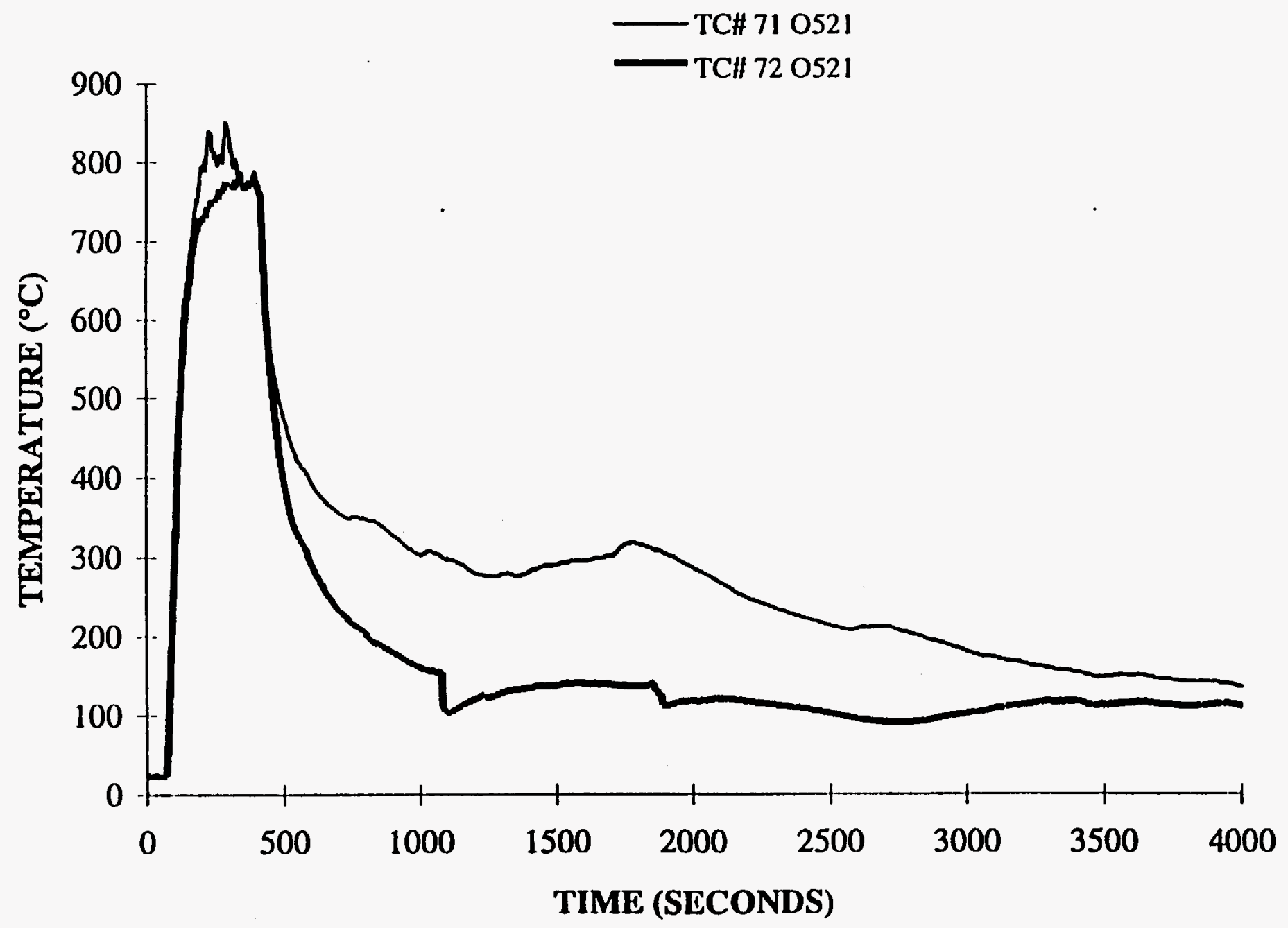

TIME (SECONDS) 
$\longrightarrow$

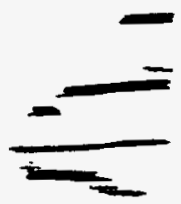

-

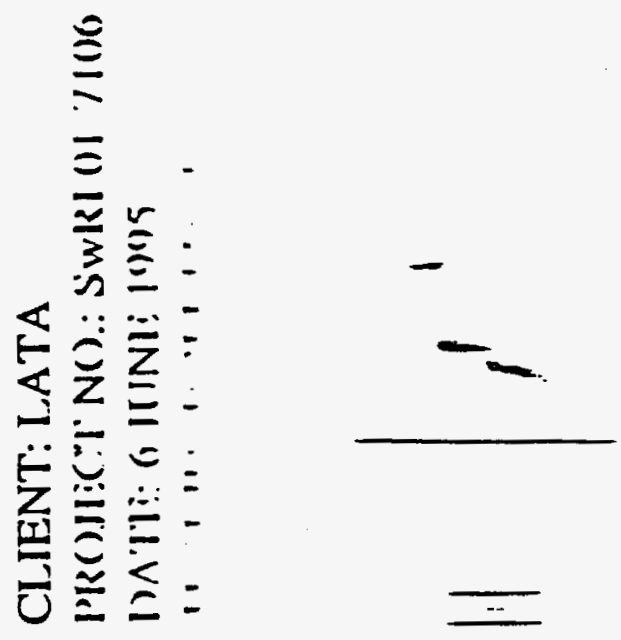

$\therefore=$ 


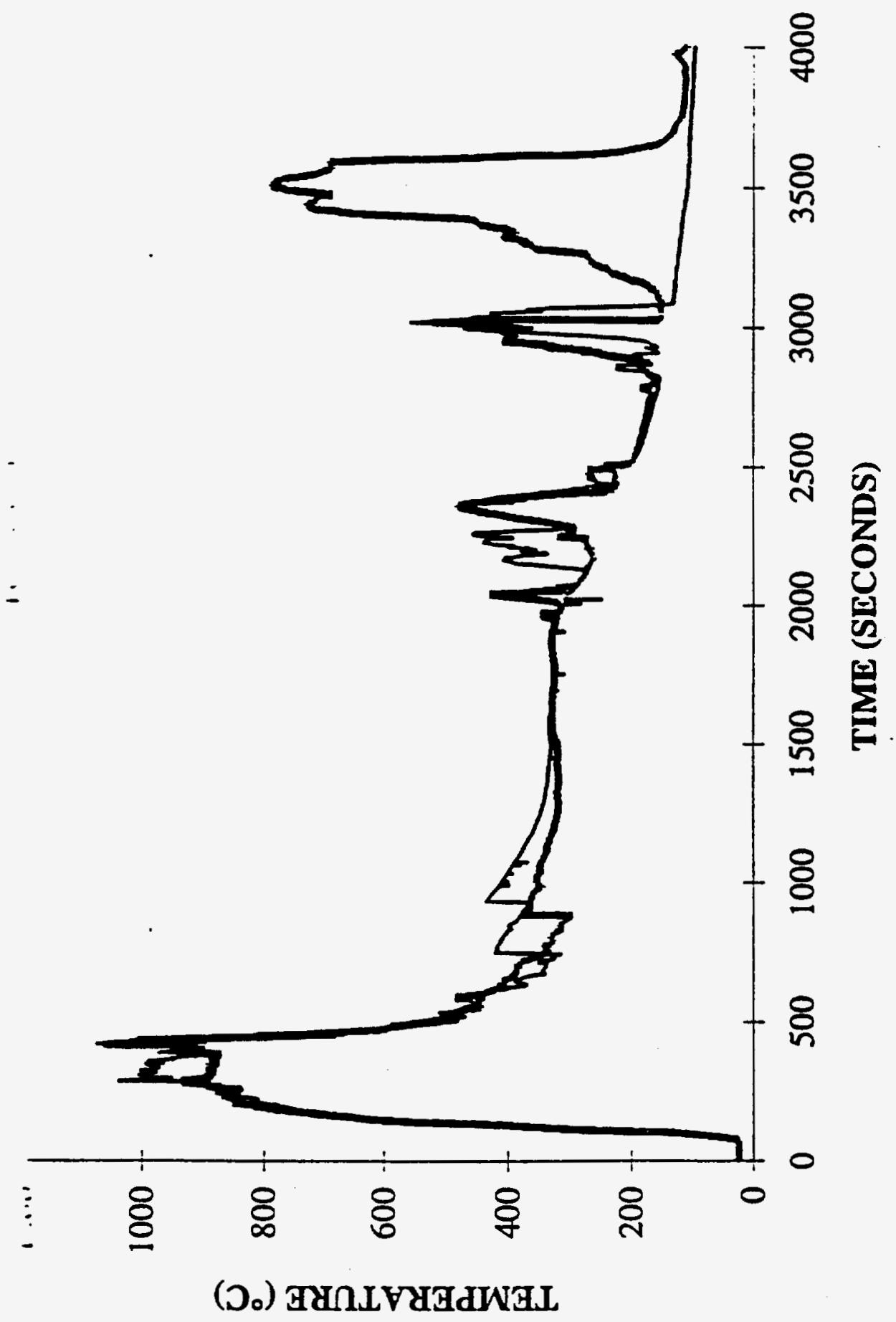


CLIENT: LATA

PROJECT NO.: SwRI 01-7106

DATE: 6 JUNE 1995

TEST ID: 163LP5.DAT

\section{PALLETIZED STORAGE TEST DRUM SURFACE TC'S}

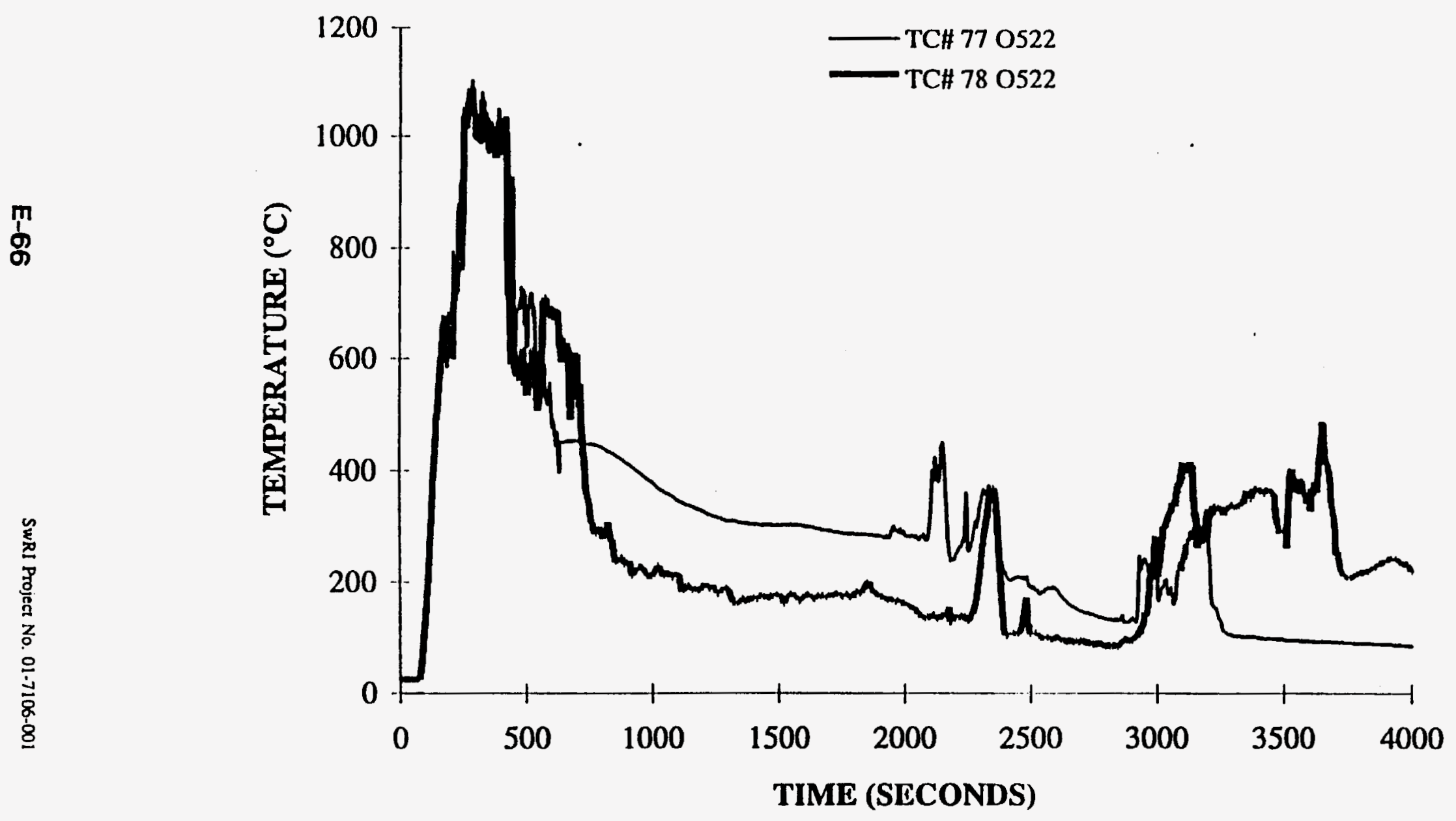


CLIENT: LATA

PROJECT NO.: SwRI 01-7106

DATE: 6 JUNE 1995

TEST ID: 163LP5.DAT

\section{PALLETIZED STORAGE TEST DRUM SURFACE TC'S}

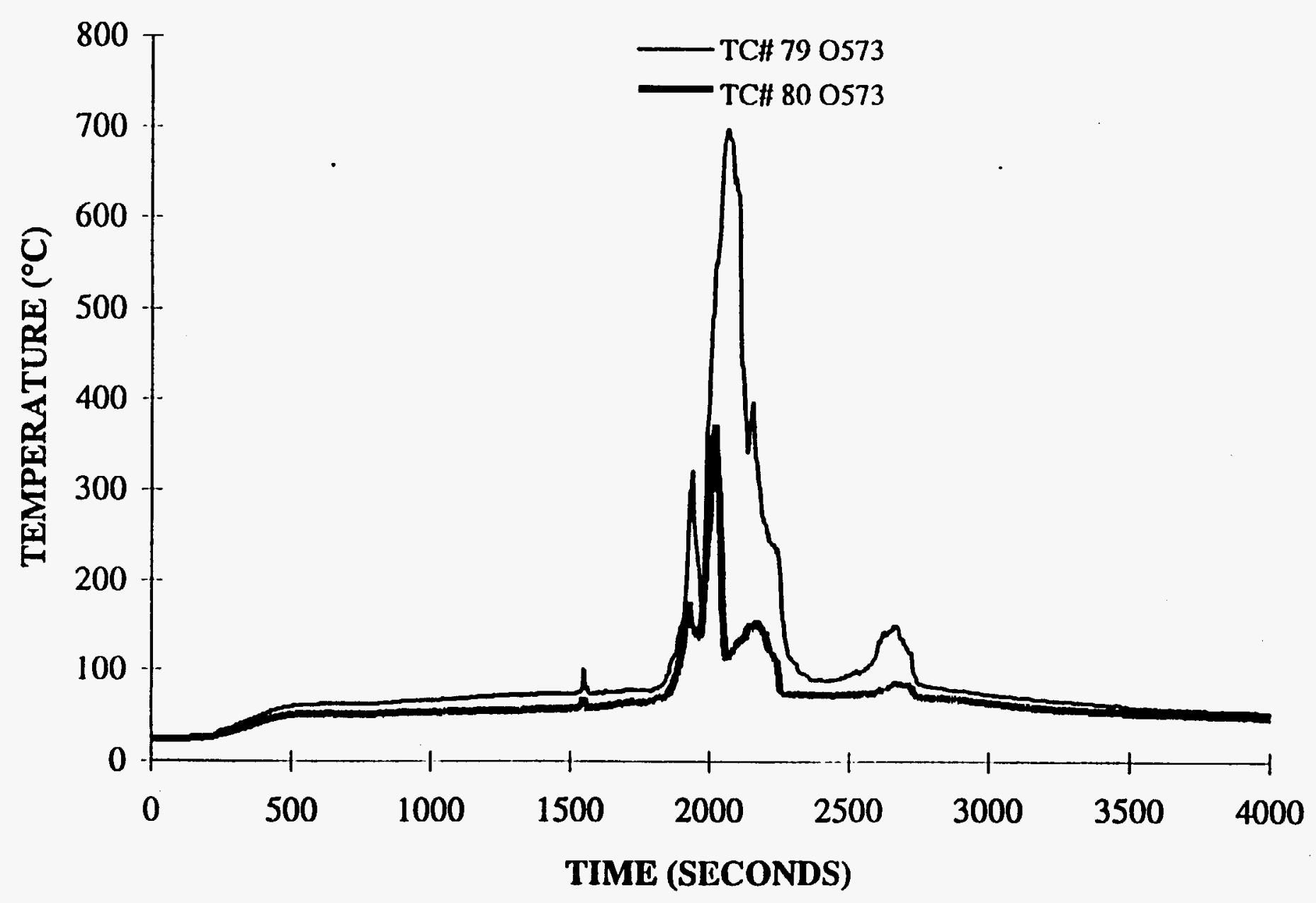

TME (SECONDS) 


\section{CLIENT: LATA}

PROJECT NO.: SwRI 01-7106

DATE: 6 JUNE 1995

TEST ID: 163LP5.DAT

\section{PALLETIZED STORAGE TEST DRUM SURFACE TC'S}

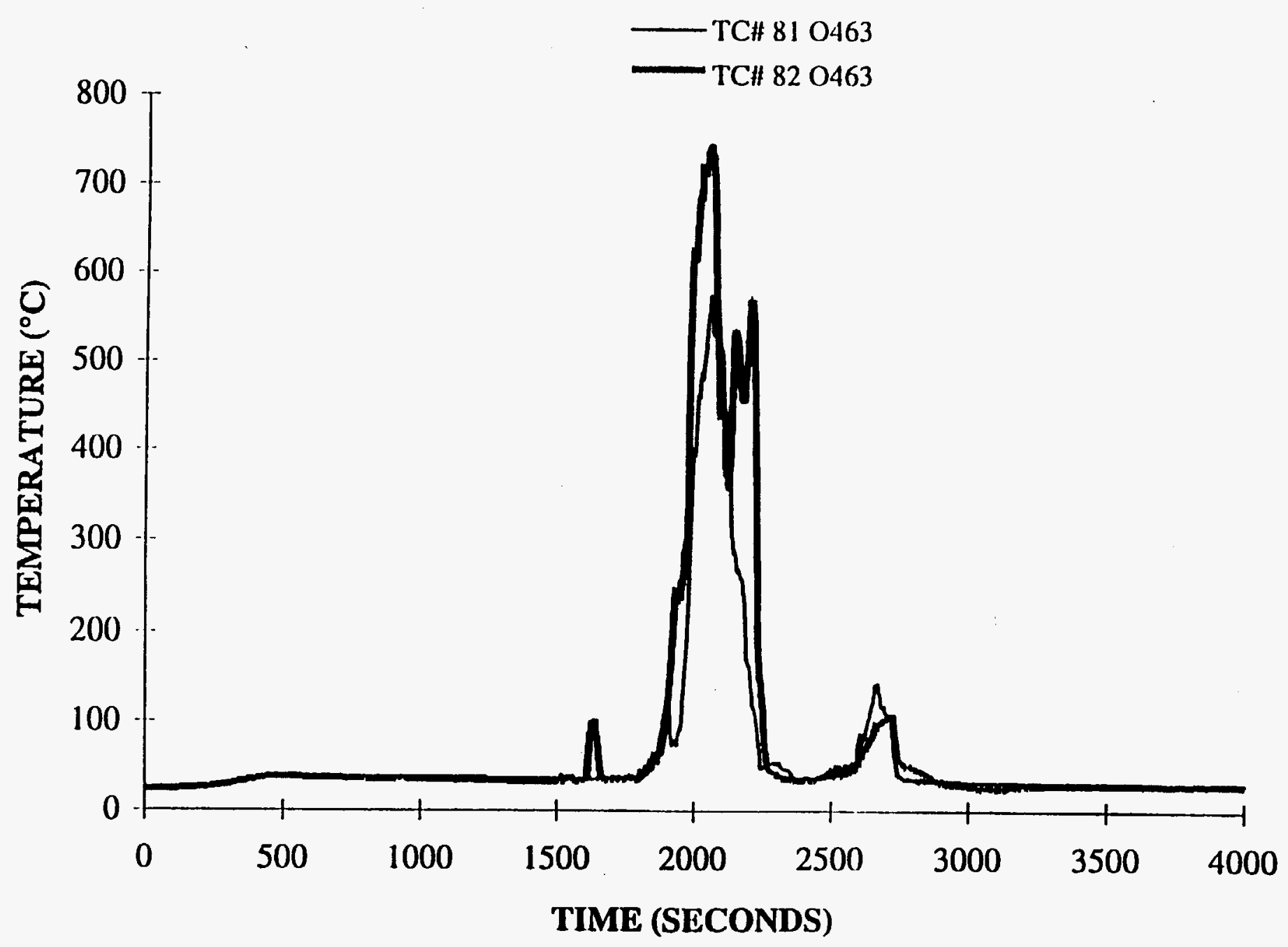

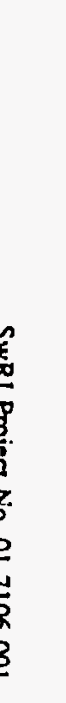

TME (SECONDS) 
CLIENT: LATA

PROJECT NO.: SwRI 01-7106

DATE: 6 JUNE 1995

TEST ID: 163LP5.DAT

\section{PALLETIZED STORAGE TEST DRUM SURFACE TC'S}

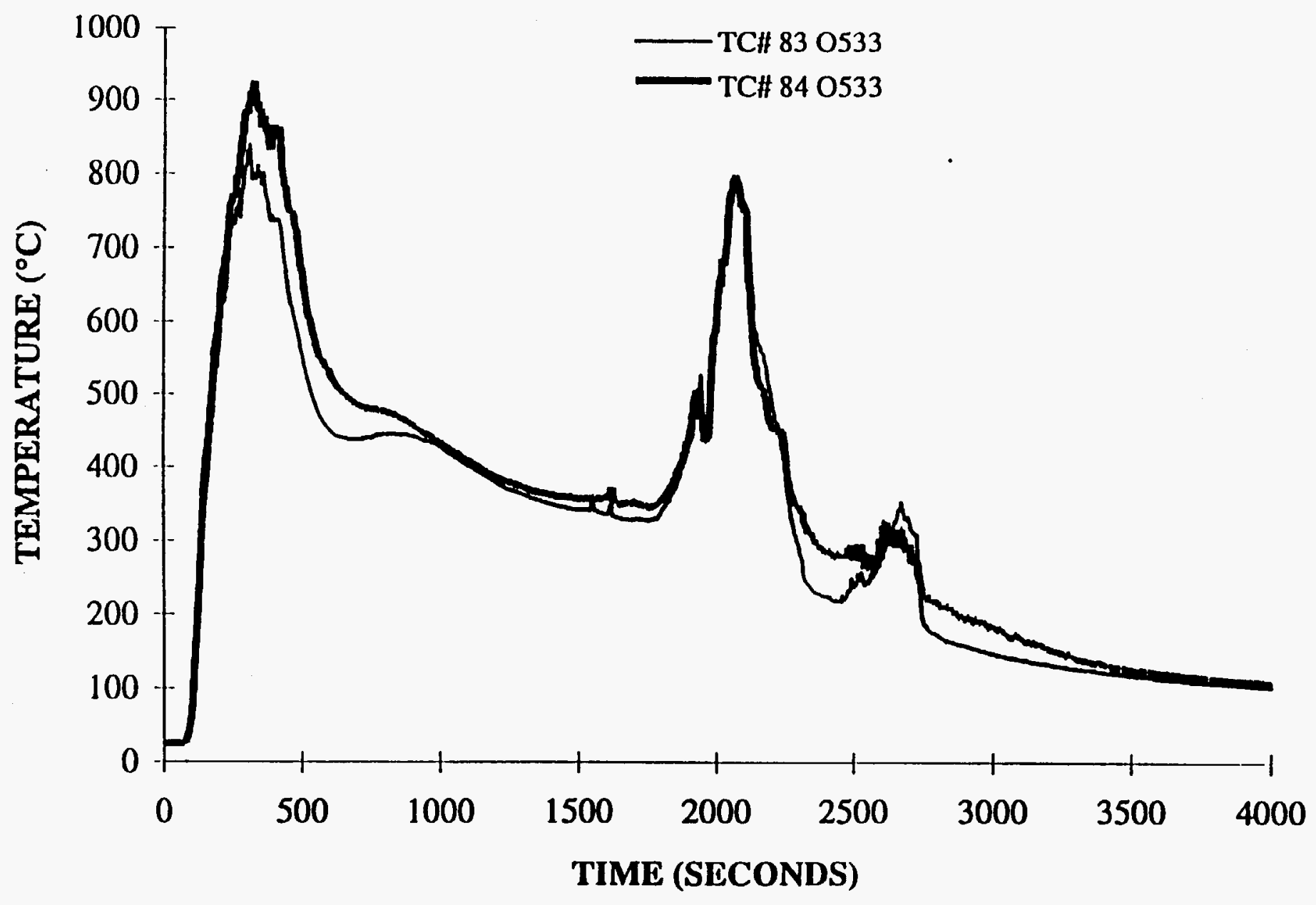


CLIENT: LATA

PROJECT NO.: SWRI 01-7106

DATE: 6 JUNE 1995

TEST ID: 157LR7.DAT

\section{PALLETIZED STORAGE TEST}

TC TREE \#1

(BETWEEN ROWS 5\&6)

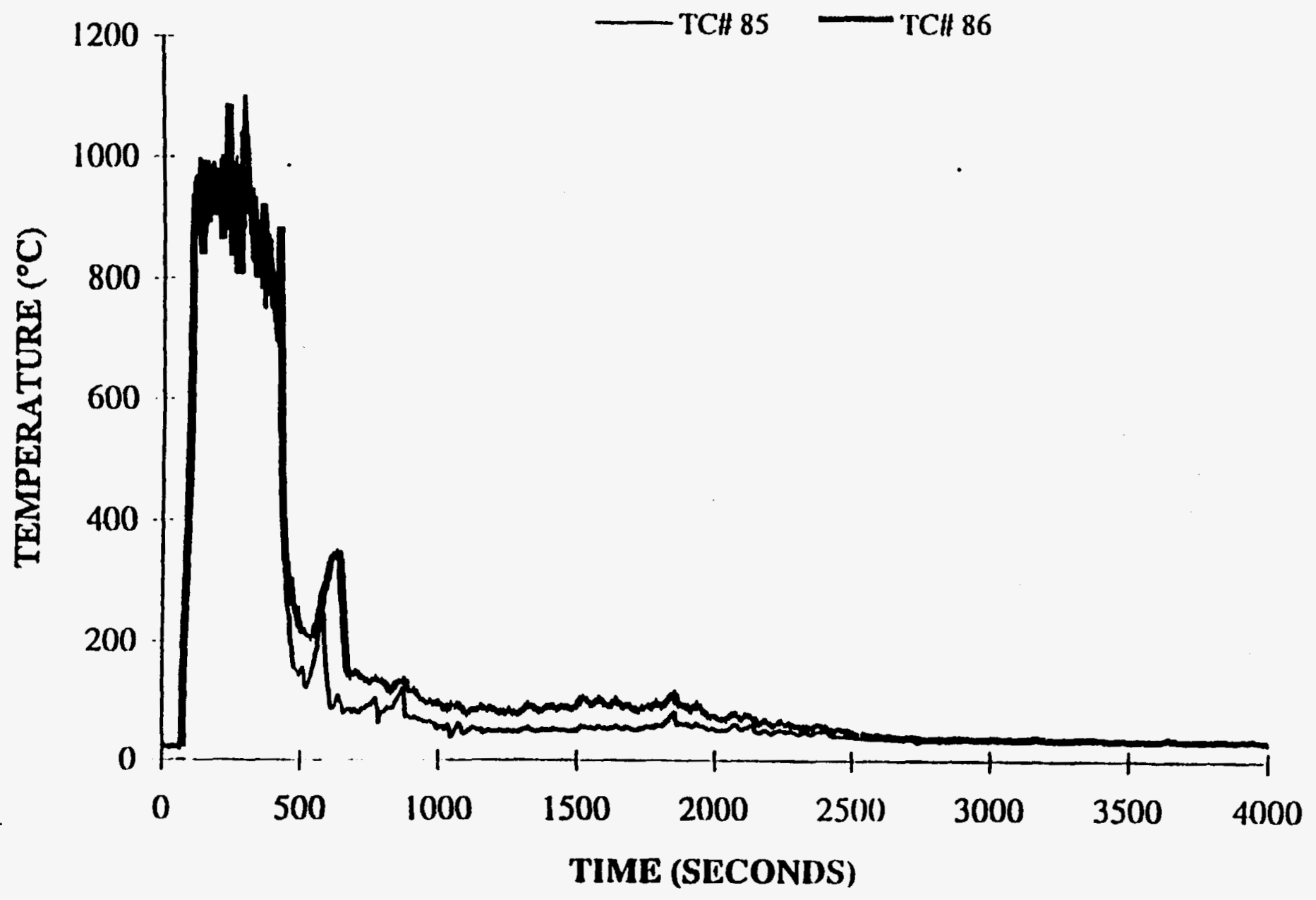




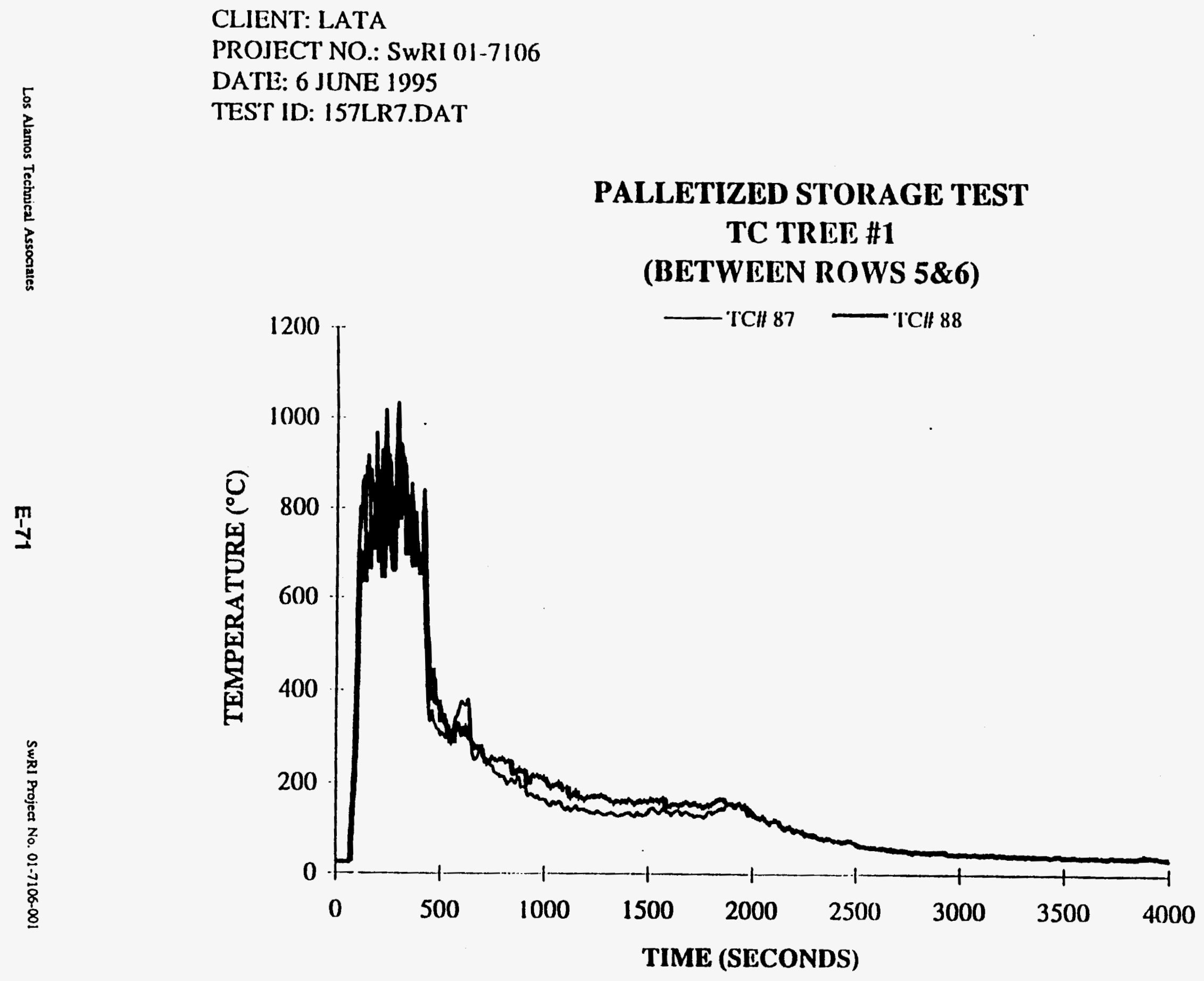


CLIENT: LATA

PROJECT NO.: SwRI 01-7106

DATE: 6 JUNE 1995

TEST ID: 157LR7.DAT

\section{PALLETIZED STORAGE TEST}

TC TREE \#1

(BETWEEN ROWS 5\&6)

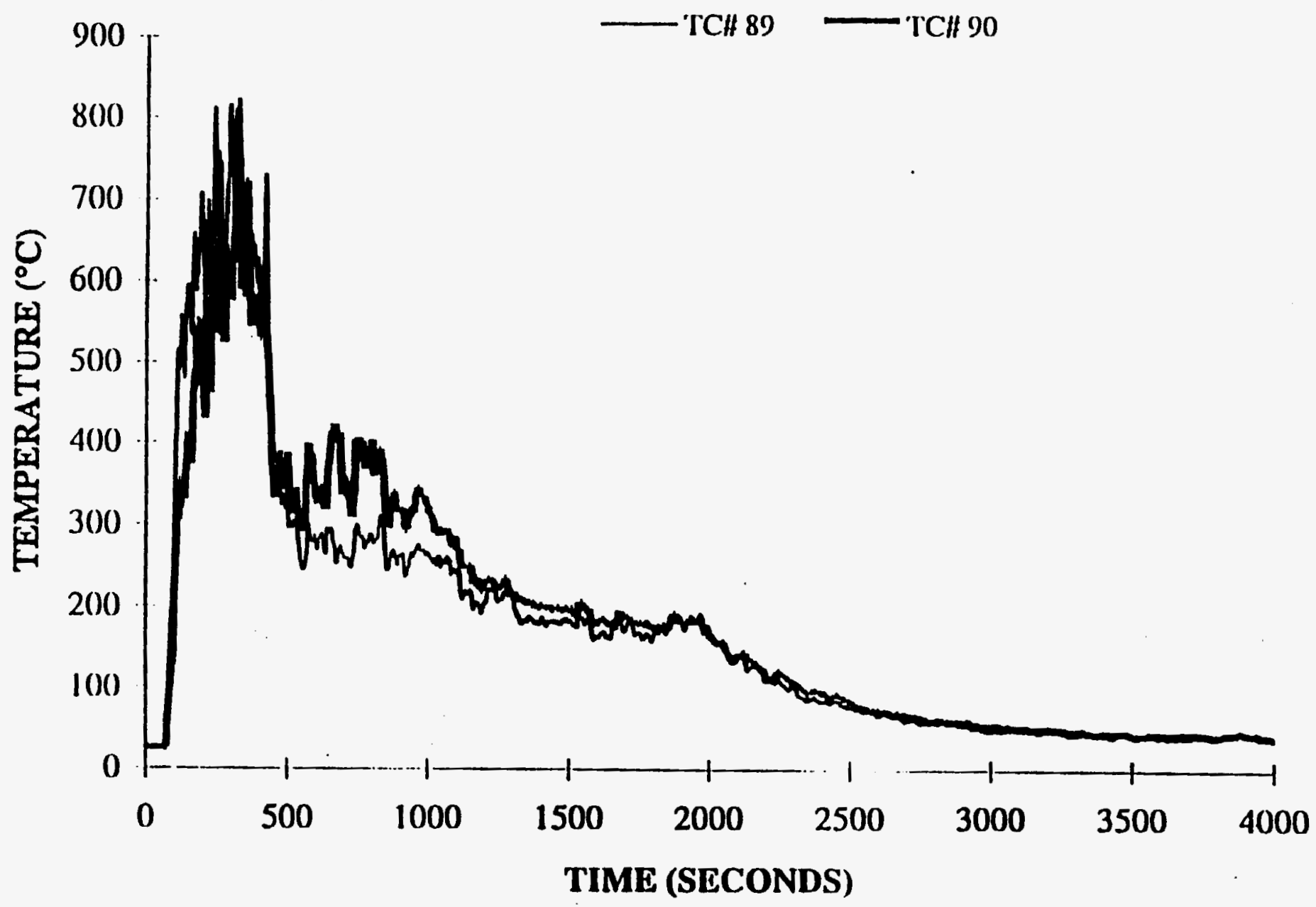


CLIENT: LATA

PROJECT NO.: SwRI 01-7106

DATE: 6 JUNE 1995

TEST ID: 157LR7.DAT

PALLETIZED STORAGE TEST

TC TREE \#1

(BETWEEN ROWS 5\&6)

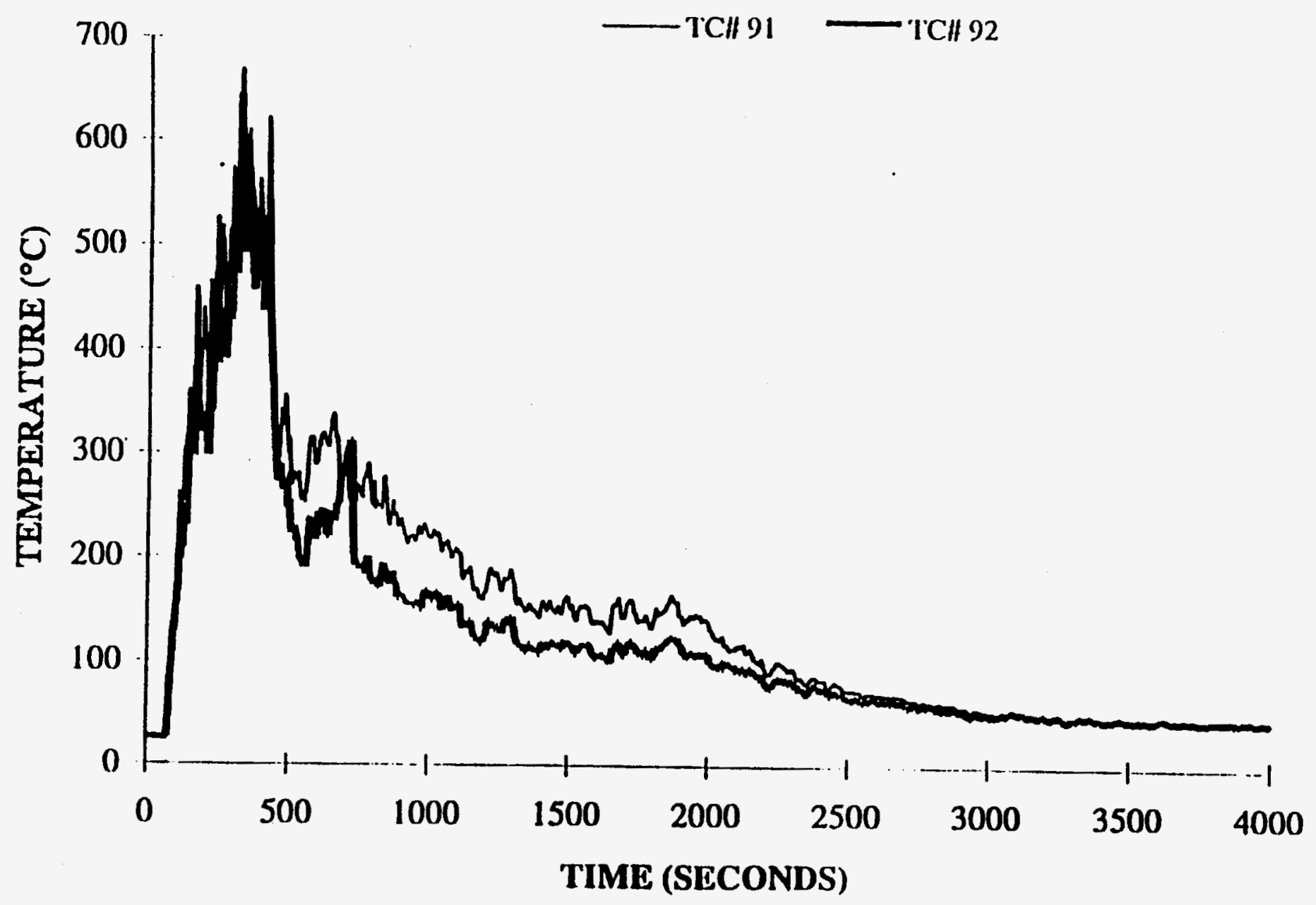


CLIENT: LATA

PROJECT NO.: SwRI 01-7106

DATE: 6 JUNE 1995

TEST ID: 157LR7.DAT

\section{PALLETIZED STORAGE TEST}

TC TREE \#1

(BETWEEN ROWS 5\&6)

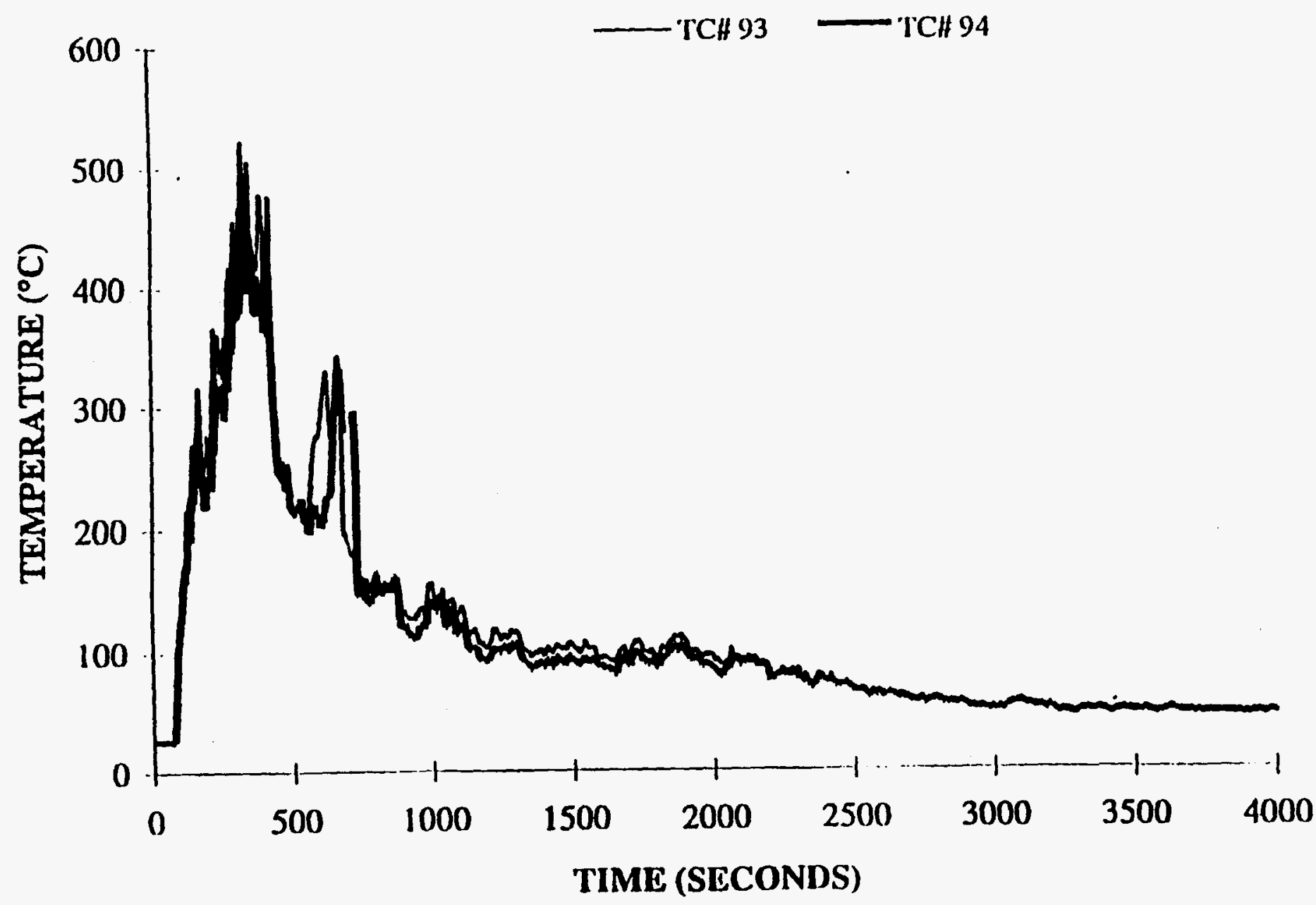


CLIENT: LATA

PROIECT NO.: SwRI 01-7106

DATE: 6 JUNE 1995

TEST ID: 157LR7.DAT

PALLETIZED STORAGE TEST

TC TREE \#1

(BETWEEN ROWS 5\&6)

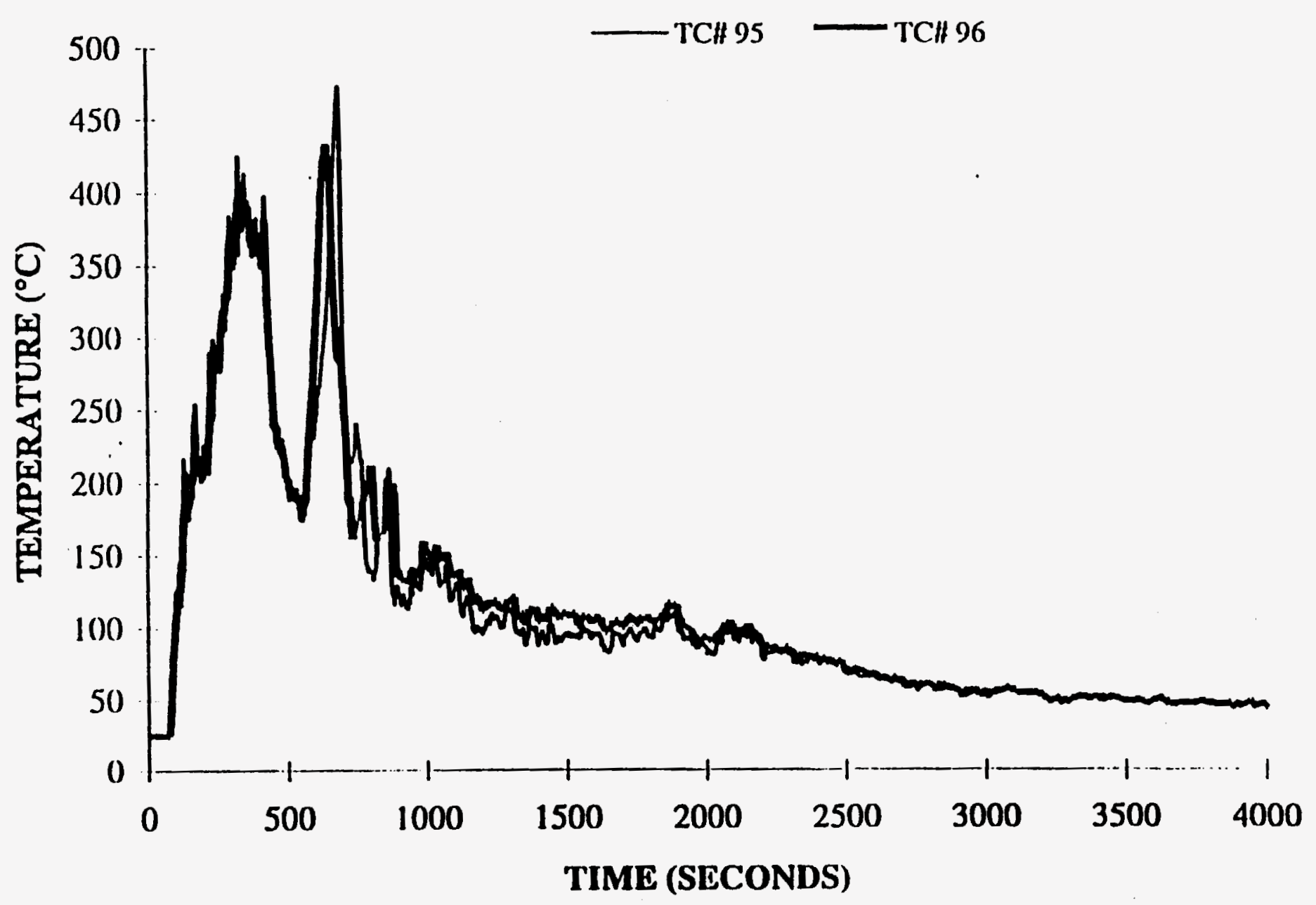


CLIENT: LATA

PROJECT NO.: SwRI 01-7106

DATE: 6 JUNE 1995

TEST ID: 157LR7.DAT

PALLETIZED STORAGE TEST

TC TREE \#1

(BETWEEN ROWS 5\&6)

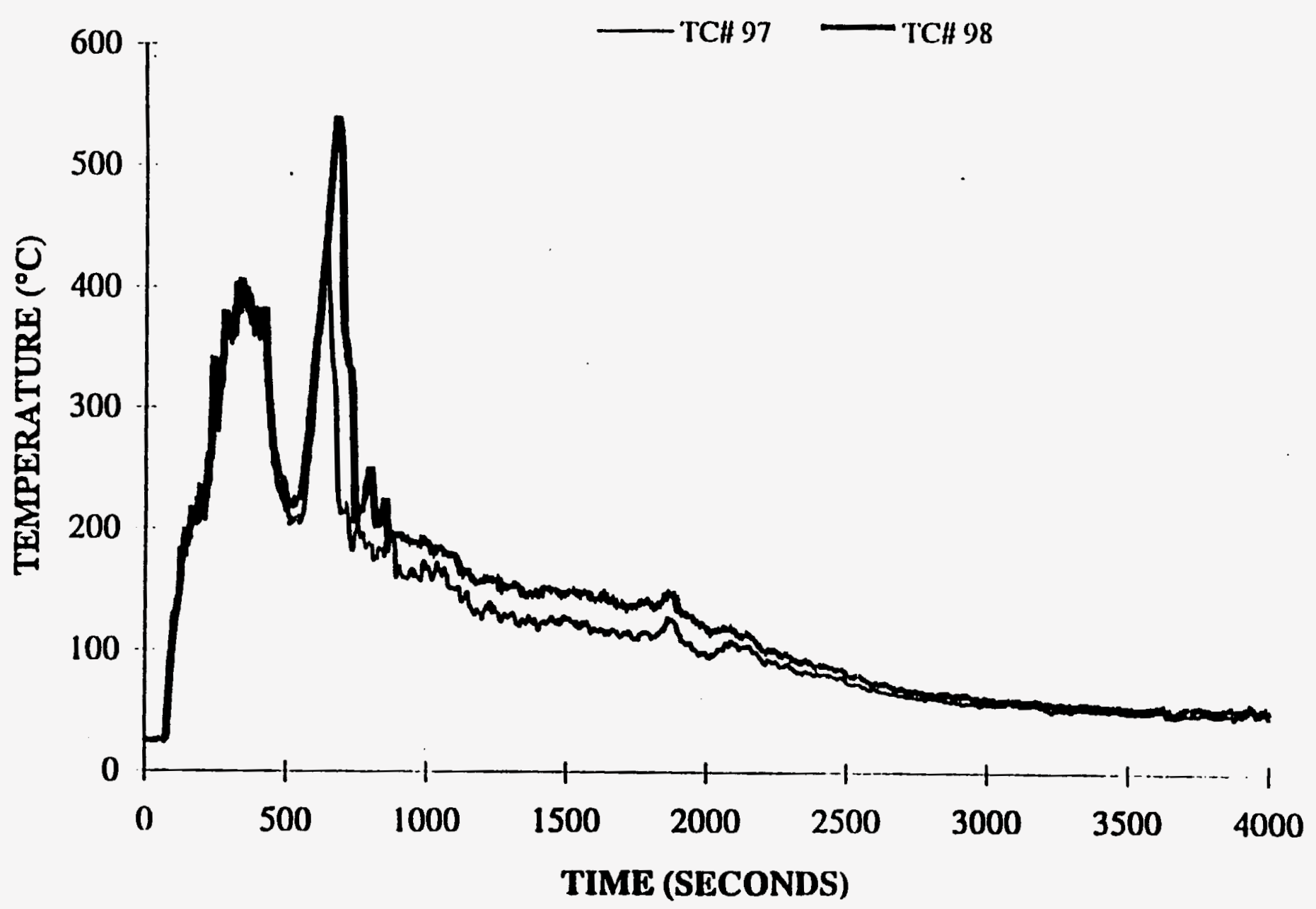




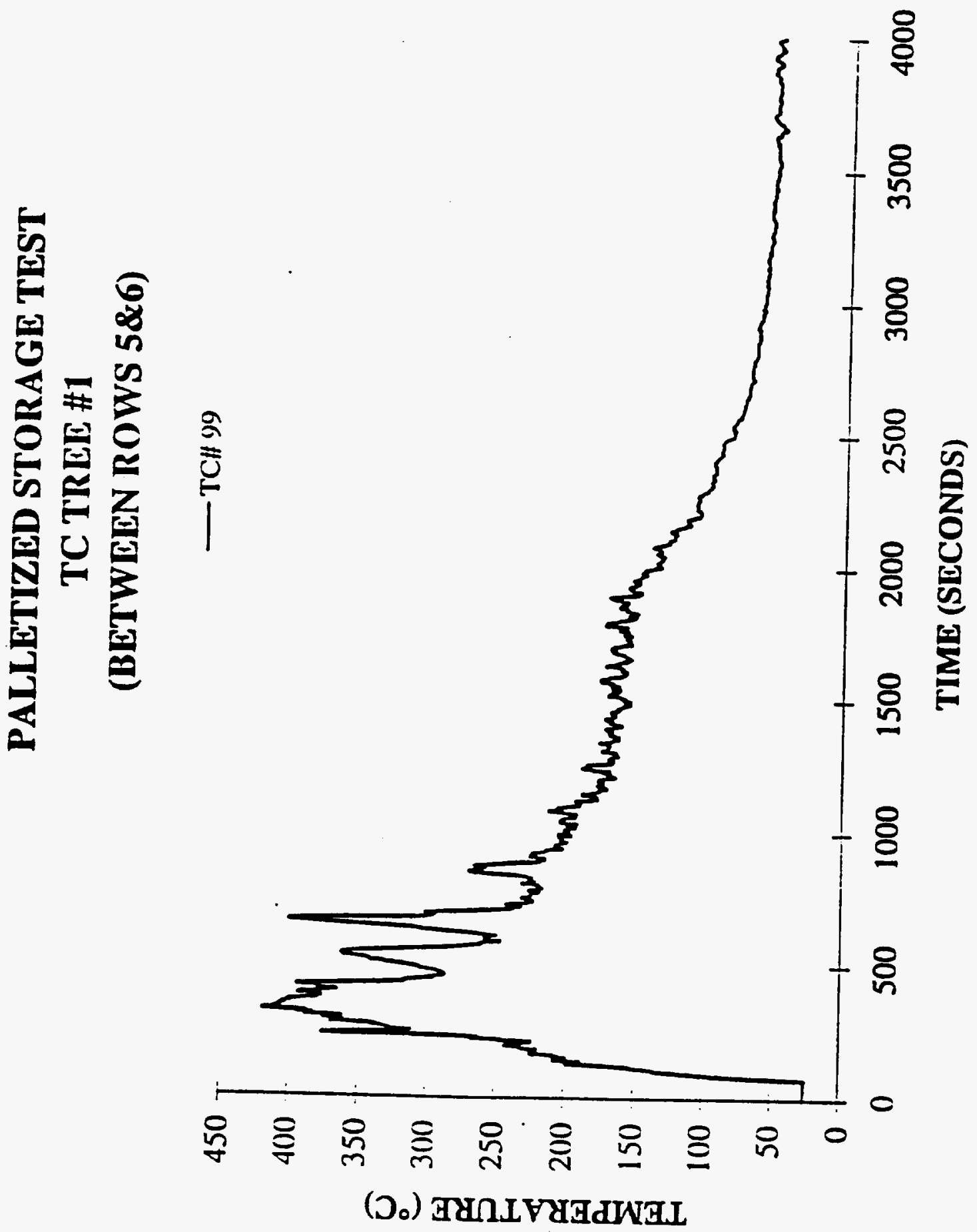




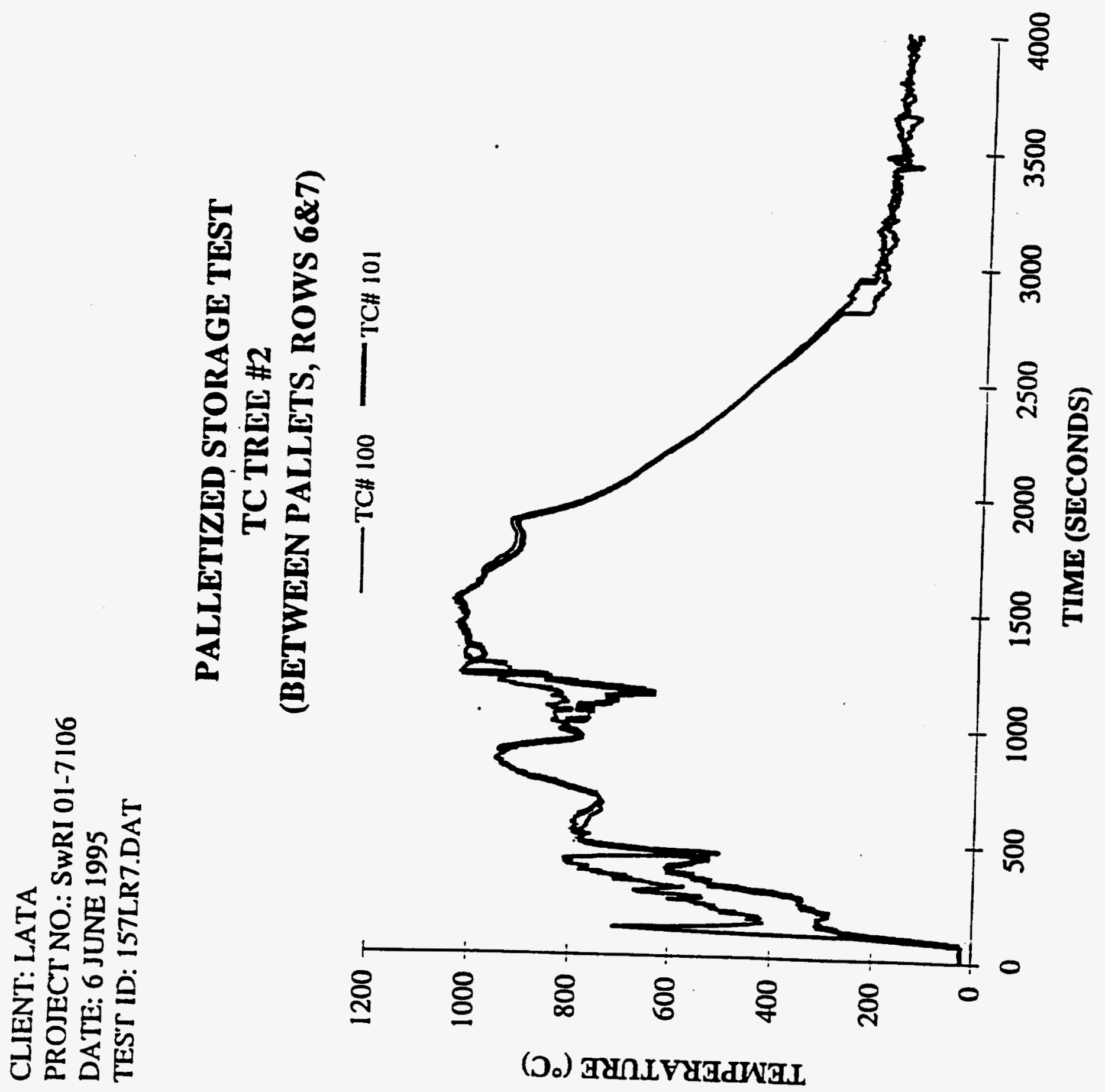


CLIENT: LATA

PROJECT NO.: SwRI 01-7106

DATE: 6 JUNE 1995

TEST ID: 157LR7.DAT

\section{PALLETIZED STORAGE TEST}

TC TREE \#2

(BETWEEN PALLETS, ROWS 6\&7)

罗

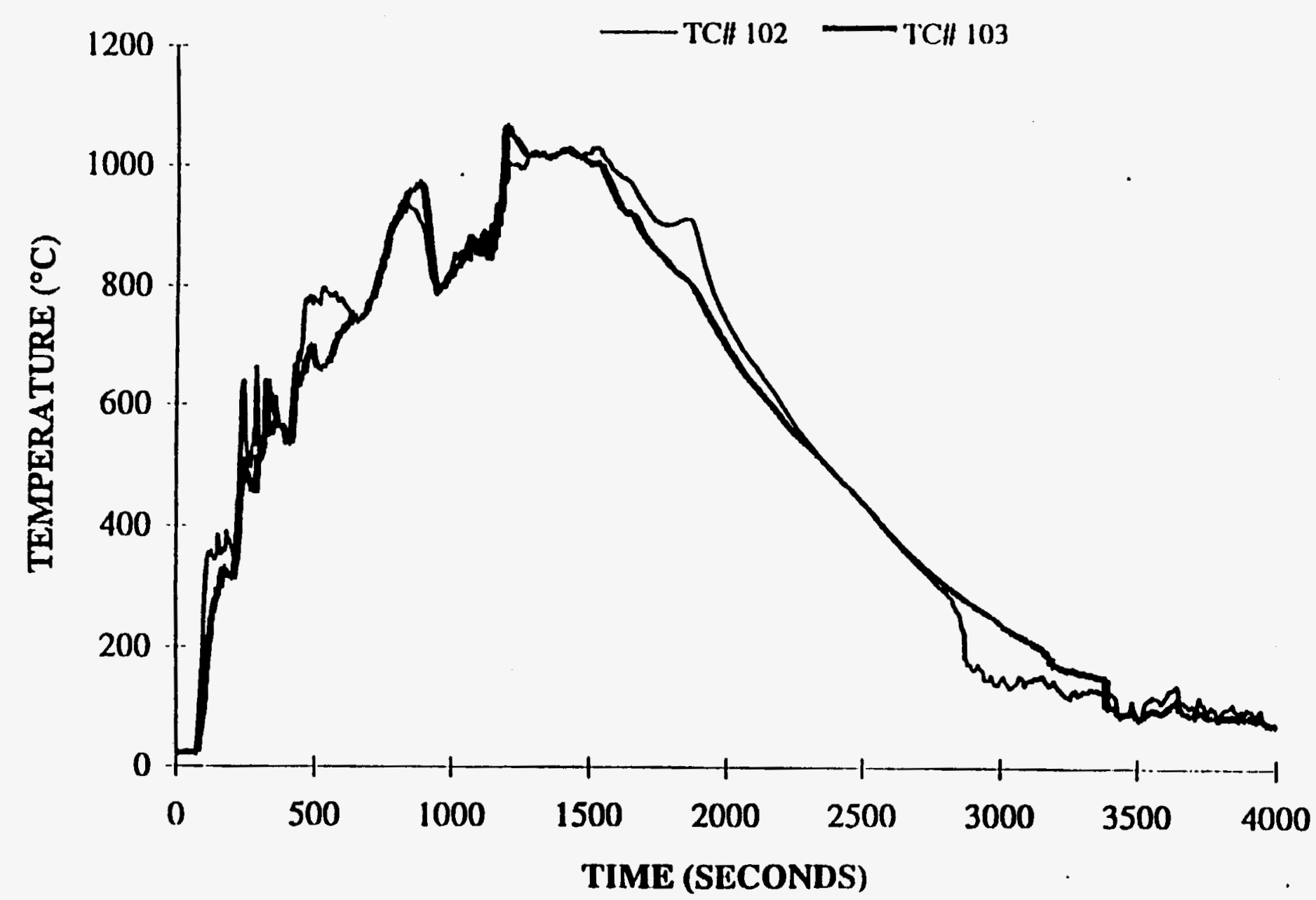

$\vdots$
1
0
0
$\vdots$
$\vdots$
$\vdots$
$\vdots 1$
0
0
0
0
0
0
0
0
0 
CLIENT: LATA

PROJECT NO.: SwRI 01-7106

DATE: 6 JUNE 1995

TEST ID: 157LR7.DAT

\section{PALLETIZED STORAGE TEST}

TC TREE \#2

(BETWEEN PALLETS, ROWS 6\&7)

点

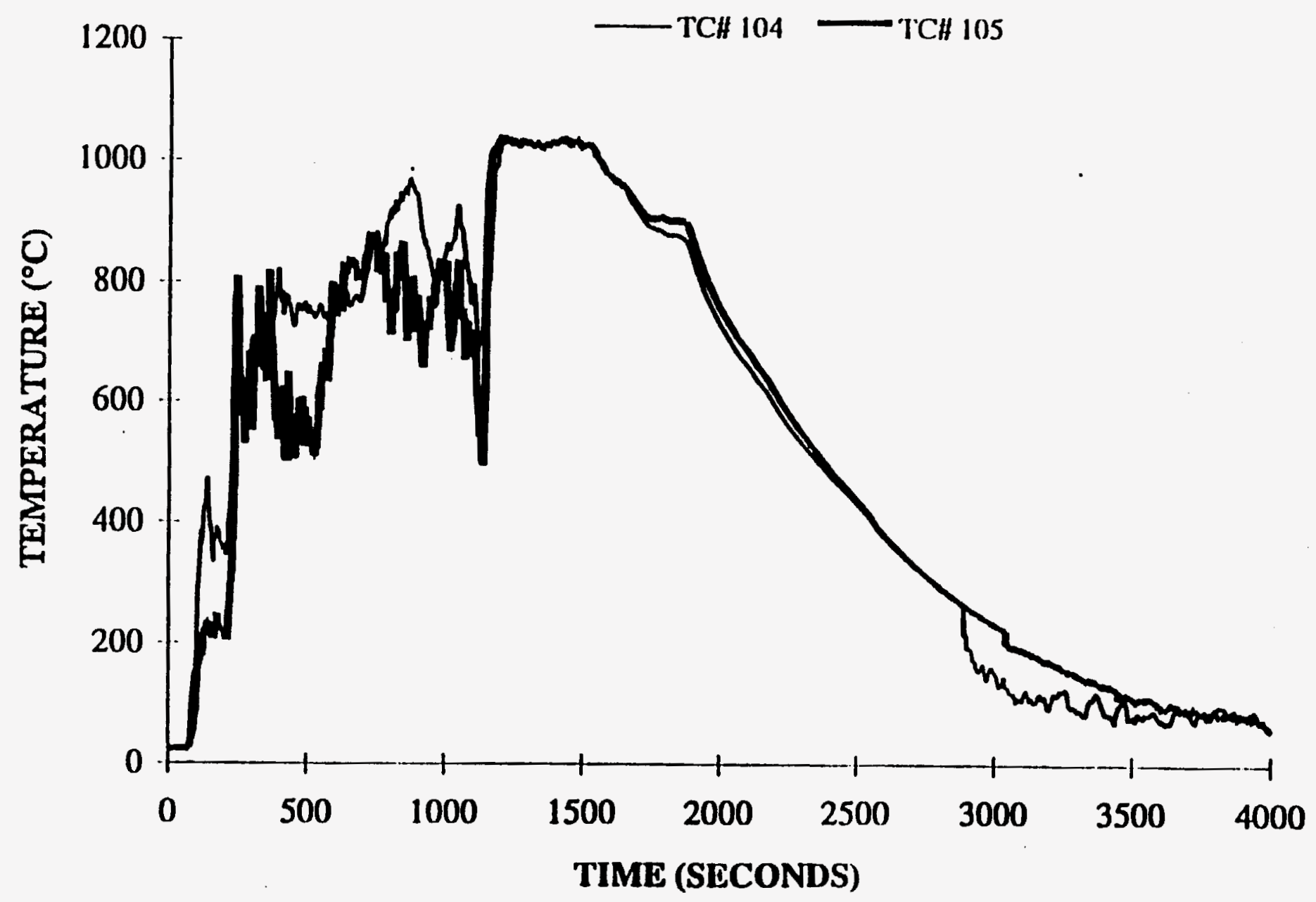




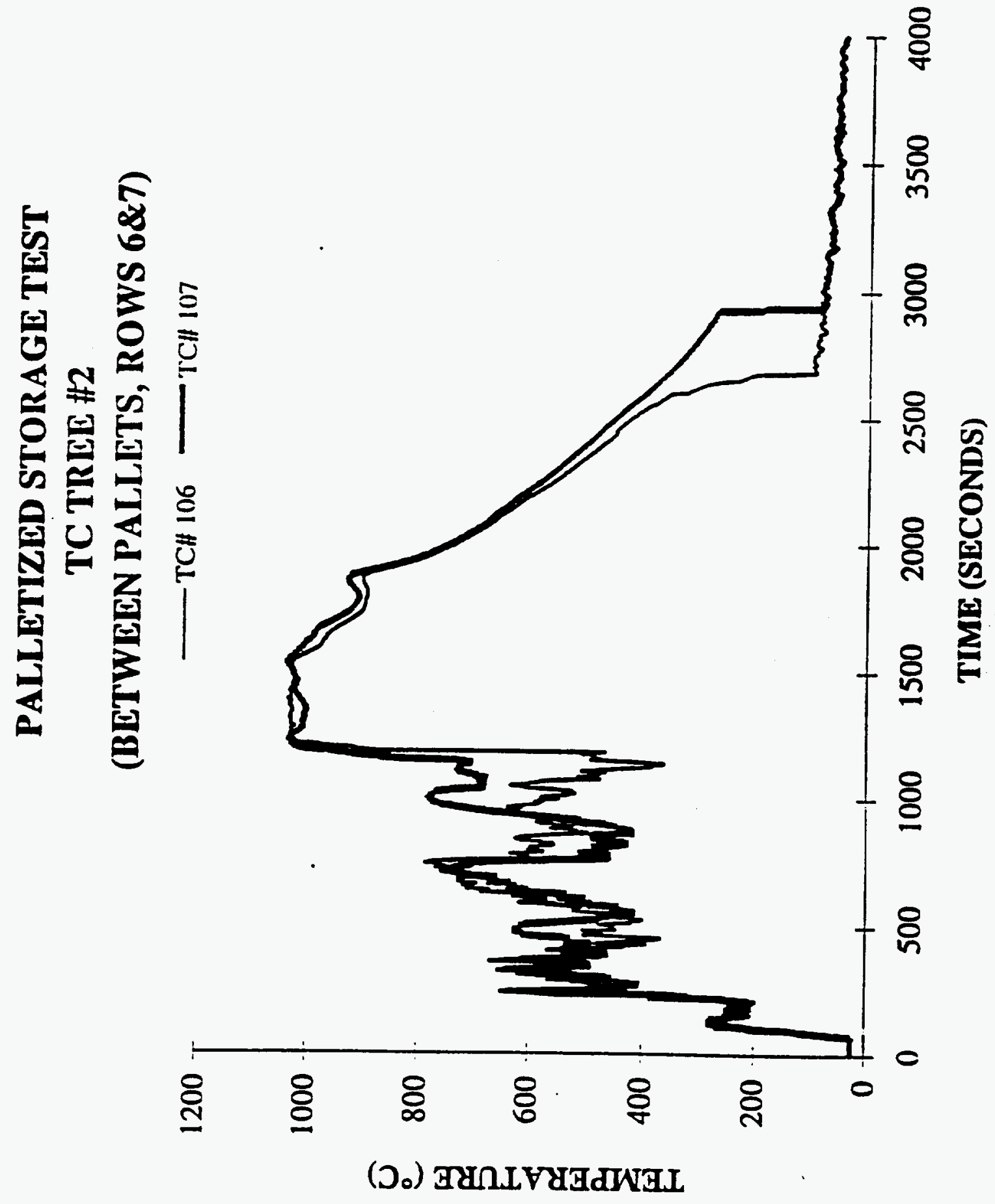


CLIENT: LATA

PROJECT NO.: SwRI 01-7106

DATE: 6 JUNE 1995

TEST ID: 157LR7.DAT

PALLETIZED STORAGE TEST

TC TREE \#2

(BETWEEN PALLETS, ROWS 6\&7)

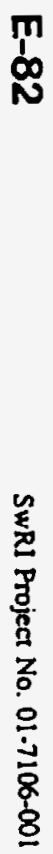

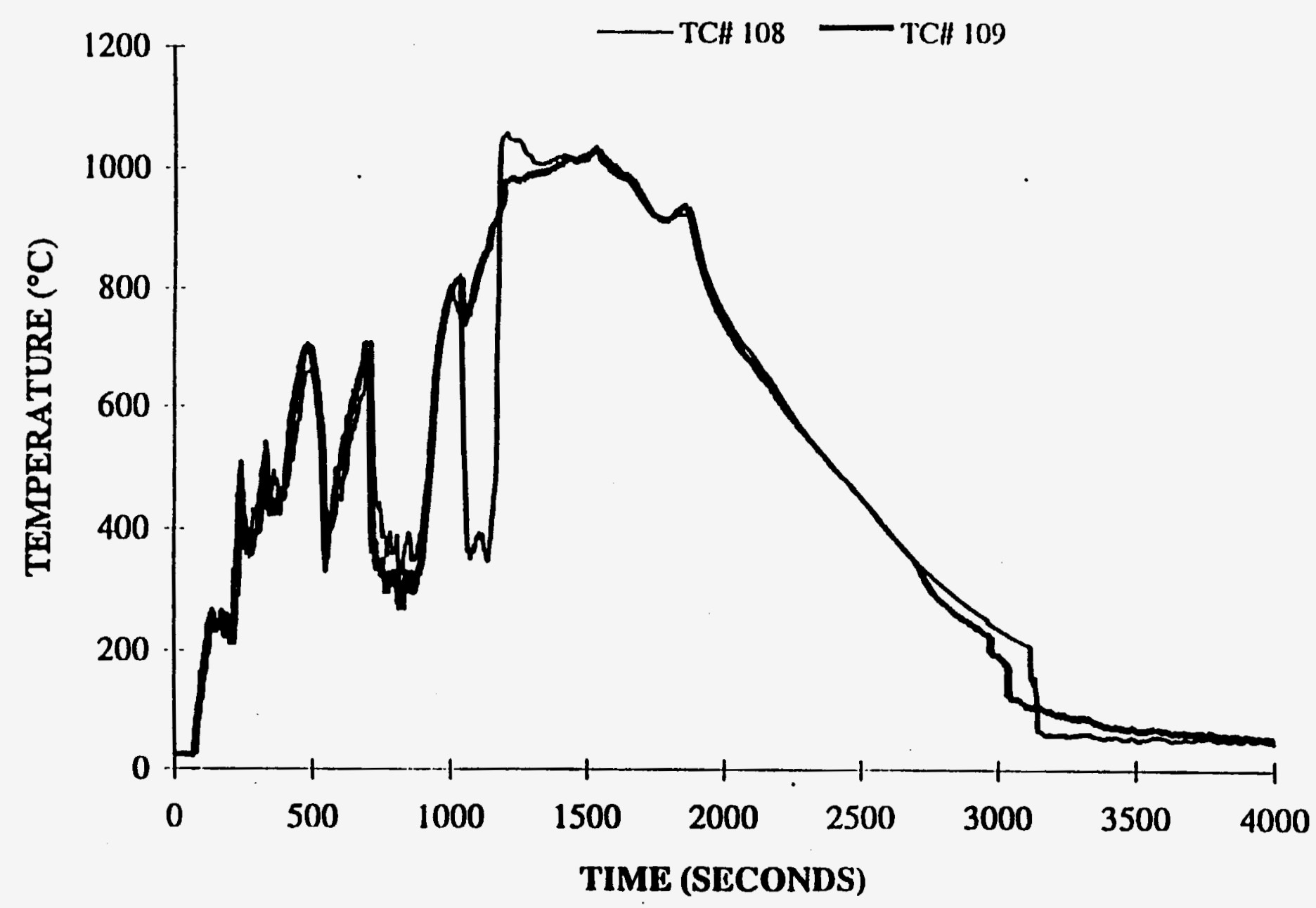




\section{CLIENT: LATA}

PROJECT NO.: SwRI 01-7106

DATE: 6 JUNE 1995

TEST ID: 157LR7.DAT

\section{PALLETIZED STORAGE TEST}

TC TREE \#2

(BETWEEN PALLETS, ROWS 6\&7)

蚛

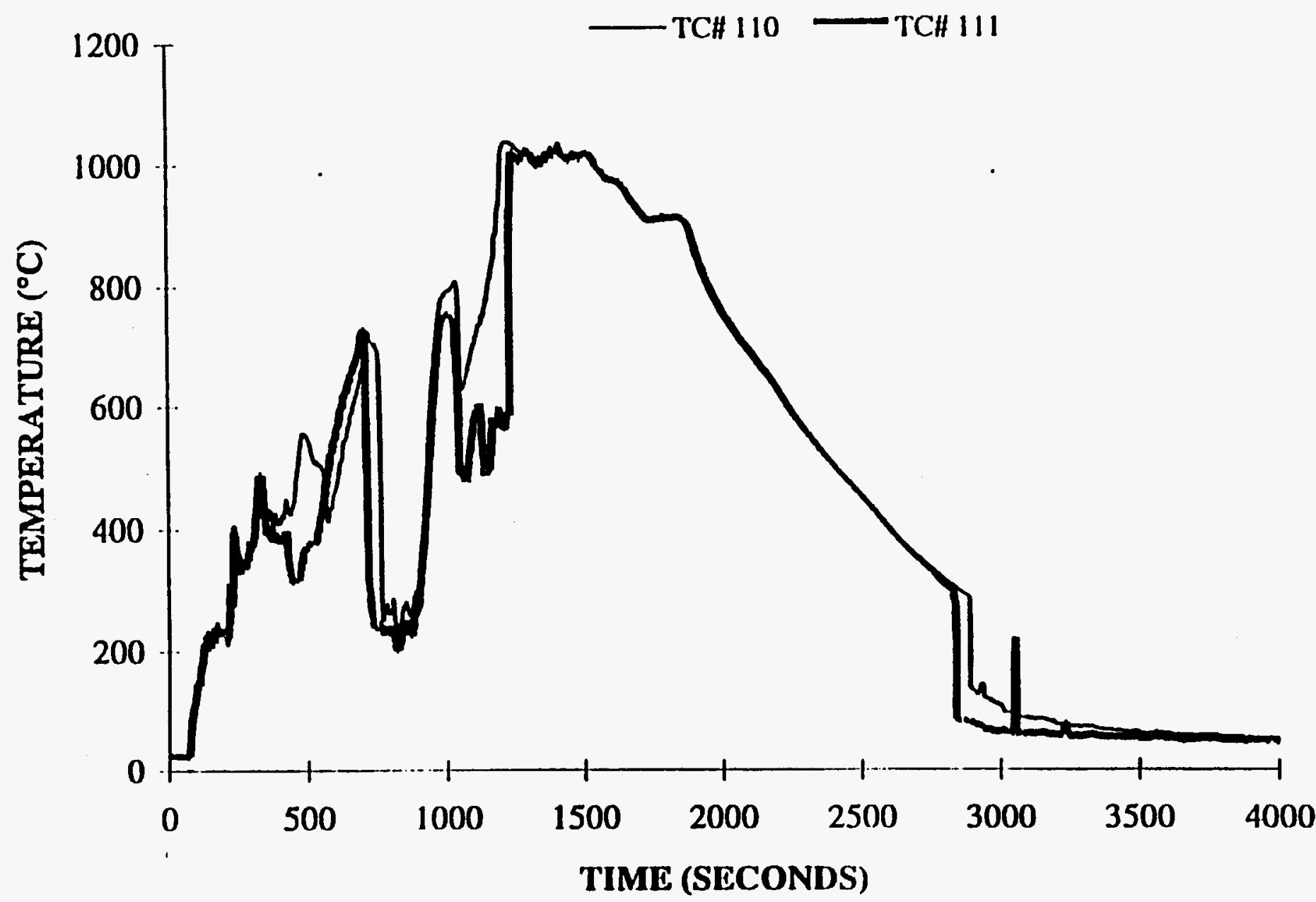


CLIENT: LATA

PROJECT NO.: SWRI 01-7106

DATE: 6 JUNE 1995

TEST ID: 157LR7.DAT

\section{PALLETIZED STORAGE TEST}

TC TREE \#2

(BETWEEN PALLETS, ROWS 6\&7)

$\underset{\substack{m \\ \infty}}{\mathrm{m}}$

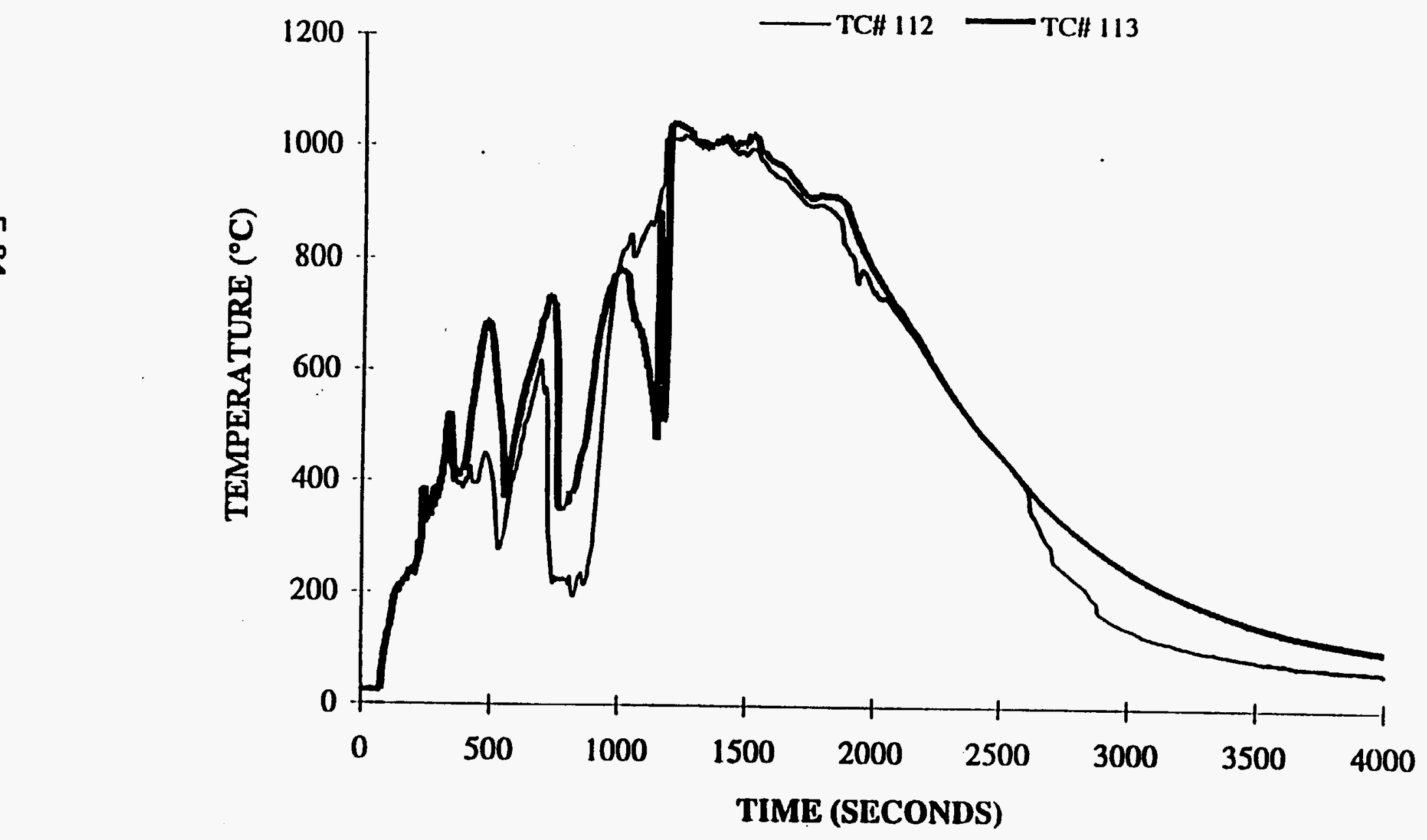


CLIENT: LATA

PROJECT NO.: SwRI 01-7106

DATE: 6 JUNE 1995

TEST ID: 157LR7.DAT

\section{PALLETIZED STORAGE TEST}

TC TREE \#2

\section{(BETWEEN PALLETS, ROWS 6\&7)}

-TCH 114

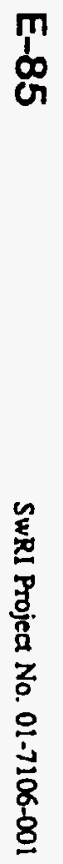

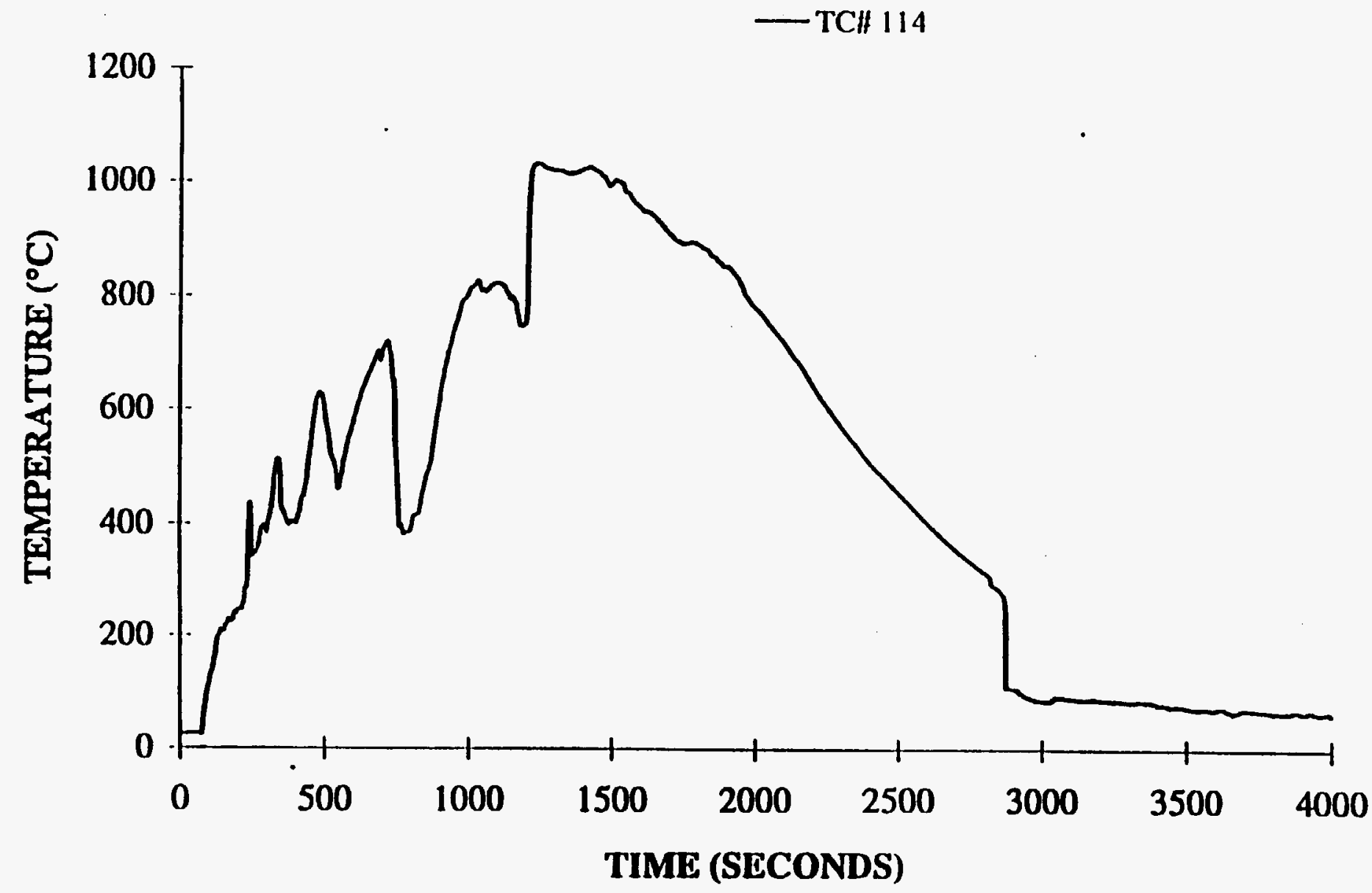

TIME (SECONDS) 
Blank 
WHC-SD-WM-TRP-246 REV. 0

Appendix $F$

Palletized Storage Drum Data Sheets

$\mathrm{F}-1$ 
Blank 


\section{Palletized Storage Drum Data Sheet}

Drum No. 0.411

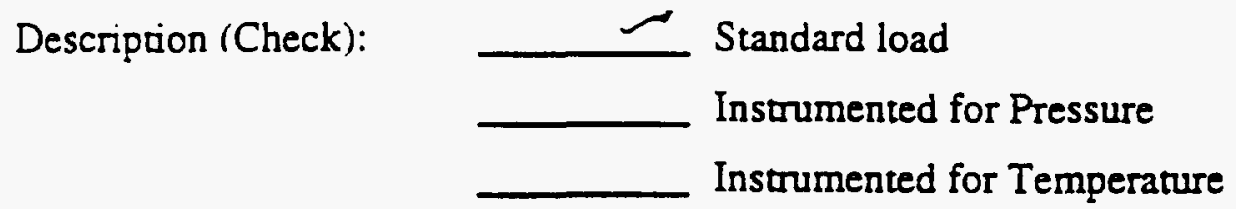

\begin{tabular}{|c|c|c|c}
\hline Loading Starting at Bottom & Layer 1 & Layer 2 & Layer 3 \\
\hline 3 pieces of steel & & N/A & N/A \\
\hline $1 / 3$ of the brown paper & & & \\
\hline $1 / 3$ of the 10-mil plastic & & & \\
\hline $1 / 3$ of the bond paper & & & \\
\hline $1 / 3$ of the towels & & & \\
\hline $1 / 3$ of the bags & & & \\
\hline $1 / 3$ of the cut paper & & & \\
\hline $1 / 3$ of the fabric & & $\checkmark$ & \\
\hline $1 / 3$ of the rubber & Go to Layer 2 & Go to Laver 3 & \\
\hline
\end{tabular}

Drum Filled:

Ring bolt placed opposite drum seam and in down position

Lid sealed and torqued to $40 \mathrm{ft} / \mathrm{b}$ :

Leak tested:

Pre-test weight (lb)

Post-test weight (lb)
Date $5-15-95$ Initial Af

Date $5-15-95$ Initial i2P

Date $5-15-95$ Initial AP

N/A or Pressure (psig)

Pressure (psig)

Date

Initial

Time (min) Time (min) 5

\subsection{Target weight $-175.6 \mathrm{lb}$}

Loss $=$ 
Drum No. 0411176.016.

Post-test Assessment

Position:

original: tipped: toppled

Lid on drum: $\quad \chi \quad$ Yes No

Gasket missing (cm) Cl sww what rompresused

Contents expeiled: _ Yes $\underline{X}$ No

Seal failure:

$$
\text { - Yes } \ \text { Not visible }
$$

Burn partern:

NONe

Photo taken:

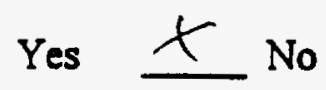

Leak tested:

A $\sqrt{A}$ or Pressure (psig) Pressure (psig)

Date
Time (min) $\quad \frac{0}{5}$

Initial

Interior assessment:

slighity metted to sall 12"

C IDec 98

Photo taken:

$X$ Yes

No

Comments: 


\section{Palletized Storage Drum Data Sheet}

Drum No. 0421

Descriprion (Check):

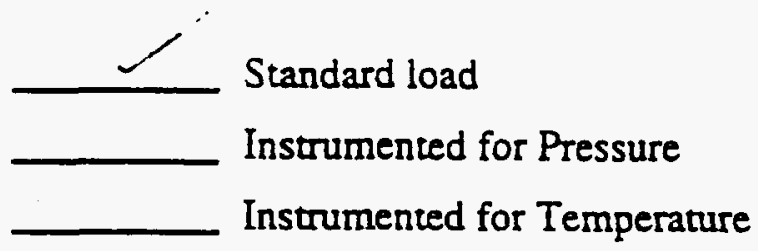

\begin{tabular}{|c|c|c|c|}
\hline Loading Starting at Bottom & Layer 1 & Layer 2 & Layer 3 \\
\hline 3 pieces of steel & & N/A & N/A \\
\hline $1 / 3$ of the brown paper & & & \\
\hline $1 / 3$ of the 10-mil plastic & & & \\
\hline $1 / 3$ of the bond paper & & & \\
\hline $1 / 3$ of the towels & & & \\
\hline $1 / 3$ of the bags & & & \\
\hline $1 / 3$ of the cut paper & & & \\
\hline $1 / 3$ of the fabric & Go to Layer 2 & Go to Layer 3 & \\
\hline $1 / 3$ of the rubber & & \\
\hline
\end{tabular}

Drum Filled:

Ring bolt placed opposite drum seam and in down position

Lid sealed and torqued to $40 \mathrm{ft} / \mathrm{b}$ :

Leak tested:

Pre-test weight (lb)

Post-test weight (lb)
Date $5-15-25$ Initial 178

Date $5-15-95$ Initial $4 P$

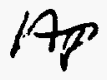

Date $5-15-95$ Initial
N/A or Pressure (psig)

Pressure (psig)

Date

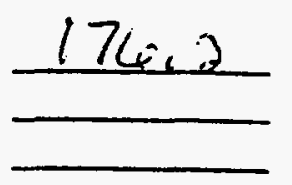

Time (min) 0

Time (min) 5
Initial

Target weight $\sim 175.6 \mathrm{lb}$ 
Drm No. $\quad 0421 \quad 176.21 b$

Post-test Assessment

Position: original: tipped: toppled

Lid on drum: $\quad x$ Yes No

Gasket missing (cm) $O 24$ "co-pressed thand

Contents expelled: __ Yes $x$ No

Seal failure: Yes $\quad x$ Not visible

Burn pattern: None

Photo taken: $\quad$ Yes $\underline{x}$ No

Leak tested: $\quad$ Th7 or Pressure (psig) Pressure (psig)

Date \begin{tabular}{lll}
- & Time (min) & 0 \\
\hline & Time (min)
\end{tabular}

Interior assessment:

bacily mithd 19" ret of sean

videc 97

Ehoto taken:

$\lambda$ Yes No

Comments: 
WHC-SD-WM-TRP-246 REV. 0

\section{Palletized Storage Drum Data Sheet}

Drum No. 043

Description (Check):

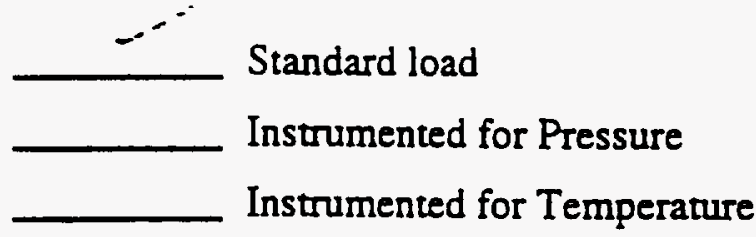

\begin{tabular}{|c|c|c|c|}
\hline Loading Starting at Bottom & Layer 1 & Layer 2 & Layer 3 \\
\hline 3 pieces of steel & & N/A \\
\hline $1 / 3$ of the brown paper & & & \\
\hline $1 / 3$ of the 10-mil plastic & & & \\
\hline $1 / 3$ of the bond paper & & & \\
\hline $1 / 3$ of the towels & & & \\
\hline $1 / 3$ of the bags & & & \\
\hline $1 / 3$ of the cut paper & & & \\
\hline $1 / 3$ of the fabric & Go to Layer 2 & Go to Layer 3 & \\
\hline $1 / 3$ of the rubber & & & \\
\hline
\end{tabular}

Drum Filled:

Ring bolt placed opposite drum seam and in down position
Leak tested:

Pre-test weight (lb)

Post-test weight (lb)

$$
\text { Loss }=
$$

Lid sealed and torqued to $40 \mathrm{ft} / 1 \mathrm{~b}:$ Date $5-15-95$ Initial 17 i

Date $5-15-95$ Initial 24

Date $5-15-55$ Initial eff
Time (min) 0

Initial
N/A or Pressure
Pressure (psig)

Date

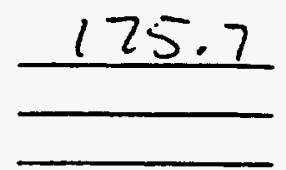

Target weight $-175.6 \mathrm{lb}$ 
WHC-SD-WM-TRP-246 REV. 0

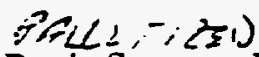

Reol Eterege Drum Data Sheer

Drum No. $\quad 0431 \quad 175.716$

Post-test Assessment

Position:

original:

tipped:

toppled

Lid on drum: $\quad x$ Yes $\quad$ No

Gasket missing (cm) _ 7 _

Contents expelled: $\quad$ Yes

Seal failure: $\quad$ Yes $\quad$ Y Not visible

Burn pattern:

Nove except 8" spit vean rim centened $28^{\prime \prime}$ Iftofsean

Photo taken: $\quad$ Yes $X$ No

Leak tested: $\quad$ (I/A or Pressure (psig)

Pressure (psig)

Date

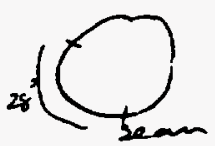

Interior assessment:

$\frac{\text { at buen spot - metting of plastic and surface chaming }}{\text { of inatual agringt wall }}$

venec ill

Photo taken:

$x$ Yes

Comments: 
WHC-SD-WM-TRP-246 REV. 0

\section{Palletized Storage Drum Data Sheet}

Drum No. $(744)$

Description (Check):

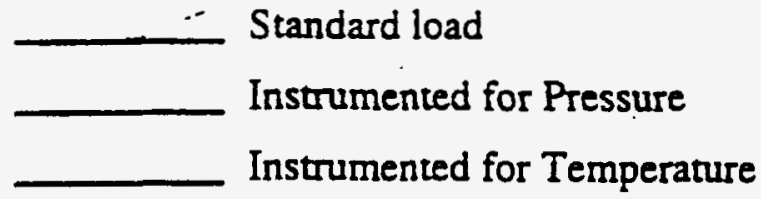

\begin{tabular}{|c|c|c|c|}
\hline Loading Starting at Bottom & Layer 1 & Layer 2 & Layer 3 \\
\hline 3 pieces of steel & N/A \\
\hline $1 / 3$ of the brown paper & & & \\
\hline $1 / 3$ of the 10 -mil plastic & & & \\
\hline $1 / 3$ of the bond paper & & & \\
\hline $1 / 3$ of the toweis & & & \\
\hline $1 / 3$ of the bags & & & \\
\hline $1 / 3$ of the cut paper & & & \\
\hline $1 / 3$ of the fabric & & Go to Layer 3 & \\
\hline $1 / 3$ of the rubber & Go to Layer 2 & \\
\hline
\end{tabular}

Drum Filled:

Ring bolt placed opposite drum seam and in down position

Lid sealed and torqued to $40 \mathrm{ft} / \mathrm{b}$ :

Leak tested:

Pre-test weight (lb)

Post-test weight (lb)
Date $5-15-25$ Initial $A P$

Date 5-15-95 Initial RP
N/A or Pressure (psig)

Pressure (psig)

Date

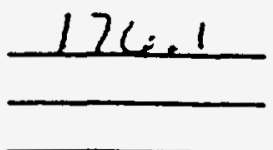

F-8
Time (min) 0

Time (min) 5

Initial

Target weight $-175.6 \mathrm{lb}$ 
Drim No. $0441 \quad 176.1$

Post-test Assessment

Position: original: tipped: toppled

Lid on drum: $\quad X$ Yes No

Gasket missing (cm) $O$ soinewhat conpressed

Contents expelled: _ Yes $x$ No

Seal failure: Yes $\$ Not visible

Burn pattern:

Nonte

Photo taken: $\quad$ Yes $\searrow$ No

Leak tested: $\quad$ N/A or Pressure (psig)

Pressure (psig)

Date

\begin{tabular}{lll} 
& Time (min) & 0 \\
\hline Initial & Time (min)
\end{tabular}

Interior assessment:
some melting at sersm - - $10 "$ span

Photo taken:

$x$ Yes

No

Comments: 
WHC-SD-WM-TRP-246 REV. 0

\section{Palletized Storage Drum Data Sheet}

Drum No. 0451

Description (Check):

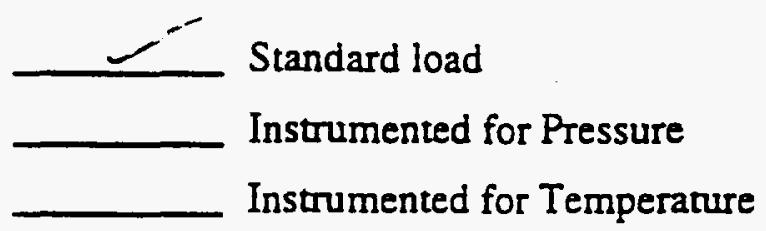

\begin{tabular}{|c|c|c|c|}
\hline Loading Starting at Bottom & Layer 1 & Layer 2 & Layer 3 \\
\hline 3 pieces of steel & & & \\
\hline $1 / 3$ of the brown paper & & & \\
\hline $1 / 3$ of the 10-mil plastic & & & \\
\hline $1 / 3$ of the bond paper & & & \\
\hline $1 / 3$ of the towels & & & \\
\hline $1 / 3$ of the bags & Go to Layer 2 & Go to Layer 3 & \\
\hline $1 / 3$ of the cut paper & $1 / 3$ of the fabric & & \\
\hline $1 / 3$ of the rubber & & \\
\hline
\end{tabular}

Drum Filled:

Ring bolt placed opposite drum seam and in down position

Lid sealed and torqued to $40 \mathrm{ft} / \mathrm{b}$ :

Leak tested:

Pre-test weight (lb)

Post-test weight (lb)

Date $5-15-95$ Initial $A P$

Date $5-15-95$ Initial if

Date $5-15=-55$ Initial AP

N/A or Pressure (psig)

Pressure (psig)

Date

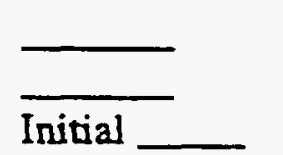

\section{$176.1 \quad$ Target weight $\sim 175.6 \mathrm{lb}$}

Time (min) 0

Time $(\min ) 5$

Loss $=$ 
Drum No. $0451 \quad 176.0$ ib.

Post-test Assessment

Position: __ originai: __ _tipped: __

Lid on drum: $\quad \quad \quad \times$ Yes No

Gasket missing (cm) O slightly compressed

Contents expelled: __ Yes $x$ No

Seal failure: $\quad$ Yes $X$ Not visible

Burn pattern:

nove

Photo taken: _ Yes $x$ No

Leak tested: $\quad$ (N/A) or Pressure (psig)

Pressure (psig)

Date

\begin{tabular}{lll}
- & Time (min) & 0 \\
\hline & Time (min)
\end{tabular}

$\overline{\text { Initial }}$

Interior assessment:

No meltury

$\checkmark$ DEC : 25

Photo taken:

$x$ Yes

No

Comments: 
WHC-SD-WM-TRP-246 REV. 0

\section{Palletized Storage Drum Data Sheet}

Drum No. (1461

Description (Check):

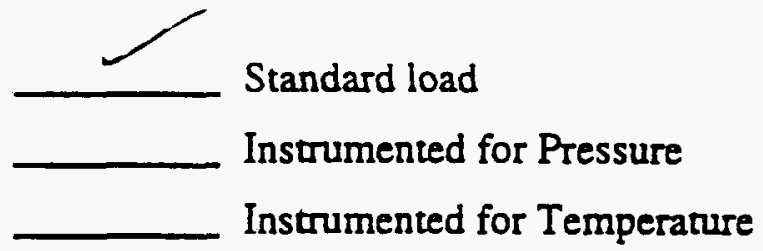

\begin{tabular}{|c|c|c|c|}
\hline Loading Starting at Bottom & Layer 1 & Layer 2 & Layer 3 \\
\hline 3 pieces of steel & - & N/A & N/A \\
\hline $1 / 3$ of the brown paper & $\sigma^{\circ}$ & 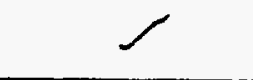 & \\
\hline \multicolumn{4}{|l|}{$1 / 3$ of the 10 -mil plastic } \\
\hline \multicolumn{4}{|l|}{$1 / 3$ of the bond paper } \\
\hline \multicolumn{4}{|l|}{$1 / 3$ of the towels } \\
\hline \multicolumn{4}{|l|}{$1 / 3$ of the bags } \\
\hline \multicolumn{4}{|l|}{$1 / 3$ of the cut paper } \\
\hline $1 / 3$ of the fabric & & - & $\nu$ \\
\hline \multicolumn{4}{|l|}{$1 / 3$ of the rubber } \\
\hline & Go to Layer 2 & Go to Layer 3 & \\
\hline
\end{tabular}

Drum Filled:

Ring bolt placed opposite drum seam and in down position

Lid sealed and torqued to $40 \mathrm{ft} / \mathrm{lb}$ :

Leak tested:

Pre-test weight (lb)

Post-test weight (lb)

Date 5-1.5-95 Initial Df

Date $5-15-95$ Initial Af

Date $5-15-95$ Initial Af

N/A or Pressure (psig)

Pressure (psig)

Date

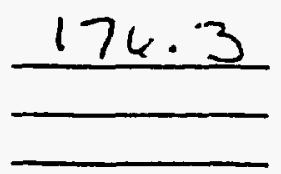

Initial
Time (min) 0

Time (min) 5

Target weight $\sim 175.6 \mathrm{lb}$ 
Drum No. $0461 \quad 176.216$.

Post-test Assessment

Position: __ original: __ tipped: __ toppled

Lid on drum: $\quad x$ Yes No

Gasket missing (cm) O $52^{4}$ coipprissed thand.

Contents expelled: _u Yes $\_$No

Seal failure: $\quad$ Yes $\quad t$ Not visible

Burn partern:

Nove

Photo taken:

Yes $\chi$ No

Leak tested:

$\mathbb{N R A D r}$ Pressure (psig) Pressure (psig)

Date

\begin{tabular}{lll} 
Time (min) & 0 \\
\hline 5
\end{tabular}

Interior assessment:

no mitting

$\checkmark$ De 1.25

Ehoto raken:

$\underline{x}$ Yes No

Comments: 


\section{Palletized Storage Drum Data Sheet}

Drum No. 0471

Description (Check):

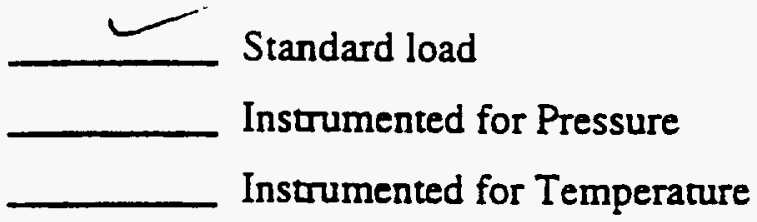

\begin{tabular}{|c|c|c|c|}
\hline Loading Starting at Bottom & Layer 1 & Layer 2 & Layer 3 \\
\hline 3 pieces of steel & 一 & N/A & N/A \\
\hline $1 / 3$ of the brown paper & - & & \\
\hline \multicolumn{4}{|l|}{$1 / 3$ of the 10 -mil plastic } \\
\hline \multicolumn{4}{|l|}{$1 / 3$ of the bond paper } \\
\hline \multicolumn{4}{|l|}{$1 / 3$ of the towels } \\
\hline \multicolumn{4}{|l|}{$1 / 3$ of the bags } \\
\hline \multicolumn{4}{|l|}{$1 / 3$ of the cut paper } \\
\hline \multicolumn{4}{|l|}{$1 / 3$ of the fabric } \\
\hline \multicolumn{4}{|l|}{$1 / 3$ of the rubber } \\
\hline & Go to Layer 2 & Go to Layer 3 & \\
\hline
\end{tabular}

Drum Filled:

Ring bolt placed opposite drum seam and in down position

Lid sealed and torqued to $40 \mathrm{ft} / \mathrm{b}$ :

Leak tested:

Pre-test weight (lb)

Post-test weight (lb)
Date $\leq 5-15-95$ Initial $A P$

Date $5-15-95$ Initial 120

Date $5-15-95$ Initial $\Lambda P$

N/A or Pressure (psig)

Pressure (psig)

Date

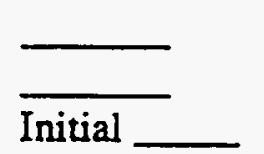

Time (min) 0

Time (min) 5

\section{Loss $=$}


Drum No. $0471,75.5$ lb.

Post-test Assessment

Position: __ original: __ tipped: __ toppled

Lid on drum: $\quad X$ Yes No

Gasket missing (cm) C slightty complinssed

Contents expelled: _ Yes $\succ$ No

Seal failure: $\quad$ Yes $X$ Not visible

Burn pattern:

- vione

Photo taken:

Yes $x$ No

Leak tested:

NFA or Pressure (psig)

Pressure (psig)

Date

\begin{tabular}{ll} 
Time (min) & 0 \\
\hline Initial & Time (min)
\end{tabular}

Interior assessment:

No chan

No sialting

rided 137

Photo taken:

$x$ Yes

No

Comments: 


\section{Palletized Storage Drum Data Sheet}

vo. .481

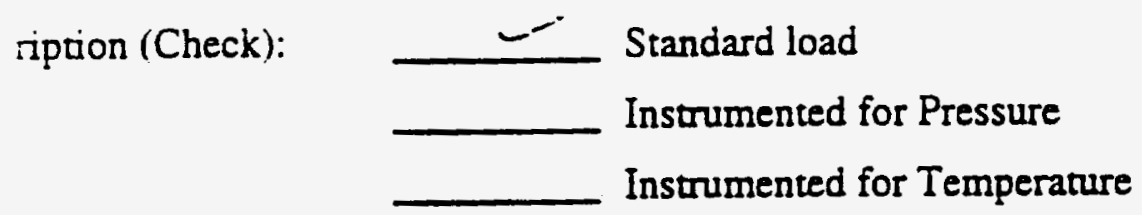

\begin{tabular}{|c|c|c|c|}
\hline Loading Starting at Bottom & Layer 1 & Layer 2 & Layer 3 \\
\hline 3 pieces of steel & & & \\
\hline $1 / 3$ of the brown paper & & & \\
\hline $1 / 3$ of the 10 -mil plastic & & & \\
\hline $1 / 3$ of the bond paper & & & \\
\hline $1 / 3$ of the towels & & & \\
\hline $1 / 3$ of the bags & & & \\
\hline $1 / 3$ of the cut paper & & & \\
\hline $1 / 3$ of the fabric & & Go to Layer 3 & \\
\hline $1 / 3$ of the rubber & & \\
\hline
\end{tabular}

n Filled:

3 bolt placed opposite drum seam in down position

sealed and torqued to $40 \mathrm{ft} / \mathrm{lb}$ :

$k$ tested:

-test weight (lb)

$t$-test weight (lb)

$$
\text { Loss }=
$$

Date $5-15-95$ Initial $A^{P}$

Date $5-15-55$ Initial RP
N/A or Pressure (psig) Pressure (psig)

Date

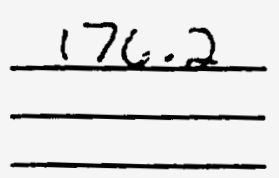

F-16
Time (min) 0

Time (min) 5

Initial

Target weight $-175.6 \mathrm{lb}$
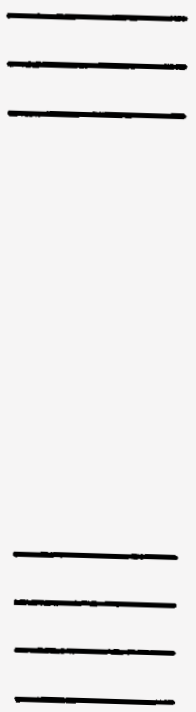


\section{Palletized Storage Drum Data Sheet}

Drum No. OS11

Descriprion (Check):

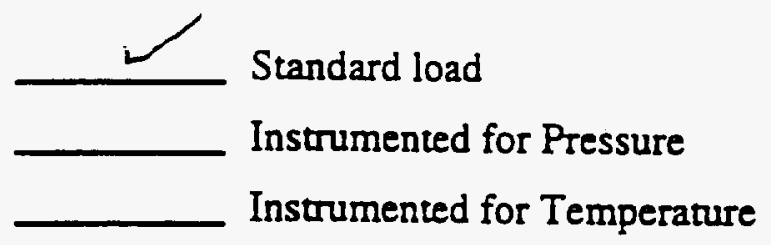

\begin{tabular}{|c|c|c|c|}
\hline Loading Starting at Bottom & Layer 1 & Layer 2 & Layer 3 \\
\hline 3 pieces of steel & & N/A & N/A \\
\hline \multicolumn{4}{|l|}{$1 / 3$ of the brown paper } \\
\hline \multicolumn{4}{|l|}{$1 / 3$ of the 10 -mil plastic } \\
\hline \multicolumn{4}{|l|}{ 1/3 of the bond paper } \\
\hline $1 / 3$ of the towels & & 1 & $\checkmark$ \\
\hline \multicolumn{4}{|l|}{$1 / 3$ of the bags } \\
\hline \multicolumn{4}{|l|}{$1 / 3$ of the cut paper } \\
\hline \multicolumn{4}{|l|}{$1 / 3$ of the fabric } \\
\hline \multirow[t]{2}{*}{$1 / 3$ of the rubber } & $d$ & $l$ & $\gamma^{\prime}$ \\
\hline & Go to Layer 2 & Go to Layer 3 & \\
\hline
\end{tabular}

Drum Filled:

Ring bolt placed opposite drum seam and in down position

Lid sealed and torqued to $40 \mathrm{ft} / \mathrm{lb}$ :

Leak tested:

Pre-test weight (lb)

Post-test weight (lb)

$$
\text { Loss }=
$$

Date $5-15-95-$ Initial $A P$

Date 5-15-95 Initial Af
N/A or Pressure (psig) Pressure (psig)

Date

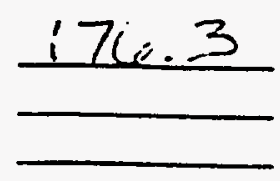

Time (min) 0

Time (min) 5 
Drum No. $0511,75.8$ ib.

Post-test Assessment

Position: ___ original: ___ tipped: ___ toppled

Lid on drum: $\quad \backslash$ Yes $\quad$ No

Gasket missing (cm) $37^{\prime \prime}$

Contents expelled: __ Yes $\underline{x}$ No

Seal failure: $\quad$ Yes $\$ Not visible

Burn pattern:

$24 "$ seine tol-ft

Photo taken: _ Yes $\underline{x}$ No

Leak tested: $\quad$ N/A or Pressure (psig)

Pressure (psig)

Date

Initial

Time (min) $\quad 0$

Interior assessment:

$\overline{\text { Initial }} \quad$\begin{tabular}{ll} 
Time (min) & 0 \\
\hline
\end{tabular}

2 "han $(\underbrace{17}_{\text {sean }})^{6}$ chan

Photo taken: $\quad x$ Yes N No

Comments: 


\section{Palletized Storage Drum Data Sheet}

Drum No. $(.5 .71(p)$

Description (Check):

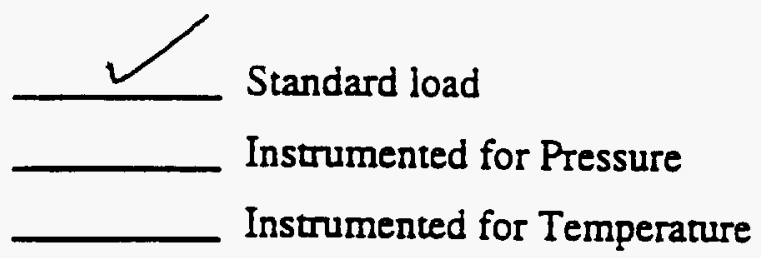

\begin{tabular}{|c|c|c|c|}
\hline Loading Starting at Bottom & Layer 1 & Layer 2 & Layer 3 \\
\hline 3 pieces of steel & & N/A & N/A \\
\hline $1 / 3$ of the brown paper & & & \\
\hline $1 / 3$ of the 10 -mil plastic & & & \\
\hline $1 / 3$ of the bond paper & & & \\
\hline $1 / 3$ of the towels & & & \\
\hline $1 / 3$ of the bags & & & \\
\hline $1 / 3$ of the cut paper & & Go to Layer 3 & \\
\hline $1 / 3$ of the fabric & Go to Layer 2 & Go \\
\hline $1 / 3$ of the rubber & & & \\
\hline
\end{tabular}

Drum Filled:

Ring bolt placed opposite drum seam and in down position

Lid sealed and torqued to $40 \mathrm{fv} / \mathrm{lb}$ :

Leak tested:

Pre-test weight (lb)

Post-test weight (lb)

\section{Loss $=$}

Date $5-15-95$

Initial $A P$

Date $.5-15-55$ Initial Af

Date 5-15-25 Initial if

N/A or Pressure (psig)

Pressure (psig)

Date

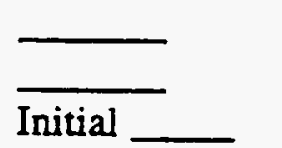

Time $(\min )$

Time $(\min )$

Target weight $-175.6 \mathrm{lb}$ 


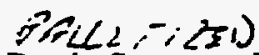

Rodictome Drum Data Sheet

Drum No. $052191.116, \mathrm{H}_{2} \mathrm{O}$

Post-test Assessment

Posicion: ___ original: ___ _ _ _ _ _

Lid on drum: $\quad$ Yes $X$ No stuck to steil pallet aboure

Gasket missing (cm) ail

Contents expelled: __ Yes $x$ No

Seal failure: $\quad X$ Yes Not visible

Burn pattern:

26 " sean te left

Photo taken: $\quad$ Yes $£$ No

$\begin{array}{lllll}\text { Leak tested: } & \text { Ponor Pressure (psig) } & \text { Time (min) } & 0 \\ & \text { Pressure (psig) } & \text { Time (min) } & -5\end{array}$

Date __

Interior assessment:

10 " of chanid contents re mainiz]

Y ioeo icc

photo taken:

$\times$ Yes

Comments: 
WHC-SD-WM-TRP-246 REV. O

\section{Palletized Storage Drum Data Sheet}

Drum No. $0: 531$

Description (Check):

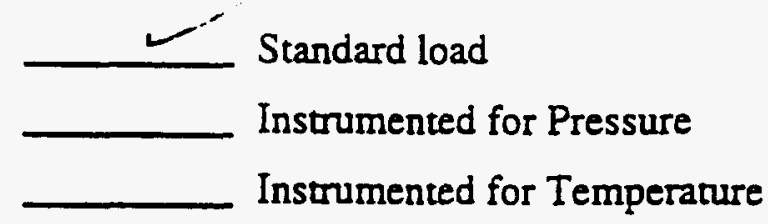

\begin{tabular}{|c|c|c|c|}
\hline Loading Starting at Bottom & Layer 1 & Layer 2 & Layer 3 \\
\hline 3 pieces of steel & & N/A & \\
\hline $1 / 3$ of the brown paper & & & \\
\hline $1 / 3$ of the 10-mil plastic & & & \\
\hline $1 / 3$ of the bond paper & & & \\
\hline $1 / 3$ of the towels & & & \\
\hline $1 / 3$ of the bags & & & \\
\hline $1 / 3$ of the cut paper & & Go to Layer 3 & \\
\hline $1 / 3$ of the fabric & Go to Layer 2 & Go & \\
\hline $1 / 3$ of the rubber & & \\
\hline
\end{tabular}

Drum Filled:

Ring bolt placed opposite drum seam and in down position

Lid sealed and torqued to $40 \mathrm{ft} / \mathrm{b}$ :

Leak tested:

Pre-test weight (lb)

Post-test weight (lb)
Date $5-15-95$ Initial Af

Date $5-15-95$ Initial iff

Date 5-15-15 Initial if
N/A or Pressure (psig) Pressure (psig)

Date

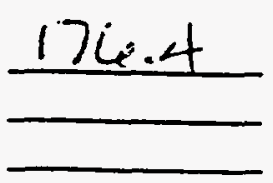

Time (min) 0 Time (min) 5
Initial

Target weight $\sim 175.6 \mathrm{lb}$ 
Drum No. $0531,73.516$.

Post-test Assessment

Position: __ original: __ tipped: __ toppled

Lid on drum: $\quad \quad \quad \quad$ Yes No Top bulged

Gasket missing (cm) all

Contents expeiled: _ Yes $\underline{x}$ No

Seal failure: $\quad X$ Yes Not visible eppewens to be a crack b/o

lidering th buige

Burn pattern:

27 "at.id Lt.

Photo taken:

Yes $x$ No

Leak tested:

NT/A-or Pressure (psig)

Pressure (psig)

Date $\begin{array}{ll}\text { Time (min) } & 0 \\ \text { Time (min) } & \underline{5}\end{array}$

Interior assessment:

Top chaned 2 4-5" "har allaround

phope ir

$x$ Yes $\quad$ No

Comments: 


\section{Palletized Storage Drum Data Sheet}

Drum No. $041(f)$

Descriprion (Check):

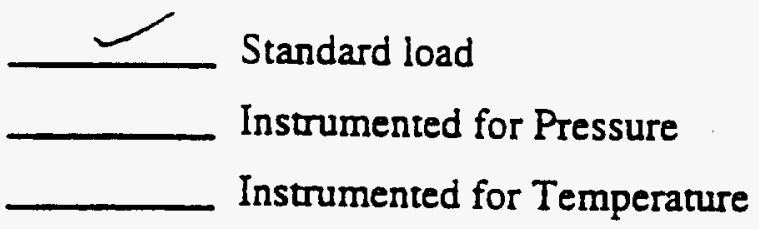

\begin{tabular}{|c|c|c|c|}
\hline Loading Starting at Bottom & Layer 1 & Layer 2 & Layer 3 \\
\hline 3 pieces of steel & & & N/A \\
\hline $1 / 3$ of the brown paper & & & \\
\hline $1 / 3$ of the 10-mil plastic & & & \\
\hline $1 / 3$ of the bond paper & & & \\
\hline $1 / 3$ of the towels & & & \\
\hline $1 / 3$ of the bags & & & \\
\hline $1 / 3$ of the cut paper & & & \\
\hline $1 / 3$ of the fabric & & Go to Layer 3 & \\
\hline $1 / 3$ of the rubber & & & \\
\hline
\end{tabular}

Drum Filled:

Ring bolt placed opposite drum seam and in down position

Leak tested:

weight (lb)

Post-test weight (lb)
Date $5-15-95$ Initial of

Date S-15-95 Initial AP
Lid sealed and torqued to $40 \mathrm{ft} / \mathrm{lb}$ :
Date $5-15-95$
Initial A?

N/A or Pressure (psig)

Pressure (psig)

Date

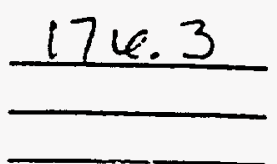

Time (min) 0

Time $(\min )+5$

Initial

Target weight $\sim 175.6 \mathrm{lb}$ 
Drum No. $0541(p)$ 175.s Ib.

\section{Post-test Assessment}

Position: originai: tipped: toppled

Lid on drum: $\quad x$ Yes $\quad$ No

Gasket missing (cm) 48

Contents expeiled: Yes $\quad x$ No

Seal failure: Yes $X$ Not visible

Burn pattern:

$19 "$ t wid wT.

lick burnegl

Photo taken: Yes $t$ No

Leak tested:

T/A or Pressure (psig) Pressure (psig)

Date

$\square \quad$\begin{tabular}{ll} 
Time (min) & 0 \\
\hline Initial & Time (min)
\end{tabular}

Interior assessment:

Top is ahaned

P้̆poro : taken.

$x$ Yes _ No

Comments: 


\section{Palletized Storage Drum Data Sheet}

Drum No. 0.551

Descriprion (Check):

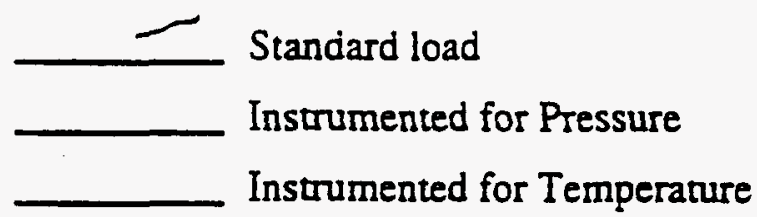

\begin{tabular}{|c|c|c|c|}
\hline Loading Starting at Bottom & Layer 1 & Layer 2 & Layer 3 \\
\hline 3 pieces of steel & & N/A & N/A \\
\hline $1 / 3$ of the brown paper & ، & & \\
\hline \multicolumn{4}{|l|}{$1 / 3$ of the 10 -mil plastic } \\
\hline \multicolumn{4}{|l|}{$1 / 3$ of the bond paper } \\
\hline \multicolumn{4}{|l|}{$1 / 3$ of the towels } \\
\hline \multicolumn{4}{|l|}{$1 / 3$ of the bags } \\
\hline \multicolumn{4}{|l|}{$1 / 3$ of the cut paper } \\
\hline \multicolumn{4}{|l|}{$1 / 3$ of the fabric } \\
\hline \multicolumn{4}{|l|}{$1 / 3$ of the rubber } \\
\hline & Go to Layer 2 & Go to Layer 3 & \\
\hline
\end{tabular}

Drum Filled:

Ring bolt placed opposite drum seam and in down position

Lid sealed and torqued to $40 \mathrm{ft} / \mathrm{lb}$ :

Leak tested:

Pre-test weight (lb)

Post-test weight (lb)

\section{Loss $=$}

Date $5-15-95=$ Initial Af

Date $5-15-55$ Initial Af

Date 5-15-55 Initial AP

N/A or Pressure (psig)

Pressure (psig)

Date

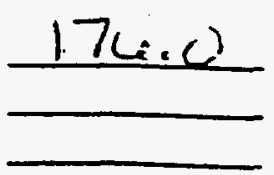

Time (min)

Time (min) 5 


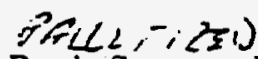

Roolt-sionge Drum Data Sheet

Drum No. clss1 175.916.

Post-test Assessment

Position: ___ originai: ___ tipped: ___ toppled

Lid on drum: $\quad \ldots$ Yes _ _ No

Gasket missing (cm) _ 19 "

Contents expelled: $\quad$ Yes $\not x$ No

Seal failure: __ Yes $X$ Not visible

Burn pattern: id it it.

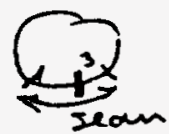

Photo taken: $\quad$ Y Yes t No

Leak tested: $\quad$ N

Pressure (psig)

Date

\begin{tabular}{lll} 
& Time $(\min )$ & 0 \\
\hline & Time (min)
\end{tabular}

Interior assessment:

4"char $28^{\prime \prime}$ p pan centered co barn area
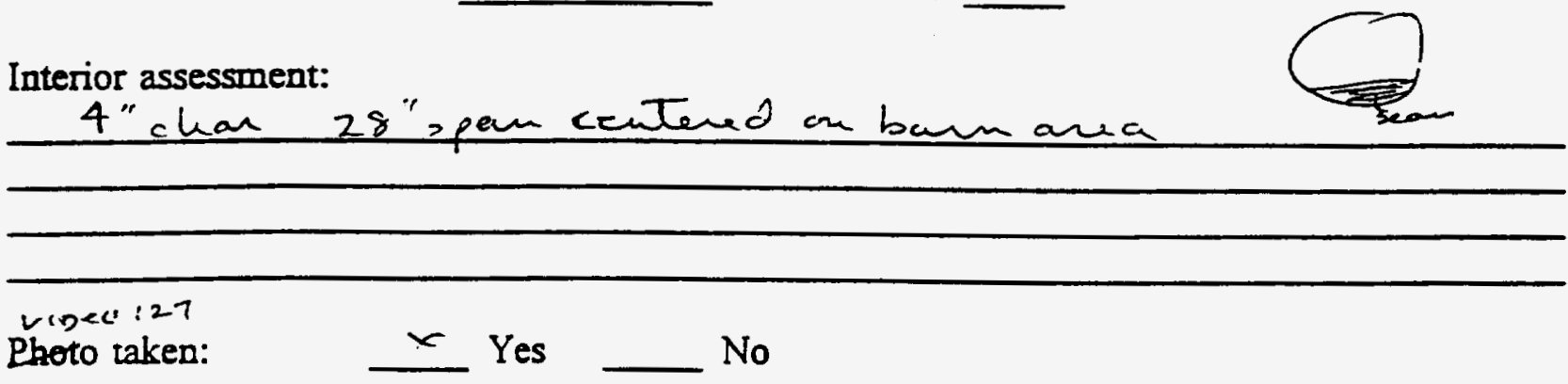

Comments: 
WHC-SD-WM-TRP-246 REV. 0

\section{Palletized Storage Drum Data Sheet}

Drum No. OSLel

Description (Check):

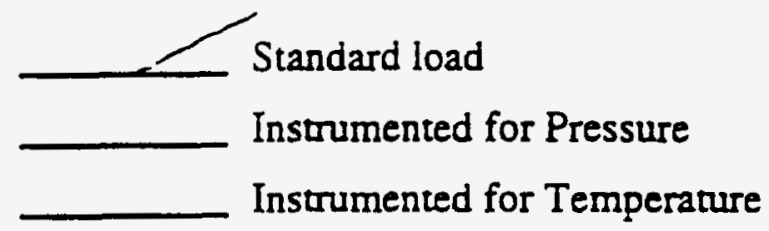

\begin{tabular}{|c|c|c|c|}
\hline Loading Starting at Bottom & Layer 1 & Layer 2 & Layer 3 \\
\hline 3 pieces of steel & & N/A & (1/A \\
\hline $1 / 3$ of the brown paper & & & \\
\hline $1 / 3$ of the 10-mil plastic & & & \\
\hline $1 / 3$ of the bond paper & & & \\
\hline $1 / 3$ of the towels & & & \\
\hline $1 / 3$ of the bags & & & \\
\hline $1 / 3$ of the cur paper & Go to Layer 2 & Go to Layer 3 & \\
\hline $1 / 3$ of the fabric & & & \\
\hline $1 / 3$ of the rubber & & \\
\hline
\end{tabular}

Drum Filled:

Ring bolt placed opposite drum seam and in down position

Lid sealed and torqued to $40 \mathrm{ft} / \mathrm{lb}$ :

Leak tested:

Pre-test weight (lb)

Post-test weight (lb)

Date $5-15-95$ Initial $A P$

Date $.5-15-55$ Initial AP

Date $5-15-9.5$ Initial $A f$

N/A or Pressure (psig)

Pressure (psig)

Date

$$
175.6
$$

Time (min) 0

Time $(\min ) 5$

Initial

Target weight $-175.6 \mathrm{lb}$ 
Drum No. 0561 is5.51b.

Post-test Assessment

Position:

originai: tipped: toppled

Lid on drum: $\quad x$ Yes _ No

Gasket missing (cm) $O$ ts" coippusud Thand

Contents expeiled: __ Yes $x$ No

Seal failure: $\quad$ Yes $\quad x$ Not visible

Burn pattern:

None

Photo taken:

Yes $x$ No

Leak tested:

N/A or Pressure (psig)

Pressure (psig)

Date

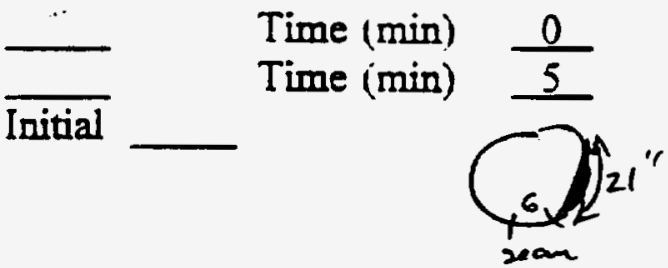

Interior assessment:

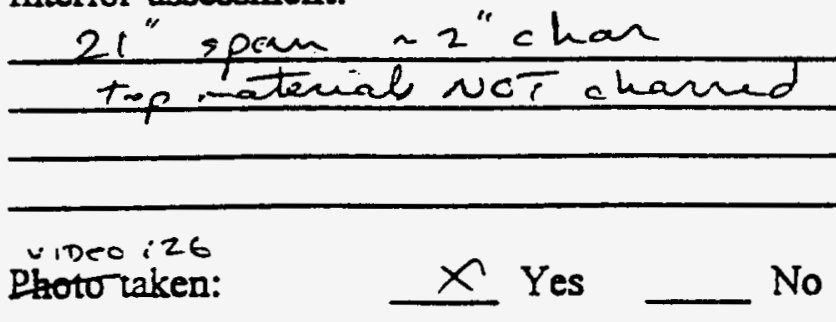

Comments: 
WHC-SD-WM-TRP-246 REV. 0

\section{Palletized Storage Drum Data Sheet}

D.um No. 0571

Description (Check):

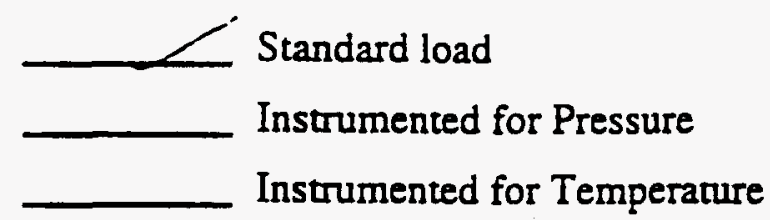

\begin{tabular}{|c|c|c|c|}
\hline Loading Starting at Bottom & Layer 1 & Layer 2 & Layer 3 \\
\hline 3 pieces of steel & & N/A & N/A \\
\hline $1 / 3$ of the brown paper & - & & \\
\hline \multicolumn{4}{|l|}{$1 / 3$ of the 10 -mil plastic } \\
\hline \multicolumn{4}{|l|}{ 1/3 of the bond paper } \\
\hline \multicolumn{4}{|l|}{$1 / 3$ of the toweis } \\
\hline \multicolumn{4}{|l|}{$1 / 3$ of the bags } \\
\hline \multicolumn{4}{|l|}{$1 / 3$ of the cut paper } \\
\hline \multicolumn{4}{|l|}{$1 / 3$ of the fabric } \\
\hline \multirow[t]{2}{*}{ 1/3 of the rubber } & & / & $\gamma$ \\
\hline & Go to Layer 2 & Go to Layer 3 & \\
\hline
\end{tabular}

Drum Filled:

Ring bolt placed opposite drum seam and in down position

Lid sealed and torqued to $40 \mathrm{ft} / \mathrm{b}$ :

Leak tested:

Pre-test weight (lb)

Post-test weight (lb)

$$
\text { Loss }=
$$

Date $5-15-55$ Initial $\mathrm{AP}$

Date $5-15-55$ Initial Af
N/A or Pressure (psig) Pressure (psig)

Date

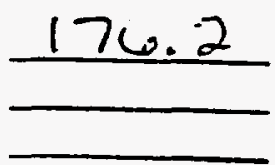

Time (min)

Time (min) 5
Initial

Target weight $-175.6 \mathrm{lb}$ 
Drum No. $0571+76.2 \mathrm{lb}$.

Post-test Assessment

Position: ___ original: ___ tipped: ___ toppled

Lid on drum: $\quad x$ Yes No

Gasket missing (cm) $O$ hlight haved rcomplessed 19 "

Contents expelled: _ـ Yes $\searrow$ No

Seal failure: $\quad$ Yes $x$ Not visible

Burn pattern:

Nont

Photo taken: $\quad$ Yes $x$ No

Leak tested: N/A or Pressure (psig)

Pressure (psig)

Date

$\bar{T} \quad$ Time (min) $\quad \frac{0}{5}$

Interior assessment:

metted to wall $14^{\prime \prime}$ spon about seam

V.D C. 140

Ptoto taken:

$x$ Yes No

Comments: 


\section{Palletized Storage Drum Data Sheet}

Drum No. 0581

Description (Check):

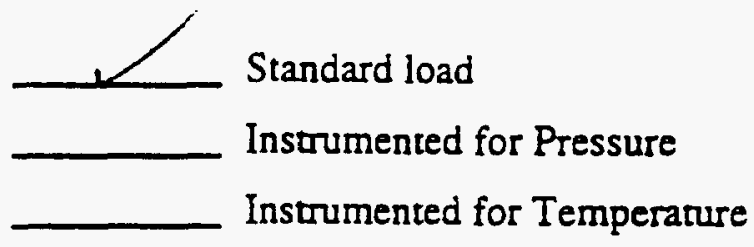

\begin{tabular}{|c|c|c|c|}
\hline Loading Starting at Bottom & Layer 1 & Layer 2 & Layer 3 \\
\hline 3 pieces of steel & & N/A & N/A \\
\hline \multicolumn{4}{|l|}{$1 / 3$ of the brown paper } \\
\hline \multicolumn{4}{|l|}{$1 / 3$ of the 10 -mil plastic } \\
\hline \multicolumn{4}{|l|}{$1 / 3$ of the bond paper } \\
\hline \multicolumn{4}{|l|}{$1 / 3$ of the towels } \\
\hline \multicolumn{4}{|l|}{$1 / 3$ of the bags } \\
\hline \multicolumn{4}{|l|}{$1 / 3$ of the cut paper } \\
\hline \multicolumn{4}{|l|}{$1 / 3$ of the fabric } \\
\hline \multicolumn{4}{|l|}{$1 / 3$ of the rubber } \\
\hline & Go to Layer 2 & Go to Layer 3 & \\
\hline
\end{tabular}

Drum Filled:

Ring bolt placed opposite drum seam and in down position

Lid sealed and torqued to $40 \mathrm{ft} / \mathrm{lb}$ :

Leak tested:

Post-test weight (lb)

$$
\text { Loss = }
$$

Date 5-15-95 Initial AP

Date 5-15-95 Initial AP

Date $5-15-35$ Initial AP

N/A or Pressure (psig)

Pressure (psig)

Date

Initial

Time $(\min )+$

Time $(\min ) 5$ 
Drum No. $02581 \quad 176.2$ b.

Post-test Assessment

Position: __ original: ___ _ _ _ _

Lid on drum: $\quad \quad \quad \quad$ Yes No

Gasket missing $(\mathrm{cm}) \mathrm{O}$ sightlly compressed

Contents expelled: __ Yes $x$ No

Seal failure: $\quad$ Yes $x$ Not visible

Burn pattern:

NONe

Photo taken: $\quad$ Yes $\underline{x}$ No

Leak tested:

NFA or Pressure (psig)

Pressure (psig)

Date

$\begin{array}{ll}\text { Time (min) } & 0 \\ \text { Time }(\min ) & \end{array}$

Initial

Interior assessment:

plastic sligltey melted 16" Rt of rean

UIDCO 139

Photo taken:

$x$ Yes No

Comments: 


\section{Palletized Storage Drum Data Sheet}

Drm No. Cle $11(A)$

Descripion (Check):

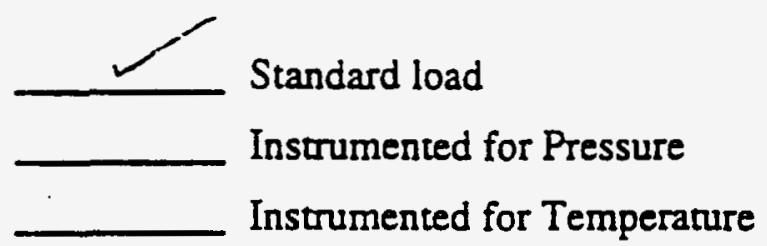

\begin{tabular}{|c|c|c|c|}
\hline Loading Starting at Bottom & Layer 1 & Layer 2 & Layer 3 \\
\hline 3 pieces of steel & & & N/A \\
\hline $1 / 3$ of the brown paper & & & \\
\hline $1 / 3$ of the 10-mil plastic & & & \\
\hline $1 / 3$ of the bond paper & & & \\
\hline $1 / 3$ of the towels & & & \\
\hline $1 / 3$ of the bags & & & \\
\hline $1 / 3$ of the cut paper & Go to Layer 2 & Go to Layer 3 & \\
\hline $1 / 3$ of the fabric & & \\
\hline $1 / 3$ of the rubber & & \\
\hline
\end{tabular}

Drum Filled:

Ring bolt placed opposite drum seam and in down position

Lid sealed and torqued to $40 \mathrm{ft} / \mathrm{b}$ :

Leak tested:

Pre-test weight (lb)

Post-test weight (lb)

$$
\text { Loss = }
$$

Date 5-15-95 Initial AP

Date $5-15-95$ Initial PP

Date $5-15-55$ Initial AP
N/A or Pressure (psig)

Pressure (psig)

Date

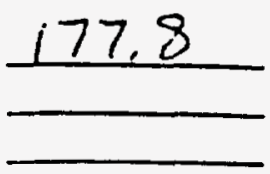

Time (min) 0

Time (min) 5
Initial 
Drum No. $06 ! 1,21 ! 71,316$.

Post-test Assessment

Position: original: tipped: toppled

Lid on drum: $\quad x$ Yes No $-\frac{11}{x} \times 22^{\prime \prime} \mathrm{jap}$

Gasket missing (cm) aif

Contents expelled: $\quad$ Yes $\longrightarrow$ No

Seal failure: $\quad \quad \quad \quad \quad$ Yes $\quad$ Not visible

Burn pattern:

al around

Photo taken:

Yes $x$ No

Leak tested:

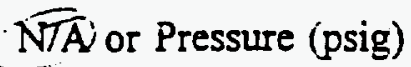

Pressure (psig)

Date

Initial

$\begin{array}{ll}\text { Time }(\min ) & 0 \\ \text { Time }(\min ) & \end{array}$

Interior assessment:

$16 "$ top then te top of ahan

U1003 37

Photo taken:

$x$ Yes No

Comments: 


\section{Palletized Storage Drum Data Sheet}

Drum No. 0121

Description (Check):

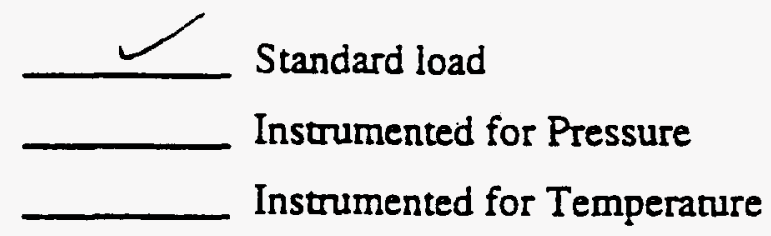

\begin{tabular}{|c|c|c|c|}
\hline Loading Starting at Bottom & Layer 1 & Layer 2 & Layer 3 \\
\hline 3 pieces of steel & & & N/A \\
\hline $1 / 3$ of the brown paper & & & \\
\hline $1 / 3$ of the 10-mil plastic & & & \\
\hline $1 / 3$ of the bond paper & & & \\
\hline $1 / 3$ of the toweis & & & \\
\hline $1 / 3$ of the bags & & & \\
\hline $1 / 3$ of the cut paper & & Go to Layer 3 & \\
\hline $1 / 3$ of the fabric & Go to Layer 2 & Go \\
\hline $1 / 3$ of the rubber & & \\
\hline
\end{tabular}

Drum Filled:

Ring bolt placed opposite drum seam and in down position

Lid sealed and torqued to $40 \mathrm{ft} / \mathrm{b}$ :

Leak tested:

Pre-test weight (lb)

Post-test weight (lb)

$$
\text { Loss }=
$$

Date $5-15-55$ Initial Af

Date $5-15-55$ Initial if

Date $5-15^{-55}$ Initial AP

N/A or Pressure (psig)

Pressure (psig)

Date

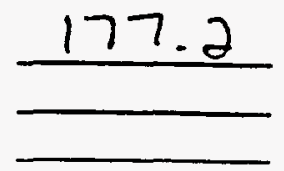

Time $(\min )$

Initial 
Drum No. $0621,4.616$

Post-test Assessment

Position: original: tipped: toppled

Lid on drum: $\quad \underline{X}$ Yes No

Gasket missing (cm)

Contents expelled: _ Yes $\backslash$ No

Seal failure: $\quad x$ Yes Not visible Top bi'ged difficult to sen rrask - i/4" $\times 4$ " spoce $=$ b/u lid r rivg

Burn pattern:

ali ancumel

Photo taken:

Yes $x$ No

Leak tested:

N/A Pressure (psig)

Date

\begin{tabular}{lll} 
& Time $(\min )$ & 0 \\
\hline & Time (min)
\end{tabular}

Interior assessment:

18 " top of dumm.te top of chen

$\because$ Dec 40

Photo taken:

$x$ Yes

No

Comments: 
WHC-SD-WM-TRP-246 REV. 0

\section{Palletized Storage Drum Data Sheet}

Dum No. $0631(p)$

Descriprion (Check):

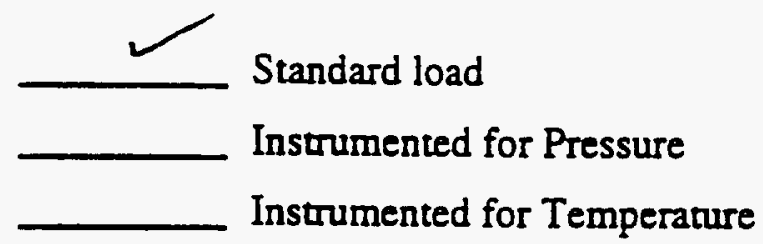

\begin{tabular}{|c|c|c|c|}
\hline Loading Starting at Bottom & Layer 1 & Layer 2 & Layer 3 \\
\hline 3 pieces of steel & & N/A & N/A \\
\hline \multicolumn{4}{|l|}{$1 / 3$ of the brown paper } \\
\hline \multicolumn{4}{|l|}{$1 / 3$ of the 10 -mil plastic } \\
\hline \multicolumn{4}{|l|}{$1 / 3$ of the bond paper } \\
\hline \multicolumn{4}{|l|}{$1 / 3$ of the towels } \\
\hline \multicolumn{4}{|l|}{$1 / 3$ of the bags } \\
\hline \multicolumn{4}{|l|}{$1 / 3$ of the cut paper } \\
\hline \multicolumn{4}{|l|}{$1 / 3$ of the fabric } \\
\hline \multirow[t]{2}{*}{$1 / 3$ of the rubber } & & & \\
\hline & Go to Layer 2 & Go to Layer 3 & \\
\hline
\end{tabular}

Drum Filled:

Ring bolt placed opposite drum seam and in down position

Lid sealed and torqued to $40 \mathrm{ft} / \mathrm{lb}$ :

Leak tested:

Pre-test weight (lb)

Post-test weight (lb)

$$
\text { Loss }=
$$

Date $5-15-95$ Initial $A P$

Date 5-15-95 Initial AP

Date 5-15-95 Initial AP

N/A or Pressure (psig)

Pressure (psig)

Date

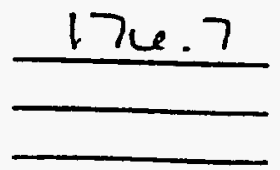

Time $(\min )$

Time (min) 5 
Drum No. Gi=31 (P) it4.; b.

Post-test Assessment

Position: original: tipped: toppled

Lid on drum: $\quad$ Yes $x$ No witifriry $z$ - wotivg of drum

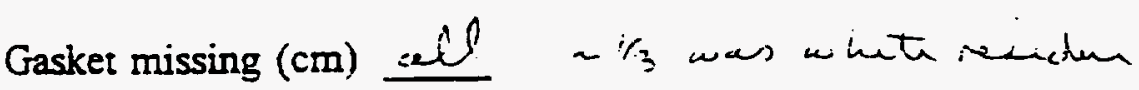

Contents expelled: __ Yes _

Seal failure: $\quad \quad \quad \times$ Yes _ Not visible

Burn pattern:

al around

Photo taken:

Y Yes X No

Leak tested: $\quad$ NIA br Pressure (psig)

Pressure (psig)

Date

Initial

Time (min)
Time (min)

Interior assessment:

it" top at deum to top -1 chur

Rioec 3:

Photo taken:

$\underset{x}{x}$ Yes No

Comments: 


\section{Palletized Storage Drum Data Sheet}

Drum No. $(1041(p)$

Descriprion (Check):

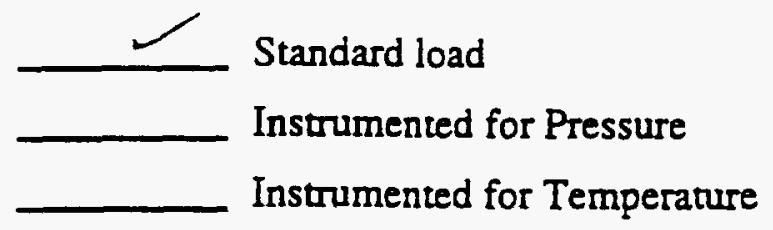

\begin{tabular}{|c|c|c|c|}
\hline Loading Starting at Bottom & Layer 1 & Layer 2 & Layer 3 \\
\hline 3 pieces of steel & & N/A & N/A \\
\hline \multicolumn{4}{|l|}{ 1/3 of the brown paper } \\
\hline \multicolumn{4}{|l|}{$1 / 3$ of the 10 -mil plastic } \\
\hline \multicolumn{4}{|l|}{ 1/3 of the bond paper } \\
\hline \multicolumn{4}{|l|}{$1 / 3$ of the towels } \\
\hline \multicolumn{4}{|l|}{$1 / 3$ of the bags } \\
\hline \multicolumn{4}{|l|}{$1 / 3$ of the cut paper } \\
\hline \multicolumn{4}{|l|}{$1 / 3$ of the fabric } \\
\hline \multicolumn{4}{|l|}{$1 / 3$ of the rubber } \\
\hline & Go to Layer 2 & Go to Layer 3 & \\
\hline
\end{tabular}

Drum Filled:

Ring bolt placed opposite drum seam and in down position

Lid sealed and torqued to $40 \mathrm{ft} / \mathrm{lb}$ :

Leak tested:

Pre-test weight (lb)

Post-test weight (lb)
Date $5-15-55$ Initial AD

Date .5-15-95 Initial AP
N/A or Pressure (psig) Pressure (psig)

Date

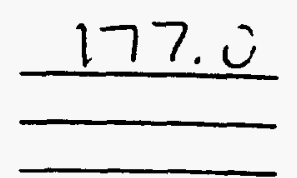

Time (min) 0

Time (min) 5 
Drum No. CE+i ir', 71.9

$\underline{\text { Post-test Assessment }}$

Position: original: tipped: toppled

Lid on drum: $x$ Yes No buige a $\left.x^{\prime(1 / 5}-1 / 4\right) \times 3 "$ jap

Gasket missing (cm)

Contents expelled: _ Yes $x$ No

Seal failure: $\quad y$ Yes $\quad$ Not visible

Burn partern:

21 at mid it. als

Photo taken:

Yes $x$ No

Leak tested:

NFA or Pressure (psig) Pressure (psig)

Date

\begin{tabular}{lll} 
& Time $(\min )$ & 0 \\
\hline
\end{tabular}

Interior assessment:

$13^{\prime \prime}$ wp of dimen re top of Chen

propo-3c

Photo taken:

$x$ Yes

Comments: 
WHC-SD-WM-TRP-246 REV. 0

\section{Palletized Storage Drum Data Sheet}

Drim No.

Description (Check):

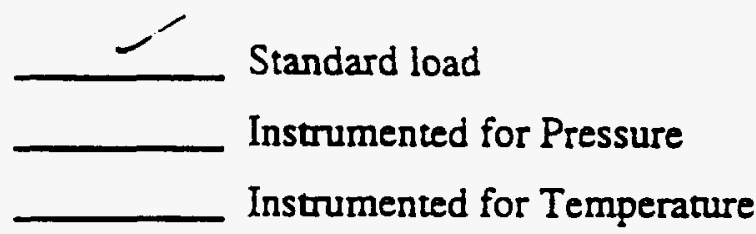

\begin{tabular}{|c|c|c|c|}
\hline Loading Starting at Bottom & Layer 1 & Layer 2 & Layer 3 \\
\hline 3 pieces of steel & $\gamma$ & N/A & N/A \\
\hline \multicolumn{4}{|l|}{$1 / 3$ of the brown paper } \\
\hline \multicolumn{4}{|l|}{$1 / 3$ of the 10 -mil plastic } \\
\hline \multicolumn{4}{|l|}{$1 / 3$ of the bond paper } \\
\hline $1 / 3$ of the toweis & $\checkmark$ & & $\gamma$ \\
\hline \multicolumn{4}{|l|}{$1 / 3$ of the bags } \\
\hline \multicolumn{4}{|l|}{$1 / 3$ of the cut paper } \\
\hline \multicolumn{4}{|l|}{$1 / 3$ of the fabric } \\
\hline \multirow[t]{2}{*}{$1 / 3$ of the rubber } & ノ & r & \\
\hline & Go to Layer 2 & Go to Layer 3 & \\
\hline
\end{tabular}

Drum Filled:

Ring bolt placed opposite drum seam and in down position

Lid sealed and torqued to $40 \mathrm{ft} / \mathrm{b}$ :

Leak tested:

Pre-test weight (lb)

Post-test weight (lb)

$$
\text { Loss }=
$$

Date $5-15-95$ Initial A?

Date $5-15.95$ Initial AP

Date $5-15-75$ Initial AP

N/A or Pressure (psig)

Pressure (psig)

Date

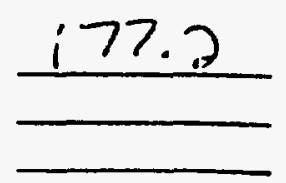

Time $(\min ) \ell$

Time (min) 5 
Drum No. 0651 175.5 16.

\section{Post-test Assessment}

Position: originai: tipped: toppled

Lid on drum: $\quad \quad \quad$ Yes No riwg belt loo:i

Gasket missing (cm) all

Contents expelled: __ Yes $x$ No

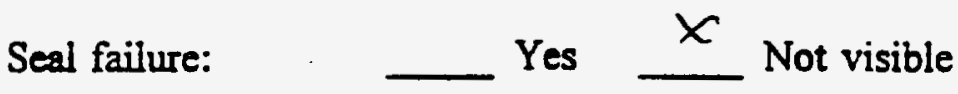

Burn pattern:

$s^{3}$ seam teleft of around rim

Photo taken: _ Yes $\chi$ No

Leak tested: N/A or Pressure (psig)

Pressure (psig)

Date

$\longrightarrow \quad$ Time $(\mathrm{min}) \quad \frac{0}{5}$

Interior assessment:

8" top to top fo char

visec i4 1

Photo taken:

$x$ Yes

Comments: 
WHC-SD-WM-TRP-246 REV. 0

\section{Palletized Storage Drum Data Sheet}

Drum No. Syelei

Descripion (Check):

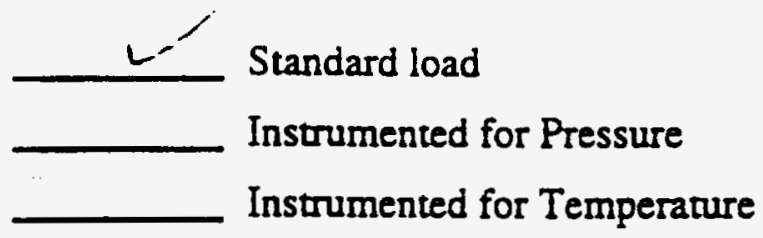

\begin{tabular}{|c|c|c|c|}
\hline Loading Starting at Bottom & Layer 1 & Layer 2 & Layer 3 \\
\hline 3 pieces of steel & & N/A & N/A \\
\hline $1 / 3$ of the brown paper & & & \\
\hline $1 / 3$ of the 10-mil plastic & & & \\
\hline $1 / 3$ of the bond paper & & & \\
\hline $1 / 3$ of the toweis & & & \\
\hline $1 / 3$ of the bags & & & \\
\hline $1 / 3$ of the cut paper & & & \\
\hline $1 / 3$ of the fabric & Go to Layer 2 & Go to Layer 3 & \\
\hline $1 / 3$ of the rubber & &
\end{tabular}

Drum Filled:

Ring boit placed opposite drum seam and in down position

Lid sealed and torqued to $40 \mathrm{ft} / \mathrm{lb}$ :

Leak tested:

Pre-test weight (lb)

Post-test weight (lb)

$$
\text { Loss = }
$$

Date 5-15-45 Initial AP

Date $=-15-95$ Initial $A P$
N/A or Pressure (psig)

Pressure (psig)

Date

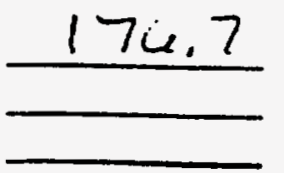

Time (min) 0

Time (min) 5

Initial

Target weight $\sim 175.6 \mathrm{lb}$ 
Drum No. $0661,75.0$

Post-test Assessment

Position: original: tipped: toppied

Lid on drum: $\quad x$ Yes No $1: \alpha$ roctes

Gasket missing (cm) all

Contents expelled: _u Yes $x$ No

Seal failure: $\quad$ Yes $\quad x$ Not visible

Burn pattern:

- 25 "RT of seam appears flames impinging on bottom thind

Photo taken:

$$
\text { Yes } \searrow \text { No }
$$

Leak tested:

N/A or Pressure (psig)

Pressure (psig)

Date $\begin{array}{ll}\text { Time }(\min ) & 0 \\ \text { Time }(\min ) & \end{array}$

Interior assessment:

7 "top to top of chan -4-5" char all around

VIDeC 143

Photo taken:

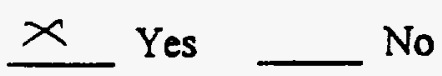

Initial

Comments: 


\section{Palletized Storage Drum Data Sheet}

Drim No. $Q X_{2} 71$

Descriprion (Check):

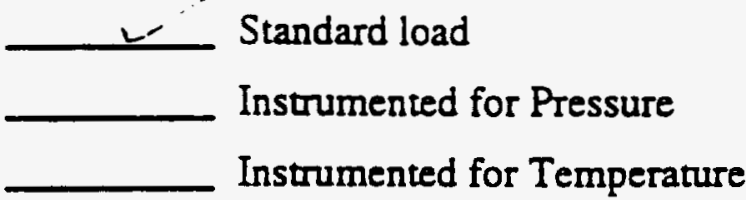

\begin{tabular}{|c|c|c|c|}
\hline Loading Starting at Bottom & Layer 1 & Layer 2 & Layer 3 \\
\hline 3 pieces of steel & & N/A & \\
\hline $1 / 3$ of the brown paper & & & \\
\hline $1 / 3$ of the 10-mil plastic & & & \\
\hline $1 / 3$ of the bond paper & & & \\
\hline $1 / 3$ of the towels & & & \\
\hline $1 / 3$ of the bags & & & \\
\hline $1 / 3$ of the cut paper & & Go to Layer 3 & \\
\hline $1 / 3$ of the fabric & Go to Layer 2 & G & \\
\hline $1 / 3$ of the rubber & & \\
\hline
\end{tabular}

Drum Filled:

Ring bolt placed opposite drum seam and in down position

Lid sealed and torqued to $40 \mathrm{ft} / \mathrm{b}$ :

Leak tested:

Pre-test weight (lb)

Post-test weight (lb)

Loss =

Date $5-15-95$ Initial 4 ?

Date $5-15 \cdot 95$ Initial Af

Date $5-15-95$ Initial $A P$

N/A or Pressure (psig)

Pressure (psig)

Date

Initial
Time $(\min ) \quad 0$

Time $(\min ) 5$

\section{$177.2 \quad$ Target weight $-175.6 \mathrm{lb}$}


Drum No. $0671,75.9$ k.

\section{Post-test Assessment}

Position: __ original: ___ tipped: ___ toppled

Lid on drum: $\quad X$ Yes No

Gasket missing (cm) al

Contents expeiled: __ Yes $x$ No

Seal failure: $\quad$ Yes $\quad x$ Not visible

Burn pattern:

Nont except arownd rim

Photo taken:

Yes $x$ No

Leak tested:

(NTA) or Pressure (psig)

Pressure (psig)

Date

\begin{tabular}{lll}
- & Time (min) & 0 \\
\hline & Time (min)
\end{tabular}

Interior assessment:

top chaved $z \approx z^{4}$ all around

viseo: 21

Photo taken:

$x$ Yes

Comments: 


\section{Palletized Storage Drum Data Sheet}

Drum No. $\alpha 681$

Description (Check):

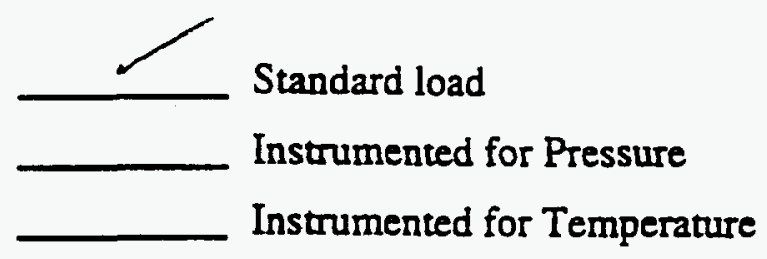

\begin{tabular}{|c|c|c|c|}
\hline Loading Staring at Bottom & Layer 1 & Layer 2 & Layer 3 \\
\hline 3 pieces of steel & & N/A \\
\hline $1 / 3$ of the brown paper & & & \\
\hline $1 / 3$ of the 10-mil plastic & & & \\
\hline $1 / 3$ of the bond paper & & & \\
\hline $1 / 3$ of the towels & & & \\
\hline $1 / 3$ of the bags & & & \\
\hline $1 / 3$ of the cut paper & & & \\
\hline $1 / 3$ of the fabric & Go to Layer 2 & Go to Layer 3 & \\
\hline $1 / 3$ of the rubber & & & \\
\hline
\end{tabular}

Drum Filled:

Ring bolt placed opposite drum seam and in down position

Lid sealed and torqued to $40 \mathrm{ft} / \mathrm{b}$ :

Leak tested:

Pre-test weight (lb)

Post-test weight (lb)

$$
\text { Loss }=
$$

Date $5-15-75$ Initial $17 ?$

Date 5-15-95 Initial Af

Date 5-15-45 Initial Af?
N/A or Pressure (psig)

Pressure (psig)

Date

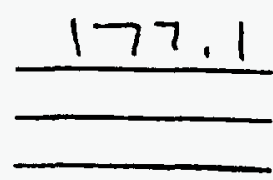

Time $(\min )$ Time (min) 5
Initial

Target weight $\sim 175.6 \mathrm{lb}$ 
Drum No. $0681176.3 \mathrm{lb}$.

\section{Post-test Assessment}

Position:

original:

tipped;

toppled

Lid on drum: $\quad x$ Yes No ringelid loose

Gasket missing (cm) all

Contents expelled: _ Yes $\searrow$ No

Seal failure: $\quad$ _ $\quad$ Yes $\quad X$ Not visible

Burn pattern:

nove except at rim

Photo taken: $\quad$ Yes $\chi$ No

Leak tested:

$\widehat{N / A}$ or Pressure (psig)

Pressure (psig)

Date

\begin{tabular}{ll} 
Time (min) & $\frac{0}{5}$ \\
\hline Initial
\end{tabular}

Interior assessment:

top chanred , 3-8" allaround $x^{\prime \prime}$ at seam, $3^{\prime \prime}$ atbott side

Litrec: $: 24$

Photo taken:

$\underline{x}$ Yes No

Comments: 


\section{Palletized Storage Drum Data Sheet}

Drum No. $0711(P)$
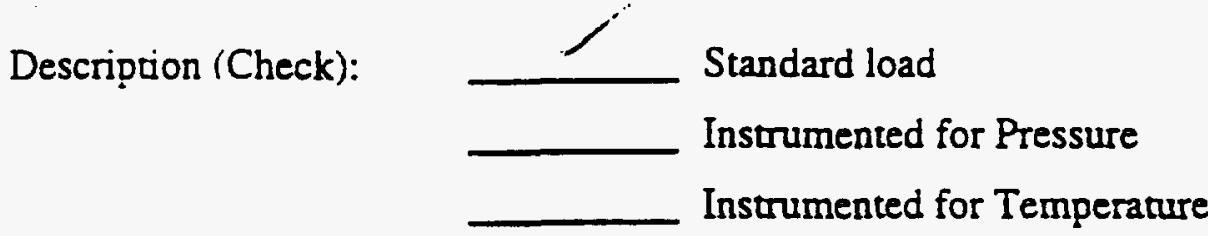

\begin{tabular}{|c|c|c|c|}
\hline Loading Starting at Bottom & Layer 1 & Layer 2 & Layer 3 \\
\hline 3 pieces of steel & & N/A & N/A \\
\hline $1 / 3$ of the brown paper & & & \\
\hline $1 / 3$ of the 10-mil plastic & & & \\
\hline $1 / 3$ of the bond paper & & & \\
\hline $1 / 3$ of the towels & & & \\
\hline $1 / 3$ of the bags & & & \\
\hline $1 / 3$ of the cut paper & & & \\
\hline $1 / 3$ of the fabric & Go to Layer 2 & Go to Layer 3 & \\
\hline $1 / 3$ of the rubber & &
\end{tabular}

Drum Filled:

Ring bolt placed opposite drum seam and in down position

Lid sealed and torqued to $40 \mathrm{ft} / \mathrm{b}$ :

Leak tested:

Pre-test weight (lb)

Post-test weight (lb)

$$
\text { Loss }=
$$

Date 5-15-95 Initial AP

Date $5-15-95$ Initial Af

Date $5=15-55$ Initial $A f$

N/A or Pressure (psig)

Pressure (psig)

Date

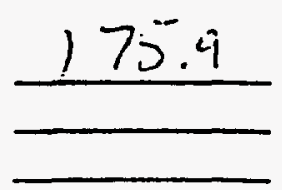

Time $(\min )$

Time (min) 5 
Drum No. $0711(p), 70 . c$ ib.

Post-test Assessment

Position: ___ original: ___ tipped: ___ toppled

Lid on drum: $\quad x$ Yes No $1 / 4^{\prime \prime} \times 4^{\prime \prime} g y$

Gasket missing (cm) 내

Contents expelled: $\quad$ Yes $x$ No

Seal failure: $\quad \quad$ Yes $\quad$ Not visible

Burn pattern:

all around

Photo taken:

Yes $\chi$ No

Leak tested: $\quad$ Nator Pressure (psig)

Pressure (psig)

Date

\begin{tabular}{lll} 
& Time (min) & 0 \\
\hline & Time (min)
\end{tabular}

Initial

Interior assessment:

15 "Top of drum Co top of cha

U:D 37

Photo taken:

$x$ Yes _ No

Comments: 


\section{Palletized Storage Drum Data Sheet}

Drim No. $C 721(\theta)$

Description (Check):

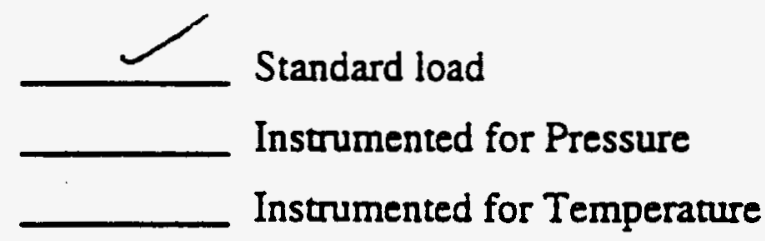

\begin{tabular}{|c|c|c|c|}
\hline Loading Starting at Bottom & Layer 1 & Layer 2 & Layer 3 \\
\hline \hline 3 pieces of steel & & N/A \\
\hline $1 / 3$ of the brown paper & & & \\
\hline $1 / 3$ of the 10-mil plastic & & & \\
\hline $1 / 3$ of the bond paper & & & \\
\hline $1 / 3$ of the towels & & & \\
\hline $1 / 3$ of the bags & & & \\
\hline $1 / 3$ of the cut paper & & Go to Layer 3 & \\
\hline $1 / 3$ of the fabric & Go to Layer 2 & Go & \\
\hline $1 / 3$ of the rubber & &
\end{tabular}

Drum Filled:

Ring bolt placed opposite drum seam and in down position

Lid sealed and torqued to $40 \mathrm{f} / \mathrm{lb}$ :

Leak tested:

Pre-test weight (lb)

Post-test weight (lb)

$$
\text { Loss }=
$$

Date $5-15.95$ Initial AP

Date $5=5=95$ Initial AP

Date $5-15-95$ Initial AP

N/A or Pressure (psig)

Pressure (psig)

Date

$$
176.7
$$

Time (min) $\frac{e}{5}$

Initial

Target weight $\sim 175.6 \mathrm{lb}$ 
Drum No. $\quad 072 i(p), 09.3$ is

Post-test Assessment

Position: $\quad$ _ original: ___ tipped: ___ toppled

Lid on drum: $\quad Y$ Yes _ No lad bul ged

Gasket missing (cm) al

Contents expelled: $\quad$ Yes $Y$ No

Seal failure: $\quad$ Yes $X$ Not visible

Burn pattern:

all around

Photo taken: Yes $x$ No

Leak tested:

Nor Pressure (psig) Pressure (psig)

Date

$+\quad \begin{array}{ll}\text { Time (min) } \\ \text { Time (min) }\end{array}$

Interior assessment:

17 "Top ofdewm te tap of hhan

videce 25

Photo taken:

$x$ Yes No

\section{Comments:}


WHC-SD-WM-TRP-246 REV. 0

\section{Palletized Storage Drum Data Sheet}

Drum No. 4731

Description (Check):

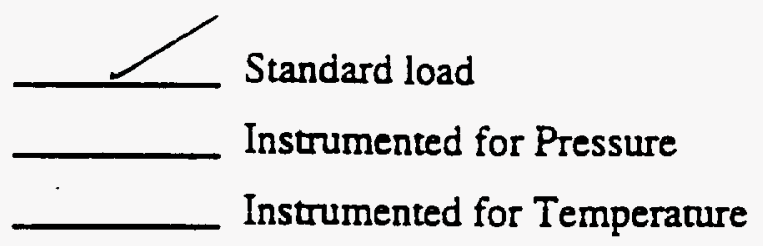

\begin{tabular}{|c|c|c|c|}
\hline Loading Starting at Bottom & Layer 1 & Layer 2 & Layer 3 \\
\hline 3 pieces of steel & & N/A & N/A \\
\hline $1 / 3$ of the brown paper & & & \\
\hline \multicolumn{4}{|l|}{$1 / 3$ of the 10 -mil plastic } \\
\hline \multicolumn{4}{|l|}{$1 / 3$ of the bond paper } \\
\hline \multicolumn{4}{|l|}{$1 / 3$ of the towels } \\
\hline \multicolumn{4}{|l|}{$1 / 3$ of the bags } \\
\hline \multicolumn{4}{|l|}{$1 / 3$ of the cut paper } \\
\hline \multicolumn{4}{|l|}{$1 / 3$ of the fabric } \\
\hline \multicolumn{4}{|l|}{$1 / 3$ of the rubber } \\
\hline & Go to Layer 2 & Go to Layer 3 & \\
\hline
\end{tabular}

Drum Filled:

Ring boit placed opposite drum seam and in down position

Lid sealed and torqued to $40 \mathrm{ft} / \mathrm{b}$ :

Leak tested:

Pre-test weight (lb)

Post-test weight (lb)

$$
\text { Loss }=
$$

Date $5-15-95$ Initial Qf

Date 5-15-55 Initial if

Date $5-15-95$ Initial $A f$
N/A or Pressure (psig)

Pressure (psig)

Date

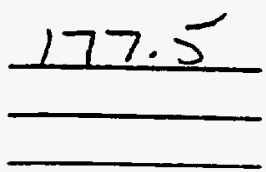

Time (min) 0

Time (min) 5
Initial

Target weight $\sim 175.6 \mathrm{lb}$ 
WHC-SD-WM-TRP-246 REV. 0

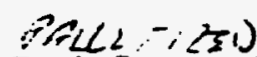

Reoi-Ciesege Drum Data Sheet

Drum No. $\quad \underbrace{073 !}$ i ?.5.5 is.

\section{Post-test Assessment}

Position: original: tipped: toppled

Lid on drum: $\quad x$ Yes ㄴ So sighthy isulged incenter

Gasket missing (cm)

Contents expelled: __ Yes

Seal failure: $\quad$ Y $\quad$ Yes $\quad x$ Not visible

Burn pattern:

-9" at ind int.

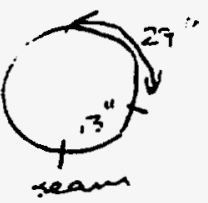

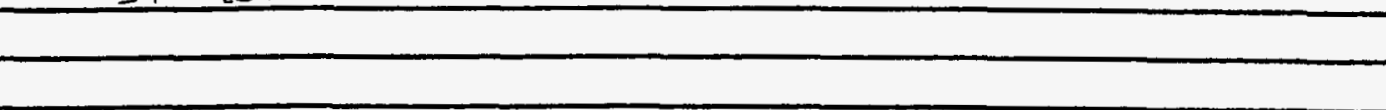

Photo taken:

Yes $x$ No

Leak tested:

$X \overparen{R}$ or Pressure (psig)

Pressure (psig)

Date

\begin{tabular}{ll} 
Time (min) & 0 \\
\hline Initial & Time (min)
\end{tabular}

Interior assessment:

$15 "$ to of dum to top of hen

i. Deo $=7$

photo taken:

$\checkmark$ Yes

No

Comments: 
WHC-SD-WM-TRP-246 REV. 0

\section{Palletized Storage Drum Data Sheet}

Drum No. $0741(p)$

Description (Check):

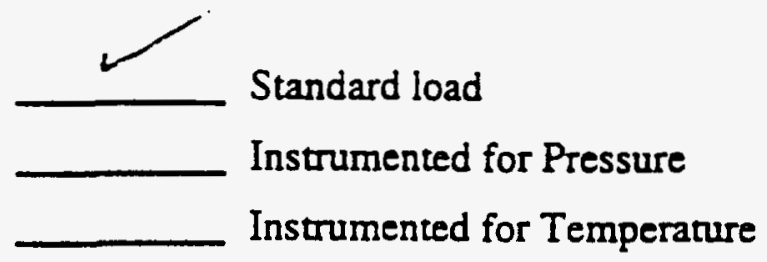

\begin{tabular}{|c|c|c|c|}
\hline Loading Starting at Bottom & Layer 1 & Layer 2 & Layer 3 \\
\hline 3 pieces of steel & & & \\
\hline $1 / 3$ of the brown paper & & & \\
\hline $1 / 3$ of the 10 -mil plastic & & & \\
\hline $1 / 3$ of the bond paper & & & \\
\hline $1 / 3$ of the towels & & & \\
\hline $1 / 3$ of the bags & & & \\
\hline $1 / 3$ of the cut paper & & & \\
\hline $1 / 3$ of the fabric & & & \\
\hline $1 / 3$ of the rubber & & & \\
\hline & Go to Layer 2 & Go to Layer 3 & \\
\hline
\end{tabular}

Drum Filled:

Ring bolt placed opposite drum seam and in down posicion

Lid sealed and torqued to $40 \mathrm{ft} / \mathrm{b}$ :

Leak tested:

Pre-test weight (lb)

Post-test weight (lb)

Date 5-15-95 Initial AP

Date 5-15-75 Initial AP

Date 5-15-95 Initial Af

N/A or Pressure (psig)

Pressure (psig)

Date

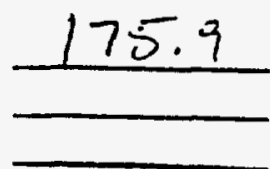

F-56
Time (min) $l$ Time (min) 5

Initial

Target weight $-175.6 \mathrm{lb}$ 
Drum No. $0 ? 4 i|p|-1.61$ is.

\section{Post-test Assessment}

Position:

original:

tipped: toppled

Lid on drum: $\quad x$ Yes No

Gasket missing (cm) a

Contents expelled: _u Yes $x$ No

Seal failure: _ Yes $\quad$ Y Not visible

Burn partern: .

iq" timd ht centeced on bitt

Photo taken: $\quad$ Yes $x$ No

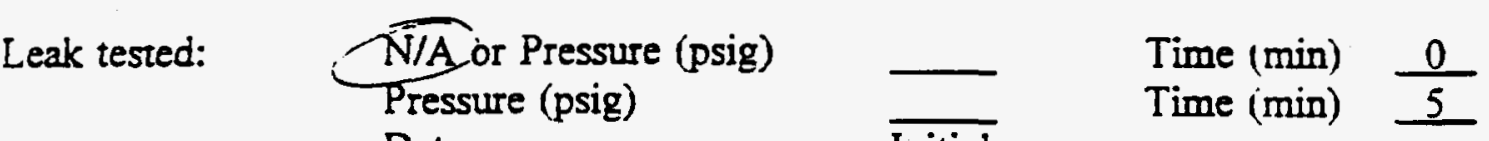

Date

Initial

Interior assessment:

-10 " tor of dum to tire of oher

virscos 2

Photo taken:

$x$ Yes

Comments: 


\section{Palletized Storage Drum Data Sheet}

Drum No. $(.151$

Description (Check):

Standard load Instrumented for Pressure Instrumented for Temperanure

\begin{tabular}{|c|c|c|c|}
\hline Loading Starting at Bottom & Layer 1 & Layer 2 & Layer 3 \\
\hline \hline 3 pieces of steel & & & N/A \\
\hline $1 / 3$ of the brown paper & & & \\
\hline $1 / 3$ of the 10 -mil plastic & & & \\
\hline $1 / 3$ of the bond paper & & & \\
\hline $1 / 3$ of the towels & & & \\
\hline $1 / 3$ of the bags & & & \\
\hline $1 / 3$ of the cut paper & Go to Layer 2 & Go to Layer 3 & \\
\hline $1 / 3$ of the fabric & & \\
\hline $1 / 3$ of the rubber & & \\
\hline \hline
\end{tabular}

Drum Filled:

Date $5-15-95$ Initial $\mu$

Ring bolt placed opposite drum seam Date $5-15-75$ Initial $A O$ and in down position

Lid sealed and torqued to $40 \mathrm{ft} / \mathrm{b}$ : Date $5-.5-95$ Initial Af

Leak tested:

N/A or Pressure (psig)

Pressure (psig)

Date

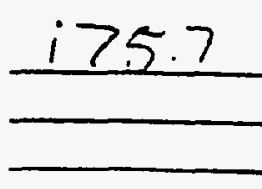

Time (min) 0

Time (min) 5
Pre-test weight (lb)

Post-test weight (lb)

$$
\text { Loss }=
$$

Los Alamos Techaical Associates
Initial

Target weight $-175.6 \mathrm{lb}$ 


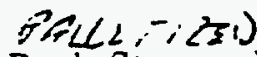

Poolt Ciamege Drum Data Sheet

Drum No. $0751 \quad 174,215$

Post-test Assessment

Position: __ original: ___ tipped: ___ toppled

Lid on drum: $\quad X$ Yes ﹎. No lid loose, ing is wet lee. doesuct

Gasket missing (cm) all

Contents expelled: _ـ Yes $\quad$ Y No

Seal failure: $\quad$ Y Yes $\$ Not visible

Burn pattern:

around rim

Photo taken:

Yes $\rightleftharpoons$ No

Leak tested:

N/A or Pressure (psig)

Pressure (psig)

Date

(1... raviks)

rotate)

Initial

Time (min) $\quad \frac{0}{5}$

Time (min)

Interior assessment:

8"top to top of chan

VIDEC i42

Photo taken:

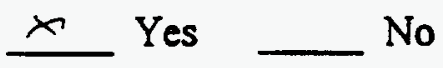

Comments: 


\section{Palletized Storage Drum Data Sheet}

Drum No. C7ies

Descriprion (Check):

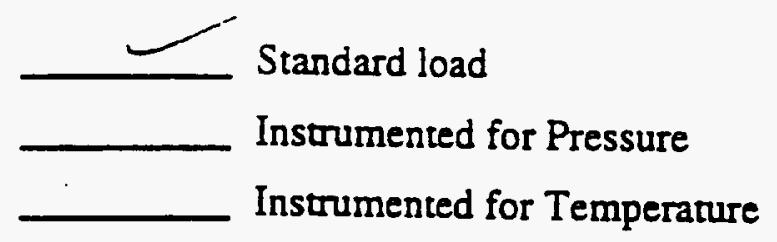

\begin{tabular}{|c|c|c|c|}
\hline Loading Staring at Bottom & Layer 1 & Layer 2 & Layer 3 \\
\hline 3 pieces of steel & & N/A & N/A \\
\hline \multicolumn{4}{|l|}{ 1/3 of the brown paper } \\
\hline \multicolumn{4}{|l|}{$1 / 3$ of the 10 -mil plastic } \\
\hline \multicolumn{4}{|l|}{$1 / 3$ of the bond paper } \\
\hline \multicolumn{4}{|l|}{$1 / 3$ of the towels } \\
\hline \multicolumn{4}{|l|}{$1 / 3$ of the bags } \\
\hline \multicolumn{4}{|l|}{$1 / 3$ of the cut paper } \\
\hline \multicolumn{4}{|l|}{$1 / 3$ of the fabric } \\
\hline \multicolumn{4}{|l|}{$1 / 3$ of the rubber } \\
\hline & Go to Layer 2 & Go to Layer 3 & \\
\hline
\end{tabular}

Drum Filled:

Ring bolt placed opposite drum seam and in down position

Lid sealed and torqued to $40 \mathrm{ft} / \mathrm{b}$ :

Leak tested:

Pre-test weight (lb)

Post-test weight (lb)

$$
\text { Loss = }
$$

Date 5-15-95 Initial AP

Date 3-15-95 Initial AP
N/A or Pressure (psig)

Pressure (psig)

Date

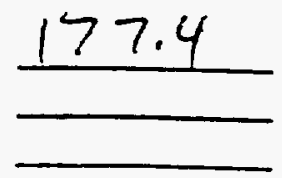

F-60
Time (min)

Time $(\min )$ 
Drum No. $0761,76.016$.

Post-test Assessment

Position: originai: tipped: toppled

Lid on drum: $\quad \underline{C}$ Yes No lid tringloose (rotate freely)

Gasket missing (cm) all

Contents expelled: _ues $x$ No

Seal failure: $\quad$ Yes $\quad x$ Not visible

Burn pattern:

around rim

Photo taken:

Yes $x$ No

Leak tested:

N/Aor Pressure (psig)

Pressure (psig)

Date

$\begin{array}{lll}\text { Time (min) } & 0 \\ \text { Initial } & \text { Time (min) }\end{array}$

Interior assessment:

$\frac{7 \text { Top to top of - han }}{\sim 6 " \text { chan ol torewd }}$

Videci4 4

Photo taken:

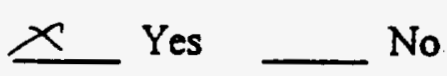

Comments: 


\section{Palletized Storage Drum Data Sheet}

Drum No. 9771

Descriprion (Check):

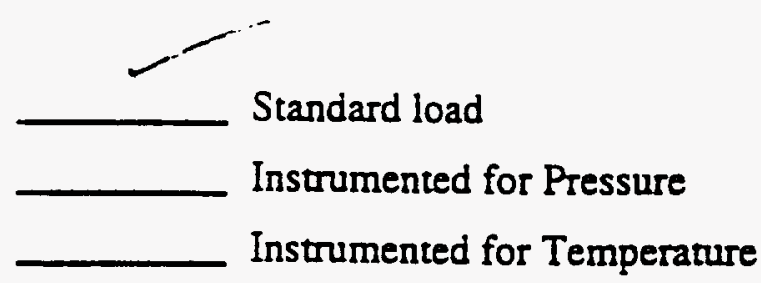

\begin{tabular}{|c|c|c|c|}
\hline Loading Starting at Bottom & Layer 1 & Layer 2 & Layer 3 \\
\hline 3 pieces of steel & & N/A & \\
\hline $1 / 3$ of the brown paper & & & \\
\hline $1 / 3$ of the 10-mil plastic & & & \\
\hline $1 / 3$ of the bond paper & & & \\
\hline $1 / 3$ of the towels & & & \\
\hline $1 / 3$ of the bags & & & \\
\hline $1 / 3$ of the cut paper & & & \\
\hline $1 / 3$ of the fabric & Go to Layer 2 & Go to Layer 3 & \\
\hline $1 / 3$ of the rubber & & \\
\hline
\end{tabular}

Drum Filled:

Date $5-15-15$ Initial $A P$

Ring boit placed opposite drum seam and in down position

Date $5-15=95$ Initial AP

Lid sealed and torqued to $40 \mathrm{ft} / \mathrm{lb}: \quad$ Date $5-1.5-5.5$ Initial $A P$

Leak tested:

N/A or Pressure (psig)

Pressure (psig)

Date

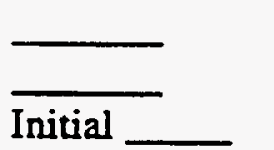

Time (min) 0

Time $(\min ) 5$

Pre-test weight (lb)

Post-test weight (lb)

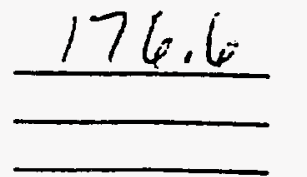

F-62 
Drum No. $0771,75.4 \mathrm{lb}$

\section{Post-test Assessment}

Position: __ original: ___ tipped: __

Lid on drum: $\quad \quad \quad$ Yes No lid a ringloose

Gasket missing (cm) all

Contents expelled: _ Yes $X$ No

Seal failure: $\quad$ Y Yes $\quad x$ Not visible

Burn pattern:

moinly aroundrim and some on bottom seam

Photo taken: $\quad$ Yes No

Leak tested: N/A gressure (psig)

Pressure (psig)

Date

$\overline{\text { Initial }}$

$\begin{array}{ll}\text { Time }(\min ) & 0 \\ \text { Time (min) }\end{array}$

Interior assessment:

To $p$ is chaned up to $-6 "$ " han all around

Videc:22

Photo taken:

$x$ Yes No

Comments: 


\section{Palletized Storage Drum Data Sheet}

Drum No. 0281

Description (Check):

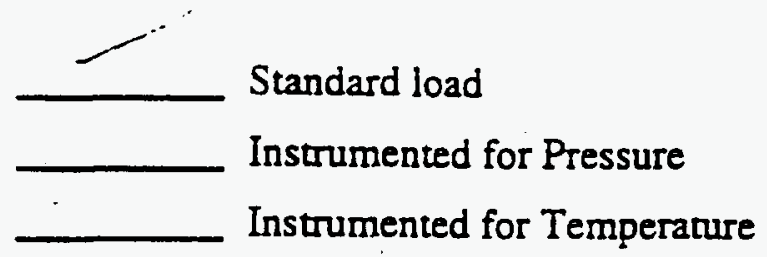

\begin{tabular}{|c|c|c|c|}
\hline Loading Starting at Bottom & Layer 1 & Layer 2 & Layer 3 \\
\hline 3 pieces of steel & & & \\
\hline $1 / 3$ of the brown paper & & & \\
\hline $1 / 3$ of the $10-$ mil plastic & & & \\
\hline $1 / 3$ of the bond paper & & & \\
\hline $1 / 3$ of the towels & & & \\
\hline $1 / 3$ of the bags & & & \\
\hline $1 / 3$ of the cut paper & & Go to Layer 3 & \\
\hline $1 / 3$ of the fabric & & \\
\hline $1 / 3$ of the rubber & Go to Layer 2 & Go & \\
\hline
\end{tabular}

Drum Filled:

Ring bolt placed opposite drum seam and in down position

Lid sealed and torqued to $40 \mathrm{f} / \mathrm{lb}$ :

Leak tested:

Pre-test weight (lb)

Post-test weight (lb)

$$
\text { Loss }=
$$

Date $5.15-95$ Initial of

Date $5-15-95$ Initial $A P$

Date $5-15-95$ Initial AP

N/A or Pressure (psig)

Pressure (psig)

Date

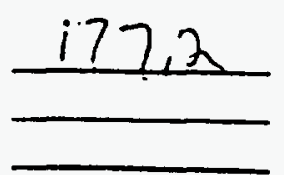

F-64
Time (min) 0 Time (min) 5

Initial

Target weight $\sim 175.6 \mathrm{lb}$ 
Drum No. $0781 \quad 176.9 \mathrm{lb}$.

Post-test Assessment

Position: original: tipped: toppled

Lid on drum: $\quad x$ Yes No ting

Gasket missing (cm) _ 52 "

Contents expelled: __ Yes $\longleftarrow$ No

Seal failure: $\quad$ Yes $x$ Not visible

Burn pattern:

None excapt enound rim

Photo taken: $\quad$ Yes $\stackrel{x}{\longrightarrow}$ No

Leak tested:

or Pressure (psig)

Time (min) $\quad \frac{0}{5}$

Pressure (psig)

Date

\begin{tabular}{lll}
- & Time $(\min )$ & 0 \\
\hline
\end{tabular}$\quad$ Time (min)

Interior assessment:

top chained, thos 4-5" all around

$\therefore 2020123$

Photo taken:

$x$ Yes

Comments: 
WHC-SD-WM-TRP-246 REV. 0

\section{Palletized Storage Drum Data Sheet}

Drum No. $2811(p)$

Descriprion (Check):

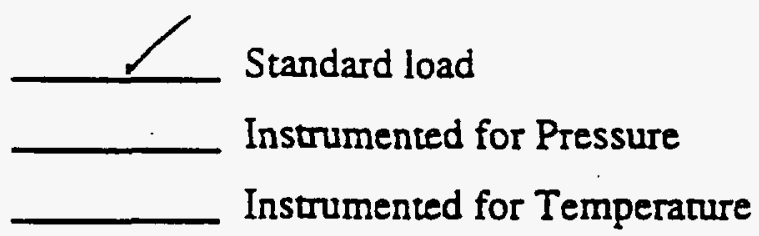

\begin{tabular}{|c|c|c|c|}
\hline Loading Starting at Bottom & Layer 1 & Layer 2 & Layer 3 \\
\hline 3 pieces of steel & & N/A & N/A \\
\hline $1 / 3$ of the brown paper & & $\checkmark$ & J \\
\hline $1 / 3$ of the 10 -mil plastic & & $\sim$ & $\mathcal{J}$ \\
\hline $1 / 3$ of the bond paper & & & \\
\hline $1 / 3$ of the towels & & $\gamma$ & $v$ \\
\hline $1 / 3$ of the bags & & $\mathcal{J}$ & \\
\hline $1 / 3$ of the cut paper & - & $\checkmark$ & \\
\hline $1 / 3$ of the fabric & & $\sqrt{ }$ & $\sqrt{ }$ \\
\hline $1 / 3$ of the rubber & 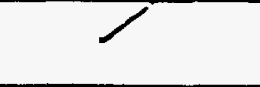 & $J$ & $\sim$ \\
\hline & Go to Layer 2 & Go to Layer 3 & \\
\hline
\end{tabular}

Drum Filled:

Ring bolt placed opposite drum seam and in down position

Lid sealed and torqued to $40 \mathrm{ft} / \mathrm{b}$ :

Leak tested:

Pre-test weight (lb)

Post-test weight (lb)

\section{Loss $=$}

Los Alamos Technical Associates
Date $5-16-95$ Initial f.8.

Date $5-16-95$ Initial $P \&$
N/A or Pressure (psig)

Pressure (psig)

Date

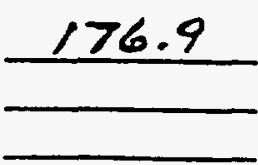

F-66
Time (min)

Time (min) 5

Initial

Target weight $-175.6 \mathrm{lb}$

SwRI Project No. 01-7106-001 
Drum No. $0 \times 11 \quad \omega t_{1}=170.9$

\section{Post-test Assessment}

Position: original: tipped: toppled

Lid on drum: $\quad x$ Yes No $\quad$ No $x 23$ gap

Gasket missing (cm)

Contents expelled: _ Yes $\$ No

Seal failure: $\quad x$ Yes Not visible

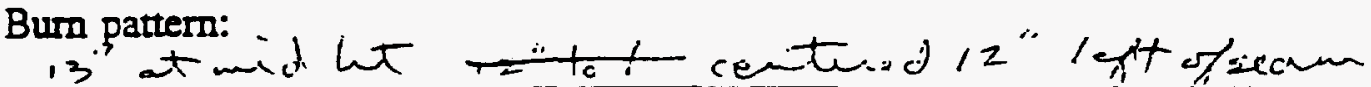
coms iwhat ael arerund.

Photo taken: $\quad$ Yes $X$

Leak tested: $\quad$ N/A or Pressure (psig)

Pressure (psig)

Date

$-\quad$\begin{tabular}{ll} 
Time $(\min )$ & 0 \\
\hline
\end{tabular}$\quad$ Time (min)

Initial

Interior assessment:

$15^{\circ}$ from top of drum to top thehan id contert

Vitpec 2

Photo taken:

$\not$ Yes No

Comments: 


\section{Palletized Storage Drum Data Sheet}

Drum No. 0821

Description (Check):

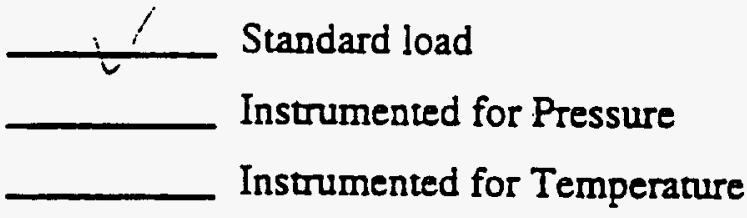

\begin{tabular}{|c|c|c|c|}
\hline Loading Starting at Bottom & Layer 1 & Layer 2 & Layer 3 \\
\hline 3 pieces of steel & & N/A & N/A \\
\hline $1 / 3$ of the brown paper & & & \\
\hline $1 / 3$ of the 10-mil plastic & & & \\
\hline $1 / 3$ of the bond paper & & & \\
\hline $1 / 3$ of the towels & & & \\
\hline $1 / 3$ of the bags & & $\checkmark$ & \\
\hline $1 / 3$ of the cut paper & & $\checkmark$ & \\
\hline $1 / 3$ of the fabric & & $\checkmark$ & \\
\hline $1 / 3$ of the rubber & Go to Layer 2 & Go to Layer 3 & \\
\hline
\end{tabular}

Drum Filled:

Ring bolt placed opposite drum seam and in down position

Lid sealed and torqued to $40 \mathrm{ft} / \mathrm{lb}$ :

Leak tested:

Pre-test weight (lb)

Post-test weight (lb)
Date 5-16-95 Initial P. \&.

Date $5-16-95$ Initial $\ell \&$
N/A or Pressure (psig)

Pressure (psig)

Date

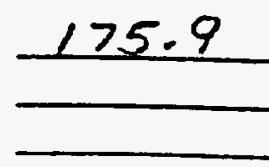

F-68
Time (min)

Time $(\min )-5$

Initial

Target weight $\sim 175.6 \mathrm{lb}$ 
WHC-SD-WM-TRP-246 REV. O

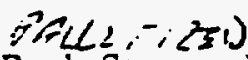

Reat Gtome Drum Data Sheet

Drum No.

\section{Post-test Assessment}

Position: __ original: ___tipped: __ toppled

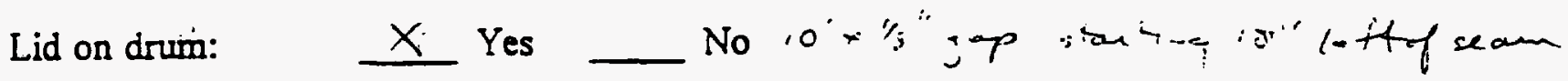

Gasket missing (cm) iel

Contents expelled: __ Yes $\stackrel{x}{\longrightarrow}$ No

Seal failure: $\quad \quad x$ Yes _ Not visible

Burn pattern:

27 "atmed lat

Yes $\underline{c}$ No

JIA or Pressure (psig) Pressure (psig)

Date $\begin{array}{ll}\text { Time }(\min ) & 0 \\ \text { Time (min) } & \underline{5}\end{array}$

$\overline{\text { Initial }}$

Interior assessment:

12 "char top to drum top $9^{\circ}$ inte center al around

ineci 4

Photo taken:

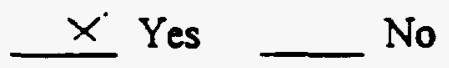

Comments: 


\section{Palletized Storage Drum Data Sheet}

Drum No. $0.831(\rho)$

Description (Check):

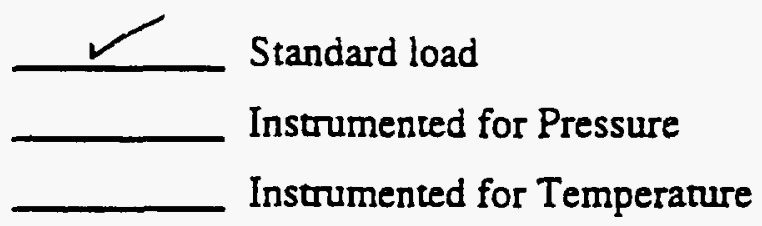

\begin{tabular}{|c|c|c|c|}
\hline Loading Starting at Bottom & Layer 1 & Layer 2 & Layer 3 \\
\hline 3 pieces of steel & & N/A & N/A \\
\hline $1 / 3$ of the brown paper & & $\checkmark$ & 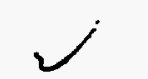 \\
\hline $1 / 3$ of the 10 -mil plastic & & ノ & $\checkmark$ \\
\hline \multicolumn{4}{|l|}{$1 / 3$ of the bond paper } \\
\hline \multicolumn{4}{|l|}{$1 / 3$ of the towels } \\
\hline \multicolumn{4}{|l|}{$1 / 3$ of the bags } \\
\hline \multicolumn{4}{|l|}{$1 / 3$ of the cut paper } \\
\hline $1 / 3$ of the fabric & & $\mathcal{J}$ & $\checkmark$ \\
\hline \multirow[t]{2}{*}{$1 / 3$ of the rubber } & $\zeta$ & $\checkmark$ & v \\
\hline & Go to Layer 2 & Go to Layer 3 & \\
\hline
\end{tabular}

Drum Filled:

Ring bolt placed opposite drum seam and in down position

Lid sealed and torqued to $40 \mathrm{ft} / \mathrm{lb}$ :

Leak tested:

Pre-test weight (lb)

Post-test weight (lb)

$$
\text { Loss = }
$$

Los Alamos Technieal Associates
Date $5-16-95$ Initial P.\&.

Date 5-16-95 Initial $P 1$

Date $5-14-95$ Initial P. 8.
N/A or Pressure (psig) Pressure (psig)

Date

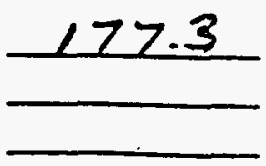

F-70
Time $(\min ) \quad l$

Time (min) 5
Initial

Target weight $\sim 175.6 \mathrm{lb}$

SwRI Project No. 01-7106-001 


\section{$P A(1,-i 251)$ \\ Rack Drum Data Sheet}

Drum No. $0831 \quad 176.91 \mathrm{~b}$.

Post-test Assessment

Position: original: tipped: toppied

Lid on drum: $\quad \quad \quad \quad$ Yes No

Gasket missing (cm) ___ al but 10 "

Contents expelled: _u Yes $X$ No

Seal failure: $\quad$ Yes $\quad X$ Not visible

Burn pattern:

Burn pattern:

$$
\text { Yes } \quad \times \text { Not visible }
$$

$\longrightarrow$

Photo taken:

$$
\text { Yes } x \text { No }
$$

Leak tested:

N/A or Pressure (psig)

Pressure (psig)

Date

\begin{tabular}{lll} 
& Time (min) & 0 \\
\hline Initial & Time (min)
\end{tabular}

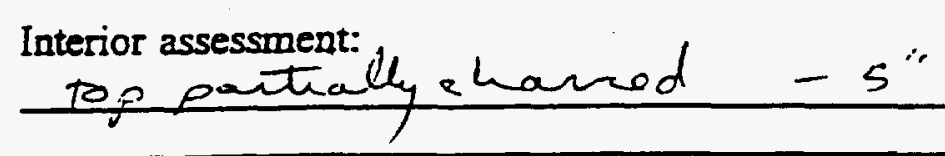

UIDeC $5 i$

Pboto taken:

$\times$ Yes

Comments: 
WHC-SD-WM-TRP-246 REV. 0

\section{Palletized Storage Drum Data Sheet}

Drum No. 0.341

Description (Check):

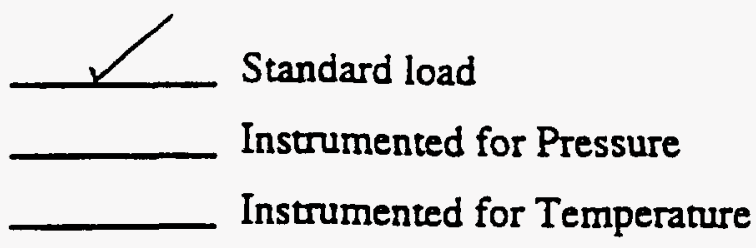

\begin{tabular}{|c|c|c|c|}
\hline Loading Starting at Bottom & Layer 1 & Layer 2 & Layer 3 \\
\hline 3 pieces of steel & & N/A \\
\hline $1 / 3$ of the brown paper & & & \\
\hline $1 / 3$ of the 10 -mil plastic & & & \\
\hline $1 / 3$ of the bond paper & & & \\
\hline $1 / 3$ of the toweis & & & \\
\hline $1 / 3$ of the bags & & & \\
\hline $1 / 3$ of the cut paper & & & \\
\hline $1 / 3$ of the fabric & & Go to Layer 3 & \\
\hline $1 / 3$ of the rubber & Go to Layer 2 & G & \\
\hline
\end{tabular}

Drum Filled:

Ring bolt placed opposite drum seam and in down position

Lid sealed and torqued to $40 \mathrm{ft} / \mathrm{b}$ :

Leak tested:

Pre-test weight (lb)

Post-test weight (lb)

Loss $=$

Date $5-16-95$ Initial $f_{.}$

Date $5-16-95$ Initial \&8.

Date $s-16-95$ Initial $\rho \&$

N/A or Pressure (psig)

Pressure (psig)

Date

176.6

Initial

Target weight $\sim 175.6 \mathrm{lb}$
Time (min) 0

Time (min) 5 
Drum No. OE41 $174.9 \mathrm{lb}$

Post-iest Assessment

Position: original: tipped: toppied

Lid on drum: $\quad X$ Yes No

Gasket missing (cm)

Contents expelled: __ Yes $X$ No

Seal failure: $\quad$ Yes $X$ Not visible

Burn partern: id Lt.

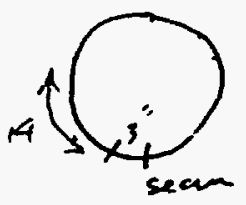

Photo taken: Yes $\underline{x}$ No

Leak tested: Pressure (psig)

Date \begin{tabular}{lll} 
Time (min) & 0 \\
\hline & Time (min)
\end{tabular}

Interior assessment:

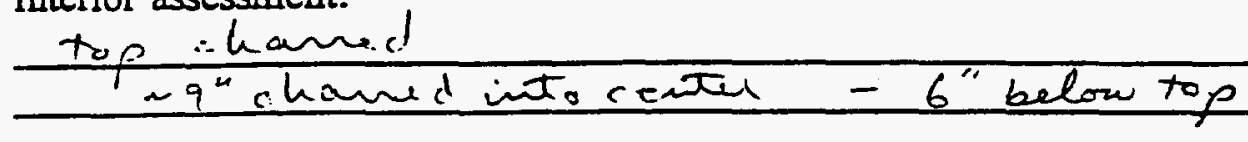

Comments: 


\section{Palletized Storage Drum Data Sheet}

Drum No. DSSI

Description (Check):

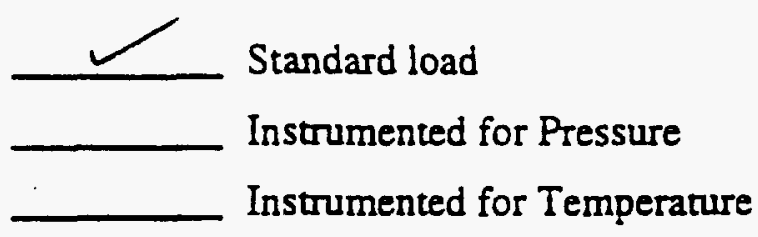

\begin{tabular}{|c|c|c|c|}
\hline Loading Starting at Bottom & Layer 1 & Layer 2 & Layer 3 \\
\hline 3 pieces of steel & 乙 & N/A & N/A \\
\hline \multicolumn{4}{|l|}{$1 / 3$ of the brown paper } \\
\hline \multicolumn{4}{|l|}{$1 / 3$ of the 10 -mil plastic } \\
\hline \multicolumn{4}{|l|}{ 1/3 of the bond paper } \\
\hline \multicolumn{4}{|l|}{$1 / 3$ of the towels } \\
\hline $1 / 3$ of the bags & & $\checkmark$ & $\mathcal{J}$ \\
\hline \multicolumn{4}{|l|}{ 1/3 of the cut paper } \\
\hline \multicolumn{4}{|l|}{$1 / 3$ of the fabric } \\
\hline \multirow[t]{2}{*}{$1 / 3$ of the rubber } & 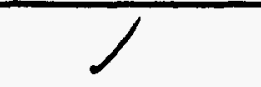 & $\checkmark$ & $\checkmark$ \\
\hline & Go to Layer 2 & Go to Layer 3 & \\
\hline
\end{tabular}

Drum Filled:

Ring bolt placed opposite drum seam and in down posicion

Lid sealed and torqued to $40 \mathrm{ft} / \mathrm{b}$ :

Leak tested:

Pre-test weight (lb)

Post-test weight (lb)

$$
\text { Loss }=
$$

Date $\leq-16-95$ Initial $\rho \&$

Date 5-16-95 Initial P\&. Date 5-16-95 Initial P.\&

N/A or Pressure (psig)

Pressure (psig)

Date

\begin{tabular}{ll}
$\square$ & Time (min) $\frac{0}{5}$ \\
\hline Initial & Time (min)
\end{tabular}


*ak RMIS View/Frint Document Cover Sheet tow

This document was retrieved from the Documentation and Records Manaqement (DRM) ISEARCH System. It is intended for Information only and may not be the most recent or updated version. Contact a Document Service Center (see Hanford Info for locations) if you need additional retrieval information.

Accession \#: D195061641

Document \#: SD-WM-TRP-246

Title/Desc:

SOLID WASTE DRUM ARRAY FIRE PERFORMANCE [SEC 6 OF 7]

Pages: 151 
THIS DOCUMENT WAS TOO LARGE TO SCAN AS A SINGLE DOCUMENT. IT REQUIRED BREAKING INTO SMALLER SECTIONS.

DOCUMENT NUMBER: SD-WM-TRP.2HW

SECTION: $\quad$ 6 OF 7

TITLE: Aolid waote Drum Array jirie Performance

AUTHOR: LoUUi RL

RECIPIENT:

DATE: $11 / 07 / 95$

KEYWORDS:

REFERENCES: EDT-612232 
Blank 
Drum No. 0551 $75.8 \mathrm{k}$.

Post-test Assessment

Position: original: ___ tipped: toppled

Lid on drum: $\quad X$ Yes No lideringloose

Gasket missing (cm) all

Contents expeiled: __ Yes $\lfloor$ No

Seal failure:

Yes $\searrow$ Not visible

Burn pattern:

only around tap rim

Photo taken:

$$
\text { Yes } \underline{\text { No }}
$$

Leak tested:

(N)A or Pressure (psig)

Pressure (psig)

Date

\begin{tabular}{lll} 
& Time (min) & 0 \\
\hline Timitial & Time (min)
\end{tabular}

Interior assessment:
to $p$ h havied $\sim s^{\prime}$ all anouned

V.peo 59

Photo taken:

$x$ Yes

Comments: 
WHC-SD-WM-TRP-246 REV. 0

\section{Palletized Storage Drum Data Sheet}

Drum No. celc1

Descriprion (Check):

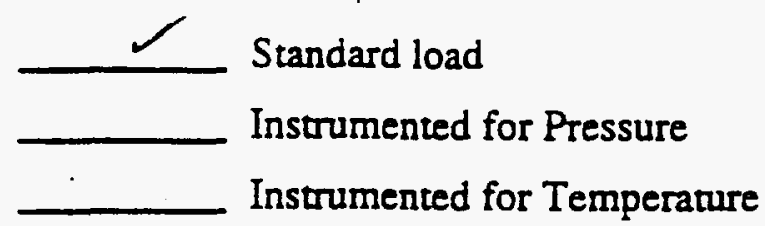

\begin{tabular}{|c|c|c|c}
\hline Loading Starting at Bottom & Layer 1 & Layer 2 & Layer 3 \\
\hline 3 pieces of steel & $\checkmark$ & N/A & N/A \\
\hline $1 / 3$ of the brown paper & $\checkmark$ & & \\
\hline $1 / 3$ of the 10-mil plastic & & $\checkmark$ & $\checkmark$ \\
\hline $1 / 3$ of the bond paper & $\checkmark$ & $\checkmark$ & $\checkmark$ \\
\hline $1 / 3$ of the toweis & $\checkmark$ & $\checkmark$ & $\checkmark$ \\
\hline $1 / 3$ of the bags & $\checkmark$ & $\checkmark$ & $\checkmark$ \\
\hline $1 / 3$ of the cut paper & $\checkmark$ & $\checkmark$ & \\
\hline $1 / 3$ of the fabric & $\checkmark$ & $\checkmark$ & \\
\hline $1 / 3$ of the rubber & Go to Layer 2 & Go to Layer 3 & \\
\hline
\end{tabular}

Drum Filled:

Ring bolt placed opposite drum seam and in down position

Lid sealed and torqued to $40 \mathrm{ft} / \mathrm{b}$ :

Leak tested:

Pre-test weight (lb)

Post-test weight (lb)

Date 5-16-95 Initial P.\&.

Date $5-16-95$ Initial P. L

Date $5-16-95$ Initial L.\&

N/A or Pressure (psig)

Pressure (psig)

Date

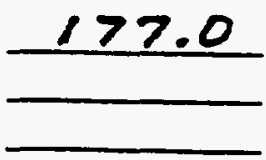

F-76
Time (min)

Time (min) 5 
Drum No. $0861 \quad 177.4 \mathrm{kr}$.

Post-test Assessment

Position: originai: tipped: toppled

Lid on drum: $\quad \quad \quad x$ Yes $\quad$ No

Gasket missing (cm) _ـ $27 "$

Contents expelled: $\quad$ Yes $x$ No

Seal failure: Yes $x$ Not visible

Burn pattern: none

Photo taken: Yes $\underline{x}$ No

Leak tested:

N/A or Pressure (psig)

Pressure (psig)

Date 
WHC-SD-WM-TRP-246 REV. 0

\section{Palletized Storage Drum Data Sheet}

Drum No. 0871

Description (Check):

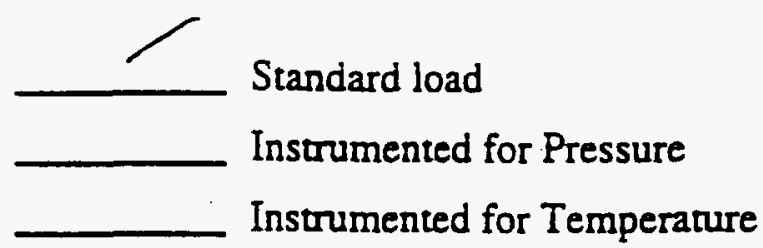

\begin{tabular}{|c|c|c|c|}
\hline Loading Starting at Bottom & Layer 1 & Layer 2 & Layer 3 \\
\hline 3 pieces of steel & $\checkmark$ & N/A & N/A \\
\hline $1 / 3$ of the brown paper & $\checkmark$ & 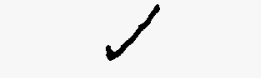 & $\checkmark$ \\
\hline $1 / 3$ of the 10 -mil plastic & $\mathcal{J}$ & 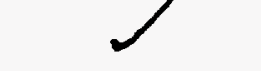 & $\checkmark$ \\
\hline $1 / 3$ of the bond paper & $\sqrt{ }$ & $\mathcal{J}$ & $\checkmark$ \\
\hline $1 / 3$ of the towels & $V$ & $\checkmark$ & \\
\hline $1 / 3$ of the bags & $\checkmark$ & $\sqrt{ }$ & $\sqrt{ }$ \\
\hline $1 / 3$ of the cut paper & $\nearrow$ & $\mathcal{J}$ & $\checkmark$ \\
\hline $1 / 3$ of the fabric & $\sqrt{ }$ & 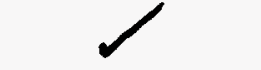 & $v$ \\
\hline $1 / 3$ of the rubber & & $\sqrt{ }$ & \\
\hline & Go to Layer 2 & Go to Layer 3 & \\
\hline
\end{tabular}

Drum Filled:

Ring bolt placed opposite drum seam and in down position

Lid sealed and torqued to $40 \mathrm{ft} / \mathrm{b}$ :

Leak tested:

Pre-test weight (lb)

Post-test weight (lb)

Date $5-16-95$ Initial Le\&

Date $5-16-95$ Initial P.d.

Date $5-16-95$ Initial P.\&

N/A or Pressure (psig) Pressure (psig)

Date

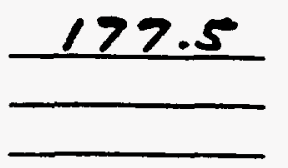

Time (min) 0 Time (min) 5

Initial

Target weight $-175.6 \mathrm{lb}$

SwRI Project No. 01-7106-001 


\section{Prfill, -1251$)$}

Rook Sienge Drum Data Sheet

Drum No. $0871,77.4 \mathrm{~h}$,

Post-test Assessment

Position: __ original: ___ tipped: ___ toppled

Lid on drum: $\quad x$ Yes No

Gasket missing (cm) $O \sim 10-1 \mathrm{~S}^{\prime \prime}$ compressed thand

Contents expeiled: _ues $x$ No

Seal failure: $\quad$ Yes $x$ Not visible

Burn pattern:

NoNhe

Photo taken:

Yes $\searrow$ No

Leak tested:

NTA 0 Pressure (psig)

Pressure (psig)

Date

\begin{tabular}{lll}
- & Time (min) & 0 \\
\hline & Time (min)
\end{tabular}

Interior assessment:

bavely metted plantic ot sean

virec $5 \gamma$

Eboto taken:

$x$ Yes _ No

Comments: 


\section{Palletized Storage Drum Data Sheet}

Drum No. 0851

Descripion (Check):

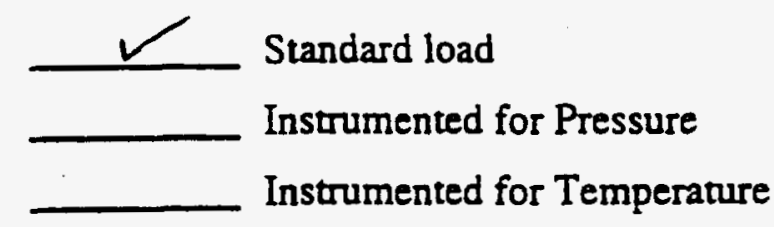

\begin{tabular}{|c|c|c|c|}
\hline Loading Staring at Bottom & Layer 1 & Layer 2 & Layer 3 \\
\hline 3 pieces of steel & & N/A & N/A \\
\hline $1 / 3$ of the brown paper & & & \\
\hline $1 / 3$ of the 10-mil plastic & & & \\
\hline $1 / 3$ of the bond paper & & & \\
\hline $1 / 3$ of the towels & & & \\
\hline $1 / 3$ of the bags & & $\checkmark$ & \\
\hline $1 / 3$ of the cut paper & & Go to Layer 3 & \\
\hline $1 / 3$ of the fabric & Go to Layer 2 & G & \\
\hline $1 / 3$ of the rubber & &
\end{tabular}

Drum Filled:

Ring bolt placed opposite drum seam and in down position

Lid sealed and torqued to $40 \mathrm{ft} / \mathrm{b}$ :

Leak tested:

Pre-test weight (lb)

Post-test weight (lb)

Date 5-16-95 Initial P\&

Date $5-16-95$ Initial P.\&.

Date $5-16-95$ Initial L.S.

N/A or Pressure (psig)

Pressure (psig)

Date

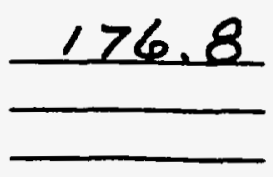

F-80
Time (min) 0

Time (min) 5

Initial

Target weight $-175.6 \mathrm{lb}$

SwRI Projea No. 01-7106-001 
Drum No. 0881176.815.

\section{Post-test Assessment}

Position: originai: tipped: toppled

Lid on drum: $\quad x$ Yes No

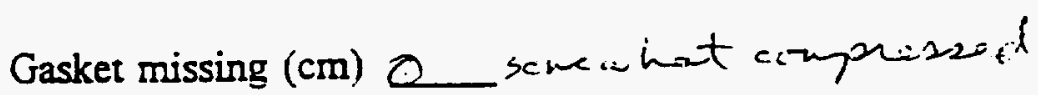

Contents expelled: $\quad$ Yes $\nsim$ No

Seal failure: $\quad$ Yes $\quad$ Not visible

Burn pattern:

Nove

Photo taken:

Yes $\longrightarrow$ No

Leak tested:

NFor Pressure (psig)

Pressure (psig)

Date

$\frac{{ }_{\text {Initial }}}{\longrightarrow} \begin{gathered}\text { Time (min) } \\ \text { Time (min) }\end{gathered} \quad$

\section{Interior assessment:}

ne melting

video 55

Photo taken:

$\ltimes$ Yes _ No

\section{Comments:}




\section{Palletized Storage Drum Data Sheet}

Drum No. 9911

Description (Check):

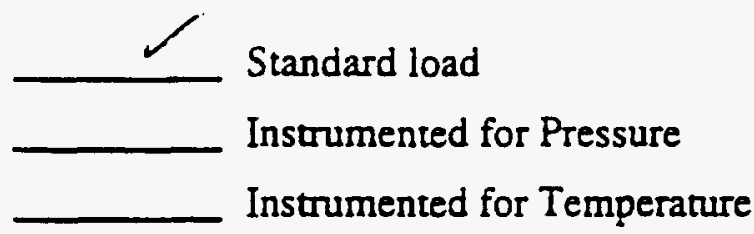

\begin{tabular}{|c|c|c|c|}
\hline Loading Staring at Bottom & Layer 1 & Layer 2 & Layer 3 \\
\hline \hline 3 pieces of steel & & N/A & N/A \\
\hline $1 / 3$ of the brown paper & $\checkmark$ & & \\
\hline $1 / 3$ of the 10-mil plastic & $\checkmark$ & & \\
\hline $1 / 3$ of the bond paper & $\checkmark$ & & \\
\hline $1 / 3$ of the towels & $\checkmark$ & & \\
\hline $1 / 3$ of the bags & $\checkmark$ & $\checkmark$ & \\
\hline $1 / 3$ of the cut paper & $\checkmark$ & & \\
\hline $1 / 3$ of the fabric & $\checkmark$ & $\checkmark$ & \\
\hline $1 / 3$ of the rubber & Go to Layer 2 & Go to Layer 3 & \\
\hline
\end{tabular}

Drum Filled:

Ring bolt placed opposite drum seam and in down position

Lid sealed and torqued to $40 \mathrm{ft} / \mathrm{b}$ :

Leak tested:

Pre-test weight (lb)

Post-test weight (lb)

\section{Loss $=$}

Date $5-17-95$ Initial $P . \&$.

Date $5-17.95$ Initial 1.8

Date $5-17-95$ Initial P.S.

N/A or Pressure (psig)

Pressure (psig)

Date

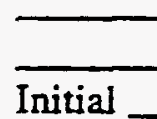

Time (min) 0

Time (min) 5

$\angle 77.1 \quad$ Target weight $-175.6 \mathrm{lb}$ 
WHC-SD-WM-TRP-246 REV. O

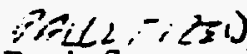

Roeiderene Drum Data Sheet

Drum No. $\quad 0911$ wt. = 175.5 ib

Post-test Assessment

Position: ___ original: ___ tipped: ___ toppled

Lid on drum: $\quad X$ Yes No

Gasket missing (cm) Al!

Contents expelled: _u Yes $x$ No

Seal failure: $\quad$ Yes $\quad X$ Not visible

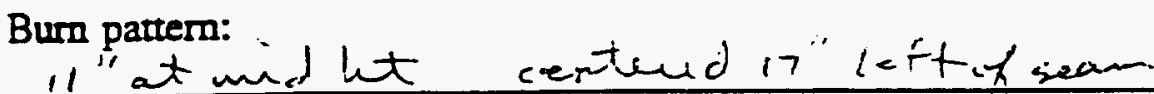
also io"widh $\times 12$ spot belsw ise it'

Photo taken:

Yes $\mathrm{X}$ No

Leak tested:

SN/A or Pressure (psig) Pressure (psig)

Date

\begin{tabular}{lll} 
& Time (min) & 0 \\
\hline Initiai & Time (min)
\end{tabular}

Interior assessment:

Top chancd

"2-5" chanid ail ancund

$v_{1}>201$

Phototaken:

$\checkmark$ Yes

No

Comments: 
WHC-SD-WM-TRP-246 REV. 0

\section{Palletized Storage Drum Data Sheet}

Drum No. 9921

Descriprion (Check):

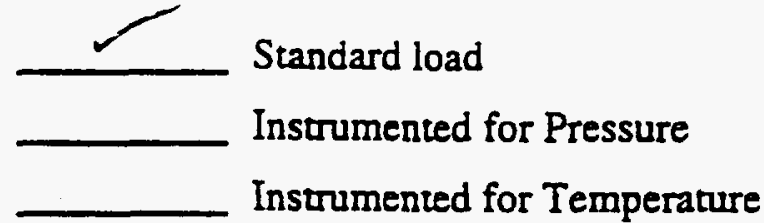

\begin{tabular}{|c|c|c|c|}
\hline Loading Starting at Bottom & Layer 1 & Layer 2 & Layer 3 \\
\hline 3 pieces of steel & & N/A \\
\hline $1 / 3$ of the brown paper & & & \\
\hline $1 / 3$ of the 10-mil plastic & & & \\
\hline $1 / 3$ of the bond paper & & & \\
\hline $1 / 3$ of the towels & & & \\
\hline $1 / 3$ of the bags & & & \\
\hline $1 / 3$ of the cut paper & & & \\
\hline $1 / 3$ of the fabric & Go to Layer 2 & Go to Layer 3 & \\
\hline $1 / 3$ of the rubber & & \\
\hline
\end{tabular}

Drum Filled:

Ring bolt placed opposite drum seam and in down position

Lid sealed and torqued to $40 \mathrm{ft} / \mathrm{lb}$ :

Leak tested:

Pre-test weight (lb)

Post-test weight (lb)

$$
\text { Loss }=
$$

Date $5-17-95$ Initial 2.8 .

Date $5-17.95$ Initial $2 \&$

Date $5-17-95$ Initial P.8.

N/A or Pressure (psig)

Pressure (psig)

Date

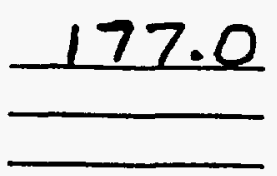

Time (min) 0

Time (min) 5 
Drum No. $09 \geq 1$ i75.7 its

\section{Post-test Assessment}

Position:

original:

tipped:

toppled

Lid on drum: $\quad x$ Yes No

Gasket missing (cm) Al!

Contents expeiled: __ Yes $x$ No

Seal failure: $\quad$ Y Yes $\quad x$ Not visible

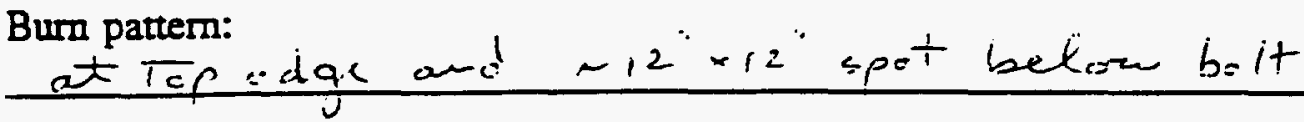

Photo taken:

$$
\text { Yes } x \text { No }
$$

Leak tested:

\section{NHA or Pressure (psig) Pressure (psig)}

Date

$\begin{array}{ll}\text { Time }(\min ) & 0 \\ \text { Time (min) } & 5\end{array}$

Initial

Interior assessment:

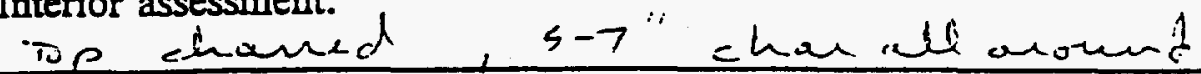

ritseo

Photo taken:

$\underline{X}$ Yes

No

Comments: 


\section{Palletized Storage Drum Data Sheet}

Drum No. 9931

Description (Check):

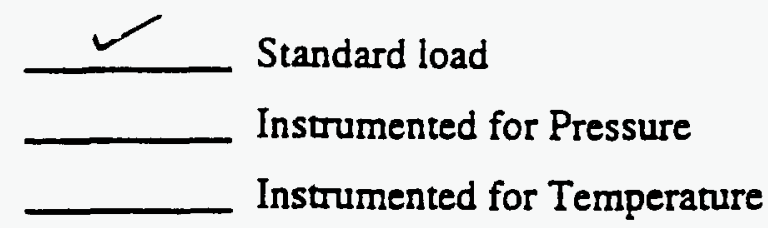

\begin{tabular}{|c|c|c|c|}
\hline Loading Starting at Bottom & Layer 1 & Layer 2 & Layer 3 \\
\hline 3 pieces of steel & $\mathcal{J}$ & N/A & N/A \\
\hline \multicolumn{4}{|l|}{$1 / 3$ of the brown paper } \\
\hline $1 / 3$ of the 10 -mil plastic & $\checkmark$ & $\mathcal{J}$ & $\checkmark$ \\
\hline $1 / 3$ of the bond paper & $\checkmark$ & $\sim$ & \\
\hline \multicolumn{4}{|l|}{$1 / 3$ of the towels } \\
\hline \multicolumn{4}{|l|}{$1 / 3$ of the bags } \\
\hline \multicolumn{4}{|l|}{$1 / 3$ of the cut paper } \\
\hline \multicolumn{4}{|l|}{$1 / 3$ of the fabric } \\
\hline \multicolumn{4}{|l|}{$1 / 3$ of the rubber } \\
\hline & Go to Layer 2 & Go to Layer 3 & \\
\hline
\end{tabular}

Drum Filled:

Ring bolt placed opposite drum seam and in down position

Lid sealed and torqued to $40 \mathrm{f} / / \mathrm{b}$ :

Leak tested:

Pre-test weight (lb)

Post-test weight (lb)

$$
\text { Loss }=
$$

Date $5-17-95$ Initial P.\&.

Date $5-17-95$ Initial $\rho . \&$. Date 5-17-95 Initial P. .

N/A or Pressure (psig) Pressure (psig)

Date

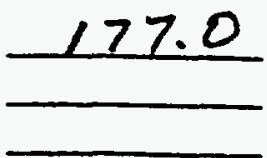

F-86
Time (min) 0

Time (min) 5
Initial

Target weight $-175.6 \mathrm{lb}$ 
WHC-SD-WM-TRP-246 REV. O

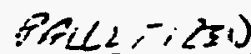

Reclestonge Drum Data Sheer

Drum No. $0931 \cdot 75.21 b$.

Post-test Assessment

Position: __ originai: ___ tipped: ___ toppled

Lid on drum: $\quad \quad \quad x$ Yes No

Gasket missing (cm) all

Contents expelled: __ Yes $X$ No

Seal failure: $\quad$ Yes $\_$Not visible

Burn partern:

Nove except around rim

Photo taken: $\quad$ Yes $\not \subset$ No

$\begin{array}{llll}\text { Leak tested: } \quad \text { Time (min) } & 0 \\ & \text { Pressure (psig) } & \text { Time (min) } & -\end{array}$

Date

$\overline{\text { Initial }}$

Interior assessment:

top charied - 5"chan all around

W.0? 2

Photo taken:

$x$ Yes

Comments: 


\section{Palletized Storage Drum Data Sheet}

Drum No. $\longdiv { 9 } 4 1$

Description (Check):

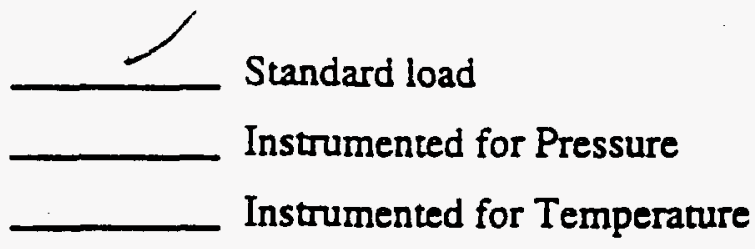

\begin{tabular}{|c|c|c|c|}
\hline Loading Starting at Bottom & Layer 1 & Layer 2 & Layer 3 \\
\hline 3 pieces of steel & $\checkmark$ & N/A & N/A \\
\hline \multirow{2}{*}{\multicolumn{4}{|c|}{$1 / 3$ of the 10 -mil plastic }} \\
\hline & & & \\
\hline \multicolumn{4}{|l|}{$1 / 3$ of the bond paper } \\
\hline \multicolumn{4}{|l|}{$1 / 3$ of the towels } \\
\hline \multicolumn{4}{|l|}{$1 / 3$ of the bags } \\
\hline \multicolumn{4}{|l|}{$1 / 3$ of the cur paper } \\
\hline \multicolumn{4}{|l|}{$1 / 3$ of the fabric } \\
\hline \multicolumn{4}{|l|}{$1 / 3$ of the rubber } \\
\hline & Go to Layer 2 & Go to Layer 3 & \\
\hline
\end{tabular}

Drum Filled:

Ring bolt placed opposite drum seam and in down position

Lid sealed and torqued to $40 \mathrm{ft} / \mathrm{b}$ :

Leak tested:

Pre-test weight (lb)

Post-test weight (lb)

$$
\text { Loss }=
$$

Date 5.17 .95 Initial P\& Date $s-17-95$ Initial $P \&$

N/A or Pressure (psig) Pressure (psig)

Date

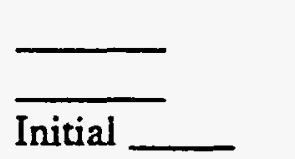

Time (min) 0 Time (min) 5

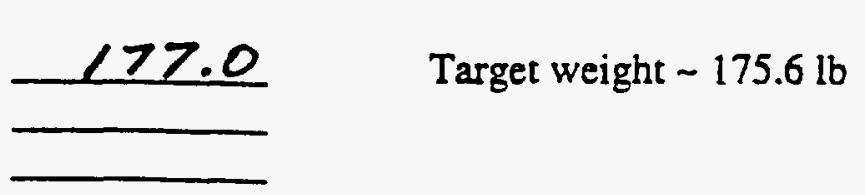


Drum No. $\quad 0941,75.7$ ib

Post-test Assessment

Position: __ original: ___ tipped: __ toppled

Lid on drum: $\quad X$ Yes No Ring - Lid Lw

Gasket missing (cm) all

Contents expeiled: __ Yes $X$ No

Seal failure: $\quad$ Yes $X$ Not visible

Burn pattern:

wotre - some flaming abcumd the ri.. I

Photo taken: Yes $\underline{x}$ No

Leak tested:

NIA or Pressure (psig) Pressure (psig)

Date

\begin{tabular}{lll} 
& Time $(\min )$ & 0 \\
\hline & Time $(\min )$
\end{tabular}

Interior assessment; $2-5^{\prime \prime}$ chat
Top chaned

$\checkmark$ itsec s,

Phote taken: $\quad X$ Yes No

Comments: 
WHC-SD-WM-TRP-246 REV. 0

\section{Palletized Storage Drum Data Sheet}

Drum No. 0951

Description (Check):

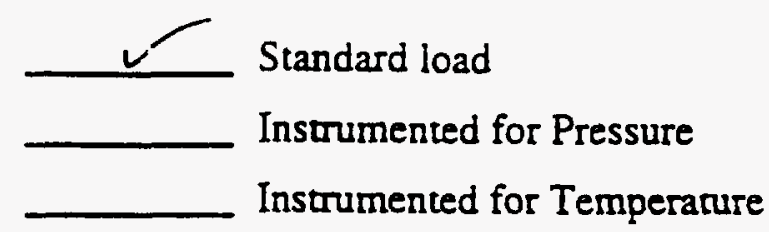

\begin{tabular}{|c|c|c|c|}
\hline Loading Starting at Bottom & Layer 1 & Layer 2 & Layer 3 \\
\hline 3 pieces of steel & & N/A & N/A \\
\hline $1 / 3$ of the brown paper & $\mathcal{J}$ & $\checkmark$ & $\checkmark$ \\
\hline \multicolumn{4}{|l|}{$1 / 3$ of the 10 -mil plastic } \\
\hline \multicolumn{4}{|l|}{$1 / 3$ of the bond paper } \\
\hline \multicolumn{4}{|l|}{$1 / 3$ of the towels } \\
\hline \multicolumn{4}{|l|}{$1 / 3$ of the bags } \\
\hline \multicolumn{4}{|l|}{$1 / 3$ of the cut paper } \\
\hline \multicolumn{4}{|l|}{$1 / 3$ of the fabric } \\
\hline \multicolumn{4}{|l|}{$1 / 3$ of the rubber } \\
\hline & Go to Layer 2 & Go to Layer 3 & \\
\hline
\end{tabular}

Drum Filled:

Ring bolt placed opposite drum seam and in down position

Lid sealed and torqued to $40 \mathrm{ftll}$ :

Leak tested:

Pre-test weight (lb)

Post-test weight (lb)

$$
\text { Loss = }
$$

Date 5-17-95 Initial P.S.

Date $5-17-95$ Initial P.8.

Date $5-17-95$ Initial P.S.

N/A or Pressure (psig)

Pressure (psig)

Date

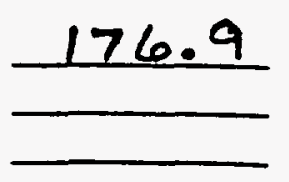

Time $(\min )$

Time (min) 5 
Drum No. 0951176.4

Post-test Assessment

Position: __ original: __ _tipped: __ toppled

Lid on drum: $\quad x$ Yes No ring tid Lerose

Gasket missing (cm) all

Contents expelled: __ Yes $\_$No

Seal failure: $\quad$ Yes $X$ Not visible

Burn pattern:

Nove - some around rim

Photo taken: $\quad$ Yes $\simeq$ No

Leak tested:

ThA or Pressure (psig)

Pressure (psig)

Date

$\longrightarrow \quad$\begin{tabular}{ll} 
Time (min) & 0 \\
\hline
\end{tabular}

Interior assessment:

top chaned, 3-5" of chan depth

Yoec Ec
Photo taken:

$x$ Yes

Comments: 


\section{Palletized Storage Drum Data Sheet}

Drum No. 0961

Descriprion (Check):

Standard load

Instrumented for Pressure

Instrumented for Temperature

\begin{tabular}{|c|c|c|c|}
\hline Loading Starting at Bottom & Layer 1 & Layer 2 & Layer 3 \\
\hline 3 pieces of steel & & N/A & N/A \\
\hline \multicolumn{4}{|l|}{$1 / 3$ of the brown paper } \\
\hline \multicolumn{4}{|l|}{$1 / 3$ of the 10 -mil plastic } \\
\hline \multicolumn{4}{|l|}{$1 / 3$ of the bond paper } \\
\hline \multicolumn{4}{|l|}{$1 / 3$ of the towels } \\
\hline \multicolumn{4}{|l|}{$1 / 3$ of the bags } \\
\hline \multicolumn{4}{|l|}{$1 / 3$ of the cut paper } \\
\hline \multicolumn{4}{|l|}{$1 / 3$ of the fabric } \\
\hline \multicolumn{4}{|l|}{$1 / 3$ of the rubber } \\
\hline & Go to Layer 2 & Go to Layer 3 & \\
\hline
\end{tabular}

Drum Filled:

Ring bolt placed opposite drum seam and in down position

Lid sealed and torqued to $40 \mathrm{ft} / \mathrm{lb}$ :

Leak tested:

Pre-test weight (lb)

Post-test weight (lb)

\section{Loss $=$}

Date $5-17-95$ Initial P.\&

Date $s-17-95$ Initial P.\&.

Date 5-17-95 Initial $P \&$.

N/A or Pressure (psig)

Pressure (psig)

Date

\begin{tabular}{ll}
$\square$ & Time $(\min ) \frac{0}{5}$ \\
\hline Initial & Time $(\min )$
\end{tabular}


Drum No. 0961 177.8

\section{Post-test Assessment}

Position: original: tipped: toppled

Lid on drum: $\quad \quad \quad \quad$ Yes _ No

Gasket missing (cm) $027 "$ compussad thand

Contents expelled: $\quad$ Yes $\perp$ No

Seal failure: $\quad$ Yes $\quad X$ Not visible

Burn pattern: NoNe

\begin{tabular}{llll} 
Photo taken: & \multicolumn{1}{c}{ Yes $\not$ No } & \\
Leak tested: & $\begin{array}{l}\text { N/A or Pressure (psig) } \\
\text { Pressure (psig) } \\
\text { Date }\end{array}$ & Time (min) & 0 \\
\hline
\end{tabular}

Interior assessment:

Interior assessment:
plastic melted to wall $\sim 20 "$, below bolt
No chor

Comments: 


\section{Palletized Storage Drum Data Sheet}

Drum No. 9.971

Description (Check):

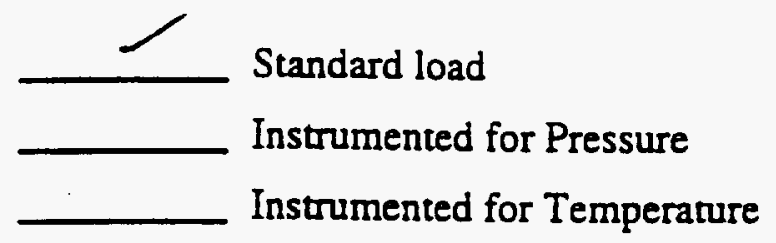

\begin{tabular}{|c|c|c|c|}
\hline Loading Starting at Bottom & Layer 1 & Layer 2 & Layer 3 \\
\hline 3 pieces of steel & & N/A & N/A \\
\hline \multicolumn{4}{|l|}{$1 / 3$ of the brown paper } \\
\hline \multicolumn{4}{|l|}{$1 / 3$ of the 10 -mil plastic } \\
\hline \multicolumn{4}{|l|}{$1 / 3$ of the bond paper } \\
\hline \multicolumn{4}{|l|}{$1 / 3$ of the towels } \\
\hline \multicolumn{4}{|l|}{$1 / 3$ of the bags } \\
\hline \multicolumn{4}{|l|}{$1 / 3$ of the cut paper } \\
\hline \multicolumn{4}{|l|}{$1 / 3$ of the fabric } \\
\hline \multicolumn{4}{|l|}{$1 / 3$ of the rubber } \\
\hline & Go to Layer 2 & Go to Layer 3 & \\
\hline
\end{tabular}

Drum Filled:

Ring bolt placed opposite drum seam and in down position

Lid sealed and torqued to $40 \mathrm{ft} / \mathrm{lb}$ :

Leak tested:

Pre-test weight (lb)

Post-test weight (lb)
Date $5-17-95$ Initial P.\&.

Date $5-17-95$ Initial 9.8 .
N/A or Pressure (psig)

Pressure (psig)

Date

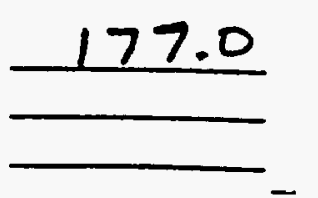

Time (min) 0

Time (min) 5

Initial

Target weight $-175.6 \mathrm{lb}$ 
Drum No. $0971 \quad 177.1 \mathrm{~b}$.

\section{Post-test Assessment}

Position:

originai:

tipped:

toppled

Lid on drum: $\quad \quad \quad X$ Yes No

Gasket missing (cm) $O$ coimplessed $\&$ hand all ancund

Contents expelled: __ Yes $x$ No

Seal failure: $\quad$ Y $\quad$ Yes $\$ Not visible

Burn pattern:

None

Photo taken:

Yes $X$ No

Leak tested:

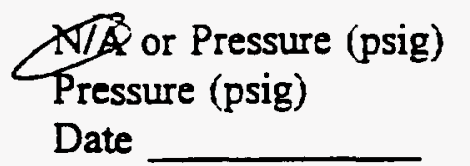

\begin{tabular}{lll} 
& Time (min) & 0 \\
\hline Initial & Time (min)
\end{tabular}

Interior assessment:

no meter

$\checkmark$ ideec $5 \%$

Photo taken:

$\not$ Yes No

Comments: 
WHC-SD-WM-TRP-246 REV. 0

Drum No. 0981

\author{
Pre-test Data Sheet Missing
}

Loading was videotaped, and pre-test weight was $176.3 \mathrm{lb}$. 
Drum No. $0981-177 .+16$.

Post-test Assessment

Position: __ original ___ _tipped: __ toppled Lid on drum: $\quad \quad \quad \quad X$ Yes No

Gasket missing (cm) O like vew

Contents expelled: _ Yes $x$ No

Seal failure:

Yes $\quad$ Not visible

Burn pattern:

NONe

Photo taken:

Yes $x$ No

Leak tested:

N/A) or Pressure (psig)

Pressure (psig)

Date 


\section{Palletized Storage Drum Data Sheet}

Drum No. 0.412

Descripion (Check):

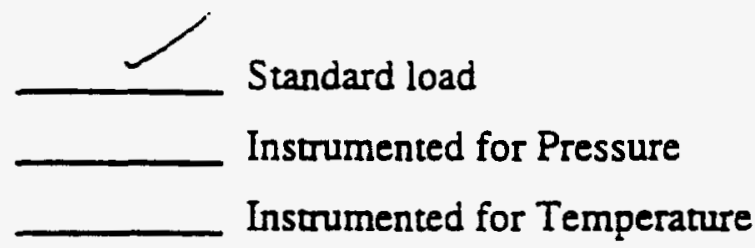

\begin{tabular}{|c|c|c|c|}
\hline Loading Starting at Bottom & Layer 1 & Layer 2 & Layer 3 \\
\hline 3 pieces of steel & 2 & N/A & N/A \\
\hline \multicolumn{4}{|l|}{$1 / 3$ of the brown paper } \\
\hline $1 / 3$ of the 10 -mil plastic & $\checkmark$ & - & \\
\hline \multicolumn{4}{|l|}{$1 / 3$ of the bond paper } \\
\hline \multicolumn{4}{|l|}{$1 / 3$ of the towels } \\
\hline \multicolumn{4}{|l|}{$1 / 3$ of the bags } \\
\hline \multicolumn{4}{|l|}{$1 / 3$ of the cut paper } \\
\hline \multicolumn{4}{|l|}{$1 / 3$ of the fabric } \\
\hline \multicolumn{4}{|l|}{$1 / 3$ of the rubber } \\
\hline & Go to Layer 2 & Go to Layer 3 & \\
\hline
\end{tabular}

Drum Filled:

Ring bolt placed opposite drum seam and in down position

Lid sealed and torqued to $40 \mathrm{ft} / \mathrm{b}$ :

Leak tested:

Pre-test weight (lb)

Post-test weight (lb)
Date $5-15-95$ Initial AP

Date $5-15-95$ Initial AR

Date $5-15-95$ Initial $\not 4$
N/A or Pressure (psig)

Pressure (psig)

Date

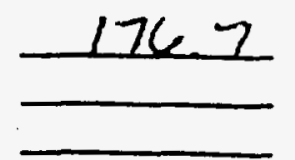

Time (min) 0

Time (min) 5
Initial

Target weight $-175.6 \mathrm{lb}$

\section{Loss =}


WHC-SD-WM-TRP-246 REV. O

$P F(L 2,-i(z)$

Reoistome Drum Data Sheet

Drum No. $04,2 \quad 176.61 b$.

Post-test Assessment

Position: __ original: ___ tipped: __

Lid on drum: $\quad x$ Yes No

Gasket missing (cm) O compressed = hand all anound

Contents expelled: __ Yes $\underline{x}$ No

Seal failure: $\quad$ Yes $\quad x$ Not visible

Burn pattern:

NoNe

Photo taken: $\quad$ Yes $x$ No

Leak tested: $\quad$ NTA or Pressure (psig)

Pressure (psig)

Date

\begin{tabular}{lll} 
Time (min) & 0 \\
\hline Initial & Time (min)
\end{tabular}

Interior assessment:

no chan, welting

VIDee 23

Ptoto taken:

$\subsetneq$ Yes

Comments: 
WHC-SD-WM-TRP-246 REV. 0

\section{Palletized Storage Drum Data Sheet}

Drum No. 422

Descriprion (Check):

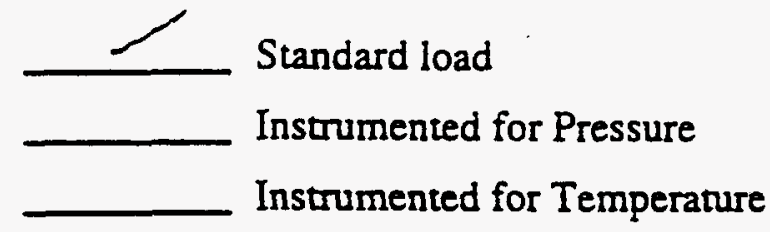

\begin{tabular}{|c|c|c|c|}
\hline Loading Starting at Bottom & Layer 1 & Layer 2 & Layer 3 \\
\hline 3 pieces of steel & N/A & \\
\hline $1 / 3$ of the brown paper & & & \\
\hline $1 / 3$ of the 10 -mil plastic & & & \\
\hline $1 / 3$ of the bond paper & & & \\
\hline $1 / 3$ of the towels & & & \\
\hline $1 / 3$ of the bags & & & \\
\hline $1 / 3$ of the cut paper & & \\
\hline $1 / 3$ of the fabric & & Go to Layer 3 & \\
\hline $1 / 3$ of the rubber & Go to Layer 2 & G \\
\hline
\end{tabular}

Drum Filled:

Ring bolt placed opposite drum seam and in down position

Lid sealed and torqued to $40 \mathrm{ft} / \mathrm{b}$ :

Leak tested:

Pre-test weight (lb)

Post-test weight (lb)

\section{Loss $=$}

Date $5-15-95$ Initial 48

Date $5-15-95 \quad$ Initial $M P$

Date $S=-15-45$ Initial AP

N/A or Pressure (psig)

Pressure (psig)

Date

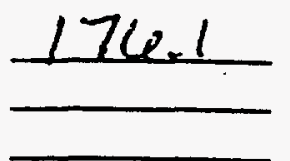

F-100
Time (min) 0

Time (min) 5

Initial

Target weight $-175.6 \mathrm{lb}$ 
Drum No. 0422175.9 lb.

\section{Post-test Assessment}

Position: original: tipped: toppied

Lid on drum: $\quad x$ Yes No

Gasket missing (cm) $13^{\prime \prime}$

Contents expelled: __ Yes \o No

Seal failure: $\quad$ Yes $\quad \times$ Not visible

Burn pattern: at rim 15" pt of sean - small spot otharuise nove

Photo taken: Yes $\simeq$ No

Leak tested:

XT/A or Pressure (psig) Pressure (psig)

Date

\begin{tabular}{lll} 
& Time (min) & 0 \\
\hline Initial & Time (min)
\end{tabular}

Interior assessment:

¿2" chan centend it barm

Photo taken:

$x$ Yes

Comments: 
WHC-SD-WM-TRP-246 REV. 0

\section{Palletized Storage Drum Data Sheet}

Drum No. 0432

Description (Check):

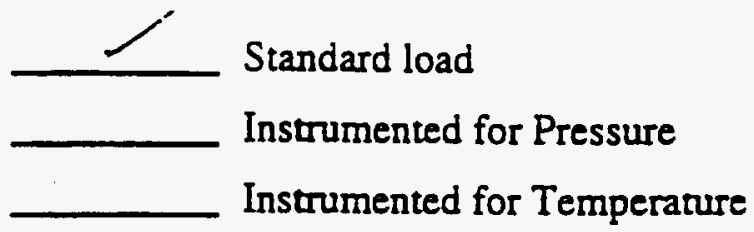

\begin{tabular}{|c|c|c|c|}
\hline Loading Starting at Bottom & Layer 1 & Layer 2 & Layer 3 \\
\hline 3 pieces of steel & & N/A \\
\hline $1 / 3$ of the brown paper & & & \\
\hline $1 / 3$ of the 10 -mil plastic & & & \\
\hline $1 / 3$ of the bond paper & & & \\
\hline $1 / 3$ of the towels & & & \\
\hline $1 / 3$ of the bags & & & \\
\hline $1 / 3$ of the cut paper & & & \\
\hline $1 / 3$ of the fabric & Go to Layer 2 & Go to Layer 3 & \\
\hline $1 / 3$ of the rubber & & \\
\hline
\end{tabular}

Drum Filled:

Date $5-15-55$ Initial $A P$

Ring boit placed opposite drum seam Date $5-15-95$ Initial AR and in down position

Lid sealed and torqued to $40 \mathrm{ft} / \mathrm{b}: \quad$ Date $5-15-95$ Initial AP

Leak tested:

Pre-test weight (lb)

Post-test weight (lb)
N/A or Pressure (psig)

Pressure (psig)

Date

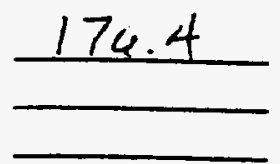

Time (min) 0

Time (min) 5

Initial

Target weight $-175.6 \mathrm{lb}$ 
Drum No. 0432,76.S K.

Post-test Assessment

Position: ___ original: ___ tipped: ___ toppled

Lid on drum: $\quad$ $\quad x$ Yes $\quad$ No

Gasket missing (cm) $0 \sim 12^{\prime \prime}$ darh bucwn

Contents expelled: _u Yes $x$ No

Seal failure: $\quad$ Yes $\quad x$ Not visible

Burn pattern:

No.ve

Photo taken:

Yes $x$ No

Leak tested:

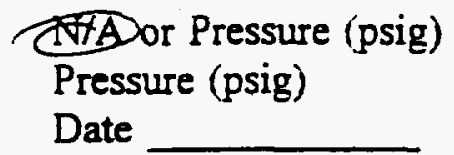

$-\quad$\begin{tabular}{ll} 
Time $(\min )$ & 0 \\
\hline
\end{tabular}$\quad$ Time $(\min ) \quad$

Date

Initial

Interior assessment:

I" melting \& surfore charring. 15"span about the bott

vioec:06

Photo taken:

$x$ Yes

No

Comments: 


\section{Palletized Storage Drum Data Sheet}

Drim No. 0442

Description (Check):

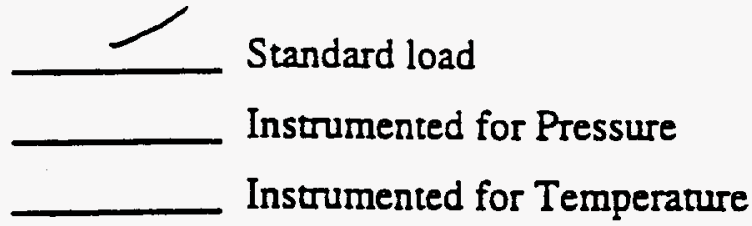

\begin{tabular}{|c|c|c|c|}
\hline Loading Starting at Bottom & Layer 1 & Layer 2 & Layer 3 \\
\hline 3 pie ees of steel & & & N/A \\
\hline $1 / 3$ of the brown paper & & & \\
\hline $1 / 3$ of the 10 -mil plastic & & & \\
\hline $1 / 3$ of the bond paper & & & \\
\hline $1 / 3$ of the towels & & & \\
\hline $1 / 3$ of the bags & & & \\
\hline $1 / 3$ of the cut paper & Go to Layer 2 & Go to Layer 3 & \\
\hline $1 / 3$ of the fabric & & & \\
\hline $1 / 3$ of the rubber & & \\
\hline
\end{tabular}

Drum Filled:

Ring bolt placed opposite drum seam and in down position

Lid sealed and torqued to $40 \mathrm{ft} / \mathrm{b}$ :

Leak tested:

Pre-test weight (lb)

Post-test weight (lb)

$$
\text { Loss = }
$$

Date $5-15-25$ Initial 128

Date 5-15-95 Initial AP

Date $5-15-8.5$ Initial MS

N/A or Pressure (psig)

Pressure (psig)

Date

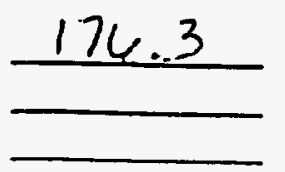

F-104
Time (min)

Time $(\min )$
Initial

Target weight $\sim 175.6 \mathrm{lb}$ 
Drum No. $0442,76=13$.

Post-test Assessment

Position: original: tipped: toppled

Lid on drum: $\quad \quad \quad \quad$ Yes $\quad$ No

Gasket missing (cm) $\mathcal{O} 46 "$ co.uprissed thand"

Contents expelled: __ Yes $x$ No

Seal failure: $\quad$ Yes $\quad x$ Not visible

Burn pattern:

wone

Photo taken: $\quad$ Yes $\quad$ No

Leak tested: $\quad$ NTIA or Pressure (psig)

Pressure (psig)

Date

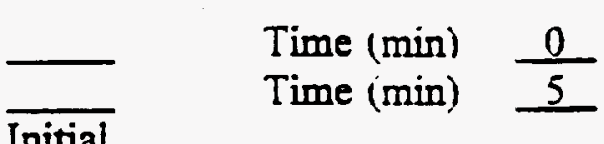

$\overline{\text { Initial }}$

Interior assessment:

n" dep melting at sean $\sim 12 "$ spon

videcics

Photo taken:

$\times$ Yes _ No

Comments: 


\section{Palletized Storage Drum Data Sheet}

Drum No. 0452

Descriprion (Check):

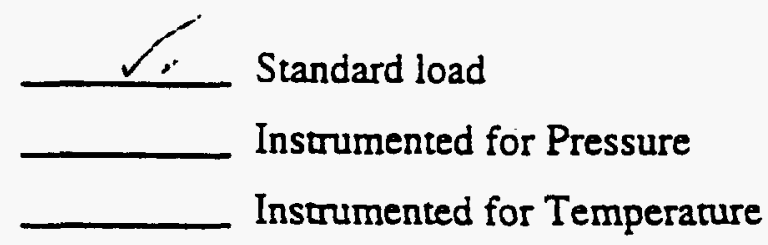

\begin{tabular}{|c|c|c|c|}
\hline Loading Starting at Bottom & Layer 1 & Layer 2 & Layer 3 \\
\hline 3 pieces of steel & & N/A & \\
\hline $1 / 3$ of the brown paper & & & \\
\hline $1 / 3$ of the 10-mil plastic & & & \\
\hline $1 / 3$ of the bond paper & & & \\
\hline $1 / 3$ of the towels & & & \\
\hline $1 / 3$ of the bags & & & \\
\hline $1 / 3$ of the cut paper & & Go to Layer 3 & \\
\hline $1 / 3$ of the fabric & Go to Layer 2 & G & \\
\hline $1 / 3$ of the rubber & &
\end{tabular}

Drum Filled:

Ring bolt placed opposite drum seam and in down position

Lid sealed and torqued to $40 \mathrm{ft} / \mathrm{b}$ :

Leak tested:

Pre-test weight (lb)

Post-test weight (lb)

Loss $=$
Date 5-15-95 Initial Af

Date $5-15-95$ Initial $4 \Omega$
N/A or Pressure (psig)

Pressure (psig)

Date

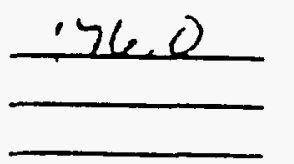

F-106
Time (min) 0

Time (min) 5

Initial

Target weight $-175.6 \mathrm{lb}$ 
Drum No. $+0452 \quad 175.9$

Post-test Assessment

Position: original: tipped: toppled

Lid on drum:

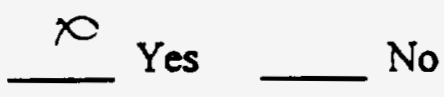

Gasket missing (cm) 0 shghtty compuessed

Contents expeiled: __ Yes $\not \subset$ No

Seal faiiure: $\quad$ Yes $\quad x$ Not visible

Burn pattern: NONT

Photo taken:

Yes $\Longrightarrow$ No

Leak tested:

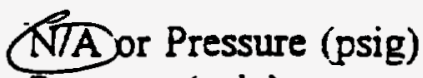

Pressure (psig)

Date

- Time (min) $\quad \frac{0}{5}$

Interior assessment:

Nochan

Line in

Rhoto taken:

$x$ Yes

Comments: 
WHC-SD-WM-TRP-246 REV. O

\section{Palletized Storage Drum Data Sheet}

Drum No. 0462

Description (Check):

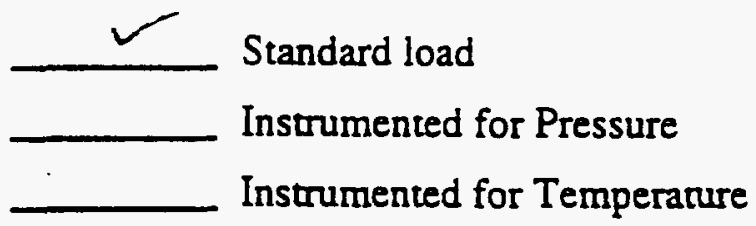

\begin{tabular}{|c|c|c|c|}
\hline Loading Starting at Bottom & Layer 1 & Layer 2 & Layer 3 \\
\hline 3 pieces of steel & 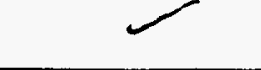 & N/A & N/A \\
\hline $1 / 3$ of the brown paper & & $\checkmark$ & \\
\hline $1 / 3$ of the 10 -mil plastic & $\checkmark$ & & \\
\hline \multicolumn{4}{|l|}{$1 / 3$ of the bond paper } \\
\hline \multicolumn{4}{|l|}{$1 / 3$ of the towels } \\
\hline \multicolumn{4}{|l|}{$1 / 3$ of the bags } \\
\hline $1 / 3$ of the cut paper & & & $\checkmark$ \\
\hline $1 / 3$ of the fabric & 工 & $\nearrow$ & - \\
\hline \multicolumn{4}{|l|}{$1 / 3$ of the rubber } \\
\hline & Go to Layer 2 & Go to Layer 3 & \\
\hline
\end{tabular}

Drum Filled:

Ring bolt placed opposite drum seam and in down position

Lid sealed and torqued to $40 \mathrm{ft} / \mathrm{b}$ :

Leak tested:

Pre-test weight (lb)

Post-test weight (lb)

Loss $=$
Date 5-15-25 Initial Af

Date 5-15-95 Initial Af

Date 5-15-95 Initial AP
N/A or Pressure (psig)

Pressure (psig)

Date

176.3

F-108
Time (min) 0

Time (min) 5
Initial

Target weight $\sim 175.6 \mathrm{lb}$ 
Drum No. $0462,76.2 \mathrm{th}$

\section{Post-test Assessment}

Position: ___ original: ___ tipped: ___ toppled

Lid on drum: $\quad \quad \quad X$ Yes $\quad$ No

Gasket missing (cm) 0 sightly eompressed

Contents expelled: _ Yes $x$ No

Seal failure: $\quad$ Yes $X$ Not visible

Burn pattern:

none

Photo taken: $\quad$ Yes $x$ No

Leak tested:

Pressure (psig)

Date

$\begin{array}{ll}\text { Time (min) } & 0 \\ \text { Time (min) } & 5\end{array}$

Initial

Interior assessment:

no wetting

No char

u. Deo 118

Photo raken:

$\underline{x}$ Yes

Comments: 


\section{Palletized Storage Drum Data Sheet}

Drum No. $\mathrm{C} \times \mathrm{i}>2$

Description (Check):

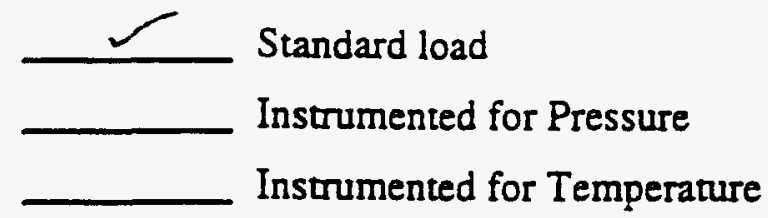

\begin{tabular}{|c|c|c|c|}
\hline Loading Starting at Bottom & Layer 1 & Layer 2 & Layer 3 \\
\hline 3 pieces of steel & & N/A & N/A \\
\hline $1 / 3$ of the brown paper & & & \\
\hline $1 / 3$ of the 10-mil plastic & & & \\
\hline $1 / 3$ of the bond paper & & & \\
\hline $1 / 3$ of the toweis & & & \\
\hline $1 / 3$ of the bags & & & \\
\hline $1 / 3$ of the cut paper & & Go to Layer 3 & \\
\hline $1 / 3$ of the fabric & Go to Layer 2 & G \\
\hline $1 / 3$ of the rubber & & \\
\hline
\end{tabular}

Drum Filled:

Date $5-15-8 s^{-}$Initial $1 \mathrm{~h}^{\mathrm{D}}$

Ring bolt placed opposite drum seam Date $5-15-95$ Initial $4 f$ and in down position

Lid sealed and torqued to $40 \mathrm{ft} / \mathrm{lb}$ : Date $\frac{5-15-5.5}{\text { Initial }}$ AP

Leak tested:

Pre-test weight (lb)

Post-test weight (lb)
N/A or Pressure (psig) Pressure (psig) Date

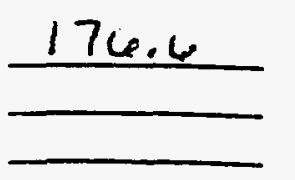

Time (min) 0 Time $(\min ) 5$

Initial

Target weight $\sim 175.6 \mathrm{lb}$ 
Drum No. $\quad 0472+76.6 \mathrm{~kb}$.

\section{Post-test Assessment}

Position: ___ originai: ___ tipped: ___ toppled

Lid on drum: $\quad x$ Yes No

Gasket missing (cm) 0

Contents expelled: __ Yes $x$ No

Seal failure: $\quad$ Yes $x$ Not visible

Burn pattern:

None

Photo taken: Yes $x$ No

Leak tested:

N/A or Pressure (psig)

Pressure (psig)

Date

\begin{tabular}{lll}
- & Time $(\min )$ & 0 \\
\hline & Time $(\min )$
\end{tabular}

Interior assessment:

$\frac{\text { no chas }}{\text { no mettur }}$

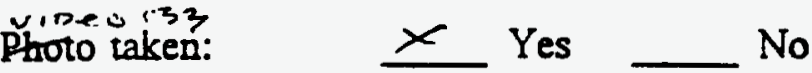

Comments: 


\section{Palletized Storage Drum Data Sheet}

Drum No. 0482

Description (Check):

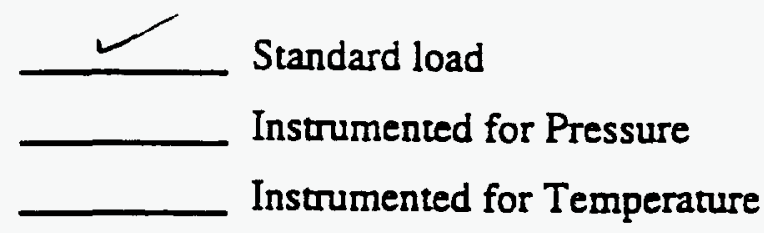

\begin{tabular}{|c|c|c|c|}
\hline Loading Starting at Bottom & Layer 1 & Layer 2 & Layer 3 \\
\hline 3 pieces of steel & & N/A & N/A \\
\hline \multicolumn{4}{|l|}{$1 / 3$ of the brown paper } \\
\hline \multicolumn{4}{|l|}{$1 / 3$ of the 10 -mil plastic } \\
\hline \multicolumn{4}{|l|}{ 1/3 of the bond paper } \\
\hline \multicolumn{4}{|l|}{$1 / 3$ of the towels } \\
\hline \multicolumn{4}{|l|}{$1 / 3$ of the bags } \\
\hline \multicolumn{4}{|l|}{$1 / 3$ of the cut paper } \\
\hline \multicolumn{4}{|l|}{$1 / 3$ of the fabric } \\
\hline \multicolumn{4}{|l|}{$1 / 3$ of the rubber } \\
\hline & Go to Layer 2 & Go to Layer 3 & \\
\hline
\end{tabular}

Drum Filled:

Ring bolt placed opposite drum seam and in down position

Lid sealed and torqued to $40 \mathrm{ft} / \mathrm{b}$ :

Leak tested:

Pre-test weight (lb)

Post-test weight (lb)

$$
\text { Loss = }
$$

Date $5-15-55$ Initial $A P$

Date $5-15-95$ Initial AP

N/A or Pressure (psig)

Pressure (psig)

Date

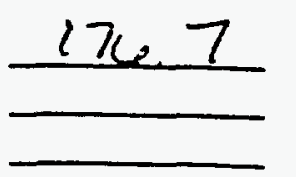

F-112
Time (min) 0

Time $(\min ) 5$

Initial

Target weight $\sim 175.6 \mathrm{lb}$

-




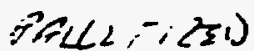 \\ Rael Sterage Drum Data Sheet}

Drum No. $0482 \quad 176.61 \mathrm{~b}$.

Post-test Assessment

Position: ___ original: ___ tipped: ___ toppled

Lid on drum: $\quad X$ Yes $\quad$ No

Gasket missing (cm) $O$ slig htte compressed

Contents expelled: $\quad$ Yes $\quad x$ No

Seal failure: $\quad$ Y Yes $\not$ Not visible

Burn pattern:

None

Photo taken:

Yes No

Leak tested:

(TA or Pressure (psig)

Pressure (psig)

Date

$+\quad \begin{array}{ll}\text { Time }(\min ) & 0 \\ - & \text { Time }(\min )\end{array}$

Initial

Interior assessment:

No shar

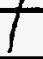

idec is 4

$\frac{\not 0}{:}$ Yes __ No

Comments: 
WHC-SD-WM-TRP-246 REV. 0

\section{Palletized Storage Drum Data Sheet}

Drum No. $05 / 2$

Descripion (Check):

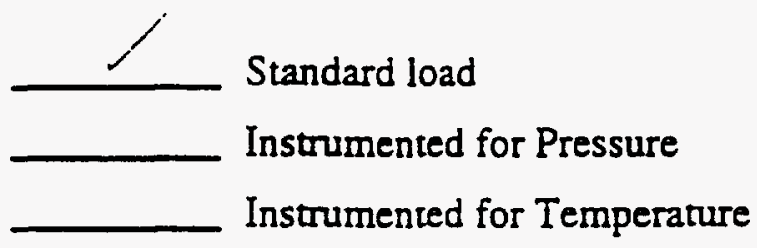

\begin{tabular}{|c|c|c|c|}
\hline Loading Starting at Bottom & Layer 1 & Layer 2 & Layer 3 \\
\hline 3 pieces of steel & & & \\
\hline $1 / 3$ of the brown paper & & & \\
\hline $1 / 3$ of the 10 -mil plastic & & & \\
\hline $1 / 3$ of the bond paper & & & \\
\hline $1 / 3$ of the towels & & & \\
\hline $1 / 3$ of the bags & & & \\
\hline $1 / 3$ of the cut paper & Go to Layer 2 & Go to Layer 3 & \\
\hline $1 / 3$ of the fabric & & \\
\hline $1 / 3$ of the rubber & & \\
\hline
\end{tabular}

Drum Filled:

Ring bolt placed opposite drum seam and in down position

Lid sealed and torqued to $40 \mathrm{ft} / \mathrm{b}$ :

Leak tested:

Pre-test weight (lb)

Post-test weight (lb)

$$
\text { Loss = }
$$

Date 5.12 .95 Initial R.K

Date 5.12 .95 Initial L.K

Date 5.12 .95 Initial R.K

N/A or Pressure (psig)

Pressure (psig)

Date

\begin{tabular}{ll} 
Time (min) & 0 \\
\hline & Time $(\min )$
\end{tabular}

Initial

Target weight $\sim 175.6 \mathrm{lb}$ 


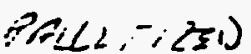

\section{Peotrestesage Drum Data Sheet}

Drum No. $0512+767.016$.

\section{Post-test Assessment}

Position:

original:

tipped:

toppled

Lid on drum: $\quad X$ Yes No

Gasket missing (cm) 22

Contents expeiled:

Yes $x$ No

Seal failure: $\quad$ Yes $\quad X$ Not visible

Burn pattern:

13" at mid lit

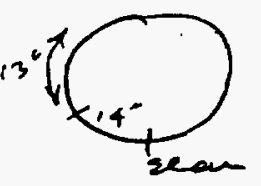

Photo taken:

\pm Yes $\leq$ No

Leak tested:

VIA or Pressure (psig)

Pressure (psig)

Date

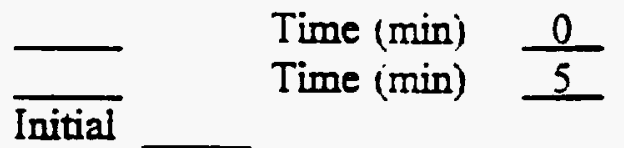

Interior assessment:

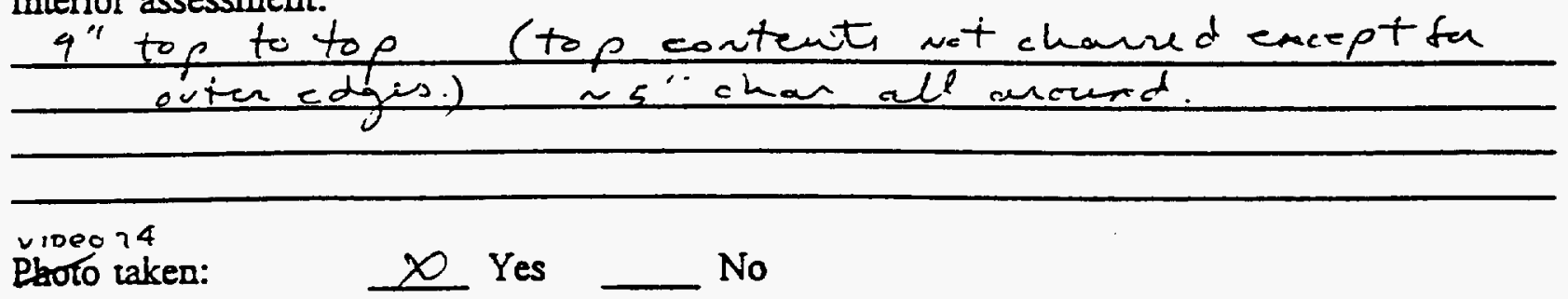

\section{Comments:}


WHC-SD-WM-TRP-246 REV. 0

\section{Palletized Storage Drum Data Sheet}

Drmino. 0522

Description (Check):

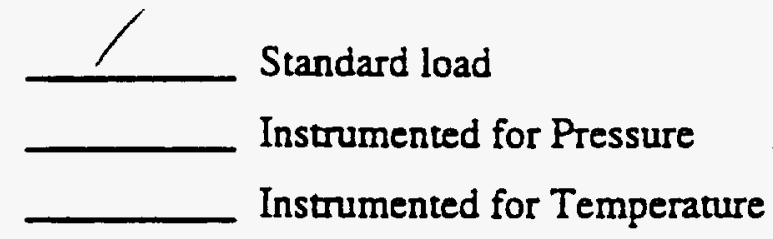

\begin{tabular}{|c|c|c|c|}
\hline Loading Starting at Bottom & Layer 1 & Layer 2 & Layer 3 \\
\hline 3 pieces of steel & & & \\
\hline $1 / 3$ of the brown paper & & & \\
\hline $1 / 3$ of the 10 -mil plastic & & & \\
\hline $1 / 3$ of the bond paper & & & \\
\hline $1 / 3$ of the towels & & & \\
\hline $1 / 3$ of the bags & & & \\
\hline $1 / 3$ of the cut paper & & & \\
\hline $1 / 3$ of the fabric & Go to Layer 2 & Go to Layer 3 & \\
\hline $1 / 3$ of the rubber & & \\
\hline \hline
\end{tabular}

Drum Filled:

Ring bolt placed opposite drum seam and in down position

Lid sealed and torqued to $40 \mathrm{ft} / \mathrm{b}$ :

Leak tested:

Pre-test weight (lb)

Post-test weight (lb)

$$
\text { Loss = }
$$

Date $5 \cdot 12 \cdot 95$ Initial

Date 5.12.95 Initial L.K

Date $5.12 .95 \quad$ Initial R.K
N/A or Pressure (psig)

Pressure (psig)

Date

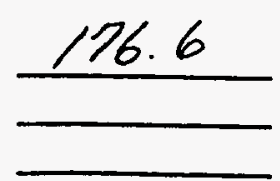

F-116
Time (min) 0 Time (min) 5
Initial

Target weight $\sim 175.6 \mathrm{lb}$ 
Drum No. $0522,73.9 \mathrm{~b}$.

\section{Post-test Assessment}

Position: original: tipped: toppled

Lid on drum: $\quad x$ Yes N_ No top rbottom, ligitty bulged

Gasket missing (cm) al

Contents expeiled: _ $\quad$ Yes $\underline{X}$ No

Seal failure: $\quad$ _ $\quad$ Yes $\quad X$ Not visible

Bum pattern:

$$
\text { -16" at mid led. }
$$

Photo taken:

$$
\text { - Yes } x \text { No }
$$

Leak tested:

\section{TIA or Pressure (psig)} Pressure (psig)

Date

\begin{tabular}{lll} 
& Time (min) & 0 \\
\hline Initial & Time (min)
\end{tabular}

Interior assessment:

$12 "$ top to top fehar

visen 76

Pboto taken:

$x$ Yes

Comments: 
WHC-SD-WM-TRP-246 REV. 0

\section{Palletized Storage Drum Data Sheet}

Drum No. 0532

Description (Check):

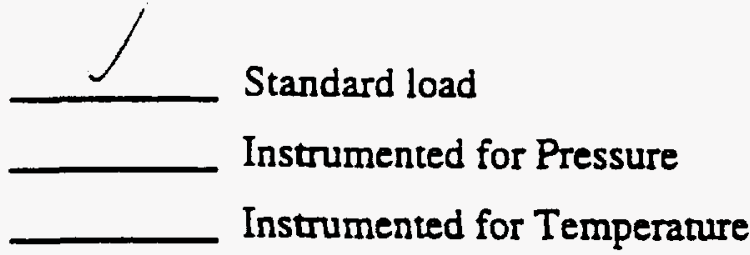

\begin{tabular}{|c|c|c|c|}
\hline Loading Staring at Bottom & Layer 1 & Layer 2 & Layer 3 \\
\hline 3 pieces of steel & & N/A & \\
\hline $1 / 3$ of the brown paper & & & \\
\hline $1 / 3$ of the 10-mil plastic & & & \\
\hline $1 / 3$ of the bond paper & & & \\
\hline $1 / 3$ of the towels & & & \\
\hline $1 / 3$ of the bags & & & \\
\hline $1 / 3$ of the cur paper & Go to Layer 2 & Go to Layer 3 & \\
\hline $1 / 3$ of the fabric & & & \\
\hline $1 / 3$ of the rubber & & \\
\hline
\end{tabular}

Drum Filled:

Ring boit placed opposite drum seam and in down position

Lid sealed and torqued to $40 \mathrm{ft} / \mathrm{b}$ :

Leak tested:

Pre-test weight (lb)

Post-test weight (lb)

$$
\text { Loss = }
$$

Date $5=12.95$ Initial L.K

Date 5.12.95 Initial L.K

Date 5.12.95 Initial R.K

N/A or Pressure (psig)

Pressure (psig)

Date

Initial

Time (min) 0

Time $(\min )$

Target weight $\sim 175.6 \mathrm{lb}$ 
Drum No. $0532(P) 173.6 \mathrm{~K}$.

Post-test Assessment

Position:

original:

tipped: toppled

Lid on drum: $\quad x$ Yes __ No top bulged

Gasket missing (cm) all

Contents expelled: $\quad$ Yes $x$ No

Seal failure:

Yes $x$ Not visible be Thom : lightty bulged

Burn pattern:

22 at mid wt. seam to left

Photo taken:

Yes $\underset{\text { No }}{ }$

Leak tested:

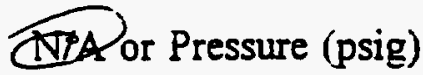

Pressure (psig)

Date

Initial

Time (min) $\quad 0$ Time (min)

Interior assessment:

15 " top to top

ن̈:oec :98

photo taken:

$\Upsilon_{\text {Yes }}$

No

Comments: 


\section{Palletized Storage Drum Data Sheet}

Drim No. 0542

Description (Check):

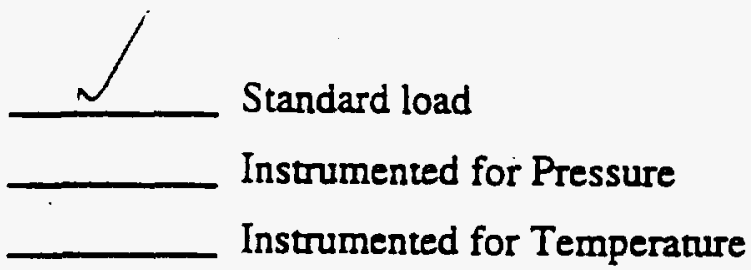

\begin{tabular}{|c|c|c|c|}
\hline Loading Starting at Bottom & Layer 1 & Layer 2 & Layer 3 \\
\hline 3 pieces of steel & - & N/A & N/A \\
\hline $1 / 3$ of the brown paper & $\gamma$ & 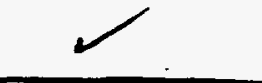 & $r$ \\
\hline $1 / 3$ of the 10 -mil plastic & $r$ & 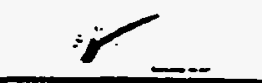 & 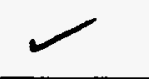 \\
\hline $1 / 3$ of the bond paper & $r$ & 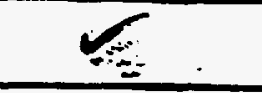 & \\
\hline $1 / 3$ of the towels & 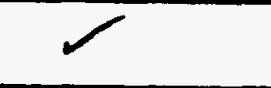 & $r$ & $\gamma$ \\
\hline $1 / 3$ of the bags & $r$ & 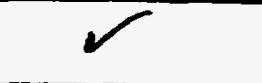 & \\
\hline $1 / 3$ of the cut paper & $r$ & $r$ & \\
\hline $1 / 3$ of the fabric & レ & $r$ & \\
\hline $1 / 3$ of the rubber & $r$ & l & \\
\hline & Go to Layer 2 & Go to Layer 3 & \\
\hline
\end{tabular}

Drum Filled:

Ring bolt placed opposite drum seam and in down position

Lid sealed and torqued to $40 \mathrm{ft} / \mathrm{lb}$ :

Leak tested:

Pre-test weight (lb)

Post-test weight (lb)

$$
\text { Loss = }
$$

Date 5-12.95 Initial L.K

Date 5-12.95 Initial L.K
N/A or Pressure (psig)

Pressure (psig)

Date

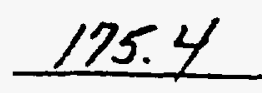

175.4

Initial

Target weight $-175.6 \mathrm{lb}$ 
Drum No. 0542 i73.6 ih.

Post-test Assessment

Position:

original: tipped: toppled

Lid on drum: $\quad x$ Yes _ No

Gasket missing (cm) $61^{\prime \prime}$

Contents expelled: $\quad$ Yes $x$ No

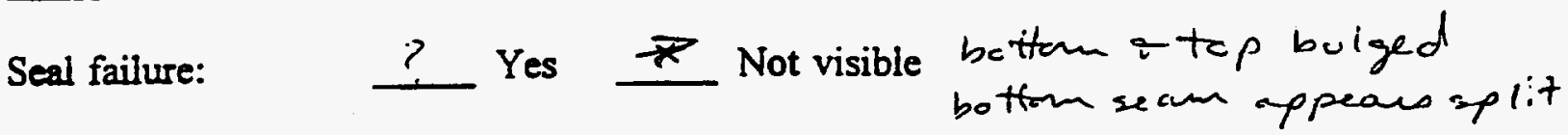

Burn pattern:

$24^{\prime \prime}$ sean $t \cdot r i g h t$

Photo taken: $\quad$ Yes $x$ No

Leak tested: $\quad$ NNAijor Pressure (psig) Pressure (psig)

Date

\begin{tabular}{ll} 
Time $(\min )$ & 0 \\
\hline & Time $(\min )$
\end{tabular}

Interior assessment:

10 " tep te tor of cha:

U: oecic7

Photo taken:

$\not \quad$ Yes No

Comments: 


\section{Palletized Storage Drum Data Sheet}

Drum No. $\underline{0552}$

Description (Check):

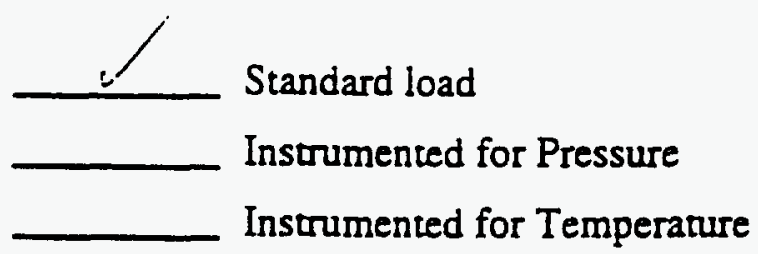

\begin{tabular}{|c|c|c|c|}
\hline Loading Starting at Bottom & Layer 1 & Layer 2 & Layer 3 \\
\hline 3 pieces of steel & & N/A \\
\hline $1 / 3$ of the brown paper & & & \\
\hline $1 / 3$ of the 10 -mil plastic & & & \\
\hline $1 / 3$ of the bond paper & & & \\
\hline $1 / 3$ of the towels & & & \\
\hline $1 / 3$ of the bags & & & \\
\hline $1 / 3$ of the cut paper & & & \\
\hline $1 / 3$ of the fabric & & Go to Layer 3 & \\
\hline $1 / 3$ of the rubber & Go to Layer 2 & G \\
\hline
\end{tabular}

Drum Filled:

Ring bolt placed opposite drum seam and in down position

Lid sealed and torqued to $40 \mathrm{ft} / \mathrm{b}$ :

Leak tested:

Pre-test weight (lb)

Post-test weight (lb)

Date $5=12.95$ Initial

Date 5.12.95 Initial l.K

Date 5-12.95

Initial L.K

N/A or Pressure (psig)

Pressure (psig)

Date

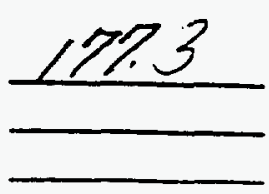

F-122
Time (min) 0

Time (min) 5

Initial

Target weight $-175.6 \mathrm{lb}$

$$
\text { Loss = }
$$




\section{$P$ Pril $(-; 21)$}

Prentage Drum Data Sheet

Drum No. $0552177.1 \mathrm{k}$.

\section{Post-test Assessment}

Position:

originai: tipped: toppied

Lid on drum: $\quad x$ Yes No

Gasket missing (cm) __ 20 "

Contents expelled: —Yes $x$ No

Seal failure: $\quad$ Yes $\longrightarrow$ Not visible

Burn partern: Burn partern: ind lot, seam to $10 \mathrm{f} T$

Photo taken: Yes $\nsim$ No

Leak tested:

Aor Pressure (psig) Pressure (psig)

Date $\begin{array}{ll}\text { Time (min) } & 0 \\ \text { Time (min) } & 5\end{array}$

$\overline{\text { Initial }}$

Interior assessment:

$\frac{5 \text { "char on burn area opposite side suffece chan }}{\text { itmettin }}$

$\frac{5 \text { "char on burn area opposte side sufore chan }}{\text { imettin }}$

U ideo: 20

Ehoto taken:

$x$ Yes No

Comments: 


\section{Palletized Storage Drum Data Sheet}

Drum No. 0562

Descriprion (Check):

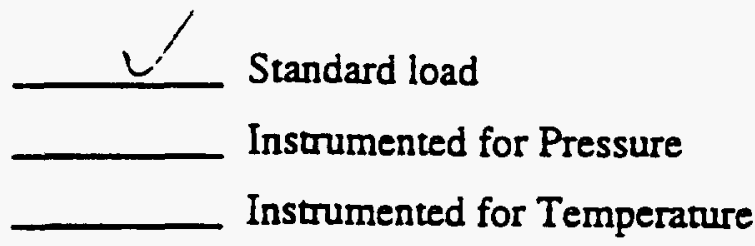

\begin{tabular}{|c|c|c|c|}
\hline Loading Starting at Bottom & Layer 1 & Layer 2 & Layer 3 \\
\hline 3 pieces of steel & $r$ & N/A & N/A \\
\hline $1 / 3$ of the brown paper & $r$ & & $r$ \\
\hline $1 / 3$ of the 10 -mil plastic & & $\angle$ & $\sim$ \\
\hline $1 / 3$ of the bond paper & 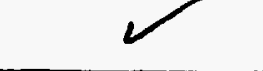 & & $\checkmark$ \\
\hline $1 / 3$ of the towels & & $\mathscr{V}$ & $\mathscr{L}$ \\
\hline $1 / 3$ of the bags & & $\nu$ & 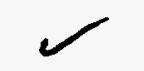 \\
\hline $1 / 3$ of the cut paper & 2 & $V$ & $\swarrow$ \\
\hline $1 / 3$ of the fabric & 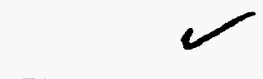 & $V$ & $r$ \\
\hline $1 / 3$ of the rubber & $\checkmark$ & $\checkmark$ & $\checkmark$ \\
\hline & Go to Layer 2 & Go to Layer 3 & \\
\hline
\end{tabular}

Drum Filled:

Ring bolt placed opposite drum seam and in down position

Lid sealed and torqued to $40 \mathrm{ft} / \mathrm{b}$ :

Leak tested:

Pre-test weight (lb)

Post-test weight (lb)

$$
\text { Loss }=
$$

Date 5.12 .95

Date $5 \cdot 12 \cdot 95$

Initial

Initial

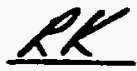

Date 5.12 .95 Initial P.K

N/A or Pressure (psig)

Pressure (psig)

Date

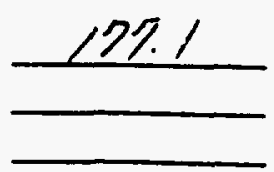

F-124
Time (min) 0

Time (min) 5
Initial

Target weight $\sim 175.6 \mathrm{lb}$ 
Drum No. 0562177.0

\section{Post-test Assessment}

Position: __ original: __ _tipped: __ toppled

Lid on drum: $\quad \quad \quad \quad$ Yes No

Gasket missing (cm) O compreased = hand all around

Contents expelled: __ Yes $X$ No

Seal failure: $\quad$ Yes $X$ Not visible

Burn pattern:

wone

Photo taken:

Leak tested:

NifA or Pressure (psig)

Pressure (psig)

Date
Yes $\varnothing$ No

$\longrightarrow \quad$\begin{tabular}{ll} 
Time (min) & 0 \\
\hline & Time (min)
\end{tabular}

Interior assessment:

"char sean to iet 28 "

$\cup . n<0.17$

Photo taken:

$x$ Yes No

Comments: 


\section{Palletized Storage Drum Data Sheet}

Drum No. 0572

Descriprion (Check):

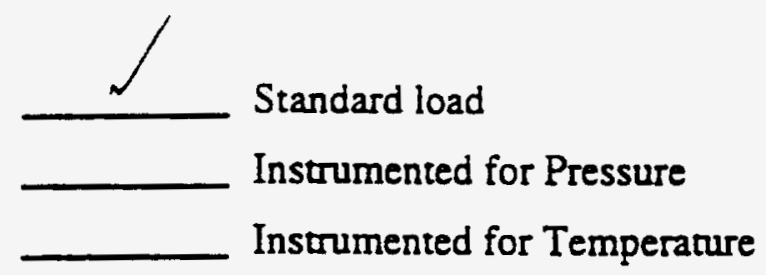

\begin{tabular}{|c|c|c|c|}
\hline Loading Starting at Bottom & Layer 1 & Layer 2 & Layer 3 \\
\hline 3 pieces of steel & $\gamma$ & N/A & N/A \\
\hline \multicolumn{4}{|l|}{$1 / 3$ of the brown paper } \\
\hline $1 / 3$ of the 10 -mil plastic & $\checkmark$ & $y=0$ & \\
\hline \multicolumn{4}{|l|}{$1 / 3$ of the bond paper } \\
\hline \multicolumn{4}{|l|}{$1 / 3$ of the toweis } \\
\hline \multicolumn{4}{|l|}{$1 / 3$ of the bags } \\
\hline \multicolumn{4}{|l|}{$1 / 3$ of the cut paper } \\
\hline $1 / 3$ of the fabric & $\checkmark$ & 2 & \\
\hline \multicolumn{4}{|l|}{$1 / 3$ of the rubber } \\
\hline & Go to Layer 2 & Go to Layer 3 & \\
\hline
\end{tabular}

Drum Filled:

Ring bolt placed opposite drum seam and in down position

Lid sealed and torqued to $40 \mathrm{ft} / \mathrm{b}$ :

Leak tested:

Pre-test weight (lb)

Post-test weight (lb)

Loss $=$

Date 5.12 .95 Initial RK

Date 5.12.95 Initial RK

Date 5.12 .95 Initial R/C

N/A or Pressure (psig)

Pressure (psig)

Date

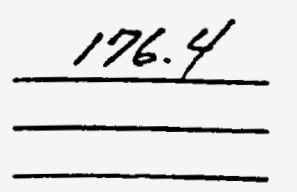

F-126
Time (min) 0

Time (min) 5

Initial

Target weight $-175.6 \mathrm{lb}$ 
Drum No. $0572 \quad 176.4 \mathrm{lb}$.

Post-test Assessment

Position: original: tipped: toppled

Lid on drum:

$$
X \text { Yes No }
$$

Gasket missing (cm) $O$ cimpressed t hand all around

Contents expelled: _ues $x$ No

Seal failure:

$$
\text { Yes } \searrow \text { Not visible }
$$

\section{Burn pattern:}

None

Photo taken:

Yes $\longleftarrow$ No

Leak tested:

Or Pressure (psig)

Pressure (psig)

Date $\overline{\text { Initial }}$
Time (min)

Time (min)

$\frac{0}{5}$

Interior assessment:

plastic melted 21" span

$\checkmark 1 D C 0136$

Photo taken:

$\underline{x}$ Yes _ No

Comments: 


\section{Palletized Storage Drum Data Sheet}

Drim No. 0582

Description (Check):

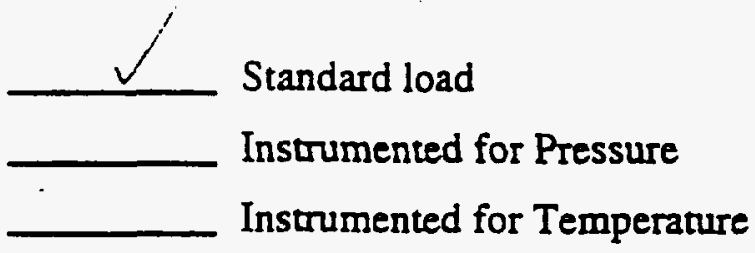

\begin{tabular}{|c|c|c|c|}
\hline Loading Starting at Bottom & Layer 1 & Layer 2 & Layer 3 \\
\hline 3 pieces of steel & & N/A \\
\hline $1 / 3$ of the brown paper & & & \\
\hline $1 / 3$ of the 10 -mil plastic & & & \\
\hline $1 / 3$ of the bond paper & & & \\
\hline $1 / 3$ of the towels & & & \\
\hline $1 / 3$ of the bags & & & \\
\hline $1 / 3$ of the cut paper & & & \\
\hline $1 / 3$ of the fabric & Go to Layer 2 & Go to Layer 3 & \\
\hline $1 / 3$ of the rubber & & & \\
\hline
\end{tabular}

Drum Filled:

Ring bolt placed opposite drum seam and in down position

Lid sealed and torqued to $40 \mathrm{ft} / \mathrm{b}$ :

Leak tested:

Pre-test weight (lb)

Post-test weight (lb)

Loss =
N/A or Pressure (psig)

Pressure (psig)

Date

Initial

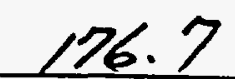

Target weight $-175.6 \mathrm{lb}$
Time (min) 0

Time (min) 5 
Drum No. $0582 \cdot 76.61 \mathrm{~b}$

Post-test Assessment

Position: originai: tipped: toppled

Lid on drum: $\quad \quad \quad \quad$ Yes $\quad$ No

Gasket missing (cm) O sliglutty compreszed

Contents expelled: $\quad$ Yes $x$ No

Seal failure: $\quad$ Yes $\longrightarrow$ Not visible

Burn partern:

Noxe

Photo taken: $\quad$ Yes XE No

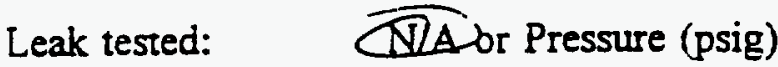
Pressure (psig)

Date

\begin{tabular}{lll}
- & Time (min) & 0 \\
\hline & Time (min)
\end{tabular}

Interior assessment:

slight metting to wall 17 " RT of zerm

V.Dec 135

Photo raken:

$\underline{X}$ Yes

Comments: 


\section{Palletized Storage Drum Data Sheet}

Drum No. $06 / 2$

Description (Check):

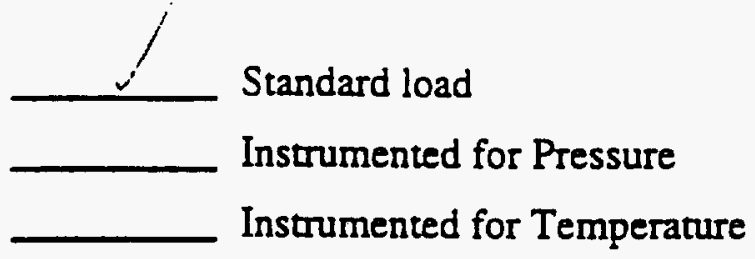

\begin{tabular}{|c|c|c|c|}
\hline Loading Starting at Bottom & Layer 1 & Layer 2 & Layer 3 \\
\hline 3 pieces of steel & & N/A & N/A \\
\hline \multicolumn{4}{|l|}{$1 / 3$ of the brown paper } \\
\hline \multicolumn{4}{|l|}{$1 / 3$ of the 10 -mil plastic } \\
\hline \multicolumn{4}{|l|}{$1 / 3$ of the bond paper } \\
\hline \multicolumn{4}{|l|}{$1 / 3$ of the toweis } \\
\hline \multicolumn{4}{|l|}{$1 / 3$ of the bags } \\
\hline \multicolumn{4}{|l|}{$1 / 3$ of the cut paper } \\
\hline \multicolumn{4}{|l|}{$1 / 3$ of the fabric } \\
\hline \multicolumn{4}{|l|}{$1 / 3$ of the nubber } \\
\hline & Go to Layer 2 & Go to Layer 3 & \\
\hline
\end{tabular}

Drum Filled:

Ring boit placed opposite drum seam and in down position

Lid sealed and torqued to $40 \mathrm{ft} / \mathrm{lb}$ :

Leak tested:

Pre-test weight (lb)

Post-test weight (lb)

$$
\text { Loss }=
$$

Date $5 \cdot 12 \cdot 95$ Initial L.R

Date $5-12.95$ Initial L.R

Date 5./2-95 Initial $R \cdot K$
N/A or Pressure (psig)

Pressure (psig)

Date

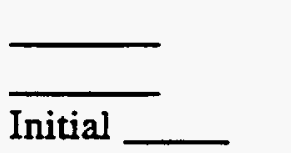

Target weight $-175.6 \mathrm{lb}$
Time (min) Time (min) 5 
WHC-SD-WM-TRP-246 REV. 0

Ifoul, - isil)

Reok- Cromage Drum Data Sheet

Drum No. $26 ! 2$, ?1.8 ib.

Post-test Assessment

Position: __ original: ___ tipped: ___ toppled

Lid on drum: $\quad X$ Yes No bulged

Gasket missing (cm) ell

Contents expelled: _u Yes $x$ No

Seal failure: $\quad$ Yes $X$ Not visible

Burn pattern:

al ancum ì

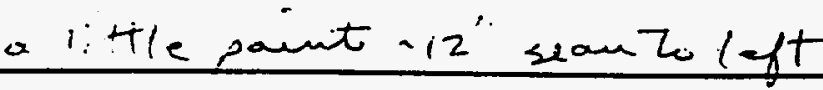

Photo taken:

Yes $\stackrel{\sim}{\longrightarrow}$ No

Leak tested:

NTS or Pressure (psig)

Pressure (psig)

Date

$\longrightarrow \quad$\begin{tabular}{lll} 
Time $(\min )$ & 0 \\
\hline & Time (min)
\end{tabular}

Interior assessment:

$18^{\prime \prime}$ top of dumm t top of chan

$V / D \leq 0 \quad \geq C$

Photo taken:

$x$ Yes

Comments: 


\section{Palletized Storage Drum Data Sheet}

Drum No. 0622

Description (Check):

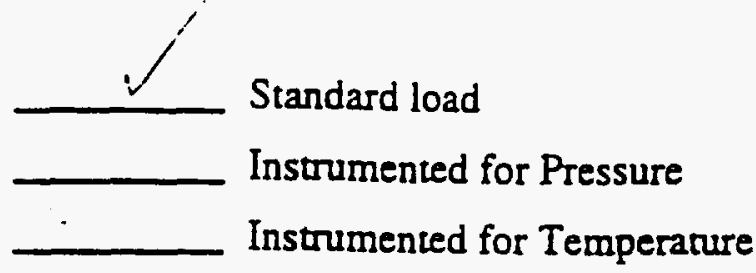

\begin{tabular}{|c|c|c|c|}
\hline Loading Starting at Bottom & Layer 1 & Layer 2 & Layer 3 \\
\hline 3 pieces of steel & & & \\
\hline $1 / 3$ of the brown paper & & & \\
\hline $1 / 3$ of the 10 -mil plastic & & & \\
\hline $1 / 3$ of the bond paper & & & \\
\hline $1 / 3$ of the towels & & & \\
\hline $1 / 3$ of the bags & & & \\
\hline $1 / 3$ of the cut paper & Go to Layer 2 & Go to Layer 3 & \\
\hline $1 / 3$ of the fabric & & \\
\hline $1 / 3$ of the rubber & & \\
\hline
\end{tabular}

Drum Filled:

Ring bolt placed opposite drum seam and in down position

Lid sealed and torqued to $40 \mathrm{ft} / \mathrm{b}$ :

Leak tested:

Pre-test weight (lb)

Post-test weight (lb)

Date $5 . / 2.95$ Initial

Date 5./2.95 Initial R.K

Date 5-12.95 Initial R.K

N/A or Pressure (psig)

Pressure (psig)

Date

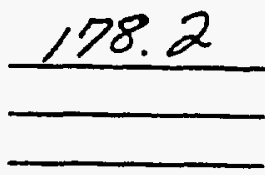

F-132
Time $(\min )$

Time (min) 5
Initial

Target weight $-175.6 \mathrm{lb}$

\section{Loss $=$}


Drum No. N.6.2? $162.5 i b$.

Post-test Assessment

Position: __ original: ___ tipped: ___ toppled

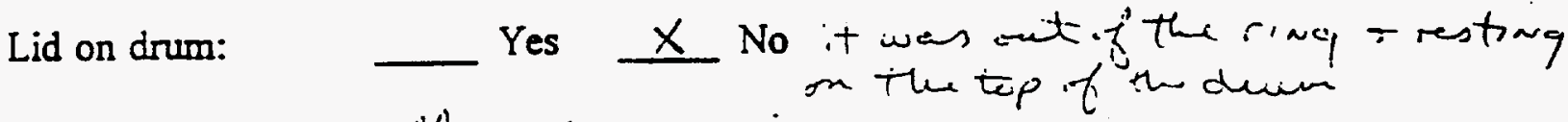

Gasket missing (cm) celf' : i/4 wus schite pouder restiun

Contents expelled: __ Yes $\underline{x}$ No

Seal failure: $\quad \quad \quad X$ Yes _ Not visible

Burm pattern:

Photo taken:

Yes $\longleftarrow$ No

Leak tested:

Ni/A or Pressure (psig)

Pressure (psig)

Date

$\longrightarrow \quad$ Time (min) $\quad \frac{0}{5}$

Interior assessment:

$20^{\prime \prime} t_{c \rho}-1$ dumm ta top of chan

iviDec is

Pboto taken:

$x$ Yes

Comments: 
WHC-SD-WM-TRP-246 REV. 0

\section{Palletized Storage Drum Data Sheet}

Drum No. 0632

Description (Check):

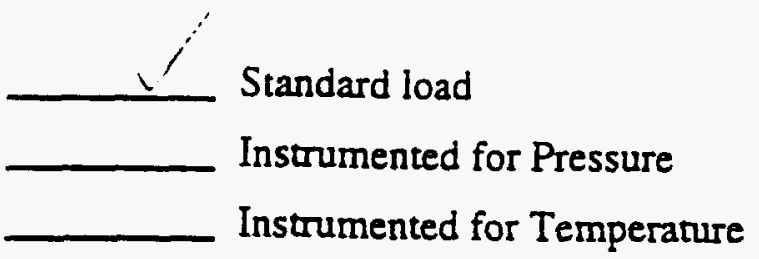

\begin{tabular}{|c|c|c|c|}
\hline Loading Starting at Bottom & Layer 1 & Layer 2 & Layer 3 \\
\hline 3 pieces of steel & & & \\
\hline $1 / 3$ of the brown paper & & & \\
\hline $1 / 3$ of the 10-mil plastic & & & \\
\hline $1 / 3$ of the bond paper & & & \\
\hline $1 / 3$ of the towels & & & \\
\hline $1 / 3$ of the bags & & & \\
\hline $1 / 3$ of the cut paper & & & \\
\hline $1 / 3$ of the fabric & & & \\
\hline $1 / 3$ of the rubber & Go to Layer 2 & Go to Layer 3 & \\
\hline
\end{tabular}

Drum Filled:

Ring bolt placed opposite drum seam and in down posicion

Lid sealed and torqued to $40 \mathrm{ft} / \mathrm{b}$ :

Leak tested:

Pre-test weight (lb)

Post-test weight (lb)

$$
\text { Loss }=
$$

Date 5-12.95 Initial LK.

Date $5=12.95$ Initial R.K
N/A or Pressure (psig)

Pressure (psig)

Date

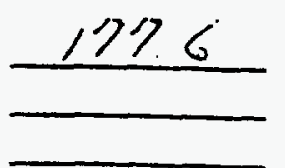

F-134
Time (min) 0

Time (min) 5 
WHC-SD-WM-TRP-246 REV. 0

PFu(L, - ; ral

Redt dierage Drum Data Sheet

Drum No. $06^{.32(i)}$

Post-test Assessment

Position: ___ original: ___ tipped: ___ toppled

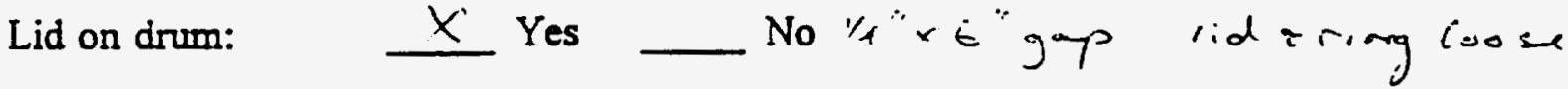

Gasket missing (cm)

Contents expelled: _ـ Yes $\quad$ Y No

Seal failure: $\quad \quad \quad$ Yes _ Not visible

Burn pattern:

all around

Photo taken:

$\downarrow$ Yes $\Perp$ No

Leak tested:

NLA or Pressure (psig)

Pressure (psig)

Date

$\longrightarrow \quad$\begin{tabular}{ll} 
Time (min) & 0 \\
\hline
\end{tabular}$\quad$ Time (min)

$\overline{\text { Initial }}$

Interior assessment:

20 " top of dum ti tep of elnse

$\because$ ter 4 is

photo taken:

$\times$ Yes

Comments: 
WHC-SD-WM-TRP-246 REV. 0

\section{Palletized Storage Drum Data Sheet}

Drum No. 0642

Description (Check):

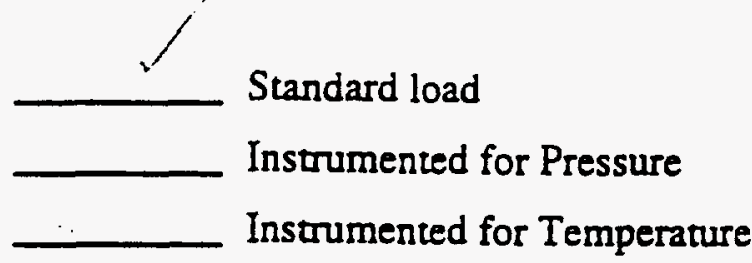

\begin{tabular}{|c|c|c|c|}
\hline Loading Starting at Bottom & Layer 1 & Layer 2 & Layer 3 \\
\hline 3 pieces of steel & N/A & N/A \\
\hline $1 / 3$ of the brown paper & & & \\
\hline $1 / 3$ of the 10-mil plastic & & & \\
\hline $1 / 3$ of the bond paper & & & \\
\hline $1 / 3$ of the towels & & & \\
\hline $1 / 3$ of the bags & Go to Layer 2 & Go to Layer 3 & \\
\hline $1 / 3$ of the cur paper & & \\
\hline $1 / 3$ of the fabric & & \\
\hline $1 / 3$ of the rubber & & \\
\hline \hline
\end{tabular}

Drum Filled:

Ring bolt placed opposite drum seam and in down position

Lid seaied and torqued to $40 \mathrm{f} / \mathrm{lb}$ :

Leak tested:

Pre-test weight (lb)

Post-test weight (lb)
Date 5.12.95 Initial R.K

Date 5.12.95 Initial R.K
N/A or Pressure (psig)

Pressure (psig)

Date

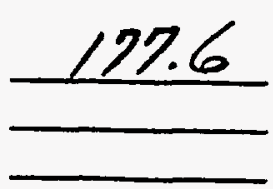

F-136
Time (min) 0

Time (min) 5

Initial

Target weight $\sim 175.6 \mathrm{lb}$ 
Drum No. $\underline{O \in} \div 2, i \in 0 . C \omega$

Post-test Assessment

Position: original: tipped: toppled

Lid on drum: $\quad x$ Yes No $1 \frac{1}{2} \times 17^{\prime} 97$

Gasket missing (cm)

Contents expelled: _ Yes \} \text { No }

Seal failure: $\quad \quad \quad X^{\prime}$ Yes $\quad$ Not visible

Burn pattern:

sell around

Photo taken: $\quad$ Yes $X$ No

Leak tested: $\quad \overline{N A}$ or Pressure (psig)

Pressure (psig)

Date

$\longrightarrow \quad$\begin{tabular}{ll} 
Time (min) & 0 \\
\hline & Time (min)
\end{tabular}

$\overline{\text { Initial }}$

Interior assessment:

24" top thum to top tehan

Videc 46

Photo taken:

$x$ Yes

No

Comments: 


\section{Palletized Storage Drum Data Sheet}

Drum No. 0652

Descriprion (Check):

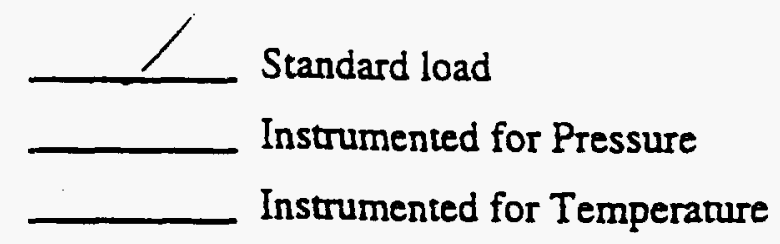

\begin{tabular}{|c|c|c|c|}
\hline Loading Starting ar Bottom & Layer 1 & Layer 2 & Layer 3 \\
\hline 3 pieces of steel & & N/A & N/A \\
\hline $1 / 3$ of the brown paper & & & \\
\hline $1 / 3$ of the 10-mil plastic & & & \\
\hline $1 / 3$ of the bond paper & & & \\
\hline $1 / 3$ of the towels & & & \\
\hline $1 / 3$ of the bags & & & \\
\hline $1 / 3$ of the cut paper & Go to Layer 2 & Go to Layer 3 & \\
\hline $1 / 3$ of the fabric & & & \\
\hline $1 / 3$ of the rubber & & & \\
\hline
\end{tabular}

Drum Filled:

Ring bolt placed opposite drum seam and in down position

Lid sealed and torqued to $40 \mathrm{ft} / \mathrm{b}$ :

Leak tested:

Pre-test weight (lb)

Post-test weight (lb)

$$
\text { Loss }=
$$

Date $5.12 \cdot 95$ Initial

Date $5 \cdot 12 \cdot 95$ Initial

Date $5 \cdot 12 \cdot 95$ Initial R.K

N/A or Pressure (psig)

Pressure (psig)

Date

$\overline{\text { Time }(\min )} \frac{0}{5}$
$\overline{\text { Initial }} \quad$ Time (min)

Target weight $\sim 175.6 \mathrm{lb}$ 
Drum No. 0652169.216.

\section{Post-test Assessment}

Position: __ original: ___ tipped: ___ toppled

Lid on drum: $\quad X$ Yes No

Gasket missing (cm) all

Contents expelled: __ Yes $X$ No

Seal failure: $\quad$ Yes $x$ Not visible $\tau_{0} /$ isittem bulged

Burn pattern:

$35 "$ at ind $t T$ seam $t$ left

Photo taken:

Yes $x$ No

Leak tested:

$\Varangle \mathbb{R}$ or Pressure (psig)

Pressure (psig)

Date $\begin{array}{ll}\text { Time }(\min ) & 0 \\ \text { Time }(\min ) & \underline{5}\end{array}$

Initial

Interior assessment:

17 "Top of dum te tor of chan

visco is

Photo taken:

$x$ Yes

Comments: 


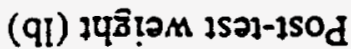

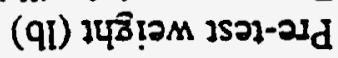

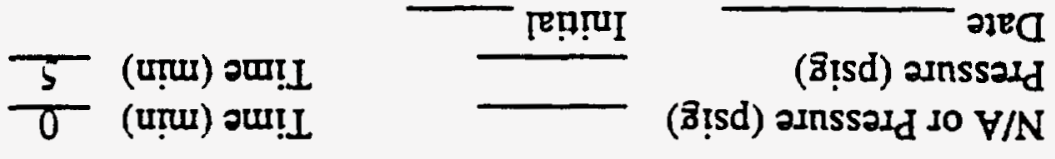

:pวาsว1 үеวТ

$\overline{y \cdot y}$ rẹ़ा $\overline{56 \cdot 6 / 5}$ med

:qI/3 0† 01 panbjol pue poreas p!T

uopisod ussop u! pue

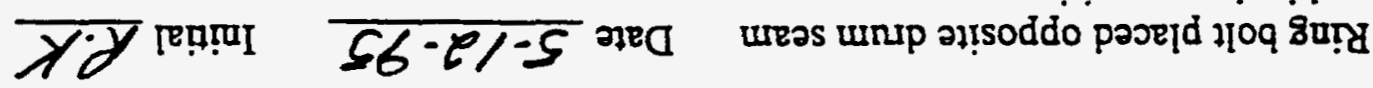
$\overline{\gamma \cdot \gamma}$ [ẹ!n $\overline{56 \cdot 6 / 5}$ med

:pगI!త una

\begin{tabular}{|c|c|c|c|}
\hline & E IDKe 7 OD OD & Z JPKET OI OD & \\
\hline & & & I2qqn ә्प] f० $\varepsilon / I$ \\
\hline & & & गफq̨द әपД J० E/I \\
\hline & & & Iaded Iחכ כน J० E/I \\
\hline & & & s8ిeq ग्पा ग० ह/I \\
\hline & & & 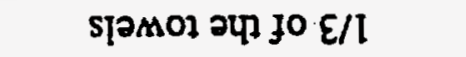 \\
\hline & & & Ioded puoq ә्प] J० $\varepsilon / l$ \\
\hline & & & 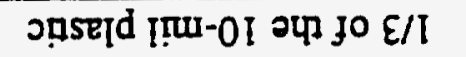 \\
\hline & & & Ioded uMOIq ग्र f० ह/l \\
\hline$\forall / N$ & $\forall / \mathbb{N}$ & & [201s fo səoว!d $\varepsilon$ \\
\hline$\varepsilon \mathrm{JOKE} T$ & $Z$ IOKE] & I IaאE] & 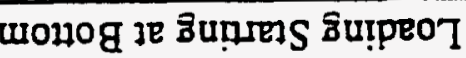 \\
\hline
\end{tabular}

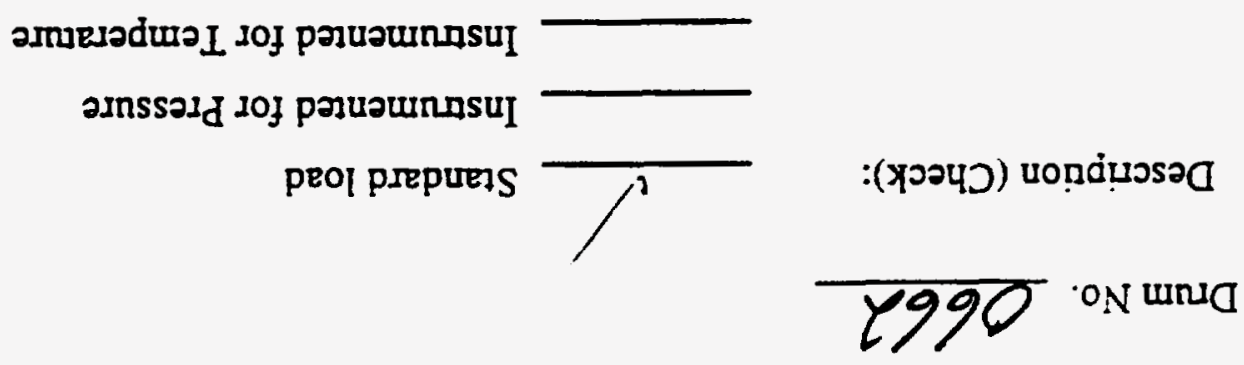


Drum No. $066.2171 .2 \mathrm{~kb}$

Post-test Assessment

Position:

original: tipped: toppied

Lid on drum: $\quad X$ Yes No

Gasket missing (cm)

Contents expeiled: __ Yes $\underline{X}$ No

Seal failure: $\quad$ Yes $\quad x$ Not visible

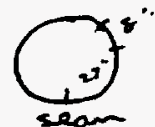

Burn parterg:

Photo taken: $\quad$ Yes $\not \subset$ No

$\begin{array}{llll}\text { Leak tested: } & \text { Time (min) } & 0 \\ & \text { Pressure (psig) } & \text { Time (min) } & -5\end{array}$

Date

Initial

Interior assessment:

is" top of than to top of dinn

inec is

Photo taken:

$x$ Yes No

Comments: 


\section{Palletized Storage Drum Data Sheet}

Drum No. 0672

Description (Check):

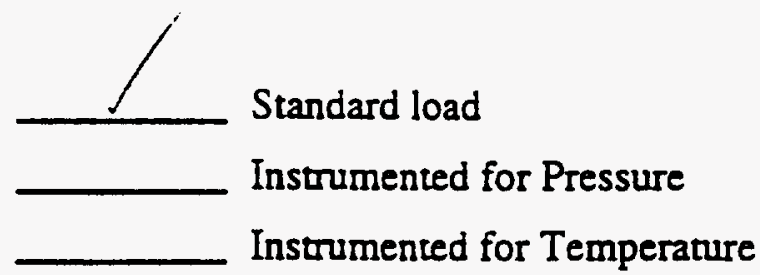

\begin{tabular}{|c|c|c|c|}
\hline Loading Starting at Bottom & Layer 1 & Layer 2 & Layer 3 \\
\hline 3 pieces of steel & & & N/A \\
\hline $1 / 3$ of the brown paper & & & \\
\hline $1 / 3$ of the 10 -mil plastic & & & \\
\hline $1 / 3$ of the bond paper & & & \\
\hline $1 / 3$ of the towels & & & \\
\hline $1 / 3$ of the bags & & & \\
\hline $1 / 3$ of the cut paper & Go to Layer 2 & Go to Layer 3 & \\
\hline $1 / 3$ of the fabric & & \\
\hline $1 / 3$ of the rubber & & \\
\hline
\end{tabular}

Drum Filled:

Ring bolt placed opposite drum seam and in down position

Lid sealed and torqued to $40 \mathrm{ft} / \mathrm{lb}$ :

Leak tested:

Pre-test weight (lb)

Post-test weight (lb)

\section{Loss $=$}

Date 5.12 .95 Initial R.K

Date 5.12.95 Initial L.K
N/A or Pressure (psig)

Pressure (psig)

Date

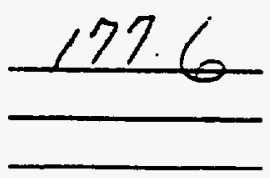

F-142
Time (min) 0

Time (min) 5

Initial

Target weight $\sim 175.6 \mathrm{lb}$ 
Drum No. $\quad 0672,71.31 \mathrm{~b}$

Post-test Assessment

Position: ___ originai: ___ tipped: ___ toppled

Lid on drum: $\quad \quad \quad$ Yes No top slightty bulged

Gasket missing (cm) all

Contents expelled: $\quad$ Yes $\longrightarrow$ No

Seal failure: $\quad$ Y $\quad$ Yes Not visible bottem bulged.

Burn partern:

sean teleft $s \times 12^{\prime}$ spot at top. small spot at botton $\sim$ sane lor cotain 2,2,

Photo taken:

Yes $\underline{x}$ No

Leak tested: $\quad$ $\quad$ Pr/Aor Pressure (psig) Pressure (psig)

Date

\begin{tabular}{lll} 
& Time (min) & 0 \\
\hline Initial & Time (min)
\end{tabular}

Interior assessment:

$14^{\prime \prime}$ top to top

-ineo 30

Photo taken:

$\checkmark$ Yes

Comments: 


\section{Palletized Storage Drum Data Sheet}

Drum No. 0682

Description (Check):

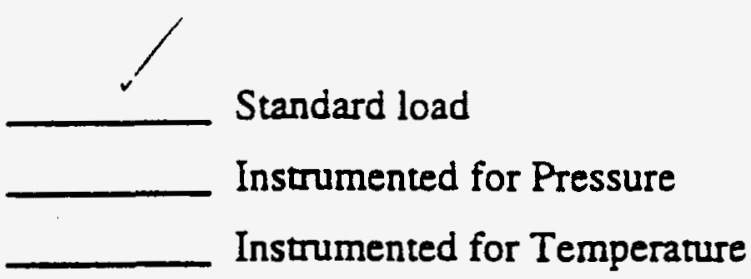

\begin{tabular}{|c|c|c|c|}
\hline Loading Starting at Bottom & Layer 1 & Layer 2 & Layer 3 \\
\hline 3 pieces of steel & & & N/A \\
\hline $1 / 3$ of the brown paper & & & \\
\hline $1 / 3$ of the 10-mil plastic & & & \\
\hline $1 / 3$ of the bond paper & & & \\
\hline $1 / 3$ of the towels & & & \\
\hline $1 / 3$ of the bags & & & \\
\hline $1 / 3$ of the cut paper & Go to Layer 2 & Go to Layer 3 & \\
\hline $1 / 3$ of the fabric & & \\
\hline $1 / 3$ of the rubber & & \\
\hline
\end{tabular}

Drum Filled:

Ring bolt placed opposite drum seam and in down position

Lid sealed and torqued to $40 \mathrm{ft} / \mathrm{b}$ :

Leak tested:

Pre-test weight (lb)

Post-test weight (lb)

$$
\text { Loss }=
$$

Date $5-12.95$ Initial

Date $5-12.95$

Initial L.K

Date 5.12 .95 Initial L.K

N/A or Pressure (psig)

Pressure (psig)

Date

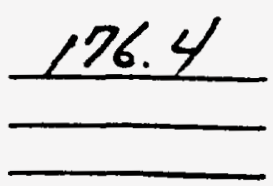

F-144
Time (min) 0

Time (min) 5

Initial 
Drum No. 0682179.516

Post-test Assessment

Position: ___ original: ___ tipped: ___ toppled

Lid on durn: $\quad x$ Yes No ring $=1$ lid latere

Gasket missing (cm) all

Contents expeiled: __ Yes $\longrightarrow x$ No

Seal failure: $\quad$ Y $\quad$ Yes $\$ Not visible

Burn pattern:

arerumed rim $\%$

arerence

Photo taken:

Yes $\$ No

Leak tested:

V/A or Pressure (psig)

Pressure (psig)

Date

Date

\section{$\overline{\text { Initial }}$}

Time (min)
Time (min)

\section{Interior assessment:}

$$
12^{\prime r} \text { tep of dume to } t_{p}, \text { of char }
$$

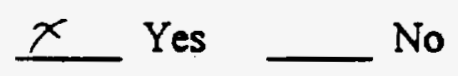

Comments: 


\section{Palletized Storage Drum Data Sheet}

Drum No. $07 / 2$

Description (Check):

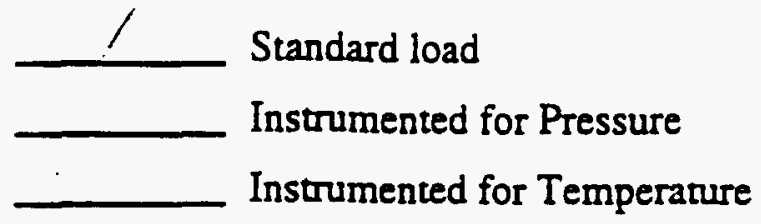

\begin{tabular}{|c|c|c|c|}
\hline Loading Starting at Bottom & Layer 1 & Layer 2 & Layer 3 \\
\hline 3 pieces of steel & $\sim$ & N/A & N/A \\
\hline $1 / 3$ of the brown paper & 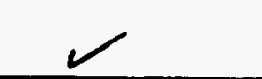 & $r$ & $\gamma$ \\
\hline $1 / 3$ of the 10 -mil plastic & $r$ & $\sim$ & ح \\
\hline $1 / 3$ of the bond paper & $\swarrow$ & $\sim$ & \\
\hline $1 / 3$ of the towels & $r$ & $\checkmark$ & $\mathcal{J}$ \\
\hline $1 / 3$ of the bags & $r$ & & \\
\hline $1 / 3$ of the cut paper & $\checkmark$ & $\sim$ & \\
\hline $1 / 3$ of the fabric & $r$ & $r$ & $\nu$ \\
\hline $1 / 3$ of the rubber & 7 & & \\
\hline & Go to Layer 2 & Go to Layer 3 & \\
\hline
\end{tabular}

Drum Filled:

Ring bolt placed opposite drum seam and in down position

Lid sealed and torqued to $40 \mathrm{ft} / \mathrm{b}$ :

Leak tested:

Pre-test weight (lb)

Post-test weight (lb)

$$
\text { Loss = }
$$

Los Alamos Technical Associates
Date $5-12.95$ Initial R.K

Date 5.12 .95 Initial R.K

Date 5-12-95 Initial R.K
N/A or Pressure (psig) Pressure (psig)

Date

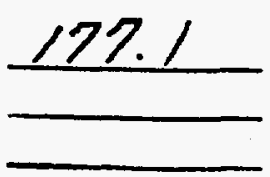

Time (min) 0

Time $(\min )-5$
Initial

Target weight $\sim 175.6 \mathrm{lb}$ 
Drum No. $07 i 2$ i6s. 4 ib.

Post-test Assessment

Position: __ original: __ tipped: __ toppled

Lid on drum: $\quad \quad \quad \quad \quad$ Yes $\quad$ No

Gasket missing (cm)

Contents expelled: _ Yes $x$ No

Seal failure: $\quad x$ Yes Not visible lid bulged bonely see $1 / 8$ " cracle -6 " long

Burn pattern:

ull around

Photo taken:

$$
\text { Yes } \simeq \text { No }
$$

Ieak tested:

N/A or Pressure (psig) Pressure (psig)

Date

\begin{tabular}{lll} 
& Time (min) & 0 \\
\hline & Time (min)
\end{tabular}

Interior assessment:

$19^{\prime \prime}$ to pof dum to top of chan

roca 17

Pैaoto taken:

$x$ Yes

\section{Comments:}




\section{Palletized Storage Drum Data Sheet}

Drum No. 0722 (P)

Description (Check):

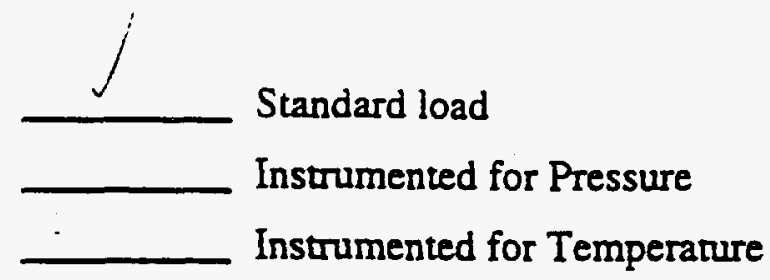

\begin{tabular}{|c|c|c|c|}
\hline Loading Starting at Bottom & Layer 1 & Layer 2 & Layer 3 \\
\hline 3 pieces of steel & & N/A & N/A \\
\hline $1 / 3$ of the brown paper & & & \\
\hline $1 / 3$ of the 10 -mil plastic & & & \\
\hline $1 / 3$ of the bond paper & & & \\
\hline $1 / 3$ of the towels & & & \\
\hline $1 / 3$ of the bags & & & \\
\hline $1 / 3$ of the cut paper & & & \\
\hline $1 / 3$ of the fabric & Go to Layer 2 & Go to Layer 3 & \\
\hline $1 / 3$ of the rubber & &
\end{tabular}

Drum Filled:

Ring bolt placed opposite drum seam and in down position

Lid sealed and torqued to $40 \mathrm{ft} / \mathrm{b}$ :

Leak tested:

Pre-test weight (lb)

Post-test weight (lb)

Date $5.12 \cdot 95$ Initial R.K

Date 5.12.95 Initial R.K

Date $5-12-95$ Initial R.K

N/A or Pressure (psig)

Pressure (psig)

Date

Initial

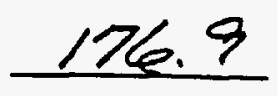

Target weight $\sim 175.6 \mathrm{lb}$
Time (min) 0

Time (min) 5 


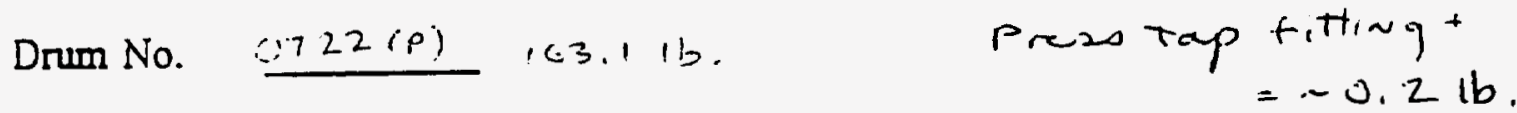

Post-iest Assessment

Position: __ original: ___ tipped: __ toppled

Lid on drum: $\quad X$ Yes No buiged

Gasket missing (cm) al

Contents expelled: _ـ Yes

Seal failure: $\quad$ Yes $\quad x$ Not visibie

Burn pattern:

Photo taken: $\quad$ Y Yes $\underset{\text { No }}{\longrightarrow}$ No

Leak tested: $\quad$ N/A or Pressure (psig)

Pressure (psig)

Date

\begin{tabular}{ll} 
Time (min) & 0 \\
\hline & Time (min)
\end{tabular}

Interior assessment:

$21^{\prime \prime ~ I 0 P . t ~ d u m ~ t o ~ t o p ~ o f ~ c h a n ~}$

i. 00019

Photo taken:

$\times$ Yes

No

Comments:

$$
\text { bottom sean sppeans ruptund }
$$




\section{Palletized Storage Drum Data Sheet}

Drum No. 0732

Description (Check):

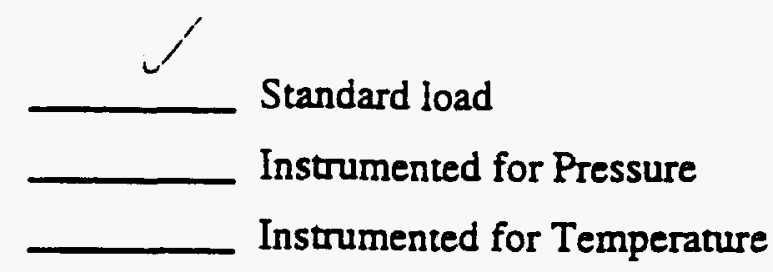

\begin{tabular}{|c|c|c|c|}
\hline Loading Starting at Bottom & Layer 1 & Layer 2 & Layer 3 \\
\hline 3 pieces of steel & $\gamma$ & N/A & N/A \\
\hline $1 / 3$ of the brown paper & $\sim$ & 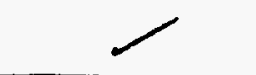 & $\checkmark$ \\
\hline $1 / 3$ of the 10 -mil plastic & & & $\checkmark$ \\
\hline $1 / 3$ of the bond paper & & & $\sim$ \\
\hline $1 / 3$ of the toweis & $\tau$ & $\checkmark$ & $\sim$ \\
\hline $1 / 3$ of the bags & & $\gamma$ & $\checkmark$ \\
\hline $1 / 3$ of the cut paper & $\checkmark$ & $\checkmark$ & 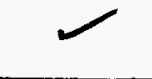 \\
\hline $1 / 3$ of the fabric & $r$ & 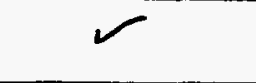 & $\checkmark$ \\
\hline $1 / 3$ of the rubber & & $\nearrow$ & 2 \\
\hline & Go to Layer 2 & Go to Layer 3 & \\
\hline
\end{tabular}

Drum Filled:

Ring bolt placed opposite drum seam and in down position

Lid sealed and torqued to $40 \mathrm{ft} / \mathrm{b}$ :

Leak tested:

Pre-test weight (lb)

Post-test weight (lb)

\section{Loss $=$}

Date 5.12.95 Initial R.K

Date 5.12 .95 Initial L.K
N/A or Pressure (psig)

Pressure (psig)

Date

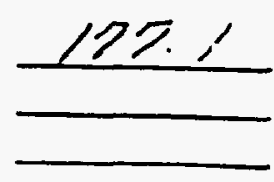

F-150
Time (min) $\frac{\theta}{5}$

Time (min) 5
Initial

Target weight $\sim 175.6 \mathrm{lb}$ 
Drum No. $0732,16: 3$ ib.

Post-test Assessment

Position: originai: tipped: toppied

Lid on drum: $\quad \longrightarrow$ Yes No

Gasket missing (cm) all

Contents expelled: _u Yes $x$ No

Seal failure: $\quad$ Yes $X$ Not visible top t bottem bulged

Burn pattern:

all around

Photo taken:

Yes $x$ No

Leak tested:

N/A or Pressure (psig) Pressure (psig)

Date

\begin{tabular}{lll}
- & Time (min) & 0 \\
\hline & Time (min)
\end{tabular}

Interior assessment:

18 " Top of drum. The tor of chan

Photo taken:

$x$ Yes No

Comments: 
WHC-SD-WM-TRP-246 REV. O

\section{Palletized Storage Drum Data Sheet}

Drum No. 0742

Descriprion (Check):

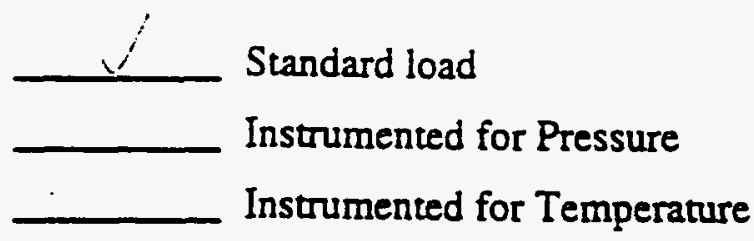

\begin{tabular}{|c|c|c|c|}
\hline Loading Starting at Bottom & Layer 1 & Layer 2 & Layer 3 \\
\hline 3 pieces of steel & - & N/A & N/A \\
\hline \multicolumn{4}{|l|}{ 1/3 of the brown paper } \\
\hline \multicolumn{4}{|l|}{$1 / 3$ of the 10 -mil plastic } \\
\hline \multicolumn{4}{|l|}{$1 / 3$ of the bond paper } \\
\hline \multicolumn{4}{|l|}{$1 / 3$ of the toweis } \\
\hline \multicolumn{4}{|l|}{$1 / 3$ of the bags } \\
\hline \multicolumn{4}{|l|}{$1 / 3$ of the cut paper } \\
\hline \multicolumn{4}{|l|}{$1 / 3$ of the fabric } \\
\hline \multicolumn{4}{|l|}{$1 / 3$ of the rubber } \\
\hline & Go to Layer 2 & Go to Layer 3 & \\
\hline
\end{tabular}

Drum Filled:

Ring bolt placed opposite drum seam and in down position

Lid sealed and torqued to $40 \mathrm{ft} / \mathrm{b}$ :

Leak tested:

Pre-test weight (lb)

Post-test weight (lb)

$$
\text { Loss }=
$$

Date $5 \cdot 12 \cdot 95$ Initial R.K

Date $5 \cdot 12.95$ Initial R.K

Date $5-12.95$ Initial R.K

N/A or Pressure (psig)

Pressure (psig)

Date

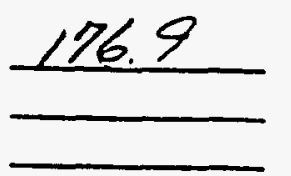

Initial

Time (min) 0

Time (min) 5

Target weight $-175.6 \mathrm{lb}$ 
Drum No. 2742 ibt. ib.

Post-test Assessment

Position:

original:

tipped:

toppled

Lid on drum: $\quad \frac{x}{n !}$ Yes No pare bulging bow

Gasket missing (cm) ell

Contents expelled: __ Yes $x$ No

Seal failure: $\quad$ ? Yes Not visible $\underset{\text { brotion? }}{\times}$ botiged, fimay be

Burn pattem:

ill but - $16^{\prime \prime}$ seam te right

Photo taken: $\quad$ Yes ${ }^{\prime}$ No

Leak tested: $\quad \widehat{\mathbb{N} / A}$ or Pressure (psig) Pressure (psig)

Date

\begin{tabular}{lll} 
Time (min) & 0 \\
\hline & Time (min)
\end{tabular}

Interior assessment:

$21 "$ top of dum te tep of ohar

UDEO 47

Photo taken:

$\times$ Yes No

Comments: 
WHC-SD-WM-TRP-246 REV. 0

\section{Palletized Storage Drum Data Sheet}

Drum No. 0752

Descripion (Check):

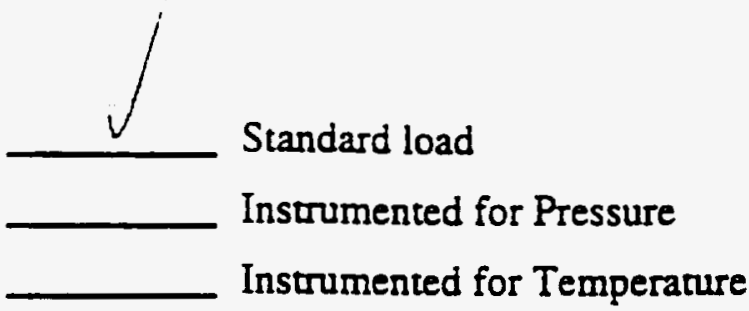

\begin{tabular}{|c|c|c|c|}
\hline Loading Starting at Bottom & Layer 1 & Layer 2 & Layer 3 \\
\hline 3 pieces of steel & & N/A & N/A \\
\hline $1 / 3$ of the brown paper & $\checkmark$ & $\nu$ & $\checkmark$ \\
\hline \multicolumn{4}{|l|}{$1 / 3$ of the 10 -mil plastic } \\
\hline \multicolumn{4}{|l|}{$1 / 3$ of the bond paper } \\
\hline \multicolumn{4}{|l|}{$1 / 3$ of the towels } \\
\hline $1 / 3$ of the bags & 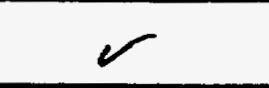 & $\nu$ & \\
\hline \multicolumn{4}{|l|}{$1 / 3$ of the cut paper } \\
\hline \multicolumn{4}{|l|}{$1 / 3$ of the fabric } \\
\hline \multirow[t]{2}{*}{$1 / 3$ of the rubber } & $\Omega$ & $\sim$ & c \\
\hline & Go to Layer 2 & Go to Layer 3 & \\
\hline
\end{tabular}

Drum Filled:

Ring bolt placed opposite drum seam and in down position

Lid sealed and torqued to $40 \mathrm{ft} / \mathrm{b}$ :

Leak tested:

Pre-test weight (lb)

Post-test weight (lb)

$$
\text { Loss }=
$$

Los Alamos Technical Associates

Date 5.12 .95 Initial L.K

Date 5.12.95 Initial R.K

Date $5 \cdot 12 \cdot 95$ Initial $2 K$

N/A or Pressure (psig)

Pressure (psig)

Date

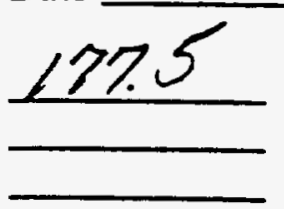

F-154
Time (min) 0

Time (min) 5

Initial

Target weight $-175.6 \mathrm{lb}$ 
Drum No. $0752,69.9$ ib.

Post-test Assessment

Position: ___ original: ___ tipped: ___ toppled

Lid on drum: $\quad x$ Yes N No ring glid Coose

Gasket missing (cm) all

Contents expeiled: _u Yes $x$ No

Seal failure: $\quad$ Yes $X$ Not visibie

Burn pattern: .

$25^{\prime \prime a t}$ ind lit $l$.

Photo taken:

Yes $x$ No

Leak tested:

NFA or Pressure (psig)

Pressure (psig)

Date

Initial

$\begin{array}{ll}\text { Time (min) } & 0 \\ \text { Time (min) } & \underline{5}\end{array}$

Interior assessment:

$17^{\prime \prime}$ tep of drem titop $t$ chan

roec 76

Photo taken:

$x$ Yes

Comments: 
WHC-SD-WM-TRP-246 REV. 0

\section{Palletized Storage Drum Data Sheet}

Drum No. 0762

Description (Check):

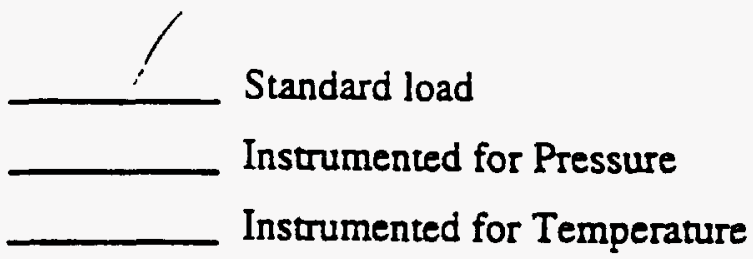

\begin{tabular}{|c|c|c|c|}
\hline Loading Starting at Bottom & Layer 1 & Layer 2 & Layer 3 \\
\hline 3 pieces of steel & & N/A & N/A \\
\hline $1 / 3$ of the brown paper & & $\gamma$ & $\sim$ \\
\hline $1 / 3$ of the 10 -mil plastic & $\sim$ & $v$ & 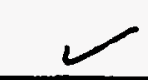 \\
\hline 1/3 of the bond paper & & $v$ & \\
\hline $1 / 3$ of the towels & & & \\
\hline $1 / 3$ of the bags & & & \\
\hline $1 / 3$ of the cut paper & & & \\
\hline $1 / 3$ of the fabric & $\sim$ & 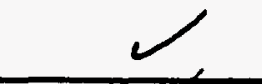 & $v$ \\
\hline $1 / 3$ of the rubber & & & 2 \\
\hline & Go to Layer 2 & Go to Layer 3 & \\
\hline
\end{tabular}

Drum Filled:

Date 5.12 .95 Initial L.K

Ring bolt placed opposite drum seam Date $5-12.95$ Initial L.K and in down position

Lid sealed and torqued to $40 \mathrm{ft} / \mathrm{lb}: \quad$ Date 5.12 .95 Initial R.K

Leak tested:

Pre-test weight (lb)

Post-test weight (lb)
N/A or Pressure (psig) Pressure (psig)

Date

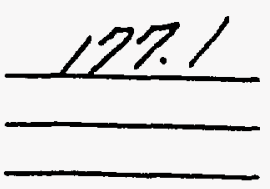

F-156
Time (min) 0 Time $(\mathrm{min})$
Initial

Target weight $\sim 175.6 \mathrm{lb}$

\section{Loss $=$}


Drum No. $0762,172.115$.

Post-test Assessment

Position: original: tipped: toppied

Lid on drum: $\quad X$ Yes No lid ringlock

Gasket missing (cm) all

Contents expelled: __ Yes $\underline{x}$ No

Seal failure: $\quad$ _ $\quad$ Yes _ $\$ Not visible

Burn pattern:
$\frac{21 \text { leffel sean tant sevenal pots }}{215^{\prime \prime} \text { wide }}$

Photo taken:

$$
\text { Yes }
$$

Leak tested:

XTA or Pressure (psig)

Pressure (psig)

Date $\begin{array}{ll}\text { Time }(\min ) & \frac{0}{5} \\ \text { Time }(\min ) & \end{array}$

$\overline{\text { Initial }}$

Interior assessment:

$15 \%$ lep of dum to top of chan

$\checkmark$ ioec 74

Photo taken:

$x$ Yes

No

Comments: 


\section{Palletized Storage Drum Data Sheet}

Drum No. 0772

Descriprion (Check):

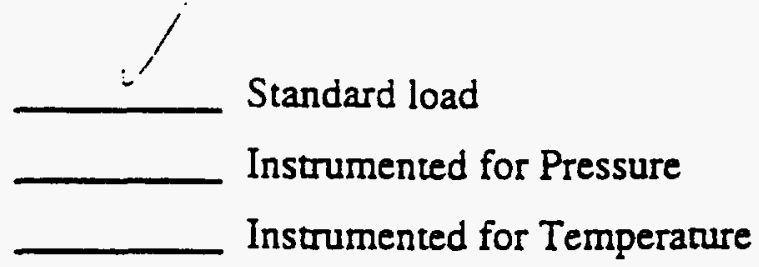

\begin{tabular}{|c|c|c|c|}
\hline Loading Starting at Bottom & Layer 1 & Layer 2 & Layer 3 \\
\hline 3 pieces of steel & & N/A & \\
\hline $1 / 3$ of the brown paper & & & \\
\hline $1 / 3$ of the 10-mil plastic & & & \\
\hline $1 / 3$ of the bond paper & & & \\
\hline $1 / 3$ of the towels & & & \\
\hline $1 / 3$ of the bags & & Go to Layer 3 & \\
\hline $1 / 3$ of the cut paper & & \\
\hline $1 / 3$ of the fabric & Go to Layer 2 & Go & \\
\hline $1 / 3$ of the rubber & & \\
\hline
\end{tabular}

Drum Filled:

Ring bolt placed opposite drum seam and in down position

Lid sealed and torqued to $40 \mathrm{ft} / \mathrm{b}$ :

Leak tested:

Pre-test weight (lb)

Post-test weight (lb)

\section{Loss $=$}

Date 5.12 .95 Initial R.K

Date 5.12 .95 Initial L.K
N/A or Pressure (psig)

Pressure (psig)

Date

Initial

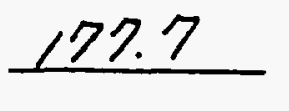

Target weight $\sim 175.6 \mathrm{lb}$
Time $(\min )-0$ Time (min) 5 
Drum No. $0772 \quad 173.4 \mathrm{~h}$.

Post-test Assessment

Position: original: tipped: toppied

Lid on drum: $\quad x$ Yes No

Gasket missing (cm) ald

Contents expeiled: __ Yes $\$ No

Seal failure: $\quad$ Yes $\quad Y$ Not visible

Burn pattern:

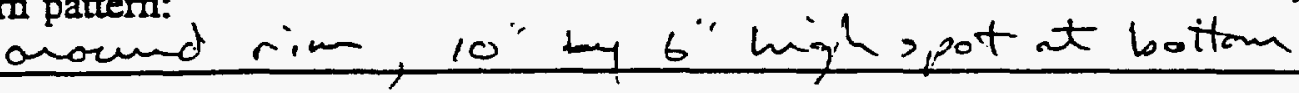

Photo taken: Yes $\infty$ No

Leak tested:

ATA pr Pressure (psig) Pressure (psig)

Date

\begin{tabular}{lll} 
& Time (min) & 0 \\
\hline Initial & Time (min) &
\end{tabular}

Interior, assessment:

15 top te top

Uinec 79

Photo taken:

$x$ Yes No

Comments: 
WHC-SD-WM-TRP-246 REV. 0

\section{Palletized Storage Drum Data Sheet}

Drum No. 0782

Description (Check):

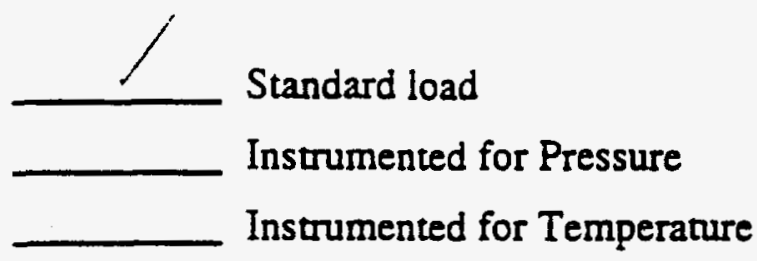

\begin{tabular}{|c|c|c|c|}
\hline Loading Starting at Bottom & Layer 1 & Layer 2 & Layer 3 \\
\hline 3 pieces of steel & 2 & N/A & N/A \\
\hline \multicolumn{4}{|l|}{$1 / 3$ of the brown paper } \\
\hline \multicolumn{4}{|l|}{$1 / 3$ of the 10 -mil plasric } \\
\hline \multicolumn{4}{|l|}{$1 / 3$ of the bond paper } \\
\hline \multicolumn{4}{|l|}{$1 / 3$ of the towels } \\
\hline \multicolumn{4}{|l|}{$1 / 3$ of the bags } \\
\hline \multicolumn{4}{|l|}{$1 / 3$ of the cut paper } \\
\hline \multicolumn{4}{|l|}{$1 / 3$ of the fabric } \\
\hline \multicolumn{4}{|l|}{$1 / 3$ of the rubber } \\
\hline & Go to Layer 2 & Go to Layer 3 & \\
\hline
\end{tabular}

Drum Filled:

Ring bolt placed opposite drum seam and in down position

Lid sealed and torqued to $40 \mathrm{f} / \mathrm{lb}$ :

Leak tested:

Pre-test weight (lb)

Post-test weight (lb)
Date 5.12 .95 Initial

Date $5 \cdot 1295$ Initial

Date 5.12.95 Initial R.K

N/A or Pressure (psig)

Pressure (psig)

Date

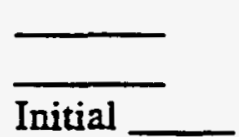

Time (min) 0

Time $(\min )-5$

177. $7 \quad$ Target weight $-175.6 \mathrm{lb}$ 
Drum No. $\quad 0782 \quad 175.8$ ib

Post-test Assessment

Position:

original: tipped: toppled

Lid on drum: $\quad x$ Yes N No ringrid loose

Gasket missing (cm) all

Contents expelled: $\quad$ Yes $\stackrel{x}{\longrightarrow}$ No

Seal failure: $\quad$ Yes $X$ Not visible

Burn pattern:
$10 " \times 10^{\prime \prime}$
spot
raround rim

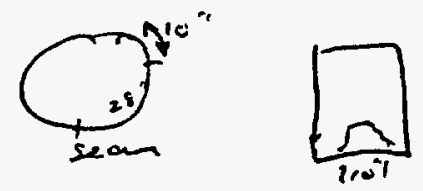

Photo taken:

Yes $x$ No

Leak tested:

N/A or Pressure (psig)

Pressure (psig)

Date

Initial
Time (min)
Time (min)

- $\quad$\begin{tabular}{ll} 
Time (min) & 0 \\
\hline
\end{tabular}

Interior assessment: $\frac{7^{\prime \prime} \text { top tu top of a har }}{n 6^{\prime \prime} \text { char on chend }}$

Viseo 78

Photo raken:

$\leftarrow$ Yes

\section{Comments:}


WHC-SD-WM-TRP-246 REV. 0

\section{Palletized Storage Drum Data Sheet}

Drum No. 0812

Descriprion (Check):

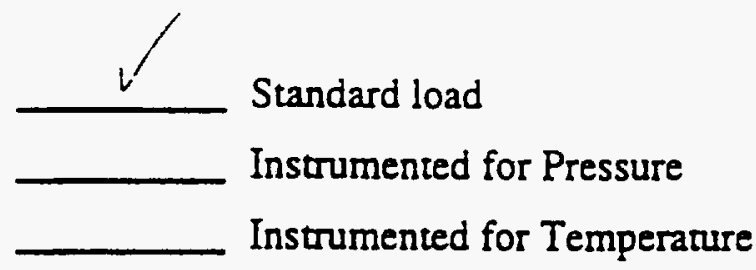

\begin{tabular}{|c|c|c|c|}
\hline Loading Starting at Bottom & Layer 1 & Layer 2 & Layer 3 \\
\hline 3 pieces of steel & $\therefore$ & N/A & N/A \\
\hline $1 / 3$ of the brown paper & $i$ & $:^{-}$ & $k^{\prime}$ \\
\hline $1 / 3$ of the 10 -mil plastic & $c$ & $b^{\prime}$ & $v^{\prime}$ \\
\hline $1 / 3$ of the bond paper & 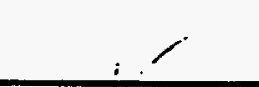 & $v^{\prime}$ & $b^{\prime}$ \\
\hline $1 / 3$ of the toweis & 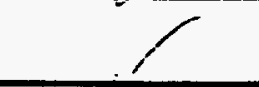 & $i^{\prime}$ & \\
\hline $1 / 3$ of the bags & $i^{\prime}$ & $\sqrt{ }$ & \\
\hline $1 / 3$ of the cut paper & $i^{\prime}$ & 11 & $V$ \\
\hline $1 / 3$ of the fabric & $v^{\prime}$ & I & \\
\hline $1 / 3$ of the rubber & $y^{\prime}$ & $! \prime$ & \\
\hline & Go to Layer 2 & Go to Layer 3 & \\
\hline
\end{tabular}

Drum Filled:

Ring bolt placed opposite drum seam and in down position

Lid sealed and torqued to $40 \mathrm{ft} / \mathrm{lb}$ :

Leak tested:

Pre-test weight (lb)

Post-test weight (lb)
Date 5.5 .95 Initial As

Date $5 \cdot 5 \cdot 95$ Initial AS
N/A or Pressure (psig) Pressure (psig)

Date

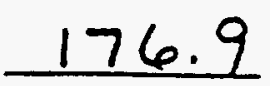

Time (min) 0 Time $(\mathrm{min}) 5$

\section{Loss $=$}


PFill, 一个I

Roal- Simoge Drum Data Sheet

Drum No. $\quad 0,812 \quad 183.5 \quad \ldots i+26$

Post-test Assessment

Position: ___ original: ___ tipped: ___ toppied

Gasket missing (cm)

Contents expelled: _ Yes $\underline{X}$ No

Seal failure: $\quad X$ Yes $\quad$ Not visible

Burn pattern:

all around

Photo taken:

- Yes $x$ No

Leak tested:

$\widehat{N / A}$ or Pressure (psig)

Pressure (psig)

Date

\begin{tabular}{lll} 
& Time $(\min )$ & 0 \\
\hline & Time $(\min )$
\end{tabular}

Initial

Interior assessment:

23 "top of them to top of chave d contests

Pेंroco 12

$x$ Yes No

Comments: 


\section{Palletized Storage Drum Data Sheet}

Drum No. 0822

Descriprion (Check):

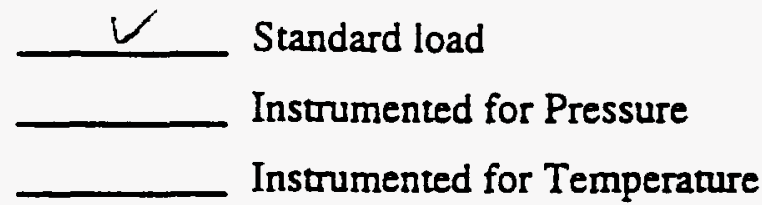

\begin{tabular}{|c|c|c|c|}
\hline Loading Staring at Bottom & Layer 1 & Layer 2 & Layer 3 \\
\hline 3 pieces of steel & $\sigma$ & N/A & N/A \\
\hline $1 / 3$ of the brown paper & $L^{\prime \prime}$ & $v^{\prime}$ & $v^{\prime}$ \\
\hline $1 / 3$ of the 10 -mil plastic & $v^{\prime}$ & 1, & $\therefore$ \\
\hline $1 / 3$ of the bond paper & 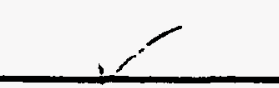 & $L^{\prime}$ & 19 \\
\hline $1 / 3$ of the towels & $v^{\prime}$ & 1 & I \\
\hline $1 / 3$ of the bags & $1<$ & $v^{\prime}$ & \\
\hline $1 / 3$ of the cut paper & $x^{\prime}$ & $1^{\prime}$ & \\
\hline $1 / 3$ of the fabric & - & 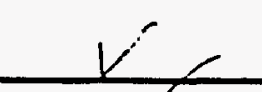 & \\
\hline $1 / 3$ of the rubber & & $V^{\prime}$ & $V$ \\
\hline & Go to Layer 2 & Go to Layer 3 & \\
\hline
\end{tabular}

Drum Filled:

Ring bolt placed opposite drum seam and in down position

Lid sealed and torqued to $40 \mathrm{ft} / \mathrm{b}$ :

Leak tested:

Pre-test weight (lb)

Post-test weight (lb)

$$
\text { Loss }=
$$

Date $5 \cdot 5 \cdot 95$ Initial $A 5$

Date 5-5.95 Initial AS

Date $5-5-95 \quad$ Initial AS

N/A or Pressure (psig)

Pressure (psig)

Date

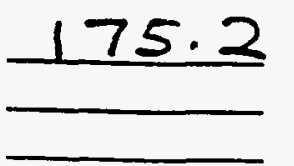

F-164
Time (min)

Time (min) 5
Target weight $-175.6 \mathrm{lb}$ 
Drum No. 05:22 164.0 B.

\section{Post-test Assessment}

Position: origina: tipped: toppled

Lid on drum: $\quad X$ Yes No No $1 / 2 \times 1 "$ gap centend $19^{\circ}$ Left of ze an

Gasket missing (cm)

Contents expeiled: _ Yes $x$ No

Seal failure: $\quad x$ Yes Not visible

Burn pattern:

$47^{\prime \prime}$ sean to teft

Photo taken:

Leak tested:

$$
\text { Yes } \underset{\text { No }}{\longrightarrow}
$$

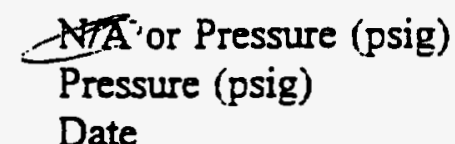

Date

\begin{tabular}{ll} 
Time $(\min )$ & 0 \\
Time $(\min )$ & 5 \\
\hline
\end{tabular}

$\overline{\text { Initial }}$

Interior assessment:

$19^{\circ}$ top t dum to top f chaned contents

Phoco il

Photo taken:

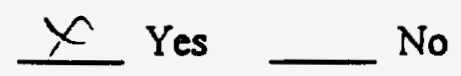

Comments: 
WHC-SD-WM-TRP-246 REV. 0

Palletized Storage Drum Data Sheet

Drum No. 0832

Descriprion (Check):

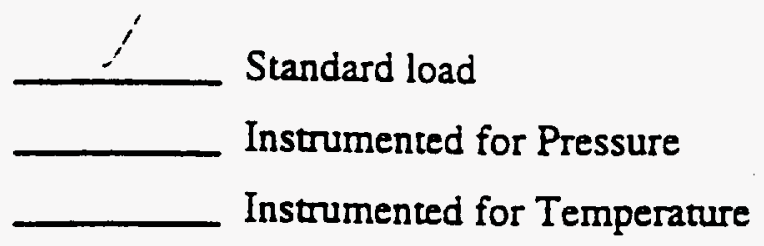

\begin{tabular}{|c|c|c|c|}
\hline Loading Starting at Bottom & Layer 1 & Layer 2 & Layer 3 \\
\hline \hline 3 pieces of steel & & N/A \\
\hline $1 / 3$ of the brown paper & & & \\
\hline $1 / 3$ of the 10 -mil plastic & & & \\
\hline $1 / 3$ of the bond paper & & & \\
\hline $1 / 3$ of the towels & & & \\
\hline $1 / 3$ of the bags & & & \\
\hline $1 / 3$ of the cut paper & & $\checkmark$ & \\
\hline $1 / 3$ of the fabric & Go to Layer 2 & Go to Layer 3 & \\
\hline $1 / 3$ of the rubber & & \\
\hline
\end{tabular}

Drum Filled:

Ring bolt placed opposite drum seam and in down position

Lid sealed and torqued to $40 \mathrm{ft} / \mathrm{b}$ :

Leak tested:

Pre-test weight (lb)

Post-test weight (lb)

Date 512.95 Initial R.K

Date $5-12-95 \quad$ Initial R.K

Date $5=12-95$ Initial L.K

N/A or Pressure (psig)

Pressure (psig)

Date

176.9

Initial

Target weight $\sim 175.6 \mathrm{lb}$ 
Drum No. 요료 i75.1 is

Post-test Assessment

Position:

original: tipped: toppled

Lid on drum: $\quad \underline{x}$ Yes No

Gasket missing (cm) a

Contents expelled: __ Yes $\$ No

Seal failure: $\quad$ Yes $\quad$ Y Not visible

Burn pattern:

IA" sein te right

Photo taken: $\quad$ Yes $\not$ No

Leak tested: TIA or Pressure (psig)

Pressure (psig)

Date

$-\quad$\begin{tabular}{ll} 
Time (min) & 0 \\
\hline & Time (min)
\end{tabular}

$\overline{\text { Initial }}$

Interior assessment:

12 "top of dum to top ichar

- 102029

Photo taken:

$x$ Yes N No

Comments: 


\section{Palletized Storage Drum Data Sheet}

Drim No. 0842

Description (Check):

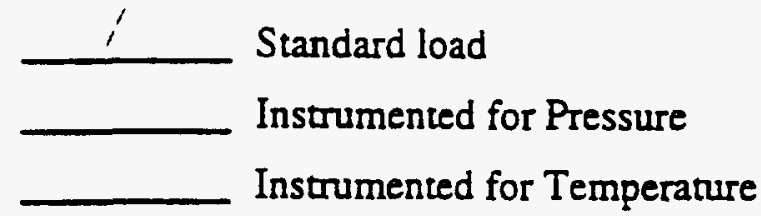

\begin{tabular}{|c|c|c|c|}
\hline Loading Staring at Bottom & Layer 1 & Layer 2 & Layer 3 \\
\hline 3 pieces of steel & 2 & N/A & N/A \\
\hline \multicolumn{4}{|l|}{$1 / 3$ of the brown paper } \\
\hline $1 / 3$ of the 10 -mil plastic & r & 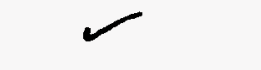 & $\sim$ \\
\hline \multicolumn{4}{|l|}{$1 / 3$ of the bond paper } \\
\hline \multicolumn{4}{|l|}{$1 / 3$ of the towels } \\
\hline \multicolumn{4}{|l|}{$1 / 3$ of the bags } \\
\hline \multicolumn{4}{|l|}{$1 / 3$ of the cut paper } \\
\hline $1 / 3$ of the fabric & 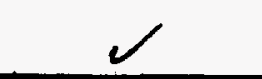 & $r$ & $r$ \\
\hline \multicolumn{4}{|l|}{$1 / 3$ of the rubber } \\
\hline & Go to Layer 2 & Go to Layer 3 & \\
\hline
\end{tabular}

Drum Filled:

Ring bolt placed opposite drum seam and in down position

Lid sealed and torqued to $40 \mathrm{ft} / \mathrm{b}$ :

Leak tested:

Pre-test weight (lb)

Post-test weight (lb)

$$
\text { Loss = }
$$

Date $5=12.95$ Initial R.K

Date 5-12.95 Initial L.K

Date S-12-95 Initial R.K

N/A or Pressure (psig)

Pressure (psig)

Date

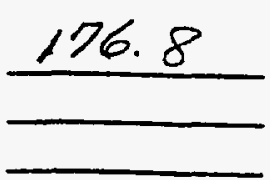

Time $(\min )$

Time (min) 5 
Drum No. $0342: 71: 16$.

\section{Post-test Assessment}

Position: __ original: ___ tipped: __ toppled

Lid on drum: $\quad \longrightarrow$ Yes No

Gasket missing (cm) all

Contents expelled: __ Yes

Seal failure: $\quad$ Yes $\quad x$ Not visible

Burn pattern:

s"at.id lt .

Photo taken: $\quad$ Yes $X$ No

Leak tested: N/A or Pressure (psig) Pressure (psig)

Date

\begin{tabular}{lll}
- & Time $(\min )$ & 0 \\
\hline & Time (min)
\end{tabular}

Interior assessment:

18" topt dum Et tepef dhan

DDeO $2 \dot{y}$

Photo raken:

$\geq$ Yes

\section{Comments:}




\section{Palletized Storage Drum Data Sheet}

Drum No. 0852

Descriprion (Check):

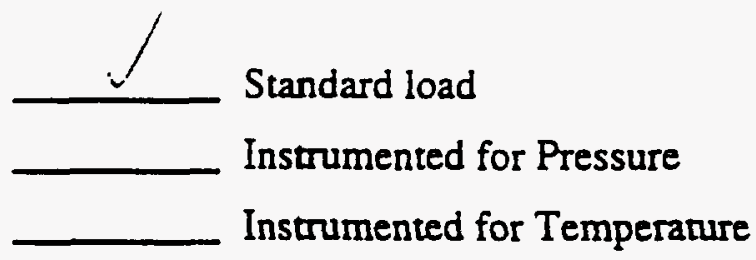

\begin{tabular}{|c|c|c|c|}
\hline Loading Starting at Bottom & Layer 1 & Layer 2 & Layer 3 \\
\hline 3 pieces of steel & & N/A & N/A \\
\hline \multicolumn{4}{|l|}{ 1/3 of the brown paper } \\
\hline $1 / 3$ of the 10 -mil plastic & $\gamma$ & $\checkmark$ & 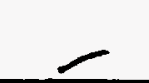 \\
\hline $1 / 3$ of the bond paper & r & 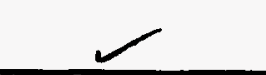 & r \\
\hline $1 / 3$ of the towels & $\sim$ & 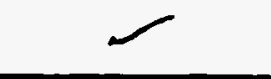 & $\alpha$ \\
\hline $1 / 3$ of the bags & 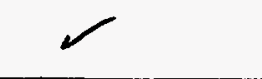 & & $\checkmark$ \\
\hline $1 / 3$ of the cut paper & 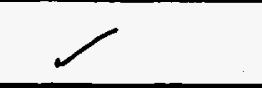 & & $\checkmark$ \\
\hline $1 / 3$ of the fabric & $\checkmark$ & $\alpha$ & r \\
\hline \multirow[t]{2}{*}{$1 / 3$ of the rubber } & $r$ & $\gamma$ & $\checkmark$ \\
\hline & Go to Layer 2 & Go to Layer 3 & \\
\hline
\end{tabular}

Drum Filled:

Ring bolt placed opposite drum seam and in down position

Lid sealed and torqued to $40 \mathrm{ft} / \mathrm{b}$ :

Leak tested:

Pre-test weight (lb)

Post-test weight (lb)
Date 5.12 .95 Initial R.K

Date $5-12.95 \quad$ Initial L.K

Date 512.95 Initial R.K
N/A or Pressure (psig)

Pressure (psig)

Date

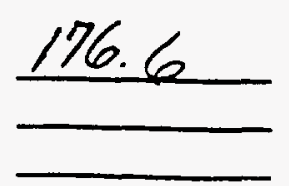

Time (min) 0

Time (min) 5
Initial

Target weight $-175.6 \mathrm{lb}$ 
Drum No. $0852 i 70.6$

Post-test Assessment

Position: originai: tipped: toppled

Lid on drum: $\quad X$ Yes No ring tid looze

Gasket missing (cm) all

Contents expelled: _u Yes $x$ No

Seal failure: $\quad$ Yes $X$ Not visible

Burn partern:

chound rim or 12" up around bittom

Photo taken:

Y Yes $x$ No

Leak tested:

NyA or Pressure (psig) Pressure (psig)

Date

\begin{tabular}{lll}
- & Time (min) & 0 \\
\hline & Time (min)
\end{tabular}

Interior assessment:

$i 7$ "top to top f - han

Photo taken:

$x$ Yes

Comments: 


\section{Palletized Storage Drum Data Sheet}

Drum No. 0862

Description (Check):

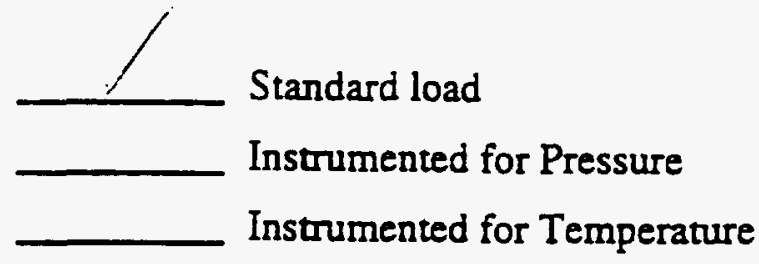

\begin{tabular}{|c|c|c|c|}
\hline Loading Starting at Bottom & Layer 1 & Layer 2 & Layer 3 \\
\hline 3 pieces of steel & & N/A & N/A \\
\hline $1 / 3$ of the brown paper & & & \\
\hline $1 / 3$ of the 10-mil plastic & & & \\
\hline $1 / 3$ of the bond paper & & & \\
\hline $1 / 3$ of the towels & & & \\
\hline $1 / 3$ of the bags & & & \\
\hline $1 / 3$ of the cut paper & & & \\
\hline $1 / 3$ of the fabric & Go to Layer 2 & Go to Layer 3 & \\
\hline $1 / 3$ of the rubber & & & \\
\hline
\end{tabular}

Drum Filled:

Ring bolt placed opposite drum seam and in down position

Lid sealed and torqued to $40 \mathrm{f} / \mathrm{b}$ :

Leak tested:

Pre-test weight (lb)

Post-test weight (lb)

$$
\text { Loss }=
$$

Date 5-12.95 Initial

Date $5=12.95$ Initial L.R

Date 5/295 Initial LF
N/A or Pressure (psig)

Pressure (psig)

Date

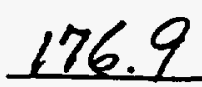

Time (min) 0

Time $(\min )-5$
Initial

Target weight $-175.6 \mathrm{lb}$ 
Drum No. $0862+75.5 \mathrm{Bb}$.

\section{Post-iest Assessment}

Position: orig :al: tipped: toppled

Lid on drum: $\quad x$ Yes No

Gasket missing (cm) all

Contents expeiled: _ Yes $\underline{x}$ No

Seal failure: $\quad$ Yes $\quad X$ Not visible

Burn pattern:

Nove except $-12^{\prime \prime} \times 12^{\prime \prime}$ spot befow bolt

Photo taken:

Yes $\quad x$ No

Leak tested: Ni/A or Pressure (psig) Pressure (psig)

Date

\begin{tabular}{lll}
- & Time (min) & 0 \\
\hline Initial & Time (min)
\end{tabular}

Interior assessment:

8" tup of dum to top of char

Pripe sto taken:

$x$ Yes

\section{Comments:}




\section{Palletized Storage Drum Data Sheet}

Drum No. 0872

Description (Check):

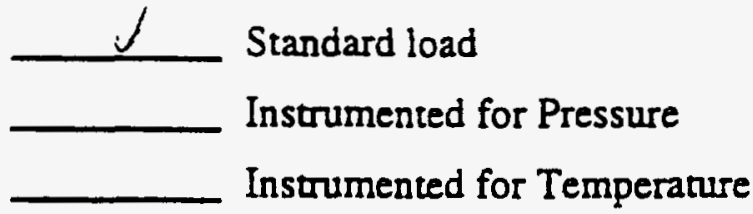

\begin{tabular}{|c|c|c|c|}
\hline Loading Starting at Bottom & Layer 1 & Layer 2 & Layer 3 \\
\hline 3 pieces of steel & & N/A & N/A \\
\hline $1 / 3$ of the brown paper & & & \\
\hline $1 / 3$ of the 10-mil plastic & & & \\
\hline $1 / 3$ of the bond paper & & & \\
\hline $1 / 3$ of the towels & & & \\
\hline $1 / 3$ of the bags & & & \\
\hline $1 / 3$ of the cut paper & & & \\
\hline $1 / 3$ of the fabric & Go to Layer 2 & Go to Layer 3 & \\
\hline $1 / 3$ of the rubber & & \\
\hline
\end{tabular}

Drum Filled:

Ring bolt placed opposite drum seam and in down position

Lid sealed and torqued to $40 \mathrm{ft} / \mathrm{lb}$ :

Leak tested:

Pre-test weight (lb)

Post-test weight (lb)
Date 5-12.95 Initial L.K

Date $5=12.95$ Initial L.K

Date S-12-95 Initial L.R
N/A or Pressure (psig)

Pressure (psig)

Date

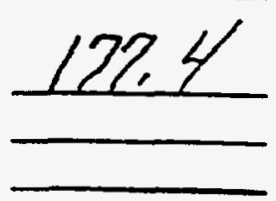

F-174
Time (min) 0

Time (min) 5
Initial

Target weight $-175.6 \mathrm{lb}$ 
Drum No. 0872177.416.

Post-test Assessment

Position: originai: tipped: toppied

Lid on drum: $\quad \quad \quad \quad$ Yes $\quad$ No

Gasket missing (cm) $49^{\prime \prime}$

Contents expelled: _ Yes $x$ No

Seal failure: $\quad$ Yes $\quad \chi$ Not visible

Burn pattern:

nove except around rim

Photo taken:

Yes $\nsim$ No

Leak tested: $\quad$ N/Autor Pressure (psig)

Pressure (psig)

Date

$\longrightarrow \quad$\begin{tabular}{ll} 
Time (min) & 0 \\
\hline
\end{tabular}

Interior assessment:

$2-\tau^{\prime \prime}$ chan ( $5^{\prime \prime}$ half ift $f$ som)

Ptroto taken:

$x$ Yes

No

Comments: 


\section{Palletized Storage Drum Data Sheet}

Drum No. 0882

Description (Check):

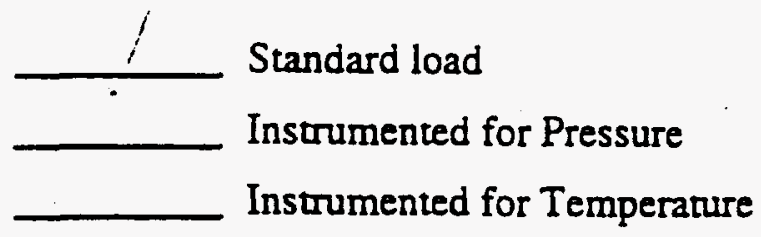

\begin{tabular}{|c|c|c|c|}
\hline Loading Starting at Bottom & Layer 1 & Layer 2 & Layer 3 \\
\hline 3 pieces of steel & $<$ & N/A & N/A \\
\hline $1 / 3$ of the brown paper & - & $\sim$ & - \\
\hline $1 / 3$ of the 10 -mil plastic & $\sim$ & 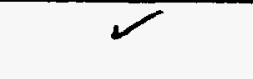 & \\
\hline $1 / 3$ of the bond paper & $r$ & $\gamma$ & \\
\hline \multicolumn{4}{|l|}{$1 / 3$ of the towels } \\
\hline $1 / 3$ of the bags & $\sim$ & 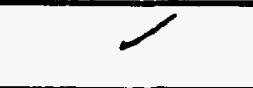 & \\
\hline \multicolumn{4}{|l|}{$1 / 3$ of the cut paper } \\
\hline \multicolumn{4}{|l|}{$1 / 3$ of the fabric } \\
\hline \multicolumn{4}{|l|}{$1 / 3$ of the rubber } \\
\hline & Go to Layer 2 & Go to Layer 3 & \\
\hline
\end{tabular}

Drum Filled:

Date $5-12.95 \quad$ Initial

Ring bolt placed opposite drum seam

Date $5=12-95$

Initial

and in down position

Lid sealed and torqued to $40 \mathrm{ft} / \mathrm{b}$ :

Date $5-12-95$

N/A or Pressure (psig)

Pressure (psig)

Date

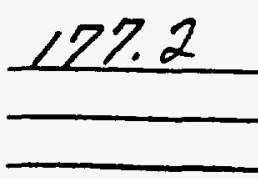

F-176

Pre-test weight (lb)

Post-test weight (lb)

Initial
Time $(\min ) \quad 0$

Time (min) 5
Initial

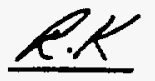

$\longleftarrow$\begin{tabular}{ll} 
Time (min) & $\frac{0}{5}$ \\
\hline & Time (min)
\end{tabular}

Target weight $\sim 175.6 \mathrm{lb}$ 
Drum No. $\quad 0882: 77.3$ is.

Post-test Assessment

Position: originai: tipped: toppled

Lid on drum: $\quad \longrightarrow$ Yes No

Gasket missing (cm) C $39^{\prime \prime}$ co ippreass d thand

Contents expeiled: _u Yes $x$ No

Seal failure: $\quad$ Yes $\quad X$ Not visible

Burn pattern:

None

Photo taken:

Leak tested: Yes $x$ No
NTA or Pressure (psig) Pressure (psig) .

Date

Yes $x$ No

\begin{tabular}{ll} 
& Time (min) \\
\hline Initial & Time (min)
\end{tabular}

Interior assessment:

bouly weitid plastec i7" ieft of sein

visec 51

Photo taken:

Yes — No

Comments:

like rew 


\section{Palletized Storage Drum Data Sheet}

Drum No. $09 / 2$

Descriprion (Check):

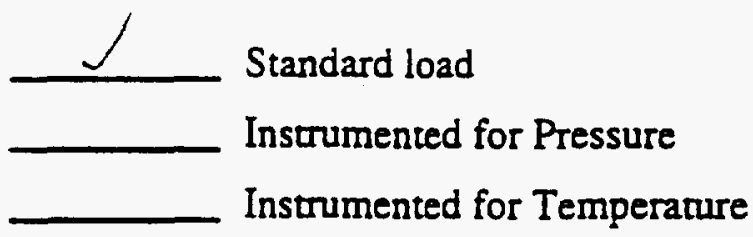

\begin{tabular}{|c|c|c|c|}
\hline Loading Staring at Bottom & Layer 1 & Layer 2 & Layer 3 \\
\hline 3 pieces of steel & $r$ & N/A & N/A \\
\hline $1 / 3$ of the brown paper & 工 & $\checkmark$ & $\checkmark$ \\
\hline $1 / 3$ of the 10 -mil plastic & r & $\gamma$ & \\
\hline $1 / 3$ of the bond paper & & $r$ & \\
\hline $1 / 3$ of the towels & & & \\
\hline $1 / 3$ of the bags & & 2 & \\
\hline $1 / 3$ of the cut paper & 2 & r & \\
\hline $1 / 3$ of the fabric & $\gamma$ & $r$ & \\
\hline $1 / 3$ of the rubber & & $\checkmark$ & 一 \\
\hline & Go to Layer 2 & Go to Layer 3 & \\
\hline
\end{tabular}

Drum Filled:

Ring bolt placed opposite drum seam and in down position

Lid sealed and torqued to $40 \mathrm{ft} / \mathrm{lb}$ :

Leak tested:

Pre-test weight (lb)

Post-test weight (lb)

$$
\text { Loss = }
$$

Date 5.3.95 Initial R.K

Date 5.3.95 Initial R.K

Date 5.3.95 Initial R./L

N/A or Pressure (psig)

Pressure (psig)

Date

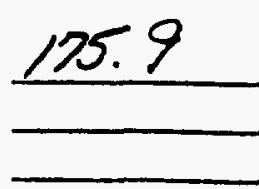

Time $(\min )$

Time (min) 5 
WHC-SD-WM-TRP-246 REV. 0

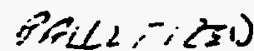

Rookstomge Drum Data Sheet

Drum No. $0912,70.9$ ib

Post-test Assessment

Position: __ originai: __ tipped: __ toppled

Lid on drum: $\quad X$ Yes No

Gasket missing $(\mathrm{cm}) \geq-1 !$

Contents expelled: $\quad$ Yes $x$ No

Seal failure: $\quad$ _ Yes $\quad x$ Not visible

Burn partern:

40" sean te RT

Photo taken:

Yes $X$ No

Leak tested:

N/A or Pressure (psig)

Pressure (psig)

Date

Initial

Time (min)

Time (min) 5

Interior assessment:

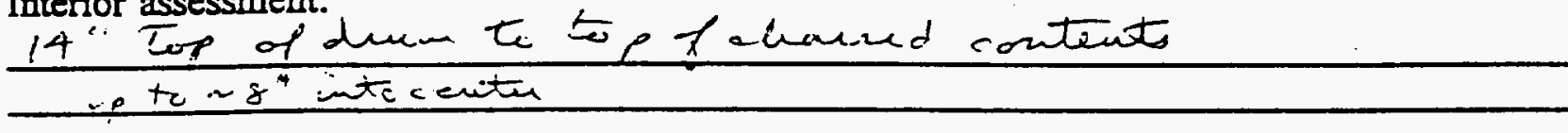

LDec 16

Photo taken:

$x$ Yes

Comments: 
WHC-SD-WM-TRP-246 REV. 0

\section{Palletized Storage Drum Data Sheet}

Drum No. 0922

Description (Check):

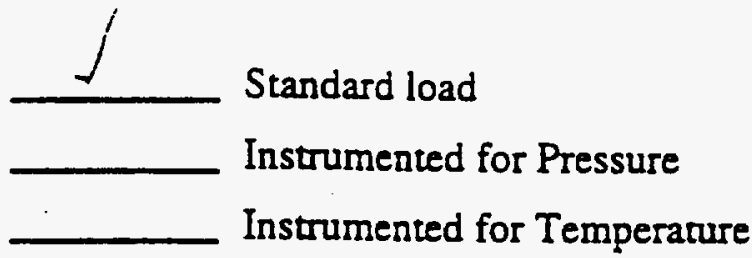

\begin{tabular}{|c|c|c|c|}
\hline Loading Starting at Bottom & Layer 1 & Layer 2 & Layer 3 \\
\hline 3 pieces of steel & - & N/A & N/A \\
\hline $1 / 3$ of the brown paper & - & & \\
\hline $1 / 3$ of the 10-mil plastic & & & \\
\hline $1 / 3$ of the bond paper & - & & \\
\hline $1 / 3$ of the towels & & & \\
\hline $1 / 3$ of the bags & & & \\
\hline $1 / 3$ of the cut paper & & & \\
\hline $1 / 3$ of the fabric & Go to Layer 2 & Go to Layer 3 & \\
\hline $1 / 3$ of the rubber & & \\
\hline
\end{tabular}

Drum Filled:

Date 5.3 .95 Initial L.K

Ring bolt placed opposite drum seam and in down position

Date 3.3 .95 Initial $L K$

Lid sealed and torqued to $40 \mathrm{ft} / \mathrm{b}$ :

Date 5-3.95 Initial R.K

Leak tested:

N/A or Pressure (psig)

Pressure (psig)

Date

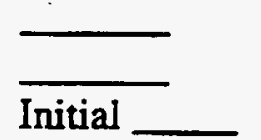

Time (min) $\ell$

Time $(\min ) 5$

Pre-test weight (lb)

Post-test weight (lb)

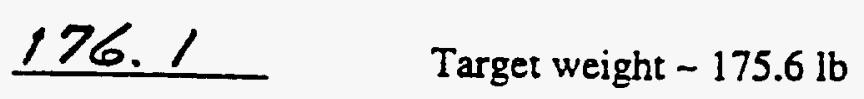

Loss $=$ 
Drum No. 5922.

\section{Post-test Assessment}

Position: originai: tipped: toppled

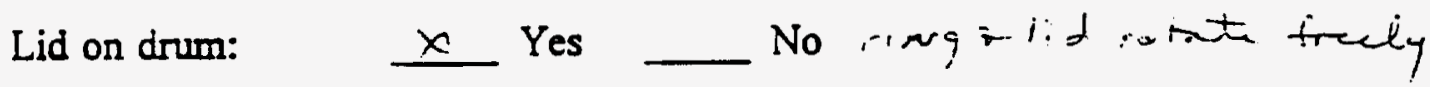

Gasket missing (cm) all

Contents expelled: $\quad$ Yes $\underline{X}$ No

Seal failure:

Yes $\quad \times$ Not visible

Burn pattern:

$10^{\circ}$ at mid lat ancund lid

Photo taken: $\quad$ Yes $\quad$ N No

Leak tested: $\quad$ N/A or Pressure (psig) stait $29^{\circ} \quad 12+f$ serem

Date

\section{Pressure (psig)}

Interior assessment:

$16 "$ is $p-1$ them to to $p$ of chas

VIDEc ;

Photo taken:

$x$ Yes

No

Comments: 


\section{Palletized Storage Drum Data Sheet}

Drum No. 0932

Description (Check):

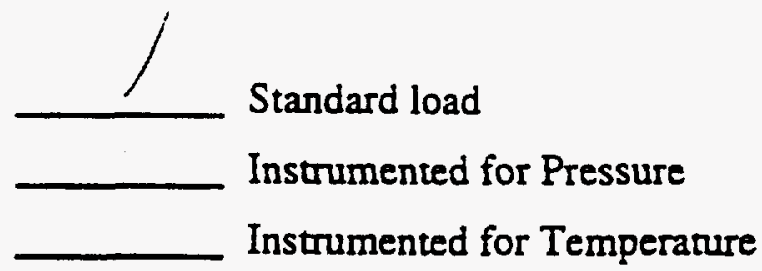

\begin{tabular}{|c|c|c|c|}
\hline Loading Staring at Bottom & Layer 1 & Layer 2 & Layer 3 \\
\hline 3 pieces of steel & 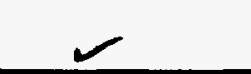 & N/A & N/A \\
\hline $1 / 3$ of the brown paper & r & $V$ & $r$ \\
\hline $1 / 3$ of the 10 -mil plastic & $\checkmark$ & $\swarrow$ & \\
\hline $1 / 3$ of the bond paper & 2 & $\gamma$ & 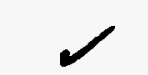 \\
\hline $1 / 3$ of the towels & $\checkmark$ & - & \\
\hline $1 / 3$ of the bags & 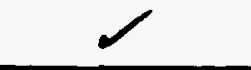 & $\checkmark$ & $\checkmark$ \\
\hline $1 / 3$ of the cut paper & & $\Omega$ & 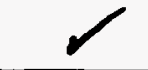 \\
\hline $1 / 3$ of the fabric & $\swarrow$ & & $\swarrow$ \\
\hline $1 / 3$ of the rubber & & $\checkmark$ & J \\
\hline & Go to Layer 2 & Go to Layer 3 & \\
\hline
\end{tabular}

Drum Filled:

Ring bolt placed opposite drum seam and in down position

Lid sealed and torqued to $40 \mathrm{ft} / \mathrm{b}$ :

Leak tested:

Pre-test weight (lb)

Post-test weight (lb)

\section{Loss $=$}

Date 5.3.55 Initial R.K

Date 5.3 .95 Initial R.K

Date 5.3.95 Initial R.K

N/A or Pressure (psig)

Pressure (psig)

Date

176.0

Initial

Target weight $-175.6 \mathrm{lb}$
Time (min) 0 Time (min) 5 
WHC-SD-WM-TRP-246 REV. 0

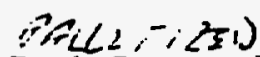

Rockstomge Drum Data Sheet

Drum No. $03952,-3,16$

Post-test Assessment

Position:

originai:

tipped:

toppled

Lid on drum: $\quad \nvdash \quad$ Yes _ No

Gasket missing (cm) 시

Contents expelled: _ـ Yes $\longrightarrow$ No

Seal failure: $\quad$ Y $\quad$ Yes $\$ Not visible

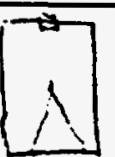

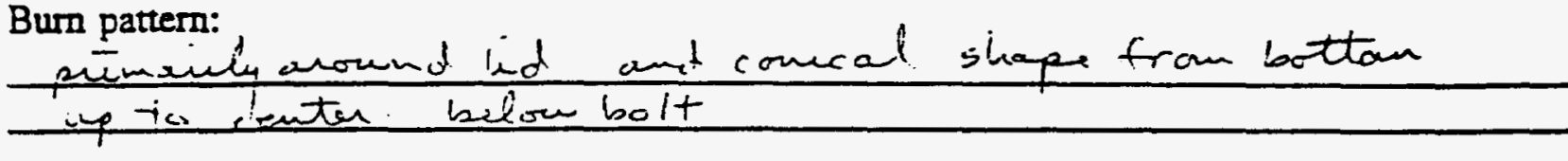

Photo taken: $\quad$ Yes $\quad X \quad$ No

Leak tested: $\quad$ NTA

Pressure (psig)

Date

$\longrightarrow \quad$\begin{tabular}{ll} 
Time (min) & 0 \\
\hline & Time (min)
\end{tabular}

Interior assessment:

15: top oh dume te top of chan

1 10ec 25

Pbotó taken:

$x$ Yes

Comments: 
WHC-SD-WM-TRP-246 REV. 0

\section{Palletized Storage Drum Data Sheet}

Drm No. 0942

Descriprion (Check):

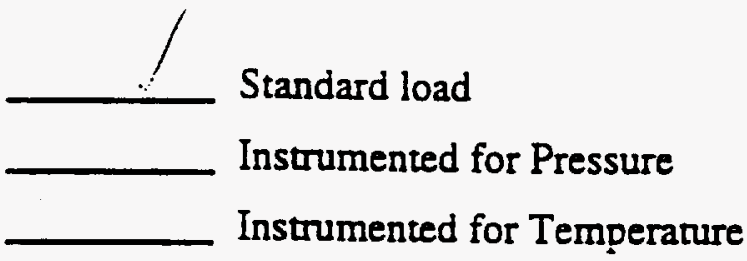

\begin{tabular}{|c|c|c|c|}
\hline Loading Starting at Bottom & Layer 1 & Layer 2 & Layer 3 \\
\hline 3 pieces of steel & - & N/A & N/A \\
\hline $1 / 3$ of the brown paper & - & $\checkmark$ & - \\
\hline $1 / 3$ of the 10 -mil plastic & - & $\sim$ & 2 \\
\hline $1 / 3$ of the bond paper & - & $\sim$ & - \\
\hline $1 / 3$ of the towels & $\sim$ & $\sim$ & $\sim$ \\
\hline $1 / 3$ of the bags & $\sim$ & $<$ & $r$ \\
\hline $1 / 3$ of the cut paper & $\sim$ & $\gamma$ & - \\
\hline $1 / 3$ of the fabric & 2 & $\gamma$ & - \\
\hline \multirow[t]{2}{*}{$1 / 3$ of the rubber } & $r$ & $\checkmark$ & $\gamma$ \\
\hline & Go to Layer 2 & Go to Layer 3 & \\
\hline
\end{tabular}

Drum Filled:

Date 5.3 .95 Initial $h . K$

Ring bolt placed opposite drum seam

Date 5.3-95 Initial R.K and in down position

Lid sealed and torqued to $40 \mathrm{ft} / \mathrm{b}$ :

Date 5.3.95 Initial $2 . K$

Leak tested:

N/A or Pressure (psig)

Pressure (psig)

Date

Initial

Time (min) 0

Time (min) 5

Pre-test weight (lb)

Post-test weight (lb)

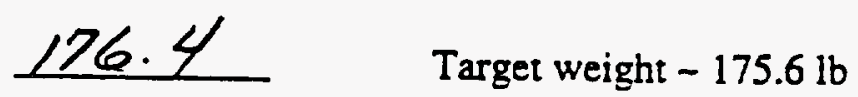

Loss $=$ 
WHC-SD-WM-TRP-246 REV. 0

IFALL, 一;

Reol-stomege Drum Data Sheet

Drum No. $\quad$ S442 $: 72 \leq 1 b$.

Post-test Assessment

Position:

originai:

tipped:

toppled

Lid on drum:

$X$ Yes No ring - lict rotate frealy

Gasket missing (cm) a

Contents expelled: __ Yes $x$ No

Seal failure: $\quad \quad$ Yes $\quad x$ Not visible

Burn partern:

$27 "$ at mid ht

Photo taken:

Yes $\underline{x}$ No

Leak tested:

Fa or Pressure (psig)

Date$$
\text { Date }
$$

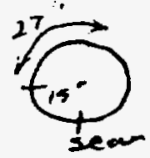

sear

\section{Pressure (psig)}

\section{Interior assessment:}

13 top ofum to top of char

\begin{tabular}{lll} 
& Time (min) & 0 \\
\hline Initial & Time (min)
\end{tabular}

VIDe: 26

Photo raken:

$X$ Yes

No

Comments: 


\section{Palletized Storage Drum Data Sheet}

Drum No. 0952

Descriprion (Check):

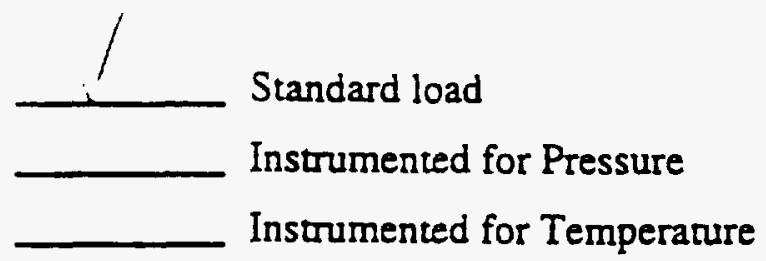

\begin{tabular}{|c|c|c|c|}
\hline Loading Starting at Bottom & Layer 1 & Layer 2 & Layer 3 \\
\hline 3 pieces of steel & $\sim$ & N/A & N/A \\
\hline $1 / 3$ of the brown paper & $\gamma$ & $\sim$ & 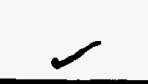 \\
\hline $1 / 3$ of the 10 -mil plastic & r & $r$ & r \\
\hline $1 / 3$ of the bond paper & 2 & 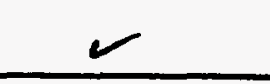 & $\checkmark$ \\
\hline $1 / 3$ of the towels & $r$ & $r$ & $\checkmark$ \\
\hline $1 / 3$ of the bags & $\checkmark$ & $r$ & $\checkmark$ \\
\hline $1 / 3$ of the cut paper & $r$ & $\swarrow$ & $>$ \\
\hline $1 / 3$ of the fabric & $\sim$ & $\checkmark$ & 工 \\
\hline $1 / 3$ of the rubber & $\gamma$ & $\checkmark$ & $\gamma$ \\
\hline & Go to Layer 2 & Go to Layer 3 & \\
\hline
\end{tabular}

Drum Filled:

Ring bolt placed opposite drum seam and in down position

Lid sealed and torqued to $40 \mathrm{ft} / \mathrm{b}$ :

Leak tested:

Pre-test weight (lb)

Post-test weight (lb)
Date $5=3.95$

Date 5.3.95

Date $5 \cdot 3 \cdot 95$

N/A or Pressure (psig)

Pressure (psig)

Date

176.4

Initial

Target weight $\sim 175.6 \mathrm{lb}$
Time (min) 0

Time (min) 5

\section{Loss $=$}


WHC-SD-WM-TRP-246 REV. 0

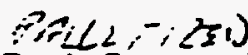

Roelt-Etonege Drum Data Sheet

Drum No. $0952 \quad 173.6 \mathrm{~h}$.

Post-test Assessment

Position:

original: tipped: toppled

Lid on drum: $\quad X$ Yes No ring $\quad$ Nlid loos

Gasket missing (cm) a

Contents expelled: __ Yes $\quad x$ No

Seal failure: $\quad$ Yes $\quad$ Y Not visible

Burn pattern: arcund rim and -12 "up from bettem, bolt side

Photo taken:

Yes $x$ No

Leak tested:

XPA or Pressure (psig)

Pressure (psig)

Date

\begin{tabular}{lll} 
Time (min) & 0 \\
\hline & Time (min)
\end{tabular}

Interior assessment:

14 " top filimen to top of chas

$\therefore$ pec 54

Photo taken:

$\chi$ Yes No

Comments: 


\section{Palletized Storage Drum Data Sheet}

Drim No. 0962

Description (Check):

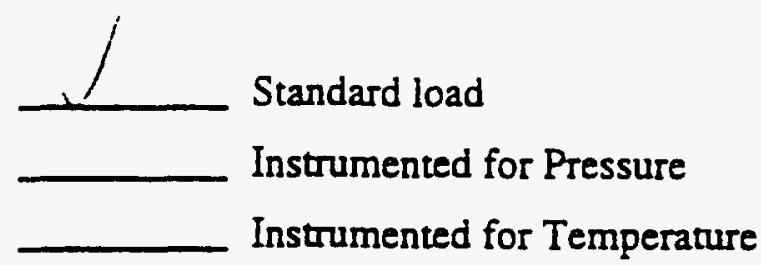

\begin{tabular}{|c|c|c|c|}
\hline Loading Starting at Bottom & Layer 1 & Layer 2 & Layer 3 \\
\hline \hline 3 pieces of steel & - & N/A & N/A \\
\hline $1 / 3$ of the brown paper & & & \\
\hline $1 / 3$ of the 10-mil plastic & & & \\
\hline $1 / 3$ of the bond paper & & & \\
\hline $1 / 3$ of the toweis & & & \\
\hline $1 / 3$ of the bags & - & $\checkmark$ & \\
\hline $1 / 3$ of the cut paper & & & \\
\hline $1 / 3$ of the fabric & Go to Layer 2 & Go to Layer 3 & \\
\hline $1 / 3$ of the rubber & & \\
\hline
\end{tabular}

Drum Filled:

Ring bolt placed opposite drum seam and in down position

Lid sealed and torqued to $40 \mathrm{ft} / \mathrm{lb}$ :

Leak tested:

Pre-test weight (lb)

Post-test weight (lb)
Date 5.3 .95 Initial R.K

Date 5.3.95 Initial R.K
N/A or Pressure (psig) Pressure (psig)

Date

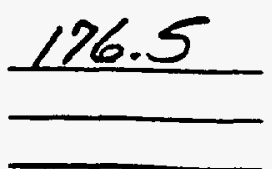

Time (min) Time $(\min )$

Initial

Target weight $\sim 175.6 \mathrm{lb}$

\section{Loss $=$}


Drum No. 0962 175.5 16

Post-test Assessment

Position: original: tipped: toppied

Lid on drum: $\quad Y$ Yes No lid r ringloose

Gasket missing (cm)

Contents expeiled: __ Yes $\underline{x}$ No

Seal failure: $\quad$ Yes $\quad X$ Not visible

Burn partern: around rim only - 6" hight spot below bitt

Photo taken: Y Yes $\underline{x}$ No

Leak tested: P1A or Pressure (psig) Pressure (psig) Date

\begin{tabular}{lll}
- & Time $(\min )$ & 0 \\
\hline
\end{tabular}$\quad$ Time (min)

Initial

Interior assessment:
top chaved - 5.6" all ar cund

Uidec S6

Photo taken:

$X$ Yes No

Comments: 


\section{Palletized Storage Drum Data Sheet}

Drum No. 0972

Description (Check):

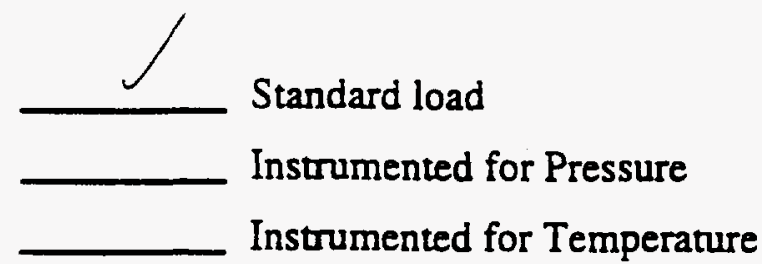

\begin{tabular}{|c|c|c|c|}
\hline Loading Starting at Bottom & Layer 1 & Layer 2 & Layer 3 \\
\hline 3 pieces of steel & - & N/A & N/A \\
\hline 1/3 of the brown paper & r & - & - \\
\hline $1 / 3$ of the 10 -mil plastic & 2 & > & r \\
\hline $1 / 3$ of the bond paper & r & $<$ & 2 \\
\hline $1 / 3$ of the toweis & 2 & 2 & 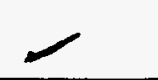 \\
\hline $1 / 3$ of the bags & r & r & $\sim$ \\
\hline $1 / 3$ of the cut paper & $<$ & 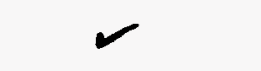 & 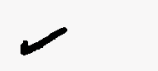 \\
\hline $1 / 3$ of the fabric & r & $r$ & $\checkmark$ \\
\hline \multirow[t]{2}{*}{$1 / 3$ of the rubber } & $\gamma$ & 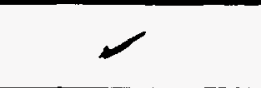 & r \\
\hline & Go to Layer 2 & Go to Layer 3 & \\
\hline
\end{tabular}

Drum Filled:

Ring bolt placed opposite drum seam and in down position

Lid sealed and torqued to $40 \mathrm{ft} / \mathrm{b}$ :

Leak tested:

Pre-test weight (lb)

Post-test weight (lb)

Date 5.3 .95 Initial R./

Date 5-3.95 Initial R.K

Date 5.3.95 Initial L.K

N/A or Pressure (psig)

Pressure (psig)

Date

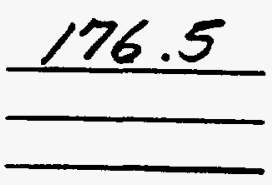

F-190
Time (min) 0

Time $(\min ) 5$ 
Drum No. $0972176.4 \mathrm{~kb}$.

\section{Post-test Assessment}

Position: original: tipped: toppled

Lid on drum: $\quad \Perp$ Yes $\quad$ No

Gasket missing (cm) $24^{\prime \prime}$

Contents expelled: $\quad$ Yes $x$ No

Seal failure: $\quad$ Y $\quad$ Yes $\quad X$ Not visible

Burn pattern:
top half of lid centered 20 'et. If sear
Nove on walls

Photo taken: Yes 즈 No

Leak tested:

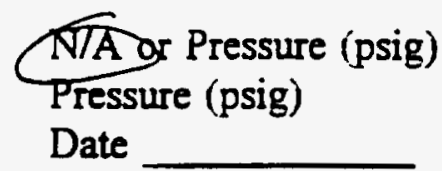

Date

\begin{tabular}{lll} 
& Time $(\min )$ & 0 \\
\hline & Time $(\min )$
\end{tabular}

Interior assessment:

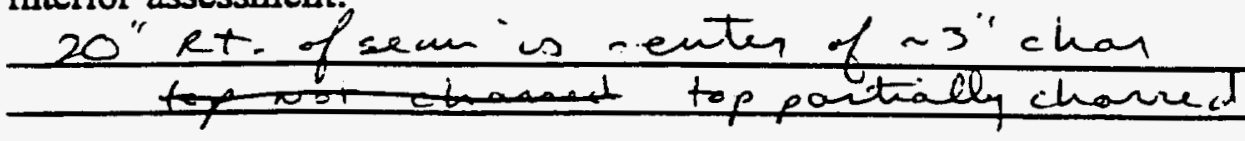

vinec 54

Photo taken:

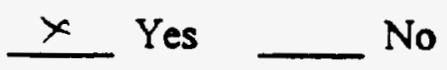

Comments: 
WHC-SD-WM-TRP-246 REV. 0

\section{Palletized Storage Drum Data Sheet}

Drum No. 0982

Description (Check):

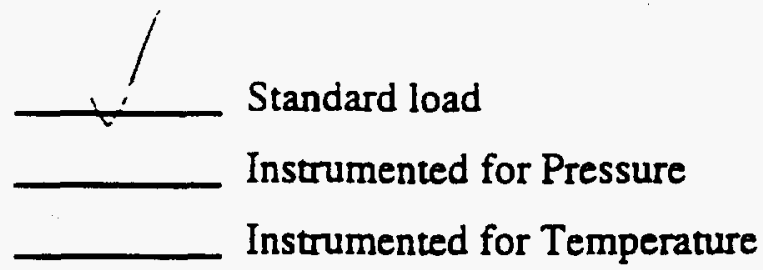

\begin{tabular}{|c|c|c|c|}
\hline Loading Starting at Bottom & Layer 1 & Layer 2 & Layer 3 \\
\hline 3 pieces of steel & $\sim$ & N/A & N/A \\
\hline \multicolumn{4}{|l|}{$1 / 3$ of the brown paper } \\
\hline \multicolumn{4}{|l|}{$1 / 3$ of the 10 -mil plastic } \\
\hline \multicolumn{4}{|l|}{$1 / 3$ of the bond paper } \\
\hline \multicolumn{4}{|l|}{$1 / 3$ of the towels } \\
\hline \multicolumn{4}{|l|}{$1 / 3$ of the bags } \\
\hline \multicolumn{4}{|l|}{$1 / 3$ of the cut paper } \\
\hline \multicolumn{4}{|l|}{$1 / 3$ of the fabric } \\
\hline \multicolumn{4}{|l|}{$1 / 3$ of the rubber } \\
\hline & Go to Layer 2 & Go to Layer 3 & \\
\hline
\end{tabular}

Drum Filled:

Ring bolt placed opposite drum seam and in down position

Lid sealed and torqued to $40 \mathrm{ft} / \mathrm{b}$ :

Leak tested:

Pre-test weight (lb)

Post-test weight (lb)
Date 5.12 .95 Initial R.R

Date $5-12.95$ Initial A.K
N/A or Pressure (psig)

Pressure (psig)

Date

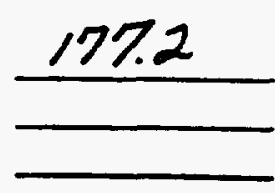

F-192
Time (min) 0

Time (min) 5

Initial

Target weight $\sim 175.6 \mathrm{lb}$

\section{Loss $=$}


Drum No. $0982,772 \mathrm{lb}$

Post-test Assessment

Position: origina: tipped: toppied

Lid on drum: $\quad \quad \quad$ Yes No

Gasket missing (cm) $O$ slighty compresse d

Contents expelled: _ Yes $X$ No

Seal failure: $\quad$ Yes $X$ Not visible

Burn pattern:

vene

Photo taken: Yes $\gg$ No

Leak tested:

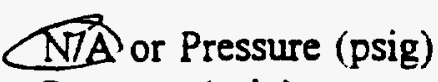

Pressure (psig)

Date

\begin{tabular}{lll}
- & Time $(\min )$ & 0 \\
\hline
\end{tabular}

Interior assessment:

\begin{tabular}{l} 
No metting \\
ve chan \\
\hline Photo taken: $\quad x$ Yes No No
\end{tabular}

Comments: 


\section{Palletized Storage Drum Data Sheet}

Drum No. $04 / 3$

Description (Check):

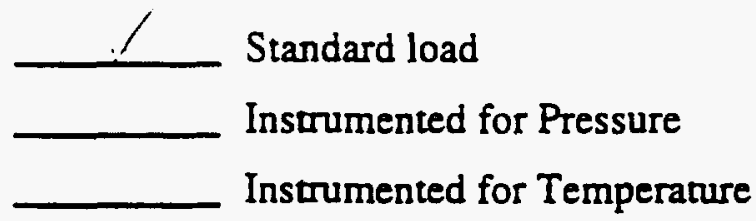

\begin{tabular}{|c|c|c|c|}
\hline Loading Staring at Bottom & Layer 1 & Layer 2 & Layer 3 \\
\hline 3 pieces of steel & $\checkmark$ & N/A & N/A \\
\hline $1 / 3$ of the brown paper & $\cdot$ & $\gamma$ & $v^{\prime}$ \\
\hline $1 / 3$ of the 10 -mil plastic & $\mathcal{L}$ & 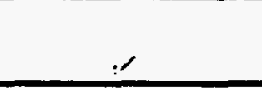 & $L^{\prime}$ \\
\hline $1 / 3$ of the bond paper & $\checkmark$ & $j$ & $\checkmark$ \\
\hline $1 / 3$ of the toweis & I & $\ell$ & $\mathscr{L}$ \\
\hline $1 / 3$ of the bags & $v$ & $\therefore$ & 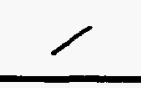 \\
\hline $1 / 3$ of the cut paper & $/$ & $\nearrow$ & $v$ \\
\hline $1 / 3$ of the fabric & $\sim$ & 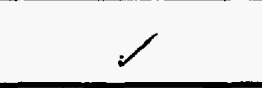 & $\zeta$ \\
\hline \multirow[t]{2}{*}{$1 / 3$ of the rubber } & $\gamma$ & ' & 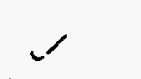 \\
\hline & Go to Layer 2 & Go to Layer 3 & \\
\hline
\end{tabular}

Drum Filled:

Ring bolt placed opposite drum seam and in down position

Lid sealed and torqued to $40 \mathrm{ft} / \mathrm{b}$ :

Leak tested:

Pre-test weight (lb)

Post-test weight (lb)
Date $5 \cdot 5 \cdot 95$ Initial AS

Date 5-5.95 Initial AS

Date $\underline{5 \cdot 5 \cdot 95}$ Initial $A 5$
N/A or Pressure (psig)

Pressure (psig)

Date

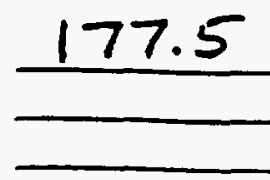

Time $(\min )$

Time (min) 5
Initial

Target weight $-175.6 \mathrm{lb}$ 
$P F(L 2,-;(51)$

Rowlesionage Drum Data Sheet

Drum No. 0413177.3 B.

Post-test Assessment

Position:

original:

tipped:

toppled

Lid on drum: $\quad \quad \quad$ Yes $\quad$ No

Gasket missing (cm) O Compressad o hand all around

Contents expelled: __ Yes $\underline{X}$ No

Seal failure: $\quad$ _ Yes $\quad X$ Not visible

Burn pattern:

NoNe

Photo taken:

Yes $\chi$ No

Leak tested:

NTA or Pressure (psig)

Pressure (psig)

Date

\begin{tabular}{lll}
- & Time (min) & 0 \\
\hline & Time (min)
\end{tabular}

Interior assessment:

No meltig

virec 92

Pheto taken:

X Yes

No

Comments: 
WHC-SD-WM-TRP-246 REV. 0

\section{Palletized Storage Drum Data Sheet}

Drum No. 0423

Description (Check):

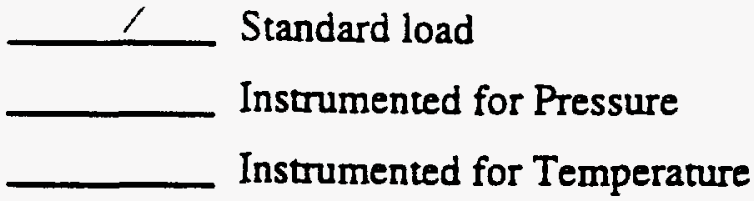

\begin{tabular}{|c|c|c|c|}
\hline Loading Starting at Bottom & Layer 1 & Layer 2 & Layer 3 \\
\hline 3 pieces of steel & J & N/A & N/A \\
\hline $1 / 3$ of the brown paper & $\iota^{\prime}$ & \llcorner & $1 ;$ \\
\hline $1 / 3$ of the 10 -mil plastic & V & $i^{\prime}$ & $l^{\prime}$ \\
\hline $1 / 3$ of the bond paper & $/$ & 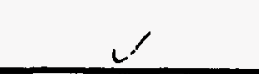 & $i^{\prime}$ \\
\hline $1 / 3$ of the towels & $\mathcal{J}$ & l & $V^{\prime}$ \\
\hline $1 / 3$ of the bags & 1 & $\checkmark$ & $\checkmark$ \\
\hline 1/3 of the cut paper & $\checkmark$ & $\checkmark$ & $V$ \\
\hline $1 / 3$ of the fabric & $\checkmark$ & $j$ & $V$ \\
\hline $1 / 3$ of the rubber & $i^{\prime}$ & $l^{\prime}$ & v \\
\hline & Go to Layer 2 & Go to Layer 3 & \\
\hline
\end{tabular}

Drum Filled:

Ring bolt placed opposite drum seam and in down position

Lid sealed and torqued to $40 \mathrm{ft} / \mathrm{lb}$ :

Leak tested:

Pre-test weight (lb)

Post-test weight (lb)

$$
\text { Loss }=
$$

Los Alamos Technical Associates
Date $5 \cdot 5 \cdot 95$ Initial AS

Date $5 \cdot 5 \cdot 95$ Initial $A S$
N/A or Pressure (psig)

Pressure (psig)

Date

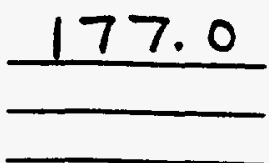

F-196
Time (min) $\quad 0$

Time (min) 5

Initial

Target weight $\sim 175.6 \mathrm{lb}$ 
WHC-SD-WM-TRP-246 REV. 0

$\because f\left(2,-i 2 \frac{2}{2} 1\right)$

Rowitstomege Drum Data Sheet

Drum No. $0423 \quad 176.9$

Post-test Assessment

Position:

originai:

tipped:

toppled

Lid on drum: $\quad x$ Yes No

Gasket missing (cm) 0 36" compressed Thave'

Contents expelled: _ Yes $\chi{ }^{-}$No

Seal failure: $\quad$ Yes $\quad x$ Not visible

Burn pattern:

None

Photo taken: _ Yes $\varnothing$ No

Leak tested: $\quad$ N/A

Pressure (psig)

Date

$-\quad$\begin{tabular}{ll} 
Time (min) & 0 \\
\hline & Time $(\min )$
\end{tabular}

Interior assessment:

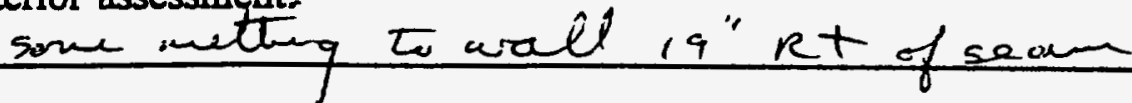

vipee 39

photo taken:

$x$ Yes _ No

Comments: 


\section{Palletized Storage Drum Data Sheet}

Drum No. 0433

Descripuion (Check):

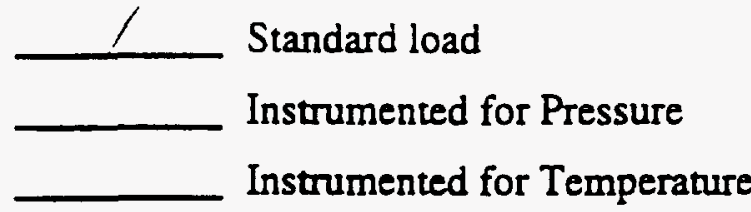

\begin{tabular}{|c|c|c|c|}
\hline Loading Starting at Bottom & Layer 1 & Layer 2 & Layer 3 \\
\hline 3 pieces of steel & 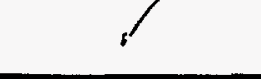 & N/A & N/A \\
\hline $1 / 3$ of the brown paper & L- & L' & $\because$ \\
\hline $1 / 3$ of the 10 -mil plastic & $r$ & $v^{\prime}$ & $L^{\prime}$ \\
\hline $1 / 3$ of the bond paper & $\nearrow$ & $\checkmark$ & $v^{\prime}$ \\
\hline $1 / 3$ of the toweis & I & $\checkmark$ & \\
\hline $1 / 3$ of the bags & 1 & $v$ & \\
\hline $1 / 3$ of the cut paper & 一 & $v^{\prime}$ & \\
\hline $1 / 3$ of the fabric & 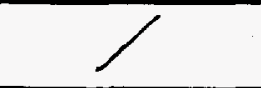 & $V$ & $v^{\prime}$ \\
\hline $1 / 3$ of the rubber & $i^{\prime}$ & $L^{\prime}$ & L- \\
\hline & Go to Layer 2 & Go to Layer 3 & \\
\hline
\end{tabular}

Drum Filled:

Ring bolt placed opposite drum seam and in down position

Lid sealed and torqued to $40 \mathrm{ft} / \mathrm{b}$ :

Leak tested:

Pre-test weight (lb)

Post-test weight (lb)

Date $5 \cdot 5 \cdot 95$ Initial AS

Date 5-5.95 Initial AS

Date 5-5.95 Initial $A 5$

N/A or Pressure (psig)

Pressure (psig)

Date

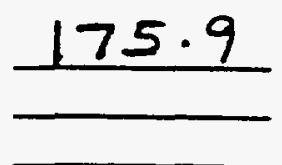

F-198
Time (min) 0

Time (min) 5
Initial

Target weight $\sim 175.6 \mathrm{lb}$

\section{Loss $=$}


WHC-SD-WM-TRP-246 REV. 0

Pru(L, - ; (2)

Rech Drange Drum Data Sheet

Drum No. 0433 '75. is ib.

Post-test Assessment

Position: ___ original: ___ tipped: ___ toppled

Lid on drum: $\quad \quad \quad$ Yes No

Gasket missing (cm) 0 co.ppussed t hand 30"

Contents expelled: _u Yes $\underline{x}$ No

Seal failure: $\quad$ Yes $\quad X$ Not visible

Burn pattern:

None

Photo taken: $\quad$ Yes $\quad x$ No

Leak tested:

N/A or Pressure (psig)

Pressure (psig)

Date

Not visible 


\section{Palletized Storage Drum Data Sheet}

Drum No. 0443

Description (Check):

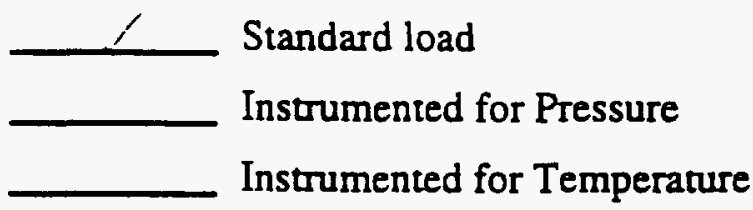

\begin{tabular}{|c|c|c|c|}
\hline Loading Starting at Bottom & Layer 1 & Layer 2 & Layer 3 \\
\hline 3 pieces of steel & $l^{\prime}$ & N/A & N/A \\
\hline $1 / 3$ of the brown paper & & $i^{\prime}$ & \\
\hline \multicolumn{4}{|l|}{$1 / 3$ of the 10 -mil plastic } \\
\hline $1 / 3$ of the bond paper & & $\checkmark$ & \\
\hline $1 / 3$ of the towels & $i^{\prime}$ & $\nearrow$ & \\
\hline $1 / 3$ of the bags & & $\checkmark$ & \\
\hline $1 / 3$ of the cut paper & $\checkmark$ & $v^{\prime}$ & \\
\hline $1 / 3$ of the fabric & $I^{\prime}$ & $\sim$ & 2 \\
\hline \multirow[t]{2}{*}{$1 / 3$ of the rubber } & $v^{\prime}$ & & \\
\hline & Go to Layer 2 & Go to Layer 3 & \\
\hline
\end{tabular}

Drum Filled:

Ring bolt placed opposite drum seam and in down position

Lid sealed and torqued to $40 \mathrm{ft} / \mathrm{lb}$ :

Leak tested:

Pre-test weight (lb)

Post-test weight (lb)

Date $5 \cdot 5 \cdot 95$ Initial AS

Date $\underline{5 \cdot 5 \cdot 95}$ Initial $A 5$

Date $5-5 \cdot 95$ Initial $A S$

N/A or Pressure (psig) Pressure (psig)

Date

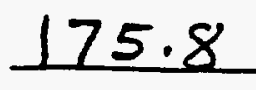

Time (min) Time (min) 5

Initial

Target weight $-175.6 \mathrm{lb}$ 
Drum No. $0443 \quad 75.7 \mathrm{~h}$

Post-test Assessment

Position:

original: tipped: toppled

Lid on drum: $\quad \quad \quad$ Yes No

Gasket missing (cm) $O$ GS" coipressed thand

Contents expeiled: __ Yes $x$ No

Seal failure: $\quad$ Yes $X$ Not visible

Bum pattern:

None

Photo taken:

Yes $\underset{\text { No }}{\longrightarrow}$

Leak tested:

NTA)or Pressure (psig)

Pressure (psig)

Date $\begin{array}{ll}\text { Time }(\min ) & 0 \\ \text { Time }(\min ) & \end{array}$

\section{Interior assessment:}

bavely melted below be It

Gedec 1C. 3

Photo taken:

$x$ Yes

Comments: 


\section{Palletized Storage Drum Data Sheet}

Drum No. 0453

Description (Check):

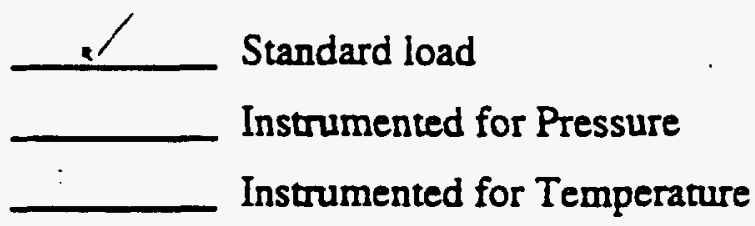

\begin{tabular}{|c|c|c|c|}
\hline Loading Starting at Bottom & Layer 1 & Layer 2 & Layer 3 \\
\hline 3 pieces of steel & $\checkmark$ & N/A & N/A \\
\hline $1 / 3$ of the brown paper & $6^{\prime}$ & $\iota^{\prime}$ & vi \\
\hline $1 / 3$ of the 10 -mil plastic & $\zeta$ & $\bar{V}$ & \\
\hline $1 / 3$ of the bond paper & , & $\sqrt{ }$ & $\checkmark$ \\
\hline $1 / 3$ of the towels & $\nearrow$ & $v$ & $V$ \\
\hline $1 / 3$ of the bags & / & $\checkmark$ & $\checkmark$ \\
\hline $1 / 3$ of the cut paper & $\checkmark$ & $\checkmark$ & $\checkmark$ \\
\hline $1 / 3$ of the fabric & $j$ & $\checkmark$ & $\sqrt{ }$ \\
\hline \multirow[t]{2}{*}{$1 / 3$ of the rubber } & $\sim^{\prime}$ & L.' & $V$ \\
\hline & Go to Layer 2 & Go to Layer 3 & \\
\hline
\end{tabular}

Drum Filled:

Ring boit placed opposite drum seam and in down position

Lid sealed and torqued to $40 \mathrm{ft} / \mathrm{b}$ :

Leak tested:

Pre-test weight (lb)

Post-test weight (lb)

Date $5 \cdot 5 \cdot 95$ Initial AS

Date 5-5.95 Initial AS

Date $5-5 \cdot 95$ Initial AS

N/A or Pressure (psig)

Pressure (psig)

Date

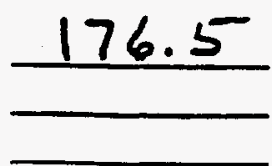

Time (min) 0 Time (min) 5

Initial

Target weight $\sim 175.6 \mathrm{lb}$ 
WHC-SD-WM-TRP-246 REV. O

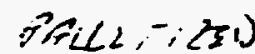

Roelt-Etonge Drum Data Sheet

Drum No. $0453 \quad 176.5 \mathrm{ib}$.

Post-test Assessment

Position: ___ original: ___ tipped: ___ toppled

Lid on drum: $\quad X$ Yes No

Gasket missing (cm) $O$ compressed and hand oll around

Contents expelled: __ Yes $x$ No

Seal failure: $\quad$ Yes $x$ Not visible

Burn pattern:

pone

Photo taken:

Yes $x$ No

Leak tested:

NTA or Pressure (psig)

Pressure (psig)

Date

\begin{tabular}{lll} 
Time (min) & 0 \\
\hline & Time (min)
\end{tabular}

Interior assessment:

wo char

. 1020116

Photo taken:

$x$ Yes

Comments: 


\section{Palletized Storage Drum Data Sheet}

Drum No. 0463

Description (Check):

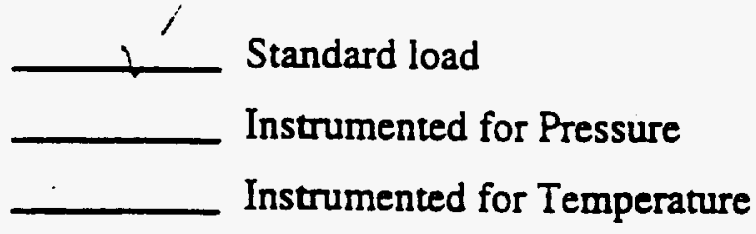

\begin{tabular}{|c|c|c|c|}
\hline Loading Starting at Bottom & Layer 1 & Layer 2 & Layer 3 \\
\hline 3 pieces of steel & $\checkmark$ & N/A & N/A \\
\hline $1 / 3$ of the brown paper & & & \\
\hline $1 / 3$ of the 10-mil plastic & $\checkmark$ & $V^{\prime}$ & \\
\hline $1 / 3$ of the bond paper & $\checkmark$ & $V^{\prime}$ & \\
\hline $1 / 3$ of the towels & $\checkmark$ & $\checkmark$ & \\
\hline $1 / 3$ of the bags & & & \\
\hline $1 / 3$ of the cut paper & $V^{\prime}$ & & \\
\hline $1 / 3$ of the fabric & $\checkmark$ & $V^{\prime}$ & \\
\hline $1 / 3$ of the rubber & Go to Layer 2 & Go to Layer 3 & \\
\hline
\end{tabular}

Drum Filled:

Date $5 \cdot 5 \cdot 95$ Initial AS

Ring boit placed opposite drum seam and in down position

Date $5-5 \cdot 95$ Initial AS

Lid sealed and torqued to $40 \mathrm{ft} / \mathrm{b}$ :

Date 5-5.95 Initial AS

Leak tested:

Pre-test weight (lb)

Post-test weight (lb)
N/A or Pressure (psig)

Pressure (psig)

Date

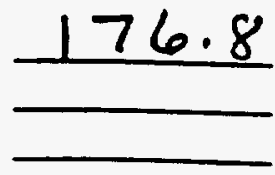

Time (min) 0

Time (min) 5

Initial

Target weight $\sim 175.6 \mathrm{lb}$ 


\section{Pre(L), - iका)}

Rook-siome Drum Data Sheet

Drum No. $0463,76.7 \mathrm{bl}$

Post-test Assessment

Position:

original:

tipped: toppled

Lid on drum: $\quad \propto$ Yes $\quad$ No

Gasket missing (cm) O somewhat compressed

Contents expelled: $\quad$ Yes $\underline{x}$ No

Seal failure: $\quad$ Y $\quad$ Yes $\quad x$ Not visible

Burn pattern: None

Photo taken:

Yes $x$ No

Leak tested:

NTA or Pressure (psig)

Pressure (psig)

Date

\begin{tabular}{lll}
- & Time $(\min )$ & 0 \\
\hline & Time (min)
\end{tabular}

Interior assessment:

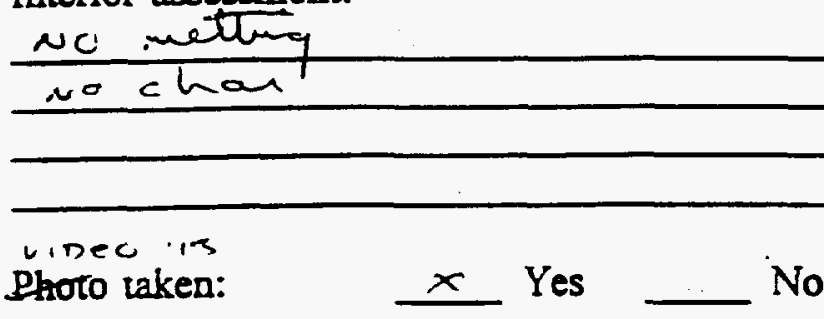

\section{Comments:}




\section{Palletized Storage Drum Data Sheet}

Drum No. 0473

Description (Check):

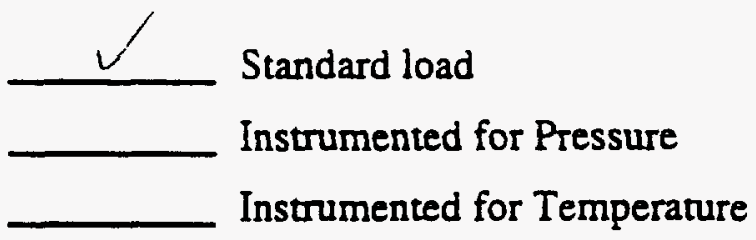

\begin{tabular}{|c|c|c|c|}
\hline Loading Staring at Bottom & Layer 1 & Layer 2 & Layer 3 \\
\hline 3 pieces of steel & $l^{\prime}$ & N/A & N/A \\
\hline $1 / 3$ of the brown paper & 1 & - & $i^{\prime}$ \\
\hline $1 / 3$ of the 10 -mil plastic & $V$ & $V^{\prime}$ & $v^{\prime}$ \\
\hline $1 / 3$ of the bond paper & $\checkmark$ & $\swarrow$ & 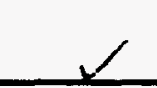 \\
\hline $1 / 3$ of the towels & $\checkmark$ & $\checkmark$ & 1 \\
\hline $1 / 3$ of the bags & $\checkmark$ & $\checkmark$ & $V^{\prime}$ \\
\hline $1 / 3$ of the cut paper & $\checkmark$ & $\checkmark$ & $r$ \\
\hline $1 / 3$ of the fabric & $i^{\prime}$ & $\checkmark$ & $V^{\prime}$ \\
\hline $1 / 3$ of the rubber & $\iota^{\prime}$ & $v^{\prime}$ & $v^{\prime}$ \\
\hline & Go to Layer 2 & Go to Layer 3 & \\
\hline
\end{tabular}

Drum Filled:

Ring bolt placed opposite drum seam and in down position

Lid sealed and torqued to $40 \mathrm{ft} / \mathrm{lb}$ :

Leak tested:

Pre-test weight (lb)

Post-test weight (lb)
Date $5 \cdot 5 \cdot 93$ Initial AS

Date $5 \cdot 5 \cdot 93$ Initial AS

Date $5 \cdot 5 \cdot 93 \quad$ Initial $A S$
N/A or Pressure (psig)

Pressure (psig)

Date

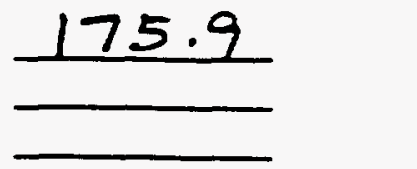

Time (min)

Time (min) 5
Initial

Target weight $-175.6 \mathrm{lb}$ 
Drum No. $0473,75.516$.

Post-test Assessment

Position: __ original: ___ tipped: __ toppled

Lid on drum: $\quad \quad \quad$ Yes No

Gasket missing (cm) O compressed \& hand $s^{\prime \prime}$

Contents expelled: _ Yes $x$ No

Seal failure: _ Yes $\quad$ Y Not visible

Burn pattern: Noxte

Photo taken: _ Yes $\$ No

$\begin{array}{lllll}\text { Leak tested: } & \text { Th/A } & \text { Time (min) } & 0 \\ \text { Pressure (psig) } & - & \text { Time (min) } & 5\end{array}$

Date

Interior assessment:

No chan
No incleting

$\checkmark 10<0$ is 2

Photo taken: $\quad x$ Yes _ No

Comments: 
WHC-SD-WM-TRP-246 REV. O

\section{Palletized Storage Drum Data Sheet}

Drum No. 0483

Description (Check):

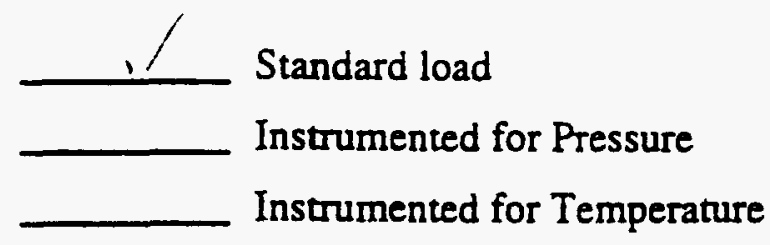

\begin{tabular}{|c|c|c|c|}
\hline Loading Starting at Bottom & Layer 1 & Layer 2 & Layer 3 \\
\hline 3 pieces of steel & $J$ & N/A & N/A \\
\hline $1 / 3$ of the brown paper & 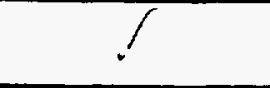 & $\mathcal{J}$ & $V^{\prime}$ \\
\hline $1 / 3$ of the 10 -mil plastic & Y & $\checkmark$ & $\sqrt{ }$ \\
\hline $1 / 3$ of the bond paper & 1 & i & $j$ \\
\hline $1 / 3$ of the towels & $j$ & $i$ & 1 \\
\hline $1 / 3$ of the bags & $v^{\prime}$ & $i$ & $r$ \\
\hline $1 / 3$ of the cut paper & $\nearrow$ & 1 & 1 \\
\hline $1 / 3$ of the fabric & $!$ & 1 & I \\
\hline $1 / 3$ of the rubber & $V$ & $i^{\prime}$ & i \\
\hline & Go to Layer 2 & Go to Layer 3 & \\
\hline
\end{tabular}

Drum Filled:

Ring bolt placed opposite drum seam and in down position

Lid sealed and torqued to $40 \mathrm{ft} / \mathrm{lb}$ :

Leak tested:

Pre-test weight (lb)

Post-test weight (lb)

$$
\text { Loss }=
$$

Date $5 \cdot 5 \cdot 95$ Initial AS

Date $5-5-95$ Initial $A S$

Date $5 \cdot 5 \cdot 95$ Initial AS

N/A or Pressure (psig)

Pressure (psig)

Date

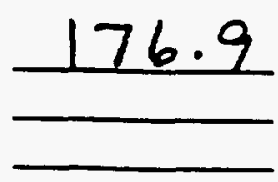

Time (min)

Time (min) 5 
Drum No. $0483,76.8$ ib.

Post-test Assessment

Position:

original:

tipped:

toppled

Lid on drum: $\quad X$ Yes _ No

Gasket missing (cm) O neppressedt hand all around

Contents expelled: $\quad$ Yes $\underline{\varnothing}$ No

Seal failure: $\quad$ Yes $\quad x$ Not visible

Burn partern:

none

Photo taken: $\quad$ Yes $x$ No

Leak tested: $\quad$ N/A or Pressure (psig)

Pressure (psig)

Date

\begin{tabular}{lll}
- & Time $(\min )$ & 0 \\
\hline & Time $(\min )$ &
\end{tabular}

Interior assessment:

we chan

ve ineting

$\therefore \sec 129$

Photo taken:

$x$ Yes _ No

\section{Comments:}




\section{Palletized Storage Drum Data Sheet}

Drum No. $05 / 3$

Description (Check):

2 Standard load Instrumented for Pressure Instrumented for Temperature

\begin{tabular}{|c|c|c|c|}
\hline Loading Starting at Bottom & Layer 1 & Layer 2 & Layer 3 \\
\hline 3 pieces of steel & $\checkmark$ & N/A & N/A \\
\hline $1 / 3$ of the brown paper & 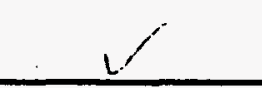 & $\zeta$ & $l$ \\
\hline $1 / 3$ of the 10 -mil plastic & $\checkmark$ & 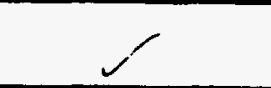 & $l^{\prime}$ \\
\hline $1 / 3$ of the bond paper & $\checkmark$ & $\checkmark$ & r \\
\hline $1 / 3$ of the toweis & $\checkmark$ & $\checkmark$ & $V$ \\
\hline $1 / 3$ of the bags & Y & $r$ & $\checkmark$ \\
\hline $1 / 3$ of the cut paper & $j^{\prime}$ & $v^{\prime}$ & $v^{\prime}$ \\
\hline $1 / 3$ of the fabric & $\gamma$ & $\checkmark$ & $v^{\prime}$ \\
\hline $1 / 3$ of the rubber & $\checkmark$ & $v^{\prime}$ & $V$ \\
\hline & Go to Layer 2 & Go to Layer 3 & \\
\hline
\end{tabular}

Drum Filled:

Ring bolt placed opposite drum seam and in down position

Lid sealed and torqued to $40 \mathrm{ft} / \mathrm{lb}$ :

Leak tested:

Pre-test weight (lb)

Post-test weight (lb)

$$
\text { Loss = }
$$

Date $5 \cdot 5 \cdot 95$ Initial AS

Date 5-5.95 Initial AS
N/A or Pressure (psig)

Pressure (psig)

Date

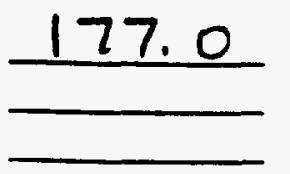

Time (min) 0

Time (min) 5 
WHC-SD-WM-TRP-246 REV. 0

Prill, - iका

Rech

Drum No. $0513: 77.0 \mathrm{~b}$.

Post-test Assessment

Position: __ original: ___ tipped: ___ toppled

Lid on drum: $\quad \leq$ Yes No

Gasket missing (cm) $21^{\prime \prime}$

Contents expelled: __ Yes $\underline{x}$ No

Seal failure: $\quad$ Yes $X$ Not visible

Burn pattern: id lat

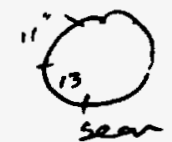

Photo taken: $\quad$ Yes $x$ No

Leak tested: $\quad$ (N/A Pressure (psig)

Date

\begin{tabular}{lll} 
Time (min) & 0 \\
\hline Initial & Time (min)
\end{tabular}

Interior assessment:

3-5" chan arreune

$\checkmark$ inec 90

Photo taken:

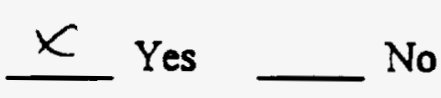

\section{Comments:}


WHC-SD-WM-TRP-246 REV. 0

\section{Palletized Storage Drum Data Sheet}

Drum No. 0523

Description (Check):

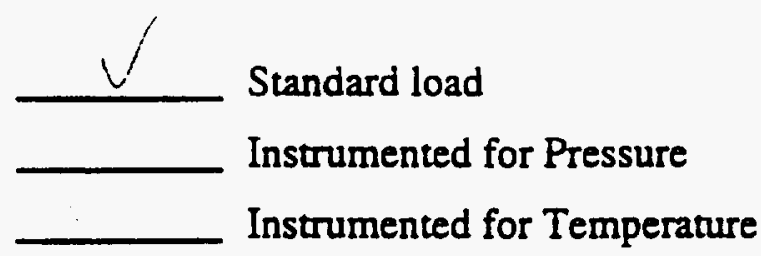

\begin{tabular}{|c|c|c|c|}
\hline Loading Starting at Bottom & Layer 1 & Layer 2 & Layer 3 \\
\hline 3 pieces of steel & $\checkmark$ & N/A & N/A \\
\hline $1 / 3$ of the brown paper & $i^{\prime}$ & $\imath^{\prime}$ & 1. \\
\hline $1 / 3$ of the 10 -mil plastic & v' & $V^{\prime}$ & $l$ \\
\hline $1 / 3$ of the bond paper & $\checkmark$ & l & $V^{\prime}$ \\
\hline $1 / 3$ of the towels & 1 & $\sqrt{ }$ & \\
\hline $1 / 3$ of the bags & 1 & $V$ & $r$ \\
\hline $1 / 3$ of the cut paper & $\sqrt{ }$ & $V^{\prime}$ & $\sqrt{ }$ \\
\hline $1 / 3$ of the fabric & $\int$ & $j^{\prime}$ & $1{ }^{\prime}$ \\
\hline \multirow[t]{2}{*}{$1 / 3$ of the rubber } & 1 & $i$ & L \\
\hline & Go to Layer 2 & Go to Layer 3 & \\
\hline
\end{tabular}

Drum Filled:

Ring bolt placed opposite drum seam and in down position

Lid sealed and torqued to $40 \mathrm{fu} / \mathrm{b}$ :

Leak tested:

Pre-test weight (lb)

Post-test weight (lb)

Date $5.5 \cdot 95$ Initial AS

Date $5 \cdot 5 \cdot 95 \quad$ Initial AS

Date $5 \cdot 5 \cdot 95 \quad$ Initial AS

N/A or Pressure (psig)

Pressure (psig)

Date

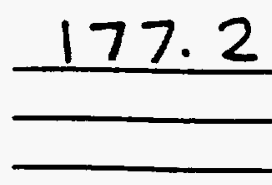

F-212
Time (min) 0

Time $(\min ) 5$

Initial

Target weight $\sim 175.6 \mathrm{lb}$ 
WHC-SD-WM-TRP-246 REV. 0

$P A(L 2,-i 251)$

Real-siomage Drum Data Sheet

Drum No. 0523 i7t 4

Post-test Assessment

Position:

original:

tipped:

toppled

Lid on drum: $\quad X$ Yes $\quad$ No

Gasket missing (cm) $37^{\prime \prime}$

Contents expelled: __ Yes $\$ No

Seal failure: $\quad$ Yes $\perp$ Not visible bottom bulged

Burn pattern:

i.t.id ht.

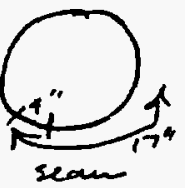

Photo taken:

Yes $\geq$ No

Leak tested:

NTA or Pressure (psig)

Pressure (psig)

Date

\begin{tabular}{lll} 
Time $(\min )$ & 0 \\
\hline & Time $(\min )$
\end{tabular}

Initial

Interior assessment:

10" top to top of chan

-10ec 9 ?

Photo taken:

$x$ Yes N No

Comments: 
WHC-SD-WM-TRP-246 REV. 0

\section{Palletized Storage Drum Data Sheet}

Drum No. 0533

Description (Check):

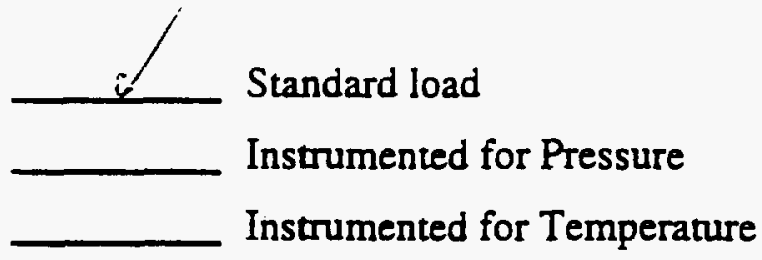

\begin{tabular}{|c|c|c|c|}
\hline Loading Starting at Bottom & Layer 1 & Layer 2 & Layer 3 \\
\hline 3 pieces of steel & 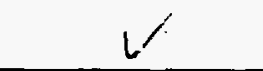 & N/A & N/A \\
\hline $1 / 3$ of the brown paper & 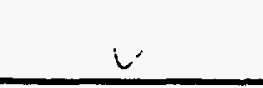 & 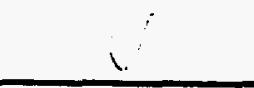 & $\iota^{\prime}$ \\
\hline $1 / 3$ of the 10 -mil plastic & $\checkmark$ & $i^{\prime}$ & $V$ \\
\hline $1 / 3$ of the bond paper & $i$ & 1 & 1 \\
\hline $1 / 3$ of the toweis & $i$ & $\prime^{\prime}$ & 1 \\
\hline $1 / 3$ of the bags & $i^{\prime}$ & 1 & 1 \\
\hline $1 / 3$ of the cut paper & $v^{\prime}$ & $v^{\prime}$ & $\checkmark$ \\
\hline $1 / 3$ of the fabric & 1 & 11 & $i^{\prime}$ \\
\hline \multirow[t]{2}{*}{$1 / 3$ of the rubber } & 1. & 9 & $l$ \\
\hline & Go to Layer 2 & Go to Layer 3 & \\
\hline
\end{tabular}

Drum Filled:

Ring boit placed opposite drum seam and in down position

Lid sealed and torqued to $40 \mathrm{ft} / \mathrm{b}$ :

Leak tested:

Pre-test weight (lb)

Post-test weight (lb)

$$
\text { Loss }=
$$

Date $5 \cdot 5 \cdot 95$ Initial AS

Date 5-5.95 Initial AS

Date $5-5.95$ Initial AS

N/A or Pressure (psig)

Pressure (psig)

Date

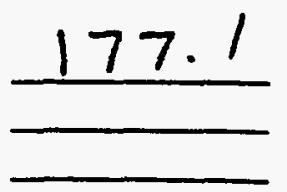

Time (min) 0 Time (min) 5 


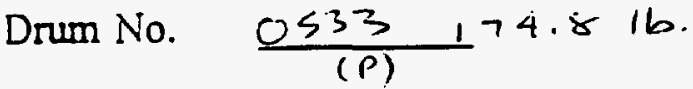

Post-test Assessment

Position: originai: tipped: toppled

Lid on drum:

$$
x \text { Yes No lidbuiged }-1 / 16 \times 2 \text { " erack }
$$

Gasket missing (cm) $47^{\prime \prime}$

Contents expelled: Yes $x$ No

Seal failure:

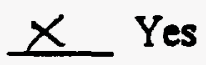
Not visible

Burn pattern:

$22 "$ ind lt. seam tu loft

Photo taken:

$\therefore$ Yes

Leak tested:

V7A or Pressure (psig) Pressure (psig)

Date

Initial

Time (min) 0

Time (min)

Interior assessment:

$14^{\circ}$ top te top fohar

Pinoto taken:

$\leftarrow$ Yes $\_$No

Comments: 


\section{Palletized Storage Drum Data Sheet}

Drum No. 0543

Description (Check):

$\begin{array}{ll}\ldots \ldots & \text { Standard load } \\ \text { Instrumented for Pressure } \\ \text { Instrumented for Temperature }\end{array}$

\begin{tabular}{|c|c|c|c|}
\hline Loading Starting at Bottom & Layer 1 & Layer 2 & Layer 3 \\
\hline 3 pieces of steel & $\checkmark$ & N/A & N/A \\
\hline $1 / 3$ of the brown paper & $\therefore$ & $\therefore-$ & $\smile$ \\
\hline $1 / 3$ of the 10 -mil plastic & - & r & 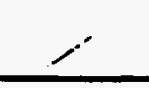 \\
\hline $1 / 3$ of the bond paper & $;$ & 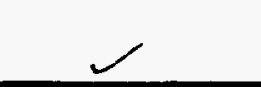 & $\sim$ \\
\hline $1 / 3$ of the towels & $\because$ & $\angle$ & $<$ \\
\hline $1 / 3$ of the bags & 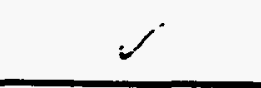 & $\mathcal{L}$ & $v^{\prime}$ \\
\hline 1/3 of the cut paper & $\because$ & - & 1 \\
\hline $1 / 3$ of the fabric & $\ddots^{\circ}$ & $\sim$ & $v^{-}$ \\
\hline $1 / 3$ of the rubber & $\omega$ & $\therefore$ & $\sim$ \\
\hline & Go to Layer 2 & Go to Layer 3 & \\
\hline
\end{tabular}

Drum Filled:

Date 5.5 .95 Initial $A 5$

Ring bolt placed opposite drum seam Date $5 \cdot 5 \cdot 95$ Initial $A S$ and in down position

Lid sealed and torqued to $40 \mathrm{ft} / \mathrm{b}: \quad$ Date $5-5.95$ Initial AS

Leak tested:

Pre-test weight (lb)

Post-test weight (lb)
N/A or Pressure (psig) Pressure (psig)

Date

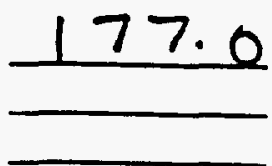

F-216
Time (min)

Time $(\min )$

Initial

Target weight $\sim 175.6 \mathrm{lb}$ 
Drum No. $0543,76.215$

Post-test Assessment

Position: original: tipped: toppled

Lid on drum:

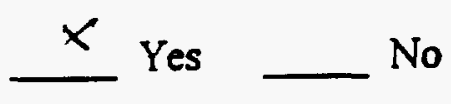

Gasket missing (cm) $39^{\prime \prime}$

Contents expelled: _ـ Yes $\not$ No

Seal failure:

Yes $x$ Not visible Lo Hom s lighttey buiged

Burn partern:

20 "at aid lt.

Photo taken:

$\therefore$ Yes $\nsim$ No

Leak tested:

\لRAOr Pressure (psig)

Pressure (psig)

Date

\begin{tabular}{lll} 
Time (min) & 0 \\
\hline Initial & Time (min)
\end{tabular}

Interior assessment:

$\frac{6^{\prime \prime} \text { te } \text { to tep }}{4-c^{\prime \prime} \text { chan all nownel }}$

$\because \cdot 7+c$ ic4

Photo taken:

$\underline{x}$ Yes

No

Comments: 
WHC-SD-WM-TRP-246 REV. 0

\section{Palletized Storage Drum Data Sheet}

Drum No. 0553

Descriprion (Check):

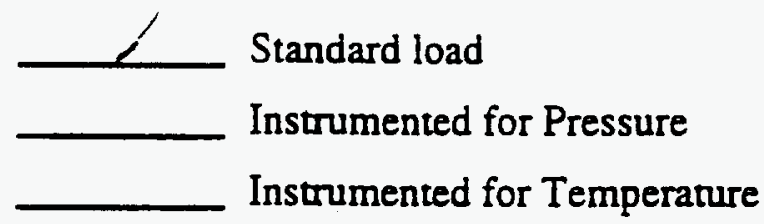

\begin{tabular}{|c|c|c|c|}
\hline Loading Starting at Bottom & Layer 1 & Layer 2 & Layer 3 \\
\hline 3 pieces of steel & N/A & N/A \\
\hline $1 / 3$ of the brown paper & & & \\
\hline $1 / 3$ of the 10-mil plastic & & & \\
\hline $1 / 3$ of the bond paper & & & \\
\hline $1 / 3$ of the towels & & & \\
\hline $1 / 3$ of the bags & & & \\
\hline $1 / 3$ of the cut paper & & & \\
\hline $1 / 3$ of the fabric & Go to Layer 2 & Go to Layer 3 & \\
\hline $1 / 3$ of the rubber & & \\
\hline
\end{tabular}

Drum Filled:

Ring bolt placed opposite drum seam and in down position

Lid sealed and torqued to $40 \mathrm{ft} / \mathrm{b}$ :

Leak tested:

Pre-test weight (lb)

Post-test weight (lb)

Date $5 \cdot 5 \cdot 95$ Initial AS

Date $5 \cdot 5 \cdot 95$ Initial AS

Date $5 \cdot 5 \cdot 95 \quad$ Initial $A 5$

N/A or Pressure (psig)

Pressure (psig)

Date

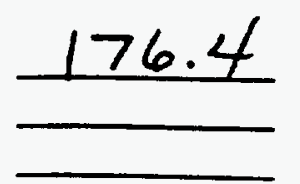

Time (min)

Time (min) 5

Initial

Target weight $\sim 175.6 \mathrm{lb}$ 
Drum No. $0553,76.1$ its.

Post-test Assessment

Position: original: tipped: toppled

Lid on drum: $\quad x$ Yes No

Gasket missing (cm) _ $20^{\prime \prime}$ centin d crm baun area

Contents expelled: __ Yes $x$ No

Seal failure: $\quad$ Yes $\quad x$ Not visible

Burn pattern:

$5 "$ mid wt. (top to bottom) seam tel-fot

Photo taken:

Leak tested:

Yes $\ldots$ No
N/A)or Pressure (psig) Pressure (psig)

Date

\begin{tabular}{lll} 
Time $(\min )$ & 0 \\
\hline
\end{tabular}

Interior assessment:

5 " dayp chan centered on berm area

inec 1/s

photo taken:

$x$ Yes _ No

Comments: 


\section{Palletized Storage Drum Data Sheet}

Drum No. 0563

Description (Check):

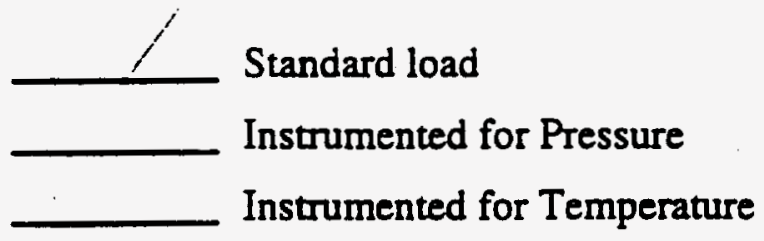

\begin{tabular}{|c|c|c|c|}
\hline Loading Starting at Bottom & Layer 1 & Layer 2 & Layer 3 \\
\hline 3 pieces of steel & j & N/A & N/A \\
\hline $1 / 3$ of the brown paper & 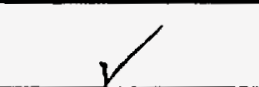 & $i$ & $v^{\prime}$ \\
\hline $1 / 3$ of the $10-\mathrm{mil}$ plastic & , & $1 ;$ & 1 \\
\hline $1 / 3$ of the bond paper & $i^{\prime}$ & it & $v^{\prime}$ \\
\hline $1 / 3$ of the towels & $\angle$ & $i^{\prime}$ & $1 \%$ \\
\hline $1 / 3$ of the bags & 5 & $\checkmark$ & $v^{\prime}$ \\
\hline $1 / 3$ of the cut paper & $1 /$ & 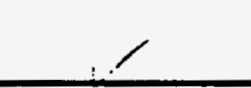 & $i^{\prime}$ \\
\hline $1 / 3$ of the fabric & $i /$ & $i$ & $r$ \\
\hline $1 / 3$ of the rubber & $i^{\prime}$ & $\because$ & $\therefore$ \\
\hline & Go to Layer 2 & Go to Layer 3 & \\
\hline
\end{tabular}

Drum Filled:

Ring bolt placed opposite drum seam and in down position

Lid sealed and torqued to $40 \mathrm{ft} / \mathrm{b}$ :

Leak tested:

Pre-test weight (lb)

Post-test weight (lb)
Date 5-5.95 Initial AS

Date $5 \cdot 5 \cdot 95$ Initial AS

Date $5 \cdot 5 \cdot 95 \quad$ Initial AS
N/A or Pressure (psig)

Pressure (psig)

Date

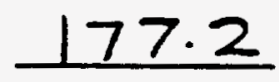

Time (min)

Time $(\mathrm{min})$
Initial

Target weight $-175.6 \mathrm{lb}$ 
Drum No. 0563 i77.1 $\mathrm{k}$

\section{$\underline{\text { Post-test Assessment }}$}

Position: __ original: ___ tipped: ___ toppled

Lid on drum: $\quad x$ Yes $\quad$ No

Gasket missing (cm) 0 compressect o hand all around, -8 "broun

Contents expelled: __ Yes $x$ No

Seal failure: $\quad$ Yes $\quad X$ Not visible

Burn pattern:

NCNe

Photo taken: $\quad$ Yes $x$ No

Leak tested:

Pressure (psig)

Date

Initial

Time $(\min ) \quad \frac{0}{5}$

Interior assessment:

16 " pan 26 " deep uelting rehan

Ploto taken:

$x$ Yes

Comments: 


\section{Palletized Storage Drum Data Sheet}

Drum No. 0573

Description (Check):

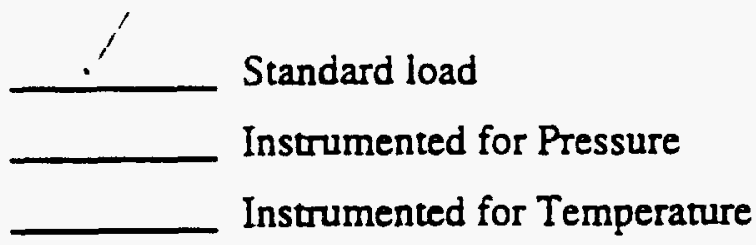

\begin{tabular}{|c|c|c|c|}
\hline Loading Starting at Bottom & Layer 1 & Layer 2 & Layer 3 \\
\hline 3 pieces of steel & $i^{\prime}$ & N/A & N/A \\
\hline $1 / 3$ of the brown paper & 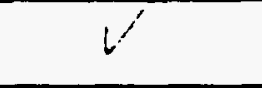 & $i$ & $i^{\prime}$ \\
\hline $1 / 3$ of the 10 -mil plastic & $v^{\prime}$ & $v^{\prime}$ & 6 \\
\hline $1 / 3$ of the bond paper & $\iota^{\prime}$ & 1 & 1 \\
\hline $1 / 3$ of the towels & 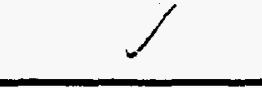 &.$!$ & $\mathrm{i}^{\prime}$ \\
\hline $1 / 3$ of the bags & $i$ & $v^{\prime}$ & $L^{\prime}$ \\
\hline $1 / 3$ of the cut paper & $\nearrow$ & $v^{\prime}$ & \\
\hline $1 / 3$ of the fabric & $i^{\prime}$ & $1 /$ & $i$ \\
\hline \multirow[t]{2}{*}{$1 / 3$ of the rubber } & $v^{\prime}$ & $v^{\prime}$ & \\
\hline & Go to Layer 2 & Go to Layer 3 & \\
\hline
\end{tabular}

Drum Filled:

Ring bolt placed opposite drum seam and in down position

Lid sealed and torqued to $40 \mathrm{ft} / \mathrm{b}$ :

Leak tested:

Pre-test weight (lb)

Post-test weight (lb)

$$
\text { Loss }=
$$

Date 5.5.95 Initial AS

Date $5 \cdot 5 \cdot 95$ Initial AS

Date $5-5 \cdot 95$ Initial AS

N/A or Pressure (psig)

Pressure (psig)

Date

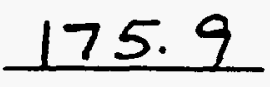

Initial

Time (min) 0

Time $(\min ) 5$ 
*ak RMIS View/Frint Document Cover Sheet tow

This document was retrieved from the Documentation and Records Manaqement (DRM) ISEARCH System. It is intended for Information only and may not be the most recent or updated version. Contact a Document Service Center (see Hanford Info for locations) if you need additional retrieval information.

Accession \#: D195061642

Document \#: SD-WM-TRP-246

Title/Desc:

SOLID WASTE DRUM ARRAY FIRE PERFORMANCE [SEC 7 OF 7]

Pages: 73 
THIS DOCUMENT WAS TOO LARGE TO SCAN AS A SINGLE

DOCUMENT. IT REQUIRED BREAKING INTO SMALLER SECTIONS.

DOCUMENT NUMBER: SD-WM.TRP. 246

SECTION: 7 OF 7

TITLE: Aolid waote Drum array firie Performance

AUTHOR: Louie RL

RECIPIENT:

DATE: $11 / 07 / 95$

KEYWORDS:

REFERENCES: EDT-612232 
Blante 
Drum No. $0573+75.8$

Post-test Assessment

Position: ___ original: ___ _tipped: ___ toppled

Lid on drum: $\quad x$ Yes No

Gasket missing (cm) $O 21$ "compressed and hand

Contents expelled: _ Yes $x$ No

Seal failure: $\quad$ Yes $\quad x$ Not visible

Burn pattern:

Noxve

Photo taken:

Yes $\propto$ No

Leak tested:

Pressure (psig)
Date

$\overline{\text { Initial }}$

Time (min) $\quad 0$

Time (min)

Interior assessment:

melting 19" span about sean

ineci3l

Photo taken:

$x$ Yes

\section{Comments:}




\section{Palletized Storage Drum Data Sheet}

Drum No. 0583

Description (Check):

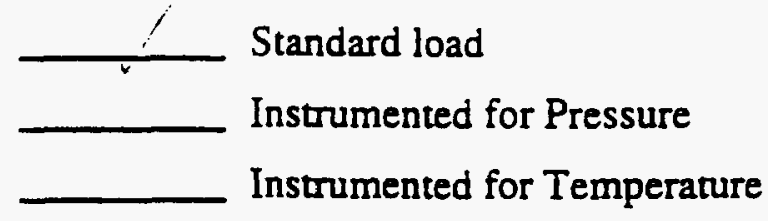

\begin{tabular}{|c|c|c|c|}
\hline Loading Starting at Bottom & Layer 1 & Layer 2 & Layer 3 \\
\hline 3 pieces of steel & $i^{\prime}$ & N/A & N/A \\
\hline $1 / 3$ of the brown paper & $\therefore$ & $i$ & $l^{\prime}$ \\
\hline $1 / 3$ of the 10 -mil plastic & 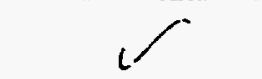 & $v^{\prime}$ & $V$ \\
\hline $1 / 3$ of the bond paper & $\swarrow$ & V & \\
\hline $1 / 3$ of the towels & $\checkmark$ & I & \\
\hline $1 / 3$ of the bags & $i$ & 1 & \\
\hline $1 / 3$ of the cut paper & $6^{\prime}$ & $y^{\prime}$ & \\
\hline $1 / 3$ of the fabric & $\checkmark$ & 1 & $v^{\prime}$ \\
\hline $1 / 3$ of the rubber & $l^{\prime}$ & $V^{\prime}$ & $\not$ \\
\hline & Go to Layer 2 & Go to Layer 3 & \\
\hline
\end{tabular}

Drum Filled:

Ring bolt placed opposite drum seam and in down position

Lid sealed and torqued to $40 \mathrm{ft} / \mathrm{lb}$ :

Leak tested:

Pre-test weight (lb)

Post-test weight (lb)

Date $5.5 \cdot 95$ Initial AS

Date $5-5 \cdot 95$ Initial AS

Date 5-5.95 Initial AS

N/A or Pressure (psig)

Pressure (psig)

Date

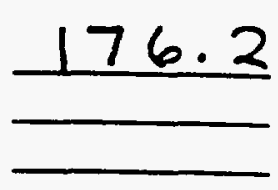

F-224
Time (min) 0

Time $(\min ) 5$

Initial

Target weight $\sim 175.6 \mathrm{lb}$ 
Drum No. $0583,76.1$

Post-test Assessment

Position: original: tipped: toppled

Lid on drum: $\quad \chi$ Yes No

Gasket missing (cm) $O 20$ "corpreased and hand

Contents expeiled: _ـ Yes $X$ No

Seal failure:

Yes $\times$ Not visible

Burn pattern:

None

Photo taken: $\quad$ Yes $\cong$ No

Leak tested: $\quad$ N/A

Pressure (psig)

Date

Time $(\min ) \quad 0$

Time (min)

$\overline{\text { Initial }}$

Interior assessment:

slighilly wettid to wall $11^{\prime \prime}$ to Rt of seam

vineo isc

Photo taken:

$x$ Yes

No

Comments: 
WHC-SD-WM-TRP-246 REV. 0

\section{Palletized Storage Drum Data Sheet}

Drum No. 0613

Description (Check):

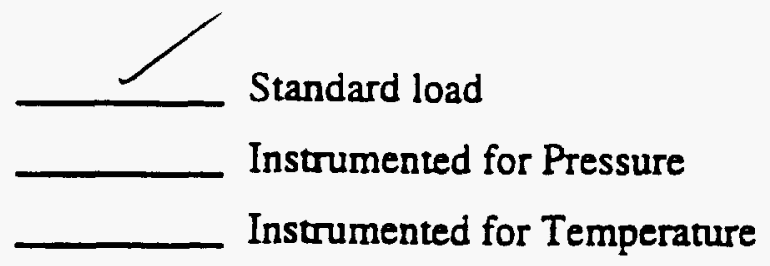

\begin{tabular}{|c|c|c|c|}
\hline Loading Starting at Bottom & Layer 1 & Layer 2 & Layer 3 \\
\hline 3 pieces of steel & & N/A & N/A \\
\hline $1 / 3$ of the brown paper & & & \\
\hline $1 / 3$ of the 10-mil plastic & & & \\
\hline $1 / 3$ of the bond paper & & & \\
\hline $1 / 3$ of the toweis & & & \\
\hline $1 / 3$ of the bags & & & \\
\hline $1 / 3$ of the cut paper & & Go to Layer 3 & \\
\hline $1 / 3$ of the fabric & Go to Layer 2 & (1/3 of the rubber & \\
\hline
\end{tabular}

Drum Filled:

Ring bolt placed opposite drum seam and in down position

Lid sealed and torqued to $40 \mathrm{ft} / \mathrm{b}$ :

Leak tested:

Pre-test weight (lb)

Post-test weight (lb)

$$
\text { Loss }=
$$

Date $5 \cdot 5 \cdot 95$ Initial AS

Date $5.5 \cdot 95$ Initial 45

Date $5-5.95$ Initial AS

N/A or Pressure (psig)

Pressure (psig)

Date
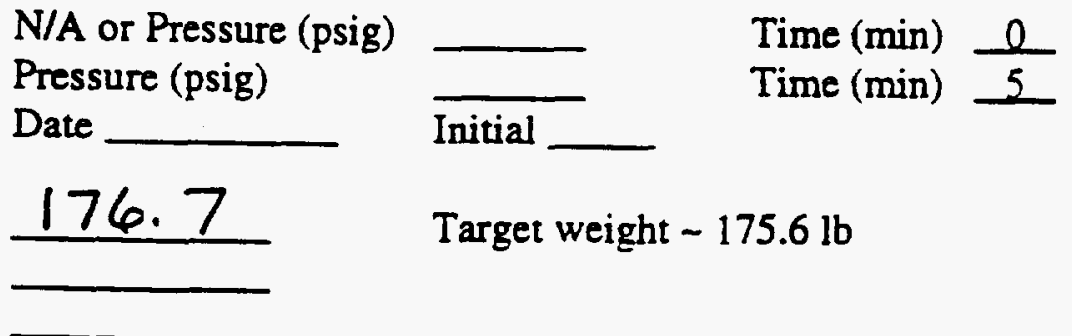

Target weight $\sim 175.6 \mathrm{lb}$ 
Drum No. C61? 16.516

\section{Post-test Assessment}

Position: original: tipped: toppled

Lid on drum: $\quad<$ Yes $\quad$ No Gasket missing (cm) Contents expelled: _ Yes $x$ No

Seal failure: $\quad$ Yes $\quad x$ Not visible buigi at belt

Burn pattern:
26 "at mid let stants, $2^{\prime \prime}$ to lept of sian

Photo taken: Yes $x$ No

Leak tested:

VIIA or Pressure (psig) Pressure (psig)

Date

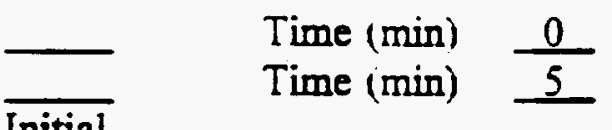

Interior assessment:

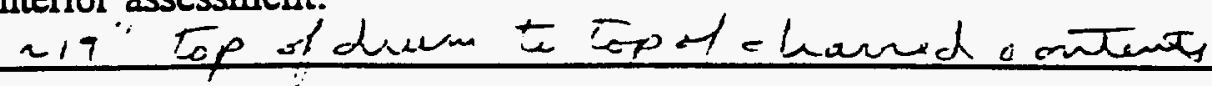

id Dec) 5

Pfoto taken:

$x$ Yes

\section{Comments:}


WHC-SD-WM-TRP-246 REV. 0

\section{Palletized Storage Drum Data Sheet}

Drum No. 0623

Description (Check):

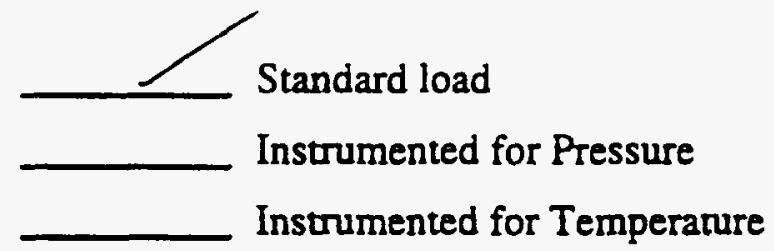

\begin{tabular}{|c|c|c|c|}
\hline Loading Starting at Bottom & Layer 1 & Layer 2 & Layer 3 \\
\hline 3 pieces of steel & & & N/A \\
\hline $1 / 3$ of the brown paper & & & \\
\hline $1 / 3$ of the 10 -mil plastic & & & \\
\hline $1 / 3$ of the bond paper & & & \\
\hline $1 / 3$ of the towels & & & \\
\hline $1 / 3$ of the bags & & & \\
\hline $1 / 3$ of the cut paper & & & \\
\hline $1 / 3$ of the fabric & & Go to Layer 3 & \\
\hline $1 / 3$ of the rubber & & & \\
\hline
\end{tabular}

Drum Filled:

Ring bolt placed opposite drum seam and in down position

Lid sealed and torqued to $40 \mathrm{ft} / \mathrm{b}$ :

Leak tested:

Pre-test weight (lb)

Post-test weight (lb)

$$
\text { Loss }=
$$

Los Alamos Technical Associates
Date $5 \cdot 5 \cdot 95$ Initial AS

Date $5-5 \cdot 95$ Initial AS

Date $5-5.95$ Initial AS
N/A or Pressure (psig)

Pressure (psig)

Date

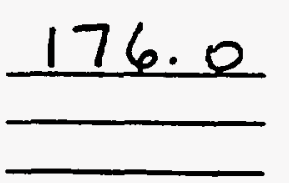

$F-228$
Time $(\min )$ Time $(\min )+5$
Initial

Target weight $\sim 175.6 \mathrm{lb}$

SwRI Projec No. 01-7106-001 
Drum No. Q623 int $=16,7916$.

\section{Post-test Assessment}

Position: ___ original: ___ tipped: ___ toppled

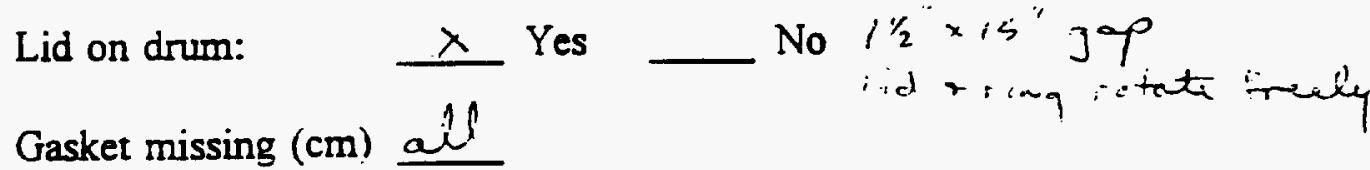

Contents expelled: $\quad$ Yes $\longleftarrow$ No

Seal failure: $\quad x$ Yes _ Not visible

Burn partern:

$35^{\prime \prime}$ at midl lt staiting

Photo taken: $\quad x$ Yes No

\begin{tabular}{llll} 
Leak tested: & $\widehat{N / A b r}$ Pressure (psig) & Time (min) & 0 \\
\hline Pressure (psig) & - & Time (min) & -5
\end{tabular}

Date $\overline{\text { Initial }}$

Interior assessment:

is" top - them to top of chand contents

ViTeco $\dot{0}$

Bhoto taken:

$X$ Yes N No

Comments: 


\section{Palletized Storage Drum Data Sheet}

Drum No. 0633

Descriprion (Check):

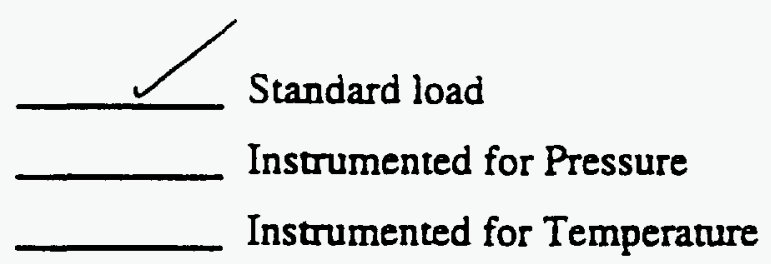

\begin{tabular}{|c|c|c|c|}
\hline Loading Starting at Bottom & Layer 1 & Layer 2 & Layer 3 \\
\hline 3 pieces of steel & & & \\
\hline $1 / 3$ of the brown paper & & & \\
\hline $1 / 3$ of the 10-mil plastic & & & \\
\hline $1 / 3$ of the bond paper & & & \\
\hline $1 / 3$ of the towels & & & \\
\hline $1 / 3$ of the bags & & & \\
\hline $1 / 3$ of the cut paper & & Go to Layer 3 & \\
\hline $1 / 3$ of the fabric & & & \\
\hline $1 / 3$ of the rubber & Go to Layer 2 & GolA \\
\hline
\end{tabular}

Drum Filled:

Ring bolt placed opposite drum seam and in down position

Lid sealed and torqued to $40 \mathrm{ft} / \mathrm{lb}$ :

Leak tested:

Pre-test weight (lb)

Post-test weight (lb)
Date $5.5 \cdot 95$ Initial A.5.

Date $5 \cdot 5-95$ Initial A.S.

Date $5 \cdot 5 \cdot 95$ Initial A.S.
N/A or Pressure (psig)

Pressure (psig)

Date

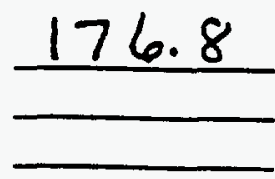

Time (min) 0

Time $(\mathrm{min})$
Initial

Target weight $\sim 175.6 \mathrm{lb}$ 
Drum No. $0.033(p): 53: 16$.

\section{Post-test Assessment}

Position: original: tipped: toppied

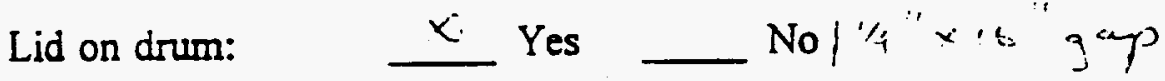

Gasket missing (cm) cill

Contents expelled: _u Yes $\underline{K}$ No

Seal failure: $\quad x$ Yes $\quad$ Not visible

Burn pattern:

al aroune

Photo taken:

Yes $\quad \times$ No

Leak tested:

N/A or Pressure (psig) Pressure (psig)

Date

\begin{tabular}{lll} 
Time (min) & 0 \\
\hline & Time (min)
\end{tabular}

Interior assessment:

-21 top fol dum titop of thas $\overline{\text { Initial }}$

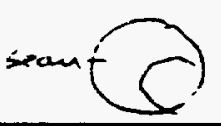

Comments: 


\section{Palletized Storage Drum Data Sheet}

Drum No. 0643

Descriprion (Check):

Standard load Instrumented for Pressure Instrumented for Temperature

\begin{tabular}{|c|c|c|c|}
\hline Loading Starting at Bottom & Layer 1 & Layer 2 & Layer 3 \\
\hline 3 pieces of steel & & N/A & N/A \\
\hline \multicolumn{4}{|l|}{ 1/3 of the brown paper } \\
\hline \multicolumn{4}{|l|}{$1 / 3$ of the 10 -mil plastic } \\
\hline \multicolumn{4}{|l|}{$1 / 3$ of the bond paper } \\
\hline \multicolumn{4}{|l|}{$1 / 3$ of the towels } \\
\hline \multicolumn{4}{|l|}{$1 / 3$ of the bags } \\
\hline \multicolumn{4}{|l|}{$1 / 3$ of the cut paper } \\
\hline \multicolumn{4}{|l|}{$1 / 3$ of the fabric } \\
\hline \multirow[t]{2}{*}{$1 / 3$ of the rubber } & & & \\
\hline & Go to Layer 2 & Go to Layer 3 & \\
\hline
\end{tabular}

Drum Filled:

Ring bolt placed opposite drum seam and in down position

Lid sealed and torqued to $40 \mathrm{ft} / \mathrm{lb}$ :

Leak tested:

Pre-test weight (lb)

Post-test weight (lb)

Date $5.5 \cdot 95$ Initial AS

Date 5.5 .95 Initial $A 5$

Date $5-5 \cdot 95$ Initial AS

N/A or Pressure (psig)

Pressure (psig)

Date

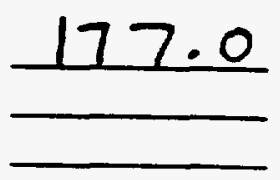

Time (min)

Initial

Target weight $-175.6 \mathrm{lb}$ 
Drum No. S54? ; 99.4

Post-test Assessment

Position: __ original: ___ tipped: ___ toppled

Lid on drum: $\quad x$ Yes No ${ }_{\text {gep }}^{3 / 5} \times s^{\prime \prime}$

Gasket missing (cm) che

Contents expelled: —_ Yes $\leq$ No

Seal failure: $\quad \quad K$ Yes _ Not visible

Burn partern:

als ocound

Photo taken:

$$
\text { Yes }, \ldots \text { No }
$$

Leak tested:

N/A or Pressure (psig)

Pressure (psig)

Date

\begin{tabular}{lll} 
Time (min) & 0 \\
\hline & Time (min)
\end{tabular}

\section{Interior assessment:}

20 "Top if dhum to tope of chat

$\checkmark 10<043$

Photo taken:

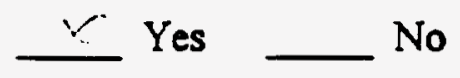

Comments: 


\section{Palletized Storage Drum Data Sheet}

Drum No. 0653

Description (Check):

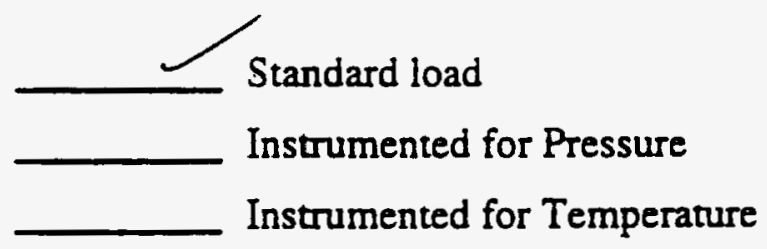

\begin{tabular}{|c|c|c|c|}
\hline Loading Starting at Bottom & Layer 1 & Layer 2 & Layer 3 \\
\hline 3 pieces of steel & & N/A & N/A \\
\hline \multicolumn{4}{|l|}{$1 / 3$ of the brown paper } \\
\hline \multicolumn{4}{|l|}{$1 / 3$ of the 10 -mil plastic } \\
\hline \multicolumn{4}{|l|}{$1 / 3$ of the bond paper } \\
\hline \multicolumn{4}{|l|}{$1 / 3$ of the towels } \\
\hline \multicolumn{4}{|l|}{$1 / 3$ of the bags } \\
\hline \multicolumn{4}{|l|}{$1 / 3$ of the cut paper } \\
\hline \multicolumn{4}{|l|}{$1 / 3$ of the fabric } \\
\hline \multicolumn{4}{|l|}{$1 / 3$ of the rubber } \\
\hline & Go to Layer 2 & Go to Layer 3 & \\
\hline
\end{tabular}

Drum Filled:

Ring bolt placed opposite drum seam and in down position

Lid sealed and torqued to $40 \mathrm{ft} / \mathrm{b}$ :

Leak tested:

Pre-test weight (lb)

Post-test weight (lb)

$$
\text { Loss = }
$$

Date $5-5 \cdot 95$ Initial $A S$

Date $5 \cdot 5 \cdot 95$ Initial AS
N/A or Pressure (psig)

Pressure (psig)

Date

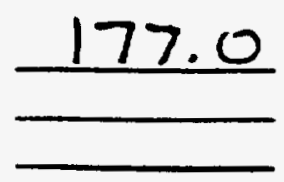

F-234
Time (min) 0

Time $(\min ) 5$

Initial

Target weight $\sim 175.6 \mathrm{lb}$ 
Drum No. $\quad 0653164.4$

Post-test Assessment

Position: original: tipped: toppled

Lid on drum: $\quad x$ Yes $\quad$ No

Gasket missing (cm) all

Contents expelled: __ Yes $x$ No

Seal failure: $\quad$ Yes $x$ Not visible top + bottom bulged

Burn partern:

41 sean to $1-f T$

Photo taken:

Leak tested:

(N/A Dor Pressure (psig) Pressure (psig)

Date

$$
\text { Yes } x \text { No }
$$

\begin{tabular}{lll}
- & Time (min) & 0 \\
\hline
\end{tabular}

Interior assessment:

19" top of dum totop fokan

Photo taken:

$x$ Yes

Comments: 


\section{Palletized Storage Drum Data Sheet}

Drum No. 0663

Description (Check):

Standard load

Instrumented for Pressure

Instrumented for Temperature

\begin{tabular}{|c|c|c|c|}
\hline Loading Starting at Bottom & Layer 1 & Layer 2 & Layer 3 \\
\hline 3 pieces of steel & & N/A & N/A \\
\hline $1 / 3$ of the brown paper & & & \\
\hline $1 / 3$ of the 10 -mil plastic & & & \\
\hline $1 / 3$ of the bond paper & & & \\
\hline $1 / 3$ of the towels & & & \\
\hline $1 / 3$ of the bags & & & \\
\hline $1 / 3$ of the cut paper & & & \\
\hline $1 / 3$ of the fabric & & Go to Layer 3 & \\
\hline $1 / 3$ of the rubber & & & \\
\hline
\end{tabular}

Drum Filled:

Ring bolt placed opposite drum seam and in down position

Lid sealed and torqued to $40 \mathrm{ft} / \mathrm{b}$ :

Leak tested:

Pre-test weight (lb)

Post-test weight (lb)

$$
\text { Loss }=
$$

Date $5 \cdot 5 \cdot 95$ Initial AS

Date $5 \cdot 5 \cdot 95$ Initial AS

Date $5-5 \cdot 95$ Initial $A S$

N/A or Pressure (psig)

Pressure (psig)

Date

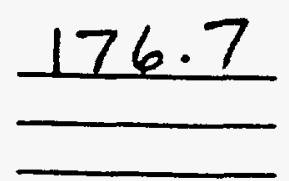

Time (min) $\varrho$ Time $(\min )$
Initial

Target weight $\sim 175.6 \mathrm{lb}$ 


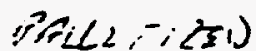

Reolerage Drum Data Sheet

Drum No. 0663166.716 .

Post-test Assessment

Position: original: tipped: toppled

Lid on drum: $\quad x$ Yes No

Gasket missing (cm) all

Contents expelled: _ Yes $\underline{X}$ No

Seal failure:

Yes $x$ Not visible top o bettem buiged

$\frac{\text { Burm pattern: }}{\text { 14"indlestats } 16 \text { "left of seam other spots atbettom }}$ vean sein

Photo taken:

Yes $\propto$ No

Leak tested:

N/Aior Pressure (psig)
Pressure (psig)

Date

\begin{tabular}{lll} 
Time $(\min )$ & 0 \\
\hline Timitial & Time $(\min )$
\end{tabular}

Interior assessment:

$20^{\circ}$ top of dum to tor of chan

$\checkmark 10+0.70$

Photo taken:

$X$ Yes

Comments: 
WHC-SD-WM-TRP-246 REV. 0

\section{Palletized Storage Drum Data Sheet}

Drum No. 0673

Description (Check):

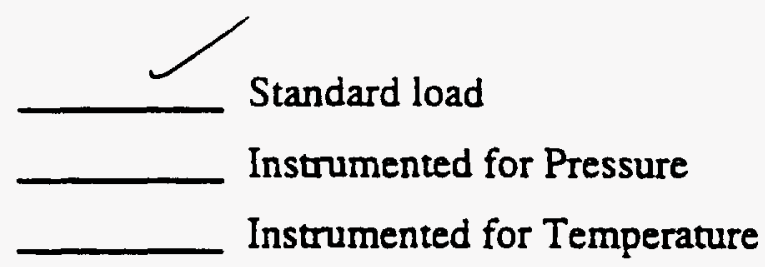

\begin{tabular}{|c|c|c|c|}
\hline Loading Starting at Bottom & Layer 1 & Layer 2 & Layer 3 \\
\hline 3 pieces of steel & & N/A & N/A \\
\hline \multicolumn{4}{|l|}{$1 / 3$ of the brown paper } \\
\hline \multicolumn{4}{|l|}{$1 / 3$ of the 10 -mil plastic } \\
\hline \multicolumn{4}{|l|}{$1 / 3$ of the bond paper } \\
\hline \multicolumn{4}{|l|}{$1 / 3$ of the towels } \\
\hline \multicolumn{4}{|l|}{$1 / 3$ of the bags } \\
\hline \multicolumn{4}{|l|}{ 1/3 of the cut paper } \\
\hline \multicolumn{4}{|l|}{$1 / 3$ of the fabric } \\
\hline \multicolumn{4}{|l|}{$1 / 3$ of the rubber } \\
\hline & Go to Layer 2 & Go to Layer 3 & \\
\hline
\end{tabular}

Drum Filled:

Ring bolt placed opposite drum seam and in down position

Lid sealed and torqued to $40 \mathrm{f} / \mathrm{lb}$ :

Leak tested:

Pre-test weight (lb)

Post-test weight (lb)

Date $5 \cdot 5 \cdot 95$ Initial AS

Date $5.5 \cdot 95$ Initial AS

Date $5-5 \cdot 95$ Initial $A S$

N/A or Pressure (psig) Pressure (psig)

Date

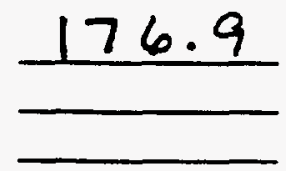

Time (min) 0

Time (min) 5

Initial

Target weight $-175.6 \mathrm{lb}$ 
Drum No. $0673 \quad 174.5 \mathrm{~h}$

Post-test Assessment

Position: original: tipped: toppled

Lid on drum: $\quad x$ Yes _ No

Gasket missing (cm) $52^{\prime \prime}$

Contents expeiled: Yes $\quad$ No

Seal failure: Yes $X$ Not visible bettoin bulged

Burn pattern:

- $1.5+T^{2}$ spet bettom of dum below bott - smallen spot betion bolt ot Top

Photo taken: Yes $\stackrel{x}{\longrightarrow}$ No

Leak tested:

N/Á or Pressure (psig)

Pressure (psig)

Date

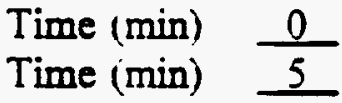

Interior assessment:

$13^{\prime \prime}$ top of ien to top of a han

Videc $\leqslant 6$

Photo taken:

$\not$ Yes No

Comments: 


\section{Palletized Storage Drum Data Sheet}

Drum No. 0683

Description (Check):

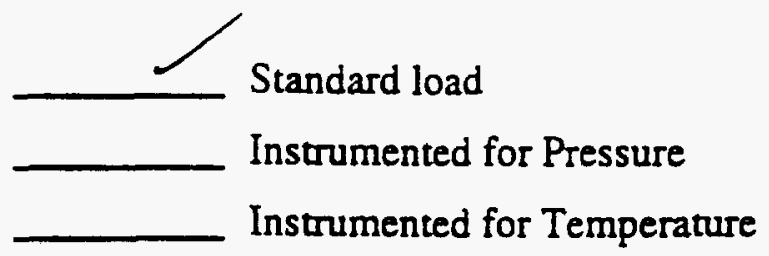

\begin{tabular}{|c|c|c|c|}
\hline Loading Starting at Bottom & Layer 1 & Layer 2 & Layer 3 \\
\hline 3 pieces of steel & $\mathcal{J}$ & N/A & N/A \\
\hline $1 / 3$ of the brown paper & Y & $\sigma$ & r \\
\hline $1 / 3$ of the 10 -mil plastic & $\zeta$ & $\checkmark$ & $\checkmark$ \\
\hline $1 / 3$ of the bond paper & l' & - & v \\
\hline $1 / 3$ of the towels & $\nearrow$ & $\checkmark$ & ك \\
\hline $1 / 3$ of the bags & 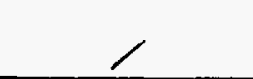 & 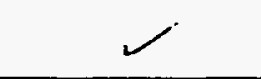 & $\tau^{2}$ \\
\hline $1 / 3$ of the cut paper & ノ & $y$ & $\checkmark$ \\
\hline $1 / 3$ of the fabric & 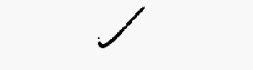 & - & 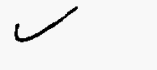 \\
\hline $1 / 3$ of the rubber & $\iota$ & $\checkmark$ & $v^{\prime}$ \\
\hline & Go to Layer 2 & Go to Layer 3 & \\
\hline
\end{tabular}

Drum Filled:

Ring bolt placed opposite drum seam and in down position

Lid sealed and torqued to $40 \mathrm{ft} / \mathrm{b}$ :

Leak tested:

Pre-test weight (lb)

Post-test weight (lb)

$$
\text { Loss = }
$$

Date $5-5.95$ Initial AS

Date $5-5 \cdot 95$ Initial $\triangle 5$
Date $5 \cdot 5 \cdot 95$ Initial AS

N/A or Pressure (psig)

Pressure (psig)

Date

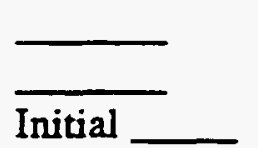

Time (min) 0

Time $(\min ) 5$

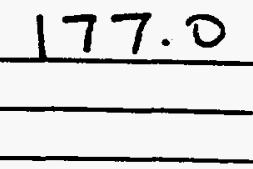


Drum No. 0683175.216.

Post-test Assessment

Position: original: tipped: toppled

Lid on drum: $\quad \chi \quad$ Yes $\quad$ No

Gasket missing (cm) $27^{\prime \prime}$

Contents expeiled: __ Yes $\$ No

Seal failure: $\quad$ Y_ Yes $\quad X$ Not visible bottam buiged son

Burn partern:

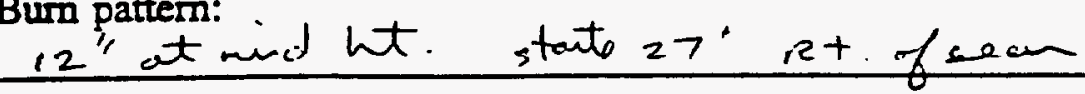

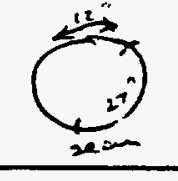

Photo taken:

Yes $X$ No

Leak tested:

NíA or Pressure (psig)

Pressure (psig)

Date $\begin{array}{ll}\text { Time (min) } & 0 \\ \text { Time (min) } & \underline{5}\end{array}$

Initial

Interior assessment:

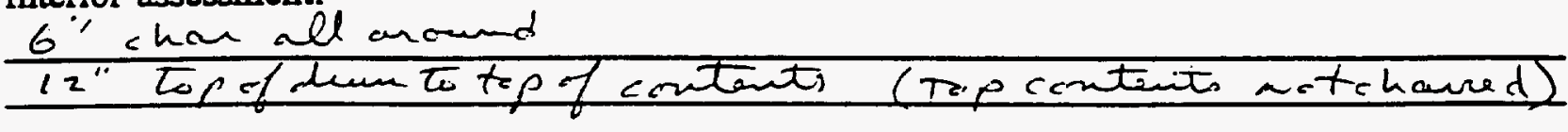

vipecis

Rhoto taken:

$\times$ Yes

No

Comments: 


\section{Palletized Storage Drum Data Sheet}

Drum No. $07 / 3$

Description (Check):

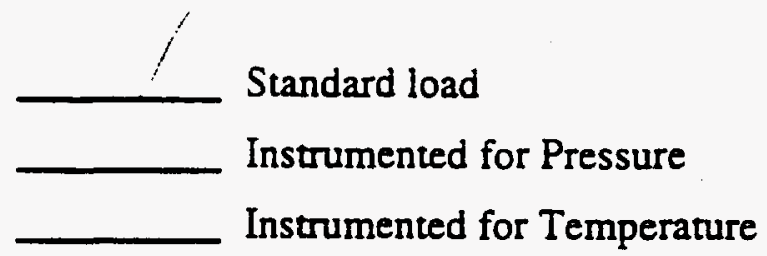

\begin{tabular}{|c|c|c|c|}
\hline Loading Starting at Bottom & Layer 1 & Layer 2 & Layer 3 \\
\hline 3 pieces of steel & & N/A & N/A \\
\hline $1 / 3$ of the brown paper & & & \\
\hline $1 / 3$ of the 10 -mil plastic & & & \\
\hline $1 / 3$ of the bond paper & & & \\
\hline $1 / 3$ of the towels & & & \\
\hline $1 / 3$ of the bags & & & \\
\hline $1 / 3$ of the cut paper & & & \\
\hline $1 / 3$ of the fabric & Go to Layer 2 & Go to Layer 3 & \\
\hline $1 / 3$ of the rubber & & & \\
\hline
\end{tabular}

Drum Filled:

Ring bolt placed opposite drum seam and in down position

Lid sealed and torqued to $40 \mathrm{ft} / \mathrm{b}$ :

Leak tested:

Pre-test weight (lb)

Post-test weight (lb)

$$
\text { Loss }=
$$

Date $5-3.95$ Initial R.K

Date 5.3 .95 Initial R.K

Date 5.3 .95 Initial R.K

N/A or Pressure (psig) Pressure (psig)

Date

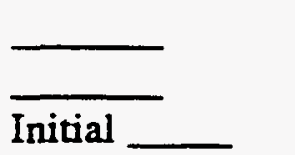

Target weight $\sim 175.6 \mathrm{lb}$
Time (min) 0

Time (min) 5 
Drum No. $0 ?, 3, t 4, i n$.

Post-test Assessment

Position: original: tipped: toppled

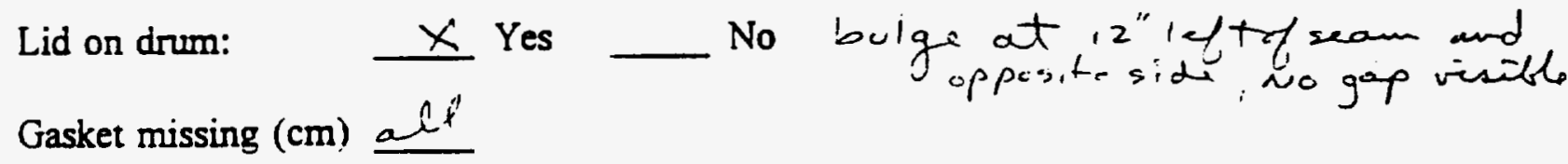

Contents expelled: $\quad$ Yes $x$ No

Seal failure: $\quad Z$ Yes $\quad$ Not visible

Burn pattern: all around

Photo taken: Yes $x$ No

Leak tested:

N/A or Pressure (psig) Pressure (psig)

Date

\begin{tabular}{ll} 
Time $(\min )$ & 0 \\
\hline Timitial & Timin
\end{tabular}

Interior assessment:

$2 i$ top of dum te top of chaund contents

purpeg s

Pheto taken:

Yes No

Comments:

$-10^{\prime \prime}$ of botlom seam un cracked geen 
WHC-SD-WM-TRP-246 REV. 0

\section{Palletized Storage Drum Data Sheet}

Drum No. 0723

Description (Check):

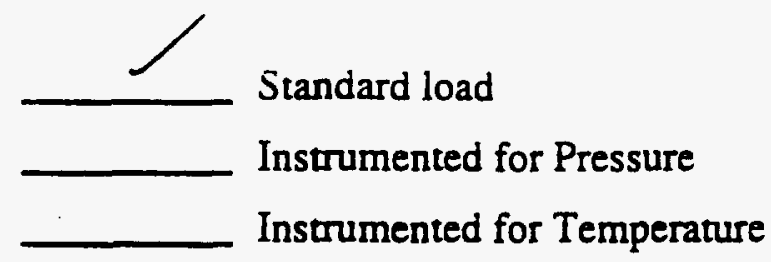

\begin{tabular}{|c|c|c|c|}
\hline Loading Starting at Bottom & Layer 1 & Layer 2 & Layer 3 \\
\hline 3 pieces of steel & & N/A & N/A \\
\hline $1 / 3$ of the brown paper & & & \\
\hline $1 / 3$ of the 10-mil plastic & & & \\
\hline $1 / 3$ of the bond paper & & & \\
\hline $1 / 3$ of the towels & & & \\
\hline $1 / 3$ of the bags & & & \\
\hline $1 / 3$ of the cut paper & & Go to Layer 3 & \\
\hline $1 / 3$ of the fabric & Go to Layer 2 & Go \\
\hline $1 / 3$ of the rubber & & \\
\hline
\end{tabular}

Drum Filled:

Ring bolt placed opposite drum seam and in down position

Lid sealed and torqued to $40 \mathrm{ft} / \mathrm{lb}$ :

Leak tested:

Pre-test weight (lb)

Post-test weight (lb)

$$
\text { Loss = }
$$

Date $5-5.95$ Initial AS

Date $5-5 \cdot 95$ Initial $A 5$

Date $5 \cdot 5 \cdot 95$ Initial AS

N/A or Pressure (psig)

Pressure (psig)

Date

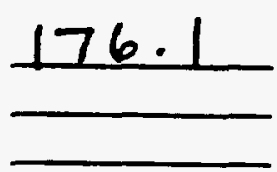

Time (min) $\frac{0}{5}$

Time (min) 5 


\section{PFALL, - Fil}

Rock Storage Drum Data Sheet

Drum No. $\quad 07 \geq 3.2613$.

Post-test Assessment

Position: __ original: ___ tipped: __ toppled

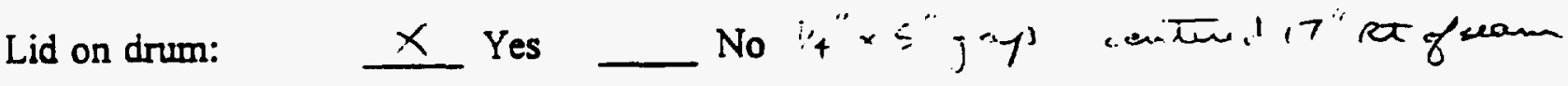

Gasket missing (cm) e

Contents expelled: $\quad$ Yes $\underline{x}$ No

Seal failure: $\quad \quad X$ Yes _ Not visible

Burn pattern:
all around

Photo taken: $\quad$ Yes $\quad$ No

Leak tested: $\quad$ (N/A or Pressure (psig)

Pressure (psig)

Date

Time $(\min )$
Time $(\min )$

Initial

Interior assessment:

is" top town te top f-han

Bhotoc taken: $\quad x$ Yes $\quad$ No

Comments: 


\section{Palletized Storage Drum Data Sheet}

Drum No. 0733

Description (Check):

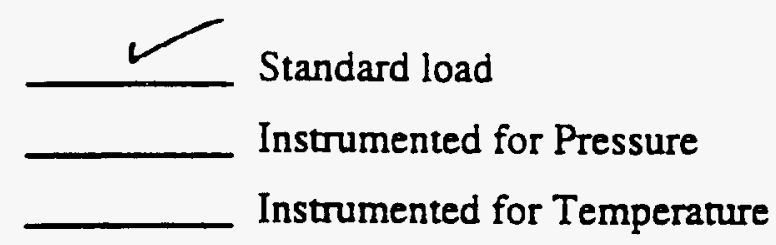

\begin{tabular}{|c|c|c|c|}
\hline Loading Starting at Bottom & Layer 1 & Layer 2 & Layer 3 \\
\hline 3 pieces of steel & $\checkmark$ & N/A & N/A \\
\hline $1 / 3$ of the brown paper & $\gamma$ & $\checkmark$ & \\
\hline \multicolumn{4}{|l|}{$1 / 3$ of the 10 -mil plastic } \\
\hline \multicolumn{4}{|l|}{$1 / 3$ of the bond paper } \\
\hline \multicolumn{4}{|l|}{$1 / 3$ of the towels } \\
\hline \multicolumn{4}{|l|}{$1 / 3$ of the bags } \\
\hline \multicolumn{4}{|l|}{$1 / 3$ of the cut paper } \\
\hline \multicolumn{4}{|l|}{$1 / 3$ of the fabric } \\
\hline \multirow[t]{2}{*}{$1 / 3$ of the rubber } & 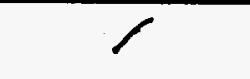 & $\checkmark$ & \\
\hline & Go to Layer 2 & Go to Layer 3 & \\
\hline
\end{tabular}

Drum Filled:

Date $5-4-25$ Initial $A P$

Ring bolt placed opposite drum seam and in down position

Date $5-4-95$ Initial AP

Lid sealed and torqued to $40 \mathrm{ft} / \mathrm{b}: \quad$ Date $5-4-95$ Initial AP

Leak tested:

N/A or Pressure (psig)

Pressure (psig)

Date

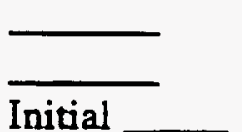

Time (min)

Time $(\mathrm{min})-5$

Pre-test weight (lb)

Post-test weight (lb)

$$
176.4 \quad \text { Target weight }-175.6 \mathrm{lb}
$$


WHC-SD-WM-TRP-246 REV. 0

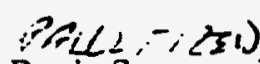

Rack-Etonage Drum Data Sheet

Drum No. $\quad 2733 \quad 1615 \mathrm{it}$.

Post-test Assessment

Position:

original:

tipped:

toppled

Lid on drum: $\quad X$ Yes No

Gasket missing (cm) axt

Contents expelled: $\quad$ Yes $\longrightarrow$ No

Seal failure: $\quad$ Yes $\quad x$ Not visible be ttom $=$ to $p$ bu lged

Burn pattern:

all around exespt $\sim 21$ " centend in zeen

Photo taken:

Yes $\chi$ No

Leak tested:

Ni

Pressure (psig)

Date \begin{tabular}{lll}
- & Time (min) & 0 \\
\hline & Time (min)
\end{tabular}

Interior assessment:

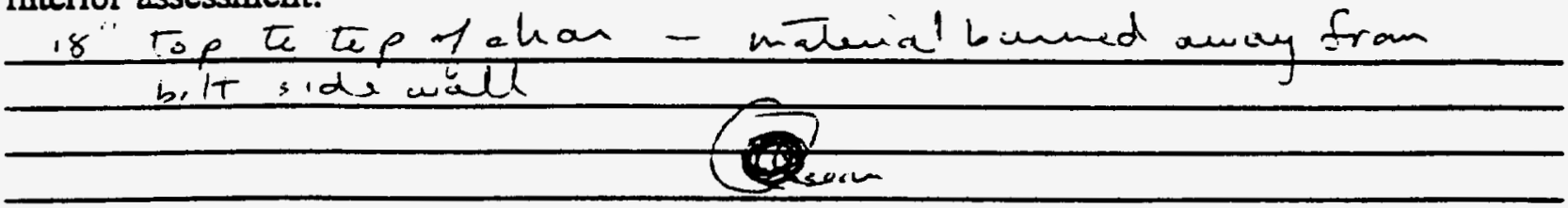

D.Des 42

photo taken:

$x$ Yes

No

Comments: 
WHC-SD-WM-TRP-246 REV. 0

$-$

Palletized Storage Drum Data Sheet

Drum No. 0743

Description (Check):

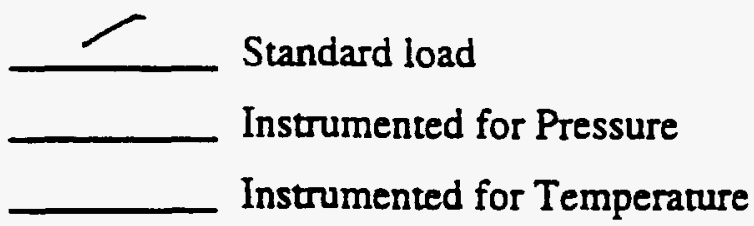

\begin{tabular}{|c|c|c|c|}
\hline Loading Starting at Bottom & Layer 1 & Layer 2 & Layer 3 \\
\hline 3 pieces of steel & N/A & N/A \\
\hline $1 / 3$ of the brown paper & & & \\
\hline $1 / 3$ of the 10-mil plastic & & & \\
\hline $1 / 3$ of the bond paper & & & \\
\hline $1 / 3$ of the towels & & & \\
\hline $1 / 3$ of the bags & & & \\
\hline $1 / 3$ of the cut paper & & & \\
\hline $1 / 3$ of the fabric & Go to Layer 2 & Go to Layer 3 & \\
\hline $1 / 3$ of the rubber & & \\
\hline
\end{tabular}

Drum Filled:

Ring bolt placed opposite drum seam and in down position

Lid sealed and torqued to $40 \mathrm{ft} / \mathrm{b}$ :

Leak tested:

Pre-test weight (lb)

Post-test weight (lb)

Loss $=$

Los Alamos Technical Associates
Date $5-4-95$ Initial AP

Date 5-4-75 Initial AP

Date 5-4-25 Initial AP

N/A or Pressure (psig)

Pressure (psig)

Date

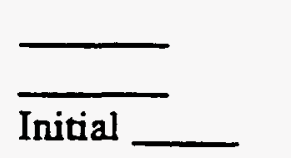

Time (min)

Time (min) 5
Target weight $-175.6 \mathrm{lb}$ 
Drum No. $\quad 0743(p) 158.2$

\section{Post-test Assessment}

Position: original: tipped: toppled

Lid on drum: $\quad x$ Yes No

Gasket missing (cm) all

Contents expelled: _ Yes $\lcm{x}$ No

Seal failure: $\quad$ Yes $X$ Not visible to $p$ bottom bulged

Burn pattern:

Photo taken: $\quad$ Yes $\chi$ No

Leak tested:

N/A or Pressure (psig)
Pressure (psig)
Date

Interior assessment:

-22 "top of dum to top of ahar

\begin{tabular}{lll}
- & Time $(\min )$ & 0 \\
\hline
\end{tabular}

Initial

viora 4 !

photo taken:

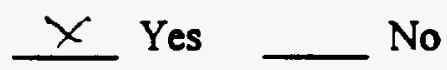

Comments: 


\section{Palletized Storage Drum Data Sheet}

Drum No. 0753

Descriprion (Check):

Standard load

Instrumented for Pressure Instrumented for Temperature

\begin{tabular}{|c|c|c|c|}
\hline Loading Starting at Bottom & Layer 1 & Layer 2 & Layer 3 \\
\hline 3 pieces of steel & $\sim$ & N/A & N/A \\
\hline $1 / 3$ of the brown paper & $\nu$ & & - \\
\hline $1 / 3$ of the 10 -mil plastic & 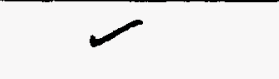 & & 2 \\
\hline \multicolumn{4}{|l|}{$1 / 3$ of the bond paper } \\
\hline $1 / 3$ of the toweis & $\sim$ & $<$ & \\
\hline $1 / 3$ of the bags & $\checkmark$ & $\checkmark$ & \\
\hline $1 / 3$ of the cut paper & $\sim$ & 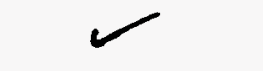 & 乙 \\
\hline $1 / 3$ of the fabric & $\sim$ & r & \\
\hline \multirow[t]{2}{*}{$1 / 3$ of the rubber } & $\checkmark$ & $\checkmark$ & - \\
\hline & Go to Layer 2 & Go to Layer 3 & \\
\hline
\end{tabular}

Drum Filled:

Ring bolt placed opposite drum seam and in down position

Lid sealed and torqued to $40 \mathrm{ftlb}$ :

Leak tested:

Pre-test weight (lb)

Post-test weight (lb)

$$
\text { Loss }=
$$

Date 5.3 .95 Initial

Date 5.3 .95 Initial R.K
N/A or Pressure (psig) Pressure (psig)

Date

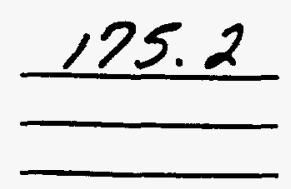

F-250
Time (min)

Time (min) 5

Initial

Target weight $\sim 175.6 \mathrm{lb}$ 
Drum No. $\quad 0753 \quad 163.7$

Post-test Assessment

Position: original: tipped: toppled

Lid on drum: $\quad x$ Yes No

Gasket missing $(\mathrm{cm})$ all

Contents expelled: _ Yes $X$ No

Seal failure: $\quad$ Yes $x$ Not visible botem o top bulged

Burn pattern:

somewikat all around, mainly seam $t$ et. side

Photo taken:

I. Yes $x$ No

Leak tested:
Pressure (psig)
Pressure (psig)
Date

\begin{tabular}{lll}
- & Time $(\min )$ & 0 \\
\hline & Time $(\min )$
\end{tabular}

Interior assessment:

17" tup of orum to top of chan

v10e0 72

Ehoto taken:

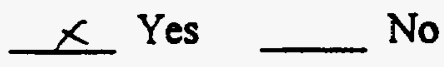

Comments: 
WHC-SD-WM-TRP-246 REV. 0

\section{Palletized Storage Drum Data Sheet}

Drum No. 0763

Description (Check):

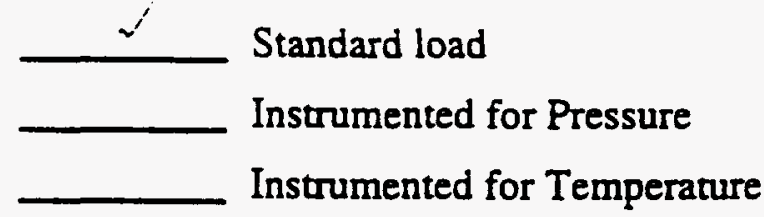

\begin{tabular}{|c|c|c|c|}
\hline Loading Starting at Bottom & Layer 1 & Layer 2 & Layer 3 \\
\hline 3 pieces of steel & $\swarrow$ & N/A & N/A \\
\hline $1 / 3$ of the brown paper & $r$ & $\sim$ & r \\
\hline $1 / 3$ of the 10 -mil plastic & 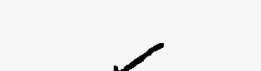 & & $N$ \\
\hline $1 / 3$ of the bond paper & 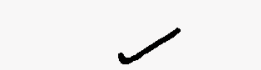 & & \\
\hline $1 / 3$ of the towels & $\checkmark$ & $\checkmark$ & \\
\hline $1 / 3$ of the bags & $\checkmark$ & $r$ & \\
\hline $1 / 3$ of the cut paper & $\checkmark$ & $\checkmark$ & \\
\hline $1 / 3$ of the fabric & $\checkmark$ & $\checkmark$ & \\
\hline $1 / 3$ of the rubber & $\checkmark$ & 7 & \\
\hline & Go to Layer 2 & Go to Layer 3 & \\
\hline
\end{tabular}

Drum Filled:

Ring bolt placed opposite drum seam and in down position

Lid sealed and torqued to $40 \mathrm{ft} / \mathrm{b}$ :

Leak tested:

Pre-test weight (lb)

Post-test weight (lb)

Loss $=$

Date $s=3.95$ Initial R.K

Date 5.3-95 Initial L.K

Date 5.3.95 Initial L.K

N/A or Pressure (psig)

Pressure (psig)

Date

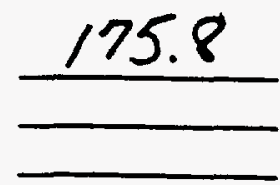

F-252
Time (min) 0

Time (min) 5

Initial

Target weight $-175.6 \mathrm{lb}$ 
WHC-SD-WM-TRP-246 REV. 0

$\because F\left(L 2,-\because x_{1}\right)$

Pact-Siemee Drum Data Sheet

Drum No. 0763166.216.

Post-test Assessment

Position:

originai:

tipped:

toppled

Lid on drum:

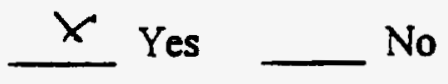

Gasket missing (cm)

$55^{\prime \prime}$

Contents expelled:

Yes $\chi$ No

Seal failure:

Yes $X$ Not visible top t hottom bulged

Burn pattern:

$33^{\prime \prime}$ at mid ht.

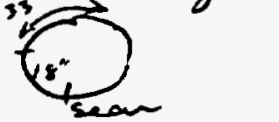

Photo taken:

Yes $\searrow$ No

Leak tested:

TPA or Pressure (psig)

Pressure (psig)

Date

$\begin{array}{lll} & \text { Time (min) } & 0 \\ \text { Initial } & \text { Time (min) }\end{array}$

Interior assessment:

$20 " 19^{\prime \prime}$ top of dum te top of char

$\sim 7 \times 071$

Photo taken:

$\times$ Yes

No

Comments: 


\section{Palletized Storage Drum Data Sheet}

Drum No. 0773

Description (Check):

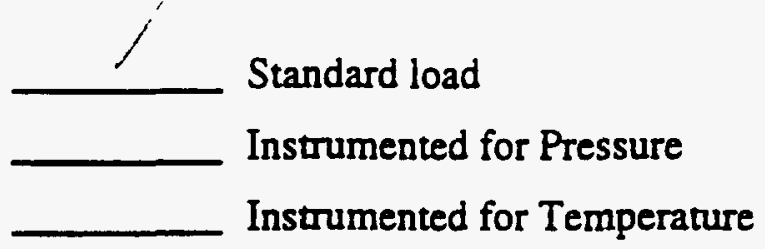

\begin{tabular}{|c|c|c|c|}
\hline Loading Starting at Bottom & Layer 1 & Layer 2 & Layer 3 \\
\hline 3 pieces of steel & & & N/A \\
\hline $1 / 3$ of the brown paper & & & \\
\hline $1 / 3$ of the 10 -mil plastic & & & \\
\hline $1 / 3$ of the bond paper & & & \\
\hline $1 / 3$ of the towels & & & \\
\hline $1 / 3$ of the bags & & & \\
\hline $1 / 3$ of the cut paper & & & \\
\hline $1 / 3$ of the fabric & Go to Layer 2 & Go to Layer 3 & \\
\hline $1 / 3$ of the rubber & & \\
\hline
\end{tabular}

Drum Filled:

Ring bolt placed opposite drum seam and in down position

Lid sealed and torqued to $40 \mathrm{ft} / \mathrm{b}$ :

Leak tested:

Pre-test weight (lb)

Post-test weight (lb)

$$
\text { Loss }=
$$

Date 5-3-95 Initial R.K

Date $5=3.95$ Initial R.L
N/A or Pressure (psig)

Pressure (psig)

Date

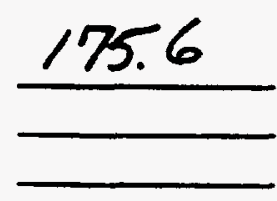

F-254
Time (min) 0

Time (min) 5

Initial

Target weight $\sim 175.6 \mathrm{lb}$ 
WHC-SD-WM-TRP-246 REV. 0

$P F(L 2,-;(2)$

Rowikgiomage Drum Data Sheet

Drum No. $0773,73.0 \mathrm{~K}$.

Post-test Assessment

Position: ___ original: ___ tipped: __ toppled

Lid on drum: $\quad x$ Yes No to $p$ t boitom buiged

Gasket missing (cm) ___ $39^{\prime \prime}$

Contents expelled: $\quad$ Yes $\quad x$ No

Seal failure: $\quad$ Yes $\quad X$ Not visible inay be at betton secum

Burn pattern:

at bott laration $\sim 1 \mathrm{fT}^{2}$ spot at Top also spot at bettom of dum 10 " migh -20 " and

Photo taken:

Yes $x$ No

Leak tested:

Pressure (psig)

Date

Initial

Time (min)
Time (min)
$\underline{5}$

Interior assessment:

17 " top of dum to top of chan

YTeC 67

Plaoto taken:

$x$ Yes

Comments: 


\section{Palletized Storage Drum Data Sheet}

Drum No. 0783

Description (Check):

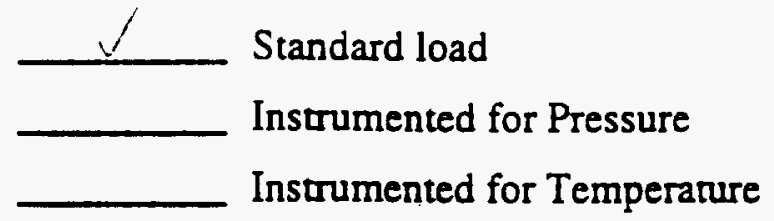

\begin{tabular}{|c|c|c|c|}
\hline Loading Staring at Bottom & Layer 1 & Layer 2 & Layer 3 \\
\hline 3 pieces of steel & & N/A & N/A \\
\hline $1 / 3$ of the brown paper & & & \\
\hline $1 / 3$ of the 10 -mil plastic & & & \\
\hline $1 / 3$ of the bond paper & & & \\
\hline $1 / 3$ of the towels & & & \\
\hline $1 / 3$ of the bags & & & \\
\hline $1 / 3$ of the cut paper & & Go to Layer 3 & \\
\hline $1 / 3$ of the fabric & Gayer 2 & Go to & \\
\hline $1 / 3$ of the rubber & & \\
\hline
\end{tabular}

Drum Filled:

Ring bolt placed opposite drum seam and in down position

Lid sealed and torqued to $40 \mathrm{ft} / \mathrm{b}$ :

Leak tested:

Pre-test weight (lb)

Post-test weight (lb)
Date $5-3.95$

Initial

Date 5.3 .95 Initial L.K
Date 5.3 .95 Initial L.K

N/A or Pressure (psig)

Pressure (psig)

Date

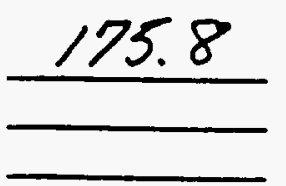

Initial
Time (min) 0

Time (min) 5

Target weight $\sim 175.6 \mathrm{lb}$ 
Drum No. $0783,174.716$.

Post-test Assessment

Position:

original: tipped: toppled

Lid on drum:

$x$ Yes No Nobulged

Gasket missing (cm) 49 " - romainieng is mstly bumid goo

Contents expelled:

Yes $x$ No

Seal failure: $?$ Yes $\backslash$ Not visible bottom bulged
seam muite?

Burn partern: 10 at mid ht. $24^{\prime \prime} 1-f+f \sec x$

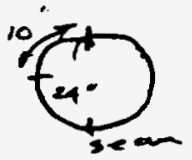

Photo taken: $x$ Yes

Leak tested:

N/A 0 Pressure ipsig) Pressure (psig)

Date

$\longrightarrow \quad$\begin{tabular}{ll} 
Time (min) & 0 \\
\hline
\end{tabular}

Interior assessment:

13 "Top of dium to top of han

\section{Comments:}




\section{Palletized Storage Drum Data Sheet}

Drum No. $08 / 3(P)$

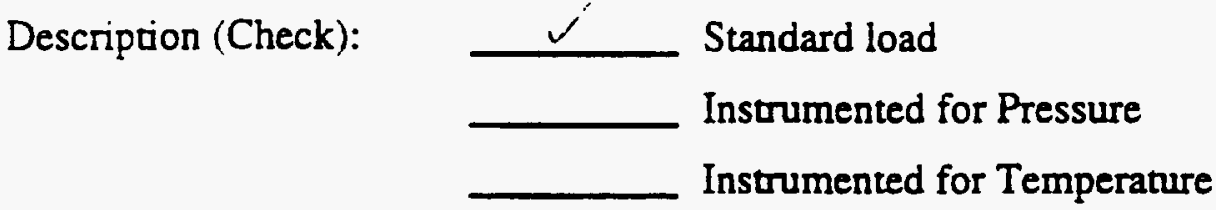

\begin{tabular}{|c|c|c|c|}
\hline Loading Starting at Bottom & Layer 1 & Layer 2 & Layer 3 \\
\hline 3 pieces of steel & $r$ & N/A & N/A \\
\hline $1 / 3$ of the brown paper & $\nu$ & $\checkmark$ & $v$ \\
\hline $1 / 3$ of the 10 -mil plastic & $r$ & $\gamma$ & $\checkmark$ \\
\hline $1 / 3$ of the bond paper & $\checkmark$ & $\checkmark$ & 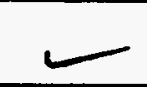 \\
\hline $1 / 3$ of the towels & $\checkmark$ & $\checkmark$ & $\sigma$ \\
\hline $1 / 3$ of the bags & $\sim$ & $\checkmark$ & $\sim$ \\
\hline $1 / 3$ of the cut paper & $\checkmark$ & $\checkmark$ & $\sim$ \\
\hline $1 / 3$ of the fabric & $\checkmark$ & $\checkmark$ & c \\
\hline \multirow[t]{2}{*}{$1 / 3$ of the rubber } & 7 & $\swarrow$ & \\
\hline & Go to Layer 2 & Go to Layer 3 & \\
\hline
\end{tabular}

Drum Filled:

Ring bolt placed opposite drum seam and in down position

Lid sealed and torqued to $40 \mathrm{ft} / \mathrm{b}$ :

Leak tested:

Pre-test weight (lb)

Post-test weight (lb)
Date 5.3 .95 Initial

Date 5.3.95 Initial L.K
Date 5.3 .95 Initial R.K
N/A or Pressure (psig)

Pressure (psig)

Date

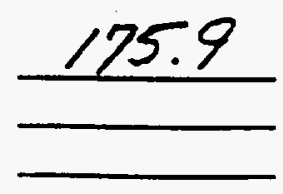

Time (min) 0

Time $(\min )+5$
Initial

Target weight $-175.6 \mathrm{lb}$ 
Drum No. ㄴ..2. -161.516

\section{Post-test Assessment}

Position: __ original: ___ tipped: __ toppled

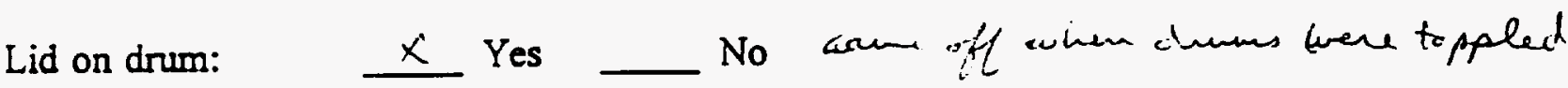

Gasket missing (cm) al

Contents expelled: — Yes $\longleftarrow$ No

Seal failure: $\quad x$ Yes _ N Not visible

Burn pattern:

all around

Photo taken:

Yes $\longrightarrow$ No

Leak tested:

N/A or Pressure (psig)
Pressure (psig)

Date
Time (min) $\quad \underline{0}$

Initial

Interior assessment:

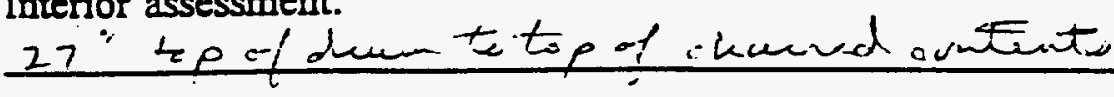

VIDCC 13

Ehoto taken:

$X$ Yes No

Comments: 


\section{Palletized Storage Drum Data Sheet}

Drum No. 0823

Description (Check):

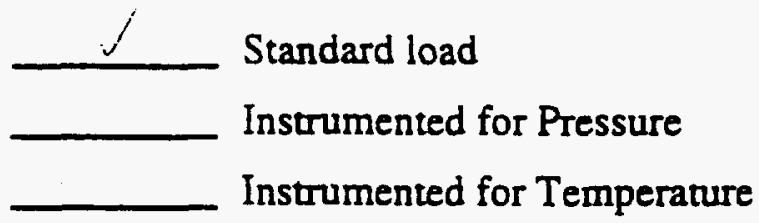

\begin{tabular}{|c|c|c|c|}
\hline Loading Starting at Bottom & Layer 1 & Layer 2 & Layer 3 \\
\hline 3 pieces of steel & $\longleftarrow$ & N/A & N/A \\
\hline $1 / 3$ of the brown paper & $\longleftarrow$ & 2 & \\
\hline $1 / 3$ of the 10 -mil plastic & & $\checkmark$ & \\
\hline $1 / 3$ of the bond paper & $\sim$ & م & \\
\hline $1 / 3$ of the towels & $\checkmark$ & $\gamma$ & \\
\hline $1 / 3$ of the bags & 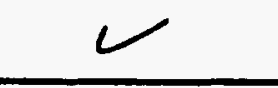 & $\checkmark$ & \\
\hline $1 / 3$ of the cut paper & $v$ & $\checkmark$ & \\
\hline $1 / 3$ of the fabric & 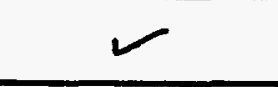 & 2 & $\mathcal{J}$ \\
\hline \multirow[t]{2}{*}{$1 / 3$ of the rubber } & $\longleftarrow$ & $\nearrow$ & $r$ \\
\hline & Go to Layer 2 & Go to Layer 3 & \\
\hline
\end{tabular}

Drum Filled:

Ring bolt placed opposite drum seam and in down position

Lid sealed and torqued to $40 \mathrm{ft} / \mathrm{b}$ :

Leak tested:

Pre-test weight (lb)

Post-test weight (lb)

$$
\text { Loss }=
$$

Date 53.95 Initial R.K

Date 5.3 .95 Initial R.K

Date $5.3 .95 \quad$ Initial R.K

N/A or Pressure (psig)

Pressure (psig)

Date

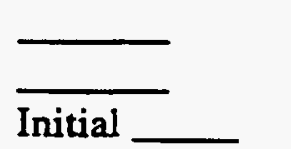

Time (min) $\frac{0}{5}$

Time (min) 5

176.2

Target weight $\sim 175.6 \mathrm{lb}$ 
Drum No. $<<23$ is $4: 410$

Post-test Assessment

Position: original: tipped: toppled

Lid on drum: $\quad X$ Yes No $4 . " 14 " g a p .7 " 1 .+4$ of sean

Gasket missing (cm) +11

Contents expelled: __ Yes $x$ No

Seal failure: $\quad X^{\prime}$ Yes Not visible

Burn pattern:

52 at mid ht.

Photo taken:

Leak tested: $\quad$ N/A or Pressure (psig)

$$
\text { Yes } t \text { No }
$$
Pressure (psig)

Date

Time (min)
Initial
$\quad$ Time (min)

Interior assessment:

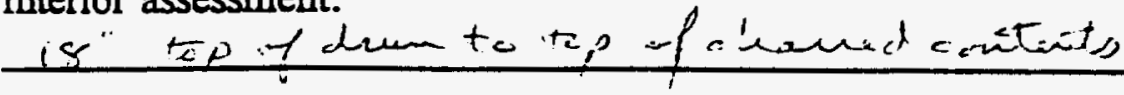

Vio 00 i

Photo taken:

$\Upsilon$ Yes No

Comments: 
WHC-SD-WM-TRP-246 REV. 0

\section{Palletized Storage Drum Data Sheet}

Drum No. 0833

Description (Check):

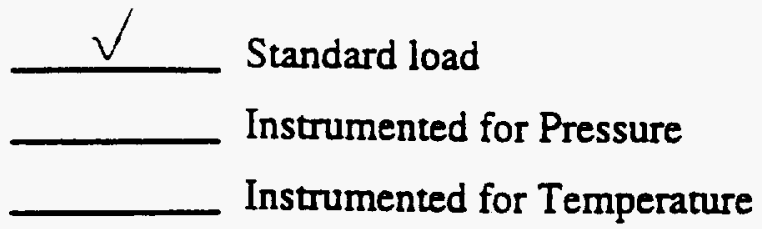

\begin{tabular}{|c|c|c|c|}
\hline Loading Starting at Bottom & Layer 1 & Layer 2 & Layer 3 \\
\hline 3 pieces of steel & & & \\
\hline $1 / 3$ of the brown paper & & & \\
\hline $1 / 3$ of the 10 -mil plastic & & & \\
\hline $1 / 3$ of the bond paper & & & \\
\hline $1 / 3$ of the towels & & & \\
\hline $1 / 3$ of the bags & & & \\
\hline $1 / 3$ of the cut paper & & & \\
\hline $1 / 3$ of the fabric & & & \\
\hline $1 / 3$ of the rubber & Go to Layer 2 & Go to Layer 3 & \\
\hline
\end{tabular}

Drum Filled:

Ring bolt placed opposite drum seam and in down position

Lid sealed and torqued to $40 \mathrm{ft} / \mathrm{lb}$ :

Leak tested:

Pre-test weight (lb)

Post-test weight (lb)

$$
\text { Loss = }
$$

Date $5-3.95$

Date $5-3.95$ Initial R.K

Date $5-3.95$ Initial

N/A or Pressure (psig)

Pressure (psig)

Date

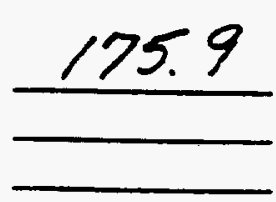

F-262

Initial
Time (min)

Time (min) 5

Target weight $\sim 175.6 \mathrm{lb}$ 
Drum No. 0837, ief,

Post-test Assessment

Position:

originai: tipped: toppled

Lid on drum: $\quad x$ Yes No tep $\quad$ No botem bulged

Gasket missing (cm) al!

Contents expelled: _ Yes $x$ No

Seal failure: $\quad$ Yes _ _ Not visible

Burn pattern:

all around

Photo taken:

Yes $\underline{x}$ No

Leak tested:

NFÀ or Pressure (psig)

Préssure (psig)

Date

\begin{tabular}{lll} 
& Time (min) & 0 \\
\hline Initial & Time (min)
\end{tabular}

Interior assessment:

22 " top of chum It top of char

VIDeC 24

Photo taken:

$\times$ Yes

No

Comments:

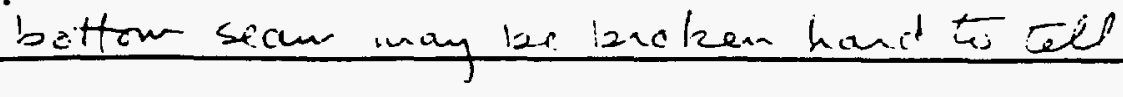


WHC-SD-WM-TRP-246 REV. 0

\section{Palletized Storage Drum Data Sheet}

Drum No. 0843

Description (Check):

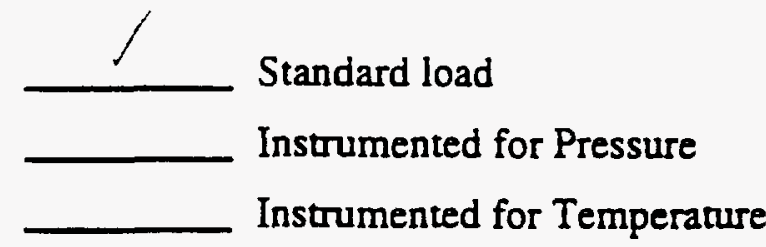

\begin{tabular}{|c|c|c|c|}
\hline Loading Starting at Bottom & Layer 1 & Layer 2 & Layer 3 \\
\hline 3 pieces of steel & & N/A & N/A \\
\hline $1 / 3$ of the brown paper & & & \\
\hline $1 / 3$ of the 10-mil plastic & & & \\
\hline $1 / 3$ of the bond paper & & & \\
\hline $1 / 3$ of the towels & & & \\
\hline $1 / 3$ of the bags & & & \\
\hline $1 / 3$ of the cut paper & Go to Layer 2 & Go to Layer 3 & \\
\hline $1 / 3$ of the fabric & & & \\
\hline $1 / 3$ of the rubber & & & \\
\hline
\end{tabular}

Drum Filled:

Date 5.3 .95 Initial R.K

Ring bolt placed opposite drum seam and in down position

Date 5.3 .95 Initial $\underline{\text { RK }}$

Lid sealed and torqued to $40 \mathrm{ft} / \mathrm{b}$ :

Date $5.3 .95 \quad$ Initial R.K

Leak tested:

Pre-test weight (lb)

Post-test weight (lb)

$$
\text { Loss }=
$$

N/A or Pressure (psig)

Pressure (psig)

Date

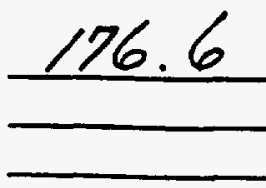

F-264
Time (min) 0

Time (min) 5 
Drum No. $\quad 0.54^{2}, 19.6$.

Post-test Assessment

Position:

originai:

tipped: toppled

Lid on drum: $\quad x$ Yes $\quad$ No

Gasket missing (cm) _

Contents expelled: $\quad$ Yes $x$ No

Seal failure: $\quad x$ Yes Not visible bitim itsp bulged

Burn pattern:

all excipt -22 " centand ein seam

Photo taken:

$X$ Yes No

Leak tested:

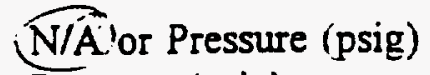

Pressure (psig)

Date
Time (min) 0

Time (min)

Initial

Interior assessment:

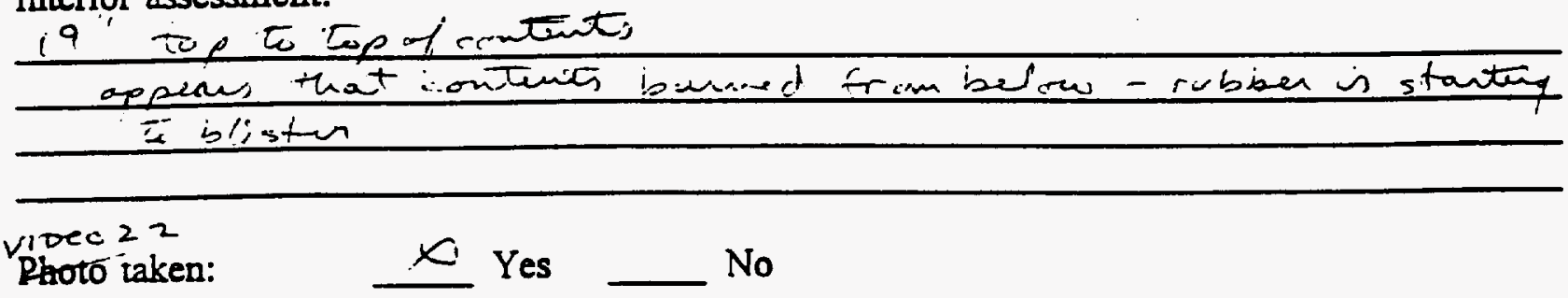

\section{Comments:}


WHC-SD-WM-TRP-246 REV. 0

\section{Palletized Storage Drum Data Sheet}

Drum No. 0853

Description (Check):

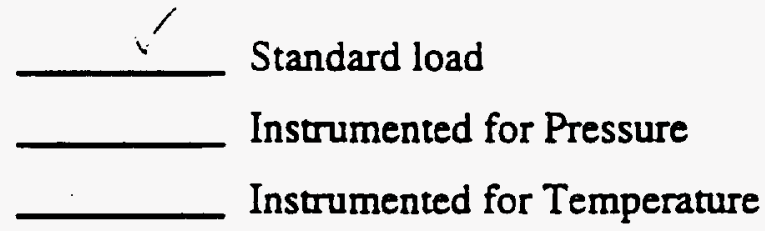

\begin{tabular}{|c|c|c|c|}
\hline Loading Starting at Bottom & Layer 1 & Layer 2 & Layer 3 \\
\hline 3 pieces of steel & & & \\
\hline $1 / 3$ of the brown paper & & & \\
\hline $1 / 3$ of the 10-mil plastic & & & \\
\hline $1 / 3$ of the bond paper & & & \\
\hline $1 / 3$ of the towels & & & \\
\hline $1 / 3$ of the bags & & & \\
\hline $1 / 3$ of the cut paper & Go to Layer 2 & Go to Layer 3 & \\
\hline $1 / 3$ of the fabric & & & \\
\hline $1 / 3$ of the rubber & & & \\
\hline
\end{tabular}

Drum Filled:

Ring bolt placed opposite drum seam and in down position

Lid sealed and torqued to $40 \mathrm{ft} / \mathrm{b}$ :

Leak tested:

Pre-test weight (lb)

Post-test weight (lb)

Date $5-3.95$ Initial

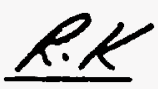

Date $5.3 .95 \quad$ Initial L.K

Date 5.3 .95 Initial L.K

N/A or Pressure (psig)

Pressure (psig)

Date

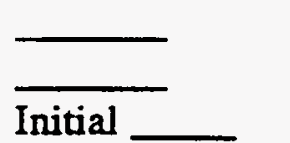

Time (min) 0

Time $(\min )$
$175.8 \quad$ Target weight $\sim 175.6 \mathrm{lb}$ 
Drim No. 0853 i 7.1 ib

Post-test Assessment

Position: __ original: ___ tipped: ___ toppled

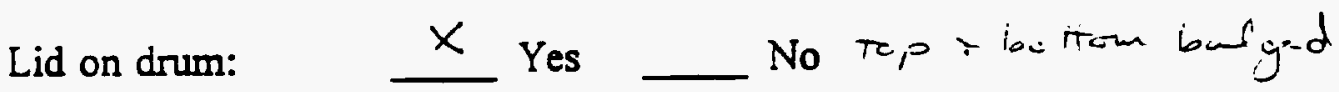

Gasket missing (cm) all

Contents expelled: __ Yes $>$ No

Seal failure: $\quad$ Y $\quad$ Yes $\quad X$ Not visible

Burn pattern:

allaround

Photo taken: $\quad$ Yes $x$ No

Leak tested: $\quad$ N/A or Pressure (psig) Pressure (psig)

Date

\begin{tabular}{lll} 
& Time (min) & 0 \\
\hline Time (min) &
\end{tabular}

Interior assessment:

22 tzp $=1$ dum te top . A char

$\therefore 100036$

Photo taken:

$\therefore$ Yes No No

Comments: 


\section{Palletized Storage Drum Data Sheet}

Drum No. 0863

Description (Check):

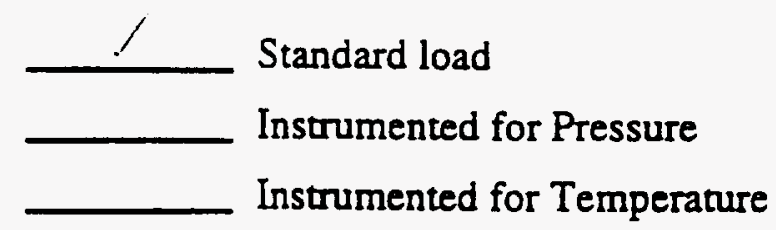

\begin{tabular}{|c|c|c|c|}
\hline Loading Starting at Bottom & Layer 1 & Layer 2 & Layer 3 \\
\hline 3 pieces of steel & N/A & N/A \\
\hline $1 / 3$ of the brown paper & & & \\
\hline $1 / 3$ of the 10 -mil plastic & & & \\
\hline $1 / 3$ of the bond paper & & & \\
\hline $1 / 3$ of the towels & & & \\
\hline $1 / 3$ of the bags & & & \\
\hline $1 / 3$ of the cut paper & Go to Layer 2 & Go to Layer 3 & \\
\hline $1 / 3$ of the fabric & & \\
\hline $1 / 3$ of the rubber & & & \\
\hline
\end{tabular}

Drum Filled:

Ring bolt placed opposite drum seam and in down position

Lid sealed and torqued to $40 \mathrm{f} / \mathrm{lb}$ :

Leak tested:

Pre-test weight (lb)

Post-test weight (lb)
Date 5.3 .95 Initial R.K

Date 5.3 .95 Initial R.K

Date 5.3 .95 Initial A.K
N/A or Pressure (psig)

Pressure (psig)

Date

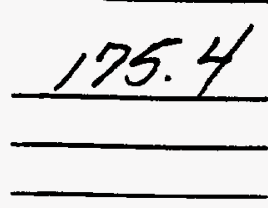

Time (min) 0

Time (min) 5
Initial

Target weight $\sim 175.6 \mathrm{lb}$ 
Drum No. 02863 is:

Post-test Assessment

Position:

original:

tipped:

toppled

Lid on drum:

$x$ Yes No :op T botifim bu

Gasket missing (cm) chl

Contents expelied: $\quad$ Yes $\longleftarrow$ No

Seal failure: $\quad$ Yes $\quad x$ Not visible

Burn pattern:

44 "at.ind it.

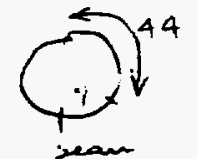

Photo taken:

Yes $\chi$ No

Leak tested:
N/A or Pressure (psig) Pressure (psig)

Date $\begin{array}{ll}\text { Time }(\min ) & 0 \\ \text { Time }(\min ) & 5\end{array}$

$\overline{\text { Initial }}$

$\left[\begin{array}{l}\text { Time (min) } \\ \text { Time (min) }\end{array} \quad \begin{array}{l}0 \\ \hline\end{array}\right.$

Interior assessment:

$17 \%$ Ep - I dum to top of o han

worc ss,

Pboto taken:

$x$ Yes

Comments: 


\section{Palletized Storage Drum Data Sheet}

Drum No. 0873

Descriprion (Check):

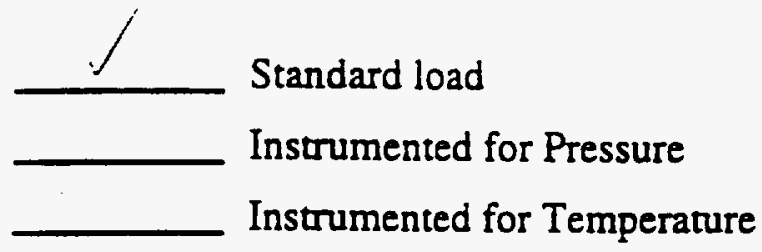

\begin{tabular}{|c|c|c|c|}
\hline Loading Starting at Bottom & Layer 1 & Layer 2 & Layer 3 \\
\hline 3 pieces of steel & $r$ & N/A & N/A \\
\hline $1 / 3$ of the brown paper & $\sim$ & 7 & $\sim$ \\
\hline $1 / 3$ of the 10 -mil plastic & & $\checkmark$ & $\checkmark$ \\
\hline $1 / 3$ of the bond paper & & 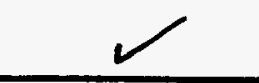 & $\sim$ \\
\hline $1 / 3$ of the towels & & $\nu$ & \\
\hline $1 / 3$ of the bags & & $\checkmark$ & $r$ \\
\hline $1 / 3$ of the cut paper & & $\checkmark$ & \\
\hline $1 / 3$ of the fabric & & $\checkmark$ & \\
\hline $1 / 3$ of the rubber & & & \\
\hline & Go to Layer 2 & Go to Layer 3 & \\
\hline
\end{tabular}

Drum Filled:

Date $5.3-95$ Initial

Ring bolt placed opposite drum seam and in down position

Date 5.3 .95 Initial S.K

Lid sealed and torqued to $40 \mathrm{ft} / \mathrm{b}$ :

Date $\underline{5-3.95}$ Initial L.K

Leak tested:

Pre-test weight (lb)

Post-test weight (lb)

$$
\text { Loss }=
$$

N/A or Pressure (psig)

Pressure (psig)

Date

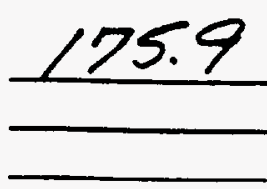

F-270
Time (min) 0

Time $(\mathrm{min})$

Initial

Target weight $\sim 175.6 \mathrm{lb}$ 
Dran No. $0973,75.0$

Post-test Assessment

Position: original: tipped: toppled

Lid on drum: $\quad X$ Yes No top r tottem bolged

Gasket missing (cm)

Contents expeiled: $\quad$ Yes $X$ No

Seal failure: ? Y Yes N_ Not visible isottom seam moy be

Burn pattern:

-9" wide $2 / 3$ up drewm

Photo taken: Yes $x$ No

Leak tested:

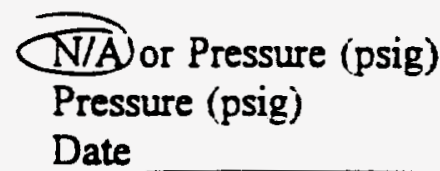

Date

$-\quad$\begin{tabular}{ll} 
Time (min) & 0 \\
\hline & Time (min)
\end{tabular}

Interior assessment:

$\frac{7^{\prime \prime} \text { ehar allariend }}{9^{\prime \prime} \text { top of dum te tep of har - top not tully chaned }}$

Y1000

$\underline{x}$ Yes __ No

Comments: 
WHC-SD-WM-TRP-246 REV. 0

\section{Palletized Storage Drum Data Sheet}

Drum No. 0883

Description (Check):

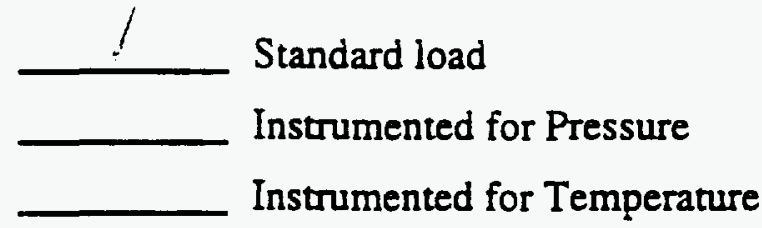

\begin{tabular}{|c|c|c|c|}
\hline Loading Starting at Bottom & Layer 1 & Layer 2 & Layer 3 \\
\hline \hline 3 pieces of steel & & N/A & N/A \\
\hline $1 / 3$ of the brown paper & & & \\
\hline $1 / 3$ of the 10-mil plastic & & & \\
\hline $1 / 3$ of the bond paper & & & \\
\hline $1 / 3$ of the towels & & & \\
\hline $1 / 3$ of the bags & & & \\
\hline $1 / 3$ of the cut paper & & $\checkmark$ & \\
\hline $1 / 3$ of the fabric & & $\checkmark$ & \\
\hline $1 / 3$ of the rubber & Go to Layer 2 & Go to Layer 3 & \\
\hline & &
\end{tabular}

Drum Filled:

Ring boit placed opposite drum seam and in down position

Lid sealed and torqued to $40 \mathrm{f} / \mathrm{lb}$ :

Leak tested:

Pre-test weight (lb)

Post-test weight (lb)

$$
\text { Loss }=
$$

Los Alamos Technical Associares
Date 5.3 .95 Initial R.K

Date $5.3 .95 \quad$ Initial $\underline{R} . K$

Date S.3.95 Initial R.K
N/A or Pressure (psig)

Pressure (psig)

Date

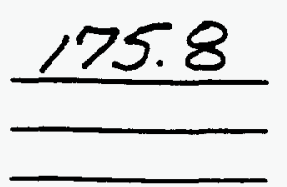

F-272
Time (min) 0

Time (min) 5
Initial

Target weight $-175.6 \mathrm{lb}$ 
WHC-SD-WM-TRP-246 REV. 0

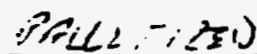

Rook- Siomge Drum Data Sheet

Drum No. 0583 i75.9 $\mathrm{h}$

Post-test Assessment

Position: ___ original: ___ tipped: ___ toppled

Lid on drum: $\quad \quad \quad \quad$ Yes $\quad$ No

Gasket missing (cm) $\mathrm{O} / 5^{\prime}$ compres20 it thand'

Contents expelled: __ Yes $\_$No

Seal failure: $\quad$ Yes $\quad X$ Not visible

Burn pattern:

Nine

Photo taken:

Yes tr No

Leak tested:

N/A; or Pressure (psig)

Pressure (psig)

Date

\begin{tabular}{lll} 
& Time (min) & 0 \\
\hline & Time (min)
\end{tabular}

Interior assessment:

plastic havely mette:i $16 " 1$ ift -1 sean

video $: 1$

Photo taken:

$y$ Yes

No

Comments: 
WHC-SD-WM-TRP-246 REV. 0

\section{Palletized Storage Drum Data Sheet}

Drum No. $99 / 3$

Description (Check):

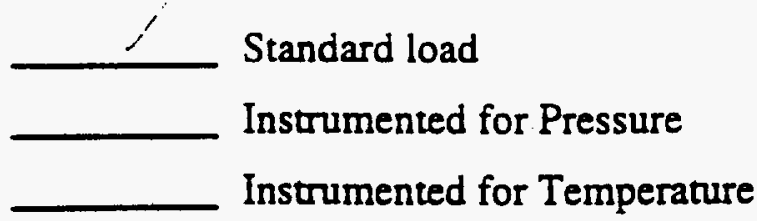

\begin{tabular}{|c|c|c|c|}
\hline Loading Starting at Bottom & Layer 1 & Layer 2 & Layer 3 \\
\hline 3 pieces of steel & N/A & \\
\hline $1 / 3$ of the brown paper & & & \\
\hline $1 / 3$ of the 10 -mil plastic & & & \\
\hline $1 / 3$ of the bond paper & & & \\
\hline $1 / 3$ of the towels & & & \\
\hline $1 / 3$ of the bags & & & \\
\hline $1 / 3$ of the cut paper & & & \\
\hline $1 / 3$ of the fabric & Go to Layer 2 & Go to Layer 3 & \\
\hline $1 / 3$ of the rubber & & \\
\hline
\end{tabular}

Drum Filled:

Ring bolt placed opposite drum seam and in down position

Lid sealed and torqued to $40 \mathrm{ft} / \mathrm{b}$ :

Leak tested:

Pre-test weight (lb)

Post-test weight (lb)
Date $5 \cdot 3 \cdot 95$

Date $5-3.95$ Initial L.K
Date 5-3.95 Initial the

N/A or Pressure (psig)

Pressure (psig)

Date

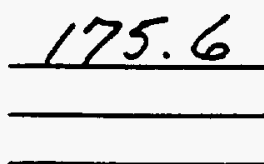

F-274
Time (min) 0

Time (min) 5

Target weight $-175.6 \mathrm{lb}$ 
WHC-SD-WM-TRP-246 REV. 0

$P(A),-i 25)$

Rachereage Drum Data Sheet

Drum No. $\quad 9912.71: 16$.

Post-test Assessment

Position: __ original: ___ tipped: ___ toppled

Lid on drum: $\quad x$ Yes No baige is" to Rt jecm

Gasket missing (cm) aU

Contents expelled: __ Yes $\underline{x}$ No

Seal failure: $\quad$ Yes $\quad X$ Not visible

Burn partern: $35^{\circ}$ t. ind Lt. stanting s" Rt. of seom

Photo taken:

Yes $x$ No

Leak tested:

- Por Pressure (psig)

Pressure (psig)

Date \begin{tabular}{lll} 
& Time (min) & 0 \\
\hline Initial & Time (min)
\end{tabular}

Interior assessment:

15

Photo taken:

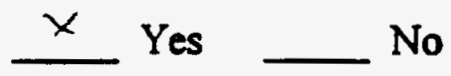

Comments: 


\section{Palletized Storage Drum Data Sheet}

Drum No. 0923

Descriprion (Check):

$\checkmark \quad$ Standard load

Instrumented for Pressure

Instrumented for Temperature

\begin{tabular}{|c|c|c|c|}
\hline Loading Starting at Bottom & Layer 1 & Layer 2 & Layer 3 \\
\hline 3 pieces of steel & & N/A & N/A \\
\hline \multicolumn{4}{|l|}{$1 / 3$ of the brown paper } \\
\hline $1 / 3$ of the 10 -mil plastic & $r$ & $\checkmark$ & $\checkmark$ \\
\hline \multicolumn{4}{|l|}{$1 / 3$ of the bond paper } \\
\hline \multicolumn{4}{|l|}{$1 / 3$ of the towels } \\
\hline \multicolumn{4}{|l|}{$1 / 3$ of the bags } \\
\hline \multicolumn{4}{|l|}{$1 / 3$ of the cut paper } \\
\hline \multicolumn{4}{|l|}{$1 / 3$ of the fabric } \\
\hline \multirow[t]{2}{*}{$1 / 3$ of the rubber } & & & $\gamma$ \\
\hline & Go to Layer 2 & Go to Layer 3 & \\
\hline
\end{tabular}

Drum Filled:

Ring bolt placed opposite drum seam and in down position

Lid sealed and torqued to $40 \mathrm{ft} / \mathrm{b}$ :

Leak tested:

Pre-test weight (lb)

Post-test weight (lb)

$$
\text { Loss }=
$$

Date 5.3 .95 Initial R.K

Date 5-3-95 Initial R.K

Date 5.3 .95 Initial R.K

N/A or Pressure (psig)

Pressure (psig)

Date

Initial

Time (min) 0

Time $(\min ) 5$

$\angle 75.3 \quad$ Target weight $-175.6 \mathrm{lb}$

F-276

SwRI Project No. 01-7106-001 
Drum No. $592 \div, 167.4 i$

Post-test Assessment

Position: ___ original: ___ tipped: ___ toppled

Lid on drum: $\quad$ Yes No

Gasket missing (cm) a

Contenis expelled: $\quad$ Yes $x$ No

Seal failure: $\quad$ Yes $\quad X$ Not visible

Burn partern:

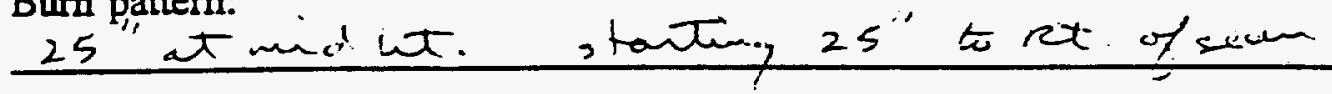

Photo taken:

Yes

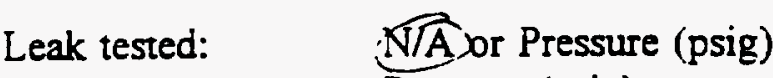

Pressure (psig)

Date

$-\quad$\begin{tabular}{ll} 
Time $(\min )$ & 0 \\
\hline & Time $(\min )$
\end{tabular}

$\overline{\text { Initial }}$

Interior assessment:

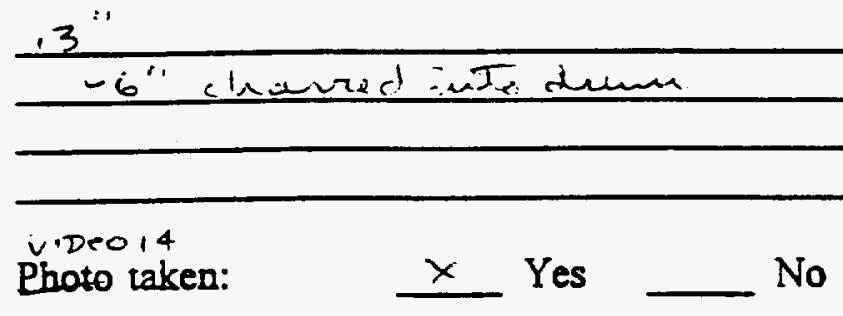

Comments: 
WHC-SD-WM-TRP-246 REV. 0

\section{Palletized Storage Drum Data Sheet}

Drum No. 0933

Description (Check): $\quad$ _ $\quad-\quad$ Standard load

Instrumented for Pressure

Instrumented for Temperature

\begin{tabular}{|c|c|c|c|}
\hline Loading Starting at Bottom & Layer 1 & Layer 2 & Layer 3 \\
\hline 3 pieces of steel & & N/A & N/A \\
\hline \multicolumn{4}{|l|}{$1 / 3$ of the brown paper } \\
\hline \multicolumn{4}{|l|}{$1 / 3$ of the 10 -mil plastic } \\
\hline \multicolumn{4}{|l|}{$1 / 3$ of the bond paper } \\
\hline \multicolumn{4}{|l|}{$1 / 3$ of the towels } \\
\hline \multicolumn{4}{|l|}{$1 / 3$ of the bags } \\
\hline \multicolumn{4}{|l|}{$1 / 3$ of the cut paper } \\
\hline \multicolumn{4}{|l|}{$1 / 3$ of the fabric } \\
\hline \multicolumn{4}{|l|}{$1 / 3$ of the rubber } \\
\hline & Go to Layer 2 & Go to Layer 3 & \\
\hline
\end{tabular}

Drum Filled:

Ring bolt placed opposite drum seam and in down position

Lid sealed and torqued to $40 \mathrm{ft} / \mathrm{lb}$ :

Leak tested:

Pre-test weight (lb)

Post-test weight (lb)
Date 5.3 .95 Initial EK

Date 5.3.95 Initial L.K

Date 5-3.95 Initial R.K

N/A or Pressure (psig)

Pressure (psig)

Date

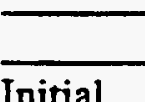

Time (min) 0

Time $(\min ) 5$

Initial

Target weight $\sim 175.6 \mathrm{lb}$ 
Drum No. $\quad 293.3 \quad 13.4$ ib

Post-test Assessment

Position:

original: tipped: toppled

Lid on drum: $\quad \times$ Yes $\quad$ No

Gasket missing (cm) _ $38^{\circ}$

Contents expelled: _ـ Yes $x$ No

Seal failure: $\quad$ Yes $\quad$ Y Not visible

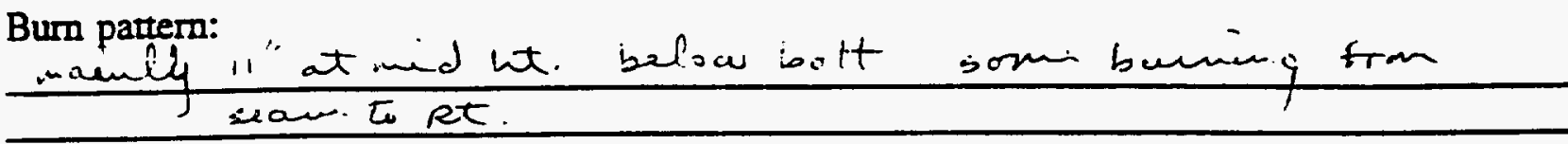

Photo taken:

Yes $\underset{x}{x}$ No

Leak tested:

N/A or Pressure (psig)

Pressure (psig)

Date \begin{tabular}{ll} 
Time (min) & 0 \\
Time (min) & $\underline{5}$ \\
\hline
\end{tabular}

$\frac{\text { Time (min) }}{\text { Initial }} \quad \begin{array}{r}0 \\ \text { Time (min) }\end{array}$

Interior assessment:

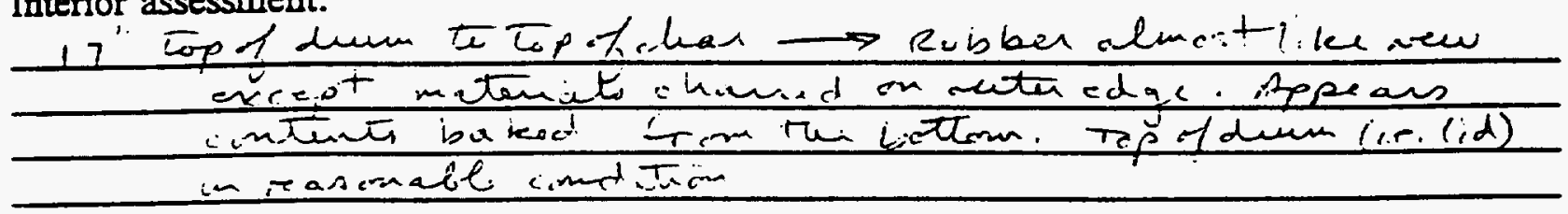

$\rightarrow$ Dee $=1$

photo taken:

$\longrightarrow$ Yes

Comments:

bunged on tactitim 
WHC-SD-WM-TRP-246 REV. 0

\section{Palletized Storage Drum Data Sheet}

Drum No. 0943

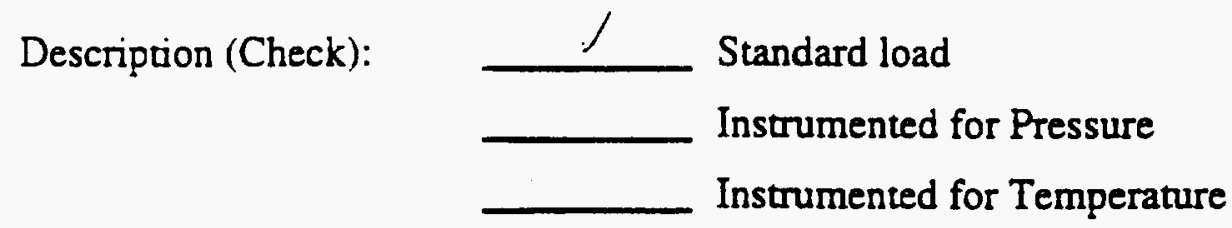

\begin{tabular}{|c|c|c|c|}
\hline Loading Starting at Bottom & Layer 1 & Layer 2 & Layer 3 \\
\hline 3 pieces of steel & 2 & N/A & N/A \\
\hline $1 / 3$ of the brown paper & $\swarrow$ & & $V$ \\
\hline $1 / 3$ of the 10 -mil plastic & - & & $\checkmark$ \\
\hline $1 / 3$ of the bond paper & $\gamma$ & & $\checkmark$ \\
\hline $1 / 3$ of the towels & $\checkmark$ & & $\checkmark$ \\
\hline $1 / 3$ of the bags & $\checkmark$ & $\checkmark$ & 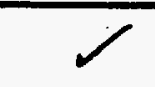 \\
\hline $1 / 3$ of the cut paper & $\checkmark$ & $\checkmark$ & $\checkmark$ \\
\hline $1 / 3$ of the fabric & 7 & $\sqrt{ }$ & $\checkmark$ \\
\hline \multirow[t]{2}{*}{$1 / 3$ of the rubber } & 7 & $\checkmark$ & $\gamma$ \\
\hline & Go to Layer 2 & Go to Layer 3 & \\
\hline
\end{tabular}

Drum Filled:

Ring bolt placed opposite drum seam and in down position

Lid sealed and torqued to $40 \mathrm{fulb}$ :

Leak tested:

Pre-test weight (lb)

Post-test weight (lb)

$$
\text { Loss }=
$$

Date $5=3.95$ Initial P.K

Date 5.3 .95 Initial A.K
N/A or Pressure (psig)

Pressure (psig)

Date

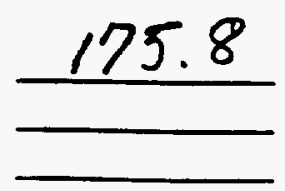

Time (min)

Time (min) 5 
WHC-SD-WM-TRP-246 REV. 0

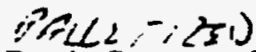

Reoit Gronage Drum Data Sheet

Drum No. 0942,1095

Post-test Assessment

Position: ___ original: ___ tipped: ___ toppled

Lid on drum: $\quad$ $\quad$ Yes $\quad$ No

Gasket missing (cm) __ i' buT 14" seam to RT

Contents expeiled: $\quad$ Yes $\longrightarrow$ No

Seal failure: $\quad$ Yes $\quad x$ Not visible botem bel gyd

Bum pattern: .

32 at mind lat

Photo taken:

Yes $\underline{x}$ No

Leak tested:

XIA or Pressure (psig)

Pressure (psig)

Date

\begin{tabular}{lll} 
Time (min) & 0 \\
\hline & Time (min)
\end{tabular}

Interior assessment:

. 7" tep to top d c han

pioes 23

Photo taken:

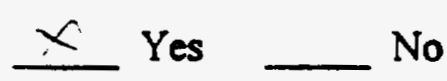

Comments:

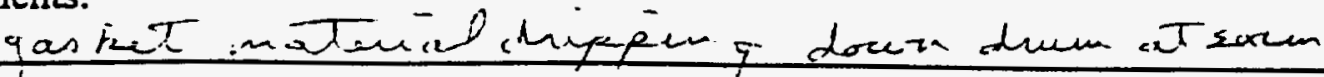


WHC-SD-WM-TRP-246 REV. 0

\section{Palletized Storage Drum Data Sheet}

Drum No. $\underline{0953}$

Descriprion (Check):

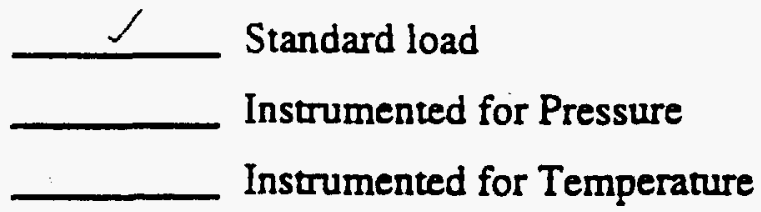

\begin{tabular}{|c|c|c|c|}
\hline Loading Starting at Bottom & Layer 1 & Layer 2 & Layer 3 \\
\hline 3 pieces of steel & & N/A & N/A \\
\hline $1 / 3$ of the brown paper & & & \\
\hline $1 / 3$ of the 10-mil plastic & & & \\
\hline $1 / 3$ of the bond paper & - & & \\
\hline $1 / 3$ of the towels & - & & \\
\hline $1 / 3$ of the bags & & & \\
\hline $1 / 3$ of the cut paper & & & \\
\hline $1 / 3$ of the fabric & Go to Layer 2 & Go to Layer 3 & \\
\hline $1 / 3$ of the rubber & & & \\
\hline
\end{tabular}

Drum Filled:

Ring bolt placed opposite drum seam and in down position

Lid sealed and torqued to $40 \mathrm{ft} / \mathrm{b}$ :

Leak tested:

Pre-test weight (lb)

Post-test weight (lb)

Date 5.3 .95 Initial R.K

Date 5.3-95 Initial R.K

Date 5.3.95 Initial L.K

N/A or Pressure (psig)

Pressure (psig)

Date

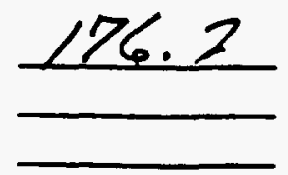

F-282
Time (min)

Time (min) 5

Initial

Target weight $\sim 175.6 \mathrm{lb}$ 


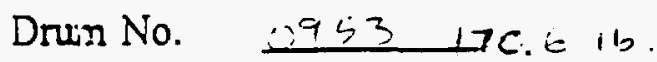

Post-test Assessment

Position:

original: tipped: toppled

Lid on drum: $\quad y$ Yes No

Gasket missing (cm) $\underline{a x} \hat{\imath}$

Contents expelled: __ Yes $\_$No

Seal failure: $\quad x$ Yes $\rightarrow$ Not visible? bettem i- to $p$ bu lged

Burn pattem:

36 " at ind ht

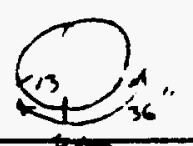
bur patten eliere bottrin seam at place suspected of faulin!

Photo taken:

Yes $\leq$ No

Leak tested:

N/A or Pressure (psig)

Pressure (psig)

Date

Initial

Time $(\min )$
Time (min)

Time (min) 5

Interior assessment:

$15 "$ top 1 them to top of char

\section{Comments:}


WHC-SD-WM-TRP-246 REV. 0

\section{Palletized Storage Drum Data Sheet}

Drum No. 0963

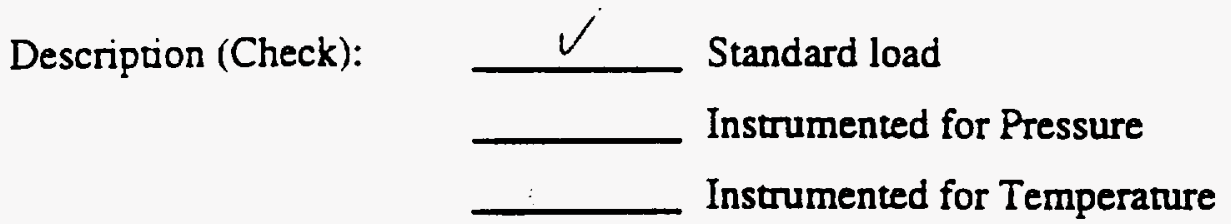

\begin{tabular}{|c|c|c|c|}
\hline Loading Starting at Bottom & Layer 1 & Layer 2 & Layer 3 \\
\hline 3 pieces of steel & & $N / A$ & N/A \\
\hline \multicolumn{4}{|l|}{$1 / 3$ of the brown paper } \\
\hline \multicolumn{4}{|l|}{$1 / 3$ of the 10 -mil plastic } \\
\hline \multicolumn{4}{|l|}{$1 / 3$ of the bond paper } \\
\hline \multicolumn{4}{|l|}{$1 / 3$ of the towels } \\
\hline \multicolumn{4}{|l|}{$1 / 3$ of the bags } \\
\hline \multicolumn{4}{|l|}{ 1/3 of the cut paper } \\
\hline \multicolumn{4}{|l|}{$1 / 3$ of the fabric } \\
\hline \multicolumn{4}{|l|}{$1 / 3$ of the rubber } \\
\hline & Go to Layer 2 & Go to Layer 3 & \\
\hline
\end{tabular}

Drum Filled:

Ring bolt placed opposite drum seam and in down position

Lid sealed and torqued to $40 \mathrm{ft} / \mathrm{b}$ :

Leak tested:

Pre-test weight (lb)

Post-test weight (lb)
Date 5.3 .95 Initial R.K

Date 5-3.95 Initial L.K

Date 5-3-95 Initial R.K
N/A or Pressure (psig)

Pressure (psig)

Date

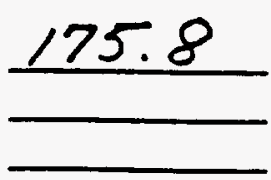

Time (min) 0

Time (min) 5
Initial

Target weight $-175.6 \mathrm{lb}$
Loss = 
Drum No. $\quad 096^{2}, 1: 9.0$ in.

Post-test Assessment

Position: original: tipped: toppled

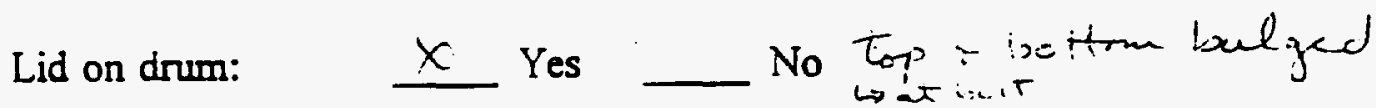

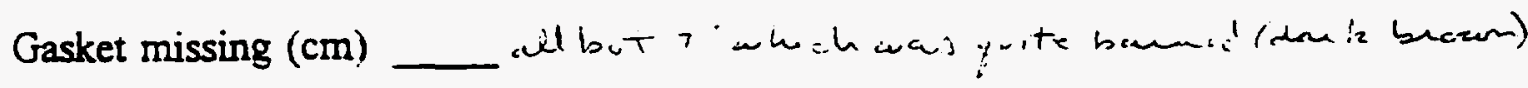

Contents expelled: _ $\quad$ Yes $x$ No

Seal failure: $\quad$ Y $\quad$ Yes $\quad x$ Not visible

Burn pattern:

44 at ind ht.

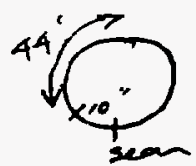

Photo taken: $x$ Yes No

Leak tested: $\quad \widehat{W A}$ or Pressure (psig) Pressure (psig)

Date

$-\quad$\begin{tabular}{ll} 
Time $(\min )$ & 0 \\
\hline & Time (min)
\end{tabular}

Interior assessment:

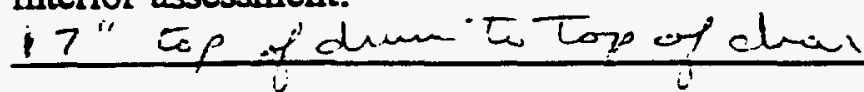

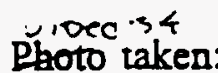

$x$ Yes

\section{Comments:}


WHC-SD-WM-TRP-246 REV. 0

\section{Palletized Storage Drum Data Sheet}

Drum No. 0973

Description (Check):

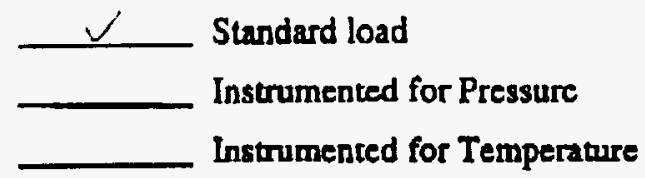

\begin{tabular}{|c|c|c|c|}
\hline Loading Starting at Bottom & Layer 1 & Layer 2 & Layer 3 \\
\hline 3 pieces of steel & & & \\
\hline $1 / 3$ of the brown paper & & & \\
\hline $1 / 3$ of the 10-mil plastic & & & \\
\hline $1 / 3$ of the bond paper & & & \\
\hline $1 / 3$ of the towels & & & \\
\hline $1 / 3$ of the bags & & & \\
\hline $1 / 3$ of the cut paper & & & \\
\hline $1 / 3$ of the fabric & Go to Layer 2 & Go to Layer 3 & \\
\hline $1 / 3$ of the rubber & & & \\
\hline
\end{tabular}

Drum Filled:

Ring bolt placed opposite drum seam and in down position

Lid senled and torqued to $40 \mathrm{fMlb}$ :

Leak tested:

Pre-test weight (lb)

Post-test weight (lb)
Dato $5=3.95$ Initial R.K

Date 5.3 .95 Initial R.K.

Date 5-3-95 Initial R.K
N/A or Pressure (psig)

Pressure (psig)

Date

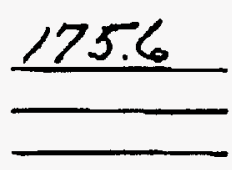

Time (min) 0

Time (min) 5
Initial

Target weight $\sim 175.6 \mathrm{lb}$ 
D.um No. $0973 \quad 175.31 \mathrm{~h}$.

Post-test Assessment

Position: original: tipped: toppled

Lid on drum: $\quad X$ Yes No

Gasket missing $(\mathrm{cm}){ }^{26} "$
Contents expelled: $\quad$ Yes $\triangle$ No

Seal failure: $\quad$ Yes $\quad x$ Not visible bottom bulged

Burn partern: centered 17 "RT of seam

Photo taken: Yes $x$ No

Leak tested:

NTA Or Pressure (psig) Pressure (psig)

Date $\begin{array}{ll}\text { Time }(\min ) & 0 \\ \text { Time }(\min ) & \end{array}$ $\overline{\text { Initial }}$

Interior assessment:

Top in good concition 3-5" chas wound

vinec 64

Photo taken:

$x$ Yes

Comments: 
WHC-SD-WM-TRP-246 REV. 0

\section{Palletized Storage Drum Data Sheet}

Drum No. 0983

Deseription (Check):

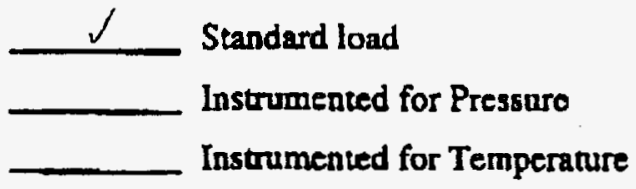

\begin{tabular}{|c|c|c|c|}
\hline Loadiog Starting at Bottom & Layer 1 & Layer 2 & Layer 3 \\
\hline 3 pieces of steel & & N/A & N/A \\
\hline $1 / 3$ of the brown paper & & & \\
\hline $1 / 3$ of the 10-mil plastic & & & \\
\hline $1 / 3$ of the bond paper & & & \\
\hline $1 / 3$ of the towels & & & \\
\hline $1 / 3$ of the bags & & & \\
\hline $1 / 3$ of the cut paper & & & \\
\hline $1 / 3$ of the fabric & & & \\
\hline $1 / 3$ of the rubber & Go to Layer 2 & Go to Layer 3 & \\
\hline
\end{tabular}

Drum Filled:

Ring bolt placed opposite drum seam and in down position

Lid sealed and corqued to $40 \mathrm{ft} / \mathrm{b}$ :

Leak rested:

Pro-test weight (lb)

Post-test weight (lb)
Date $5.3 \cdot 95$ Initial L.K

Date $5.3 .95 \quad$ Initial R.K
N/A or Pressure (psig)

Pressure (psig)

Date

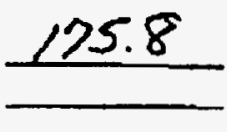

Time (min) 0

Time (min) s

Loss =

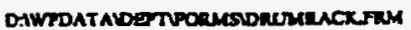


Drum No. $0983 \quad 176.0 \mathrm{k}$.

Post-test Assessment

Position: originai: tipped: toppled

Lid on drum: $\quad X$ Yes No

Gasket missing (cm) $O \quad 30$ " conforessedt hand

Contents expelled: $\quad$ Yes $x$ No

Seal failure:

Yes $\quad X$ Not visible

Burn pattern:

nowe

Photo taken:

Yes $\underline{x}$ No

Leak tested:

K/A gr Pressure (psig)

Pressure (psig)

Date $\begin{array}{ll}\text { Time (min) } & 0 \\ \text { Time (min) } & \underline{5}\end{array}$

Interior assessment:
$\frac{1--10 i^{2} \text { spot } 2 \text { lightly melted }}{\text { No chan }}$

vroeo 62

Photo taken:

$x$ Yes

Comments: 
blank 


\begin{tabular}{|c|c|c|c|c|c|}
\hline \multicolumn{6}{|c|}{ DISTRIBUTION SHEET } \\
\hline To & \multirow{2}{*}{\multicolumn{3}{|c|}{$\begin{array}{l}\text { From } \\
\text { SWP }\end{array}$}} & \multicolumn{2}{|c|}{ Page 1 of 1} \\
\hline SWP & & & & \multicolumn{2}{|c|}{ Date $10 / 12 / 95$} \\
\hline \multirow{2}{*}{\multicolumn{4}{|c|}{$\begin{array}{l}\text { sroject Title/Work Order } \\
\text { SOLID WASTE DRUM ARRAY FIRE PERFORMANCE }\end{array}$}} & \multicolumn{2}{|c|}{ EDT No. 612232} \\
\hline & & & & \multicolumn{2}{|c|}{ ECN No. N/A } \\
\hline Name & MSIN & $\begin{array}{l}\text { Text } \\
\text { With All } \\
\text { Attach. }\end{array}$ & Text Only & $\begin{array}{l}\text { Attach./ } \\
\text { Appendix } \\
\text { Only }\end{array}$ & $\begin{array}{l}\text { EDT/ECN } \\
\text { Only }\end{array}$ \\
\hline J. R. Bell & B1-10 & $x$ & & & \\
\hline T. L. Erickson & H5-71 & $x$ & & & \\
\hline D. J. Hart & $S 2-42$ & & & & $x$ \\
\hline J. R. Keene & $S 2-42$ & $x$ & & & \\
\hline R. L. Louie (3) & G3-15 & $x$ & & & \\
\hline D. R. Lucas & G3-15 & & & & $x$ \\
\hline K. M. McDonald & T4-03 & $x$ & & & \\
\hline J. R. McGee & G3-15 & & & & $x$ \\
\hline W. W. 01 son & $\mathrm{H} 4-70$ & $x$ & & & \\
\hline . B. Powe11 & T4-03 & $x$ & & & \\
\hline K. M. Quigley & T3-01 & $x$ & & & $x$ \\
\hline J. B. Sepahpur & B1-10 & $x$ & & & \\
\hline WRAP 1 DMC & G3-15 & $x$ & & & \\
\hline
\end{tabular}


Blanke 